\title{
OASIS \&
}

\section{Open Document Format for Office Applications (OpenDocument) v1.0}

\section{OASIS Standard, 1 May 2005}

\author{
Document identifier: \\ OpenDocument-v1.0-os.sxw
}

Location:

http://docs.oasis-open.org/office/v1.0

\section{Editors:}

Michael Brauer, Sun Microsystems <michael.brauer@sun.com>

Patrick Durusau, Society of Biblical Literature <Patrick.Durusau@sbl-site.org>

Gary Edwards < garyedwards@yahoo.com>

David Faure, <faure@kde.org>

Tom Magliery, Blast Radius <tom.magliery@blastradius.com>

Daniel Vogelheim, Sun Microsystems <daniel.vogelheim@sun.com>

\section{Contributors:}

Doug Alberg, Boeing <doug.alberg@boeing.com>

Simon Davis, National Archive of Australia <simond@naa.gov.au>

Paul Grosso, Arbortext <paul@arbortext.com>

Phil Boutros, Stellent <pboutros@stellent.com>

John Chelsom, CSW Informatics <john.chelsom@csw.co.uk>

Jason Harrop <jharrop@speedlegal.com>

Mark Heller, New York State Office of the Attorney General

<Mark.Heller@oag.state.ny.us>

Paul Langille, Corel <paul.langille@corel.com>

Monica Martin, Drake Certivo <mmartin@certivo.net>

Uche Ogbuji <uche.ogbuji@fourthought.com>

Lars Oppermann, Sun Microsystems <lars.oppermann@sun.com>

Lauren Wood <lauren@textuality.com>

\section{Abstract:}

This is the specification of the Open Document Format for Office Applications

(OpenDocument) format, an open, XML-based file format for office applications, based on OpenOffice.org XML [OOo].

\section{Status:}

This document was last revised or approved by the membership of OASIS on the above date. The level of approval is also listed above.

Check the current location noted above for possible later revisions of this document.

This document is updated periodically on no particular schedule. 
Technical Committee members should send comments on this specification to the Technical Committee's email list. Others should send comments to the Technical Committee by using the "Send A Comment" button on the Technical Committee's web page at

www.oasis-open.org/committees/office.

For information on whether any patents have been disclosed that may be essential to implementing this specification, and any offers of patent licensing terms, please refer to the Intellectual Property Rights section of the Technical Committee web page

(www.oasis-open.org/committees/office/ipr.php.

The non-normative errata page for this specification is located at www.oasisopen.org/committees/office. 


\section{Table of Contents}

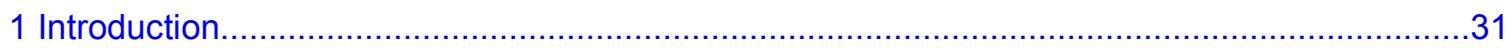

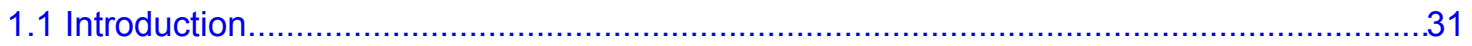

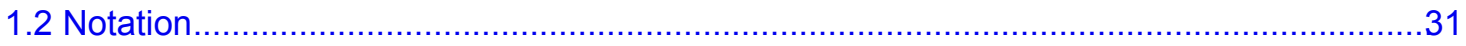

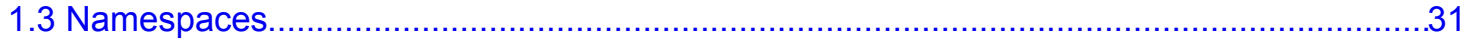

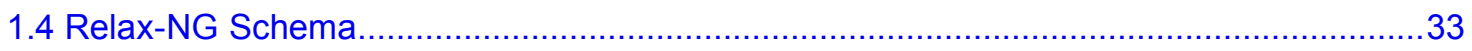

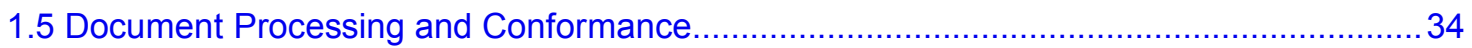

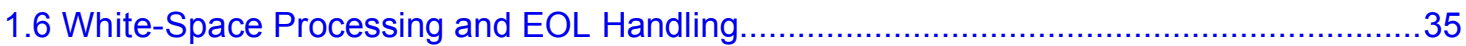

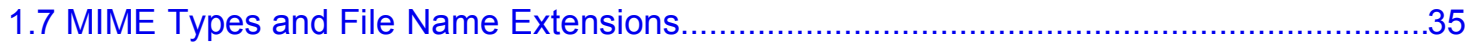

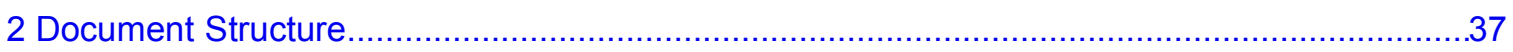

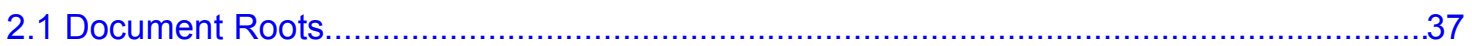

2.1.1 Document Root Element Content Models........................................................... 38

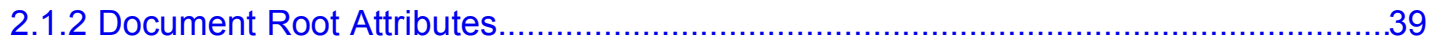

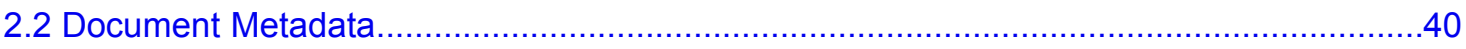

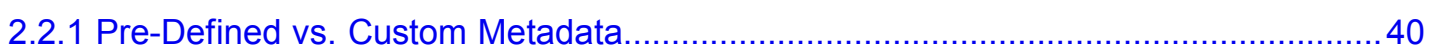

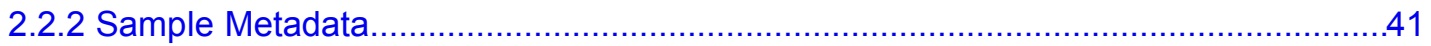

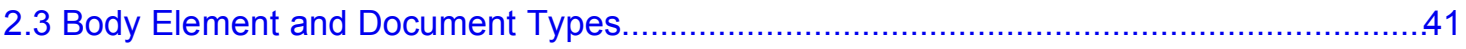

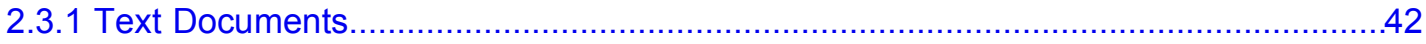

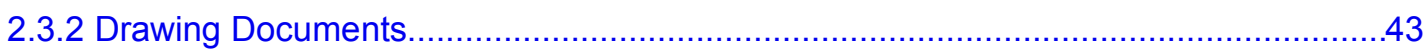

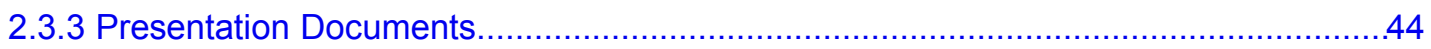

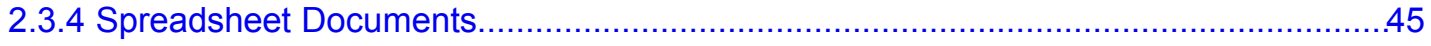

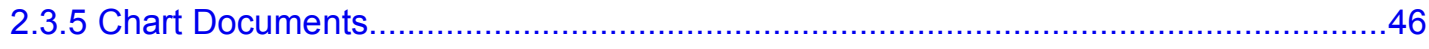

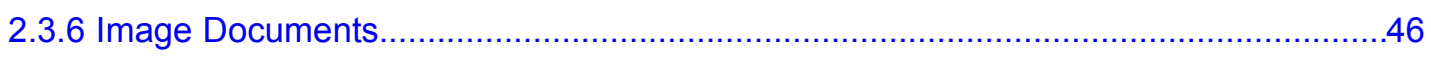

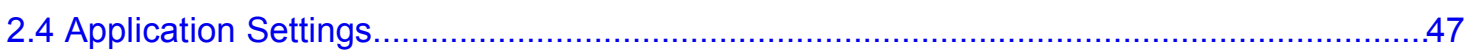

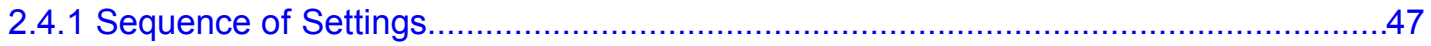

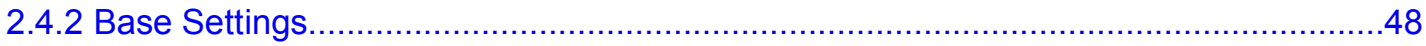

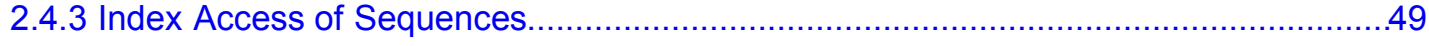

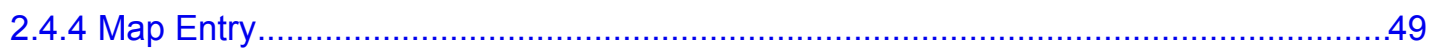

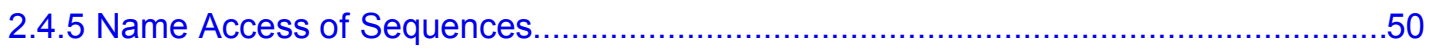

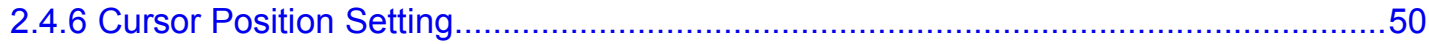

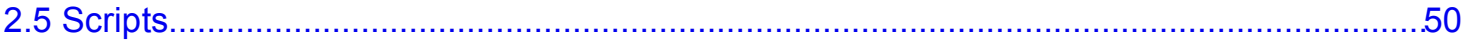

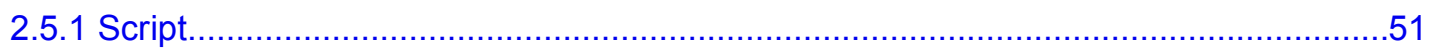

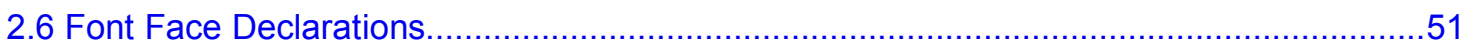

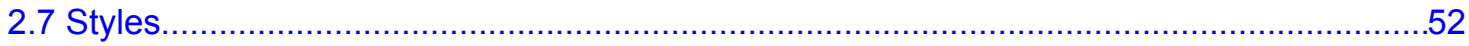

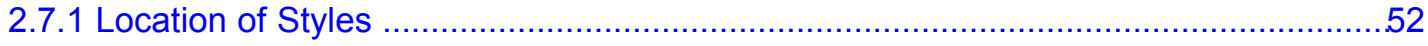




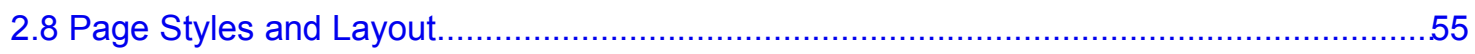

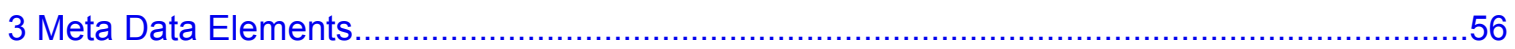

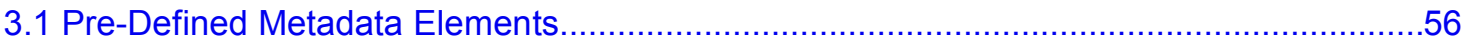

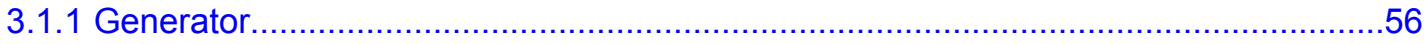

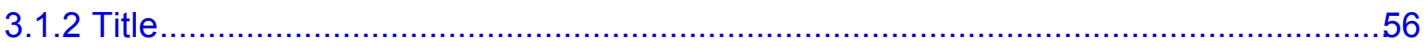

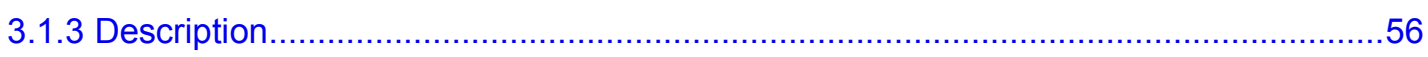

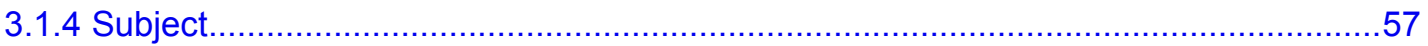

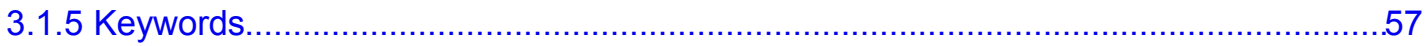

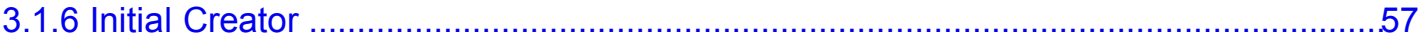

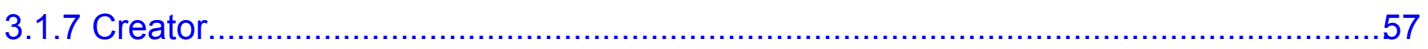

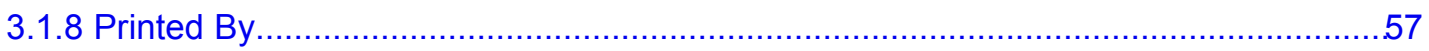

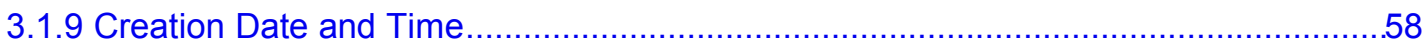

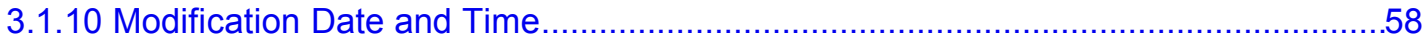

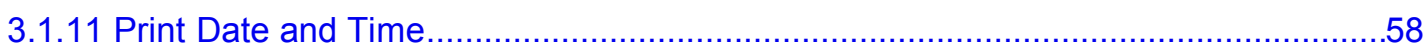

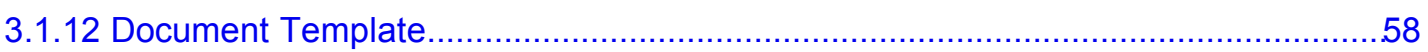

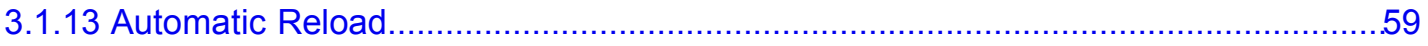

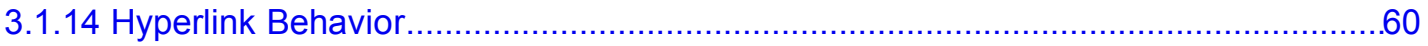

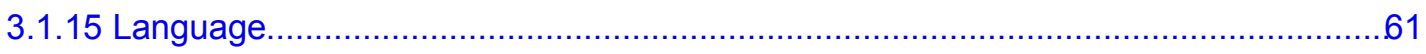

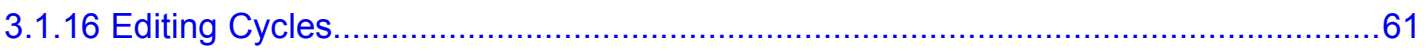

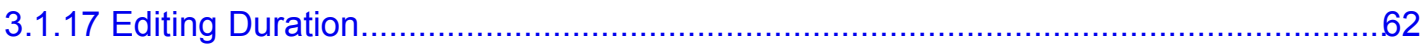

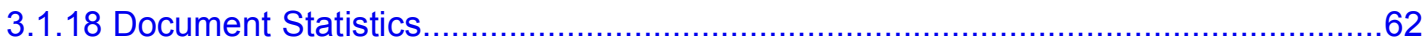

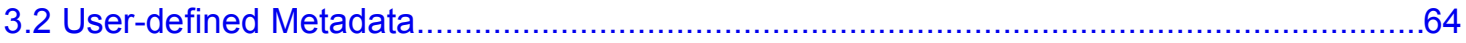

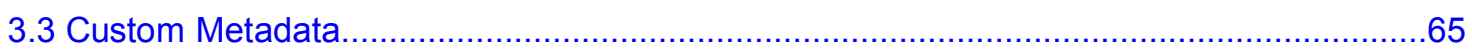

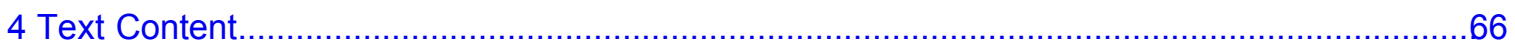

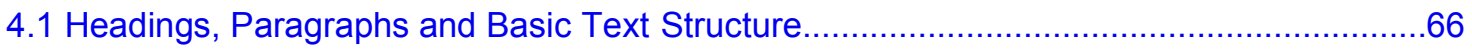

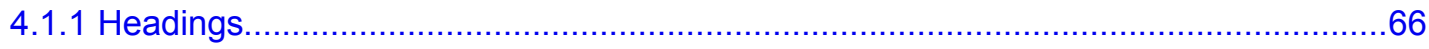

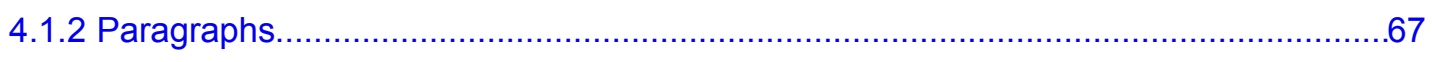

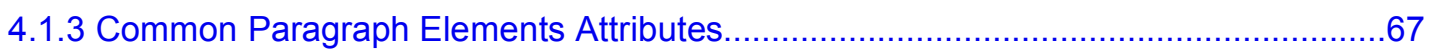

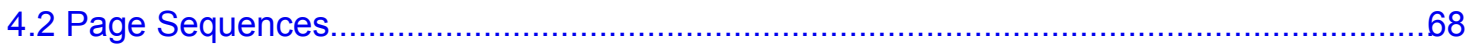

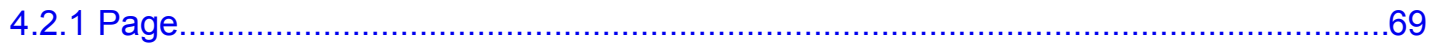

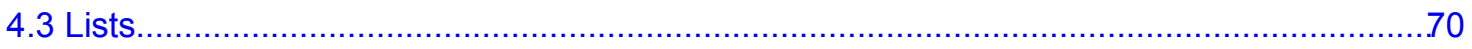

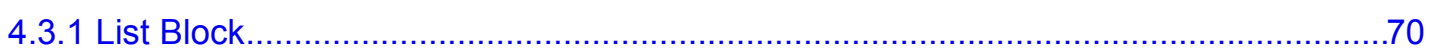

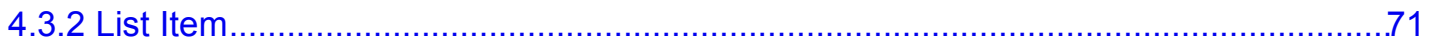

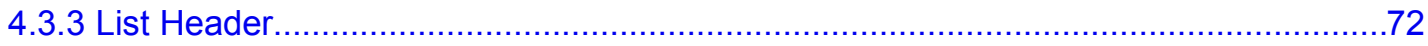

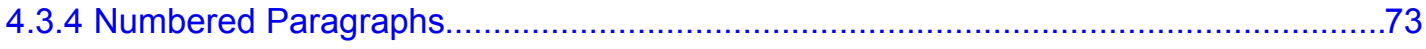

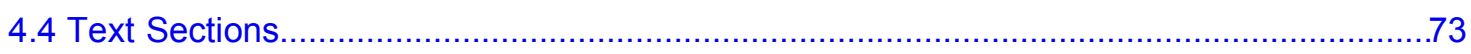

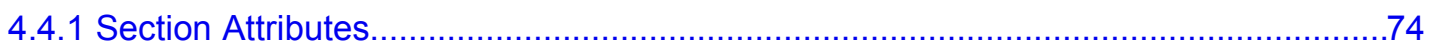




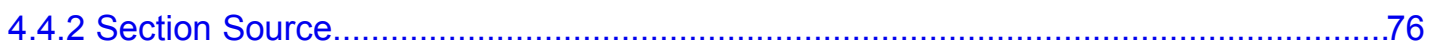

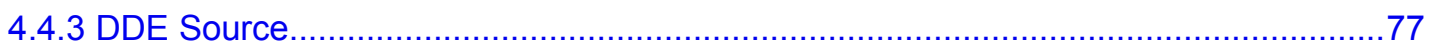

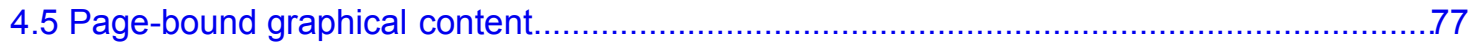

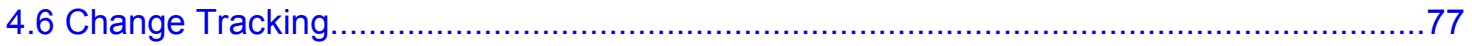

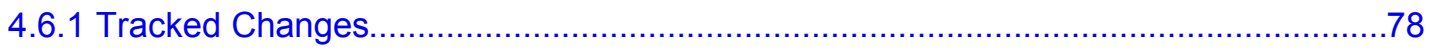

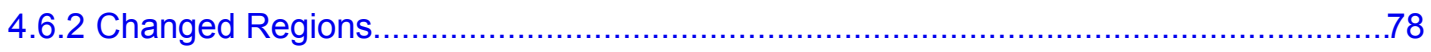

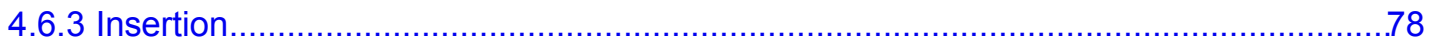

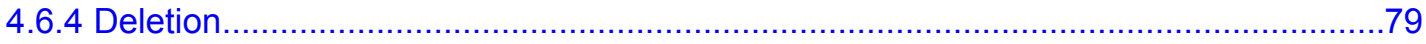

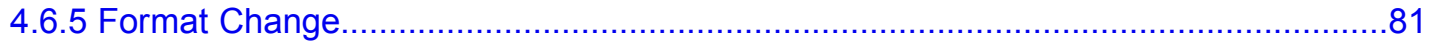

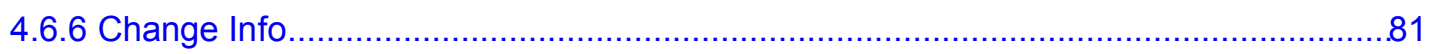

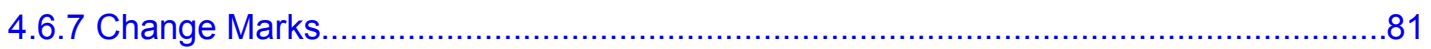

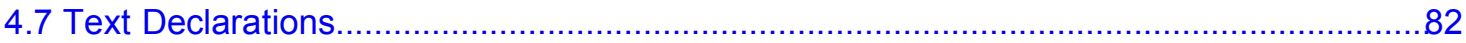

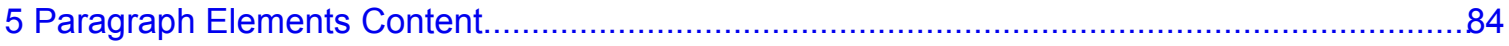

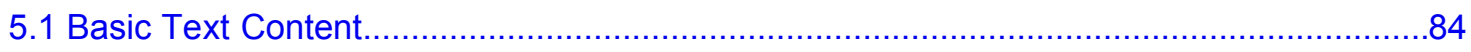

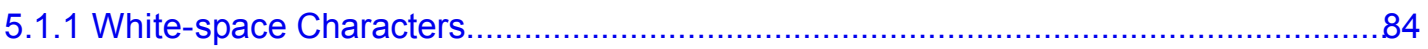

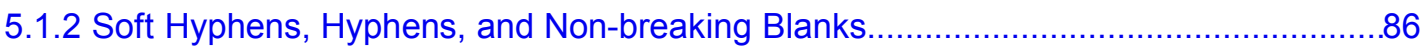

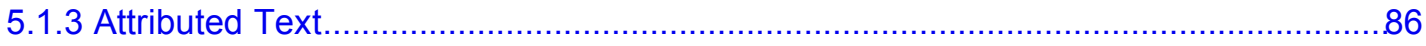

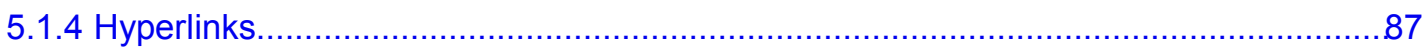

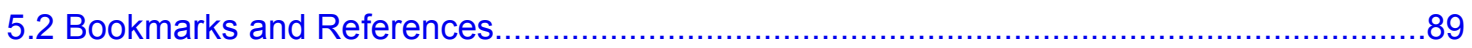

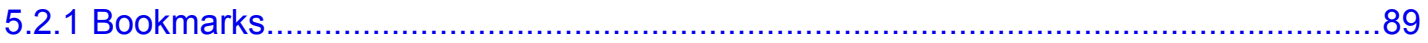

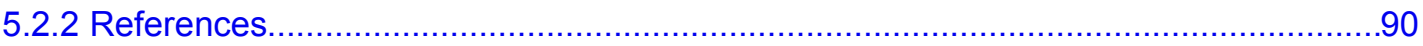

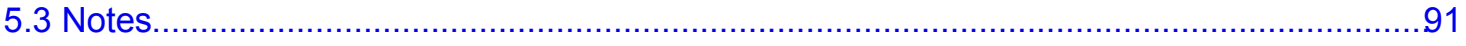

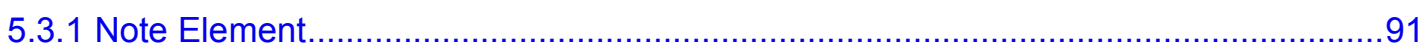

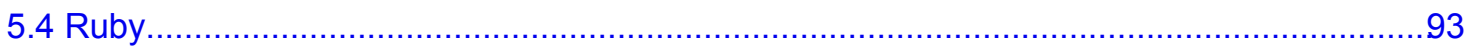

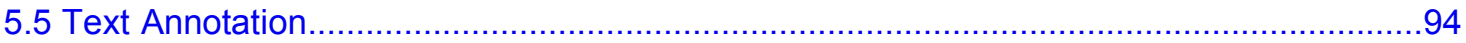

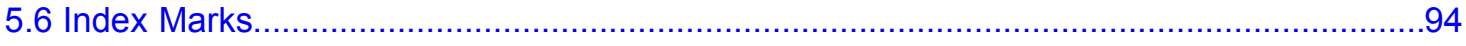

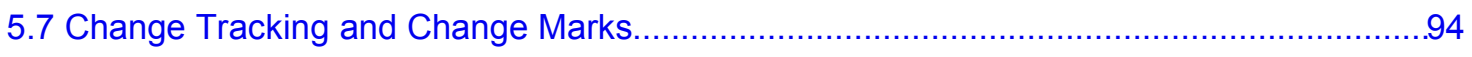

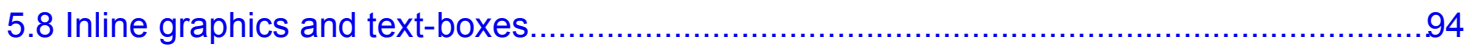

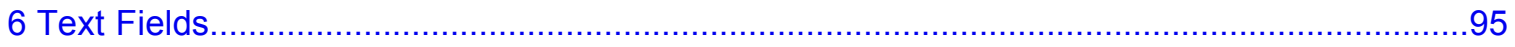

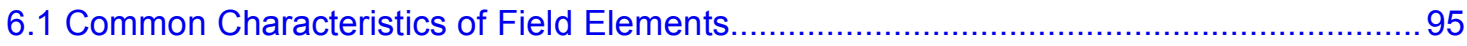

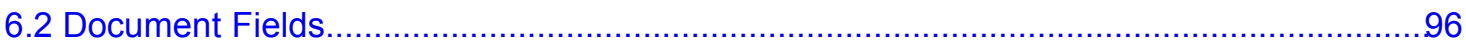

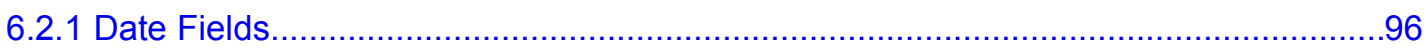

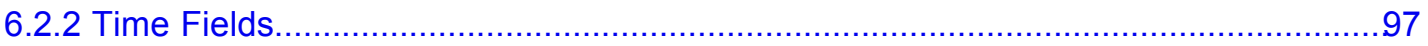

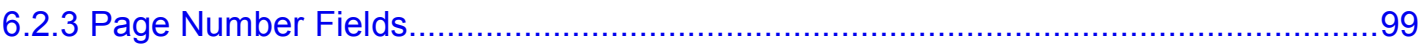

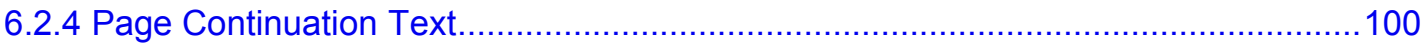

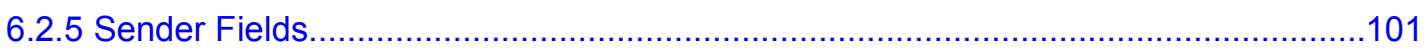

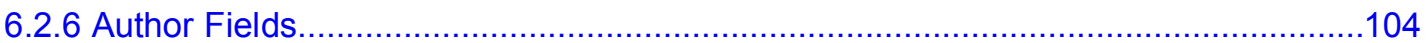




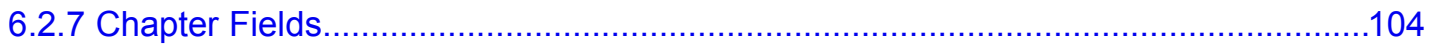

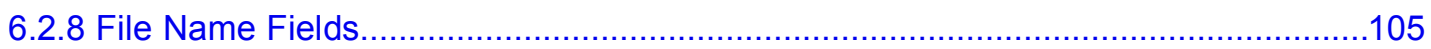

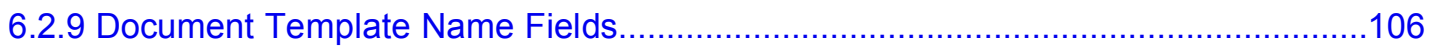

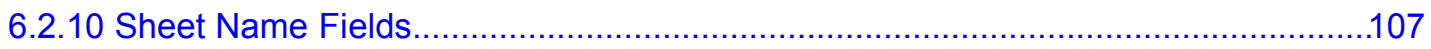

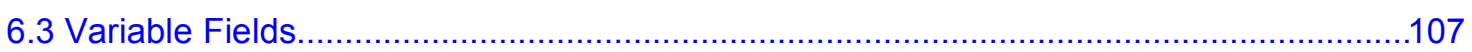

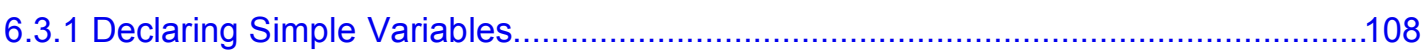

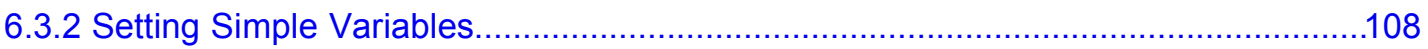

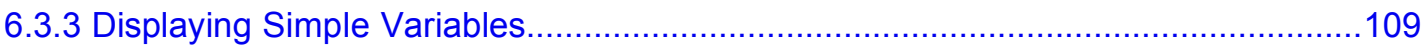

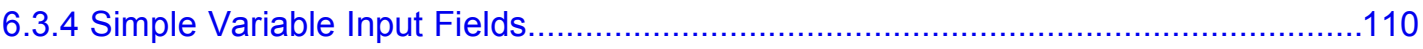

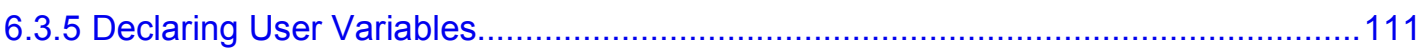

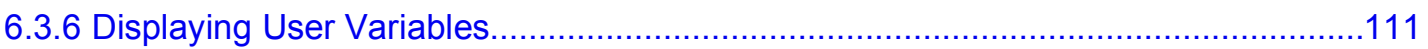

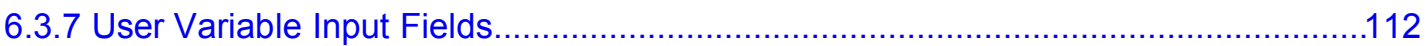

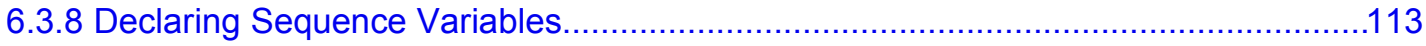

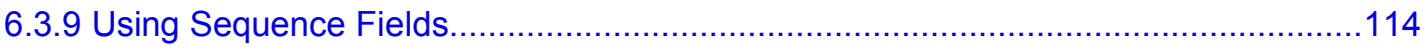

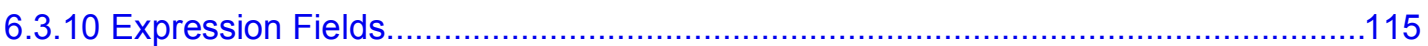

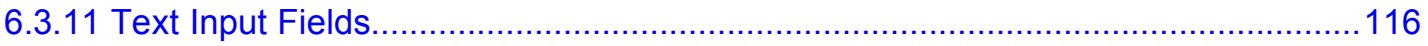

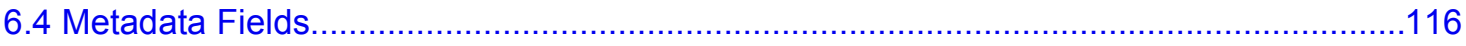

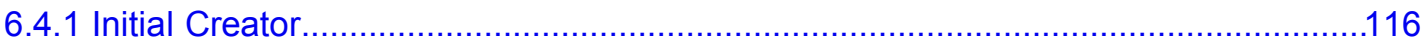

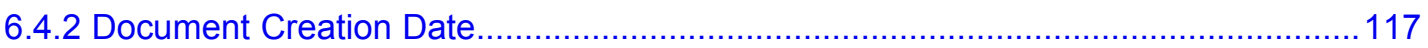

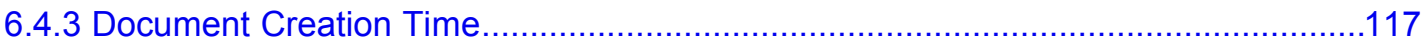

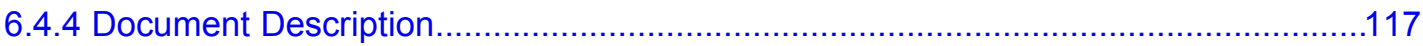

6.4.5 User-Defined Document Information................................................................ 117

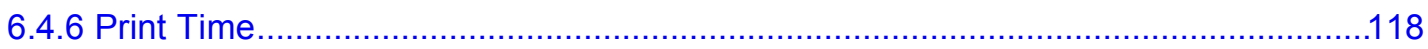

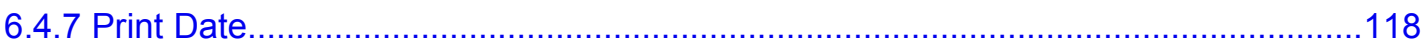

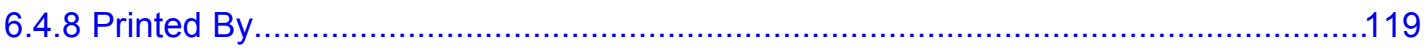

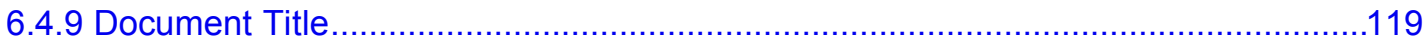

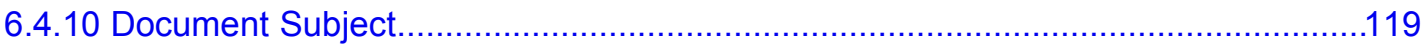

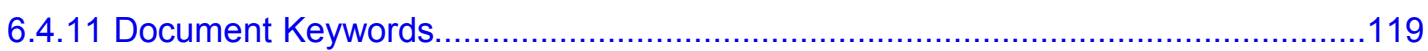

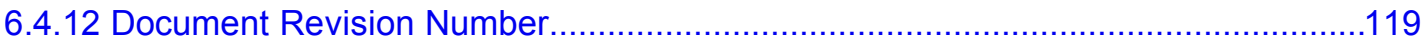

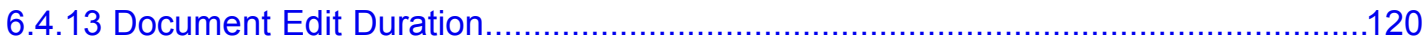

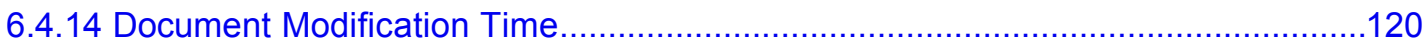

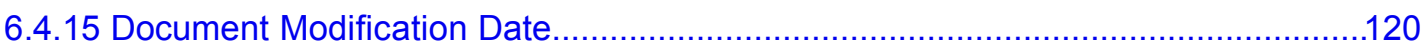

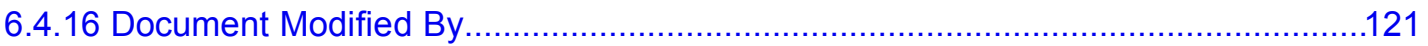

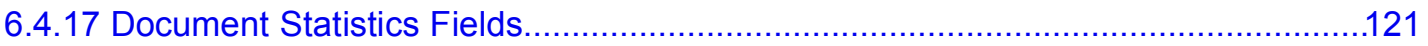

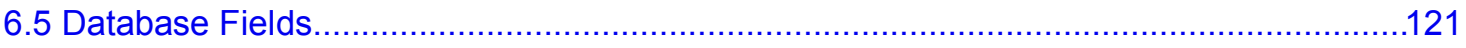

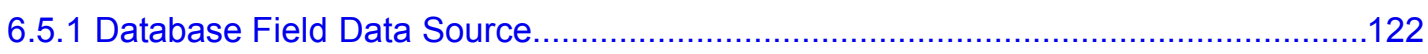

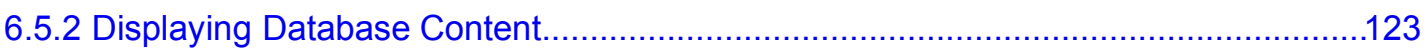




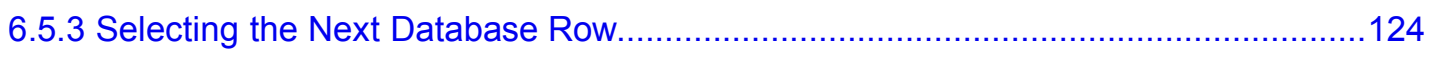

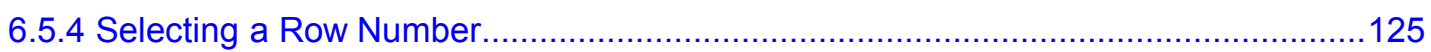

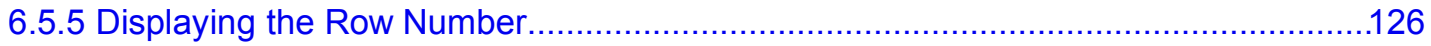

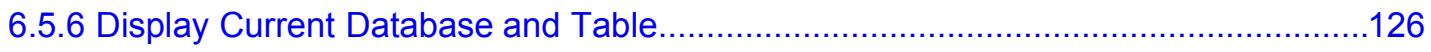

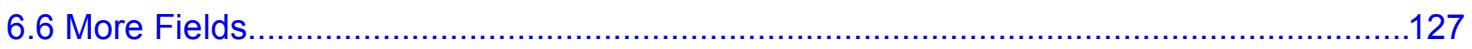

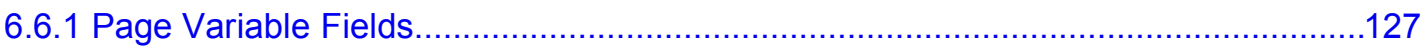

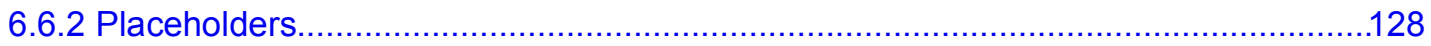

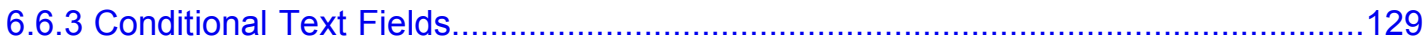

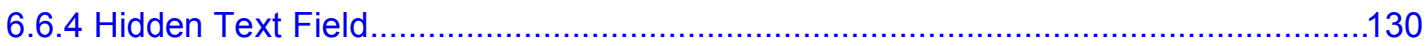

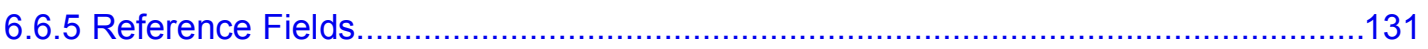

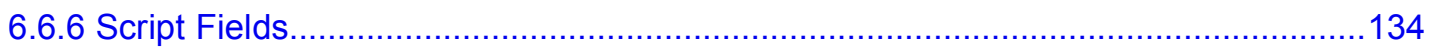

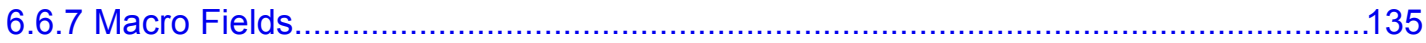

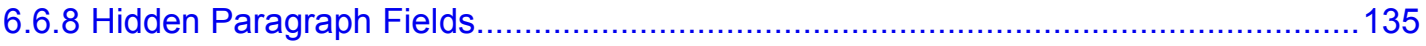

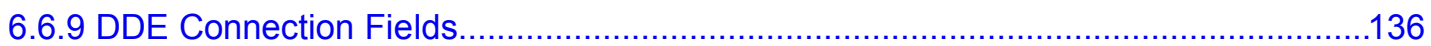

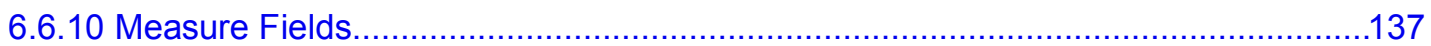

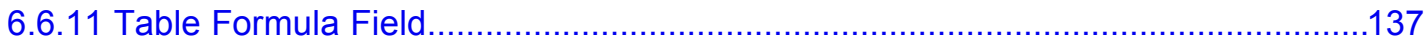

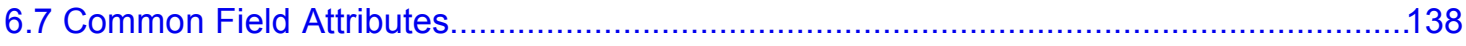

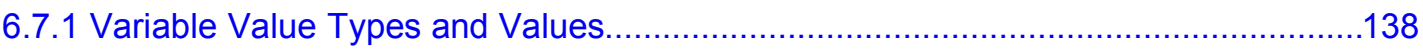

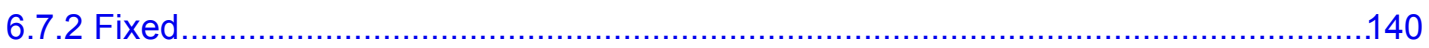

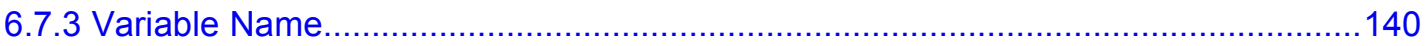

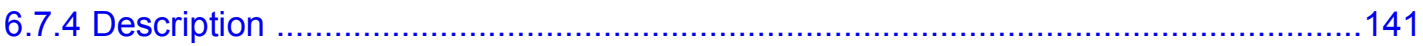

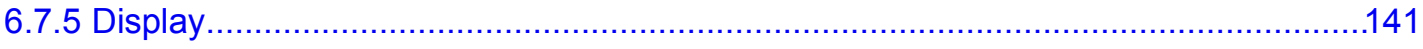

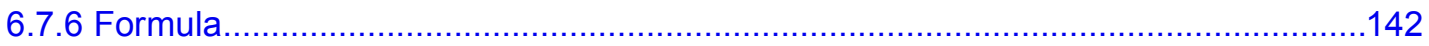

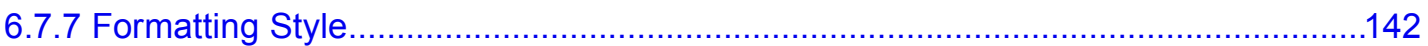

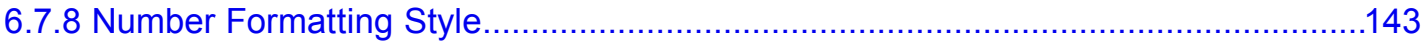

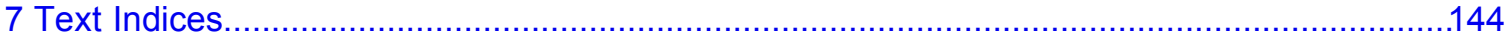

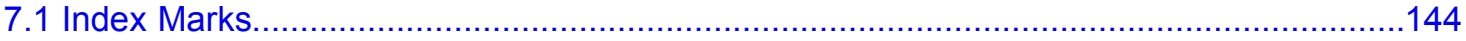

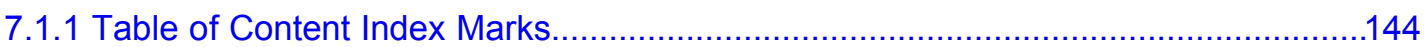

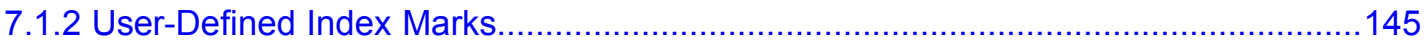

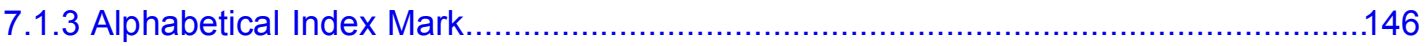

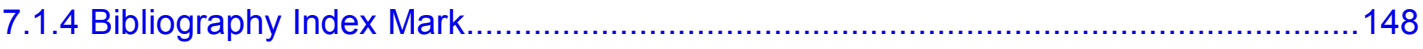

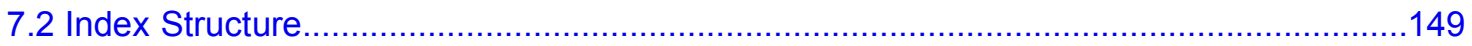

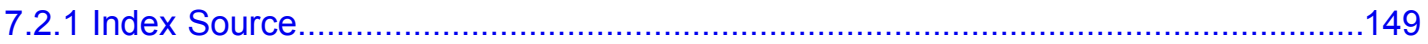

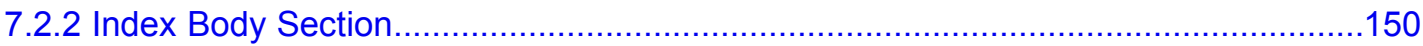

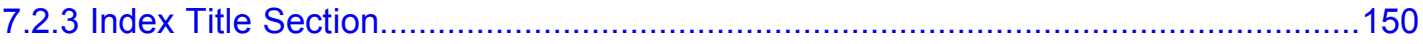

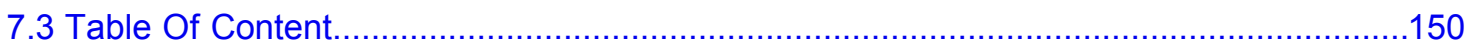

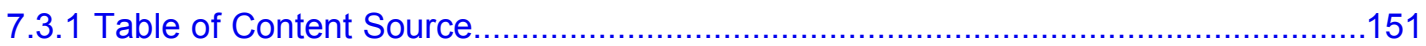




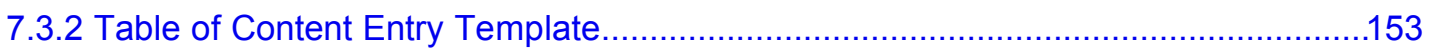

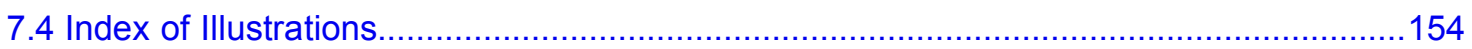

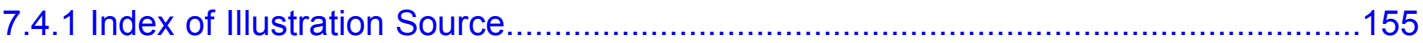

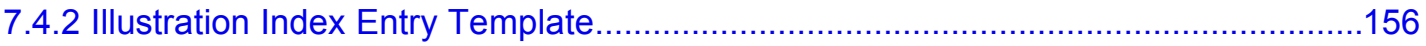

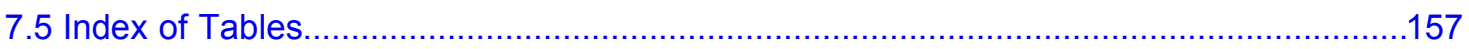

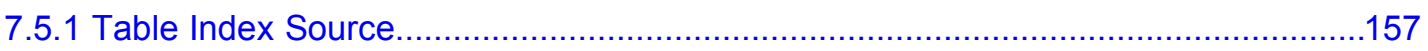

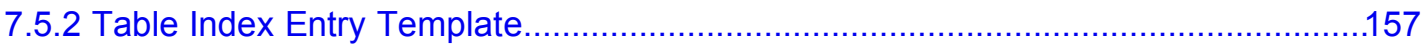

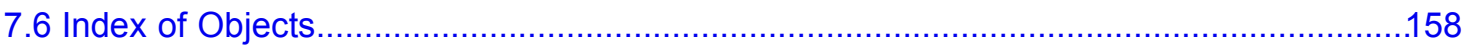

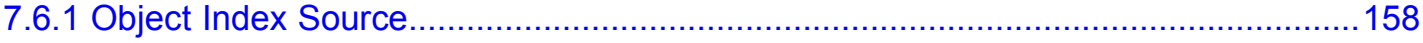

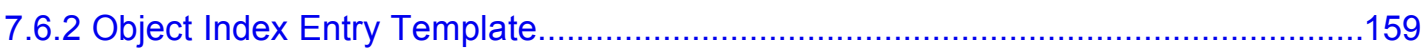

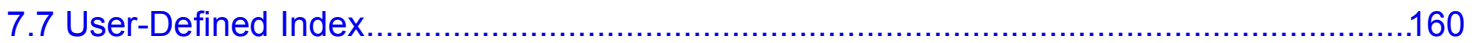

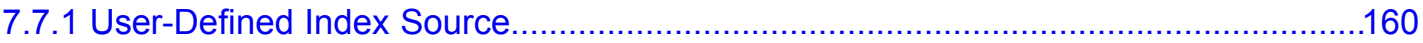

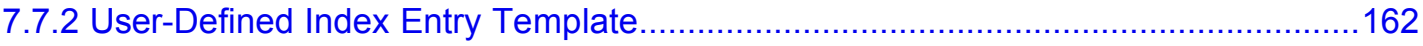

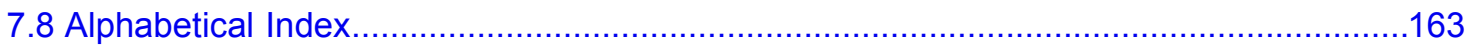

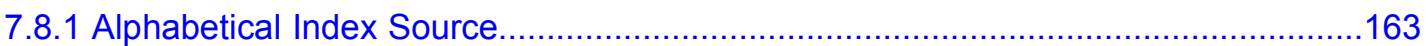

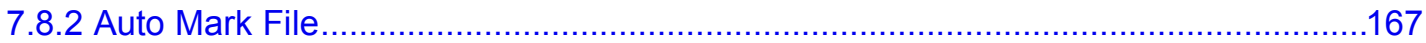

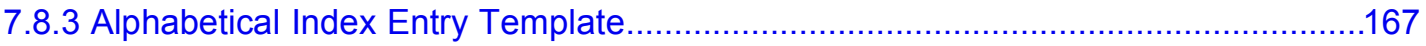

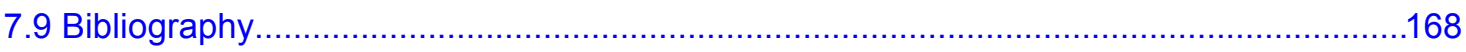

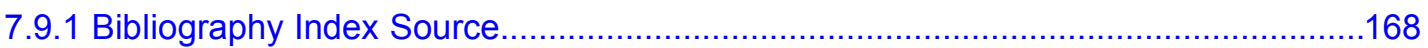

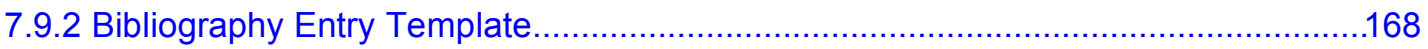

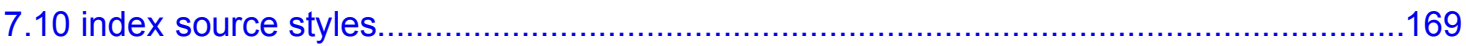

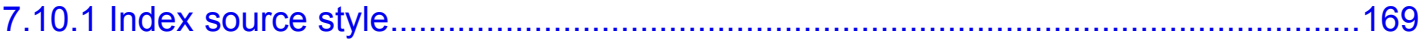

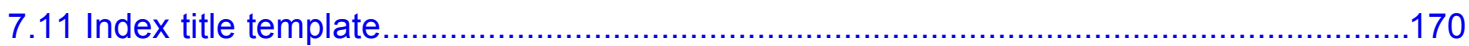

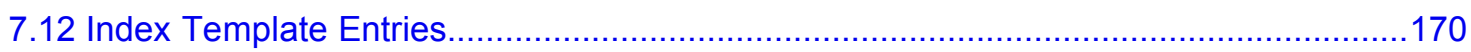

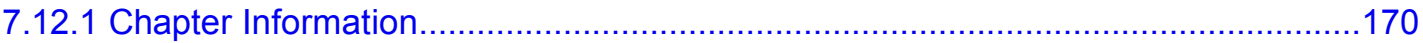

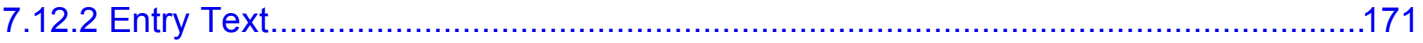

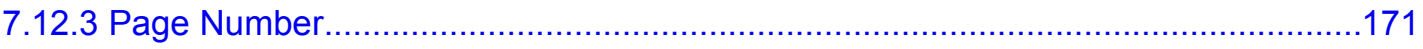

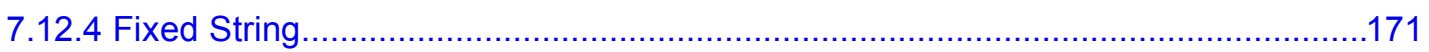

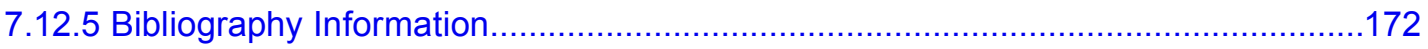

7.12.6 Tab Stop

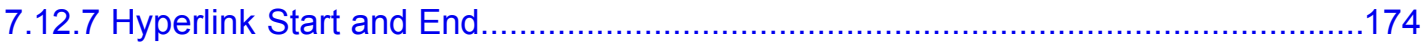

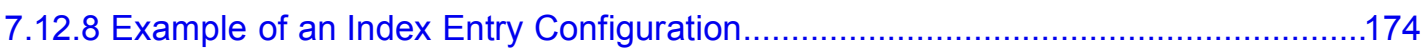

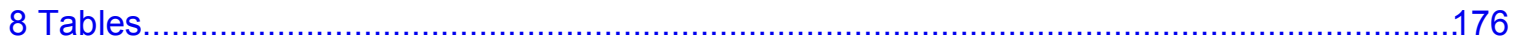

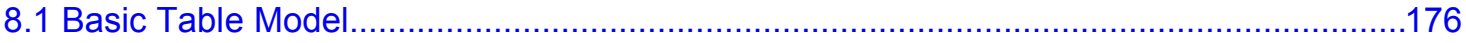

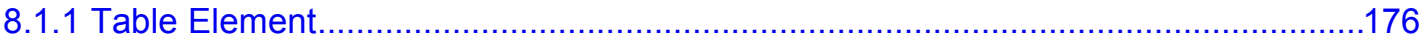

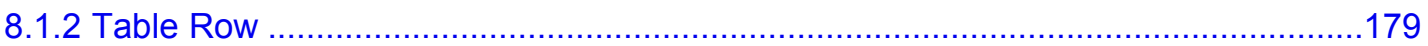

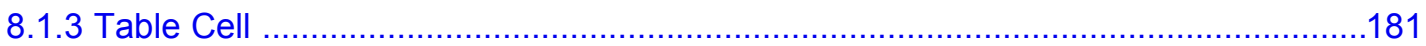




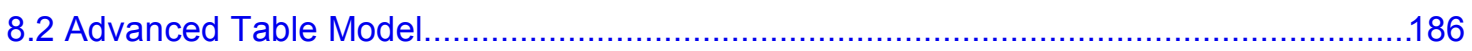

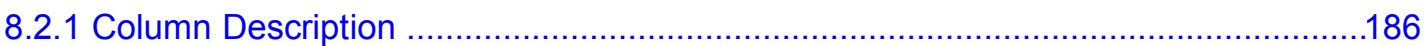

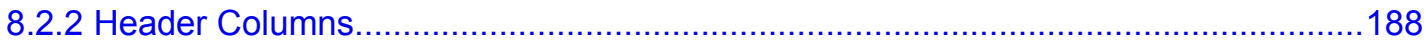

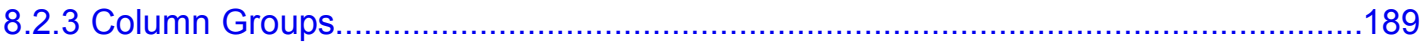

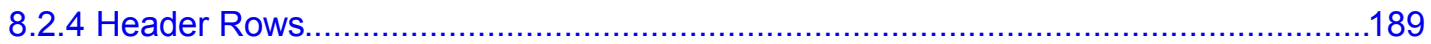

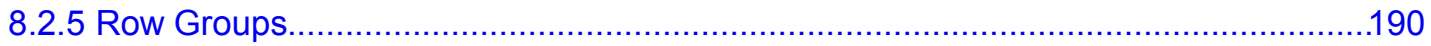

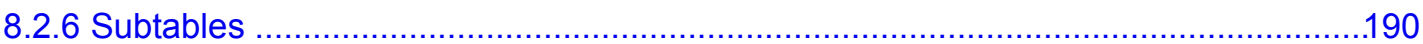

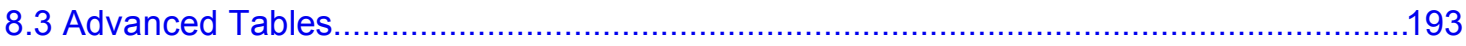

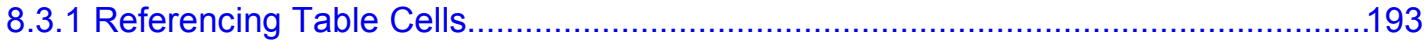

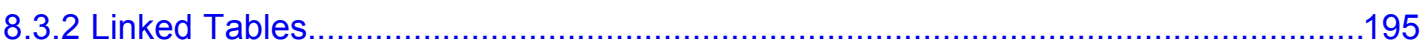

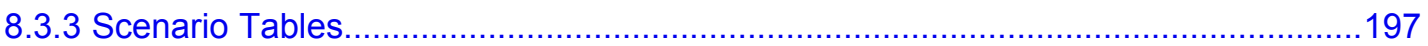

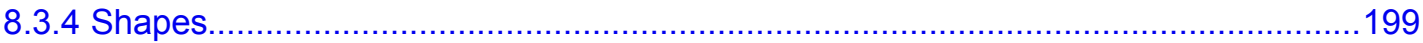

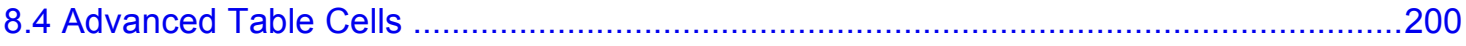

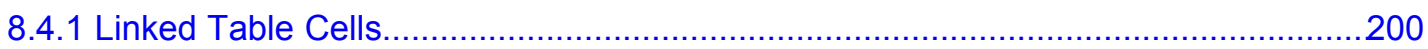

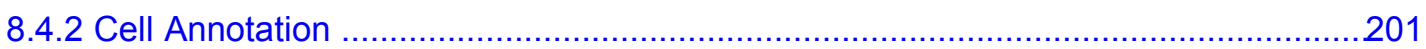

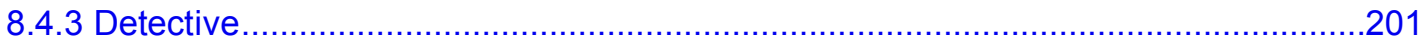

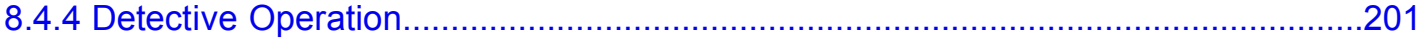

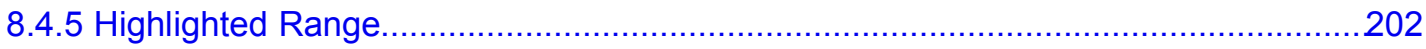

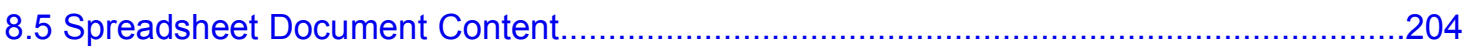

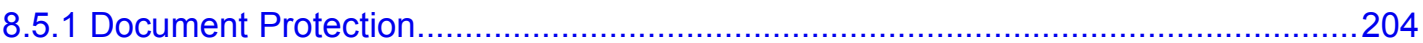

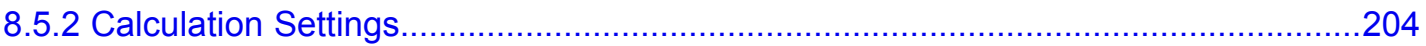

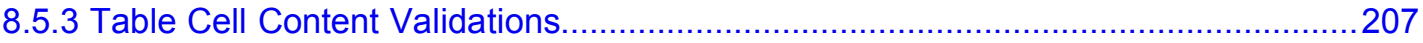

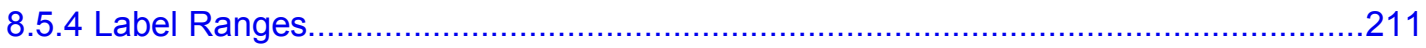

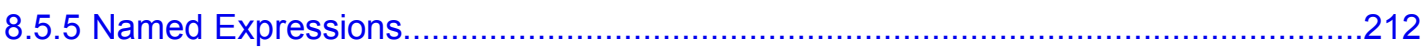

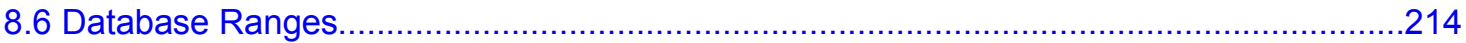

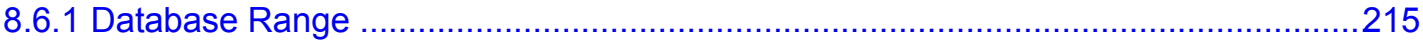

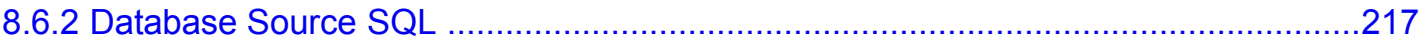

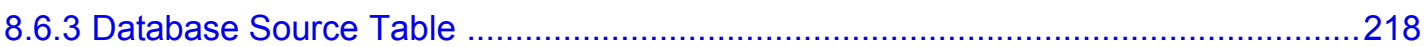

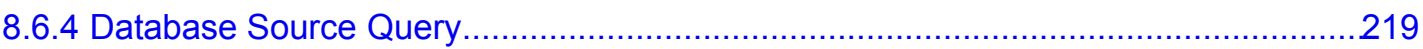

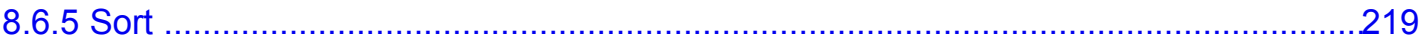

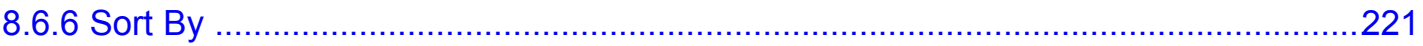

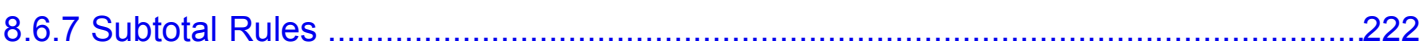

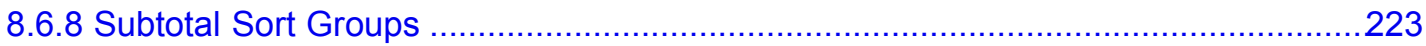

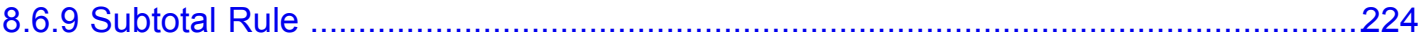

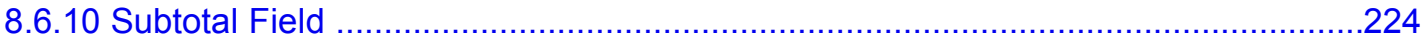

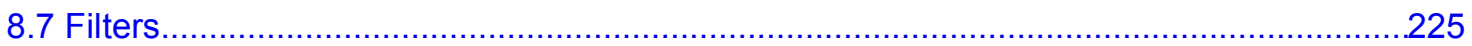

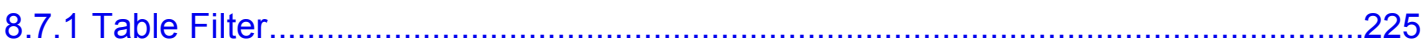




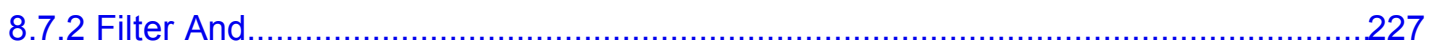

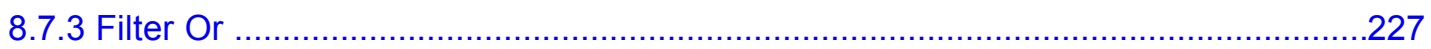

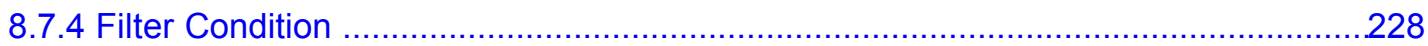

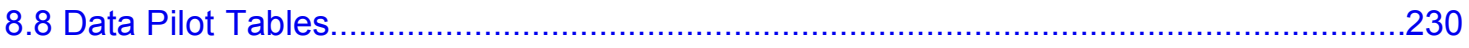

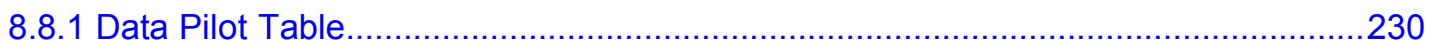

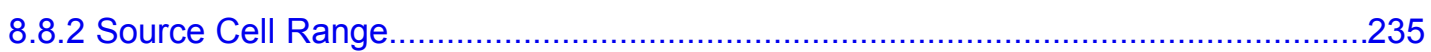

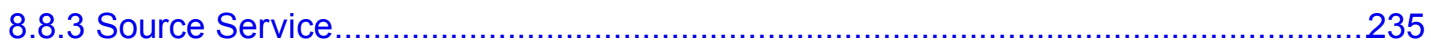

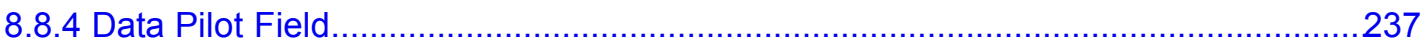

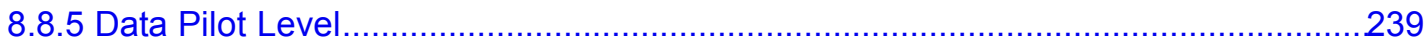

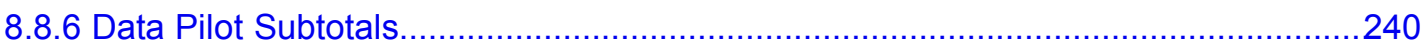

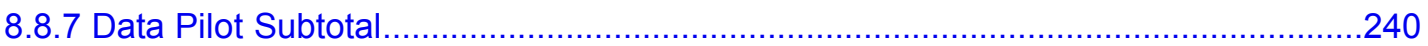

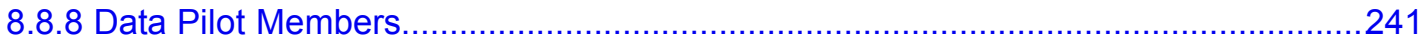

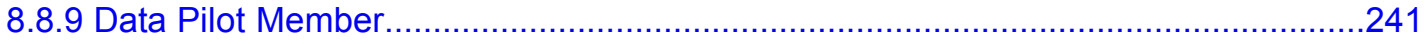

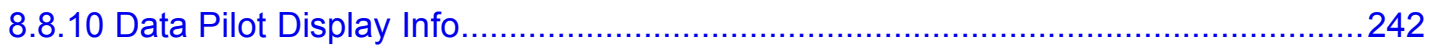

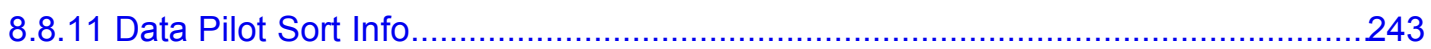

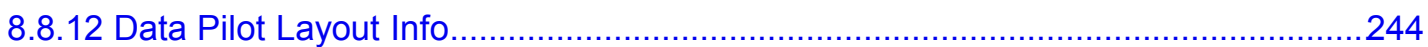

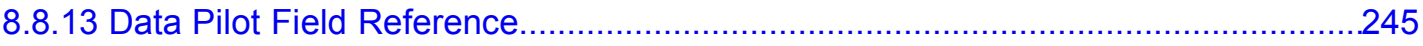

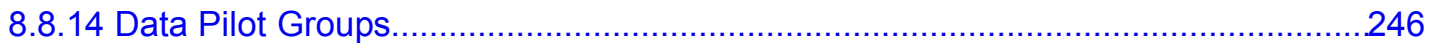

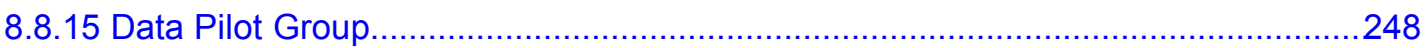

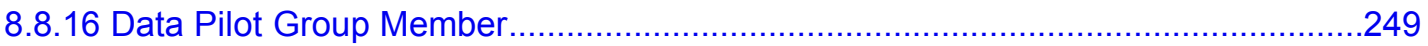

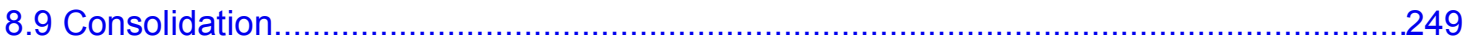

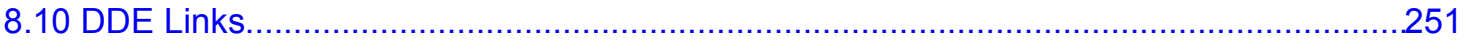

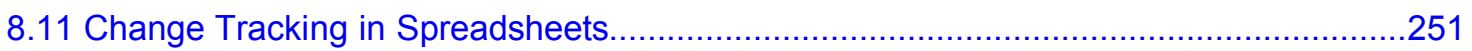

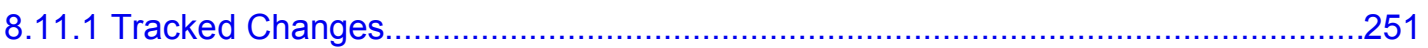

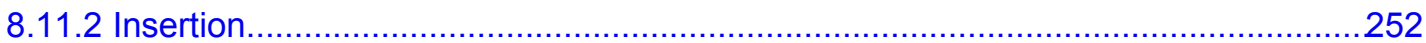

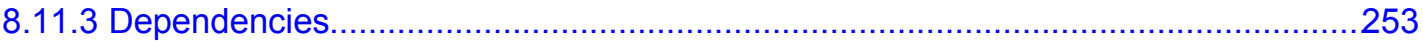

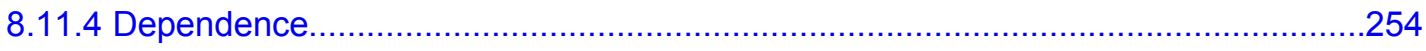

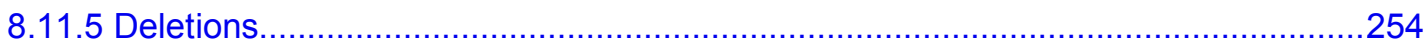

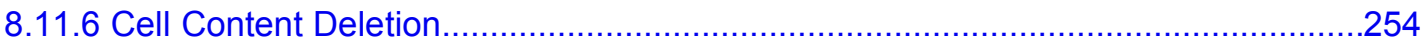

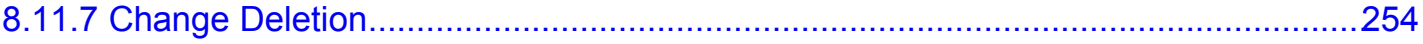

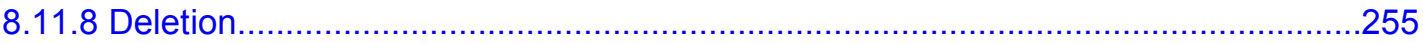

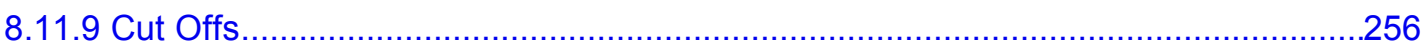

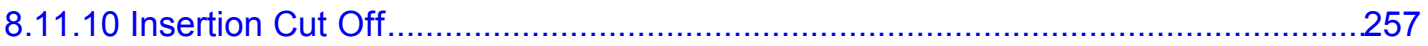

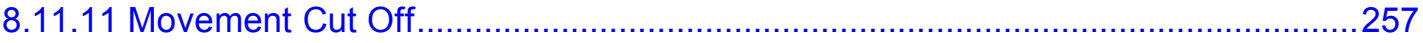

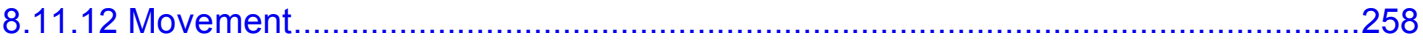

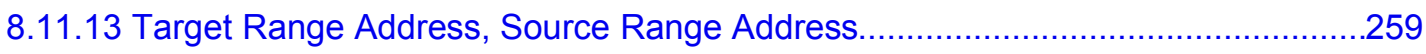

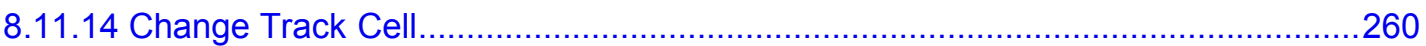




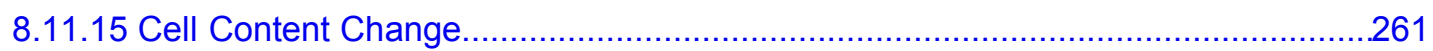

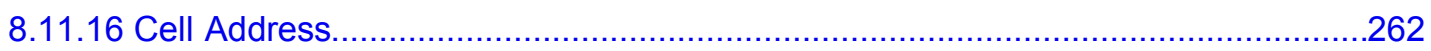

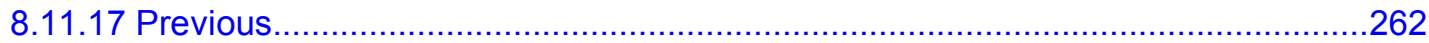

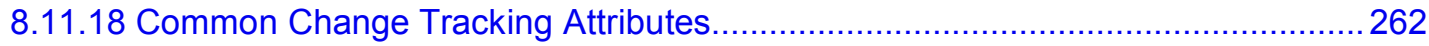

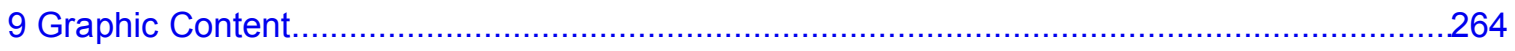

9.1 Enhanced Page Features for Graphical Applications...................................................264

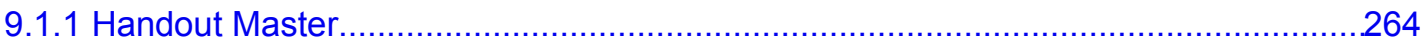

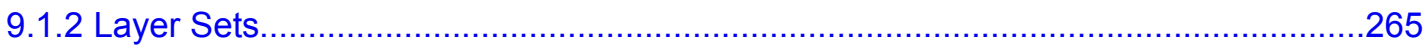

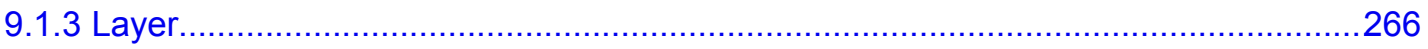

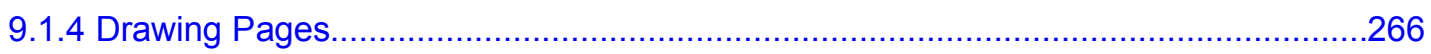

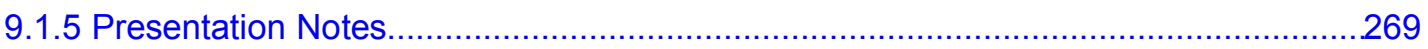

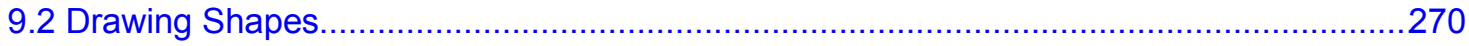

9.2.1 Rectangle

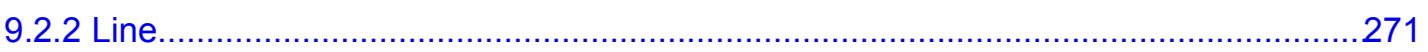

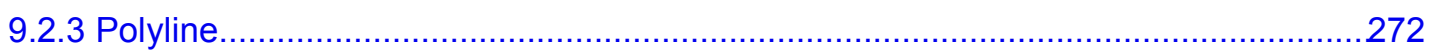

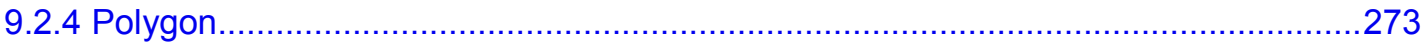

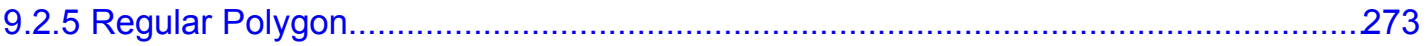

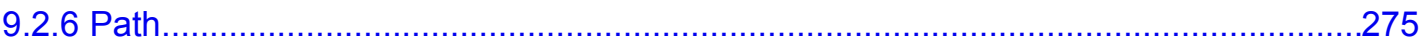

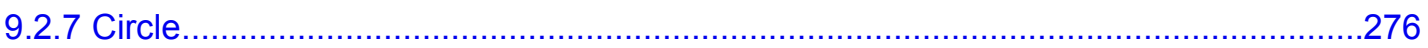

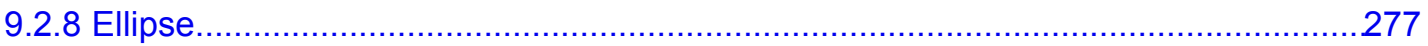

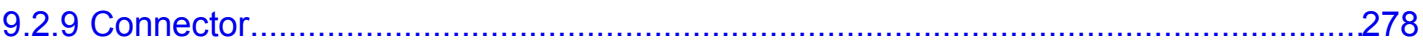

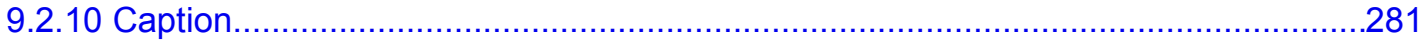

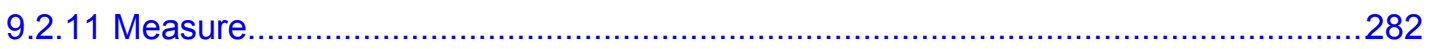

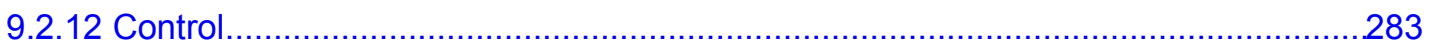

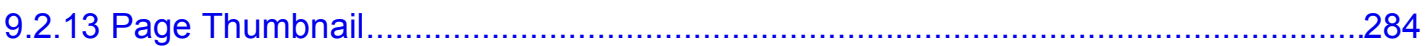

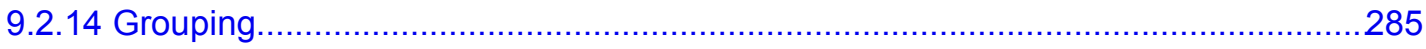

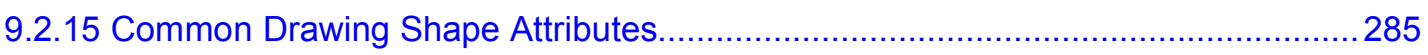

9.2.16 Common Shape Attributes for Text and Spreadsheet Documents..........................289

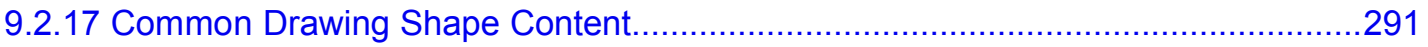

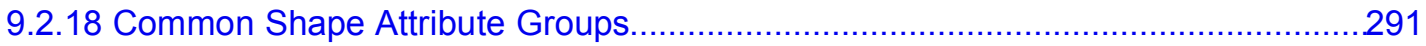

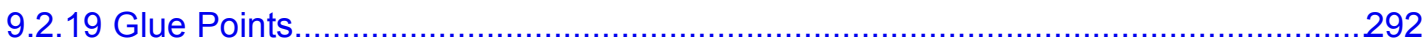

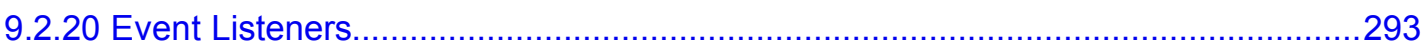

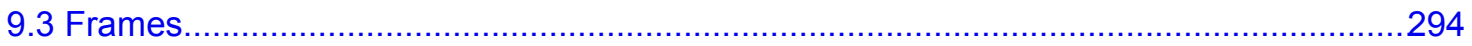

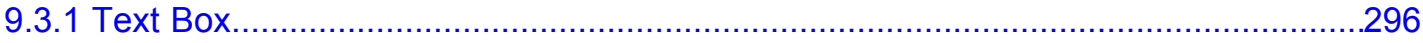

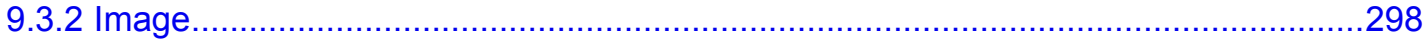

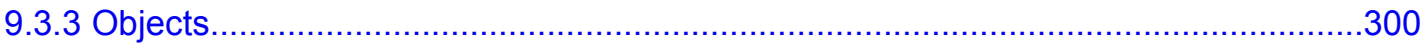

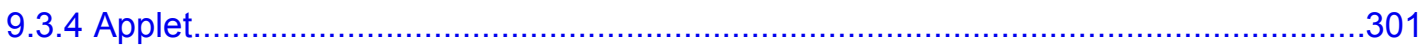




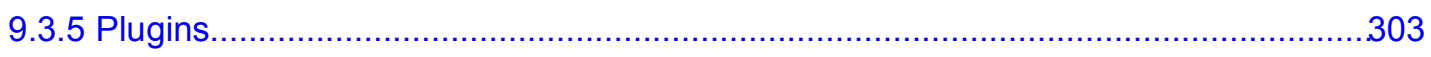

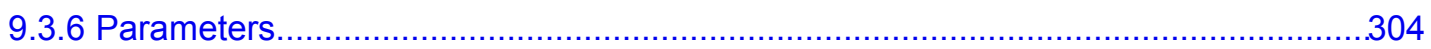

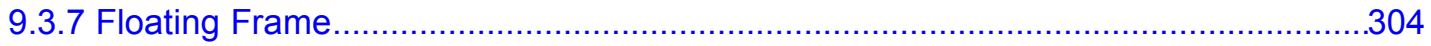

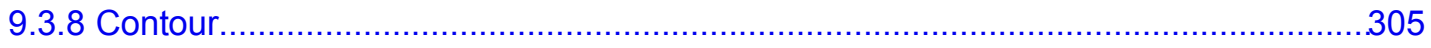

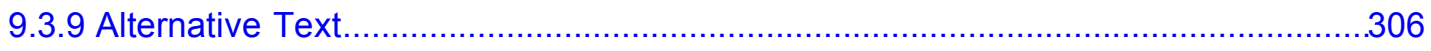

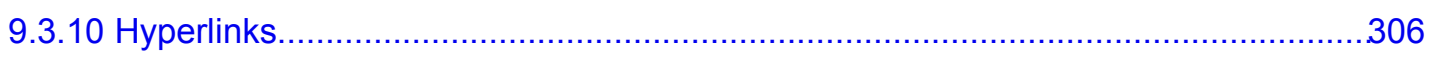

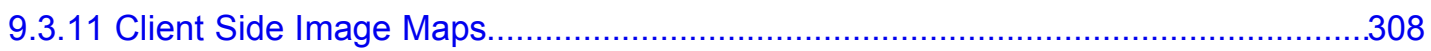

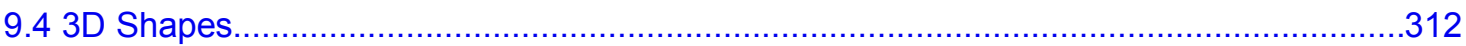

9.4.1 Scene

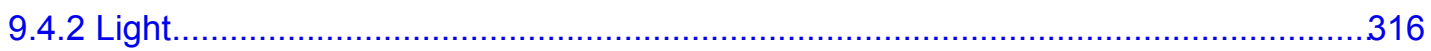

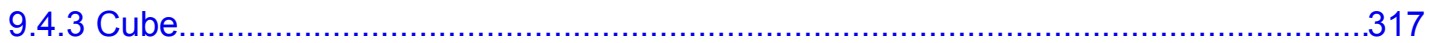

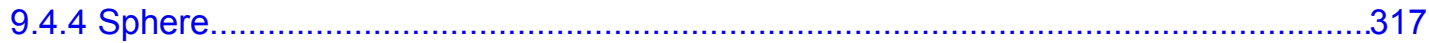

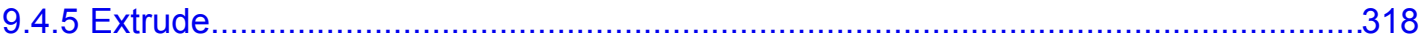

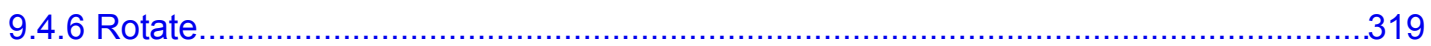

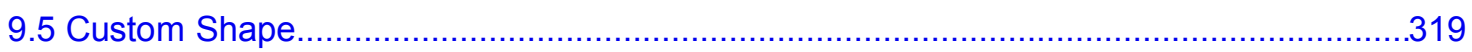

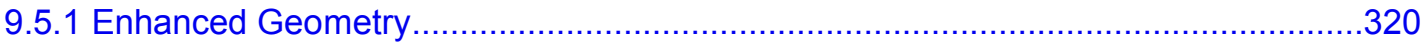

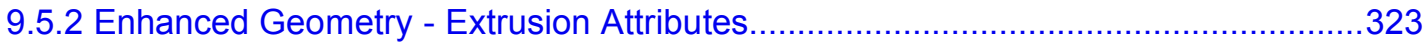

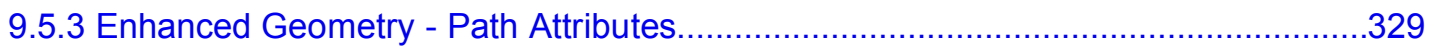

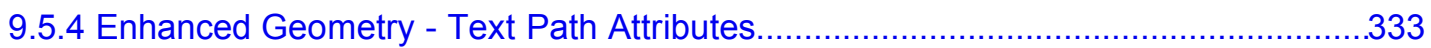

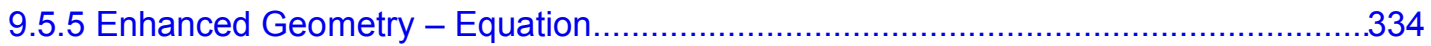

9.5.6 Enhanced Geometry - Handle Attributes..............................................................336

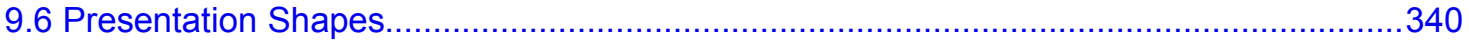

9.6.1 Common Presentation Shape Attributes......................................................... 340

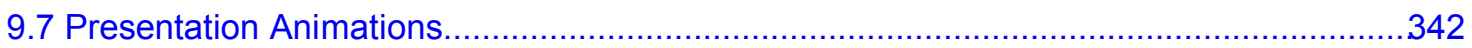

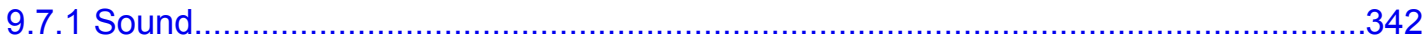

9.7.2 Show Shape

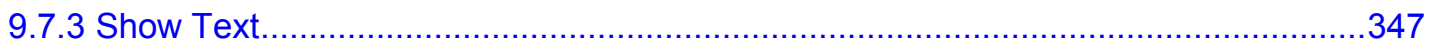

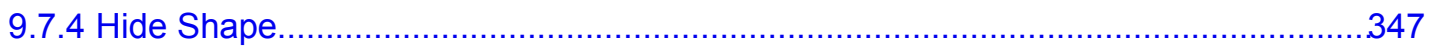

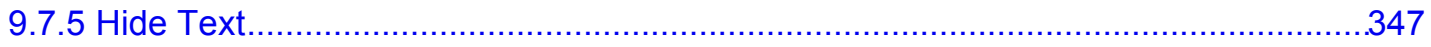

9.7.6 Dim

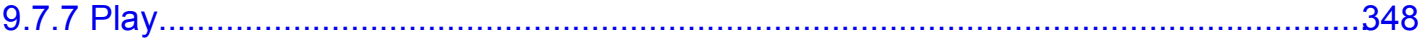

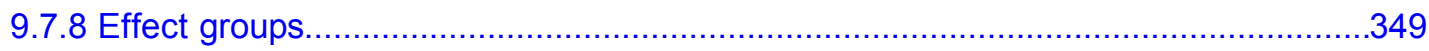

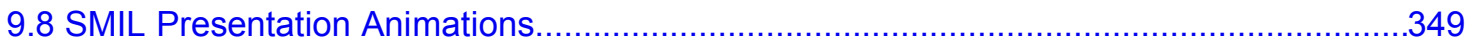

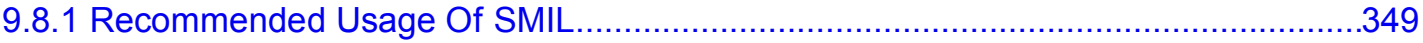

9.8.2 Document Dependent SMIL Animation Attribute Values........................................ 350

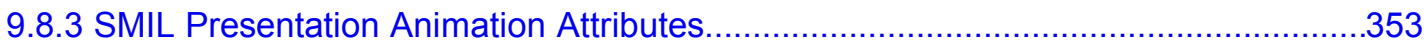

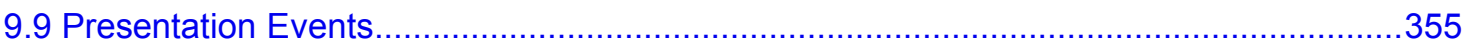




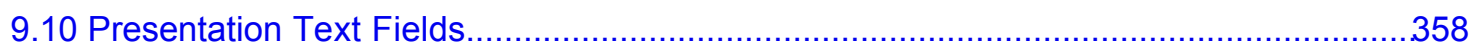

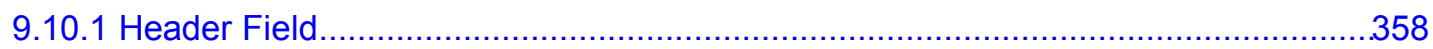

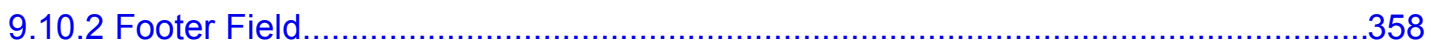

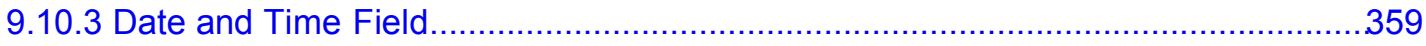

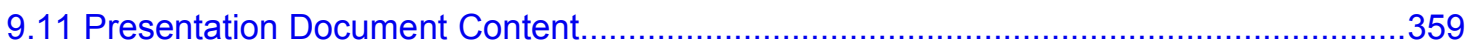

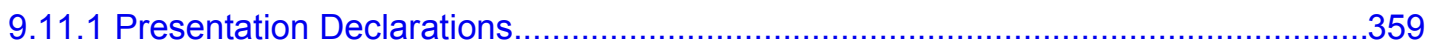

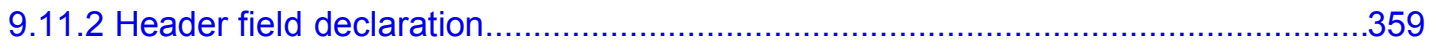

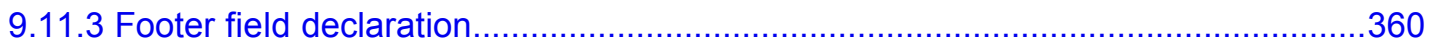

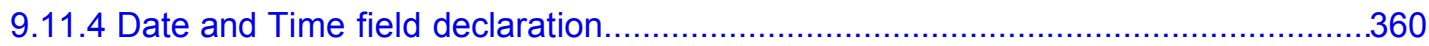

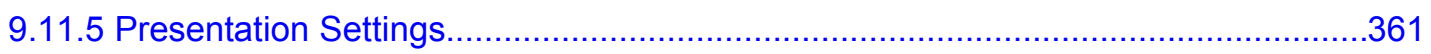

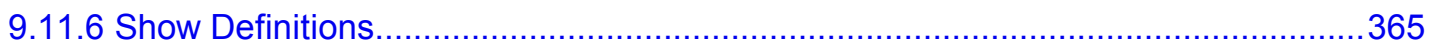

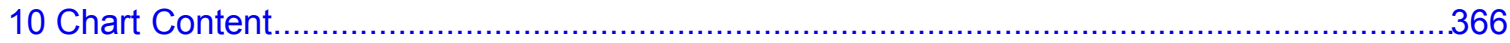

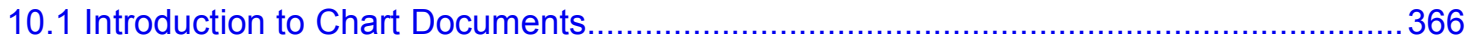

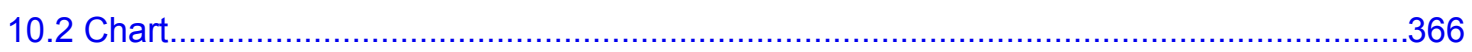

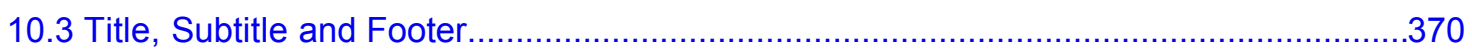

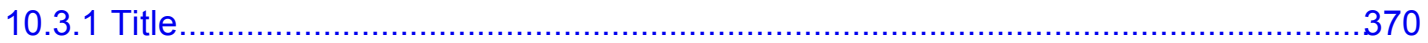

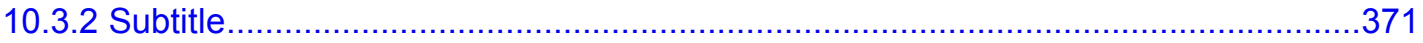

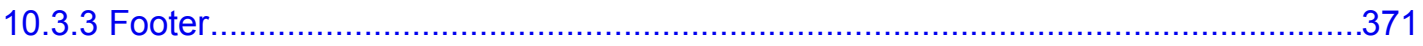

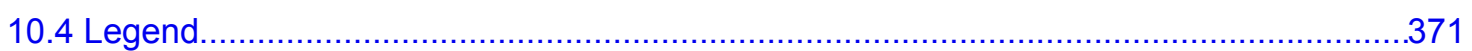

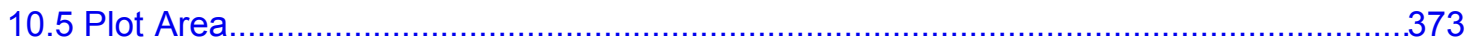

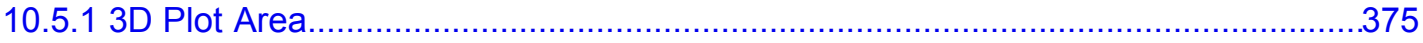

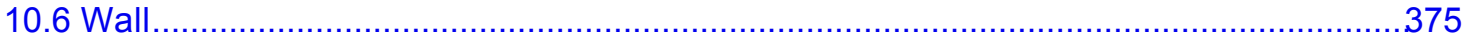

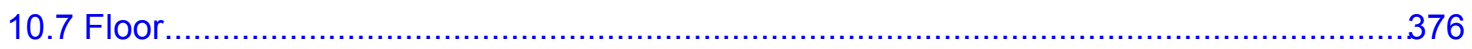

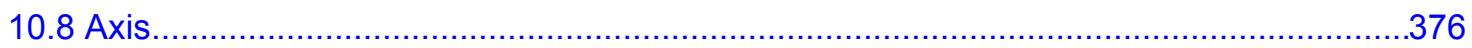

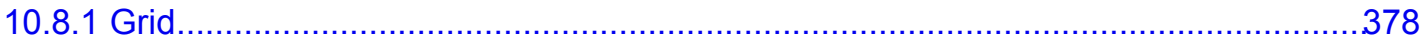

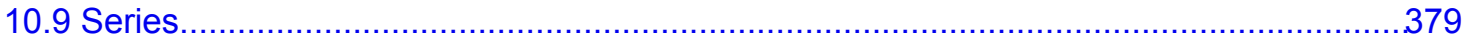

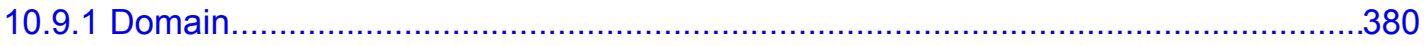

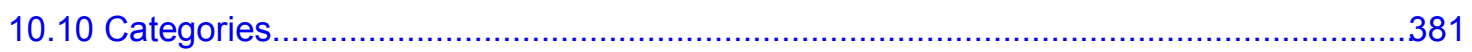

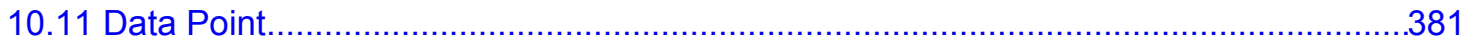

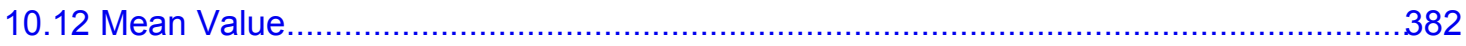

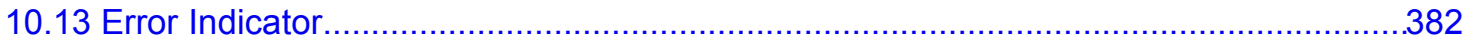

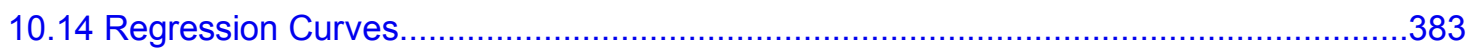

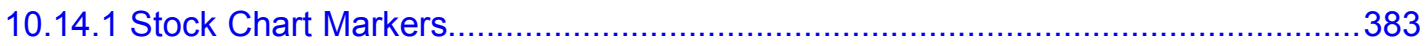

11 Form Content

11.1 Form

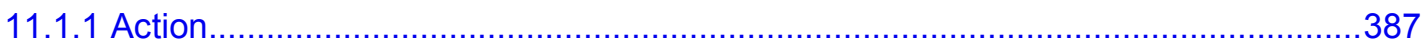

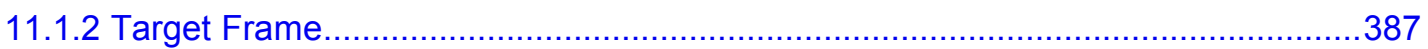




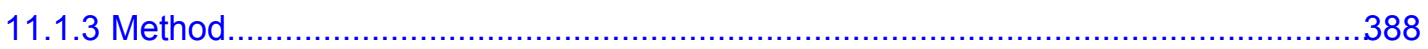

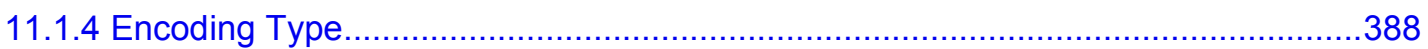

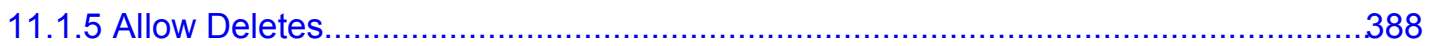

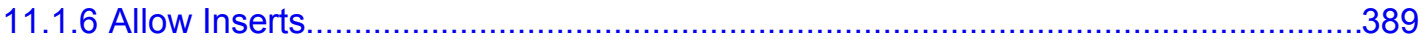

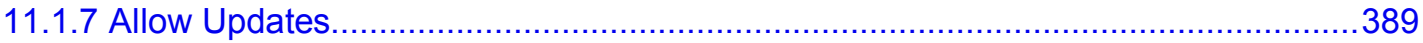

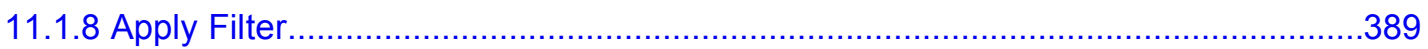

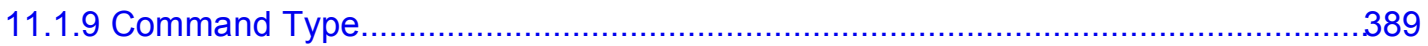

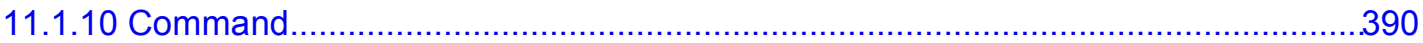

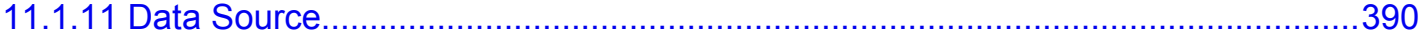

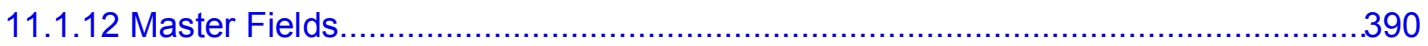

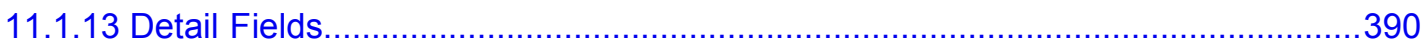

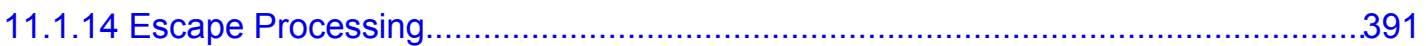

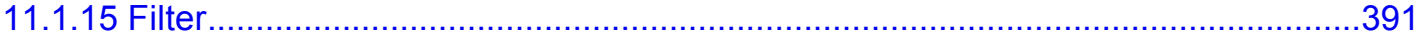

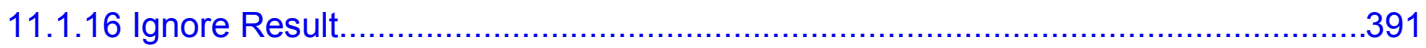

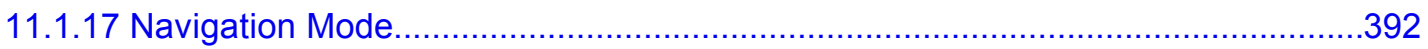

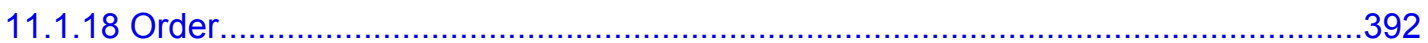

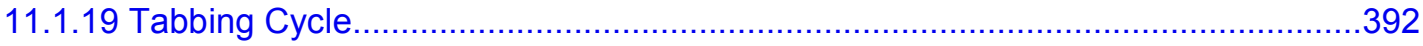

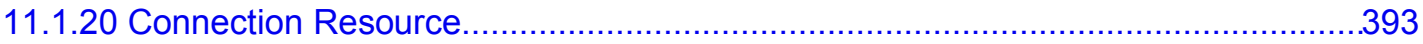

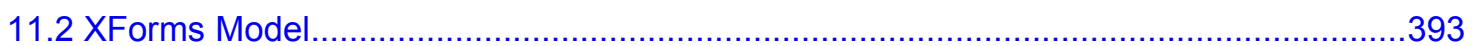

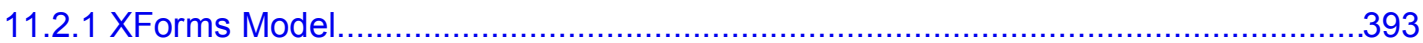

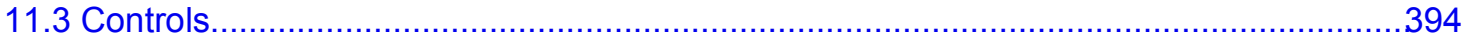

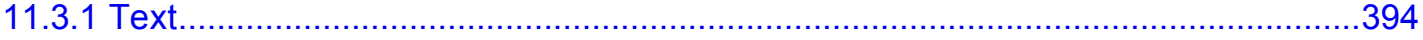

11.3.2 Text Area

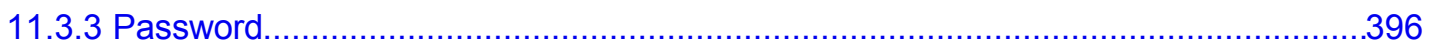

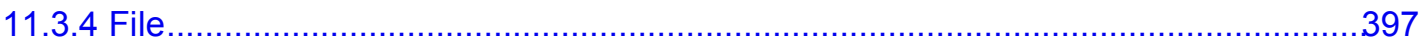

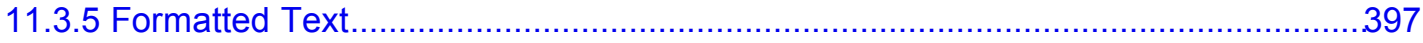

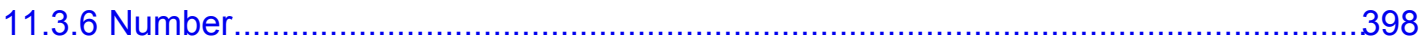

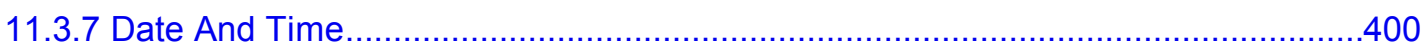

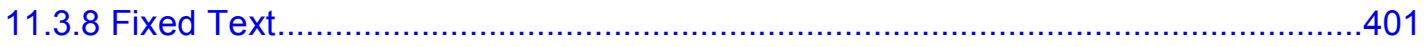

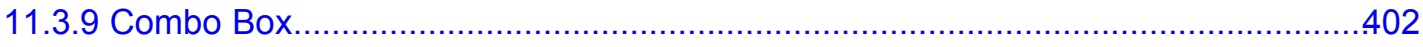

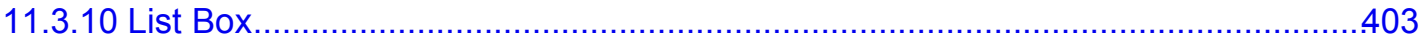

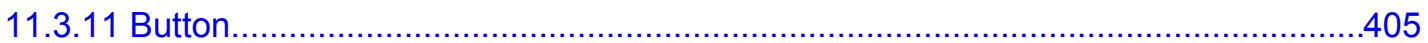

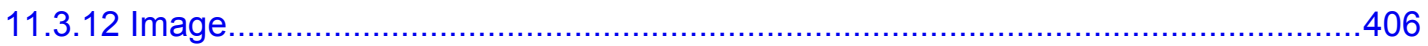

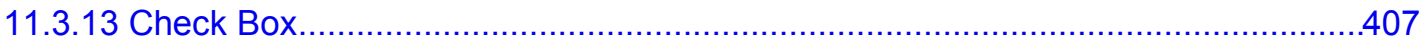

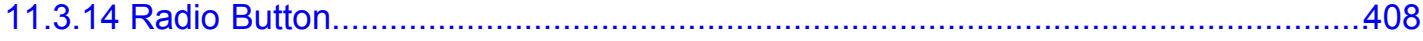

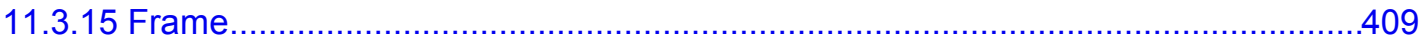

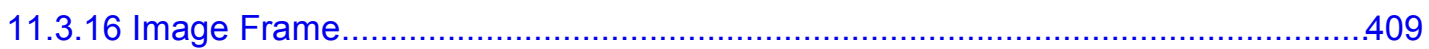




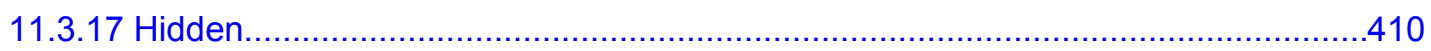

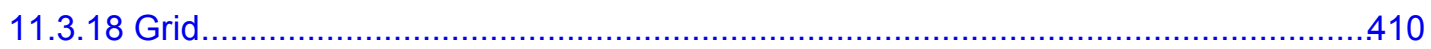

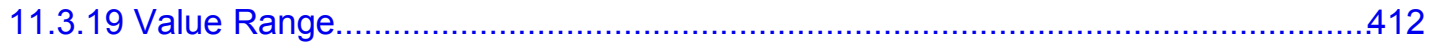

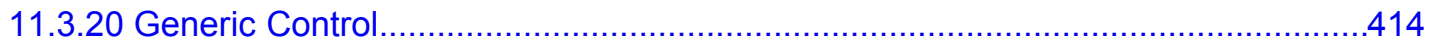

11.4 Common Form and Control Attributes.................................................................... 414

11.4.1 Name

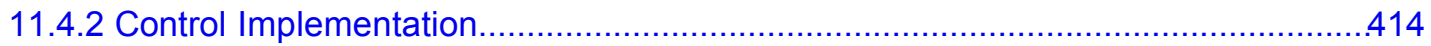

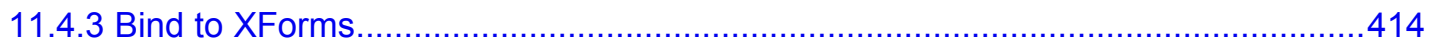

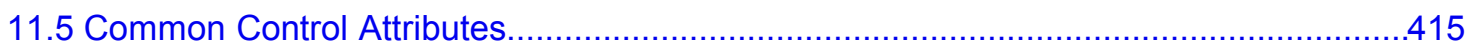

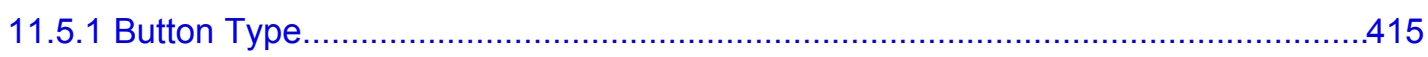

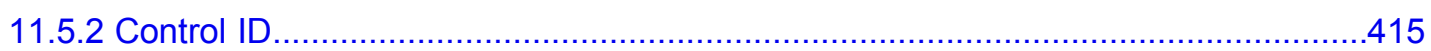

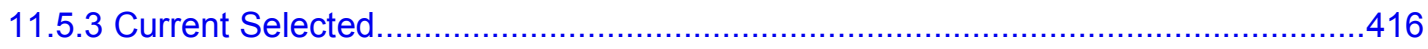

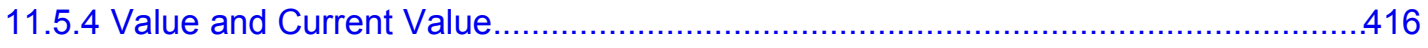

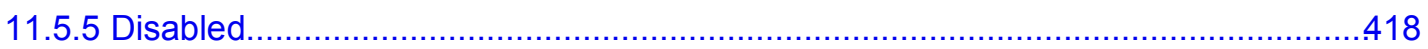

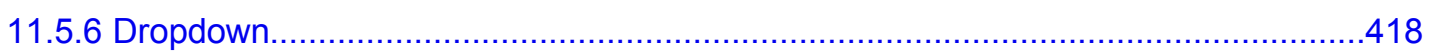

11.5.7 For

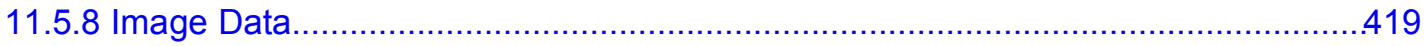

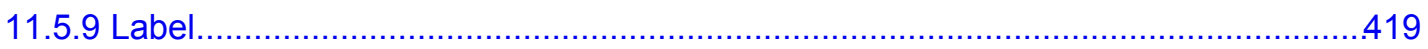

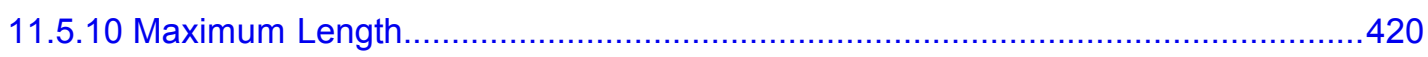

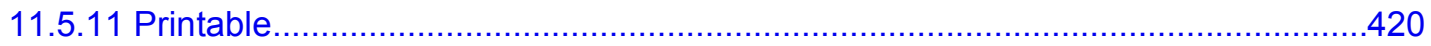

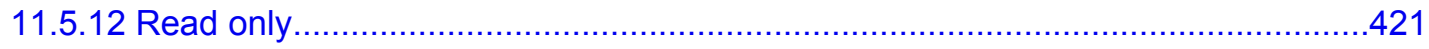

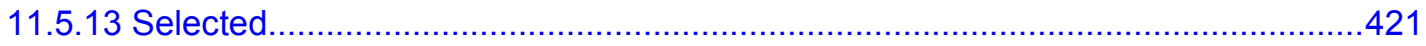

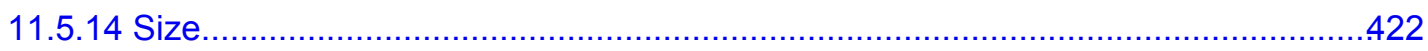

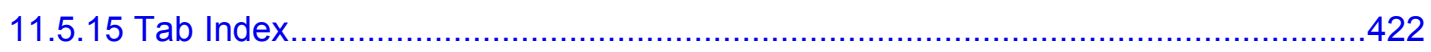

11.5.16 Tab Stop

11.5.17 Target Frame

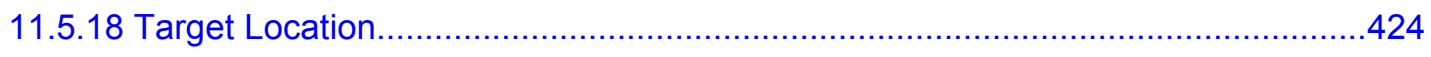

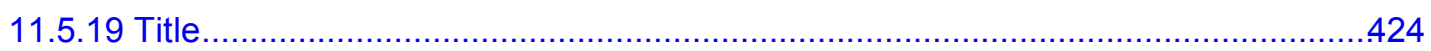

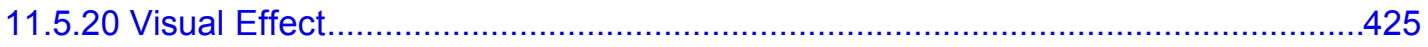

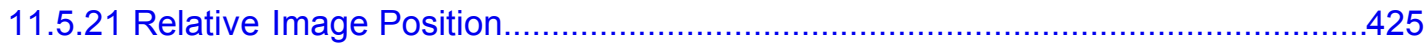

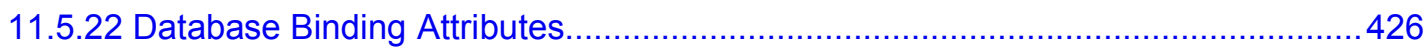

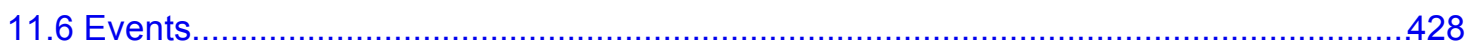

11.6.1 Events with an Equivalent HTML Event Type.................................................428

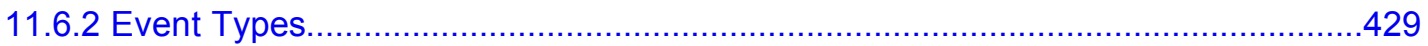

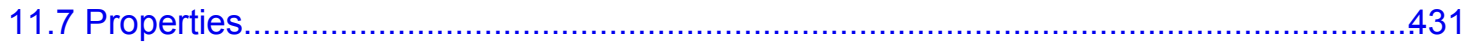

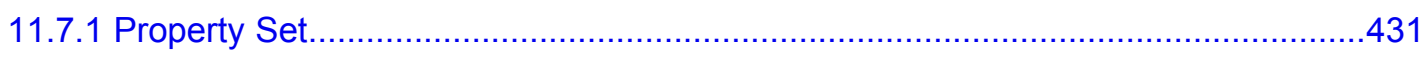

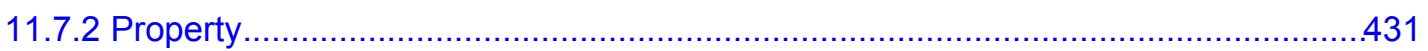




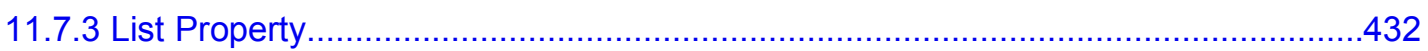

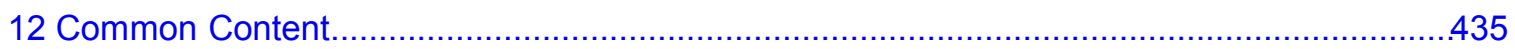

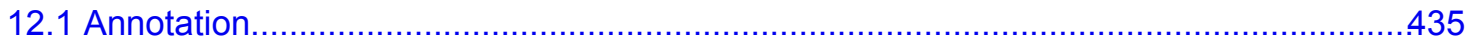

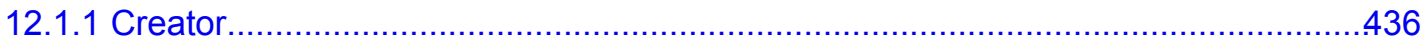

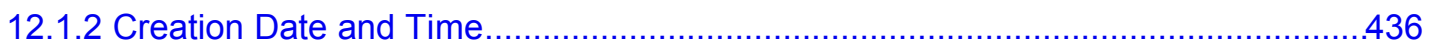

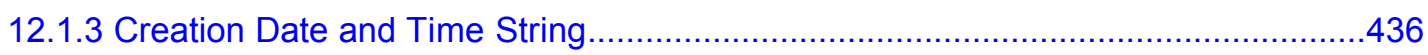

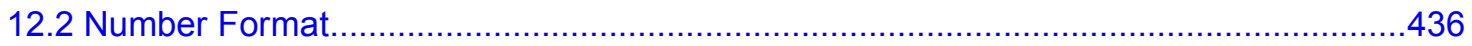

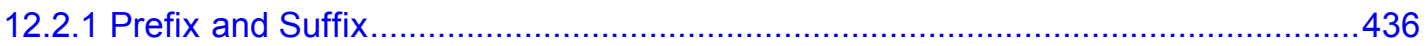

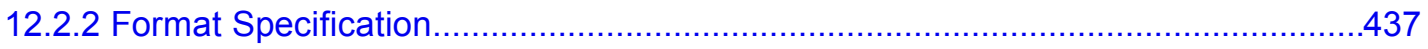

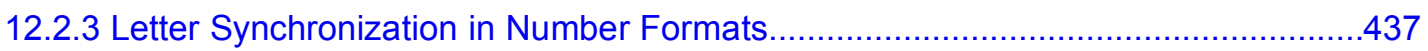

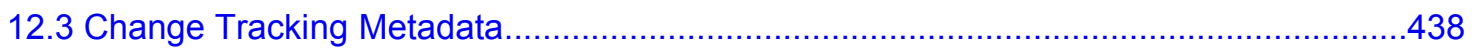

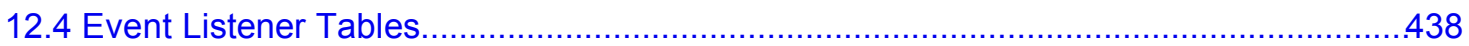

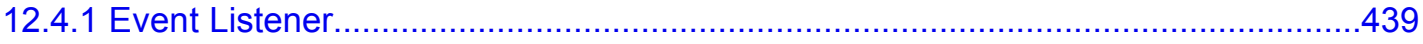

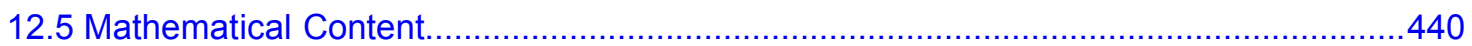

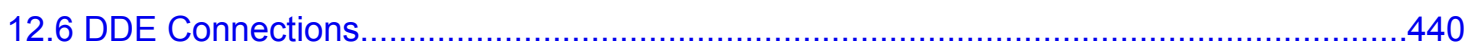

12.6.1 Container for DDE Connection Declarations..................................................... 441

12.6.2 Declaring DDE Connections for Text Fields......................................................441

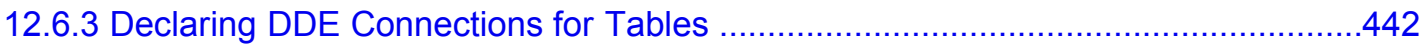

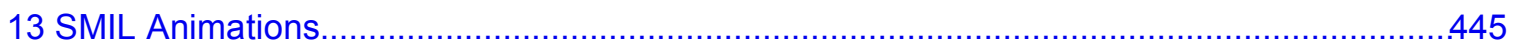

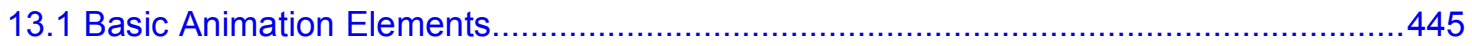

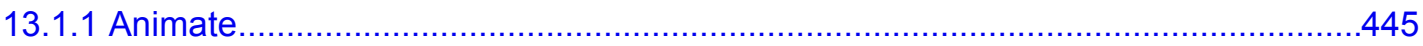

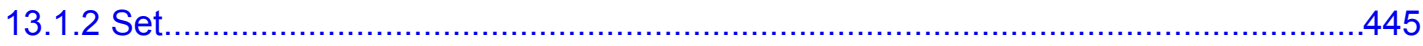

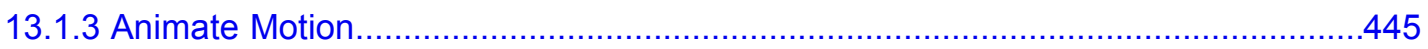

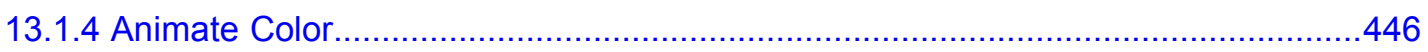

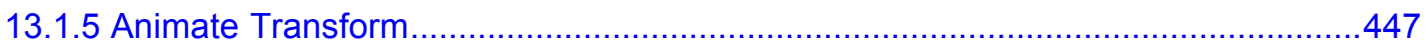

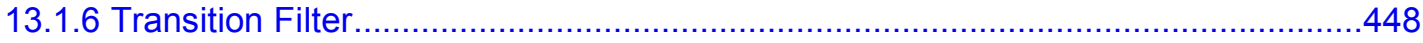

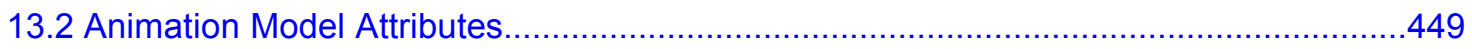

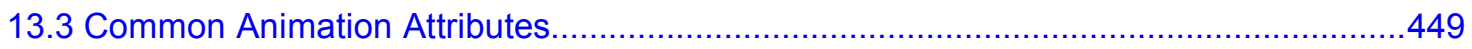

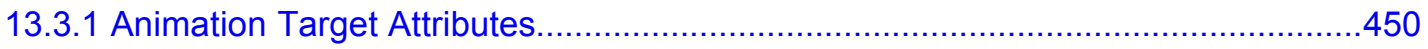

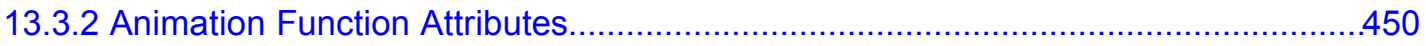

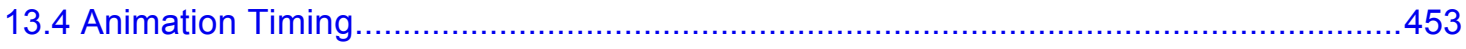

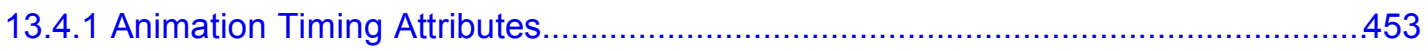

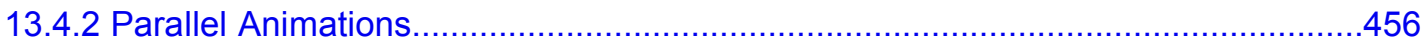

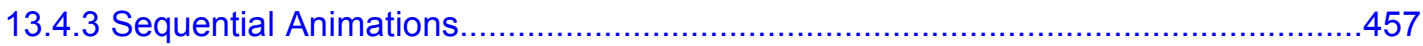

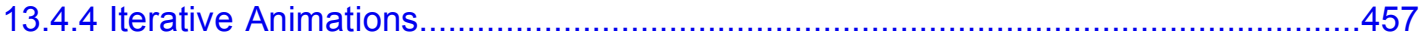

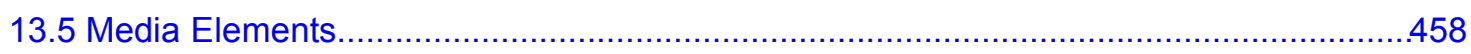

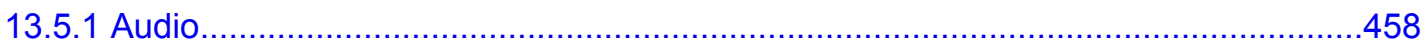




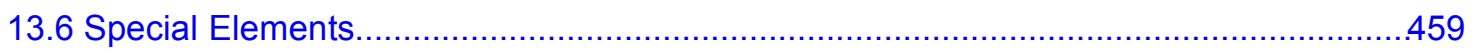

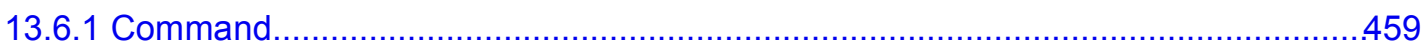

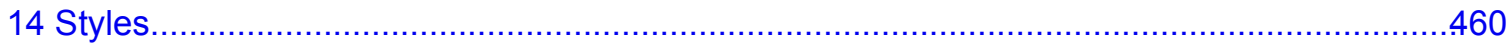

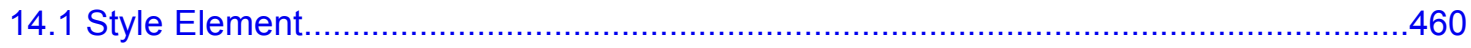

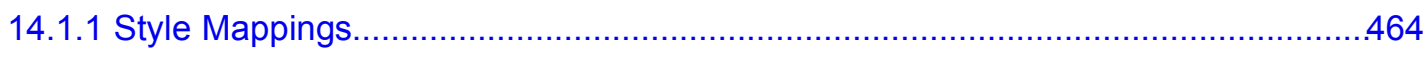

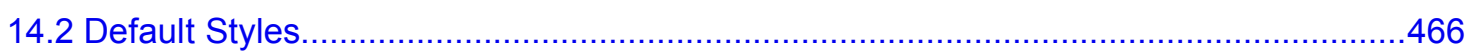

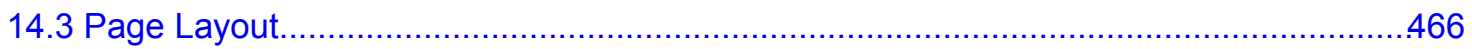

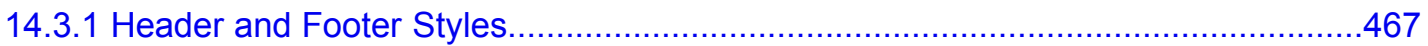

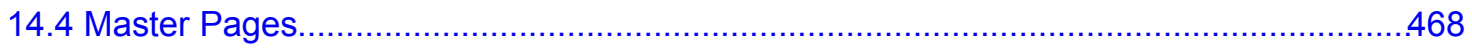

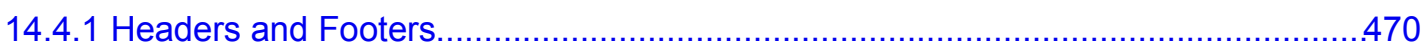

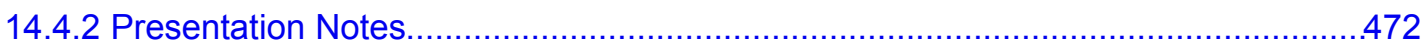

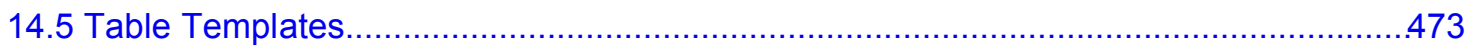

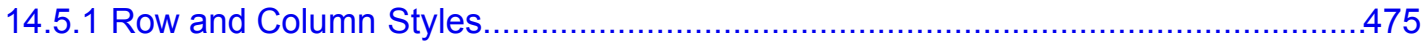

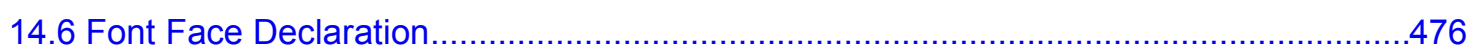

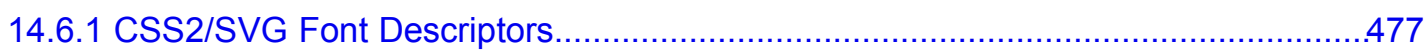

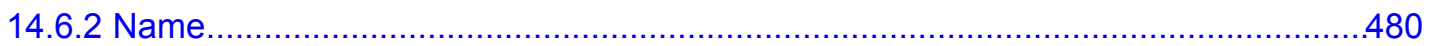

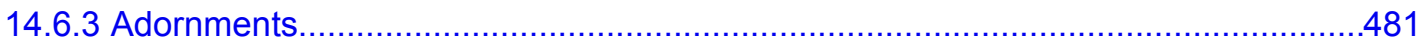

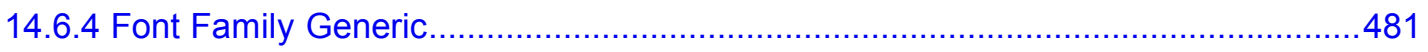

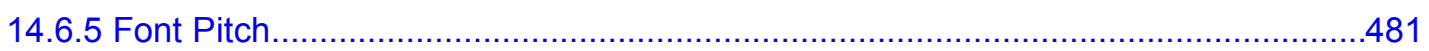

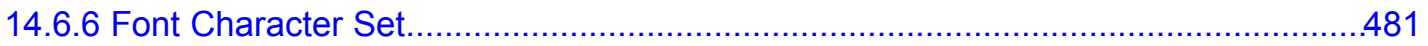

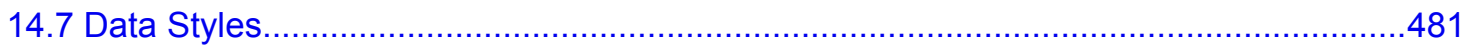

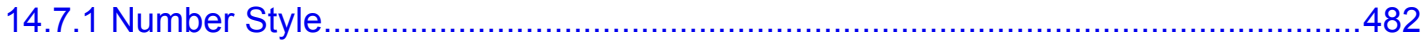

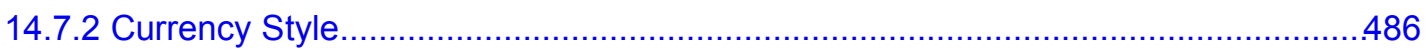

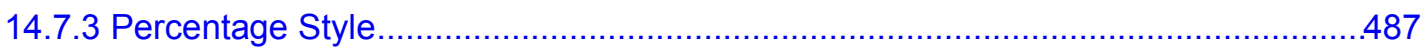

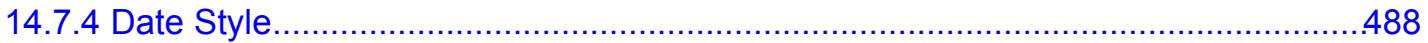

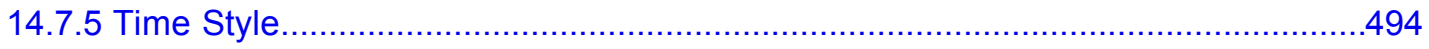

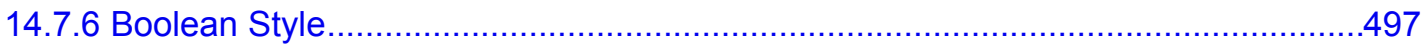

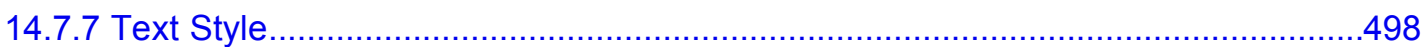

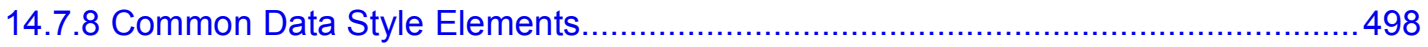

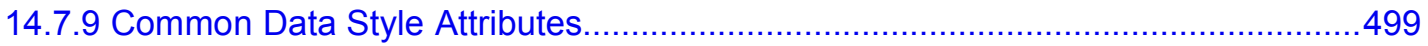

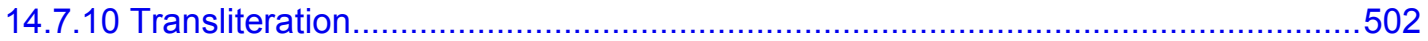

14.7.11 Common Data Style Child Element Attributes..................................................503

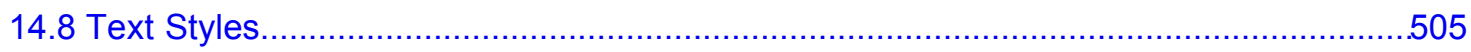

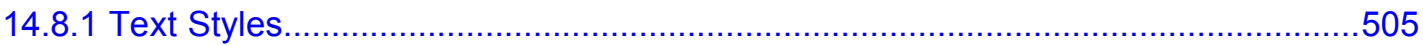

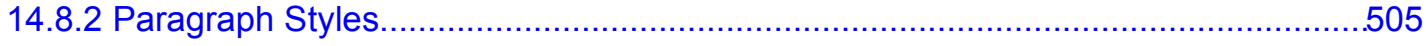

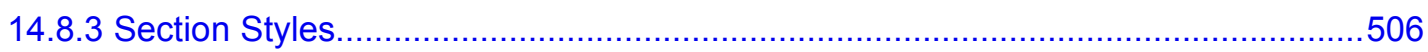

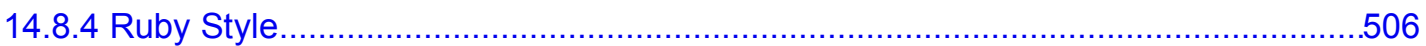




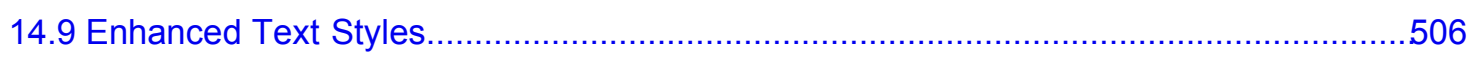

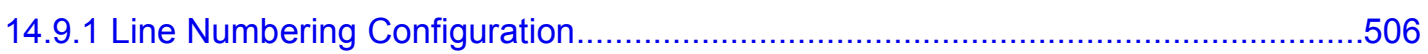

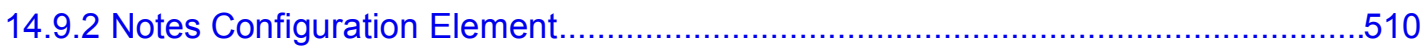

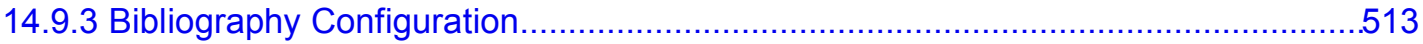

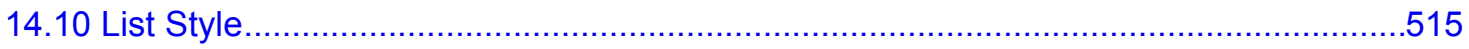

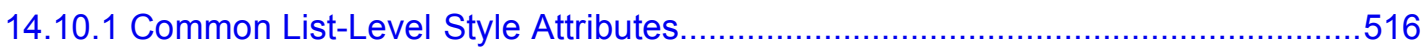

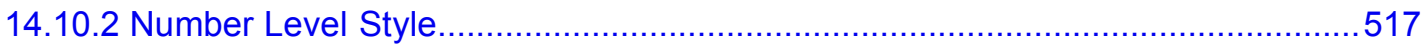

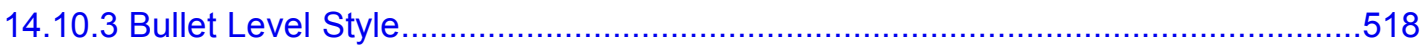

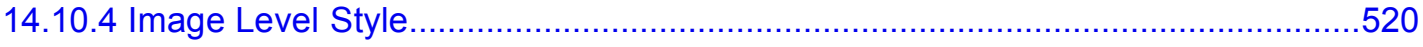

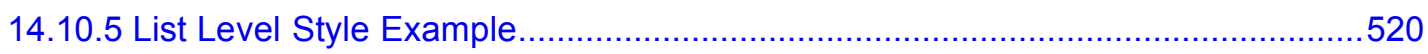

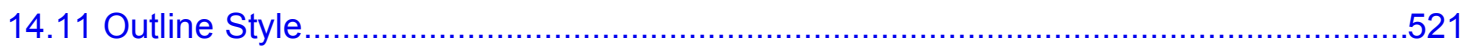

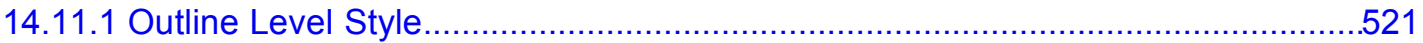

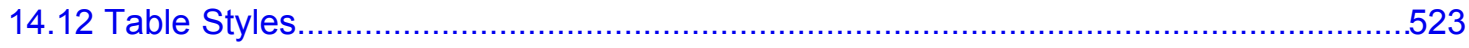

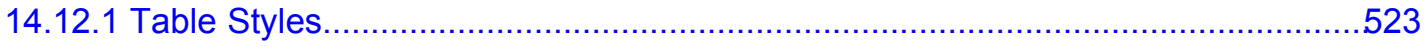

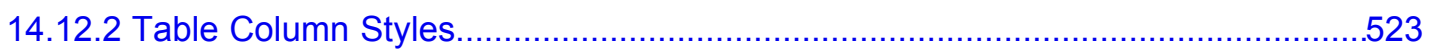

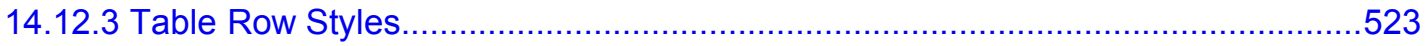

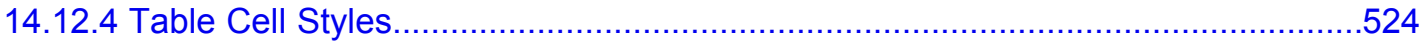

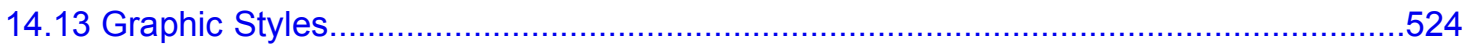

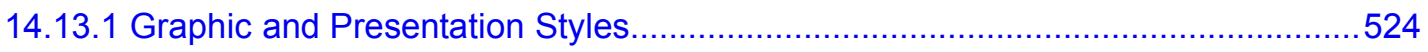

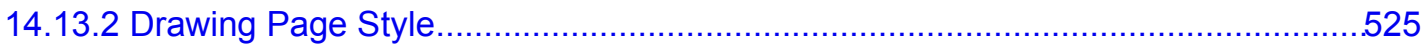

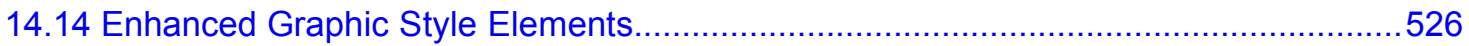

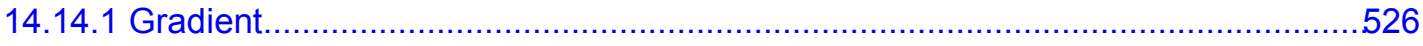

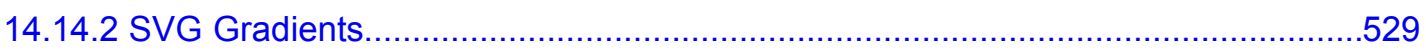

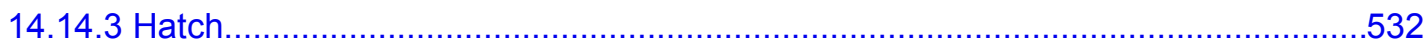

14.14.4 Fill Image

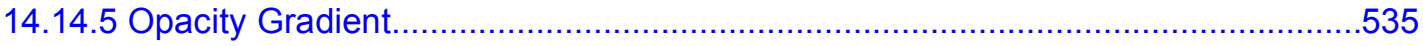

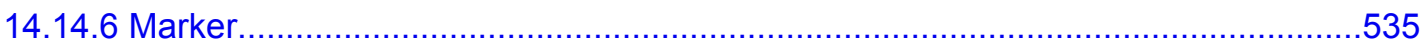

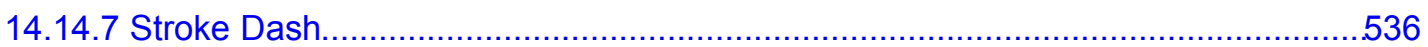

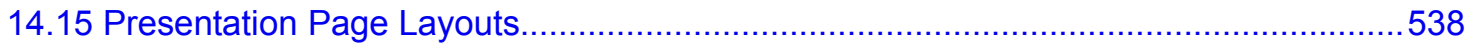

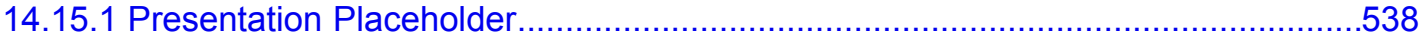

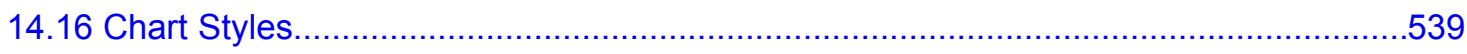

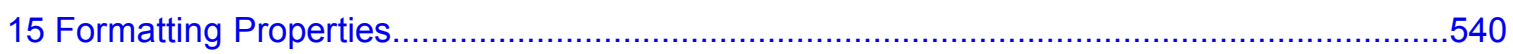

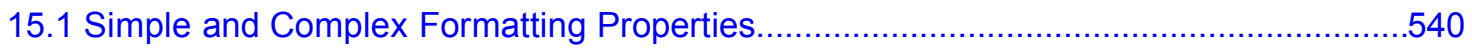

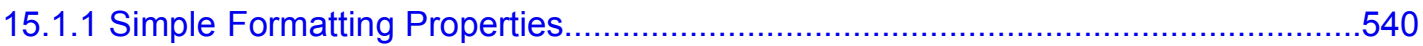

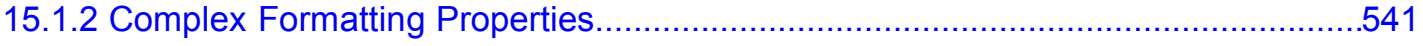

15.1.3 Processing Rules for Formatting Properties.......................................................

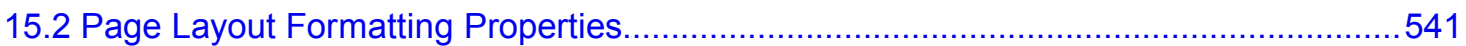




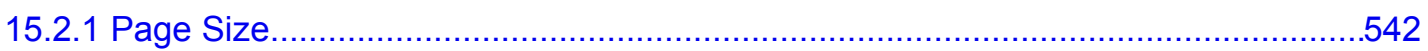

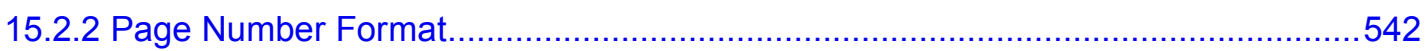

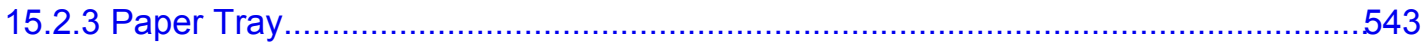

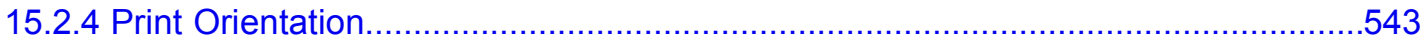

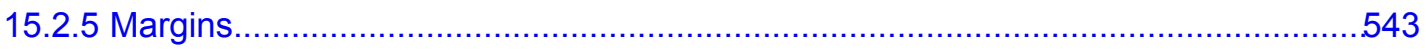

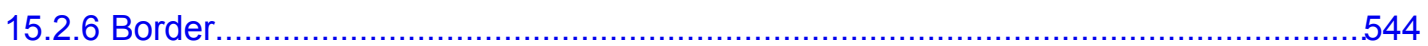

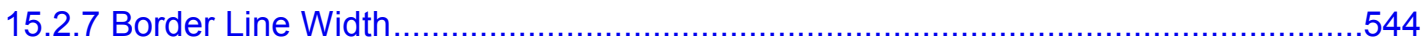

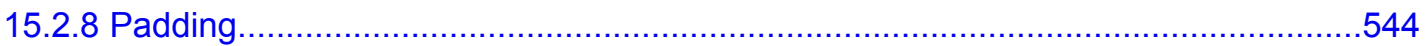

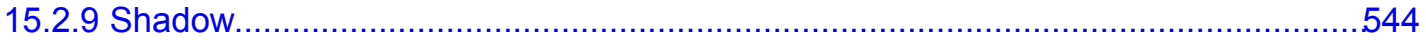

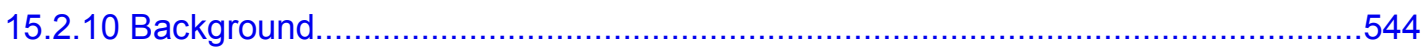

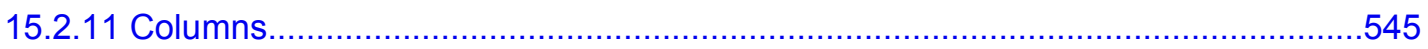

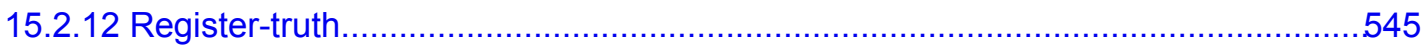

15.2.13 Print

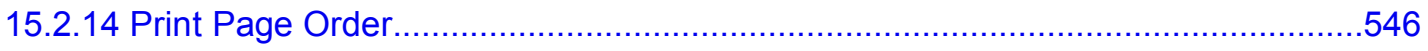

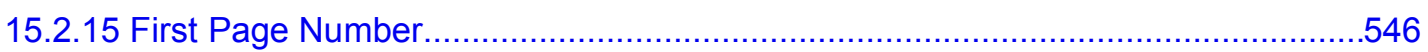

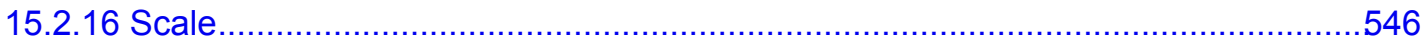

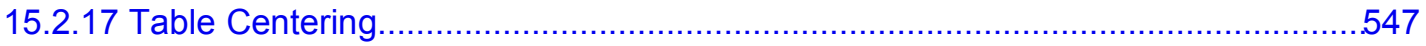

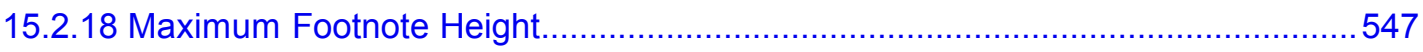

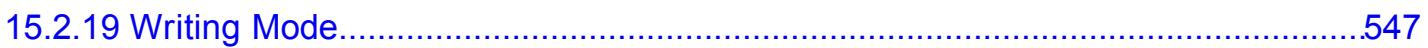

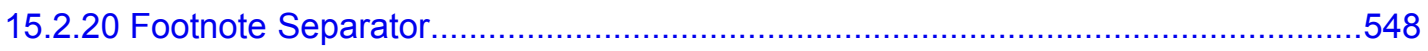

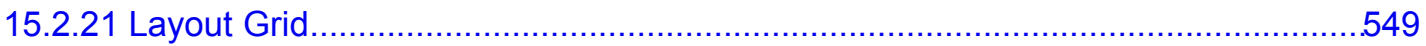

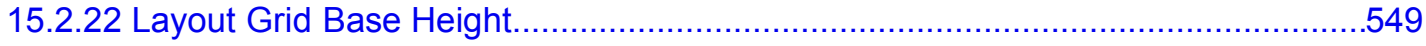

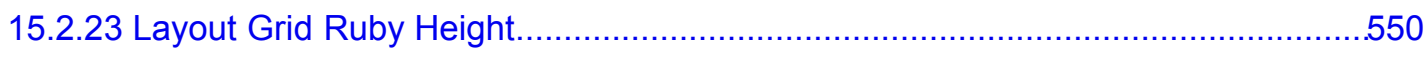

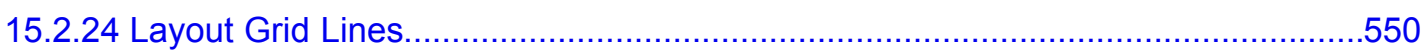

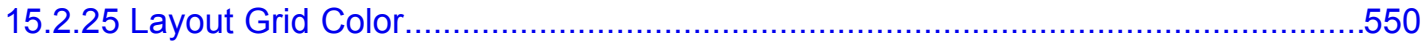

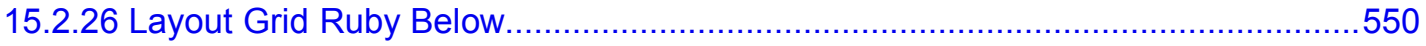

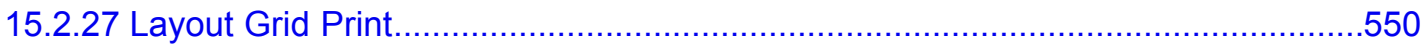

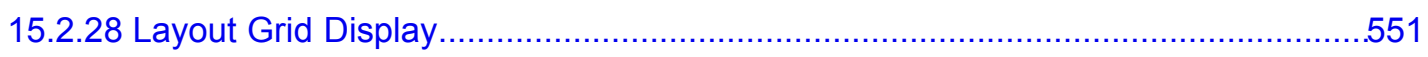

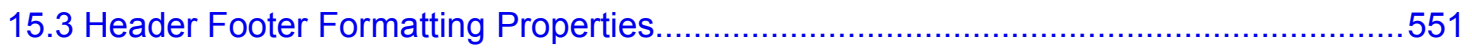

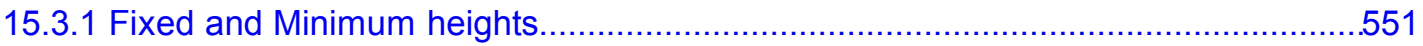

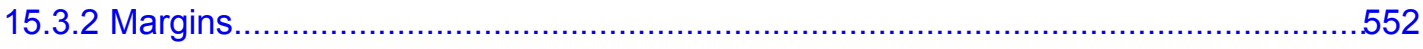

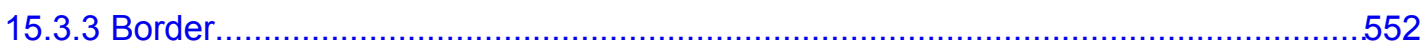

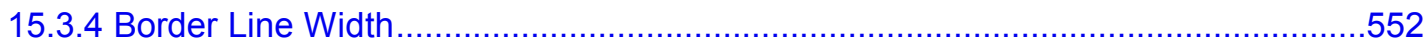

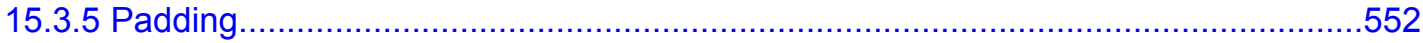

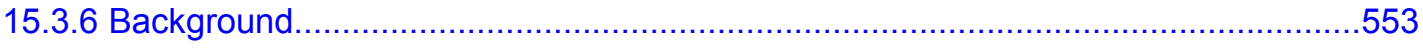

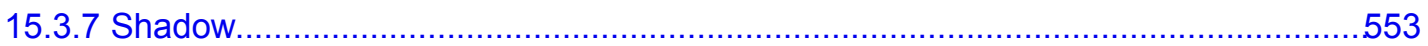

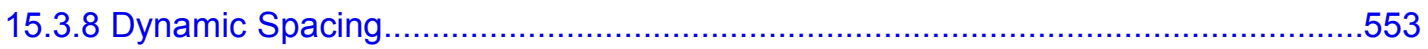




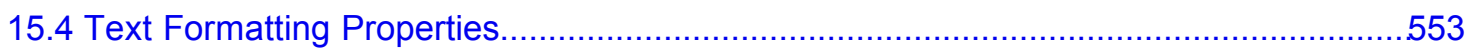

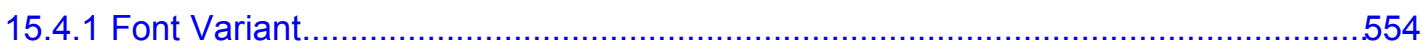

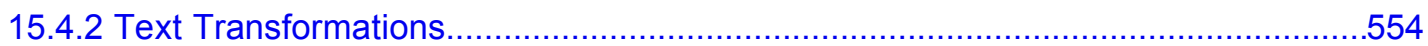

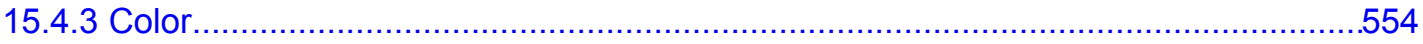

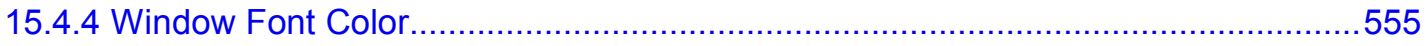

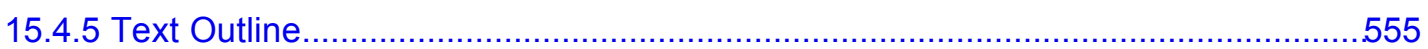

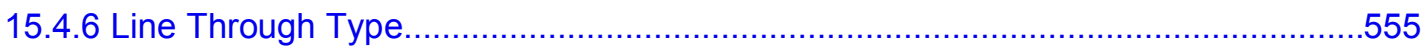

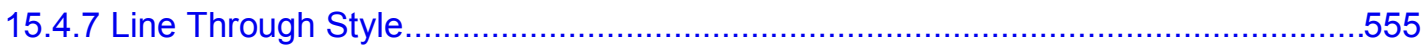

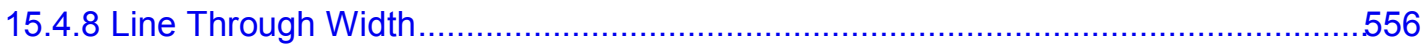

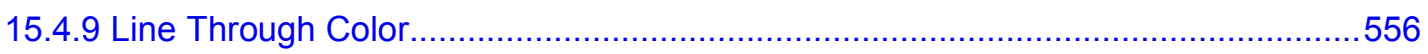

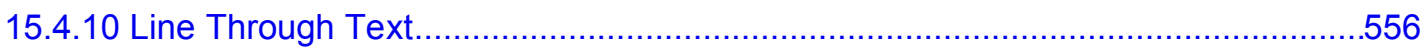

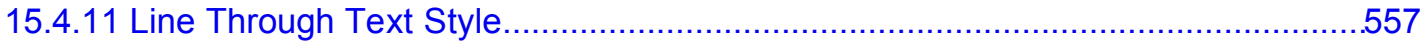

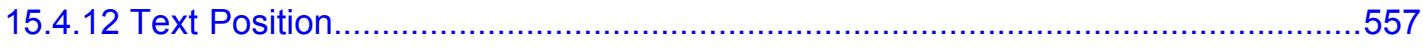

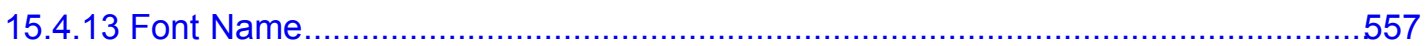

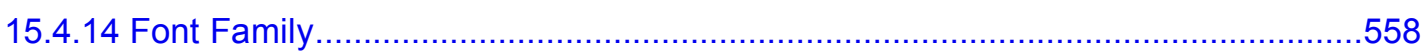

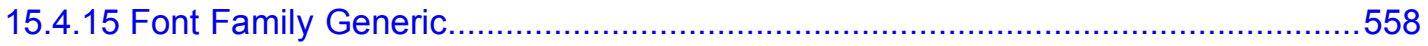

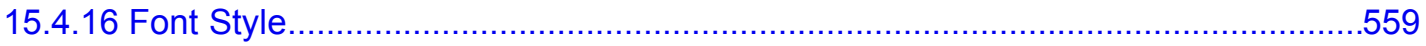

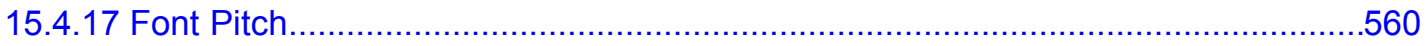

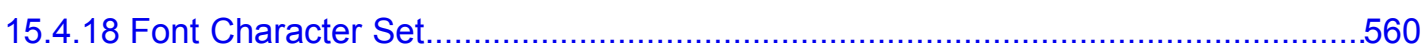

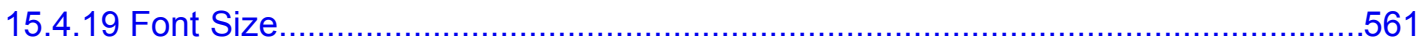

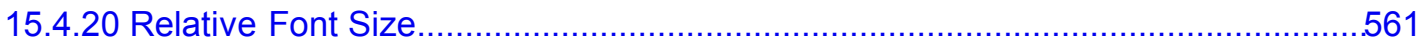

15.4.21 Script Type

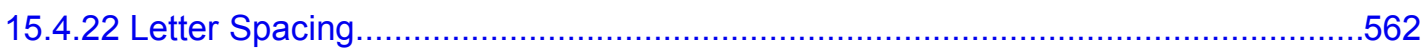

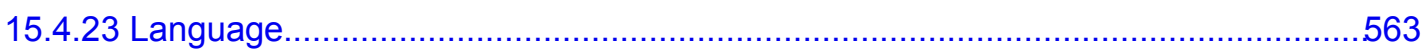

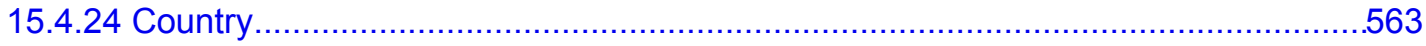

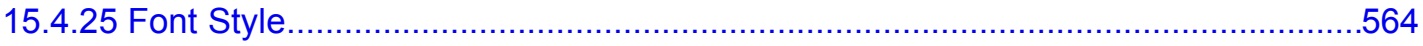

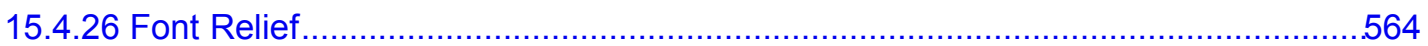

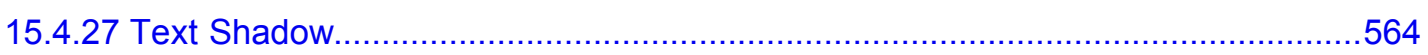

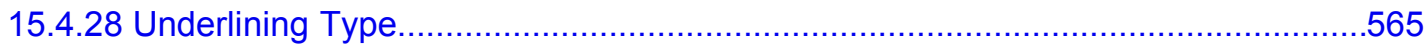

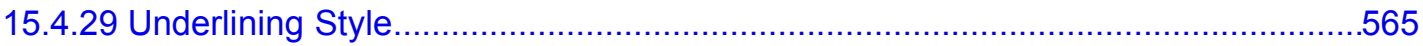

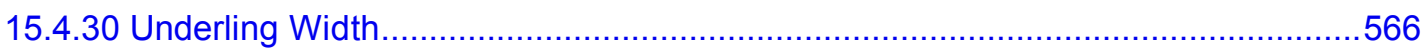

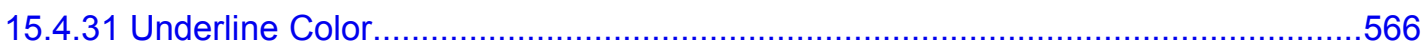

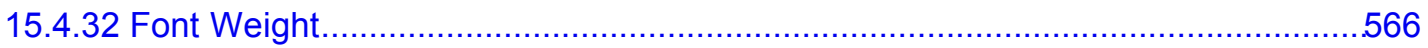

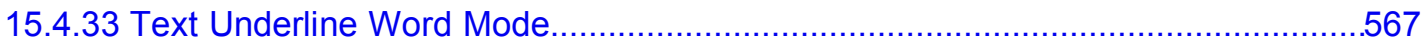

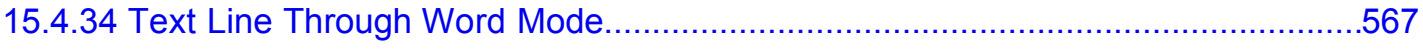

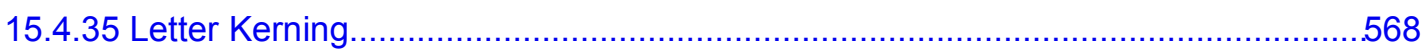

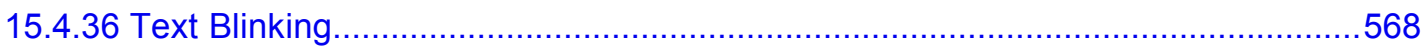




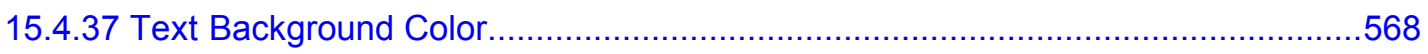

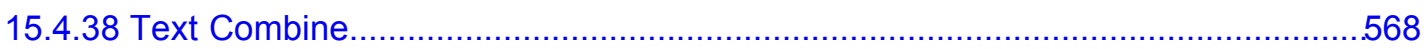

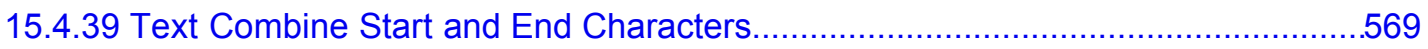

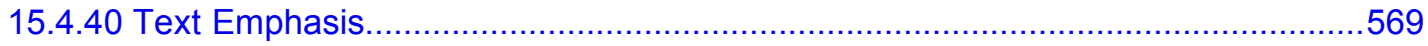

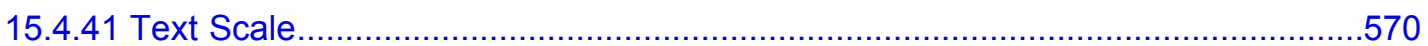

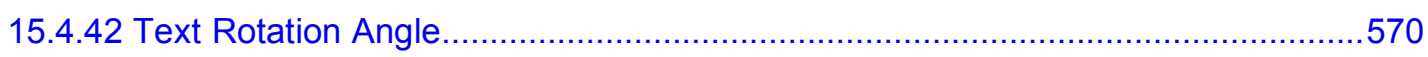

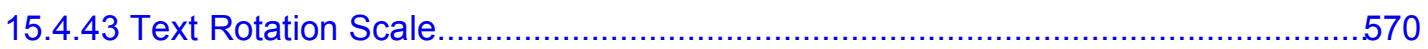

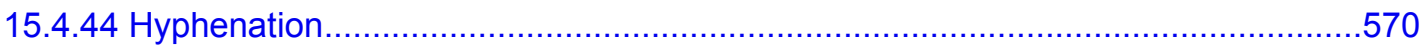

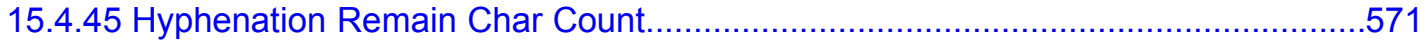

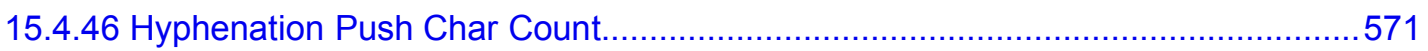

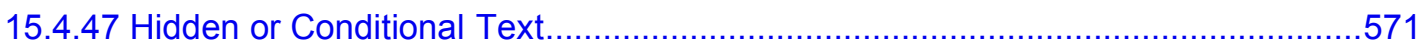

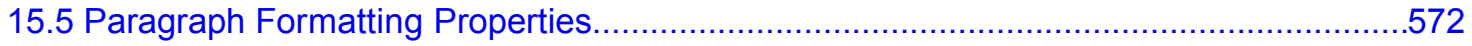

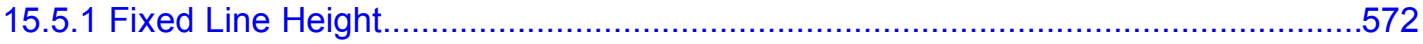

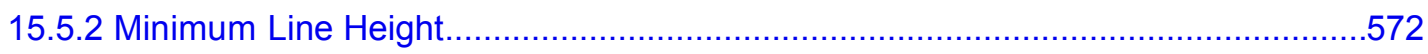

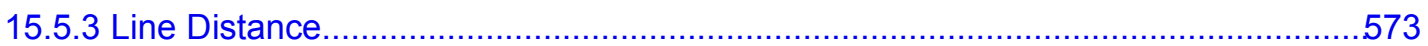

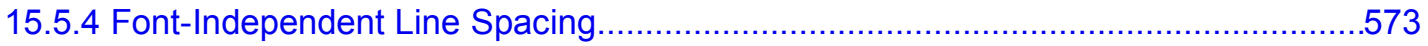

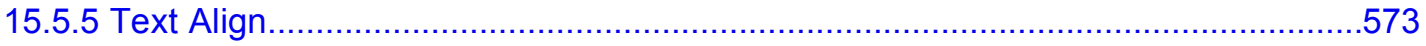

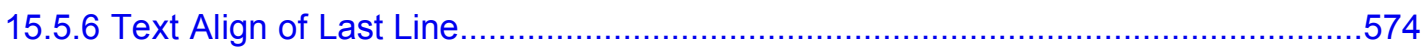

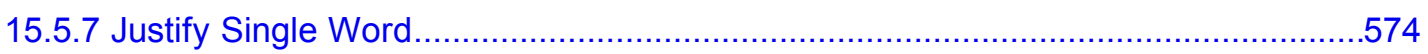

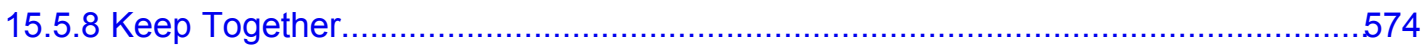

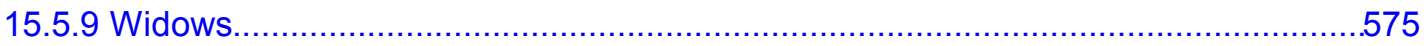

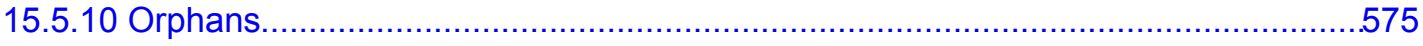

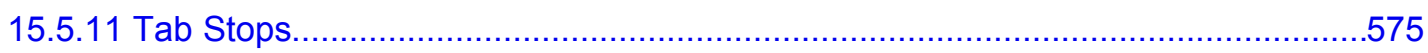

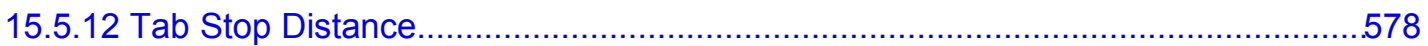

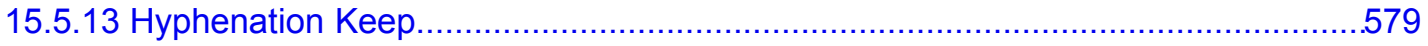

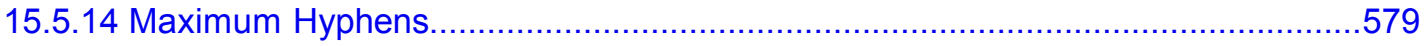

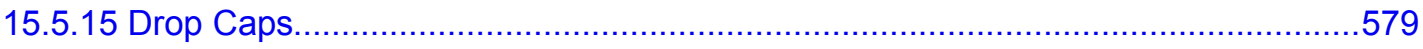

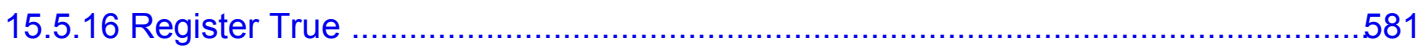

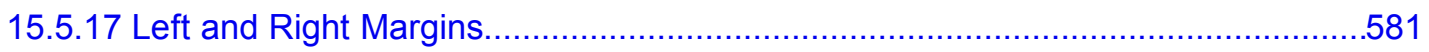

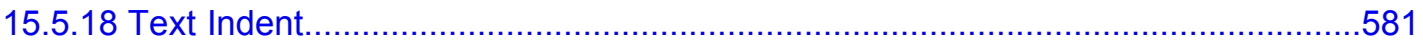

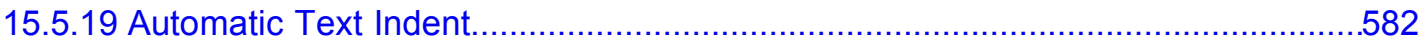

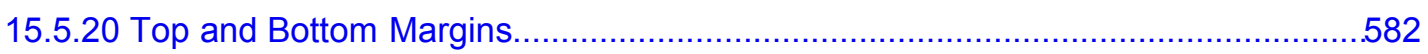

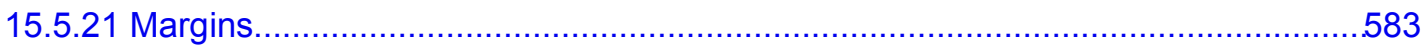

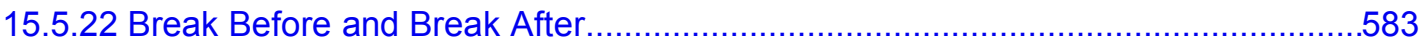

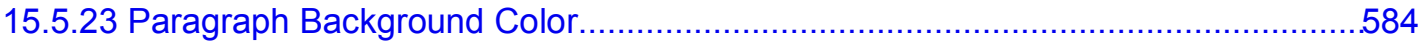

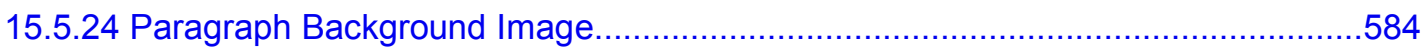

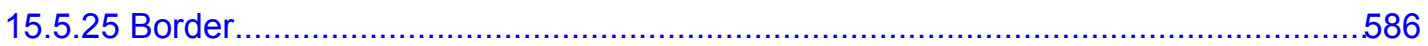




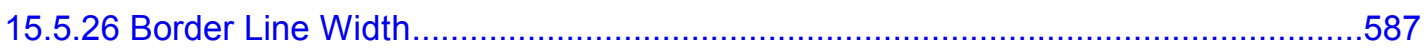

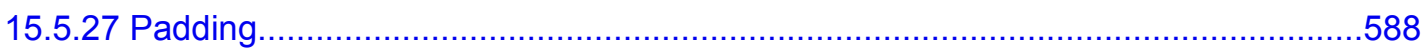

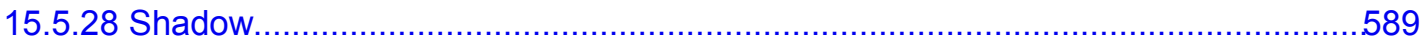

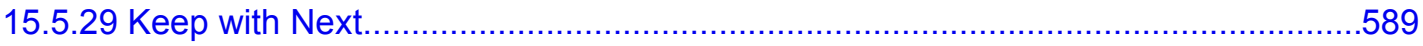

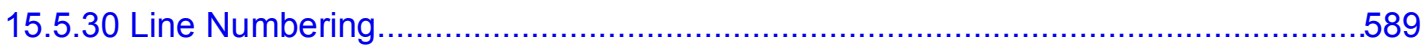

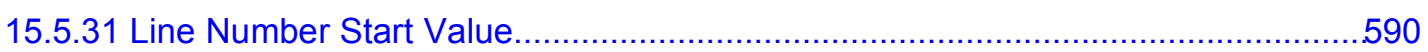

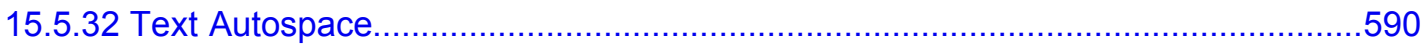

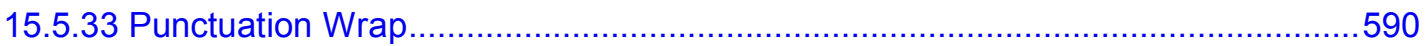

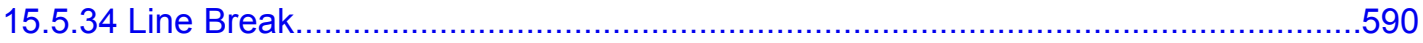

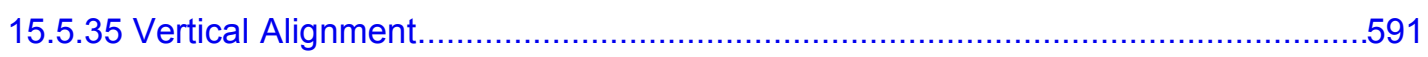

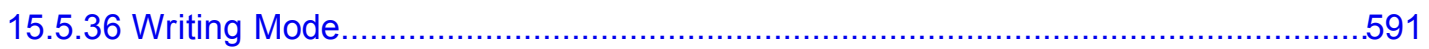

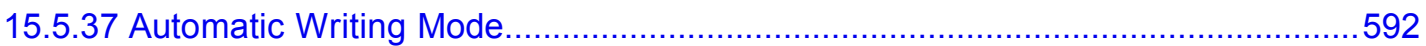

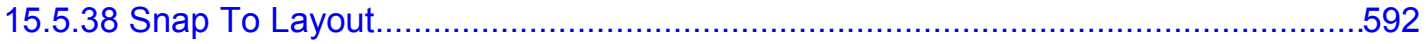

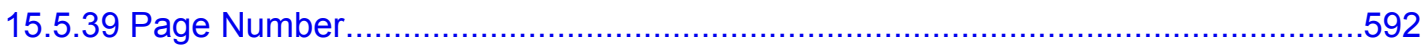

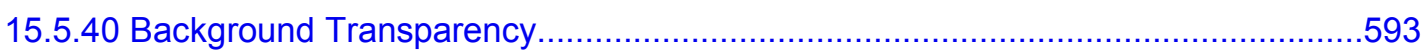

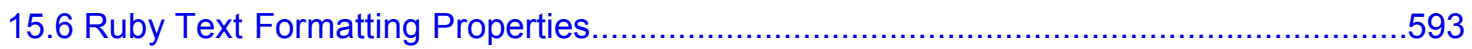

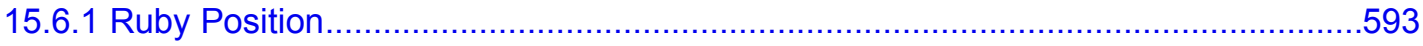

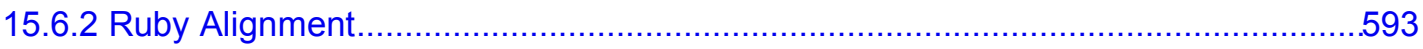

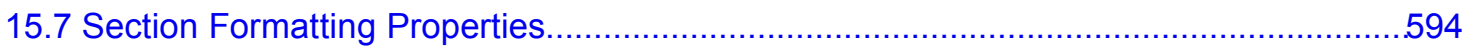

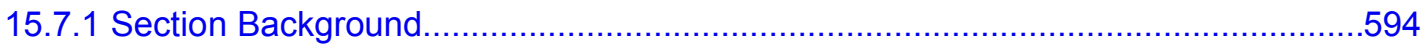

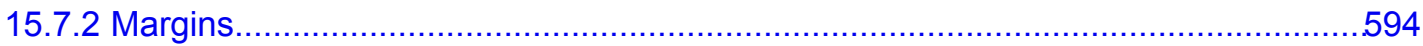

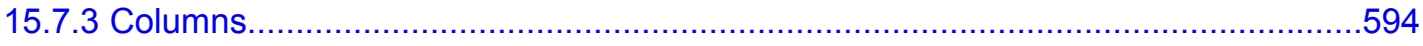

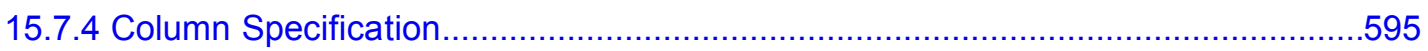

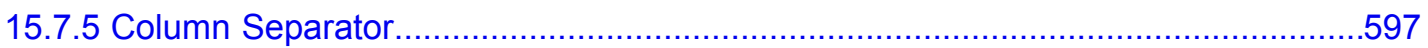

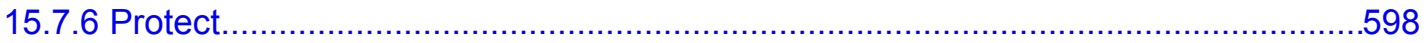

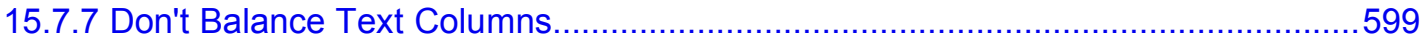

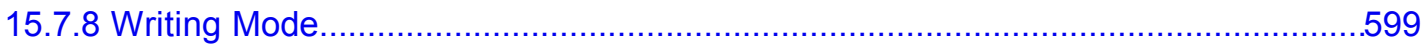

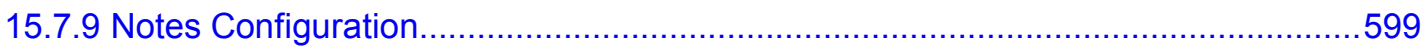

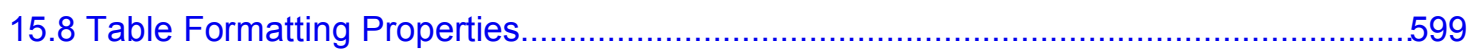

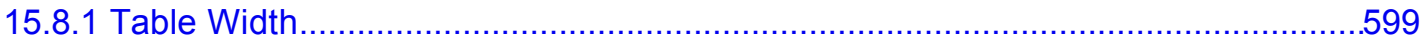

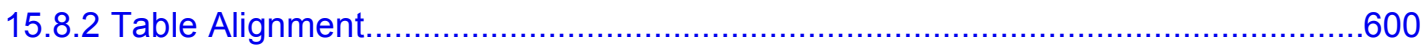

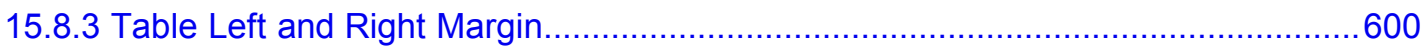

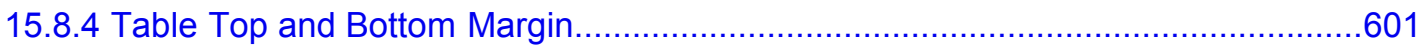

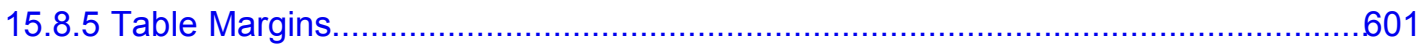

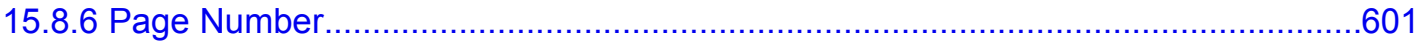

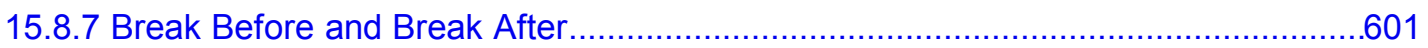

15.8.8 Table Background and Background Image .....................................................601 


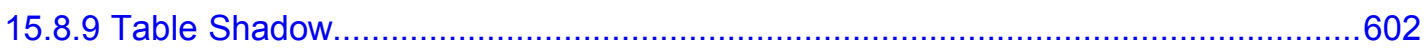

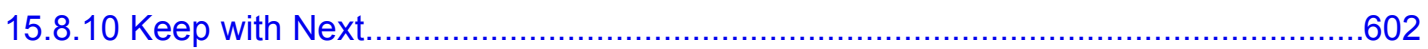

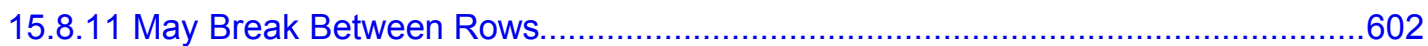

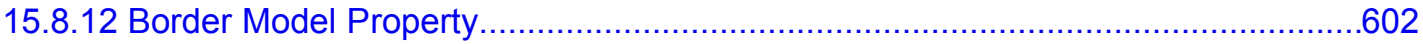

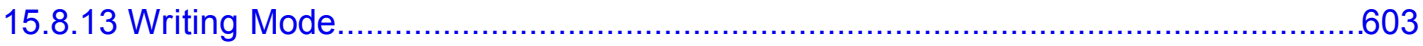

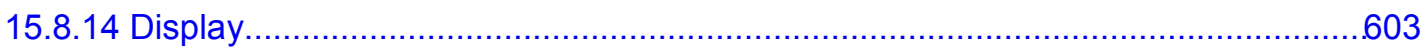

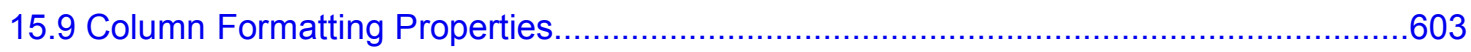

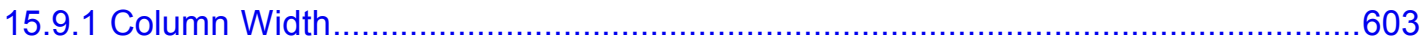

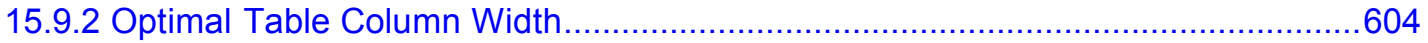

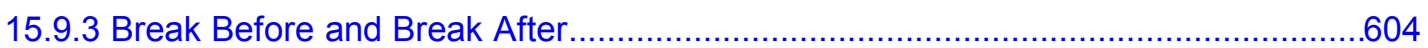

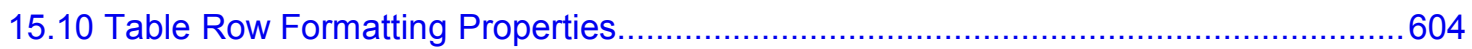

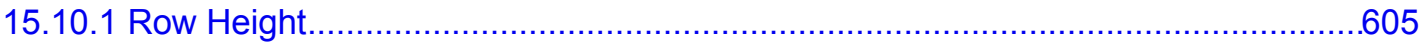

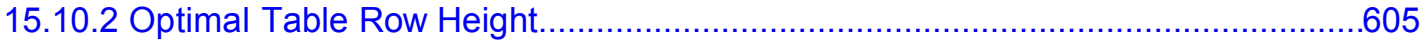

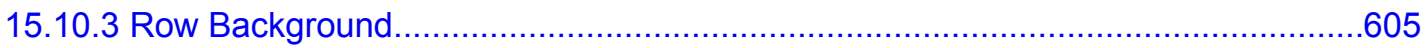

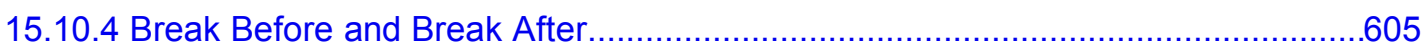

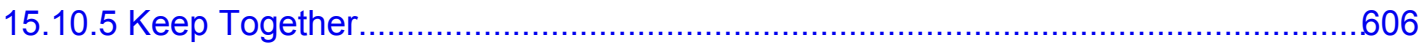

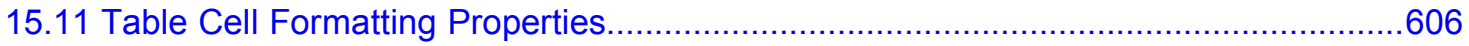

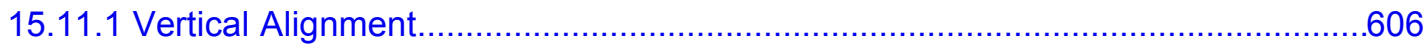

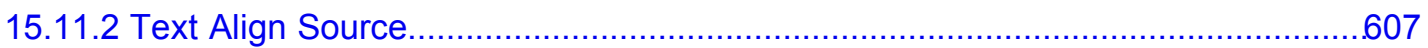

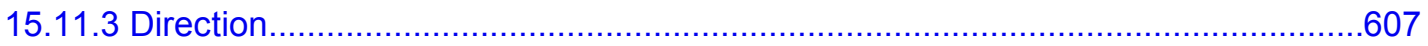

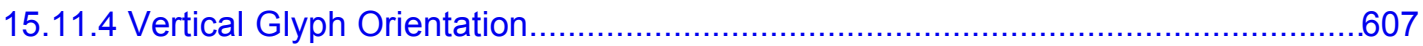

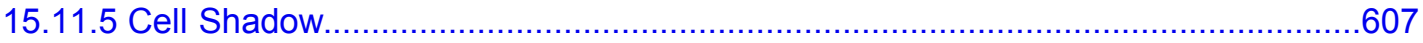

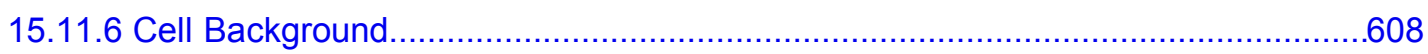

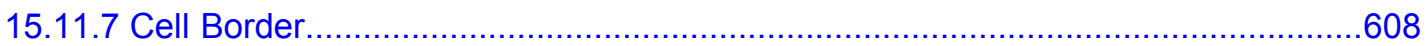

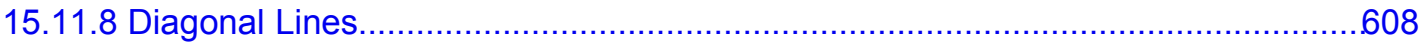

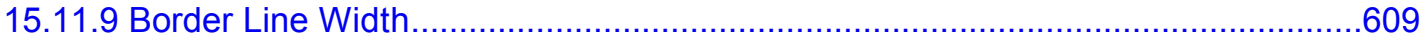

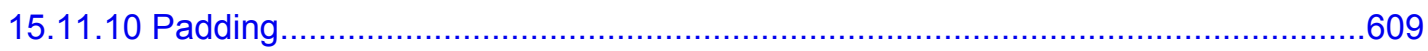

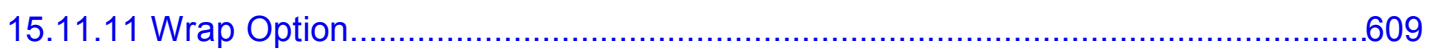

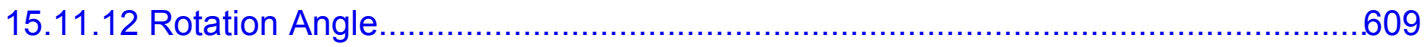

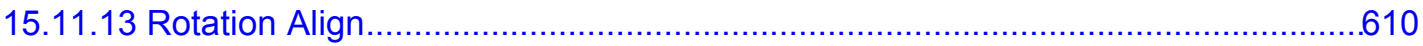

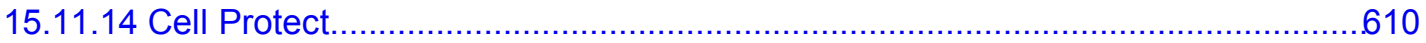

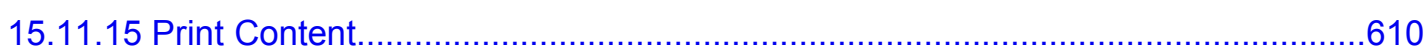

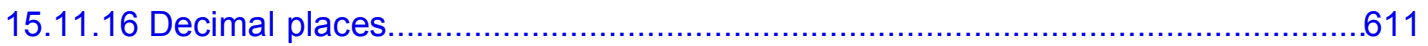

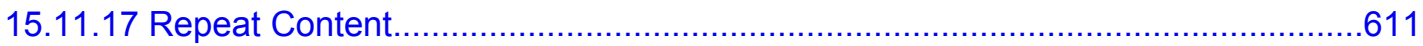

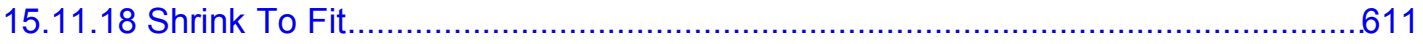

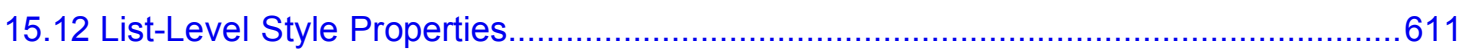

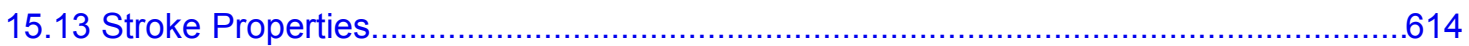




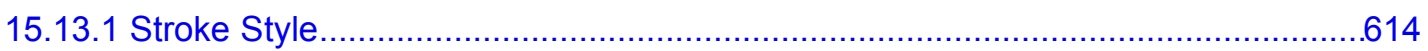

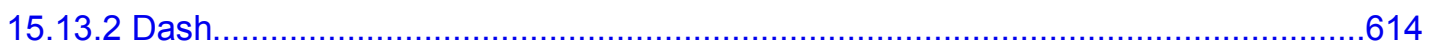

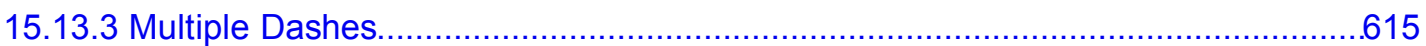

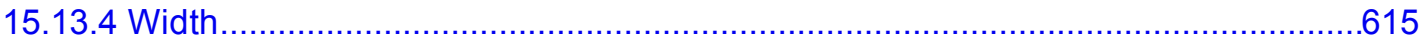

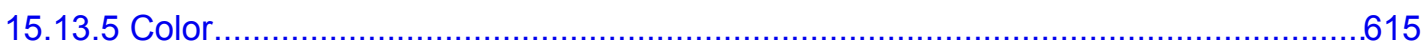

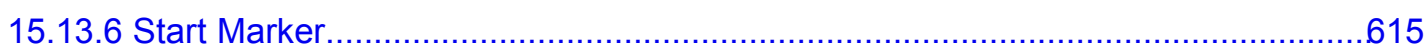

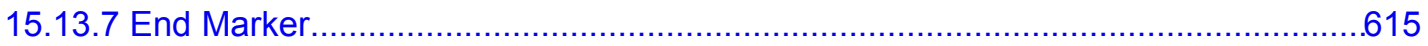

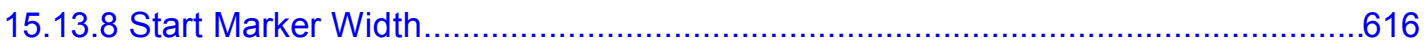

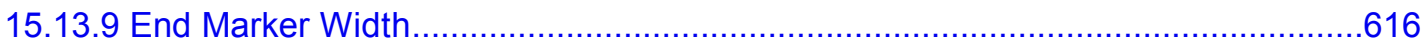

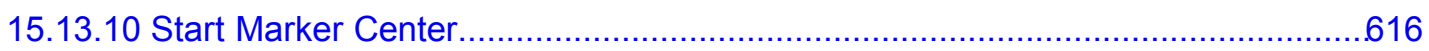

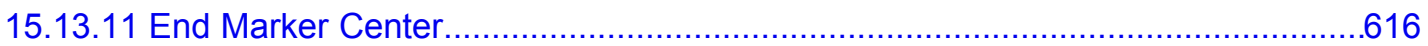

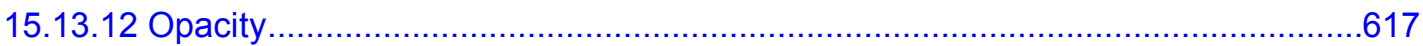

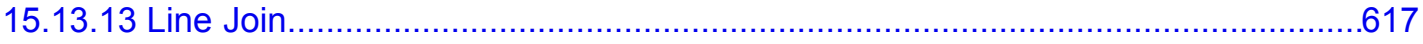

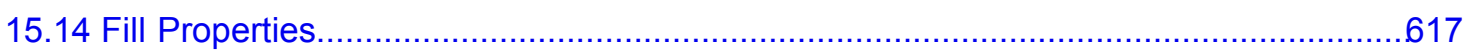

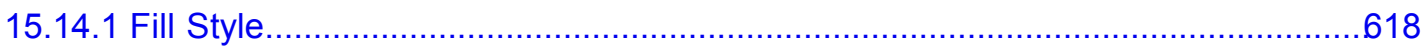

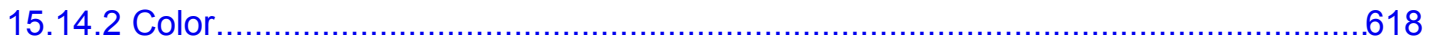

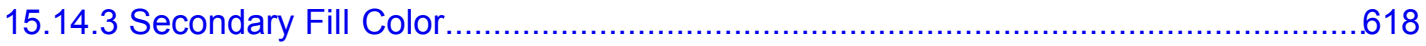

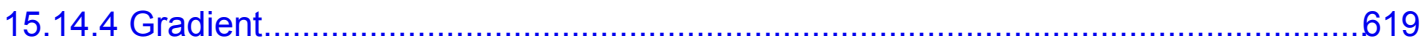

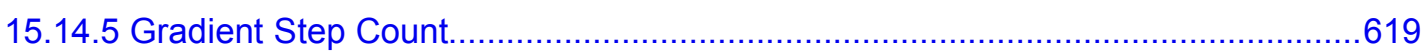

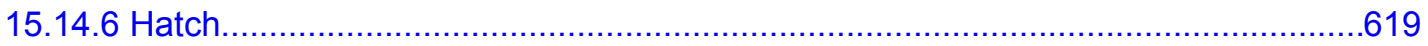

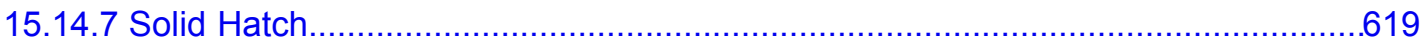

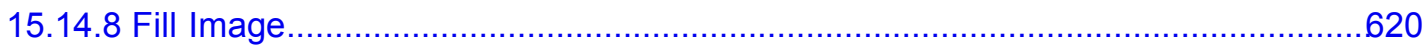

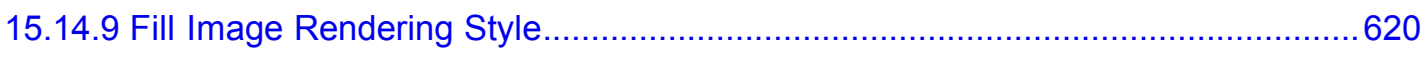

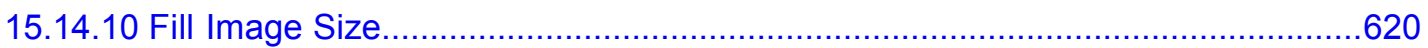

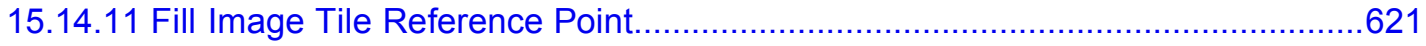

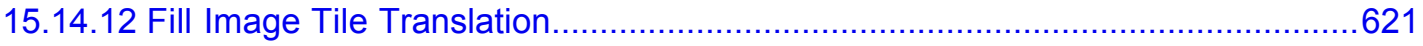

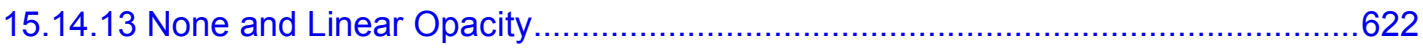

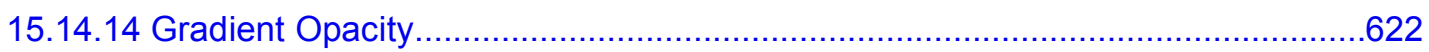

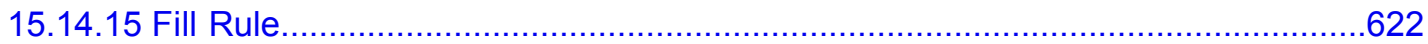

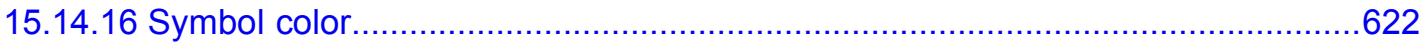

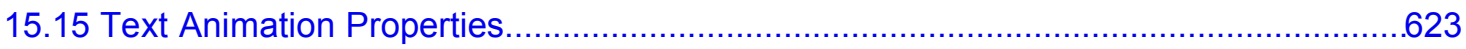

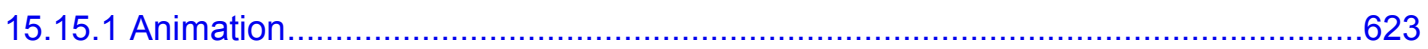

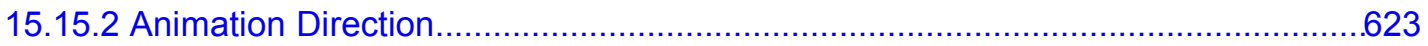

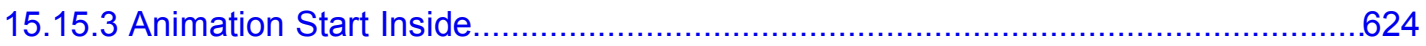

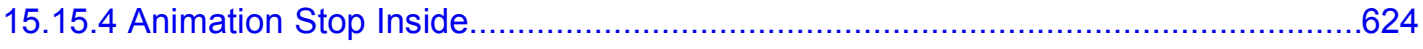

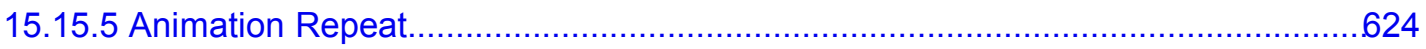

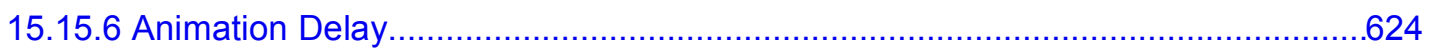




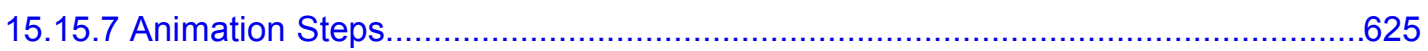

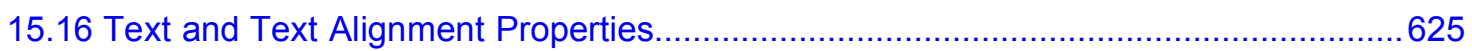

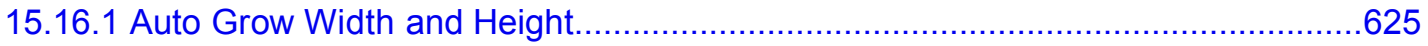

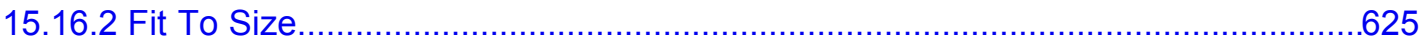

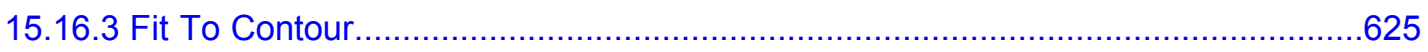

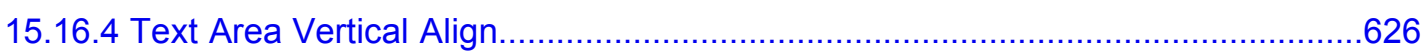

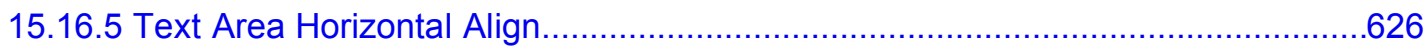

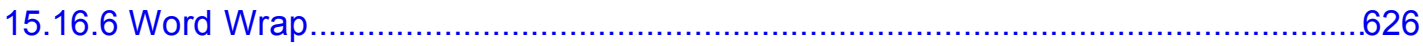

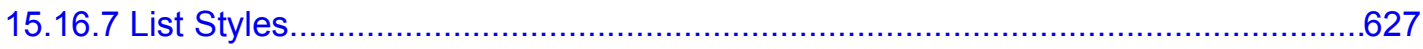

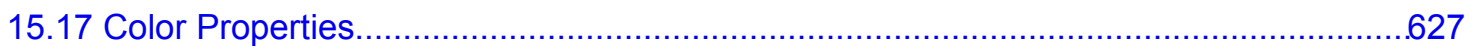

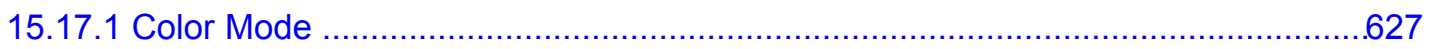

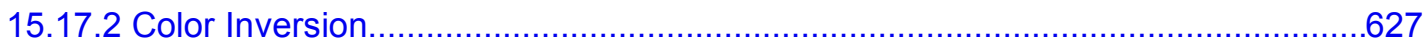

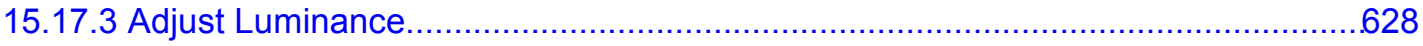

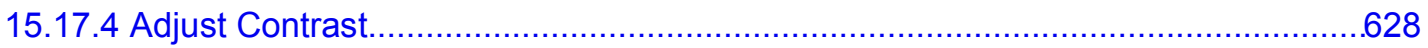

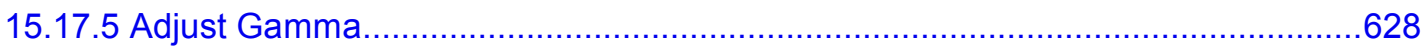

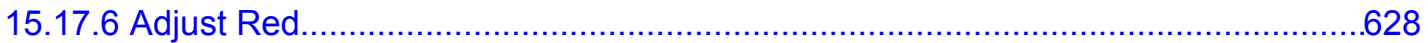

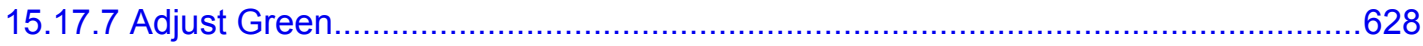

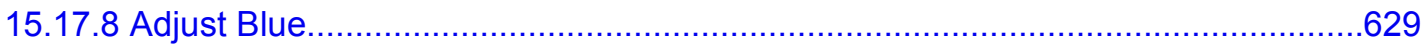

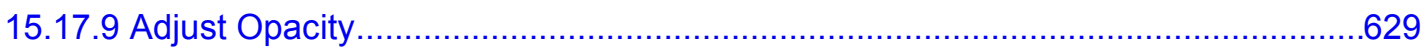

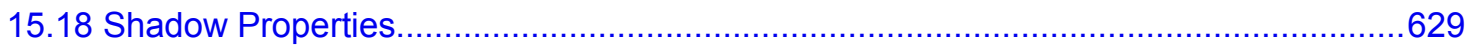

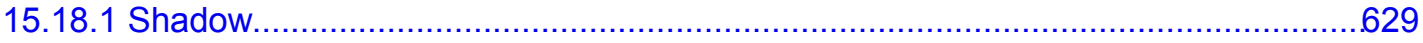

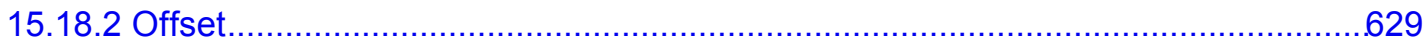

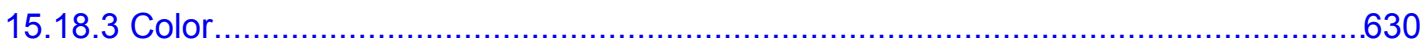

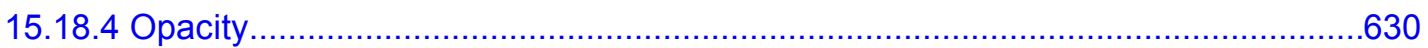

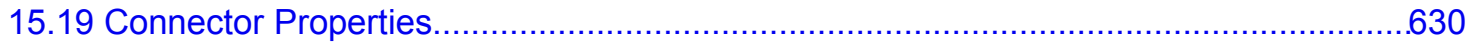

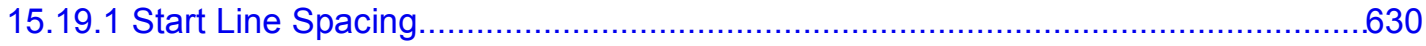

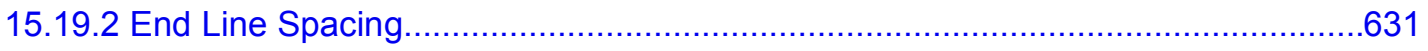

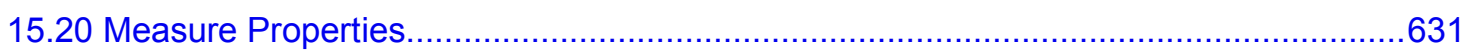

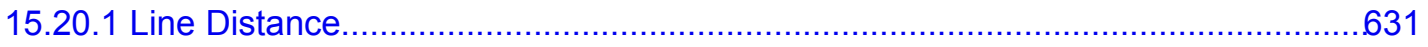

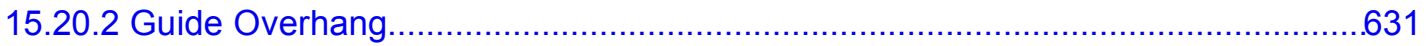

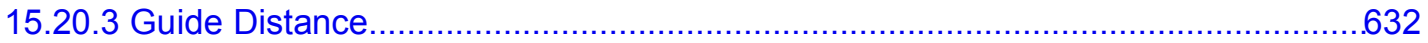

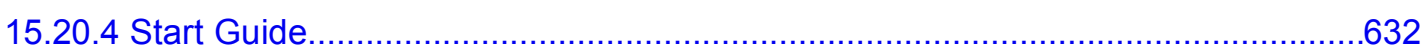

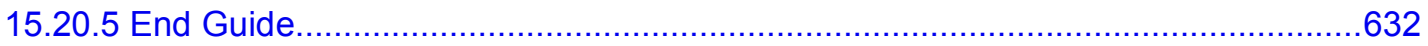

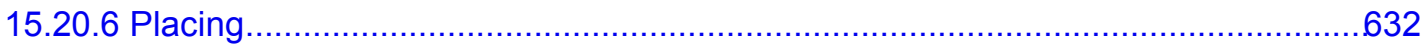

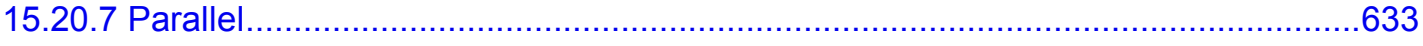

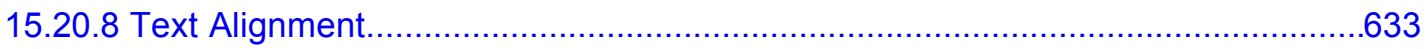

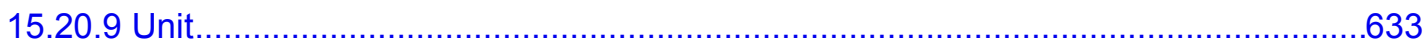




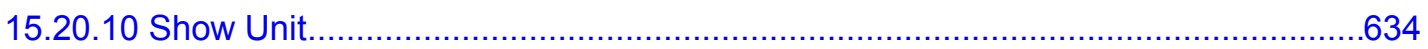

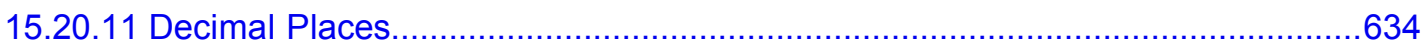

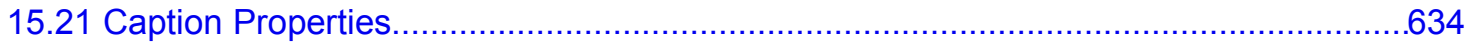

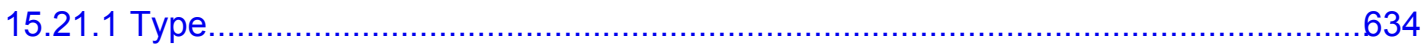

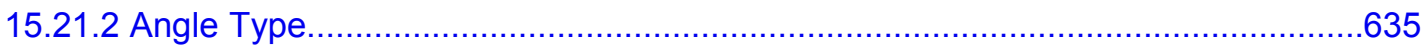

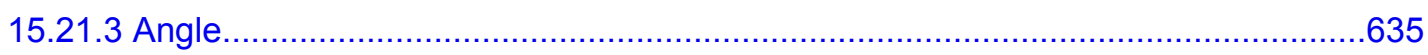

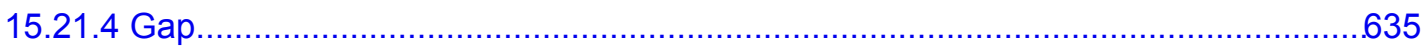

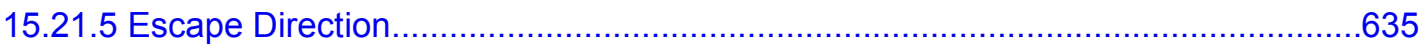

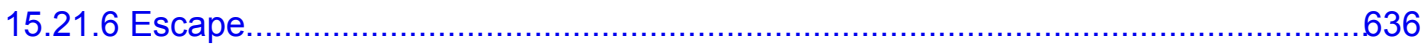

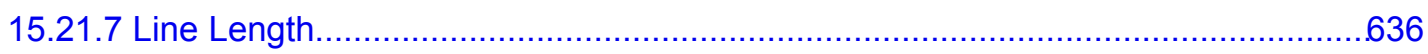

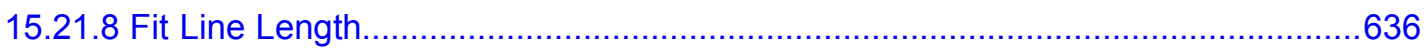

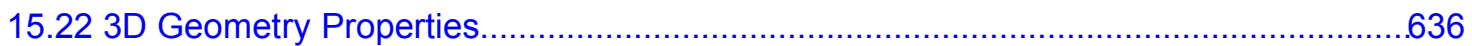

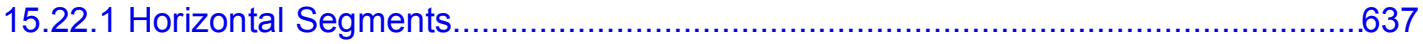

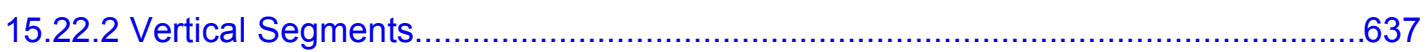

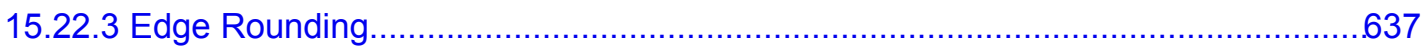

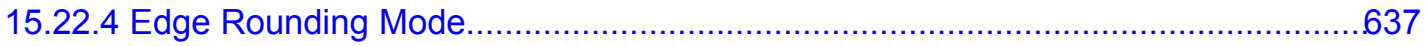

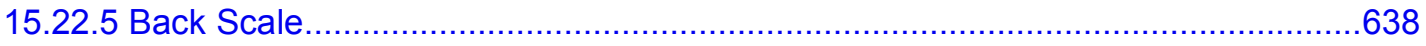

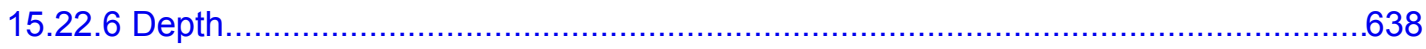

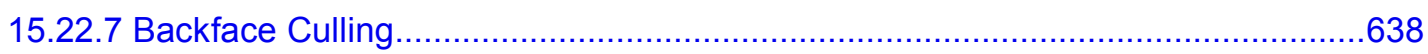

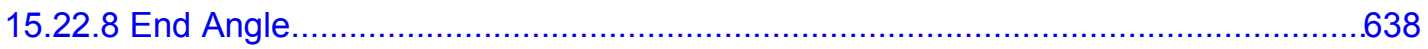

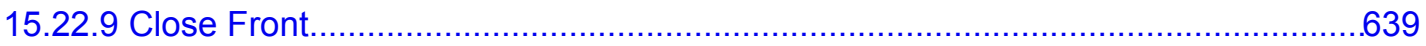

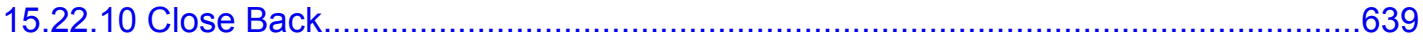

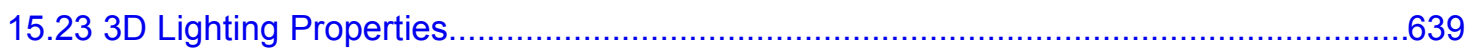

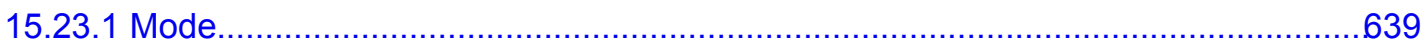

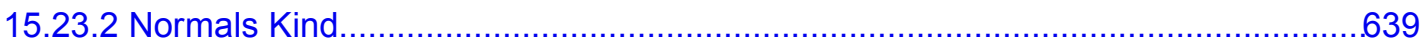

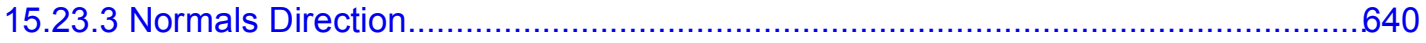

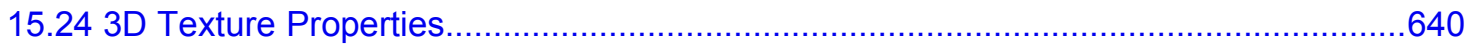

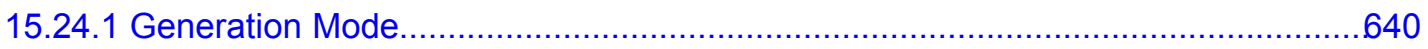

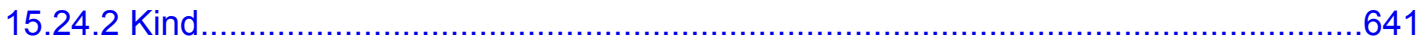

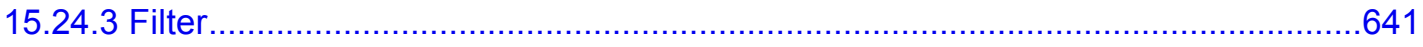

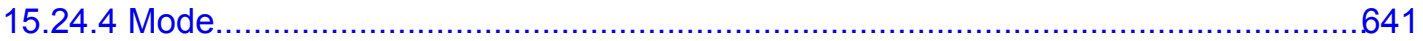

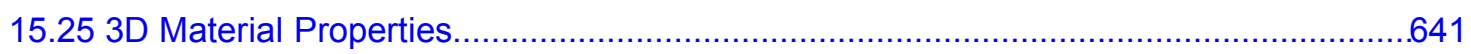

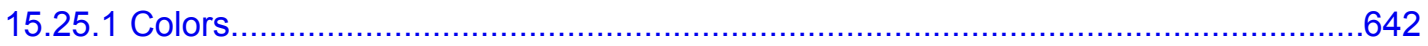

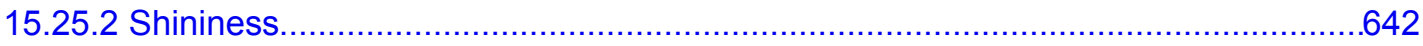

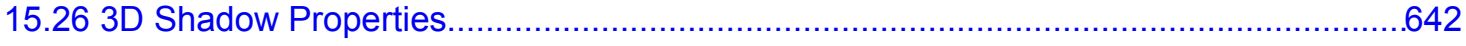

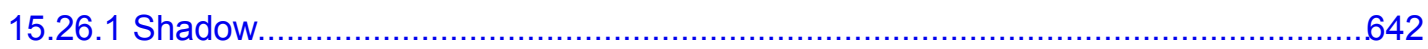

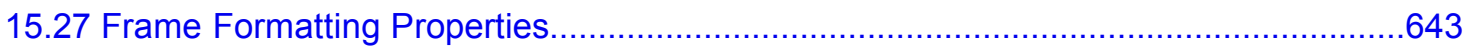




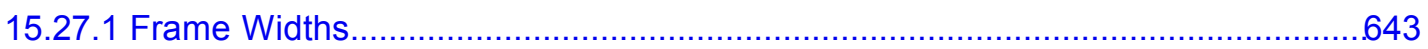

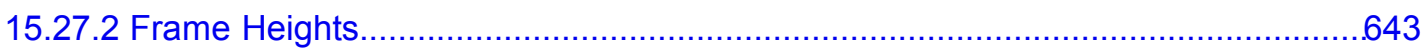

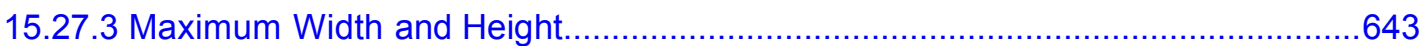

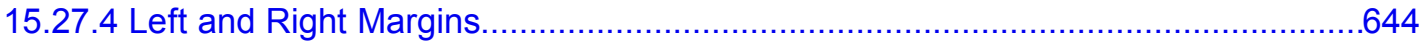

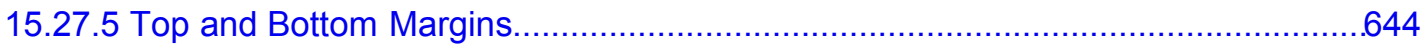

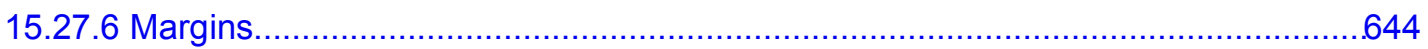

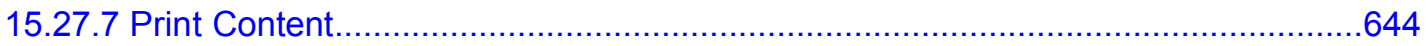

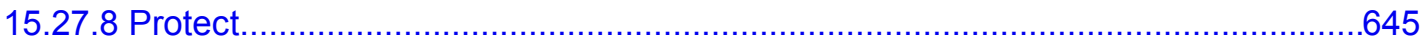

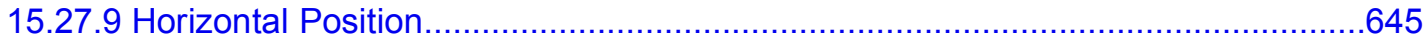

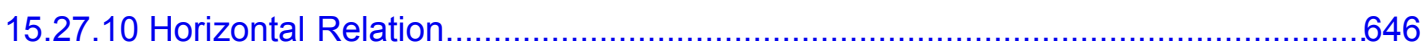

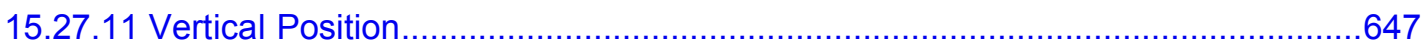

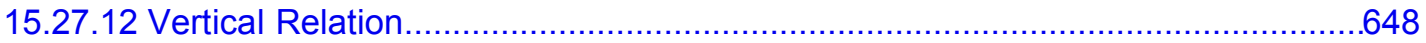

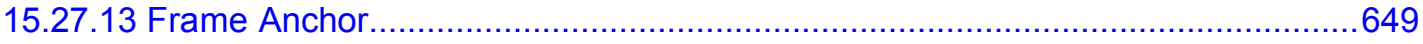

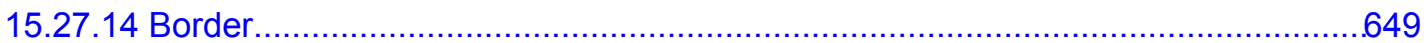

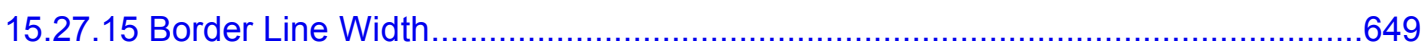

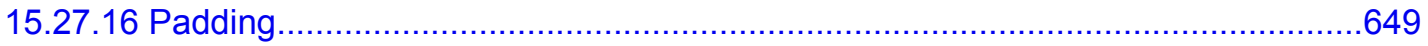

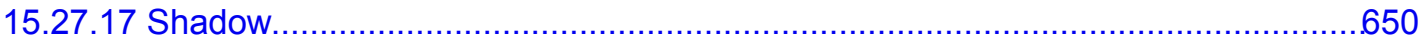

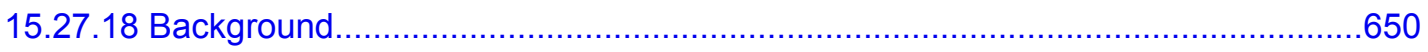

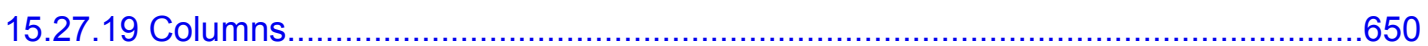

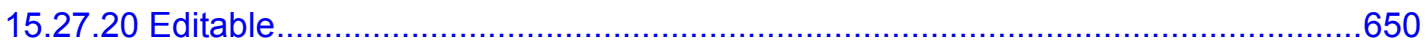

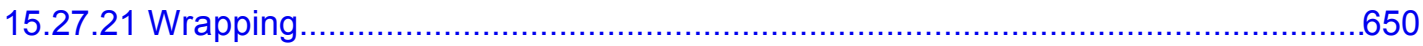

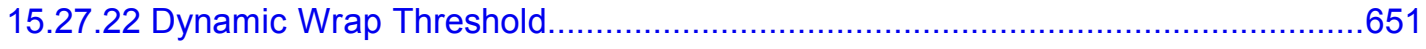

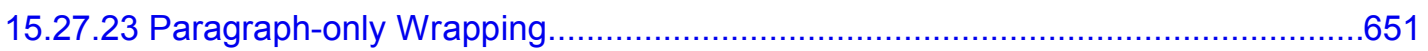

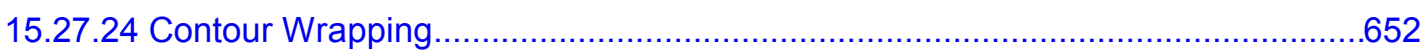

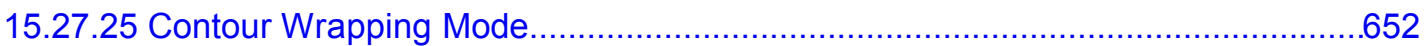

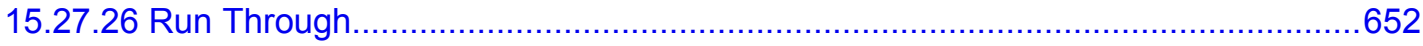

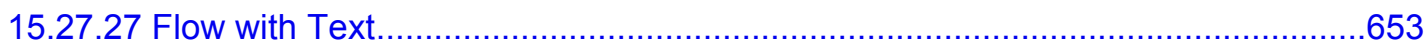

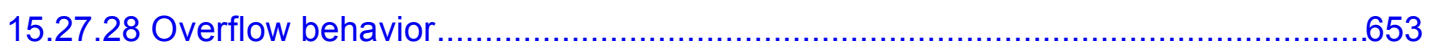

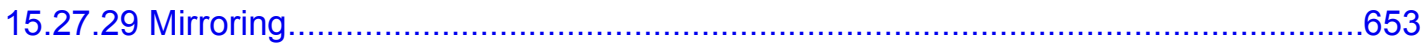

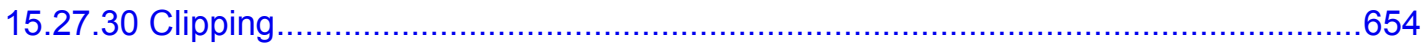

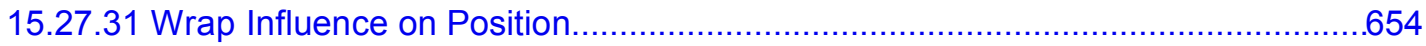

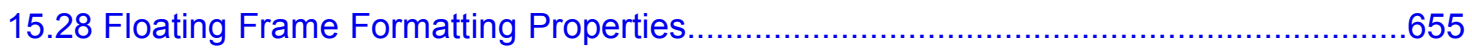

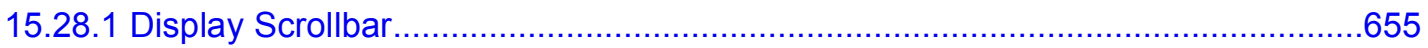

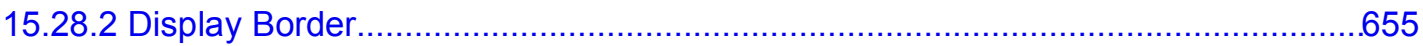

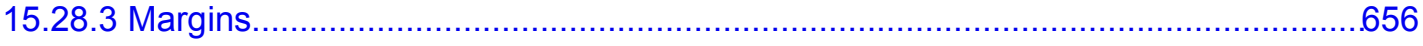

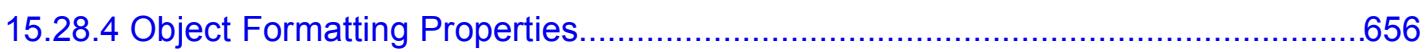

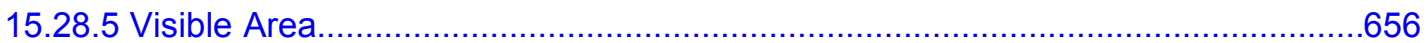




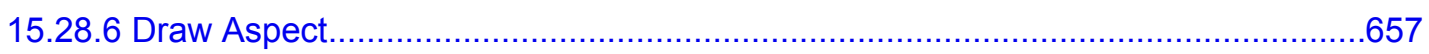

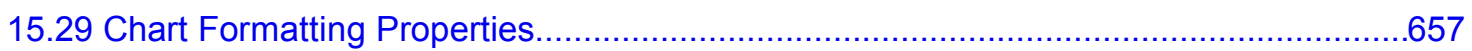

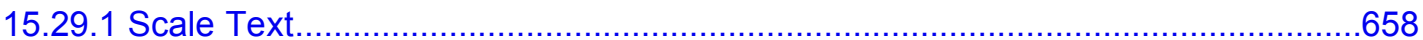

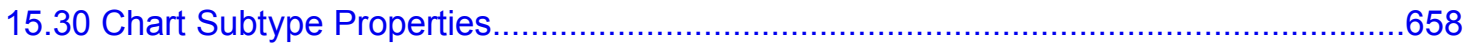

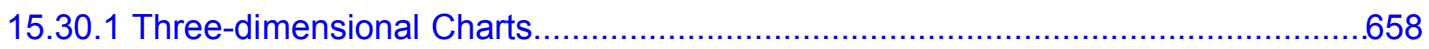

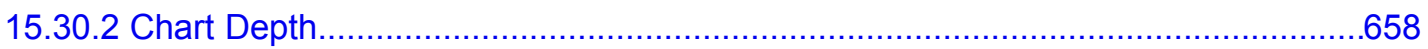

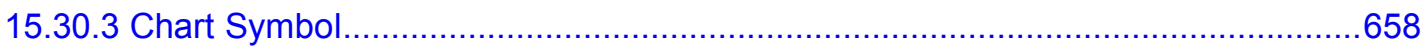

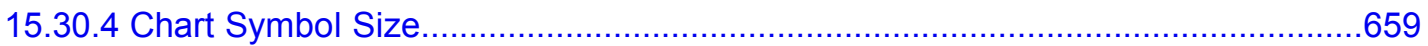

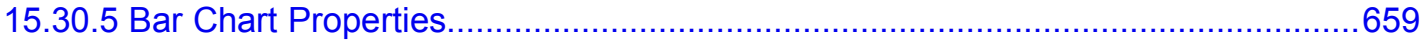

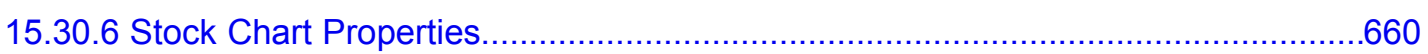

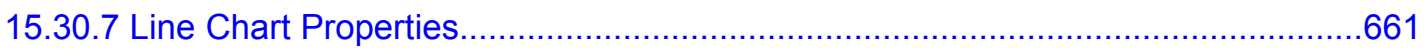

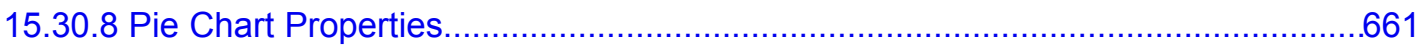

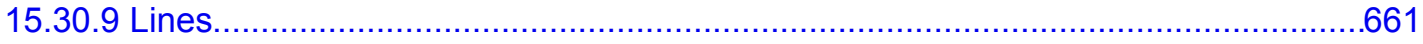

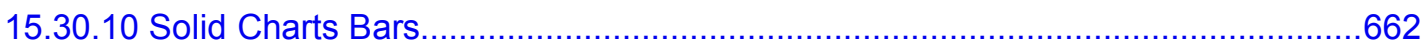

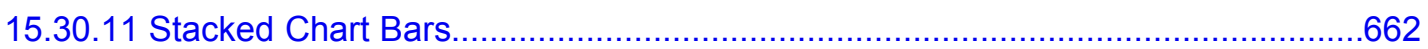

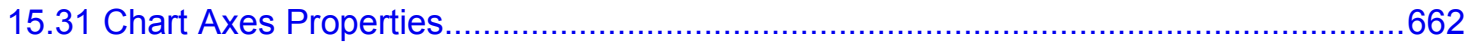

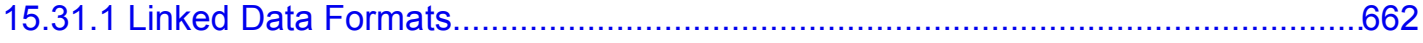

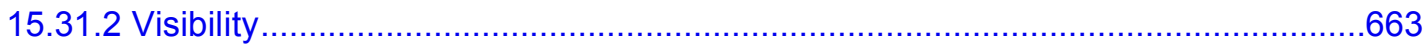

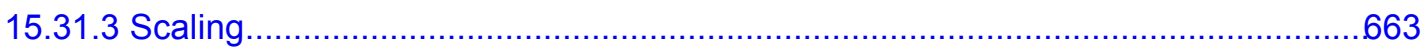

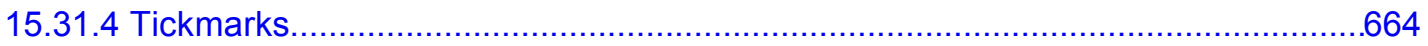

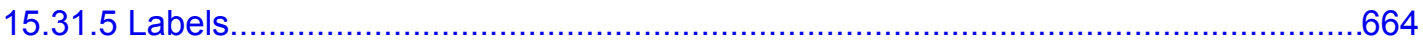

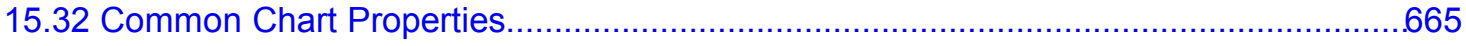

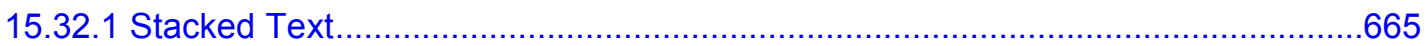

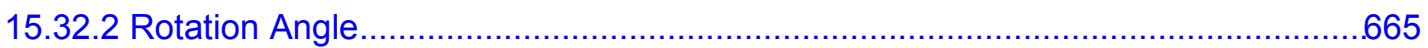

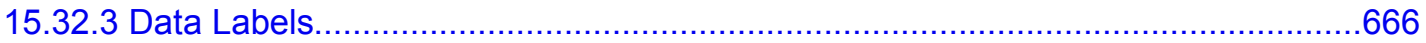

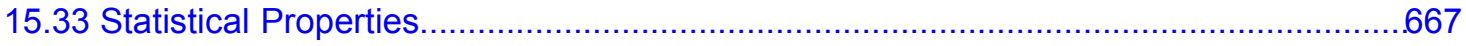

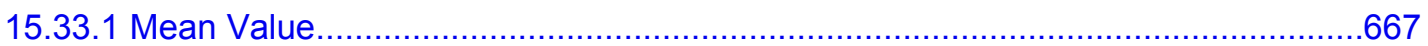

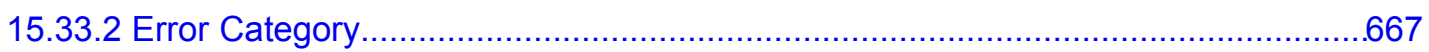

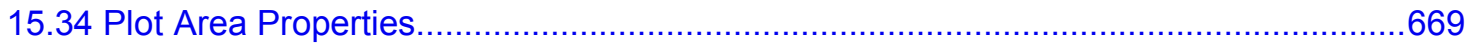

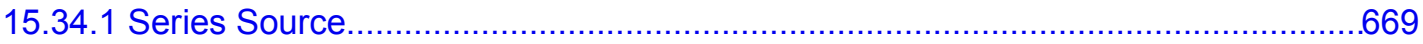

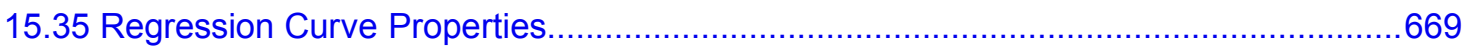

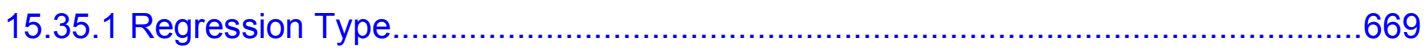

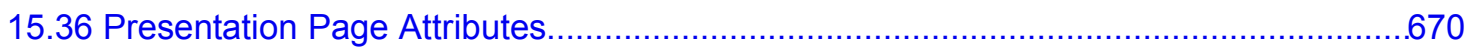

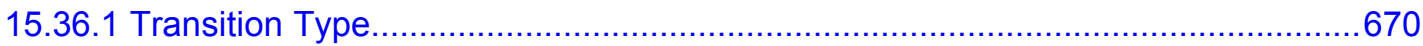

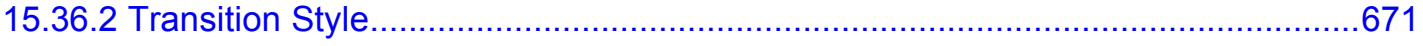

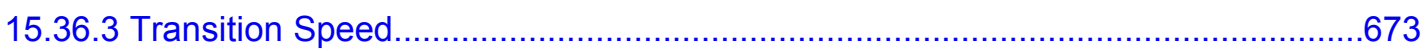

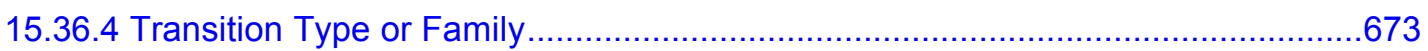




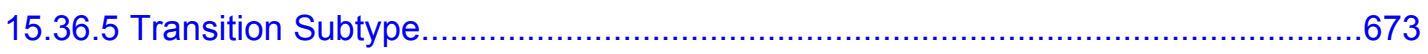

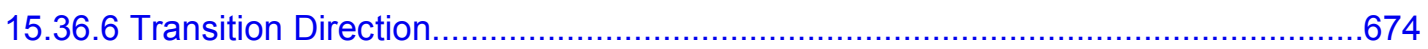

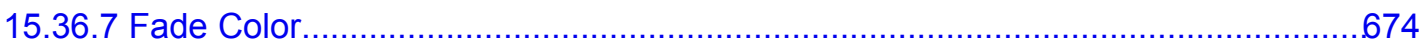

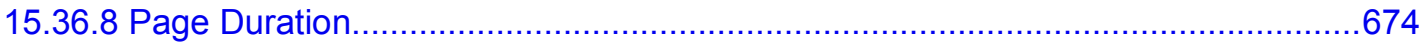

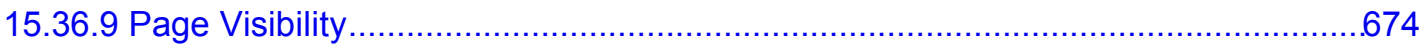

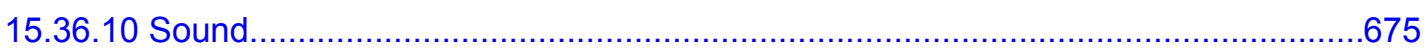

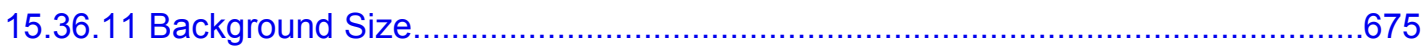

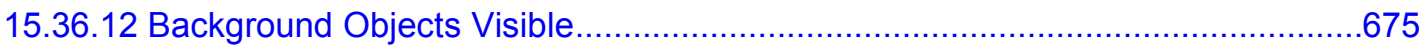

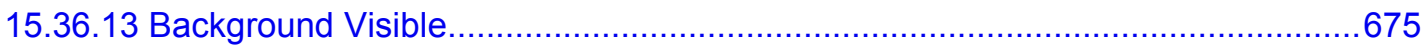

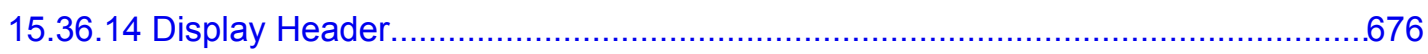

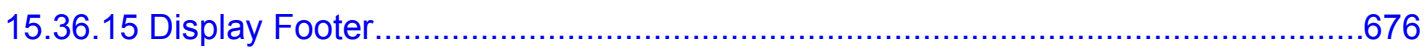

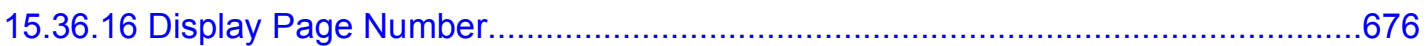

15.36.17 Display Date And Time

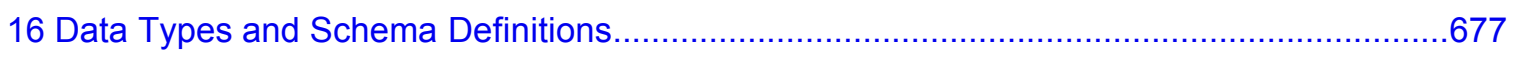

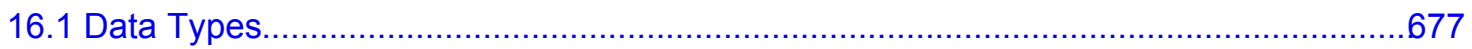

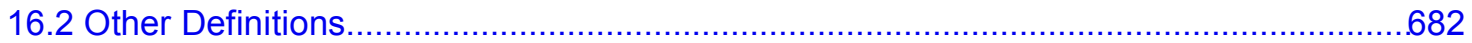

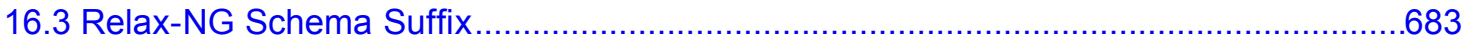

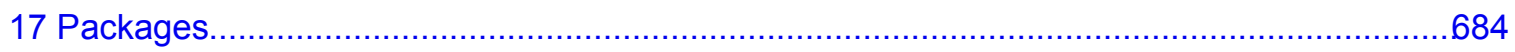

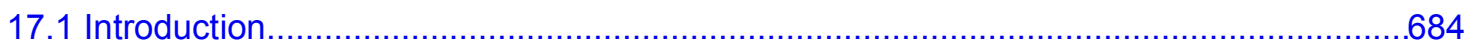

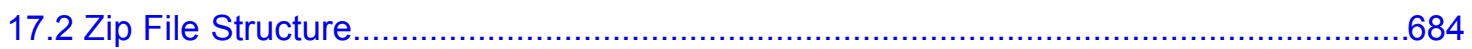

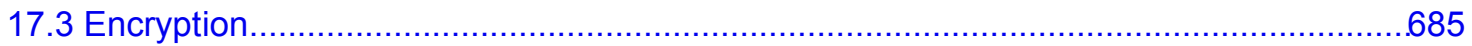

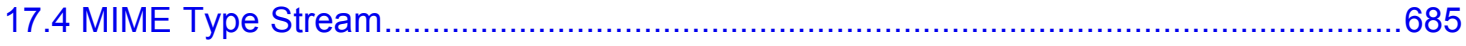

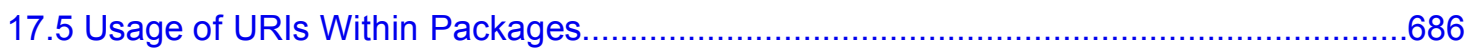

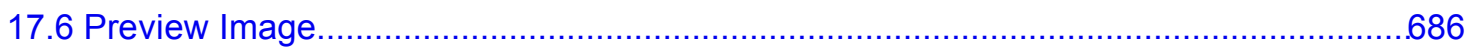

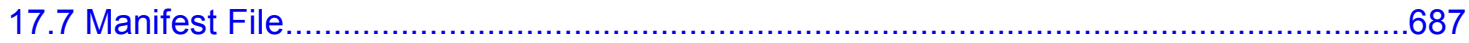

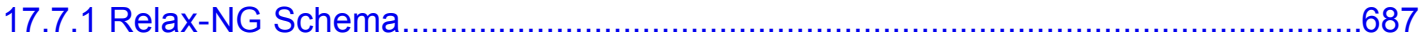

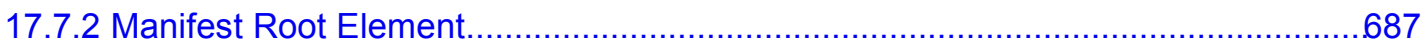

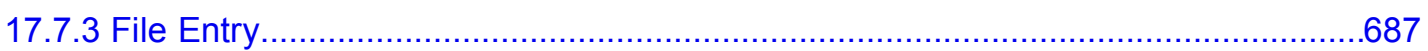

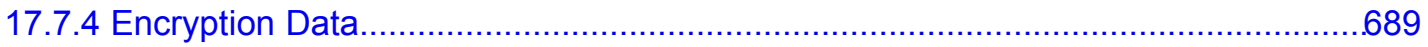

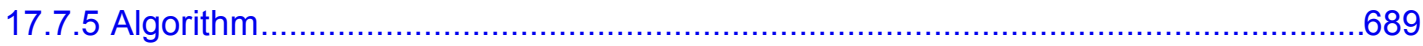

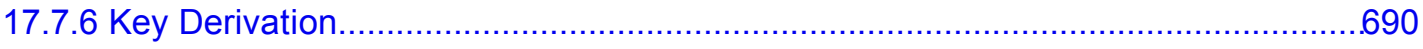

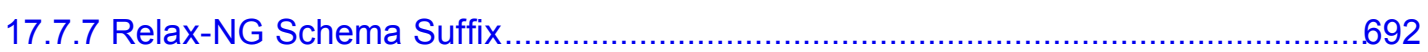

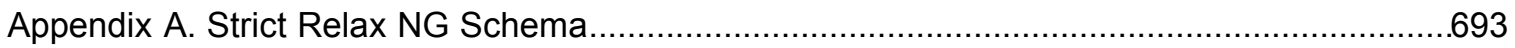

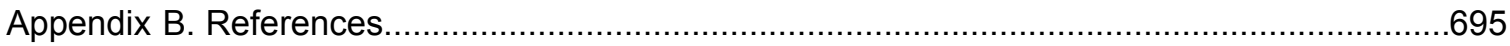

Appendix C. MIME Types and File Name Extensions (Non Normative) ..................................697

Appendix D. Core Features Sets (Non Normative)............................................................699

Appendix E. Changes From Previous Specification Versions (Non Normative)......................... 704 
E.1. Changes from "Open Office Specification 1.0 Committee Draft 1"

E.2. Changes from "Open Document Format for Office Applications (OpenDocument) 1.0

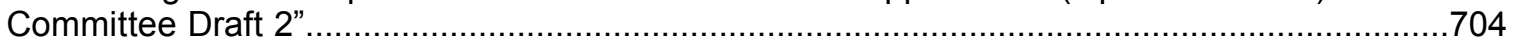

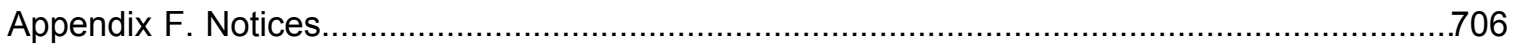




\section{Introduction}

\subsection{Introduction}

This document defines an XML schema for office applications and its semantics. The schema is suitable for office documents, including text documents, spreadsheets, charts and graphical documents like drawings or presentations, but is not restricted to these kinds of documents.

The schema provides for high-level information suitable for editing documents. It defines suitable XML structures for office documents and is friendly to transformations using XSLT or similar XML-based tools.

Chapter 1 contains an introduction to the OpenDocument format. The structure of documents that conform to the OpenDocument specification is explained in chapter 2. Chapter 3 described the meta information that can be contained in such documents. Chapters 4 and 5 describe their text and paragraph content. Text Fields are described in chapter 6, text indices in chapter 7.

Chapter 8 describes the table content of an document in OpenDocument format, chapter 9 its graphical content, chapter 10 its chart content, and chapter 11 its form content. Content that is common to all documents is described in chapter 12. The integration of SMIL animation markup into the OpenDocument schema is described in chapter 13. Chapter 14 explains style information content, chapter 15 specifies formatting properties that are can be used within styles. The data types used by the OpenDocument schema are described in chapter 16.

The OpenDocument format makes use of a package concept. These packages are described in chapter 17.

\subsection{Notation}

Within this specification, the key words "MUST", "MUST NOT", "REQUIRED", "SHALL", "SHALL NOT", "SHOULD", "SHOULD NOT", "RECOMMENDED", "MAY", and "OPTIONAL" are to be interpreted as described in [RFC2119] if they appear in uppercase bold letters.

\subsection{Namespaces}

Table 1 lists the namespaces that are defined by the OpenDocument format and their default prefixes. For more information about XML namespaces, please refer to the Namespaces in XML specification [xml-names].

Table 1: XML Namespaces defined by the OpenDocument schema

\begin{tabular}{|l|l|l|}
\hline \multicolumn{1}{|c|}{ Prefix } & \multicolumn{1}{|c|}{ Description } & \multicolumn{1}{c|}{ Namespace } \\
\hline office & $\begin{array}{l}\text { For all common pieces of information } \\
\text { that are not contained in another, } \\
\text { more specific namespace. }\end{array}$ & $\begin{array}{l}\text { urn:oasis:names:tc:opendocument:xmlns: } \\
\text { office:1.0 }\end{array}$ \\
\hline meta & $\begin{array}{l}\text { For elements and attributes that } \\
\text { describe meta information. }\end{array}$ & $\begin{array}{l}\text { urn:oasis:names:tc:opendocument:xmlns: } \\
\text { meta:1.0 }\end{array}$ \\
\hline config & $\begin{array}{l}\text { For elements and attributes that } \\
\text { describe application specific settings. }\end{array}$ & $\begin{array}{l}\text { urn:oasis:names:tc:opendocument:xmlns: } \\
\text { config:1.0 }\end{array}$ \\
\hline
\end{tabular}




\begin{tabular}{|c|c|c|}
\hline Prefix & Description & Namespace \\
\hline text & $\begin{array}{l}\text { For elements and attributes that may } \\
\text { occur within text documents and text } \\
\text { parts of other document types, such } \\
\text { as the contents of a spreadsheet cell. }\end{array}$ & $\begin{array}{l}\text { urn:oasis:names:tc:opendocument:xmlns: } \\
\text { text:1.0 }\end{array}$ \\
\hline table & $\begin{array}{l}\text { For elements and attributes that may } \\
\text { occur within spreadsheets or within } \\
\text { table definitions of a text document. }\end{array}$ & $\begin{array}{l}\text { urn:oasis:names:tc:opendocument:xmlns: } \\
\text { table:1.0 }\end{array}$ \\
\hline drawing & $\begin{array}{l}\text { For elements and attributes that } \\
\text { describe graphic content. }\end{array}$ & $\begin{array}{l}\text { urn:oasis:names:tc:opendocument:xmlns: } \\
\text { drawing:1.0 }\end{array}$ \\
\hline presentation & $\begin{array}{l}\text { For elements and attributes that } \\
\text { describe presentation content. }\end{array}$ & $\begin{array}{l}\text { urn:oasis:names:tc:opendocument:xmlns: } \\
\text { presentation:1.0 }\end{array}$ \\
\hline$d r 3 d$ & $\begin{array}{l}\text { For elements and attributes that } \\
\text { describe 3D graphic content. }\end{array}$ & $\begin{array}{l}\text { urn:oasis:names:tc:opendocument:xmlns: } \\
\text { dr3d:1.0 }\end{array}$ \\
\hline anim & $\begin{array}{l}\text { For elements and attributes that } \\
\text { describe animation content. }\end{array}$ & $\begin{array}{l}\text { urn:oasis:names:tc:opendocument:xmlns: } \\
\text { animation:1.0 }\end{array}$ \\
\hline chart & $\begin{array}{l}\text { For elements and attributes that } \\
\text { describe chart content. }\end{array}$ & $\begin{array}{l}\text { urn:oasis:names:tc:opendocument:xmlns: } \\
\text { chart:1.0 }\end{array}$ \\
\hline form & $\begin{array}{l}\text { For elements and attributes that } \\
\text { describe forms and controls. }\end{array}$ & $\begin{array}{l}\text { urn:oasis:names:tc:opendocument:xmlns: } \\
\text { form:1.0 }\end{array}$ \\
\hline script & $\begin{array}{l}\text { For elements and attributes that } \\
\text { represent scripts or events. }\end{array}$ & $\begin{array}{l}\text { urn:oasis:names:tc:opendocument:xmlns: } \\
\text { script:1.0 }\end{array}$ \\
\hline style & $\begin{array}{l}\text { For elements and attributes that } \\
\text { describe the style and inheritance } \\
\text { model used by the OpenDocument } \\
\text { format as well as some common } \\
\text { formatting attributes. }\end{array}$ & $\begin{array}{l}\text { urn:oasis:names:tc:opendocument:xmlns: } \\
\text { style:1.0 }\end{array}$ \\
\hline number & $\begin{array}{l}\text { For elements and attributes that } \\
\text { describe data style information. }\end{array}$ & $\begin{array}{l}\text { urn:oasis:names:tc:opendocument:xmlns: } \\
\text { data style:1.0 }\end{array}$ \\
\hline manifest & $\begin{array}{l}\text { For elements and attribute contained } \\
\text { in the package manifest. }\end{array}$ & $\begin{array}{l}\text { urn:oasis:names:tc:opendocument:xmlns: } \\
\text { manifest:1.0 }\end{array}$ \\
\hline
\end{tabular}

Table 2lists the namespaces that are defined by the OpenDocument format, but contain elements and attributes whose semantics are compatible to elements and attributes from other specifications.

Table 2: XML Namespaces defined by the OpenDocument schema that include elements and attributes that are compatible to elements and attributes of other standards.

\begin{tabular}{|c|l|l|}
\hline \multicolumn{1}{|c|}{ Prefix } & \multicolumn{1}{|c|}{ Description } & \multicolumn{1}{c|}{ Namespace } \\
\hline fo & $\begin{array}{l}\text { For attributes that are compatible to } \\
\text { attributes defined in [XSL]. }\end{array}$ & $\begin{array}{l}\text { urn:oasis:names:tc:opendocument:xmlns: } \\
\text { xsl-fo-compatible:1.0 }\end{array}$ \\
\hline
\end{tabular}




\begin{tabular}{|l|l|l|}
\hline \multicolumn{1}{|c|}{ Prefix } & \multicolumn{1}{|c|}{ Description } & \multicolumn{1}{c|}{ Namespace } \\
\hline svg & $\begin{array}{l}\text { For elements and attributes that are } \\
\text { compatible to elements or attributes } \\
\text { defined in [SVG]. }\end{array}$ & $\begin{array}{l}\text { urn:oasis:names:tc:opendocument:xmlns: } \\
\text { svg-compatible:1.0 }\end{array}$ \\
\hline smil & $\begin{array}{l}\text { For attributes that are compatible to } \\
\text { attributes defined in [SMIL20]. }\end{array}$ & $\begin{array}{l}\text { urn:oasis:names:tc:opendocument:xmlns: } \\
\text { smil-compatible:1.0 }\end{array}$ \\
\hline
\end{tabular}

Table 3 lists the namespaces that are imported into the OpenDocument format and their default prefixes.

Table 3: XML Namespaces used by the OpenDocument schema

\begin{tabular}{|l|l|l|}
\hline \multicolumn{1}{|c|}{ Prefix } & \multicolumn{1}{|c|}{ Description } & \multicolumn{1}{c|}{ Namespace } \\
\hline dc & $\begin{array}{l}\text { The Dublin Core Namespace (see } \\
{[\text { DCMI]). }}\end{array}$ & http://purl.org/dc/elements/1.1/ \\
\hline xlink & The XLink namespace (see [XLink]). & http://www.w3.org/1999/xlink \\
\hline math & MathML Namespace (see [MathML]) & http://www.w3.org/1998/Math/MathML \\
\hline xforms & $\begin{array}{l}\text { The XForms namespace (see } \\
{[\text { XForms]). }}\end{array}$ & http://www.w3.org/2002/xforms \\
\hline
\end{tabular}

\subsection{Relax-NG Schema}

The normative XML Schema for the OpenDocument format is embedded within this specification. It can be obtained from the specification document by concatenating all schema fragments contained in chapters 1 to 16 . All schema fragments have a gray background color and line numbers.

The schema language used within this specification is Relax-NG (see [RNG]).

The schema provided in this specification permits arbitrary content within meta information elements and formatting properties elements as described in section 1.5. Appendix A contains a schema that restricts the content within these elements to the attributes and elements defined in this specification.

Prefix for the normative Relax-NG schema:

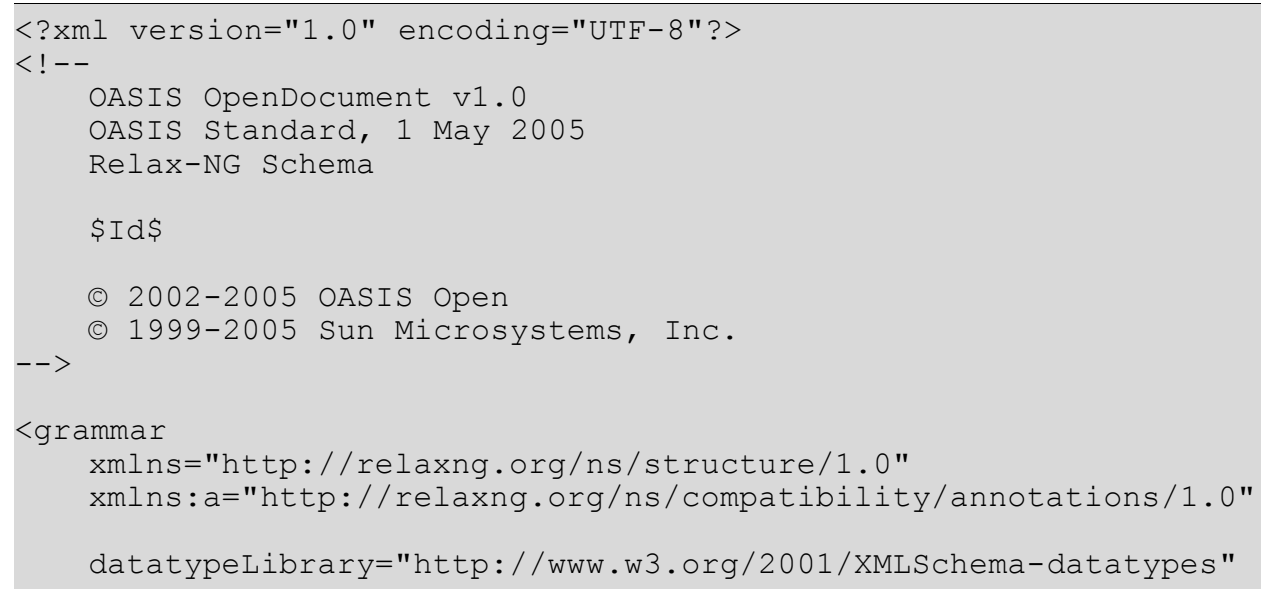


xmlns:office="urn:oasis:names:tc:opendocument:xmlns:office:1.0" xmlns:meta="urn:oasis:names:tc:opendocument:xmlns:meta: $1.0 "$ xmlns: config="urn:oasis:names: tc:opendocument:xmlns:config: $1.0 "$ xmlns: text="urn:oasis:names: tc:opendocument: xmlns: text: $1.0 "$ xmlns: table="urn:oasis:names: tc:opendocument:xmlns: table:1.0" xmlns: draw="urn:oasis:names:tc:opendocument:xmlns:drawing:1.0" xmlns:presentation="urn:oasis:names:tc:opendocument:xmlns:presentation: $1.0 "$ xmlns: $d r 3 d=" u r n: o a s i s:$ names: tc:opendocument:xmlns:dr3d: $1.0 "$ xmlns: chart="urn:oasis:names: tc:opendocument:xmlns: chart:1.0" xmlns: form="urn:oasis:names: tc:opendocument:xmlns: form: $1.0 "$ xmlns:script="urn:oasis:names:tc:opendocument:xmlns:script:1.0" xmlns: style="urn:oasis:names: tc:opendocument:xmlns:style: $1.0 "$ xmlns: number="urn:oasis: names: tc:opendocument: $x$ mlns: datastyle: $1.0 "$ xmlns:anim="urn:oasis:names:tc:opendocument:xmlns:animation: $1.0 "$

xmlns:dc="http://purl.org/dc/elements/1.1/" xmlns:xlink="http://www.w3.org/1999/xlink" xmlns:math="http: //www.w3.org/1998/Math/MathML" xmlns:xforms="http://www.w3.org/2002/xforms"

xmlns:fo="urn:oasis:names:tc:opendocument:xmlns:xsl-fo-compatible: $1.0 "$ xmlns:svg="urn:oasis:names: tc:opendocument:xmlns:svg-compatible: $1.0 "$ xmlns:smil="urn:oasis:names:tc:opendocument:xmlns:smil-compatible:1.0"

\subsection{Document Processing and Conformance}

Documents that conform to the OpenDocument specification MAY contain elements and attributes not specified within the OpenDocument schema. Such elements and attributes must not be part of a namespace that is defined within this specification and are called foreign elements and attributes.

Conforming applications either MUST read documents that are valid against the OpenDocument schema if all foreign elements and attributes are removed before validation takes place, or MUST write documents that are valid against the OpenDocument schema if all foreign elements and attributes are removed before validation takes place.

Conforming applications that read and write documents MAY preserve foreign elements and attributes.

In addition to this, conforming applications should preserve meta information and the content of styles. This means:

- The various <style: ${ }^{*}$-properties $>$ elements (see section 15) MAY have arbitrary attributes attached and MAY have arbitrary element content. All attributes attached to these elements and elements contained within these elements SHOULD be preserved (see section 15.1.3);

- elements contained within the <office:meta> element MAY have arbitrary element content and SHOULD be preserved (see section 2.2.1).

Foreign elements MAY have an office:process-content attribute attached that has the value true or false. If the attribute's value is true, or if the attribute does not exist, the element's content SHOULD be processed by conforming applications. Otherwise conforming applications SHOULD NOT process the element's content, but MAY only preserve its content. If the element's content should be processed, the document itself MUST be valid against the OpenDocument schema if the unknown element is replaced with its content only.

Conforming applications MUST read documents containing processing instructions and SHOULD preserve them. 
There are no rules regarding the elements and attributes that actually have to be supported by conforming applications, except that applications should not use foreign elements and attributes for features by the OpenDocument schema. See also appendix D.

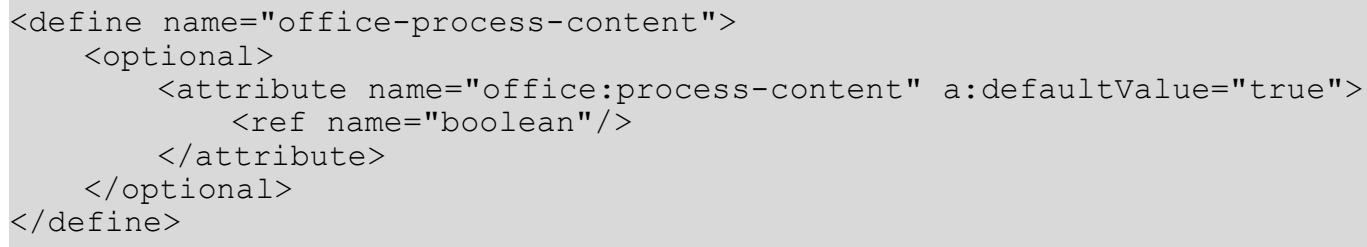

\subsection{White-Space Processing and EOL Handling}

In conformance with the W3C XML specification [XML1.0], optional white-space characters that are contained in elements that have element content (in other words that must contain elements only but not text) are ignored. This applies to the following white-space and end-of-line (EOL) Unicode characters:

- HORIZONTAL TABULATION (0x0009)

- LINE FEED (0x000A)

- CARRIAGE RETURN (0x000D)

- SPACE (0x0020)

For any other element, white-spaces are preserved by default. Unless otherwise stated, there is no special processing for any of the four white-space characters. For some elements, different white-space processing may take place, for example the paragraph element.

The XML specification also requires that any of the four white-space characters that is contained in an attribute value is normalized to a SPACE character.

One of the following characters may be used to represent line ends:

- $\quad$ LINE FEED

- CARRIAGE RETURN

- The sequence of the characters CARRIAGE RETURN and LINE FEED

Conforming to the XML specification, all the possible line ends are normalized to a single LINE FEED character.

As a consequence of the white-space and EOL processing rules, any CARRIAGE RETURN characters that are contained either in the text content of an element or in an attribute value must be encoded by the character entity \&\#xOD;. The same applies to the HORIZONTAL TABULATION and LINE FEED characters if they are contained in an attribute value.

\subsection{MIME Types and File Name Extensions}

Appendix C contains a list of MIME types and file name extensions to be used for office documents that conform to this specification and that are contained in a package (see section 2.1). This MIME types and extensions either have been registered following the procedures described in [RFC2048], or a registration is in progress.

For office documents that conform to this specification but are not contained in a package, it is RECOMMENDED to use the MIME type text/xml. 
It is RECOMMENDED that only MIME types and extensions that have been registered according to [RFC2048] are used for office documents that conform to this specification. It is also RECOMMENDED that the MIME types and extensions listed in appendix $C$ are used where appropriate. 


\section{Document Structure}

This chapter introduces the structure of the OpenDocument format. The chapter contains the following sections:

- Document Roots

- Document Metadata

- Body Element and Document Types

- Application Settings

- Scripts

- Font Face Declarations

- Styles

- Page Styles and Layout

In the OpenDocument format, each structural component is represented by an element, with associated attributes. The structure of a document in OpenDocument format applies to all document types. There is no difference between a text document, a spreadsheet or a drawing, apart from the content. Also, all document types may contain different styles. Document content that is common to all document types can be exchanged from one type of document to another.

\subsection{Document Roots}

A document root element is the primary element of a document in OpenDocument format. It contains the entire document. All types of documents, for example, text documents, spreadsheets, and drawing documents use the same types of document root elements.

The OpenDocument format supports the following two ways of document representation:

- As a single XML document.

- As a collection of several subdocuments within a package (see section 17), each of which stores part of the complete document. Each subdocument has a different document root and stores a particular aspect of the XML document. For example, one subdocument contains the style information and another subdocument contains the content of the document. All types of documents, for example, text and spreadsheet documents, use the same document and subdocuments definitions.

There are four types of subdocuments, each with different root elements. Additionally, the single $\mathrm{XML}$ document has its own root element, for a total of five different supported root elements. The root elements are summarized in the following table:

\begin{tabular}{|l|l|l|}
\hline \multicolumn{1}{|c|}{ Root Element } & \multicolumn{1}{|c|}{ Subdocument Content } & $\begin{array}{l}\text { Subdoc. Name in } \\
\text { Package }\end{array}$ \\
\hline <office: document> & $\begin{array}{l}\text { Complete office document in a single } \\
\text { XML document. }\end{array}$ & $\mathrm{n} / \mathrm{a}$ \\
\hline <office: document-content> & $\begin{array}{l}\text { Document content and automatic } \\
\text { styles used in the content. }\end{array}$ & content.xml \\
\hline
\end{tabular}




\begin{tabular}{|l|l|l|}
\hline \multicolumn{1}{|c|}{ Root Element } & \multicolumn{1}{|c|}{ Subdocument Content } & $\begin{array}{c}\text { Subdoc. Name in } \\
\text { Package }\end{array}$ \\
\hline <office:document-styles> & $\begin{array}{l}\text { Styles used in the document content } \\
\text { and automatic styles used in the } \\
\text { styles themselves. }\end{array}$ & styles.xml \\
\hline <office:document-meta> & $\begin{array}{l}\text { Document meta information, such as } \\
\text { the author or the time of the last save } \\
\text { action. }\end{array}$ & meta.xml \\
\hline <office: document-settings> & $\begin{array}{l}\text { Application-specific settings, such as } \\
\text { the window size or printer information. }\end{array}$ & settings.xml \\
\hline
\end{tabular}

The definitions of the root elements described in the table above are analogous to the definition of $<$ office: document>, except that the child element specification is suitably restricted.

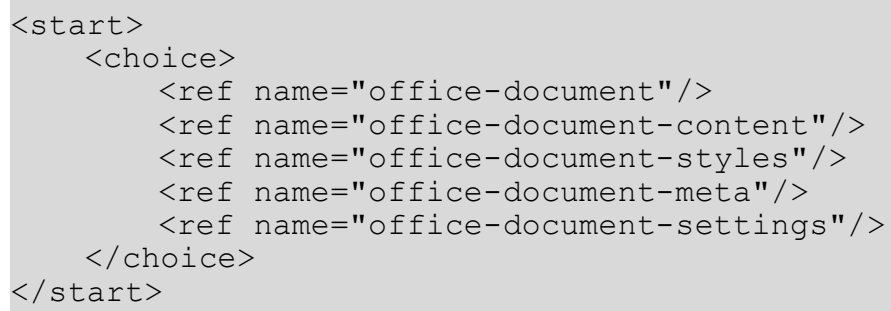

\subsubsection{Document Root Element Content Models}

The content models of the five root elements is summarized in the following table. Note that <office:document> may contain all supported top-level elements. None of the four subdocument root elements contain the complete data, but four combined do.

\begin{tabular}{|l|c|c|c|c|c|c|c|c|}
\hline \multicolumn{1}{|c|}{ Root Element } & $\begin{array}{c}\text { meta } \\
\text { data }\end{array}$ & $\begin{array}{l}\text { app. } \\
\text { sett. }\end{array}$ & script & $\begin{array}{c}\text { font } \\
\text { decls }\end{array}$ & style & $\begin{array}{c}\text { auto } \\
\text { style }\end{array}$ & $\begin{array}{c}\text { mast } \\
\text { style }\end{array}$ & body \\
\hline <office:document> & $\checkmark$ & $\checkmark$ & $\checkmark$ & $\checkmark$ & $\checkmark$ & $\checkmark$ & $\checkmark$ & $\checkmark$ \\
\hline <office:document-content> & & & $\checkmark$ & $\checkmark$ & & $\checkmark$ & & $\checkmark$ \\
\hline <office:document-styles> & & & & $\checkmark$ & $\checkmark$ & $\checkmark$ & $\checkmark$ & \\
\hline <office:document-meta> & $\checkmark$ & & & & & & & \\
\hline <office:document-settings> & & $\checkmark$ & & & & & & \\
\hline
\end{tabular}

The <office: document> root contains a complete document:

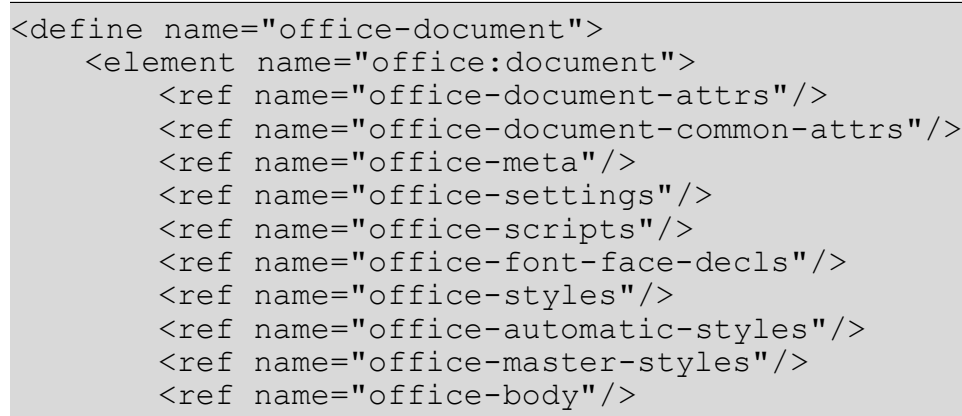


The <office: document-content> root contains only the document content, along with the automatic styles needed for the document content:

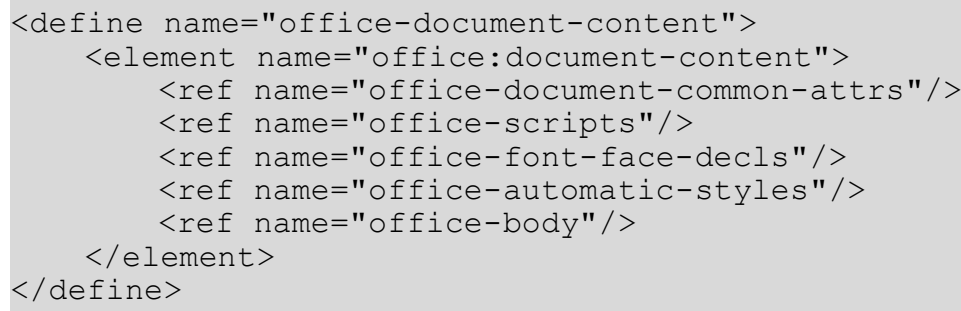

The <office: document-styles> root contains all named styles of a document, along with the automatic styles needed for the named styles:

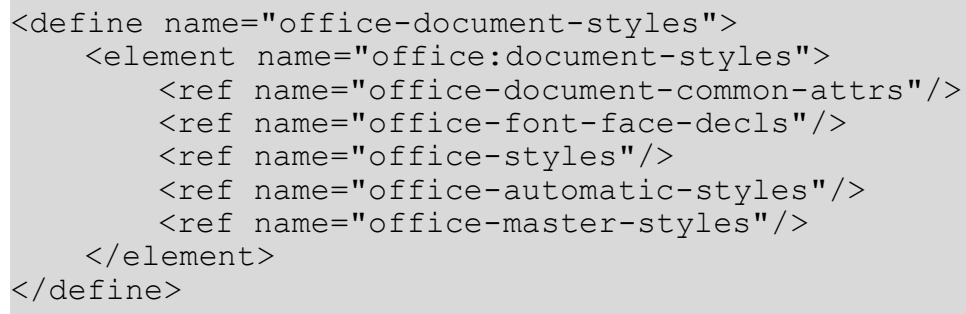

The <office: document-meta> root contains the meta information about a document.

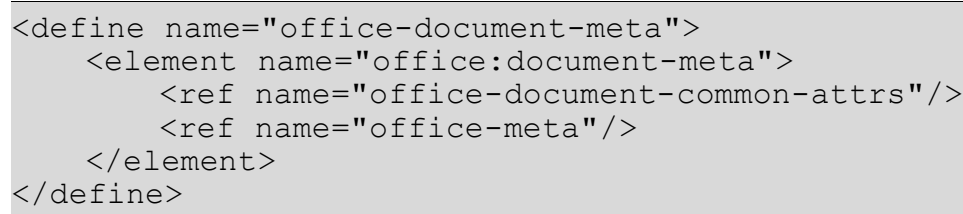

The <office:document-settings> root contains application specific settings to be applied when processing this document.

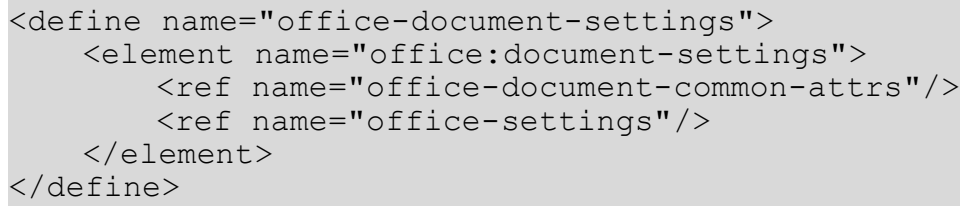

\subsubsection{Document Root Attributes}

\section{Version}

All root elements take an office:version attribute, which indicates which version of this specification it complies with. The version number is in the format revision.version. If the file has a version known to an XML processor, it may validate the document. Otherwise, it's optional to validate the document, but the document must be well formed. 


\section{MIME Type}

The <office: document> element takes an office:mimetype attribute, which indicates the type of document (text, spreadsheet etc.). This attribute is especially important for flat XML files, where this is the only way the type of document can be detected (in a package, the MIME type is also present in a separate file, see section 17.4). Its values are the MIME types that are used for the packaged variant of office documents (see section 1.7).

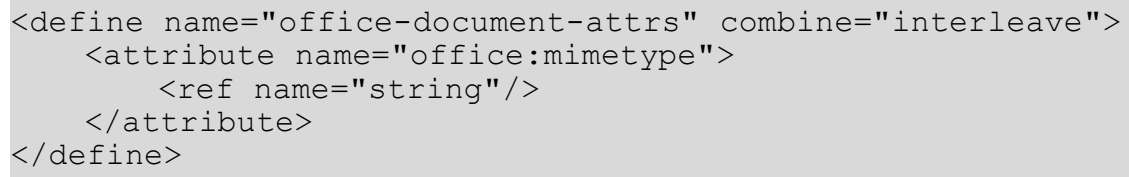

\subsection{Document Metadata}

Metadata is general information about a document. In the OpenDocument format, all of the metadata elements are contained in an <office:meta> element, usually located at start of the document. Metadata elements may be omitted or occur multiple times. It is application-specific how to update multiple instances of the same elements.

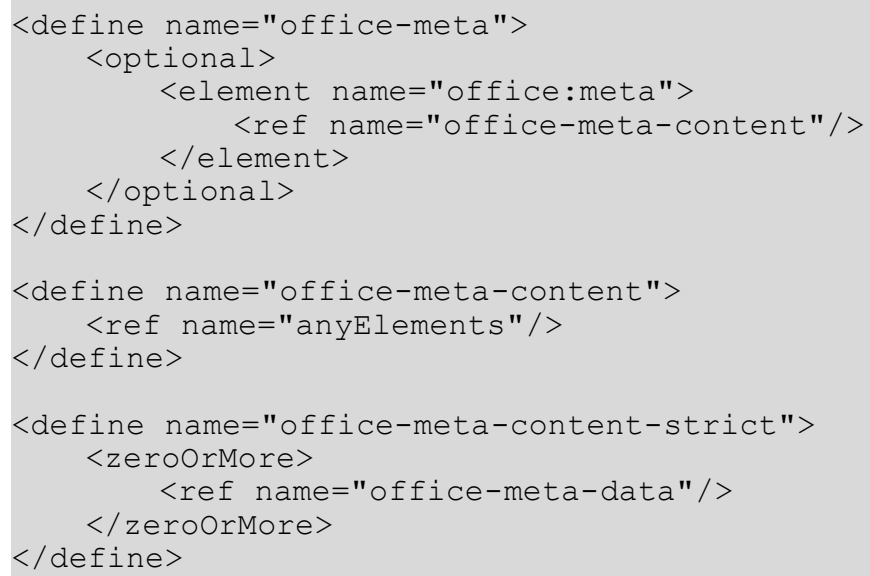

\subsubsection{Pre-Defined vs. Custom Metadata}

In the OpenDocument schema the metadata is comprised of pre-defined metadata elements, user defined metadata, as well as custom metadata elements. The pre-defined metadata elements have defined semantics. They SHOULD be processed and updated by editing applications. They can be referenced from within the document through the use of suitable text fields.

User-defined metadata is a more generic mechanism which specifies a triplet of name, type, and value. Supporting applications can present these value to the user, making use of the supplied data type. The user-defined metadata can be referenced from within the document through the use of suitable text fields.

Custom metadata are arbitrary elements inside $<$ office:meta $>$. Since their semantics is not defined in this specification, conforming applications in general cannot process or display this data. Applications SHOULD preserve this data when editing the document. 


\subsubsection{Sample Metadata}

Example: Sample metadata of a document in OpenDocument format

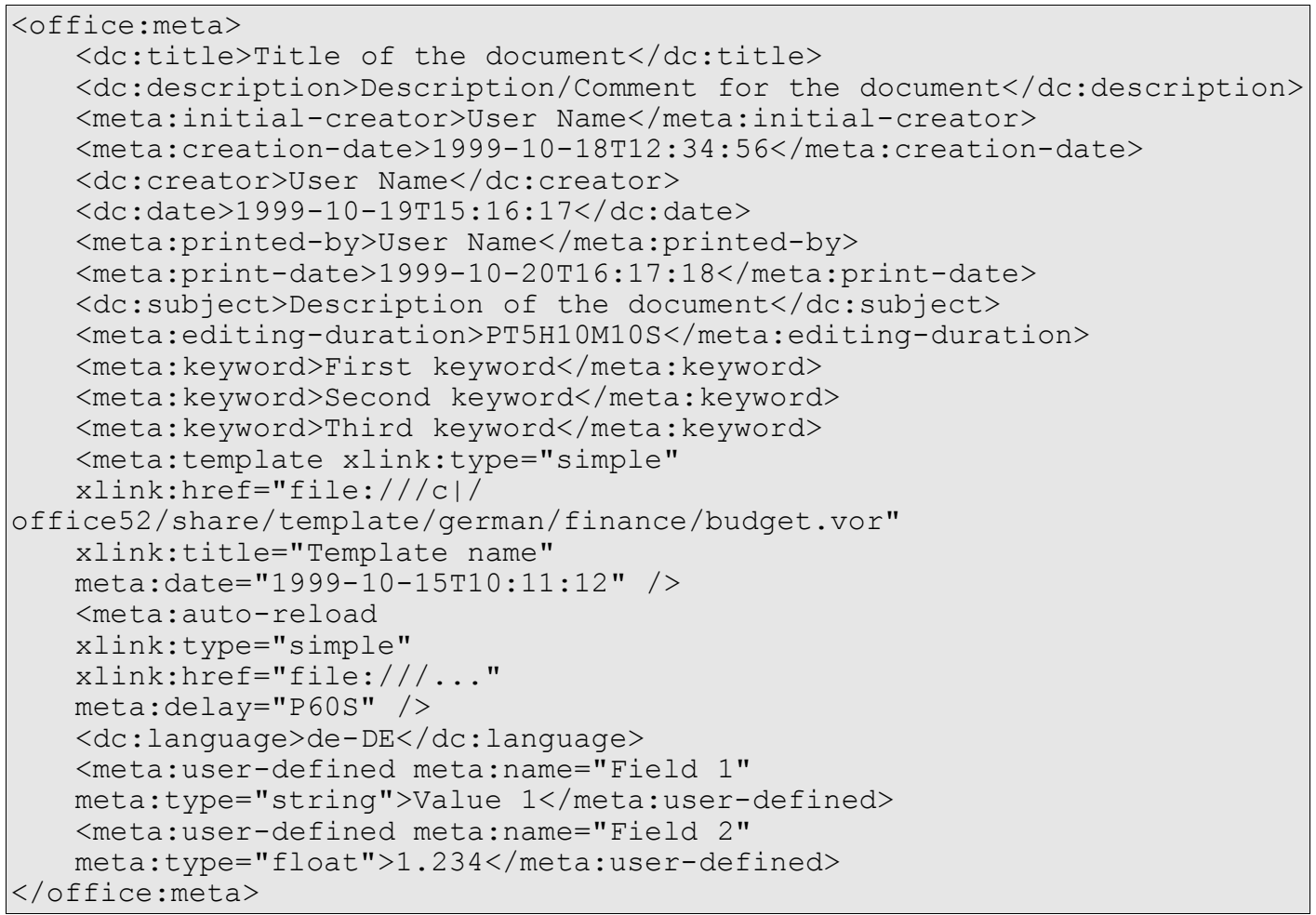

\subsection{Body Element and Document Types}

The document body contains an element to indicate which type of content this document contains. Currently supported document types are:

- text documents

- drawing documents

- presentation documents

- spreadsheet documents

- chart documents

- image documents

All document types share the same content elements, but different document types place different restrictions on which elements may occur, and in what combinations. The document content is typically framed by a prelude and epilogue, which contain additional information for a specific type of document, like form data or variable declarations.

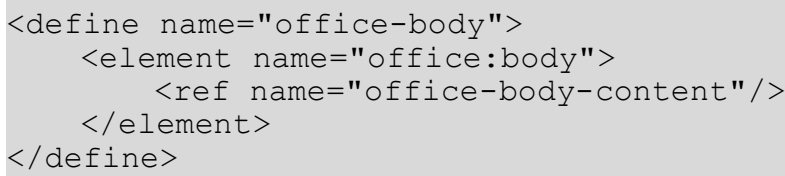




\subsubsection{Text Documents}

The content of text documents mainly consists of a sequence containing any number of paragraphs, tables, indices, text frames, text sections, and graphical elements. Additionally, a text document may contain forms, change tracking information and variable declarations. Each of these is defined in the document prelude, and may be referenced from the document content.

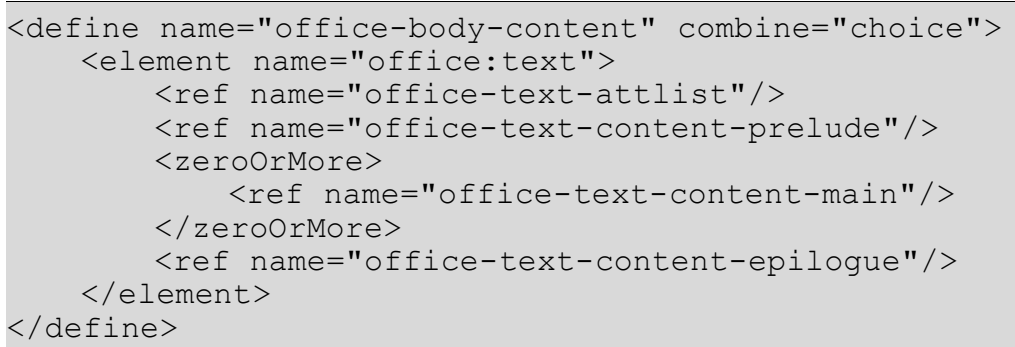

\section{Text Document Content Model}

The text document prelude contains the document's form data, change tracking information, and variable declarations. To allow office applications to implement functionality that usually is available in spreadsheets for text documents, it may also contain elements that implement enhanced table features. See also section 2.3.4.

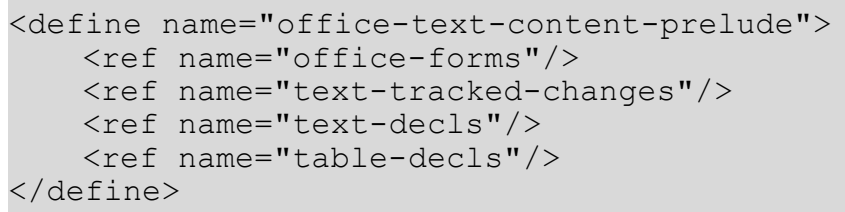

The main document content contains any sequence of text content elements, which includes paragraphs (and headings), text sections (and indices), tables, and graphical shapes. As an alternative, a text document may contain of a single page sequence.

It is not required that a text document contains a paragraph. A text document may consist of a sequence frames only.

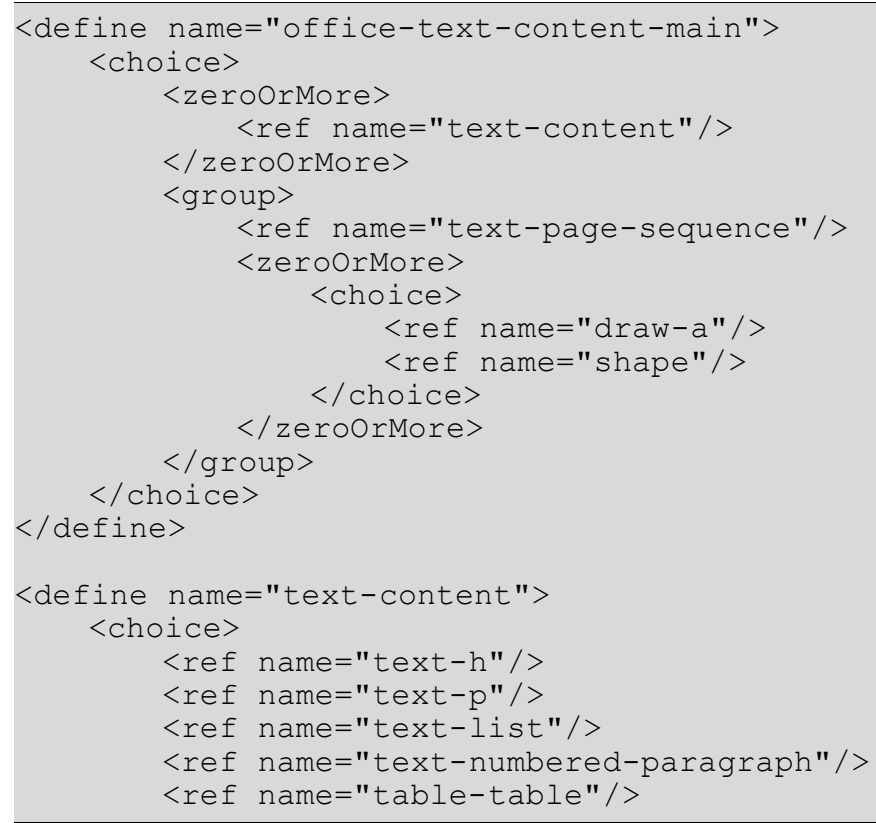




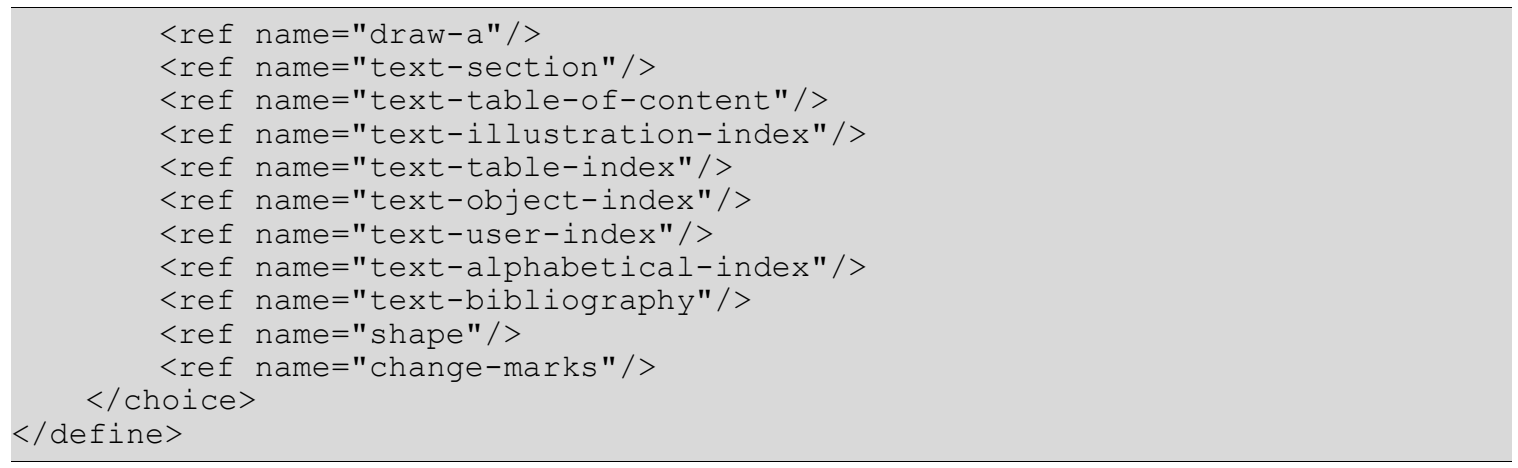

There are no text documents specific epilogue elements, but the epilogue may contain elements that implement enhanced table features. See also section 2.3.4.

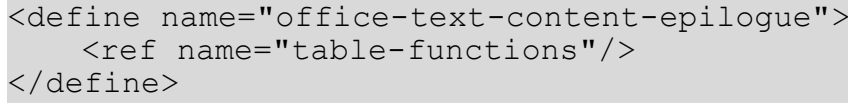

\section{Global Text Documents}

There is a common use case for large documents to be edited in separate entities, such that there is a 'global' document, containing several linked constituent subdocuments. This can be implemented by using linked text sections (see section 4.4). To facilitate an editing application adapting the user interface to better support the notion of 'global' document with constituent parts (as opposed to a document with arbitrary linked content), the text: global flag can be used. If set to true, it informs applications that linked sections in this document have part-of semantics. The actual XML representation of the sections does not change.

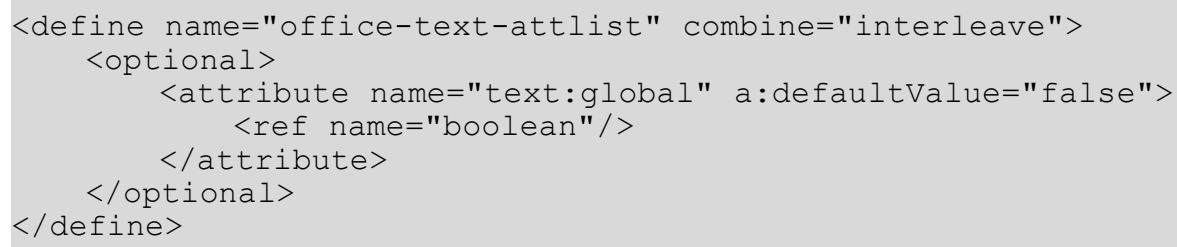

\subsubsection{Drawing Documents}

The content of drawing document consists of a sequence of draw pages.

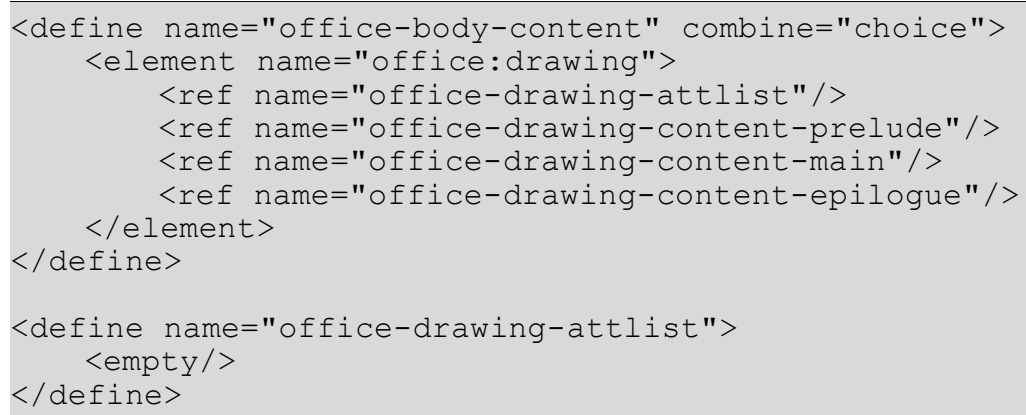

\section{Drawing Document Content Model}

The drawing document prelude may contain text declarations only. To allow office applications to implement functionality that usually is available in spreadsheets for drawing documents, it may also contain elements that implement enhanced table features. See also section 2.3.4. 


\section{Presentation Document Content Model}

The presentation document prelude equals the one of a drawing document, but may contain some additional declarations. See also section 2.3.2.

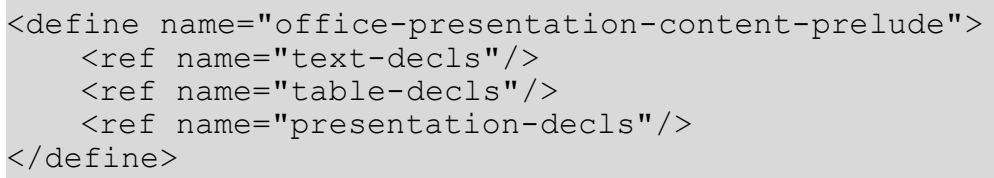

The main document content contains a sequence of draw pages.

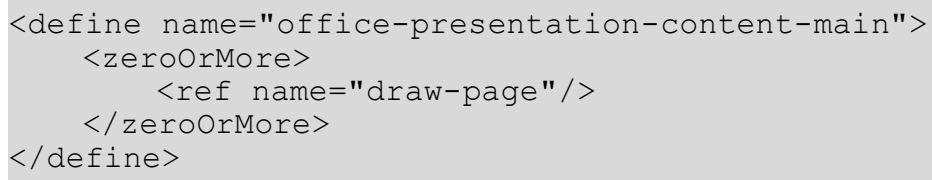

The epilogue of presentation documents may contain presentation settings. Additionally, it may contain elements that implement enhanced table features. See also section 2.3.4.

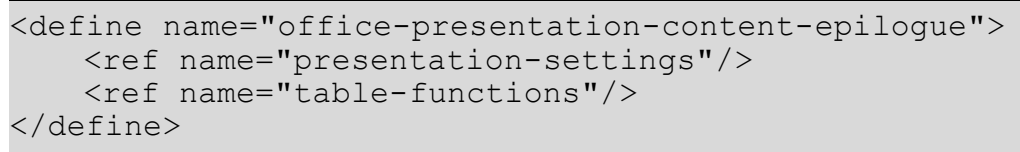




\subsubsection{Spreadsheet Documents}

The content of spreadsheet documents mainly consists of a sequence of tables. Additionally, a spreadsheet document may contain forms, change tracking information and various kinds of declarations that simplify the usage of spreadsheet tables and their analysis. Each of these are contained in either the document prelude, or the document epilogue.

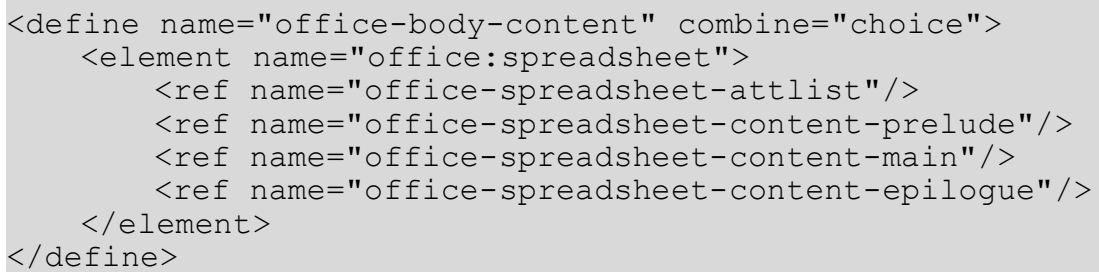

\section{Spreadsheet Document Content Model}

The spreadsheet document prelude contains the document's form data, change tracking information, calculation setting for formulas, validation rules for cell content and declarations for label ranges.

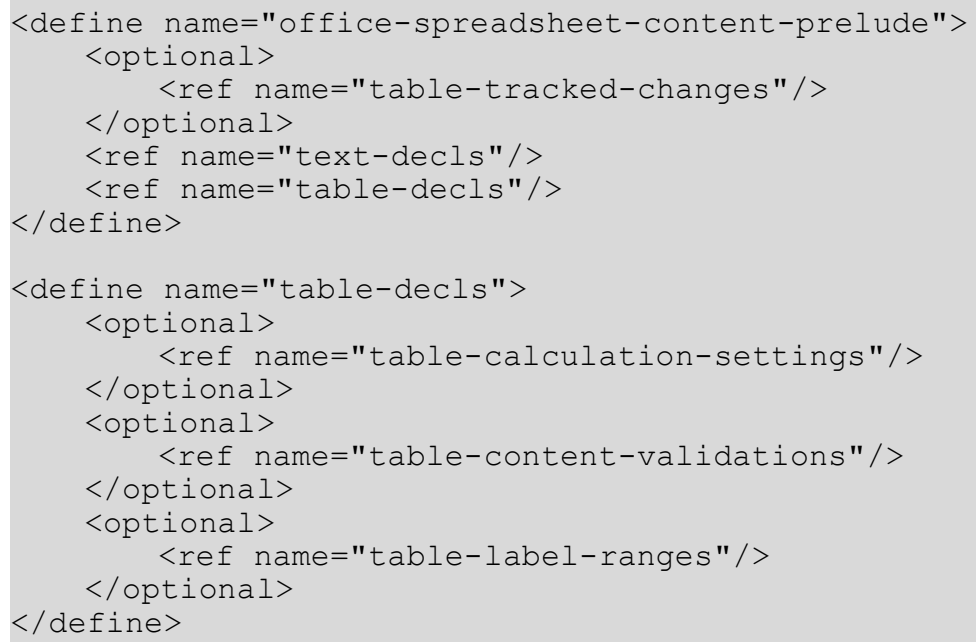

The main document is a list of tables.

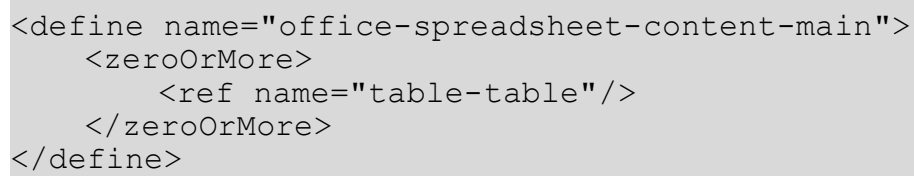

The epilogue of spreadsheet documents contains declarations for named expressions, database ranges, data pilot tables, consolidation operations and DDE links.

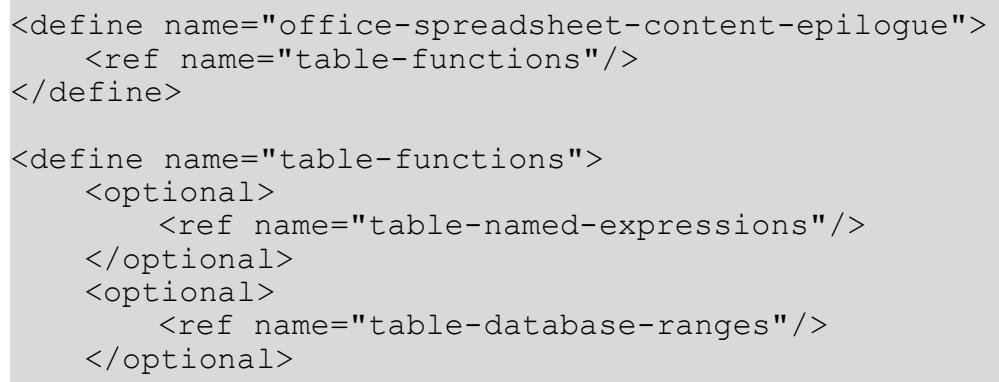


$</$ define $>$

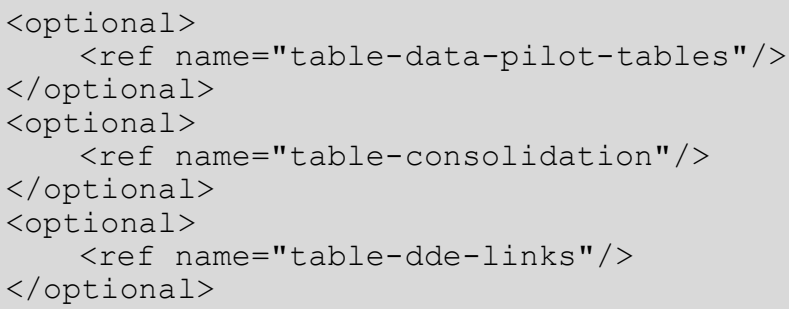

\subsubsection{Chart Documents}

The content of chart documents mainly consists of a chart element.

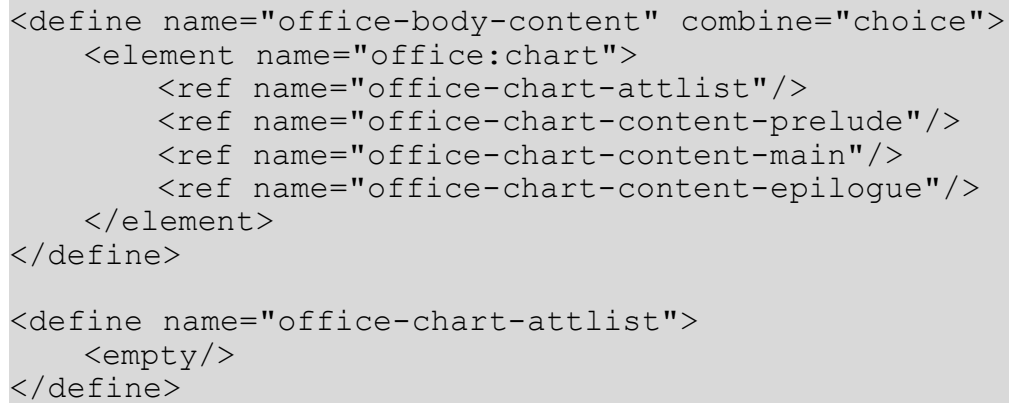

\section{Chart Document Content Model}

To allow office applications to implement functionality that usually is available in spreadsheets for the table that may be contained in a chart, the chart document prelude may contain elements that implement enhanced table features. See also section 2.3.4.

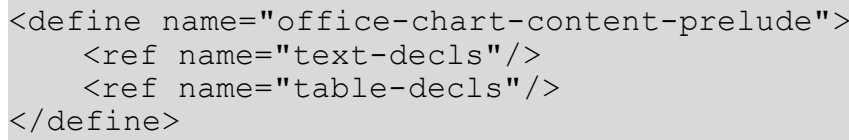

The main document is a chart element only.

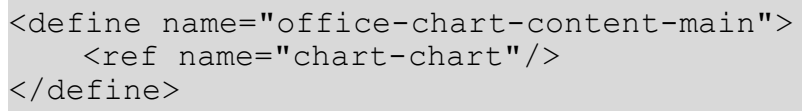

There are no chart documents specific epilogue elements, but the epilogue may contain elements that implement enhanced table features. See also section 2.3.4.

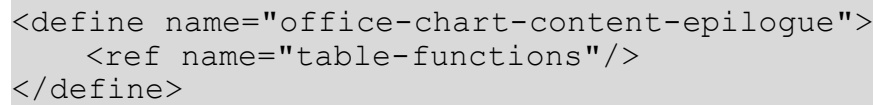

\subsubsection{Image Documents}

The content of an image document is a frame element only. The frame element must contain a single image element.

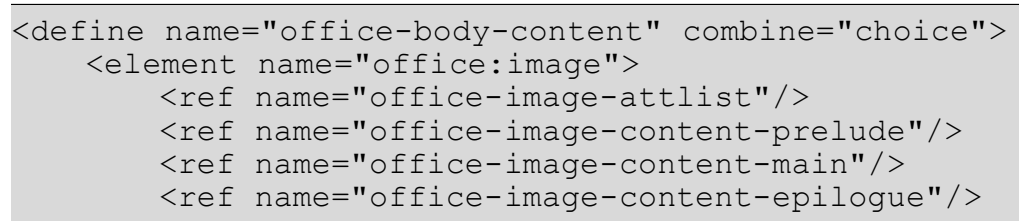




\section{Image Document Content Model}

The image document prelude is empty.

<define name="office-image-content-prelude" > <empty/>

$</$ define $>$

The main document content contains a frame only.

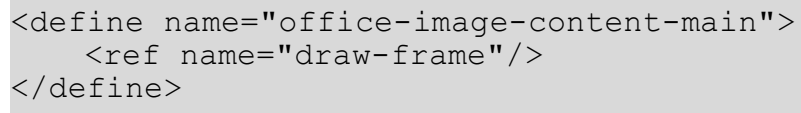

There are no image documents specific epilogue elements.

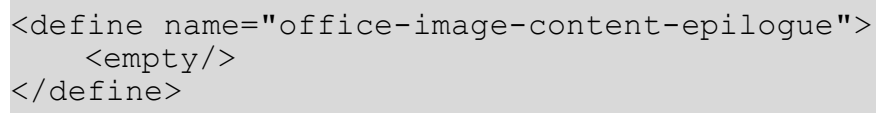

\subsection{Application Settings}

Application settings are contained in a <office: settings $>$ element.

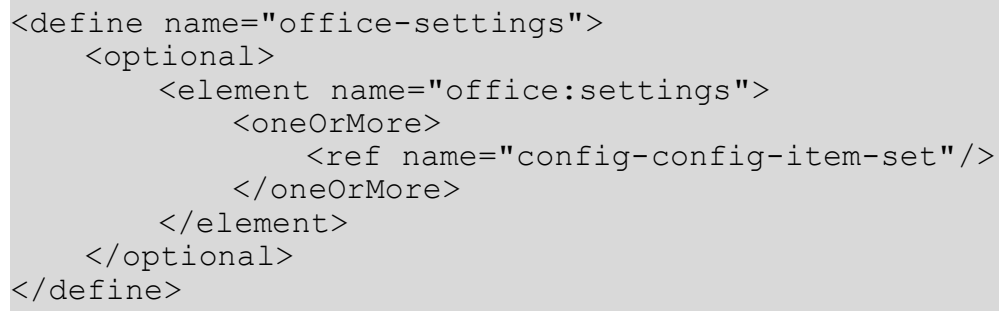

The settings for office applications may be divided into several categories each represented by a <config:config-item-set> element. For instance the following two categories may exist:

- Document settings, for example default printer.

- View settings, for example zoom level.

\subsubsection{Sequence of Settings}

The <config:config-item-set> element is a container element for all types of setting elements. The settings can be contained in the element is any order.

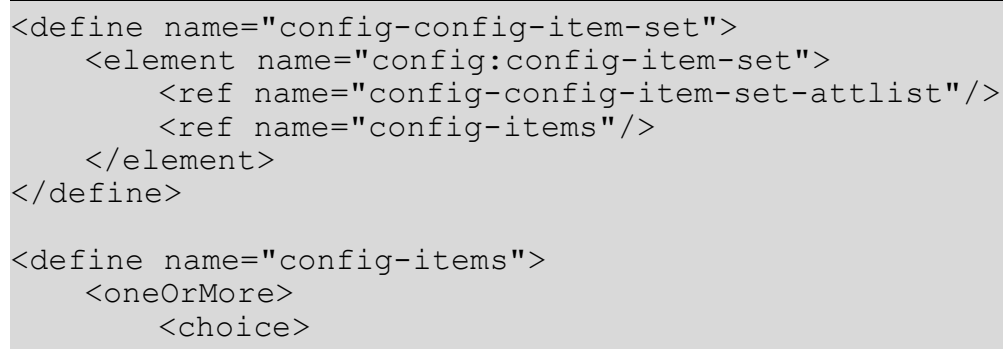




\section{Example:}

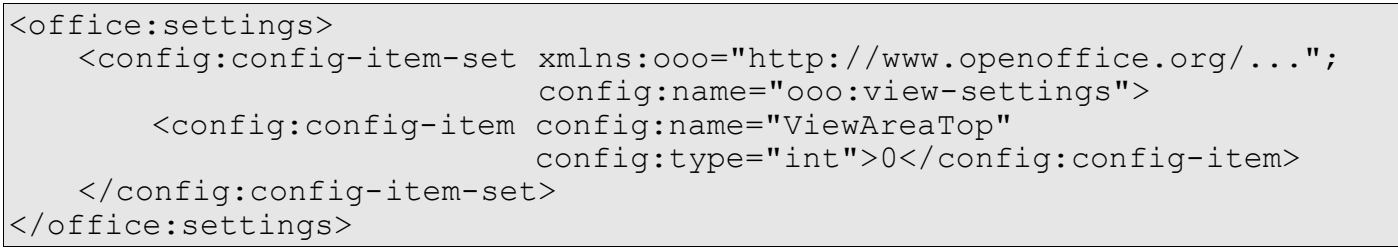

\subsubsection{Base Settings}

The <config:config-item> element contains all base settings. The value of the setting is stored in the element.

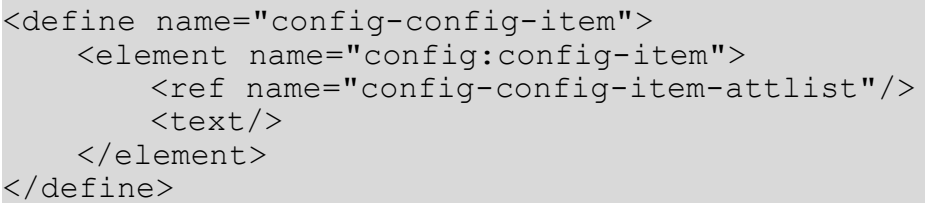

\section{Config Name}

The config: name attribute identifies the name of the setting.

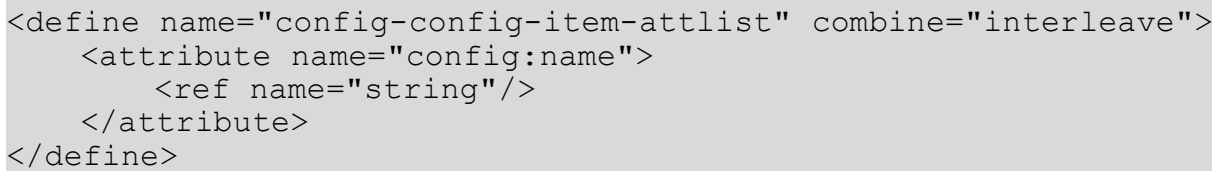

\section{Config Type}

The config:type attribute identifies the data type of setting. 


\section{Config Name}

The config: name attribute identifies the name of the setting sequence.

\subsubsection{Map Entry}

The <config: config-item-map-entry> element represents an entry in an indexed or named settings sequence. It is a container element for all types of setting elements.

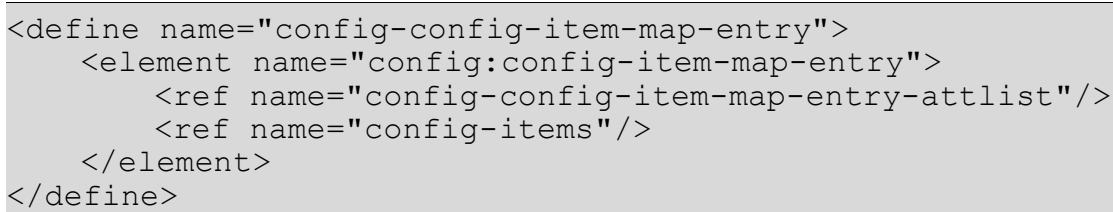

\section{Config Name}

The config: name attribute identifies the name of the setting sequence.

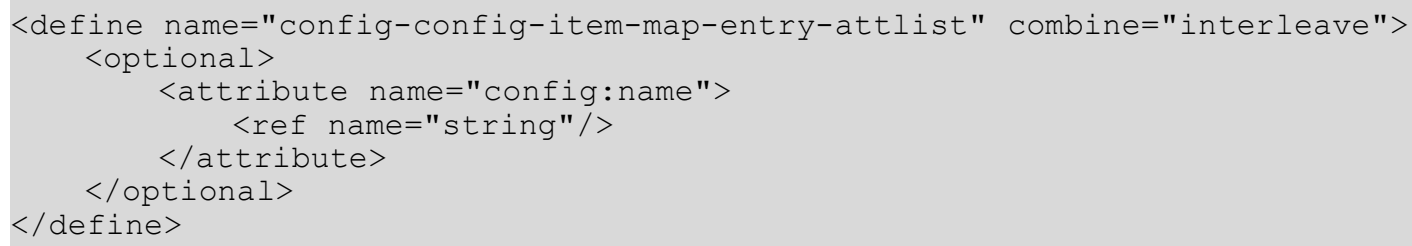




\subsubsection{Name Access of Sequences}

The <config:config-item-map-named> element is a container element for sequences, where each setting in the sequence is identified by its name.

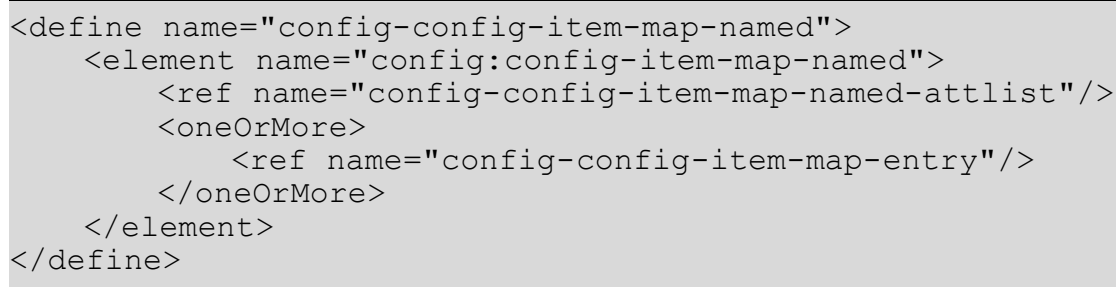

\section{Config Name}

The config:name attribute identifies the name of the setting sequence.

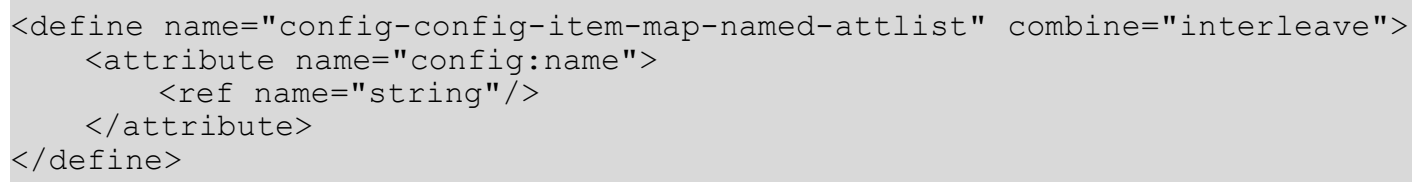

\subsubsection{Cursor Position Setting}

A common view setting for editing applications is the position where the text cursor was while saving the document. For WYSIWYG applications, this usually will be a position within a paragraph only. For applications that provide an XML based view of the document, the cursor position could be also between arbitrary elements, or even within tags.

To represent a text cursor position within a document, a processing instruction with PITarget opendocument (see §2.6 of [XML1.0]) SHOULD be used. The name of the cursor position processing instruction, cursor-position, MUST follow the PITarget opendocument. The processing instruction may have arbitrary application specific attributes, for instance to connect the cursor position with a certain view of the document, where the views themselves are specified as application specific settings. The syntax for these attributes MUST be the same as for attributes within XML start tags.

Where a text cursor position is not sufficient to recreate a document view, applications may use arbitrary document specific settings in addition to the cursor position processing instruction. They may also use arbitrary document specific settings if the cursor position is not a text cursor position, but for instance a selection of drawing objects.

Example: cursor position processing instruction

<text:p>This is<?opendocument cursor-position view-id="view1"?> an example.</text:p>

\subsection{Scripts}

A document may contain several scripts in different scripting languages. Each script is represented by a <office:script> element. All these script elements are contained in a single <office:scripts> element.

Scripts do not imply a scripting language or an object model. A script can operate on the Document Object Model (DOM) of a document in OpenDocument format or on an application specific API. 
Scripts cannot modify a document while the document is loading. However, some events are called immediately after the document is loaded.

In addition to <office: script> elements, the <office:scripts> element may also contain an <office:event-listeners> element which contains the events assigned to the document itself. Examples for these are events called when the document is opened or closed. See section 12.4 for more information on the <office:event-listeners> element.

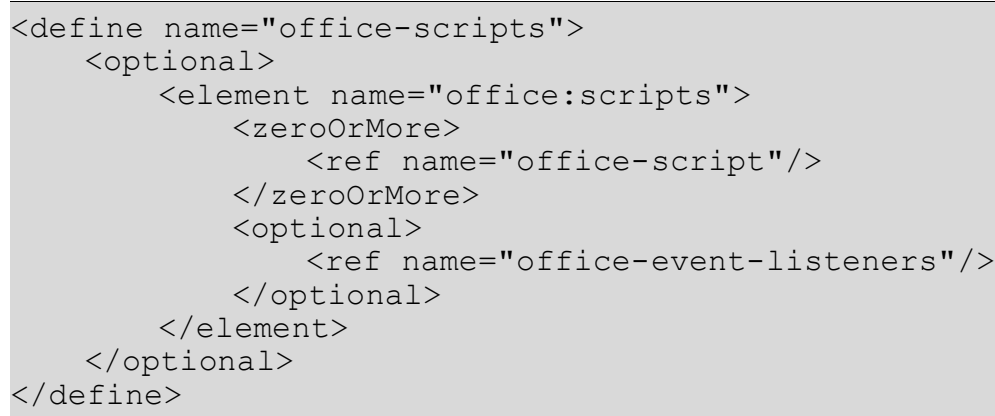

\subsubsection{Script}

The <office: script> element contains script language specific content. In most situations, the element contains the source code of the script, but it may also contain a compiled version of the script or a link to some external script code.

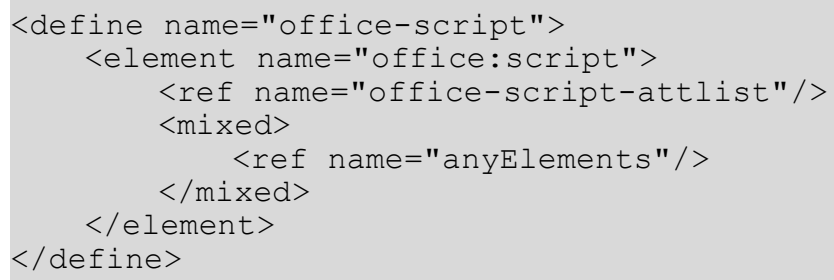

\section{Script Language}

The attribute script: language specifies the language of the script by its name. Since script language names are application specific, the name should be preceded by a namespace prefix.

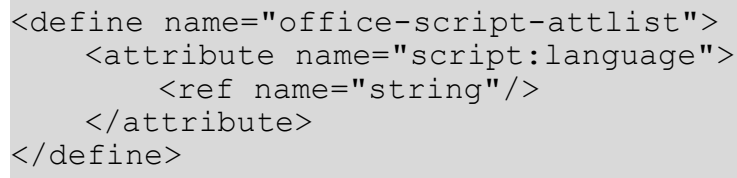

\subsection{Font Face Declarations}

A document in OpenDocument format may contain font face declarations. A font face declaration provides information about the fonts used by the author of a document, so that these fonts or fonts that are very close to these fonts may be located on other systems. See section 14.6 for details.

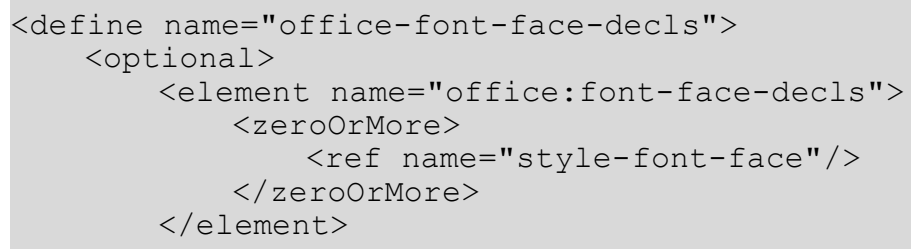




\subsection{Styles}

The OpenDocument format supports the following types of styles:

- Common styles

Most office applications support styles within their user interface. Within this specification, the XML representations of such styles are referred to as styles. When a differentiation from the other types of styles is required, they are referred to as common styles. The term common indicates that this is the type of style that an office application user considers to be a style.

\section{- Automatic styles}

An automatic style contains formatting properties that, in the user interface view of a document, are assigned to an object such as a paragraph. The term automatic indicates that the style is generated automatically. In other words, formatting properties that are immediately assigned to a specific object are represented by an automatic style. This way, a separation of content and layout is achieved.

\section{- Master styles}

A master style is a common style that contains formatting information and additional content that is displayed with the document content when the style is applied. An example of a master style are master pages. Master pages can be used in graphical applications. In this case, the additional content is any drawing shapes that are displayed as the background of the draw page. Master pages can also be used in text documents. In this case, the additional content is the headers and footers. Please note that the content that is contained within master styles is additional content that influences the representation of a document but does not change the content of a document.

As far as the office application user is concerned, all types of styles are part of the document. They represent the output device-independent layout and formatting information that the author of a document has used to create or edit the document. The assumption is that the author of the document wants this formatting and layout information to be preserved when the document is reloaded or displayed on any device, because this is common practice for documents created by word processors.

This type of style information differs from [CSS2] or [XSLT] style sheets that are used to display a document. An additional style sheet for CSS, XSLT, and so on, is required to display an document in OpenDocument format on a certain device. This style sheet must take into account the styles in the document as well as the requirements and capabilities of the output device. The ideal case is that this style sheet depends on the output device only.

See section 14 for more information on styles.

\subsubsection{Location of Styles}

Common and automatic styles have the same XML representation, but they are contained within two distinct container elements, as follows:

- <office:styles> for common styles

- <office:automatic-styles> for automatic styles

- Master styles are contained within a container element of its own:

- <office:master-styles> 


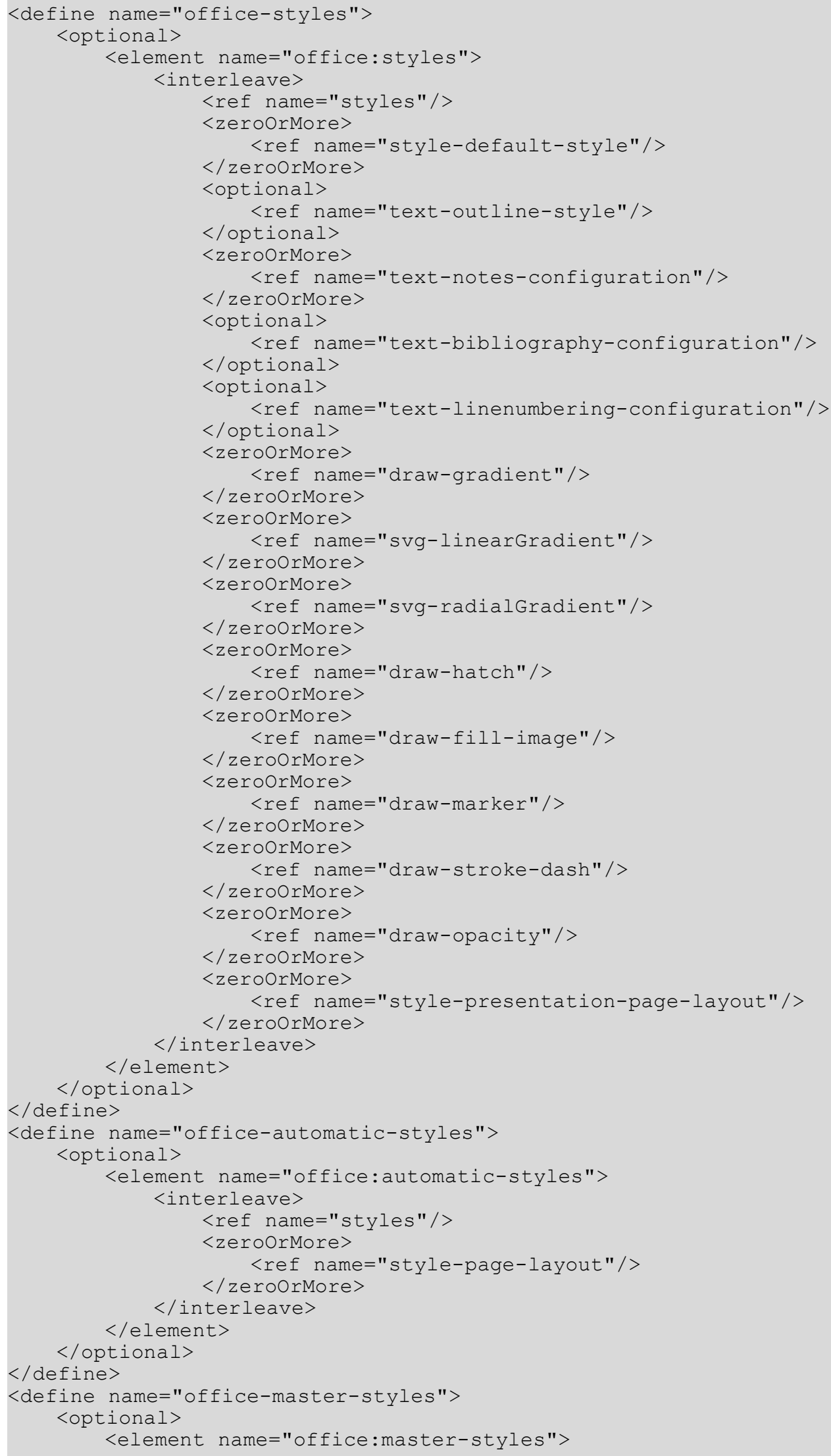




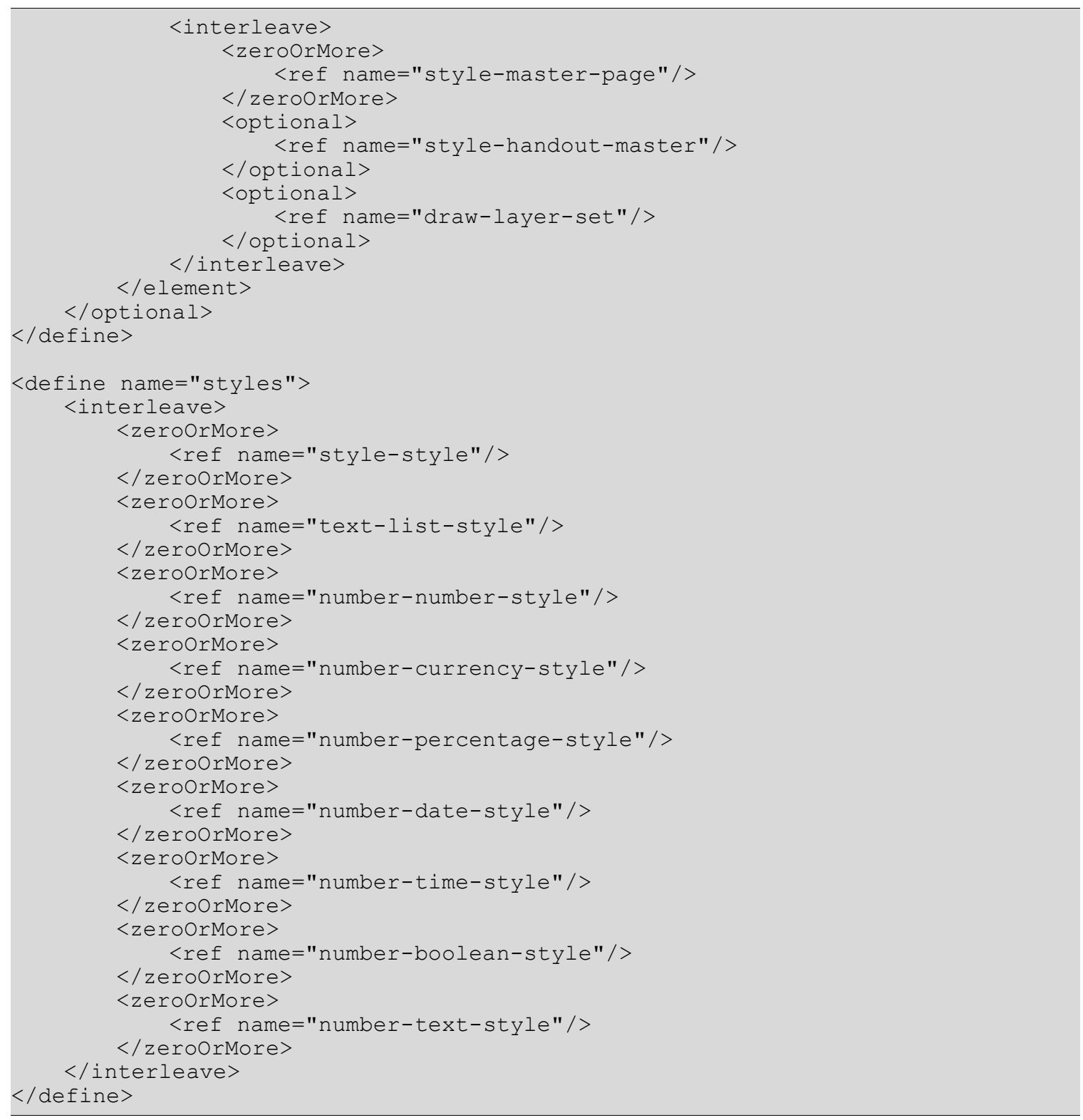

The following examples illustrate the different types of OpenDocument styles.

\section{Example: OpenDocument styles}

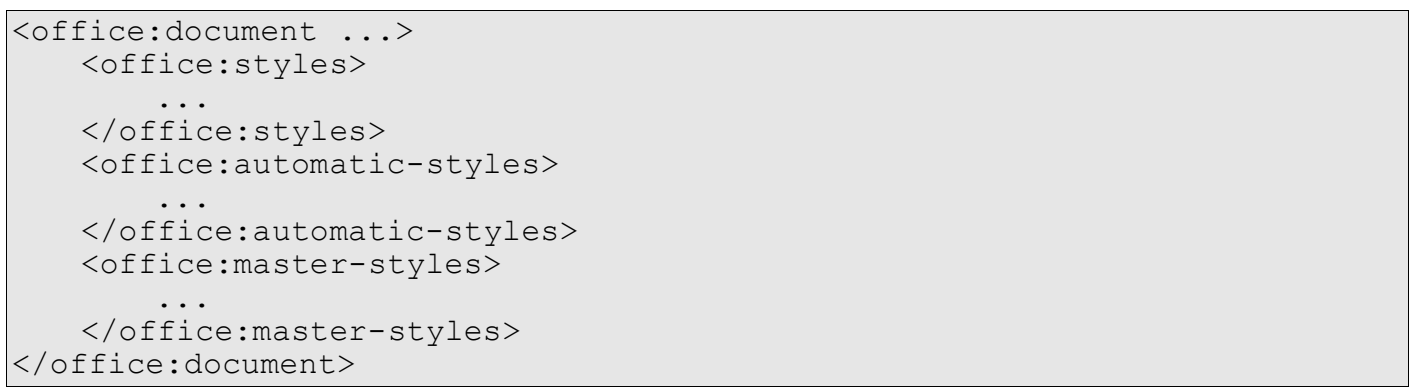




\subsection{Page Styles and Layout}

The style and layout of the pages in a document is determined by:

- Page Layouts

- Master Pages

A page layout describes the physical properties or geometry of a page, for example, page size, margins, header height, and footer height.

A master page is a template for pages in a document. It contains a reference to a page layout which specifies the physical properties of the page and can also contain static content that is displayed on all pages in the document that use the master page. Examples of static content are headers, footers, or background graphics.

If a text or spreadsheet document is displayed in a paged layout, the master pages are instantiated to generate a sequence of pages containing the document content. When a master page is instantiated, an empty page is generated with the properties of the page master and the static content of the master page. The body of the page is then filled with content. If multiple pages in a document use the same master page, the master page can be instantiated several times within the document.

In text and spreadsheet documents, a master page can be assigned to paragraph and table styles using a style:master-page-name attribute. Each time the paragraph or table style is applied to text, a page break is inserted before the paragraph or table. The page that starts at the page break position uses the specified master page.

In drawings and presentations, master pages can be assigned to drawing pages using a style:parent-style-name attribute.

Note: The OpenDocument paging methodology differs significantly from the methodology used in [XSL]. In XSL, headers and footers are contained within page sequences that also contain the document content. In the OpenDocument format, headers and footers are contained in page styles. With either approach, the content of headers and footers can be changed or omitted without affecting the document content.

Page layouts are described in section 14.3. Master pages are described in section 14.4. 


\section{Meta Data Elements}

The metadata elements borrow heavily upon the metadata standards developed by the Dublin Core Metadata Initiative (http://www.dublincore.org). Metadata elements drawn directly from the Dublin Core work use its namespace prefix (see section 1.3).

\subsection{Pre-Defined Metadata Elements}

There is a set of pre-defined metadata elements which should be processed and updated by the applications. Metadata elements may be omitted or occur multiple times. It is application-specific how to update multiple instances of the same elements.

\subsubsection{Generator}

The <meta: generator $>$ element contains a string that identifies the application or tool that was used to create or last modify the XML document. This string SHOULD match the definition for user-agents in the HTTP protocol a specified in section 14.43 of [RFC2616]. The generator string SHOULD allow product versions to differ between all released versions of a user agent, for instance by including build ids or patch level information.

Conforming applications MAY use the generator string to work around bugs that exist or existed in certain applications, but MUST NOT deliberately implement a different behavior depending on a certain generator string.

If the application that created the document could not provide an identifier string, the application does not export this element. If another application modifies the document and it cannot provide a unique identifier, it MUST NOT export the original identifier belonging to the application that created the document.

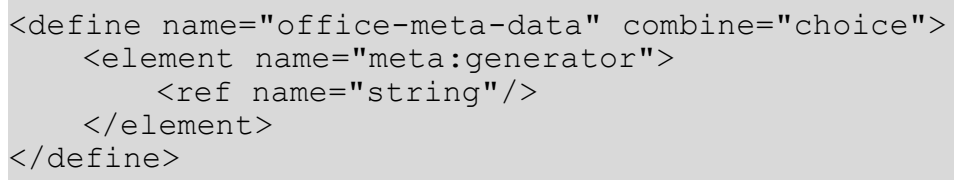

\subsubsection{Title}

The $<$ dc:title $>$ element specifies the title of the document.

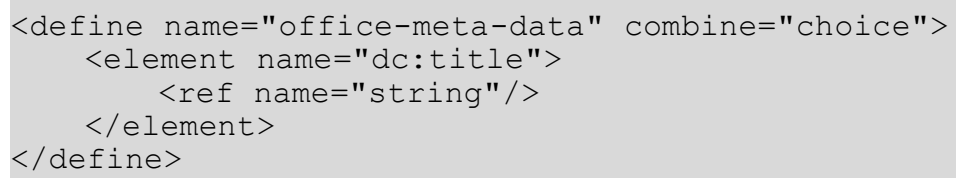

\subsubsection{Description}

The $<\mathrm{dc}$ : description $>$ element contains a brief description of the document.

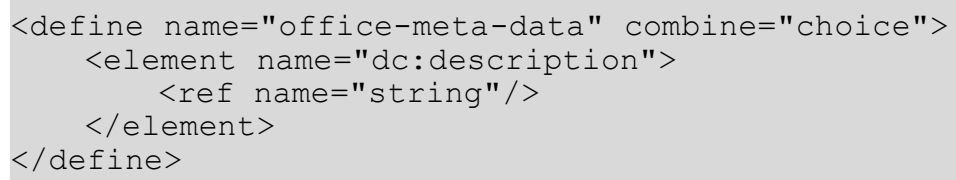




\subsubsection{Subject}

The $<d c$ : subject $>$ element specifies the subject of the document.

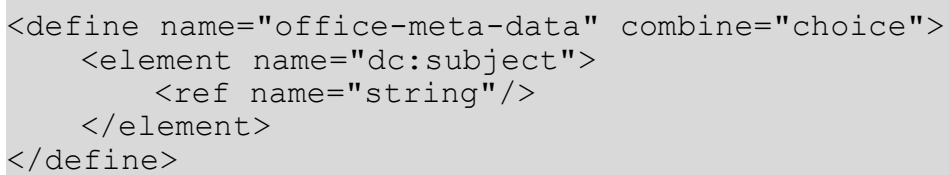

\subsubsection{Keywords}

The <meta: keyword $>$ element contains a keyword pertaining to the document. The metadata can contain any number of <meta: keyword > elements, each element specifying one keyword.

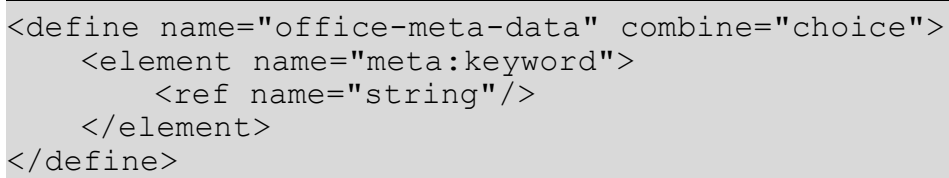

\subsubsection{Initial Creator}

The <meta:initial-creator $>$ element specifies the name of the person who created the document initially.

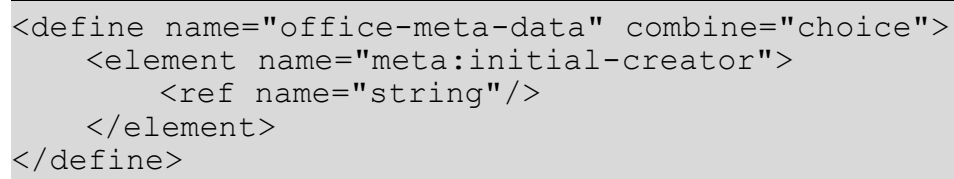

\subsubsection{Creator}

The $<\mathrm{dc}$ : creator $>$ element specifies the name of the person who last modified the document. The name of this element was chosen for compatibility with the Dublin Core, but this definition of "creator" used here differs from Dublin Core, which defines creator as "An entity primarily responsible for making the content of the resource." In OpenDocument terminology, the last person to modify the document is primarily responsible for making the content of the document.

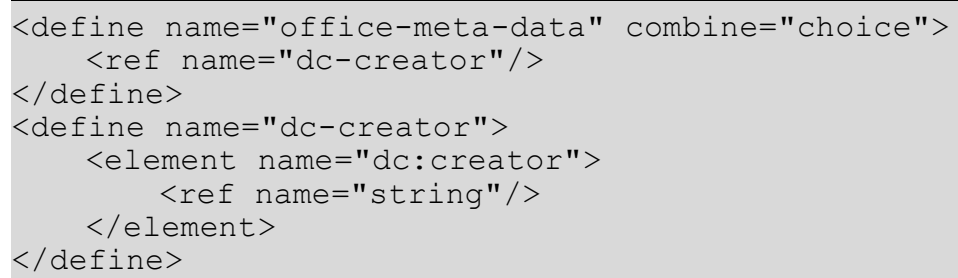

\subsubsection{Printed By}

The $<$ meta:printed-by $>$ element specifies the name of the last person who printed the document.

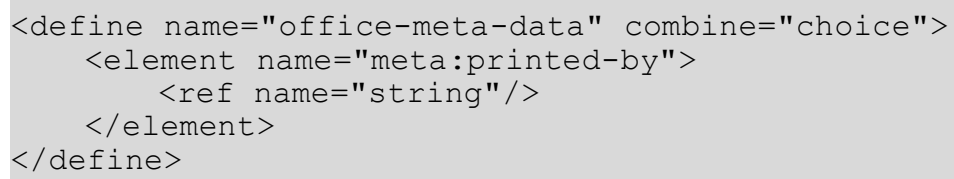




\subsubsection{Creation Date and Time}

The <meta: creation-date> element specifies the date and time when the document was created initially.

To conform with [xmlschema-2], the date and time format is YYYY-MM-DDThh:mm:ss.

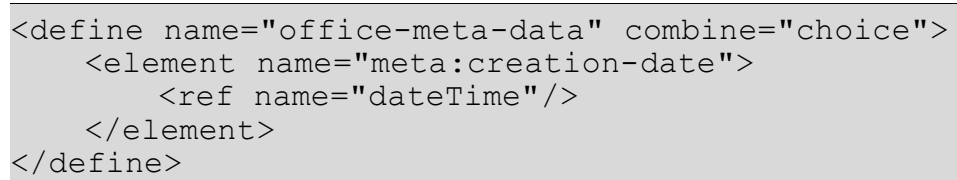

\subsubsection{Modification Date and Time}

The $<\mathrm{dc}$ : date $>$ element specifies the date and time when the document was last modified.

To conform with [xmlschema-2], the date and time format is YYYY-MM-DDThh:mm:ss.

The name of this element was chosen for compatibility with the Dublin Core.

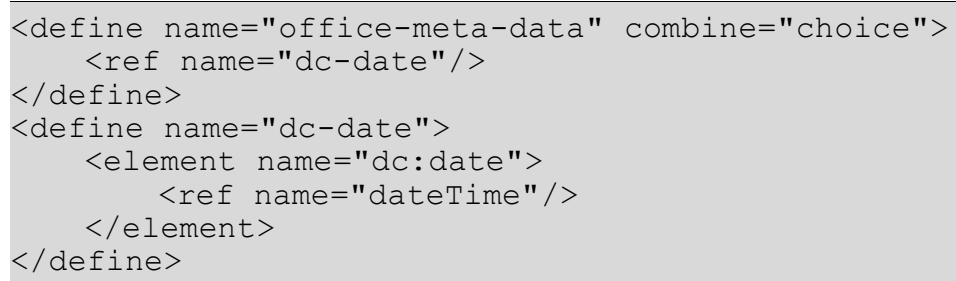

\subsubsection{Print Date and Time}

The <meta: print-date $>$ element specifies the date and time when the document was last printed.

To conform with [xmlschema-2], the date and time format is YYYY-MM-DDThh:mm:ss.

\subsubsection{Document Template}

The $<$ meta: template $>$ element contains a URL for the document template that was used to create the document. The URL is specified as an XLink.

This element conforms to the XLink Specification. See [XLink].

The attributes that may be associated with the <meta:template $>$ element are:

- Template location

- Template title

- Template modification date and time 


\section{Template Location}

An xlink: href attribute specifies the location of the document template.

\section{Template Title}

The xlink: title attribute specifies the name of the document template.

\section{Template Modification Date and Time}

The meta: date attribute specifies the date and time when the template was last modified, prior to being used to create the current document.

To conform with [xmlschema-2], the date and time format is YYYY-MM-DDThh:mm:ss.

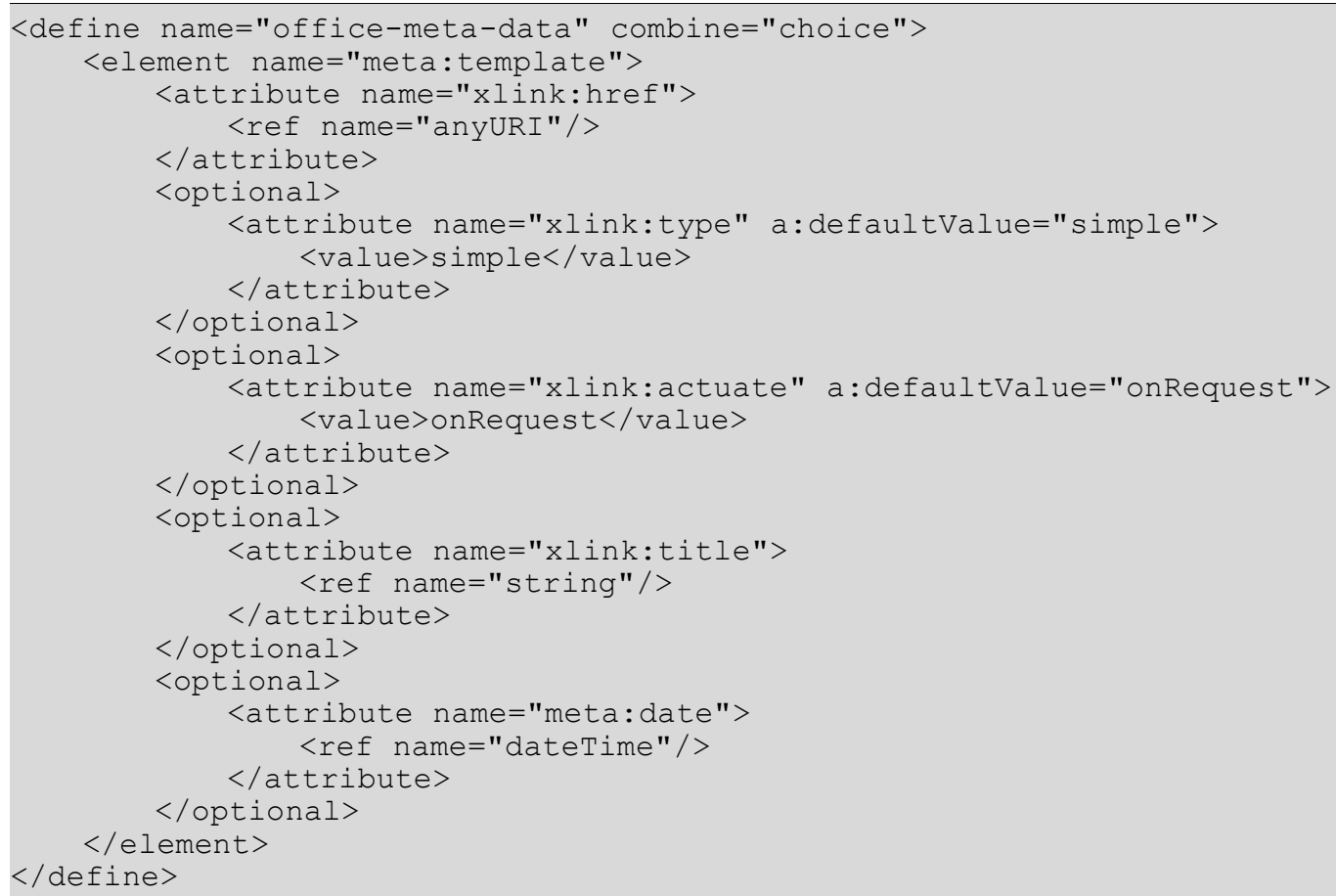

\subsubsection{Automatic Reload}

The <meta: auto-reload> element specifies whether a document is reloaded or replaced by another document after a certain period of time has elapsed.

The attributes that may be associated with the <meta: auto-reload $>$ element are:

- Reload URL

- Reload delay

\section{Reload URL}

If a loaded document should be replaced by another document after a certain period of time, the $<$ meta: auto-reload> element is presented as an XLink. An xlink:href attribute identifies the URL of the replacement document. 


\section{Reload Delay}

The meta: delay attribute specifies the reload delay.

To conform with the duration data type of [xmlschema-2], the format of the value of this attribute is PnYnMnDTnHnMnS. See §3.2.6 of [xmlschema-2] for more detailed information on this duration format.

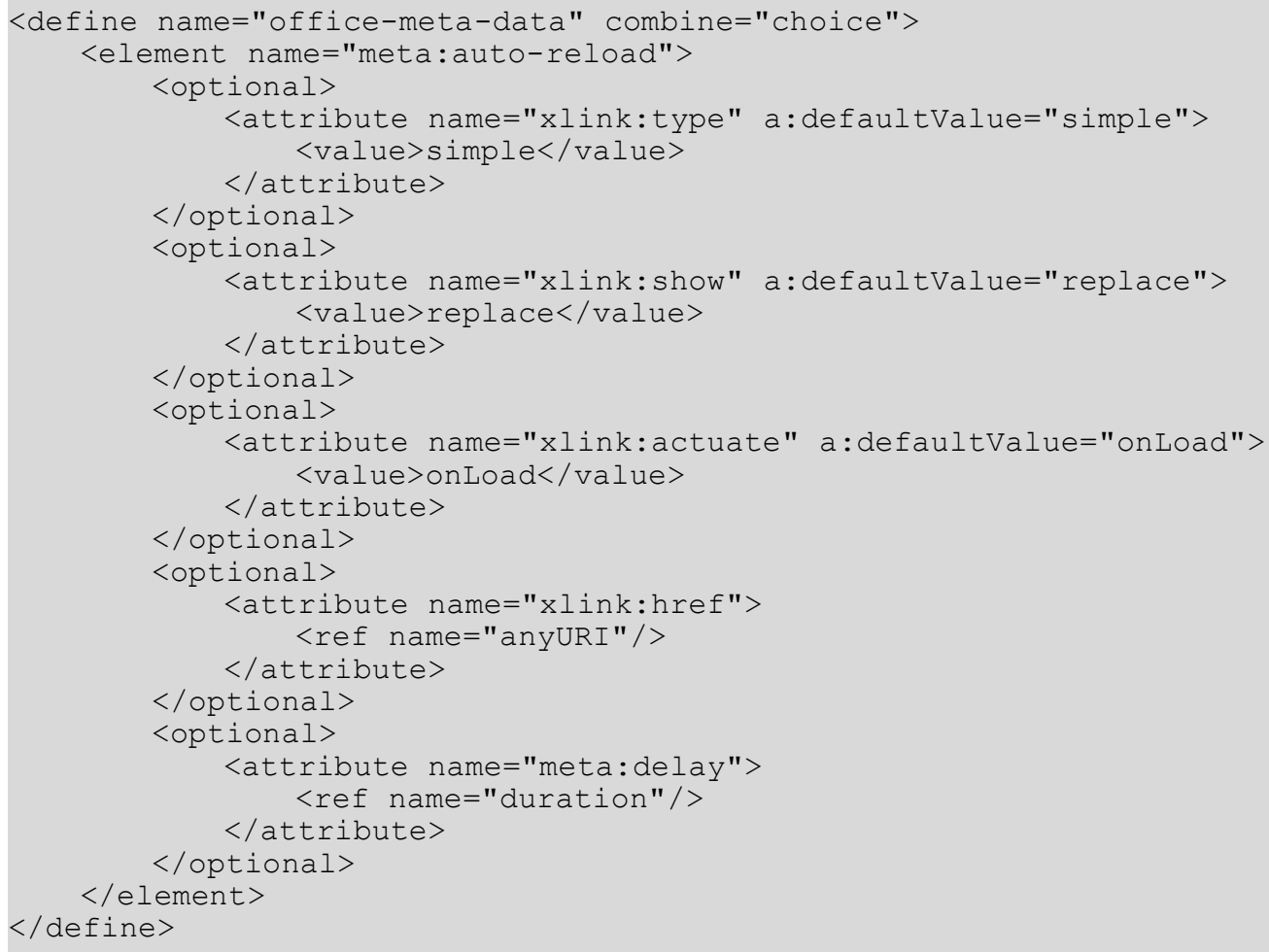

\subsubsection{Hyperlink Behavior}

The <meta: hyperlink-behaviour> element specifies the default behavior for hyperlinks in the document.

The only attribute that may be associated with the <meta: hyperlink-behaviour $>$ element is:

\section{- Target frame}

\section{Target Frame}

The meta:target-frame-name attribute specifies the name of the default target frame in which to display a document referenced by a hyperlink.

This attribute can have one of the following values:

- _ _self : The referenced document replaces the content of the current frame.

- _blank : The referenced document is displayed in a new frame.

- _ parent : The referenced document is displayed in the parent frame of the current frame. 
- _top : The referenced document is displayed in the topmost frame, that is the frame that contains the current frame as a child or descendent but is not contained within another frame.

- A frame name : The referenced document is displayed in the named frame. If the named frame does not exist, a new frame with that name is created.

To conform with the XLink Specification, an additional xlink: show attribute is attached to the $<$ meta:hyperlink-behaviour> element. If the value of the meta:target-frame-name attribute is_blank, the xlink: show attribute value is new. If the value of the meta:targetframe-name attribute is any of the other value options, the value of the $x l i n k:$ show attribute is replace.

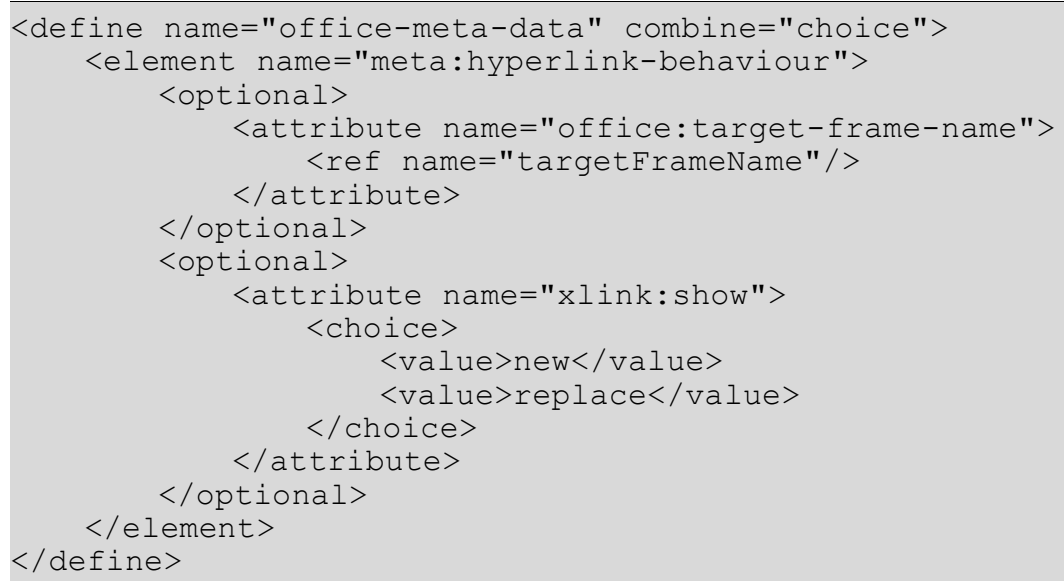

\subsubsection{Language}

The $<\mathrm{dc}$ : language $>$ element specifies the default language of the document.

The manner in which the language is represented is similar to the language tag described in [RFC3066]. It consists of a two or three letter Language Code taken from the ISO 639 standard optionally followed by a hyphen (-) and a two-letter Country Code taken from the ISO 3166 standard.

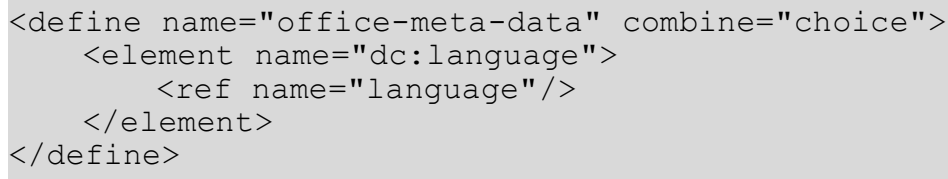

\subsubsection{Editing Cycles}

The <meta: editing-cycles $>$ element specifies the number of editing cycles the document has been through.

The value of this element is incremented every time the document is saved. The element contains the number of editing cycles as text.

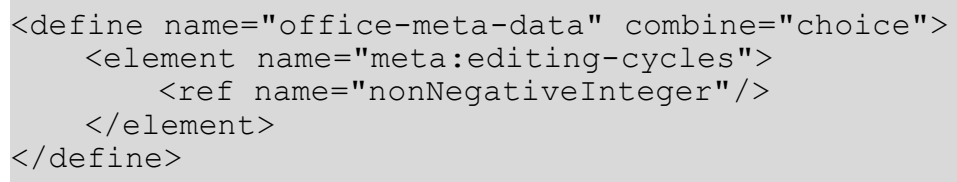




\subsubsection{Editing Duration}

The <meta:editing-duration> element specifies the total time spent editing the document.

The duration is represented in the duration data type of [xmlschema-2], that is PnYnMnDTnHnMnS. See §3.2.6 of [xmlschema-2] for more detailed information on this duration format.

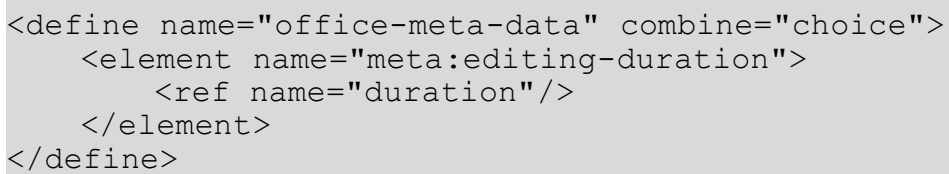

\subsubsection{Document Statistics}

The <meta: document-statistic $>$ element specifies the statistics of the document, for example, the page count, word count, and so on. The statistics are specified as attributes of the $<$ meta: document-statistic $>$ element and the statistics that are exported with the document depend on the document type and the application used to create the document.

\begin{tabular}{|c|c|}
\hline $\begin{array}{c}\text { Document } \\
\text { Type }\end{array}$ & Document Statistics Attributes \\
\hline Text & $\begin{array}{l}\text { meta: page-count } \\
\text { meta: table-count } \\
\text { meta: draw-count } \\
\text { meta: image-count } \\
\text { meta: ole-object-count } \\
\text { meta: paragraph-count } \\
\text { meta: word-count } \\
\text { meta: character-count } \\
\text { meta: row-count } \\
\text { meta: frame-count } \\
\text { meta: sentence-count } \\
\text { meta: syllable-count } \\
\text { meta: non-whitespace-character-count }\end{array}$ \\
\hline Spreadsheet & $\begin{array}{l}\text { meta: page-count } \\
\text { meta:table-count } \\
\text { meta: image-count } \\
\text { meta:cell-count } \\
\text { meta: object-count }\end{array}$ \\
\hline Graphic & $\begin{array}{l}\text { meta: page-count } \\
\text { meta: image-count } \\
\text { meta:object-count }\end{array}$ \\
\hline
\end{tabular}

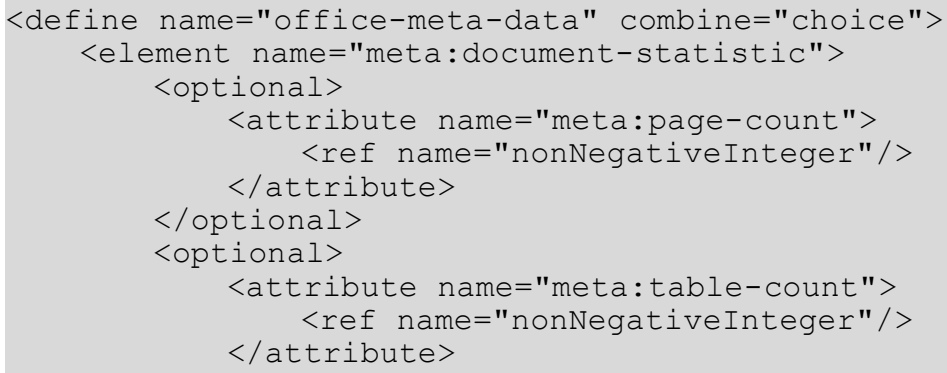




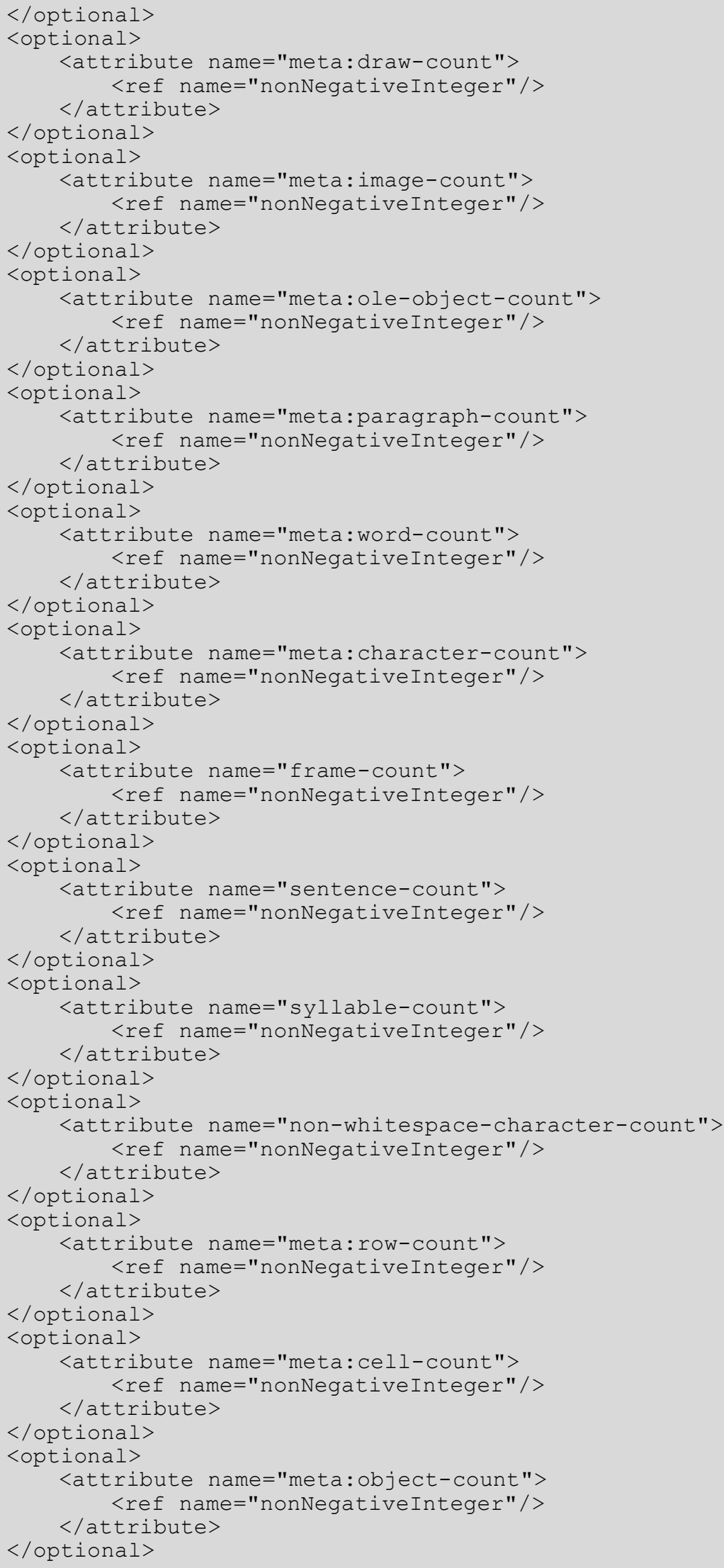




\subsection{User-defined Metadata}

The <meta: user-defined> element specifies any additional user-defined metadata for the document. Each instance of this element can contain one piece of user-defined metadata. The element contains:

- A meta: name attribute, which identifies the name of the metadata element.

- An optional meta: type attribute, which identifies the type of the metadata element. The allowed meta types are float, date, time, boolean and string (see also section 6.7.1).

- $\quad$ (see section 6.7.1).

- The value of the element, which is the metadata in the format described in section 6.7.1 as value of the office:value attributes for the various data types.

The default type for meta-data elements is string.

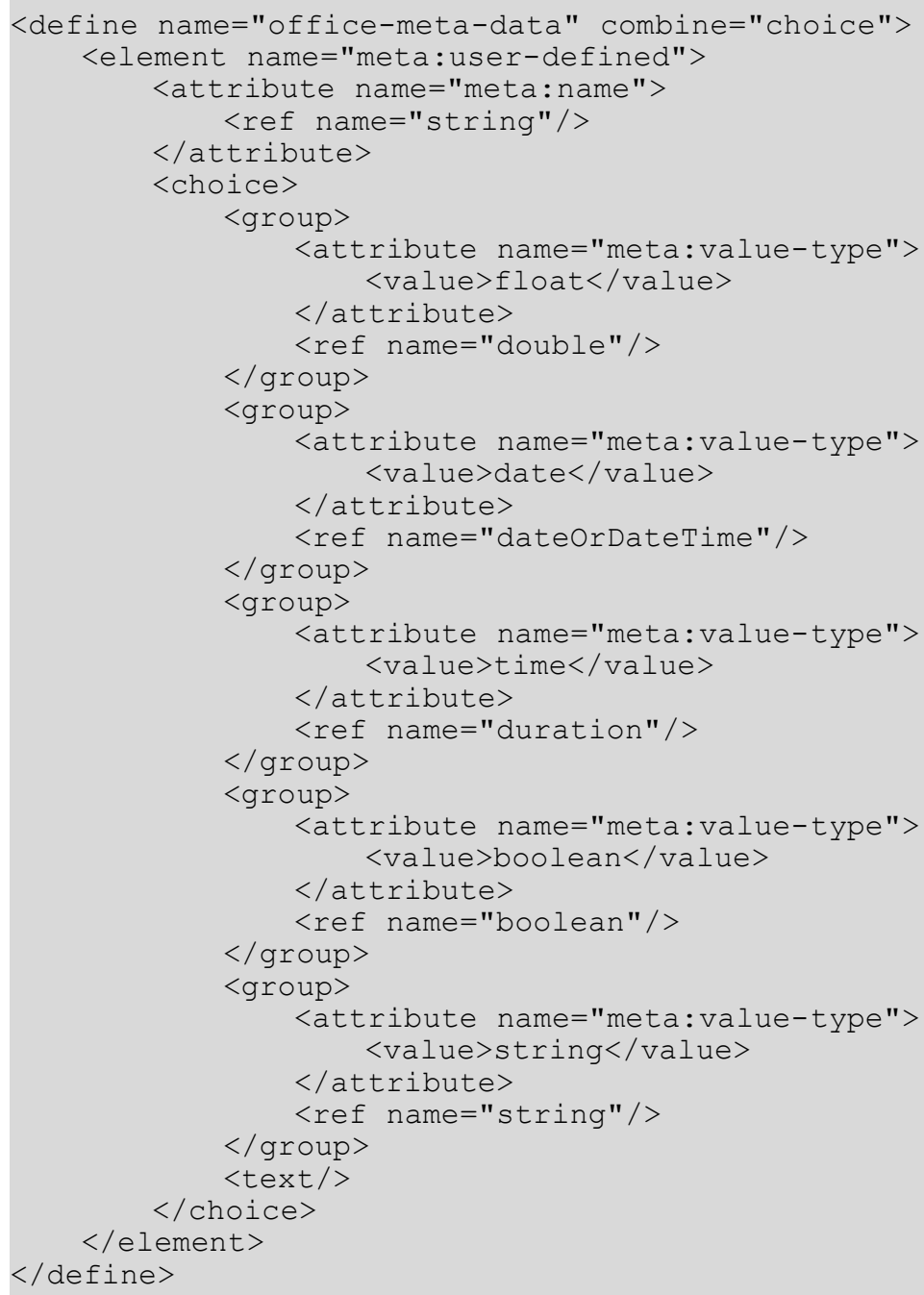




\subsection{Custom Metadata}

In addition to the pre-defined metadata elements, applications should also preserve any additional content found inside the <office:meta> element. As there is no semantics specified for such foreign content, applications need not process this information other than to preserve it when editing the document. 


\section{Text Content}

\subsection{Headings, Paragraphs and Basic Text Structure}

This section describes the XML elements and attributes that are used to represent heading and paragraph components in a text document.

The elements $<$ text: $h>$ and $<$ text $: p>$ represent headings and paragraphs, respectively, and are collectively referred to as paragraph elements. All text content in an OpenDocument file must be contained in either of these elements.

\subsubsection{Headings}

Headings define the chapter structure for a document. A chapter or subchapter begins with a heading and extends to the next heading at the same or higher level.

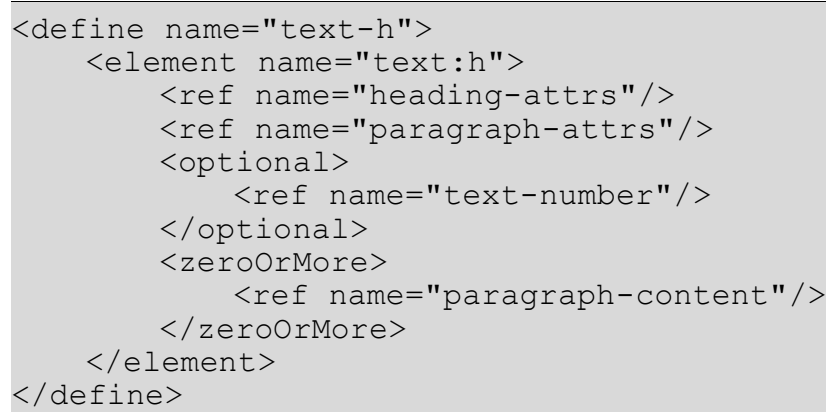

\section{Heading Level}

The text: outline-level attribute associated with the heading element determines the level of the heading, starting with 1 . Headings without a level attribute are assumed to be at level 1.

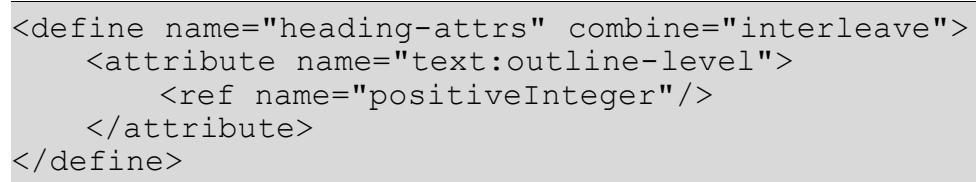

\section{Heading Numbering}

Header numbering can be changed by additional attributes, similar to those on list items (see section 4.3.2, below). The numbering of headers can be restarted by setting the text:restart-numbering attribute to true.

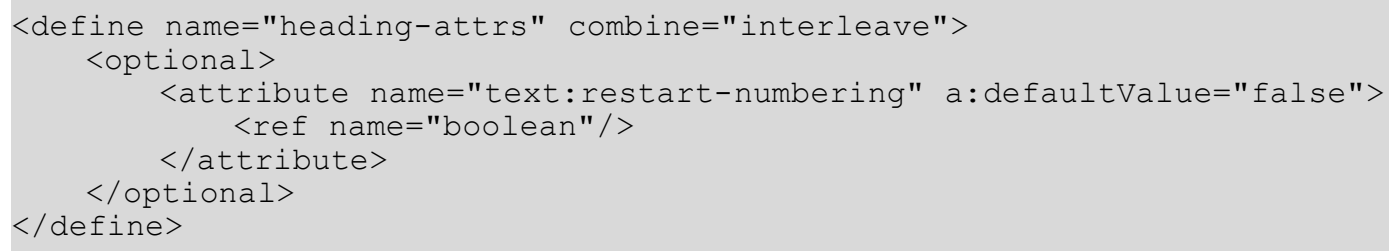




\section{Start Value}

The attribute text:start-value may be used to restart the numbering of headers of the current header's level, by setting a new value for the numbering.

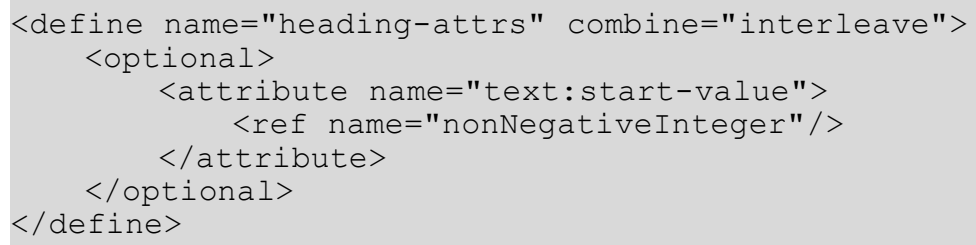

\section{Suppress Header Numbering}

It is sometimes desired to have a specific heading which should not be numbered. This corresponds to unnumbered list headers in lists (see sections 4.3). To facilitate this, an optional attribute text: is-list-header can be used. If true, the given header will not be numbered, even if an explicit list-style is given.

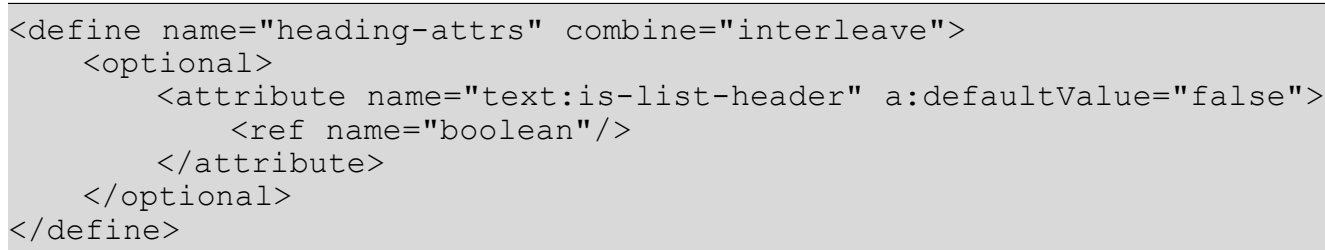

\section{Formatted Heading Number}

If a heading has a numbering applied, the text of the formatted number can be included in a $<$ text: number > element. This text can be used by applications that do not support numbering of headings, but it will be ignored by applications that support numbering.

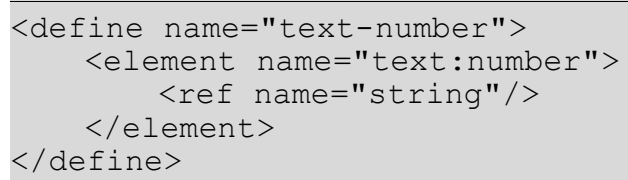

\subsubsection{Paragraphs}

Paragraphs are the basic unit of text.

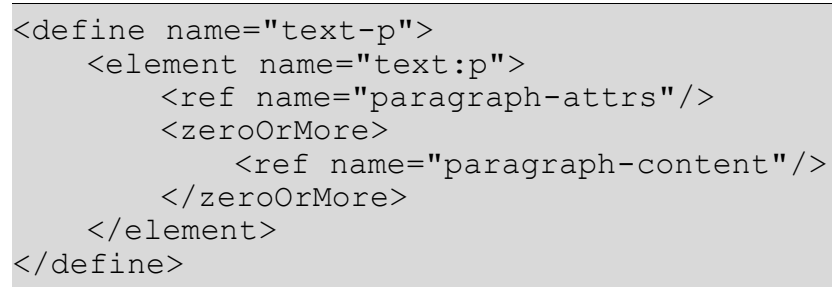

\subsubsection{Common Paragraph Elements Attributes}

The paragraph elements have text:style-name, text:class-names and text:condstyle-name attributes. These attributes must reference paragraph styles.

A text:style-name attribute references a paragraph style, while a text:cond-style-name attribute references a conditional-style, that is, a style that contains conditions and maps to other 
styles (see section 14.1.1). If a conditional style is applied to a paragraph, the text:stylename attribute contains the name of the style that was the result of the conditional style evaluation, while the conditional style name itself is the value of the text:cond-style-name attribute. This XML structure simplifies [XSLT] transformations because XSLT only has to acknowledge the conditional style if the formatting attributes are relevant. The referenced style can be a common style or an automatic style.

A text:class-names attribute takes a whitespace separated list of paragraph style names. The referenced styles are applied in the order they are contained in the list. If both, text:style-name and text:class-names are present, the style referenced by the text:style-name attribute is as the first style in the list in text:class-names. If a conditional style is specified together with a style:class-names attribute, but without the text:style-name attribute, then the first style in the style list is used as the value of the missing text: style-name attribute.

Conforming applications should support the text:class-names attribute and also should preserve it while editing.

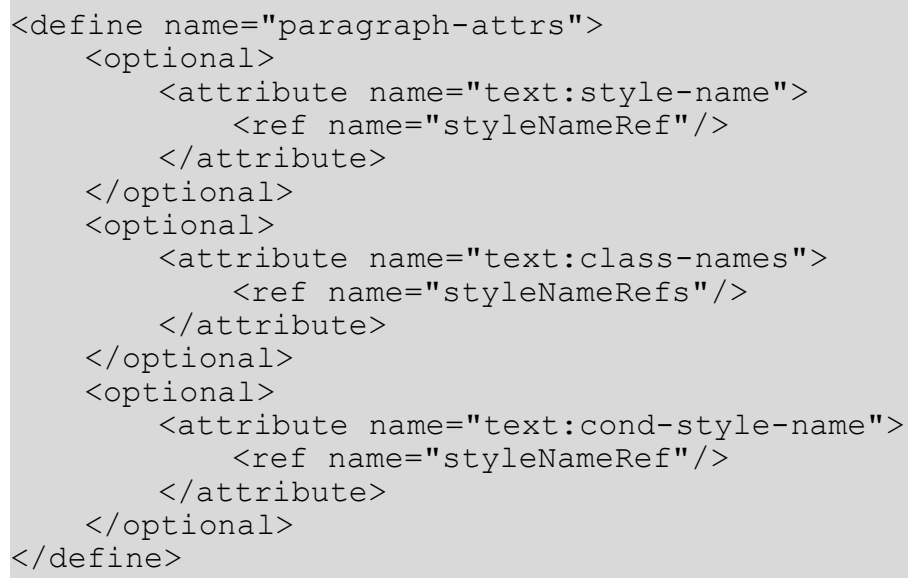

\section{Example: Styles and conditional styles}

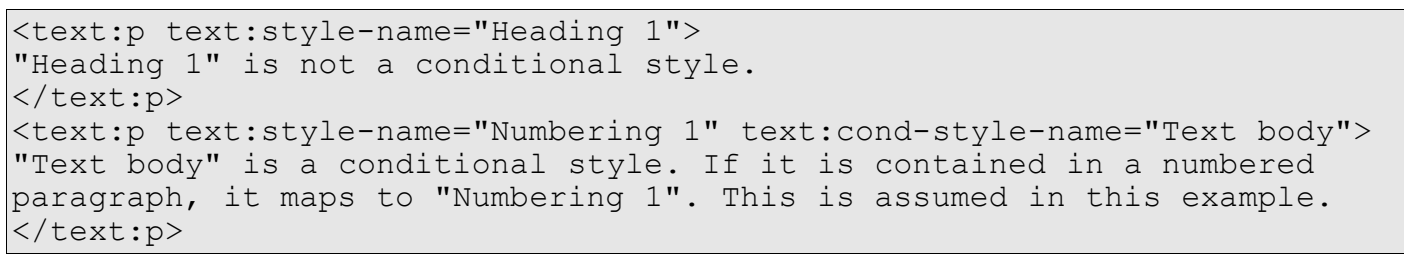

A paragraph may have an ID. This ID can be used to reference the paragraph from other elements.

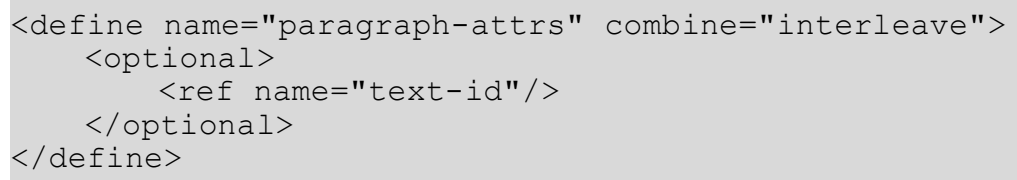

\subsection{Page Sequences}

A page sequence element $<$ text: page-sequence $>$ specifies a sequence of master pages that are instantiated in exactly the same order as they are referenced in the page sequence. If a text document contains a page sequence, it will consist of exactly as many pages as specified. Documents with page sequences do not have a main text flow consisting of headings and paragraphs as is the case for documents that do not contain a page sequence. Text content is 
included within text boxes for documents with page sequences. The only other content that is permitted are drawing objects.

\section{Example: Page Sequence}

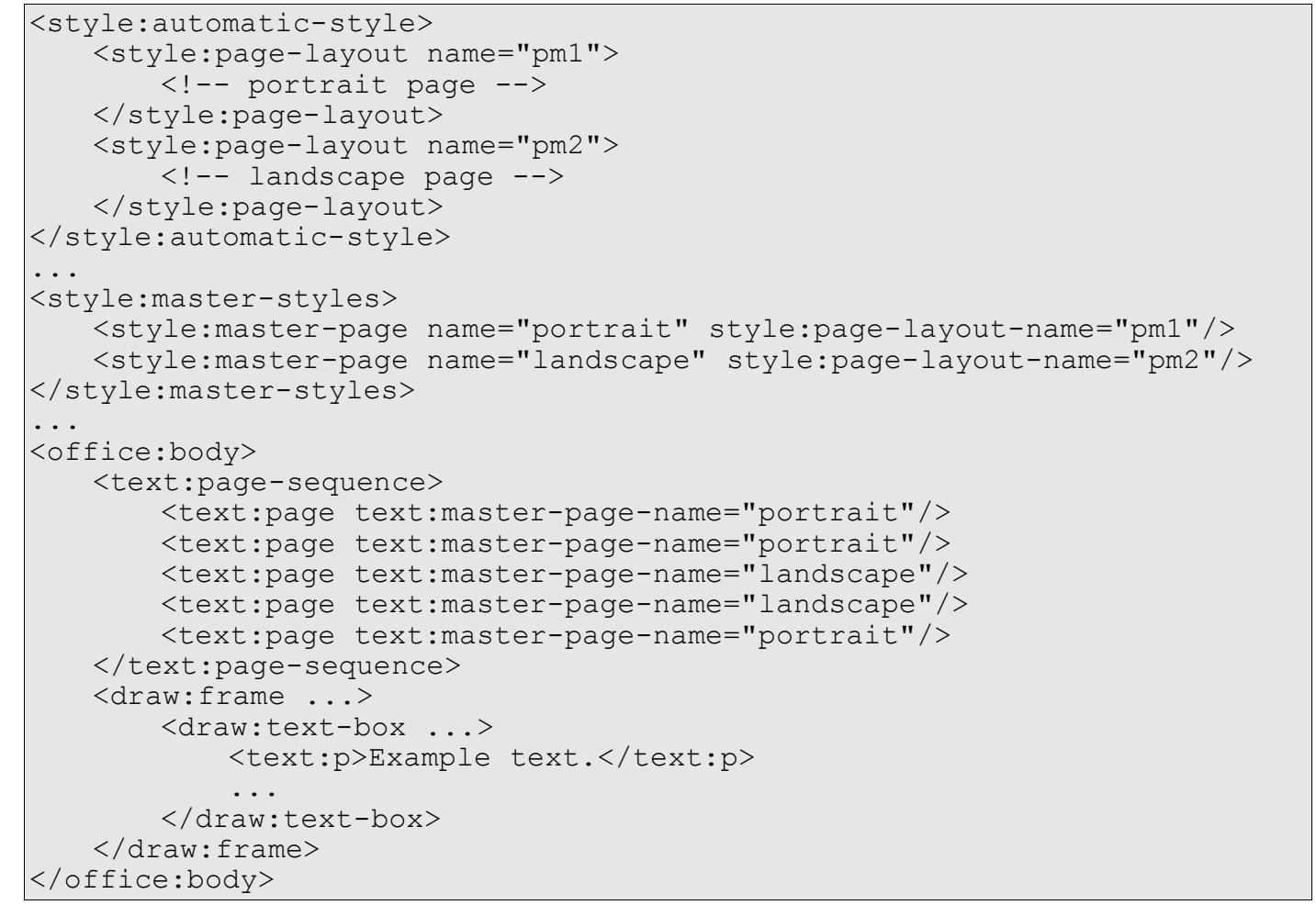

\subsubsection{Page}

The <text:page $>$ element specifies a single page within a page sequence.

\section{Master Page Name}

The text:master-page-name attribute specifies the master page that is instantiated.

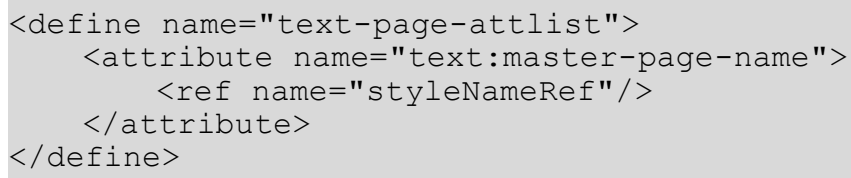




\subsection{Lists}

The OpenDocument format supports list structures, similar to those found in [HTML4]. A list is a paragraph-level element, which contains an optional list header, followed by a sequence of list items. The list header and each list item contains a sequence of paragraph or list elements. Lists can be nested.

Lists may be numbered. The numbering may be restarted with a specific numbering at each list item. Lists may also continue numbering from other lists, allowing the user to merge several lists into a single, discontinuous list. Note that whether the list numbering is displayed depends on a suitable list style being used.

In addition to this structural information, lists can have list styles associated with them, which contain the relevant layout information, such as

- the type of list item label, such as bullet or number,

- list item label width and distance,

- bullet character or image (if any),

- number format for the bullet numbering (if any),

- paragraph indent for list items.

\subsubsection{List Block}

A list is represented by the <text: list $>$ element. It contains an optional list header, followed by any number of list items.

Every list has a list level, which is determined by the nesting of the <text: 1 ist $>$ elements. If a list is not contained within another list, the list level is 1 . If the list in contained within another list, the list level is the list level of the list in which is it contained incremented by one. If a list is contained in a table cell or text box, the list level returns to 1 , even though the table or textbox itself may be nested within another list.

The attributes that may be associated with the list element are:

- Style name

- Continue numbering

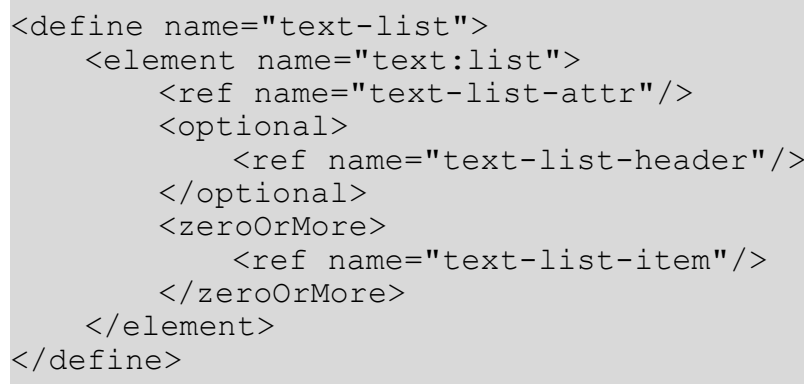

\section{Style Name}

The optional text:style-name attribute specifies the name of the list style that is applied to the list. 
If this attribute is not included and therefore no list style is specified, one of the following actions is taken:

- If the list is contained within another list, the list style defaults to the style of the surrounding list.

- If there is no list style specified for the surrounding list, but the list contains paragraphs that have paragraph styles attached specifying a list style, this list style is used for any of these paragraphs.

- A default list style is applied to any other paragraphs.

To determine which formatting properties are applied to a list, the list level and list style name are taken into account. See section $\mathbf{1 4 . 1 0}$ for more information on list formatting properties.

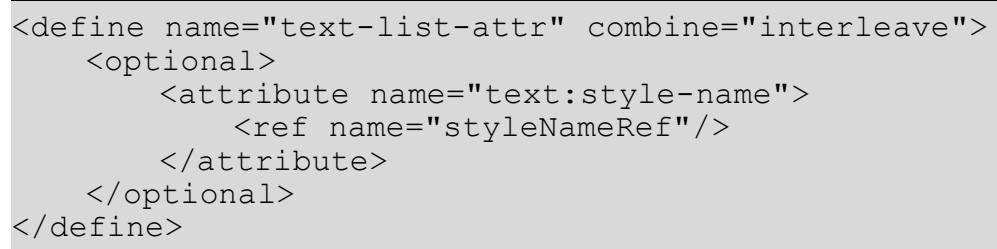

\section{Continue Numbering}

By default, the first list item in a list starts with the number specified in the list style. The continue numbering attribute can be used to continue the numbering from the preceding list.

This attribute can be used with the <text: list> element and can have a value of true or false.

If the value of the attribute is true and the numbering style of the preceding list is the same as the current list, the number of the first list item in the current list is the number of the last item in the preceding list incremented by one.

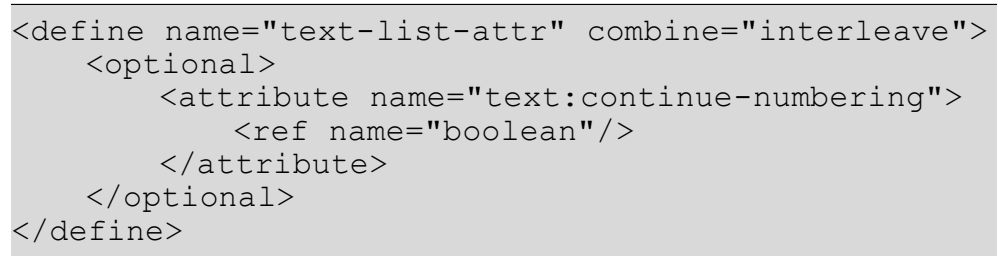

\subsubsection{List Item}

List items contain the textual content of a list. $A<$ text: list-item> element can contain paragraphs or lists. A list item cannot contain headings or tables.

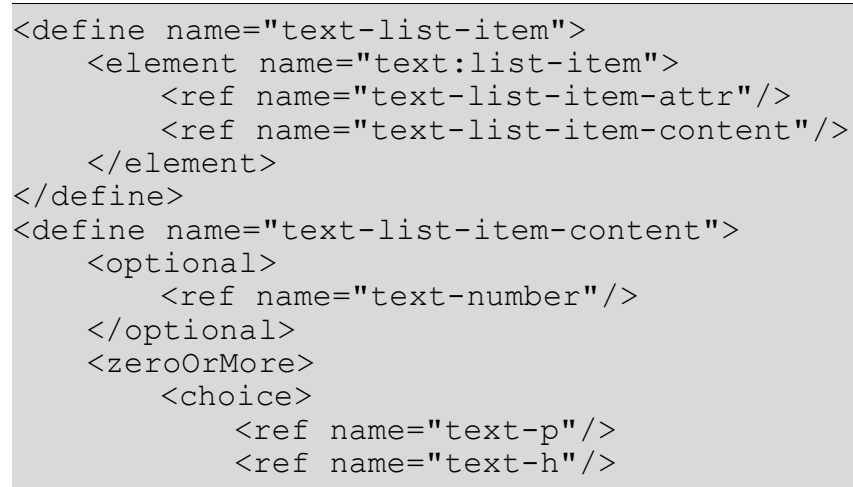




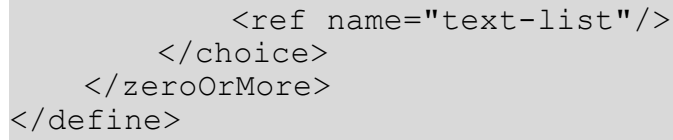

The first line in a list item is preceded by a bullet or number, depending on the list style assigned to the list. If a list item starts another list immediately and does not contain any text, no bullet or number is displayed.

The only attribute that may be associated with the <text: list-item> element is:

\section{- Start value}

\section{Start Value}

The numbering of the current list can be restarted at a certain number. The text: startvalue attribute is used to specify the number with which to restart the list.

This attribute can only be applied to items in a list with a numbering list style. It restarts the numbering of the list at the current item.

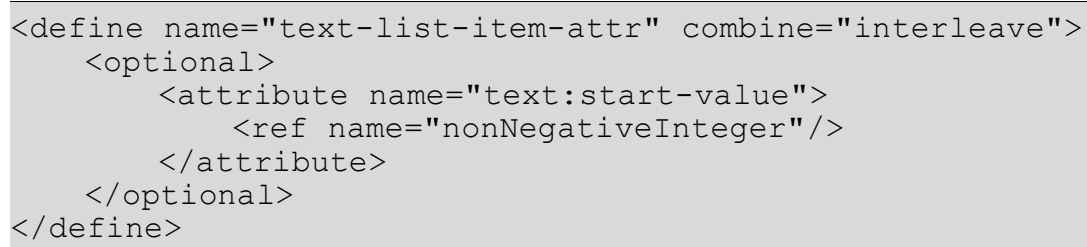

\section{Formatted Number}

If a list item has a numbering applied, the text of the formatted number can be included in a $<$ text: number> element. This text can be used by applications that do not support numbering, but it will be ignored by applications that support numbering. See also section 4.1.1.

\section{Example: Lists and sublists}

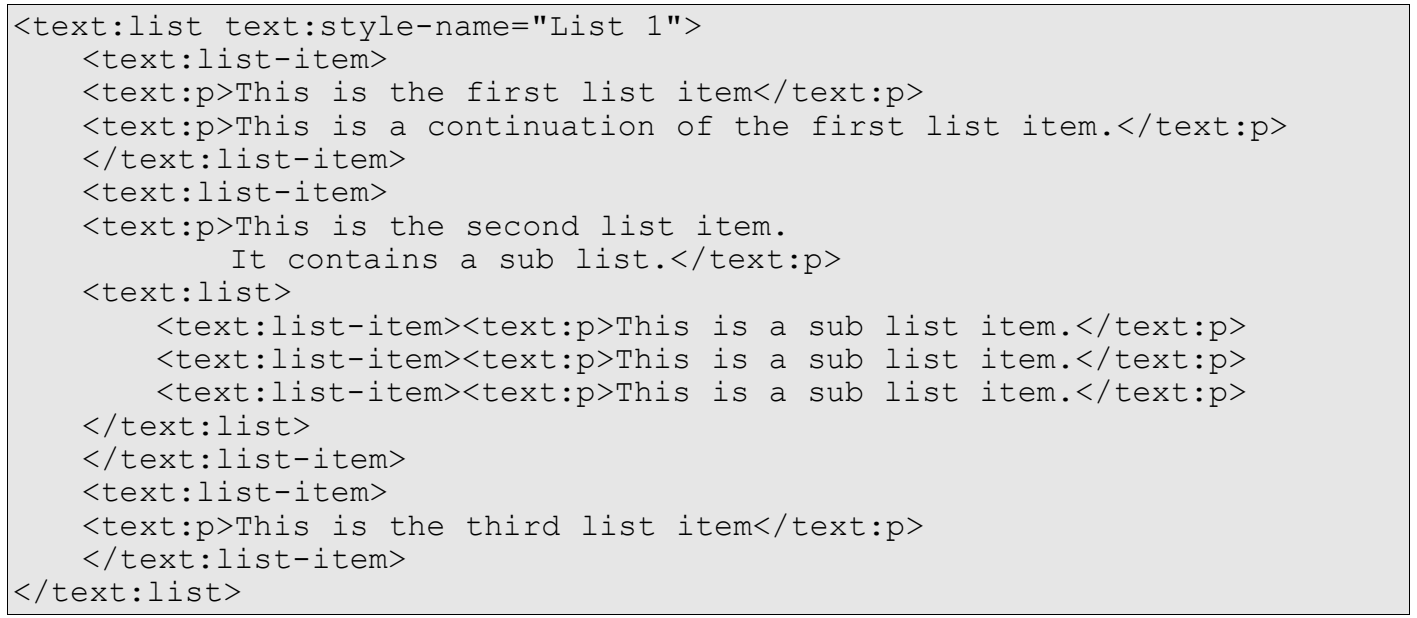

\subsubsection{List Header}

A list header is a special kind of list item. It contains one or more paragraphs that are displayed before a list. The paragraphs are formatted like list items but they do not have a preceding number or bullet. The list header is represented by the list header element. 


\subsubsection{Numbered Paragraphs}

In some instances, it is desirable to specify a list not as a structural element comprising of several list items, but to determine on a per-paragraph level whether the paragraph is numbered, and at which level. To facilitate this, the <text: numbered-paragraph> element allows the numbering of an individual paragraph, as if it was part of a list at a specified level.

Numbered paragraphs may use the same continuous numbering properties that list items use, and thus form an equivalent, alternative way of specifying lists. A list in <text: list> representation could be converted into a list in <text: numbered-paragraph $>$ representation and vice versa.

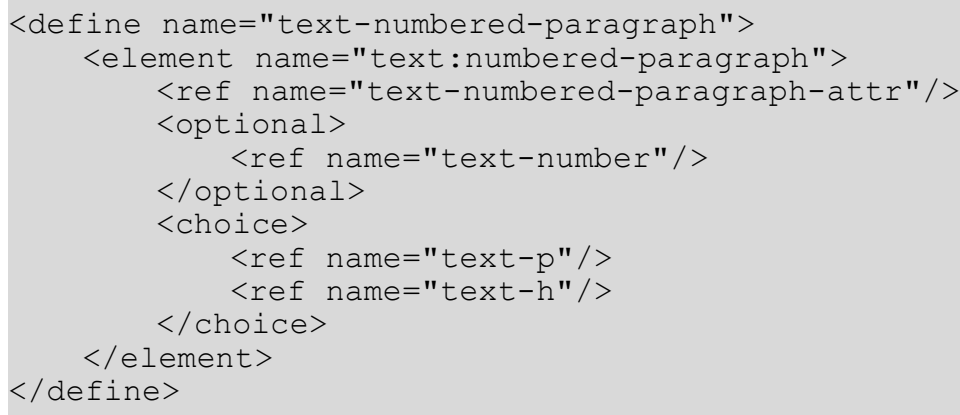

A numbered paragraph can be assigned a list level. A numbered paragraph is equivalent to a list nested to the given level, containing one list item with one paragraph. If no level is given, the numbered paragraph is interpreted as being on level 1.

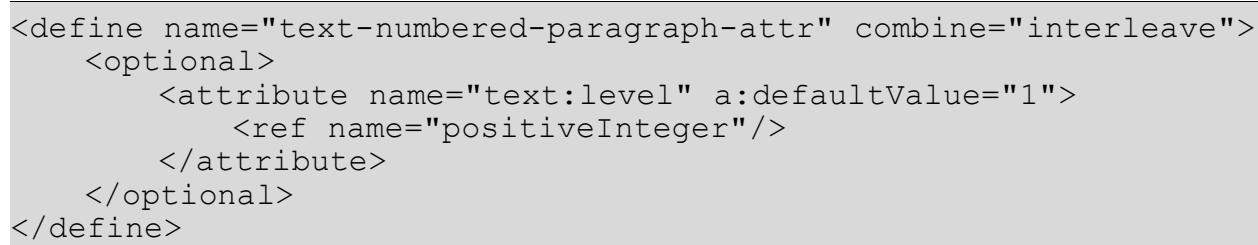

As a numbered paragraph combines the functionality of a (possibly nested) list with a single list item, it can also use the attributes of those elements.

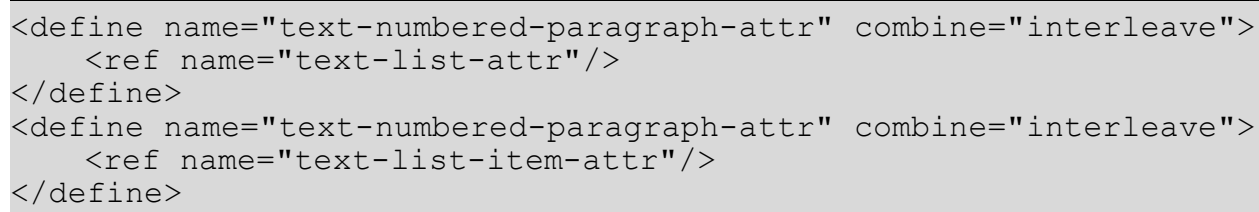

The text of a formatted number can be included in a <text: number $>$ element. This text can be used by applications that do not support numbering, but it will be ignored by applications that support numbering. See also section 4.1.1.

\subsection{Text Sections}

A text section is a named region of paragraph-level text content. Sections start and end on paragraph boundaries and can contain any number of paragraphs. 
Sections have two uses in the OpenDocument format: They can be used to assign certain formatting properties to a region of text. They can also be used to group text that is automatically acquired from some external data source.

In addition to Sections can contain regular text content or the text can be contained in another file and linked to the section. Sections can also be write-protected or hidden.

Sections can have settings for text columns, background color or pattern, and notes configuration. These settings form the section style, which is represented in a style:style> element. See section 14.8.3 for details.

The formatting properties for sections are explained in section 15.7.

Sections support two ways of linking to external content. If a section is linked to another document, the link can be through one of the following:

- A resource identified by an XLink, represented by a text: section-source element

- Dynamic Data Exchange (DDE), represented by a office: dde-source element

Linking information for external content is contained in the section element's first child. A section that links to external content contains the full representation of the data source, so that processors need to understand the linking information only if they wish to update the contents of the section.

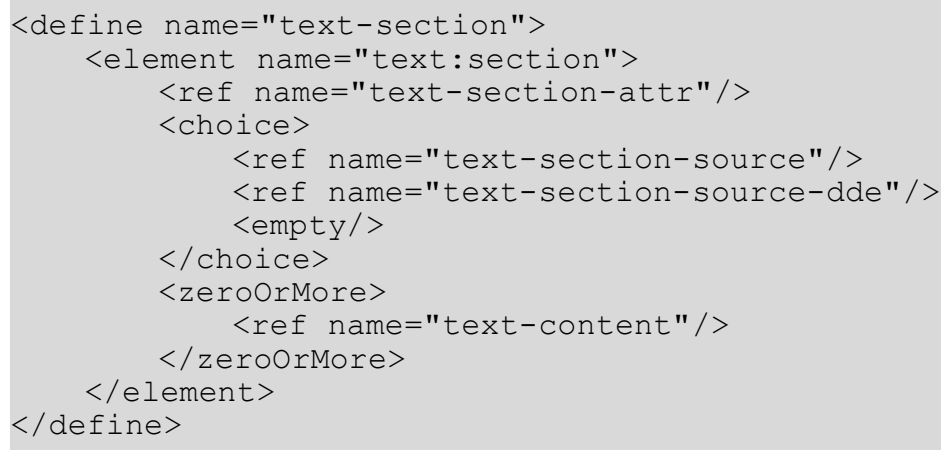

Note: List items may not contain sections. Thus, lists may only be wholly contained within section elements. If it is desired to achieve the effect of overlapping lists and sections, or of sections contained within lists, the lists must be split into several lists, each of which would then be wholly contained within a section. When splitting the list, suitable attributes for continuos numbering should be set such that display and behavior are the same as with the original list not interrupted by sections.

\subsubsection{Section Attributes}

Text indices, described in chapter 7 , may be considered a special kind of text section, as they share the same general structure as well as certain attributes. These are combined in the following definition:

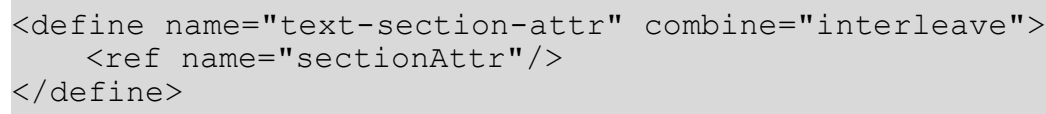

The remaining attributes in this section are specific to the $<$ text: section $>$ element.

\section{Section Style}

The text:style-name attribute refers to a section style. 


\section{$</$ define $>$}

\section{Protected Sections}

A section can be protected, which means that a user can not edit the section. The text:protected attribute indicates whether or not a section is protected. The user interface must enforce the protection attribute if it is enabled.

A user can use the user interface to reset the protection flag, unless the section is further protected by a password. In this case, the user must know the password in order to reset the protection flag. The text:protection-key attribute specifies the password that protects the section. To avoid saving the password directly into the XML file, only a hash value of the password is stored.

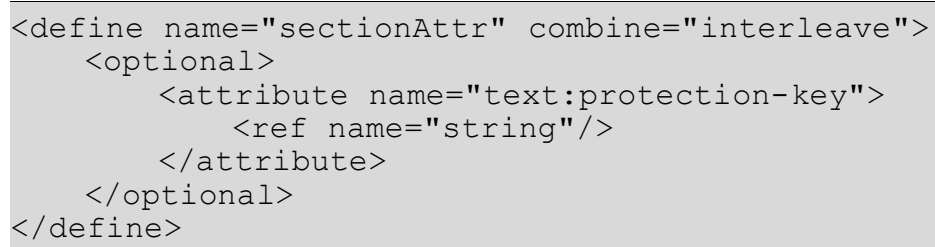

\section{Hidden Sections and Conditional Sections}

Sections can be hidden based on a condition or they can be hidden unconditionally.

The text: display attribute specifies whether or not the section is hidden. The value of this attribute can be:

- true, the section is displayed. This is the default setting.

- none, the section is hidden unconditionally.

- condition, the section is hidden under the condition specified in the text:condition attribute. 
The text: condition attribute specifies the condition under which the section is hidden. The condition is encoded as a string. If the value of text: display is condition, the text: condition attribute must be present.

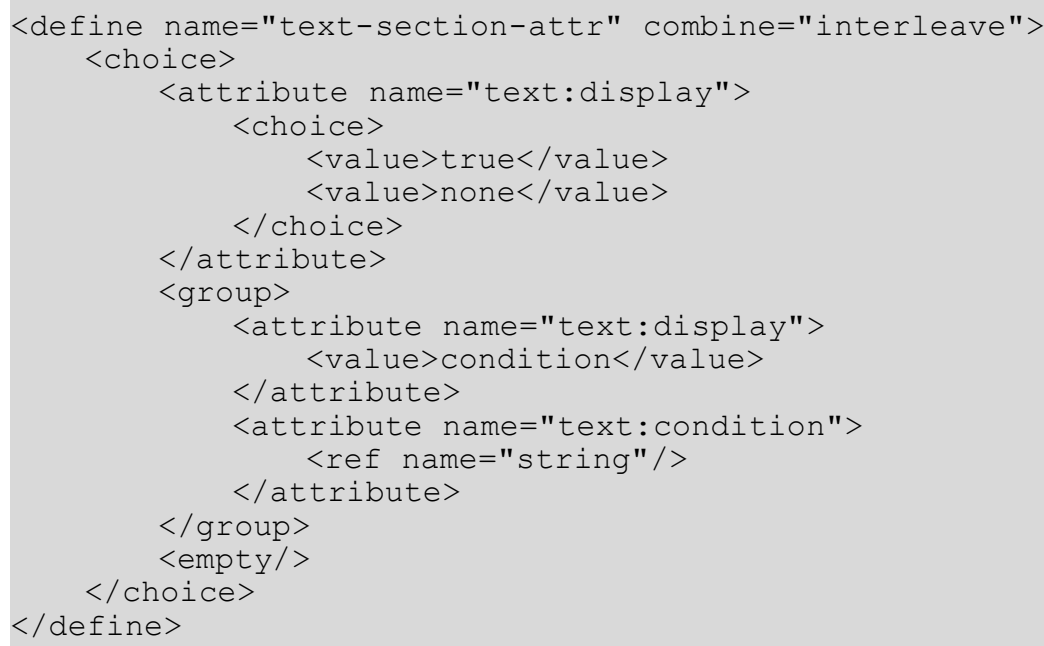

\subsubsection{Section Source}

The <text: section-source> element indicates that the enclosed section is a linked section. If this element is used, it must be the first element in the <text: section> element.

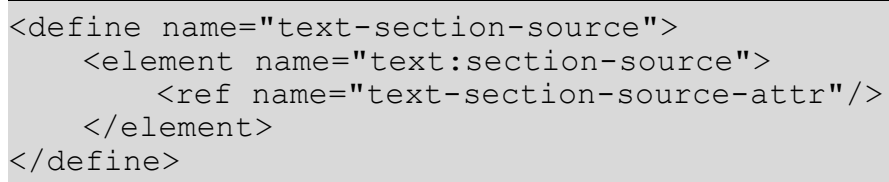

The attributes that may be associated with the <text: section-source> attribute are:

- Section source URL

- Name of linked section

- Filter name

\section{Section Source URL}

These attributes identify the document or section to which the section is linked. The name of the target section is identified by the local part of the URL, following the hash mark. The xlink: href attribute is implied because <text:section-source> elements may also link to internal sections.

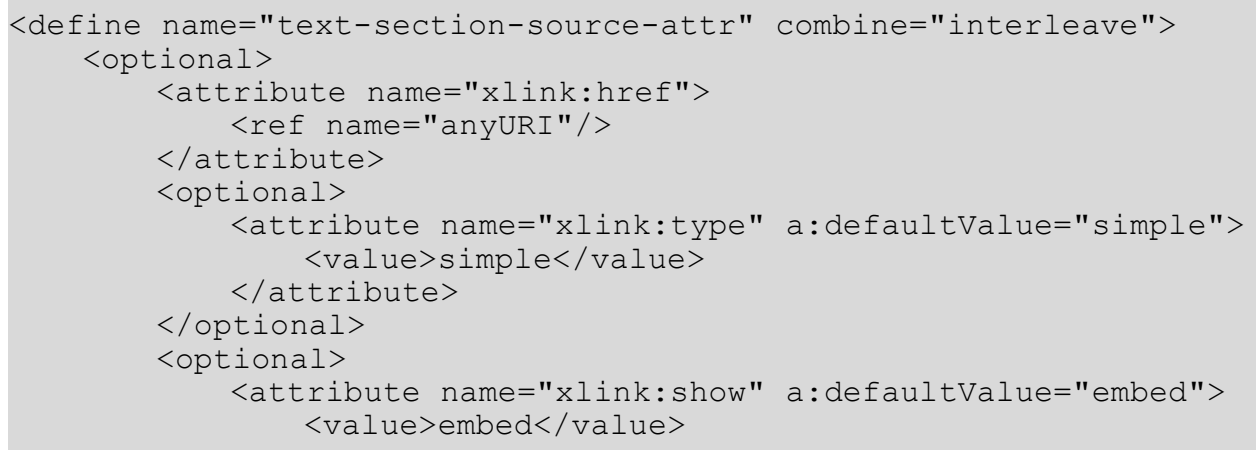




\section{Name of Linked Section}

If the link targets a section of a document, the attribute text: section name contains the name of the target section. If the attribute is not present, the link targets the entire document.

\section{Filter Name}

The text:filter-name attribute specifies which filter type was used to import the link target. The value of this attribute is implementation dependent.

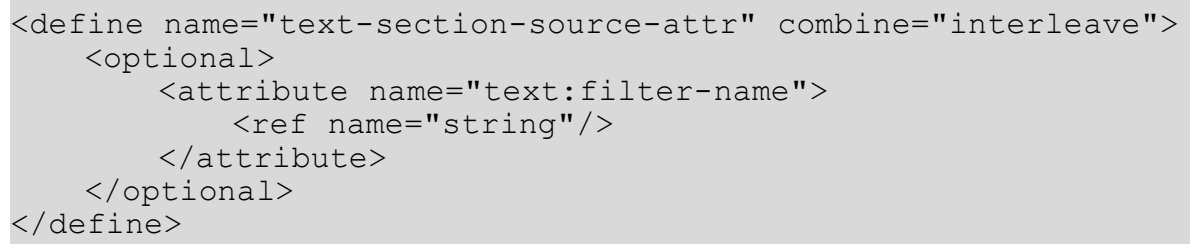

\subsubsection{DDE Source}

If sections are linked via DDE, their linking information is represented by <office: ddesource> elements. It contains attributes that specify the application, topic and item of the DDE connection. Note that because the section contains the XML rendition of the DDE link's content, this information only needs to be processed if updated data from the DDE link are desired.

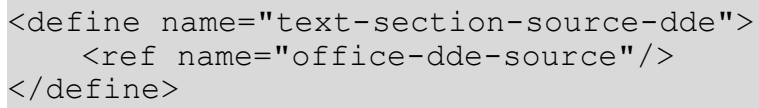

\subsection{Page-bound graphical content}

Within text documents, images, embedded objects and other drawing objects appear at the level of a paragraph if they are anchored to a page rather than to a paragraph or a character position within a paragraph. See section 9.2 for details on drawing objects, and section 9.2.16 for their anchoring.

\subsection{Change Tracking}

This section describes how changes in text documents can be represented. 


\subsubsection{Tracked Changes}

All tracked changes to text documents are stored in a list. The list contains an element for each change made to the document. If the <text:tracked-changes> element is absent, change tracking is not enabled.

\section{$</$ define $>$}

\section{Track Changes}

This attribute determines whether or not user agents should track and record changes for this document.

\section{Change ID}

Every element has an ID. The elements that mark the start and end of a region use this ID to identify the region to which they belong.

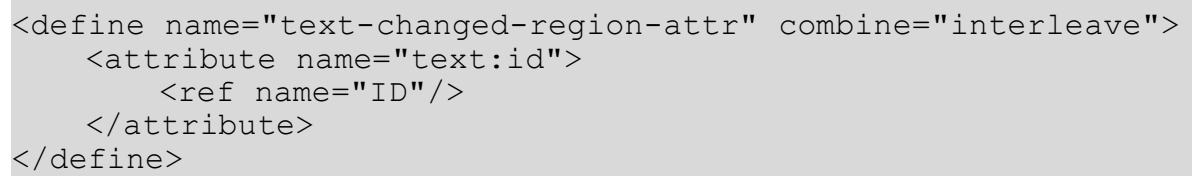

\subsubsection{Insertion}

The <text: insertion> element contains the information that is required to identify any insertion of content. This content can be a piece of text within a paragraph, a whole paragraph, or a whole table. The inserted content is part of the text document itself and is marked by a change start and a change end element. 


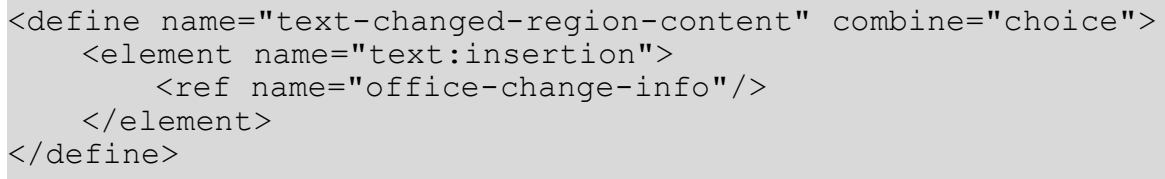

Example: Insertion of text

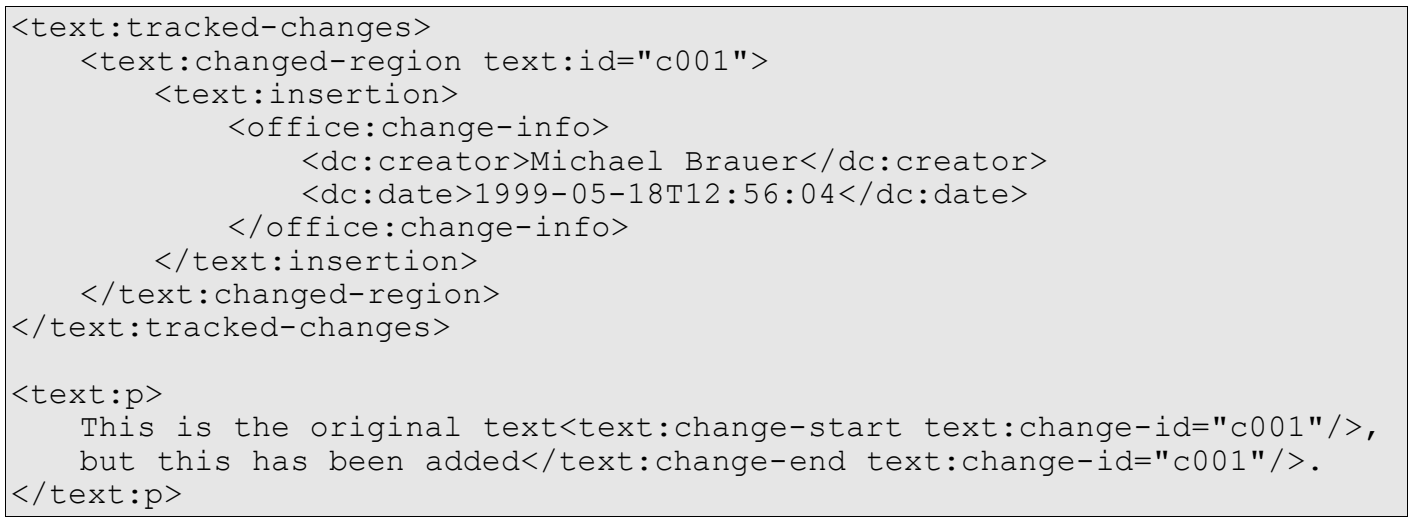

\subsubsection{Deletion}

A <text: deletion> element contains content that was deleted while change tracking was enabled. The position where the text was deleted is marked by the change position element.

If part of a paragraph was deleted, the text that was deleted is contained in this element as a paragraph element. If the deleted text is reinserted into the document, the paragraph is joined with the paragraph where the deletion took place.

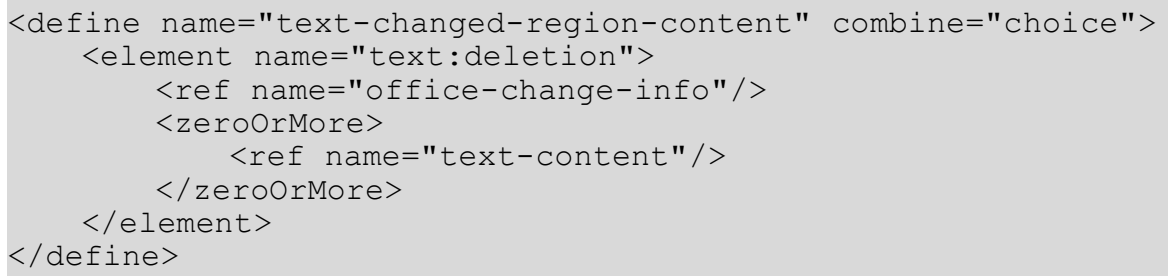

\section{Example: Deletion of text}

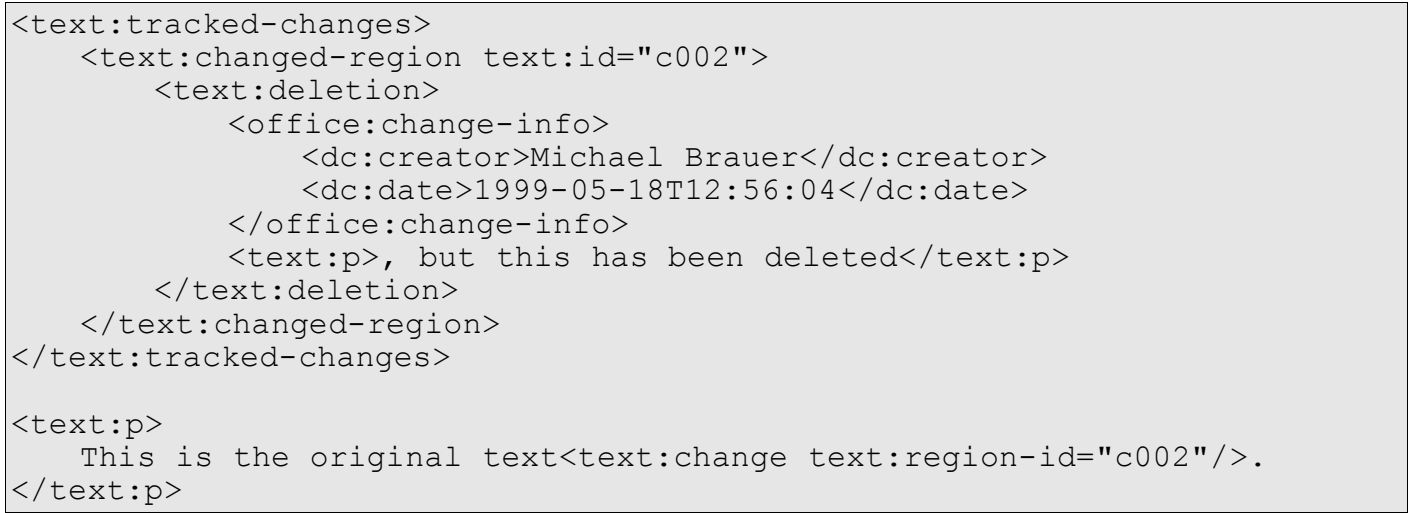

This example shows:

- $\quad$ Deleted text $=$, but this has been deleted

This text is contained in the $<$ text: $p>$ element within the $<$ text: deletion $>$ element. 
- Current text $=$ This is the original text.

This text is contained in the <text: $p>$ element at the end of the example.

- Original text before deletion took place $=$ This is the original text, but this

has been deleted.

Note that the deleted text, like all text in the OpenDocument format, is contained in a paragraph element. To reconstruct the original text, this paragraph is merged with its surrounding. In other words, a deletion consisting of only a single word would be represented as a paragraph containing the word.

To reconstruct the text before the deletion took place, do:

- If the change mark is inside a paragraph, insert the text content of the <text:deletion> element as if the beginning <text:p> and final $</$ text:p> tags were missing.

- If the change mark is inside a header, proceed as above, except adapt the inserted tags to math their new counterparts.

- Otherwise, simply copy the text content of the <text:deletion> element in place of the change mark.

Example: Given the following change:

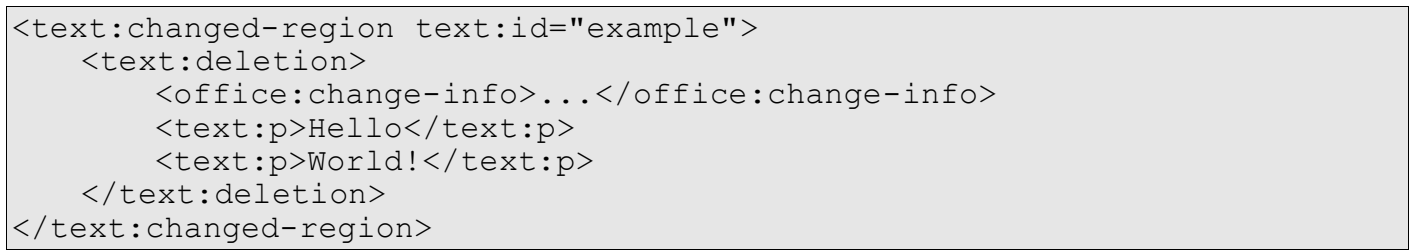

The first (and most common) case occurs if a change mark is inside a regular paragraph:

<text:p>abc<text:change text:id="example/>def</text:p>

To reconstruct the original text, the two $<$ text:p> elements are copied to replace the change mark, except the beginning and ending tags are missing:

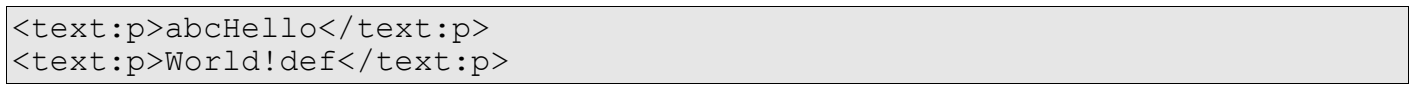

If the change mark occurred inside a header, the same procedure is followed, except the copied tags are adapted to make sure we still have well-formed XML.

<text:h>abc<text:change text:id="example/>def</text:h>

becomes:

$<$ text: $\mathrm{h}>\mathrm{abcHello} /$ text $: \mathrm{h}>$

$<$ text: $h>$ World! def $</$ text $: h>$

The third case occurs when a change occurs outside of a paragraph. In this case, the deleted text is simply copied verbatim.

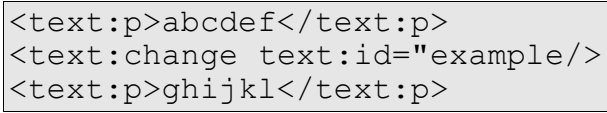

This becomes:

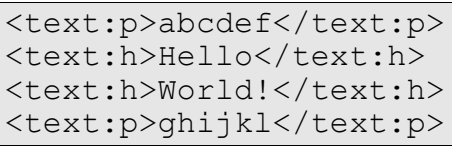

If, in the first two cases, the deletion contains complete paragraphs, then additional empty paragraphs must be put into the <text:deletion> element to achieve the desired result. 
The change that took place from

$<$ text:p>abc</text:p>

$<$ text:h>Hello</text: $h>$

$<$ text: $\mathrm{h}>$ World $!</$ text $:$ h $>$

$<$ text:p>def</text:p>

to

\section{<text:p>abc<text:change text:id="example/>def</text:p >}

would be represented as:

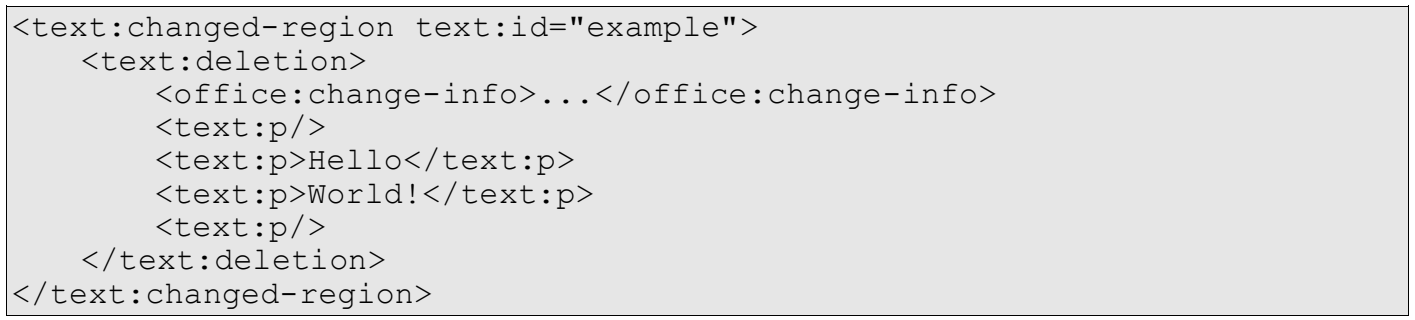

\subsubsection{Format Change}

A format change element represents any change in formatting attributes. The region where the change took place is marked by a change start and a change end element.

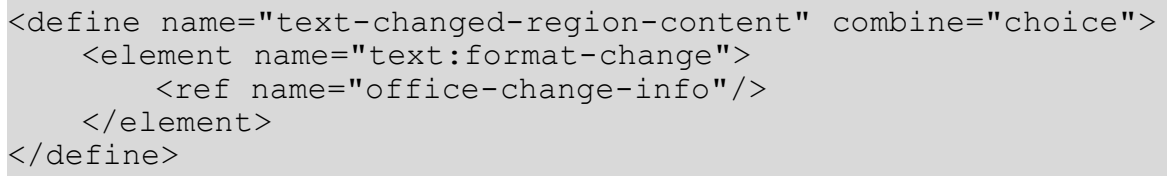

Note: A format change element does not contain the actual changes that took place.

\subsubsection{Change Info}

The change info element contains meta information who made the change and when. It is also used for spreadsheet documents, and thus described in a section 12.3 (Change Tracking Metadata).

\subsubsection{Change Marks}

There are three elements that mark the start and the end of a changed region, as follows:

- Change start element - <text: change-start>

This element marks the start of a region with content where text has been inserted or the format has been changed.

- Change end element - <text: change-end >

This element marks the end of a region with content where text has been inserted or the format has been changed.

- Change position element $-<$ text: change $>$

This element marks a position in an empty region where text has been deleted.

All three elements have an attribute that specifies the ID of the region to which they belong.

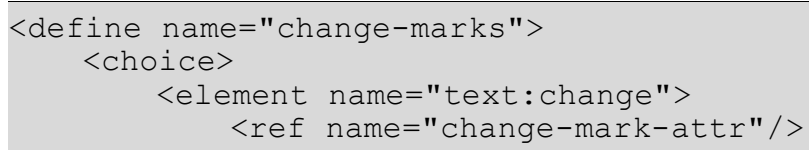




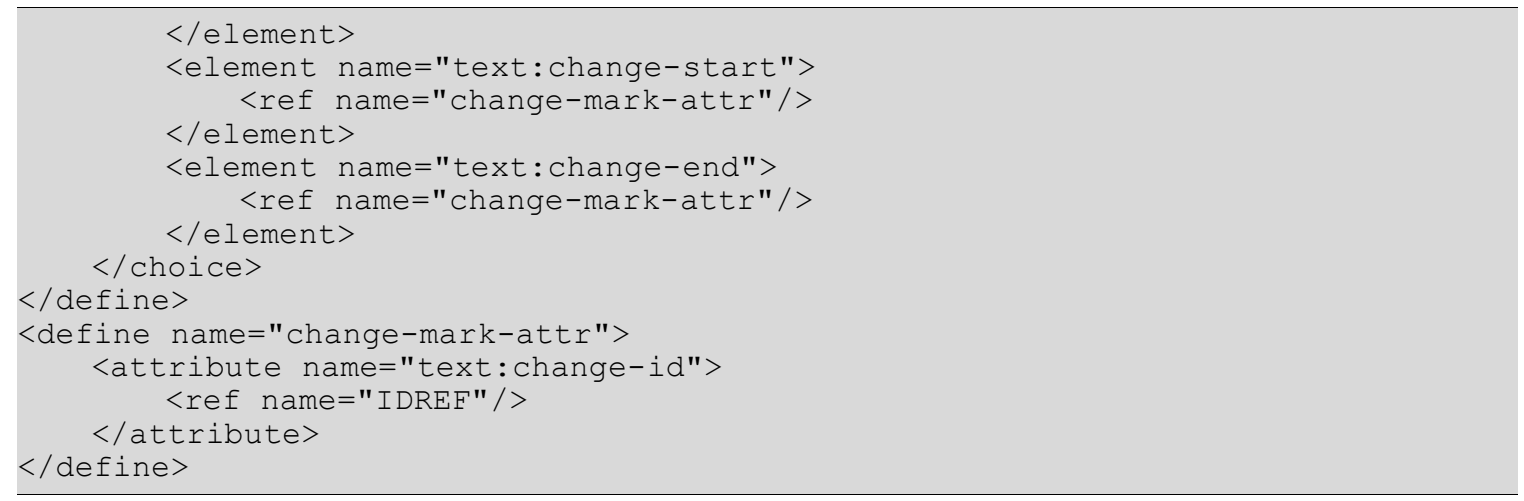

\subsection{Text Declarations}

Several text elements need per-document declarations before they can be used. For example, variable fields require that the variables used are being declared at the beginning of the document. These declarations are collected at the beginning of a text document. All such declarations are optional. The detailed description for each declaration can be found in the appropriate chapter.

The supported text declarations are:

- variable declarations - These declarations are used for variable fields. (cf. section 6.3.1).

- user field declarations - These declarations are used for user-defined fields (cf. section 6.3.5).

- sequence declarations - These declarations are used for sequence fields (cf. section 6.3.8).

- DDE connections - These declarations are used for DDE fields and DDE sections (cf. sections 6.6.9 and 4.4.3, respectively).

- auto mark file - This declaration is used for generation of alphabetical indices (cf. section 7.8.2).

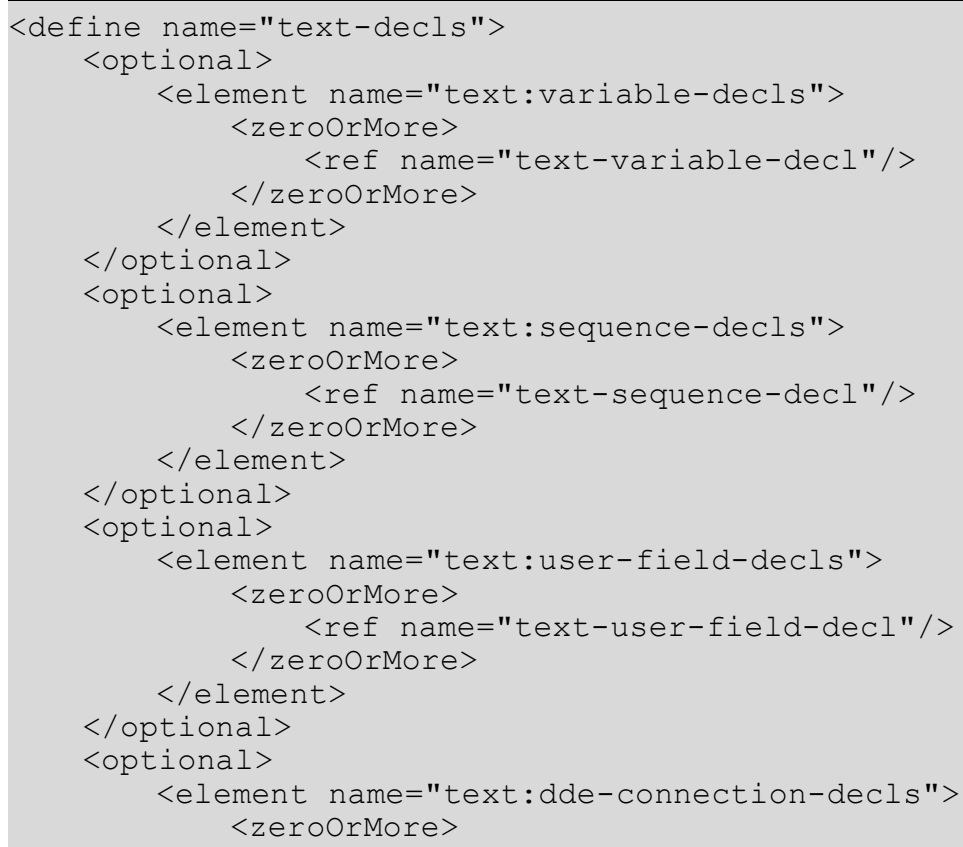




$\quad$ <ref name="text-dde-connection-decl"/>
$\quad</$ zeroorMore $>$
$</$ element>
$</$ optional>
$<$ optional>
$<$ ref name="text-alphabetical-index-auto-mark-file"/>
$</$ optional>
$</$ define>




\section{Paragraph Elements Content}

\subsection{Basic Text Content}

Paragraph element's children make up the text content of any document. All text contained in a paragraph element or their children is text content, with few exceptions detailed later. This should significantly ease transformations into other formats, since transformations may ignore any child elements of paragraph elements and only process their text content, and still obtain a faithful representation of text content.

Text content elements that do not contain in-line text children are:

- (foot- and end-)notes (see section 5.3)

Foot- and endnotes contain text content, but are typically displayed outside the main text content, e.g. at the end of a page or document.

- rubies (see section 5.4)

Ruby texts are usually displayed above or below the main text.

- $\quad$ annotations (see section 5.5)

Annotations are typically not displayed.

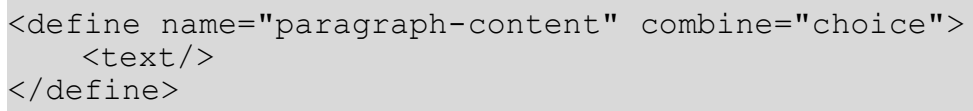

\subsubsection{White-space Characters}

If the paragraph element or any of its child elements contains white-space characters, they are collapsed, in other words they are processed in the same way that [HTML4] processes them. The following Unicode characters are normalized to a SPACE character:

- HORIZONTAL TABULATION (0x0009)

- CARRIAGE RETURN (0x000D)

- LINE FEED (0x000A)

- SPACE (0x0020)

In addition, these characters are ignored if the preceding character is a white-space character. The preceding character can be contained in the same element, in the parent element, or in the preceding sibling element, as long as it is contained within the same paragraph element and the element in which it is contained processes white-space characters as described above.

White-space processing takes place within the following elements:

- $\quad<$ text $: p>$

- $\quad$ text $: h>$

- <text:span>

- <text:a> 
- $\quad$ text:ref-point>

- $\quad$ text:ref-point-start>

- <text:ref-point-end>

- <text:bookmark>

- $\quad$ text:bookmark-start>

- $\quad$ <text:bookmark-end>

Note: In [XSL], white-space processing of a paragraph of text can be enabled by attaching an fo: white-space="collapse" attribute to the $\langle$ fo:block $\rangle$ element that corresponds to the paragraph element.

\section{Space Character}

In general, consecutive white-space characters in a paragraph are collapsed. For this reason, there is a special XML element used to represent the Unicode character SPACE (0x0020).

This element uses an optional attribute called text: $c$ to specify the number of SPACE characters that the element represents. A missing text: $c$ attribute is interpreted as meaning a single SPACE character.

This element is required to represent the second and all following SPACE characters in a sequence of SPACE characters. It is not an error if the character preceding the element is not a white-space character, but it is good practice to use this element for the second and all following SPACE characters in a sequence. This way, an application recognizes a single space character without recognizing this element.

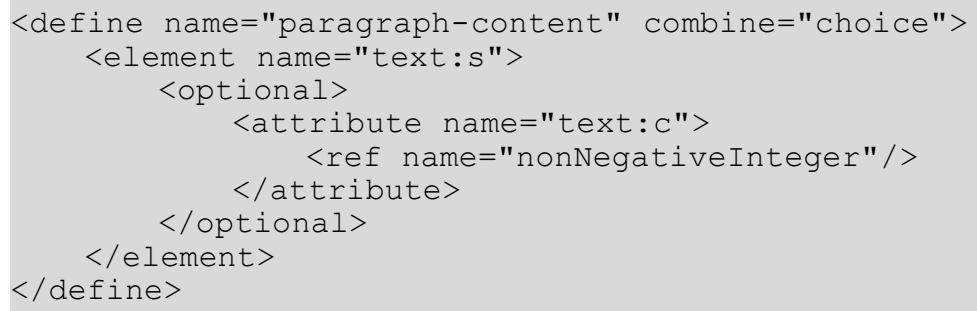

\section{Tab Character}

The <text: $t a b>$ element represents the Unicode tab character HORIZONTAL TABULATION (0x0009) in a heading or paragraph. A <text: tab> element reserves space from the current position up to the next tab-stop, as defined in the paragraph's style information.

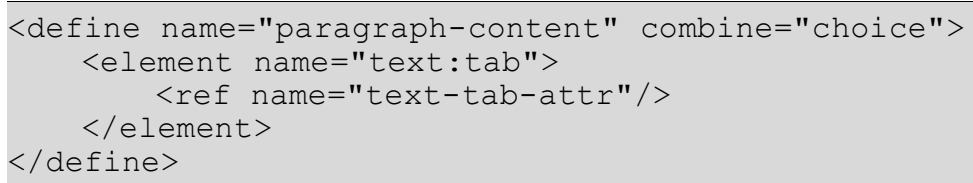

To determine which tab-stop a tab character will advance to requires layout information. To make it easier for non-layout oriented processors to determine this information, applications may generate a text:tab-ref attribute as a hint that associates a tab character with a tab-stop in the current paragraph style. It contains the number of the tab-stop that the tab character refers to. The position 0 has a special meaning and signifies the start margin of the paragraph. 


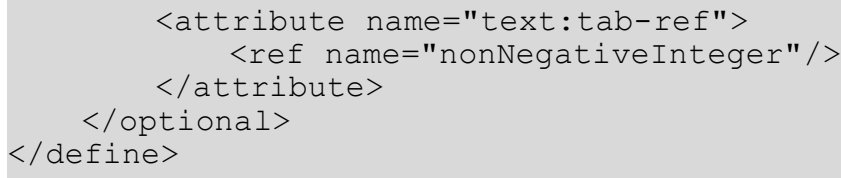

Note: The text:tab-ref attribute is only a hint to help non-layouting processors to determine the tab/tab-stop association. Layouters should determine the tab positions solely based on the style information.

\section{Line Breaks}

The <text: line-break> element represents a line break in a heading or paragraph.

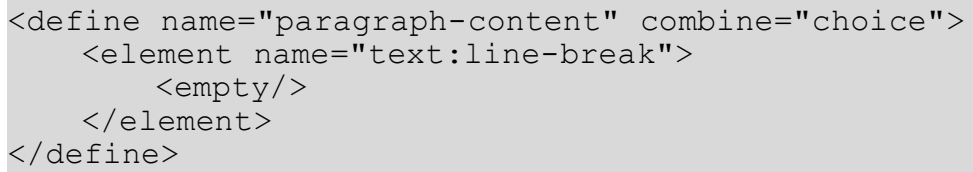

\subsubsection{Soft Hyphens, Hyphens, and Non-breaking Blanks}

Soft hyphens, hyphens, and non-breaking blanks are represented by UNICODE characters.

\begin{tabular}{|l|l|}
\hline \multicolumn{1}{|c|}{ The UNICODE character... } & \multicolumn{1}{c|}{ Represents... } \\
\hline SOFT HYPHEN (OOAD) & soft hyphens \\
\hline $\begin{array}{l}\text { NON-BREAKING HYPHEN } \\
(2011)\end{array}$ & non-breaking hyphens \\
\hline NO-BREAK SPACE (00A0) & non-breaking blanks \\
\hline
\end{tabular}

\subsubsection{Attributed Text}

The <text:span> element represents portions of text that are attributed using a certain text style or class. The content of this element is the text that uses the text style.

The name of the a text style or text class is the value of a text:style-name or text:classnames attributes, respectively, attached to the <text: span> element. These attributes must refer to text styles or classes.

A text:style-name attribute references a single text style. A text:class-names attribute takes a whitespace separated list of text style names. The referenced text styles are applied in the order they are contained in the list. If both, text:style-name and text:class-names are present, the style referenced by the text:style-name attribute is treated as the first style in the list in text:class-names. Conforming application should support the text:class-names attribute and also should preserve it while editing.

<text:span> elements can be nested.

White-space characters contained in this element are collapsed.

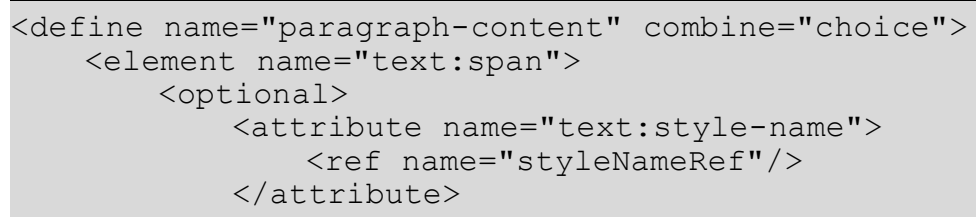




\section{Example: Text style in OpenDocument documents:}

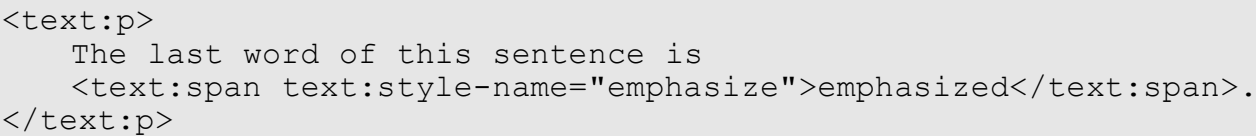

\subsubsection{Hyperlinks}

Hyperlinks in text documents are represented by $a<$ text $: a>$ element.

This element also contains an event table element, <office: event-listeners $>$, which contains the events assigned to the hyperlink. See section 12.4 for more information on the event table element.

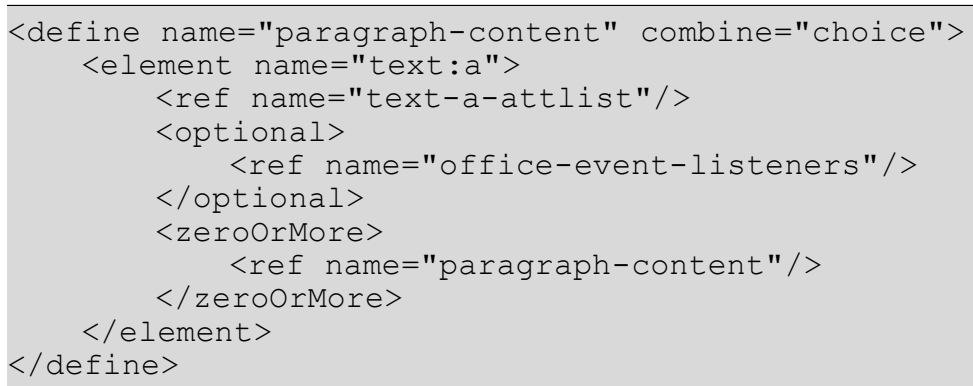

The attributes that may be associated with the <text:a> element are:

- Name

- Link location

- Target frame

- Text styles

\section{Name}

A hyperlink can have a name, but it is not essential. The office: name attribute specifies the name of the hyperlink if one exists. This name can serve as a target for some other hyperlinks.

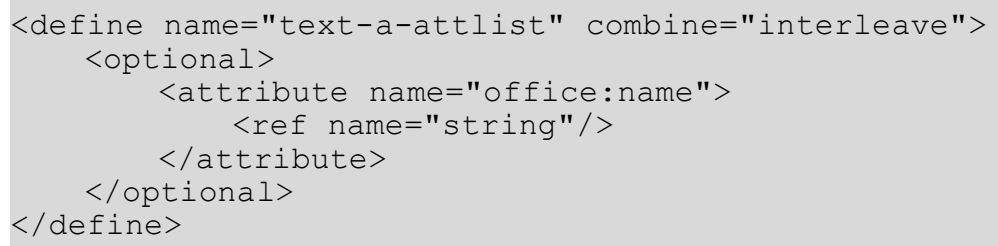




\section{Link Location}

The $x$ link: href attribute specifies the URL for the target location of the link.

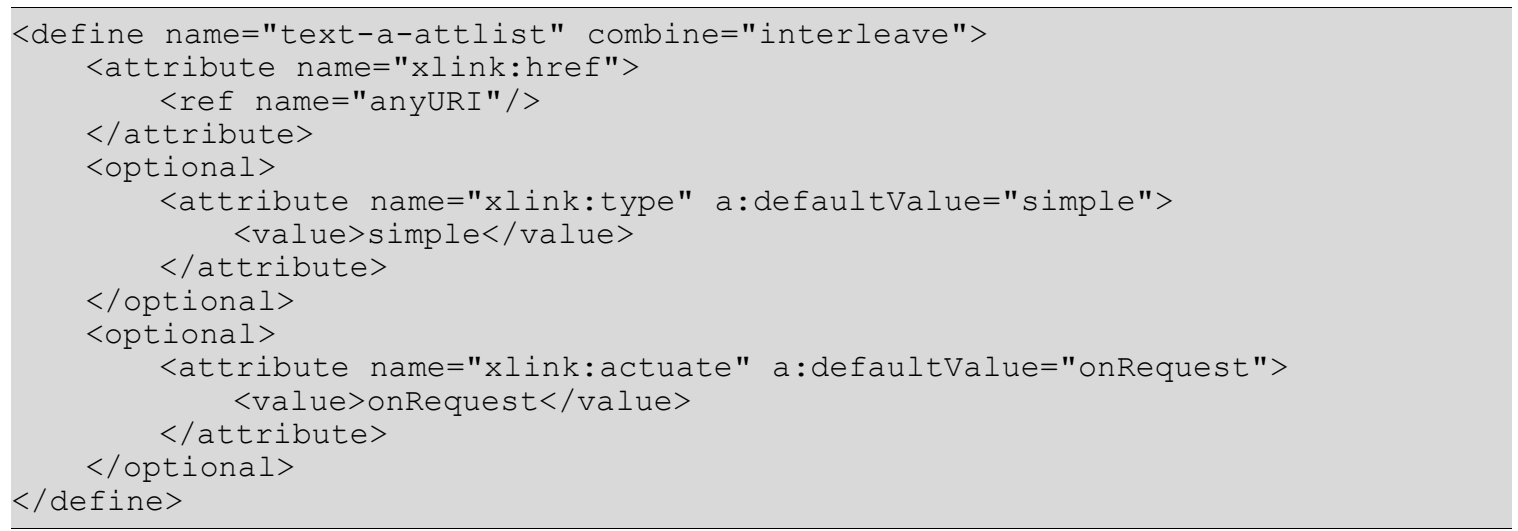

\section{Target Frame}

The office:target-frame-name attribute specifies the target frame of the link. This attribute can have one of the following values:

- _ _ self - The referenced document replaces the content of the current frame.

- _ blank - The referenced document is displayed in a new frame.

- _ parent - The referenced document is displayed in the parent frame of the current frame.

- top - The referenced document is displayed in the uppermost frame, that is the frame that contains the current frame as a child or descendent but is not contained within another frame.

- A frame name - The referenced document is displayed in the named frame. If the named frame does not exist, a new frame with that name is created.

To conform with the XLink Specification, an additional xlink: show attribute is attached to the <text:a> element. If the value of the attribute is blank, the xlink:show attribute value is new. If the value of the attribute is any of the other value options, the value of the xlink:show attribute is replace. See [XLink].

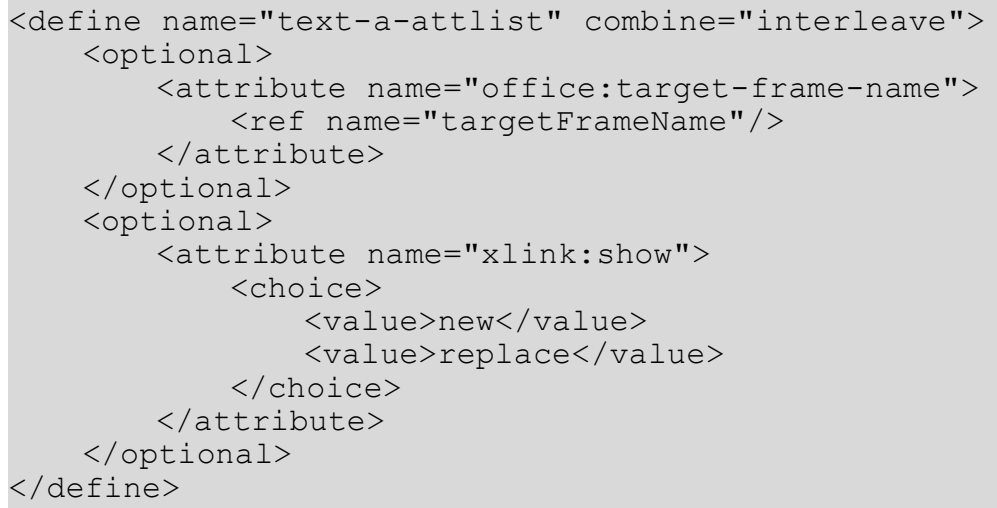

\section{Text Styles}

Every hyperlink has two text styles as follows: 
- If the link location of the hyperlink was not visited, the text style specifies by the text:style-name attribute is applied to the text of the hyperlink.

- If the link location of the hyperlink was already visited, the text style specified by the text:visited-style-name attribute is applied to the text of the hyperlink

\subsection{Bookmarks and References}

\subsubsection{Bookmarks}

Bookmarks can either mark a text position or a text range. A text range can start at any text position and end at another text position. In particular, a bookmark can start in the middle of one paragraph and end in the middle of another paragraph. The XML element used to represent a bookmark varies depending on the type of bookmark, as follows:

- <text:bookmark> - to mark one text position

- <text:bookmark-start>- to mark the start position in a text range

- <text:bookmark-end> - to mark the end position in a text range

For every <text:bookmark-start> element, there must be a <text:bookmark-end> element in the same text flow using the same text: name attribute, and vice versa. The <text:bookmark-start> element must precede the <text:bookmark-end> element.

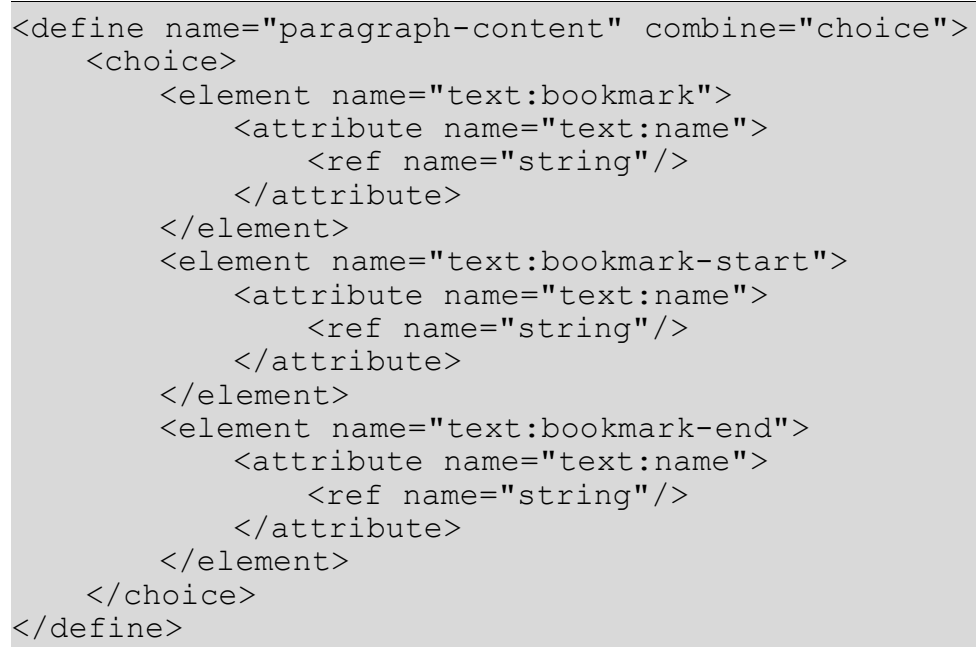

Example: Bookmarks

$<$ text:p>

<text:bookmark text:name="Mark 1"/>There is a text mark in front of this 


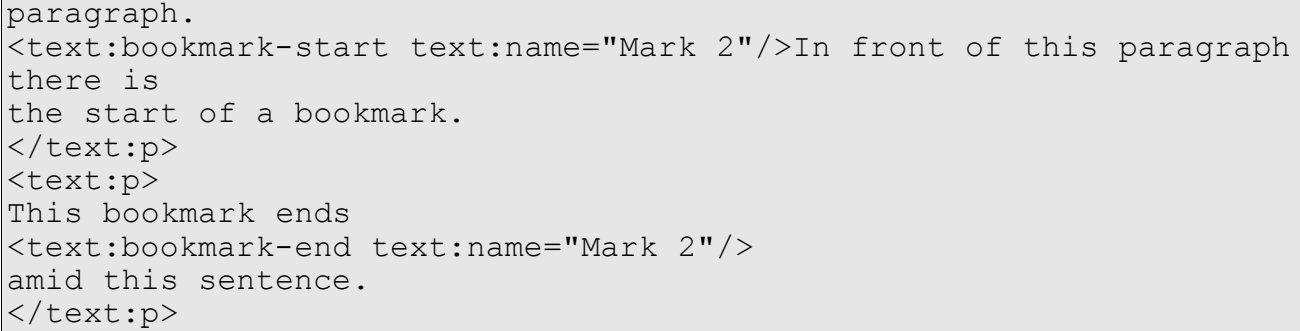

\subsubsection{References}

The representation of references is modeled on the XML representation of bookmarks. There are two types of reference marks, as follows:

- A point reference

A point reference marks a particular position in text and is represented by a single <text:reference-mark> element.

- A range reference

A range reference marks a range of characters in text and is represented by two elements; $<$ text:reference-mark-start> to mark the start of the range and <text:referencemark-end $>$ to mark the end of the range.

Every reference is identified by its name, which must be unique. In a range reference, the start and end elements must use the same reference name.

\section{Point References}

The <text: reference-mark> element represents a point reference.

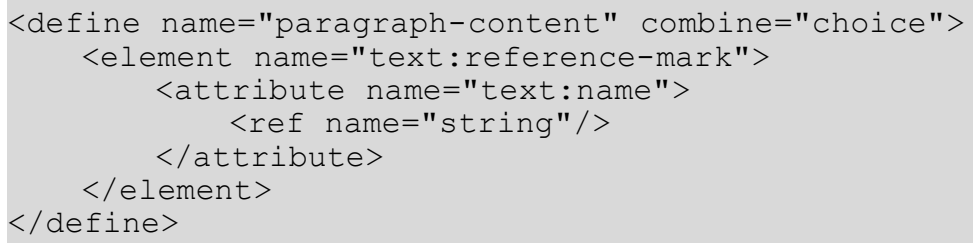

\section{Range References}

The <text:reference-mark-start> and <text:reference-mark-end> elements represent a range reference.

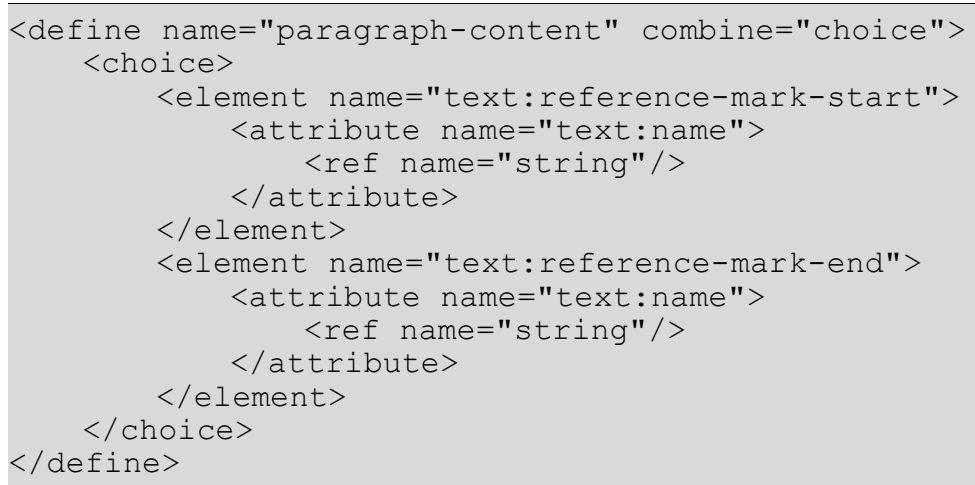


In the OpenDocument schema, three elements are used to represent references instead of one element because references represented as a single XML element:

- Cannot support overlapping references

- Do not interact well with other elements

Take the following example:

Example: Overlapping range references

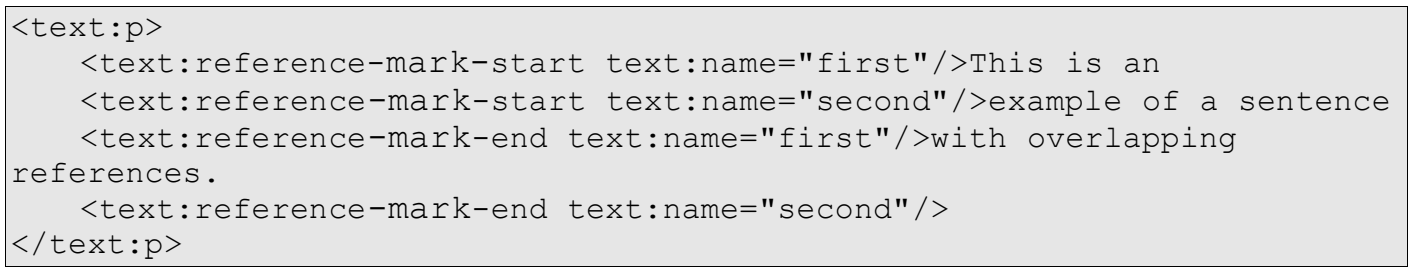

The example paragraph shows two references that cover the following text:

\begin{tabular}{|l|l|}
\hline reference "first" & "This is an example of a sentence" \\
\hline reference "second" & $\begin{array}{l}\text { "example of a sentence with overlapping } \\
\text { references." }\end{array}$
\end{tabular}

This overlapping structure cannot be represented using a single reference element to contain the referenced text. Similarly, a reference spanning multiple paragraphs creates the same situation as two overlapping XML elements, as does character formatting either starts or ends, but not both, within the referenced text.

\subsection{Notes}

Notes consist of a <text: note> element which occurs in the text stream at the position to which the note is anchored. How notes are numbered and rendered is determined by <text: notes-configuration> element, which occurs inside the <office: styles> section.

\subsubsection{Note Element}

The note element represents text notes which are attached to a certain text position. A common implementation of this concept are the footnotes and endnotes found in most word processors. A note contains a note citation element and a note body elements, which contains the note's content.

In OpenDocument documents, notes are represented in a similar fashion to footnotes in [XSL]. In $X S L$, the first child of the note element contains the citation in the form of an $<$ fo: inline $>$ element. The OpenDocument schema uses the same structure but introduces a <text: notecitation> element. The second child contains the note body, just as in XSL.

Additionally, OpenDocument features <text: notes-configuration> elements. To achieve a similar effect to the note configuration in XSL, every note citation element must be formatted appropriately.

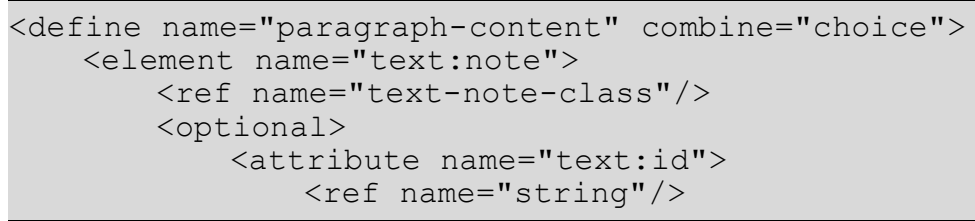




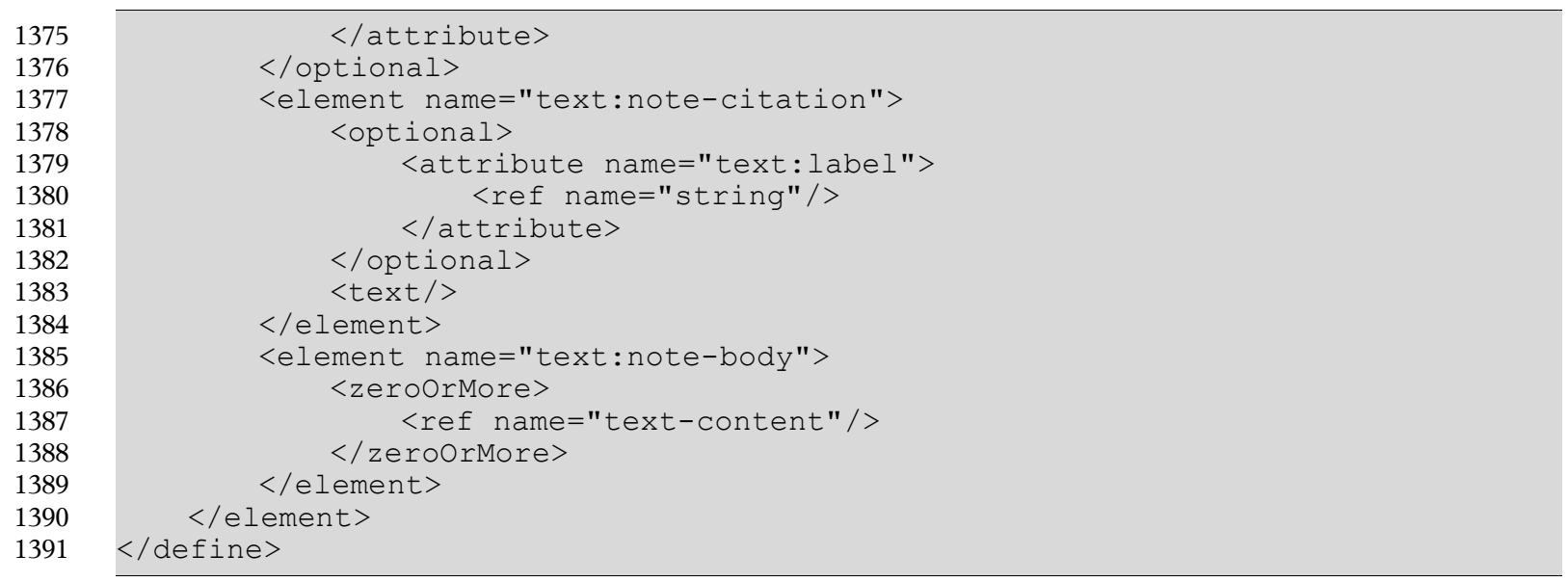

\section{Note Class}

Each note belongs to a class which determines how the note is expected to be rendered. Currently, two note classes are supported: Footnotes and endnotes.

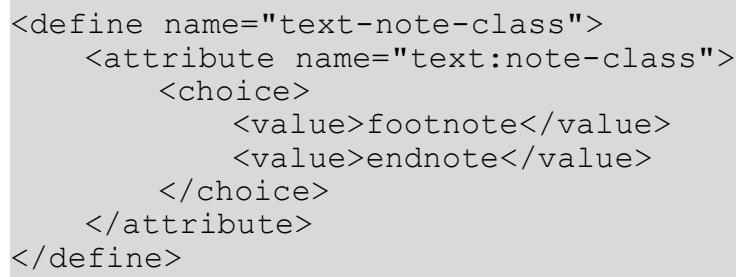

\section{Footnote Reference ID}

The footnote reference ID is used by references to footnotes to identify the footnote that is referenced.

\section{Note Citation Element}

The <text: note-citation> element contains the formatted note citation element, either as a formatted number or a string.

\section{Note Label}

Note citation elements can be labeled or numbered. If they are numbered, the number is chosen and formatted automatically according to the notes configuration element. If they are labeled, the user must supply a label for every note he/she inserts into the document. This label is stored in the text: label attribute of the <text: note-citation> element.

\section{Note Body}

The <text: note-body> element contains the actual content of the footnote. It does not have any attributes.

The schema allows for the inclusion of notes into the note body. While this may be reasonable for some future note types, it is not reasonable for footnotes and endnotes. Conforming applications may or may not support such nested notes. 


\section{Footnote example}

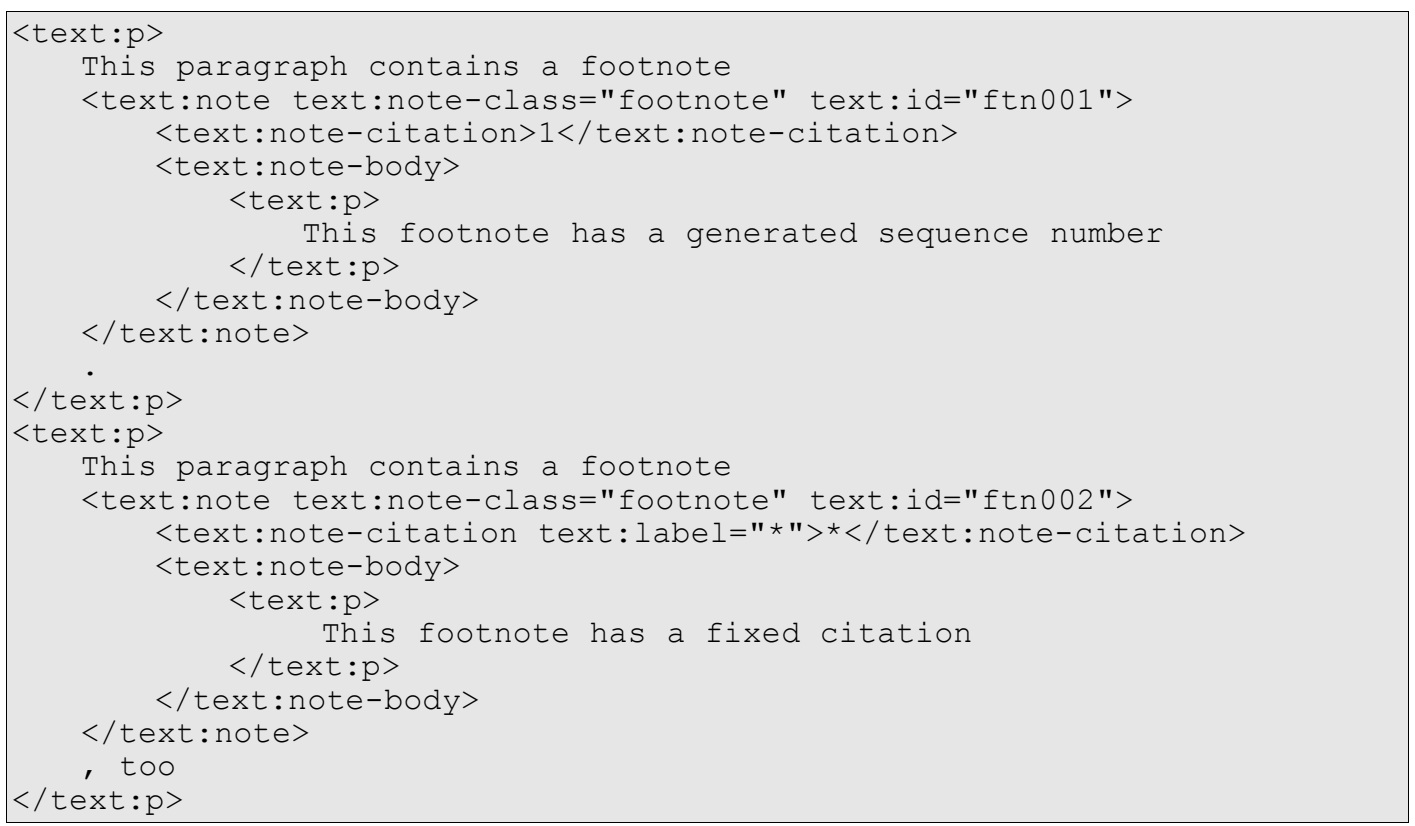

\subsection{Ruby}

A ruby is additional text that is displayed above or below some base text. The purpose of ruby is to annotate the base text or provide information about its pronunciation.

There are two elements that can be contained in the <text: ruby $>$ element:

- Ruby base

\section{- Ruby text}

The <text:ruby-base> element contains the text that is to be annotated. It contains any paragraph element content, like text spans. The element's text:style-name attribute references a ruby style that specifies further formatting attributes of the ruby. See section 14.8.4 for details.

The <text: ruby-text > element contains the annotation text. It may contain only plain text. The element's text:style-name attribute references a text style that specifies further formatting attributes used for the text.

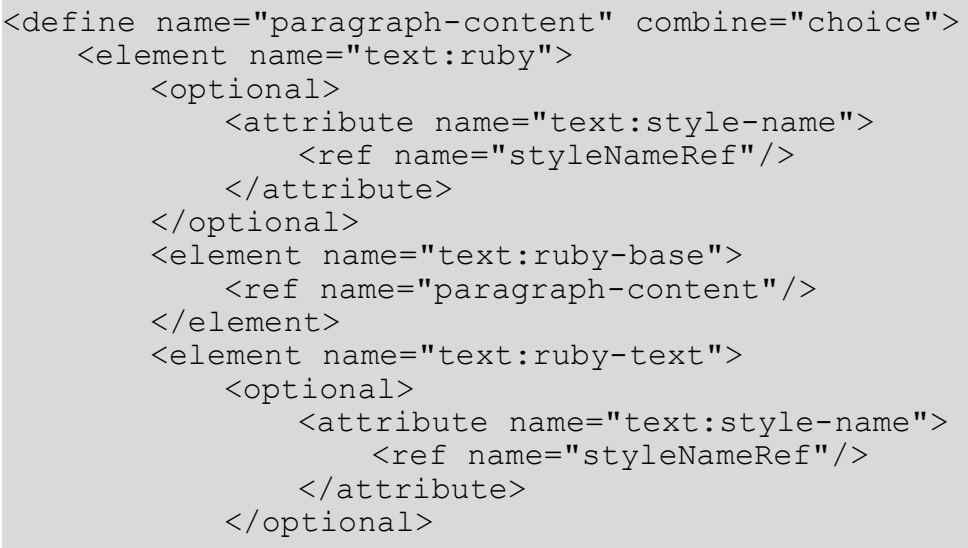




\subsection{Text Annotation}

The OpenDocument format allows annotation to appear within a paragraph element. See section 12.1 for details on annotations.

\subsection{Index Marks}

Index marks are used to mark text areas for inclusion into text indices. They are similar in structure to bookmarks and references. They are discussed in detail section 7.1, together with text indices.

\subsection{Change Tracking and Change Marks}

Paragraphs may also contain change tracking marks. These have already been explained in the chapter on change tracking (section 4.6), and are referenced here for completeness.

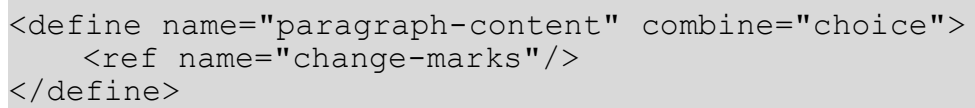

\subsection{Inline graphics and text-boxes}

Within text documents, images, embedded objects and other drawing objects may be anchored to a paragraph, to a character, or as an character. If they are anchored to a paragraph, they appear within a paragraph at an arbitrary position. If they are anchored to or as a character, they appear within a paragraph at exactly the character position they are anchored to or as. See section 9.2 for details on drawing objects, and section 9.2.16 for their anchoring.

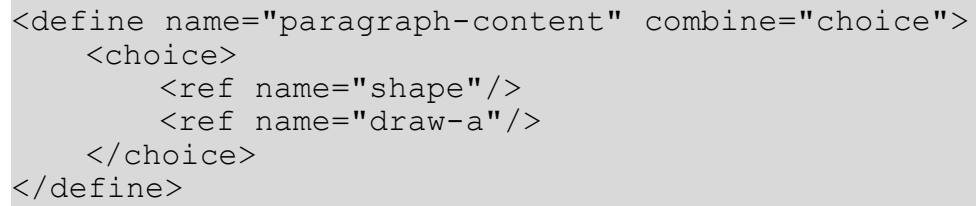




\section{Text Fields}

OpenDocument text documents or OpenDocument text content embedded in other types of documents can contain variable text elements called fields. There are several different types of field, each of which implements a different type of variable text element. Fields are most commonly used for:

- Page numbers

A page number field displays the number of the page it appears on. This field is useful for footers. For every page on which the footer appears, the field assumes the current page number so that all pages are numbered correctly.

- Creation dates

A creation date field displays the date on which the current document was created. This field is useful for document templates. Every document created using the template contains the date when it was created.

- Number ranges

A number range field allows the user to number certain elements, for example, images or tables. A number range field displays its own position in relation to the other number range fields for the same range. Therefore, if an image and its associated number range field are moved within a document, the fields are automatically updated to reflect the new order.

This section describes how fields are represented in the OpenDocument file format.

\subsection{Common Characteristics of Field Elements}

Each field type is represented by a corresponding element type. A field in a document is encoded as a single element of the appropriate type. The content of the element is the textual representation of the current field value as it would be displayed or printed. Therefore, ignoring all field elements and displaying only the textual content of the elements provides an approximate text-only version of the document.

The value of a field is usually stored in an attribute. It is necessary to store the value so that the presentation of the field can be recomputed if necessary, for example, if the user decides to change the formatting style of the field. It is also necessary to store the presentation style of the element content, to facilitate easy processing of the XML document. For example, if complete processing of a field is impossible or undesirable, the application can ignore the field and use only the content in this situation. For string values, if the value is identical to the presentation, the value attribute is omitted to avoid duplicate storage of information.

For fields that can store different types of content, for example, numbers, strings, or dates, a value type is stored in addition to the actual value. The value and value type attributes are explained later in section 6.7.1. If more information is needed to restore a field, it is stored in additional attributes.

The most common attributes of field elements are:

- Fixed fields

Many fields have a variant where the content does not change after the initial value is assigned. These fields are generally marked by the attribute text: fixed. See section 6.7.2 for more information on this attribute.

- Formatting style

Several field types, particularly those representing number, date, or time data, contain a 
formatting style. In the OpenDocument format, this formatting style is represented by a style:data-style-name attribute. Since the user can change the presentation style for fields, applications must be able to recompute a new representation of the field content at any time. See section 6.7.7 for more information on this attribute.

\subsection{Document Fields}

OpenDocument fields can display information about the current document or about a specific part of the current document, such as the author, the current page number, or the document creation date. These fields are collectively referred to as document fields.

Document fields are often fixed. A field can be marked fixed to indicate that its content is preserved, rather than re-evaluated, when the document is edited. For example, a date field shows the current date. If the date field is marked fixed, the value of the field is preserved during subsequent edits and always reflects the original date on which the field was inserted into the document. If the field is not marked fixed, its value changes whenever the document is edited. In the same way, the author field can show the original author or the last author of a document, depending on whether the field is marked fixed or not.

The group of document fields includes:

- Date and time fields

- Page number fields

- Sender and author fields

- Chapter fields

- $\quad$ File name fields

- Document template fields

\subsubsection{Date Fields}

Date fields display the current date. The date can be adjusted to display a date other than the current date. For example, the date can be changed on a document that was edited late at night so that it displays the date of the following day or several days later.

This element contains the presentation of the date field value, depending on the data style specified. The default date is the current date. The value of this element can be preserved using the text: fixed attribute described in section 6.7.2.

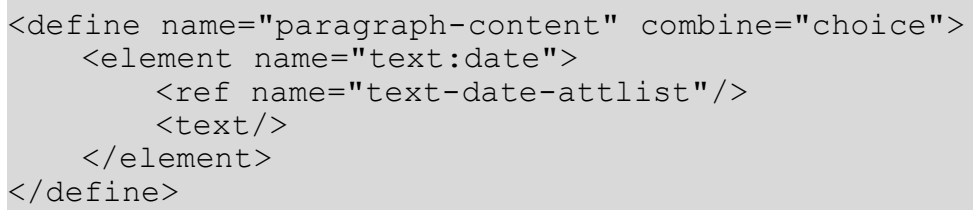

The attributes that may be associated with the <text: date> element are:

- Date value

- Date adjustment

- $\quad$ Fixed (see section 6.7.2)

- Formatting style (see section 6.7.7). The formatting style must be a date data style, see section 14.7 for more information. 


\section{Date Adjustment}

The value of a date field can be adjusted by a certain time period, which is specified using the text: date-adjust attribute. If the time period is negative, it gets subtracted from the value of the date field, yielding a date before the current date.

The value of this attribute must conform to the time period format described in $\S 3.2 .6$ of [xmlschema-2]. The value can be preceded by an optional minus sign to indicate a negative time duration.

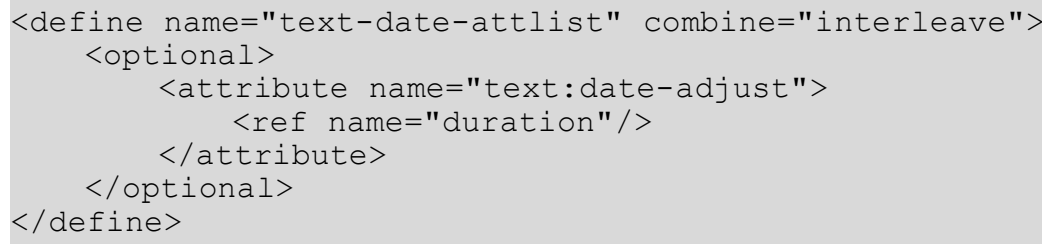

\subsubsection{Time Fields}

Time fields display the current time. They are very similar to the date fields described in the previous section, supporting the same attributes except that for time fields, they are called text: time-value and text: time-adjust attributes.

This element contains the presentation of the time field value, depending on the data style specified. The default time is the current time. The value of this element can be preserved using the text: fixed attribute described in section 6.7.2.

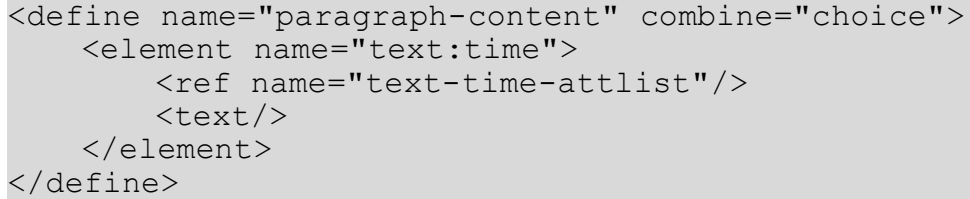


The attributes that may be associated with the <text: time> element are:

- Time value

- Time adjustment

- $\quad$ Fixed (see section 6.7.2)

- Formatting style (see section 6.7.7). The formatting style must be a time data style, see section 14.7 for more information.

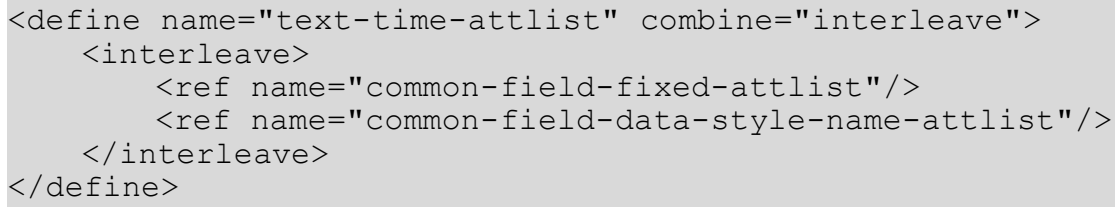

\section{Time Value}

The text: time-value attribute records the time at which the document was last edited.

Some applications support date and time in addition to date-only values.

The value of this attribute must conform with either the "dateTime" or "time" data types described in §3.2.7 and §3.2.8 of [xmlschema-2]. If no value is specified, the current time is assumed, even if the field is marked fixed.

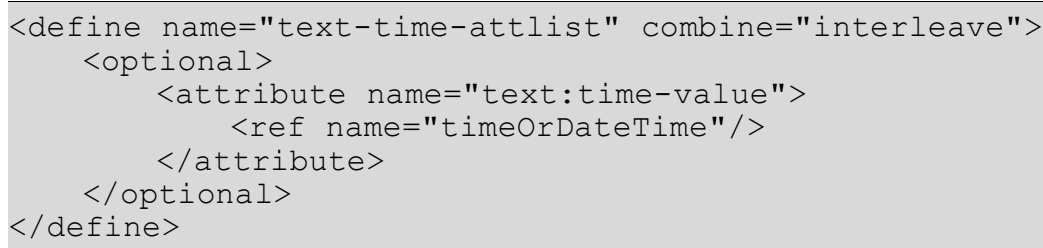

\section{Time Adjustment}

The value of a time field can be adjusted by a certain time period, which is specified using the text: time-adjust attribute.

The value of this attribute must conform to the time period format described in $\S 3.2 .6$ of [xmlschema-2]. The value can be preceded by an optional minus sign to indicate a negative time duration. Positive values adjust the time to a time in the future, while negative values adjust the time to a time in the past. The duration is truncated to full minutes.

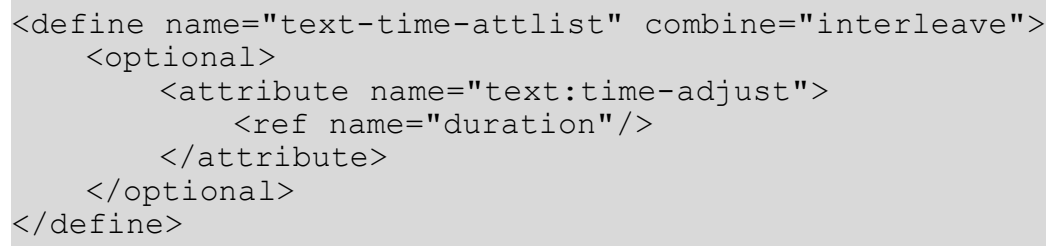

\section{Example: Time adjust attributes and their effects}

If the attribute text:time-adjust="PTM15", the time field displays a time which is 15 minutes later than the actual time specified by the time field value.

If the attribute text:time-adjust="-PTH1", the time field displays a time which is one hour before the actual time specified by the time field value. 


\subsubsection{Page Number Fields}

Page number fields display the current page number. These fields are particularly useful in headers and footers. E.g., if a page number field is inserted into a footer, the current page number is displayed on every page on which the footer appears.

The attributes that may be associated with the <text: page-number $>$ element are:

- Page adjustment

- Display previous or following page numbers

- $\quad$ Fixed (see section 6.7.2)

- $\quad$ Formatting style (see section 6.7.8)

Page numbers can be formatted according to the number format described in section 2.9. If a number style is not specified, the page numbers are formatted according to the number style defined in the current page style.

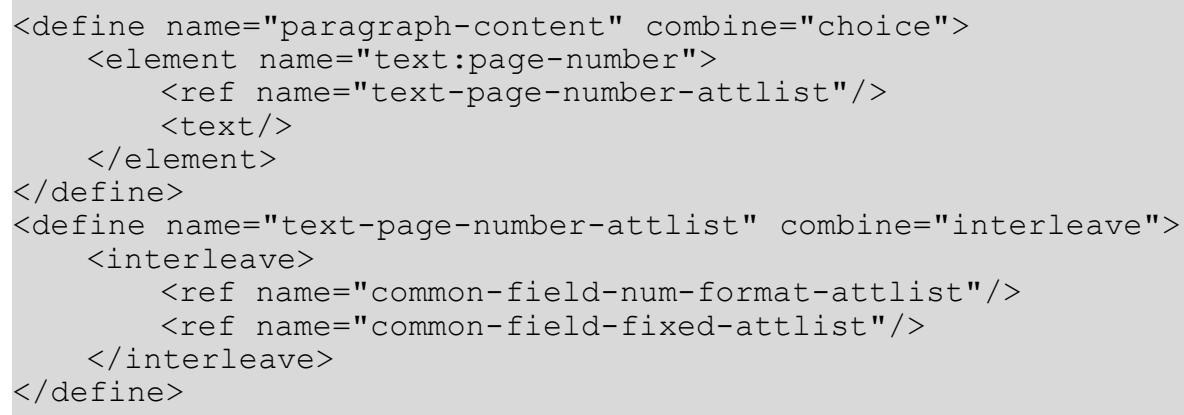

Note: To display the total number of pages in a document, use the <text:page-count/> field described in section 6.4.17.

\section{Page Adjustment}

The value of a page number field can be adjusted by a specified number, allowing the display of page numbers of following or preceding pages. The adjustment amount is specified using the text:page-adjust attribute. When this attribute is used, the application:

1. Adds the value of the attribute to the current page number.

2. Checks to see if the resulting page exists.

3. If the page exists, the number of that page is displayed.

4. If the page does not exist, the value of the page number field remains empty and no number is displayed.

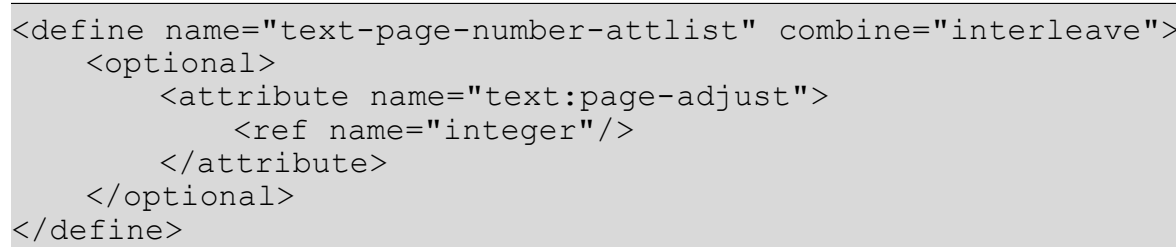




\section{Display Previous or Following Page Numbers}

The text: select-page attribute is used to display the number of the previous or the following page rather than the number of the current page.

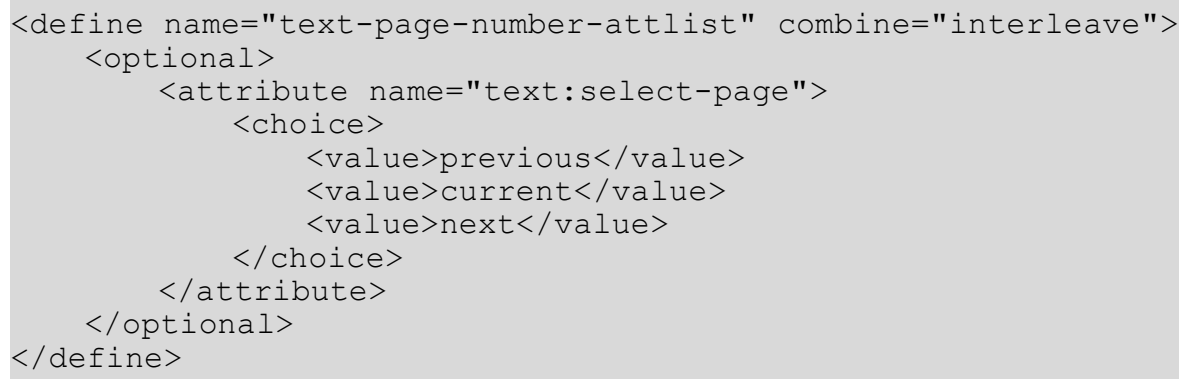

Note: To display the current page number on all pages except the first or last page, use a combination of the text: select page and text:page adjust attributes.

Example: Displaying the current page number on all pages except the first page

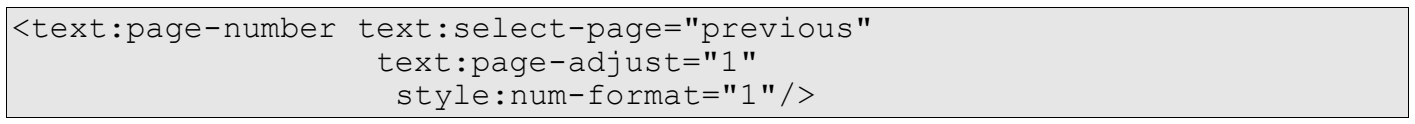

\subsubsection{Page Continuation Text}

In some publications, a continuation reminder is printed at the bottom of the page in addition to the page number. To include a continuation reminder, use the <text:page-continuation> element.

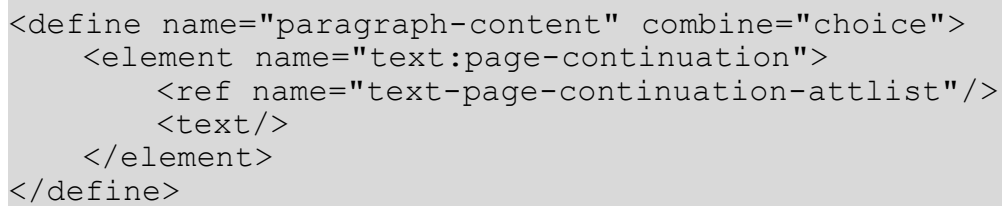

The attributes associated with the <text: page-continuation> element are:

- Previous or following page

- String value

\section{Previous or Following Page}

This attribute specifies whether to check for a previous or next page and if the page exists, the continuation text is printed.

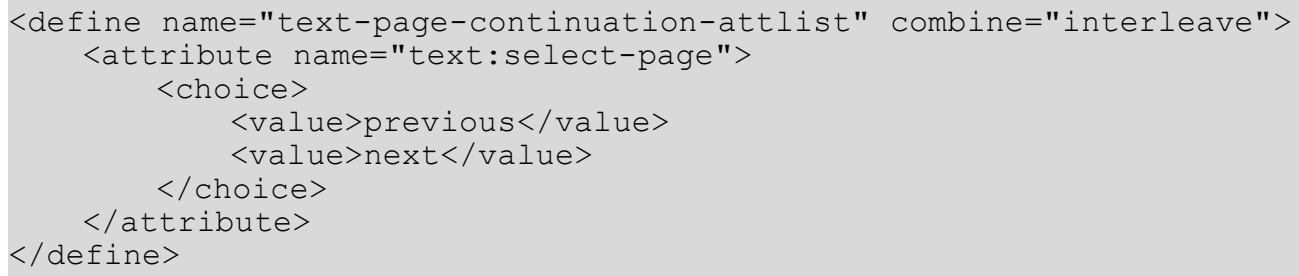




\section{String Value}

This attribute specifies the continuation text to display. If this attribute is omitted, the element content is used.

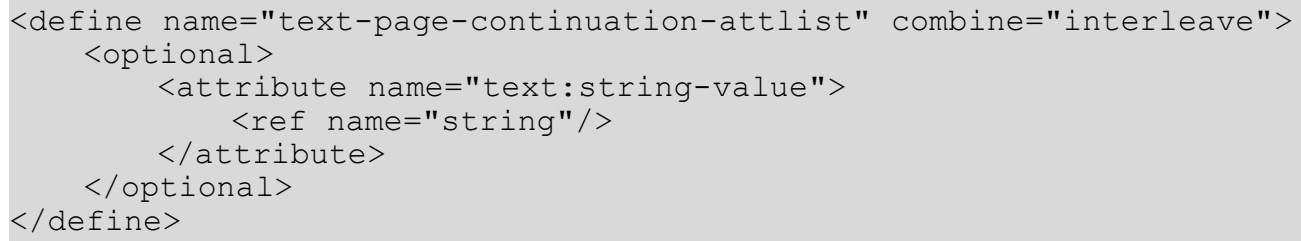

\subsubsection{Sender Fields}

There are several fields which contain information about the sender of the current document, for example, name and email address. The information about the sender is taken from the OpenDocument user information dialog. If a sender field is marked fixed using the text: fixed attribute, the original sender information in the sender fields is preserved. (cf. section 6.7.2) Otherwise, the information is updated each time the file is edited, causing the fields to change value when the document is edited by a different user.

\section{First Name}

This element represents the first name of the sender.

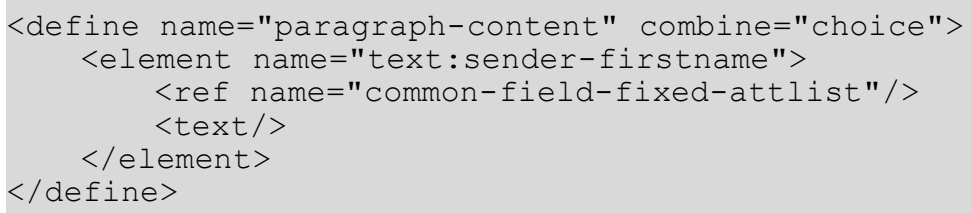

\section{Last Name}

This element represents the last name of the sender.

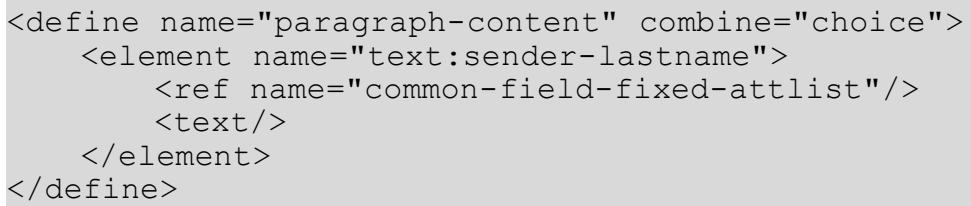

\section{Initials}

This element represents the initials of the sender.

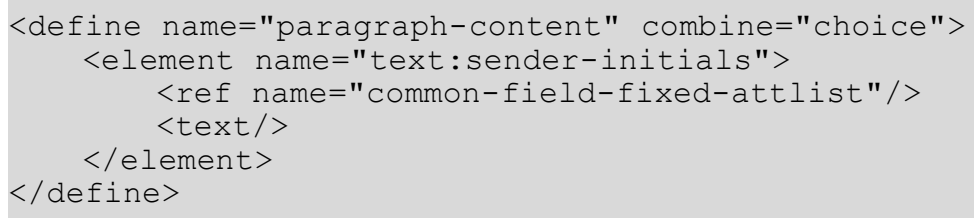

\section{Title}

This element represents the title of the sender. 


\section{Position}

This element represents the position of the sender.

\section{Email Address}

This element represents the email address of the sender.

\section{Fax Number}

This element represents the facsimile number of the sender.

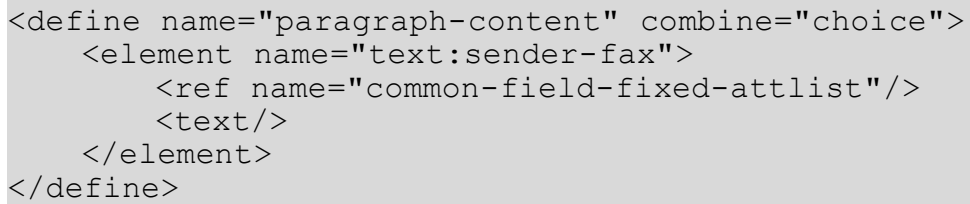

\section{Company Name}

This element represents the name of the company that employs the sender. 


\section{Office Telephone Number}

This element represents the office telephone number of the sender.

\section{Street}

This element represents the street name of the address of the sender.

\section{City}

This element represents the city name of the address of the sender.

\section{Postal Code}

This element represents the postal code of the address of the sender.

\section{Country}

This element represents the country of the address of the sender.

\section{State or Province}

This element represents the state or province of the address of the sender, if applicable. 


\subsubsection{Author Fields}

There are two elements available to display the author of a document. One element displays the full name of the author and the other element displays the initials of the author.

The value of author fields can be fixed using the text: fixed attribute. Marking an author field as fixed preserves the original field content. Otherwise, the field content changes each time the document is updated, to reflect the last author of the document.

\section{Name of the Author}

This element represents the full name of the author.

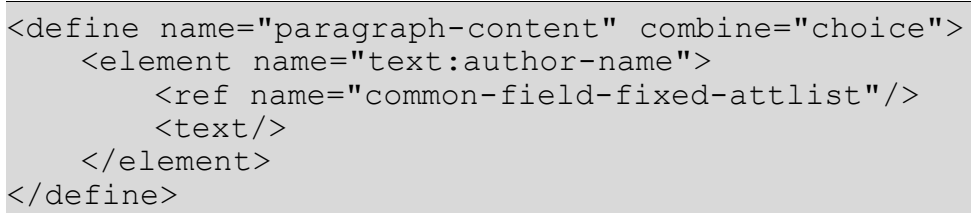

\section{Initials of the Author}

This element represents the initials of the author.

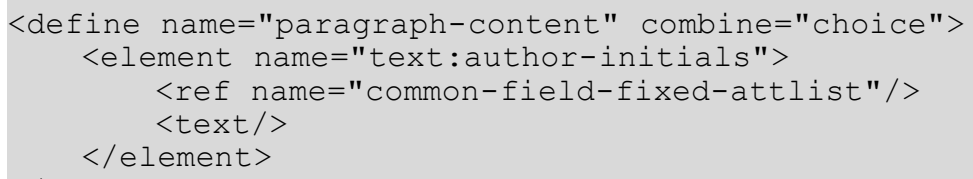

\subsubsection{Chapter Fields}

Chapter fields display one of the following:

- The name of the current chapter

- The number of the current chapter

- Both the name and number of the current chapter

If the chapter field is placed inside a header or footer, it displays the current chapter name or number on every page.

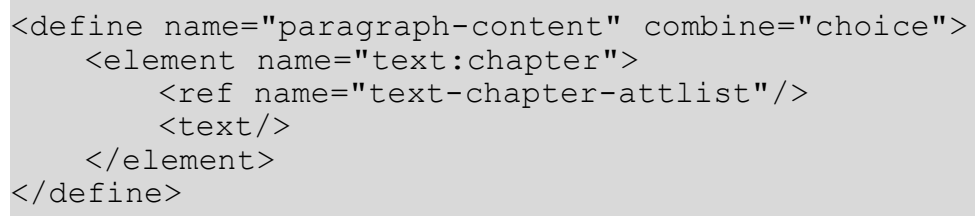

The attributes that may be associated with the <text: chapter $>$ element are:

- Display

- Outline level 


\section{Display}

The text: display attribute specifies the information that the chapter field should display.

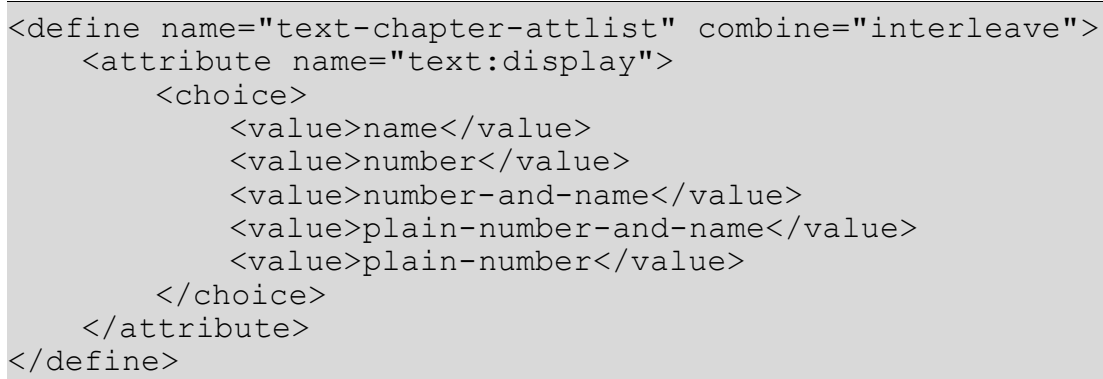

Example: If the current chapter number is 2.4 , the chapter title is Working with Tables, the prefix is [, and suffix is ], the possible display options and results are as follows:

\begin{tabular}{|c|c|}
\hline $\begin{array}{c}\text { Value of text: display } \\
\text { attribute }\end{array}$ & $\begin{array}{l}\text { Field content } \\
\text { displayed }\end{array}$ \\
\hline number & [2.4] \\
\hline name & Working with Tables \\
\hline number-and-name & $\begin{array}{l}\text { [2.4] Working with } \\
\text { Tables }\end{array}$ \\
\hline plain-number & 2.4 \\
\hline plain-number-and-name & 2.4 Working with Tables \\
\hline
\end{tabular}

\section{Outline Level}

This attribute is used to specify the outline level to use. The chapter field displays the chapter number or title up to the specified outline level.

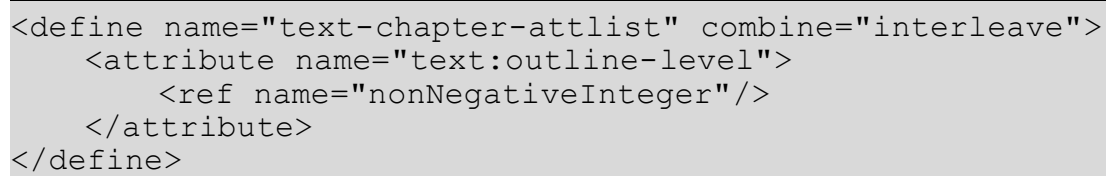

\subsubsection{File Name Fields}

File name fields display the name of the file that is currently being edited.

The attributes that may be associated with the <text:file-name> element are:

- Display

- $\quad$ Fixed

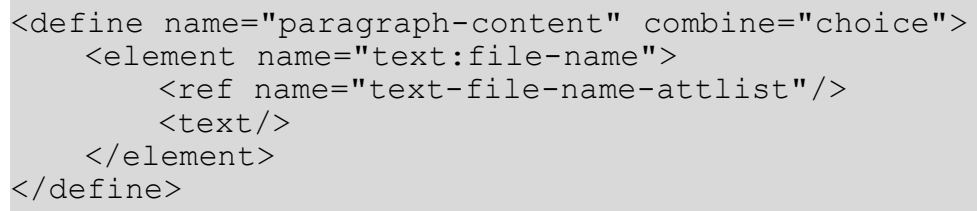




\section{Display}

The text: display attribute specifies how much of the file name to display. The following display options are allowed:

- The full file name including the path and the extension

- The file path only

- The file name only

- The file name and the extension

The filename might be an URI, either because an URI has been used to retrieve the file, or the application internally uses URIs and therefor converts even system specific paths into an URI. If this is the case, and if the the path, the name or the extension cannot be evaluated from the URI, then the URI should be displayed unmodified.

\section{Fixed File Name Fields}

If a file name field is fixed, its value does not change when the file is edited.

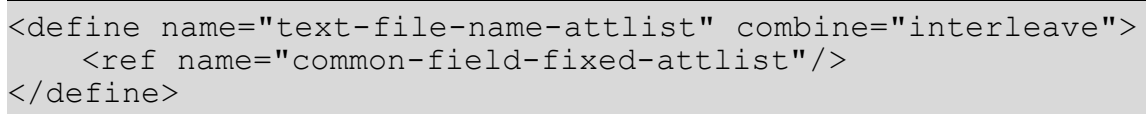

\subsubsection{Document Template Name Fields}

The document template name field displays information about the document template in use, such as the template title or the file name.

The only attribute that may be associated with the <text:template-name> element is:

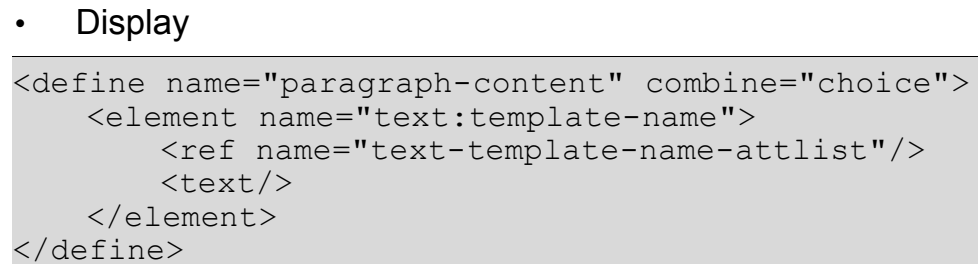

\section{Display}

This attribute specifies which information about the document template to display. The following display options are allowed:

- The full file name including the path and the extension 
- The file path only

- The file name only

- The file name and the extension

- The title

- The area of the document template

The latter two values can be used for template dialogs. The values are a superset of the display values available for the <text: file-name> element.

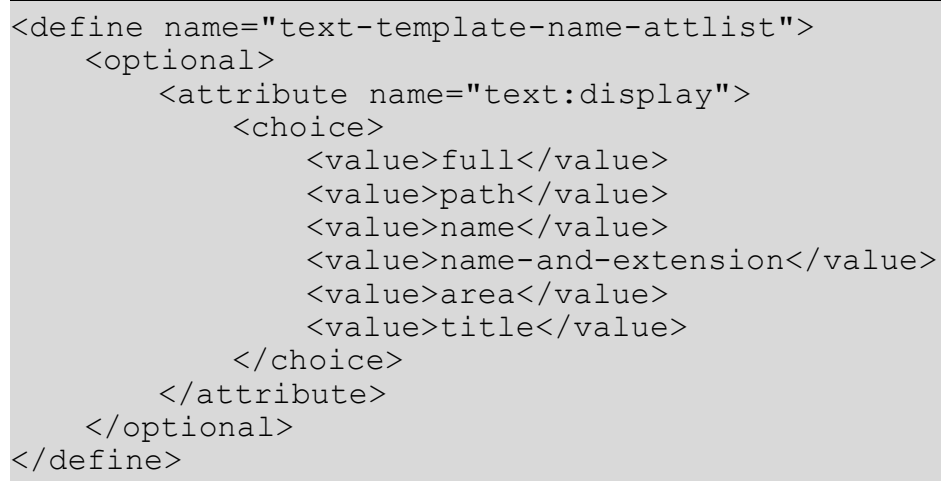

$</$ define $>$

\subsubsection{Sheet Name Fields}

For Spreadsheet documents, sheet name fields display the name of the sheet that is currently being edited.

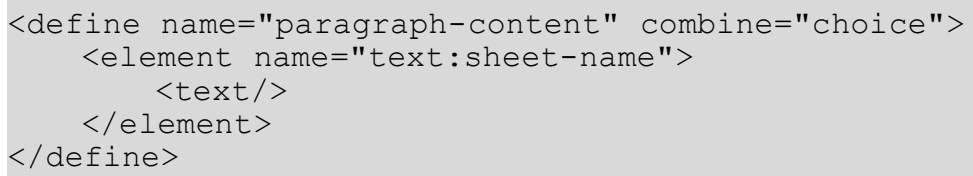

\subsection{Variable Fields}

OpenDocument text documents can contain variables, which are processed or displayed using variable fields. A variable is a name/value pair. The variable name is used throughout the document to identify a particular variable, and therefore variable names cannot be reused for different types of variables. Most variable fields support different value types, such as numbers, dates, strings, and so on. In the OpenDocument file format, a variable must be declared at the beginning of a document.

There are three types of variables:

\section{- Simple variables}

Simple variables, usually called variables, can take different values at different positions throughout a document. Simple variables can be set using either setter or input fields. Setter fields contain an expression, which is used to compute the new value of the variable. Input fields prompt the user for the new value. Simple variables can be used to display different text in recurring elements, such as headers or footers.

\section{- User variables}

User variables have the same value throughout a document. If a user variable is set anywhere within the document, all fields in the document that display the user variable have 
the same value. In the office application user interface, a user variable can be set at any occurrence of a user field, or by using user variable input fields. In the OpenDocument file format, the value of the user variable can only be set after the variable is declared.

\section{- Sequence variables}

Sequence variables are used to number certain items in an OpenDocument text document, for example, images or tables.

Expression and text input fields are also variable fields, but they are not associated with any particular variables. Since their functionality is closely related to that of the variable fields, they are also described in this section of the manual.

Variables must be declared before they can be used. The variable declarations are collected in container elements for the particular variable type. The OpenDocument code for declaring variables is described in the following section.

\subsubsection{Declaring Simple Variables}

Simple variables are declared using <text:variable-decl> elements. The declaration specifies the name and the value type of the variable.

To specify the name and value type of the simple variable, the following attributes are attached to the <text:variable-decl> element:

- text:name

The name of the variable must be unique. The name cannot already be used for any other type of variable. See section 6.7.3 for information on using this attribute.

- office:value-type

See section 6.7.1 for information on using this attribute.

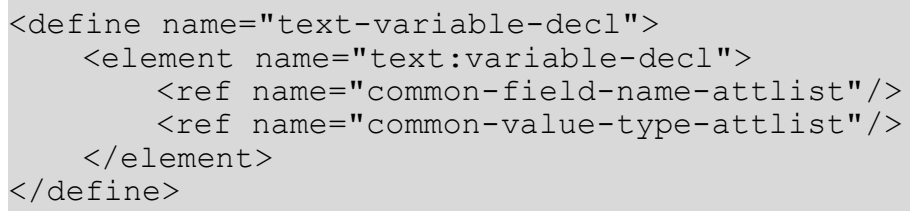

\subsubsection{Setting Simple Variables}

Simple variables can be set using variable setter elements. This element contains the presentation of the value of the variable, which can be empty if the text: display attribute is set to none.

The attributes that may be associated with the <text:variable-set $>$ element are:

- text: name

This attribute specifies the name of the variable to set. It must match the name of a variable that has already been declared. See section 6.7 .3 for information on using this attribute.

- text:formula

This attribute contains the formula to compute the value of the variable field. If the formula equals the content of the field element, this attribute can be omitted. See section 6.7 .6 for information on using this attribute. 
- office:value-type and the appropriate value attribute

See section 6.7.1 for information on using these attributes.

Note: A simple variable should not contain different value types at different places in a document. However, an implementation may allow the use of different value types for different instances of the same variable. In the case of the numeric value types float, percentage, and currency, the value is automatically converted to the different value type. For value types that are stored internally as numbers, such as date, time, and boolean types, the values are reinterpreted as numbers of the respective types. If a variable is used for both string and non-string types, the behavior is undefined, therefore this practice is not recommended.

- text:display

This attribute can be used to specify whether or not to display the value of the <text:variable-set> element. If the text:display attribute is set to value, the value of the variable is displayed. If the attribute is set to none, the value is not displayed. See section 6.7.5 for information on using this attribute.

- style:data-style-name

This attribute specifies the data style to use to format a numeric, Boolean, or date/time variable. If a data style is not specified, a standard data style is used. See section 6.7.7 for information on using this attribute.

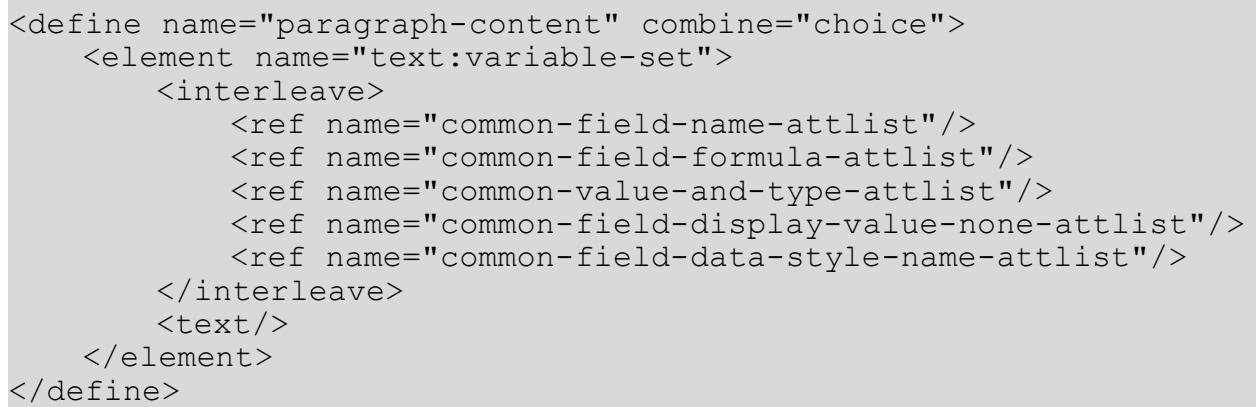

\subsubsection{Displaying Simple Variables}

The <text:variable-get> element reads and displays the value of a simple variable. The value of this element is the value of the last preceding <text:variable-set> element with an identical text: name attribute. The element determines how the value of the variable is presented, in accordance with the chosen formatting style.

The attributes that may be associated with the <text:variable-get> element are:

- text:name

This attribute specifies the name of the variable to display. The name must match the name of a preceding <text:variable-del> element. See section 6.7.3 for information on using this attribute.

- text:display

This attribute can be used to specify whether to display the formula for a simple variable or the computed value of the variable. See section 6.7 .5 for information on using this attribute.

- style:data-style-name 
This attribute specifies the data style to use to format a numeric, Boolean, or date/time variable. If a data style is not specified, a standard data style is used. See section 6.7 .7 for information on using this attribute.

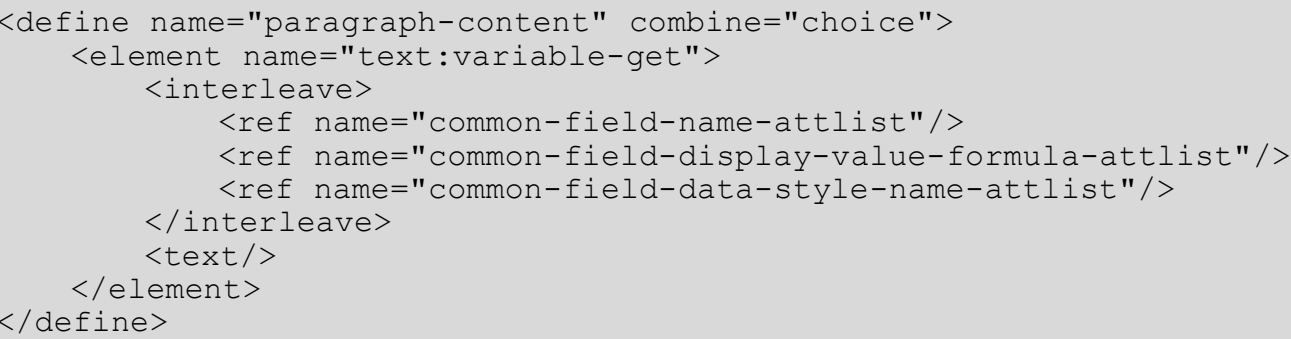

\subsubsection{Simple Variable Input Fields}

As an alternative to setting simple variables using formulas in variable setter elements, the user can be prompted for variable values. To do this, use the <text:variable-input $>$ element. This element contains the presentation of the variable's value according to the chosen formatting style. The presentation can be empty if the text: display attribute is set to none.

The attributes that may be associated with the <text:variable-input> element are:

- text:name

This attribute specifies the name of the variable to display. It must match the name of a variable that was already declared. See section 6.7.3 for information on using this attribute.

- text:description

This optional attribute contains a brief message that is presented to users when they are prompted for input. The message should give users enough information about the variable or the use of the value within the document to enable them to choose an appropriate value. See section 6.7.4 for information on using this attribute.

- office:value-type and the appropriate value attribute

See section 6.7.1 for information on using these attributes.

- text:display

This attribute can be used to specify whether to display or hide the value of the variable through the variable input field. See section 6.7.5 for information on using this attribute.

- style:data-style-name

This attribute specifies the data style to use to format a numeric, Boolean, or date/time variable. If a data style is not specified, a standard data style is used. See section 6.7.7 for information on using this attribute.

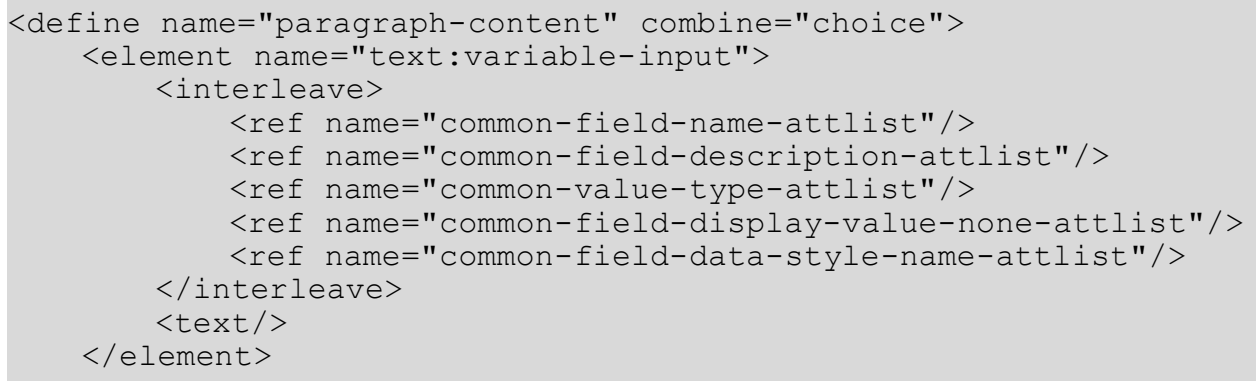




\subsubsection{Declaring User Variables}

User variables contain values that are displayed using appropriate fields. Unlike simple variables, user variables have the same value throughout a document. For this reason, the value of user variables is stored in the variable declaration itself.

The attributes that may be associated with the <text:user-field-del> element are:

- text:name

This attribute specifies the name of the variable to be declared. The name must be unique. It cannot already be used for any other type of variable including simple and sequence variables. See section 6.7.3 for information on using this attribute.

- text:formula

This attribute contains the formula to compute the value of the user variable field. If the formula is the same as the content of the field element, this attribute can be omitted. See section 6.7.6 for information on using this attribute.

- office:value-type and the appropriate value attribute

See section 6.7.1 for information on using these attributes.

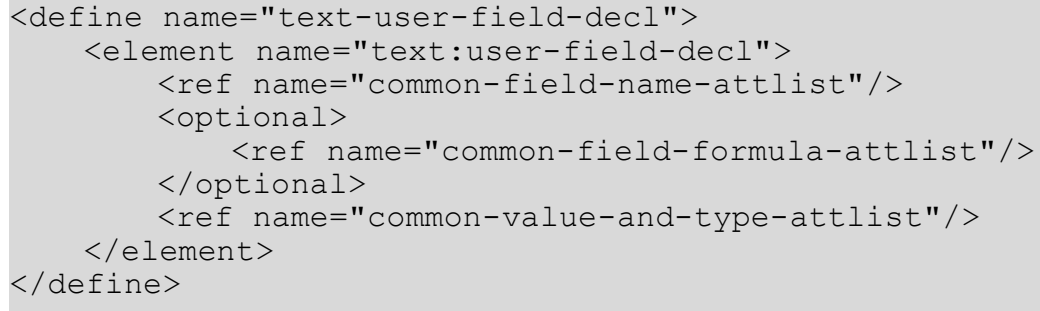

\subsubsection{Displaying User Variables}

The content of user variables can be displayed using <text: user-field-get> elements.

The attributes that may be associated with the <text: user-field-get> element are:

- text: name

This attribute specifies the name of the variable to display. The name must match the name of a preceding <text: user-field-del> element. See section 6.7.3 for information on using this attribute.

- text:display

This attribute can be used to specify whether to:

- Display the formula used to compute the value of the user variable.

- Display the value of the user variable.

- Hide the user variable fields.

- See section 6.7.5 for information on using this attribute. 
Note: Since the office application user interfaces usually allow users to edit a user field variable by clicking on any user field, a hidden <text: user-field-get> element can be used as an anchor to allow easy access to a particular user field variable.

- style:data-style-name

This attribute specifies the data style to use to format a numeric, Boolean, or date/time variable. If a data style is not specified, a standard data style is used. See section 6.7 .7 for information on using this attribute.

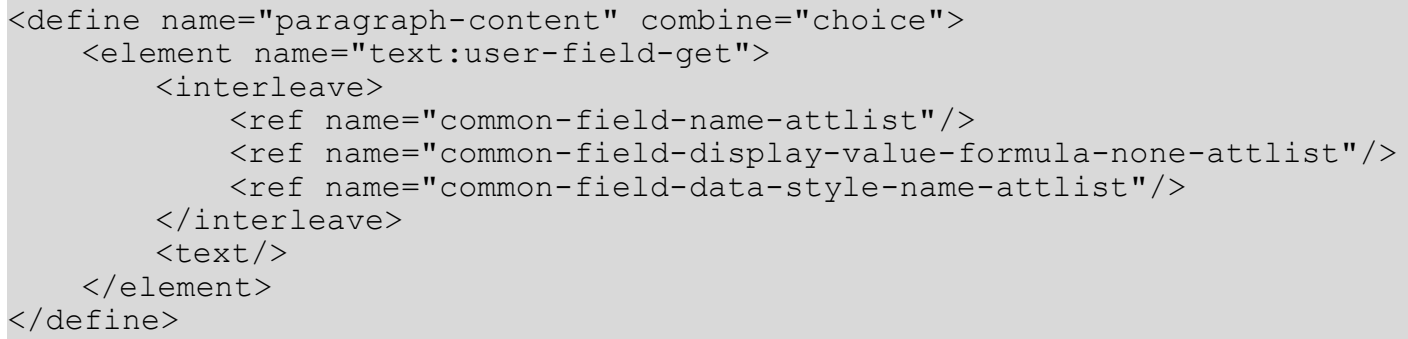

\subsubsection{User Variable Input Fields}

An alternative method of setting user variables is to use input fields, similar to the input fields for simple variables. A user variable can be set in this way using the <text: user-field-input> element. Since the value of a user field variable is stored in the <text: user-field-del> element, the <text:user-field-input> element does not contain the value and value type attributes from the <text:variable-input> field.

The presentation can be empty if the text: display attribute is set to none.

The attributes that may be associated with the <text:user-field-input> element are:

- text:name

This attribute specifies the name of the variable to set. It must match the name of a variable that has already been declared. See section 6.7 .3 for information on using this attribute.

- text:description

This optional attribute contains a brief message that is presented to users when they are prompted for input. The message should give users enough information about the variable or the use of the value within the document, to enable them to choose an appropriate value.

See section 6.7.4 for information on using this attribute.

- style:data-style-name

This attribute specifies the data style to use to format a numeric, Boolean, or date/time variable. If a data style is not specified, a standard data style is used. See section 6.7.7 for information on using this attribute.

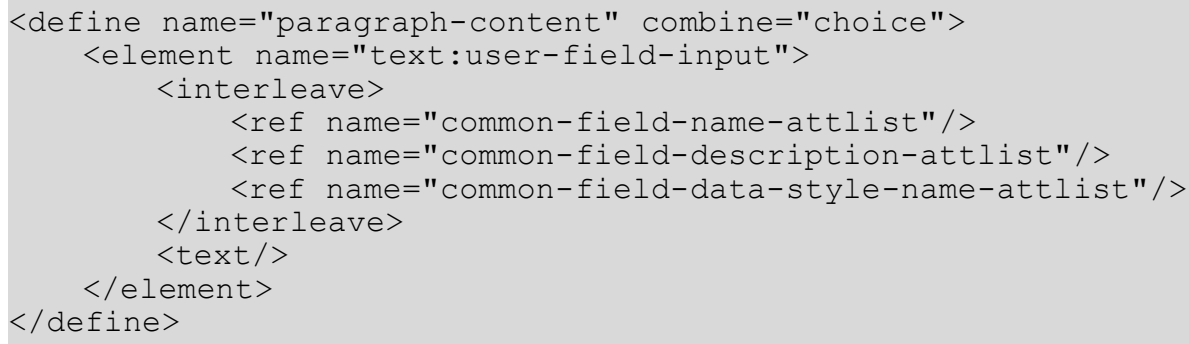




\subsubsection{Declaring Sequence Variables}

Sequence variables are used to number items within an OpenDocument text document. Sequence variables are most commonly used for sequential numbering. However, expression formulas can be included in sequence fields to support more advanced sequences. See section 6.3.9 for more information on Using Sequence Fields and their uses.

Sequence variables are declared using the $<$ text: sequence-del $>$ element.

To facilitate chapter-specific numbering, attributes can be attached to a sequence variable to specify a chapter level and a separation character. The attributes that may be associated with the <text: sequence-del> element are:

- text:name

This attribute specifies the name of the variable to be declared. The name must be unique. It cannot already be used for any other type of variable including simple and user variables.

See section 6.7.3 for information on using this attribute.

- text:display-outline-level

See following section Outline Level for information about this attribute.

- text:separation-character

See following section Separation Character for information about this attribute.

\section{Outline Level}

Sequences can be numbered by chapter. To use this feature, use the text: displayoutline-level attribute to specify an outline level that determines which chapters to reference for the chapter-specific numbering. All chapters that are at or below the specified outline level reset the value of the sequence to zero, the default value. Also, the chapter number of the last chapter at or below the specified outline level is prepended to the sequence number. Choosing an outline level of zero results in a straight sequence of all sequence elements for that sequence variable.

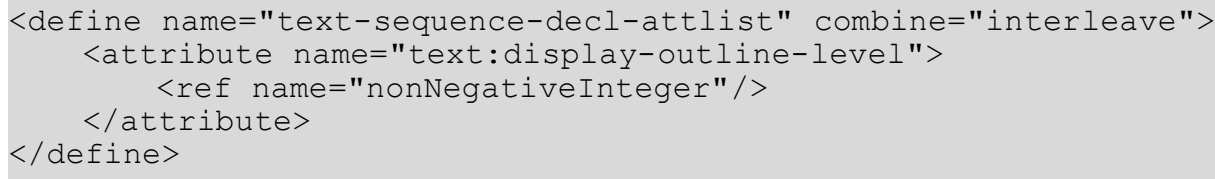

\section{Separation Character}

If sequences are numbered by chapter, this attribute is used to choose a character to separate the chapter number from the sequence number.

If the value of the text:display-outline-level attribute is a non-zero value, a separation character may be specified. The default separation character is ". ".Otherwise, if the value of text:display-outline-level is zero, this attribute must be omitted. 


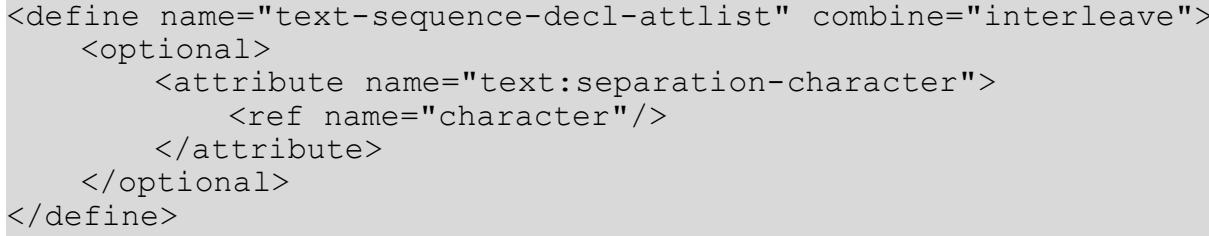

\section{Example: Sequence variable}

The sequence variable $3.7 .36 \# 5$ with a value of 5 is declared using:

\begin{tabular}{|c|l|}
\hline \multicolumn{1}{|c|}{ Attribute } & Value \\
\hline text:display-outline-level & 3 \\
\hline text:separation-character & $\#$ \\
\hline
\end{tabular}

\subsubsection{Using Sequence Fields}

Once a sequence variable is declared, it can be used in sequence fields throughout the document. Most sequence fields simply increment and display the sequence variable. However, sequence fields can also assume a new start value at any given position in a document. This start value is computed using a formula which is contained in the sequence field. If a sequence field without a start value is added, the office application software automatically inserts an expression of the type variable+1.

Sequence fields are most commonly used for simple counting sequences. However, the ability to provide arbitrary expressions supports more complex sequences. To form a sequence of even numbers, all sequence elements for that particular variable need to contain a formula incrementing the value by two, for example, variable +2 . A sequence with a starting value of 1 and all subsequent elements using the formula variable*2 yields all powers of two. Since different sequence elements for the same sequence variable may contain different formulas, complex sequences may be constructed.

The attributes that may be associated with the <text: sequence> element are:

- text:name

This attribute specifies the name of the variable that the field is to display. It must match the name of a sequence variable that was already declared. See section 6.7.3 for information on using this attribute.

- text:formula

This optional attribute contains a formula to compute the value of the sequence field. If this attribute is omitted, an expression containing the content of the element is used. See section 6.7.6 for information on using this attribute.

- $\quad$ style:num-format and style:num-letter-sync

These attributes specify the numbering style to use. If a numbering style is not specified, the numbering style is inherited from the page style. See section 6.7 .8 for information on these attributes.

- text:ref-name

See the following section Reference Name for more information about this attribute. 


\section{Reference Name}

Sequence fields can be the target of references, as implemented using reference fields. See section 6.6.5 for more information about reference fields. To enable a reference field to identify a particular sequence field, the sequence field must contain an additional attribute containing a name. No two sequence fields can have the same reference name.

If the sequence field is not the target of a reference, this attribute can be omitted.

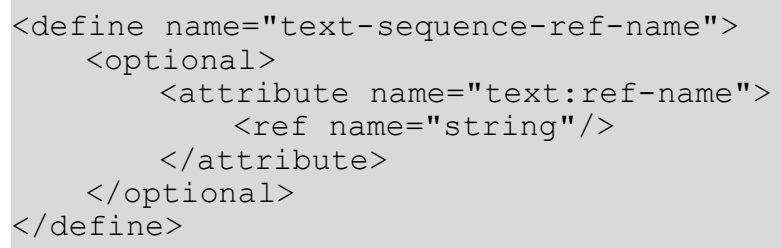

\subsubsection{Expression Fields}

Expression fields contain expressions that are evaluated and the resulting value is displayed. The value of the expression is formatted according to the chosen formatting style.

The attributes that may be associated with the <text:expression> element are:

- text:formula

This attribute contains the actual expression used to compute the value of the expression field. See section 6.7.6 for information on using this attribute.

- office:value-type and the appropriate value attribute

See section 6.7.1 for information on using these attributes.

- text:display

Use this attribute to specify one of the following:

- To display the value of the field.

- To display the formula used to compute the value.

See section 6.7 .5 for information on using this attribute.

- style:data-style-name

This attribute specifies the data style to use to format a numeric, Boolean, or date/time variable. If a data style is not specified, a standard data style is used. See section 6.7.7 for information on using this attribute. 


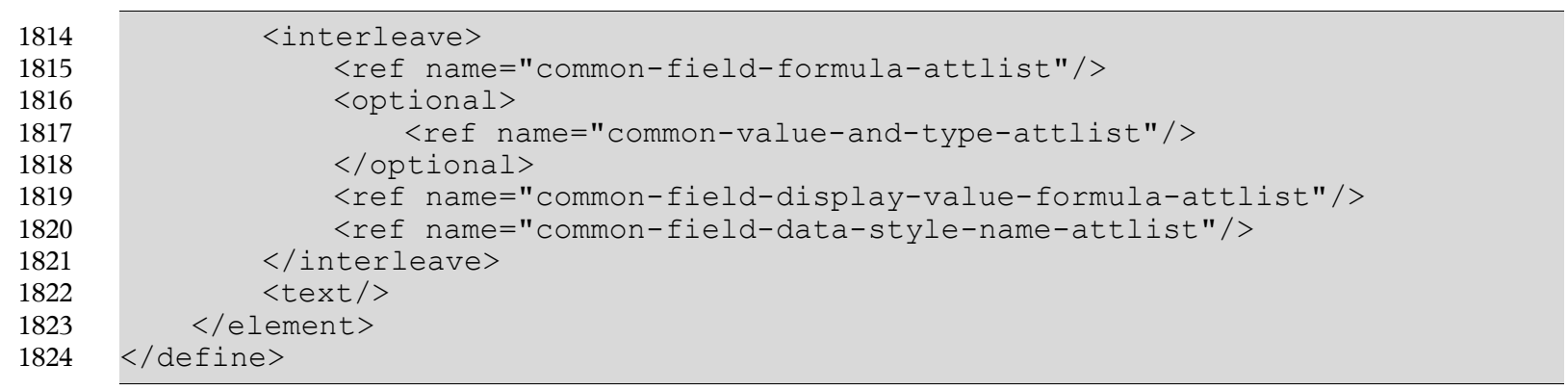

\subsubsection{Text Input Fields}

A text input field is a variable field. From the point of view of the user interface, a text input field is similar to the <text:variable-input> and <text: user-field-input> fields. However, the text input field does not change the value of any variables.

The only attribute that may be associated with the <text:text-input> element is:

- text:description

This attribute contains a brief message that is presented to users when they are prompted for input. The message should give users enough information about the purpose of the field and how it is used within the document, to enable them to choose an appropriate value. See section 6.7.4 for information on using this attribute.

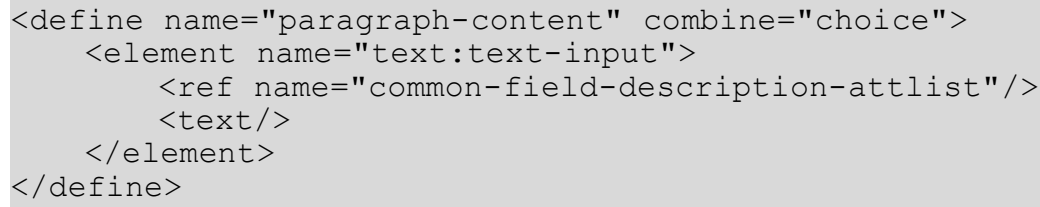

\subsection{Metadata Fields}

Metadata fields display meta information about the document, such as, the document creation date or the time at which the document was last printed. The names of the metadata field elements correspond to the metadata elements described in Chapter 3.

All metadata field elements can be marked as fixed using the text: fixed attribute. (Cf. section 6.7.2)

Several metadata fields display a date or a time. The elements for these fields require an associated text:date-value or a text:time-value attribute, and optionally, they can also have a style:data-style-name attribute. See section 6.7.1 for more information on these attributes.

\subsubsection{Initial Creator}

This element represents the name of the author who created the original document.

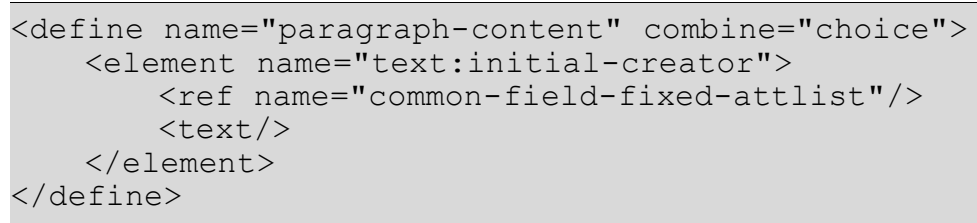




\subsubsection{Document Creation Date}

This element represents the date on which the document was created.

\subsubsection{Document Description}

This element contains a brief description of the document.

\subsubsection{User-Defined Document Information}

This element contains user-defined information about the document. It displays the information provided within a <meta: user-defined $>$ element that has the same name. 


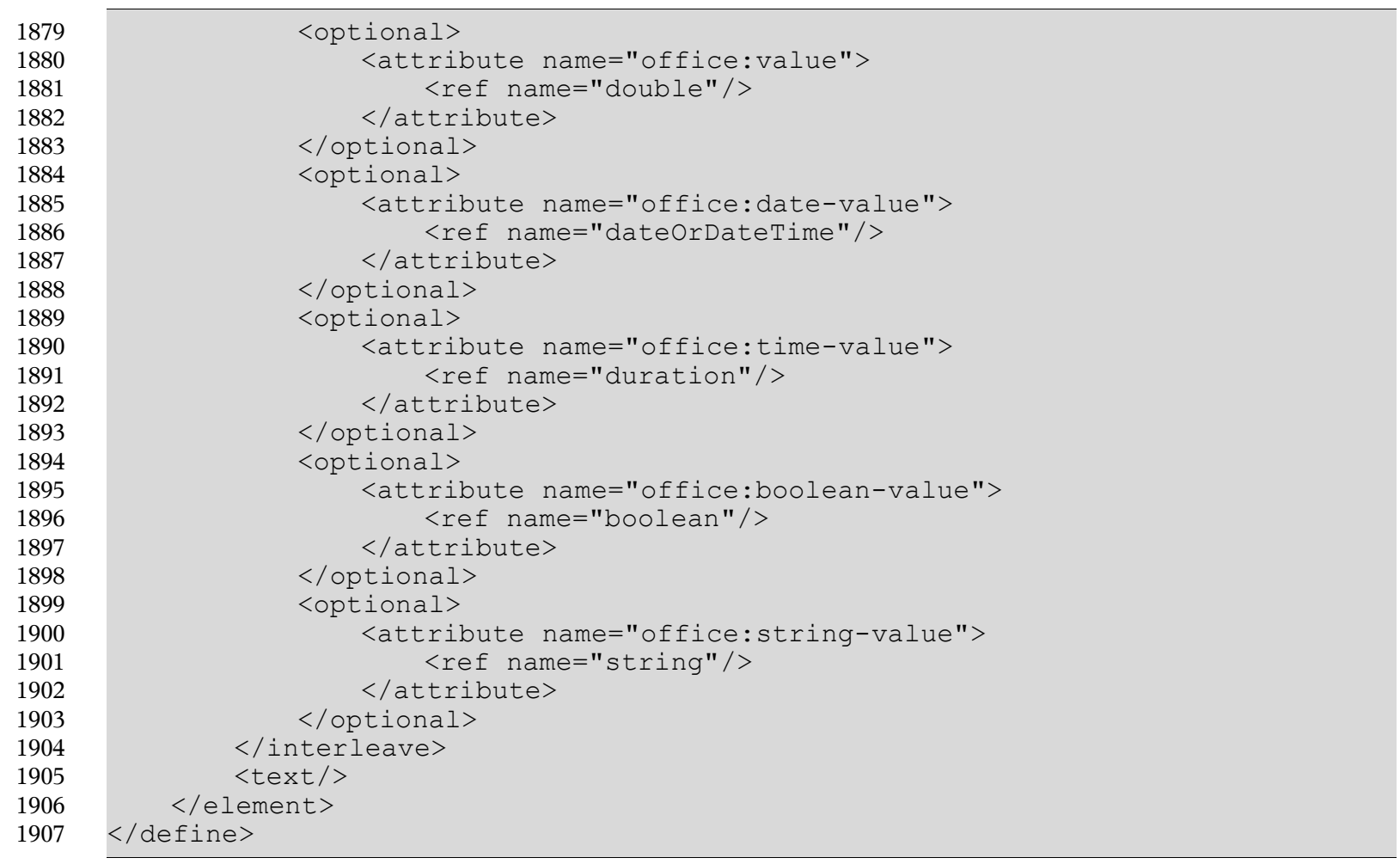

\subsubsection{Print Time}

This element represents the time at which the document was last printed.

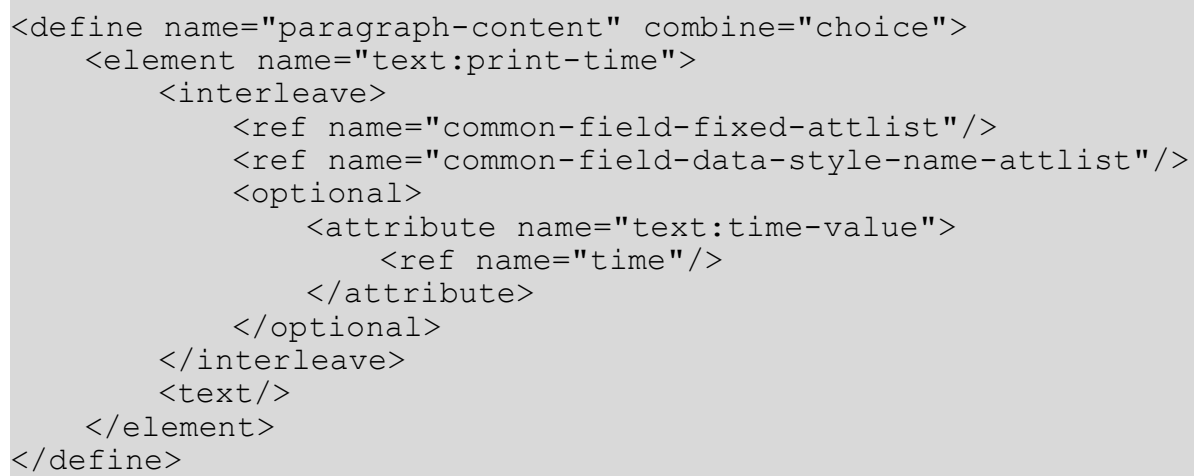

\subsubsection{Print Date}

This element represents the date on which the document was last printed.

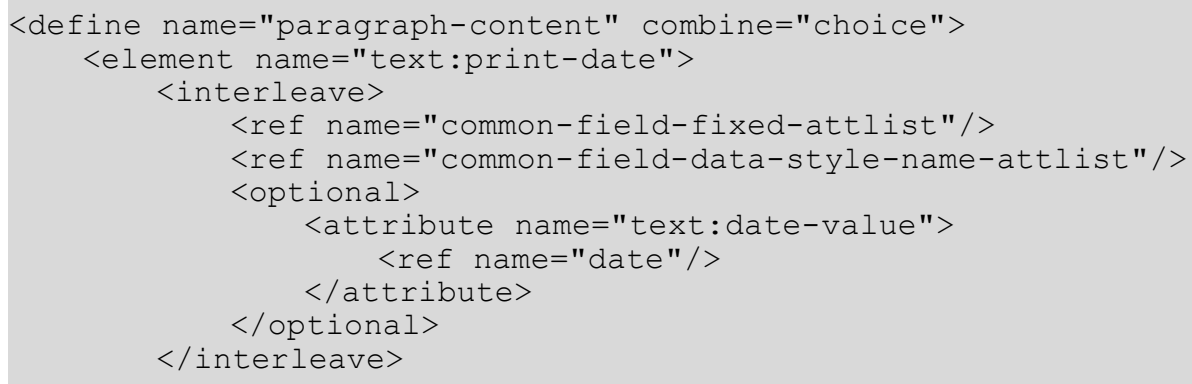




\subsubsection{Printed By}

This element represents name of the last person who printed the document.

\subsubsection{Document Subject}

This element represents the subject of the document.

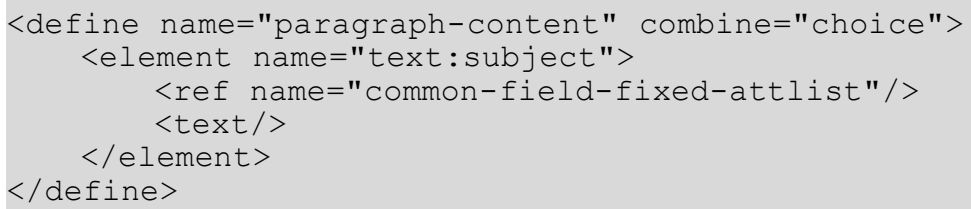

\subsubsection{Document Keywords}

This element contains a list of keywords used to describe the document.

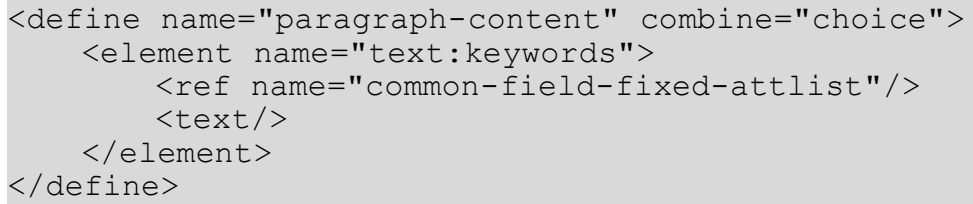

\subsubsection{Document Revision Number}

This element contains the document revision number. When the document is created, the revision number is set to 1 . Each time the document is saved, the document revision number is incremented.

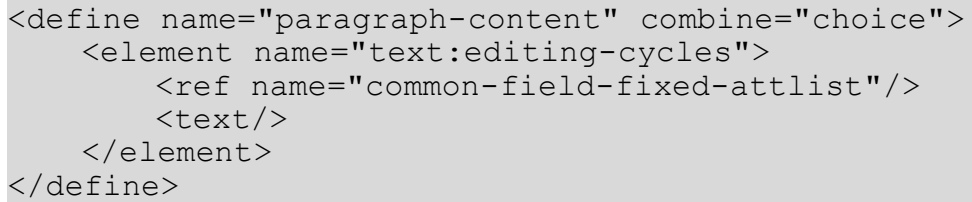


Note: Since the <text: editing-cycles $>$ field can not be formatted, the revision number can be read from the element content. Therefore, no extra attribute is needed.

\subsubsection{Document Edit Duration}

Every time a document is edited, the office application records the duration between the time the document is opened and the time the document is closed. It then adds the duration to an internal counter, thereby keeping track of the total time that has been spent editing the document.

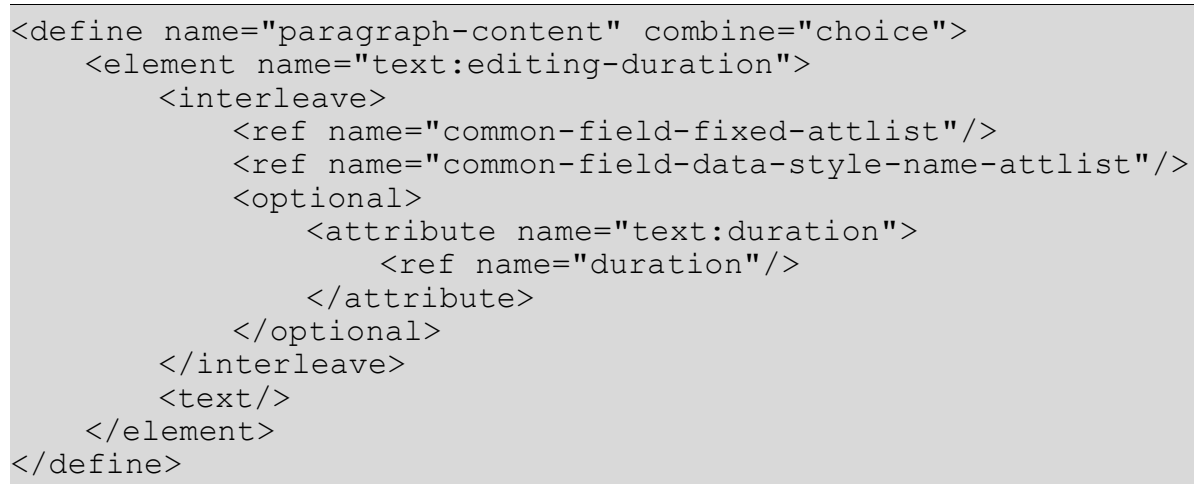

\subsubsection{Document Modification Time}

This element represents the time at which the document was last modified.

This element displays the information from the $<$ meta: date> element. The name was chosen to avoid confusion with $<$ text : date $>$ fields.

\subsubsection{Document Modification Date}

This element represents the date on which the document was last modified.

This element displays the information from the $<$ meta: date $>$ element. The name was chosen to avoid confusion with $<$ text: date $>$ fields.

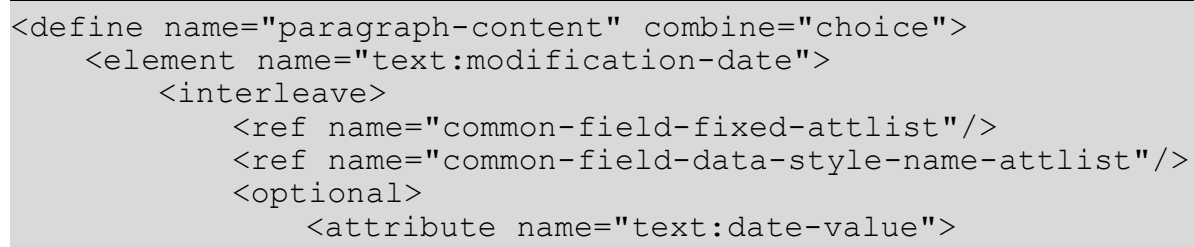




\subsubsection{Document Modified By}

This element represents the name of the person who last modified the document.

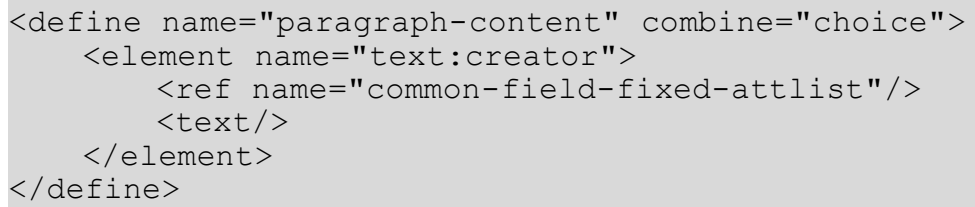

\subsubsection{Document Statistics Fields}

These fields display how many objects of a certain type a document contains. They can be used to display the number of

- pages,

- paragraphs,

- words,

- characters,

- tables,

- images, or

- embedded objects.

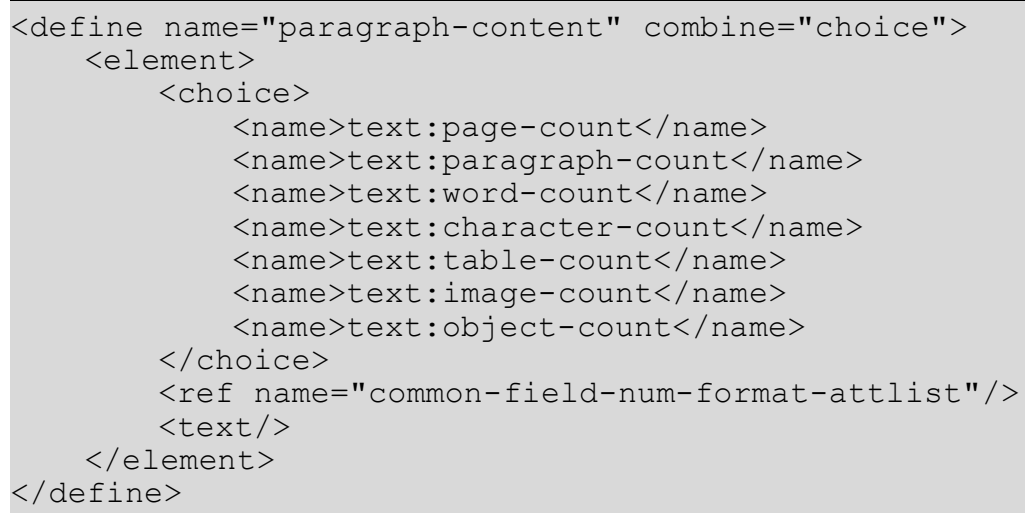

\subsection{Database Fields}

Documents can reference databases and display database information as text content. To display database information, the OpenDocument schema uses a group of text fields, collectively called database fields. Office applications may use database tables from SQL servers, therefore database fields can be used to access any SQL database, provided that the appropriate drivers are available. 
A database may contain the following components:

- Tables, which store the actual data.

- Queries, which extract a subset of data from one or more tables.

- Forms, which present the data.

- Reports, which summarize the database content.

Database forms and reports are not relevant to text content, therefore they are not discussed in this chapter. From the point of view of embedding database information in OpenDocument text documents, queries and tables are considered the same. Therefore for the remainder of this section, the phrase database table refers to both database tables and database queries.

Database fields alone do not retrieve information from a database. In addition to the database fields, a set of database rows is also added to the document. When new data is added to the document, all database fields belonging to the added database table are updated. Using the office application user interface, database rows can be added in one of the following ways:

- Manually, using a data source browser and the data to fields function.

- Using the Form Letter menu item on the File menu. This menu item adds each row in the chosen data set into a newly created copy of the form letter.

To display data from a database table use the $<$ text: database-display $>$ element. The $<$ text: database-select> and <text: database-next> elements can be used to determine which row within the current selection should be displayed. The current row number for a particular table can be displayed using the <text: database-row-number $>$ element. Finally, the <text: database-name $>$ field displays the name of the most recently used database, which is the address book file database by default.

\subsubsection{Database Field Data Source}

A database field's source can either be the name of a database, or an URI containing database connection resource data. If the source is a database name, then this name is used by all of the office application components to identify a database. All database fields contain a database name or connection resource, and most database fields also contain the name of a database table, which must be stored in the database. An additional attribute determines whether the database table refers to an SQL table, an OpenDocument query, or the result of a SQL command.

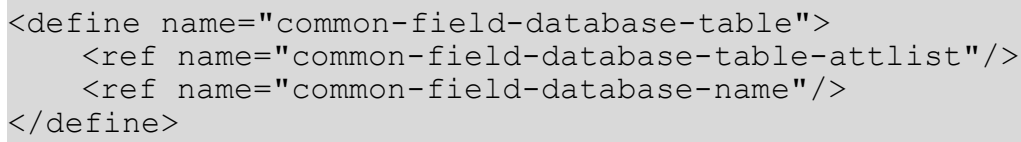

\section{Database Name}

The text: database-name attribute specifies the source database by its name.

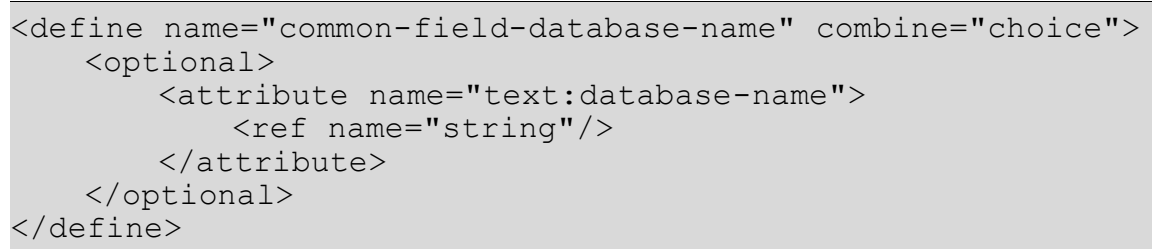




\section{Connection Resource}

The <form: connection-resource> element specifies the source database by an [XLink]. Its xlink: href attribute either references a file containing a database, or it contains information on how to make a connection to a database, for instance a [JDBC] URL. See also section 11.1.20.

\subsubsection{Displaying Database Content}

The <text: database-display> element displays data from a database. When a new data set is added to a document, all fields that display data from that database table update their content.

The attributes that may be associated with the <text:database-display $>$ element are:

- text:database-name, text:table-name and text:table-type

These attributes specify the database and database table that this field uses.

- text:database-column-name

See following section Column Name for information about this attribute.

- style:data-style-name

If the column specifies a numeric, Boolean, date, or time value, the data is formatted according to the appropriate data style. If no data style is specified, the data style assigned to this column in is used. See section 6.7 .7 for more information about using this attribute. 


\section{Column Name}

The text: column-name attribute specifies the column from which to display the data. The value of this attribute must be a column contained in the specified database.

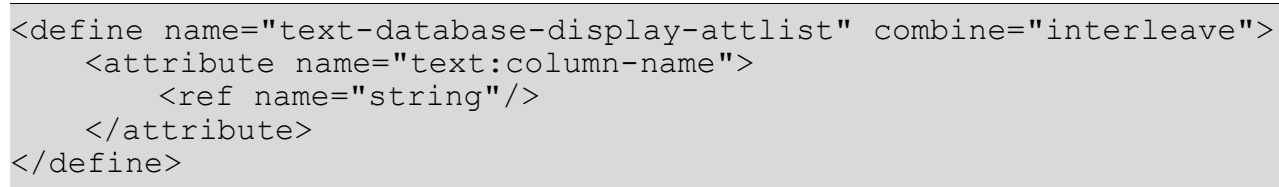

\subsubsection{Selecting the Next Database Row}

The <text: database-next> element changes the row in the current selection which is used for display in all following <text: database-display> fields. The next row from the current selection is chosen if it satisfies a given condition. If the next row is wanted regardless of any condition, the condition may be omitted or set to true.

The attributes that may be associated with the <text: database-next $>$ are:

- text:database-name, text:table-name and text:table-type

These attributes specify the database and the database table that this field uses.

- text:condition

See following section Condition for information about this attribute.

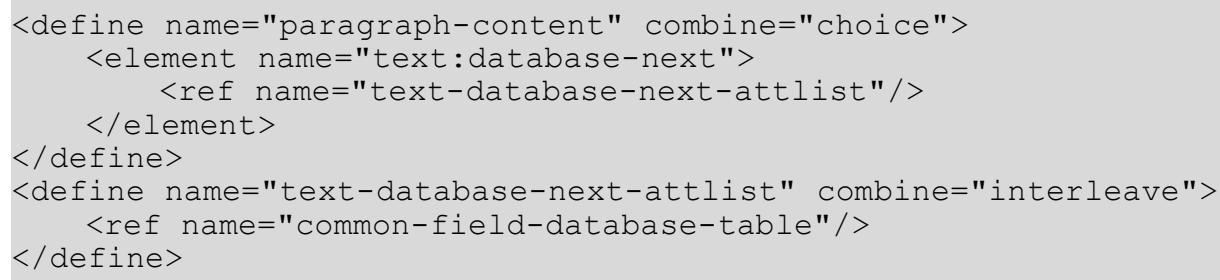

\section{Condition}

The text: condition attribute specifies the condition expression. The expression is evaluated and if the result interpreted as a Boolean value is true, the next row is used as the new current row. Database field values can be used in the expression by enclosing in square brackets the database name, the table name, and the column name, separated by dots.

If the text:condition attribute is not present, it is assumes that the formula true, meaning that the next row is selected unconditionally. 


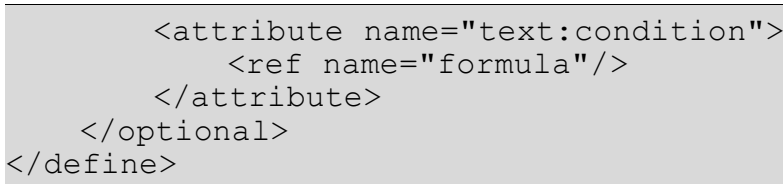

Example:

text:formula='ooo-w: [address book file.address.FIRSTNAME] == "Julie"'

This example specifies a condition that is true if the current row from an address book database table is the address for a person named Julie. If the condition shown in this example is used in a <text: database-next> element, the following happens:

- The <text:database-display> elements display the data from the first row of the current selection.

- If the FIRSTNAME column of the current row reads Julie, the current row is changed. Otherwise, nothing happens.

- If the first row is Julie, the following <text:database-display> elements display data from the second row. Otherwise, they display data from the first row.

See section 6.7.6 for more information on the formula syntax of a text: condition attribute, which is the same as that of the text: formula attribute.

\subsubsection{Selecting a Row Number}

The <text: database-row-select> element selects a specific row from the current selection. As with the <text: database-row-next> element, a condition can be specified so that the given row is only selected if the condition is true.

The attributes that may be associated with the <text: database-row-select $>$ are:

- text:database-name, text:table-name and text:table-type

These attributes determine the database and the database table that this field uses.

- text:condition

This attribute specifies the condition expression. See section 6.5 .3 for a full explanation of how to use this attribute.

- text:row-number

See the following section Selecting the Row Number about this attribute.

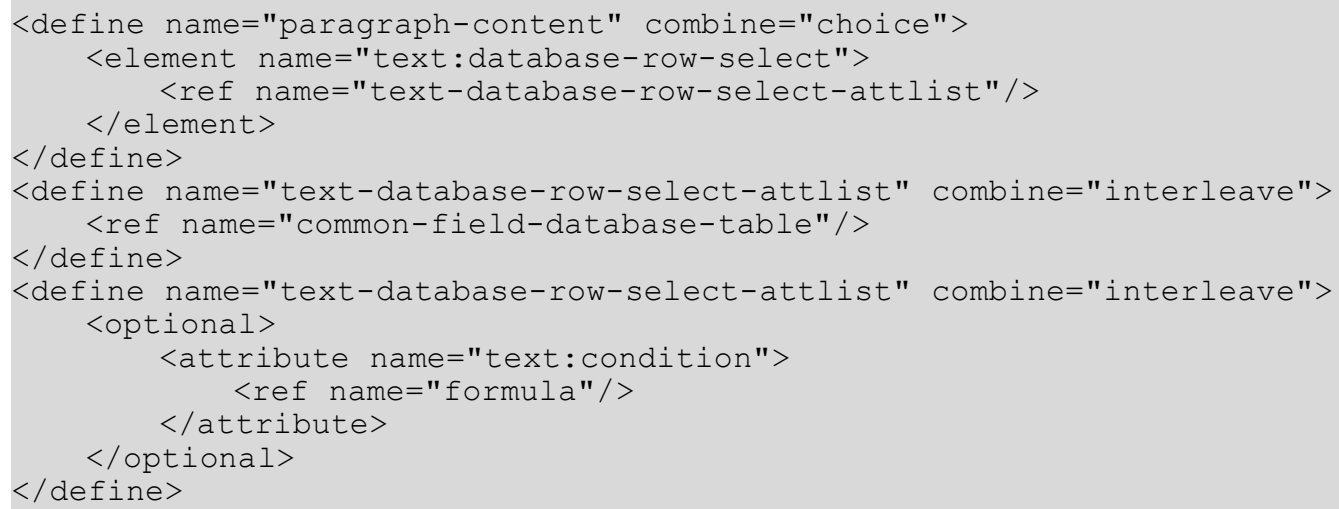




\section{Selecting the Row Number}

This attribute specifies the row number to select when a condition is true.

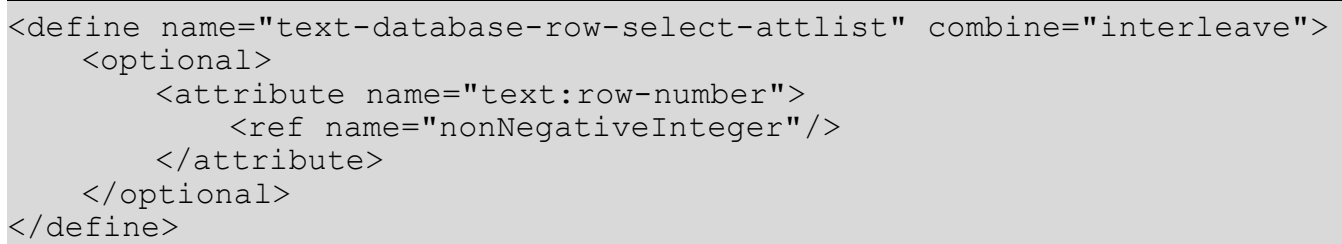

\subsubsection{Displaying the Row Number}

The <text: database-row-number > element displays the current row number for a given table. Note that the element displays the actual row number from the database and not the row number of the current selection that is used as an attribute value in the <text: database-rowselect> element.

The attributes that may be associated with the <text: database-row-number $>$ are:

- text:database-name, text:table-name and text:table-type

These attributes determine the database and the database table that this field uses.

- style:num-format and style:num-letter-sync

These attributes determine how the number should be formatted. See section 6.7 .8 for more information on how to use this attribute.

- text:value

This attribute specifies the current row number. The number changes when new data is added to the current document.

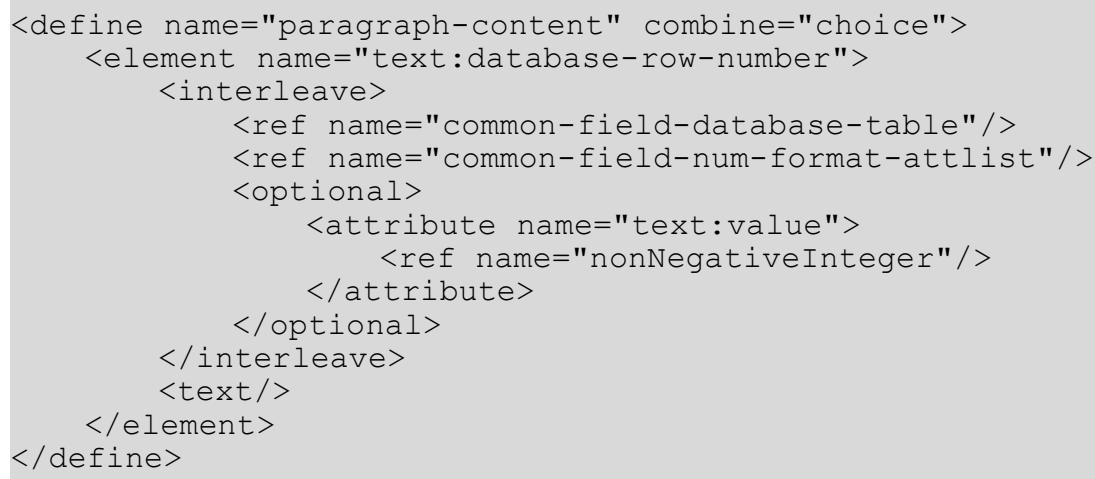

\subsubsection{Display Current Database and Table}

Office applications may keeps track of the last database and table that was used in the document. In other words, the table that is used by the last field that was inserted into the document. The <text:database-name> element displays the database and table name of the most recently used table.

The attributes that may be associated with the <text: database-name> element are:

- text:database-name, text:table-name and text:table-type 
These attributes determine the database and the database table that this field uses.

\subsection{More Fields}

\subsubsection{Page Variable Fields}

Page variables allow an alternative page numbering scheme to be defined. There is only one page variable, and it is set by any set page variable field in the document. The value of the page variable is increased on each page, in the same way as regular page numbers.

\section{Setting Page Variable Fields}

To set a page variable field, use the <text:variable-page-set> element.

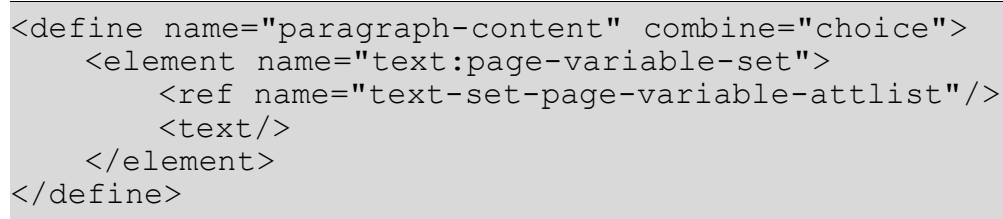

\section{Turning Page Variables On or Off}

At the beginning of a document, the page variable is inactive. The text:active attribute can be used to disable a page variable after it was used in the document.

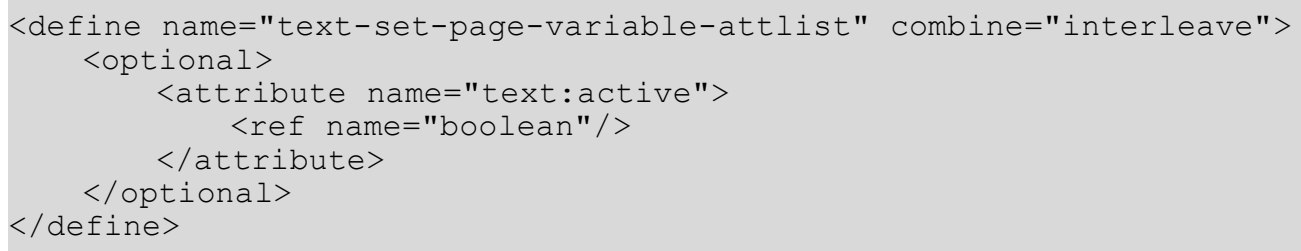

\section{Page Variable Adjustment}

The text:page-adjust attribute determines the page adjustment. The value of the active page variable is the current page number plus the closest page adjustment value that was previously set.

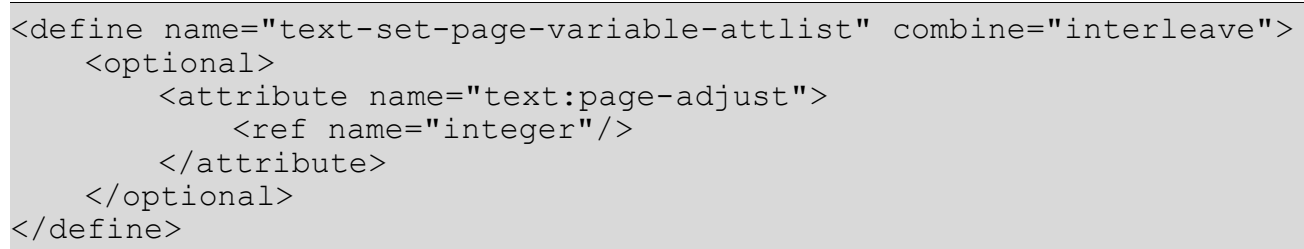




\section{Displaying Page Variable Fields}

The <text:variable-page-get> element displays the value of the page variable. The field can be formatted in the same way as regular page number fields.

\subsubsection{Placeholders}

The OpenDocument format uses placeholder fields to indicate locations in a document where the user must fill in some information. For example in a letter template, a section of the document can be reserved for the address of the recipient. A placeholder field displays text informing the user about the purpose of the placeholder and sometimes includes a description. Placeholder fields can represent different text elements, such as text or tables.

This element contains some brief text which is displayed with the placeholder.

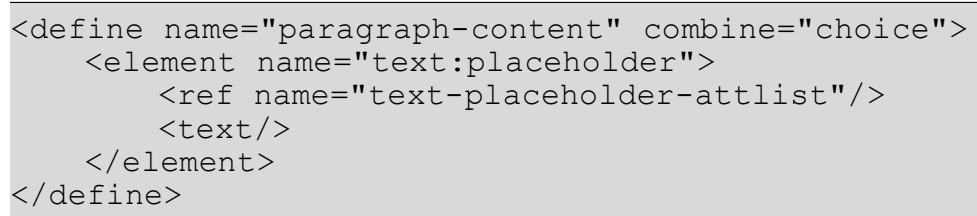

The attributes that may be associated with the <text: placeholder $>$ element are:

- Placeholder type

- Placeholder description

\section{Placeholder Type}

There are five different types of placeholder, representing the five possible types of content: text, tables, text boxes, images, or objects. The text:placeholder-type attribute represents the content type. This attribute is mandatory and it indicates which type of text content the placeholder represents. The value of the attribute can be text, text-box, image, table, or object.

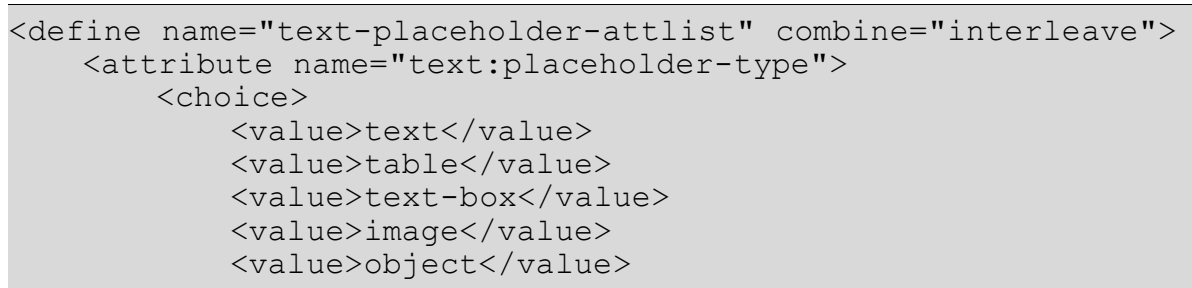




\section{Placeholder Description}

In addition to the brief text stored in the element content, may be associated a text: description attribute with the placeholder element. This attribute is optional. The purpose of the attribute is to contain a more elaborate description of the purpose of the placeholder than the description stored in the element content. See section 6.7.4 for information on using the text: description attribute.

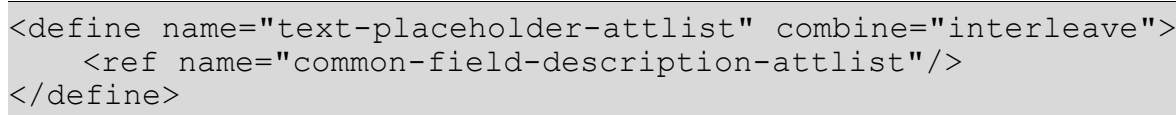

\subsubsection{Conditional Text Fields}

Text fields can be used to display one text or another, depending on a condition. Conditional text fields are given a condition and two text strings. If the condition is true, one of the text strings is displayed. If the condition is false, the other text string is displayed.

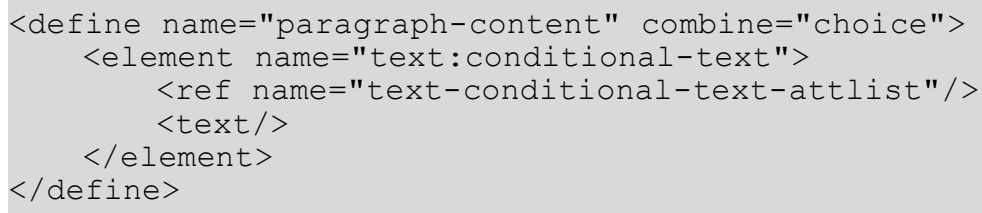

The attributes that may be associated with the <text: conditional-text $>$ element are:

- Condition

- Text to display if the condition is true

- Text to display if the condition is false

- Current condition

The text:condition attribute contains a Boolean expression. Depending on the result, the value of the text:display-if-true or text:display-if-false attribute is displayed.

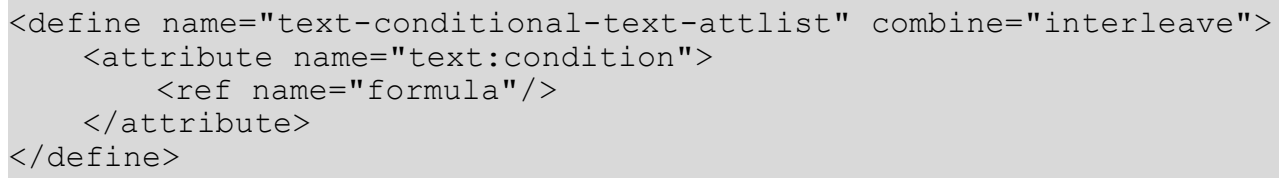

\section{Text to Display if the Condition is True}

The text:string-value-if-true attribute contains the text string to display if the condition is true.

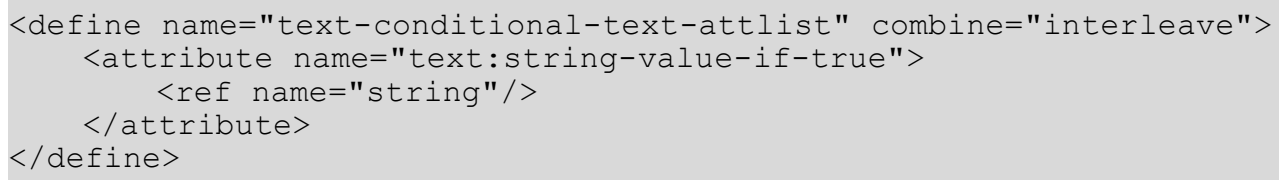




\section{Text to Display if the Condition is False}

The text:string-value-if-false attribute contains the text string to display if the condition is false.

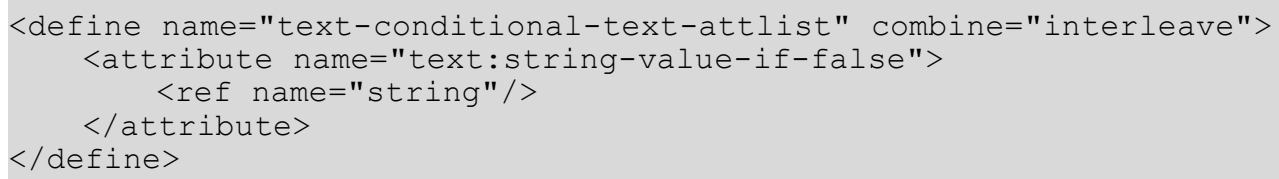

\section{Current Value and Condition}

The text: current-value attribute contains the evaluation result of the condition given by the expression in the text:condition attribute. Explicitly giving the result allows applications to delay evaluating the result until necessary. This attribute is valuable for the following reasons:

- If the expression is costly to evaluate, for example, the expression contains references to several databases.

- To allow transformations to correctly display the state of the document without having to parse and evaluate the condition.

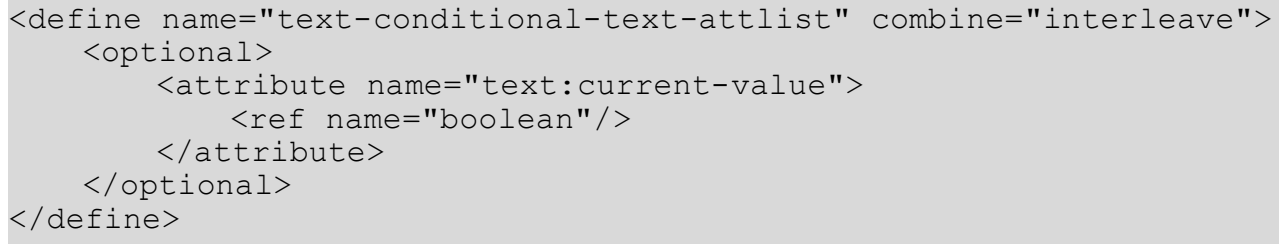

Note: The value of this attribute is overwritten with a new value as soon as the application evaluates the expression. This attribute has no function other than to ease transformation or initially display the document.

\subsubsection{Hidden Text Field}

The hidden text field is closely related to the conditional text field. It displays fixed text, except when the condition is true when it does not display anything.

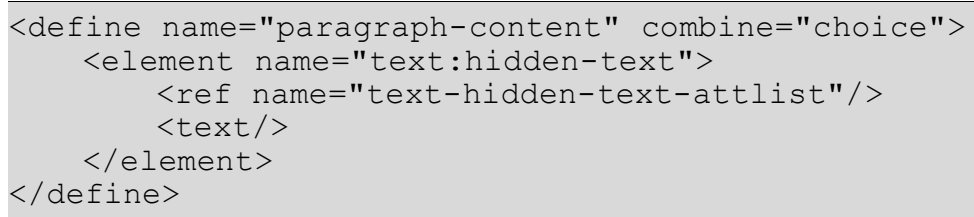

The attributes that may be associated with the <text: hidden-text $>$ element are:

- Condition

- Text

- Is hidden

\section{Condition}

The text: condition attribute contains a Boolean expression. If the expression evaluates to true, the text is hidden. 


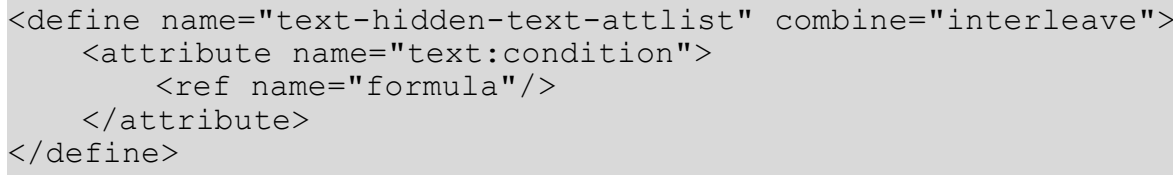

\section{Text}

The text:string-value attribute specifies the text to display if the condition is false.

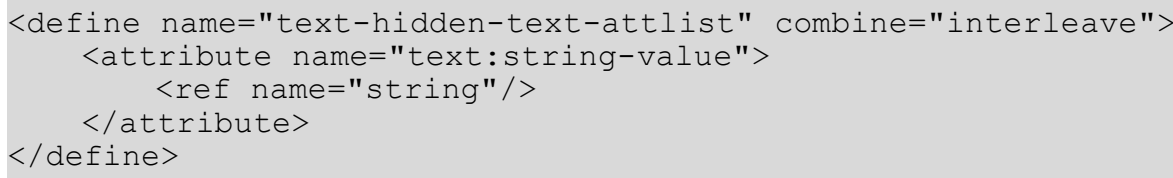

\section{Is Hidden}

The text: is-hidden attribute specifies whether or not the field is currently visible. The purpose of this attribute is similar to that of the text:current-value attribute in the text:condition field. Recording the result allows transformations to correctly represent the document without having to parse the condition expression or evaluate the condition when loading the document.

Note: The value of this attribute is overwritten with a new value as soon as the application evaluates the expression. This attribute has no function other than to ease transformation or initially display the document.

\subsubsection{Reference Fields}

The OpenDocument format uses four types of reference field and each type is represented by its own element. The reference field types are based on the type of element they refer to; notes, bookmarks, references, and sequences. Every reference contains a reference format which determines what information about the referenced target is displayed. For example, references can display:

- The page number of the referenced target

- The chapter number of the referenced target

- Wording indicating whether the referenced target is above or below the reference field

In addition, each reference field must identify its target which is usually done using a name attribute. Bookmarks and references are identified by the name of the respective bookmark or reference. Footnotes, endnotes, and sequences are are assigned names by the application used to create the OpenDocument file format automatically.

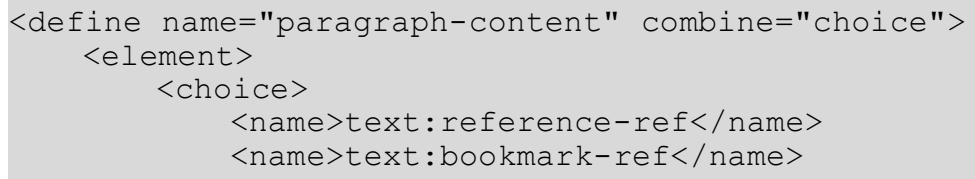




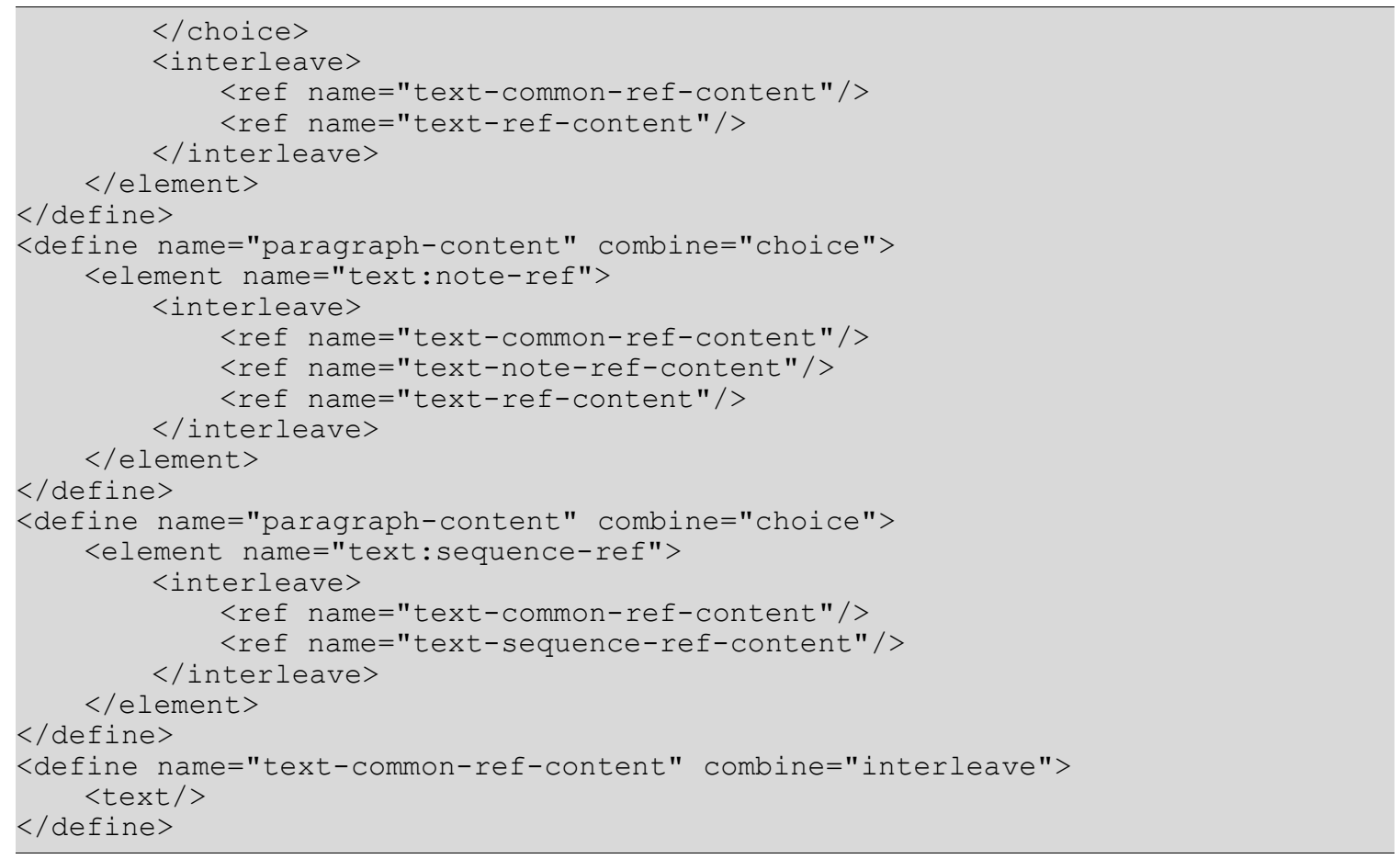

The attributes that may be associated with the reference field elements are:

- Reference name

- Reference format

\section{Reference Name}

The text:ref-name attribute identifies the referenced element. Since bookmarks and references have a name, this name is used by the respective reference fields. Footnotes, endnotes, and sequences are are identified by a name that is usually generated automatically.

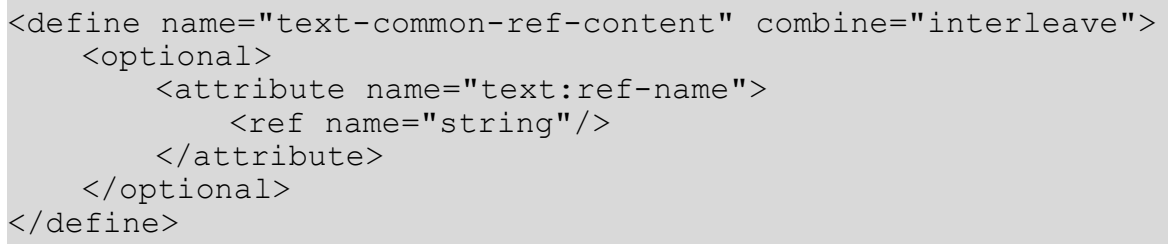

\section{Note Class}

For <text:note-ref> elements, the text:note-class attribute determines whether the field references a foot- or an endnote.

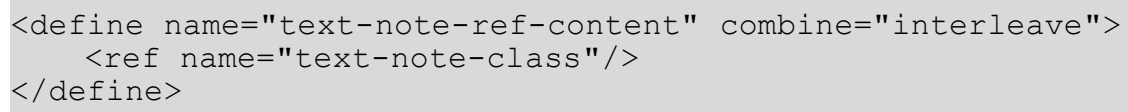

\section{Reference Format}

The text:reference-format attribute determines what information about the reference is displayed. If the reference format is not specified, the page format is used as the default. 
All types of reference fields support the following values for this attribute formats:

- page, which displays the number of the page on which the referenced item appears.

- chapter, which displays the number of the chapter in which the referenced item appears.

- direction, which displays whether the referenced item is above or below the reference field.

- text, which displays the text of the referenced item.

References to sequence fields support the following three additional values:

- category-and-value, which displays the name and value of the sequence.

- caption, which displays the caption in which the sequence is used.

- value, which displays the value of the sequence.

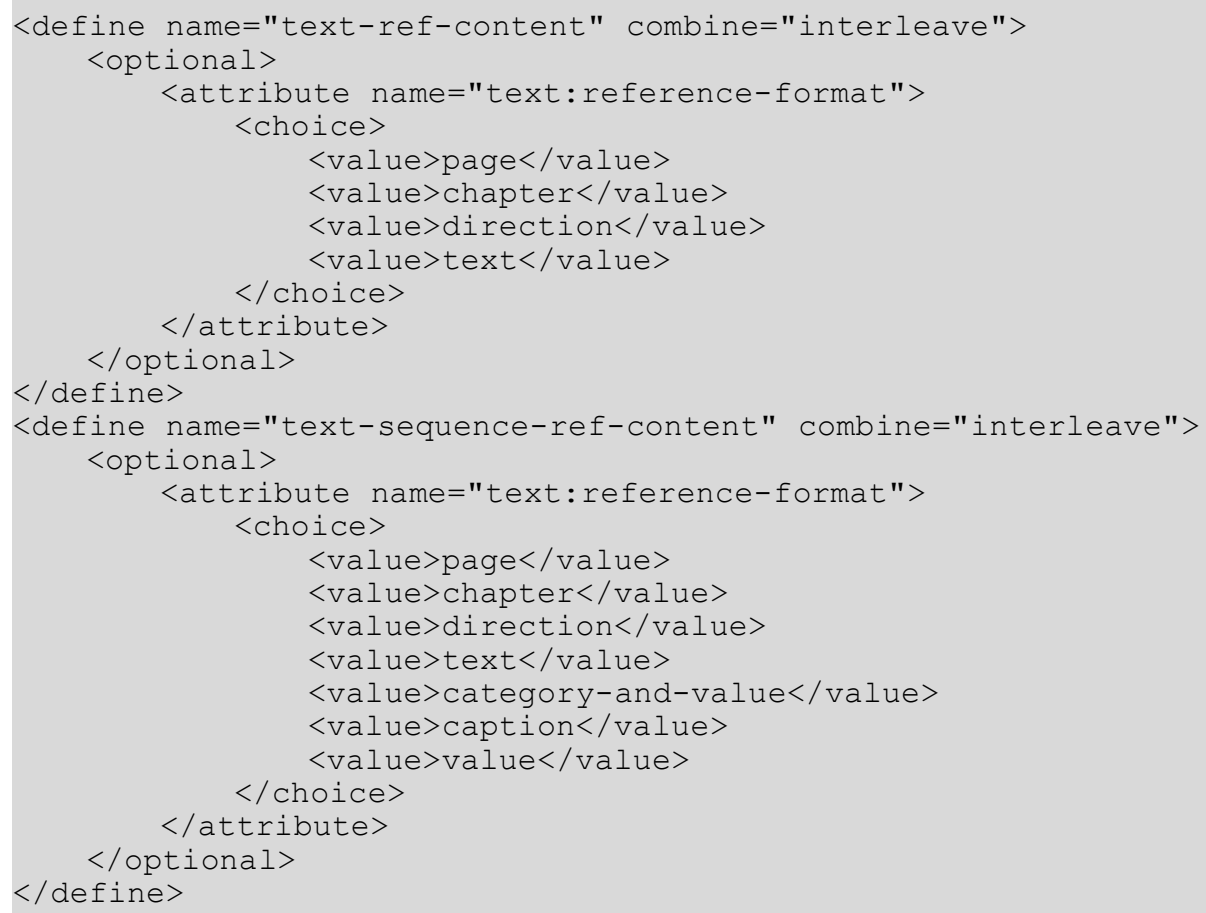

\section{Example: Different reference formats and displays}

The following table shows all possible reference formats and the resulting reference display that can be used to refer to the table itself. The left column lists the value of the text:referenceformat attribute and the right column 


\begin{tabular}{|l|l|}
\hline \multicolumn{1}{|c|}{ Reference format } & \multicolumn{1}{c|}{ Reference display } \\
\hline page & 134 \\
\hline chapter & 3.7 .27 \\
\hline text & $\begin{array}{l}\text { Table 2: Examples of reference } \\
\text { formats }\end{array}$ \\
\hline direction & above \\
\hline category-and-value & Table 1 \\
\hline caption & Examples of reference formats \\
\hline value & 1 \\
\hline
\end{tabular}

\subsubsection{Script Fields}

A script field stores scripts or sections of scripts. The field can be used to store and edit scripts that are attached to the document. The primary purpose of this field is to provide an equivalent to the <script> element in [HTML4], so that the content of a <script> element in HTML can be imported, edited, and exported using an office application software.

The source code for the script can be stored in one of the following ways:

- The <text:script> element contains the source code.

- The source code is stored in an external file. Use the xlink: href attribute to specify the location of the source file.

The element should have either a xlink: href attribute or content, but not both.

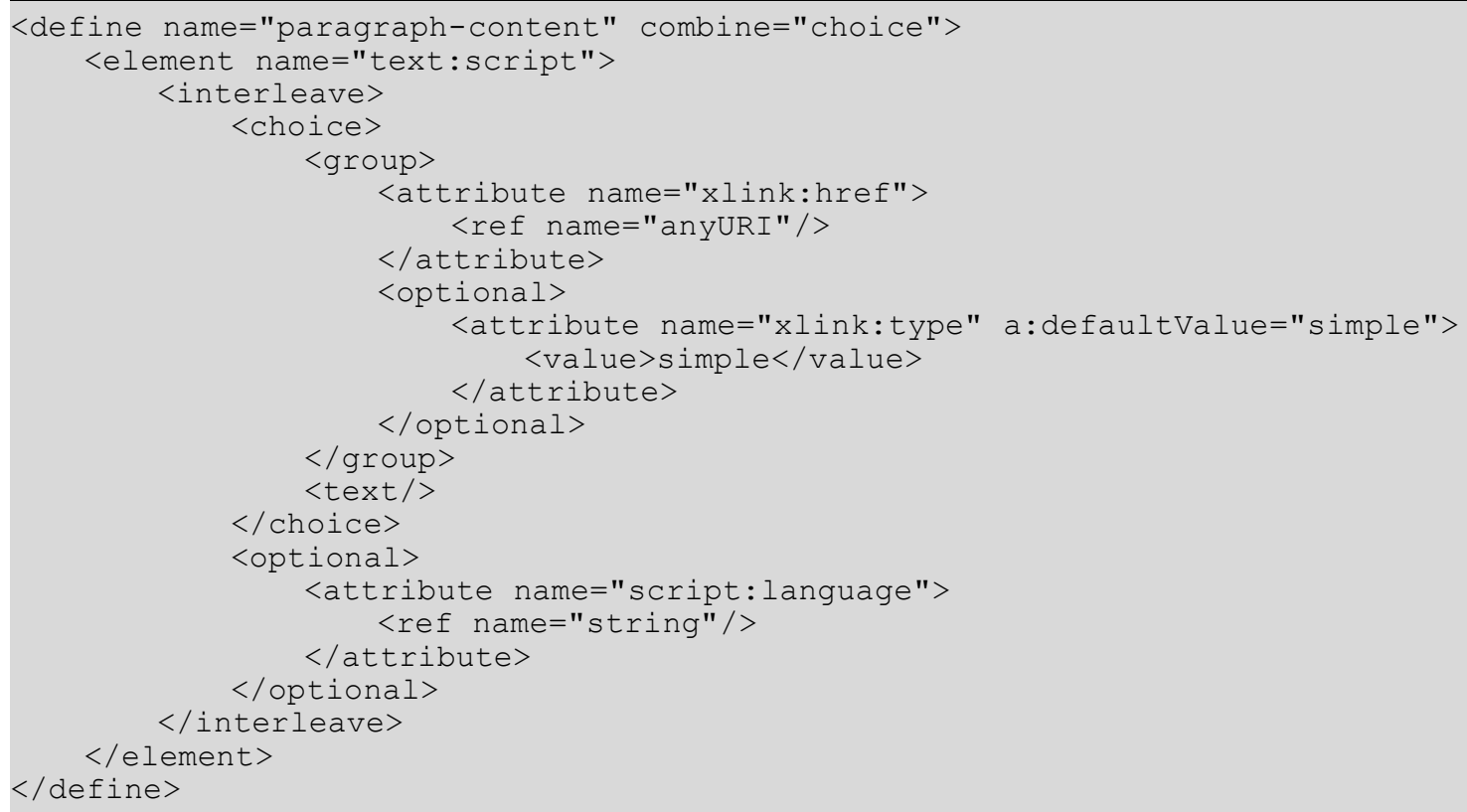




\section{Script URL}

The $x l$ ink: href attribute specifies the location of the file that contains the script source code. The script field should have either an URL attribute or content, but not both.

\section{Script Language}

The script: language attribute specifies the language in which the script source code is written, for example, JavaScript.

\subsubsection{Macro Fields}

The macro field contains the name of a macro that is executed when the field is activated. The field also contains a description that is displayed as the field content.

The only attribute that may be associated with the <text: execute-macro> element is:

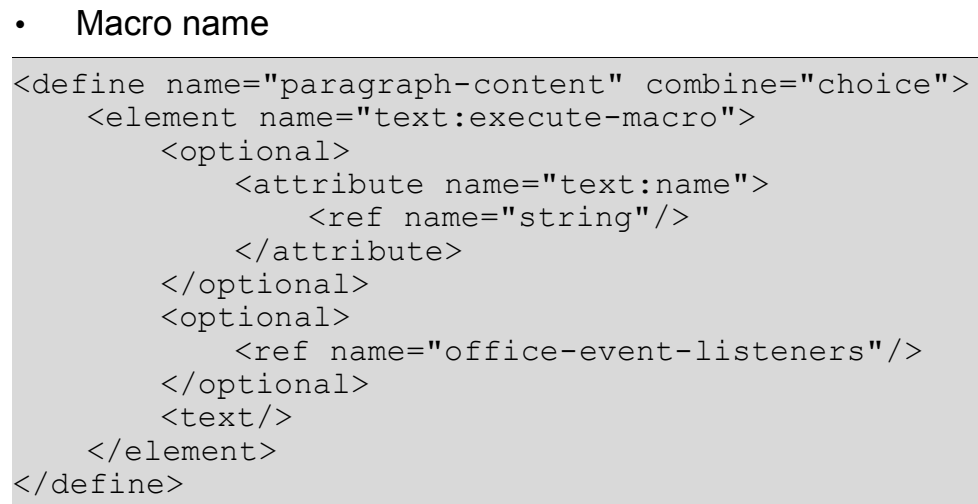

\section{Macro Name}

The text: name attribute specifies the macro to invoke when the field is activated.

\subsubsection{Hidden Paragraph Fields}

The hidden paragraph field has a similar function to the hidden text field. However, the hidden paragraph field does not have any content. It hides the paragraph in which it is contained. This allows a paragraph of formatted text to be hidden or displayed depending on whether a condition is true or false.

Hidden paragraph fields are often used together with form letters. For example, if a condition depends on a database field, a hidden paragraph field can be used to selectively include paragraphs in the form letter depending on the database content. Multiple paragraph fields can be contained one paragraph. The paragraph is displayed if the condition associated with at least one hidden paragraph field is false. Alternatively, the conditions associated with several hidden paragraph fields can be combined into a single condition for a single field using logical operations on the conditions.

Note: Unlike most fields, this field does not display text, but it affects the entire paragraph in which it is contained.

The attributes that may be associated with the <text:hidden-paragraph> element are: 
- Condition

- Is hidden

\section{Condition}

The text: condition attribute contains a Boolean expression. If the condition is true, the paragraph is hidden. If the condition is false, the paragraph is displayed.

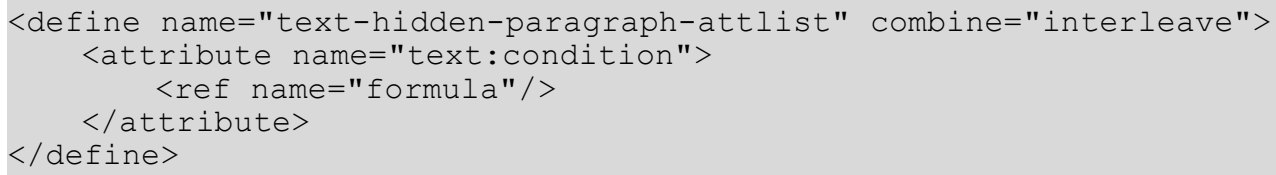

\section{Is Hidden}

The text:is-hidden attribute records whether the paragraph is currently visible or not. It has the same purpose as the corresponding attribute of the hidden text field, namely to allow correct display of the paragraph without having to evaluate the condition first. The value of this attribute is overwritten with a new value as soon as the application evaluates the expression.

Note: This attribute has no function other than to ease transformation or initially display the document.

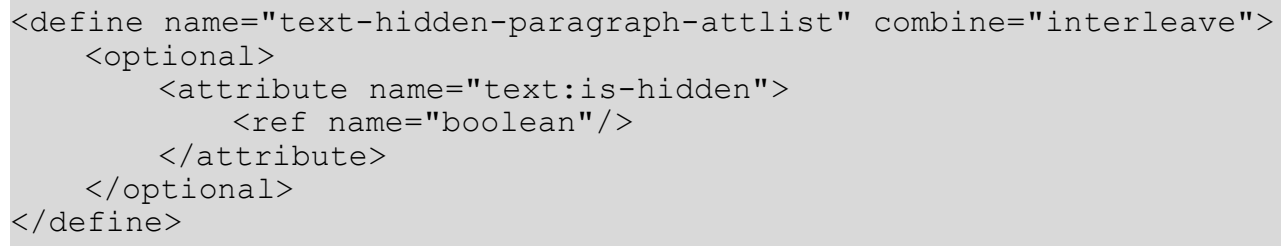

\subsubsection{DDE Connection Fields}

A DDE field allows information from a DDE connection to be displayed. The only parameter required for the DDE field is the name of the DDE connection that supplies the data to this field. This DDE connection element specifies the actual DDE field that appears in the text body.

The field element contains the content of the most recent data that was received from the DDE connection. This may be used to render the document if the DDE connection cannot be accessed.

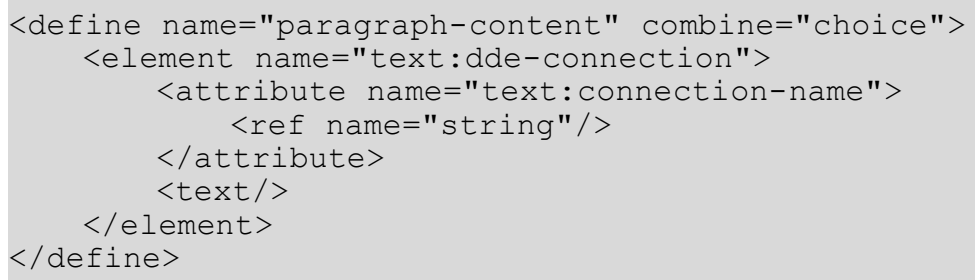

The only attribute that may be associated with the <text:dde-connection> element is: 
- DDE connection name

\section{DDE Connection Name}

The text: name attribute specifies the name of the DDE connection to which the field refers.

\subsubsection{Measure Fields}

Within the text contained in measure drawing objects (see section 9.2.11), a <text: measure $>$ field displays the current measure. The draw: kind attribute specifies which part of the measure is displayed. It my have one of the following values:

- value: The measure's value is displayed, for instance "12"

- unit: The measure's unit is displayed, for instance "inch"

- gap: A gap or blank is displayed if and only if the measure text's writing direction is perpendicular to the measure line. The purpose of this value is add some space between the measure line and the text if the text is displayed perpendicular to the measure line.

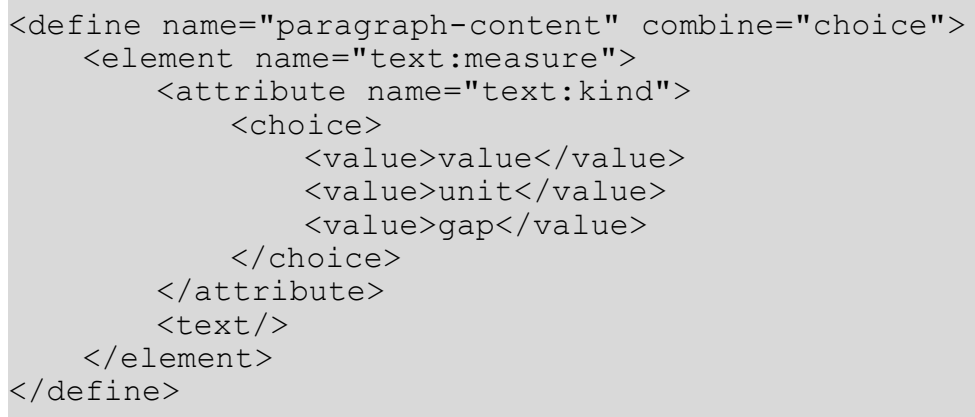

\subsubsection{Table Formula Field}

The table formula field is a legacy from previous versions of current office applications. It should not be used in new documents. It stores a formula to be used in tables, a function that is better performed by the table:formula attribute of the table cell.

Note: This element should not be used in new documents.

The table formula field can take the following attributes:

- text:formula

This attribute contains the actual expression used to compute the value of the table formula field. See section 6.7.6 for information on using this attribute.

- text:display

Use this attribute to specify one of the following:

- To display the value of the field.

- To display the formula used to compute the value.

See section 6.7 .5 for information on using this attribute.

- style:data-style-name 
This attribute specifies the data style to use to format a numeric, Boolean, or date/time variable. If a data style is not specified, a standard data style is used. See section 6.7.7 for information on using this attribute.

\subsection{Common Field Attributes}

The attributes described in this section can be used with several field elements.

\subsubsection{Variable Value Types and Values}

Variables and most variable fields have a current value. Every variable has a value type that must be specified when the field supports multiple value types. The value type is specified using the office:value-type attribute.

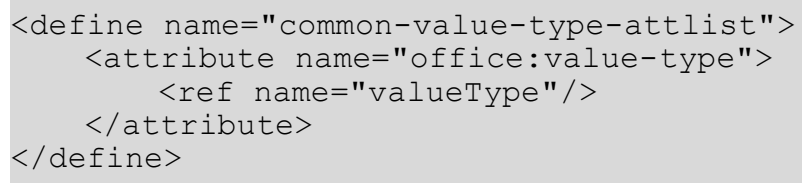

Depending on the value type, the value itself is written to different value attributes. The supported value types, their respective value attributes, and how the values are encoded are described in the following table:

\begin{tabular}{|c|c|c|c|}
\hline Value Type & Value Attribute(s) & Encoded as... & Example \\
\hline float & office:value & Numeric value & $" 12.345 "$ \\
\hline percentage & office:value & Numeric value & "0.50" \\
\hline currency & $\begin{array}{l}\text { office: value and } \\
\text { office: currency }\end{array}$ & $\begin{array}{l}\text { Numeric value and } \\
\text { currency symbol }\end{array}$ & $\begin{array}{l}\text { "100" } \\
\text { "USD" }\end{array}$ \\
\hline date & office:date-value & $\begin{array}{l}\text { Date value as specified in } \$ 3.2 .9 \\
\text { of [xmlschema-2], or date and } \\
\text { time value as specified in } \S 3.2 .7 \\
\text { of [xmlschema-2] }\end{array}$ & "2003-04-17" \\
\hline time & office:time-value & $\begin{array}{l}\text { Duration, as specified in } § 3.2 .6 \\
\text { of [xmlschema-2] }\end{array}$ & $\begin{array}{l}\text { "РT03H30M00S } \\
\text { " }\end{array}$ \\
\hline boolean & office:boolean-value & true or false & "true" \\
\hline string & office:string-value & Strings & "abc def" \\
\hline
\end{tabular}

The OpenDocument concept of field values and value types and their encoding in $\mathrm{XML}$ is modeled on the corresponding XML for table cell attributes. See section 8.1.3 for information on table cells and their attributes. 
The definition of the entity $\frac{\mathrm{v} a l u e-a t t l i s t ;}{\text { is as follows: }}$

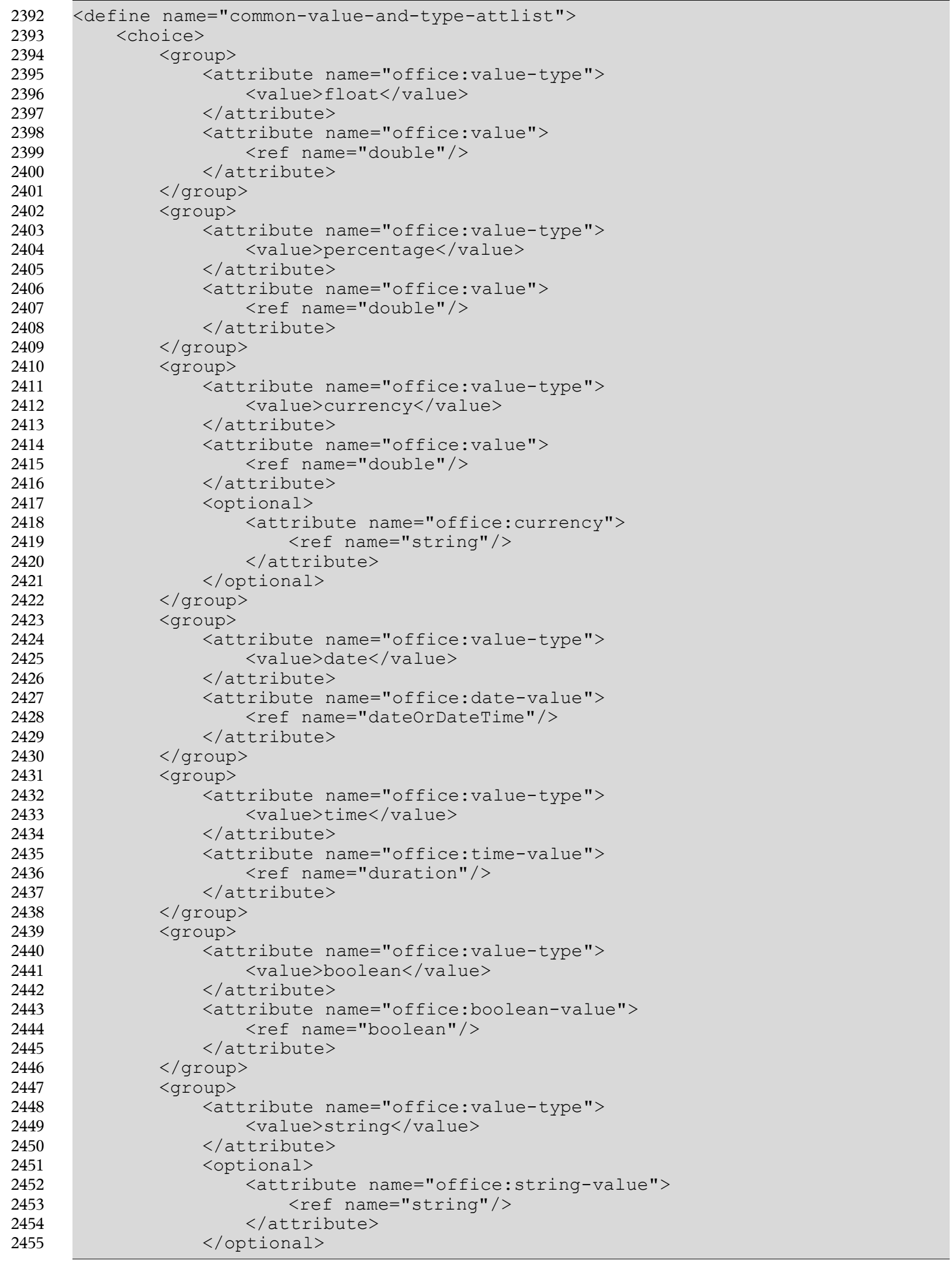




\subsubsection{Fixed}

The text: fixed attribute specifies whether or not the value of a field element is fixed. If the value of a field is fixed, the value of the field element to which this attribute is attached is preserved in all future edits of the document. If the value of the field is not fixed, the value of the field may be replaced by a new value when the document is edited.

This attribute can be used with:

- Date fields

- Time fields

- Page number fields

- All sender fields

- All author fields

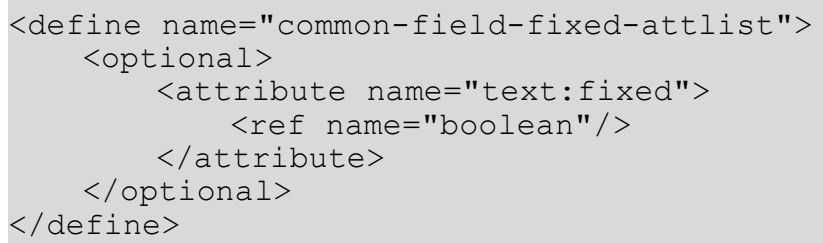

\subsubsection{Variable Name}

Use the text: name attribute to specify the name of a variable when it is being declared, set, or displayed a variable. This attribute can be used with any of the following elements:

- <text:variable-del>

- <text:variable-set>

- <text:variable-get>

- <text:variable-input>

- $\quad$ text:user-field-del>

- <text:user-field-get>

- <text:user-field-input>

- <text:sequence-del>

- <text: sequence>

When this attribute is being used to specify the name of a variable to display, a variable of the appropriate type with the same name must already have been declared.

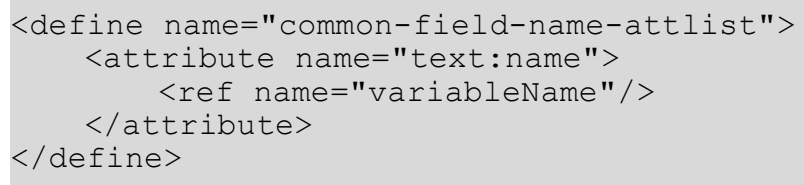




\subsubsection{Description}

The text: description attribute contains a brief message that is displayed when users are prompted for input. This attribute can be used with any of the following elements:

- $\quad$ <ext:placeholder>

- <text:variable-input>

- $\quad$ text:user-field-input>

- $\quad$ <text: text-input>

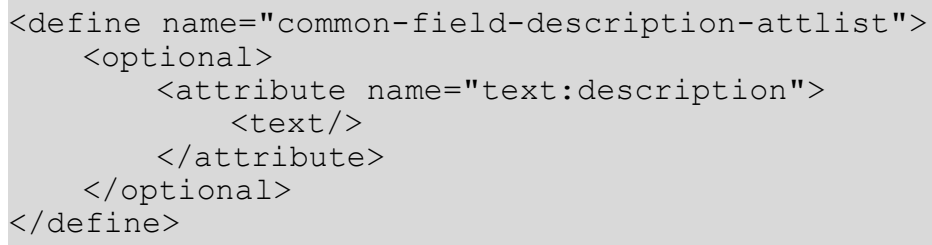

\subsubsection{Display}

The text: display attribute supports up to three values as follows:

- value

This value displays the value of the field. Some fields do not support this value. In these cases, the text:display attribute only takes the values value or none, and value or formula, respectively.

- formula

This value allows the display of the formula rather than the value of the field. Some fields do not support this value. In these cases, the text: display attribute only takes the values value or none, and value or formula, respectively.

- none

Several variable fields support this value, which hides the field content. This allows variables to be set in one part of the document and displayed in another part of the document.

This attribute can be used with any of the following elements:

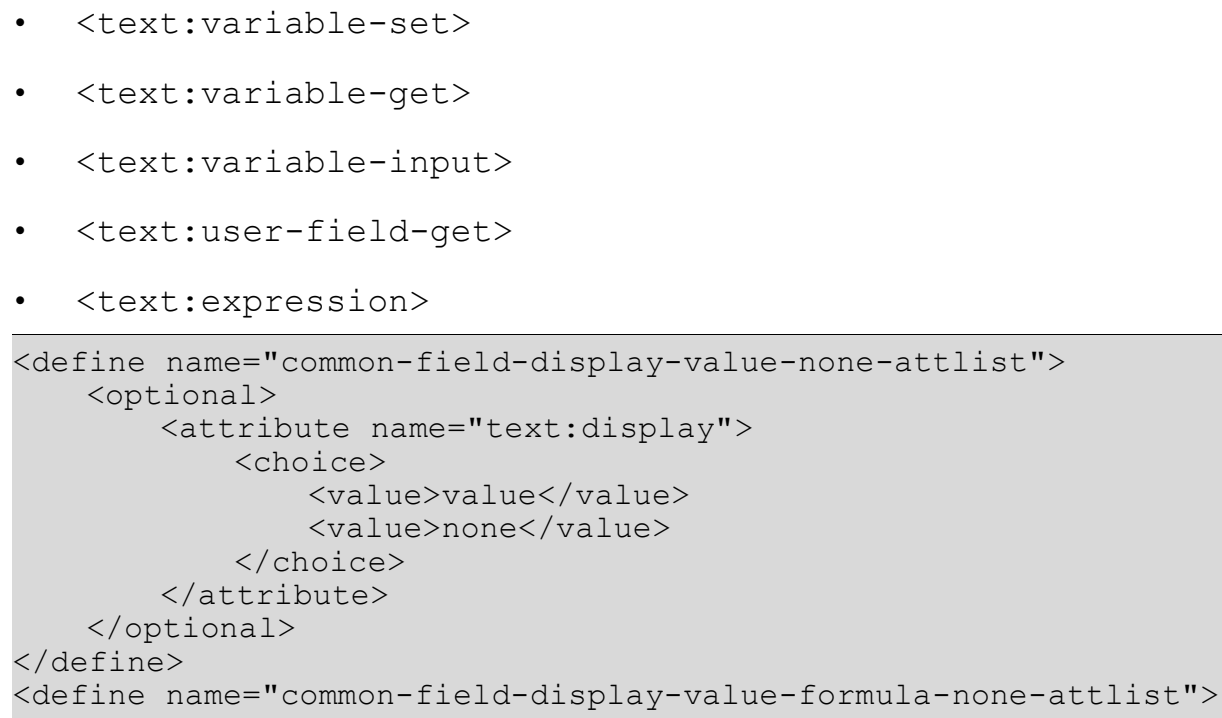




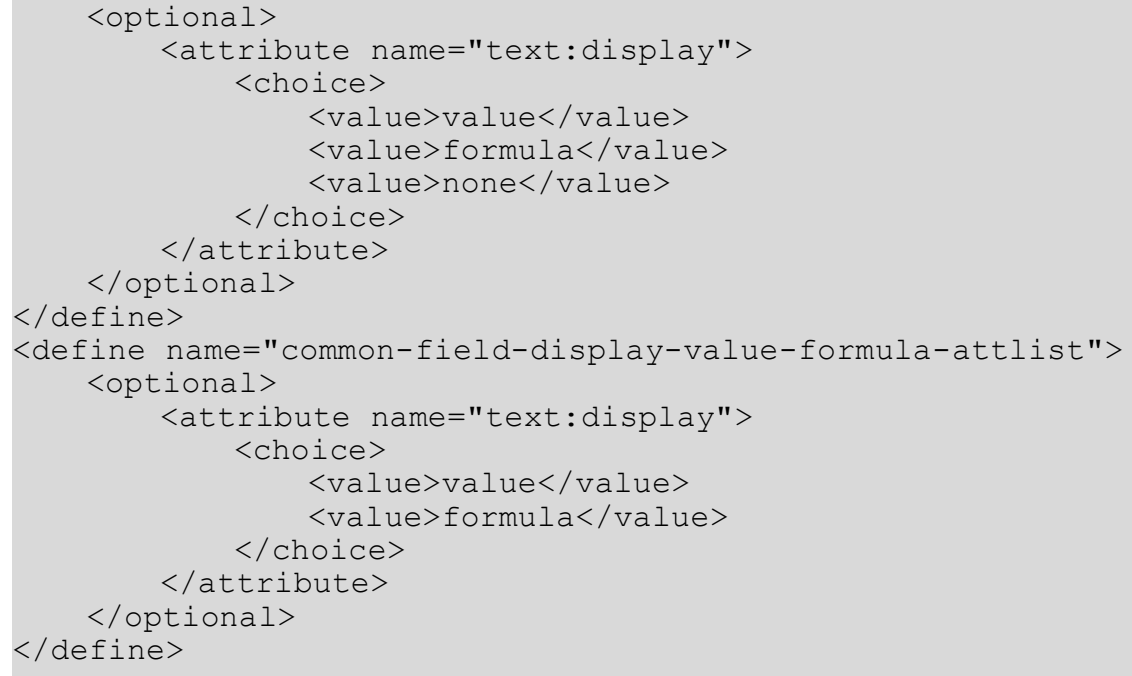

\subsubsection{Formula}

The text: formula attribute contains the formula or expression used to compute the value of the field. This attribute can be used with any of the following elements:

- <text:variable-set>

- <text:user-field-del>

- <text:sequence>

- <text:expression>

The formula should start with a namespace prefix hat indicates the syntax and semantic used within the formula.

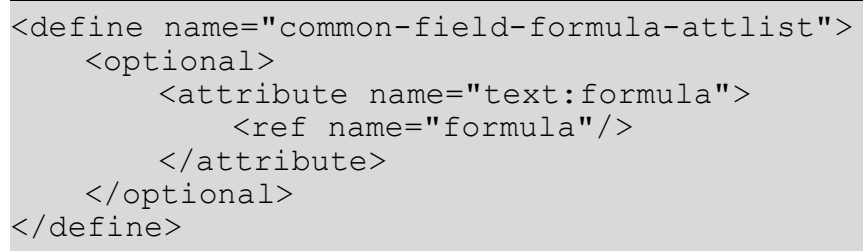

\subsubsection{Formatting Style}

The style:data-style-name attribute refers to the data style used to format the numeric value. For general information about styles, see Chapter 14 . For more information about data styles, see section 14.7 .

For string variables this attribute must be omitted. Otherwise, this attribute is required.

The name must match the name of a data style.

This attribute can be used with any of the following elements:

- $\quad$ text:date>

- $\quad$ text:time>

- <text:page-number> 
- <text:variable-set>

- <text:variable-get>

- <text:variable-input>

- <text:user-field-get>

- <text:user-field-input>

- <text:expression>

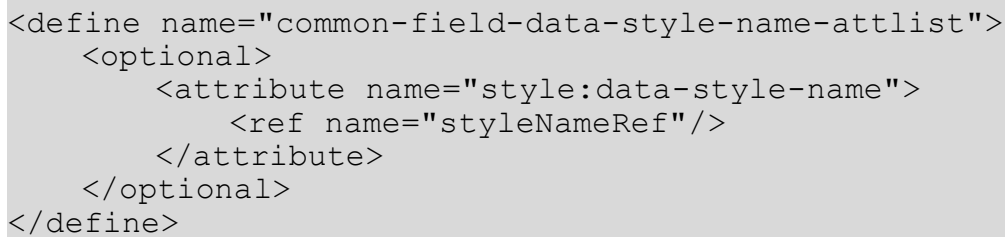

\subsubsection{Number Formatting Style}

Numbers that are used for number sequences such as page numbers or sequence fields can be formatted according to the number styles described in section 12.2. The number styles supported are as follows:

- Numeric: $1,2,3, \ldots$

- Alphabetic: $a, b, c, \ldots$ or $A, B, C, \ldots$

- Roman: i, ii, iii, iv, ... or I, II, III, IV, ...

Note: The value of this attribute can be any of the [XSLT] number format keys 1 , i, I, a, or A.

Alphabetic number styles need an additional attribute to determine how to display numbers that cannot be represented by a single letter. The OpenDocument format supports:

- Synchronized letter numbering, where letters are used multiple times, for example aa, bb, $\mathrm{cc}$, and so on.

- Non-synchronized letter numbering, for example aa, ab, ac, and so on.

See section 12.2 for more information.

<define name="common-field-num-format-attlist">

<optional>

<ref name="common-num-format-attlist"/>

$</ o p t i o n a l>$

2526

2527

$</$ define $>$ 


\section{Text Indices}

OpenDocument text documents may contain automatically generated indices. An index generally contains a sorted list of all items of a certain types, where the sorting (document position, alphabetical, etc.) and the type of items (chapter headings, tables, etc.) are determined by the specific type of index.

\subsection{Index Marks}

There are three types of index marks that correspond to the three types of index that make use of index marks. The three types of index marks are:

- Table of content index marks

- User-defined index marks

- Alphabetical index marks

The XML code for index marks is similar to the code for Bookmarks and References. The following are some basic rules about index marks:

- Each index mark is represented by a start and an end element.

- Both elements use an ID attribute to match the appropriate start and end elements.

- The start and end elements for an index mark must be contained in the same paragraph, with the start element occurring first.

- The attributes associated with the index mark are attached to the start element.

- The text between the start and end elements is the text the index entry.

- The formatting attributes for index marks can overlap.

\subsubsection{Table of Content Index Marks}

The <text: toc-mark-start> element marks the start of a table of content index entry. The ID specified by the text:id attribute must be unique except for the matching index mark end element. There must be an end element to match the start element located in the same paragraph, with the start element appearing first.

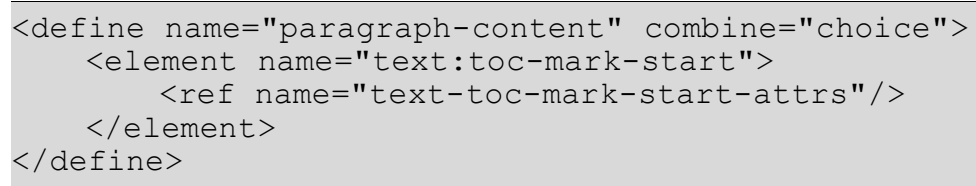

The attributes associated with the <text:toc-mark-start $>$ element are:

- A text: id attribute to allow the start and end elements to be matched.

- A text:outline-level attribute to specify the outline level of the resulting table of content index entry.

<define name="text-toc-mark-start-attrs">

$<$ ref name="text-id"/>

<ref name="text-outline-level"/> 


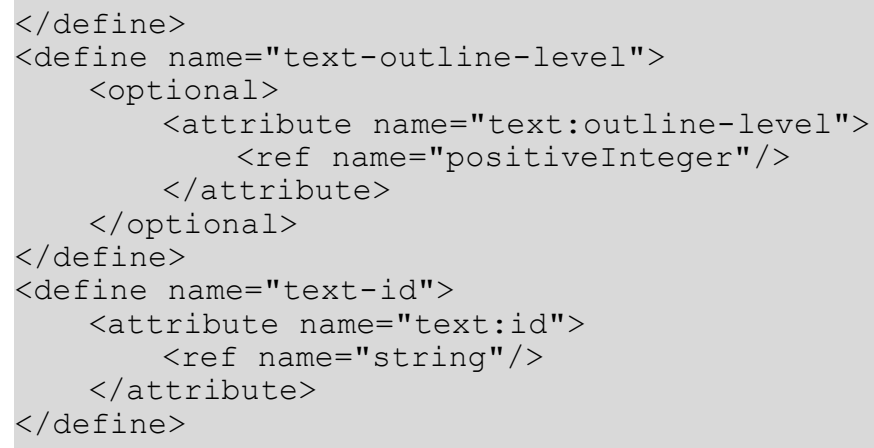

The <text: toc-mark-end > element marks the end of a table of contents index entry. There must be a start element with the same text:id value to match the end element located in the same paragraph, with the start element appearing first.

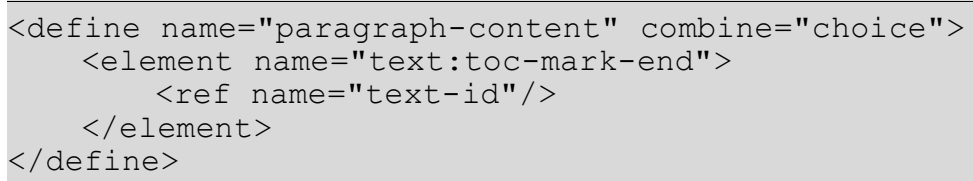

Table of content index marks also have a variant that does not enclose the text to be indexed. This is represented using the <text: toc-mark> element which contains a text:stringvalue attribute for the text of the index entry. In this situation, a text:id attribute is not necessary because there are no start and end elements to match.

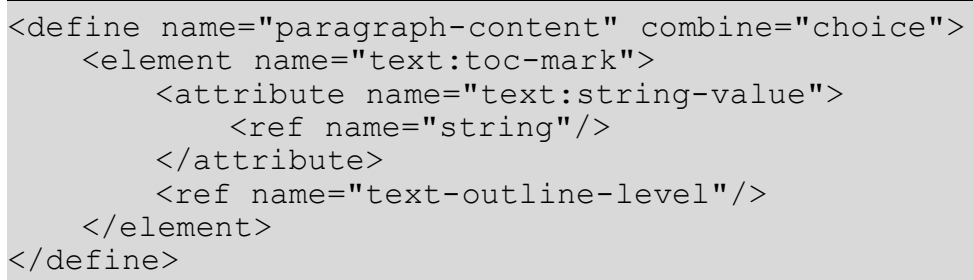

\subsubsection{User-Defined Index Marks}

The <text: user-index-mark-start> element marks the start of a user-defined index entry. The ID specified by the text:id attribute must be unique except for the matching index mark end element. There must be an end element to match the start element located in the same paragraph, with the start element appearing first.

The <text: user-index-mark-end > element marks the end of the user-defined index entry. There must be a start element with the same text:id value to match the end element located in the same paragraph, with the start element appearing first.

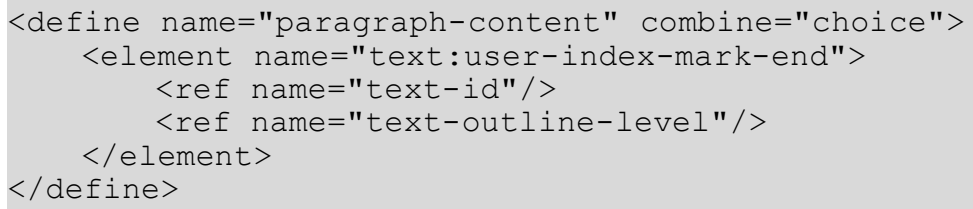


User index marks also have a variant that does not enclose the text to be indexed. This is represented by the <text: user-index-mark> element which contains a text:stringvalue attribute for the text of the index entry. In this situation, the text:id attribute is not necessary because there are no start and end elements to match.

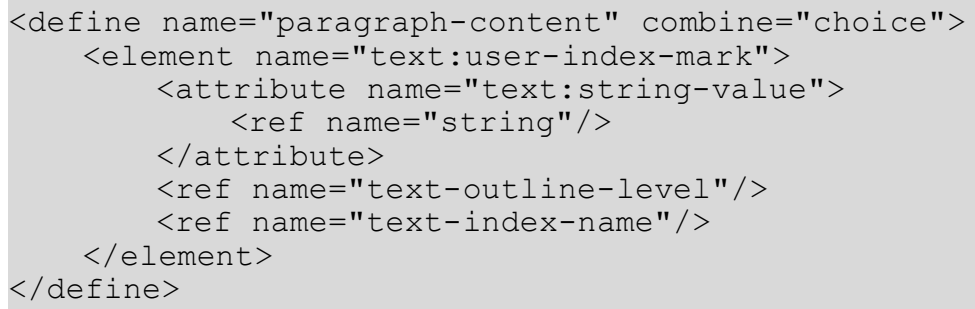

\section{Name of User Index}

There can be more than one user-defined index. In this case, the user index must be named using the text: index-name attribute. This attribute determines to which user-defined index an index mark belongs. If no name is given, the default user-defined index is used.

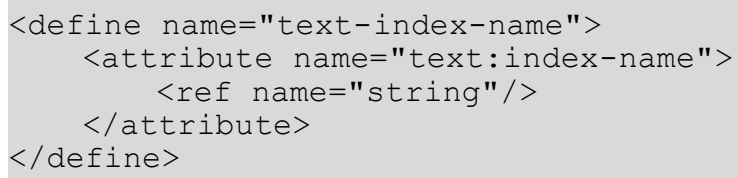

\subsubsection{Alphabetical Index Mark}

The <text:alpha-index-mark-start> element marks the start of an alphabetical index entry. There are two optional attributes that may contain keys for alphabetical entries, which allows structuring of entries. There is also a Boolean attribute that determines if this entry is intended to be the main entry, if there are several equal entries.

The ID specified by the text:id attribute must be unique except for the matching index mark end element. There must be an end element to match the start element located in the same paragraph, with the start element appearing first.

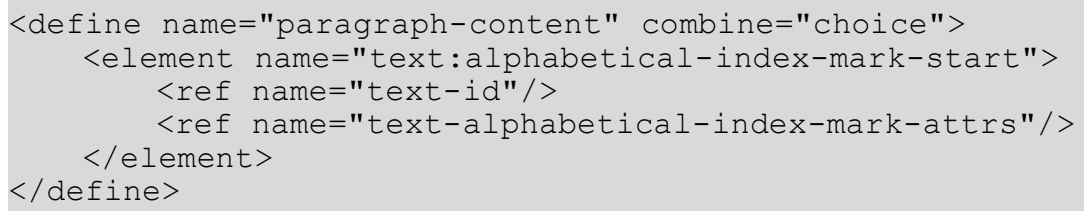

The attributes associated with the <text:toc-mark-start> element are:

- A text:id attribute to allow the start and end elements to be matched.

- Additional keys

- Main entry

The <text:alpha-index-mark-end > element marks the end of an alphabetical index entry. There must be a start element with the same text:id value to match the end element located in the same paragraph, with the start element appearing first.

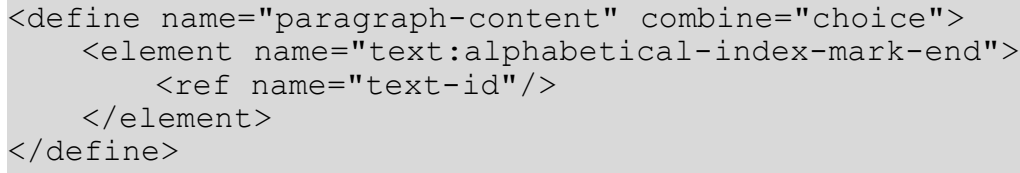


Alphabetical index marks also have a variant that does not enclose the text to be indexed. This is represented using the <text:alpha-index-mark> element which contains a

text:string-value attribute for the text of the index entry. In this situation, a text:id attribute is not necessary because there are no start and end elements to match.

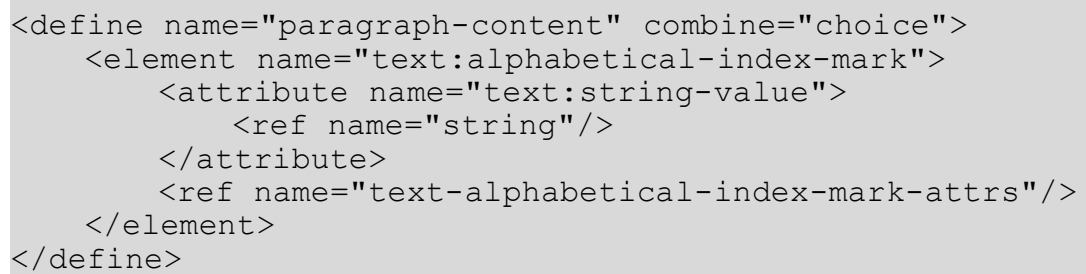

\section{Additional Keys}

The text: key 1 and text:key2 attributes specify additional keys for the alphabetical index mark. If only one key is used, it must be contained in the text: key1 attribute.

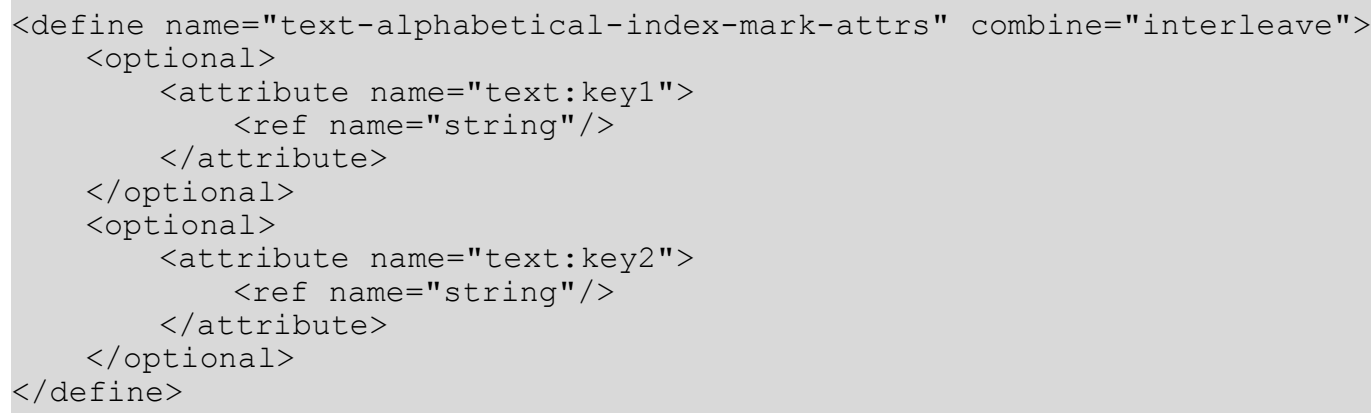

\section{Phonetic Keys}

For ideographic languages, there sometimes is no obvious or common sorting of the language's characters. One common scheme to facilitate an alphabetical index in such languages is to sort according to a phonetic description of the search time. To achieve this in the OpenDocument file format, there are additional attributes for the string value and the two keys for phonetic descriptions. The original value and key attributes are for display, but if phonetic variants are present, they should be used for sorting the index.

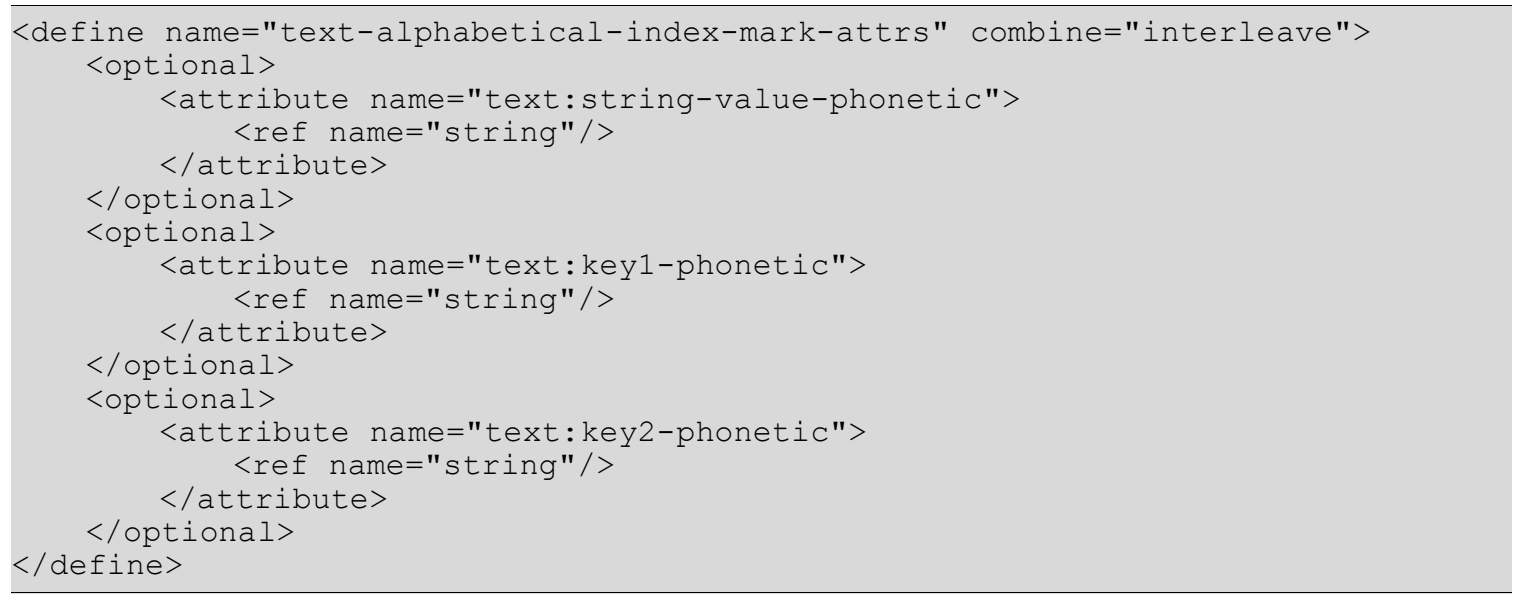




\section{Main Entry}

If there are several index marks for the same entry, one of these entries may be declared as the main entry using the text:main-entry attribute.

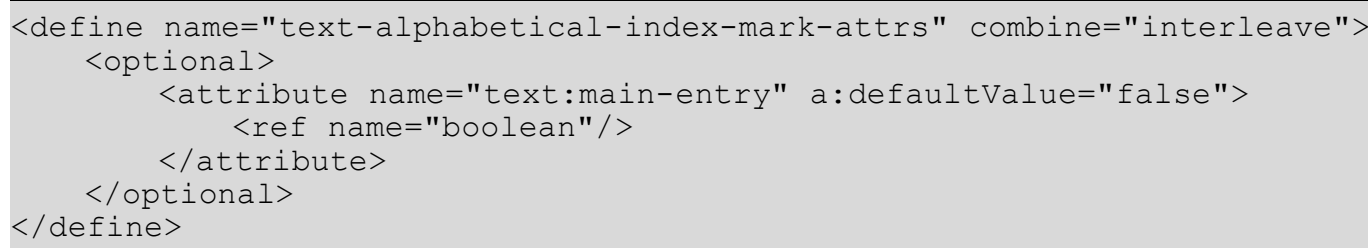

\subsubsection{Bibliography Index Mark}

The <text:bibliography-mark> element contains the text and information for a bibliography index entry. It supports attributes for each type of bibliographical data that a bibliography index may contain.

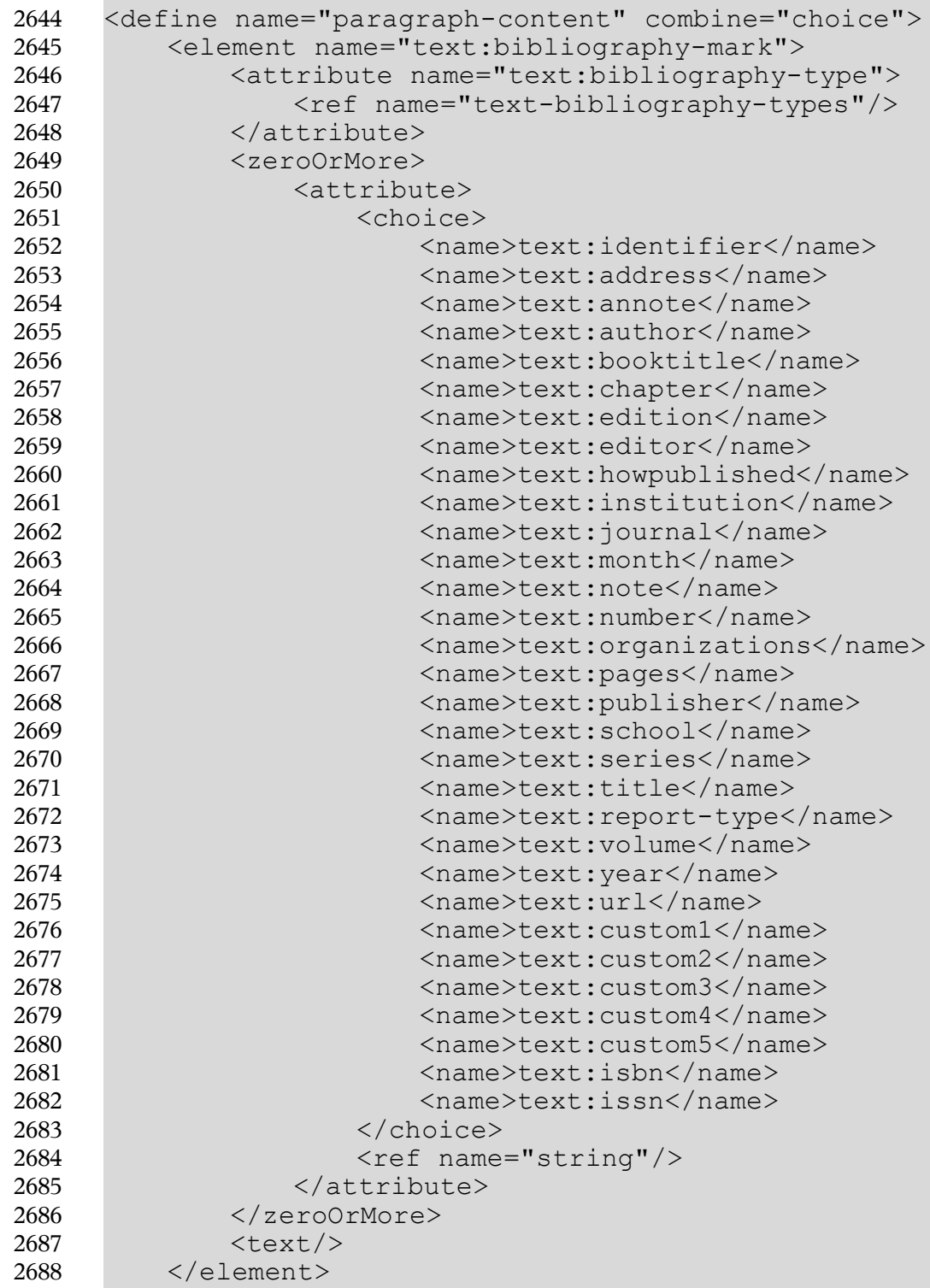

2688 


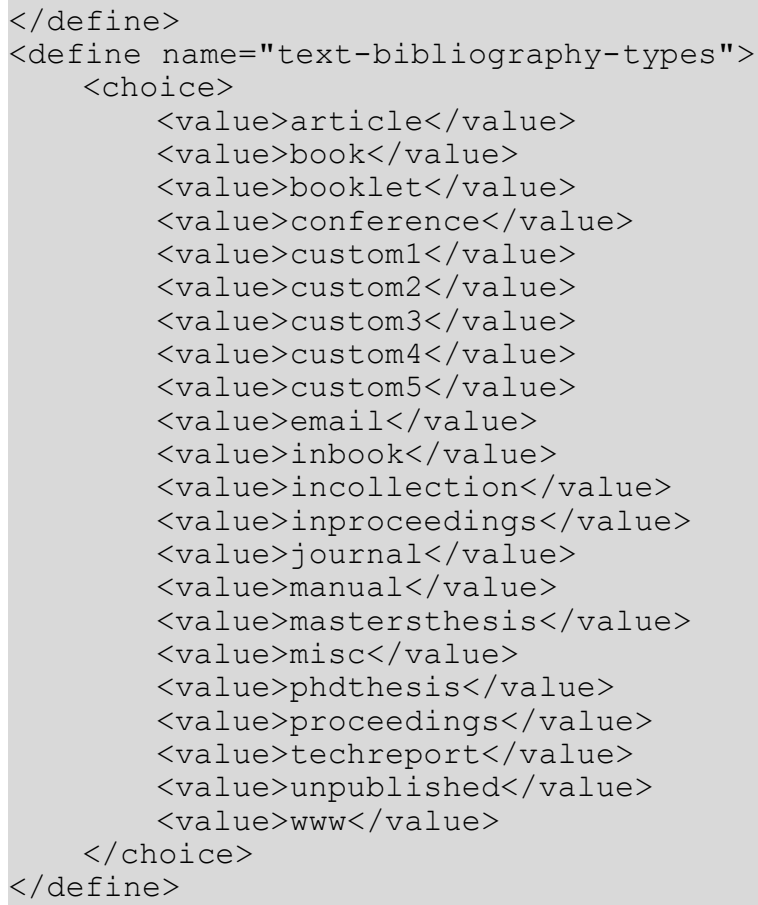

\subsection{Index Structure}

An index consists of two parts: The index source, and the index body. Both of these are contained in an element of their own, which in turn form the two child elements for the index element itself.

The index source is specific to the type of index it is being used for. It contains the information necessary to generate the index content. An index source has no graphical rendition.

The index body is the same for all types of indices. It contains the text generated from the information in the index source. The text contained in an index body is in no way special or different from text used elsewhere in this specification.

The content of the index body can be regenerated at any time from the information contained in the index source and the remainder of the document. One could say that the index source contains all the logical information about an index, while the index body contains the rendition of the index. A tool extracting structure information about a document might look only at the index source, while a rendering program might look only at an index body.

\subsubsection{Index Source}

An index source element contains the information necessary to generate the index body. In addition to a set of flags that determine which information to include in an index, the index source contains a set of index templates. Such a template determines how an item to be contained in the index is to be rendered.

For example, a table of content might look as follows:

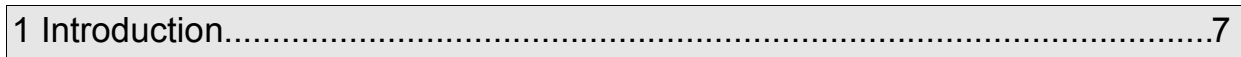

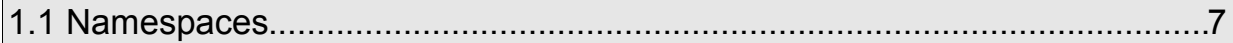

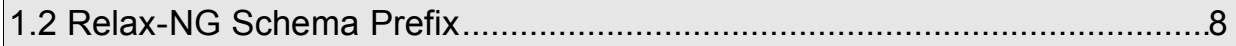


An index source for this index would contain flags indicating that chapter headers at least up to level 2 are to be included. The contained index templates would define that an entry consists of the chapter number, a space, the chapter name, a tab (with a '.' leader) and the page number.

The various index templates are described together with their index elements. The index templates elements in use are described in section 7.12.

The different index source elements are described together with their corresponding index elements.

\subsubsection{Index Body Section}

The index body contains the current textual rendition of the index. The format is the same as for regular text within this specification, e.g. text sections, except that it also allows index title sections.

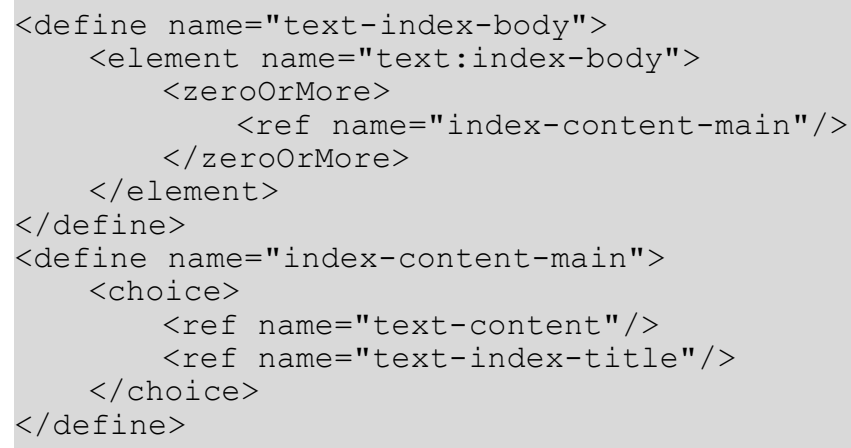

\subsubsection{Index Title Section}

The index title is usually contained in a section of its own. The reason for this enclosure is to enable the popular layout of having an index title across the entire page, but having the index itself in a two column layout.

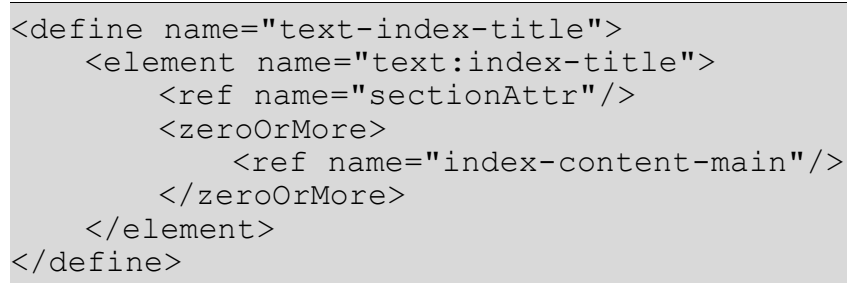

\subsection{Table Of Content}

A table of contents provides the user with a guide through the content of the document. It is typically found at the beginning of a document, contains the chapter headings with their respective page numbers. An example for a table of content may be found at the beginning of this document.

The items that can be listed in a table of content are:

- Headers (as defined by the outline structure of the document), up to a selectable level

- Table of content index marks

- Paragraphs formatted with a set of selectable paragraph styles 
The table of contents is represented by the <text:table-of-content $>$ element. The <text: table-of-content> element supports the same style (and class) attributes as a text section (see section 4.4).

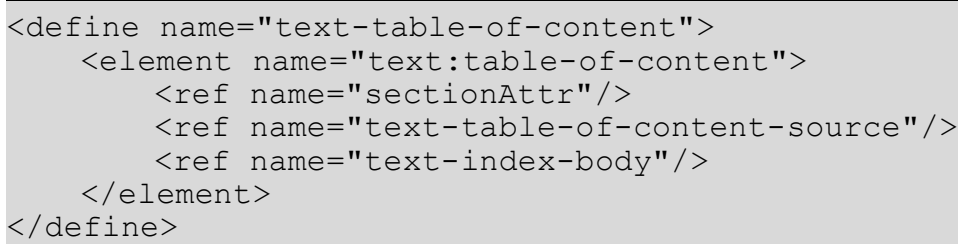

\subsubsection{Table of Content Source}

The <text: table-of-content-source> element specifies how the table of contents is generated. It specifies how the entries are gathered.

The <text: table-of-content-source> element contains

- an optional template for the index title

- optional templates for index entries, one per level

- optionally a list of styles to be used for gathering index entries

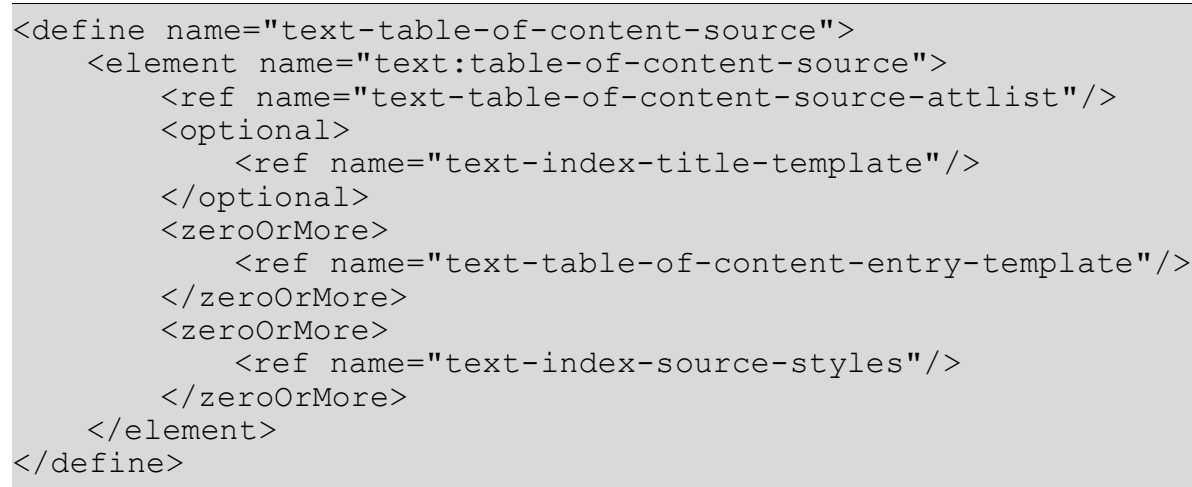

The attributes that may be associated with the <text:table-of-content-source> element are:

- Outline level

- Use outline

- Use index marks

- Use index source styles

- Index source

- Relative tab stop position

\section{Outline Level}

The text: outline-level attribute specifies which outline levels are used when generating the table of contents.

The value of this attribute must be an integer greater than zero. If this attribute is omitted, all outline levels are used by default. 


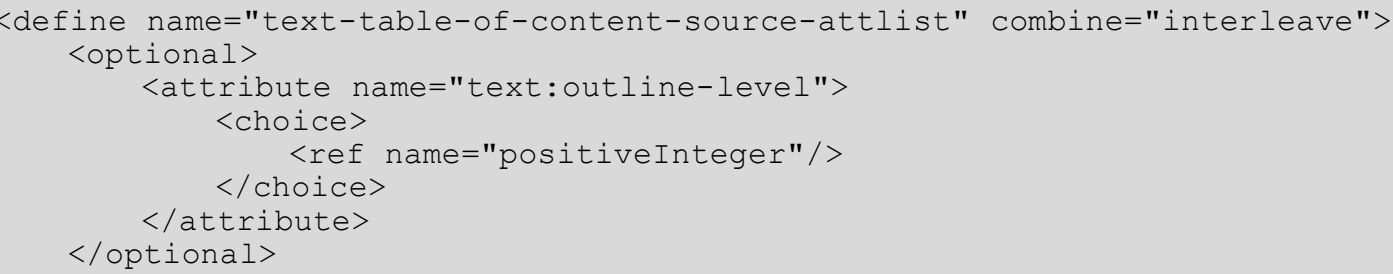

\section{Use Outline}

The text:use-outline-level attribute determines whether headings are used to generate index entries. If the value is true, the table of contents includes entries generated from headings. The text: outline-level attribute specifies up to which level headings are being included. See section 7.1 for more information on index marks.

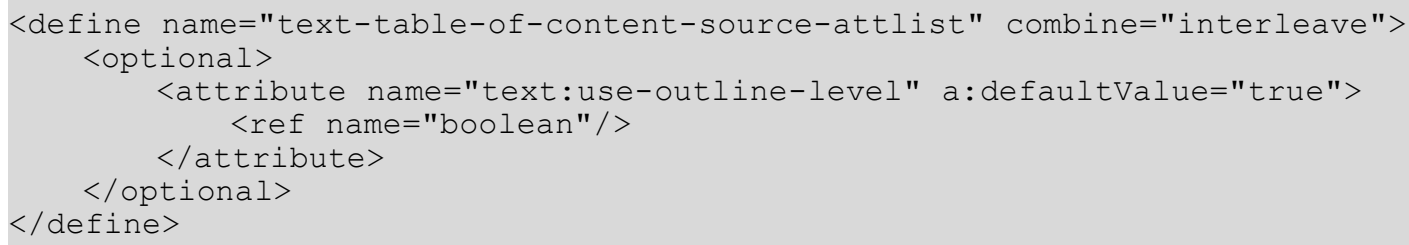

\section{Use Index Marks}

The text: use-index-marks attribute determines whether or not index marks are used to generate index entries. If the value is true, the table of contents includes entries generated from table of content index marks. The text: outline-level attribute specifies up to which level index marks are being included. See section 7.1 for more information on index marks.

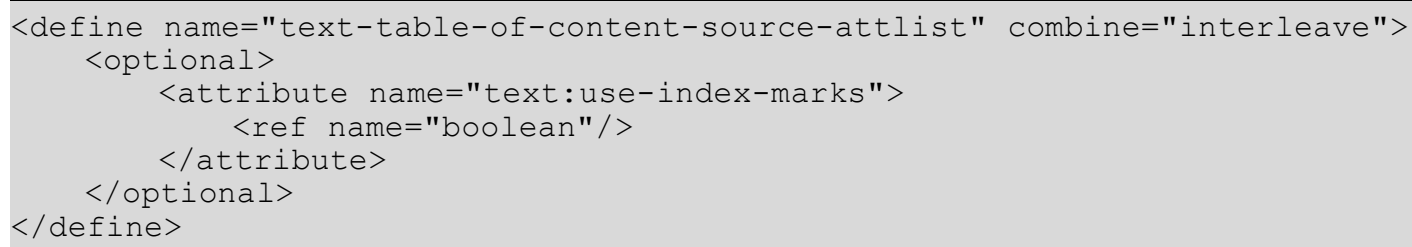

\section{Use Index Source Styles}

The text:use-index-source-styles attribute determines whether or not index entries are generated for paragraph formatted using certain paragraph styles. If the value is true, the table of contents includes an entry for every paragraph formatted with one of the styles specified in a <text: index-source-style> element. The text: outline-level attribute specifies up to which level index source styles are being included.

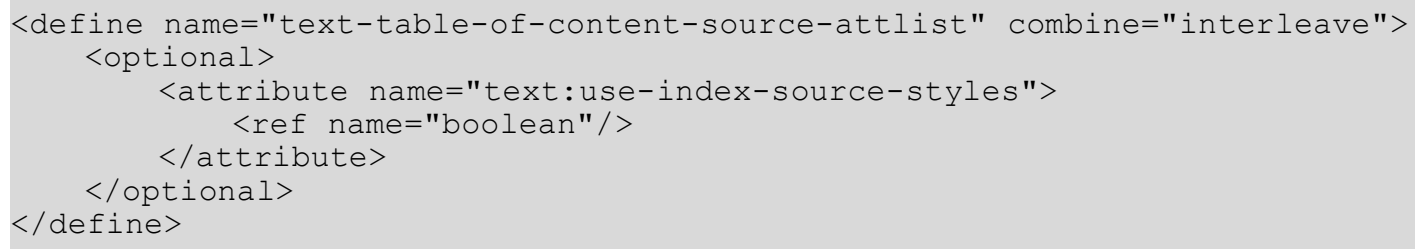




\section{Index Scope}

The text: index-scope attribute determines whether the table-of-content is generated for the whole document, or only for the current chapter.

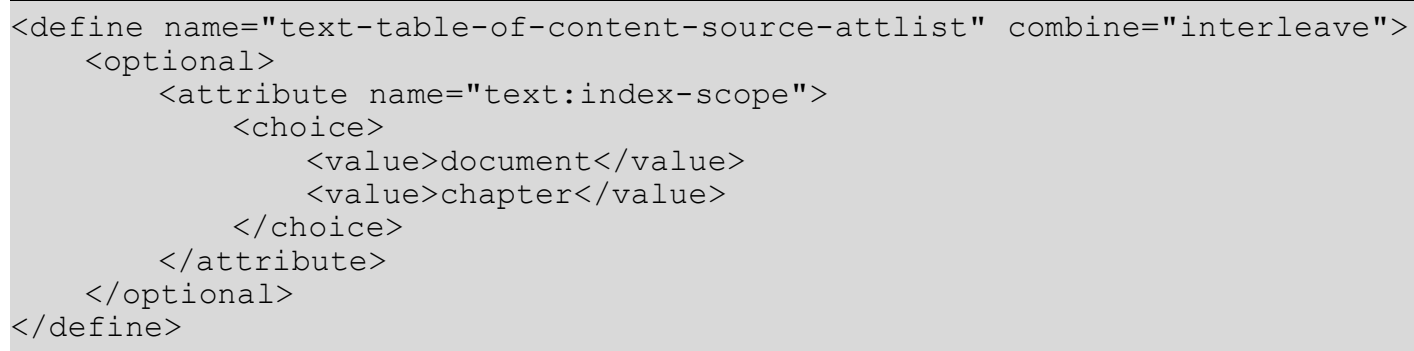

\section{Relative Tab-Stop Position}

The text:relative-tab-stop-position attribute determines whether the position of tab stops is relative to the left margin or to the left indent as determined by the paragraph style. This is useful for copying the same entry configuration for all outline levels because with relative tab stop positions the tabs do not need to be adjusted to the respective paragraph format.

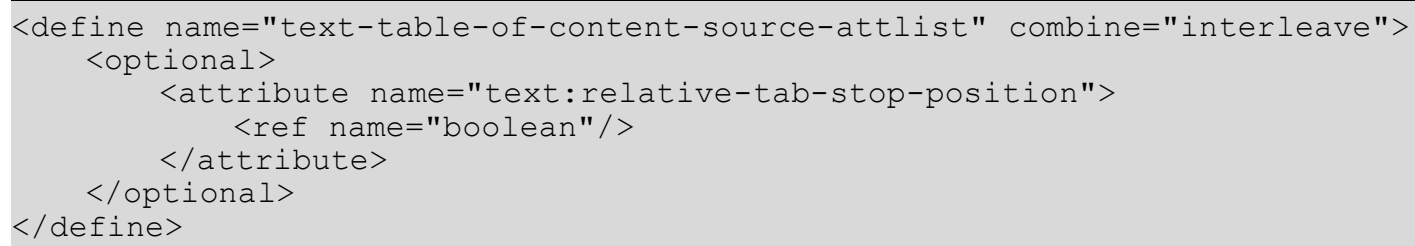

\subsubsection{Table of Content Entry Template}

The <text:table-of-content-entry-template> element determines the format of an index entry for a particular outline level. For each table of content, there must not be more than one element for any outline level. (See below.)

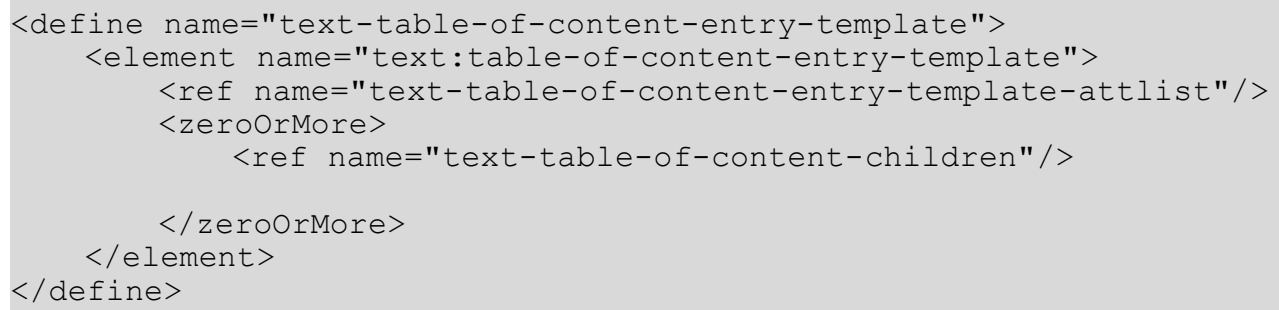

A table of content entry template supports the following kinds of text elements:

- Chapter and Page Number

- Reference Text

- Text Span

- Tab

- Hyperlink start and end

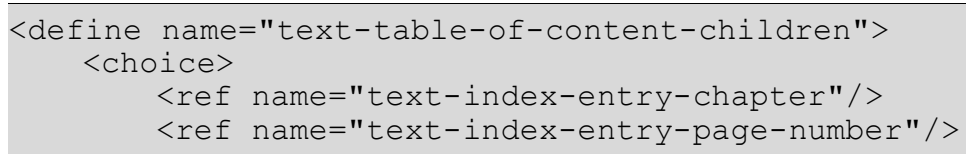




\section{$</$ define $>$}

<ref name="text-index-entry-text" $/>$

$<$ ref name="text-index-entry-span"/>

<ref name="text-index-entry-tab-stop"/>

<ref name="text-index-entry-link-start"/>

<ref name="text-index-entry-link-end"/>

The attributes that may be associated associate with the <text:table-of-content-entrytemplate> element are:

- Template outline level

- Paragraph style

\section{Template Outline Level}

This attribute specifies to which outline level the entry configuration applies. Outline levels must be unique for the template elements in one index source.

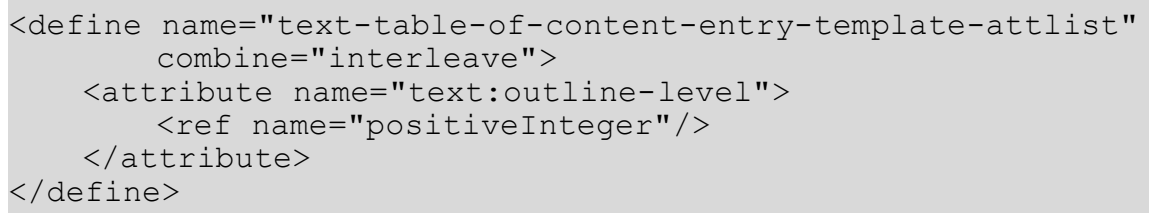

\section{Paragraph Style}

The text:style-name attribute specifies the paragraph style to use for this template.

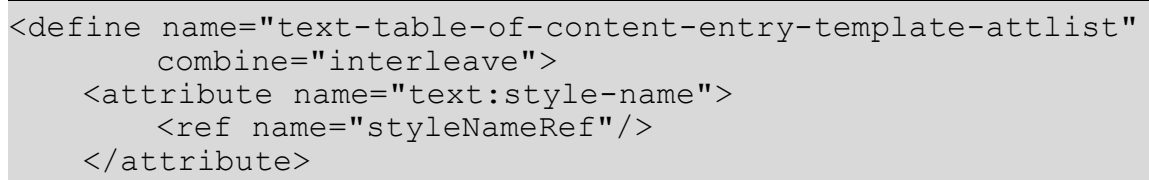

\subsection{Index of Illustrations}

The index of illustrations lists all images and graphics in the current document or chapter. The index entries can be derived from the caption of the illustration or the name of the illustration.

The attribute that may be attached to the <text:illustration-index> element is:

- text:style-name

This attribute specifies the section style to use for the index of illustrations.

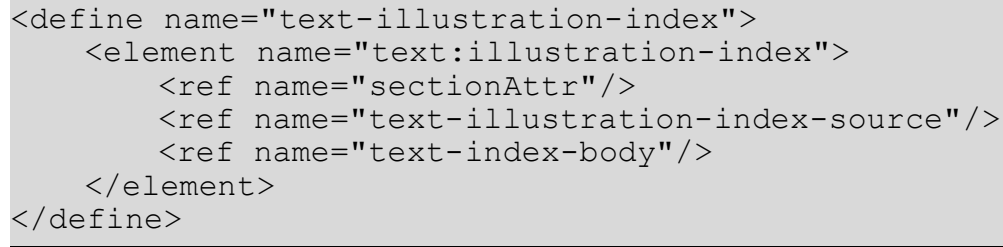




\subsubsection{Index of Illustration Source}

The <text:illustration-index-source> element specifies how the index of illustrations is generated.

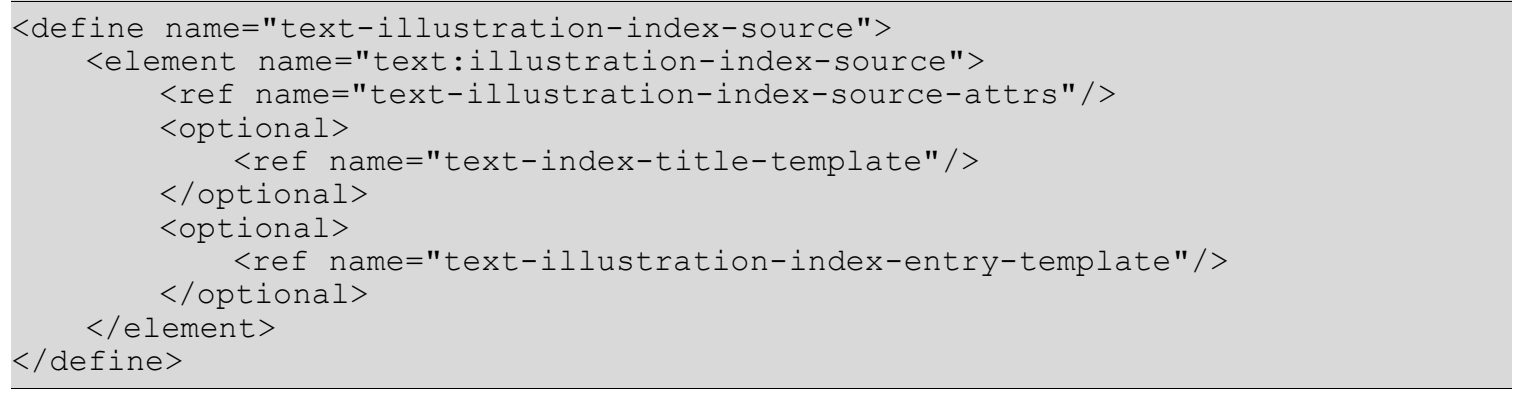

The attributes that may be associated with a <text:illustration-index-source $>$ element are:

- Use caption

- Caption sequence name

- Caption sequence format

- Index scope

This attribute specifies whether the index applies to the entire document or only the the current chapter.

- text:relative-tab-stop-position

This attribute specifies whether the position of tab stops are interpreted relative to the left margin or the left indent.

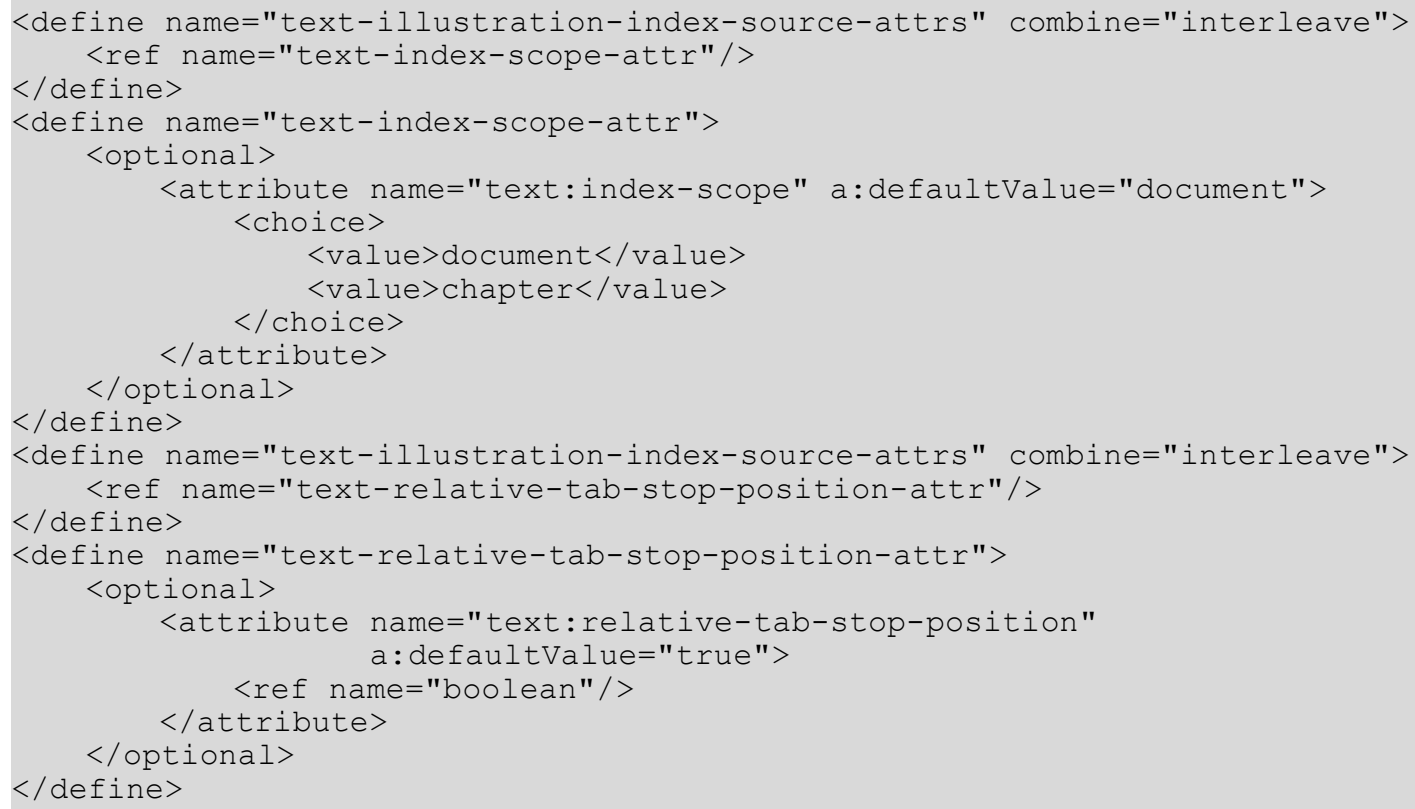




\section{Use Caption}

Each object contained in a text document has a name. In addition, images also have a caption. The image caption or the image name can be gathered for the index of illustrations.

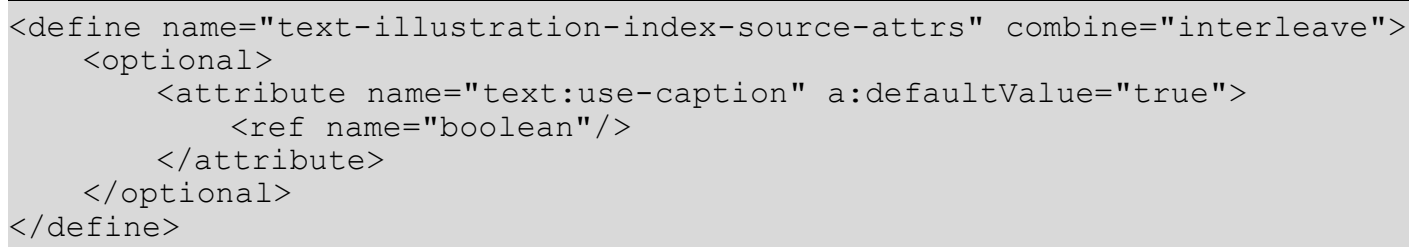

\section{Caption Sequence Name}

Captions are associated with a sequence name. If the text: use-caption attribute is set to true, this attribute must be used to specify the sequence with which the captions are associated.

If this attribute is omitted, the default sequence for the object type is used, for example the sequence "Illustration" is used for illustrations.

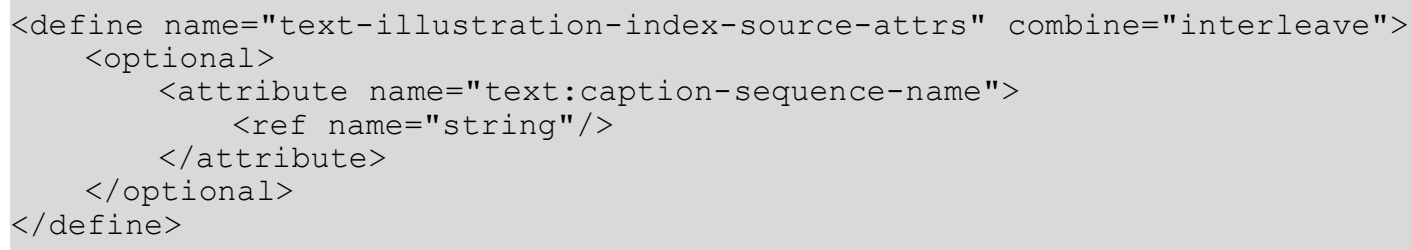

\section{Caption Sequence Format}

If the entries for the index of illustrations are obtained from the image captions, this attribute must be used to specify the format for the entries.

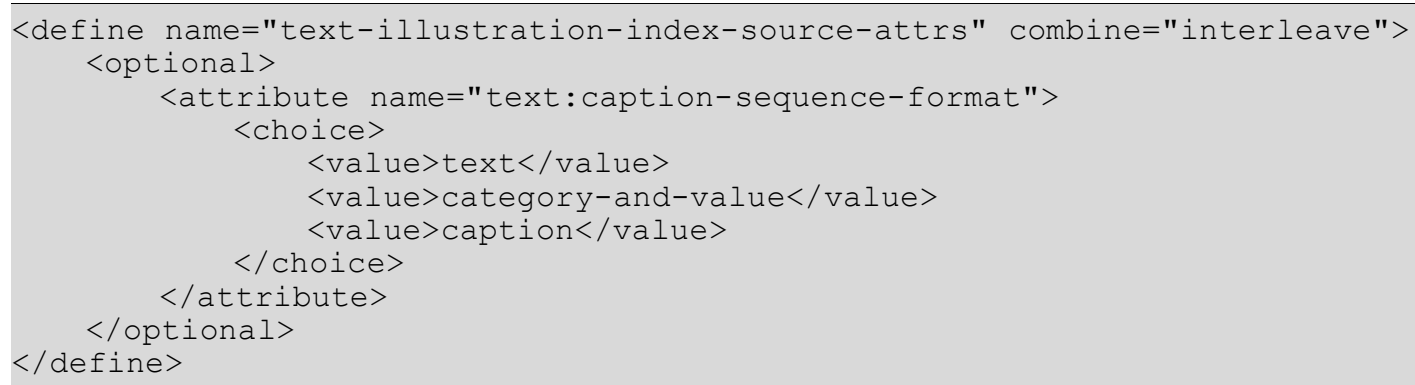

\subsubsection{Illustration Index Entry Template}

The illustration index entry template element determines the format of an index entry for a particular outline level.

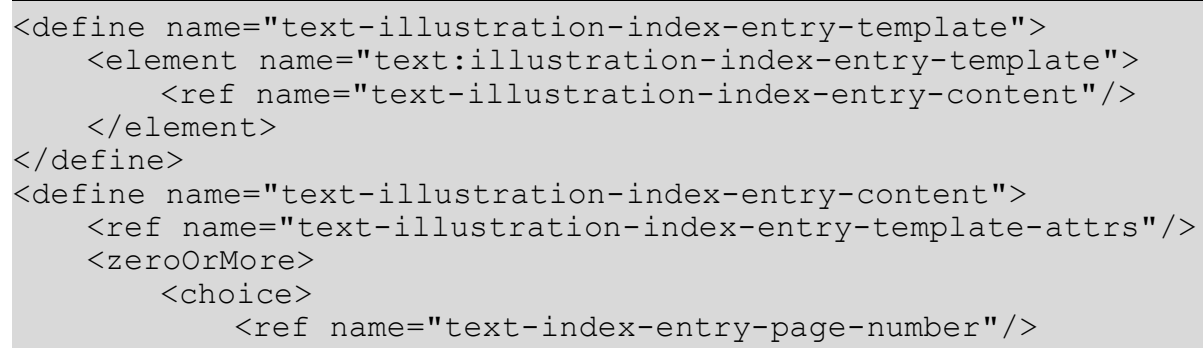


$</$ define $>$

<ref name="text-index-entry-text"/>

<ref name="text-index-entry-span"/>

<ref name="text-index-entry-tab-stop"/>

$</$ choice $>$

$</$ zeroorMore $>$

The attribute that may be associated with the <text:illustration-index-entrytemplate> element is:

- Paragraph style

\section{Paragraph Style}

This attribute identifies the paragraph style to use for this template.

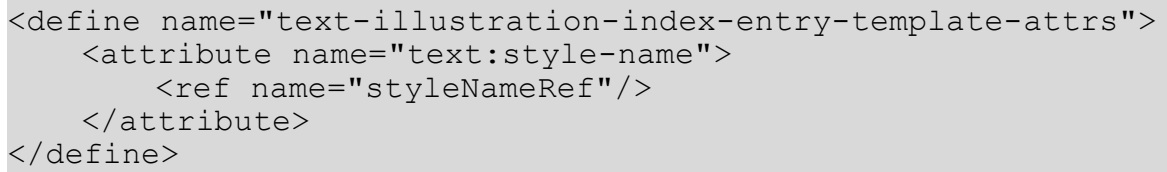

\subsection{Index of Tables}

The index of tables lists all of the tables in the current document or chapter. It works in exactly the same way as the index of illustrations.

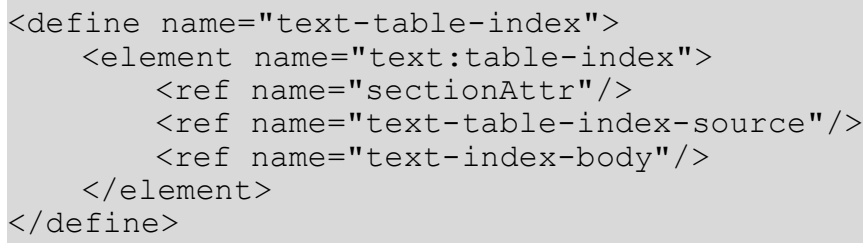

\subsubsection{Table Index Source}

The <text:table-index-source> element specifies how the index of tables is generated.

The attributes that may be associated with this element are the same as those that can be associated with the <text: illustration-index-source> element. See section 7.4.1 for detailed information about these attributes.

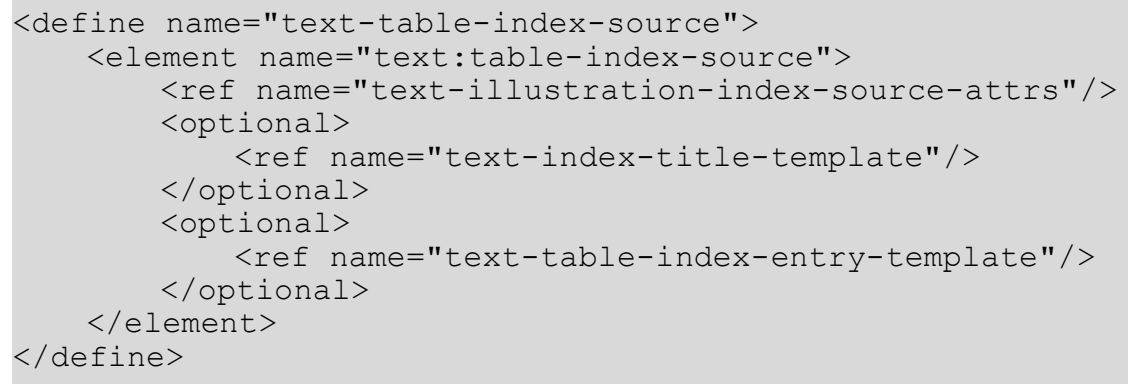

\subsubsection{Table Index Entry Template}

The table index entry template element determines the format of an index entry for a particular outline level. 
The attributes that may be associated with this element are the same as those that can be associated with the <text: illustration-index-entry-template> element. See section 7.4.2 for detailed information about these attributes.

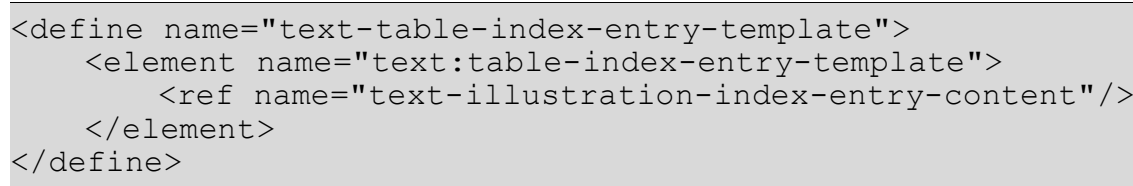

\subsection{Index of Objects}

The index of objects lists all of the objects in the current document or chapter. It gathers its entries from the known object types.

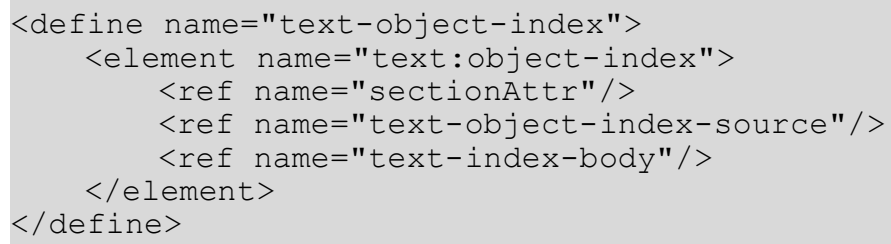

\subsubsection{Object Index Source}

The <text: object-index-source> element determines which object types to include in the index of objects. It also supports the standard index source attributes.

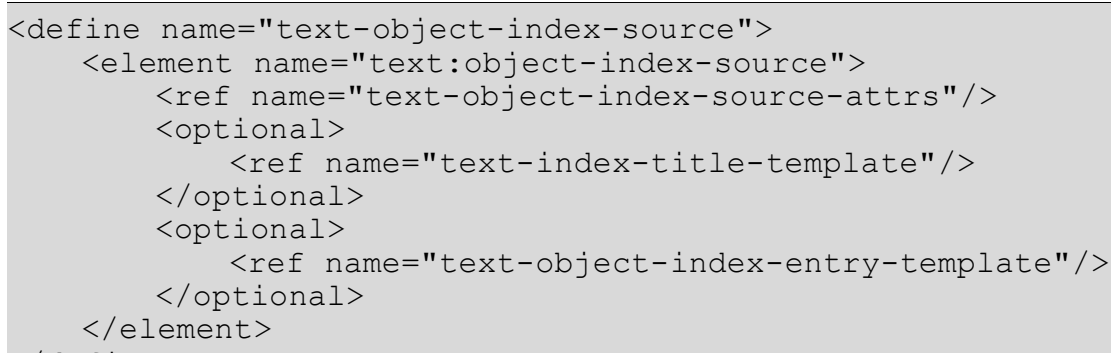

The attributes that may be associated with the <text:object-index-source $>$ element are:

- Use attributes, text: use-*-objects

- Index scope (see section 7.4.1)

This attribute specifies whether the index applies to the entire document or only the the current chapter.

- Relative tab stop position (see section 7.4.1)

This attribute specifies whether the position of tab stops are interpreted relative to the left margin or the left indent.

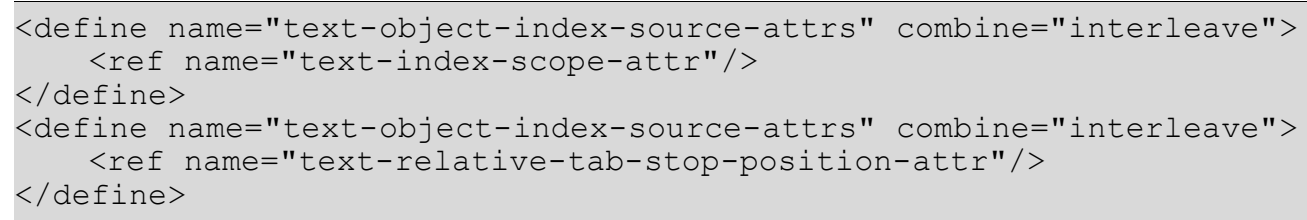




\section{Use Attributes}

The text: use-*-objects attributes specify which types of objects to include in the index of objects. There is an attribute for each type of object as follows:

- text:use-spreadsheet-objects

- text:use-draw-objects

- text:use-chart-objects

- text:use-math-objects

Other objects are included or omitted using the following attribute:

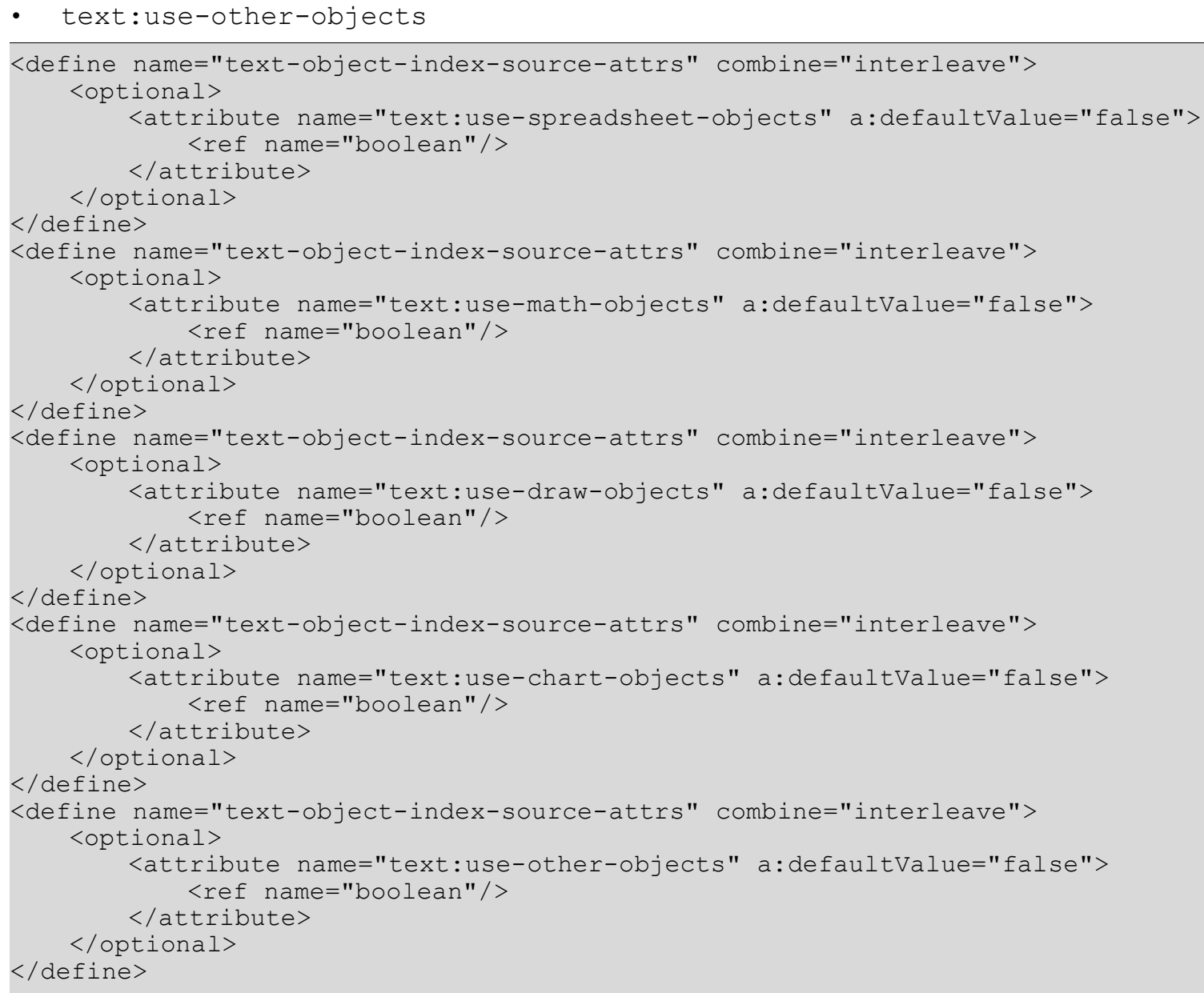

\subsubsection{Object Index Entry Template}

The object index entry template element determines the format of an index entry for a particular outline level.

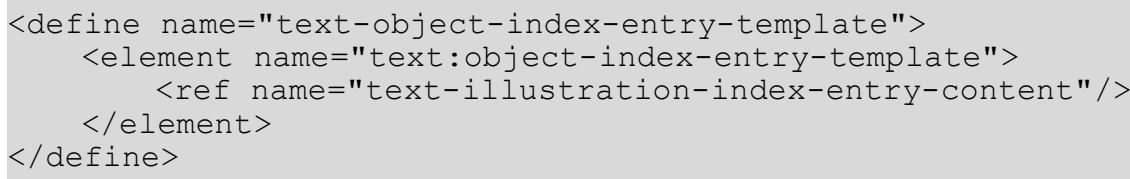


The attributes that may be associated with this element are the same as those that can be associated with the <text: illustration-index-entry-template> element. See section 7.4.2 for detailed information about these attributes.

\subsection{User-Defined Index}

A user-defined index combines the capabilities of the indexes discussed earlier in this chapter. A user-defined index can gather entries from the following sources:

- Index marks

- Paragraphs formatted using particular paragraph styles

- Tables, images, or objects

- Text frames

The <text: user-index> element represents a user-defined index.

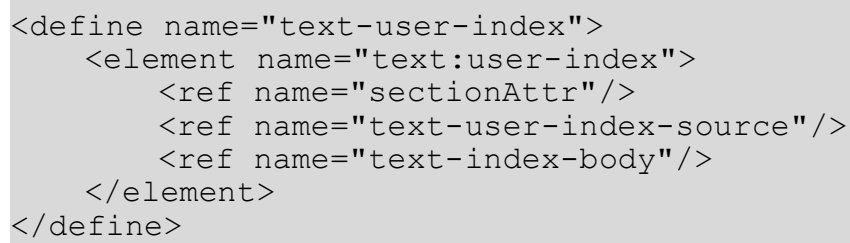

\subsubsection{User-Defined Index Source}

The <text: user-index-source> element can contain several attributes that determine how the index entries are gathered. It also supports an attribute that determines how the outline levels of the index entries are gathered.

The paragraph formats that are used as index marks are encoded in $<$ text: index-sourcestyles> elements, just like in <text:table-of-content-source> elements.

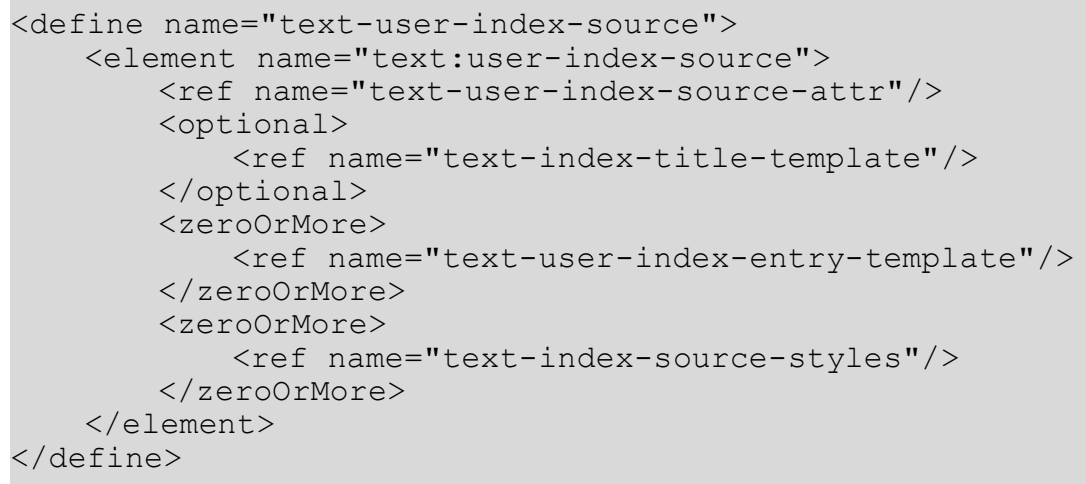

The attributes that may be associated with <text: user-index-source> elements are:

- Use attributes, text: use-*

- Copy outline level

- Index scope (see section 7.4.1)

This attribute specifies whether the index applies to the entire document or only to the current chapter. 


\section{- Index name}

In order to support several user-defined indexes with different contents, user index marks have a text: index-name attribute. The same attribute can be used with a <text: userindex-source> element to specify which index marks apply to the current index.

- $\quad$ Relative tab stop position (see section 7.4.1)

This attribute specifies whether the position of tab stops are interpreted relative to the left margin or the left indent.

3033

3034

3035

3036

3037

3038

3039

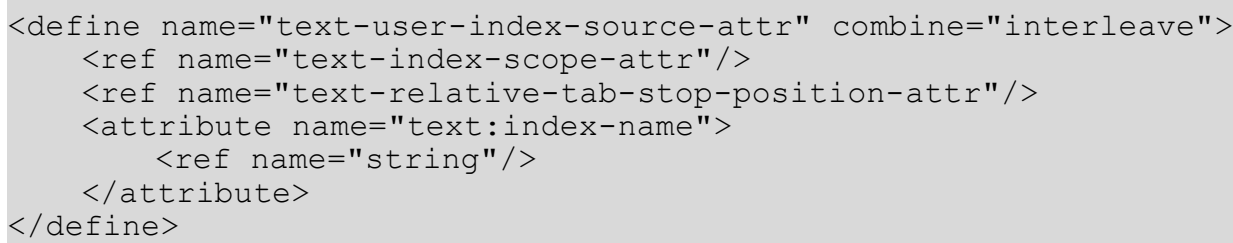

\section{Use Attributes}

The text: use-* attributes specify which entries to include in the user-defined index. The following attributes exist:

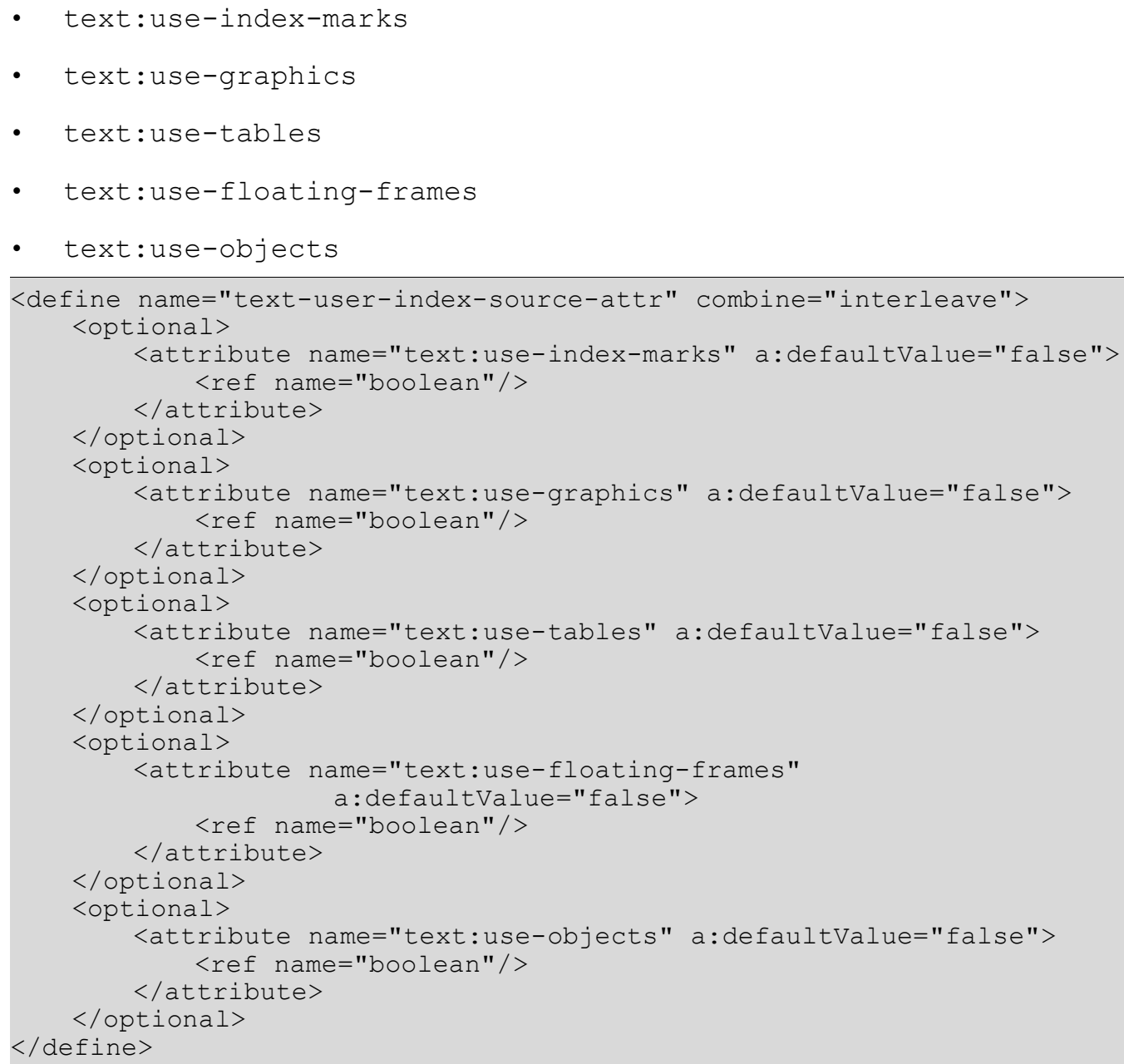




\section{Copy Outline Levels}

This attribute can have a value of true or false.

If the value is true, the entries are gathered at the outline level of the source element to which they refer.

If the value is false, all index entries gathered are at the top outline level. For example, if an image appears in section 1.2.3, the entry for the image is located at outline level 3.

\subsubsection{User-Defined Index Entry Template}

User index entry templates support entry elements for chapter number, page number, entry text, text spans, and tab stops.

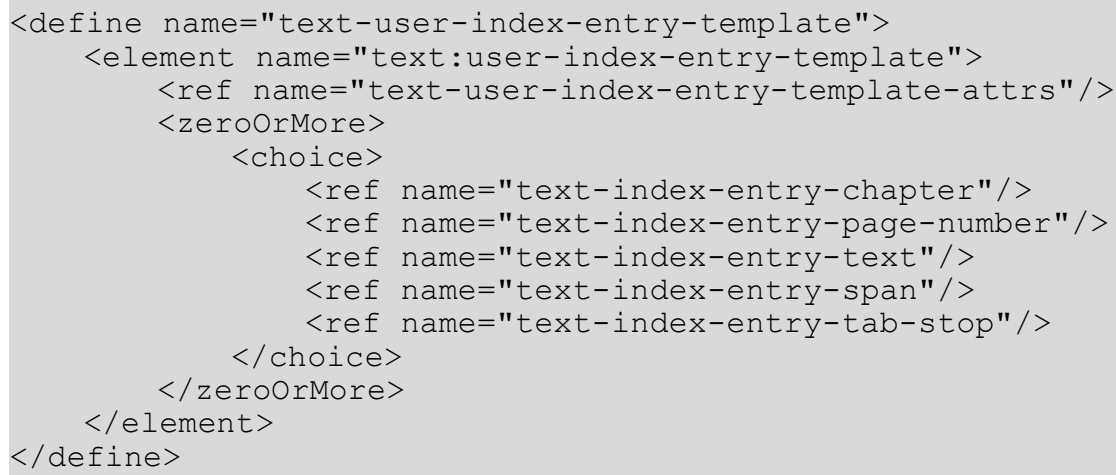

The attributes that may be associated with the <text: user-index-entry-template> elements are:

- Template outline level

- Paragraph style

\section{Template Outline Level}

The text:outline-level attribute specifies to which outline level this entry configuration applies.

All <text: outline-level> elements that are contained in the same parent element must specify different outline levels.

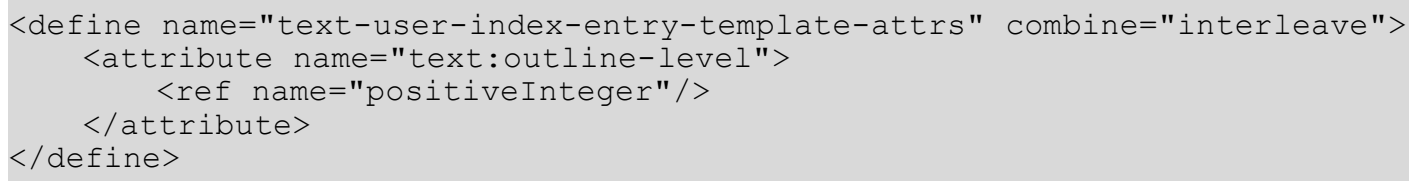




\section{Paragraph Style}

The text:style-name attribute specifies the paragraph style to use for the template.

3095

3096

3097

3098

3099

\subsection{Alphabetical Index}

An alphabetical index gathers its entries solely from index marks.

\subsubsection{Alphabetical Index Source}

The <text:alphabetical-index-source> element specifies how the alphabetical index is generated.

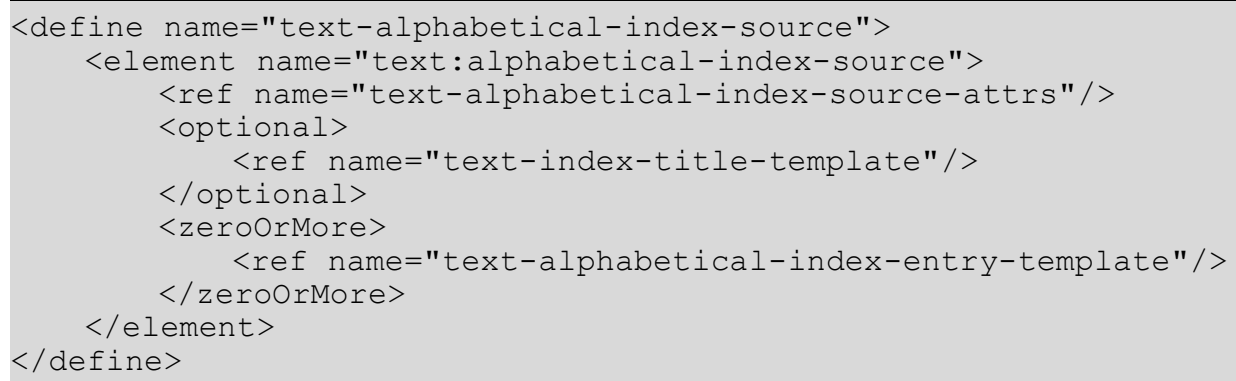

The attributes that may be associated with <text:alphabetical-index-source $>$ elements are:

- Ignore case

- Main entry style name

- Alphabetical separators

- Combine entries attributes

- Use keys as entries

- Capitalize entries

- Comma separated entries

- Sort language, country and algorithm

- Index scope (see section 7.4.1)

This attribute specifies whether the index applies to the entire document or only to the current chapter. 
- $\quad$ Relative tab stop position (see section 7.4.1)

This attribute specifies whether the position of tab stops are interpreted relative to the left margin or the left indent.

\section{Alphabetical Separators}

The text:alphabetical-separators attribute determines whether or not entries beginning with the same letter are grouped and separated from the entries beginning with the next letter, and so on.

The value of this attribute can be true or false.

If the value is true, all entries beginning with the same letter are grouped together. The index contains headings for each section, for example, A for all entries starting with the letter A, B for all entries starting with the letter $\mathrm{B}$, and so on.

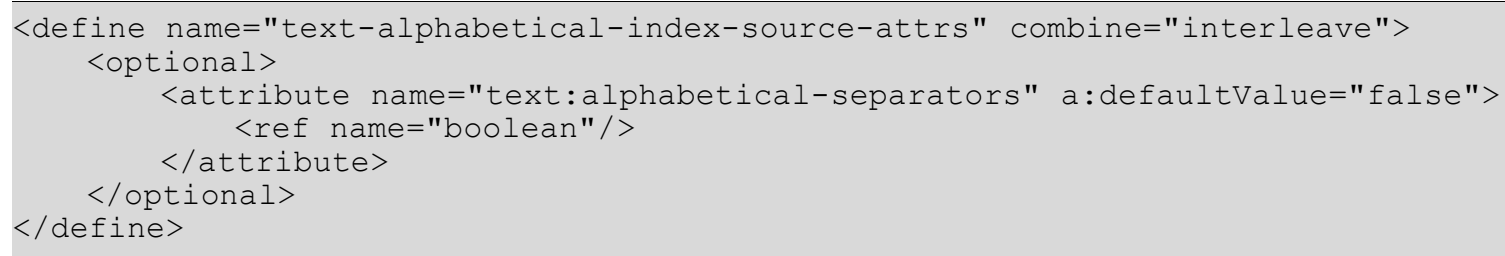




\section{Combining Entries}

There are several options for dealing with the common situation where there are multiple index entries for the same word or phrase, as follows:

- Multiple entries for the same word can be combined into a single entry using the text: combine-entries attribute.

- The pages referenced by a combined entry can be formatted as:

- As a range of numbers separated by a dash using the text:combine-entrieswith-dash attribute

- As the start number with a pp label, or the appropriate label for the chosen language, using the text:combine-entries-with-pp attribute

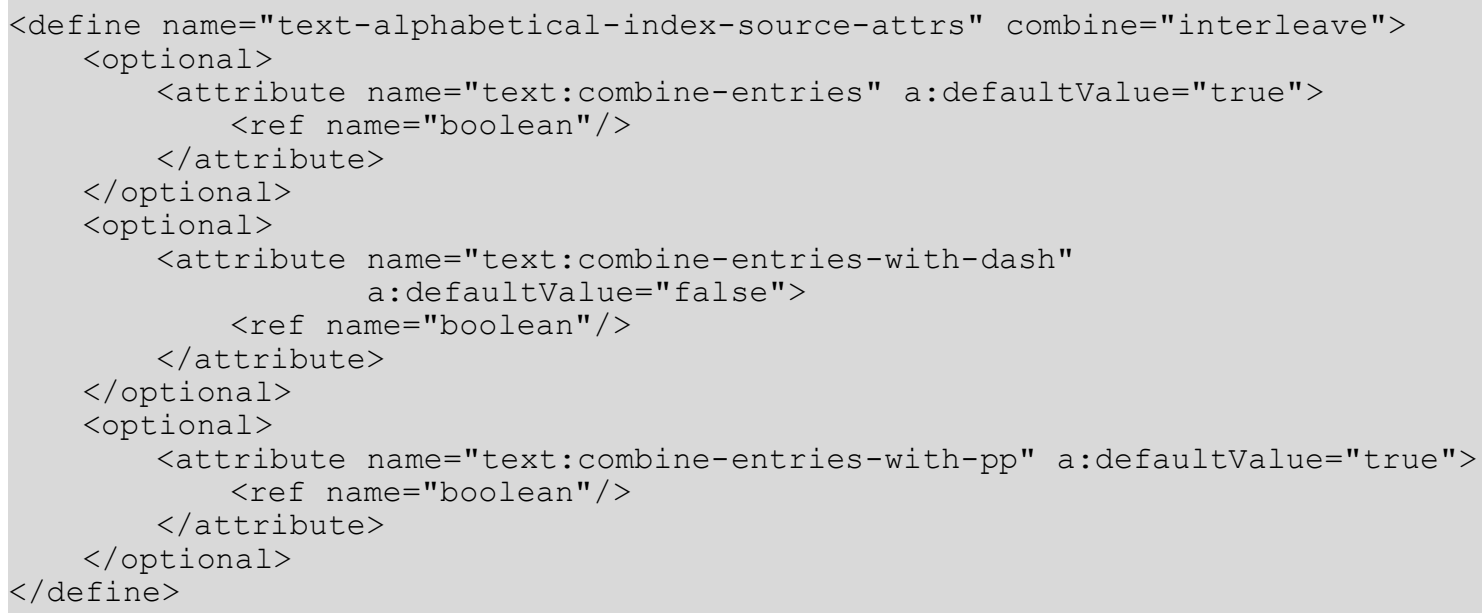

Example: Combining index entries

An index mark for the word "XML" occurs on pages $45,46,47$, and 48 . The entries can be formatted as follows:

\begin{tabular}{|l|l|}
\hline Entry formatted as & Result \\
\hline Separate entries & $\begin{array}{l}\text { XML 45 } \\
\text { XML 46 } \\
\text { etc. }\end{array}$ \\
\hline Simple combined entries & XML 45, 46, 47, 48 \\
\hline Entries combined with dash & XML 45-48 \\
\hline Entries combined with pp & XML 45pp \\
\hline
\end{tabular}

\section{Use Keys as Entries}

In addition to a keyword, index marks can have up to two keys. If the value of this attribute is true, the keys are used as additional entries. If the value of this attribute is false, the keys are used as subentries.

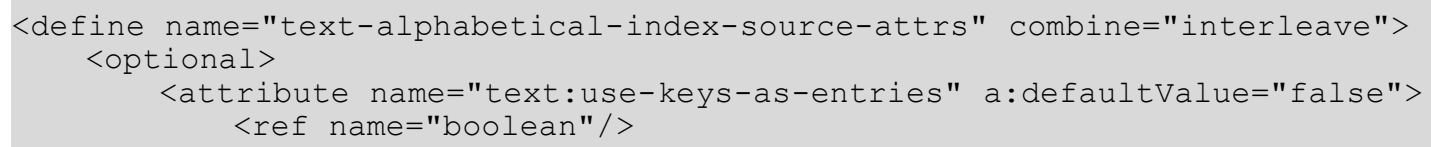




\section{Capitalize Entries}

The text:capitalize-entries attribute determines whether or not the entries in the index are to be capitalized.

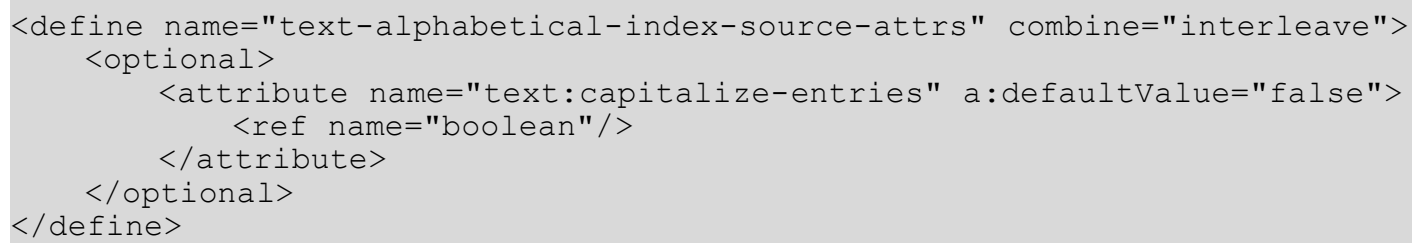

\section{Comma Separated Entries}

The text:comma-separated attribute specifies how to treat multiple index entries. Instead of listing each index entry on a separate line, multiple entries can be listed on a single line separated by a comma. If the value of this attribute is true, multiple entries are listed on a single line separated by a comma. By default, the value of this attribute is false and each index entry is displayed on a separate line.

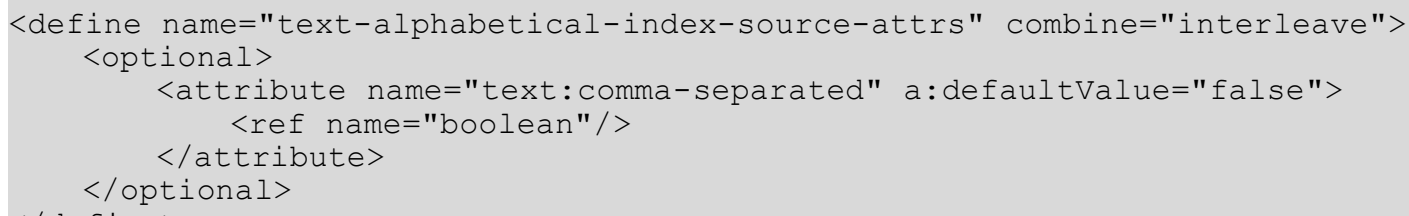

\section{Sort country, Language, and Algorithm}

If index entries are to be sorted, these attributes can be used to specify the sorting. The attributes country and language specify the sorting locale. For some locales, there are multiple sorting algorithms in use. In this case, the algorithm attribute can be used to specify an algorithm by name.

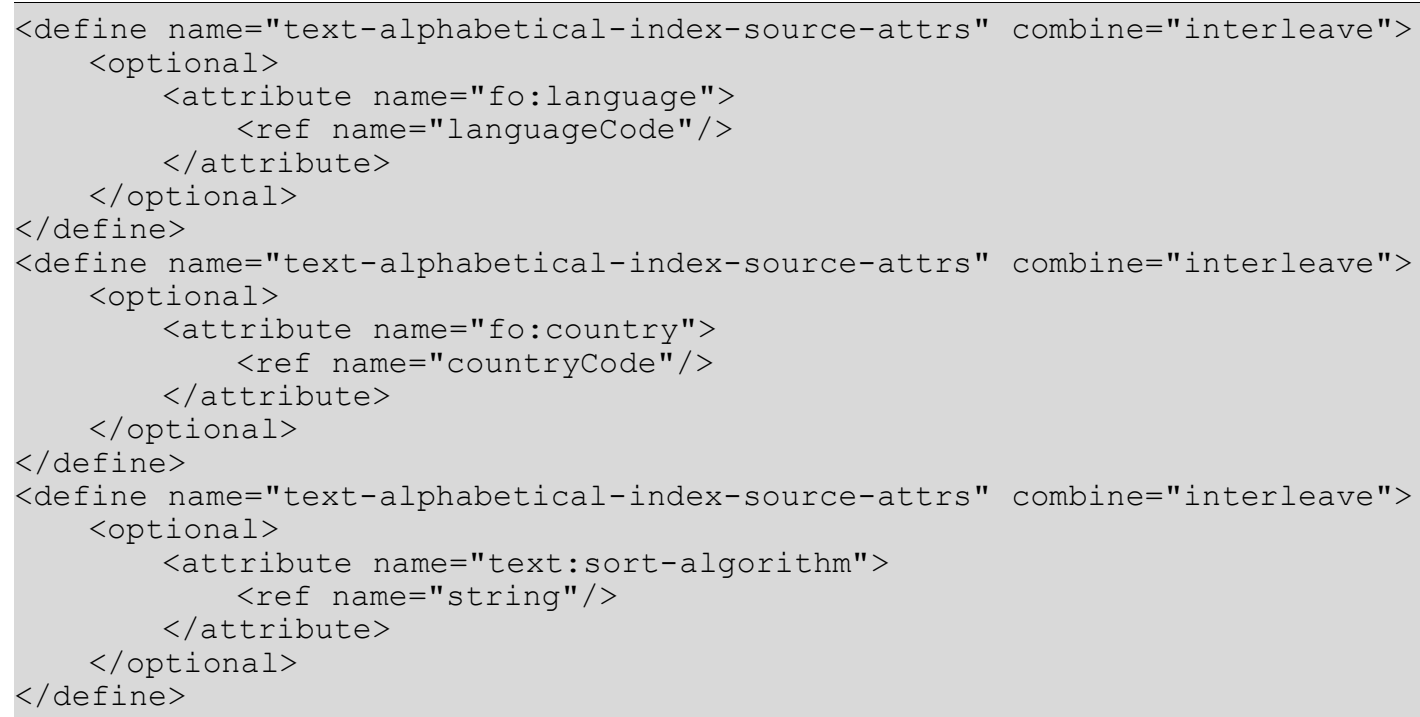




\subsubsection{Auto Mark File}

The alphabetical index supports a so-called auto mark file. Such a file contains a list of terms, and each occurrence of such a term is to be included in the alphabetical index. The alphabetical index mark file is declared as part of the text declarations (see section 4.7). The declaration element in an XLink, which points to the resource containing the list of terms.

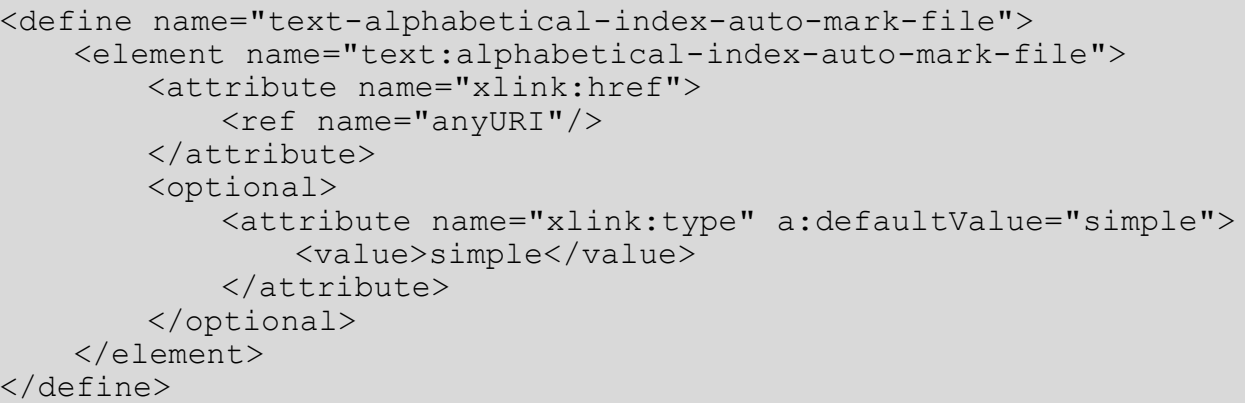

\subsubsection{Alphabetical Index Entry Template}

Alphabetical indexes support three levels; one level for the main index entry, and up to two additional levels for keys associated with the index entries. Alphabetical indexes also use an entry template for the alphabetical separator.

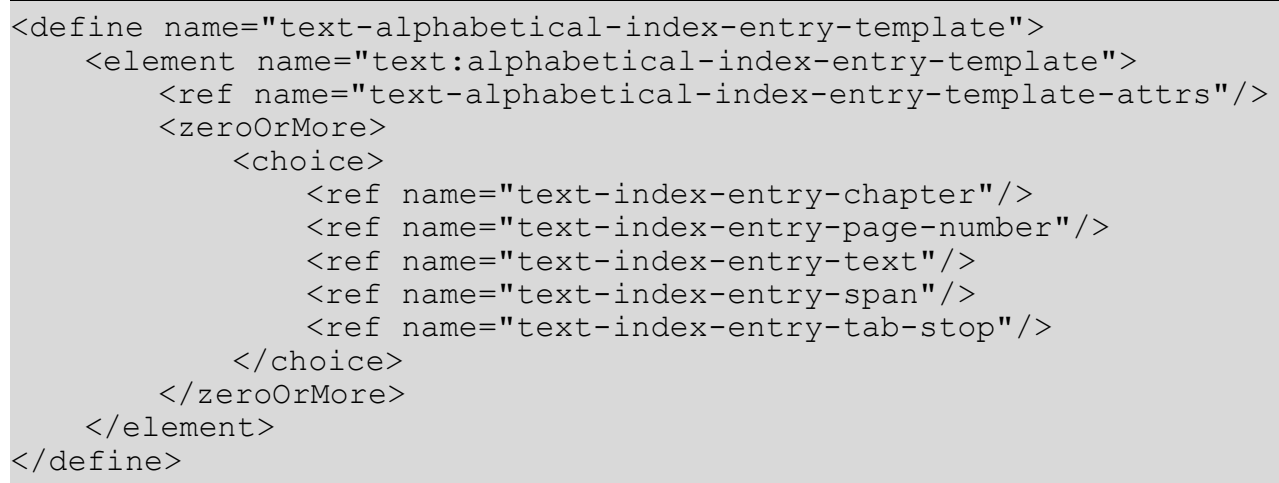

The attributes that may be associated with the <text:alphabetical-index-entrytemplate> elements are:

- Template outline level

- Paragraph style

\section{Template Outline Level}

This attribute specifies whether the template applies to:

- One of the three levels 1,2 ,or 3

or

- The alphabetical separator 


\section{$</$ define $>$}

$<$ value $>1</$ value $>$

$<$ value $>2</$ value $>$

$<$ value $>3</$ value $>$

$<$ value>separator</value>

$</$ choice $>$

$</$ attribute $>$

\section{Paragraph Style}

The text:style-name attribute specifies the paragraph style to use for the template.

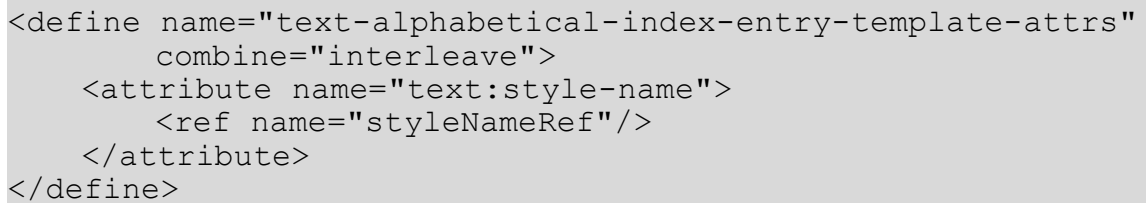

\subsection{Bibliography}

A bibliography index gathers its entries from bibliography index marks. The <text:bibliography> element represents a bibliography.

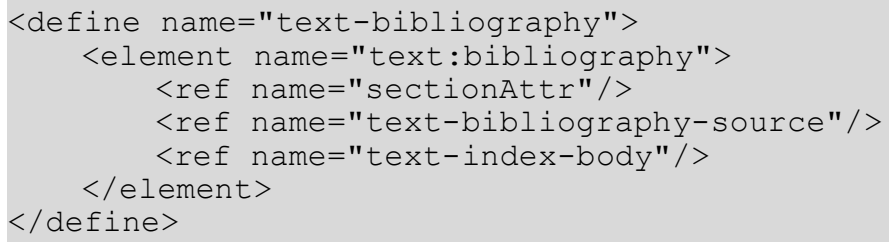

\subsubsection{Bibliography Index Source}

The <text:bibliography-source> element specifies how the bibliography is generated.

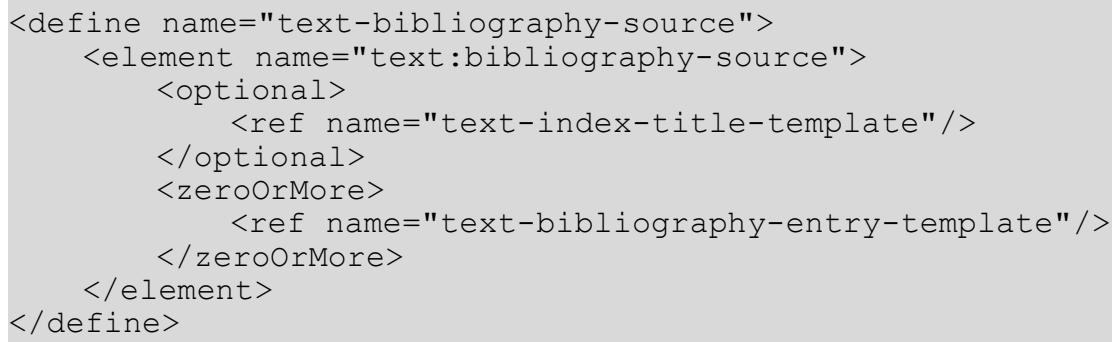

\subsubsection{Bibliography Entry Template}

Bibliography entry templates support entry elements for bibliography data, text spans, and tab stops. There is one entry template element for each type of entry. 
$</$ define $>$

The attributes that may be associated with the <text:bibliography-entry-template> elements are:

- Bibliography type

- Paragraph style

\section{Bibliography Type}

This attribute specifies to which type of bibliographical entry the template applies. This attribute must be unique among all <text:bibliography-type> elements within the same parent element.

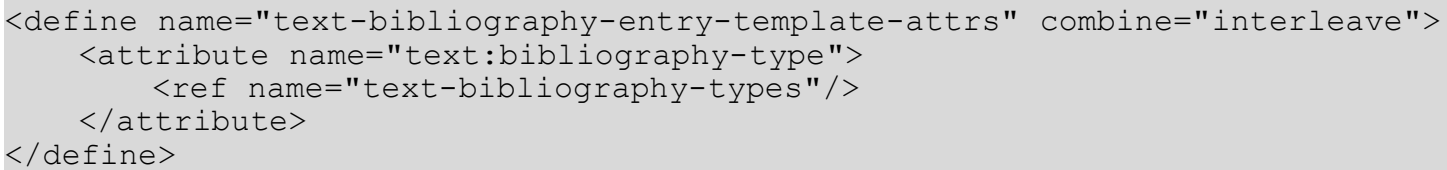

\section{Paragraph Style}

The text:style-name attribute specifies the paragraph style to use for this template.

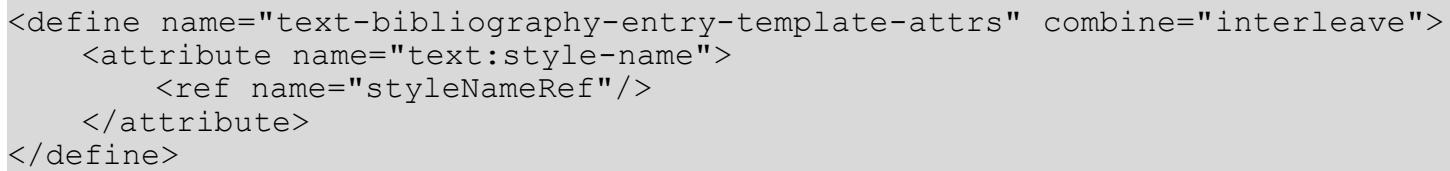

\subsection{0 index source styles}

Some indices can gather index entries from paragraphs formatted using certain paragraph styles. The <text: index-source-styles> element contains all of the <text: indexsource-style> elements for a particular outline level. The text:outline-levels attribute determines at which outline level to list the index entries gathered from the respective paragraph styles. There can only be one <text: index-source-style> element for each outline level.

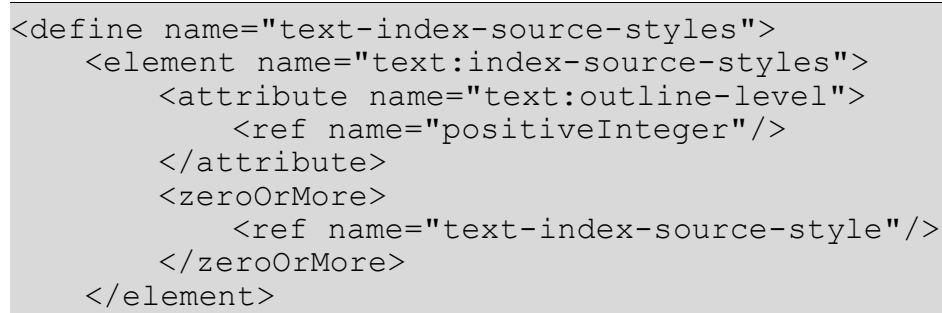

\subsubsection{Index source style}

All paragraphs formatted using the style or class specified in the <text: index-sourcestyle> element are included in the index.

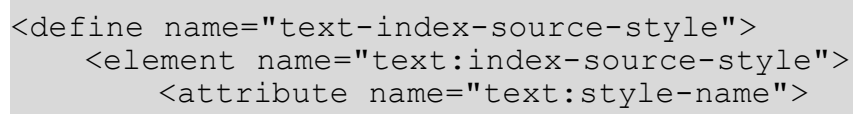




\subsection{Index title template}

The <text: index-title-template> element determines the style and content of the index title. There can only be one <text: index-title-template> element contained in a <text: table-of-content-source> element.

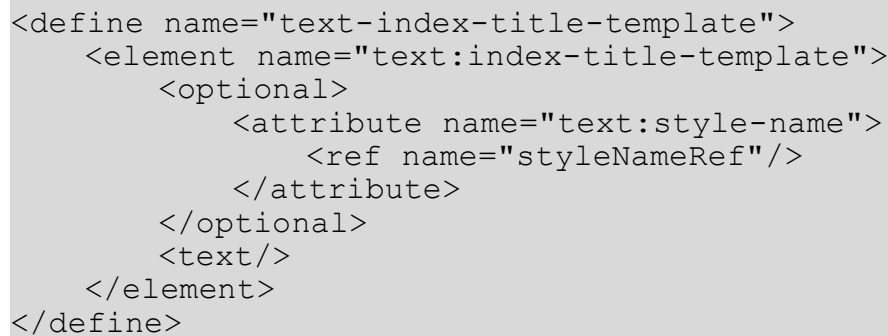

\subsection{Index Template Entries}

There are eight types of index entries, as follows:

- Chapter information

- Entry text

- Page number

- $\quad$ Fixed string

- Bibliography information

- Tab stop

- Hyperlink start and end

\subsubsection{Chapter Information}

The <text: index-entry-chapter> element displays the chapter number of the index entry. The character style for the chapter number can be included in the index entry element as a text:style-name attribute.

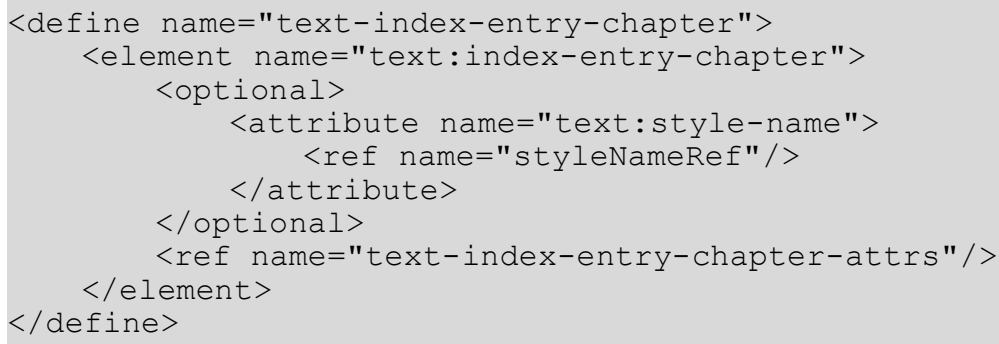

Note: This element can only display the chapter number. To display the chapter name, the <text: index-entry-text> elements must be used. 


\section{Display Chapter Format}

The text: display attribute displays either the chapter number, the chapter name, or both.

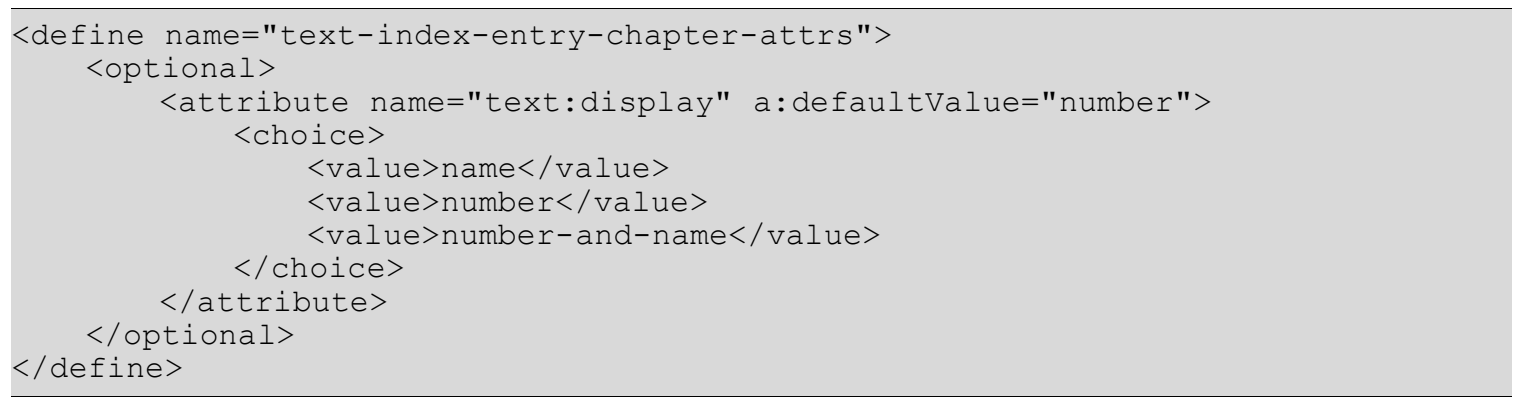

\subsubsection{Entry Text}

The <text: index-entry-text> element displays the text of the index entry, for example, the chapter name if the entry is derived from a header or the phrase contained in the index mark if the entry is derived from an index mark. The character style for the entry text can be included in the index entry element as a text:style-name attribute.

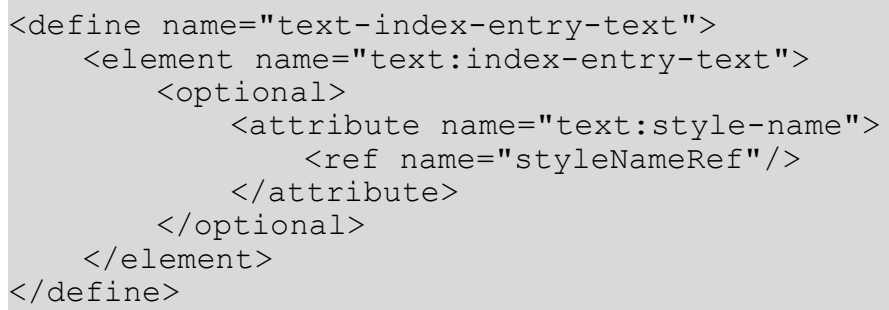

\subsubsection{Page Number}

The <text: index-entry-page-number $>$ element displays the page number on which the index entry is located. The character style for the page number can be included in the index entry element as a text:style-name attribute.

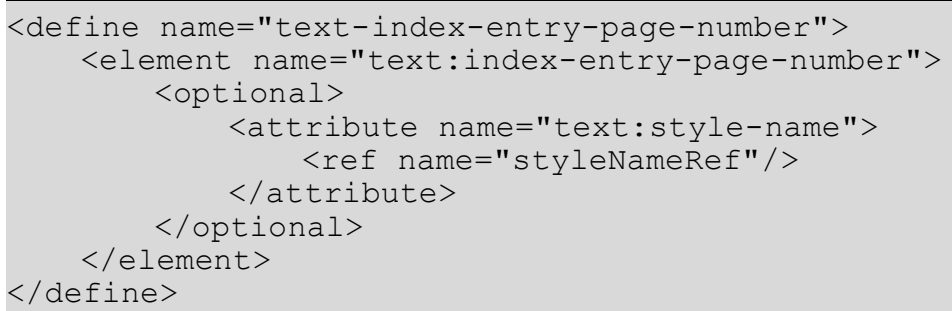

\subsubsection{Fixed String}

The <text: index-entry-span> element represents a fixed string within an index entry. The character style for the entry text can be included in the index entry element as a text:stylename attribute. Unlike the <text:span> element, the <text: index-entry-span> element does not have any child elements. 


\subsubsection{Bibliography Information}

The <text: index-entry-bibliography> element introduces bibliography data into index entry templates.

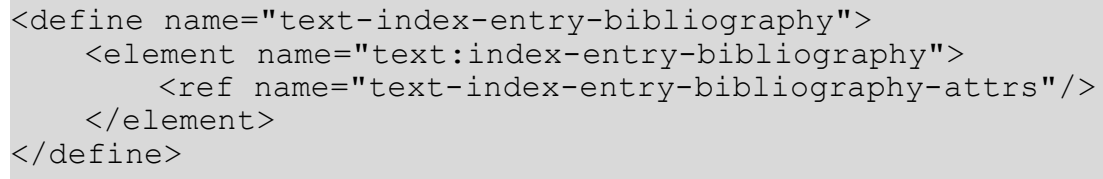

The attributes that may be associated with the <text: index-entry-bibliography> element are:

- text:style-name attribute

- text:bibliography-data-field attribute

\section{Text Style Name}

The text:style-name attribute determines the style for display of the entry.

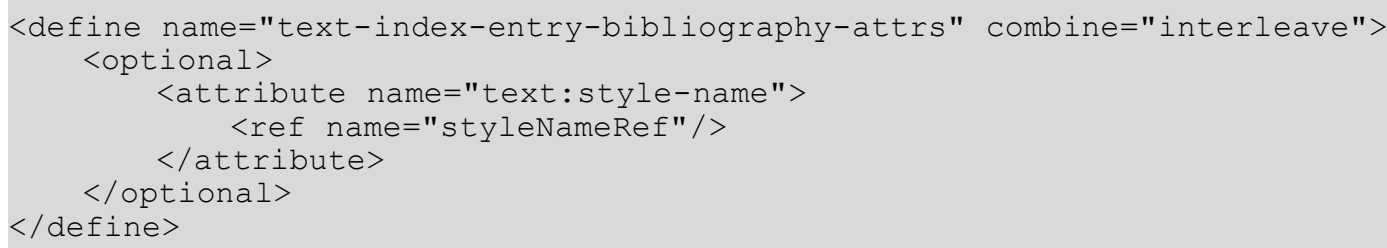

\section{Bibliography Data Field Identifier}

The text:bibliography-data-field attribute determines which part of the bibliography data field will be displayed.

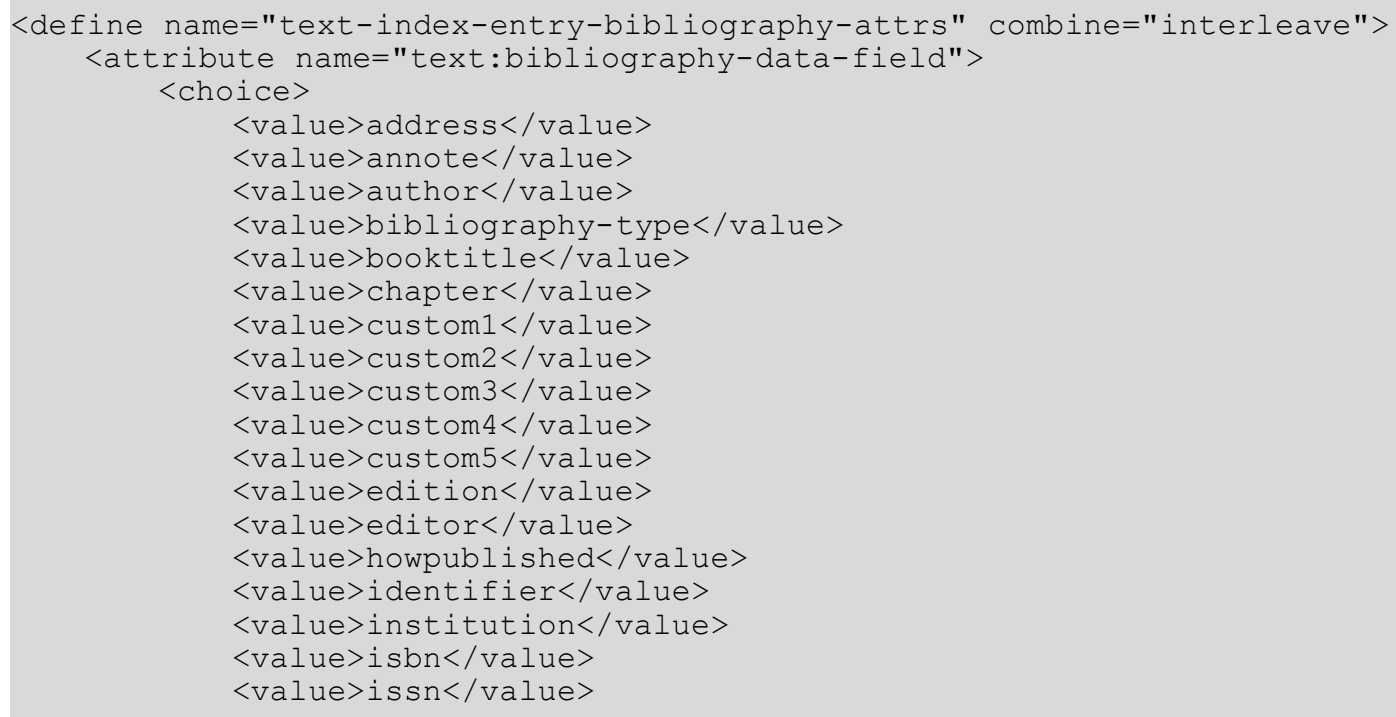


<value>journal</value $>$

$<$ value $>$ month $</$ value $>$

$<$ value $>$ note $</$ value $>$

$<$ value>number</value $>$

$<$ value>organizations</value>

$<$ value>pages</value $>$

$<$ value>publisher</value>

$<$ value>report-type</value $>$

$<$ value $>$ school</value $>$

$<$ value>series</value $>$

$<$ value>title</value $>$

$<$ value $>$ url</value $>$

$<$ value $>$ volume</value $>$

$<$ value $>$ year $</$ value $>$

$</$ attribute $>$

$</$ define $>$

\subsubsection{Tab Stop}

The <text: index-entry-tab-stop> element represents a tab stop within an index entry. It also contains the position information for the tab stop.

\section{$</$ define $>$}

The attributes that may be associated with the <text: index-entry-tab-stop> element are:

- style:leader-char

- style:type

- $\quad$ style:position

\section{Leader Char}

The style: leader-char attribute specifies the leader character.

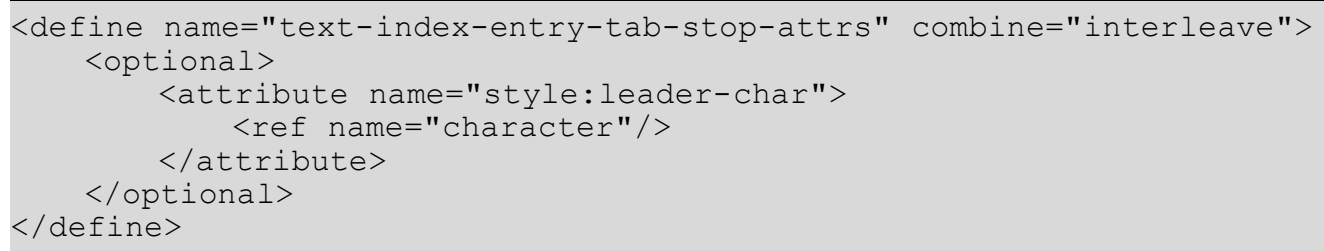

\section{Tab Type and Position}

The style:type attribute specifies the tab stop type. The <text:index-entry-tab-stop> element only supports two types of tab: left and right.

If the value of this attribute is left, the style:position attribute must also be used.

Otherwise, this attribute must be omitted. The style:position attribute specifies the position 
of the tab. Depending on the value of the text:relative-tab-stop-position attribute in the <text: index-entry-config> element, the position of the tab is interpreted as being relative to the left margin or the left indent.

\subsubsection{Hyperlink Start and End}

The <text: index-entry-link-start> and <text: index-entry-link-end> elements mark the start and end of a hyperlink index entry. The character style for the hyperlink can be included in the index entry element as a text:style-name attribute.

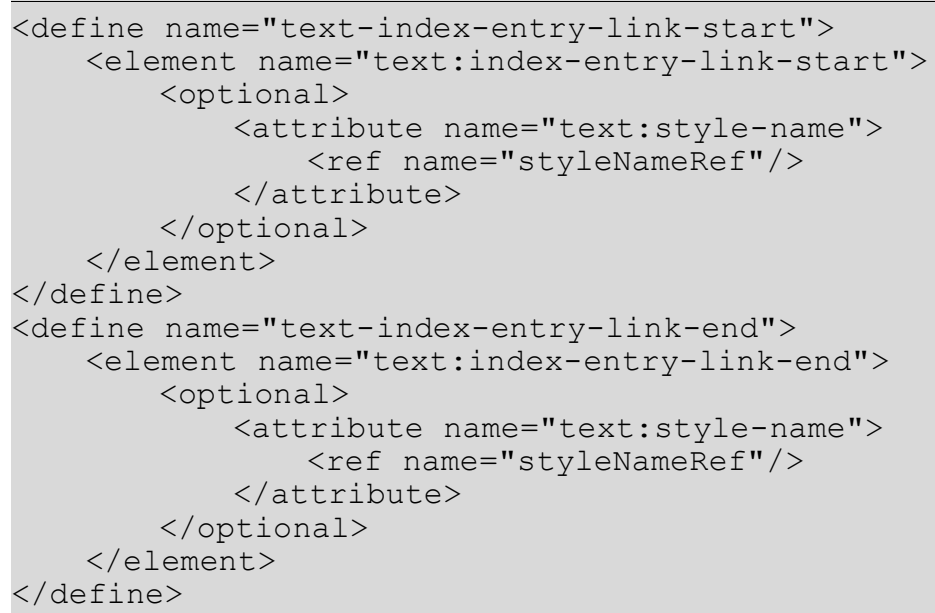

\subsubsection{Example of an Index Entry Configuration}

The following is an example of the XML code for a table of contents called Table of Content with the following characteristics:

- It uses the top two outline levels.

- Each entry consists of the chapter number, a closing parenthesis, the chapter title, a tab stop, and the page number.

- For the top outline level, the page number is formatted using a style called Bold.

- For the second outline level, a bracket is used instead of a closing parenthesis.

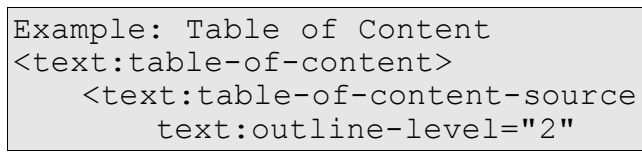




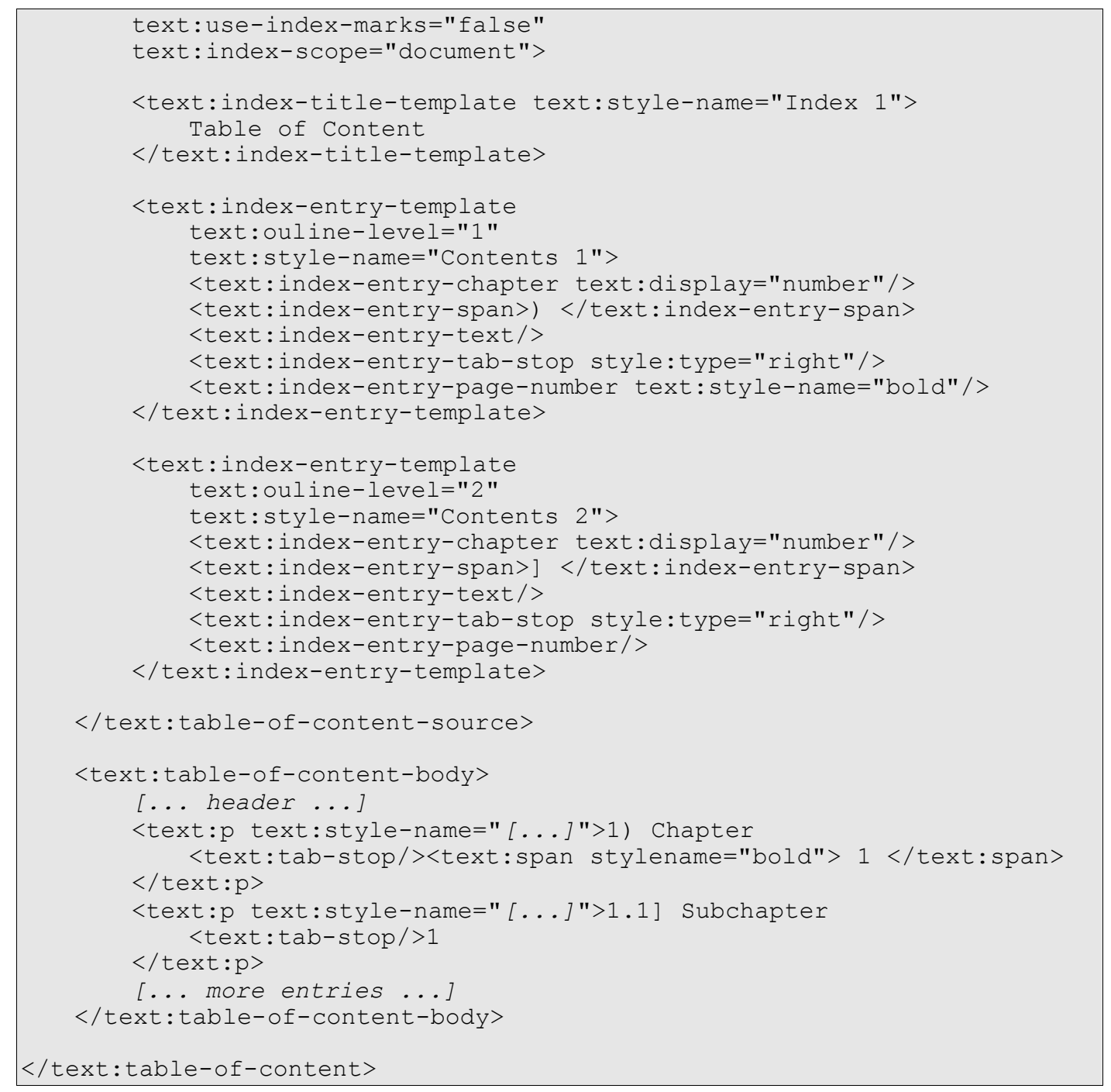




\section{Tables}

This chapter describes the table structure that is used for tables that are embedded within text documents and for spreadsheets.

\subsection{Basic Table Model}

The structure of OpenDocument tables is similar to the structure of [HTML4] or [XSL] tables, and like these tables, they can be nested.

The representation of tables is based on a grid of rows and columns. Rows take precedence over columns. The table is divided into rows and the rows are divided into cells. Each column includes a column description, but this description does not contain any cells.

Table rows may be empty, and different rows might contain a different number of table cells. This is not an error, but applications might resolve this in different ways. Spreadsheet applications typically operate on large tables that have a fixed application dependent row and column number, but may have an unused area. Only the used area of the table is saved in files. When loading a table with empty or incomplete rows into a spreadsheet application, empty rows typically introduce a default row (just as in an empty sheet), and incomplete rows are filled with empty cells (just like in an empty sheet). All other applications typically have fixed size tables. Incomplete rows are basically rendered as if they had the necessary number of empty cells, and the same applies to empty rows. Empty cells typically occupy the space of an empty paragraph.

Rows and columns appear in row groups and column groups. These groups specify whether or not to repeat a row or column on the next page.

\subsubsection{Table Element}

The table element is the root element for tables.

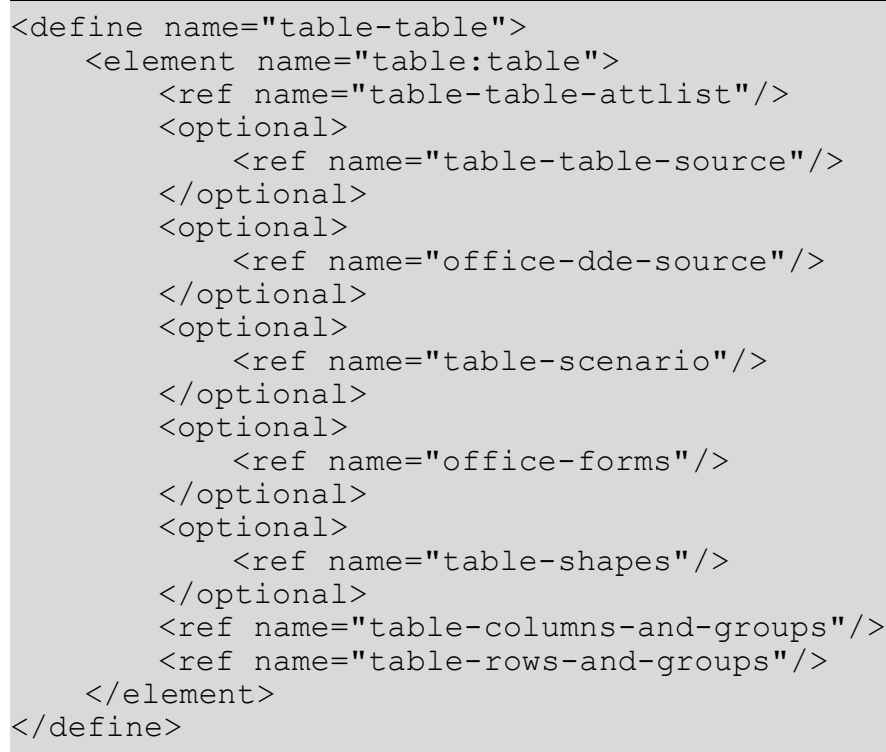

The content models for tables is rather complex. The details are explained in the section 8.2. For the moment, it can be assumed that table element's content are columns and row elements. 


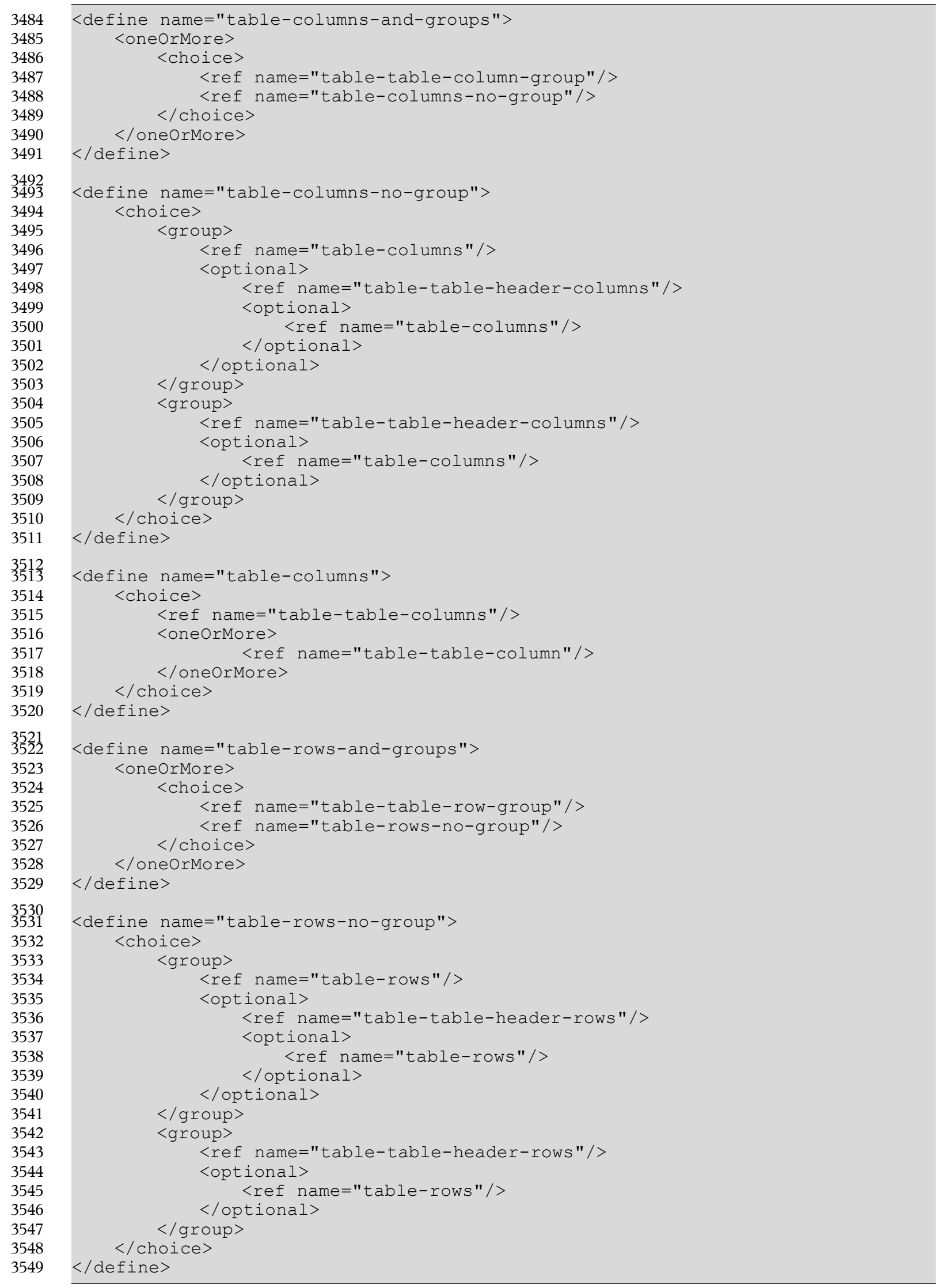




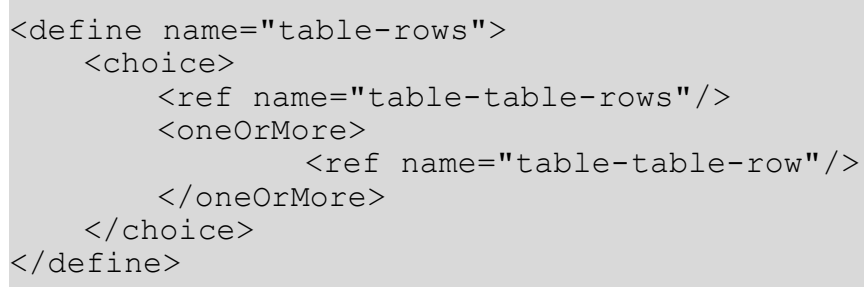

\section{Table Name}

The table: name attribute specifies the name of a table.

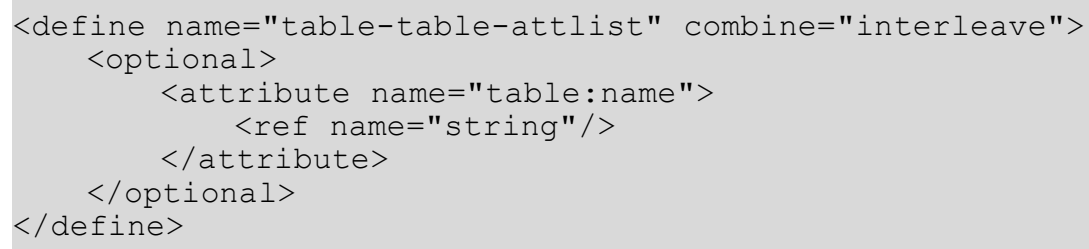

\section{Table Style}

The table:style-name attribute references a table style, i.e. an <style:style $>$ element of type "table". The table style describes the formatting properties of the table, such as width and background color. The table style can be either an automatic or common style.

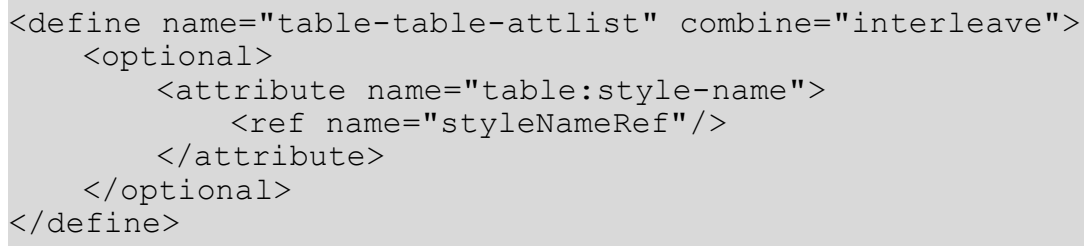

\section{Example: Table Style}

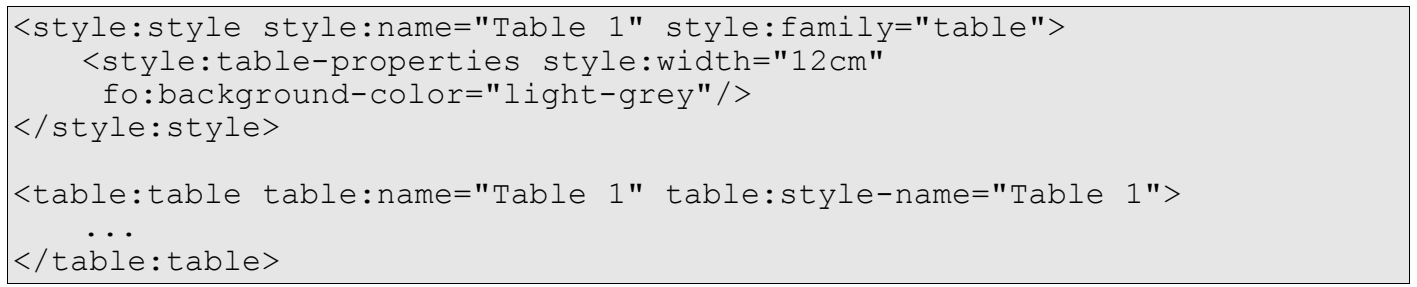

\section{Protected}

The table:protected attribute specifies whether or not a table is protected from editing. If the table is protected, the table:protection-key attribute can specify a password to prevent a user from resetting the protection flag to enable editing. If a table is protected, all of the table elements and the cell elements with a style:cell-protect attribute set to true are protected.

To avoid saving the password directly into the XML file, only a hash value of the password is stored within the table:protection-key attribute.

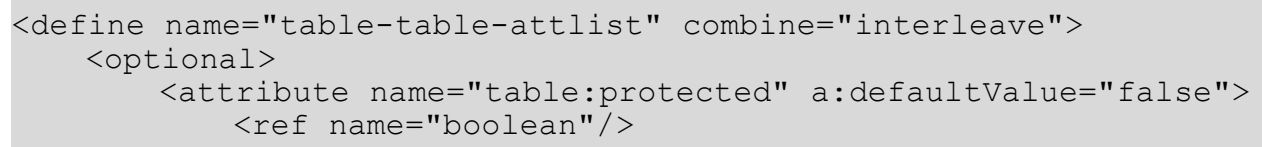




\section{Print}

The table:print attribute specifies if a table is printed. It takes a Boolean value. If its value is true, the table is printed, if its value is false, the table is not printed. The default value is true. The table:print attribute will be overwritten by the table: display attribute described in section 15.8.14. That is, if the table is not displayed, it also will not be printed.

If the table is printed, the table range that actually is printed can be specified by table:printrange attribute (see following section). If this attribute is not existing, the used area of the table will be printed.

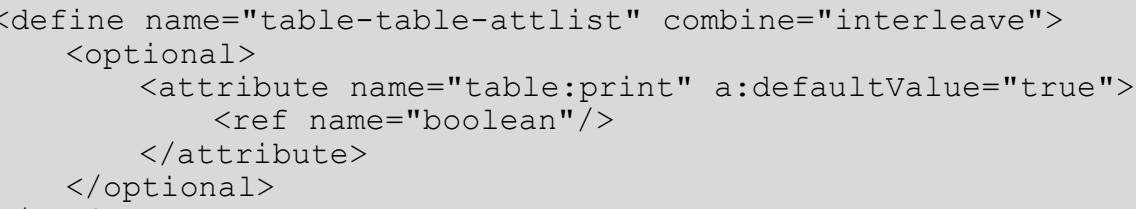

\section{Print Ranges}

The table:print-ranges attribute specifies the print ranges of the table, i.e. the cells that should be printed. It contains a list of cell addresses or cell range addresses as described in section 8.3.1.

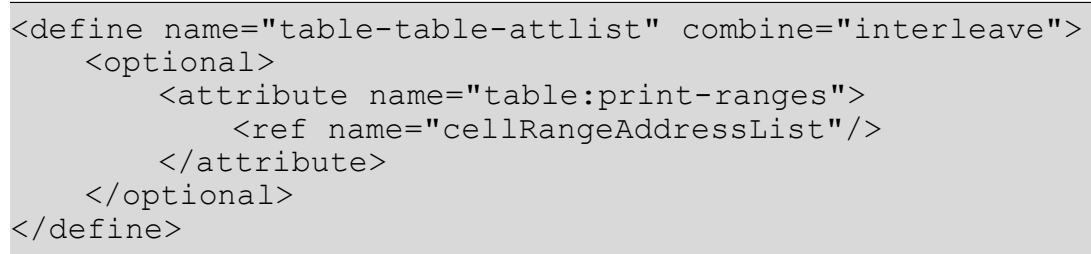

\subsubsection{Table Row}

The $<$ table:table-row $>$ element represents a row in a table. It content are elements that specify the cells of the table row.

The $<$ table:table-row $>$ element is similar to the $[X S L]<f o: t a b l e-r o w>$ element.

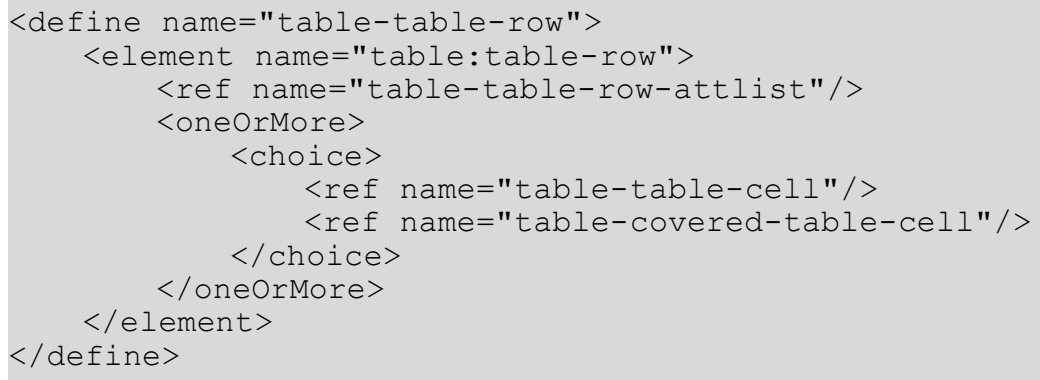




\section{Number of Rows Repeated}

The table: number-rows-repeated attribute specifies the number of rows to which a row element applies. If two or more rows are adjoining, and have the same content and properties, and do not contain vertically merged cells, they may be described by a single $<$ table: tablerow $>$ element that has a table:number-rows-repeated attribute with a value greater than 1.

\section{Visibility}

The table:visibility attribute specifies whether the row is visible, filtered, or collapsed. Filtered and collapsed rows are not visible. Filtered rows are invisible, because a filter is applied to the table that does not select the table row. Collapsed rows have been made invisible by invisible in the UI directly.

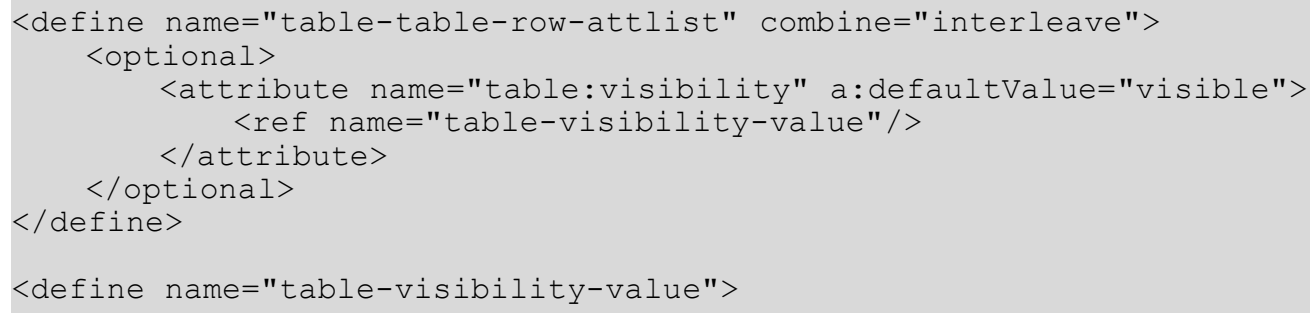




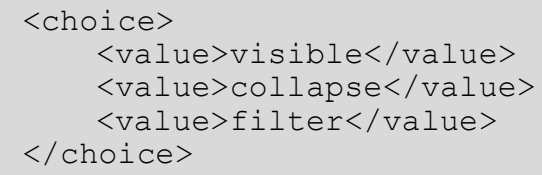

\section{Example: Table with three rows and three columns}

This example shows the OpenDocument code for a table with three rows and three columns. The first two rows of the table have a blue background.

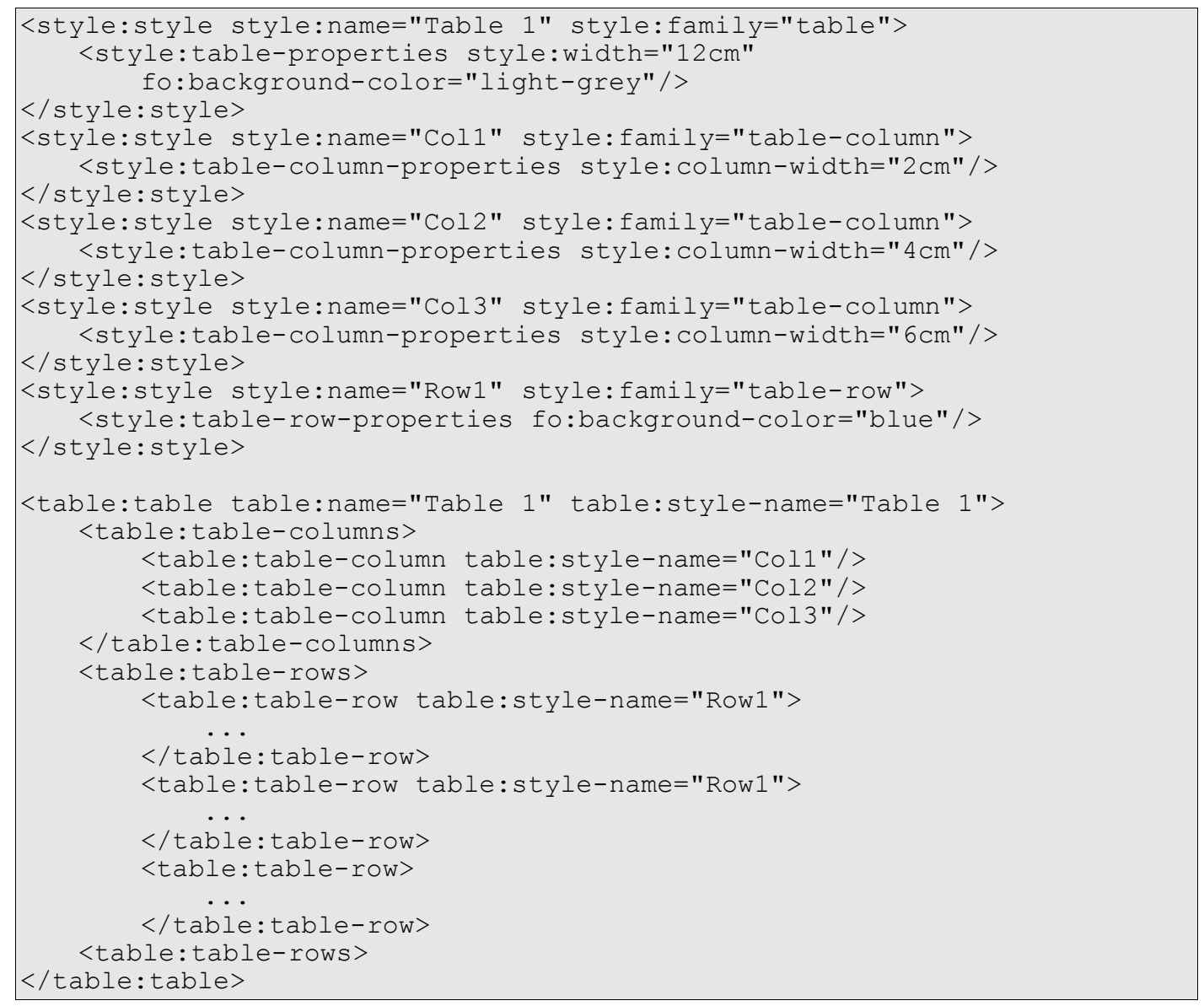

\subsubsection{Table Cell}

The <table:table-cell> and <table:covered-table-cell> elements specify the content of a table cells. They are contained in table row elements. A table cell can contain paragraphs and other text content as well as sub tables. Table cells may be empty.

The <table:table-cell> element is very similar to the table cell elements of [XSL] and [HTML4], and the rules regarding cells that span several columns or rows that exist in HTML and XSL apply to the OpenDocument specification as well. This means that there are no $<$ table:table-cell> elements in the row/column grid for positions that are covered by a merged cell, that is, that are covered by a cell that spans several columns or rows. To be able to specify cells for such positions as well, the $<t a b l e$ : covered-table-cell $>$ element exists. It has to appear wherever a position in the row/column grid is covered by a cell spans several rows or columns. Its position in the grid is calculated by a assuming a column and row span of 1 for all cells regardless whether they are specified by a $<$ table:table-cell $>$ or a 
<table:covered-table-cell> element. The <table:covered-table-cell> is especially used by spreadsheet applications, where it is a common use case that a covered cell contains content.

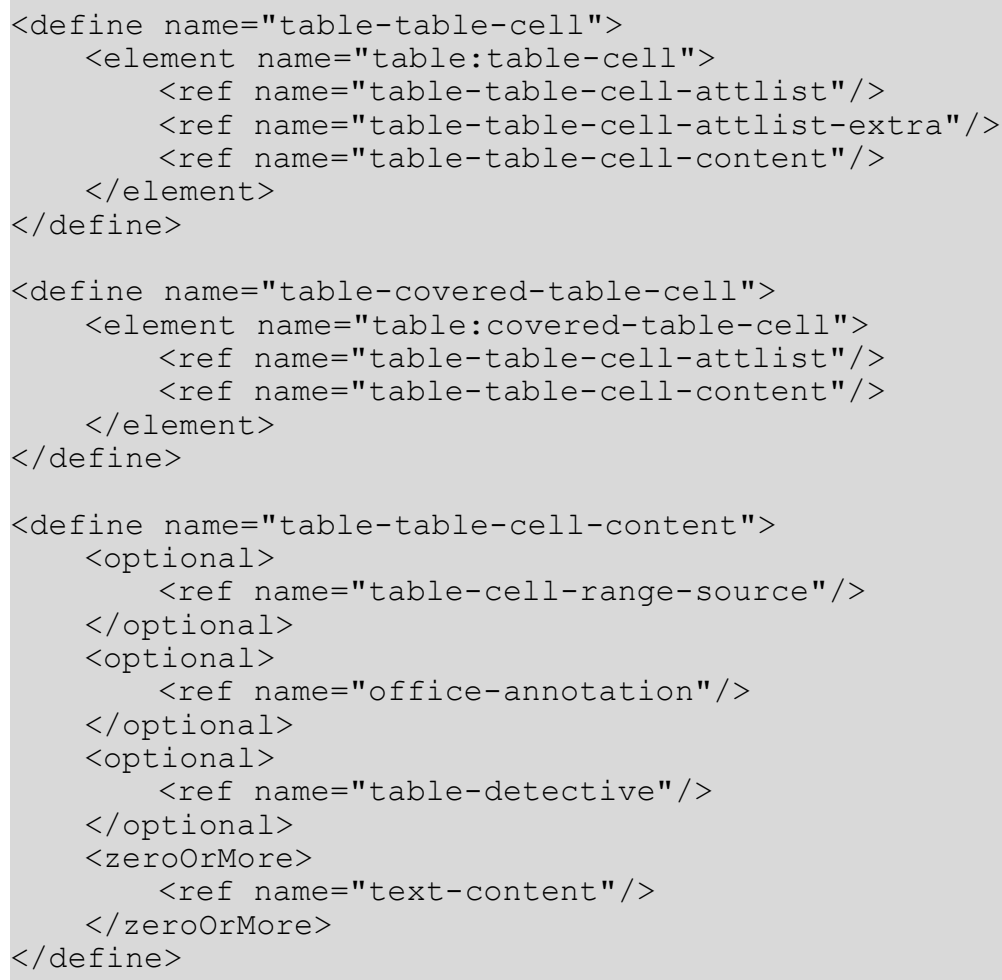

\section{Number of Cells Repeated}

The table:number-columns-repeated attribute specifies the number of successive columns in which a cell is repeated. It can be used to describe two or more adjoining cells with a single cell element, if they meet the following conditions:

- The cells contain the same content and properties.

- The cells are not merged horizontally or vertically.

In this case, a table:number-columns-repeated attribute must be used to specify the number of successive columns in which the cell is repeated. This attribute is specified with either the $<$ table:table-cell> element or the $<$ table:covered-table-cell $>$ element.

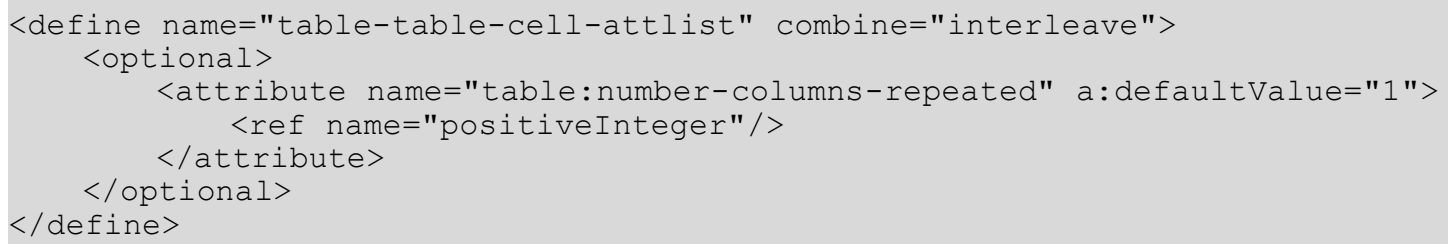

\section{Number of Rows and Columns Spanned}

These attributes specify the number of rows and columns that a cell spans. These attributes cane be used with the $<$ table: table-cell > element only. 
When a cell covers another cell because of a column or row span value greater than one, a $<$ table:covered-table-cell> element must appear in the table to represent the covered cell.

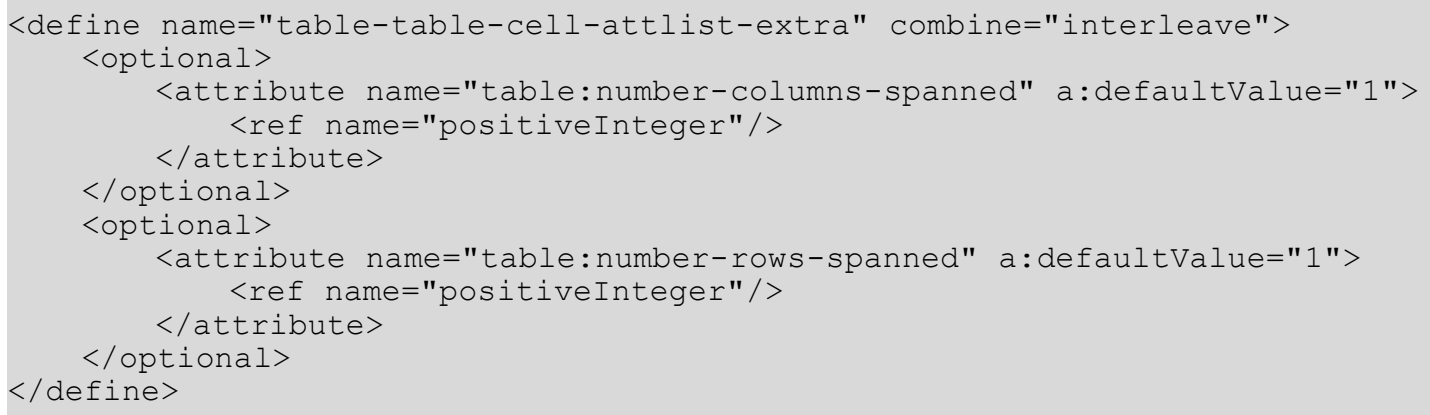

\section{Cell Style}

A table cell style stores the formatting properties of a cell, such as the following:

- Background color

- Number format

- Vertical alignment

\section{- Borders}

The table cell style can be either an automatic or a common style. The style is specified with a table:style-name attribute. If a cell does not have a cell style assigned, the application checks if a the current row has a default cell style assigned. If the current row does not have a default cell assigned style as well, the application checks if the current column has a default cell style assigned.

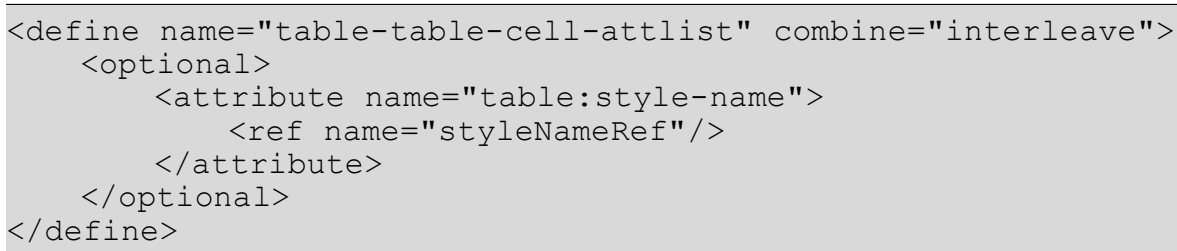

\section{Cell Content Validation}

The table: content-validation-name attribute specifies if a cell contains a validity check. The value of this attribute is the name of $a<t a b l e: c e l l-c o n t e n t-v a l i d a t i o n>$ element. If the attribute is not present, the cell may have arbitrary content.

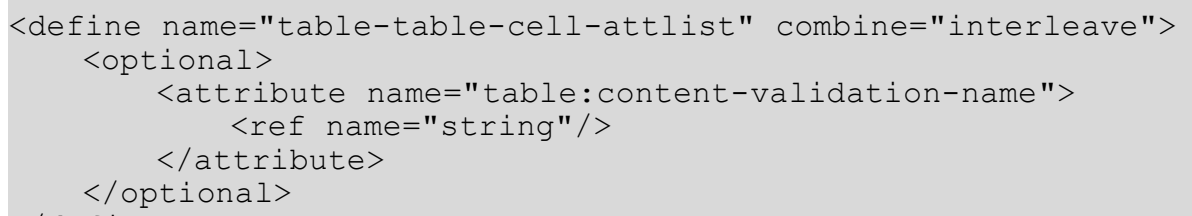

See section 8.5.3 for more information on cell content validation and the <table:cellcontent-validation> element. 


\section{Formula}

Formulas allow calculations to be performed within table cells. Every formula should begin with a namespace prefix specifying the syntax and semantics used within the formula. Typically, the formula itself begins with an equal (=) sign and can include the following components:

- Numbers.

- Text.

- Named ranges.

- Operators.

- Logical operators.

- Function calls.

- Addresses of cells that contain numbers. The addresses can be relative or absolute, see section 8.3.1. Addresses in formulas start with a "[" and end with a "]". See sections 8.3.1 and 8.3.1 for information about how to address a cell or cell range.

The following is an example of a simple formula:

$$
=\operatorname{sum}([\cdot A 1: . A 5])
$$

This formula calculates the sum of the values of all cells in the range ".A1: .A 5 ". The function is "sum". The parameters are marked by a " " at the start and a ") " at the end. If a function contains more than one parameter, the parameters are separated by a ";".

The following is a variation of the formula shown above:

$$
=\operatorname{sum}([. A 1] ;[. A 2] ;[. A 3] ;[. A 4] ;[. A 5])
$$

The result of this formula is the same. The components used in the formula depend on the application being used.

The table:formula attribute contains a formula for a table cell.

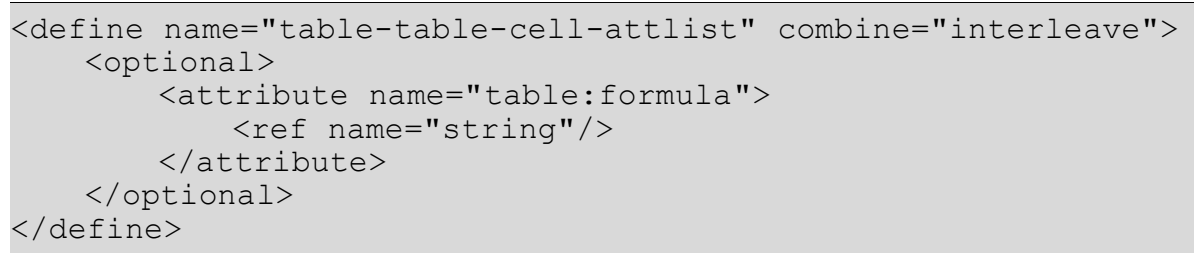

In addition to this, the calculated value of the formula is available as well. One of the following attributes represents the current value of the cell:

- office:value

- office:date-value

- office:time-value

- office:boolean-value

- office:string-value 


\section{Matrix}

When an application is performing spreadsheet calculations, a connected range of cells that contains values is called a matrix. If the cell range contains $m$ rows and $n$ columns, the matrix is called an $m \times n$ matrix. The smallest possible matrix is a $1 \times 2$ or $2 \times 1$ matrix with two adjacent cells. To use a matrix in a formula, include the cell range address of the matrix in the formula. In a matrix formula, only special matrix operations are possible.

The number of rows and columns that a matrix spans are represented by the table: numbermatrix-rows-spanned and table:number-matrix-columns-spanned attributes, which are attached to the cell elements.

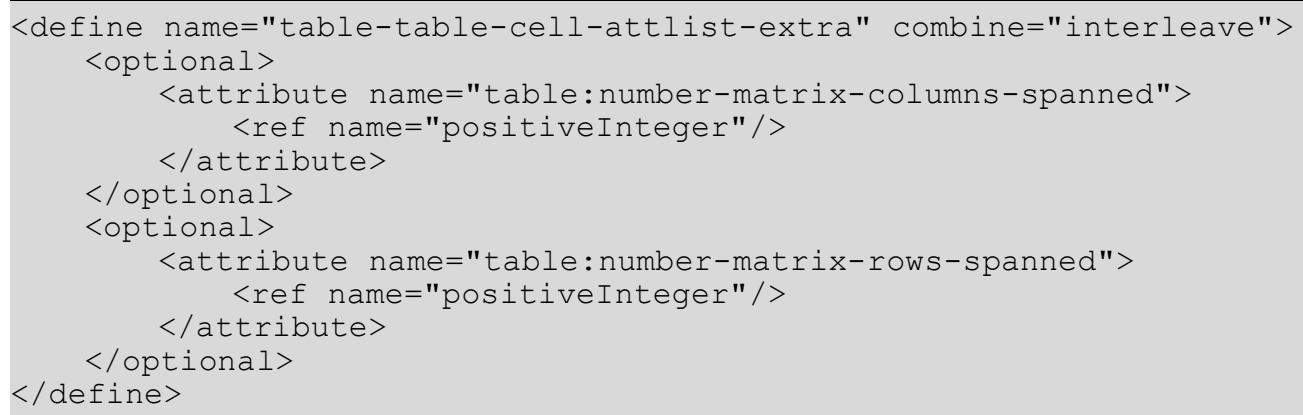

\section{Value Type}

The table:value-type attribute specifies the type of value that can appear in a cell. It may contain one of the following values:

- float, percentage or currency (numeric types)

- date

- $\quad$ time

- boolean

- $\quad$ string

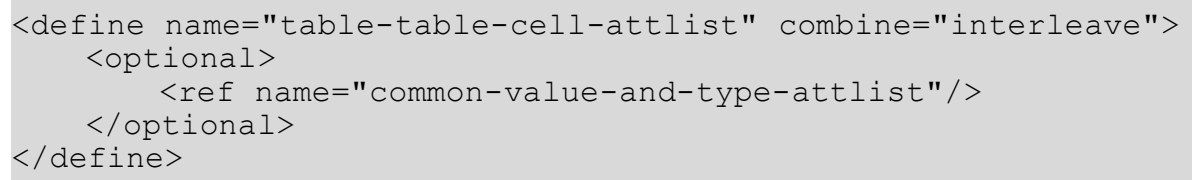

\section{Cell Current Numeric Value}

The office:value attribute specifies the current numeric value of a cell. This attribute is only evaluated for cells that contain the following data types:

- float

- percentage

- currency 


\section{Cell Current Currency}

The tableoffice:currency attribute specifies the current currency value of a cell. The value of this attribute is usually currency information such as DEM or EUR. This attribute is only evaluated for cells whose data type is currency.

\section{Cell Current Date Value}

The office:date-value attribute specifies the current date value of a cell. This attribute is only evaluated for cells whose data type is date.

Some application support date and time values in addition to dates.

\section{Cell Current Time Value}

The office:time-value attribute specifies the current time value of a cell. This attribute is only evaluated for cells whose data type is time.

\section{Cell Current Boolean Value}

The office:boolean-value attribute specifies the current Boolean value of a cell. This attribute is only evaluated for cells whose data type is boolean.

\section{Cell Current String Value}

The office:string-value attribute specifies the current string value of a cell. This attribute is only evaluated for cells whose data type is string.

\section{Table Cell Protection}

The table:protected attribute protects the table cells. Users can not edit the content of a cell that is marked as protected.

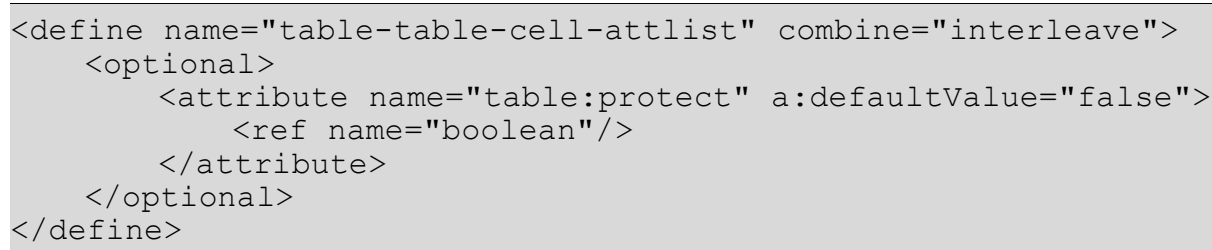

This attribute is not related to the table: protected attribute for table elements (see section 8.1.1) and the table: cell-protect attribute for table cell styles (see section 15.11.14).

\subsection{Advanced Table Model}

\subsubsection{Column Description}

Every column in a table has a column description element $\langle$ table:table-column $\rangle$. It is similar to the $[\mathrm{XSL}]<\mathrm{fo}_{\mathrm{O}}$ : table-column> element, and it's primary use is to reference a table column style that for instance specifies the table column's width. 


\section{Number of Columns Repeated}

The table:number-columns-repeated attribute specifies the number of columns to which a column description applies. If two or more columns are adjoining, and have the same properties, this attribute allows to describe them with a single $<$ table:table-column> element.

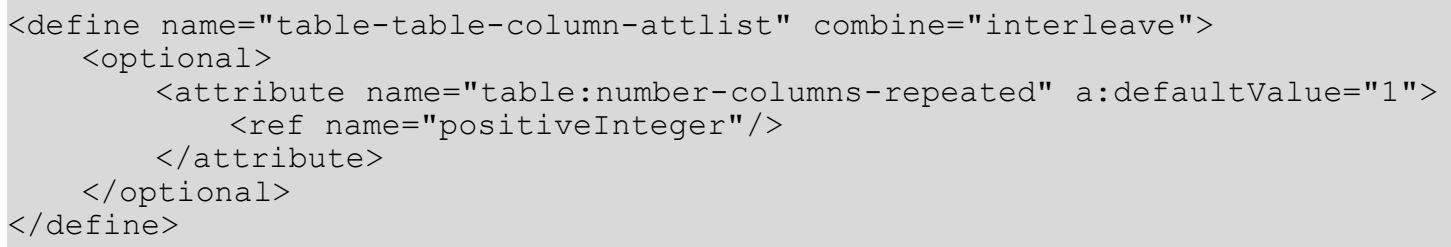

\section{Column Style}

A table column style stores the formatting properties of a table column, such as width and background color. It is specified by a <style:style $>$ element with a family attribute value of table-column and can be either an automatic or a common style. The style of a column is specified using a table:style-name attribute.

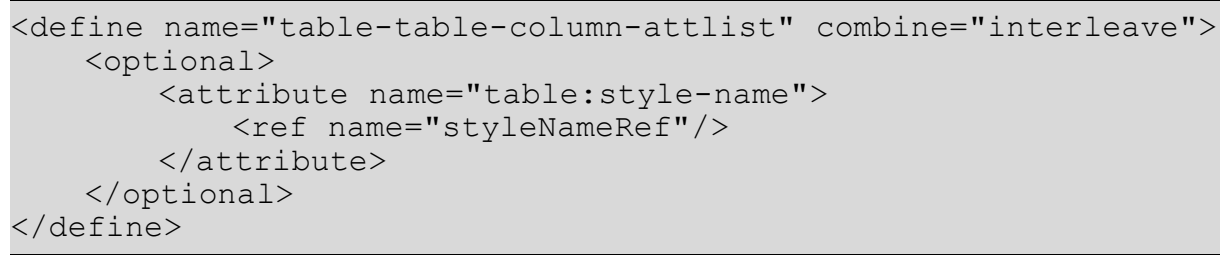

\section{Visibility}

The table:visibility attribute specifies whether the column is visible, filtered, or collapsed. See section 8.1 .2 for more details.

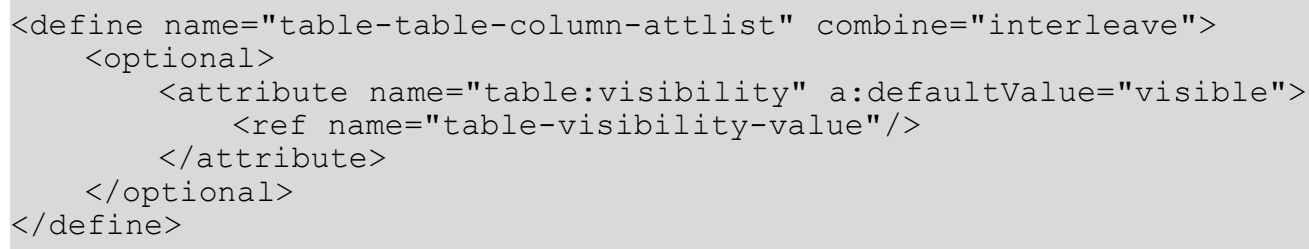

\section{Default Cell Style}

The table:default-cell-style-name attribute specifies the default cell style. Cells without a style use this style when there is no default cell style specified for the cell's row as well.

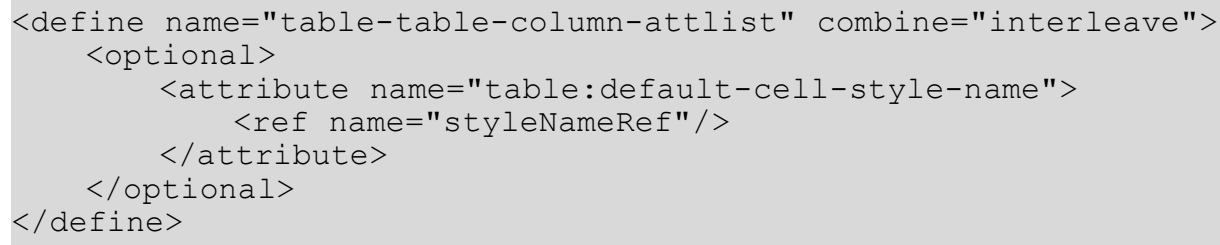

Example: Table with three columns 
This example shows the OpenDocument code for a table with three columns.

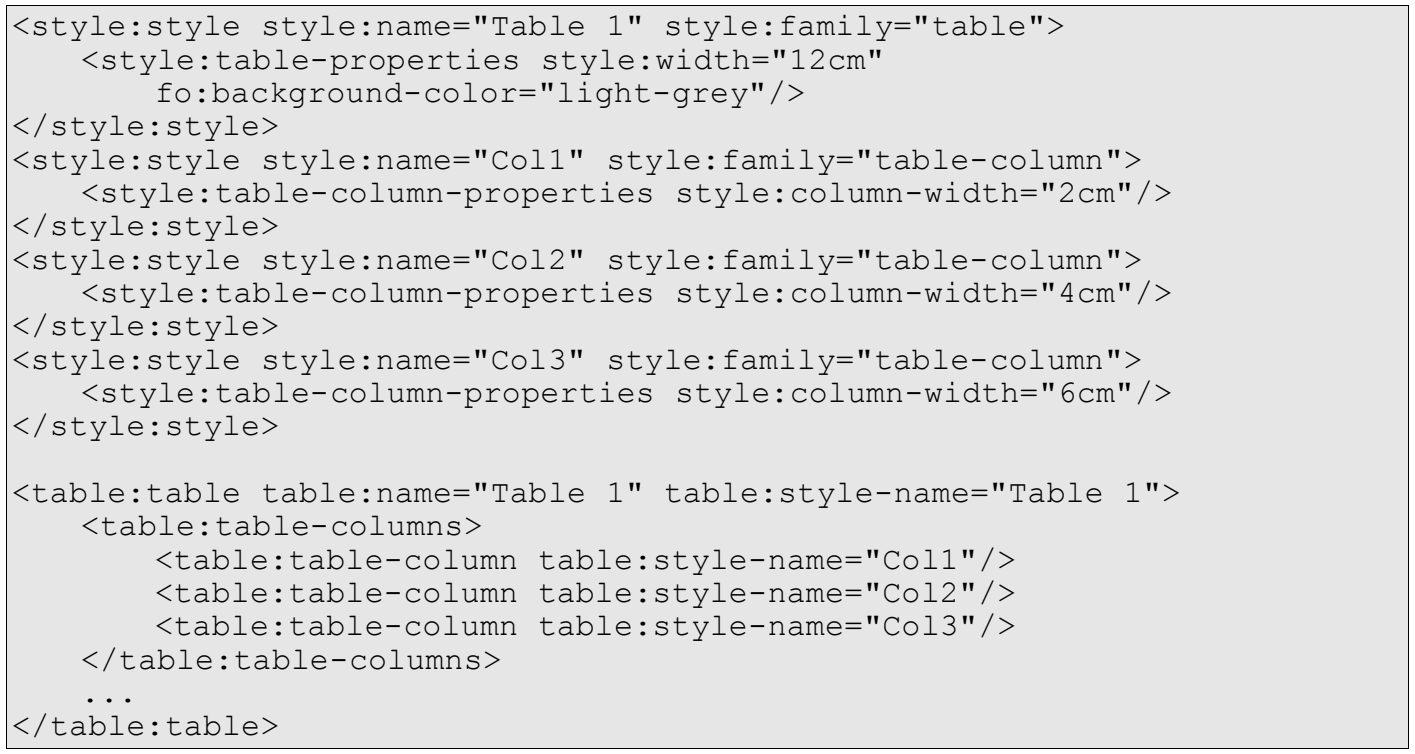

\subsubsection{Header Columns}

If a table does not fit on a single page, a set of adjacent table columns can be automatically repeated on every page. To do so, their columns descriptions have to be included in a $<$ table:table-header-columns> element. Descriptions of columns that shall not be repeated on every page can be included into a $\langle$ table:table-columns $>$ element, but don't have to. A table must not contain more than one $<$ table:table-header-columns $>$ element, and $a<t a b l e: t a b l e-c o l u m n s>$ must not follow another $<$ table: table-columns $>$ element. The only exception are tables that contain grouped columns (see 8.2.3). Such tables contain more than one $<$ table:table-header-columns> element, provided that they are contained in different column groups and the columns contained in the elements are adjacent.

Applications that do not support header columns have to process header column descriptions the same way as non header column descriptions.

The <table: table-header-columns> and <table:table-columns> element are very similar to [HTML4]'s <THEAD> and <TBODY $>$ elements for rows.

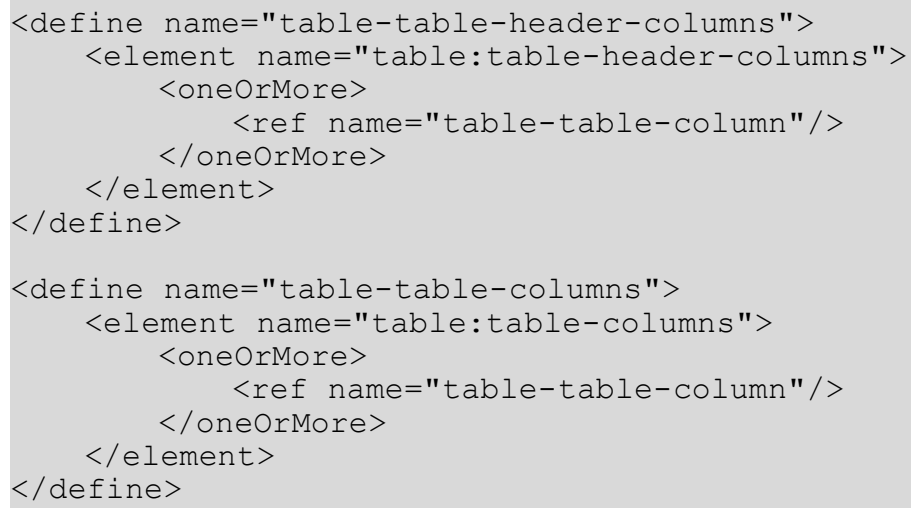




\subsubsection{Column Groups}

Adjacent table columns can be grouped with the <table:table-column-group $>$ element. Every group can contain a new group, columns, and column headers. A column group can be visible or hidden. Column groups can for instance used by spreadsheet applications to group columns that are summarized, so that the individual columns that contribute to the sum can be made invisible easily, but the sum remains visible.

If a set of header columns and a column group overlap, the header column group breaks the column header set. That is, the <table:table-column-group > may contain

<table:table-header-columns> elements, but not vice versa.

\subsubsection{Header Rows}

If a table does not fit on a single page, a set of adjacent table rows can be automatically repeated on every page. To do so, their row elements have to be included in a t table:tableheader-rows > element. Rows that shall not be repeated on every page can be included into a $<$ table:table-rows > element, but don't have to. A table must not contain more than one $<$ table:table-header-rows $>$ element, and a $<$ table:table-rows $>$ must not follow another $<$ table: table-rows $>$ element. The only exception are tables that contain grouped rows (see 8.2.5). Such tables contain more than one $\langle$ table: $t a b l e-h e a d e r-r o w s>$ element, provided that they are contained in different row groups and the rows contained in the elements are adjacent.

Applications that do not support header rows have to process header rows the same way as non header rows.

The $<$ table: table-header-rows $>$ and $<$ table:table-rows $>$ element are very similar to [HTML4]'s $<$ THEAD $>$ and $<$ TBODY $>$ elements.

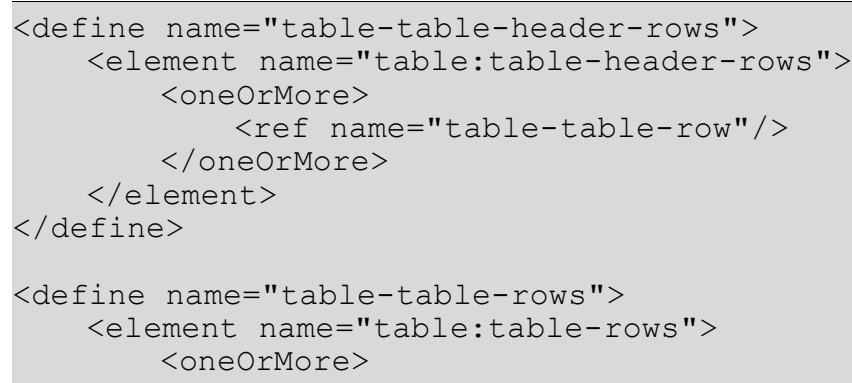




\subsubsection{Row Groups}

Adjacent table rows can be grouped with the <table:table-row-group> element. Every group can contain a new group, rows, and row headers. A row group can be visible or hidden. Row groups can for instance used by spreadsheet applications to group rows that are summarized, so that the individual rows that contribute to the sum can be made invisible easily, but the sum remains visible.

If a set of header rows and a row group overlap, the header row group breaks the row header set. That is, the <table:table-row-group> may contain <table:table-header-rows> elements, but not vice versa.

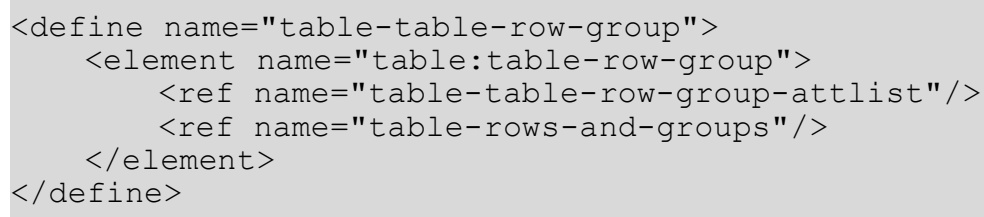

\section{Display}

The table:display attribute specifies whether or not the group is visible.

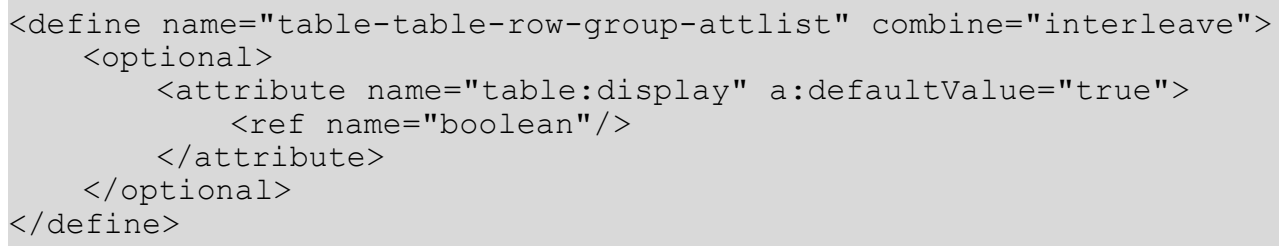

\subsubsection{Subtables}

If a table cell only contains a single table but no paragraphs or other content, this table can be specified as subtable. It then occupies the whole cell and no other content can appear in this cell.

The borders of a subtable merge with the borders of the cell that it resides in. A subtable does not contain any formatting properties. A subtable is essentially a container for some additional table rows that integrate seamlessly with the parent table.

A nested table is turned into a subtable with the attribute table:is-subtable that is attached to the table element. A nested table that is not a specified to be a subtable appears as a table within a table, that is, it has borders distinct from those of the parent cell and respects the padding of the parent cell. 


\section{Example of Representation of subtable}

In the OpenDocument schema, this table can be represented in either of the ways detailed in Sample 1 and Sample 2.

\begin{tabular}{|c|r|l|}
\hline \multicolumn{1}{|c|}{$\boldsymbol{A 1}$} & \multicolumn{1}{c|}{ B1 } & \multicolumn{1}{c|}{ C1 } \\
\hline A2 & B2.1.1 & B2.2.1 \\
\cline { 2 - 3 } & B2.1.2 & \\
\hline
\end{tabular}

\section{Sample 1}

Using cells that span several rows, the preceding table is specified as follows:

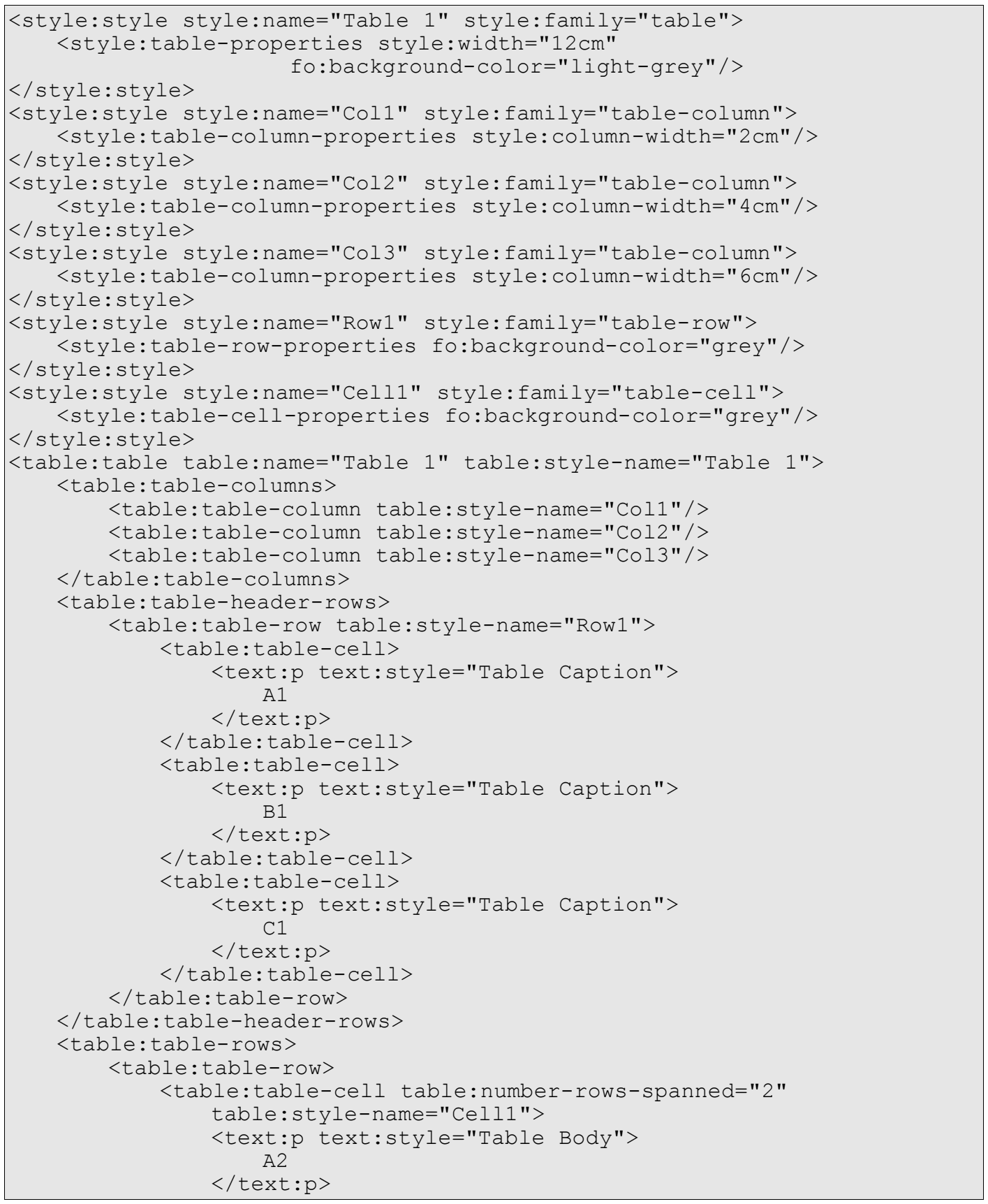




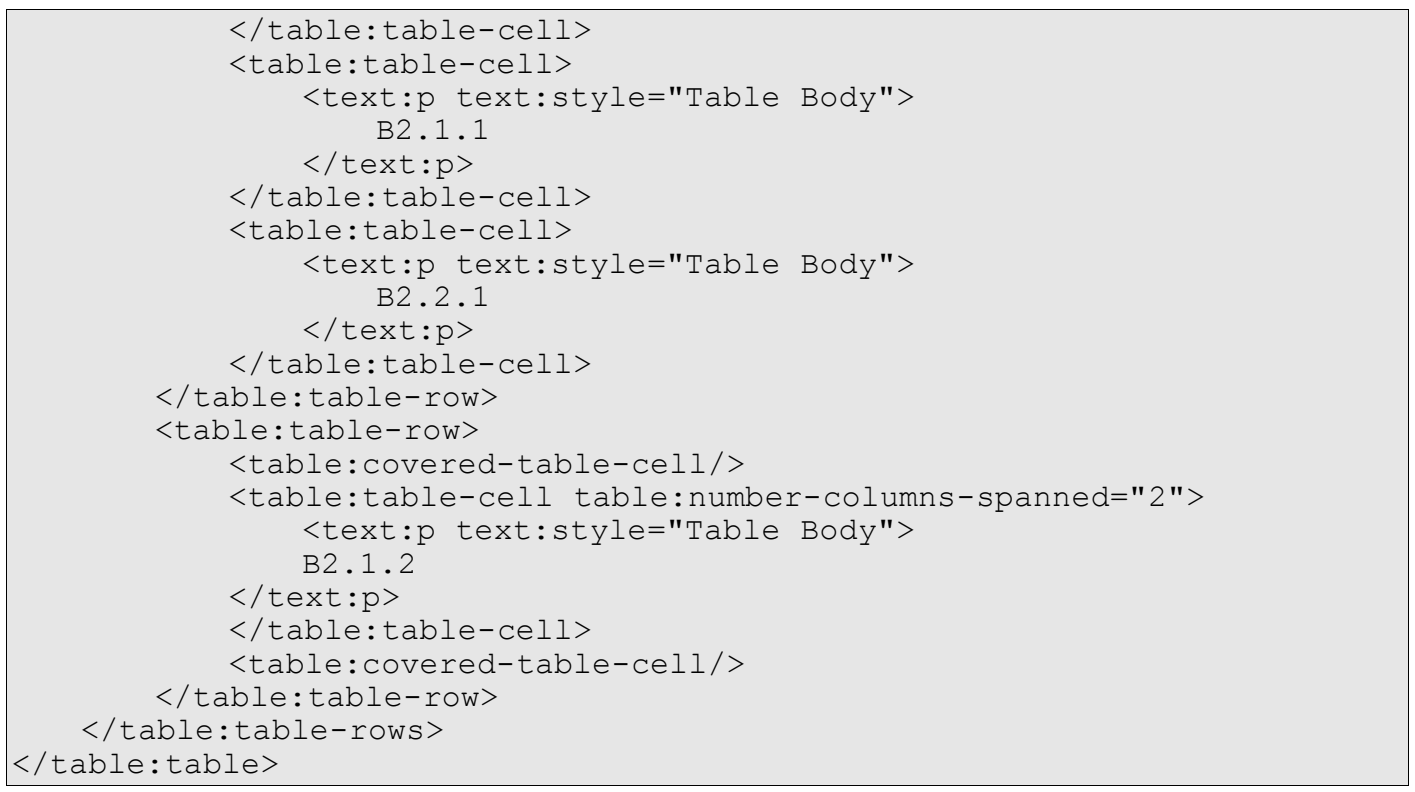

\section{Sample 2}

Using sub tables, the preceding table is specified as follows:

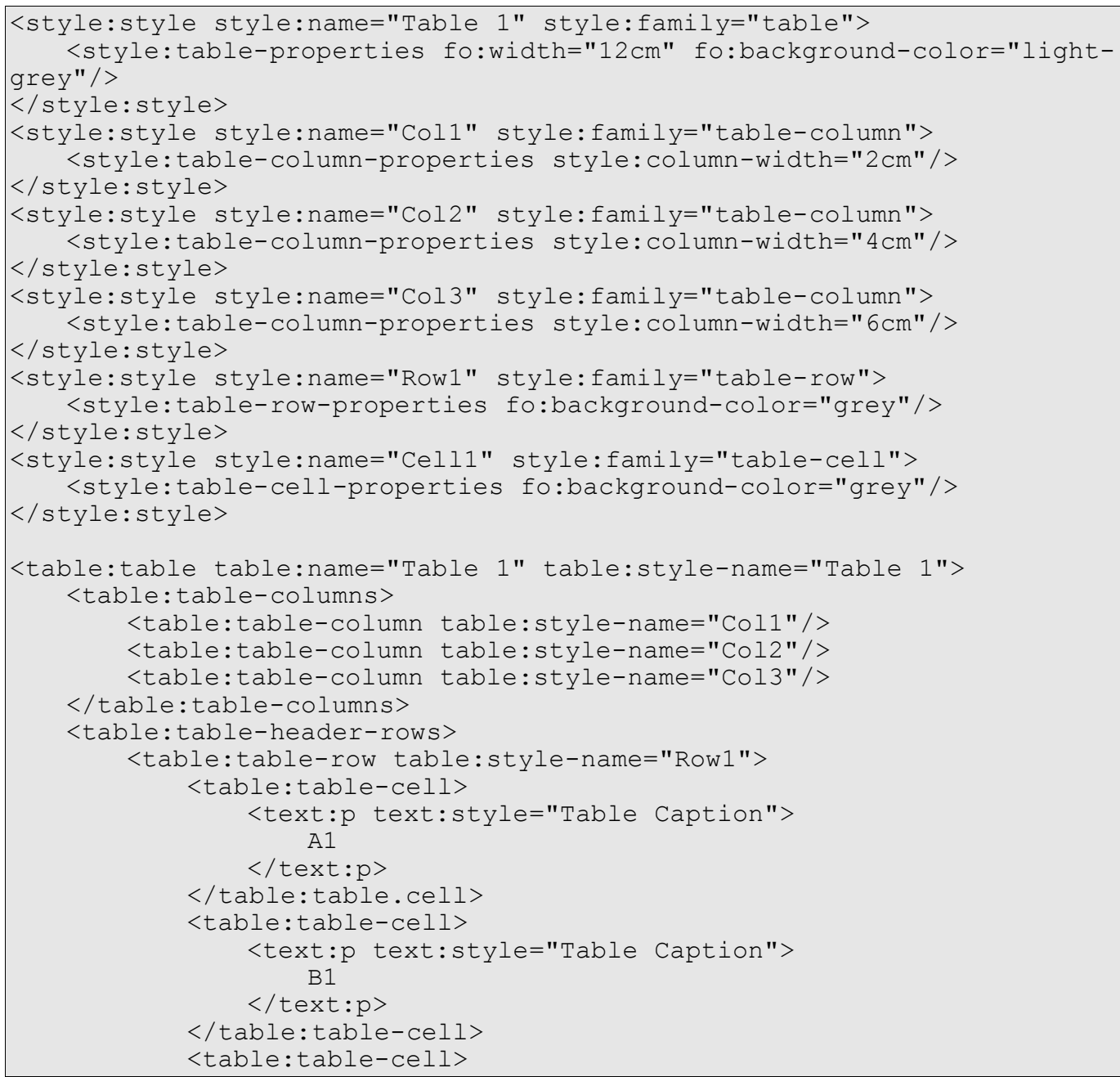




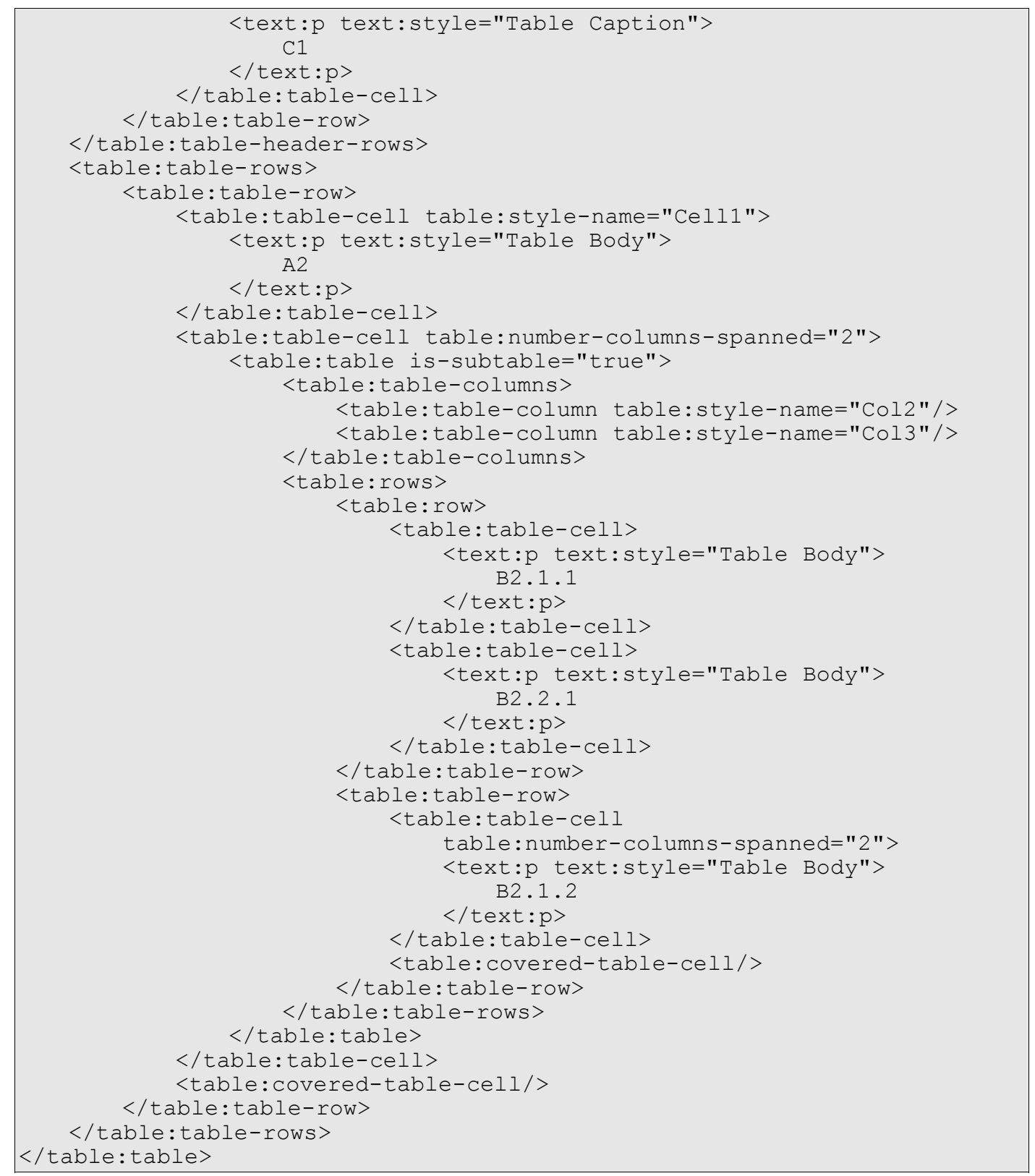

\subsection{Advanced Tables}

\subsubsection{Referencing Table Cells}

To reference table cells so called cell addresses are used. The structure of a cell address is as follows:

1. The name of the table.

2. $A \operatorname{dot}($.$) .$

3. An alphabetic value representing the column. The letter $A$ represents column $1, B$ represents column 2, and so on. AA represents column $27, A B$ represents column 28 , and so on. 
4. A numeric value representing the row. The number 1 represents the first row, the number 2 represents the second row, and so on.

This means that $A 1$ represents the cell in column 1 and row 1 . B1 represents the cell in column 2 and row 1 . A2 represents the cell in column 1 and row 2 .

For example, in a table with the name SampleTable the cell in column 34 and row 16 is referenced by the cell address SampleTable.AH16. In some cases it is not necessary to provide the name of the table. However, the dot must be present. When the table name is not required, the address in the previous example is .AH16.

The structure of the address of a cell in a subtable is as follows:

1. The address of the cell that contains the subtable.

2. $A \operatorname{dot}($.$) .$

3. The address of the cell in the subtable.

For example, to reference the cell in column 1 and row 1 in a subtable that is called Subtable, and that is in column 34 and row 16 of the table SampleTable, the address is

SampleTable.AH16.A1. If the name of the table contains blanks, the name should be quoted with apostrophes (').

\section{Absolute and relative cell addressing}

Cells can be referenced by using either absolute addresses or relative addresses. When an operation is performed on a table cell, for example when a formula is copied, absolute cell references do not change; In contrast to this, relative cell references are adapted to the address of target cell of the copy operation. The previous example uses relative addressing.

To create an absolute address, a dollar sign (\$) has to be placed before each table name, column reference, and row reference. For example, the absolute address of the previous example is $\$$ SampleTable. $\$ A H \$ 16$. Absolute and relative references can be mixed within a single cell address. For example, SampleTable.AH $\$ 16$ refers to a relative table and column, but to an absolute row. Absolute addresses must contain a table name. The differentiation between absolute and relative addressing is only necessary in some situations. Where a differentiation is not required, a cell reference without the dollar signs is used.

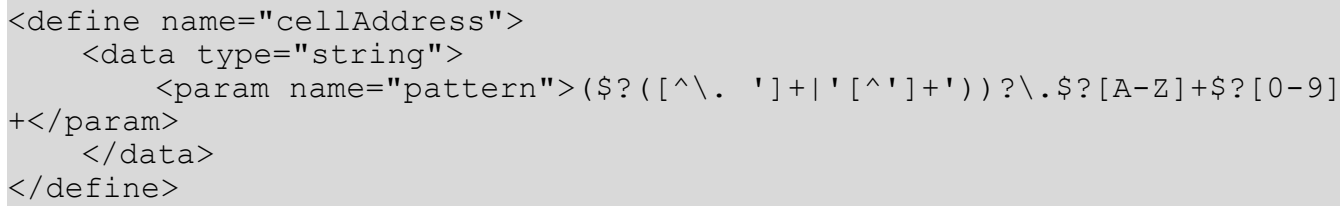

\section{Cell Range Address}

A cell range is a number of adjacent cells forming a rectangular shape. The rectangle stretches from the cell on the top left to the cell on the bottom right.

A cell range address references a cell range. It is constructed as follow:

1. The address of the cell at the top left of the range.

2. A colon (:).

3. The address of the cell at the bottom right of the range. 
For example, the address .A1 : . B2 references the cell range of cells from column 1 and row 1 to column 2 and row 2 . The smallest range one can specify is a single cell. In this case, the range address is the same as the cell address.

\subsubsection{Linked Tables}

If a table is linked to an original table, the information about the source table is contained in a $<$ table:table-source> element. The attributes that may be associated with the $<$ table:table-source> element are:

- Mode

- Table name

- URL

- Filter name

- Filter options

- Refresh delay

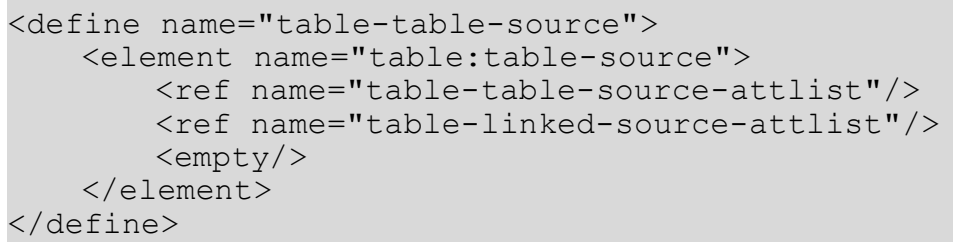

\section{Mode}

The table:mode attribute specifies what data should be copied from the source table to the destination table. If the attribute's value is "copy-all" formulas and styles are copied. If the attribute's value is "copy-results-only", only formula results and non calculated cell content will be copied.

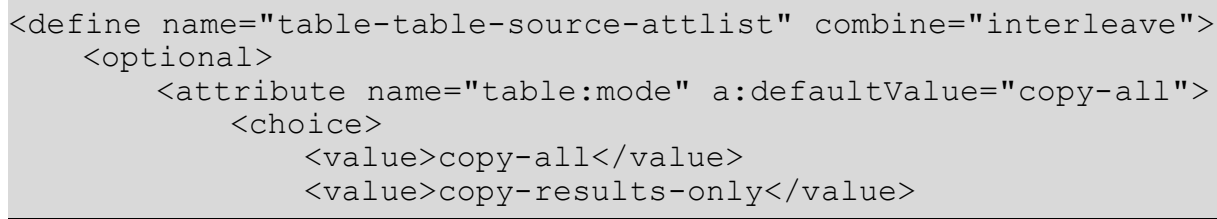




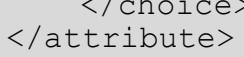

\section{Table Name}

The table:table-name attribute specifies the name of the table in the original document. If the table name is not specified, the first table in the document is used.

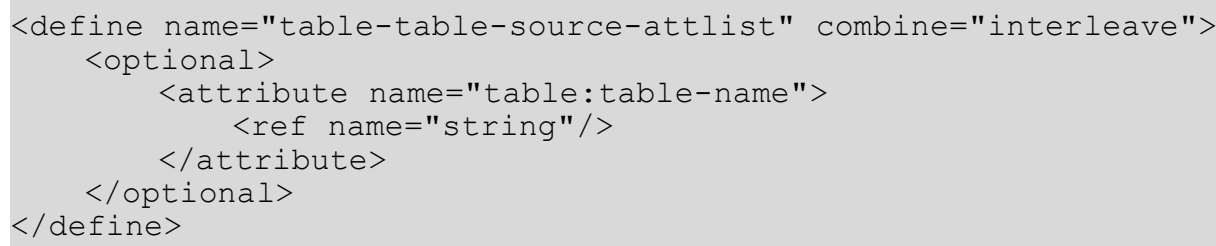

\section{URL}

The original table is specified by a an XLink, where the xlink: href attribute specifies the URL of the document containing the original table.

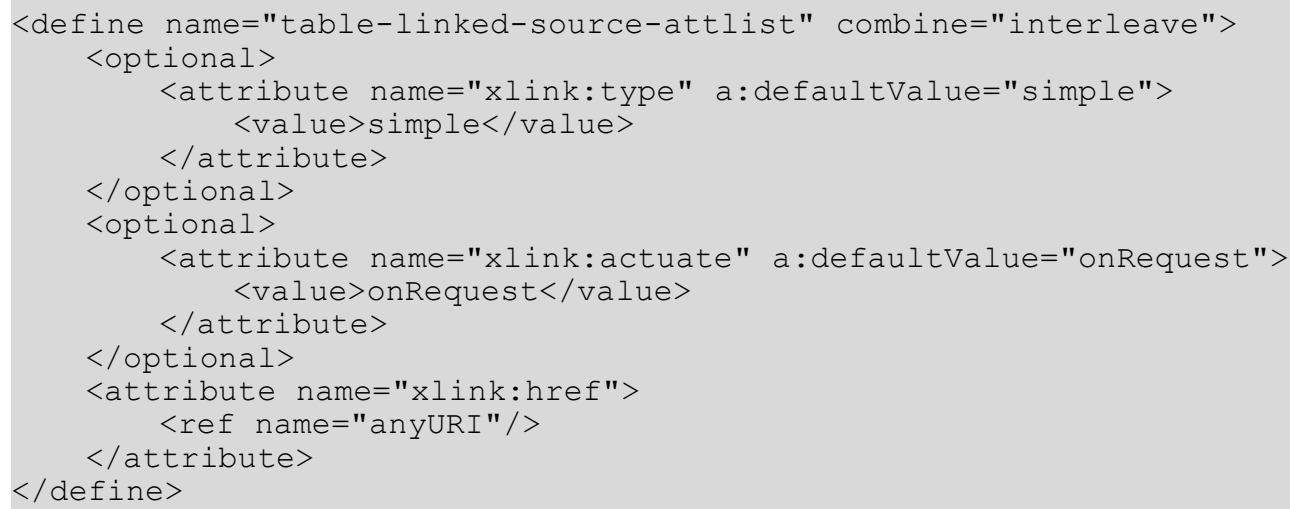

\section{Filter Name}

The table:filter-name attribute specifies the file type of the document containing the original table. The value of this attribute is application-specific.

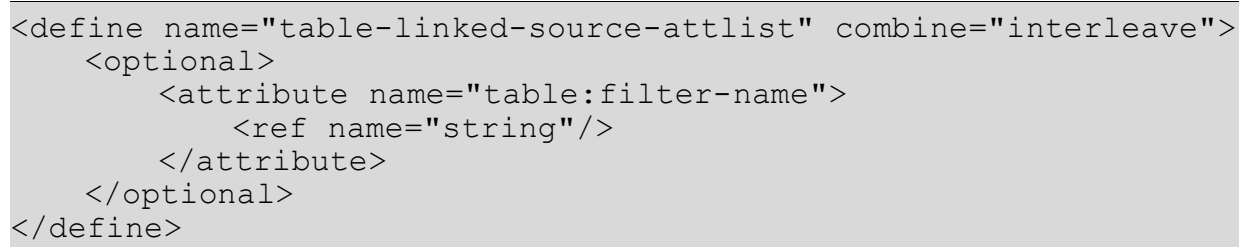

\section{Filter Options}

The table:filter-options attribute specifies optional settings about the file type. The value of this attribute is application-specific.

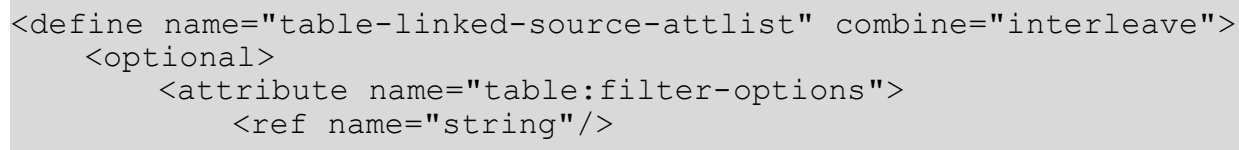




\section{Refresh Delay}

The table:refresh-delay attribute specifies the time delay between refresh actions for the linked table.

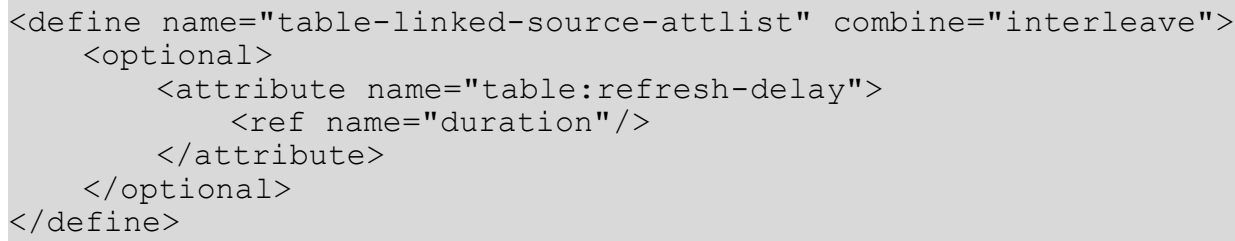

\subsubsection{Scenario Tables}

A scenario is an area of a table where data from other, so called scenario tables, is linked to temporarily. If several scenarios are defined for the same area, an user might choose between the scenarios. Whether a scenario table is visible itself is controlled by table's style. Only one scenario table can be active per table.

A table that contains a $<$ table: scenario $>$ represents a scenario table. The name of the table and the name of the scenario are the same. The scenario is displayed in the regular table preceding the scenario table. If a scenario table is existing for a table, a scenario is displayed on that table automatically. These means the the existence of a scenario table implies the existence of a scenario.

The attributes that may be associated with this element are:

- Scenario Ranges

- Is Active

- Display Border

- Border Color

- Copy Back

- Copy Styles

- Copy Formulas

- Comment

- Protected

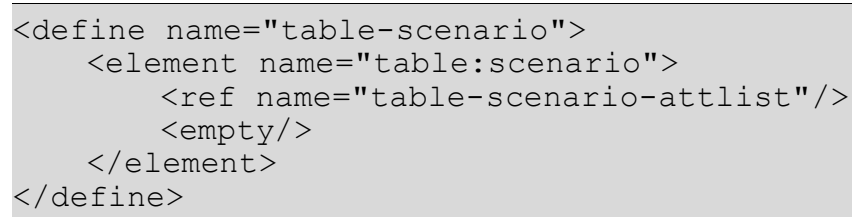

\section{Scenario Ranges}

The table:scenario-ranges attribute specifies the table range that is displayed as a scenario. The value of this attribute is a list of cell range addresses. 


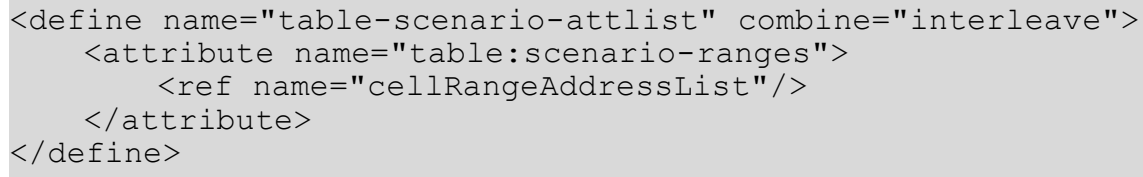

\section{Is Active}

The table: is-active attribute specifies whether or not the scenario that belongs to the scenario table is active.

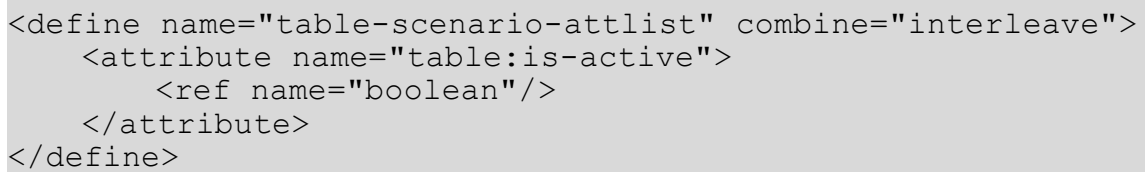

\section{Display Border}

The table:display-border attribute specifies whether or not to display a border around the scenario that belongs to the scenario table.

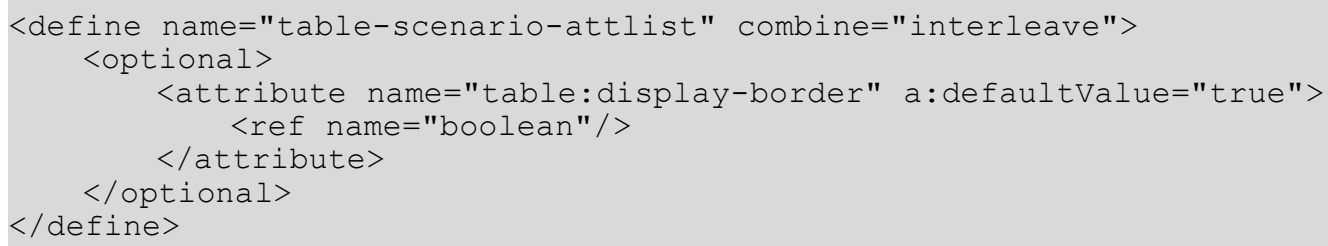

\section{Border Color}

The table:border-color attribute specifies the color of the border that is displayed around the scenario that belongs to the scenario table.

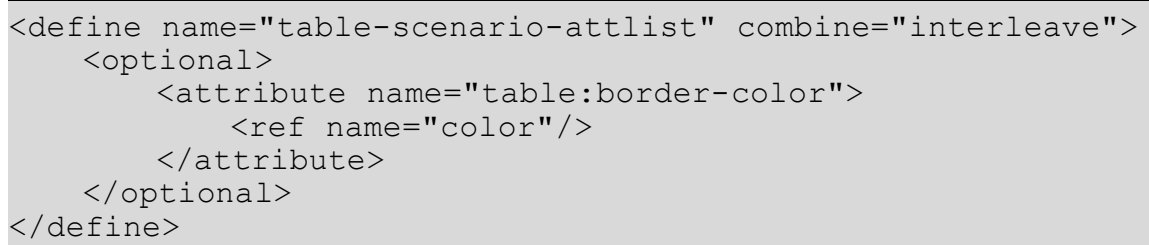

\section{Copy Back}

The table:copy-back attribute specifies whether or not data is copied back into the scenario table if another scenario is activated.

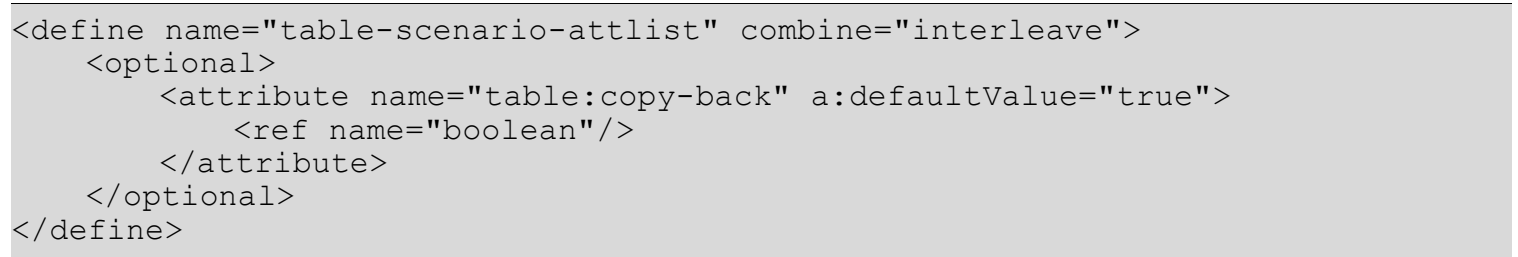

\section{Copy Styles}

The table:copy-styles attribute specifies whether or not styles are copied from the scenario table to the destination table together with the data. 


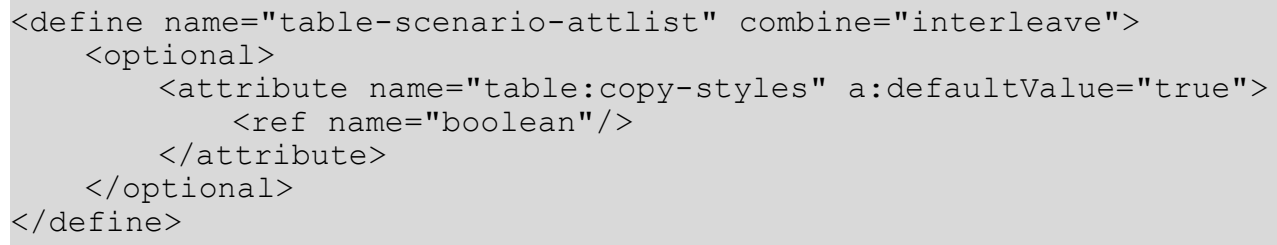

\section{Copy Formulas}

The table:copy-formulas attribute specifies whether or not formulas are copied from the scenario table to the destination table. The value of this attribute can be true or false. If the value is true, the formulas are copied. If the value is false, only the values resulting from the formulas are copied.

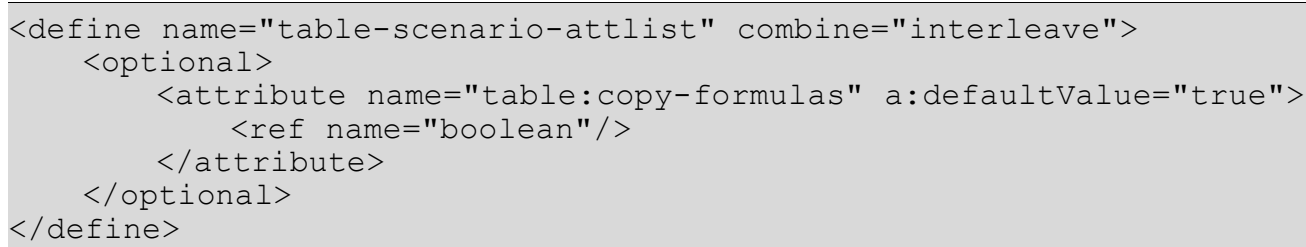

\section{Comment}

The table:comment attribute contains a comment about the scenario.

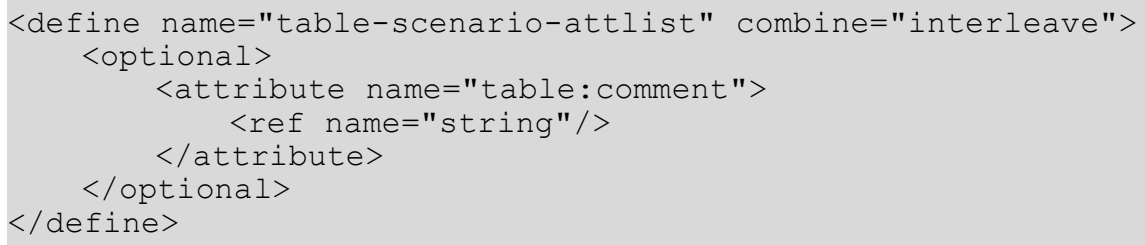

\section{Protected}

The table:protected attribute specifies whether or not the data that is displayed within the scenario is protected from being edited. The attribute is only evaluated if the table on which the scenario displayed is also protected (see section 8.1.1).

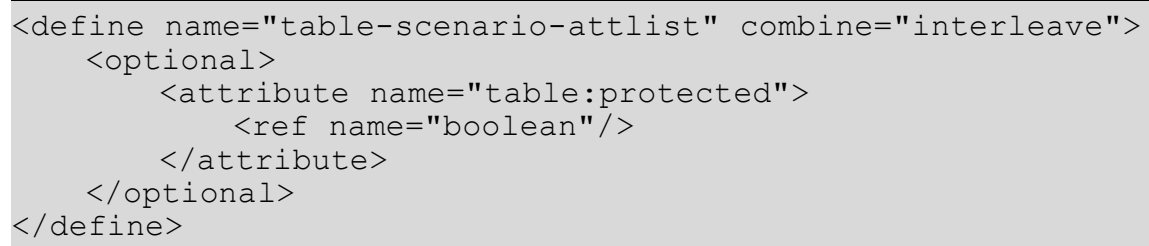

\subsubsection{Shapes}

The <table: shapes $>$ element contains all graphic shapes with an anchor on the table this element is a child of. It is a container element and does not have any associated attributes.

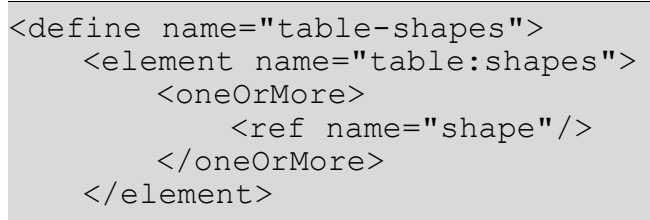




\subsection{Advanced Table Cells}

\subsubsection{Linked Table Cells}

A cell range can be linked to a database range or named range of another file. In this case the information about the original database range or named range is contained in a t table:cellrange-source> element that is contained in the element of the first cell of the range. The attributes that may be associated with this element are:

- Name

- $\quad$ Last size

- URL

- Filter name

- Filter options

- Refresh delay

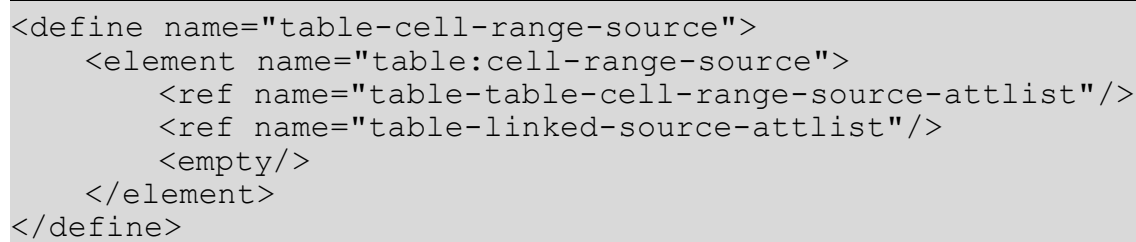

\section{Name}

The table: name attribute specifies the name of the source database range or named range.

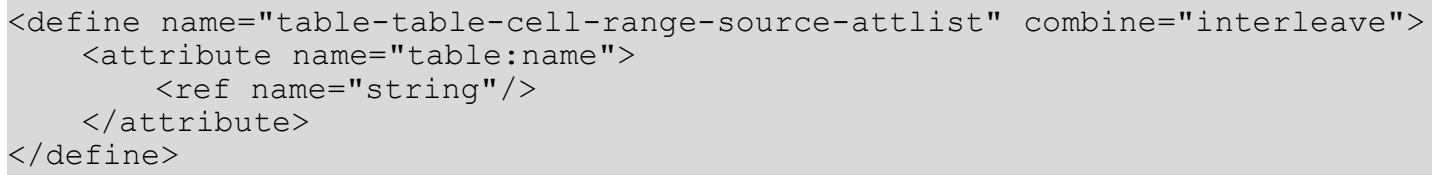

\section{Last Size}

The table:last-column-spanned and table:last-row-spanned attributes specify the last known size of the range. If the size of the range is changed since the last operation, the values of these attributes are incorrect.

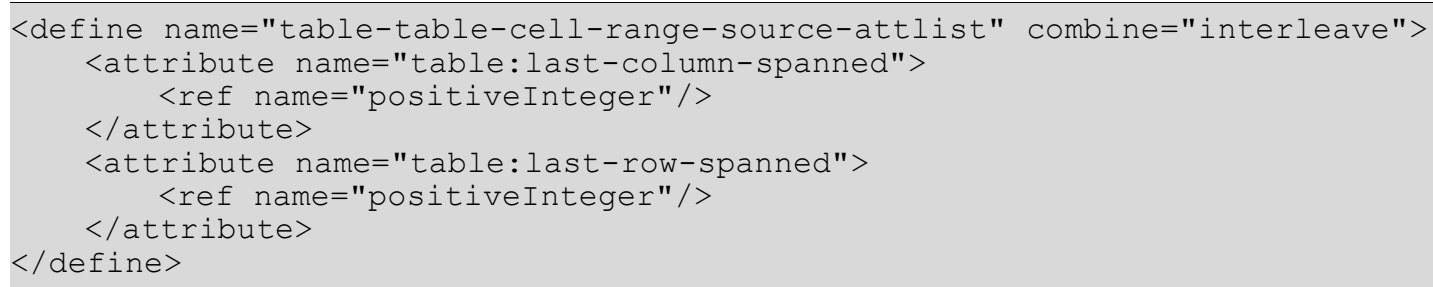




\section{URL, Filter Name, Filter Options and Refresh Delay}

The attributes $x l i n k:$ href, xlink:type, xlink:actuate, table:filter-name and table: filter-options are the same as for linked tables. See section 8.3.2 for details.

\subsubsection{Cell Annotation}

The OpenDocument format allows annotation to appear within table cells. See section 12.1 for details on annotations.

\subsubsection{Detective}

The $<$ table: detective> element has two purposes. One the one hand, it contains information about relations that exist between table cells because of formulas and that should be highlighted in the UI. On the other hand, the element contains information about cells that are highlighted currently in the UI either because of the relations mentioned above or because of error conditions.

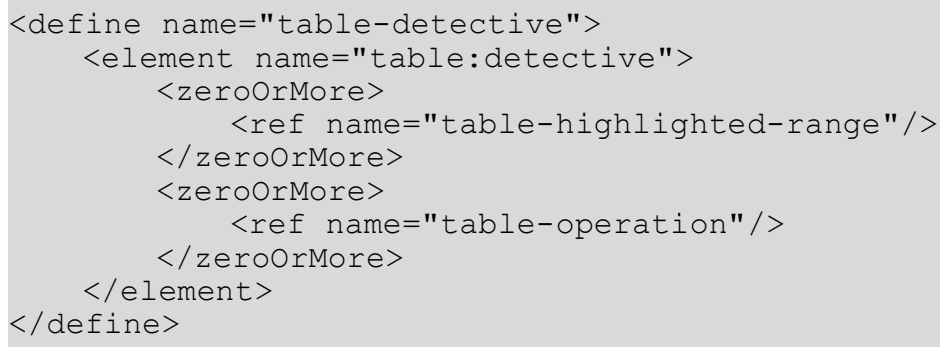

The elements that can be contained in the $<$ table: detective> element are:

- Detective Operation

- Highlighted range

\subsubsection{Detective Operation}

The $<$ table: operation $>$ element specifies that certain relations that exist between the cell the element is a child of and other cells should be made visible or invisible in the UI. One and the same detective operation can be applied multiple times to the same cell. In this case, the 2nd operation is applied to the resulting cells of the first operation and so on. This means that an operation not necessarily is applied to the cell the operation is defined in, but also to other cells, and that it therefor can interact with operations defined in other cells. This especially applies to operations that make relations invisible. To get a determinate behavior, operations have an index and are applied in the order of that index. The attributes associated with the $<$ table: operation> element are:

- Name

- Index

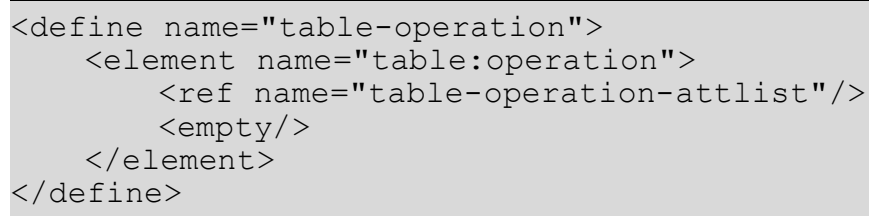




\section{Name}

The table: name attribute specifies the name of the detective operation. Possible names are trace-dependents, remove-dependents, trace-precedents, remove-precedents and trace-errors. trace-dependents and remove-dependents displays or hides cells that use the value of the current cell in their formula. Trace-precedents and removeprecedents displays or hides cells whose value is used in the formula of the current cell. Trace-errors displays cells that cause an error while calculating the result of the current cell's formula.

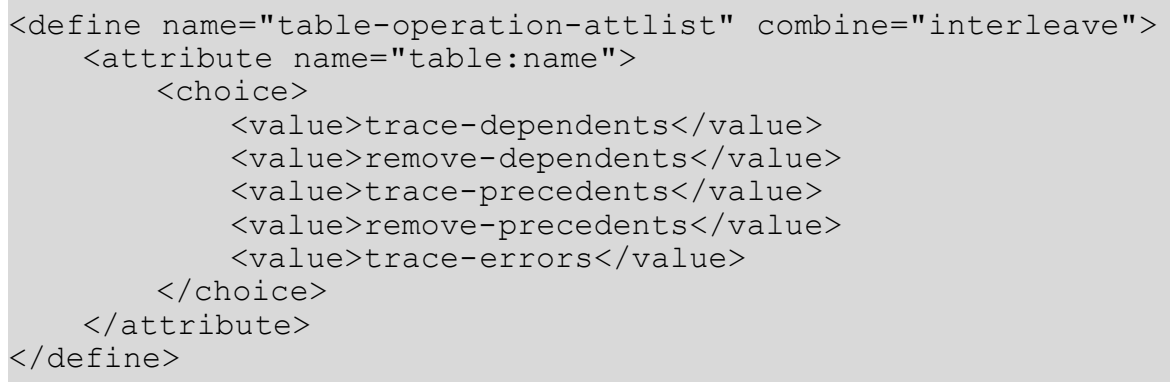

\section{Index}

The table: index attribute specifies the the order in which detective operations are applied.

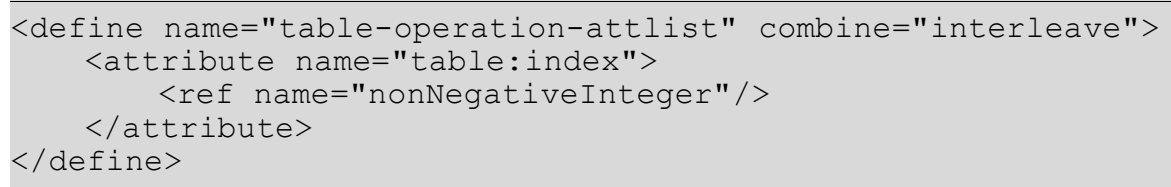

\subsubsection{Highlighted Range}

The <table:highlighted-range> element specifies a cell range that is highlighted in the UI either because of an detective operations described above or because it contains an error or invalid data.

The information contained in this element is not guaranteed to be up to date but reflects the state that at the time the detective operations or error conditions have been calculated.

The attributes that can be associated with the <table:highlighted-range> element are:

- Cell Range Address

- Direction

- Contains Error

- Marked Invalid

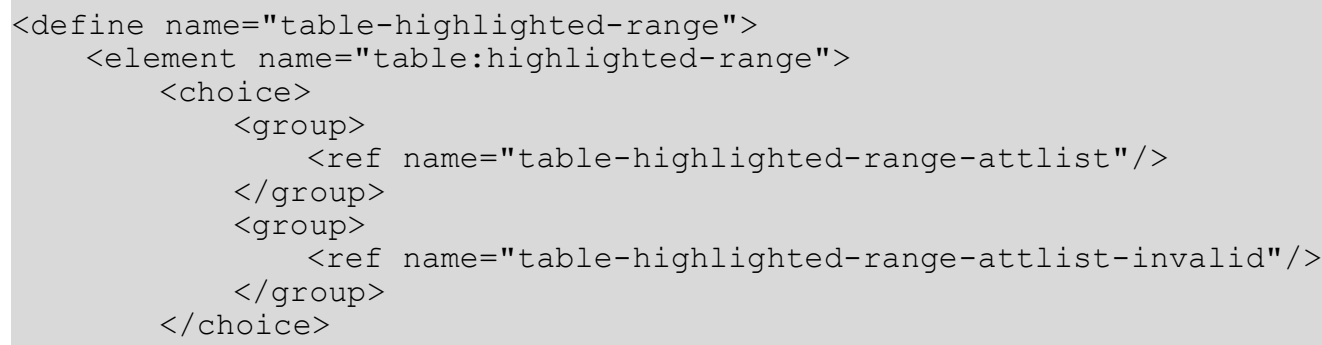




\subsection{Spreadsheet Document Content}

\subsubsection{Document Protection}

The structure of a spreadsheet document may be protected by using the table: structureprotected attribute, so that users can not insert, delete, move or rename the tables in the document. The optional table:protection-key attribute may be used to specify a password that prevents users from resetting the table protection flag to allow editing. To avoid saving the password directly into the XML file, only a hash value of the password is stored.

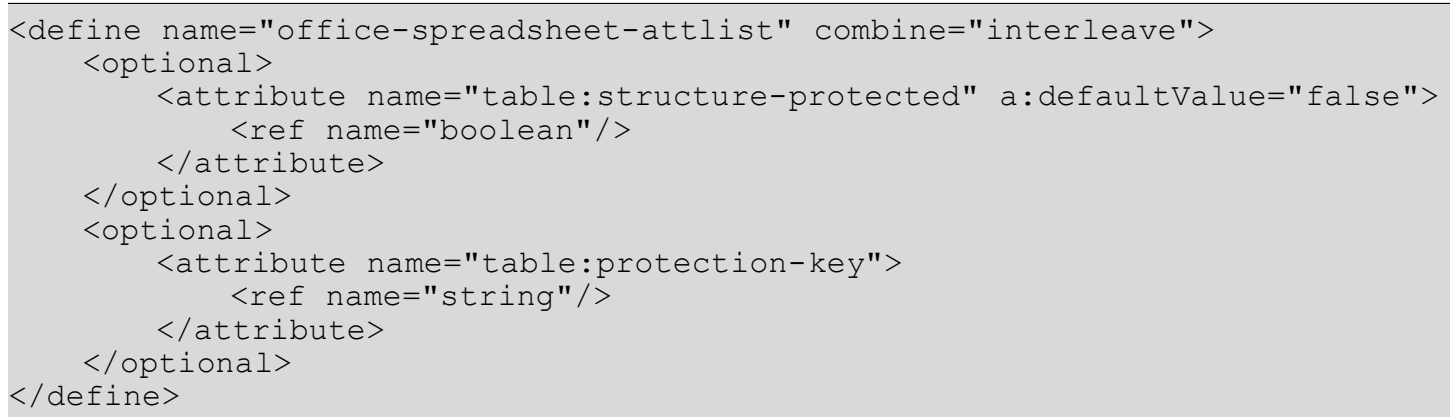

\subsubsection{Calculation Settings}

Spreadsheet documents contain settings that affect the calculation of formulas, for example the null date or iteration settings. These settings must be saved in the document in the <table:calculation-settings> element.

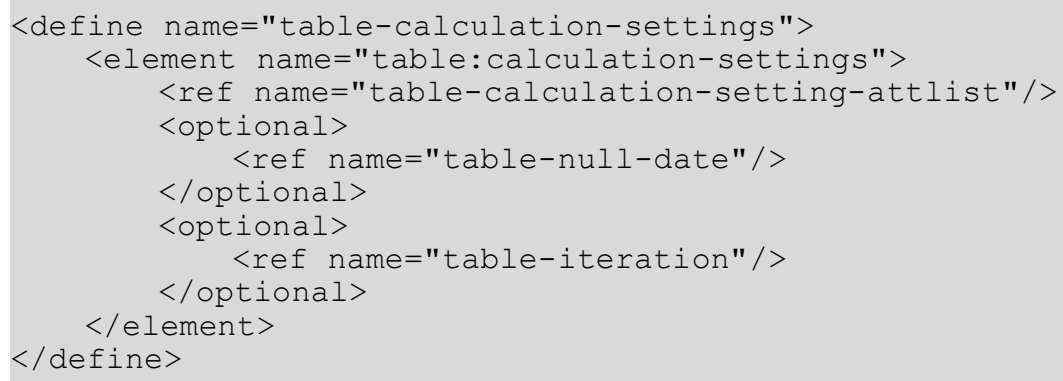

The attributes that may be associated with the $<$ table:calculation-settings $>$ element are:

- Case sensitive

- Precision as shown

- Search criteria must apply to whole cell

- Automatic find labels

- Use regular expression

- Null year

- Null date

- Iteration 


\section{Case Sensitive}

The table:case-sensitive attribute specifies whether or not to distinguish between upper and lower case when comparison operators are applied to cell content.

\section{Automatic Find Labels}

The table:automatic-find-labels attribute specifies whether or not to automatically find the labels of rows and columns.

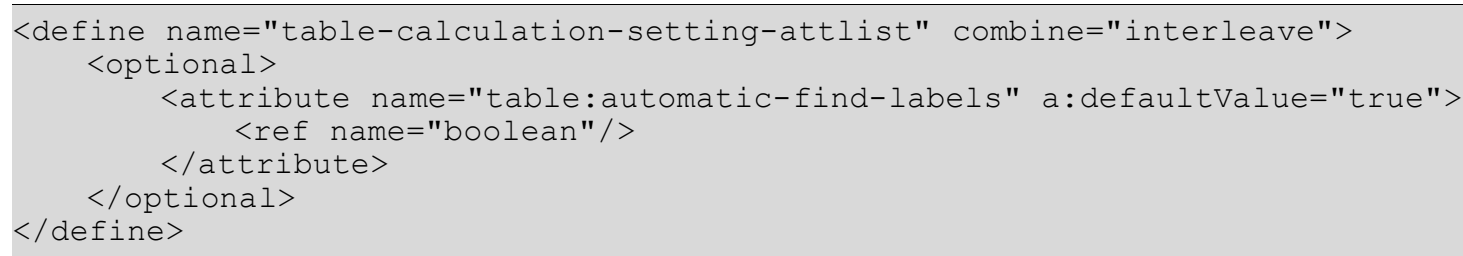

\section{Precision as Shown}

The table:precision-as-shown attribute specifies whether to perform a calculation using the rounded values displayed in the spreadsheet or using all of the digits in a number. If the value of this attribute is true, calculation are performed using the rounded values displayed in the spreadsheet. If the value of this attribute is false, calculations are performed using all of the digits in the number, but the result is still displayed as a rounded number.

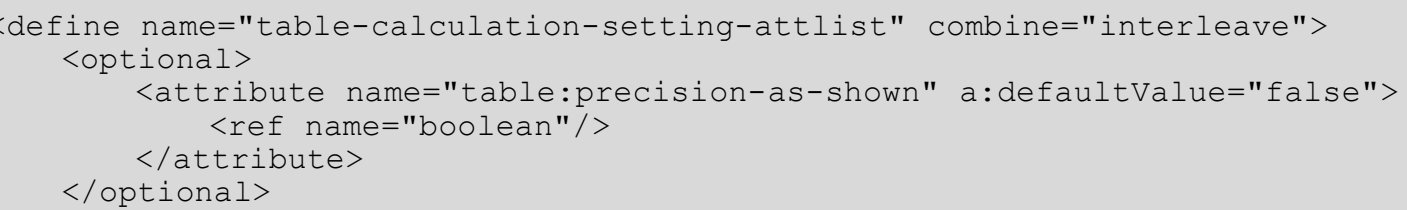

\section{Search Criteria Must Apply to Whole Cell}

The table:search-criteria-must-apply-to-whole-cell attribute specifies whether or not the specified search criteria, according to the regular expression used, must apply to the entire cell contents.

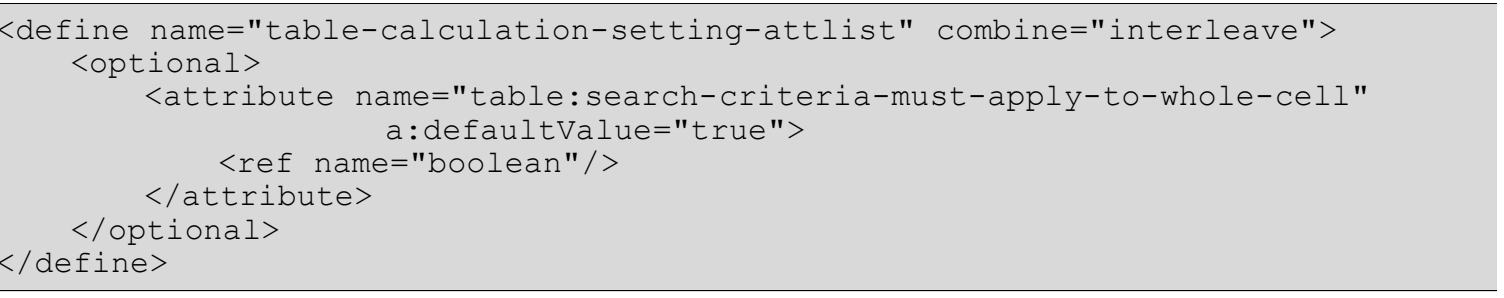




\section{Use Regular Expressions}

The table: use-regular-expressions attribute specifies whether regular expressions are enabled for character string comparisons and when searching.

\section{Null Date}

The <table:null-date> element specifies the null date. The null date is the date that results in the value " 0 " if a date value is converted into a numeric value. The null date is specified in the element's table: date-value attribute. Commonly used values are 12/30/1899, $01 / 01 / 1900$, and 01/01/1904

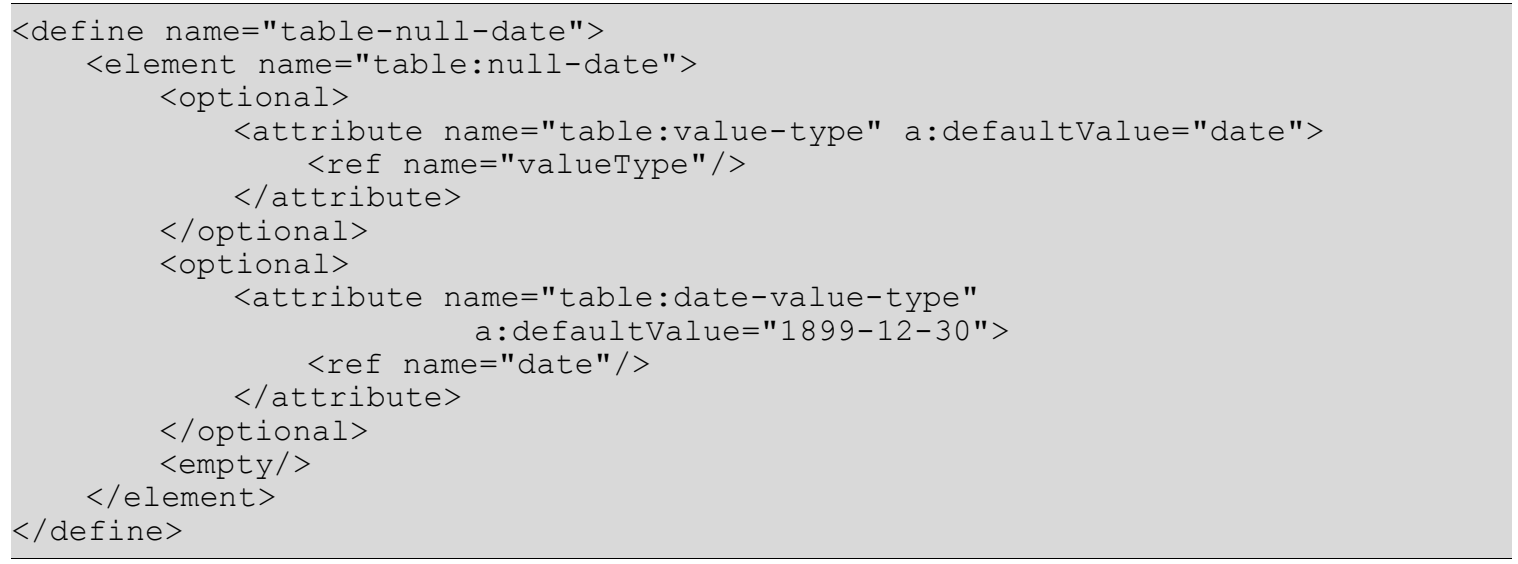

\section{Iteration}

The <table: iteration> element enables formulas with iterative (or cyclic) references to be calculated after a specific number of iterations. Formulas with iterative references are repeated until the problem is solved. If this iterative calculations are not enabled, a formula with an iterative reference in a table causes an error message.

Iterative calculations are enabled and disabled with the table:status attribute. If iterative calculations are enabled, the table:steps attribute specifies the maximum number of 
iterations allowed. The table:maximum-difference attribute specifies the maximum difference allowed between two calculation results. The iteration is stopped if the result is less than the value of this attribute.

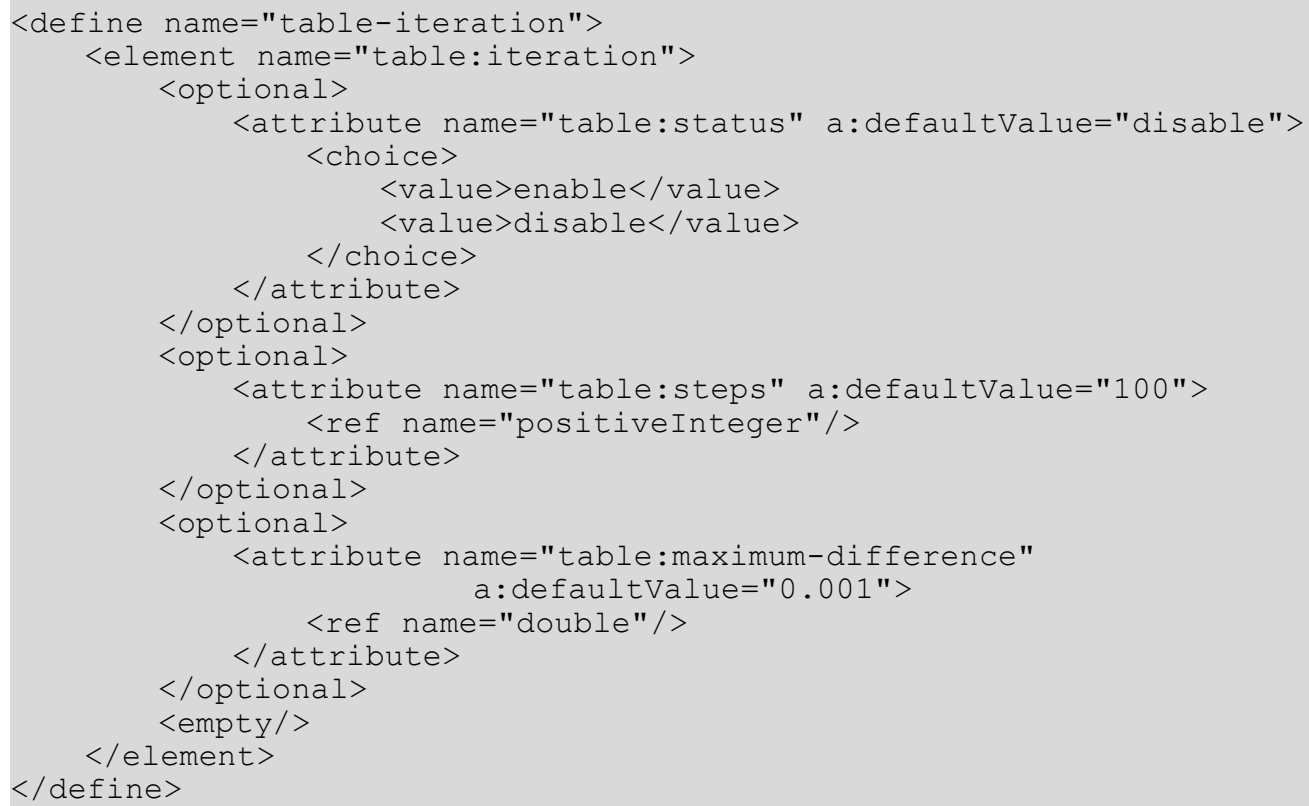

\subsubsection{Table Cell Content Validations}

Table cell content validations specify validation rules for the content of table cells. The $<$ table: content-validation> element specifies such a validation rule. All validation rules that exist in a document are contained <table: content-validations > element. The validation rules themselves are named and referenced from the table cell by its name.

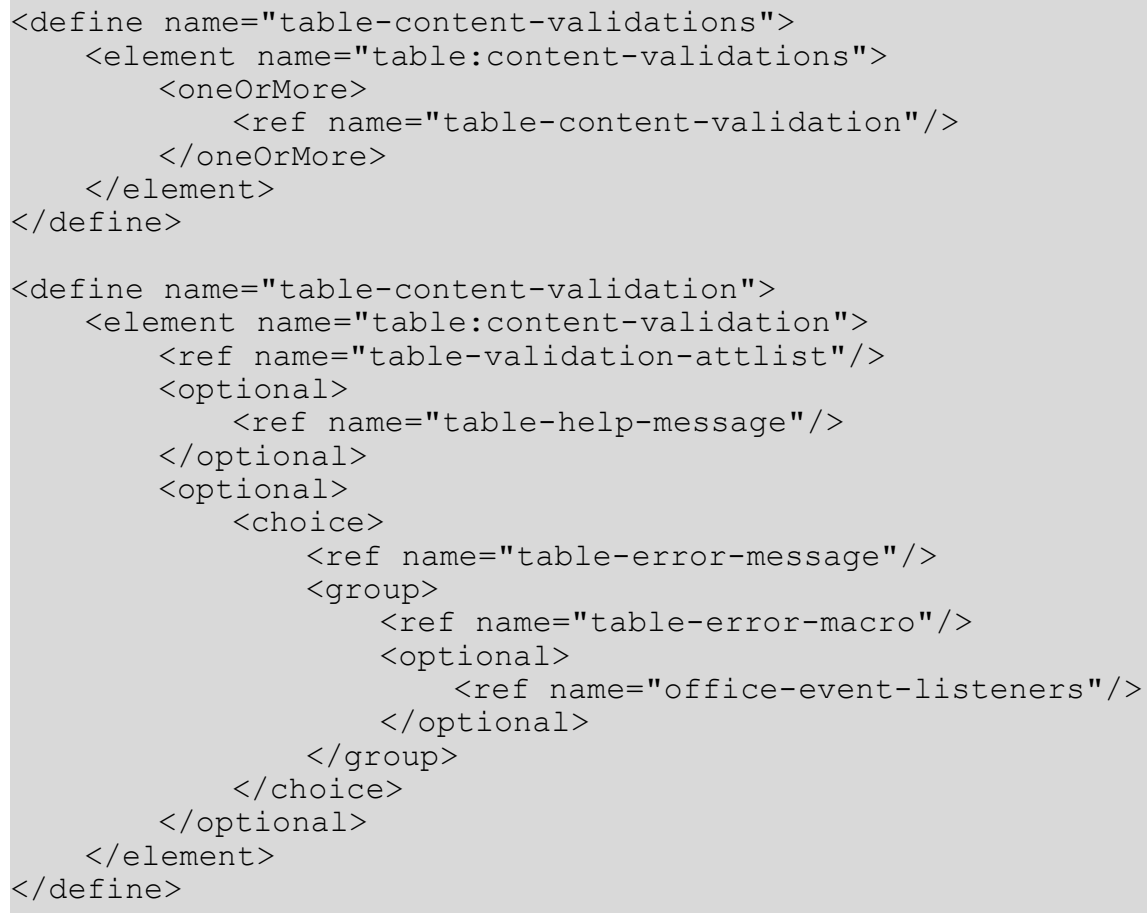


The attributes that may be associated with the $<$ table: content-validation $>$ element are:

- Name

- Condition

- Base cell address

- Allow empty cell

- Display list

\section{Name}

The table: name attribute specifies the name of the content validation. It is used to reference the validation rule from the cell the rule should applied to. The name is created automatically by the application.

4211

4212

4213

4214

4215

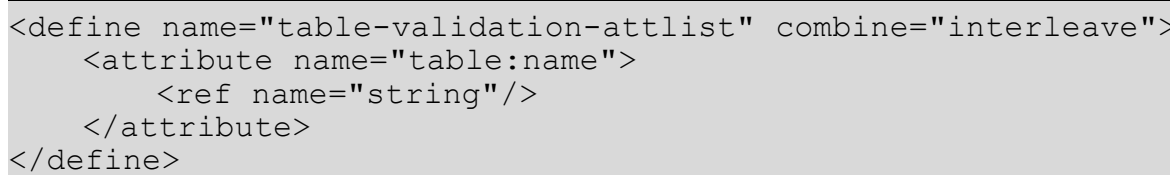

\section{Condition}

The table:condition attribute specifies the condition that must evaluate to "true" for all cells the validation rule is applied to. The value of this attribute should be a namespace prefix, followed by an Boolean expression.

A typical syntax of the expression may be similar to the XPath syntax. The following are valid conditions:

- Condition : := ExtendedTrueCondition | TrueFunction 'and' TrueCondition

- Truefunction : := cell-content-is-whole-number() | cell-content-isdecimal-number() | cell-content-is-date() | cell-content-is-time() cell-content-is-text ()

- ExtendedTrueCondition : := ExtendedGetFunction | cell-content-textlength() Operator Value

- TrueCondition : := GetFunction | cell-content() Operator Value

- GetFunction : := cell-content-is-between(Value, Value) | cellcontent-is-not-between (Value, Value)

- ExtendedGetFunction : := cell-content-text-length-is-between(Value, Value) | cell-content-text-length-is-not-between(Value, Value) | cell-content-is-in-list ( Stringlist )

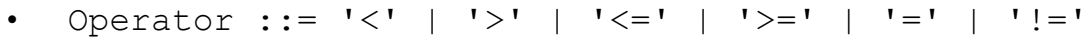

- Value : := NumberValue | String | Formula

- Stringlist : := StringList ' $;$ ' String | String

- A Formula is a formula without an equals (=) sign at the beginning. See section 8.1.3 for more information. 
- A string comprises one or more characters surrounded by quotation marks.

- A NumberValue is a whole or decimal number. It must not contain comma separators for numbers of 1000 or greater.

4216

4217

4218

4219

4220

4221

4222

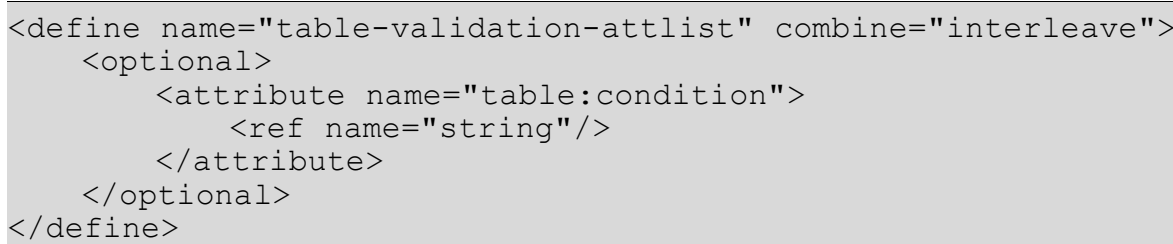

\section{Base Cell Address}

The table:base-cell-address attribute specifies the address of the base cell for relative addresses in formulas that occur within a condition. This attribute is only necessary when the condition contains a formula. The value of this attribute must be an absolute cell address that contains a table name.

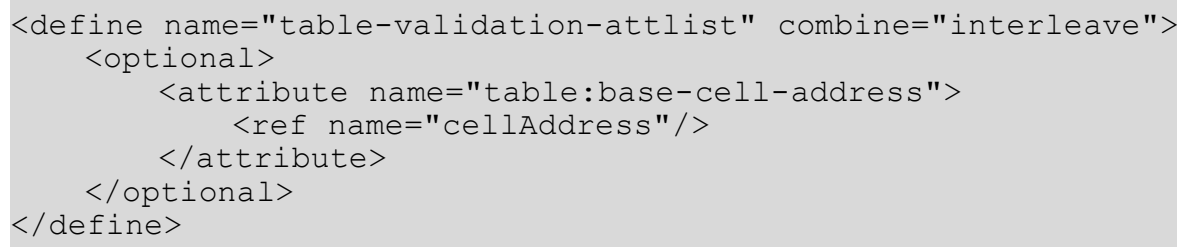

\section{Allow Empty Cell}

The table:allow-empty-cell attribute specifies whether or not a cell can be empty.

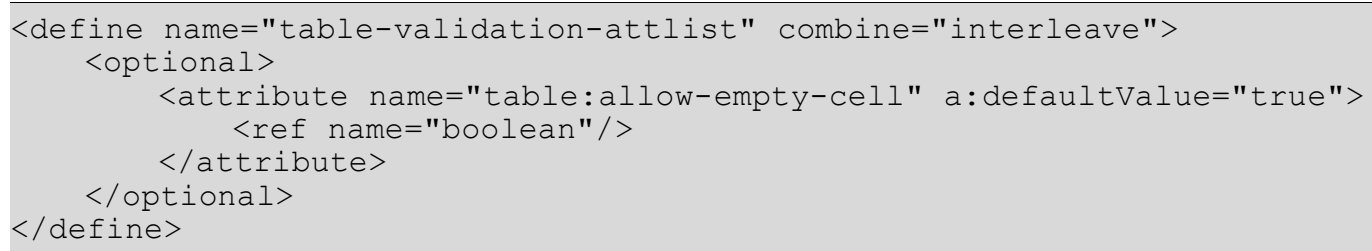

\section{Display List}

The table:display-list attribute specifies whether a list of values that occurs within a condition is displayed in the UI wile entering a cell value. The value of this attribute can be none, unsorted or sort-ascending.

- none: the list values are not displayed.

- unsorted: the list values are displayed in the order they occur in the condition.

- sort-ascending: the list values are displayed in ascending order.

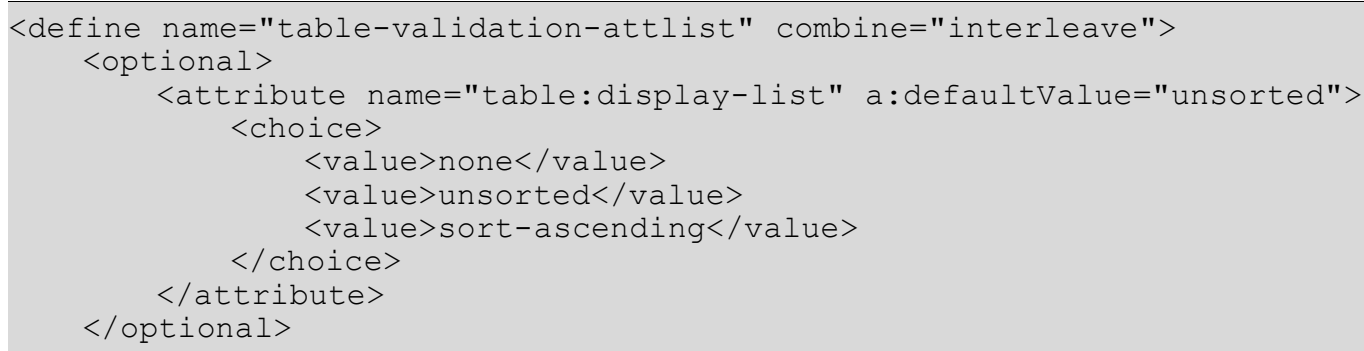




\section{Help Message}

The <table: help-message $>$ element specifies a message to display if a user selects the cell. The element has an optional table:title attribute that specifies a title of the help message. It further has an optional table:display attribute that can be used to suppress the display of the message.

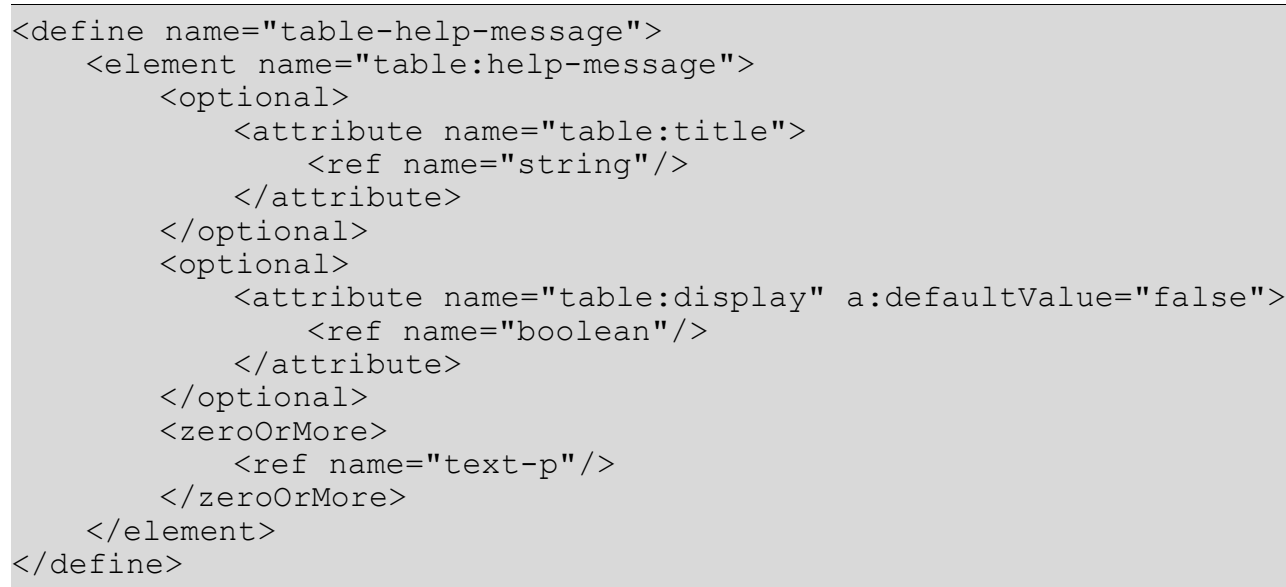

\section{Error Message}

The <table:error-message $>$ element specifies a message to display if a user tries to enter invalid content into a cell i.e. content where the validation rule's condition evaluates to "false".

The element has an optional table:title attribute that specifies a title of the help message. It further has an optional table: display attribute that can be used to suppress the display of the message. The table:message-type attribute, that can take the values stop, warning, or information, specifies whether the message should be displayed as error (stop), warning (warning) or information only (information). In case the message is displayed as error, the operation that caused the validation check (for instance a cursor travel to leave the cell) is stopped.

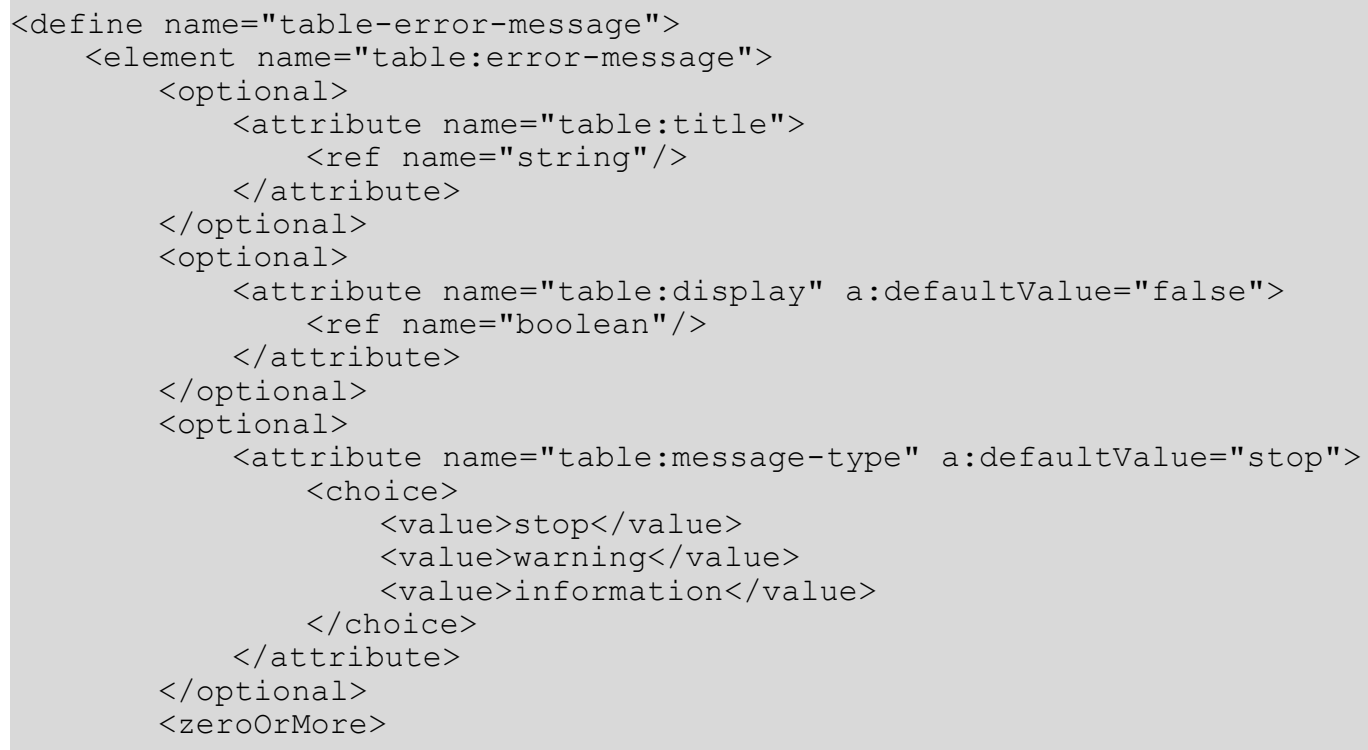




\section{Error Macro}

As an alternative to displaying a message, a macro might be called if a cell contains invalid content. The macro in this case is specified by an <office:event-listeners $>$ element as specified in section 12.4. The event name must be one that specifies an event that is called on invalid user input.

In addition to the <office: event-listeners> element, the <table:error-macro> element specifies whether the macro should be executed or not.

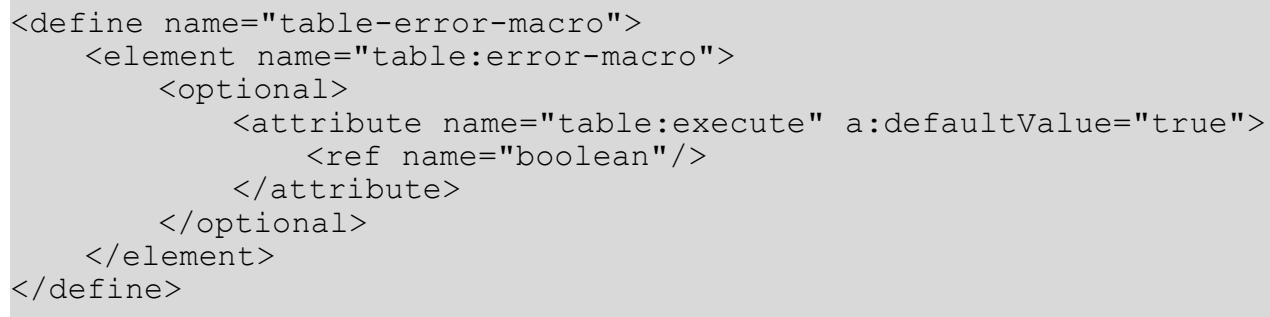

\subsubsection{Label Ranges}

Label ranges can be used to assign names to rows and columns, or to parts of rows and columns, where the names themselves are specified as the content of table cells. More precisely, the label range element $<t a b l e:$ label-range $>$ specifies a label cell range which contain the labels, and data cell range which specifies the rows or columns whose content is referenced by the labels.

There are two types of label ranges.

- Label ranges for columns

- Label ranges for rows.

The data cell range should have the same height and vertical position like the label cell range if row labels are specified, or should have the same width and horizontal position like the label range if column labels are specified. For information on defining a cell range, see section 8.3.1.

Labels can be used within formula like any other name. All label ranges that exist in a document are contained within a single <table: label-ranges $>$ element.

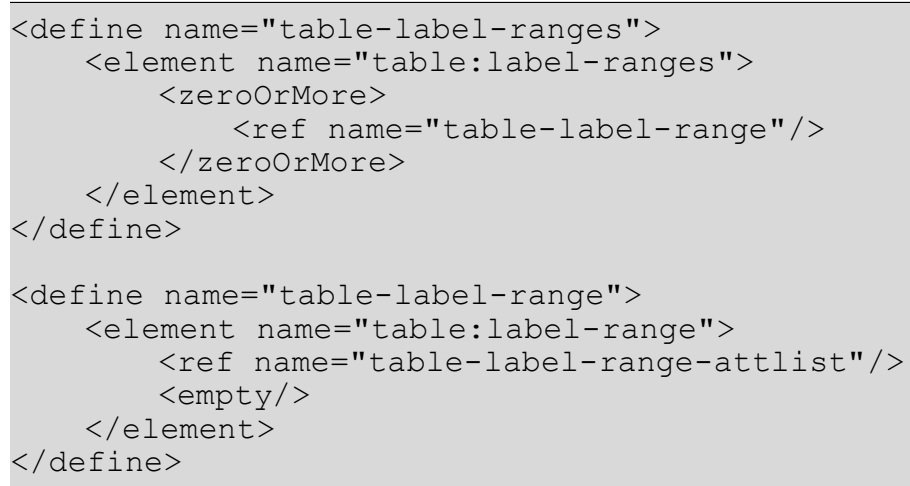




\section{Label Cell Range Address}

The table:label-cell-range-address attribute specifies the cell range address of the labels.

4314

4315

4316

4317

4318

\section{Data Cell Range Address}

The table:data-cell-range-address attribute specifies the cell range address of the data.

\section{Orientation}

The table:orientation attribute specifies the orientation of the label range. This attribute can have a value of column or row.

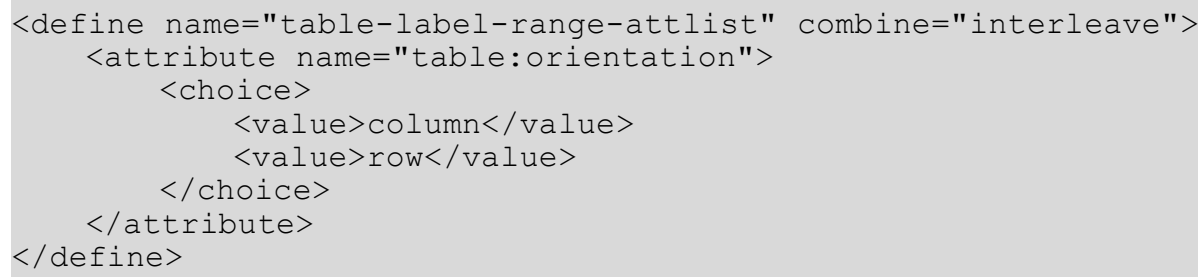

\subsubsection{Named Expressions}

The named expressions element $<$ table: named-expressions $>$ contains a collection of assignments of names to expressions, so that the names can be use to refer to the expression.

The following expression can get names:

- cell ranges.

- Other expressions, for example, parts of a formula. 


\section{Named Range}

The named range element $<$ table: named-range $>$ specifies a cell range that has a name assigned. For information on defining a cell range, see section 8.3.1.

The table: name attribute specifies the name of the range, and the table:cell-rangeaddress attribute its address. The address can be either absolute or relative. If the cell range address is relative, the table:base-cell-address attribute must exist additionally. It specifies the base cell address for the cell range. This address must be absolute. Therefore a table name in the address is required, but the dollar signs that indicate an absolute address can be omitted.

An additional table:range-usable-as attribute specifies whether the name of the range can be used within the specification of a print range, a filter, a repeating row, or a repeat column. The value of this attribute can be either:

- none, or

- a space-separated list that consists of any of the values print-range, filter, repeatrow or repeat-column.

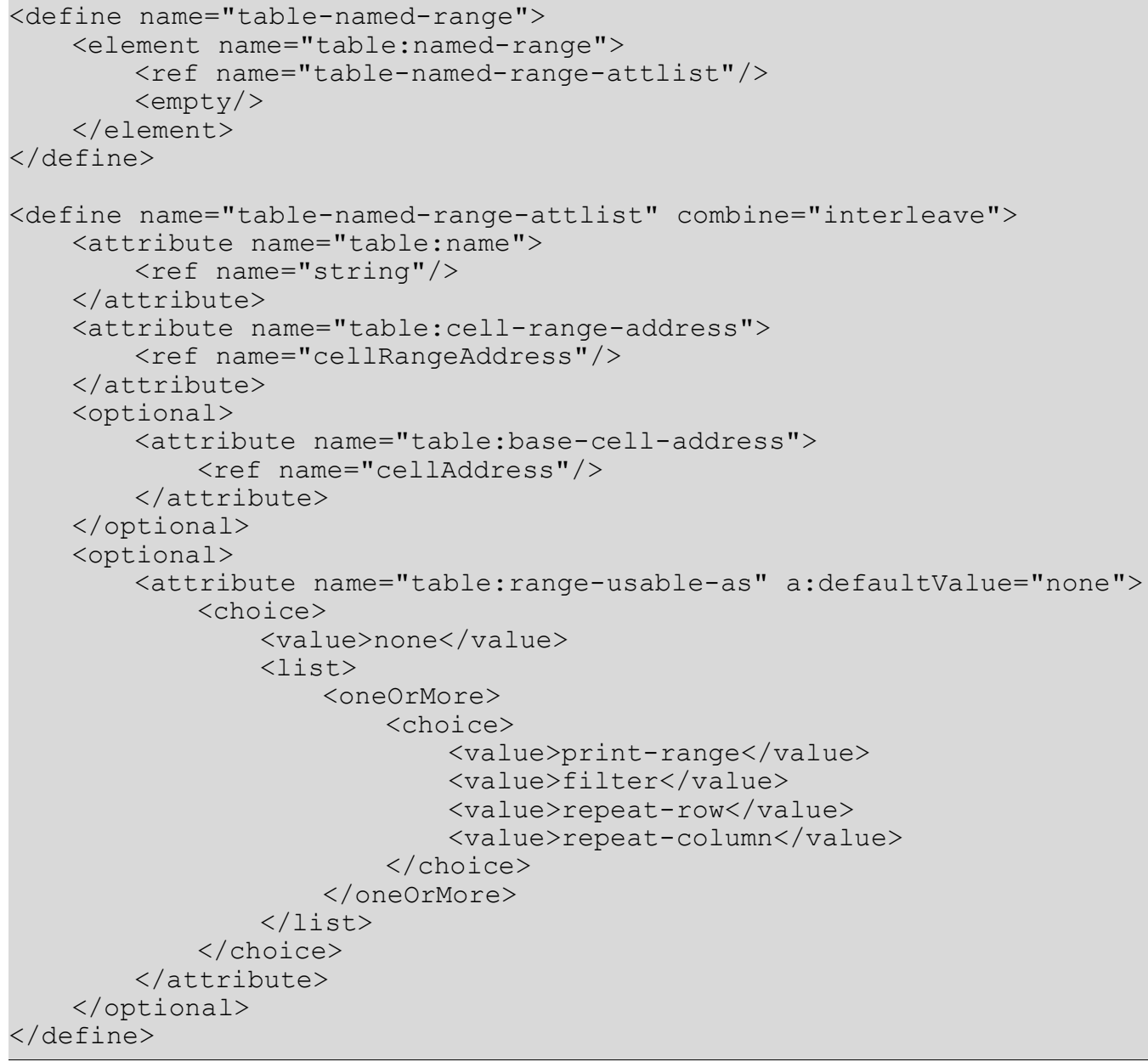




\section{Named Expression}

The named expression element $<$ table: named-expression $>$ contains an expression with a name, for example, a part of a formula.

The table: name attribute specifies the name of the expression, and the table:expression attribute the expression itself. The expressions do not support the equal (=) sign as the first character. If the expression contains a named range or another named expression, the named range or named expression must be specified first, before the containing expression. If the expression contains a relative cell range address, the table:base-cell-address attribute must exist additionally. It specifies the base cell address for the cell range. This address must be absolute. Therefore a table name in the address is required, but the dollar signs that indicate an absolute address can be omitted.

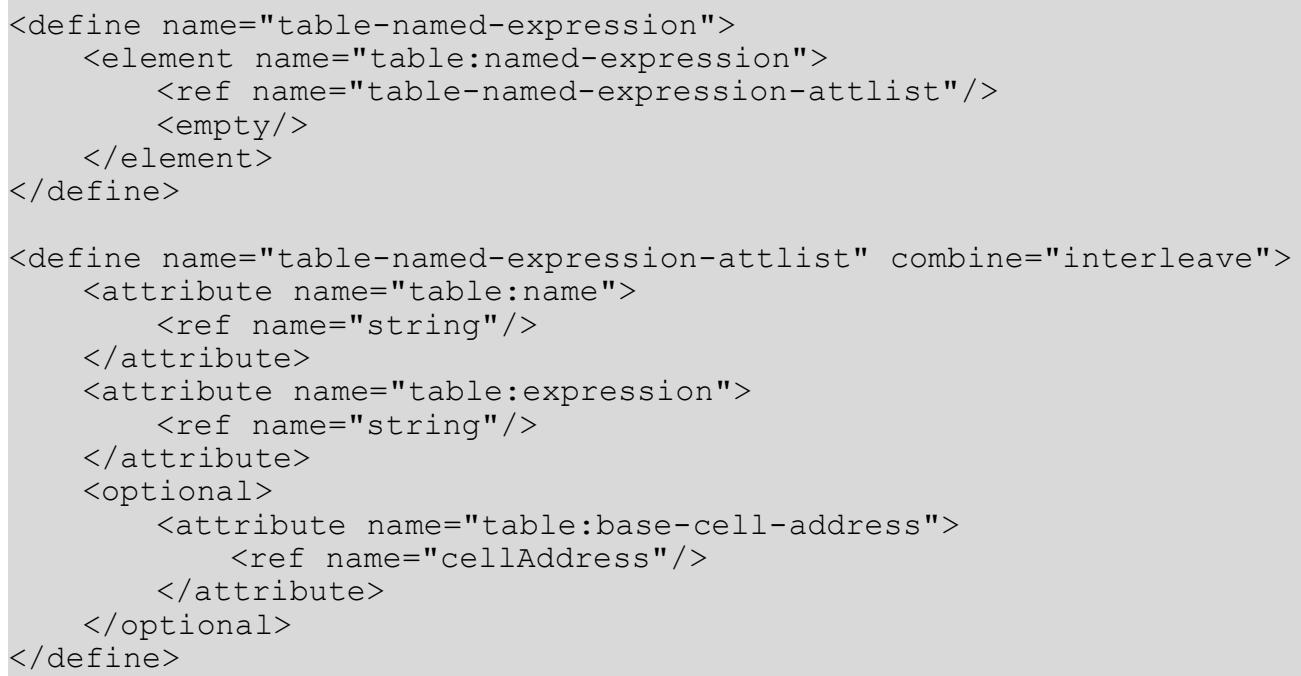

Example: Named expressions element with a named range and a named expression

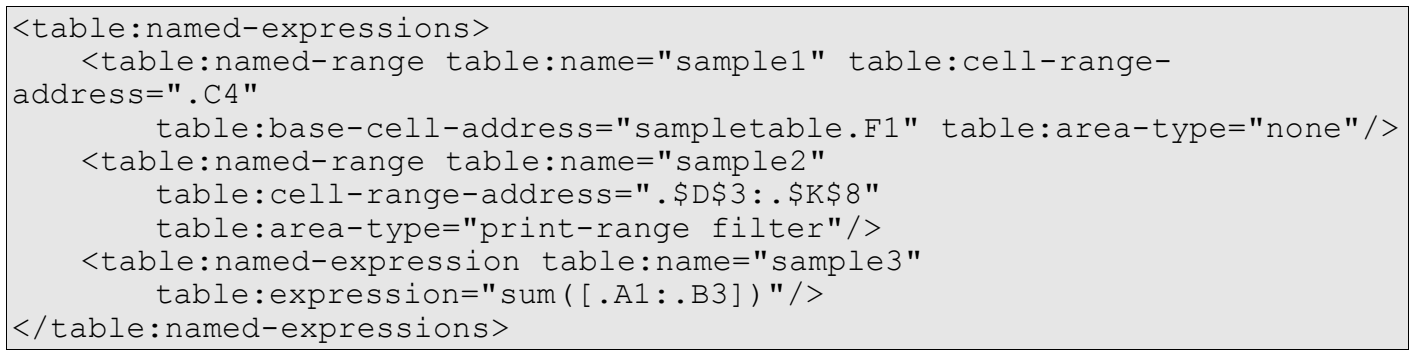

\subsection{Database Ranges}

A database range is a named area in a table where database operations, but also some other kind of operations like filtering and sorting, can be performed. The Database Ranges element $<$ table: database-ranges $>$ contains a collection of all database ranges defined in a document.

4400

4401

4402

4403

4404

4405

4406

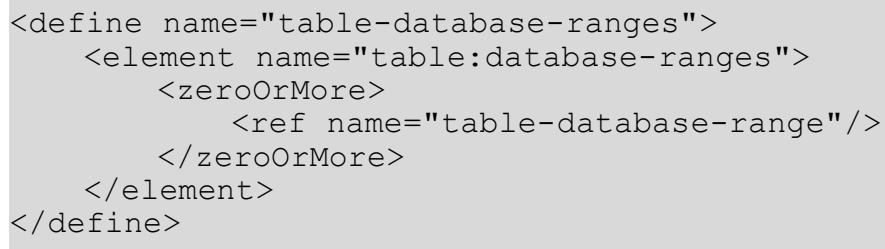




\subsubsection{Database Range}

The $<$ table: database-range $>$ defines a single database range.

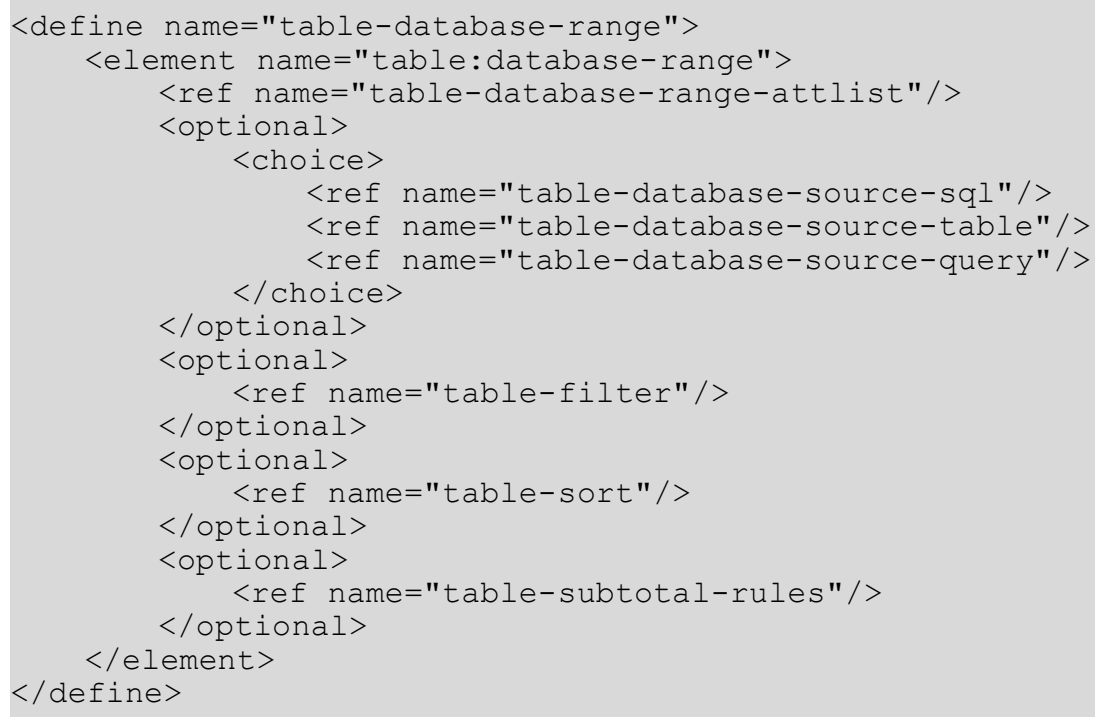

\section{Database Range Name}

The table: name attribute specifies the name of the database range on which to perform operations. Within a single document, only one database range is allowed to have no name. This database range is usually automatically created by the application and is used to filter or sort data in a cell ranges without the user explicitly creating a database range.

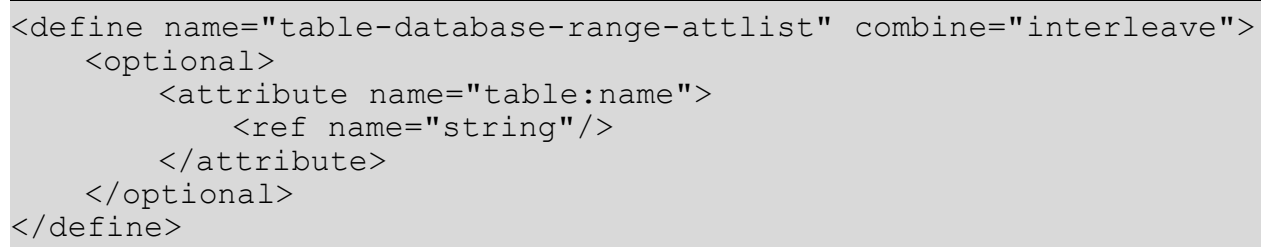

\section{Is Selection}

The table:is-selection attribute specifies whether the database range includes a complete database, or a selection of records from a database only.

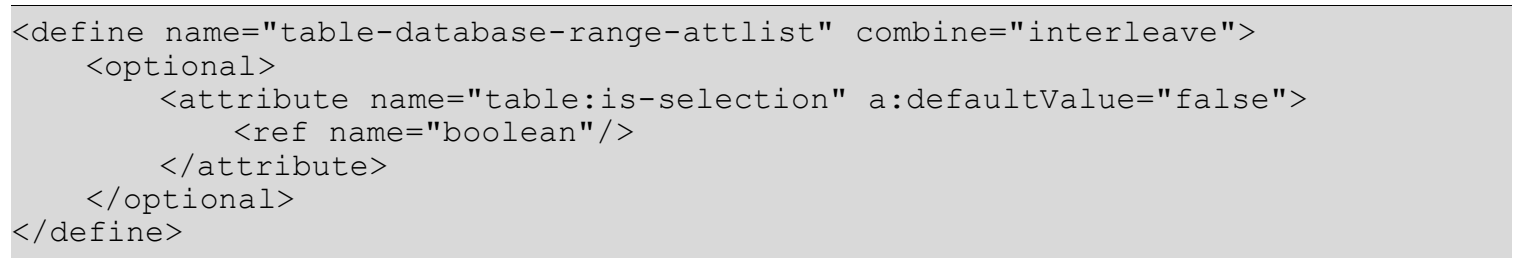

\section{On Update Keep Styles}

The table:on-update-keep-styles attribute specifies the behavior if the database range is updated. If the attribute value is "true", the cell styles that are assigned to the cells in the first non-label row of the database range are used for all rows with in the database range. If the attribute value is "false", all cells in the database range get the default cell style of the document assigned. 


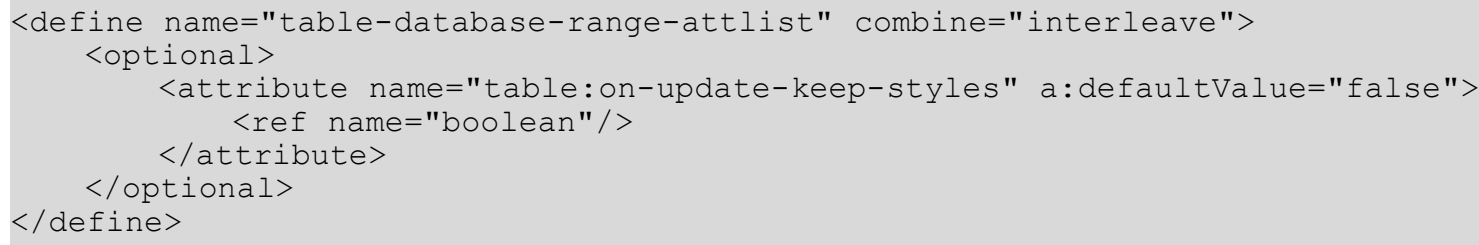

\section{On Update Keep Size}

The table:on-update-keep-size attribute specifies the behavior of the database range if the size of the data in the data source changes. If the attribute value is true, the range retains its size. If the attribute value is false, the range does not retain its size.

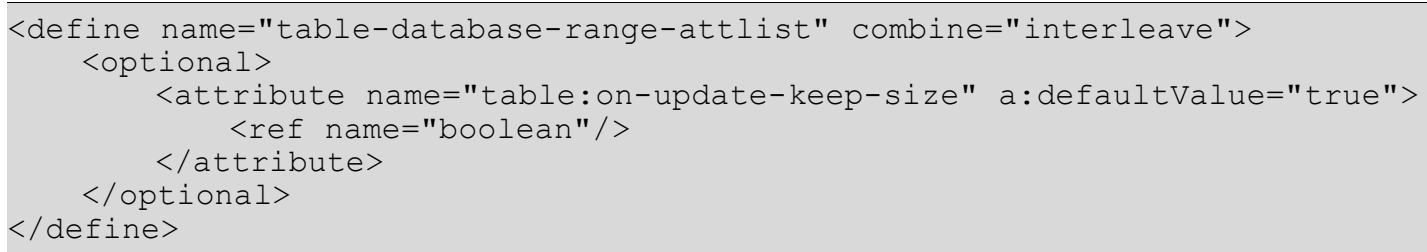

\section{Has Persistent Data}

The table:has-persistent-data attribute specifies whether the current data in a database range is saved when the document itself is saved.

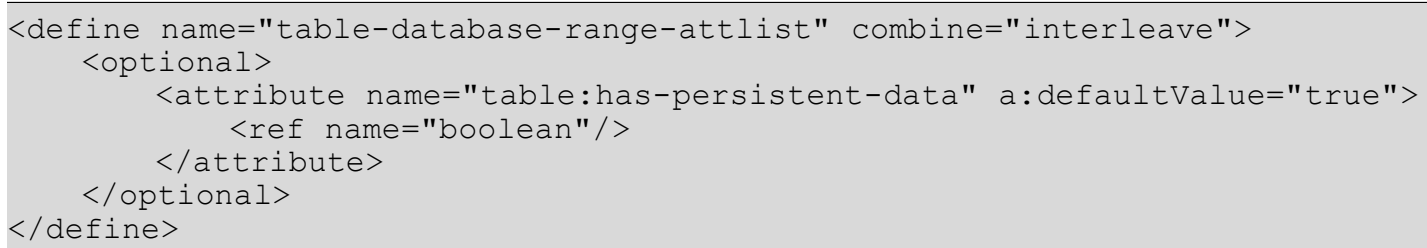

\section{Orientation}

The table: orientation attribute specifies the orientation of the database range. The values of this attribute are row and column. The orientation is for instance used when sorting database ranges (see 8.6.5). If the orientation is row, the sorting takes places for rows, otherwise for columns.

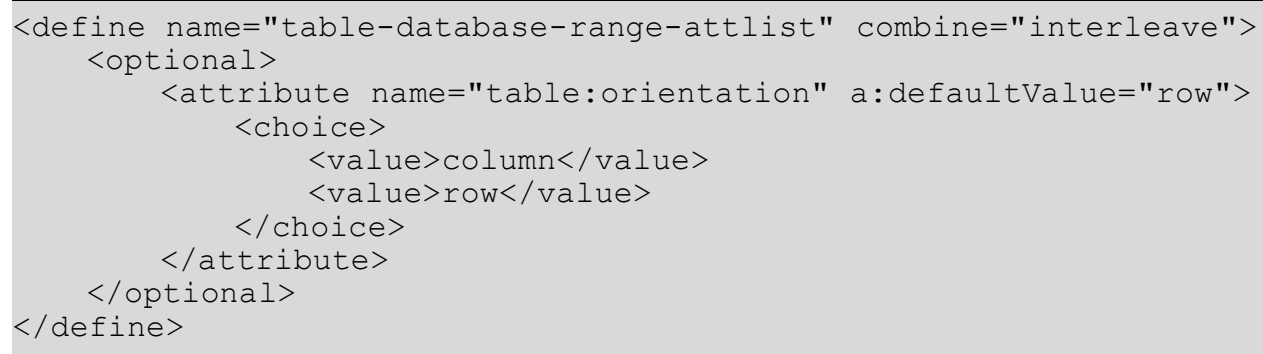

\section{Contains Header}

The table:contains-header attribute specifies whether or not the the content of the database range's first row or column should be used to specify labels. If the attribute's value is true, the content of the first cell within a row or column can be used to reference the whole row or column within many spreadsheet operations, for instance from within data pilots. 


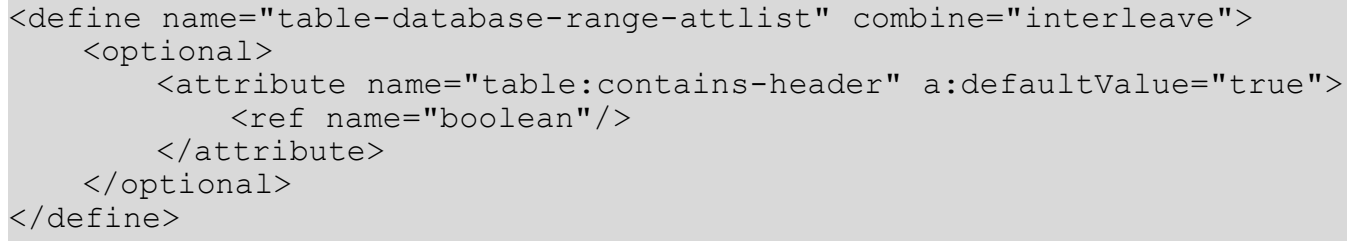

\section{Display Filter Buttons}

The table:display-filter-buttons buttons attribute specifies whether or not to display filter buttons. Filter buttons are list box controls displayed in the label cells whose list entries are the values that exist in the labeled row or column. Selecting one of these entries equals applying a filter to the database range that selects all row or columns where the cells in the labeled row or column have the selected value.

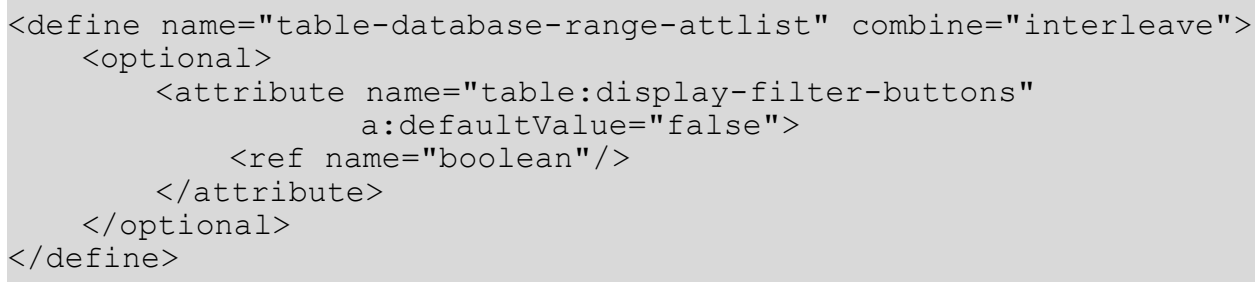

\section{Target Range Address}

The table:target-range-address attribute specifies the cell range address of the database range. A differentiation between absolute and relative addresses is not possible. Therefore, a table name must be specified in the address and dollar signs are ignored.

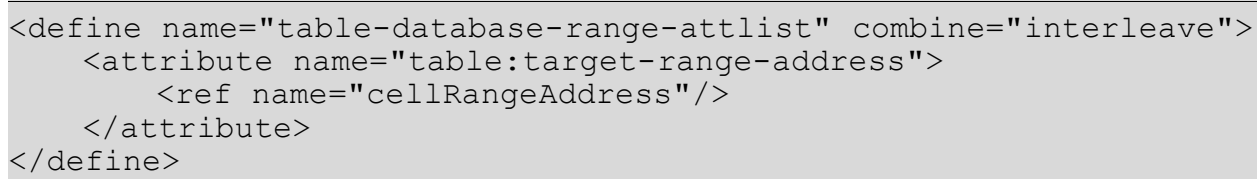

\section{Refresh Delay}

The table:refresh-delay attribute specifies a time delay between automatic refresh actions.

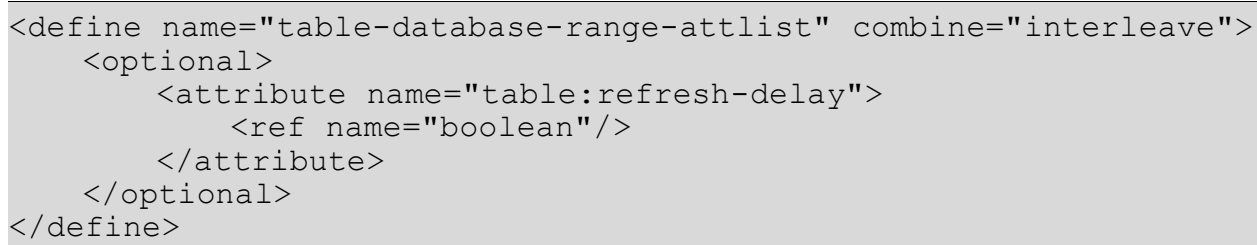

\subsubsection{Database Source SQL}

The <table: database-source-sql> element describes an SQL database that contains the source data of the database range.

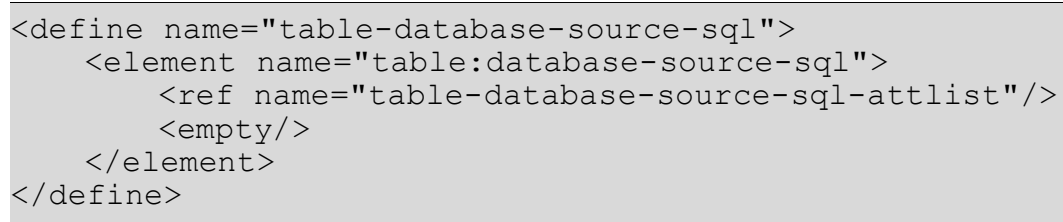




\section{Database Name}

A table: database-name attribute specifies the name of the SQL database where the data is imported from.

4506

4507

4508

4509

4510

\section{SQL Statement}

An table:sql-statement attribute specifies the SQL statement to use when importing data from an SQL database.

4511

4512

4513

4514

4515

\section{Parse SQL Statement}

A table:parse-sql-statement attribute specifies whether or not the application will parse SQL statements.

\subsubsection{Database Source Table}

The database source table element $<$ table: database-source-table $>$ specifies that the source data of the database range is stored in a database table.

\section{Database Name}

The table:database-name name attribute specifies the name of the database where the data is imported from. 


\section{Table Name}

A table:database-table-name attribute specifies the database table that data is imported from.

\subsubsection{Database Source Query}

The database source query element $<$ table: database-source-query $>$ specifies that the source data of the database range is is the result of a database query.

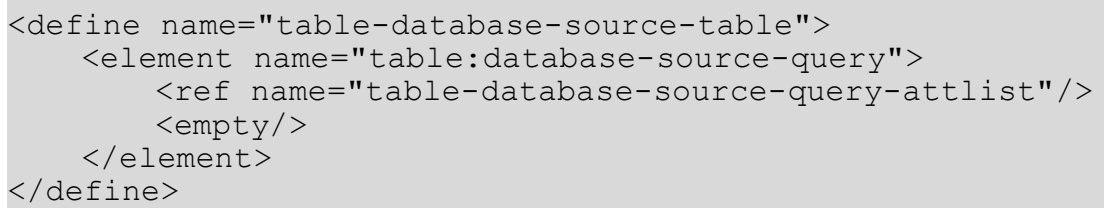

\section{Database Name}

A table: database-name attribute specifies the name of the database that data is imported from.

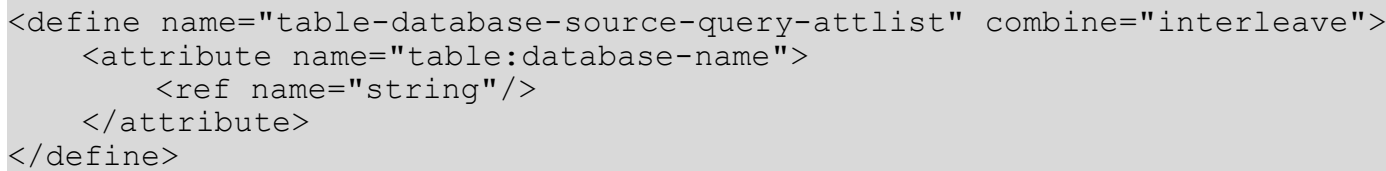

\section{Query Name}

A table:query-name attribute specifies the query to perform on the database whose data is being imported.

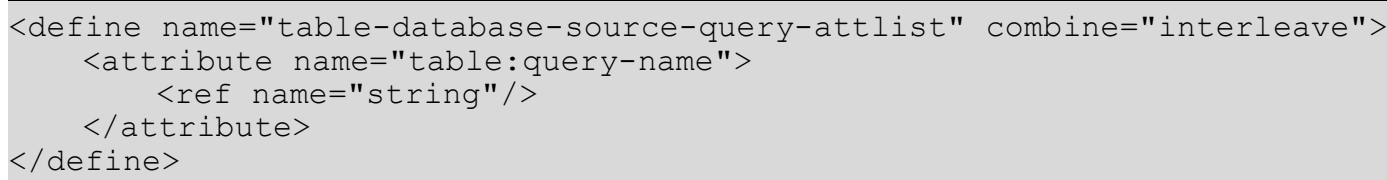

\subsubsection{Sort}

The sort element $<$ table: sort $>$ describes the sort keys that should be applied to a database range.

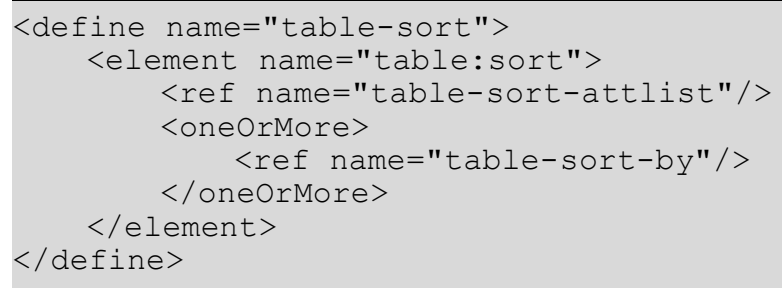




\section{Bind Styles to Content}

The table:bind-styles-to-content attribute specifies whether or not cells retain their style attributes after a sort operation.

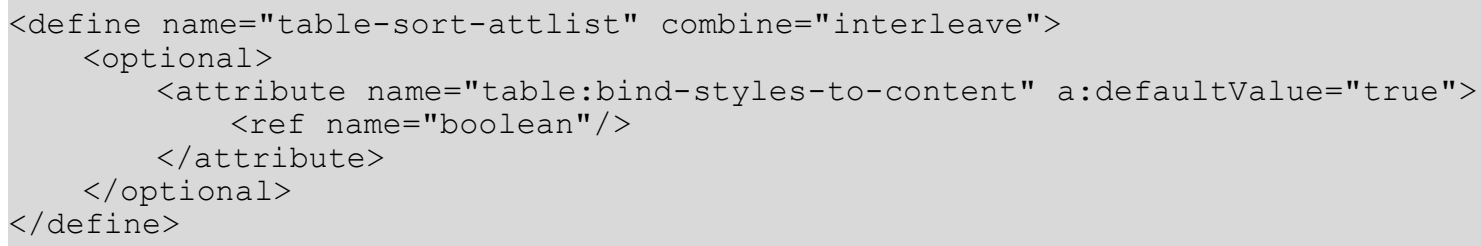

\section{Target Range Address}

If the optional table:target-range-address attribute is present, the result of the sort is copied into the specified cell range rather than in the source cell range specified by the database range. A differentiation between absolute and relative addresses is not possible. Therefore, a table name has to exist in the address and dollar signs are ignored.

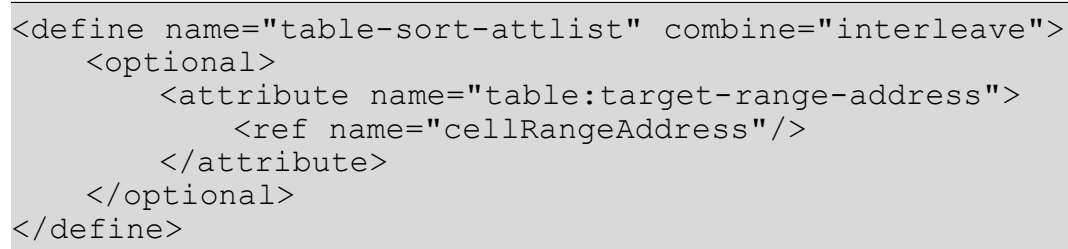

\section{Case Sensitive}

The table:case-sensitive attribute specifies whether or not the sort operation is case sensitive.

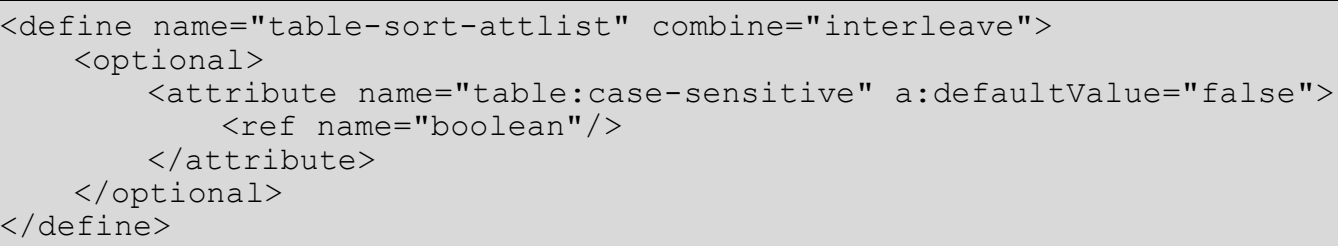

\section{Language}

The table: language attribute specifies the natural language in which the comparison will occur.

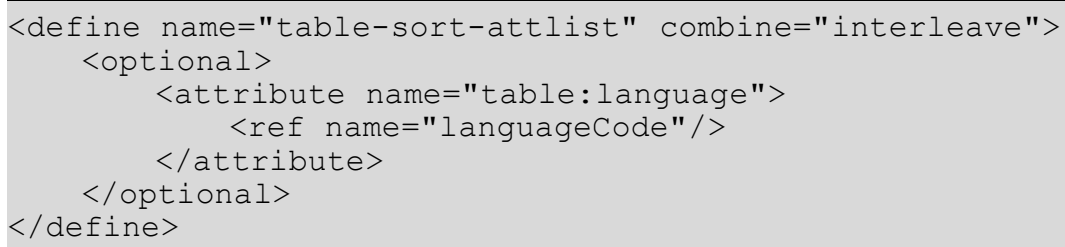

\section{Country}

The table:country attribute specifies the country specific rules to be used in string comparisons for a particular natural language. 


\section{Data Type}

The table:data-type attribute specifies the data type of the field to be sorted. It's value can be text, number, automatic or the name of user defined sort order. If the attribute value is automatic, the application must determine what type of data is in the field. User defined sort orders are for instance lists of names of months. Specifying user defined sort orders is application specific.

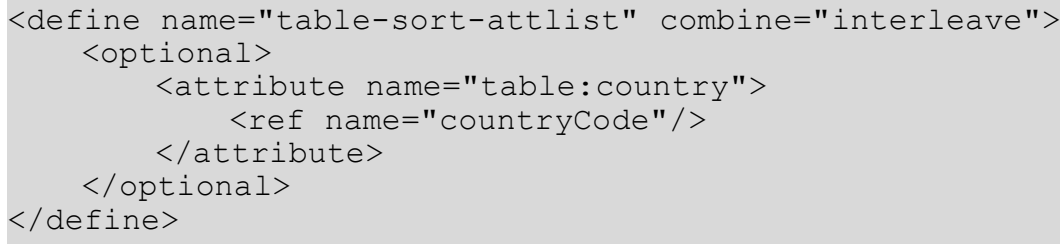

\section{Algorithm}

The table:algorithm attribute specifies the algorithm used to compare sort keys. The attribute's value is a an application but also language and country specific sort algorithm name like "phonetic (alphanumeric first)". To avoid name clashed between different applications, the name should start with a namespace prefix

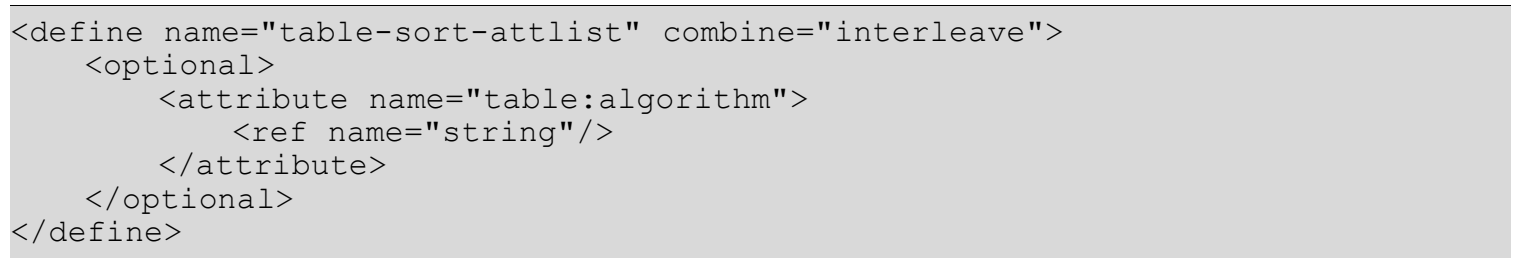

\subsubsection{Sort By}

The sort by element $<t a b l e:$ sort-by $>$ specifies a key or field to sort, the data type of this field, and how to sort it.

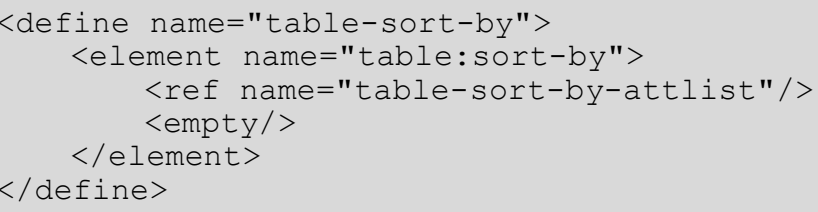

\section{Field Number}

The table:field-number number attribute specifies the row or column number to sort by. It is the number of a row or column within the database range.

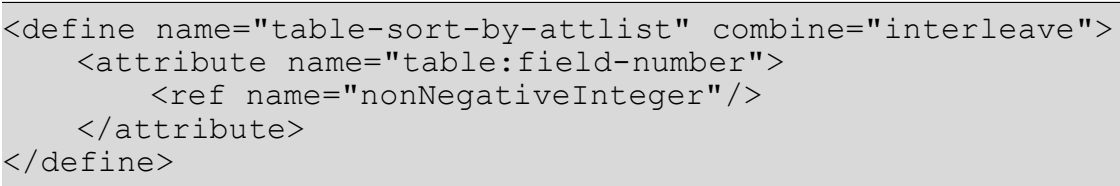




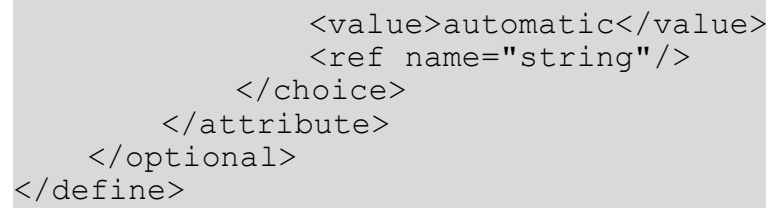

\section{Order}

The table: order attribute specifies whether to sort the data in ascending or descending order.

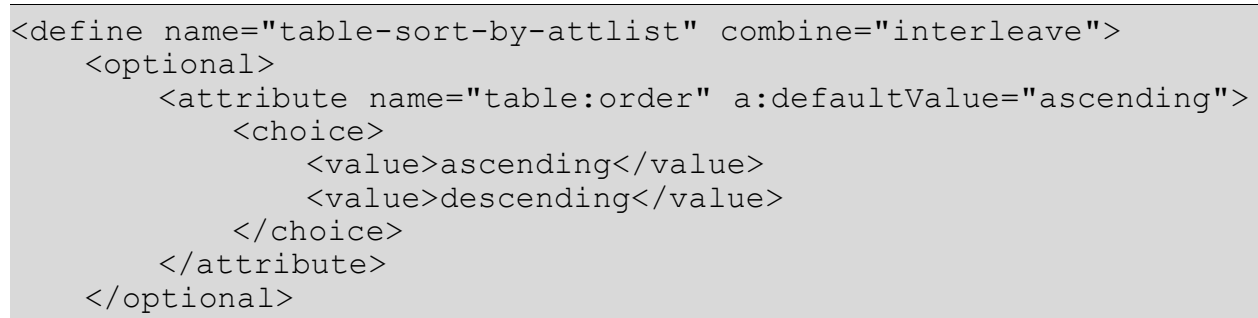

\subsubsection{Subtotal Rules}

The subtotal rules element <table:subtotal-rules $>$ specifies that provisional results (called subtotals) should be calculated for a database range. It contains information about the row or column provisional results should be calculated for, and also how these results are calculated. To calculate provisional results, the cell values of a row or column a grouped by their value, that is, all cells with the same content in the same field form a group. A provisional result is calculated and displayed at the end of each group.

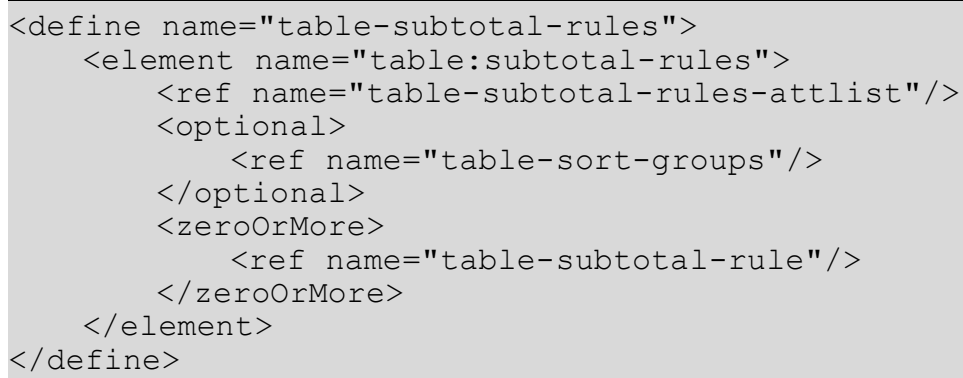

\section{Bind Styles To Content}

The table:bind-styles-to-content attribute specifies whether or not cells retain their style after a subtotal calculation. This attribute is only evaluated if the table:sort-groups element is present.

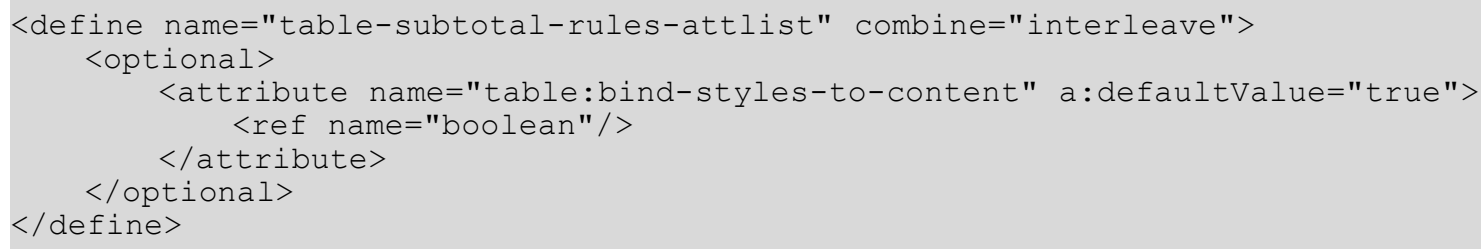




\section{Case Sensitive}

The table:case-sensitive attribute specifies whether or not the case of characters is important when comparing entries, for example, when sorting groups.

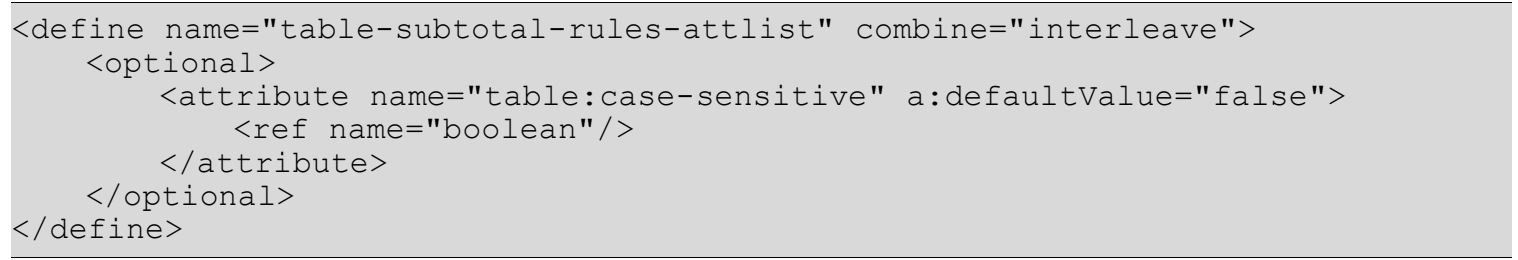

\section{Page Breaks On Group Change}

The table:page-breaks-on-group-change on group change attribute specifies whether or not to insert a page break after the subtotal for each group.

4663

4664

4665

4666

4667

4668

4669

4670

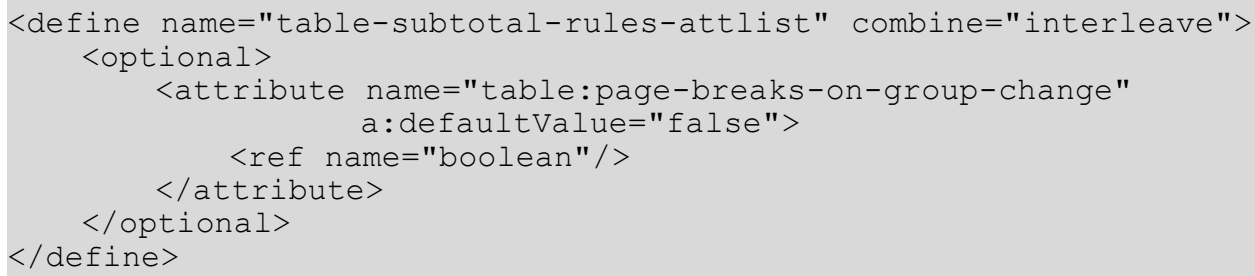

\subsubsection{Subtotal Sort Groups}

The optional sort groups element <table:sort-groups> specifies that columns or rows are sorted before grouping them, and how to sort them. It belongs to the subtotal rules element, see the previous section.

4671

4672

4673

4674

4675

4676

\section{Data Type}

The table:data-type attribute specifies the data type of the column or row group to sort. See section 8.6.6 for details.
4677
4678
4679
4680
4681
4682
4683
4684
4685
4686
4687
4688 


\section{Order}

The table:order attribute specifies whether to sort the group data in ascending or descending order. See section 8.6.6 for details.

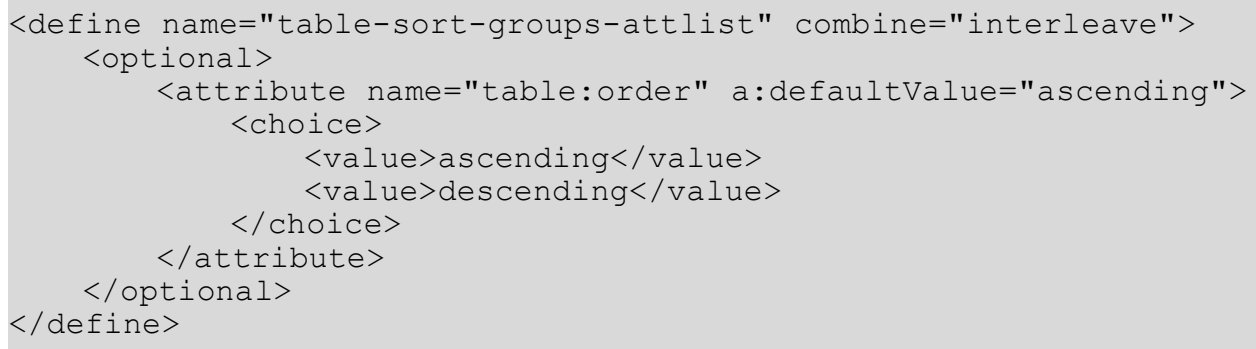

\subsubsection{Subtotal Rule}

The subtotal rule element $<$ table: subtotal-rule $>$ describes how to calculate the subtotals for a certain row or column. The rule contains the group field number, which specifies the column group for which the rule is used, and one or more subtotal fields, which specify a row a column where subtotals should be calculated as well as the function to use for the calculation.

\subsubsection{Subtotal Field}

The subtotal field element $\langle t a b l e$ : subtotal-field $>$ contains the field number and the function that is used to calculate a provisional result.

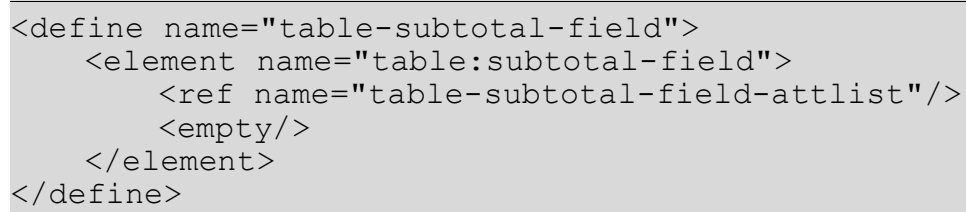

\section{Field Number}

The table:field-number attribute specifies the row or column a subtotal should be calculated for. It is the number of a row or column within the database range. 


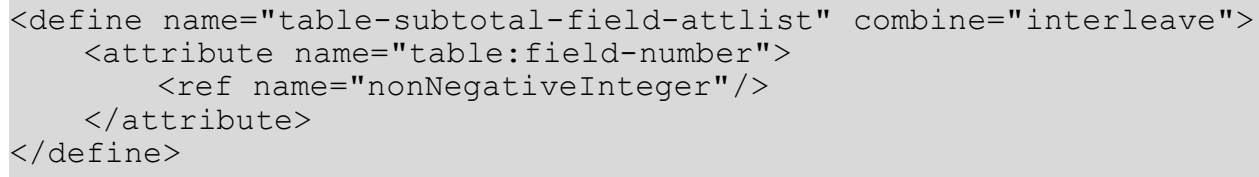

\section{Function}

The table: function attribute specifies what kind of subtotals to calculate. The following are possible values for this attribute: auto, average, count, countnums, max, min, product, stdev, stdevp, sum, var and varp.

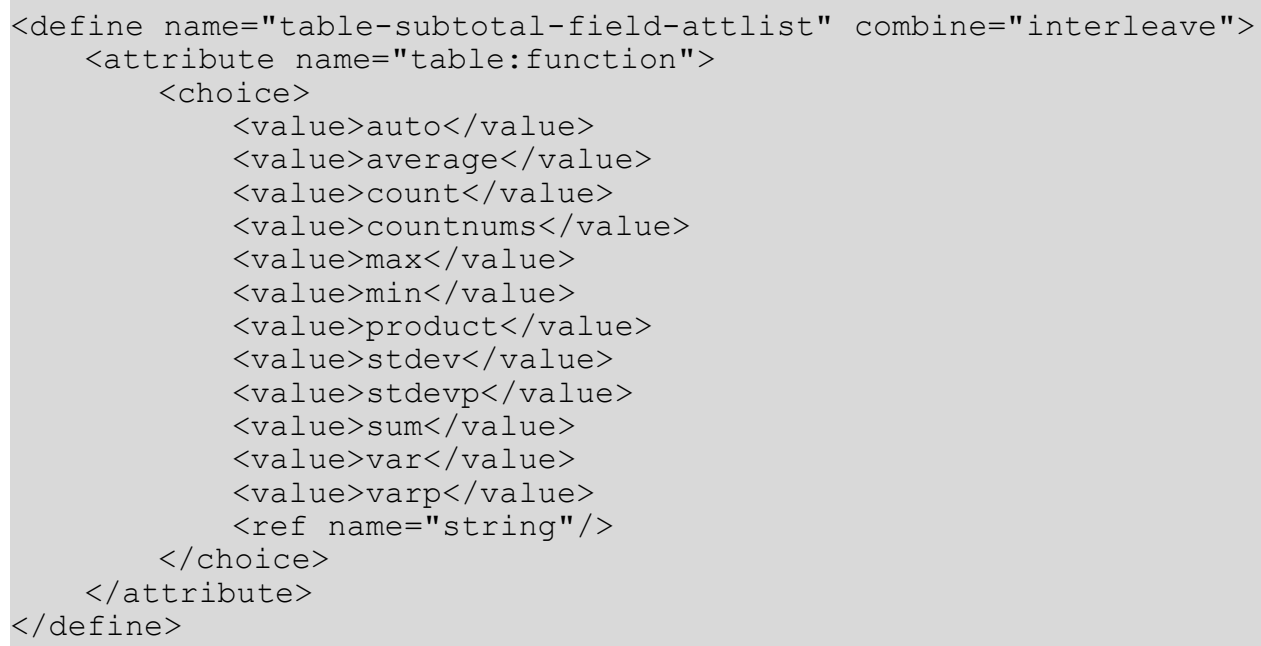

\section{Example: Subtotal field}

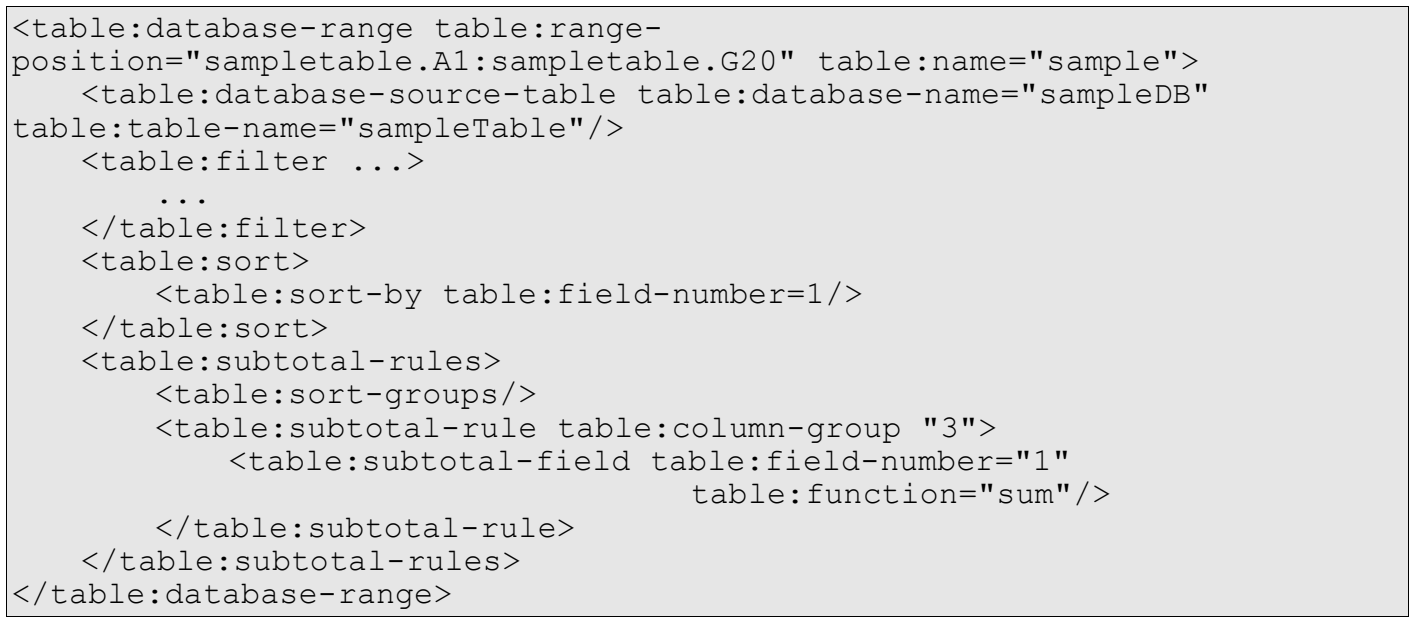

\subsection{Filters}

Filters specify that only rows that match certain conditions should be visible

\subsubsection{Table Filter}

The table filter element $\langle$ table:filter $>$ describes how the data contained in a database range or data pilot tables is filtered. The condition specified in the element are applied to all rows 
specified in the database range or the data pilot table. Rows where the condition does not evaluate to true are made invisible.

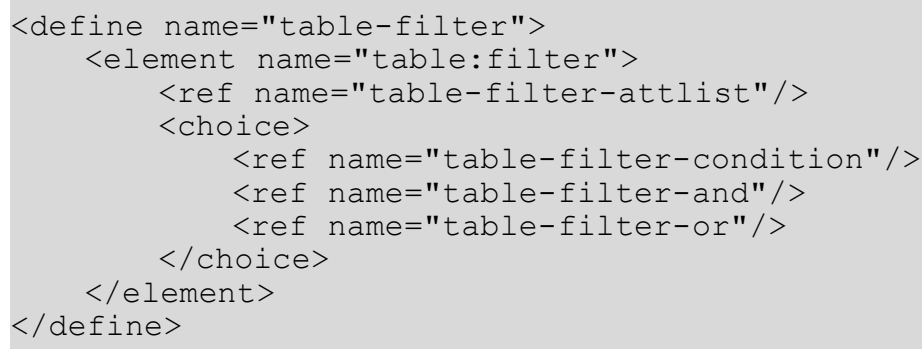

\section{Target Range Address}

If the optional table:target-range-address attribute is present, the result of the filter is copied into the specified cell range but all table rows remain visible. If the attribute is not present, the rows that do not match the filter conditions are not displayed. A differentiation between absolute and relative addresses is not possible. Therefore, a table name has to exist in the address and dollar signs are ignored.

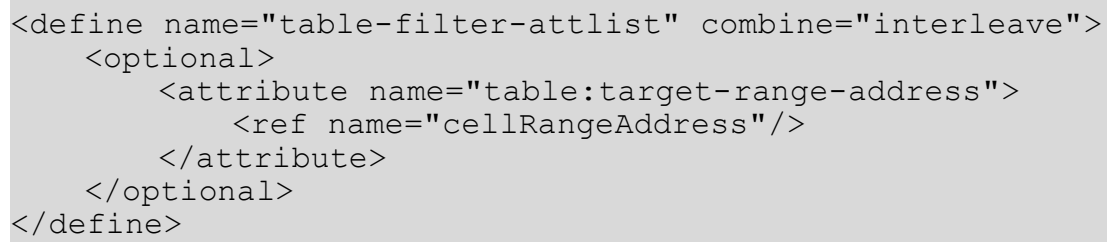

\section{Condition Source}

The table:condition-source attribute specifies whether the condition is contained in the filter or encoded in a table range. If the value is self the condition is specified by the $<$ table:filter> element's child elements. If the value is cell-range the condition is encoded into the cell range specified by the table:condition-source-range-address attribute.

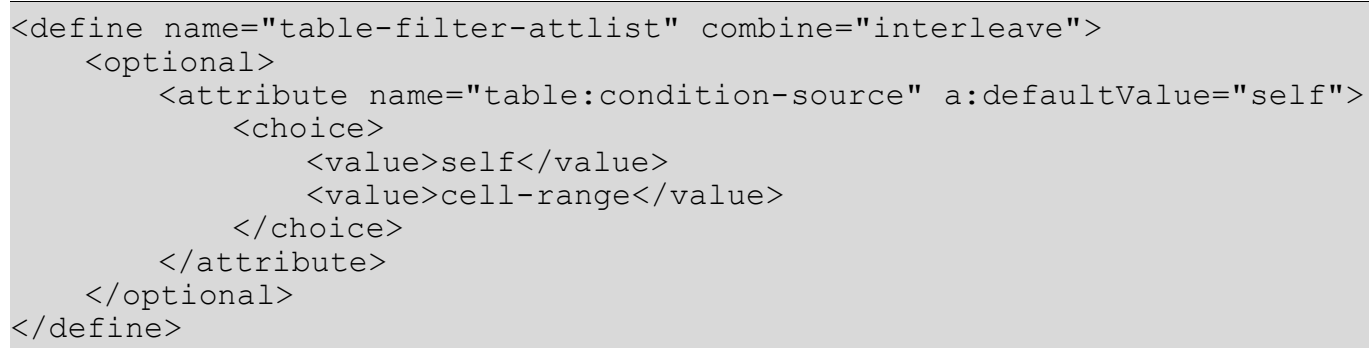

\section{Condition Source Range Address}

The table:condition-source-range-address attribute specifies a cell range that contains encoded conditions. The first row of the cell range has to contain the labels of the columns whose content should be filtered. The following rows contain conditions that have to evaluate to true for the cells contained in the columns. The conditions in each row are connected by an "and" operation, while the rows are connected by an "or" operation. This means that a row is of the source table is displayed if there is at least one row in the condition range where all conditions evaluate to true if they are applied to the columns specified in the first row of the condition range. 
Example: If the condition source range is $E 1: F 3$ (shown yellow) and the source range is $A 1: C 3$ (shown green), only rows 2 and 3 are displayed.

\begin{tabular}{|r|r|r|r|r|l|l|l|l|l|}
\hline & \multicolumn{1}{|c|}{$\boldsymbol{A}$} & \multicolumn{1}{|c|}{$\boldsymbol{B}$} & $\boldsymbol{C}$ & $\boldsymbol{D}$ & $\boldsymbol{E}$ & $\boldsymbol{F}$ & $\boldsymbol{G}$ & $\boldsymbol{G}$ & $\boldsymbol{I}$ \\
\hline 1 & 1 & 3 & 4 & & A & B & & & \\
\hline 2 & 1 & 5 & 6 & & $=1$ & $=5$ & & & \\
\hline 3 & 2 & 8 & 9 & & $>=2$ & & & & \\
\hline
\end{tabular}

Row 2 is displayed because the cell in column $A$ has the value 1 and the cell in column $B$ the value 5 , so all conditions of the $2^{\text {nd }}$ row of the condition range evaluate to true. Row 3 is displayed because the cell in column $\mathrm{A}$ is larger or equal than 2 , and therefor the only condition in the the $3^{\text {rd }}$ row of the condition range evaluates to true.

\subsubsection{Filter And}

The <table:filter-and $>$ element specifies that the logical operator AND is applied to the conditions specified by the element's child elements.

\subsubsection{Filter Or}

The <table:filter-or $>$ element specifies that the logical operator OR is applied to the conditions specified by the element's child elements. 


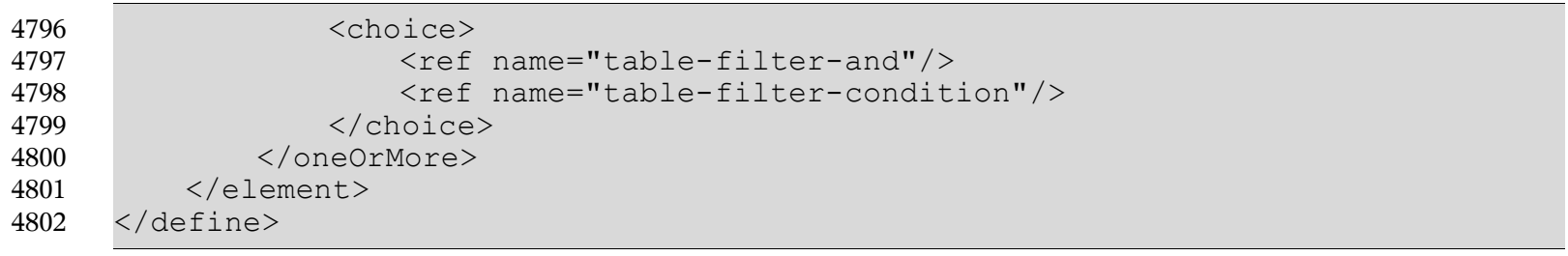

\subsubsection{Filter Condition}

The table <table:filter-condition> element describes a single condition to apply in a filter operation.

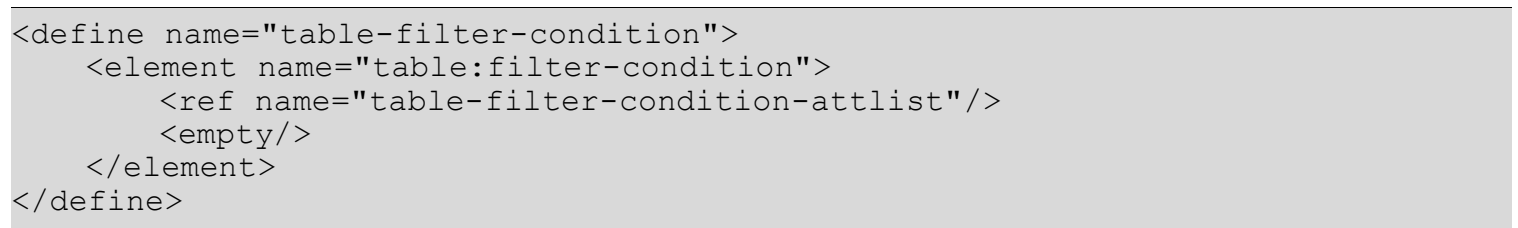

\section{Field Number}

The field number attribute table: field-number specifies which field to use for the condition. A field number is the number of a row or column in the source range of the filter.

\section{Value}

The table:value attribute specifies a value for the filter condition.

\section{Operator}

The operator attribute table: operator specifies what operator to use in the filter condition. This means that each cell contained in the columns specified by the field number (i.e. the table: field-number attribute) is compared with the value (i.e. the table:value attribute) using the given operator. The result of this comparison is the result of the filter sub conditions specified by the <table: filter-condition> element.

The operators may or may not make use of regular expressions. The operators that use regular expressions are the following:

- match (matches)

- $\quad$ match (does not match)

In both case, the table:value attribute contains the regular expression that the table cells have to match or must not match.

The relational operators that do not use regular expressions are: 


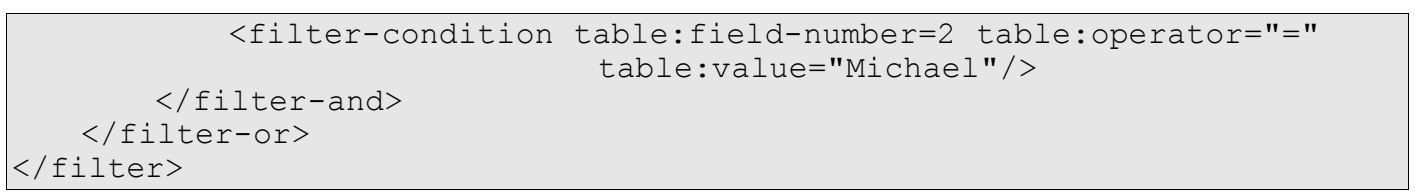

\subsection{Data Pilot Tables}

Data pilot tables allow it to analyze and evaluate data contained in spreadsheet tables. The data pilot tables element $<$ table: data-pilot-tables $>$ contains the collection of all data pilot tables within a document.

4841

4842

4843

4844

4845

4846

4847

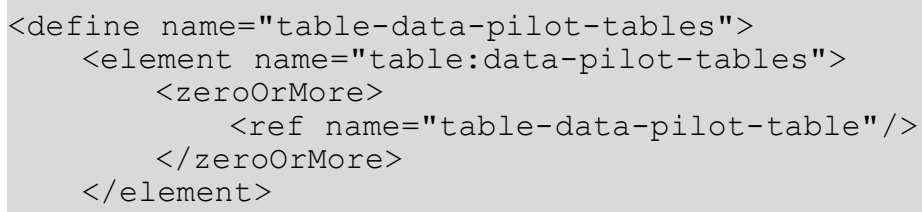

\subsubsection{Data Pilot Table}

The <table:data-pilot-table> specifies a single data pilot table. Within data pilot tables, all combinations of values that exist in selected columns are collected, and for each of these combinations a formula is applied to the cells of other columns.

Example: Given is the following source table

\begin{tabular}{|r|l|l|l|l|r|}
\hline \multicolumn{1}{|l|}{ A } & B & C & D & \\
\hline 1 & Article & City & Country & Amount & Price \\
\hline 2 & Main Unit & Hamburg & Germany & 1 & 12 \\
\hline 3 & Monitor & Hamburg & Germany & 2 & 15 \\
\hline 4 & Printer & Paris & France & 4 & 13 \\
\hline 5 & Monitor & Paris & France & 2 & 14 \\
\hline 6 & Main Unit & Paris & France & 1 & 12 \\
\hline 7 & Monitor & Hamburg & Germany & 2 & 10 \\
\hline 8 & Printer & Paris & France & 2 & 16 \\
\hline
\end{tabular}


The following data pilot table groups the source table by the columns "County", "City" and "Article" and calculates the sum of the "Amount" as well as of the "Price" columns for each combinations of values of these three columns. The values of the Country and City columns are shown in columns, while the ones of the Article columns are shown in rows.

\begin{tabular}{|c|c|c|c|c|c|c|c|}
\hline & & & Article & & & & \\
\hline Country & City & Data & $\begin{array}{l}\text { Main } \\
\text { Unit }\end{array}$ & Monitor & Printer & Total & \\
\hline France & Paris & $\begin{array}{l}\text { Sum - } \\
\text { Amount }\end{array}$ & & 1 & 6 & & 9 \\
\hline & & Sum - Price & 12 & 14 & 29 & & 55 \\
\hline Germany & Hamburg & $\begin{array}{l}\text { Sum - } \\
\text { Amount }\end{array}$ & & 1 & 4 & & 5 \\
\hline & & Sum - Price & 12 & 25 & 5 & & 37 \\
\hline Total sun & & & & 2 & 6 & & 14 \\
\hline Total sun & & & 24 & 39 & 29 & & 92 \\
\hline
\end{tabular}

The columns that are used for grouping (here "County", "City" and "Article") are called category columns. The columns for which a formula is calculated based on the value combinations of the category columns (here "Amount" and "Price") are called data columns. The individual values that exists within a category column are called members.

In general, the behavior of a data pilot is specified by fields, where each field has a name and a so called orientation. The category columns are specified by fields with the orientation "row" or "column" and the data columns are specified by fields that have the orientation "data". In the above example, "Article" is a field with the orientation column, while "Country" and "City" are fields with the orientation row. "Amount" and "Price" are fields with "data" orientation.

A third kind of fields are data layout fields. Data layout fields are not connected to a column in the source table, but have the only the purpose to change the layout of the data pilot table. In the example, "Data" is a data layout field.

The order in which fields are specified is of relevance. It specified the order in which the data of category columns is grouped and results are displayed. The data pilot table below displays how the data pilot table changes if for instance the data layout field is specified before the category column fields.

Example: A data pilot with a modified layout

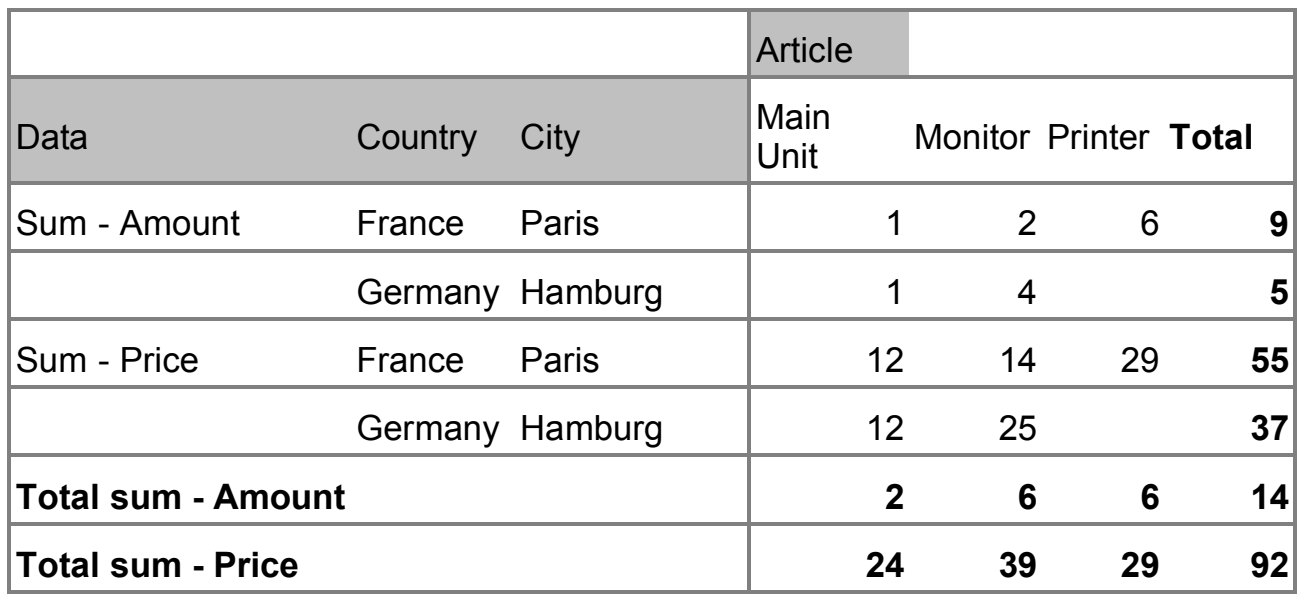


The attributes associated with the data pilot table element are:

- Data pilot table name

- Application data

- Grand total

- Ignore empty rows

- Identify categories

- Target range address

- Show Filter Button

- Drill Down On Double Click

4848

4849

4850

4851

4852

4853

4854

4855

4856

4857

4858

4859

4860

4861

4862

4863

4864

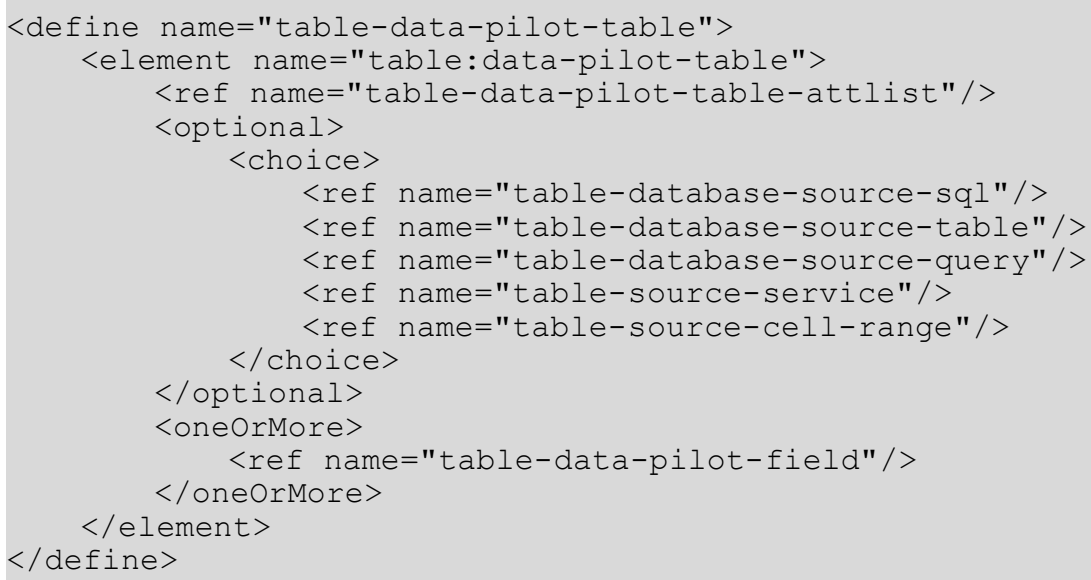

\section{Data Pilot Table Source}

The source of the data pilot table is either stored in a database, that is, a database table itself, a SQL query or a named query, or it is a cell range located within the same document. It can also be provided by an external component in an implementation dependent way.

The source of the data pilot table is specified by one of the following elements that are contained in the <table: data-pilot-table> element:

- $\quad<$ table: database-source-sql> (see section 8.6.2)

- <table: database-source-table> (see section 8.6.3)

- <table: database-source-query> (see section 8.6.4)

- <table: source-cell-range> (see section 8.8.2)

- <table: source-service> (see section 8.8.3)

\section{Data Pilot Table Name}

The table: name attribute specifies the name of the data pilot table.

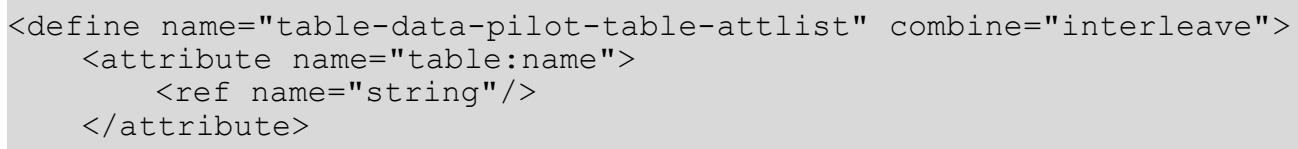




\section{Application Data}

The table:application-data attribute specifies extra information about the data pilot table, which can be used by the application, for instance within macros. This data does not influence the behavior of the data pilot.

4870

4871

4872

4873

4874

4875

4876

4877

4878

4879

4880

4881

4882

4883

4884

4885

4886

4887

4888

\section{Ignore Empty Rows}

The table:ignore-empty-rows attribute specifies whether or not empty rows in the source range should be ignored.

\section{Identify Categories}

The table:identify-categories attribute specifies whether rows that do not contain a value in one of the category columns should use the value of the nearest ancestor row that has a value, or whether such rows should be moved into a group (or category) of its own. If the attribute's value is false, empty values form a category of its own. 


\section{Target Range Address}

The table:target-range-address attribute specifies where the target range of the data pilot table output, that is, where the data pilot table is displayed. A differentiation between absolute and relative addresses is not possible, that is, the address is interpreted as an absolute address even if it contains dollar signs. The range address must contain a table name.

\section{Drill Down On Double Click}

The table:drill-down-on-double-click attribute specifies how the data pilot table reacts on a double click into the data pilot table. If the attribute's value is false, a double click on a member label or the empty area next to it starts the edit mode of the table cell, like for cells outside of the data pilot table. This can then be used to rename group fields or members. If the attribute's value is true, a double click on an member label or the empty area next to it shows or hides details for that member. A double click elsewhere in a data pilot table has no effect. 


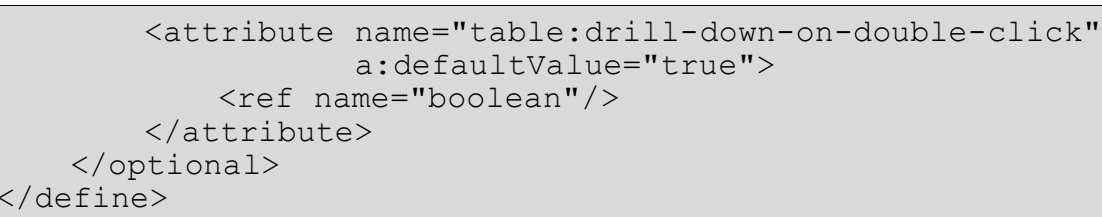

\subsubsection{Source Cell Range}

If the source of a data pilot table is a cell range, the $<$ table: source-cell-range $>$ element contains information about the cell range and how the data pilot table gets the data from the range. Before the source data is processed by the data pilot data, a filter may be applied to it. This filter has to be specified by a <table: filter $>$ child element.

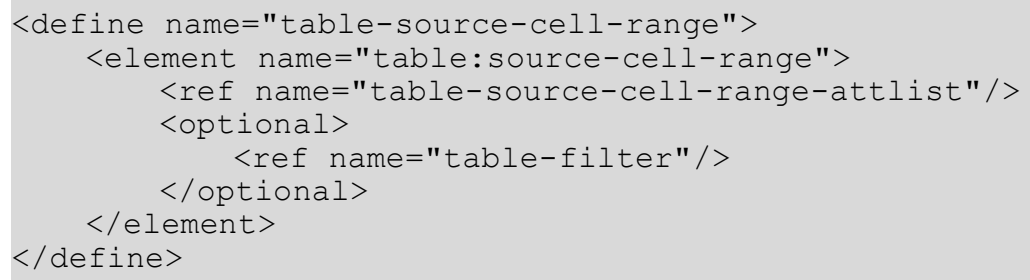

The only attribute that may be associated with the source cell range element is:

- Cell range address

\section{Cell Range Address}

The table:cell-range-address attribute specifies the cell range containing the source data. The source cell range's address must be absolute. Therefore, the cell range address must contain a table name and dollar signs are ignored.

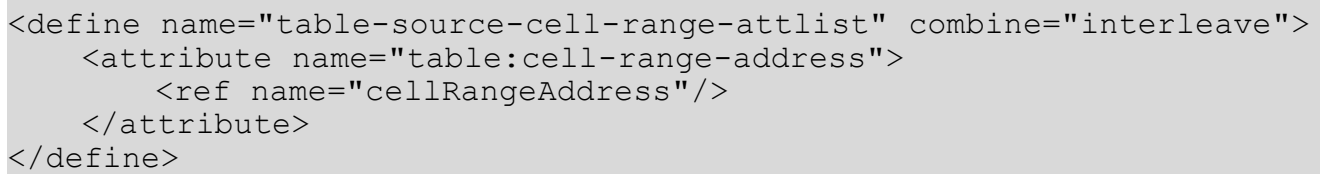

\subsubsection{Source Service}

The source of a data pilot table can be "service", that is, it can be provided by an external component. The source service element $<$ table: source-service> contains information about the service which is used to create the data pilot table.

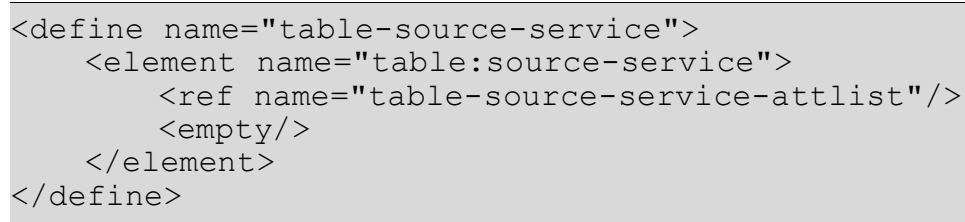

The attributes that may be associated with this element are:

- Service name

- Source name

- Object name

- Source username 
- Source password

\section{Service Name}

The table: name attribute specifies the name of the service. The value of this attribute is implementation specific.

4949

4950

4951

4952

4953

4954

4955

4956

4957

4958

\section{Object Name}

The table:object-name attribute specifies the name of the object in the source which contains the data and is passed to the service implementation. Its value is application and service specific.

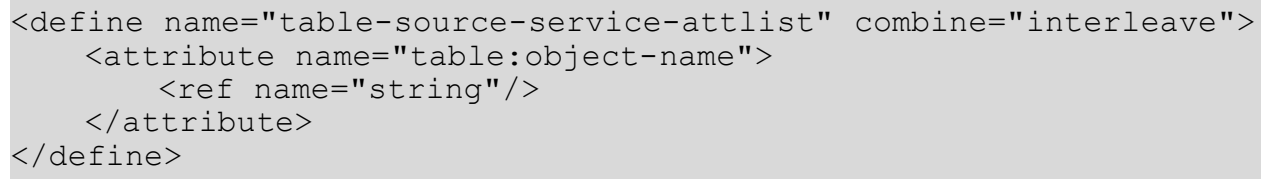

\section{Source Username}

The table: user-name attribute specifies the username required to access the source. It is passed to the service implementation. Its value is application and service specific.

\section{Source Password}

The table:password attribute specifies the password required to access the source. It is passed to the service implementation. Its value is application and service specific.

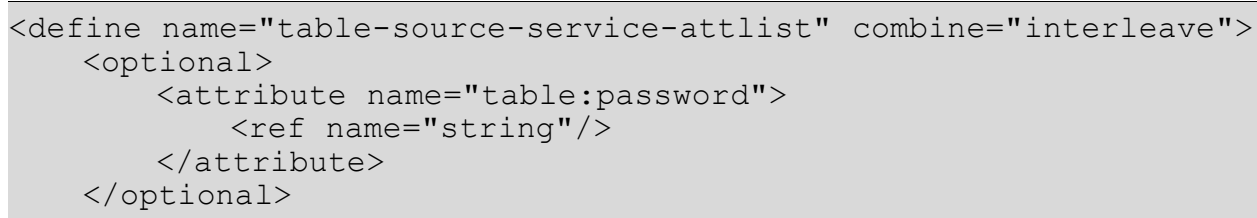




\subsubsection{Data Pilot Field}

A data pilot table's fields are specified by $<t a b l e:$ data-pilot-field $>$ elements.

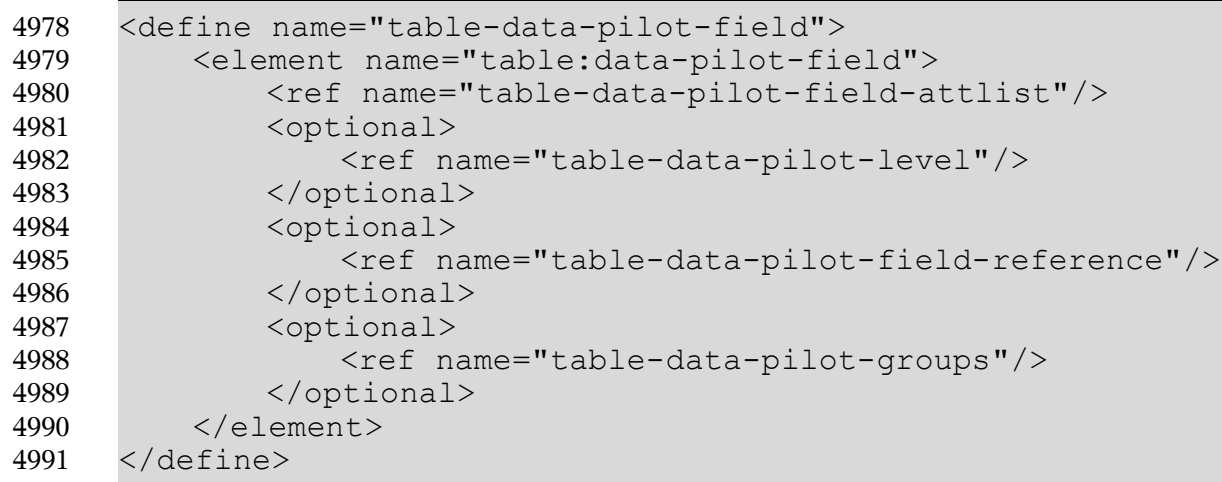

The attributes that may be associated with the data pilot field element are:

- Source field name

- Orientation

- Is data layout field

- Function

- Used hierarchy

\section{Source Field Name}

For fields that specify category or data columns, the table:source-field-name attribute specifies the name or label of the column the field is connected to. If the source of the data pilot table is for instance a cell range, then the attribute's value has to be the column's label.

There can be multiple $<$ table:data-pilot-field> elements with the same value for this attribute.

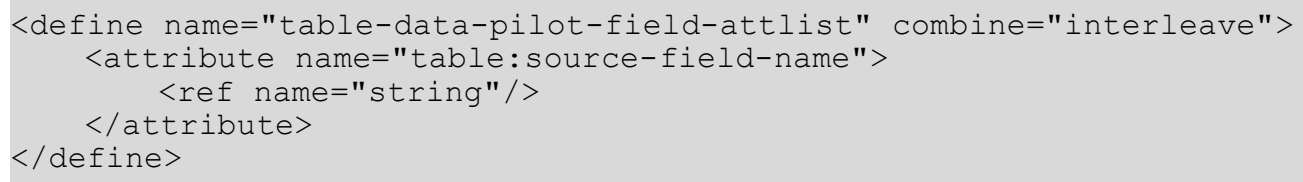

\section{Orientation}

The table:orientation attribute specifies the orientation of the source field. If the value is data, then the field specifies a data column. If the value is row or column, then the field specifies a category column. The value hidden is used for fields that have a corresponding column in the data pilot's source, but are not visible within the data pilot table. The value page indicates that an automatic filter (i.e. one that allows to choose one of the values that are contained in the column) should be generated for the corresponding column. In this case, an additional field with row, column or data orientation has to exist for the column.

If the attribute value is page, the table:selected-page attribute can be used to specify which value is selected for the filter. 


\section{Is Data Layout Field}

The table: is-data-layout-field attribute specifies whether a field is an data layout field (see section 8.8.1). Data layout fields usually don't have a name.

\section{Function}

The table: function attribute specifies the function which is applied to the cell values of data columns. It is only evaluated if the value of the table:orientation attribute is data.

Possible values for this attribute are: auto, average, count, countnums, max, min, product, stdev, stdevp, sum, var and varp. For category columns the attribute's value auto can be used that specifies that no function is applied at all.

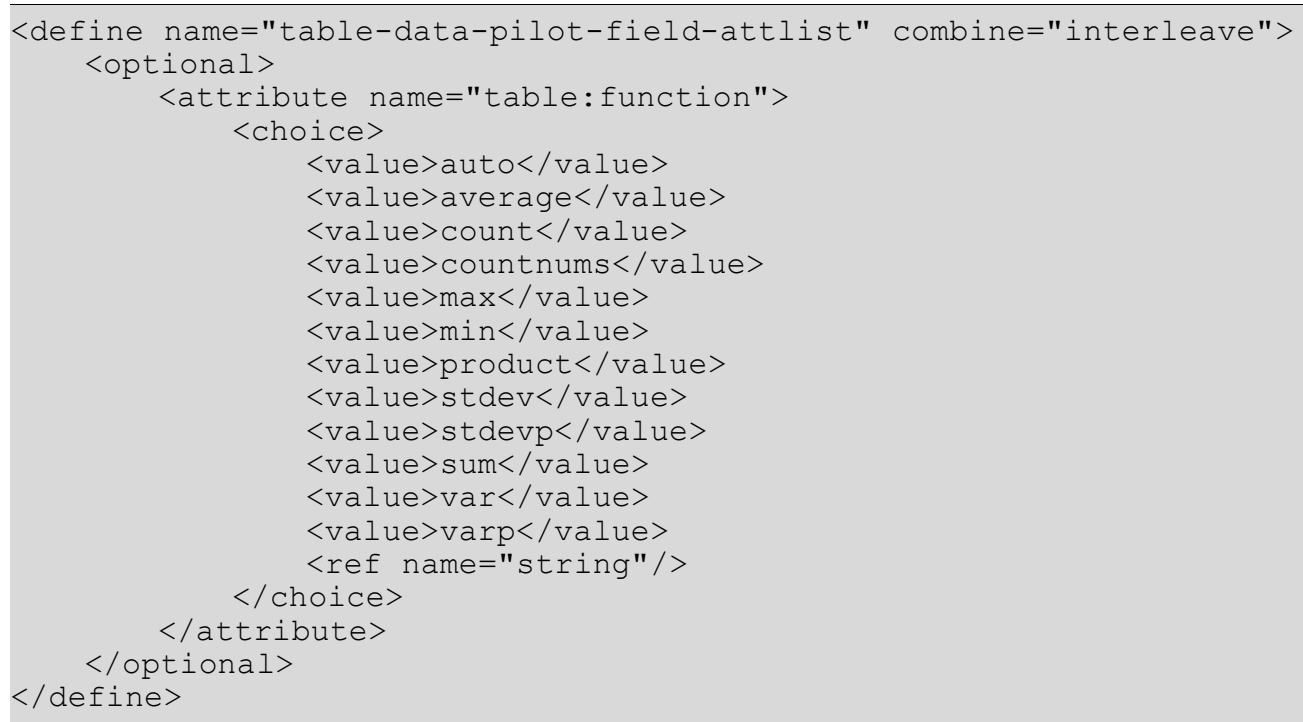




\section{Used Hierarchy}

If the data pilot source is provided by an external component or service, the data contained within category columns may not only grouped by its value, but it may be further divided into sub groups or hierarchies. A date value for instance might be grouped by

- "year", "month" and "day of month", or

- "year", "week" and "day of week"

If an external components supports hierarchies, it has to assign unique numbers to it. These numbers can be used in the table: used-hierarchy attribute to select the hierarchy that should be applied to the source field. The value means that no hierarchy should be applied at all.

\subsubsection{Data Pilot Level}

The data pilot level element $<t a b l e:$ data-pilot-level> contains additional information about a data pilot field.

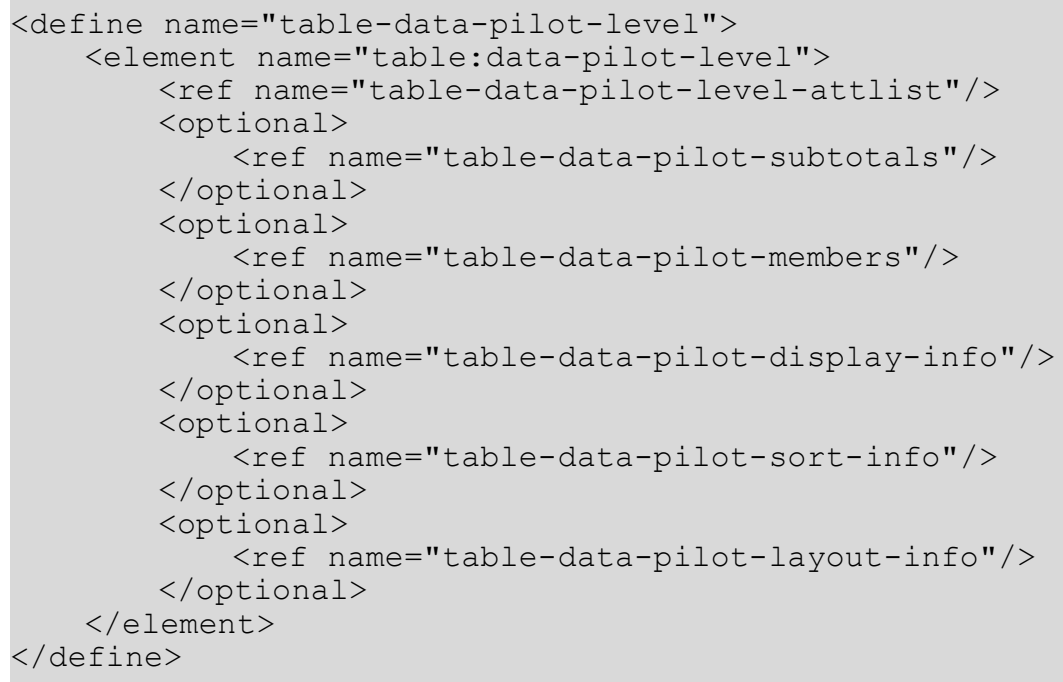

The attribute that may be associated associate with the data pilot level element is:

\section{- Show empty}

\section{Show Empty}

The table:show-empty attribute specifies whether or not fields that don't have any members should be displayed. If this attribute is not present, the application might or might not display such fields.

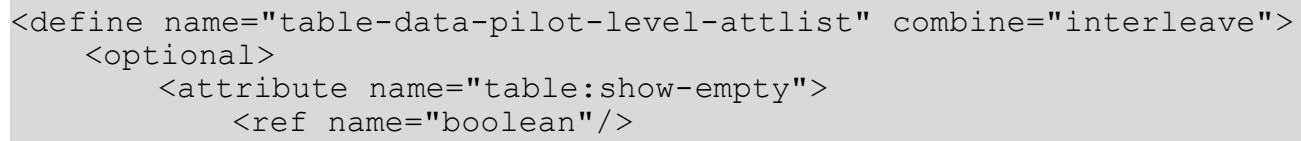




\subsubsection{Data Pilot Subtotals}

The data pilot subtotals element $<$ table: data-pilot-subtotals $>$ contains information about the provisional results that are displayed for every member of a field and the function used to calculate the result. Several provisional results can be calculated simultaneously. If the element is not present, the application might or might not display provisional results.

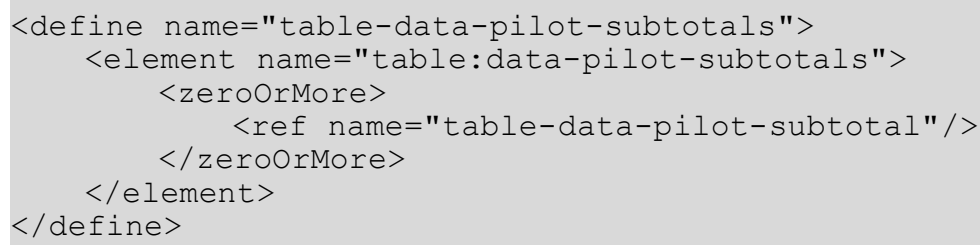

\subsubsection{Data Pilot Subtotal}

The data pilot subtotal element <table: data-pilot-subtotal>contains information about a single provision result calculation.

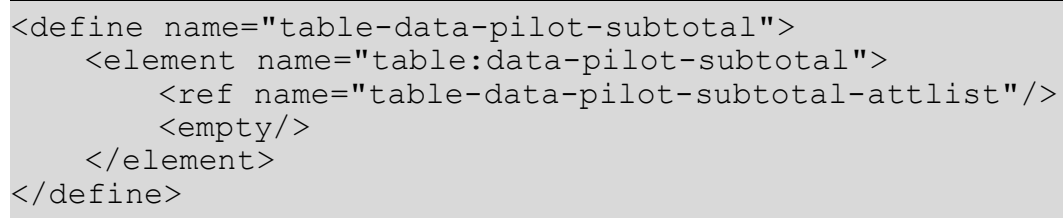

The attribute that may be associated associate with the data pilot subtotal element is:

- Function

\section{Function}

The table: function attribute specifies the function used for the subtotal. Possible functions are auto, average, count, countnums, max, min, product, stdev, stdevp, sum, var and varp.

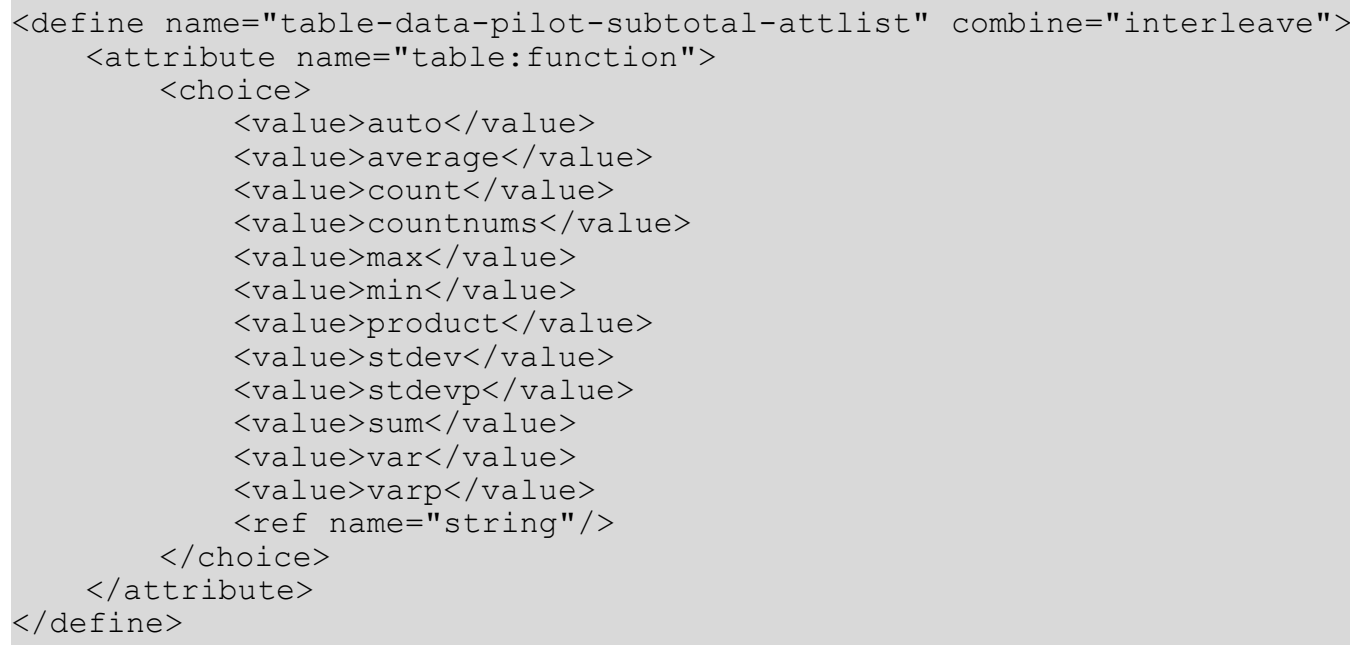




\subsubsection{Data Pilot Members}

For category columns, it can be controlled whether certain members themselves or the information displayed for a certain member actually is displayed or not. The <table: datapilot-members > element contains such information.

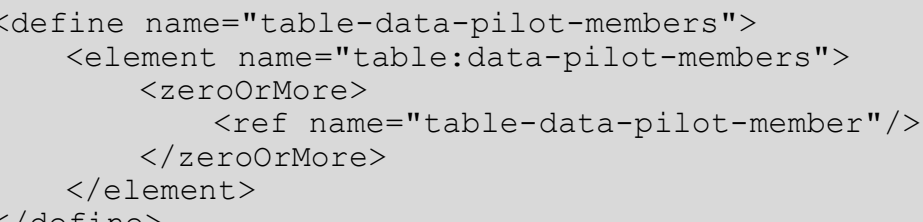

\section{$</ e l e m e n t>$}

\subsubsection{Data Pilot Member}

The data pilot member element $<$ table: data-pilot-member $>$ specifies which information is displayed for a certain member.

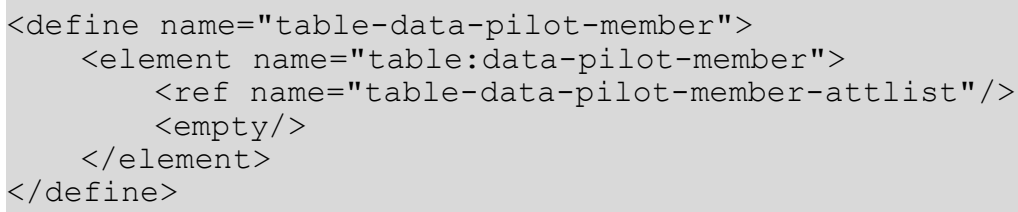

The attributes that may be associated with the data pilot member element are:

- Member name

- Display

- Show details

\section{Member Name}

The table: name attribute specifies the value for which display information is specified.

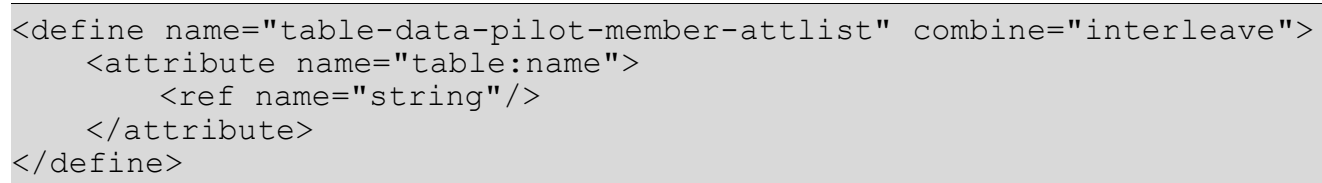

\section{Display}

The table: display attribute specifies whether or not a data pilot member is visible at all. If this attribute is not present, the application might or might not display the member.

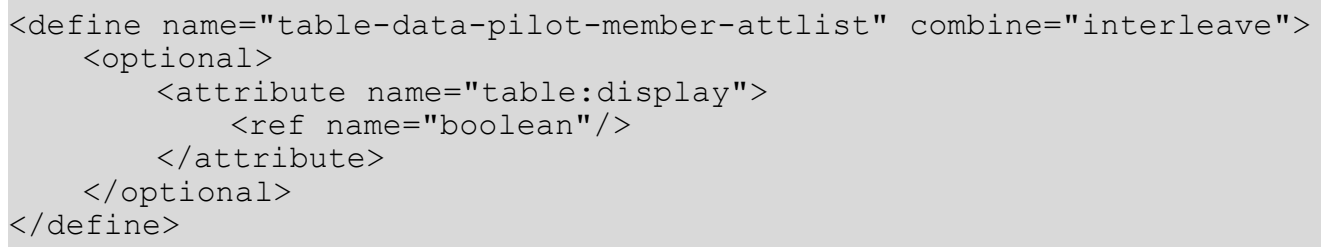




\section{Show Details}

The table:show-details attribute specifies whether additional fields are displayed for a member. This attribute changes the behavior of a data pilot only if there are several fields with the orientation row or column. If this is the case, and if the attribute's value is false for a field with row or column orientation that is not the last field with this orientation, then no members are displayed for all following fields with the same orientation. Instead of this, the data displayed for these fields will be summarized.

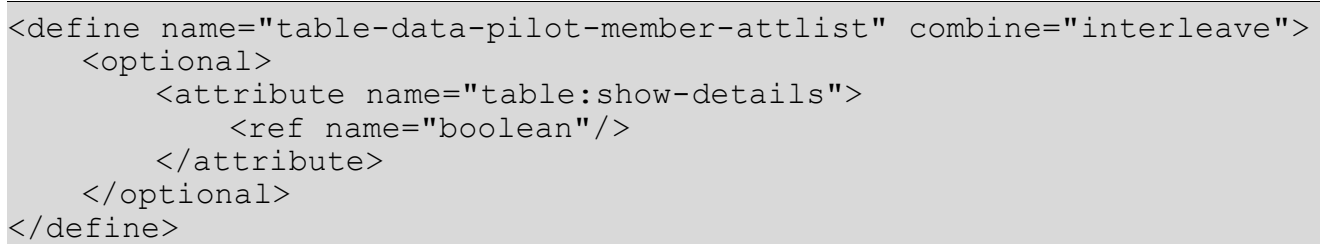

\subsubsection{Data Pilot Display Info}

The <table:data-pilot-display-info> element restricts the number rows that are displayed for a category field to a specific number of values of a data field.

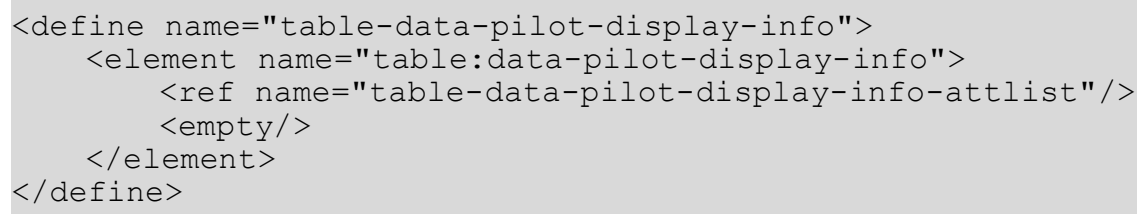

\section{Enabled}

The table:enabled attribute specifies whether the <table:data-pilot-display-info> element is evaluated or not.

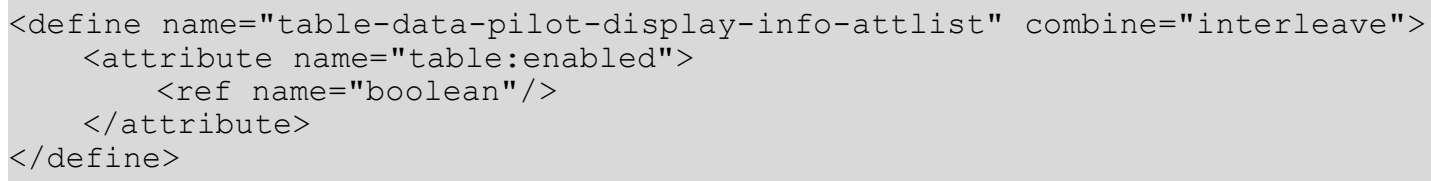

\section{Data Field}

The table:data-field attribute specifies the data field whose values are taken into account.

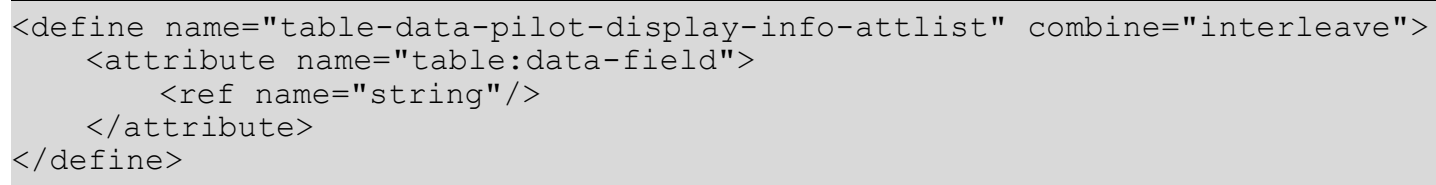

\section{Member Count}

The table:member-count attribute specifies how many values from the top or from the bottom of data field's column are shown.

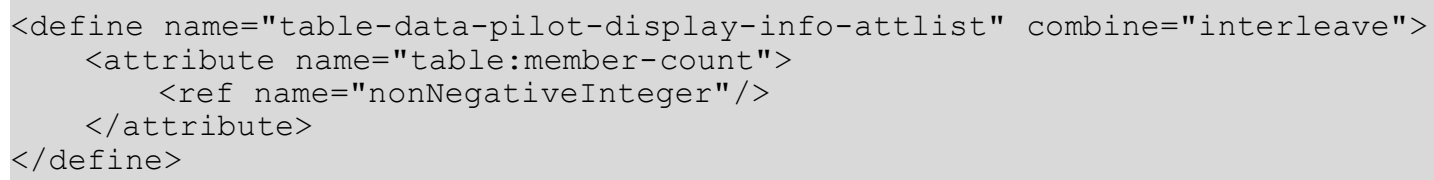




\section{Display Member Mode}

The table:display-member-mode attribute specifies whether the values specified by table:member-count should be taken from the top or from the bottom of a data field's column.

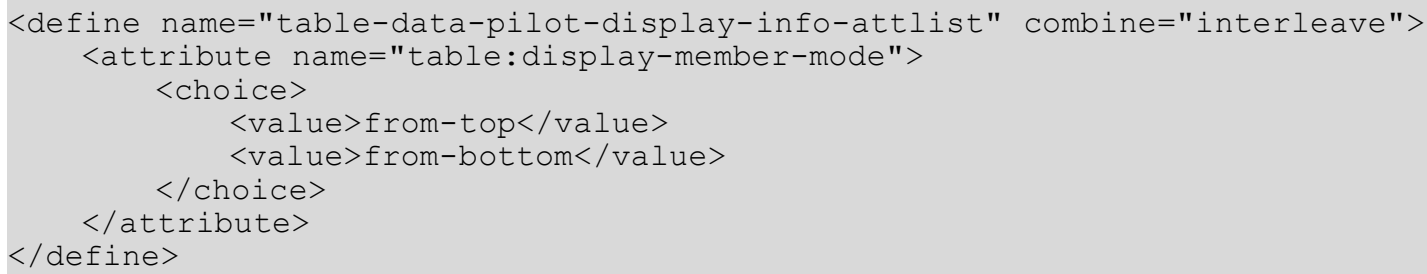

\subsubsection{Data Pilot Sort Info}

The <table:data-pilot-sort-info> element specifies how the members of a category field are sorted.

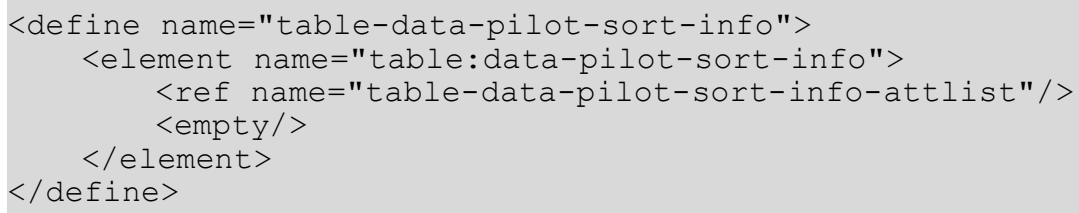

\section{Sort Mode}

The table:sort-mode attribute describes how to sort the members of a single data pilot field. If the mode is data, then the members of the current category field a sorted according to their values in the data field specified by the table:data-field attribute. If the mode is manual, the user can sort the members in the field manually. If the mode is name, the members in the field are sorted by their name.

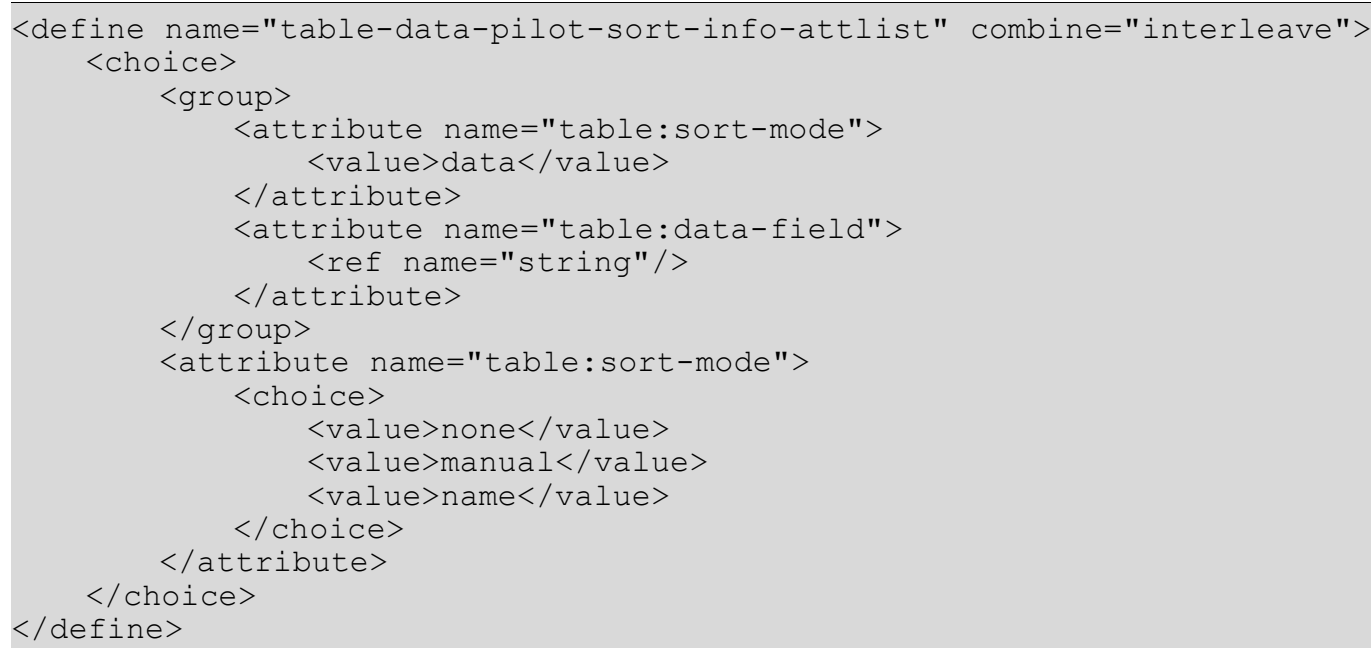

\section{Sort Order}

The table:sort-order attribute specifies whether to sort the members ascending or descending. 


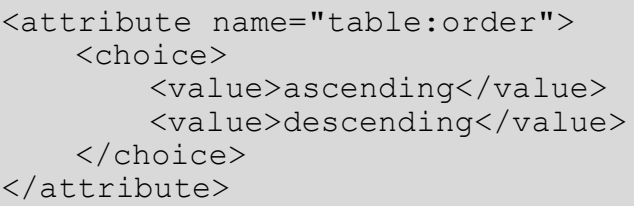

\subsubsection{Data Pilot Layout Info}

The <table:data-pilot-layout-info> element describes how to layout the field.

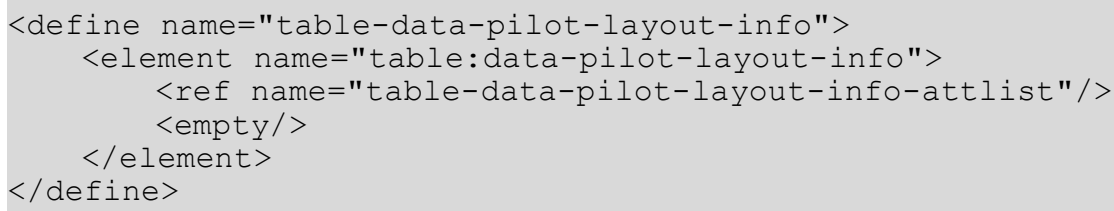

\section{Layout Mode}

The table: layout-mode attribute describes how to layout the field. It may have the following values:

- tabular-layout: Tabular layout mode is the layout, where each member's name is on the same row as the first member from the following field. Subtotals are always shown below an member's data in this mode.

- outline-subtotals-top: In outline layout mode, the members from the following field start in the row below a member's name, like in traditional database reports. Subtotals are shown at the top (in the same row as the member's name). When the subtotals take up more than one row (manually selected, or because there are several data fields), they are always shown below the member's data, regardless of the setting.

- outline-subtotals-bottom: Like outline-subtotals-top, except that subtotals are shown at the bottom (below the member's data, as in tabular layout mode).

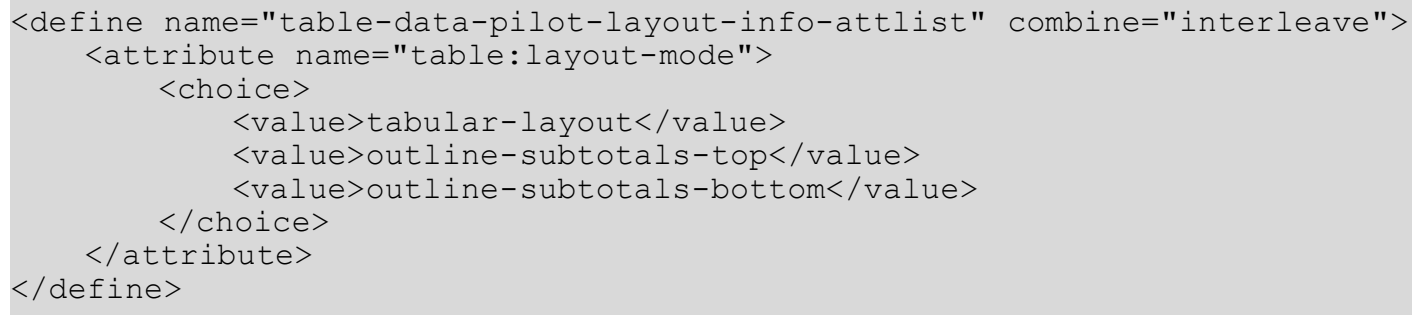

\section{Add empty lines}

If the attribute table:add-empty-lines has the value true, an empty row is inserted in the data pilot table after the data (including the subtotals) for each member of the field.

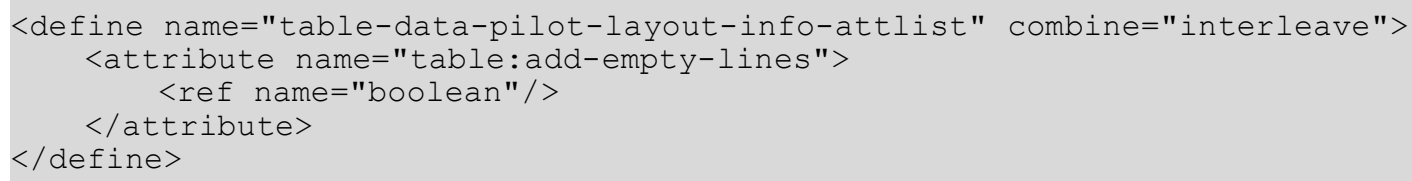




\subsubsection{Data Pilot Field Reference}

The <table:data-pilot-field-reference> element describes data which can be used to modify the displayed values of data fields.

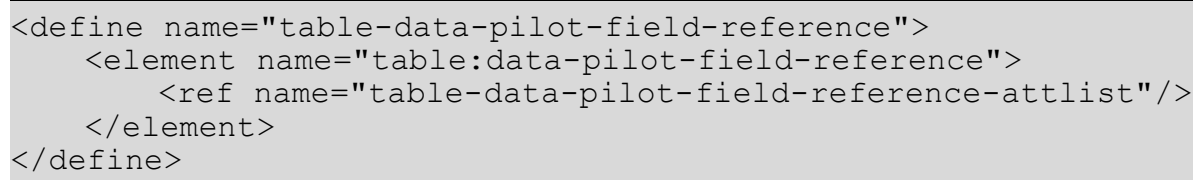

\section{Reference Field}

The table:field-name attribute references a category field whose members influence the displayed values of the data field the <table:data-pilot-field-reference $>$ is part of.

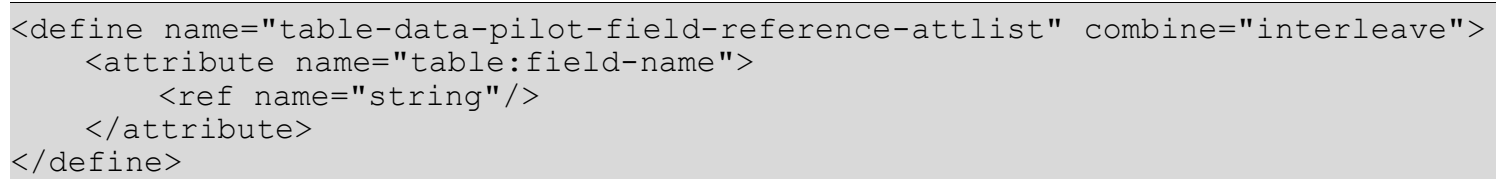

\section{Reference Member Type}

The table:member-type attribute specifies the member of the referenced category field, whose value within the current data field has to be taken into account. If its value is next (previous) then the value of the data field for the next (previous) visible member of the referenced category field will be taken into account. If its value is named, then the table:member-name specifies the member whose value within the data field is taken into account.

For previous and next, empty members are skipped.

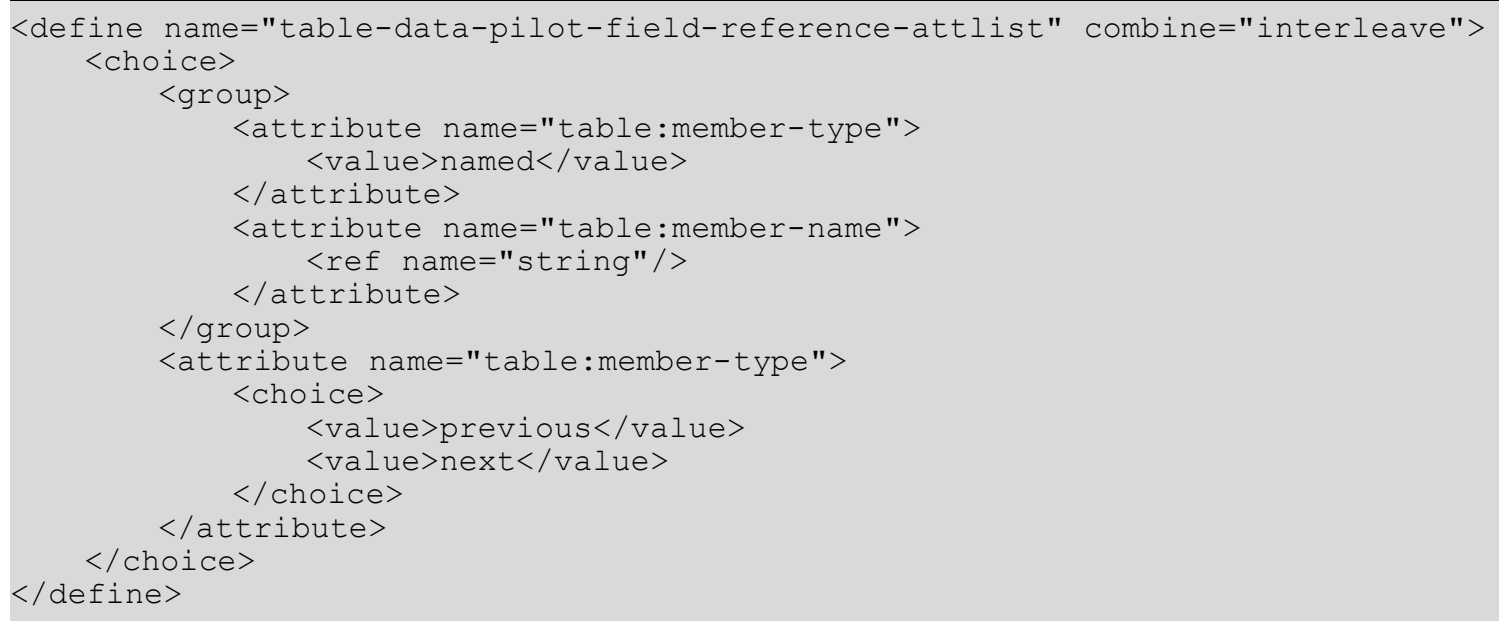

\section{Reference Type}

The table:type attribute specifies the how the referenced category field influences the displayed values of the data field. It may have one of the following values:

- none: This value means that the results in the data fields are displayed unmodified. 
- member-difference: From each result, the value calculated for the category field member specified by the table:member-type and table:member-name attributes is subtracted.

- member-percentage: Each result is divided by the value calculated for the category field member specified by the table:member-type and table:member-name attributes.

Division by zero results in an error. Empty results are shown as "0". If the table:membertype attribute has the value previous, " 1 " is displayed as first value. If the table:member-type attribute has the value next, "1" is displayed as last value.

- member-percentage-difference: From each result, the value calculated for the category field member specified by the table:member-type and table:member-name attributes is subtracted, and the result is divided by this value again. Division by zero results in an error. Otherwise, the rules for member-difference apply.

- running-total: Each result is added to the sum of the results for preceding members in the referenced category field, in the reference field's sort order, and the total sum is shown.

- row-percentage: Each result is divided by the total result for its row in the data pilot table. If there are several data fields, the total for the result's data field is used. If there are subtotals with manually selected summary functions, the total is calculated with the data field's summary function. Division by zero results in an error.

- column-percentage: Same as row-percentage, but the total for the result's column is used.

- total-percentage: Same as row-percentage, but the grand total for the result's data field is used.

- index: The row and column totals and the grand total are calculated as described above, and then are used to calculate the following expression: (original result * grand total ) / ( row total ${ }^{*}$ column total ).Division by zero results in an error.

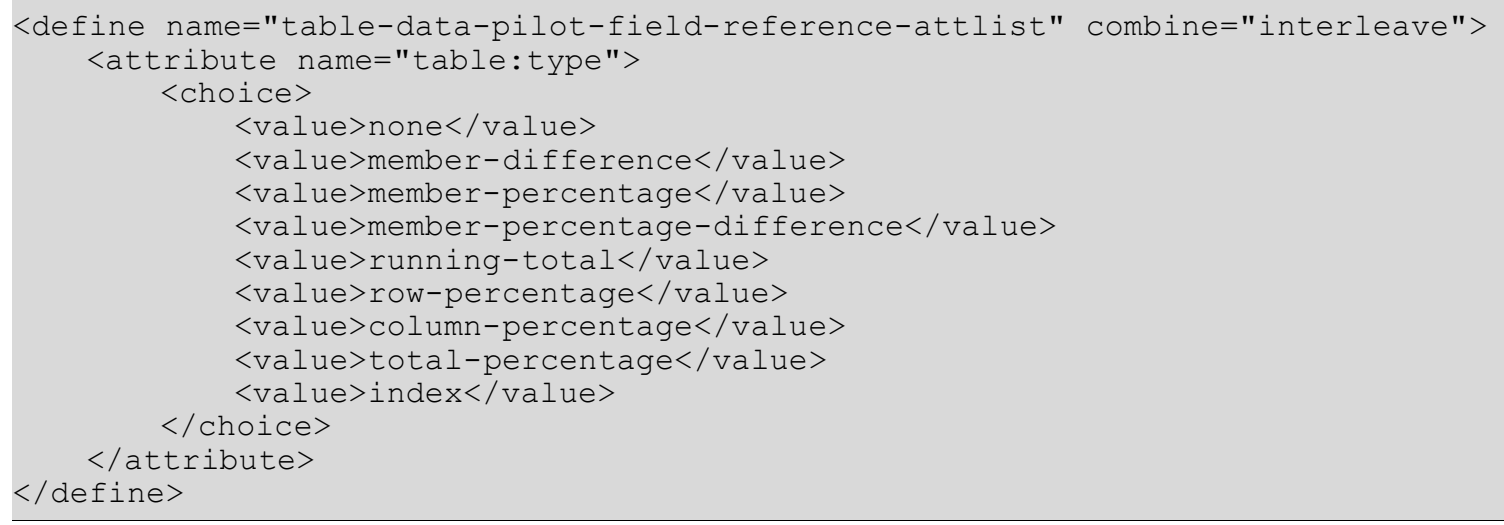

\subsubsection{Data Pilot Groups}

The <table:data-pilot-groups> element specifies that a data pilot field is a group field. A group field allows grouping of other fields. For example, if a data pilot table contains a column field with the name "city" which has the members "Berlin", "Munich", "Frankfurt", "Hamburg", "London", "Manchester", "Hastings" and "Liverpool", then one may want to group the cities by their countries. To do so, a group field with name "city2" could be added to the data pilot table, that contains two groups called "England" and "Germany". Each group here contains a list of the names of its members. In this example, the group "England" would contain "London", "Manchester", "Hastings" and "Liverpool". The group "Germany" would contain "Berlin", "Munich", "Frankfurt" and "Hamburg". 
Grouping may also take place for numeric or date values.

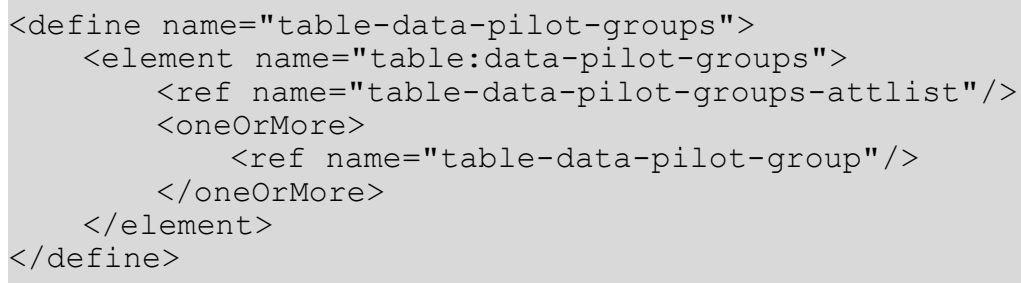

\section{Source Field Name}

The table:source-field-name attribute references the field containing the data that is grouped, if this data differs from the data that is referenced by the field itself.

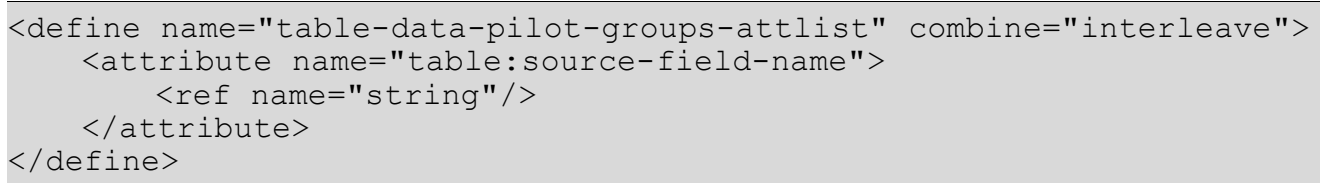

\section{Start}

If numeric or date values are grouped, the table:date-start and table:start attributes specify the start value for the grouping. All values that are lower than the start value are contained in a single group, while values that are equal to or higher than the start value are grouped as specified by the table: grouped-by and table:step attributes.

If the attribute's value is auto, the lowest value of the field is taken as start value.

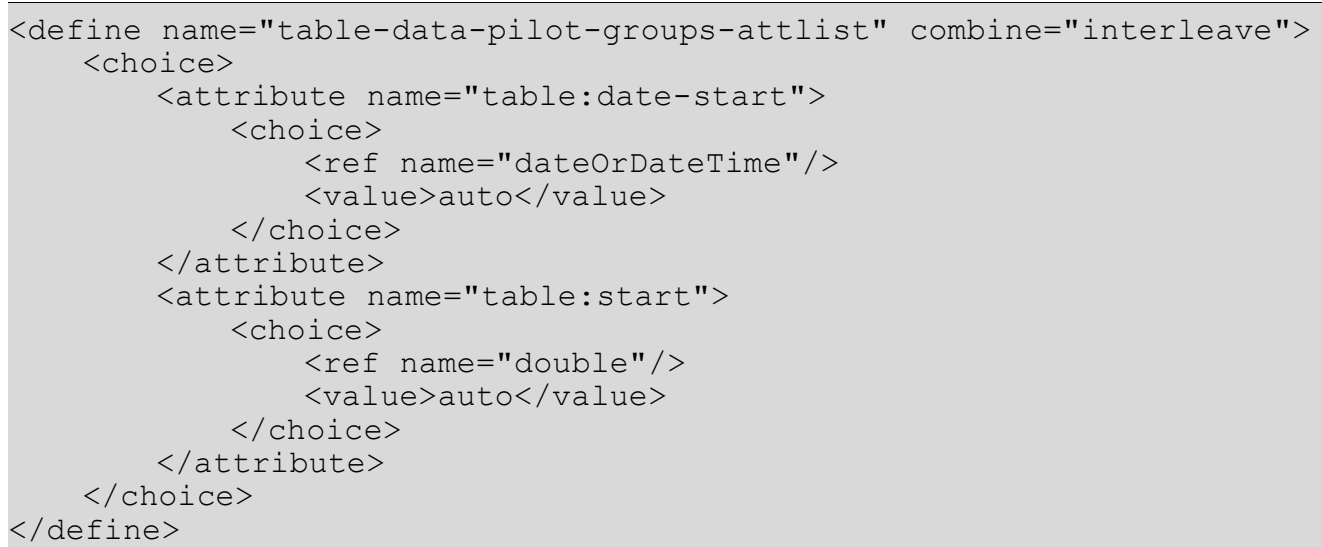

\section{End}

If numeric or date values are grouped, the table: date-end and table: end attributes specify the end value for the grouping. All values that are higher than the end value are contained in a single group, while values that are equal to or lower than the end value are grouped as specified by the table: grouped-by and table:step attributes.

If the attribute's value is auto, the highest value of the field is taken as end value.

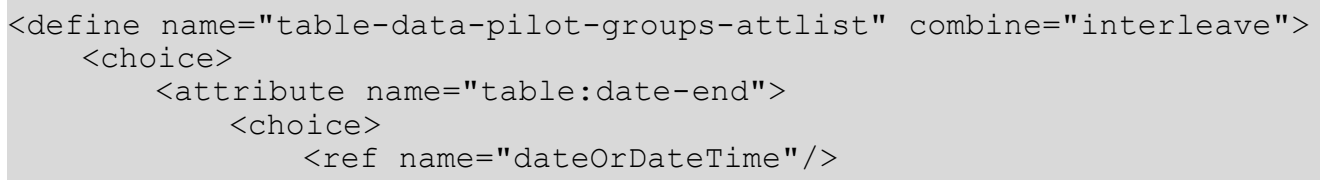




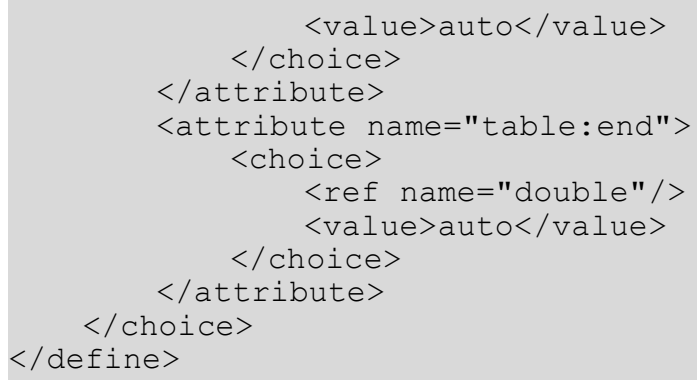

$</$ choice $>$

\section{Step}

The table:step attribute specifies the grouping of numeric values, by specifying the distance between the groups. For example, if the table:start attribute for the grouping has the value 5 , and the table:step attribute has the value 2, all values that are equal to or higher than 5 , but also lower than 7 are in one group. All values that are equal to or higher than 7, but also lower than 9 are in next group, and so on, until the end value is reached.

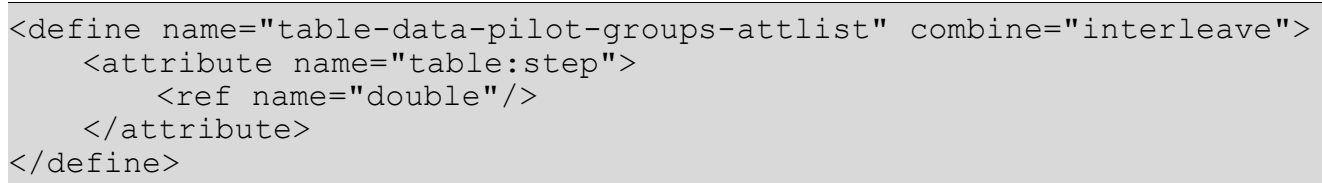

\section{Grouped By}

The table:grouped-by attribute specifies the grouping of the date values. Date values can be grouped by seconds, minutes, hours, days, months, quarters or years. It date values are for instance grouped by minutes, all dates or times that are within the same minute are within one group. That, is if the dates 2004-08-27T12:34:46,2004-08-27T12:34:56 and 2004-08$27 \mathrm{~T} 12: 35: 46$ are given, the first two would be within one group, while the last date would be a group of its own.

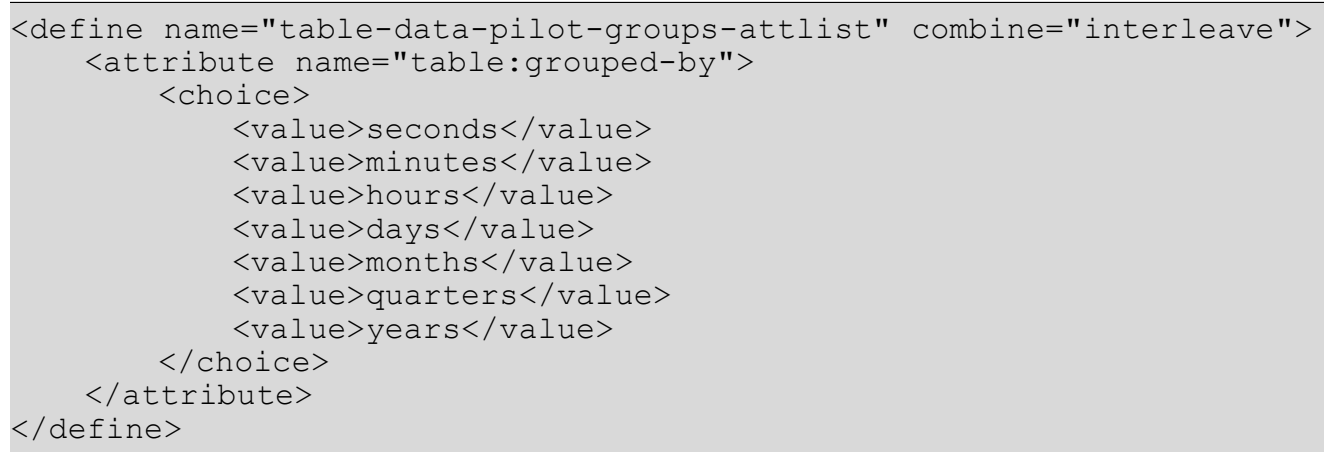

\subsubsection{Data Pilot Group}

If grouping takes place by specifying the member names, then the $<$ table: data-pilotgroup $>$ element specifies the member names of a single group.

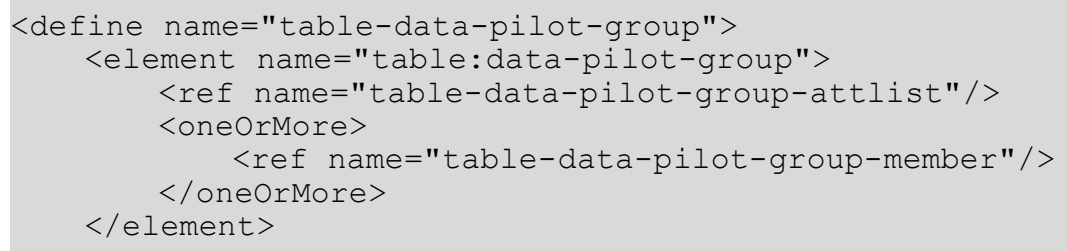




\section{Name}

The table: name attribute specifies the name of the group.

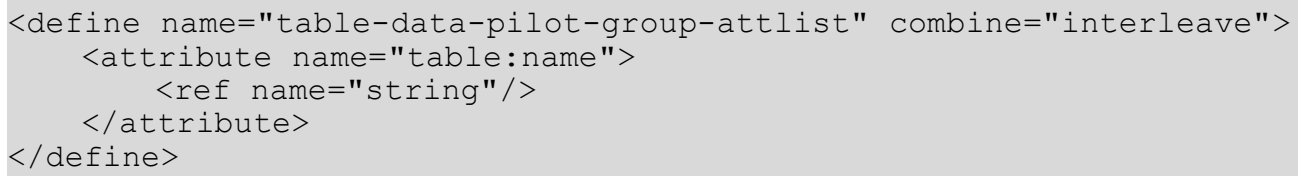

\subsubsection{Data Pilot Group Member}

The <table:data-pilot-group-member $>$ element specifies the name of a single group member.

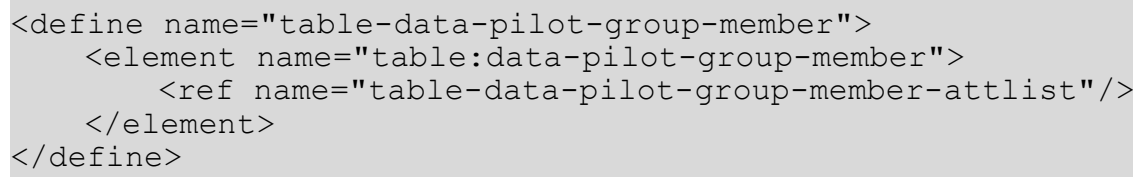

\section{Name}

The table: name attribute specifies the name of the member.

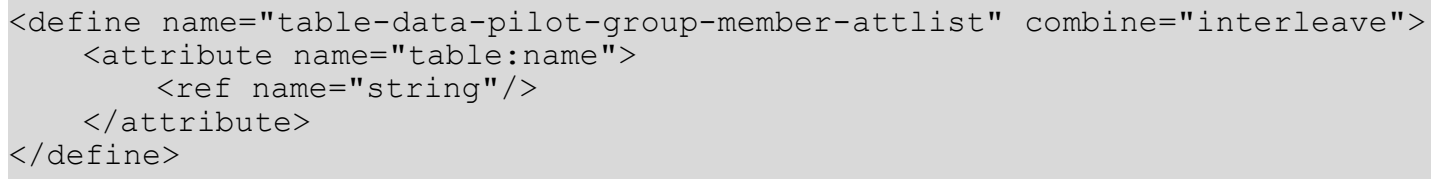

\subsection{Consolidation}

A consolidation combines data from several independent table ranges. A new table range is calculated by applying a mathematical function to all cells in the source table ranges that have the same relative address within these ranges. A consolidation is defined by the $<$ table:consolidation> element.

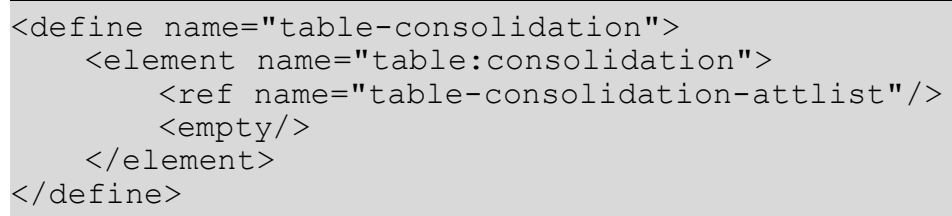

The attributes that may be associated with this element are:

- Function

- Source cell range addresses

- Target cell address

- Use label

- Link to source data 


\section{Link to Source Data}

The table:link-to-source-data attribute specifies whether the data in the consolidation table range should be linked to the source data, so that it is automatically updated if any changes are made to the source data.

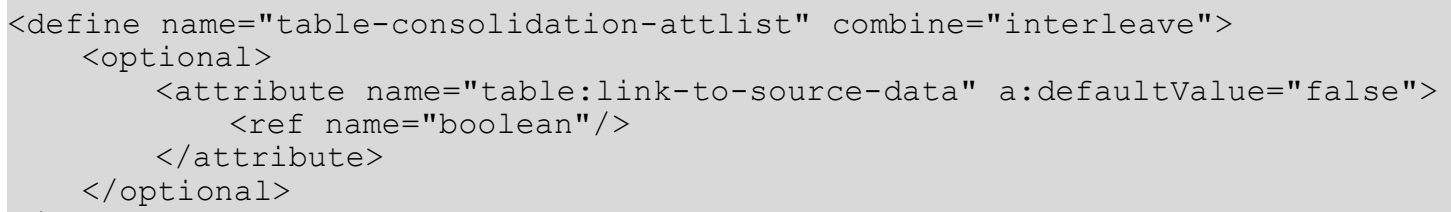

\subsection{DDE Links}

The $<$ table: dde-links $>$ container element stores all DDE links within a spreadsheet document. Every link contains the DDE Source and the data of the last connection. See section 12.6.3 for details.

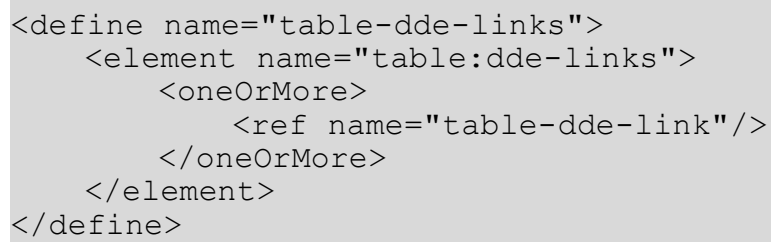

\subsection{Change Tracking in Spreadsheets}

Within spreadsheet documents, changes to tables can be tracked. This section describes how this change tracking information is represented.

Change tracking of tables is not supported for text documents.

\subsubsection{Tracked Changes}

All changes that have been applied to a spreadsheet document are stored in a list. The list contains an element for each change made to the document. To track the changes to a spreadsheet document, the <table:tracked-changes> element must be present.

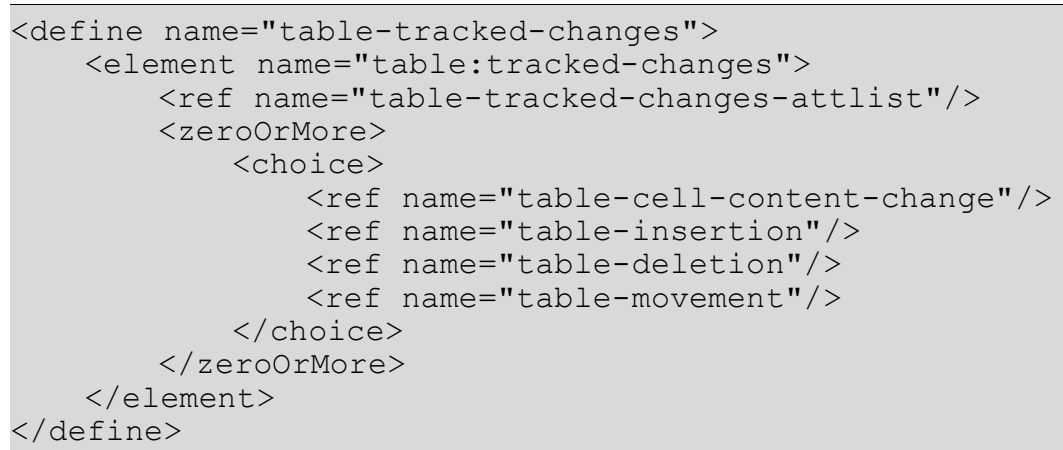




\section{Track Changes}

The table:track-changes attribute specifies whether or not the change tracking is enabled.

\subsubsection{Insertion}

The <table: insertion> element contains the information that is required to identify any insertion of content. This content can be one or more rows, one or more columns, or a table.

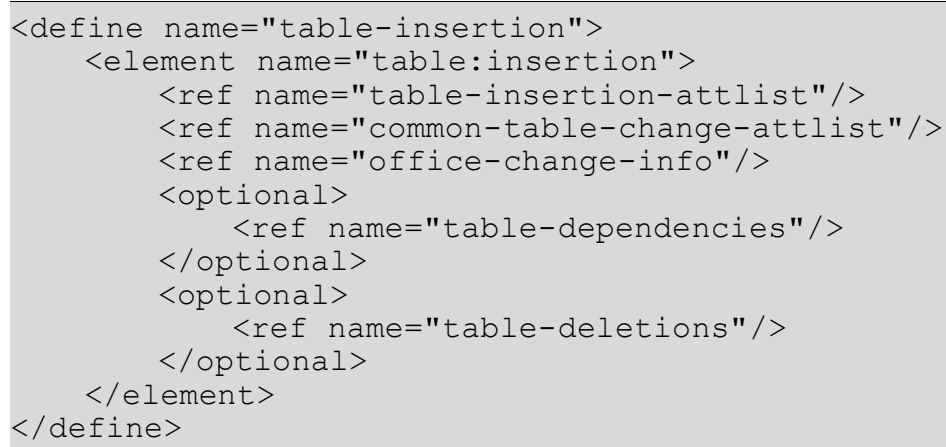

The attributes that may be associated with this element are:

- ID (see section 8.11.18)

- Acceptance State (see section 8.11.18)

- $\quad$ Rejecting Change ID (see section 8.11.18)

- Type

- Position

- Count

- Table

\section{Type}

The table:type attribute specifies the type of the insertion. It can be row, column or table. 


\section{Position}

The table:position attribute specifies the position where the insertion was made in the table. Depending on the insertion type, It is either the number of a row, a column or a table.

\section{Example: Insertion of text in a cell}

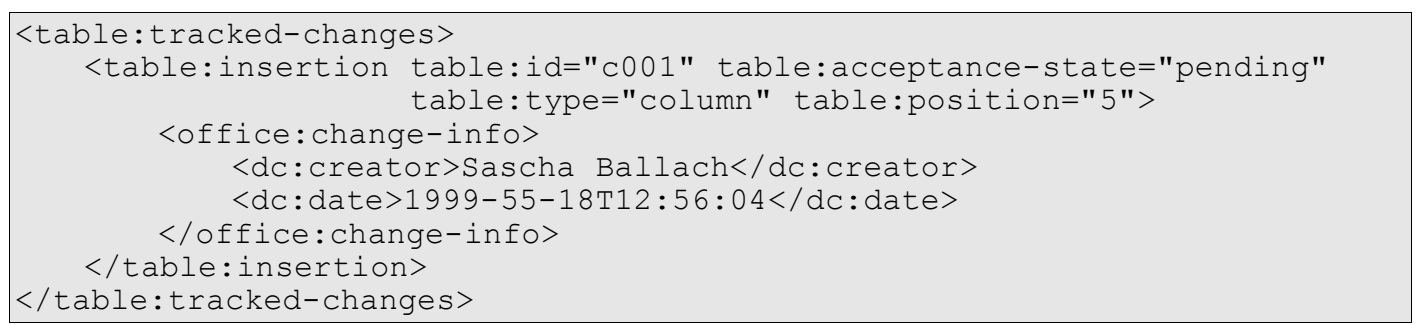

\subsubsection{Dependencies}

The $<$ table: dependencies $>$ element contains the information on which other tracked changes a tracked change depends. Every element of the tracked-changes can contain a $<$ table: dependencies> element.

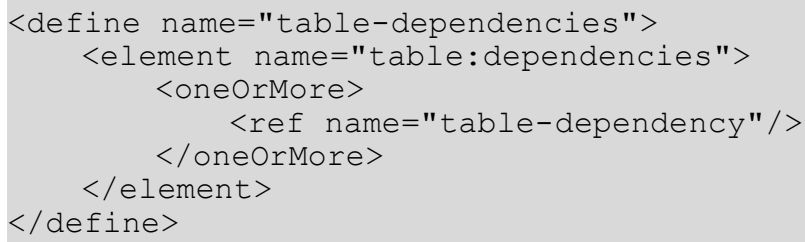




\subsubsection{Dependence}

The $<$ table: dependency $>$ element contains the information about one change action on which the parent element depends. The change action on which the current depends is referenced by an id.

\subsubsection{Cell Content Deletion}

The <table:cell-content-deletion> element specifies that a cell content has been deleted. It contains the address of the effected cell and its former content. If a text: id attribute is present, it specifies the id of a previously tracked change for the cell that gets deleted by the current change.

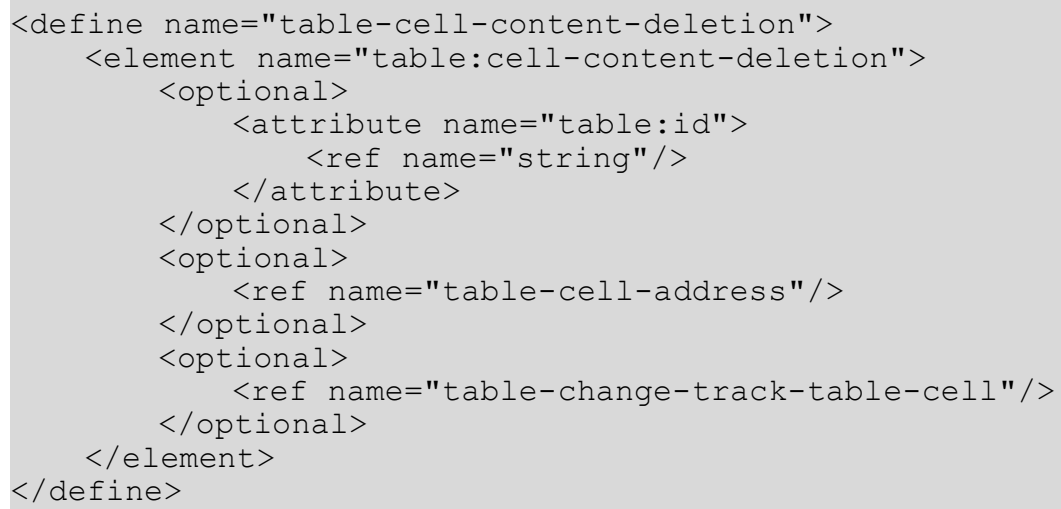

\subsubsection{Change Deletion}

The <table: change-deletion> element specified the id of a previously tracked change that gets deleted by the current change. 


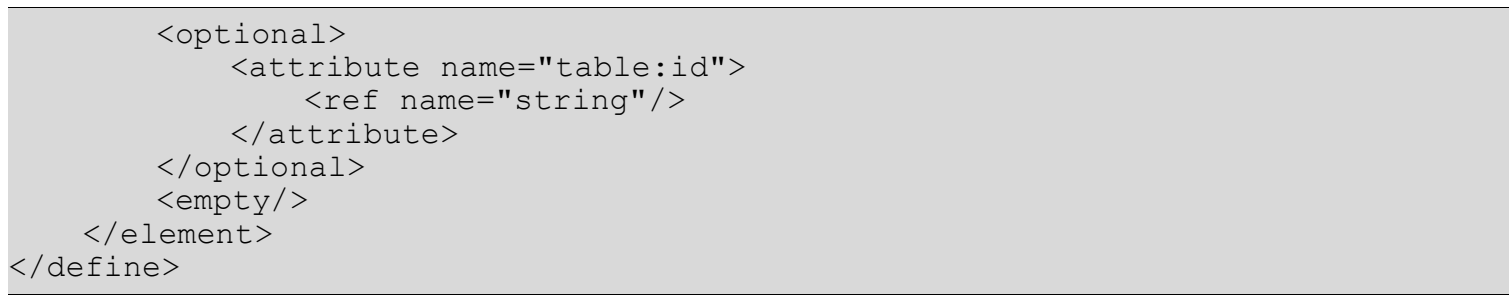

\subsubsection{Deletion}

$\mathrm{A}<$ table: deletion> element contains content that was deleted while change tracking was enabled. The content of a cell that was deleted is either contained in the $<$ table: dependencies>, or in the <table: deletions > element.

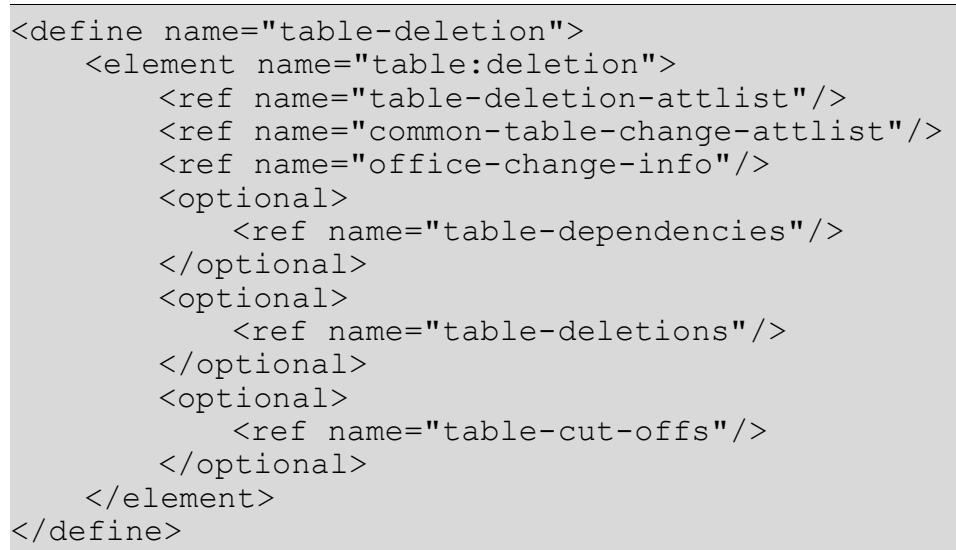

The attributes that may be associated with this element are:

- ID (see section 8.11.18)

- Acceptance State (see section 8.11.18)

- $\quad$ Rejecting Change ID (see section 8.11.18)

- Type

- Position

- Table

- Multi Deletion Spanned

\section{Type}

The table:type attribute specifies the type of the deletion. It can be row, column or table. 


\section{Position}

The table:position attribute specifies the position where the deletion was made in the table. Depending on the deletion type, It is either the number of a row, a column or a table.

5551

5552

5553

5554

5555

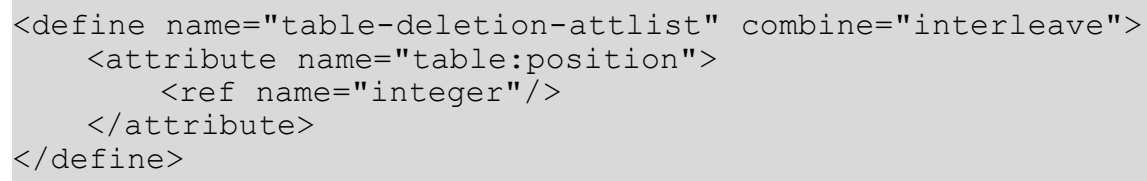

\section{Table}

The table:table attribute specifies the number of the table where the deletion took place. This attribute only exists for column and row deletions.

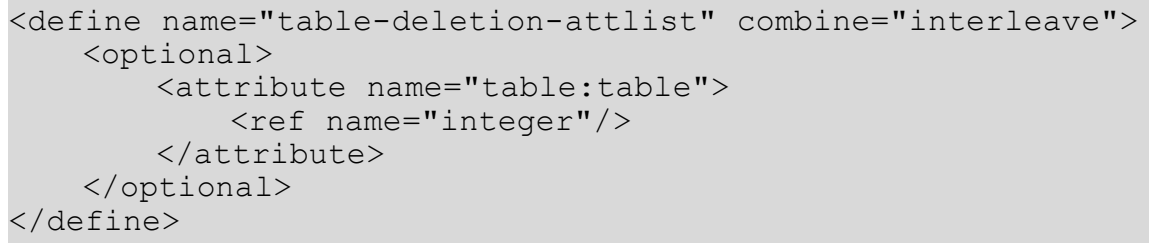

\section{Multi Deletion Spanned}

If multiple columns or rows were deleted simultaneously, each deleted row or column gets its own $<$ table: deletion> element. The element of the first deleted row or column in this case has to carry a table:multi-deletion-spanned attribute that specifies the total number of deleted rows or columns.

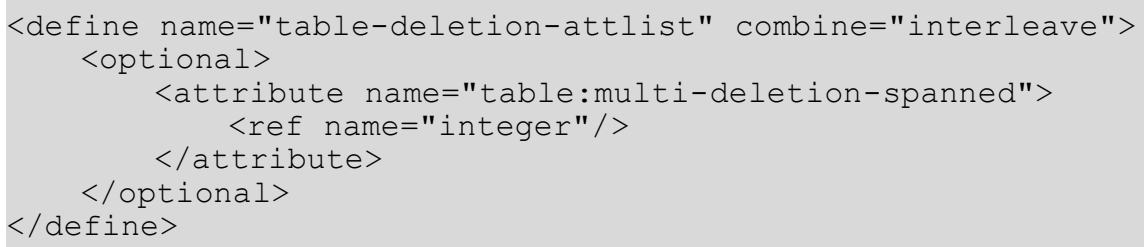

\subsubsection{Cut Offs}

A <table: cut-offs> element contains information about previously tracked insertions or movements where parts of the new content created by this operation now gets deleted. An example for this might be a cell range that has previously been moved and that now overlaps with a row that gets deleted.

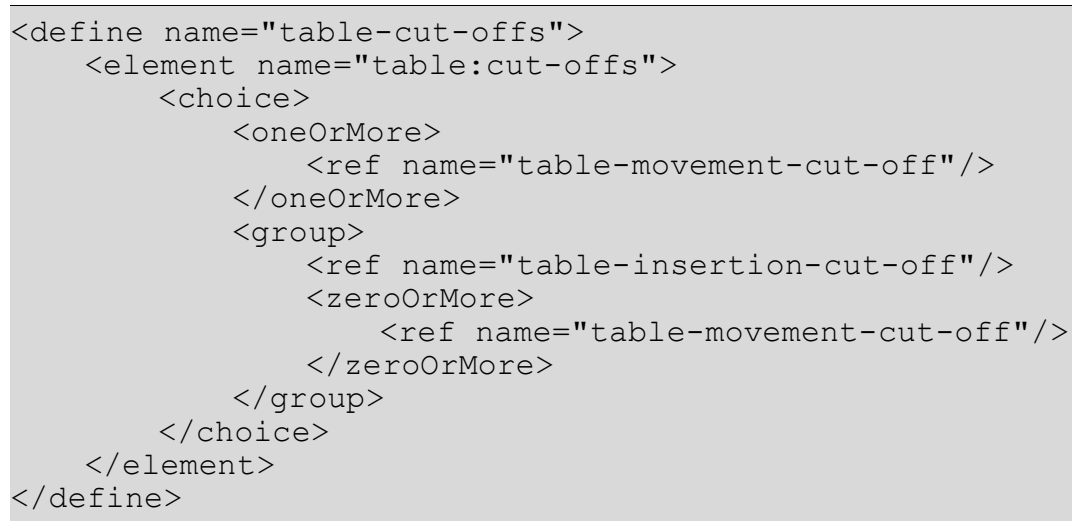




\subsubsection{Insertion Cut Off}

The <table: insertion-cut-off $>$ element contains the information where a insertion was deleted and which.

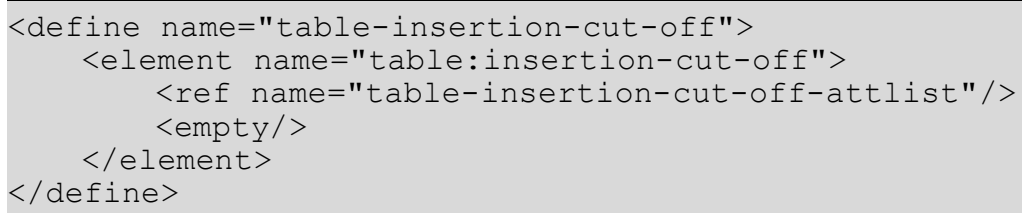

The attributes that may be associated with this element are:

- ID (see section 8.11.18)

- position

Id

The table: id attribute contains the id of the insertion where parts of now get deleted.

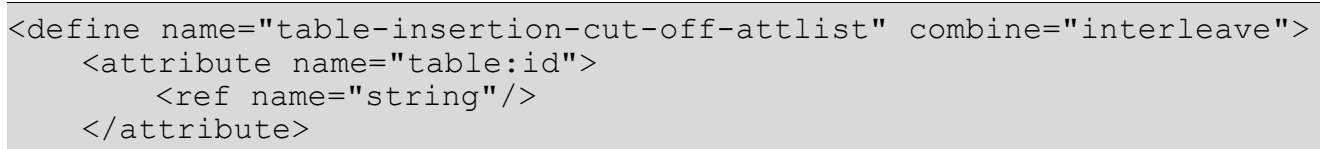

\section{Position}

The table:position attribute specifies the number of the row or column within the insertion that gets deleted.

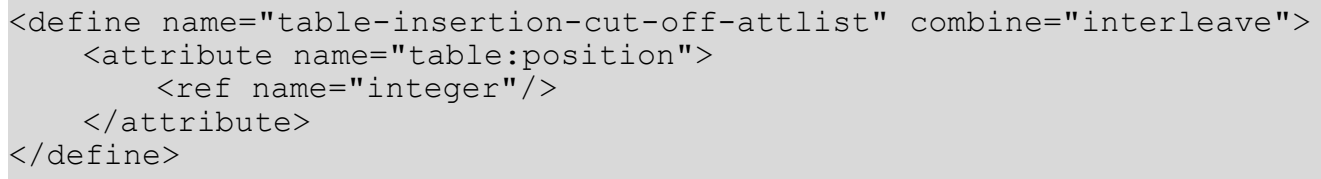

\subsubsection{Movement Cut Off}

The $<t a b l e$ :movement-cut-off $>$ element contains the information where a movement was deleted and which.

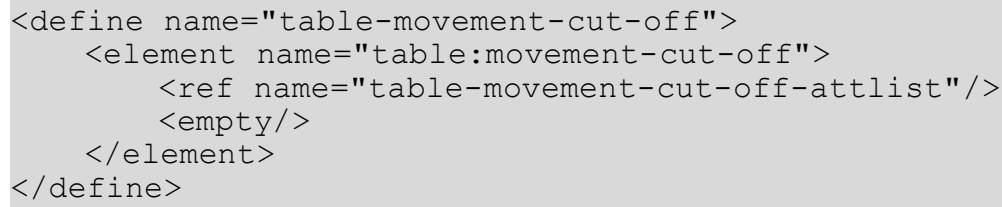

The attributes that may be associated with this element are:

- ID (see section 8.11.18)

- start position, end position, position 


\section{Start Position, End Position, Position}

The table:start-position, table:end-position and table:position attributes specify the position within the movement that gets deleted. If a single row or column gets deleted, the table:position attribute contains its number. If multiple rows or columns get deleted, the table:start-position and table:end-position attributes contain the number of the first (inclusive) and last (exclusive) deleted rows or columns.

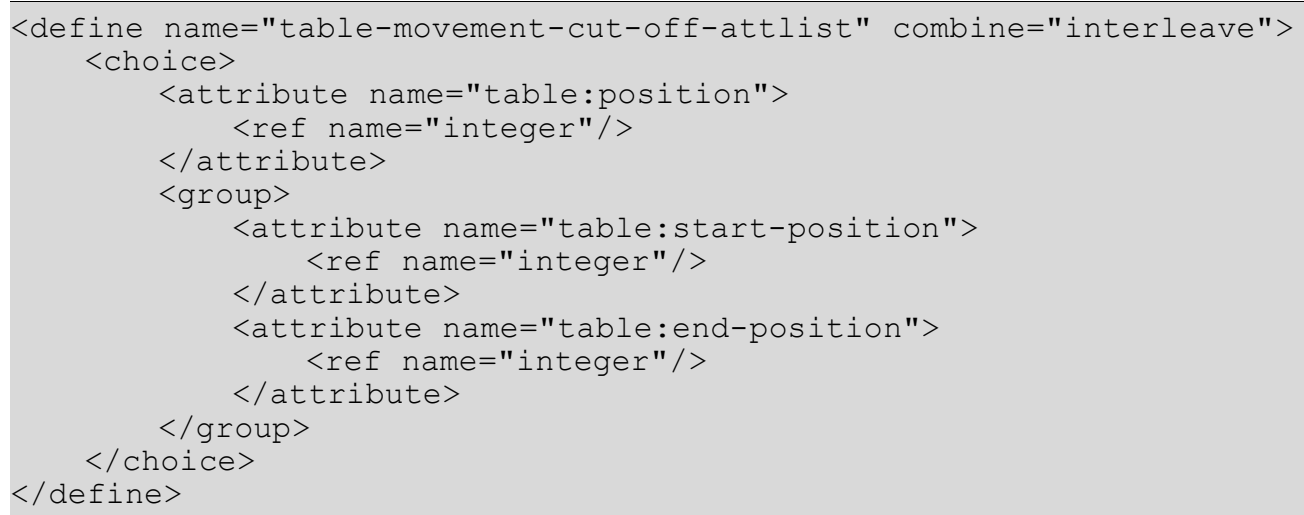

Example: Deletion of a column which do not contain content

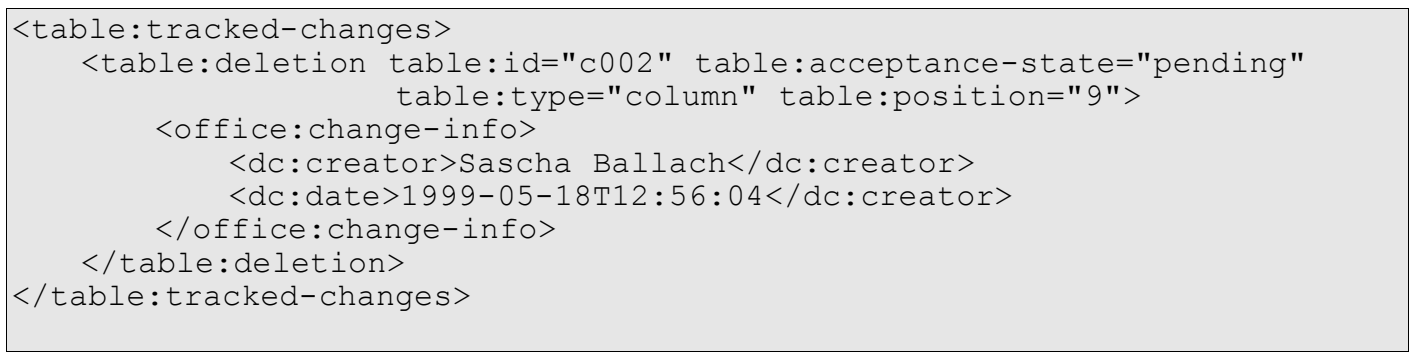

\subsubsection{Movement}

$\mathrm{A}<$ table:movement $>$ element contains the information that is required to identify any movement of content. This content can be a cell content or a cell range content.

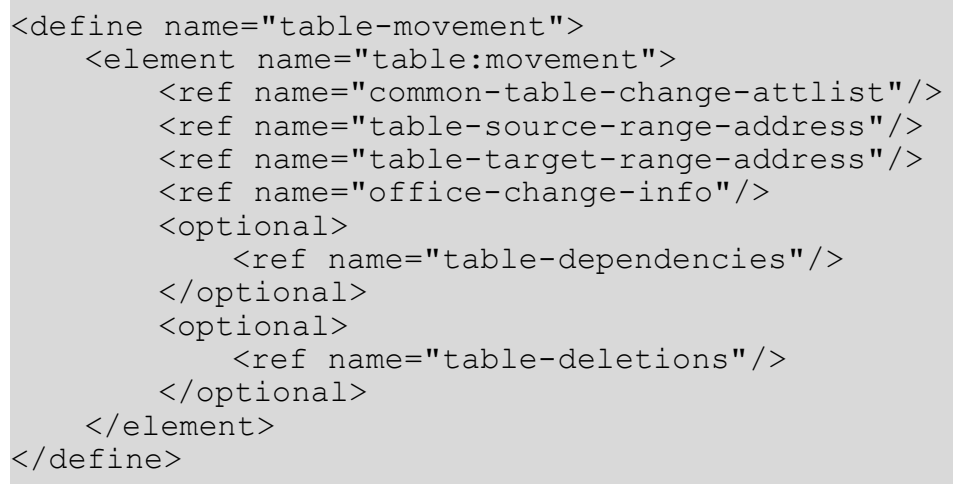

The attributes that may be associated with this element are:

- $\quad$ ID (see section 8.11.18)

- Acceptance State (see section 8.11.18)

- $\quad$ Rejecting Change ID (see section 8.11.18) 


\subsubsection{Target Range Address, Source Range Address}

The <table: source-range-address $>$ and $<$ table: target-range-address $>$ specify the source and target cell address or cell range address of a movement.

\section{Start Column, End Column, Start Row, End Row, Start Table, and End Table \\ If the range address is a cell range address instead of a cell address, the attributes table:start-column, table:end-column, table:start-row, table:end-row, table:start-table and table:end-table specify the start and end columns, rows and tables of the range. Start and end numbers both are inclusive.}

The attributes that may be associated with these elements are either

- Column, Row, and Table, or

- Start column, End column, Start row, End row, Start table, and End table

\section{Column, Row, and Table}

If the range address is a cell address then the three attributes table:column, table:row and table: table specify the column, row and table number of the cell.

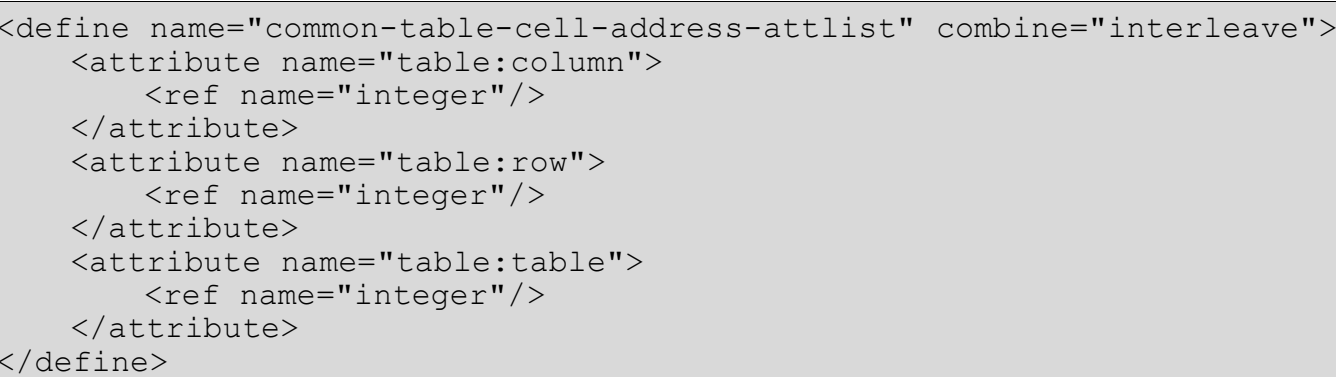




\section{Matrix Covered}

If the cell is a matrix cell and not the base of the matrix the, table:matrix-covered attribute is necessary and its value has to be true to indicate that the cell is contained in a matrix.

\subsubsection{Cell Content Change}

A <table:cell-content-change> element contains the information that is required to identify changes of the cell content.

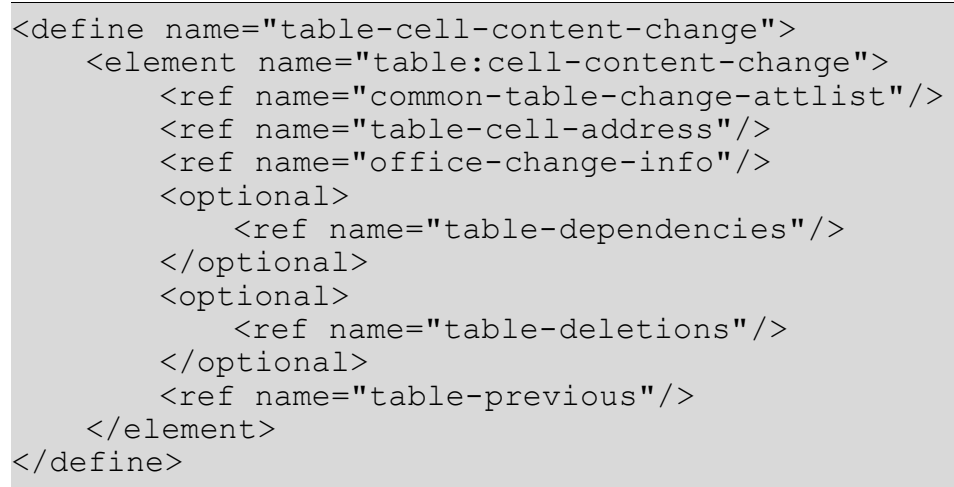

The attributes that may be associated with this element are: 
- ID (see section 8.11.18)

- Acceptance State (see section 8.11.18)

- Rejecting Change ID (see section 8.11.18)

\subsubsection{Cell Address}

The <table:cell-address $>$ element contains the address of cell that is changed. Unlike other cell addresses, the address consists of the row, column and table number of the cell. This allows specifying addresses that are outside the valid cell address range, for instance have a negative column number.

The attributes that may be associated with this element are:

- Column, Row, and Table number (see section 8.11.13)

\subsubsection{Previous}

The table:previous element contains the previous cell content which is overwritten by the current change. If a text: id attribute is present, it specifies the id of a previously tracked change for the cell that gets changed again by the current change.

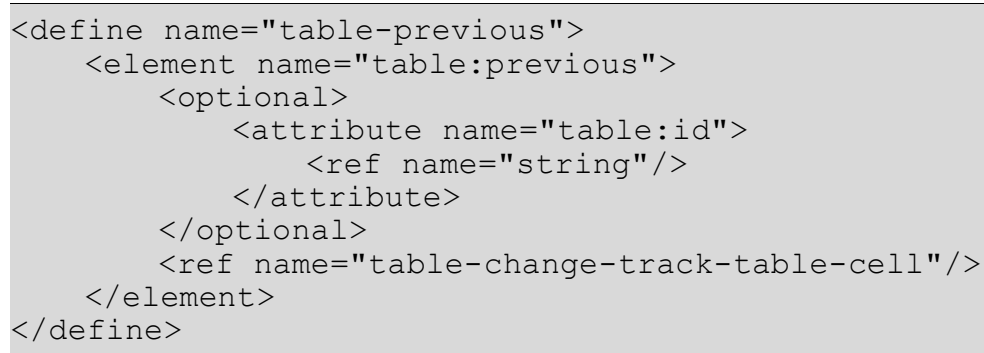

\subsubsection{Common Change Tracking Attributes}

Id

The table: id attribute specifies the id of the tracked change.

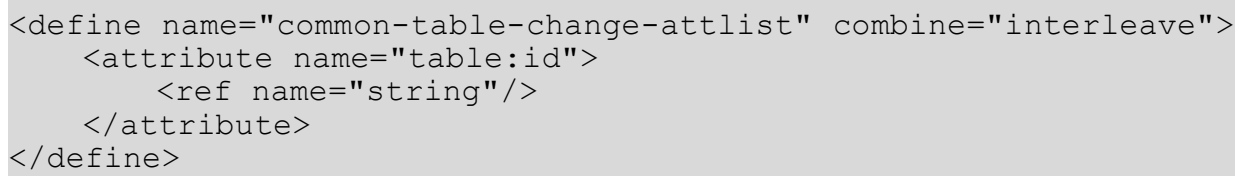

\section{Acceptance state}

The table:acceptance-state attribute specifies whether the tracked change has been accepted or rejected already, or whether an acceptance or rejection is still pending. 
5771

5772

5773

5774

5775

5776

5777

5778

5779

\section{Rejecting Change Id}

If the table:rejecting-change-id attribute is present, then the current change has been made to the table to implement the rejection of another previously tracked change. The attribute's value is the id of this previously tracked change that has been rejected. 


\section{Graphic Content}

This chapter provides the specification for the core elements of graphic applications like drawing or presentation applications, and for graphical objects contained in non-graphical applications, like word processor or spreadsheet applications.

\subsection{Enhanced Page Features for Graphical Applications}

\subsubsection{Handout Master}

For applications that support printing handout pages, this element is a template for automatically generating the handout pages. The element $\langle$ style: handout-master $>$ can contain any types of shapes. The most useful shape is the <draw: page-thumbnail $>$, which is replaced by actual pages from the document. The <style: handout-master $>$ element is contained in the <office:master-styles> element. The <office:master-styles> must not contain more than one $<$ style: handout-master $>$ element.

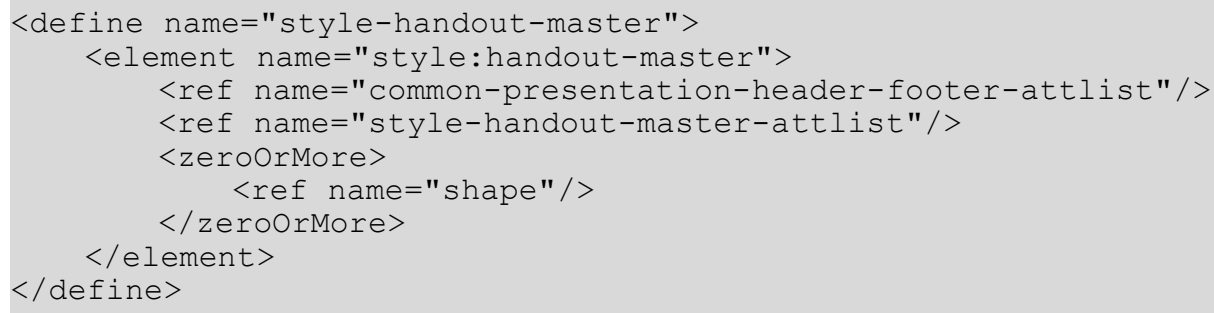

The attributes that may be associated with the <style: handout-master $>$ element are:

- Presentation Page Layout (placeholder objects)

- Page Layout (page size, margins etc.)

- Page Style

- Header Declaration

- Footer Declaration

- Date and Time Declaration

\section{Presentation Page Layout}

The attribute presentation:presentation-page-layout-name links to a <style:presentation-page-layout> element. See section 14.15 for information on the presentation page layout element. This attribute is optional.

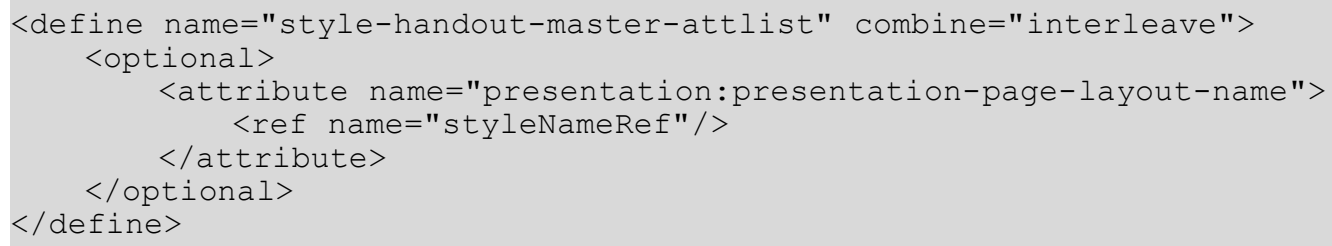




\section{Page Layout}

The style:page-layout-name attribute specifies a page layout which contains the sizes, border and orientation of the handout master page. See section 14.3 for details on page layouts.

\section{Header Declaration}

The presentation: use-header-name attribute specifies the name of the header field declaration (see section 9.11.2) that is used for all header fields (see section 9.10.1) that are displayed on the handout master page. See also section 9.1.4.

\section{Footer Declaration}

The presentation: use-footer-name attribute specifies the name of the footer field declaration (see section 9.11.3) that is used for all footer fields (see section 9.10.2) that are displayed on the handout master page. See also section 9.1.4.

\section{Date and Time Declaration}

The presentation: use-date-time-name attribute specifies the name of the date-time field declaration (see section 9.11.4) that is used for all date-time fields (see section 9.10.3) that are displayed on the handout master page. See also section 9.1.4.

\subsubsection{Layer Sets}

The element <draw: layer-set $>$ may be contained in the master styles of graphical applications. It defines a set of layers. Layers group drawing objects. Drawing objects may be assigned to these layers with the help of their draw: layer-name attribute. 


\subsubsection{Layer}

The <draw: layer $>$ element defines a single layer.

\section{Display}

The draw: display attribute specifies whether the drawing objects contain in the layer are visible on the screen and/or printed.

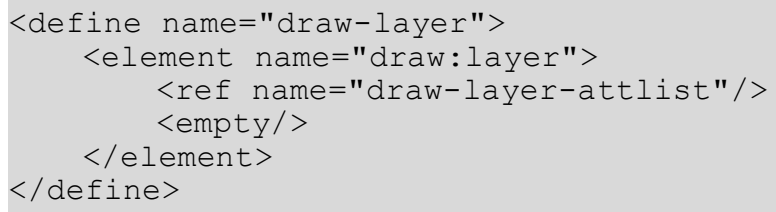

\section{Name}

Each element <draw: layer $>$ is defined and referenced by its name that is contained in the draw: name attribute. Each drawing object inside a drawing or presentation document can be assigned to a layer. Layers virtually group the object. Each object that is assigned to a layer inherits the settings of the layer.

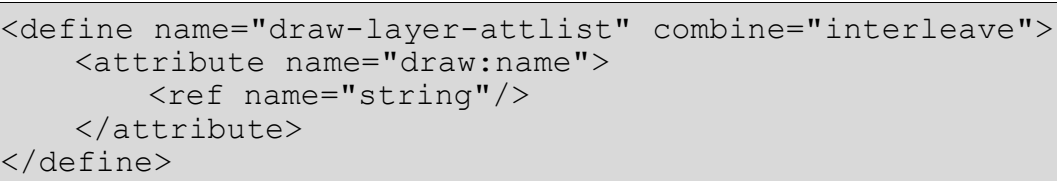

\section{Protection}

The draw: protected attribute specifies whether the drawing objects contain in the layer are protected from being modified.

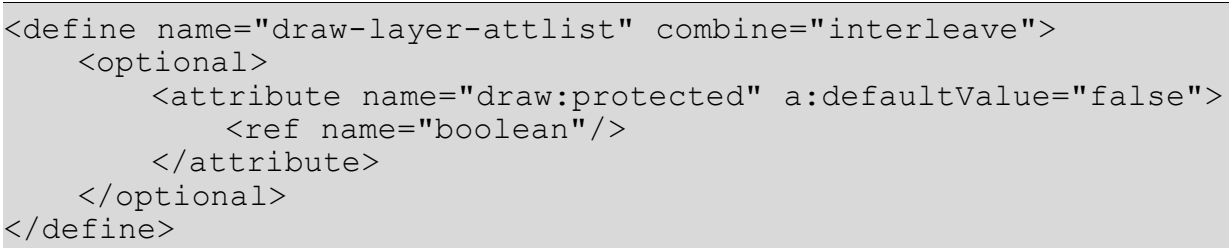

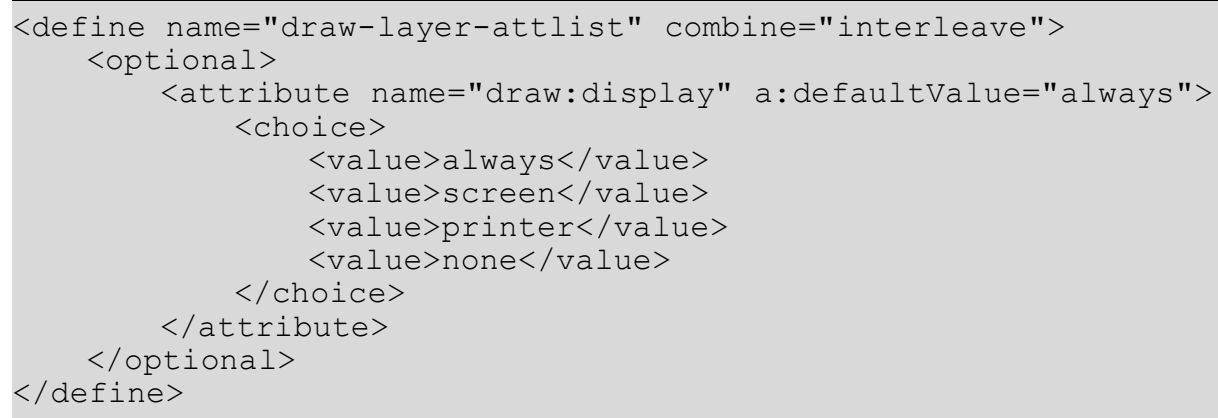

\subsubsection{Drawing Pages}

The element <draw:page > is a container for content in a drawing or presentation document. Drawing pages are used for the following: 
- Forms (see section 11.1)

- Drawings (see section 9.2)

- Frames (see section 9.3)

- Presentation Animations (see section 9.7)

- Presentation Notes (see section 9.1.5)

A master page must be assigned to each drawing page.

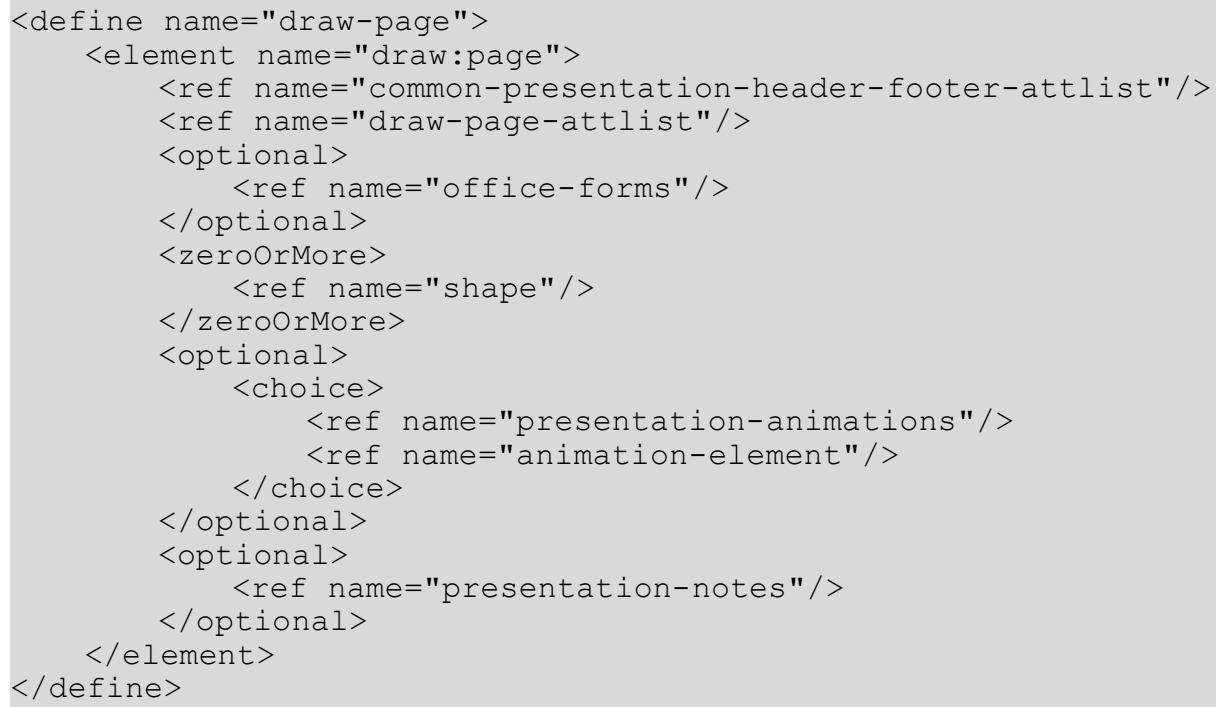

The attributes that may be associated with the <draw: page $>$ element are:

- Page name

- Page style

- Master page

- Presentation page layout

- Header declaration

- Footer declaration

- Date and time declaration

- ID

The elements that my be included in the <draw: page > element are:

- Forms

- Shapes

- Animations

- Presentation notes 


\section{Page Name}

The draw: name attribute specifies the name of a drawing page. This attribute is optional; if it is used, the name must be unique. If it is not used, the application may generate a unique name.

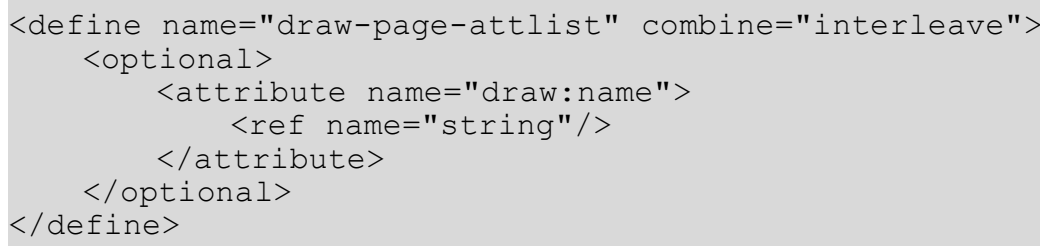

\section{Page Style}

The attribute draw:style-name assigns an additional formatting attributes to a drawing page by assigning a drawing page style. This attribute is optional. The fixed family for page styles is drawing-page.

For pages inside a presentation document, attributes from Presentation Page Attributes can also be used.

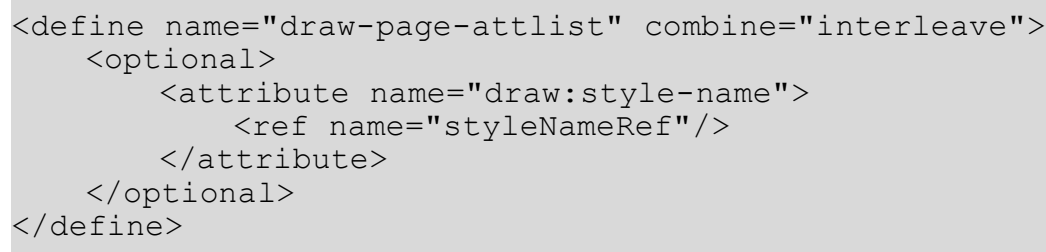

\section{Master Page}

Each drawing page must have one master page assigned to it. The master page:

- Defines properties such as the size and borders of the drawing page

- Serves as a container for shapes that are used as a common background

The draw:master-page-name attribute specifies the name of the master page assigned to the drawing page. This attribute is required.

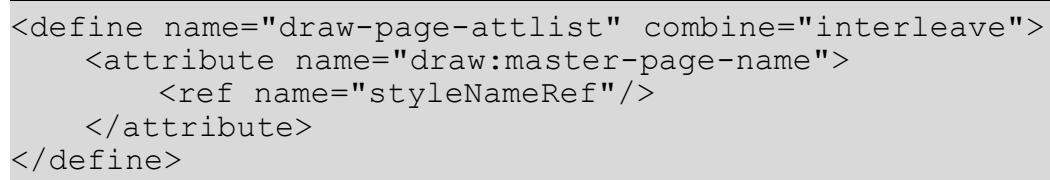

\section{Presentation Page Layout}

If the drawing page was created using a presentation page layout, the attribute presentation:presentation-page-layout-name links to the corresponding <style:presentation-page-layout> element. See section 14.15 for information on the presentation page layout element. This attribute is optional.

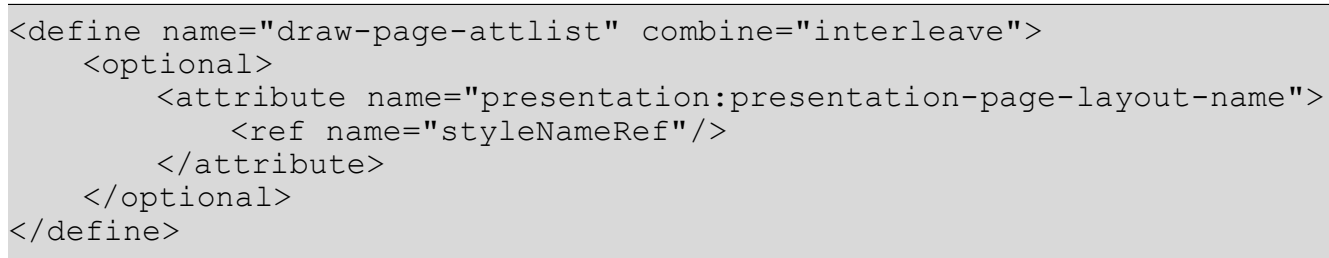


Example: Drawing page

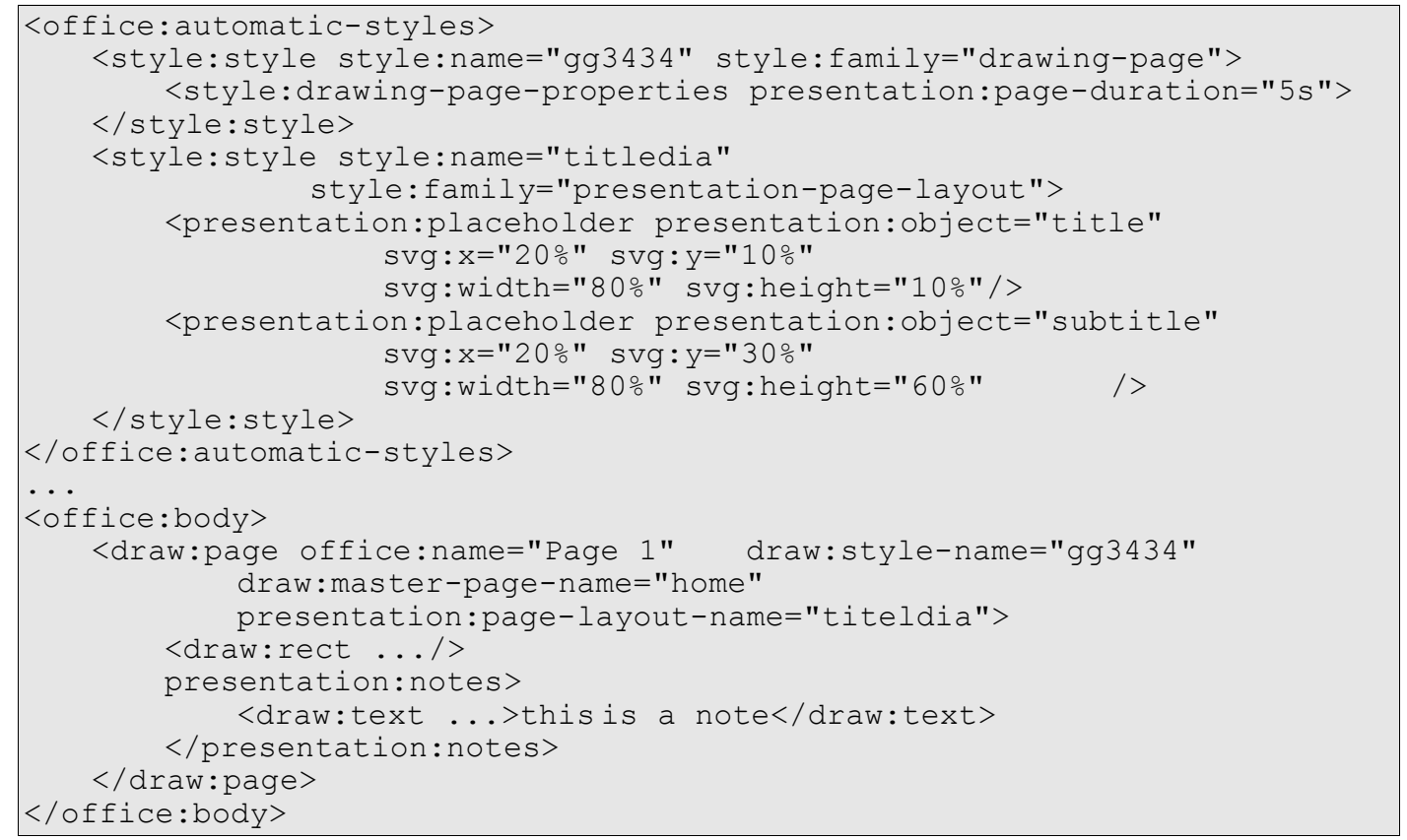

\subsection{Drawing Shapes}

This section describes drawing shapes that might occur within all kind of applications.

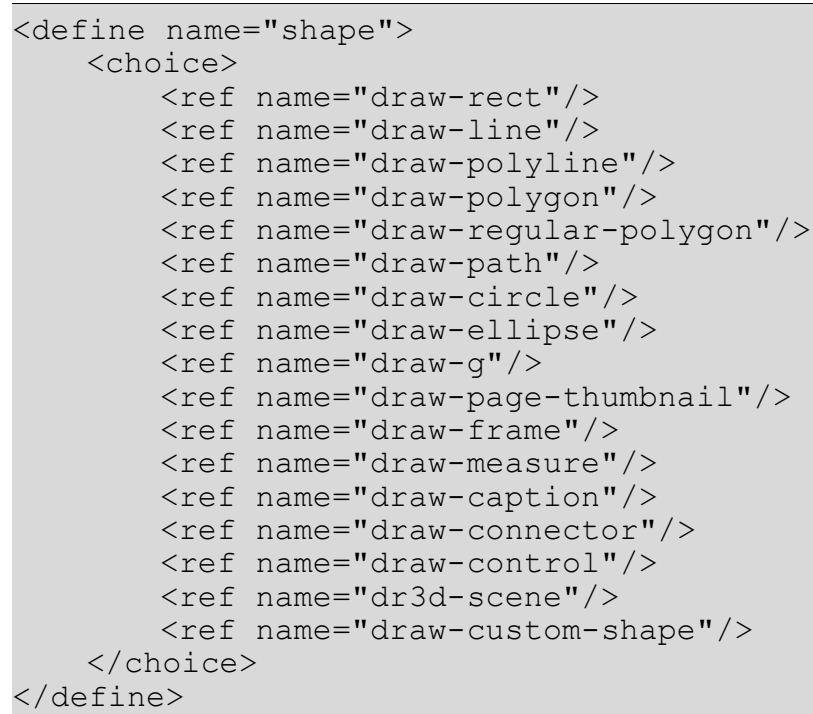

\subsubsection{Rectangle}

The <draw: rect $>$ element represents a rectangular drawing shape.

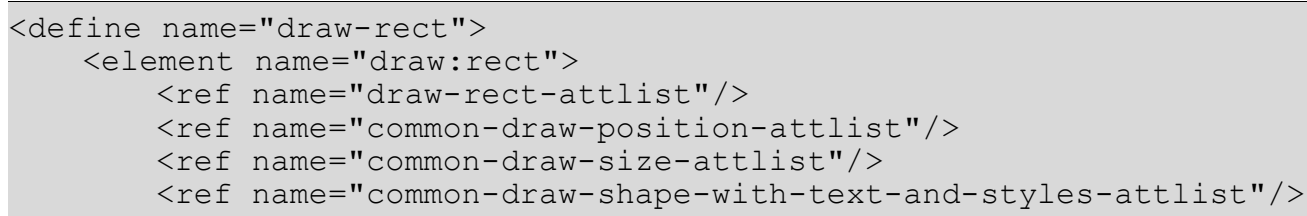




\section{$</$ define $>$}

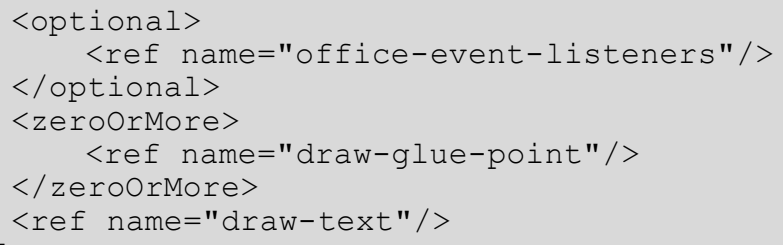

The attributes that may be associated with the <draw: rect > element are:

- $\quad$ Position, Size, Style, Layer, Z-Index, ID, and Transformation - see section 9.2.15.

- $\quad$ Text anchor, table background, draw end position - see section 9.2.16.

- Round corners

\section{Round Corners}

The attribute draw: corner-radius specifies the radius of the circle used to round off the corners of the rectangle.

Example: Rectangular drawing shape

$<$ draw: rect svg:x=" $2 \mathrm{~cm} " \mathrm{svg}: \mathrm{y}=" 3 \mathrm{~cm} " \mathrm{svg}:$ width=" $10 \mathrm{~cm}$ " svg:height=" $20 \mathrm{~cm} "$

svg:transform="rotate (45)" draw: style-name="object-with-shadow">

\subsubsection{Line}

The $<$ draw: line $>$ element represents a line.

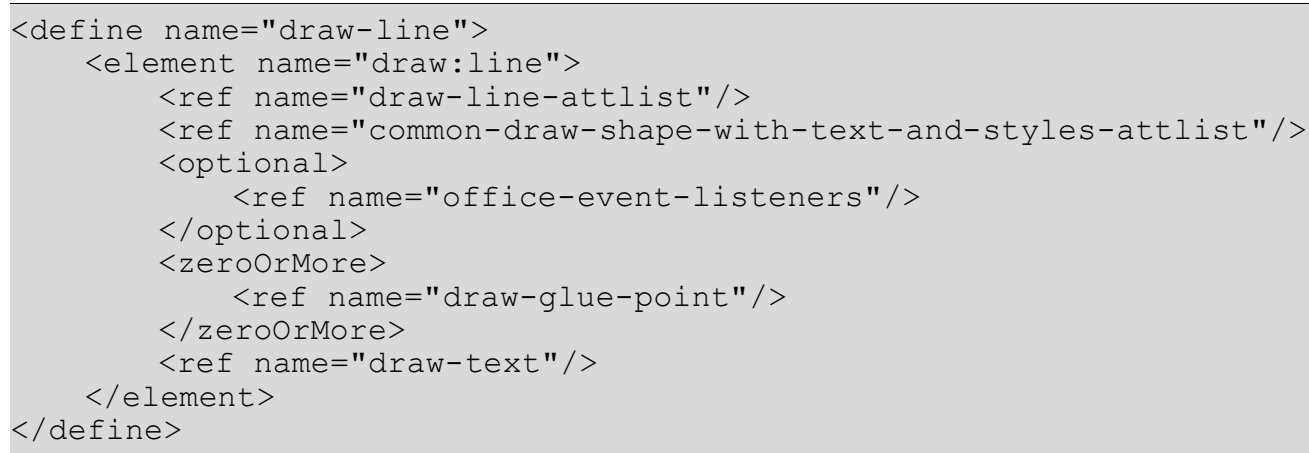

The attributes that may be associated with the <draw: line $>$ element are:

- Style, Layer, Z-Index, ID, and Transformation - see section 9.2.15.

- Text anchor, table background, draw end position- see section 9.2.16.

- Start point

- End point 


\section{Start Point}

The start point attributes $\mathrm{svg}: \mathrm{x} 1$ and $\mathrm{svg}: \mathrm{y} 1$ specify the start coordinates of the line.

\section{End Point}

The end point attributes $\mathrm{svg}: \mathrm{x} 2$ and $\mathrm{svg}: \mathrm{y} 2$ specify the end coordinates of the line.

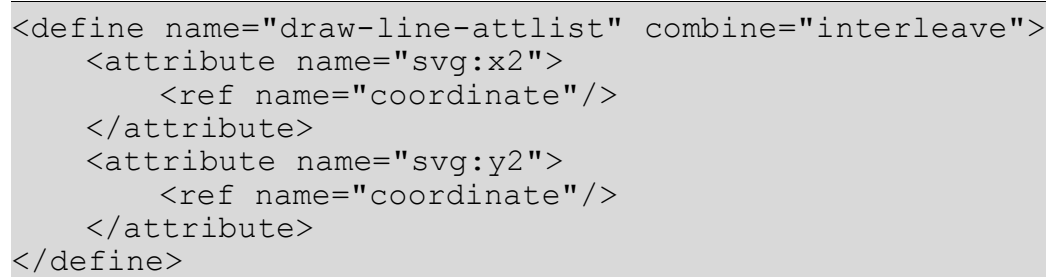

\subsubsection{Polyline}

The <draw:polyline $>$ element represents a polyline drawing shape.

Some implementations may ignore the size attribute, and instead determine the size of a shape exclusively from the shape data (i.e. polygon vertices).

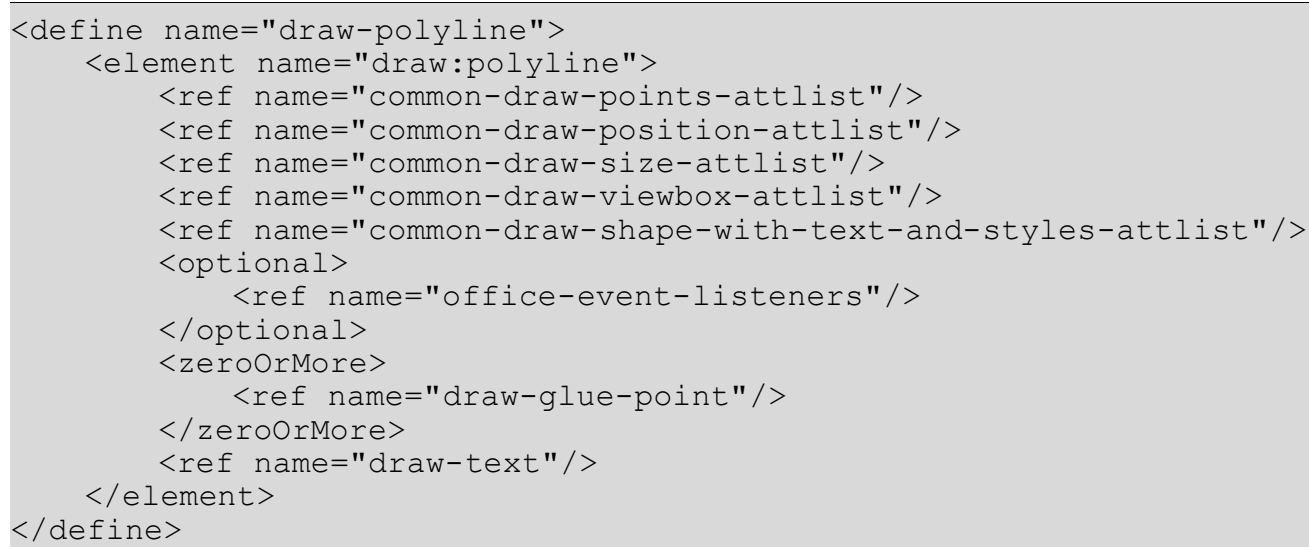

The attributes that may be associated with the <draw: polyline> element are:

- Position, Size, View box, Style, Layer, Z-Index, ID, and Transformation - see section 9.2.15

- Text anchor, table background, draw end position - see section 9.2.16

- Points 


\section{Points}

The svg:points attribute stores a sequence of points, which are connected by straight lines. Each point consists of two coordinates. The coordinates are separated by a comma and the points are separated by white spaces.

6015

6016

6017

6018

6019

\subsubsection{Polygon}

The <draw: polygon $>$ element represents a polygon. A polygon is a closed set of straight lines.

Some implementations may ignore the size attribute, and instead determine the size of a shape exclusively from the shape data (i.e. polygon vertices).

6020

6021

6022

6023

6024

6025

6026

6027

6028

6029

6030

6031

6032

6033

6034

6035

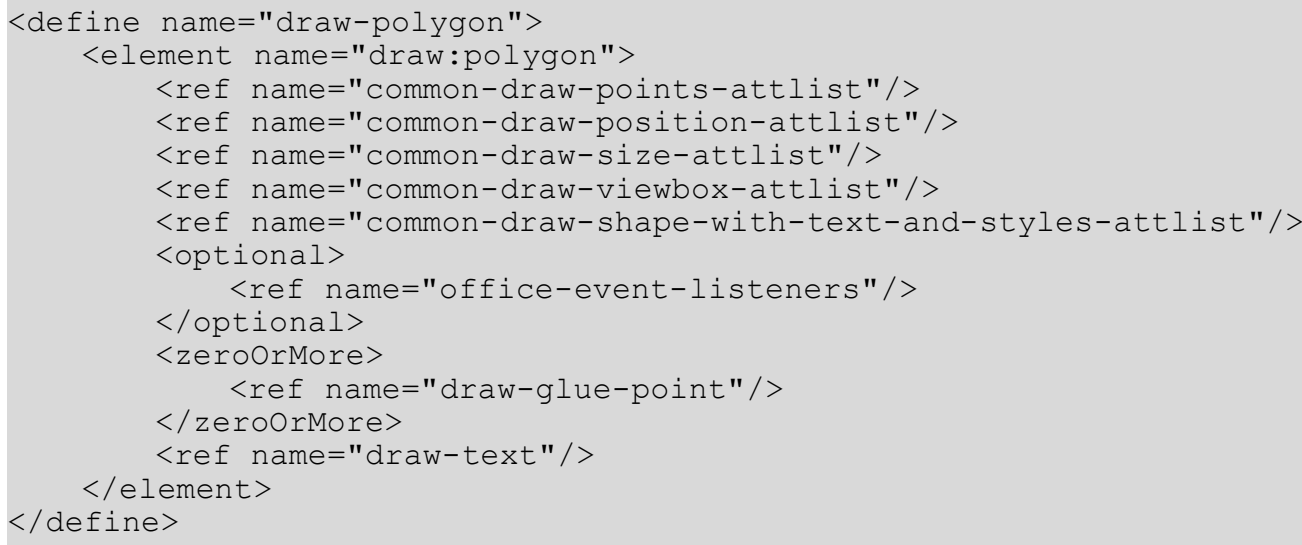

The attributes that may be associated with the <draw: polygon> element are:

- Position, Size, View box, Style, Layer, Z-Index, ID, and Transformation - see section 9.2.15

- Text anchor, table background, draw end position - see section 9.2.16

- $\quad$ Points - see section 9.2 .3

\subsubsection{Regular Polygon}

The <draw: regular-polygon> element represents a regular polygon. A regular polygon is a polygon that is specified by its number of edges (that is equal to the number of its corners), rather than by arbitrary points.

6036

6037

6038

6039

6040

6041

6042

6043

6044

6045

6046

6047

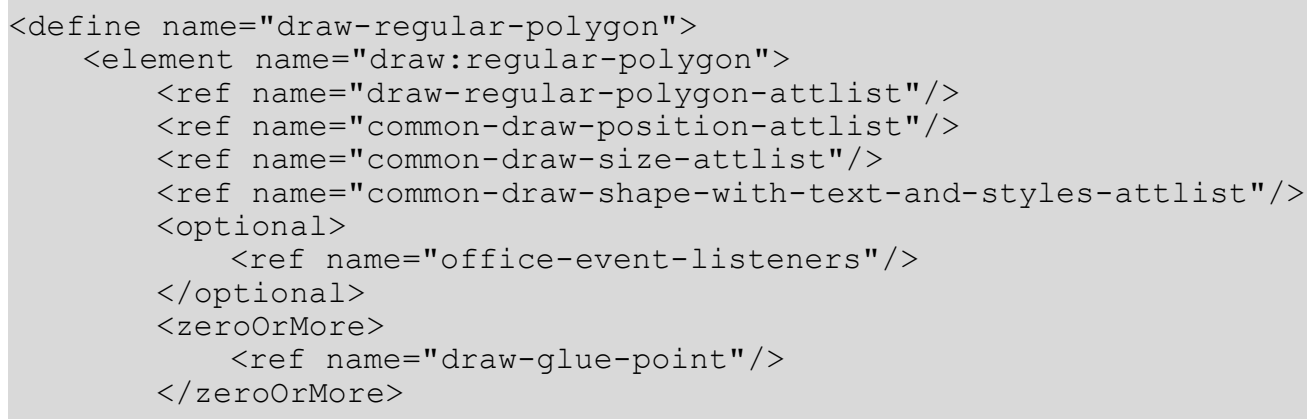


$</$ define $>$

<ref name="draw-text"/>

$</ e l e m e n t>$

The attributes that may be associated with the <draw: polygon $>$ element are:

- Position, Size, Style, Layer, Z-Index, ID, and Transformation - see section 9.2.15

- Text anchor, table background, draw end position - see section 9.2.16

- Concave

- Corners

- Sharpness

\section{Concave}

The draw: concave attribute specifies whether the polygon is convex or concave. For a convex polygon, the polygon corners are located on a single ellipse which has its center in the center of the polygon. In a concave polygon, two such ellipses are required, and corners that are located next to each other are located on different ellipses. An example for a convex polygon is a hexagon. An example for a concave polygon is a star. For concave polygons, an additional draw: sharpness attribute is required.

6051

6052

6053

6054

6055

6056

6057

6058

6059

6060

6061

6062

6063

\section{Corners}

The draw: corners attribute specifies the number of polygon corners.

6064

6065

6066

6067

6068

\section{Sharpness}

For concave attributes, the draw: sharpness attribute specifies the radius of the ellipse on which the inner polygon corners are located. The value is a percentage, where $0 \%$ means that all corners are located on a single ellipse, while $100 \%$ means that the inner corners are located at the center point of the polygon. In general, if $r$ is the radius of the polygon, and $s$ is the sharpness, the inner corners a located on a ellipse that's radius is $r(100-s) / 100$. 


\subsubsection{Path}

The <draw: path $>$ element represents a path. A path is a shape with a user-defined outline. The shape is built using multiple drawing actions such as:

- moveto - set a new current point

- lineto - draw a straight line

- curveto - draw a curve using a cubic Bezier

- $\quad$ arc - draw an elliptical or circular arc

- closepath - close the current shape by drawing a line to the last moveto

Compound paths are paths with subpaths, each subpath consisting of a single moveto followed by one or more line or curve operations. Compound paths can be used for effects such as holes in objects.

Some implementations may ignore the size attribute, and instead determine the size of a shape exclusively from the shape data (i.e. polygon vertices).

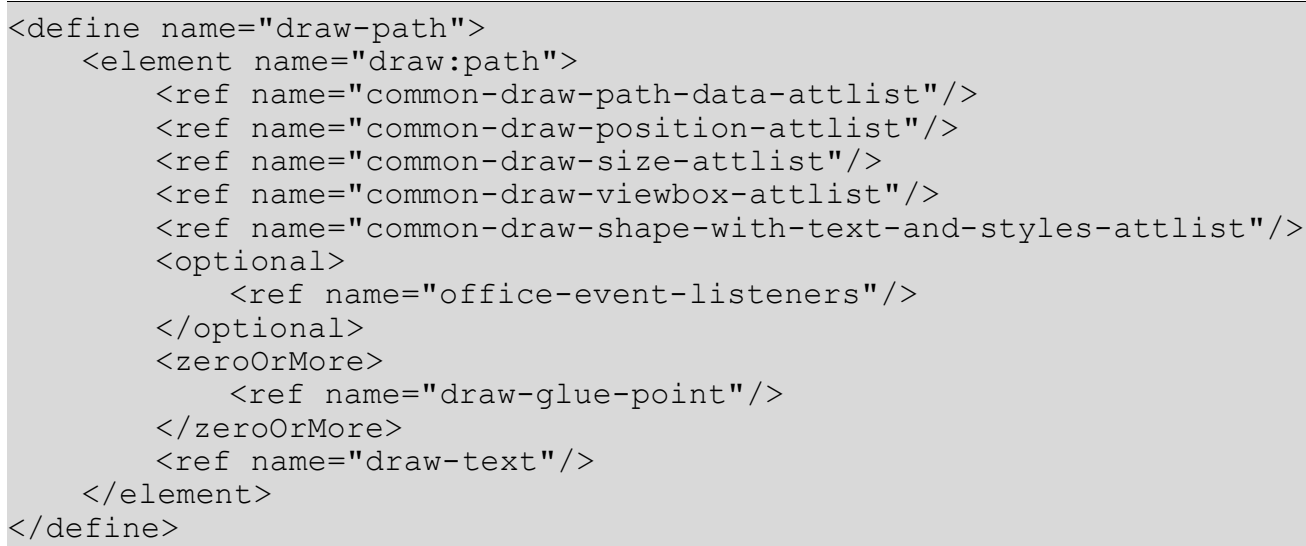

The attributes that may be associated with the <draw: path> element are:

- Position, Size, View box, Style, Layer, Z-Index, ID, and Transformation - see section 9.2.15

- Text anchor, table background, draw end position - see section 9.2.16

- Path data

\section{Path Data}

The syntax for the attribute $\mathrm{svg}: \mathrm{d}$ is described in $\S 8$ of the Scalable Vector Graphics (SVG) 1.1 Specification [SVG].

Some implementations may only supports a subset of the SVG path specification, for instance no mixtures of open and closed curves for one shape, or no elliptical arc command.

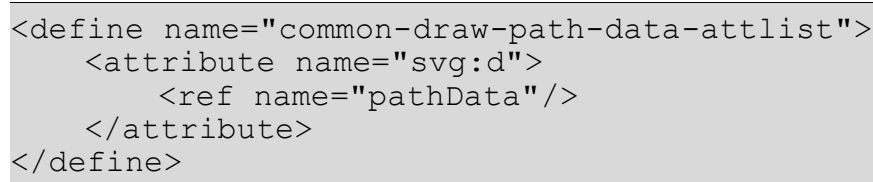

6093

6094 


\subsubsection{Circle}

The $<$ draw: circle $>$ element represents a circular drawing shape.

The attributes that may be associated with the <draw: circle $>$ element are:

- Position, Size, Style, Layer, Z-Index, ID, and Transformation - see section 9.2.15

- Text anchor, table background, draw end position - see section 9.2.16

- Center point

- Radius

- Kind

- Start angle

- End angle

\section{Center Point}

The center point attributes svg: $\mathrm{cx}$ and svg: cy specify the coordinates of the center point of the circle. If these optional attributes are not set, the position and size attributes are used to create them.

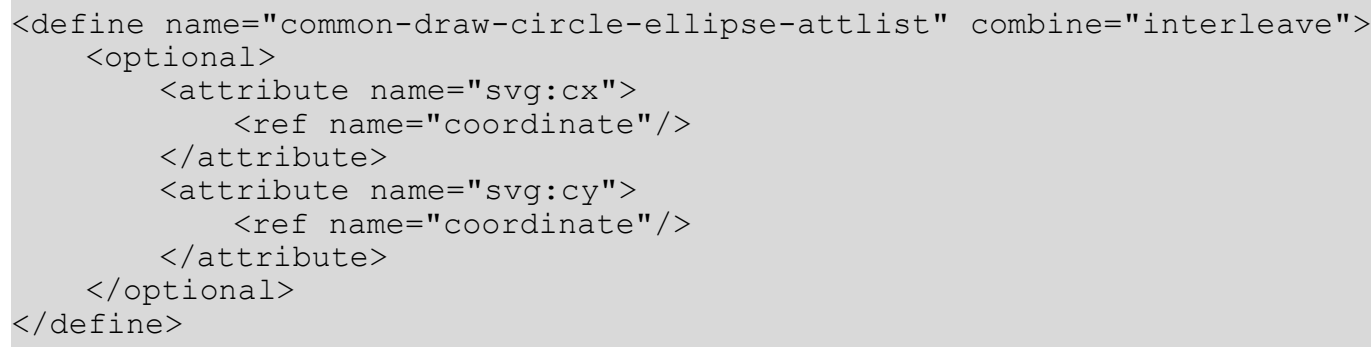

\section{Radius}

The svg: $r$ attribute specifies the radius of the circle. If this optional attribute are not set, the position and size attributes are used to create circle.

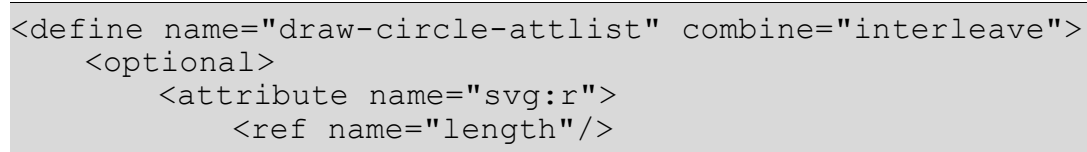




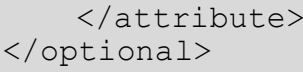

\section{Kind}

The draw: kind attribute specifies the appearance of the circle.

- full specifies a full circle or ellipse, like $\bigcirc$.

- $\quad$ section specifies a section of a circle or ellipse, like $\zeta$.

- cut specifies a circle or ellipse with a cut, like

- $\quad \operatorname{arc}$ specifies a circle or ellipse arc, like $C$.

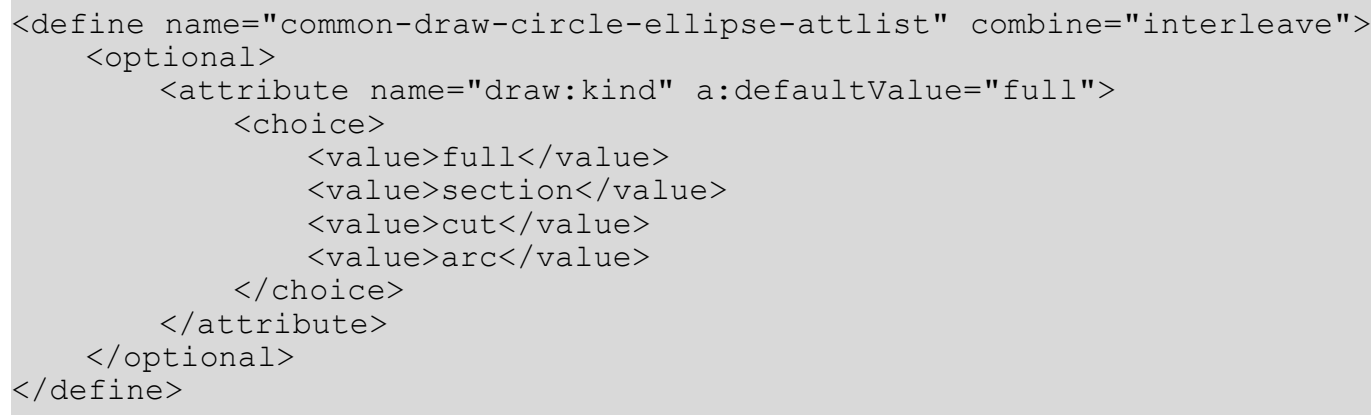

\section{Start Angle}

For circles where the draw: kind attribute value is section, cut or arc, the svg:startangle attribute specifies the start angle of the section, cut, or arc.

6140

6141

6142

6143

6144

6145

6146

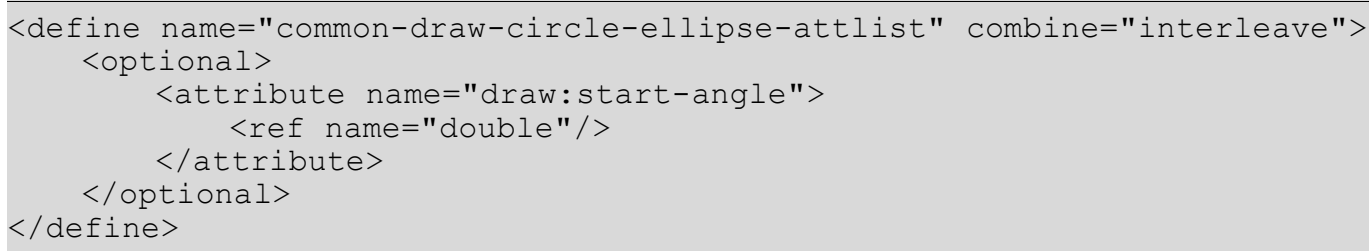

\section{End Angle}

For circles where the draw: kind attribute value is section, cut or arc, the svg:end-angle attribute specifies the end angle of the section, cut, or arc.

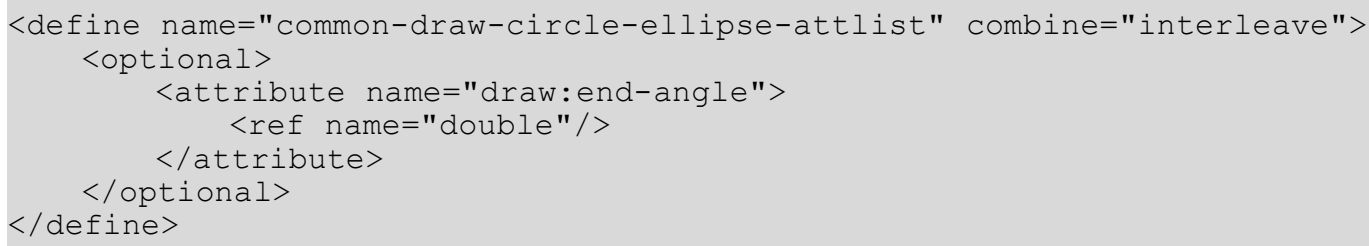

\subsubsection{Ellipse}

The <draw:ellipse> element represents an ellipse. 


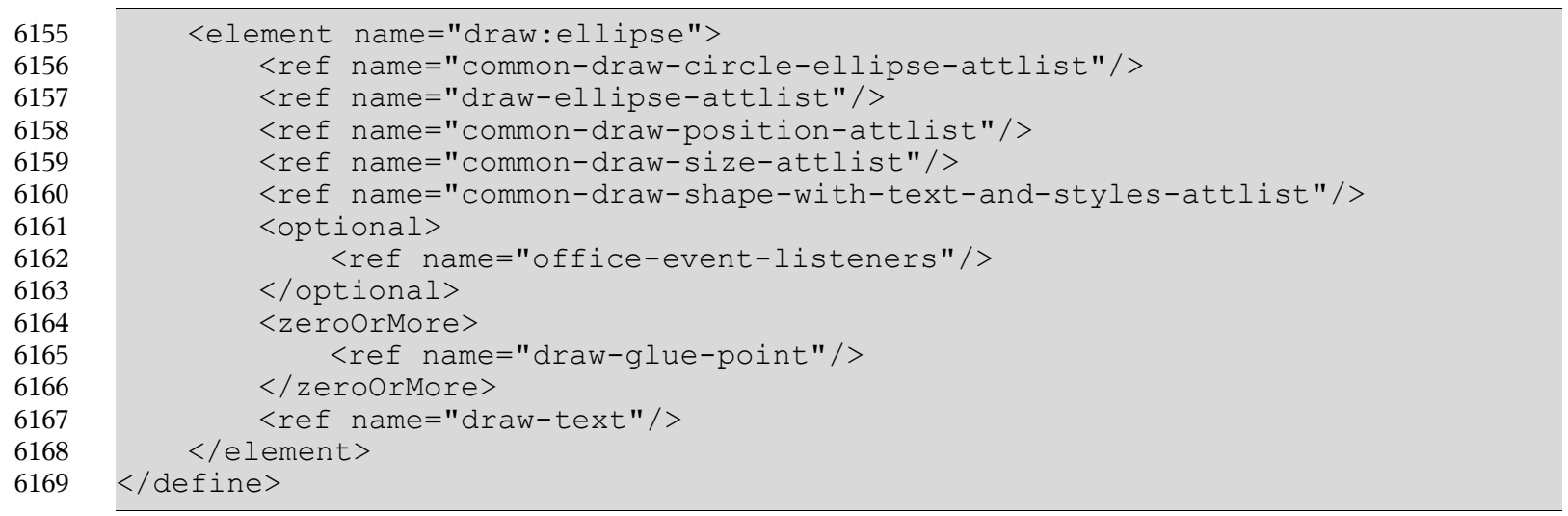

The attributes that may be associated with the <draw:ellipse> element are:

- Position, Size, Style, Layer, Z-Index, ID, and Transformation - see section 9.2.15

- Text anchor, table background, draw end position - see section 9.2.16

- $\quad$ Center point, Kind, Start angle, End angle - see section 9.2.7

- Radius

\section{Radius}

The svg: $r x$ and svg: $r x$ attribute specify the horizontal and vertical radius of the ellipse. If these optional attributes are not set, the position and size attributes are used to create the ellipse.

\subsubsection{Connector}

The <draw: connector $>$ element represents a series of lines that are connected to the glue points of two other shapes.

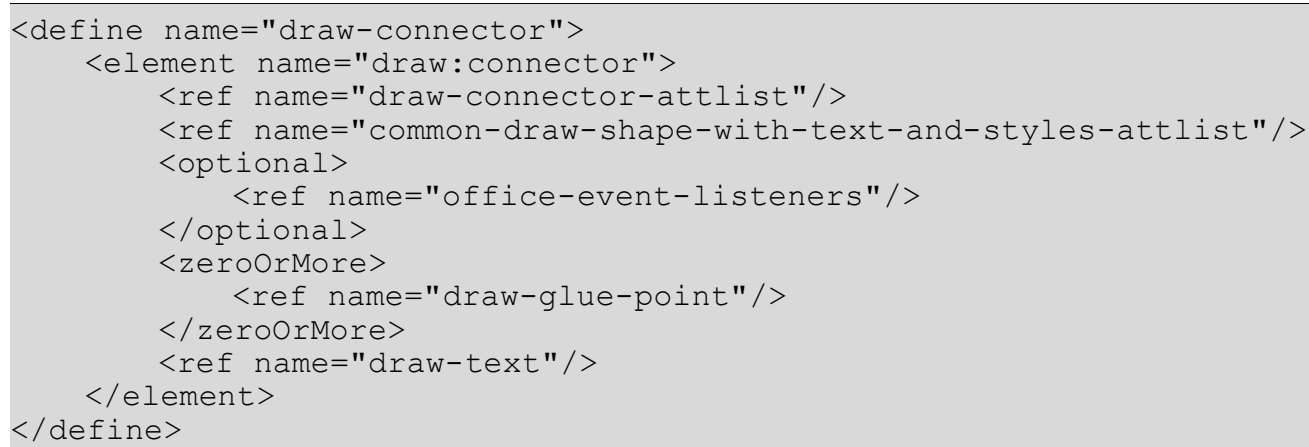

The attributes that may be associated with the $<$ draw: connector $>$ element are: 
- Style, Layer, Z-Index and ID - see section 9.2.15

- Text anchor, table background, draw end position - see section 9.2.16

- Type

- Start position

- Start shape

- Start glue point

- End position

- End shape

- End glue point

- Line skew

\section{Type}

The draw: type attribute specifies how the connection between two points is rendered. The value of this attribute can be standard, lines, line, or curve.

- standard: a standard connector escapes the two connecting objects with straight lines and connects them with a straight perpendicular line.

- lines: a lines connector escapes the two connecting objects with straight lines and connects them with a straight (not necessarily perpendicular) line.

- line: a line connector draws one straight line between the two escape points of the connected objects.

- curve: a curve connector draws a single curved line between the two escape points of the connected objects.

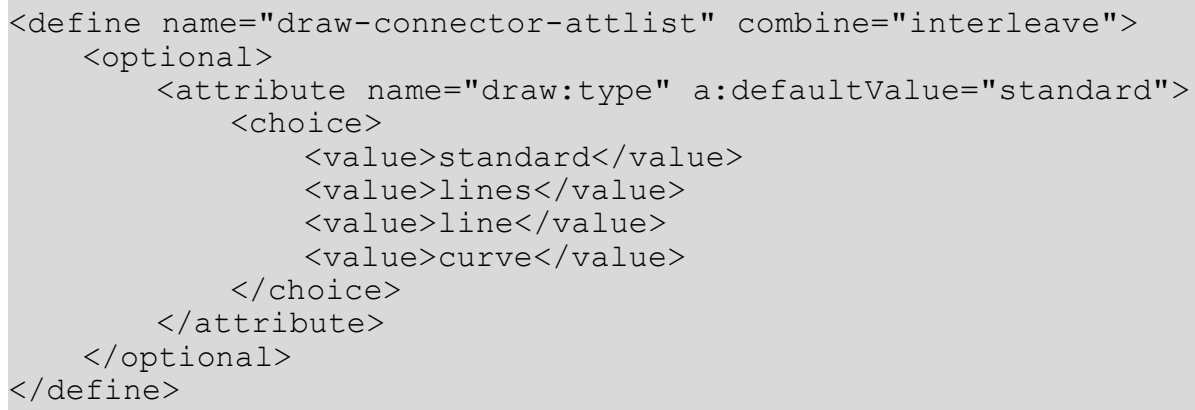

\section{Start Position}

The start position attributes $\mathrm{svg}: \mathrm{x} 1$ and $\mathrm{svg}: \mathrm{y} 1$ specify the start position of a connector.

If the start position is connected to a shape, these attributes are optional because the start position defaults to the corresponding glue point on the target shape. 
6210

6211

6212

6213

6214

6215

6216

6217

6218

6219

6220

6221

\section{Start Glue Point}

The draw: start-glue-point attribute identifies the glue point in the start shape of the connector by its number. See section 9.2.19 for details on glue points.

If this attribute is not set and the start of the connector is connected to a shape, the application may choose the glue point. If the start of the connector is not connected to a shape, this attribute is ignored.

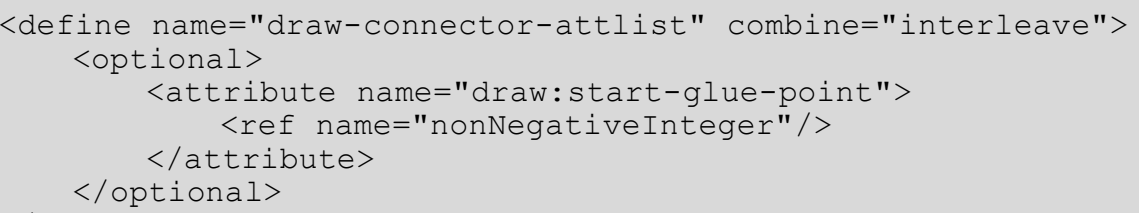

\section{End Position}

The end position attributes $\mathrm{svg}: \mathrm{x} 2$ and $\mathrm{svg}: \mathrm{y} 2$ specify the end position of a connector.

If the end position is connected to a shape, these attributes are optional because the end position defaults to the corresponding glue point on the target shape. 


\section{End Shape}

The draw: end-shape attribute identifies the drawing shape to which the end of the connector is connected by its name.

If a shape is connected to the end of a connector, the end position defaults to the corresponding glue point on the target shape.

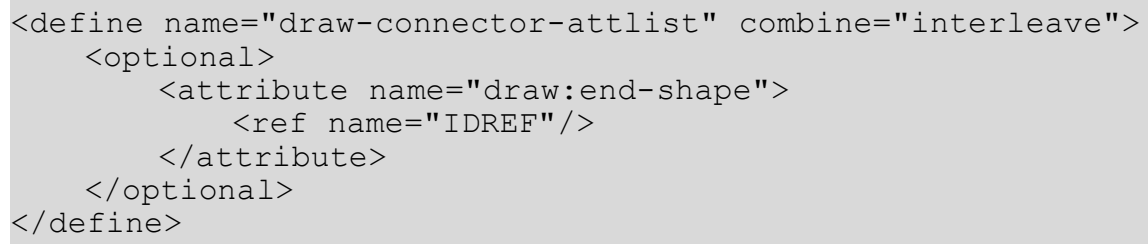

\section{End Glue Point}

The draw: end-glue-point attribute identifies the glue point in the end shape of the connector by its number. See section 9.2.19 for details on glue points.

If this attribute is not set and the end of the connector is connected to a shape, the application may choose the glue point. If the end of the connector is not connected to a shape, this attribute is ignored.

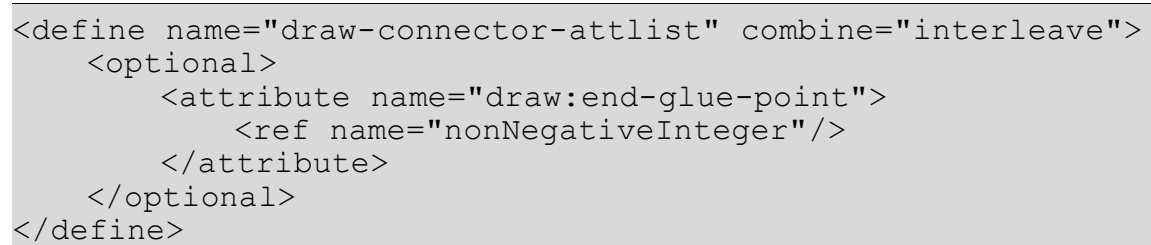

$</$ define $>$

\section{Line Skew}

The draw: line-skew attribute controls the generation of the lines that connect the start and end points. Depending on the type of connector, this can vary from one to three distances that move the connector lines relative to their normal position.

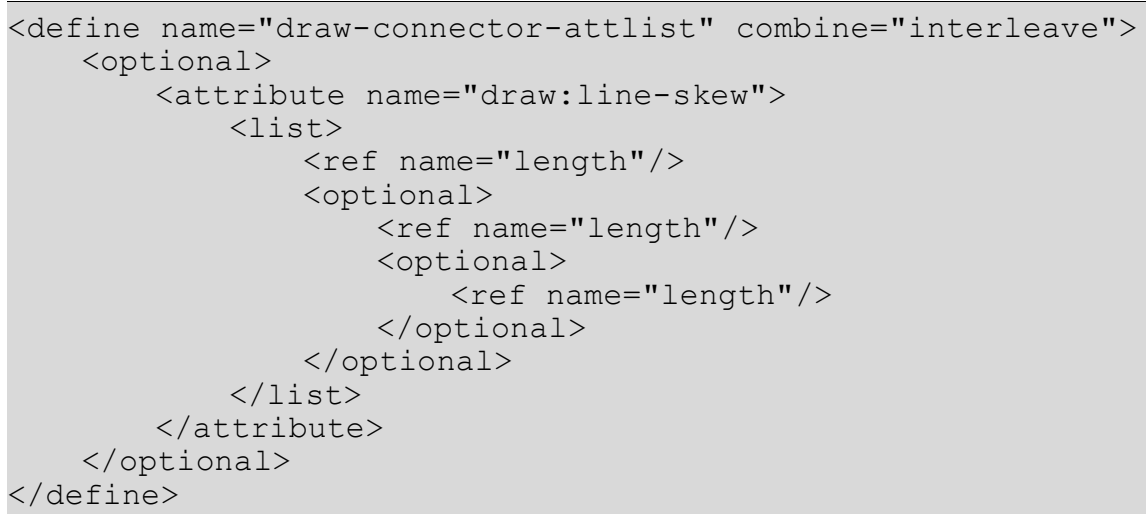

\subsubsection{Caption}

The <draw: caption> element represents a rectangular drawing shape with an additional set of lines. It can be used as a description for a fixed point inside a drawing. 
$</$ define $>$

The attributes that may be associated with the <draw: caption> element are:

- Position, Size, Style, Layer, Z-Index, ID, and Transformation - see section 9.2.15

- Text anchor, table background, draw end position - see section 9.2.16

- Caption point

- Round corners

\section{Caption Point}

The caption point attributes draw: caption-point-x and draw:caption-point-y specify the position of the point that is captioned. A set of lines are rendered from the caption area.

6283

6284

6285

6286

6287

6288

6289

6290

6291

6292

\section{Round Corners}

The draw: corner-radius attribute specifies the radius of the circle used to round off the corners of the caption.

\subsubsection{Measure}

The $<$ draw: measure $>$ element represents a shape that is used to measure distances in drawings. 


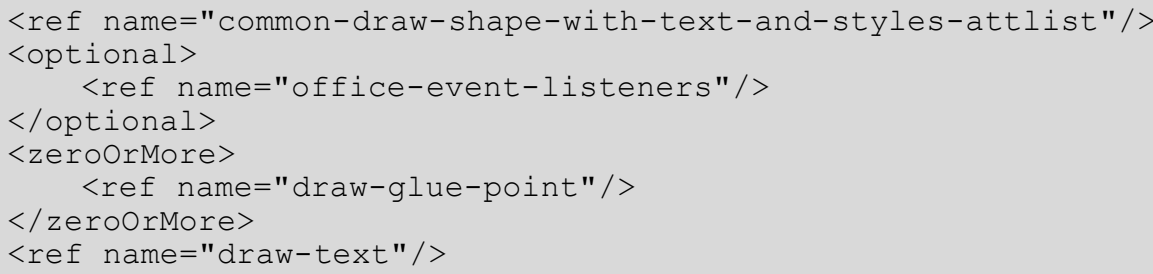

The attributes that may be associated with the $<$ draw : measure $>$ element are:

- Style, Layer, Z-Index, ID, and Transformation - see section 9.2.15

- Text anchor, table background, draw end position - see section 9.2.16

- Start position

- End position

\section{Start Position}

The attributes $\mathrm{svg}: \mathrm{x} 1$ and $\mathrm{svg}: \mathrm{y} 1$ specify the start point of the measured distance.

6313

6314

6315

6316

6317

6318

6319

6320

\section{Draw End Position}

The attributes $\mathrm{svg}: \mathrm{x} 2$ and $\mathrm{svg}: \mathrm{y} 2$ specify the end point of the measured distance.

6321

6322

6323

6324

6325

6326

6327

6328

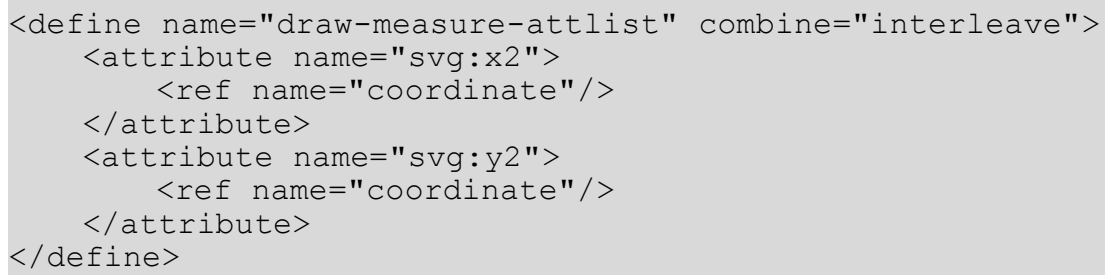

\subsubsection{Control}

The <draw: control $>$ element represents a shape that is linked to a control inside an <office: forms> element (see section 11.1).

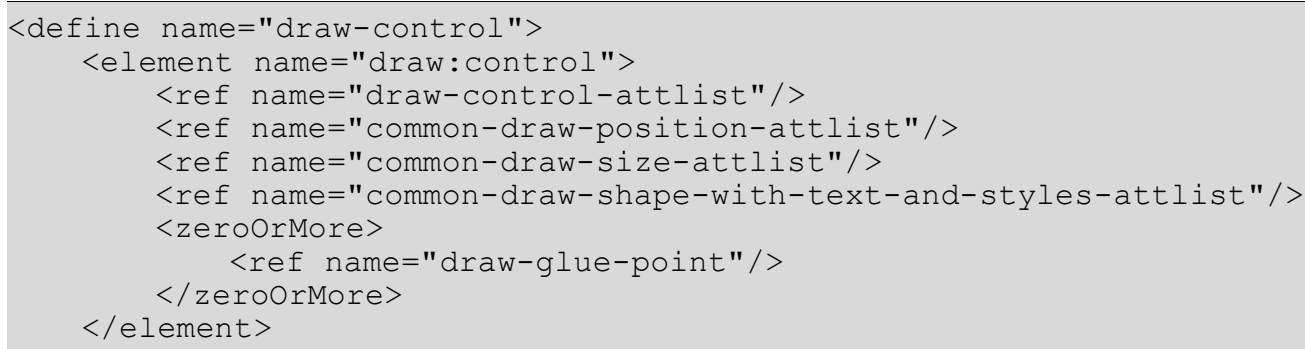


The attributes that may be associated with the <draw: control $>$ element are:

- Position, Size, Style, Layer, Z-Index, ID, and Transformation - see section 9.2.15.

- Text anchor, table background, draw end position - see section 9.2.16

- Control

\section{Control}

The attributes draw: control attribute specifies the control within a form (see section 11.5.2) that is linked to the control shape.

\subsubsection{Page Thumbnail}

The <draw: page-thumbnail $>$ element represents a rectangular area that displays the thumbnail of a drawing page.

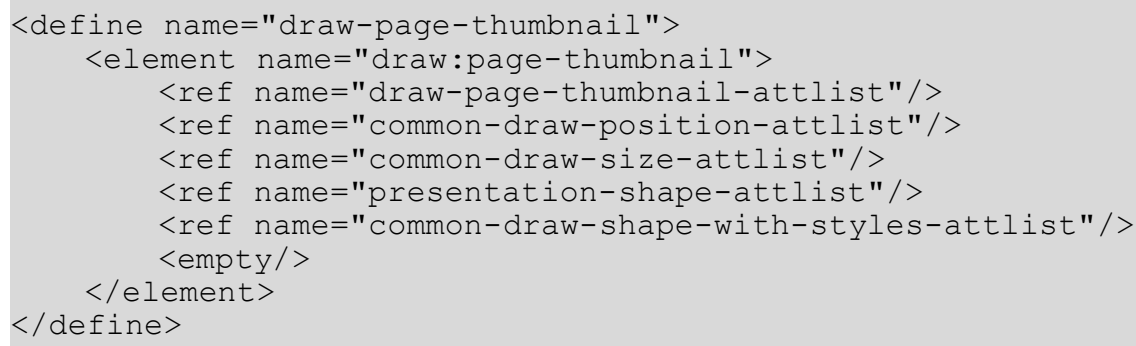

The attributes that may be associated with the <draw:page-thumbnail> element are:

- Position, Size, Style, Layer, Z-Index, ID, and Transformation - see section 9.2.15.

- Text anchor, table background, draw end position - see section 9.2.16

- $\quad$ Presentation class - see section 9.6.1

- Page number

\section{Page Number}

The draw:page-number attribute specifies the number of the page that is displayed as a thumbnail. For thumbnails on notes pages, the value of this attribute is fixed to the drawing page of the notes page. For thumbnails on handout master pages, the value of this attribute is the order in which the pages are previewed on the handout. For example, on a handout page with 4 thumbnails, the thumbnail with the lowest page number renders the first page when printing the first handout page and the fifth page when printing the second handout page and so on.

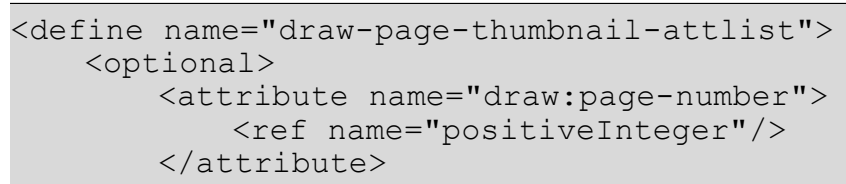




\subsubsection{Grouping}

The $<$ draw: $g>$ element represents a group of drawing shapes.

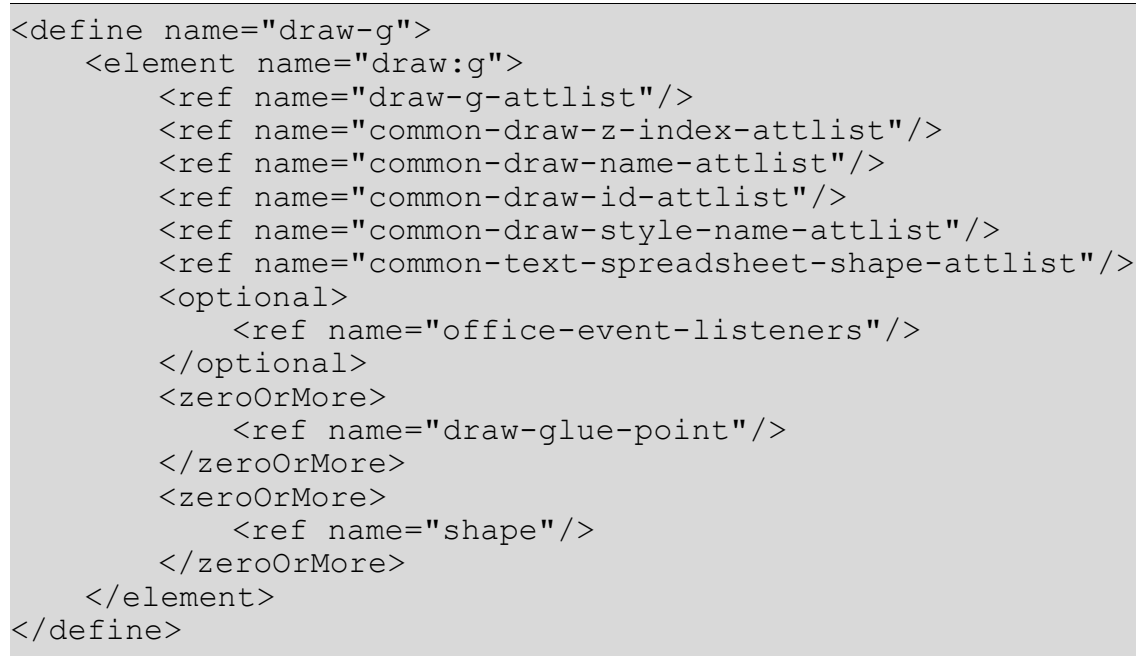

The attributes that may be associated with the <draw: $g>$ element are:

- $\quad$ Style, Z-Index and ID - see section 9.2.15.

- Text anchor, table background, draw end position - see section 9.2.16

- Position

\section{Position}

For group shapes that are contained in text documents and anchored as character, the svg:y attribute specifies the vertical position of the shape.

6381

6382

6383

6384

6385

6386

6387

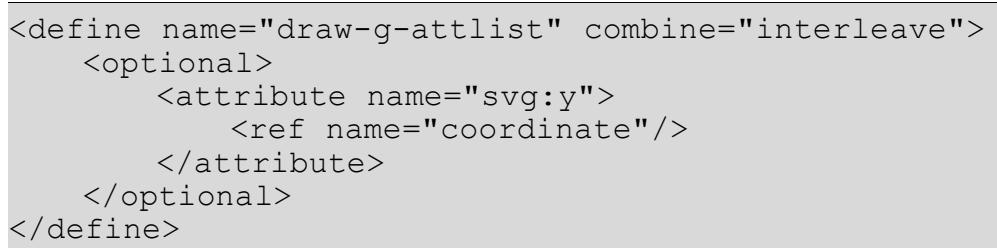

\subsubsection{Common Drawing Shape Attributes}

The attributes described in this section are common to all drawing shapes.

\section{Name}

The attribute draw: name assigns a name to the drawing shape.

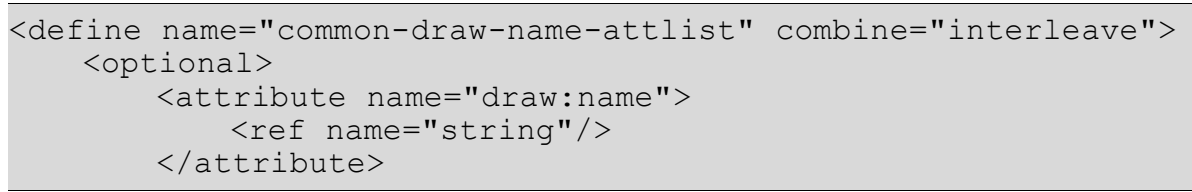




\section{Position}

The position attributes $\mathrm{Svg}: \mathrm{x}$ and $\mathrm{Svg}: \mathrm{y}$ specify the $\mathrm{x}$ and $\mathrm{y}$ coordinates of the start position of the drawing shape.

\section{Size}

The attributes svg:width and svg:height specify the width and height of the drawing shape.

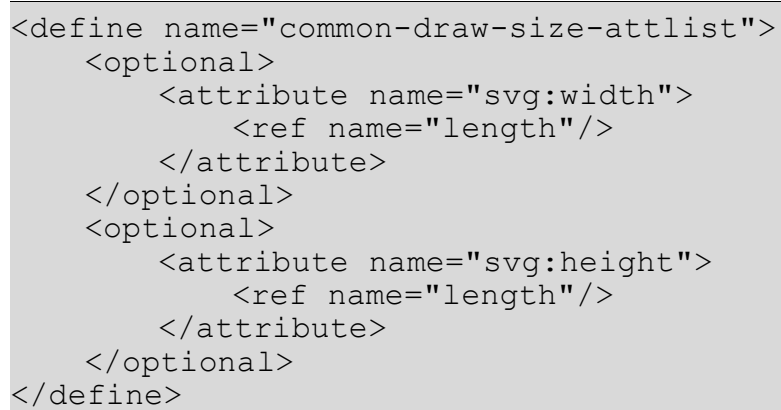

\section{Transformation}

The draw: transform attribute specifies a list of transformations that can be applied to a drawing shape.

The value of this attribute is a list of transform definitions, which are applied to the drawing shape in the order in which they are listed. The transform definitions in the list must be separated by a white space and/or a comma. The types of transform definitions available include:

- matrix $(\langle\mathrm{a}\rangle\langle\mathrm{b}\rangle\langle\mathrm{c}\rangle\langle\mathrm{d}\rangle\langle\mathrm{e}\rangle\langle\mathrm{f}\rangle)$, which specifies a transformation in the form of a transformation matrix of six values. matrix $(a, b, c, d, e, f)$ is the equivalent of applying the transformation matrix $[a b c c c c c c$.

- translate $(\langle t x\rangle[\langle t y\rangle])$, which specifies a translation by tx and ty.

- $\operatorname{scale}(\langle s x\rangle[\langle s y\rangle])$, which specifies a scale operation by $s x$ and $s y$. If $\langle s y\rangle$ is not provided, it is assumed to be equal to $\langle s x\rangle$.

- $\operatorname{rotate}(<$ rotate-angle $>)$, which specifies a rotation by <rotate-angle $>$ about the origin of the shapes coordinate system.

- skewX (<skew-angle $>)$, which specifies a skew transformation along the $\mathrm{X}$ axis. 
- $\quad$ skewY $(<$ skew-angle $>)$, which specifies a skew transformation along the $Y$ axis.

6419

6420

6421

6422

6423

6424

6425

\section{View Box}

The svg:viewBox attribute establishes a user coordinate system inside the physical coordinate system of the shape specified by the position and size attributes. This user coordinate system is used by the svg:points attribute and the <draw: path> element.

The syntax for using this attribute is the same as the [SVG] syntax. The value of the attribute are four numbers separated by white spaces, which define the left, top, right, and bottom dimensions of the user coordinate system.

Some implementations may ignore the view box attribute. The implied coordinate system then has its origin at the left, top corner of the shape, without any scaling relative to the shape.

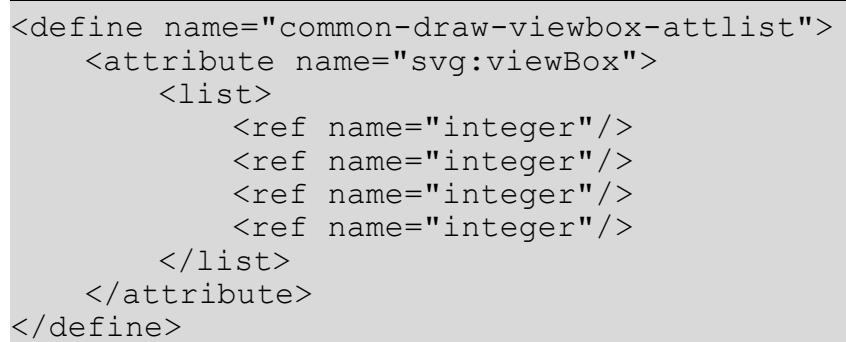

\section{Style}

The draw:style-name and presentation:style-name attributes specify a style for the drawing shape. If draw: style-name is used, the shape is a regular graphic shape. If presentation:style-name is used, the shape is a presentation shape as described in section 9.6.

The value of both attributes is the name of a style:style> element. If the draw:stylename attribute is used, the style must have a family value of graphic. If the presentation:style-name is used, the style must have a family value of presentation. The formatting properties of the specified style and its optional parent styles are used to format the shape. See also section 14.13.1.

The draw:class-names and presentation:class-names attributes take a whitespace separated list of either graphic or presentation style names. The referenced styles are applied in the order they are contained in the list. If both, draw: style-name and draw:class-names, or both presentation:style-name and presentation:class-names are present, the style referenced by the style-name attribute is treated as the first style in the list in the classnames attribute. Conforming application should support the class-names attribute and also should preserve it while editing.

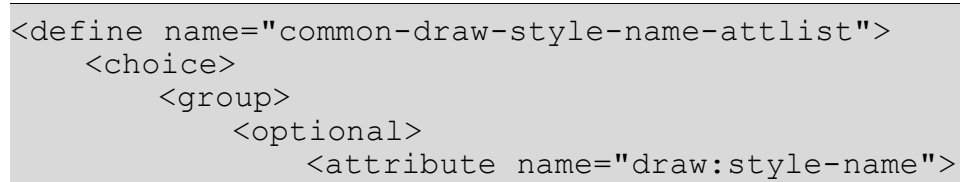




\section{Text Style}

The draw:text-style-name attribute specifies a style for the drawing shape that is used to format the text that can be added to this shape.

The value of this attribute is the name of a style:style $>$ element with a family value of paragraph.

6464

6465

6466

6467

6468

6469

6470

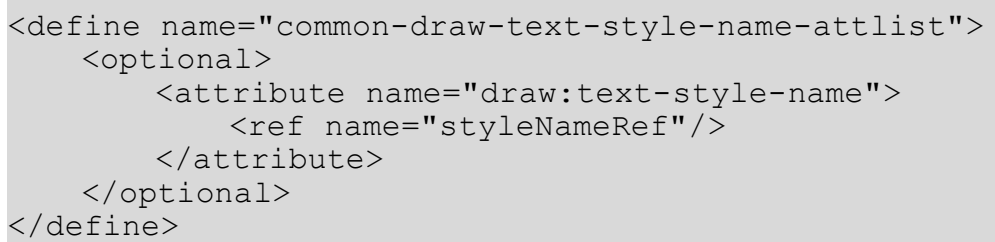

\section{Layer}

The attribute draw: layer can assign each shape to a layer. The value of this attribute must be the name of a layer inside the layer-set of the document.

6471

6472

6473

6474

6475

6476

6477

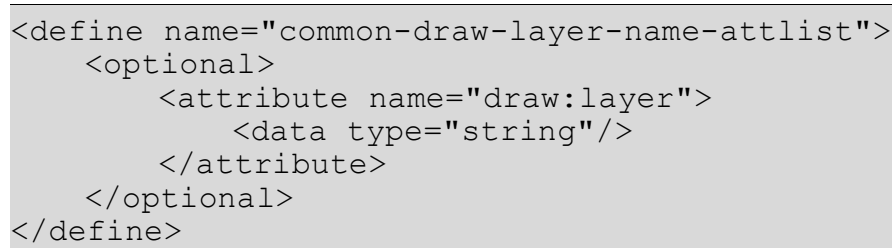

ID

The draw: id attribute assigns an unique ID to a drawing shape that can be used to reference the shape. 


\section{Z-Index}

Drawing shapes are rendered in a specific order. In general, the shapes are rendered in the order in which they appear in the XML document. To change the order, use the svg:width and svg: height attribute.

This attribute is optional.

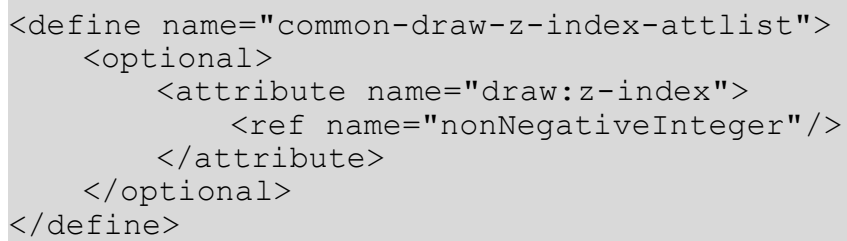

\subsubsection{Common Shape Attributes for Text and Spreadsheet Documents}

The attributes described in this section are common to all drawing shapes contained in text and spreadsheet documents.

\section{End Position}

If a drawing shape is included in a spreadsheet document and if the anchor of the shape is in a cell, then the attributes table:end-cell-address, table:end-x and table:end-y specify the end position of the shape and the size attributes are ignored. The end position is specified using the cell address of the cell in which the end position is located, and the $\mathrm{x}$ and $\mathrm{y}$ coordinates of the end position relative to the top left edge of the cell.

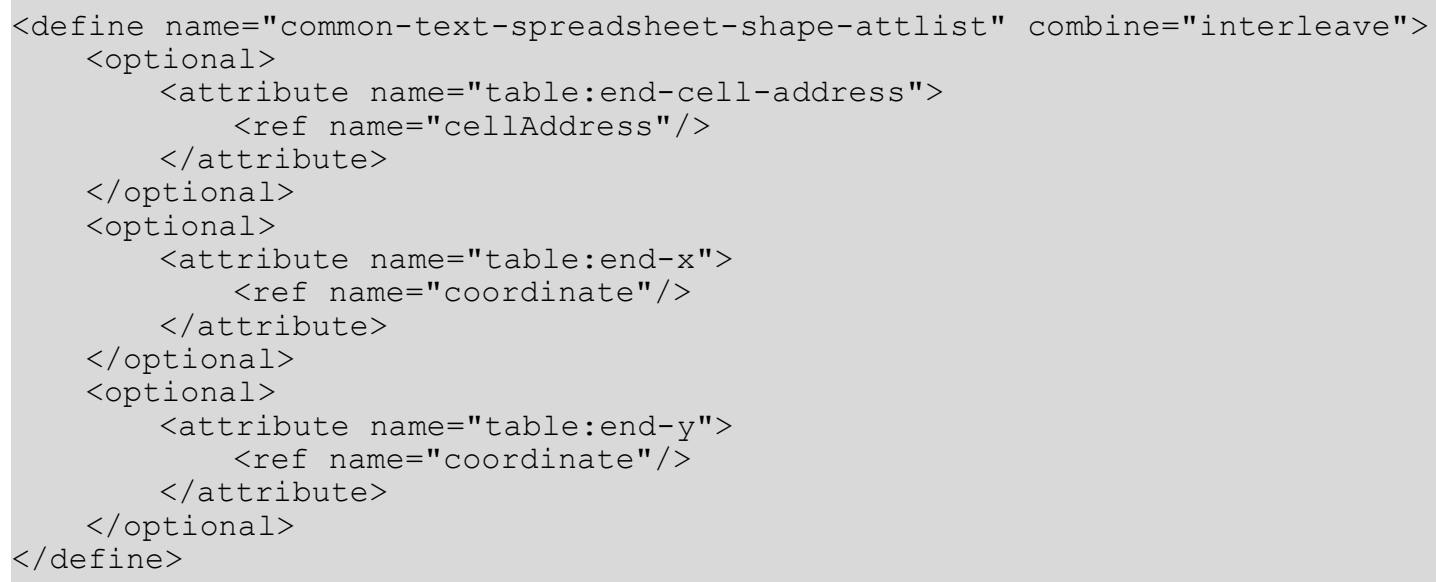

\section{Table Background}

If a drawing shape is included in a spreadsheet document, then the table:tablebackground attribute specifies whether or not the shape is in the table background. If the attribute is not existing, the shape is included in the foreground of the table. 


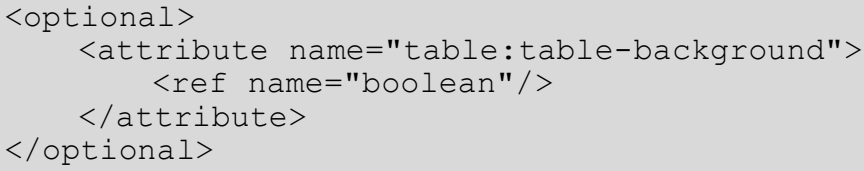

\section{Text Anchor}

Within text documents, the anchor type attribute text:anchor-type specifies how a frame is bound to the text document. The anchor position is the point at which a frame is bound to a text document. The anchor position depends on the anchor type as explained in the following table.

\begin{tabular}{|c|c|c|c|}
\hline $\begin{array}{c}\text { If the value of } \\
\text { the } \\
\text { text:anchor- } \\
\text { type attribute } \\
\text { is ... }\end{array}$ & The anchor position is... & $\begin{array}{l}\text { The drawing shape } \\
\text { element appears ... }\end{array}$ & Notes \\
\hline page & $\begin{array}{l}\text { The page that has the } \\
\text { same physical page } \\
\text { number as the value of } \\
\text { the text: anchor-page- } \\
\text { number attribute that is } \\
\text { attached to the drawing } \\
\text { shape element. If no } \\
\text { text: anchor-page- } \\
\text { number attribute is given, } \\
\text { the anchor position is the } \\
\text { page at which the } \\
\text { character behind the } \\
\text { drawing object element } \\
\text { appears. }\end{array}$ & $\begin{array}{l}\text { Either } \\
\text { - At the start of the } \\
\text { document body, } \\
\text { outside any } \\
\text { paragraph or frame, } \\
\text { provided a } \\
\text { text: anchor- } \\
\text { page-number } \\
\text { attribute is given. } \\
\text { Or } \\
\text { Inside any paragraph } \\
\text { element that is not } \\
\text { contained in a } \\
\text { header, footer, } \\
\text { footnote, or text box, } \\
\text { if a text: anchor- } \\
\text { page-number } \\
\text { attribute is not given. }\end{array}$ & $\begin{array}{l}\text { The physical page } \\
\text { number is the } \\
\text { number assigned to } \\
\text { the page if all } \\
\text { pages in the } \\
\text { document are } \\
\text { counted starting } \\
\text { with page } 1 .\end{array}$ \\
\hline frame & $\begin{array}{l}\text { The parent text box that } \\
\text { the current drawing shape } \\
\text { element is contained in. }\end{array}$ & $\begin{array}{l}\text { In the element } \\
\text { representing the text box } \\
\text { to which the drawing } \\
\text { object is bound. For } \\
\text { example, if an image is } \\
\text { bound to a text box, the } \\
\text { image element is located } \\
\text { in the text box element. }\end{array}$ & \\
\hline paragraph & $\begin{array}{l}\text { The paragraph that the } \\
\text { current drawing shape } \\
\text { element is contained in. }\end{array}$ & $\begin{array}{l}\text { At the start of the } \\
\text { paragraph element. }\end{array}$ & \\
\hline char & $\begin{array}{l}\text { The character after the } \\
\text { drawing shape element. }\end{array}$ & Just before the character. & \\
\hline
\end{tabular}




\begin{tabular}{|c|l|l|l|}
\hline $\begin{array}{c}\text { If the value of } \\
\text { the } \\
\text { text:anchor- } \\
\text { type attribute } \\
\text { is ... }\end{array}$ & The anchor position is... & $\begin{array}{c}\text { The drawing shape } \\
\text { element appears ... }\end{array}$ & Notes \\
\hline as-char & $\begin{array}{l}\text { There is no anchor } \\
\text { position. The drawing } \\
\text { shape behaves like a } \\
\text { character. }\end{array}$ & $\begin{array}{l}\text { At the position where the } \\
\text { character appears in the } \\
\text { document. }\end{array}$ & \\
\hline
\end{tabular}

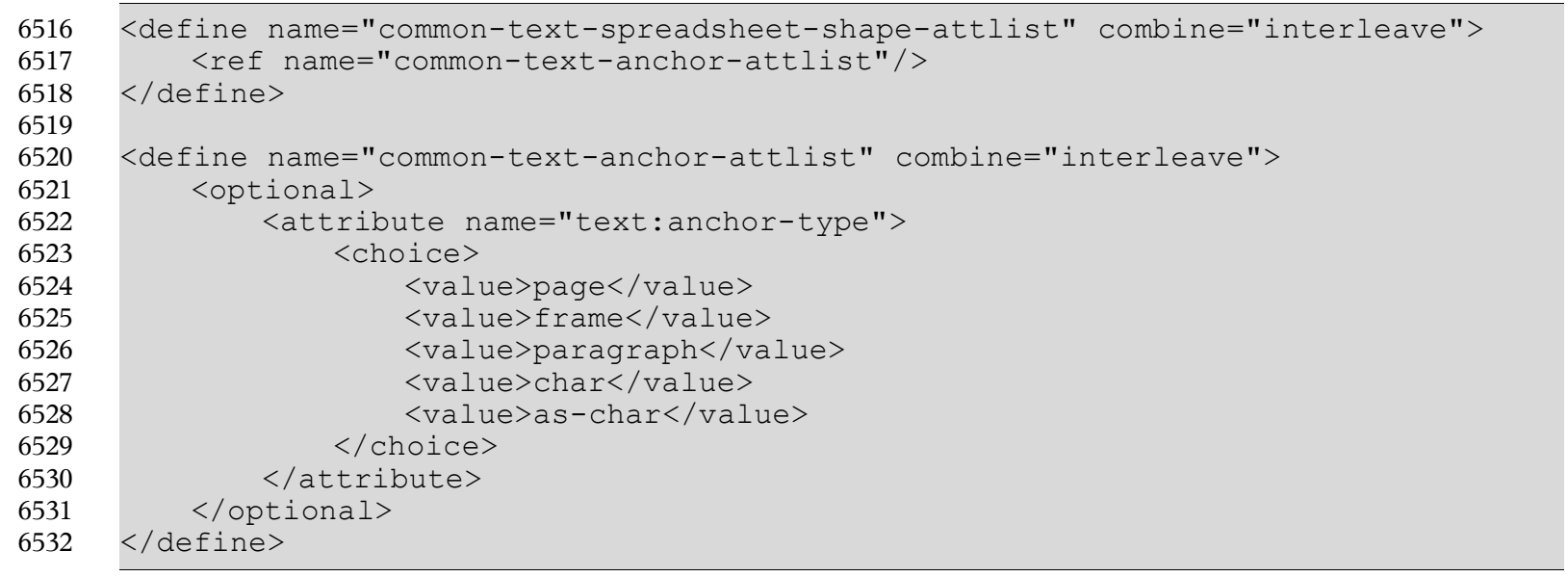

\section{Anchor Page Number}

Within text documents, the text:anchor-page-number attribute specifies the physical page number of an anchor if the drawing object is bound to a page.

\subsubsection{Common Drawing Shape Content}

Most drawing shapes may contain text content. The text content may contain paragraphs (see section 4.1.2) as well as lists (see section 4.3).

\subsubsection{Common Shape Attribute Groups}

The following defined attributes are common for all shapes that supports styles and no text. 
6580

6581

6582

6583

6584

6585

\section{Al}

\section{Align}

The attribute draw: align specifies the alignment behavior of the glue point if the drawing object is resized and the shape edge to which the glue point's position relates. A missing vertical or horizontal position in the attribute's value means that the glue point is horizontally or vertically centered.

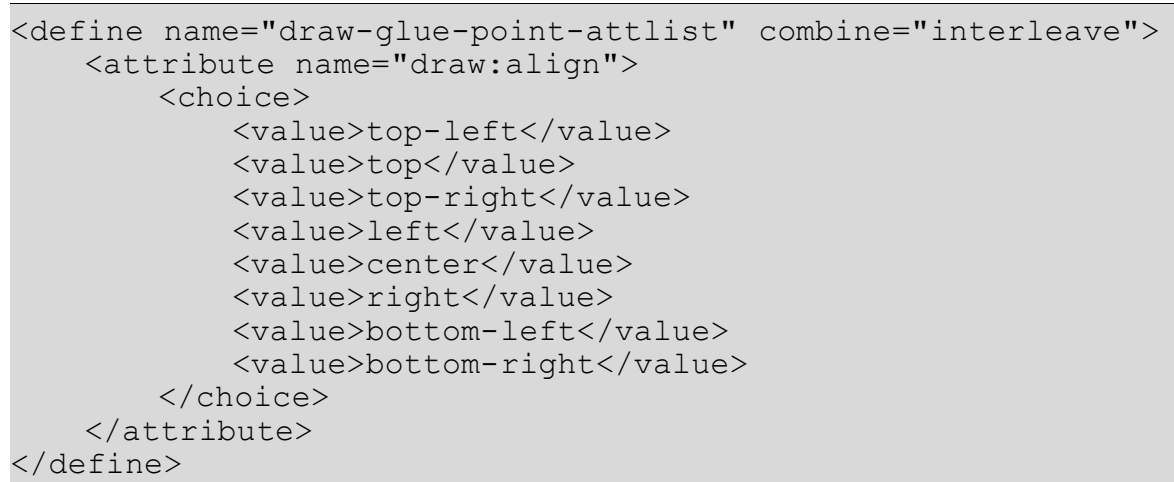

\section{Escape Direction}

The attribute draw:escape-direction specifies the direction in which the connection line escapes from the drawing object if a connector connects to the glue point. The value horizontal means the the connection line may escape to the left or to the right, the value vertical means that the connection line may escape up or down. The value auto means that the connection line may escape in all four directions.

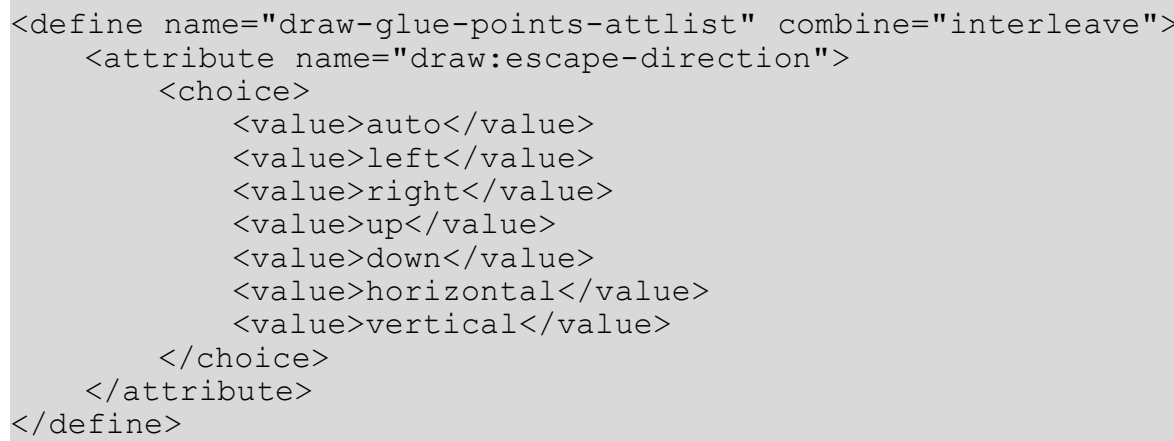

\subsubsection{Event Listeners}

Drawing shapes may have event listeners attached. The event listeners that are attached to, for example, a text box or an image, are represented by an event element as described in section 12.4. This element is contained within the drawing object element, for example, the $<$ draw: text-box> element or the <draw: image $>$ element. 


\subsection{Frames}

A frame is a rectangular container where that contains enhanced content like text boxes, images or objects. Frames are very similar to regular drawing shapes, but support some features that are not available for regular drawing shapes, like contours, image maps and hyperlinks. In particular, a frame allows to have multiple renditions of an object. That is, a frame may for instance contain an object as well as an image. In this case, the application may choose the content that it supports best. If the application supports the object type contained in the frame, it probably will render the object. If it does not support the object, it will render the image.

In general, an application must not render more than one of the content elements contained in a frame. The order of content elements dictates the document author's preference for rendering, with the first child being the most preferred. This means that applications should render the first child element that it supports. A frame must contain at least one content element. The inclusion of multiple content elements is optional. Application may preserve the content elements they don't render, but don't have to.

Within text documents, frames are also used to position content outside the default text flow of a document.

Frames can contain:

- Text boxes

- Objects represented either in the OpenDocument format or in a object specific binary format

- Images

- Applets

- Plug-ins

- Floating frames

Like the formatting properties of drawing shapes, frame formatting properties are stored in styles belonging to the graphic family. The way a frame is contained in a document also is the same as for drawing shapes.

6613

6614

6615

6616

6617

6618

6619

6620

6621

6622

6623

6624

6625

6626

6627

6628

6629

6630

6631

6632

6633

6634

6635

6636

6637

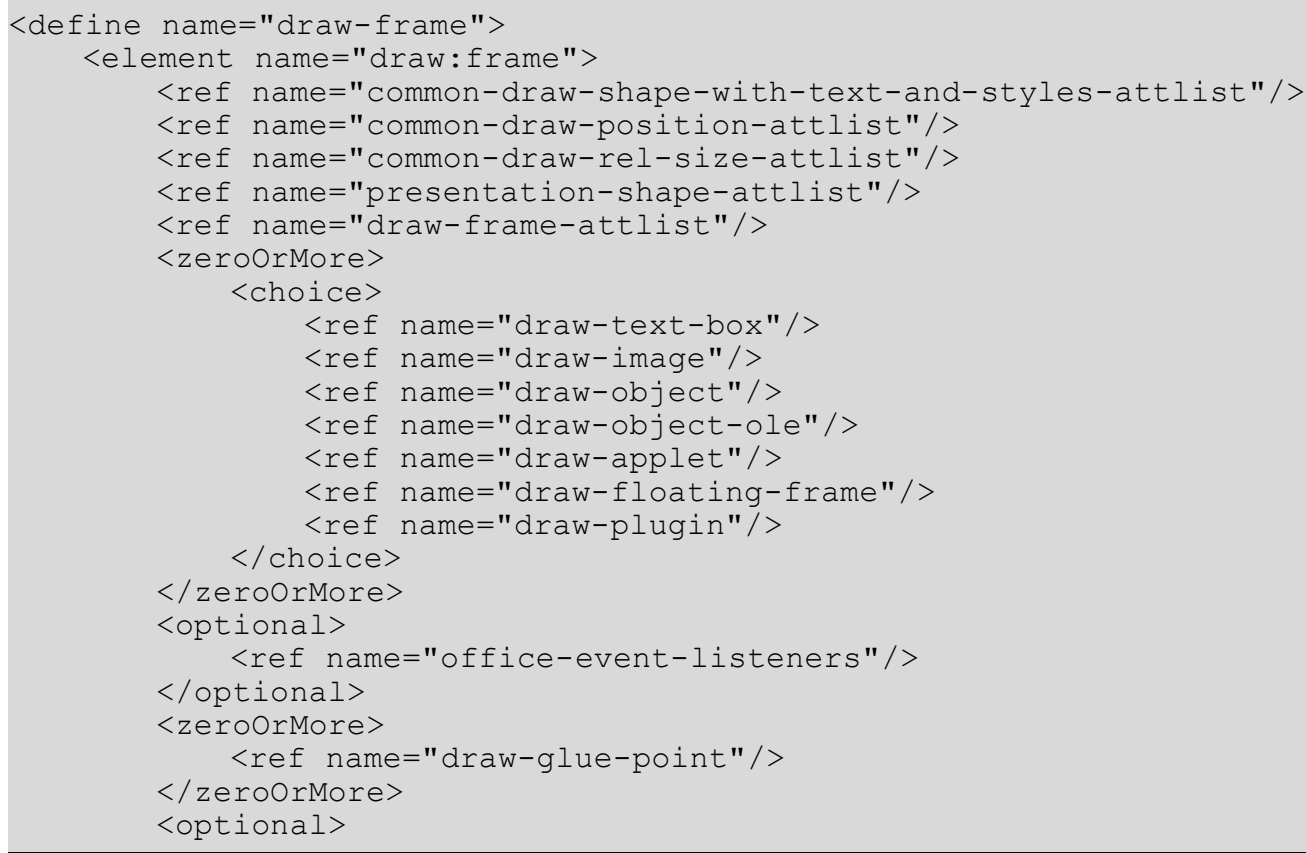




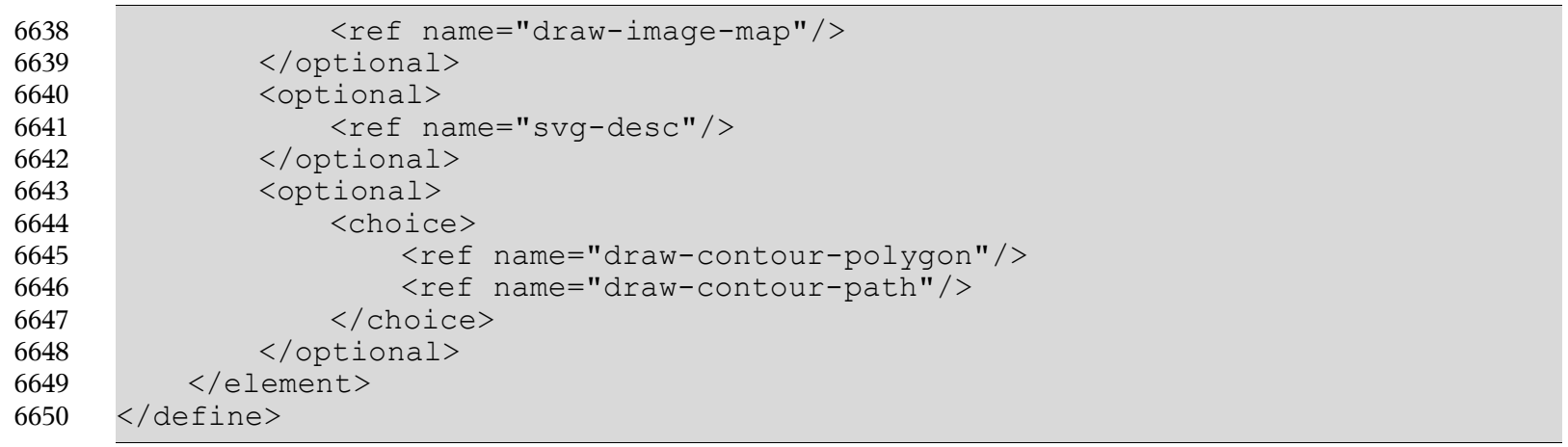

The attributes that may be associated with the <draw: frame> element are:

- Position, Size (relative sizes, see below), Style, Layer, Z-Index, ID, and Transformation see section 9.2.15.

- Text anchor, table background, draw end position - see section 9.2.16

- Presentation class - see section 9.6.1

- Copy frames

The following elements may be contained in the image element:

- Event Listeners - see section 12.4.

- Glue Points - see section 9.2.19.

- Image Map - see section 9.3.11.

- Alternative Text - see section 9.3.9.

- $\quad$ Contour - see section 9.3.8.

\section{Relative Sizes}

For frames, the width and height of the drawing object may be specified as an relative value using the style:rel-width and style:rel-height attributes. The relative value either is a percentage value, the special value scale, or the special value scale-min.

The interpretation of relative values depends on the anchor of the drawing object. If the anchor for the drawing object is in a table cell, the percentage value relates to the surrounding table box. If the anchor for the drawing object is in a text box, the percentage value relates to the surrounding text box. In other cases, the percentage values relate to the width of the page or window.

The value scale for the width means that the width should be calculated depending on the height, so that the ratio of with and height of the original image or object size is preserved.

The value scale for the height means that the height should be calculated depending on the width, so that the ratio of with and height of the original image or object size is preserved.

The value scale-min equals the value scale, except that the calculated width or height is a minimum height rather than an absolute one.

To support application that don't support relative with and heights, applications that save the attributes style:rel-width or style:rel-height should also provide the real width and heights in the svg:width and svg:height/fo:min-height attributes. 


\section{Copy Frames}

Multiple frames can be set to display the exact same underlying data: for instance for a company logo, that must appear somewhere on every page, without being part of a header or footer.

A frame can be set to display the contents of another frame, referenced by the draw: copy-of attribute. This does not effect style and position information. This is, the frame that has the draw: copy-of attribute has its own style and position information and does not use the one of the referenced frame.

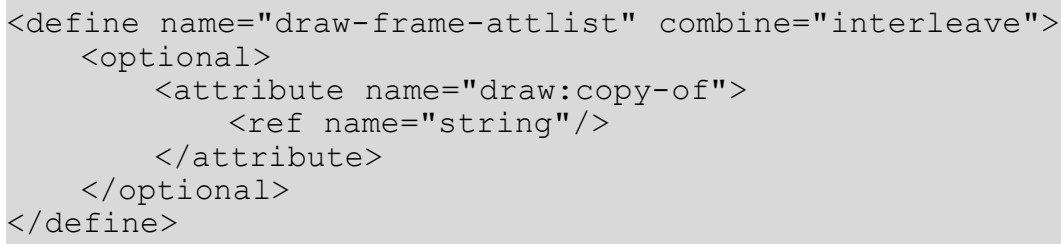

\subsubsection{Text Box}

The <draw: text-box >element represents a text box. A text box may be used to place text in a container that is outside of the normal flow of the document.

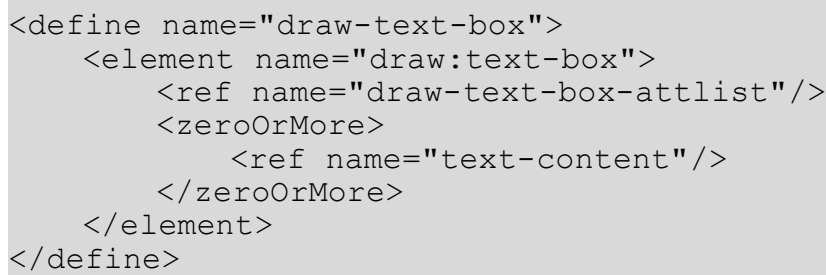

The attributes that may be associated with the <draw: text-box> element are:

- Chain

- Round Corners

- Minimum Height and Width

- Maximum Height and Width 
Text boxes don't support contours as described in section 9.3.8 and alternative texts as described in section 9.3.9.

\section{Chain}

Text boxes can be chained, in other words, if the content of a text box exceeds its capacity, the content flows into the next text box in the chain. To chain text boxes, the attribute draw: chainnext-name is used, The value of this attribute is the name of the next text box in the chain. Chained text boxes usually are supported by text documents only.

6687

6688

6689

6690

6691

6692

6693

6694

6695

6696

6697

6698

6699

6700

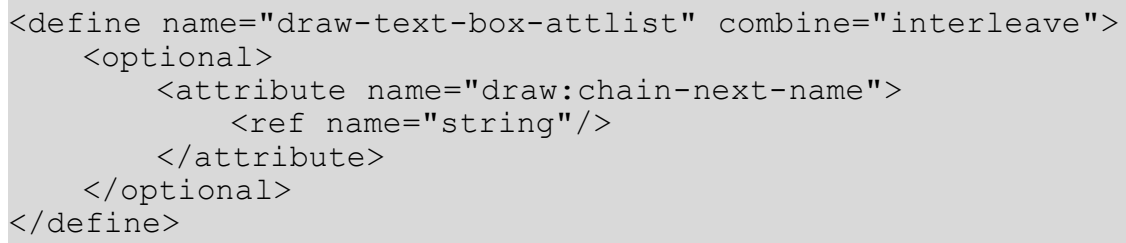

\section{Round Corners}

The attribute draw: corner-radius specifies the radius of the circle used to round off the corners of the text-box.

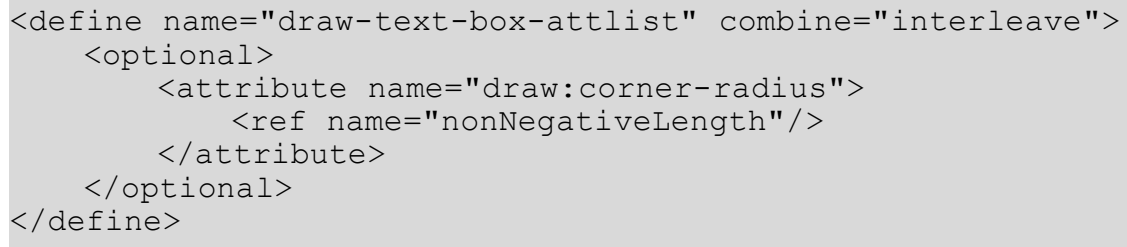

\section{Minimum Height and Width}

The fo:min-height and fo:min-width attributes specify a minimum height or width for a text box. If they are existing, they overwrite the height or width of a text box specified by the svg: height and svg:width attributes of the surrounding <draw: frame> element. Their value can be either a length or a percentage. If the anchor for the text box is in a table cell, the percentage value relates to the surrounding table box. If the anchor for the text box is in a text box, the percentage value relates to the surrounding text box. In other cases, the percentage values relate to the height of the page or window.

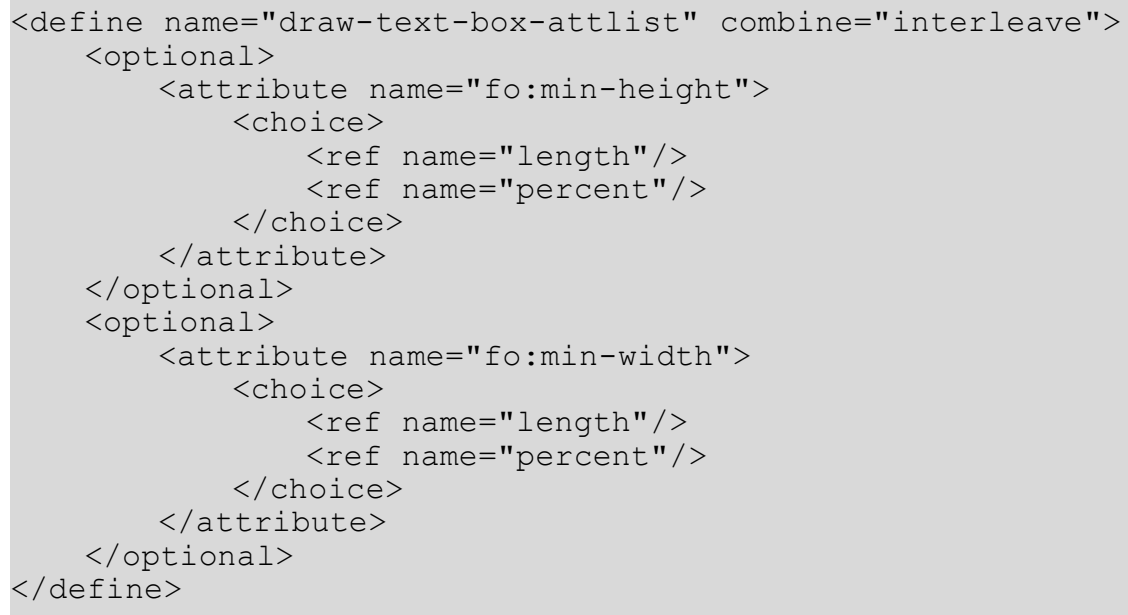




\section{Maximum Height and Width}

If the width or height of a text box is specified as a minimum width or height (using the fo:minwidth or fo:min-height attributes), then the fo:max-width and fo:max-height attributes specify a maximum width and height for the text box. When these maximum values are reached, the text box stops increasing in size. The attributes' value can be either a length or a percentage. If the anchor for the text box is in a table cell, the percentage value relates to the size of the surrounding table cell. If the anchor for the text box is in a text box, the percentage value relates to the size of the surrounding text box. In other cases, the percentage values relate to the width or height of the page or window.

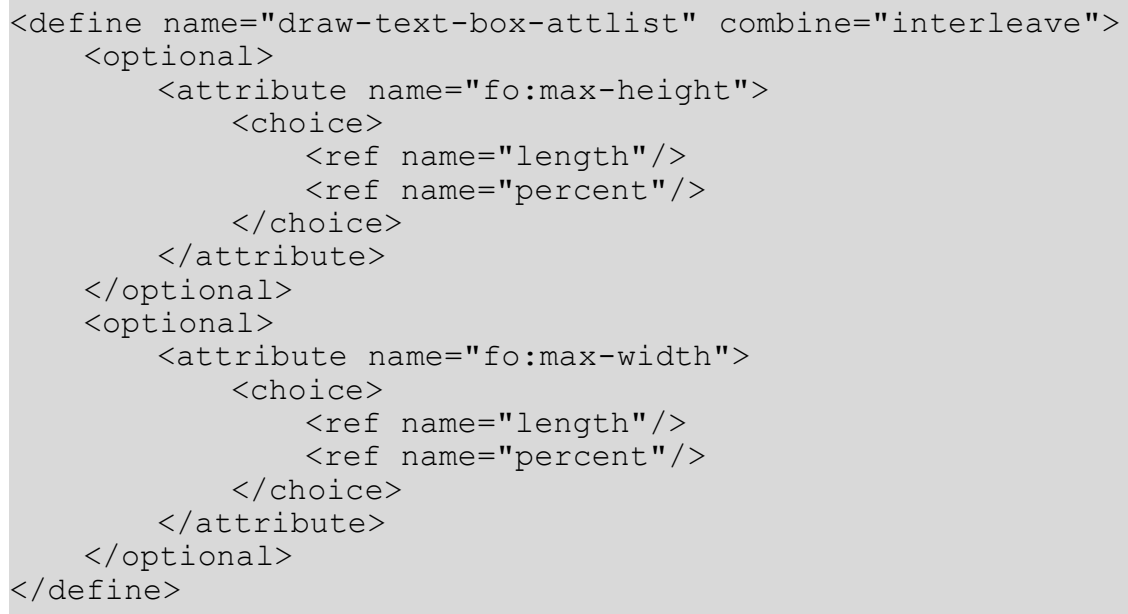

\subsubsection{Image}

The <draw: image $>$ element represents an image. An image can be either:

- Contained in a document as a link to an external resource

or

\section{- Embedded in a document}

This element can be an [XLink], in which case the element contains some attributes with fixed values that describe the link semantics.

While the image data may have an arbitrary format, it is recommended that vector graphics are stored in the [SVG] format and bitmap graphics in the [PNG] format.

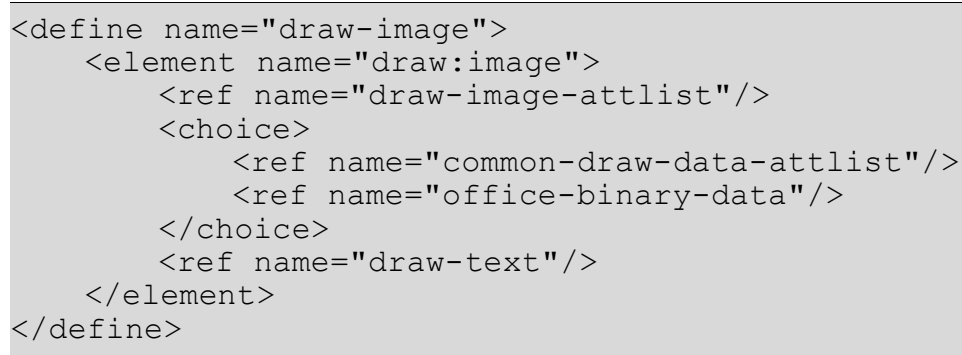

The attributes that may be associated with the <draw: image> element are:

- Image data

- Filter name 
Like most other drawing shapes, image drawing shapes may have text content. It is displayed in addition to the image data.

\section{Image Data}

The image data can be stored in one of the following ways:

- The image data is contained in an external file. Use the xlink: href and associated attributes described below to link to the external file.

- The image data is contained in the <draw: image $>$ element. The <draw: image $>$ then element contains an <office:binary-data> element that contains the image data in BASE64 encoding. In this situation the $x l i n k:$ href attribute is not required.

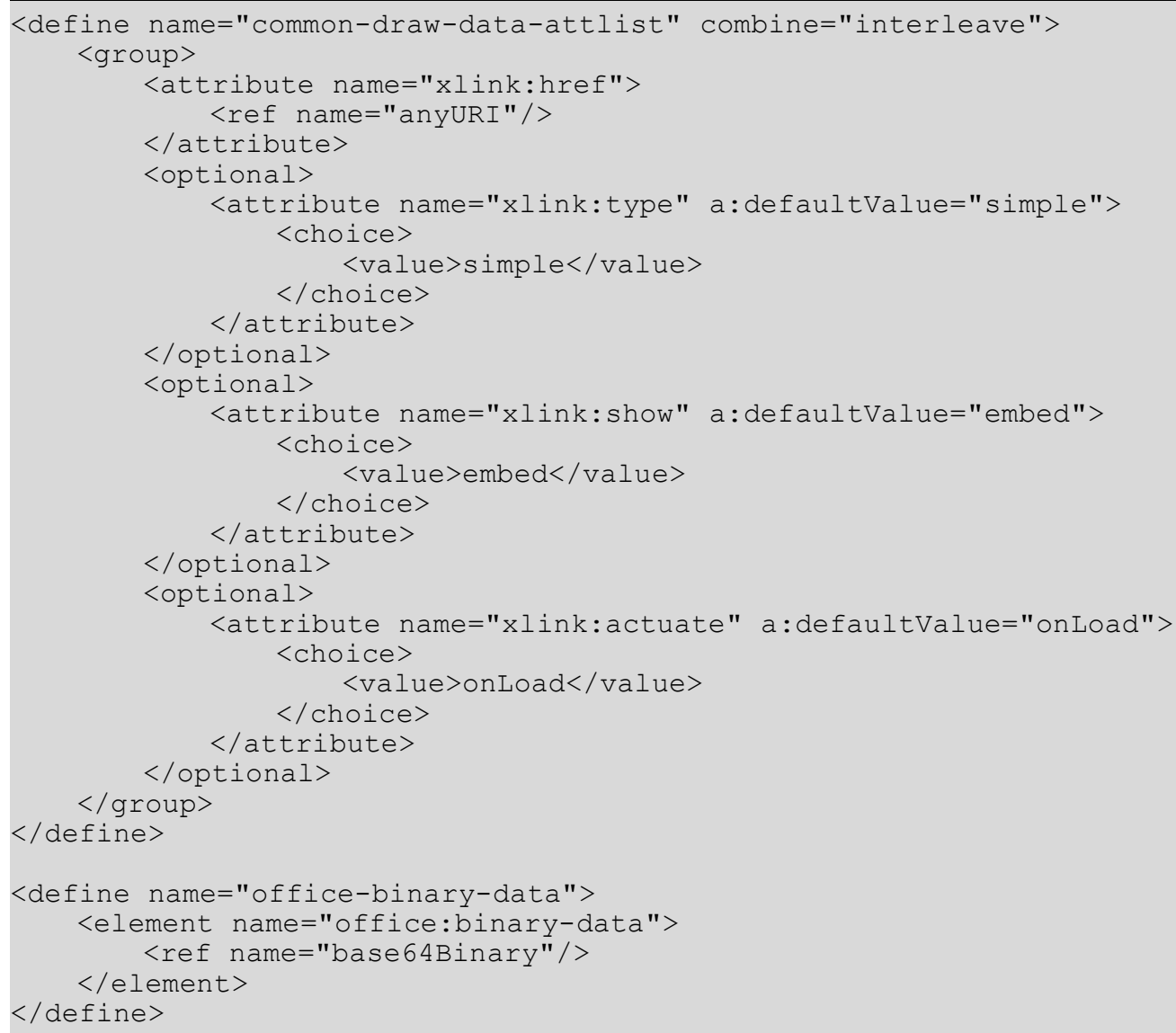

\section{Filter Name}

If required, the draw: filter-name attribute can represent the filter name of the image. This attribute contains the internal filter name that the office application software used to load the graphic. 


\subsubsection{Objects}

A document in OpenDocument format can contain two types of objects, as follows:

- Objects that have an OpenDocument representation. These objects are:

- Formulas (represented as [MathML])

- Charts

- Spreadsheets

- Text documents

- Drawings

- Presentations

- Objects that do not have an XML representation. These objects only have a binary representation, An example for this kind of objects OLE objects (see [OLE]).

The <draw: object $>$ element represents objects that have a XML representation. The $<$ draw:object-ole> element represents objects that only have a binary representation.

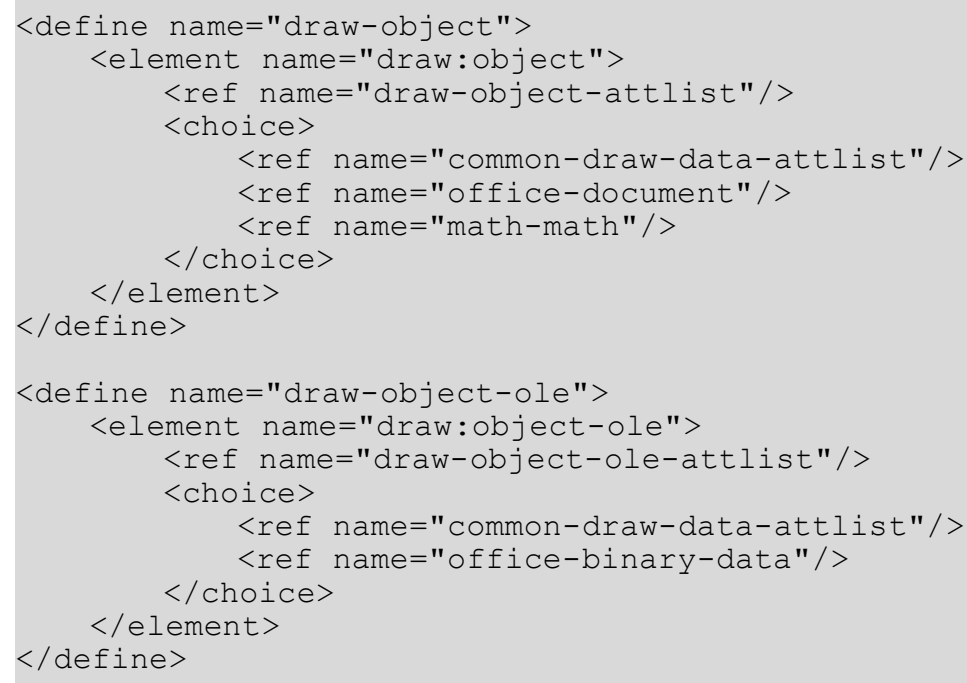

The attributes that may be associated with the <draw: object $>$ and <draw: object-ole $>$ elements are:

- Object data

- Table Change Notifications

- Class Id

Objects do not support transformations as described in section 9.2.15.

\section{Object Data}

The object data can be called in one of the following ways:

- The xlink: href attribute links to the object representation, as follows: 
- For objects that have an XML representation, the link references the sub package of the object. The object is contained within this sub page exactly as it would as it is a document of its own.

- For objects that do not have an XML representation, the link references a sub stream of the package that contains the binary representation of the object.

Application that support objects should support linking to objects that are contained within the same package. They may also support linking to object located outside the package.

- The object data is contained in the <draw:object $>$ or $<$ draw:object-ole $>$ element, as follows:

- The <draw: object $>$ element contains the XML representation of the object, for example, an <office:document> or a <math:math> element.

- The <draw:object-ole> element contains an <office:binary-data> element, which contains the binary data for the object in BASE64 encoding.

In these situations, the xlink: href attributes are not required.

The xlink: href attribute is described in section 9.3.2.

It is recommended to include an image representation of the object into the frame in addition to the object itself.

\section{Notification on Table Change}

Some objects, especially charts, may require a notification when a table in the document changes. To enable this notification, use the draw: notify-on-change-of-table attribute, which contains the name of the table. This attribute can be associated with the <draw: object $>$ element.

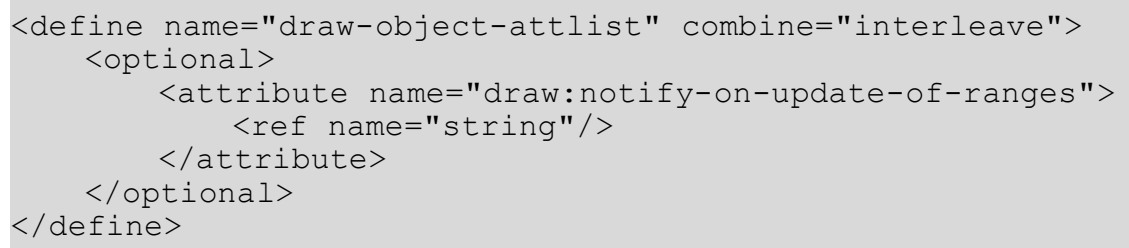

$</$ optional>

\section{Class Id}

The draw: class-id optionally contains the OLE class id of the object (see also [OLE]).

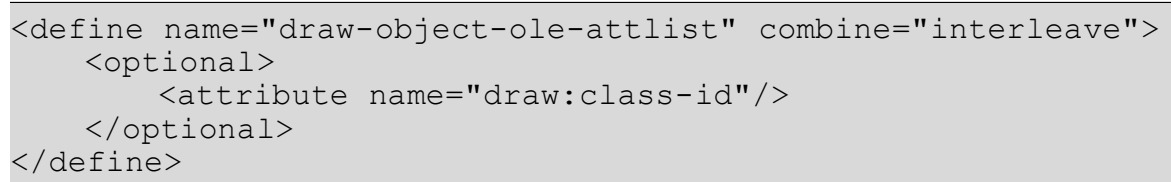

\subsubsection{Applet}

An applet is a small Java-based program that is embedded in a document. The <draw: applet> element is based on the <applet> tag in [HTML4]. This element must contain either the draw: code or draw: object attribute.

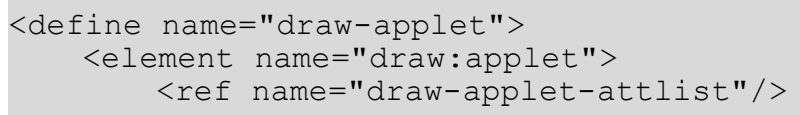




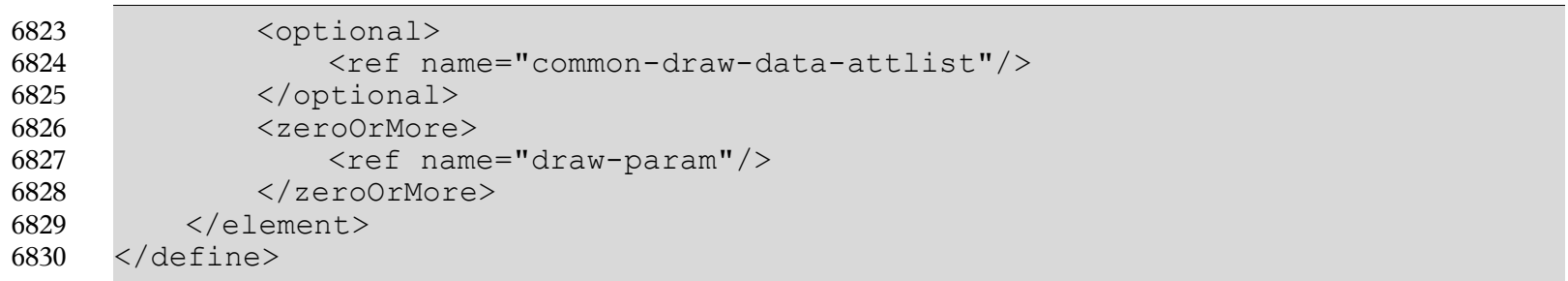

The attributes that may be associated with the <draw: applet $>$ element are:

- Codebase

- Code

- Object

- Archive

- Mayscript

The only element that may be contained in the <draw: applet> element is:

- Parameter (see section 9.3.6)

Applets do not support transformations as described in section 9.2.15.

\section{Codebase}

The codebase specifies the base URI for the applet. If this attribute is not specified, then it defaults the same base URI as for the current document. The codebase is represented be the [XLink] attributes $x l i n k:$ href, xlink:type, xlink: show, and xlink:actuate. The $\mathrm{xlink}$ : href attribute is described in section 9.3.2.

\section{Code}

The draw: code attribute specifies one of the following:

- The name of the class file that contains the compiled applet subclass.

- The path to the class, including the class file itself.

Either this attribute or the draw:object attribute is required. The value of this attribute is interpreted in relation to the codebase for the applet.

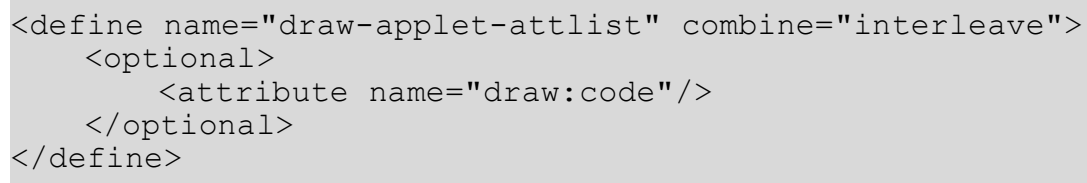

\section{Object}

The draw: object attribute specifies a resource that contains a serialized representation of the state of the applet. The serialized data contains the class name of the applet but not the implementation. The value of this attribute is interpreted in relation to the codebase for the applet. 


\section{Archive}

The draw: archive attribute specifies a comma-separated list of URLs for archives that contain classes and other resources that are preloaded.

6841

6842

6843

6844

6845

\section{Mayscript}

The draw:mayscript attribute specifies whether or not the applet can be scripted.

6846

6847

6848

6849

6850

6851

6852

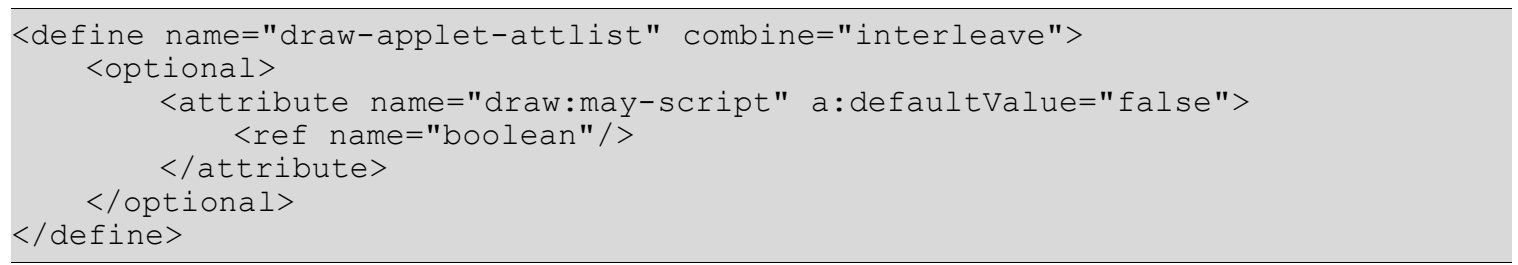

\subsubsection{Plugins}

A plugin is a binary object that is plugged into a document to represent a media-type that usually is not handled natively by office application software. Plugins are represented by the

$<$ draw:plugin> element

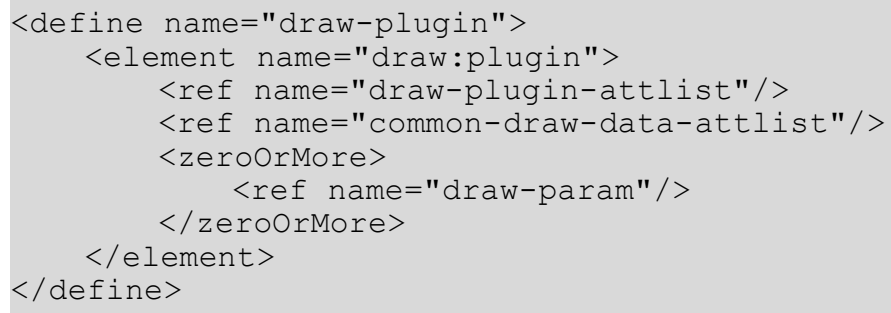

The attributes that may be associated with the <draw:plugin> element are:

- Mime type

- Source

The only element that may be contained in the <draw: plugin> element is:

- Parameter (see section 9.3.6)

Plugins do not support transformations as described in section 9.2.15.

\section{Mime type}

The draw:mimetype attribute specifies the MIME type to which this plugin should be registered.

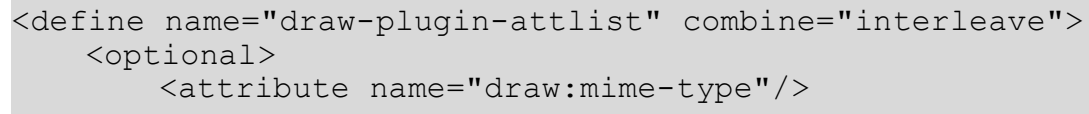




\section{Source}

The [XLink] attributes $x l i n k:$ href, $x l i n k:$ type, $x l i n k:$ show, and $x l i n k$ :actuate specify the source of the plugin. The xlink:href attribute is described in section 9.3.2.

\subsubsection{Parameters}

The <draw: param> element contains parameters that are passed to an applet or plugin when they are initialized.

6867

6868

6869

6870

6871

6872

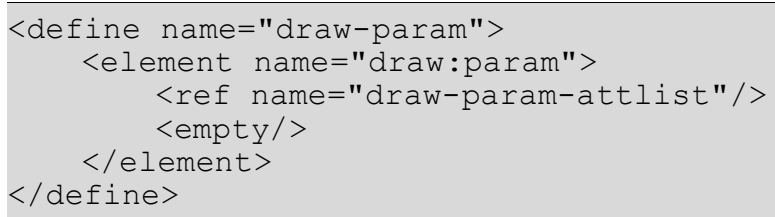

The attributes that may be associated with the <draw: param $>$ element are:

- Name

- Value

\section{Name}

The draw: name attribute specifies the name of a runtime parameter.

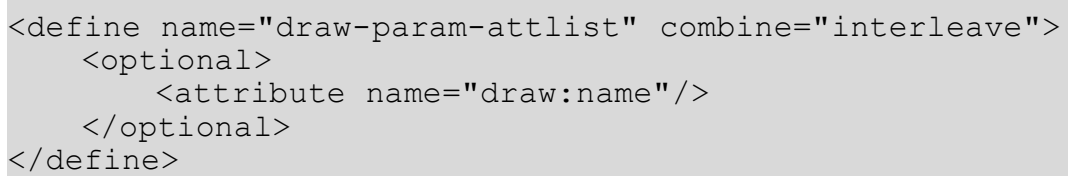

\section{Value}

The draw:value attribute specifies the value of the runtime parameter specified by the name.

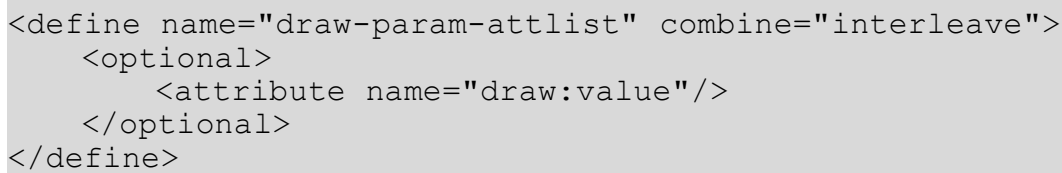

\subsubsection{Floating Frame}

A floating frame is a frame embedded in a document, which may contain, for example, a text document or spreadsheet. A floating frame is represented by the <draw: floating-frame> element.

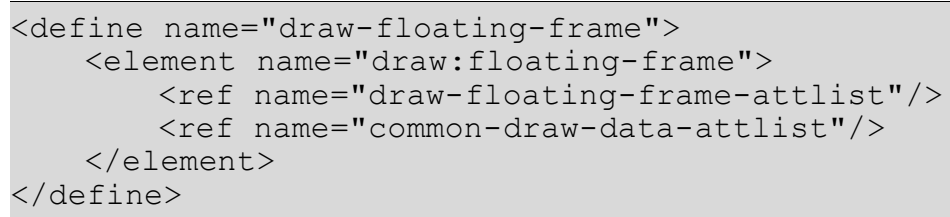


The attributes that may be associated with the <draw: floating-frame> element are:

\section{- Source}

\section{- Frame Name}

Floating frames do not support transformations as described in section 9.2.15.

\section{Source}

The [XLink] attributes $\mathrm{xlink}$ : href, $x$ link: type, $\mathrm{xlink}$ : show, and xlink: actuate specify the source of the floating frame. The xlink: href attribute is described in section 9.3.2.

\section{Frame Name}

The draw: frame-name specifies the name of the frame. This name can be used as target from within hyperlinks.

6889

6890

6891

6892

6893

6894

6895

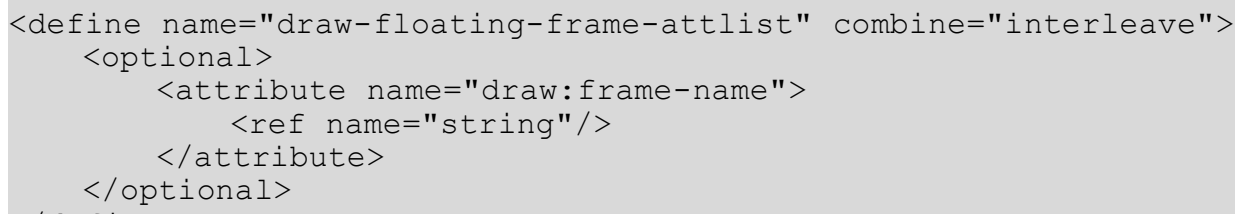

\subsubsection{Contour}

The <draw: contour-polygon> and <draw: contour-path> elements may be contained in the following elements:

- <draw:image>

- <draw:object>

- <draw:object-ole>

- <draw:applet>

- <draw:plugin>

- <draw:floating-frame>

These elements describe the contour of an image or object.

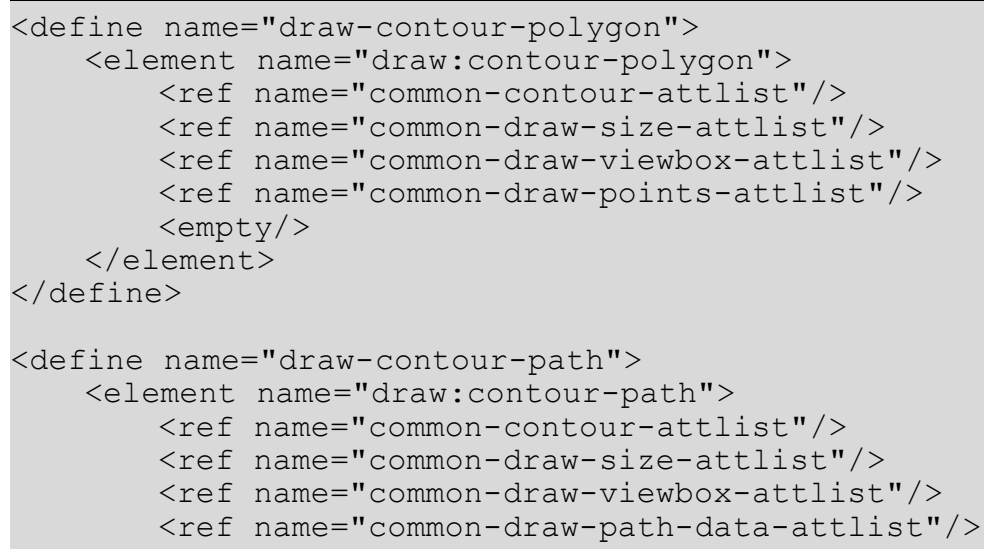


The elements are similar to the <draw: polygon $>$ (see section 9.2.4) and <draw: path $>$ (see section 9.2.6) elements, except that they specify a contour rather than a drawing shape. The attributes they support are the ones for the size, the viewbox, the points (contour polygon only) and the path (contour path only).

In contrast to any other element the $\mathrm{svg}: \mathrm{width}$ and $\mathrm{svg}:$ height attributes may have a pixel length (i.e. $20 \mathrm{px}$ ) as value (as well as traditional lengths like $2 \mathrm{~cm}$ ).

\section{Recreate on Edit}

The draw: recreate-on-edit attribute specifies if the contour of the image or object should be recreated automatically when the image or object is edited.

6915

6916

6917

6918

6919

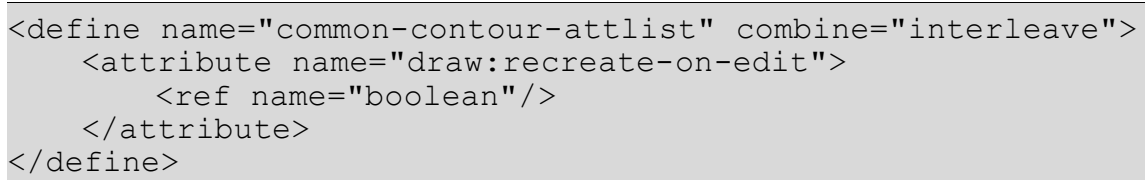

\subsubsection{Alternative Text}

The <svg: desc > element specifies an alternative text as specified in $§ 5.4$ of [SVG]. It can be used with the following elements:

- <draw:image>

- <draw:object>

- <draw:object-ole>

- <draw:applet>

- <draw:floating-frame>

- <draw:plugin>

- <draw:object-ole>

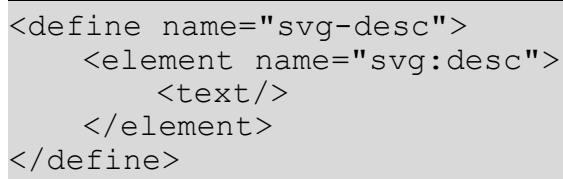

\subsubsection{Hyperlinks}

Frames may behave like hyperlinks. Such hyperlinks are represented by the <draw: a> element, where. the element's content is the frame that should be the source of the link.

This element is an [XLink] and has some attributes with fixed values and describe the semantics of the link.

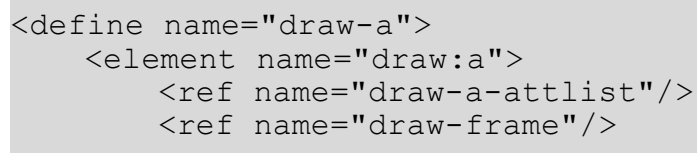


The attributes that may be associated with the <draw: a> element are:

- Link location

- Link target frame

- Name

- Server side image map

\section{Link Location}

The $x l$ ink: href attribute specifies the target location of the link.

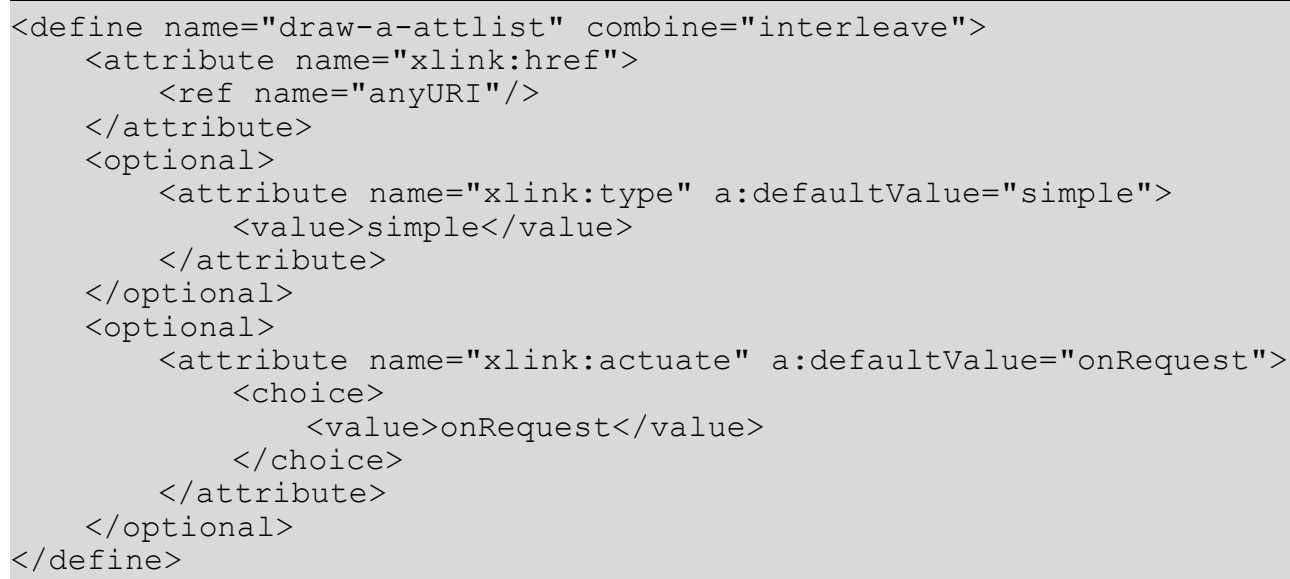

\section{Link Target Frame}

The office:target-frame attribute specifies the target frame of the link.

This attribute can have one of the following values:

- _ _self: The referenced document replaces the content of the current frame.

- _blank: The referenced document is displayed in a new frame.

- _ parent : The referenced document is displayed in the parent frame of the current frame.

- t top : The referenced document is displayed in the topmost frame, that is the frame that contains the current frame as a child or descendent but is not contained within another frame.

- A frame name : The referenced document is displayed in the named frame. If the named frame does not exist, a new frame with that name is created.

To conform with the [XLink] specification, an additional $x l i n k$ : show attribute is attached to the <draw: $a>$ element. If the value of the this attribute is blank, the xlink: show attribute value is new. If the value of the this attribute is any of the other value options, the value of the xlink: show attribute is replace.

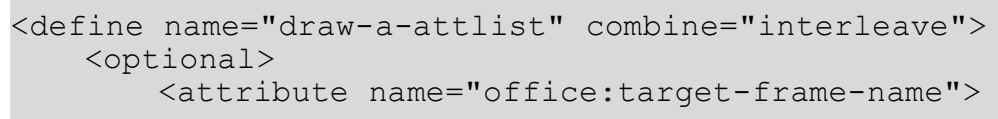




\section{Name}

A hyperlink can have a name, but it is not essential. The office: name attribute specifies the name of the link. The name can serve as a target for other hyperlinks. The name does not have to be unique.

This attribute is specified for compatibility with [HTML4] only, where an $<a>$ element may serve as a link source and target simultaneously. We strongly recommend that this attribute not be used for any purpose other than to represent links that originally came from a HTML document.

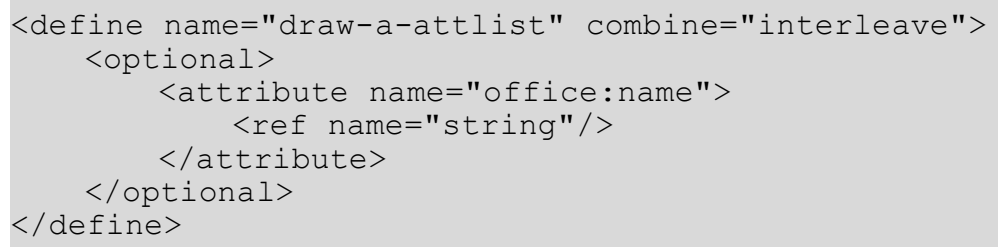

\section{Server Side Image Map}

A link can be a server side image map. If the office:server-map attribute is present, the mouse coordinates of the click position of the graphic shape are appended to the URI of the link. The coordinates may be used by the server to determine which link to activate within the image map.

\subsubsection{Client Side Image Maps}

An client side image map is a collection of hyperlinks that are associated with graphic elements. The image map is a sequence of image map elements. Each image map element associates a hyperlink with an area. The area can be one of the following shapes:

- Rectangular

- Circular

- Polygonal

The <draw: image-map > element represents an image map. 


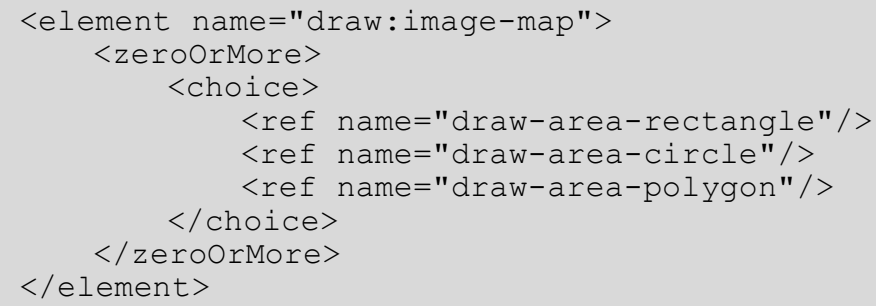

The <draw: image-map > element can contain three types of image map elements, which represent the three types of image map areas as follows:

- Rectangular image map elements

- Circular image map elements

- Polygonal image map elements

Image map elements are described in terms of absolute positions. When loading the XML file, the office application must map the image map onto its associated graphical element, for example an image, in its original size. The application then must scale the image map to match the current size of the image, but in the file format the image is always saved in its unscaled version, matching the dimensions of the unscaled image.

\section{Rectangular Image Map Areas}

The <draw: area-rectangle> element describes a rectangular image map area by an $\mathrm{x}, \mathrm{y}$ position (svg:x and svg:y attributes) as well as a width and the height (svg:width and svg: height attributes). These attributes are required. In addition to this, the attributes described in the Common Image Map Attributes and Elements section below are optionally supported.

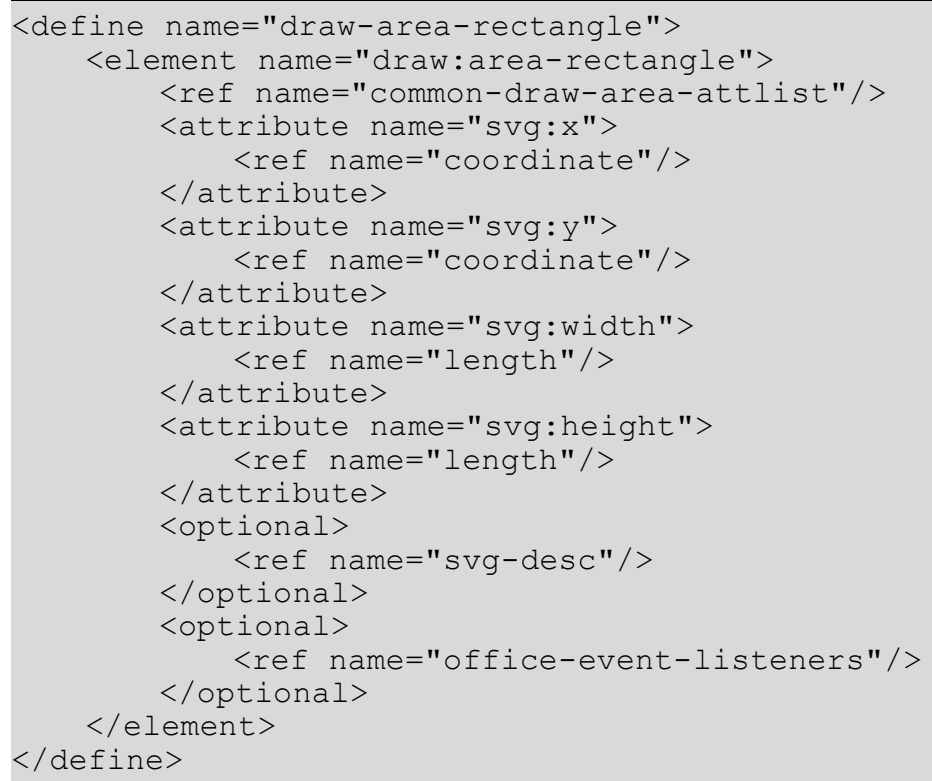

\section{Circular Image Map Areas}

The <draw: area-circle> element describes a circular image map area. The additional attributes for circular image maps are described below in the common attributes section. 
The required attributes $\mathrm{svg}: \mathrm{cx}$ and $\mathrm{svg}: \mathrm{cy}$ specify the center point of the circle. The required $s v g: r$ attribute specifies the radius of the circle.

The attributes described in the Common Image Map Attributes and Elements section are optional.

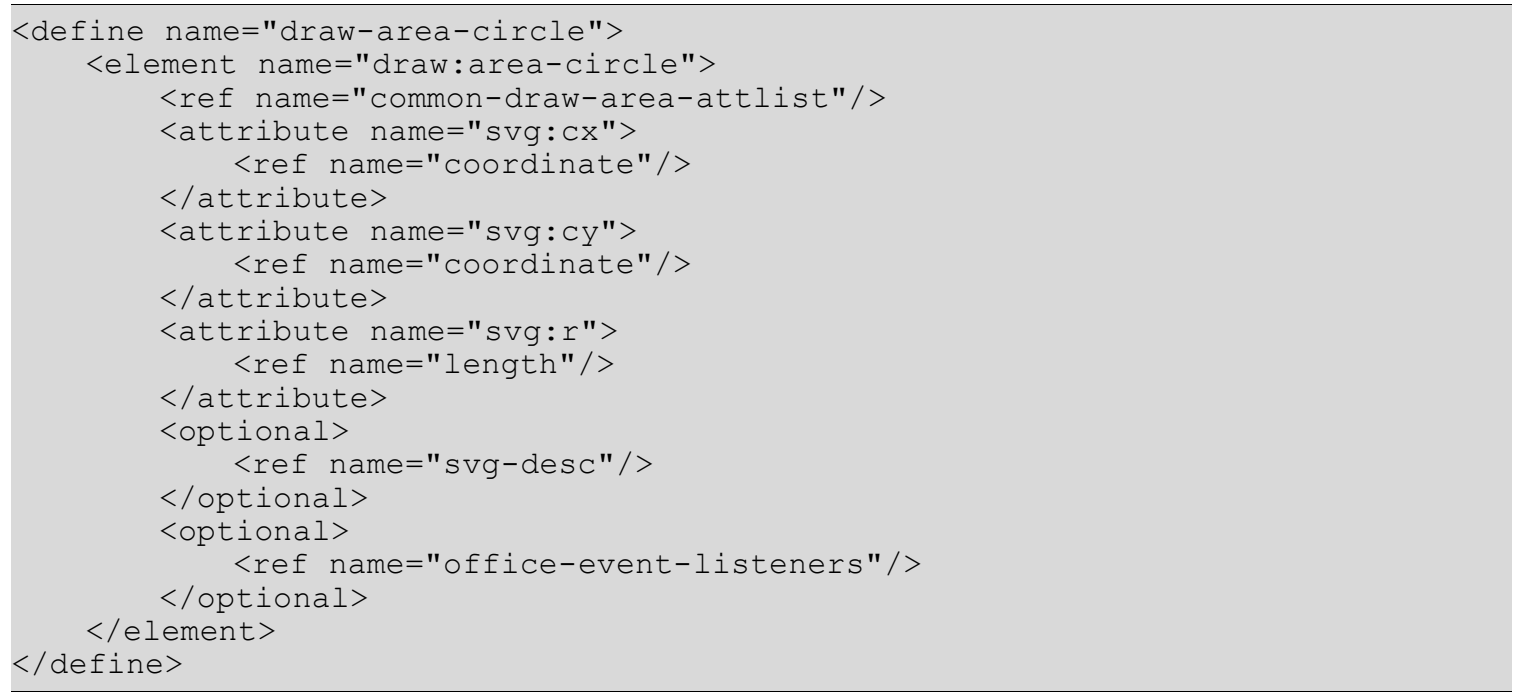

\section{Polygonal Image Map Areas}

The <draw: area-polygon> element describes a polygonal image map area. A polygonal image map area is comprised of the following components:

- A bounding box.

The bounding box, which is represented in the same way as a rectangular image map area using the svg:x, svg:y, svg:width, and svg:height attributes, establishes the reference frame for the view box and the polygon point sequence. The reference frame enables the coordinates to be translated into absolute coordinates.

- A view box.

The view box attribute svg:viewBox establishes a coordinate system for the point sequence. The view box obviates the need to record every point of the point sequence as absolute coordinates with length and unit of measurement.

- A sequence of points in view box coordinates in the svg:points attribute.

For more information about how to represent polygons, see section 9.2.4.

The attributes above are required. The attributes described in the Common Image Map Attributes and Elements section are optional.

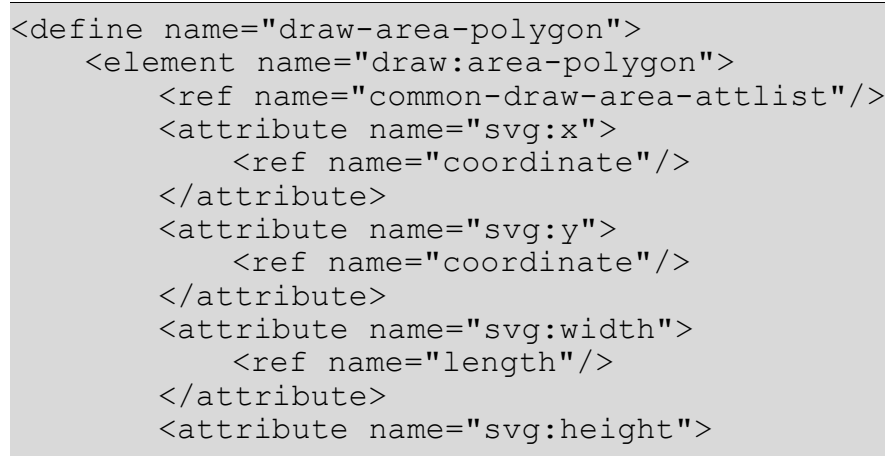




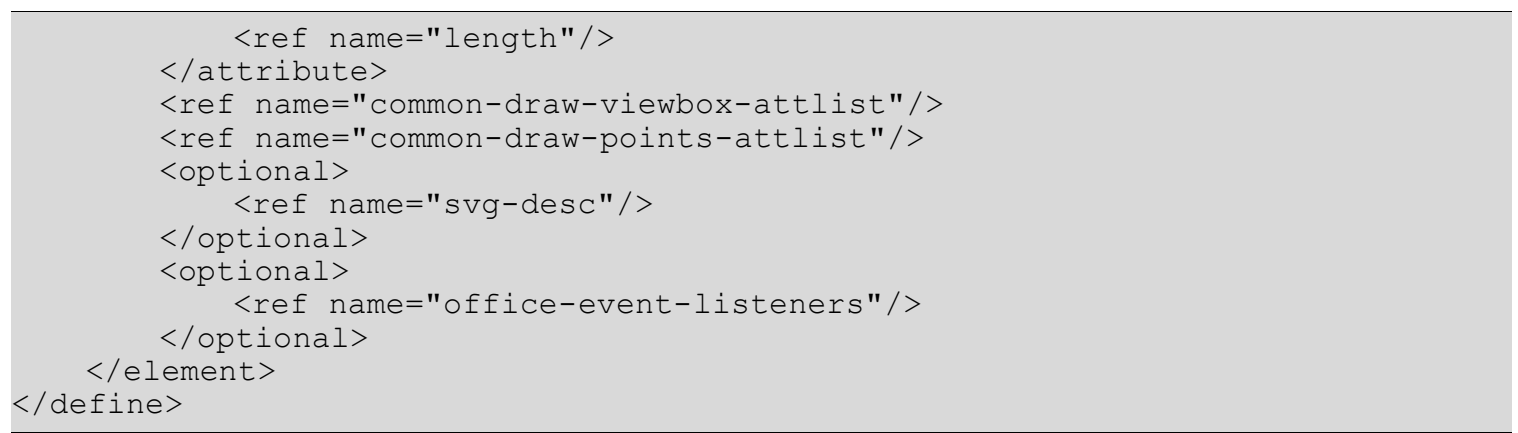

Example: Polygonal image map area

The element shown in the following example defines a triangle that is located in the middle of a $2 \mathrm{~cm}$ by $2 \mathrm{~cm}$ image. The bounding box covers an area of $2 \mathrm{~cm}$ by $1.5 \mathrm{~cm}$. One view box unit corresponds to $0.01 \mathrm{~mm}$.

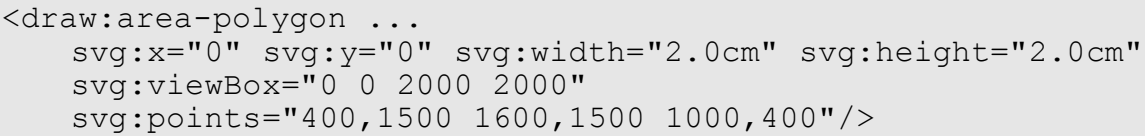

\section{Common Image Map Attributes and Elements}

In addition to the shape attributes, each image map element can contain the following information:

- Link, including a URI and link target frame.

- Name.

- Inactive flag.

- Description. Use the $\langle\mathrm{svg}$ : desc $>$ child element as described in section 9.3.9.

- Events associated with the area. Use the <office:event-listeners $>$ child element as described in section 12.4 .

Other attributes of the image maps are taken from the HTML image map representation.

Each image map element identifies a hyperlink and uses the [XLink] href, type, and show attributes, and the office:target-frame-name attribute to describe the link.

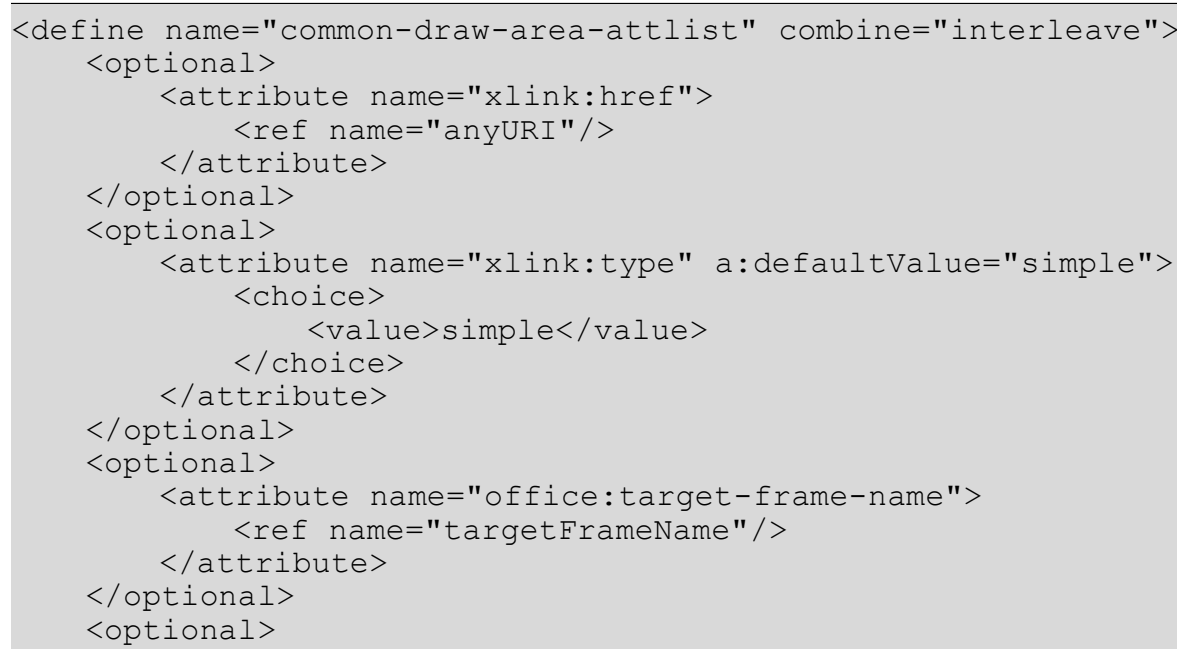




\subsection{D Shapes}

\subsubsection{Scene}

The $<\mathrm{dr} 3 \mathrm{~d}$ : scene $>$ element is the only element that can contain three-dimensional shapes. A scene is like a group, but it also defines the projection, lighting, and other render details for the shapes inside the scene.

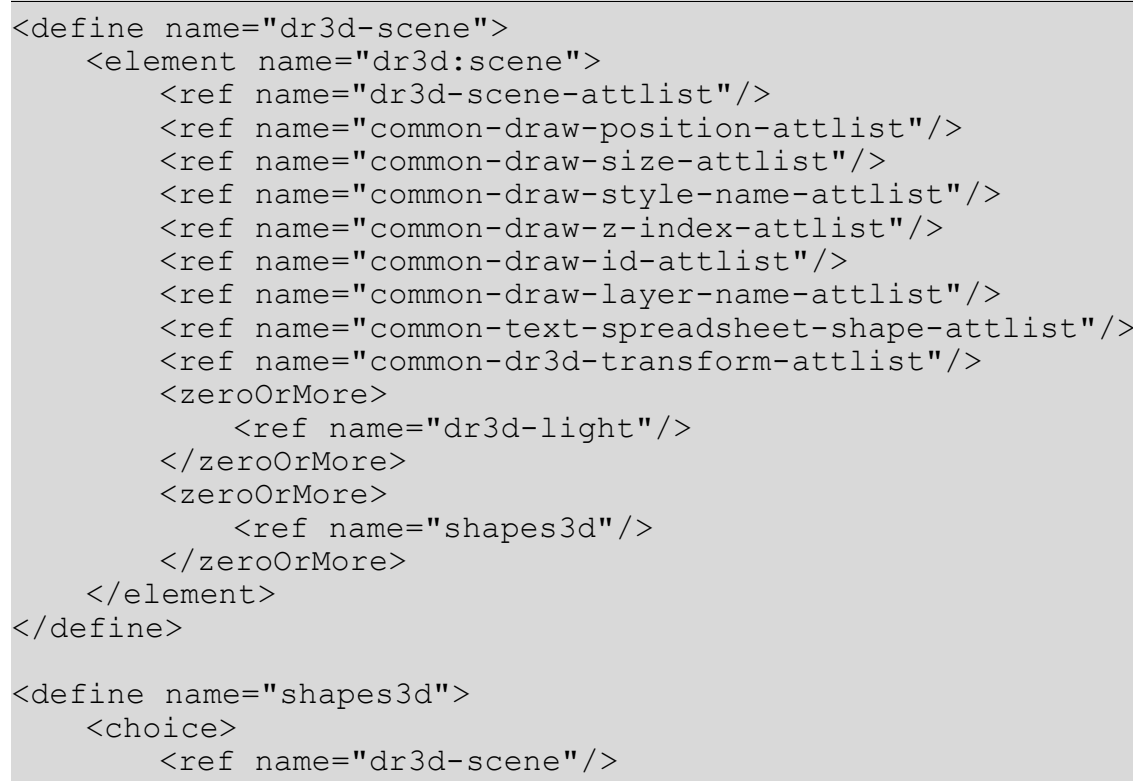


7123

7124

7125

7126

7127

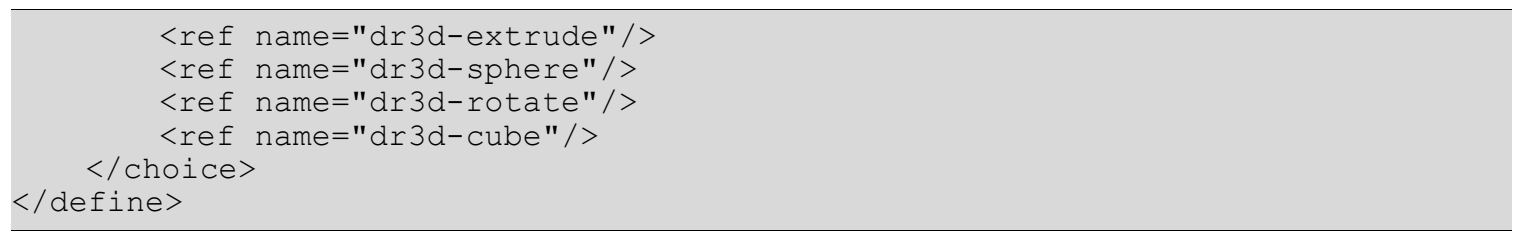

- Position, Size, Style, Layer, Z-Index, and ID - see section 9.2.15

- Text anchor, table background, draw end position - see section 9.2.16

- Camera vectors

- Projection

- Distance

- Focal length

- Shadow slant

- Shade mode

- Ambient color

- Lighting mode

\section{Camera Vectors}

The camera vectors define a viewing volume. The $d r 3 d: \operatorname{vrp}$ attribute specifies the origin, the $\mathrm{dr} 3 \mathrm{~d}:$ vpn attribute points towards the projected objects, and the $\mathrm{dr} 3 \mathrm{~d}:$ vup attribute defines the up vector.

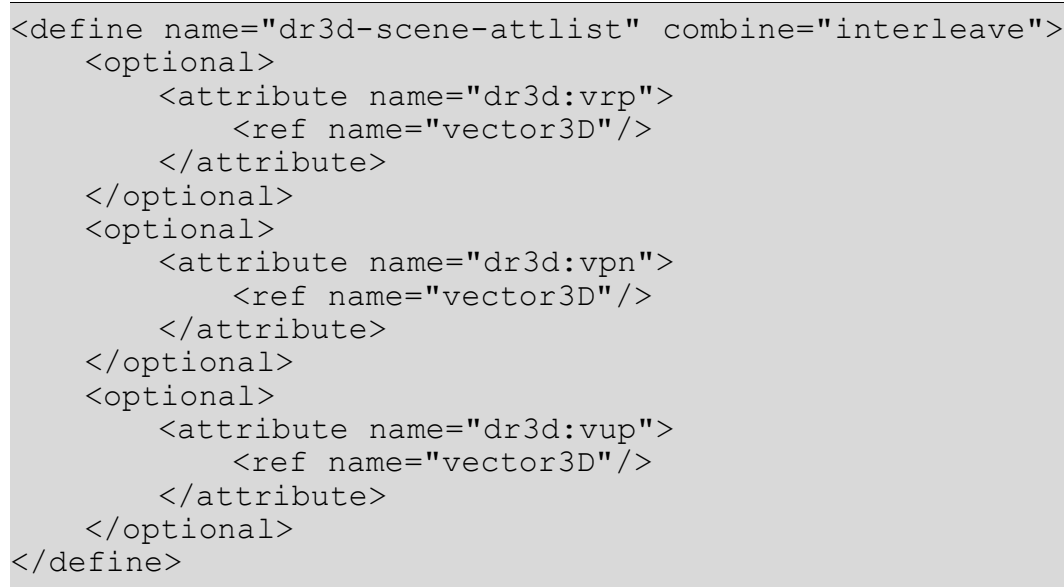

\section{Projection}

The $d r 3 d: p r o j e c t i o n$ attribute specifies the projection. The projection can be perspective or parallel. In perspective mode, objects become smaller in the distance.

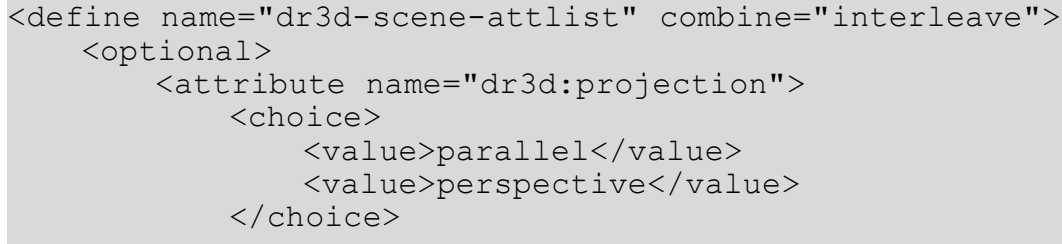




\section{Distance}

The $d r 3 d$ : distance attribute specifies the distance between the camera and the object.

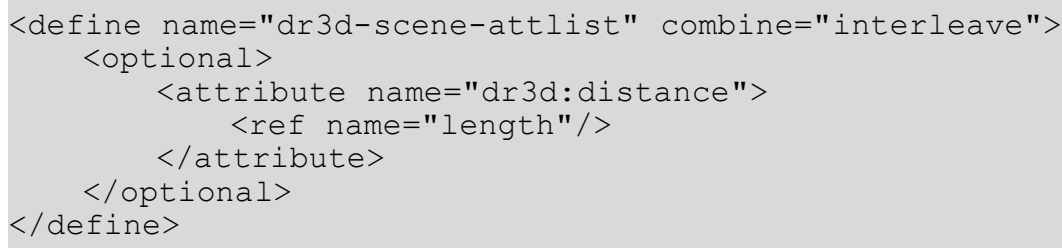

\section{Focal Length}

The $d r 3 d$ : focal-length attribute specifies the length of the focus for the virtual camera of this scene.

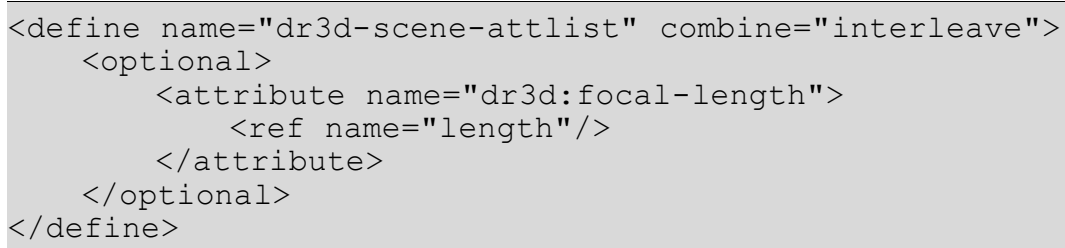

\section{Shadow Slant}

The $d r 3 d$ : shadow-slant attribute defines the angle from the three-dimensional scene to a virtual paper on which the shadow is casted.

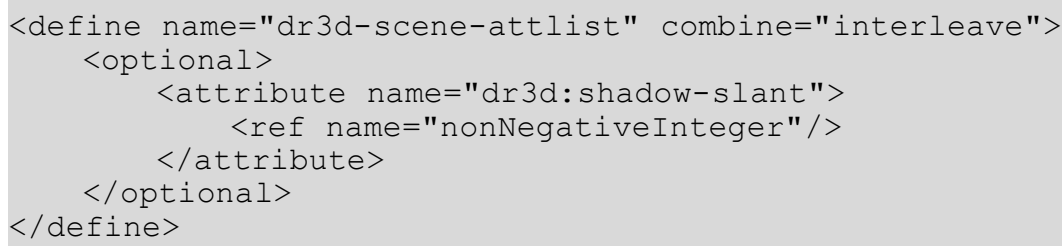

\section{Shade Mode}

The shade mode defines how the lighting is calculated for rendered surfaces

- flat: lighting is calculated by one surface normal.

- phong: lighting is calculated by interpolating the surface normals over the surface.

- gouraud: lighting is calculated by interpolating the color calculated with the surface normals at each edge.

- draft: surfaces are not lit and drawn as wireframe only.

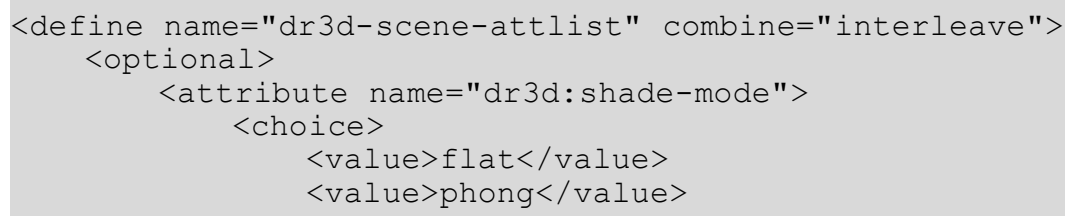




\section{Lighting Mode}

The attribute $d r 3 d$ :lighting-mode enables or disables the use of lighting in the threedimensional scene.

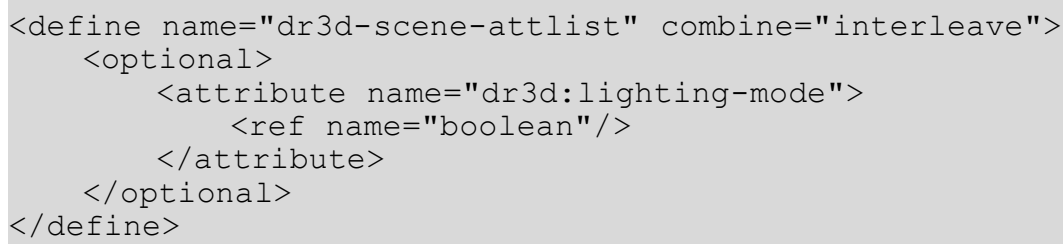

\section{D Transformation}

The value of the $d r 3 d$ :transform attribute is a list of transform definitions, which are applied in the order provided. The individual transform definitions are separated by whitespace. The available types of transform definitions include:

- matrix $(\langle\mathrm{a}\rangle\langle\mathrm{b}\rangle\langle\mathrm{c}\rangle\langle\mathrm{d}\rangle\langle\mathrm{e}\rangle\langle\mathrm{f}\rangle\langle\mathrm{g}\rangle\langle\mathrm{h}\rangle\langle\mathrm{i}\rangle\langle\mathrm{j}\rangle\langle\mathrm{k}\rangle\langle\mathrm{l}\rangle$ ), which specifies a transformation in the form of a transformation matrix of six values. matrix

$(a, b, c, d, e, f, g, h, i, j, k, l)$ is equivalent to applying the transformation matrix [a b c d e f g h i j k l].

- translate $(\langle t x\rangle\langle t y\rangle\langle t z\rangle)$, which specifies a translation by tx, ty and tz.

- scale $(\langle\mathrm{sx}\rangle\langle\mathrm{sy}\rangle\langle\mathrm{sz}\rangle)$, which specifies a scale operation by sx, sy and sz.

- rotatex (<rotate-angle>) , which specifies a rotation by <rotate-angle> degrees along the $x$-axis.

- rotatey (<rotate-angle> ), which specifies a rotation by <rotate-angle> degrees along the $y$-axis.

- rotatez (<rotate-angle> ), which specifies a rotation by <rotate-angle> degrees along the $y$-axis.

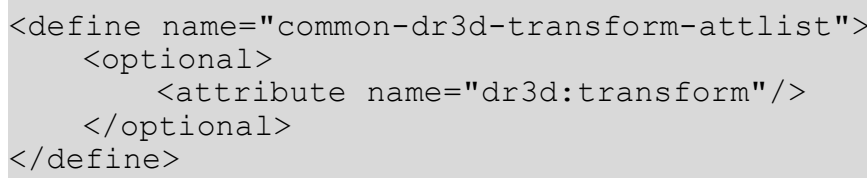




\subsubsection{Light}

The $<\mathrm{dr} 3 \mathrm{~d}:$ light $>$ element represents a light inside a scene.

This element must be the first element contained in $a<d r 3 d$ : scene $>$ element. There may be several lights, but applications may only support a limited number per scene. A typical limitation are 8 lights per scene.

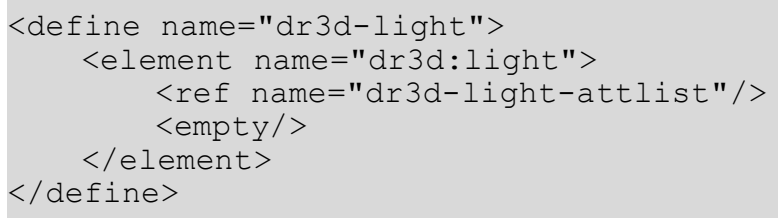

The attributes that may be associated with the $\langle\mathrm{dr} 3 \mathrm{~d}$ : light $>$ element are:

- Diffuse color

- Direction

- Enabled

- Specular

\section{Diffuse Color}

The $d r 3 d$ :diffuse-color attribute specifies the base color that the light is emitting.

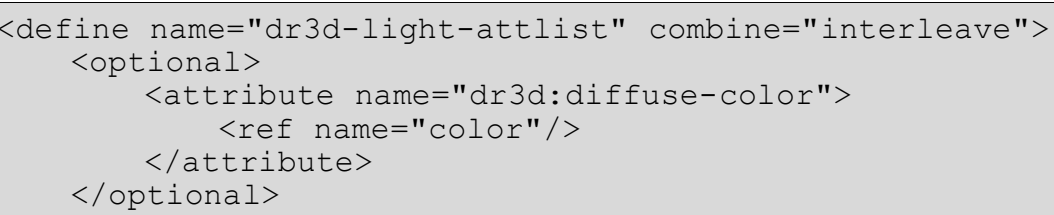

\section{Direction}

The $d r 3 d$ :direction attribute specifies the direction in which the light is emitted.

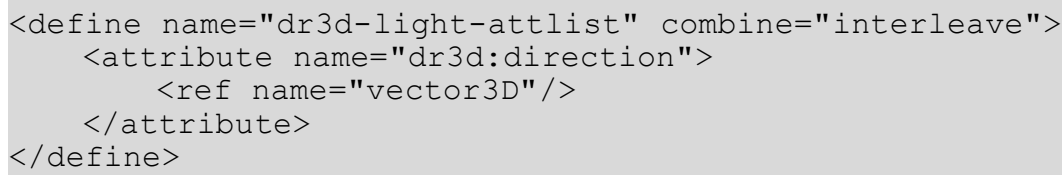

\section{Enabled}

The $d r 3 d$ : enabled attribute specifies whether or not the light is enabled. If a light is not enabled, it does not emit any light.

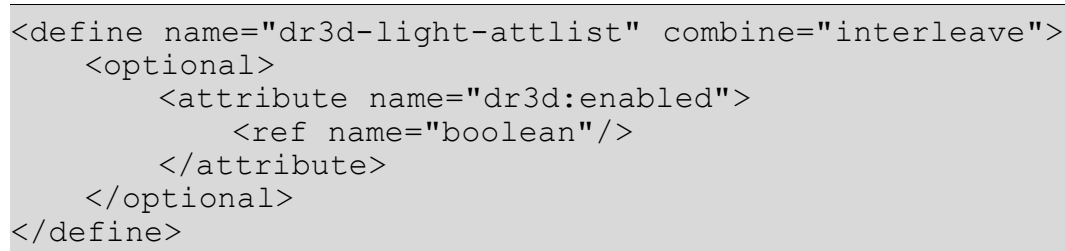




\section{Specular}

The $\mathrm{dr} 3 \mathrm{~d}$ : specular attribute specifies whether or not the light causes a specular reflection on the objects. Applications may evaluate this attribute only for the first light in a scene.

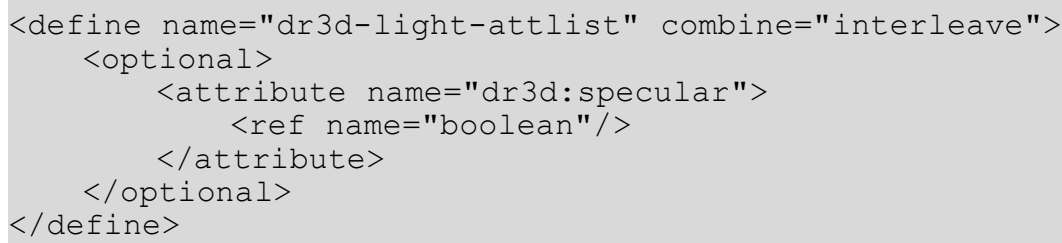

\subsubsection{Cube}

The $\langle\mathrm{dr} 3 \mathrm{~d}$ : cube $>$ element represents a three-dimensional cube shape.

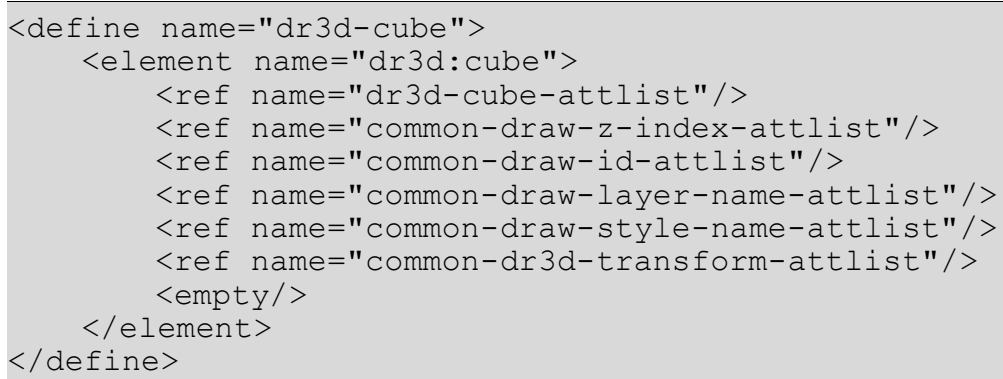

The attributes that may be associated with the $\langle\mathrm{dr} 3 \mathrm{~d}$ : cube $>$ element are:

- Style, Layer, Z-Index and ID - see section 9.2.15

- Minimum and Maximum Edge

\section{Minimum and Maximum Edge}

The attributes $d r 3 d:$ min-edge and $d r 3 d$ :max-edge specify the minimum and maximum edge of the cube in a 3D space.

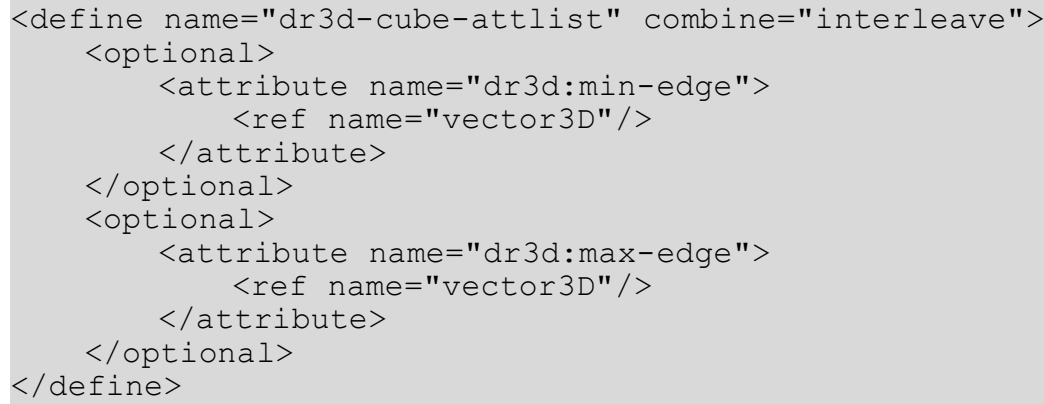

\subsubsection{Sphere}

The $\langle d r 3 d$ : sphere $>$ element represents a three-dimensional sphere shape.

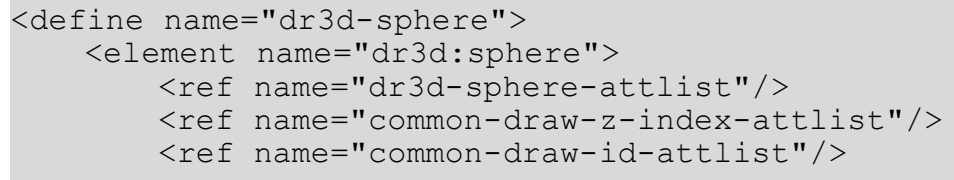


$</$ define $>$

<empty/>

- Center

- Size

\section{Center}

<ref name="common-draw-layer-name-attlist" />

<ref name="common-draw-style-name-attlist" />

$<$ ref name="common-dr3d-transform-attlist" $/>$

The attributes that may be associated with the $\langle\mathrm{dr} 3 \mathrm{~d}$ : sphere $>$ element are:

- $\quad$ Style, Layer, Z-Index, and ID - see section 9.2.15

The $\mathrm{dr} 3 \mathrm{~d}$ : center attribute defines the center of the sphere in a three-dimensional space.

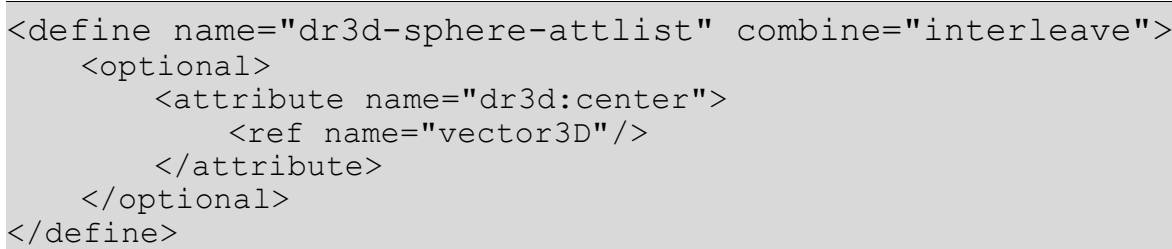

\section{$</ o p t i o n a l>$}

\section{Size}

The $d r 3 d:$ size attribute defines the size of the sphere in a three-dimensional space.

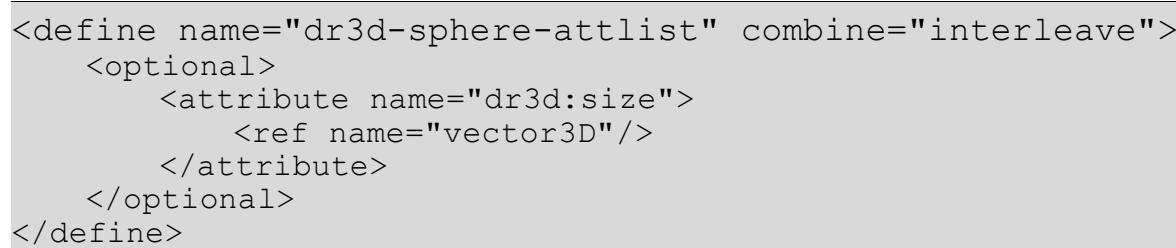

\subsubsection{Extrude}

The $<\mathrm{dr} 3 \mathrm{~d}$ : extrude $>$ element represents a three-dimensional extrude based on a polygon.

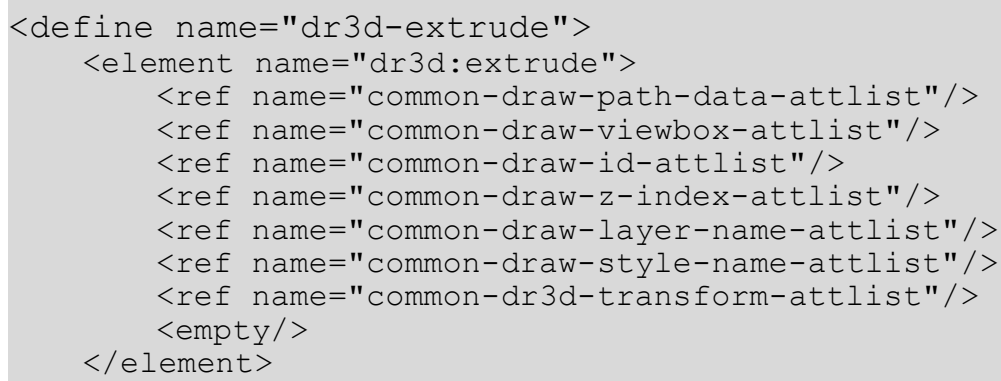

The attributes that may be associated with the $\langle d r 3 d$ : extrude $>$ element are:

- Viewbox, Style, Layer, Z-Index, and ID - see section 9.2.15

- $\quad$ Path Data - see section 9.2.6 


\subsubsection{Rotate}

The $<\mathrm{dr} 3 \mathrm{~d}$ : rotate $>$ element represents a three-dimensional rotation shape based on a polygon.

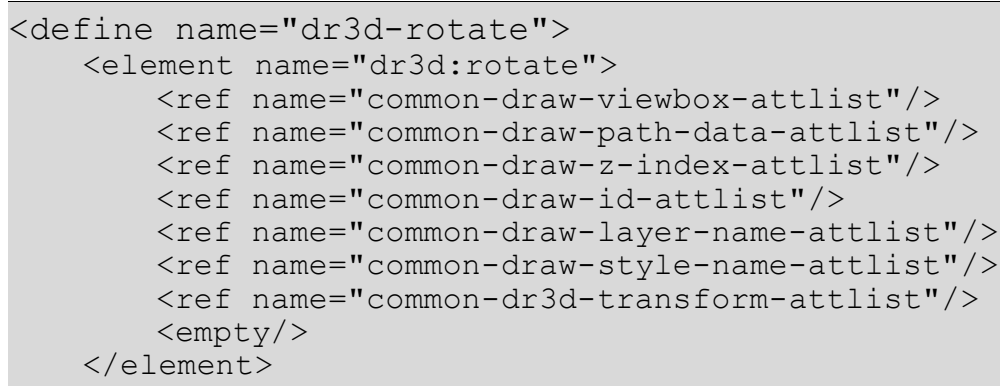

The attributes that may be associated with the $\langle\mathrm{dr} 3 \mathrm{~d}$ : rotate $>$ element are:

- Viewbox, Style, Layer, Z-Index, and ID - see section 9.2.15

- $\quad$ Path Data - see section 9.2.6

\subsection{Custom Shape}

A < draw: custom-shape $>$ represents a shape that is capable of rendering complex figures. It is offering font work and extrusion functionality. A custom shape may have a geometry that influences its shape. This geometry may be visualized in office application user interfaces, for instance by displaying interaction handles, that provide a simple way to modify the the geometry.

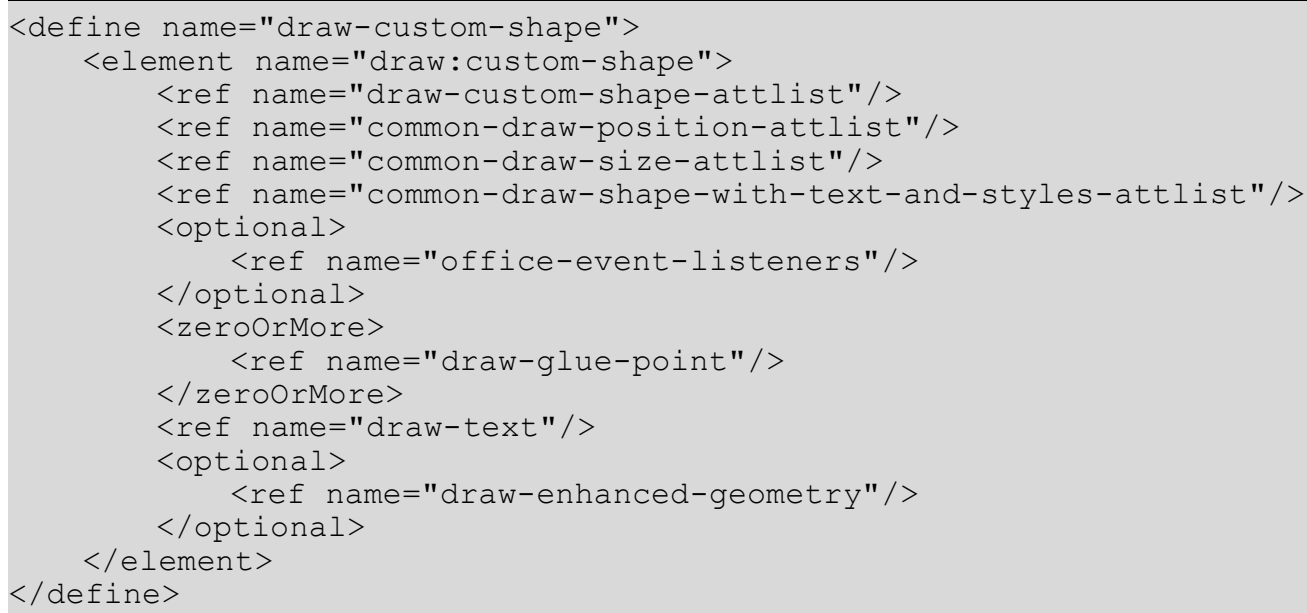

The attributes that may be associated with the <draw: custom shape> element are:

- $\quad$ Position, Size, Style, Layer, Z-Index, ID, and Transformation - see section 9.2.15.

- Text anchor, table background, draw end position - see section 9.2.16.

- Draw engine

- Draw data 


\section{Draw Engine}

The optional draw:engine attribute specifies the name of a rendering engine that can be used to render the custom shape. The attribute's value is a namespaced token, meaning an identifier prefixed by an XML namespace prefix, just like any attribute or element name in this specification. The drawing engine may get its data either from the draw: data attribute, or it may evaluate the <draw: enhanced-geometry> child element.

If the draw: engine attribute is omitted, the office application's default enhanced custom shape rendering engine will be used. This engine gets its geometry data from the $<$ draw: enhancedgeometry> element only.

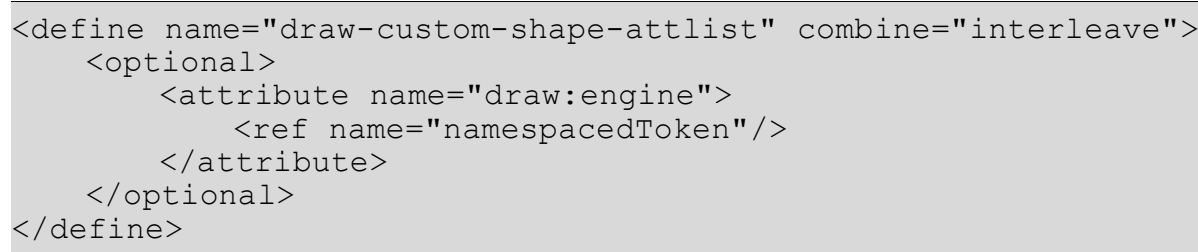

\section{Draw Data}

The draw: data attribute contains rendering engine specific data that describes the geometry of the custom shape. This attribute is only evaluated if a non default rendering engine is specified by the draw: engine attribute.

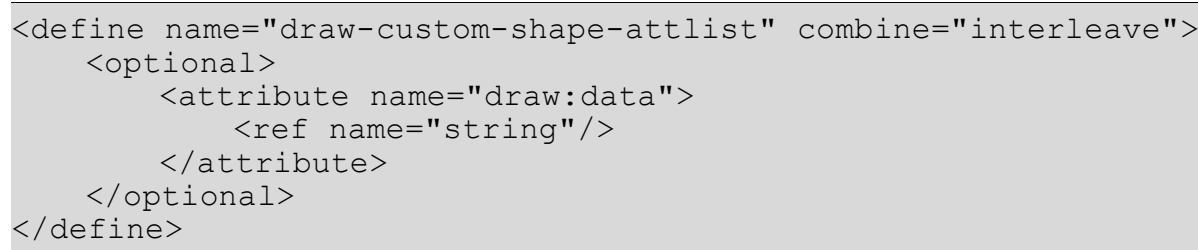

\subsubsection{Enhanced Geometry}

The <draw: enhanced-geometry $>$ element contains the geometry for a <draw: customshape> element if its draw:engine attribute has been omitted.

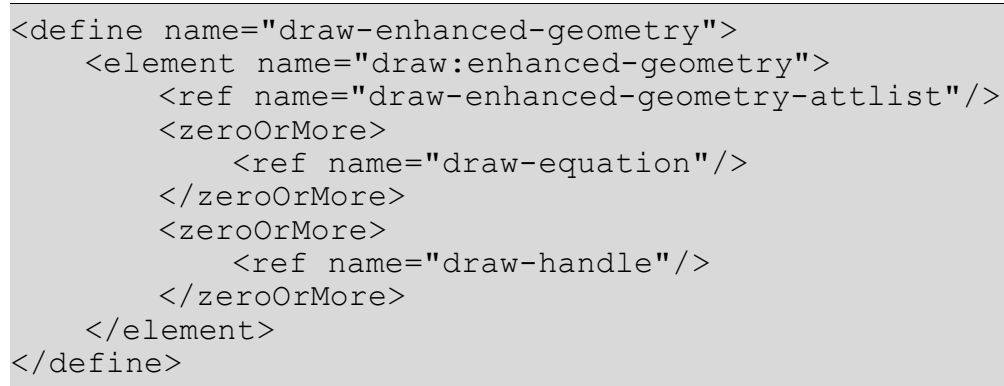

The attributes that may be associated with the <draw: enhanced-geometry> element are

- Type

- View Box

- Mirror

- Text Rotate Angle

- Extrusion Allowed 
- Text Path Allowed

- Concentric Gradient Fill Allowed

- Enhanced Geometry - Extrusion Attributes (see section 9.5.2)

- Enhanced Geometry - Path Attributes (see section 9.5.3)

- Enhanced Geometry - Text Path Attributes (see section 9.5.4)

- Enhanced Geometry - Equation (see section 9.5.5)

- $\quad$ Enhanced Geometry - Handle Attributes (see section 9.5.6)

\section{Type}

The draw: type attribute contains the name of a shape type. This name can be used to offer specialized user interfaces for certain classes of shapes, like for arrows, smileys, etc.

The shape type is rendering engine dependent and does not influence the geometry of the shape. If the value of the draw:type attribute is non-primitive, then no shape type is available.

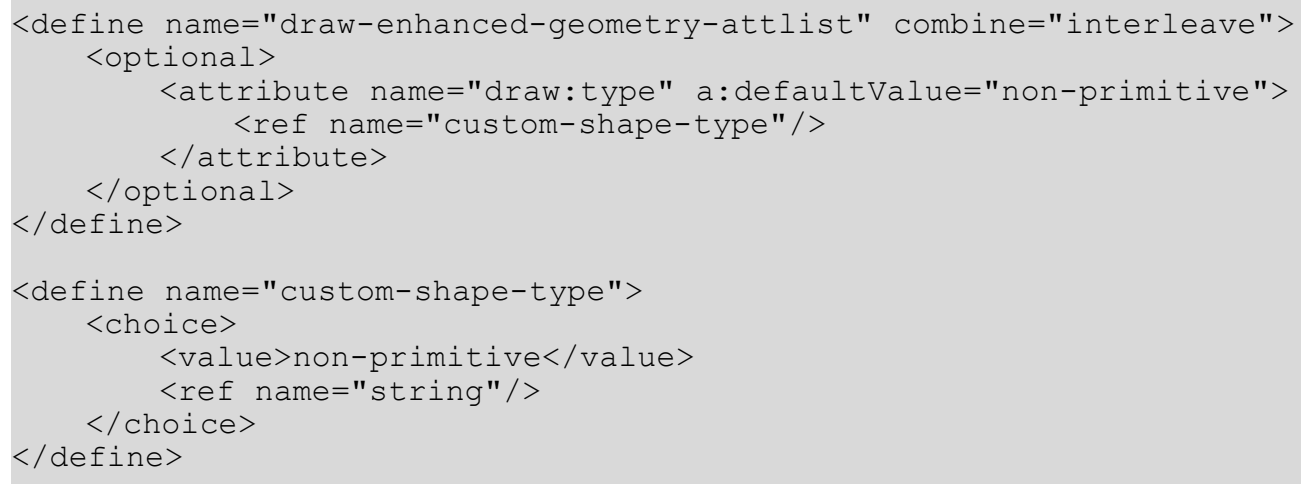

\section{View Box}

The svg:viewBox attribute establishes a user coordinate system inside the physical coordinate system of the shape specified by the position and size attributes. This user coordinate system is used by the <draw: enhanced-path> element.

The syntax for using this attribute is the same as the [SVG] syntax. The value of the attribute are four numbers separated by white spaces, which define the left, top, right, and bottom dimensions of the user coordinate system.

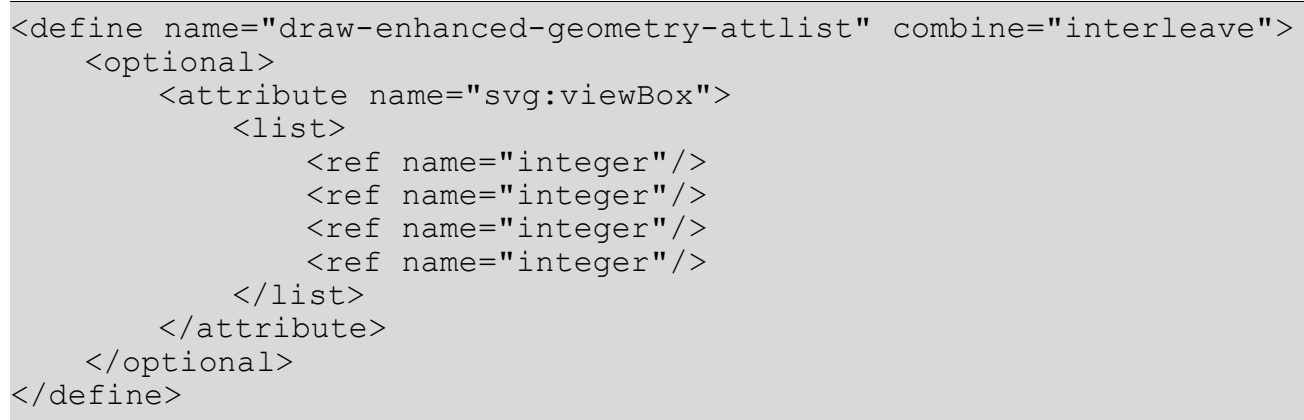




\section{Mirror}

The draw:mirror-vertical and draw:mirror-horizontal attributes specify if the geometry of the shape is to be mirrored.

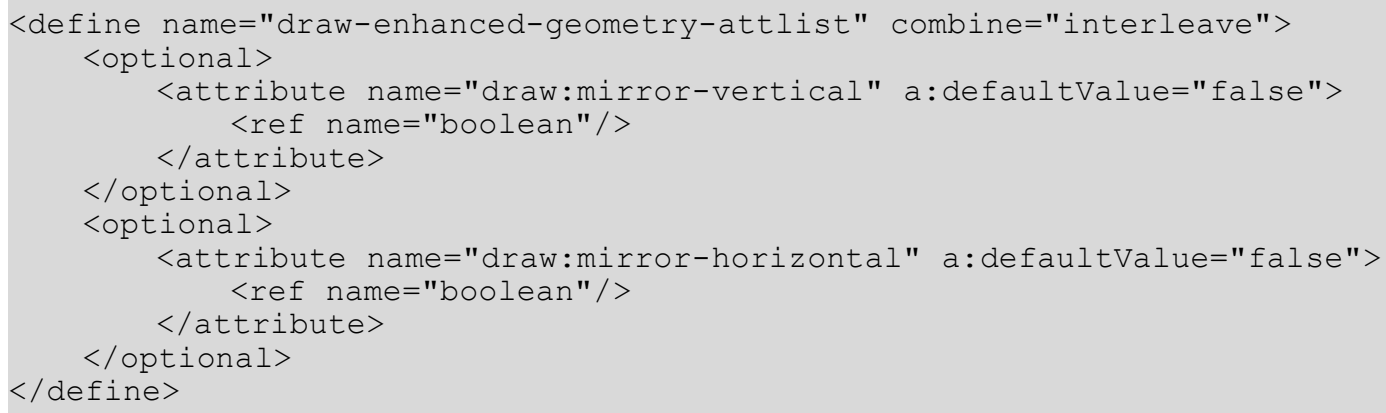

\section{Text Rotate Angle}

The draw: text-rotate-angle attribute specifies the angle by which the text within the custom shape is rotated in addition to the rotation included in the shape's draw: transform attribute.

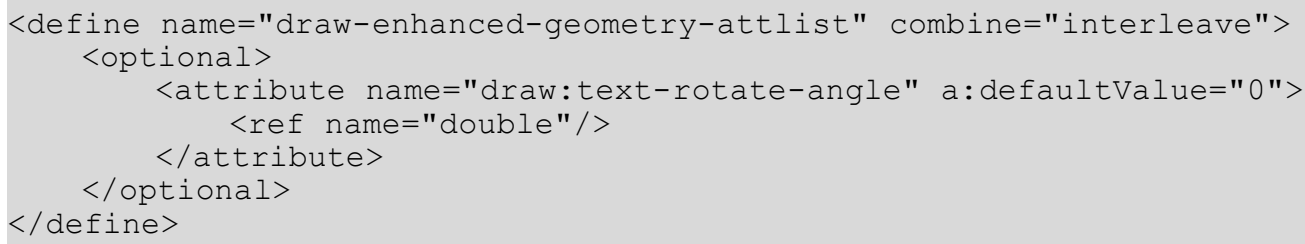

\section{Extrusion Allowed}

The draw:extrusion-allowed attribute specifies whether the shape is capable to be rendered as extrusion object.

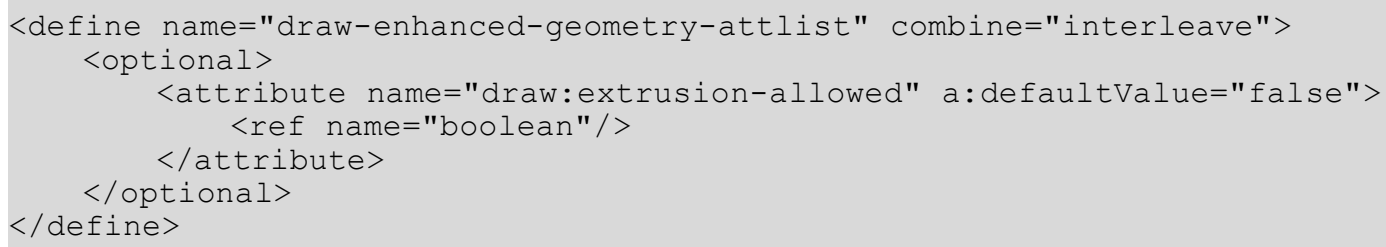

\section{Text Path Allowed}

The draw: text-path-allowed attribute specifies if the shape is capable of being rendered as Fontwork object. The text of a Fontwork object is distinguished from normal text objects by being able to render text along or between lines that are specified by the draw:enhanced-path attribute. Fontwork objects are capable to support standard graphic attributes such as fill, shadow and or line styles.

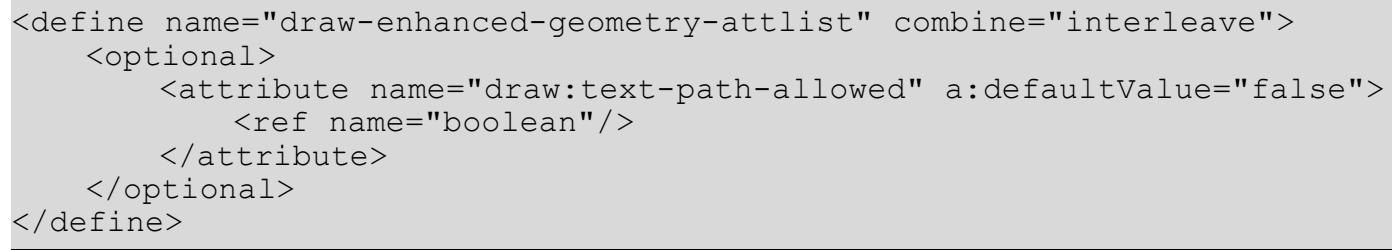




\section{Concentric Gradient Fill Allowed}

The draw: concentric-gradient-fill-allowed attribute specifies if the shape is capable being rendered with a concentric gradient that uses the custom shape path.

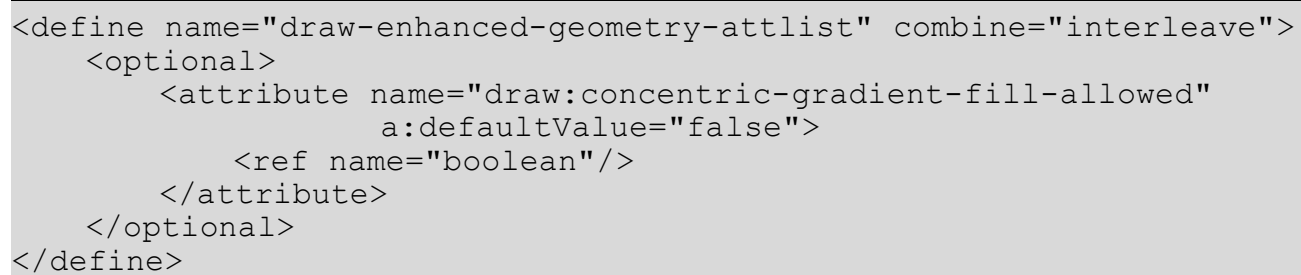

\subsubsection{Enhanced Geometry - Extrusion Attributes}

\section{Extrusion}

The draw:extrusion attribute determines if an extrusion is displayed.

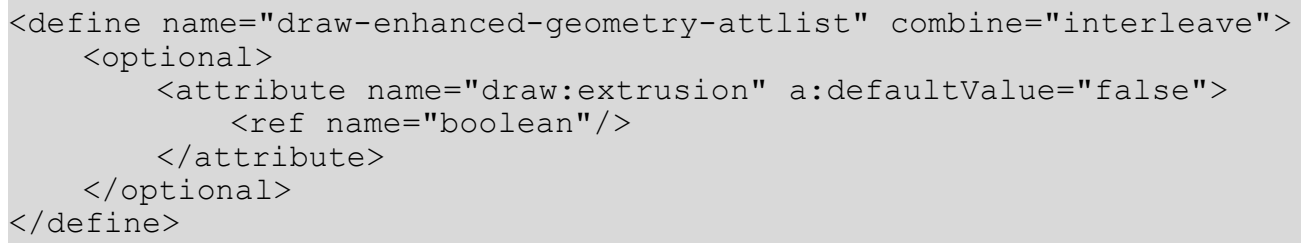

\section{Extrusion Brightness}

The draw:extrusion-brightness attribute specifies the brightness of a scene.

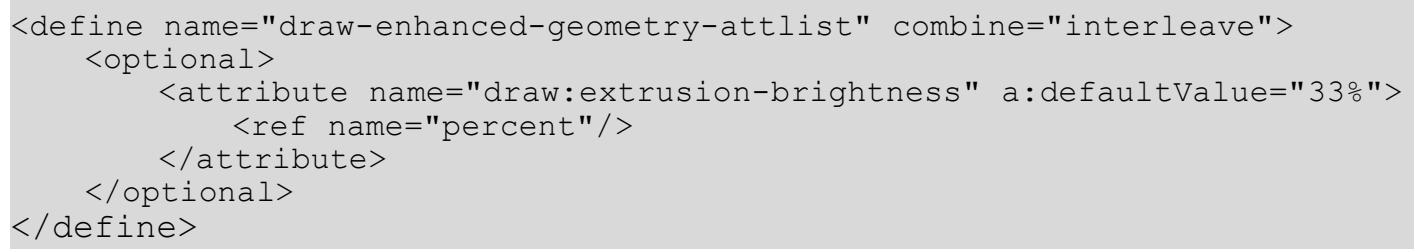

\section{Extrusion Depth}

The draw:extrusion-depth attribute specifies the depth of the extrusion. It takes two space separated values. The first value specifies the depth of the extrusion, the second value specifies the fraction of the extrusion that lies before the shape. It must be in the range $[0,1]$. A value of 0 is default.

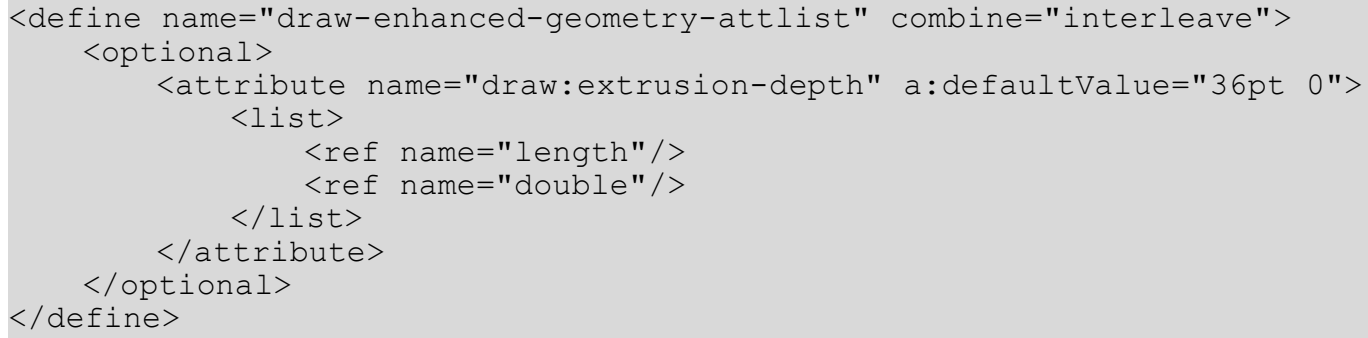




\section{Extrusion Metal}

The draw:extrusion-metal attribute specifies if the surface of the extrusion object looks like metal.

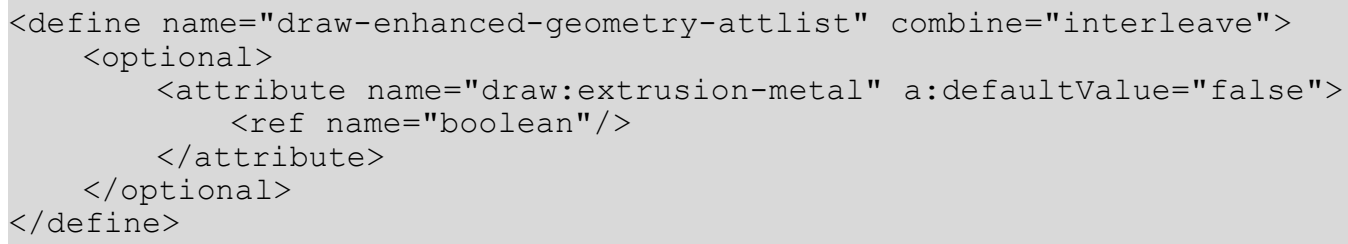

\section{Extrusion Shade Mode}

The $d r 3 d$ : shade-mode attribute defines how the lighting is calculated for rendered surfaces

- flat: lighting is calculated by one surface normal.

- phong: lighting is calculated by interpolating the surface normals over the surface.

- gouraud: lighting is calculated by interpolating the color calculated with the surface normals at each edge.

- draft: surfaces are not lit and drawn as wireframe only.

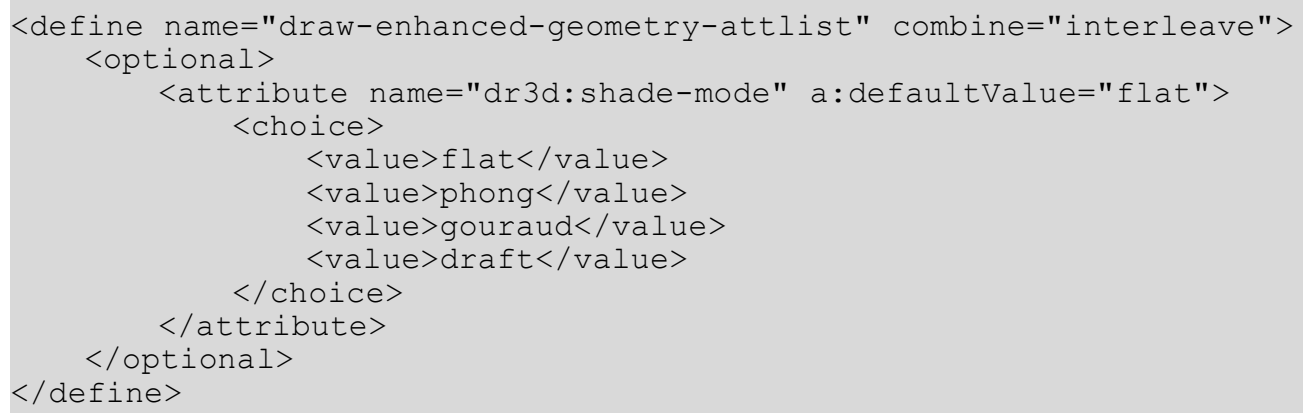

\section{Extrusion Rotation Angle}

The first value of the draw: extrusion-rotation-angle specifies the rotation about the $x$ axis. The second value of the draw:extrusion-rotation-angle specifies the rotation about the y-axis. The rotation about the z-axis is specified by the rotate angle of the draw: transform attribute.

The order of the rotation is: $z$-axis, $y$-axis and then $x$-axis.

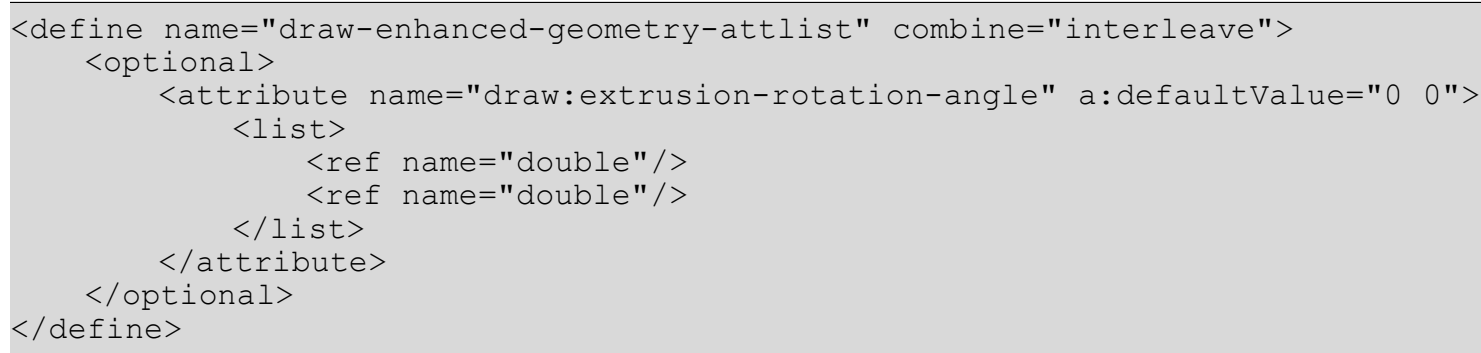




\section{Extrusion Rotation Center}

The draw:extrusion-rotation-center attribute specifies the position of the rotation center in terms of shape size fractions, if it is omitted then the geometrical center of the shape is used.

\section{Extrusion Skew}

The draw: extrusion-skew attribute specifies the skew amount and skew angle of an extrusion. Skew settings are only applied if the attribute $d r 3 d$ :projection has the value parallel.

The first parameter represents the skew amount in percent, the second parameter specifies the skew angle.

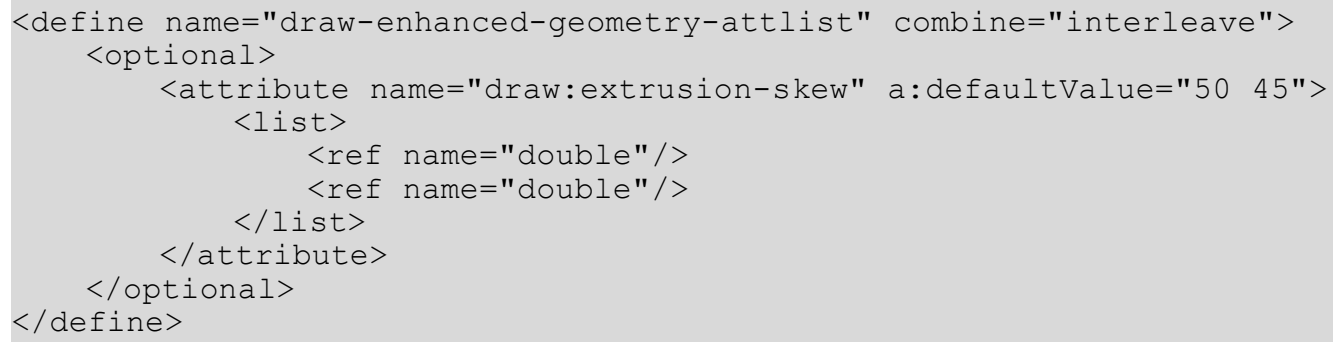

\section{Extrusion Specularity}

The draw:extrusion-specularity attribute specifies the specularity of an extrusion object.

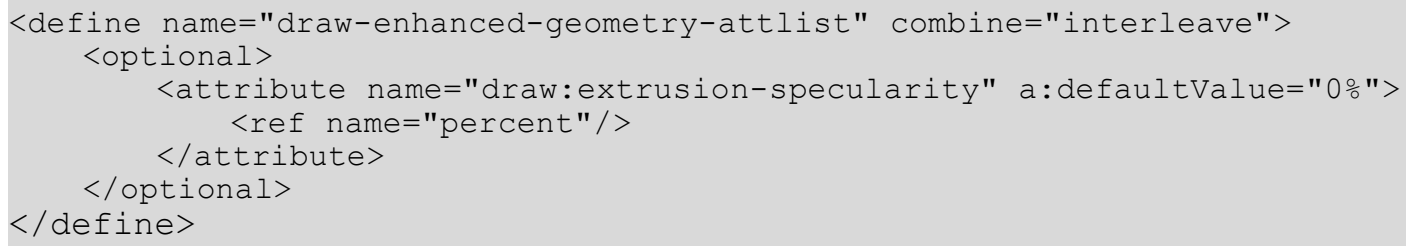

\section{Extrusion Projection Mode}

The $d r 3 d: p r o j e c t i o n$ attribute specifies if the projection mode is perspective or parallel. 


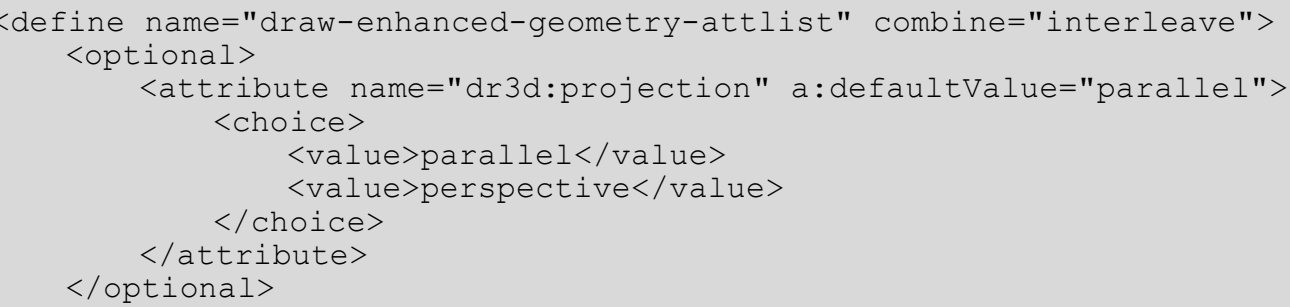

\section{Extrusion Viewpoint}

The draw: extrusion-viewpoint attribute specifies the viewpoint of the observer as an 3D point. The attribute's value syntax is similar to vector3D, solely a unit is following each parameter. An example for a 3D poin is: " $(1 \mathrm{~cm} 1 \mathrm{~cm} 0 \mathrm{~m})$ ".

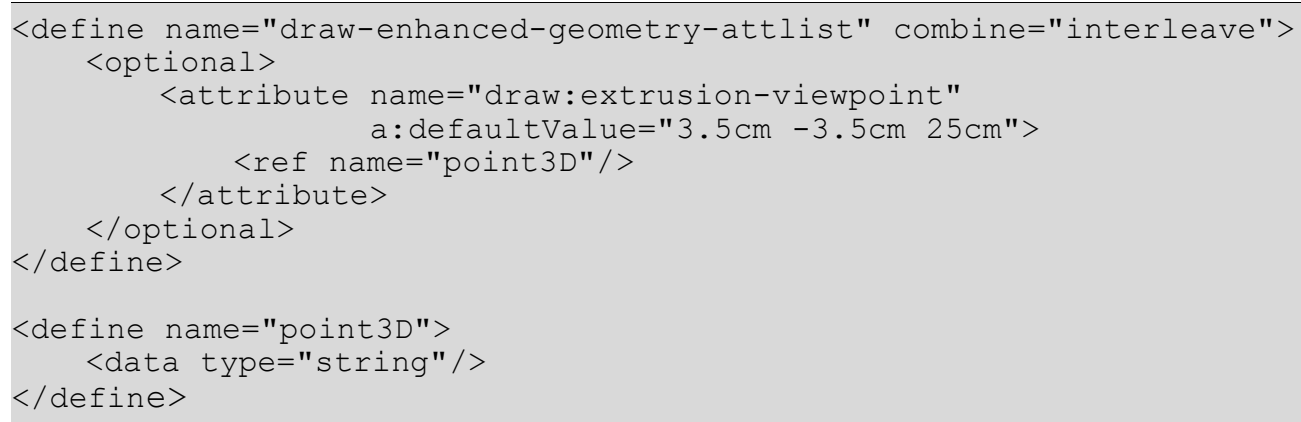

\section{Extrusion Origin}

The draw:extrusion-origin attributes specifies the origin within the bounding box of the shape in terms of the shape size fractions.

The first parameter represents the horizontal origin, a value of -0.5 represents the left side of the shape, a value of 0 represents the center of the shape, a value of 0.5 represents the right side of the shape.

The second parameter represents the vertical origin, a value of -0.5 represents the top side of the shape, a value of 0 represents the center of the shape, a value of 0.5 represents the bottom side of the shape.

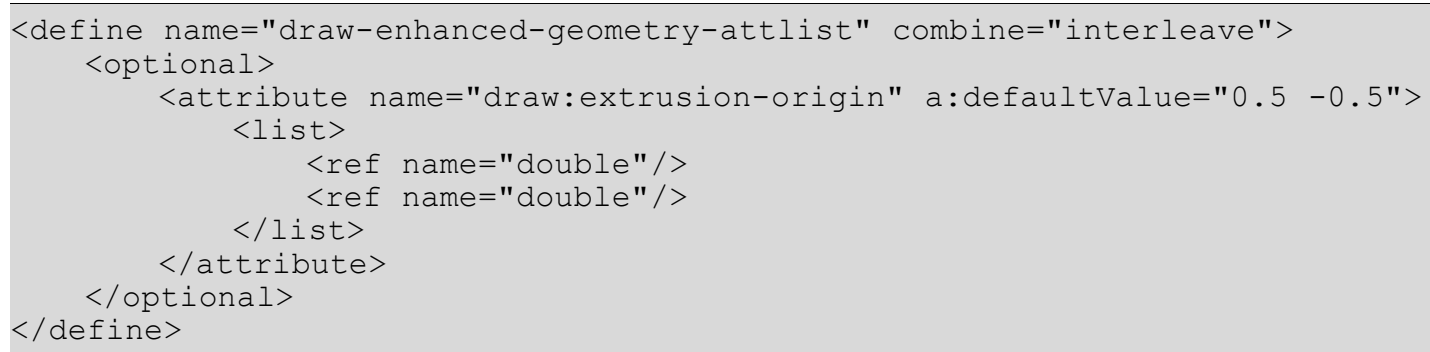

\section{Extrusion Color}

The draw: extrusion-color attribute specifies if an extrusion color is used. The extrusion color is then defined by the draw: secondary-fill-color attribute specified in the custom shape's graphic style. 


\subsubsection{Enhanced Geometry - Path Attributes}

\section{Enhanced Path}

The draw: enhanced-path attribute specifies a path similar to the svg:d attribute of the <svg:path> element. Instructions such as moveto, lineto, arcto and other instructions together with its parameter are describing the geometry of a shape which can be filled and or stroked. Relative commands are not supported.

The syntax of draw: enhanced-path attribute is as follows:

- Instructions are expressed as one character (e.g. a moveto is expressed as an M).

- A prefix notation is being used, that means that each command is followed by its parameter.

- Superfluous white space and separators such as commas can be eliminated. (e.g. "M 1010 L 2020 L 30 20" can also be written: "M10 10L20 20L30 20"

- If the command is repeated multiple times, only the first command is required. (e.g. "M 10 10 L 2020 L 30 20" can also be expressed as followed "M 1010 L 202030 20"

- Floats can be used, therefore the only allowable decimal point is a dot (“.")

The above mentioned rules are the same as specified for the $<\mathrm{svg}:$ path $>$ element.

A parameter can also have one of the following enhancements:

- A "?" is used to mark the beginning of a formula name. The result of the element's draw: formula attribute is used as parameter value in this case.

- If " $\$$ " is preceding a integer value, the value is a indexing a draw:modifiers attribute. The corresponding modifier value is used as parameter value then.

Following notation is used in the table below:

- (): grouping of parameters

- $+: 1$ or more of the given parameter(s) is required

Example for a custom-shape that uses the draw:enhanced-path to describe a pie-chart whose top right quarter segment is taken out:

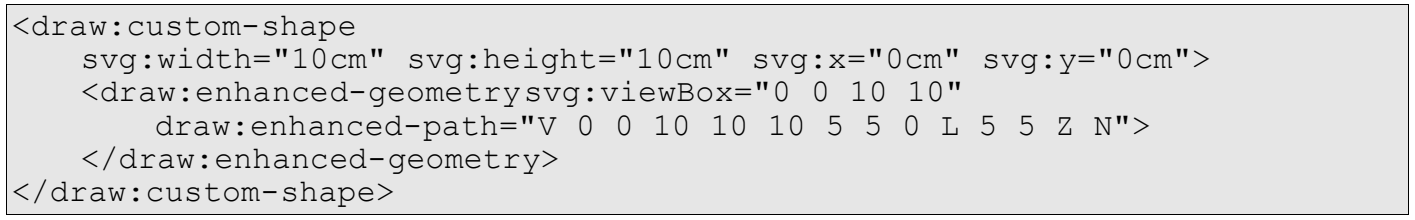

The following commands are supported: 


\begin{tabular}{|c|c|c|c|}
\hline Command & Name & Parameters & Description \\
\hline M & moveto & $(x$ y) + & $\begin{array}{l}\text { Start a new sub-path at the given }(x, y) \\
\text { coordinate. If a moveto is followed by multiple } \\
\text { pairs of coordinates, they are treated as } \\
\text { lineto. }\end{array}$ \\
\hline L & lineto & $(x$ y $)+$ & $\begin{array}{l}\text { Draws a line from the current point to }(x, y) \text {. If } \\
\text { multiple coordinate pairs are following, they } \\
\text { are all interpreted as lineto. }\end{array}$ \\
\hline C & curveto & $(x 1 y 1 x 2 y 2 x y)+$ & $\begin{array}{l}\text { Draws a cubic Bézier curve from the current } \\
\text { point to }(x, y) \text { using }(x 1, y 1) \text { as the control point } \\
\text { at the beginning of the curve and }(x 2, y 2) \text { as } \\
\text { the control point at the end of the curve. }\end{array}$ \\
\hline Z & closepath & (none) & $\begin{array}{l}\text { Close the current sub-path by drawing a } \\
\text { straight line from the current point to current } \\
\text { sub-path's initial point. }\end{array}$ \\
\hline$N$ & endpath & (none) & $\begin{array}{l}\text { Ends the current set of sub-paths. The sub- } \\
\text { paths will be filled by using eofill. Other } \\
\text { following sub-pathes will be filled } \\
\text { independently. }\end{array}$ \\
\hline $\mathrm{F}$ & nofill & (none) & $\begin{array}{l}\text { Specifies that the current set of sub-paths } \\
\text { won't be filled. }\end{array}$ \\
\hline$S$ & nostroke & (none) & $\begin{array}{l}\text { Specifies that the current set of sub-paths } \\
\text { won't be stroked. }\end{array}$ \\
\hline $\mathrm{T}$ & $\begin{array}{l}\text { angle- } \\
\text { ellipseto }\end{array}$ & $(x$ y w h t0 t1 $)+$ & $\begin{array}{l}\text { Draws a segment of an ellipse. The ellipse is } \\
\text { specified by the center }(x, y) \text {, the size }(w, h) \\
\text { and the start-angle t } 0 \text { and end-angle } t 1 .\end{array}$ \\
\hline$U$ & $\begin{array}{l}\text { angle- } \\
\text { ellipse }\end{array}$ & $(x$ y wh t0 t1) + & $\begin{array}{l}\text { The same as the "T" command, except that a } \\
\text { implied moveto to the starting point is done. }\end{array}$ \\
\hline A & arcto & $\begin{array}{l}(x 1 y 1 x 2 y 2 x 3 y 3 x y) \\
+\end{array}$ & $\begin{array}{l}(x 1, y 1) \text { and }(x 2, y 2) \text { is defining the bounding } \\
\text { box of a ellipse. A line is then drawn from the } \\
\text { current point to the start angle of the arc that } \\
\text { is specified by the radial vector of point }(x 3 \text {, } \\
y 3) \text { and then counter clockwise to the end- } \\
\text { angle that is specified by point ( } x 4, y 4) \text {. }\end{array}$ \\
\hline B & arc & $\begin{array}{l}(x 1 y 1 x 2 y 2 x 3 y 3 x y) \\
+\end{array}$ & $\begin{array}{l}\text { The same as the "A" command, except that a } \\
\text { implied moveto to the starting point is done. }\end{array}$ \\
\hline W & $\begin{array}{l}\text { clockwis } \\
\text { earcto }\end{array}$ & $\begin{array}{l}(x 1 y 1 x 2 y 2 x 3 y 3 x y) \\
+\end{array}$ & $\begin{array}{l}\text { The same as the "A" command except, that } \\
\text { the arc is drawn clockwise. }\end{array}$ \\
\hline V & $\begin{array}{l}\text { clockwis } \\
\text { earc }\end{array}$ & $(x 1 y 1 x 2 y 2 x 3 y 3 x y)+$ & $\begin{array}{l}\text { The same as the "A" command, except that a } \\
\text { implied moveto to the starting point is done } \\
\text { and the arc is drawn clockwise. }\end{array}$ \\
\hline$x$ & $\begin{array}{l}\text { elliptical- } \\
\text { quatrantx }\end{array}$ & $(x y)+$ & $\begin{array}{l}\text { Draws a quarter ellipse, whose initial segment } \\
\text { is tangential to the } x \text {-axis, is drawn from the } \\
\text { current point to }(x, y) \text {. }\end{array}$ \\
\hline
\end{tabular}




\begin{tabular}{|c|c|c|c|}
\hline Command & Name & Parameters & Description \\
\hline Y & $\begin{array}{l}\text { elliptical- } \\
\text { quadranty }\end{array}$ & $(x y)+$ & $\begin{array}{l}\text { Draws a quarter ellipse, whose initial segment } \\
\text { is tangential to the y-axis, is drawn from the } \\
\text { current point to }(x, y) \text {. }\end{array}$ \\
\hline Q & $\begin{array}{l}\text { quadratic- } \\
\text { curveto }\end{array}$ & $(x 1 y 1 x y)+$ & $\begin{array}{l}\text { Draws a quadratic Bézier curve from the } \\
\text { current point to }(x, y) \text { using }(x 1, y 1) \text { as the } \\
\text { control point. }(x, y) \text { becomes the new current } \\
\text { point at the end of the command. }\end{array}$ \\
\hline \multicolumn{4}{|c|}{$\begin{array}{l}\text { <define name="draw-enhanced-geometry-attlist" } \\
\text { <optional> } \\
\quad<\text { attribute name="draw:enhanced-path" }> \\
\quad<\text { ref name="string"/> } \\
\quad</ \text { attribute }> \\
</ \text { optional> } \\
</ \text { define }>\end{array}$} \\
\hline
\end{tabular}

\section{Path Stretchpoint}

The draw:path-stretchpoint-x and draw:path-stretchpoint-y attributes specifies the stretchpoint of a shape.

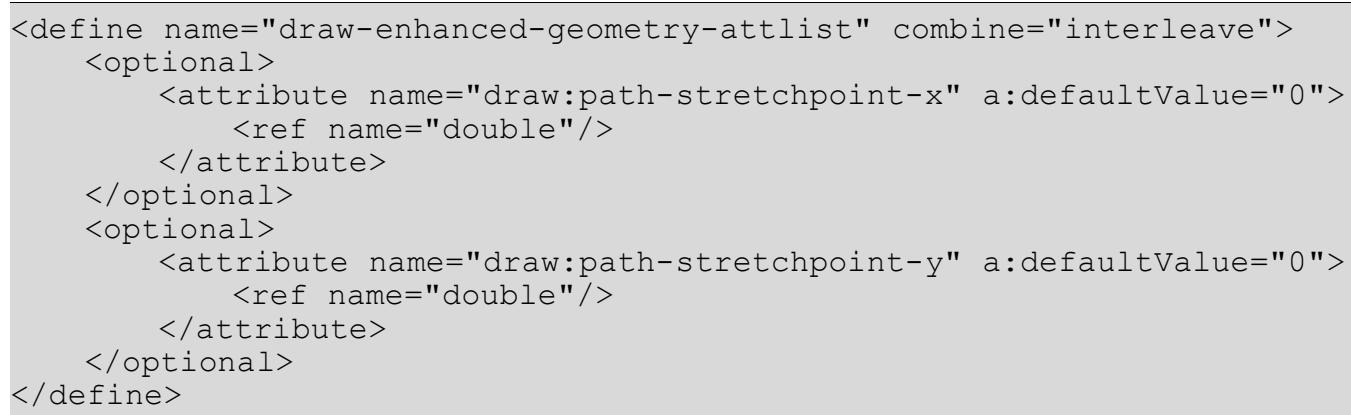

\section{Text Areas}

The draw: text-areas attribute specifies a list of text areas. The text area is used to position and align the text. If no text area is omitted, the area of the shape itself is used. If a second text area is available it is used for vertical text.

An area consists of four parameters:

The first parameter specifies the left side of the text area.

The second parameter specifies the top side of the text area.

The third parameter specifies the right side of the text area.

The fourth parameter specifies the bottom side of the text area.

A parameter can also have one of the following enhancements:

- A "?" is used to mark the beginning of a formula name. The result of the element's draw: formula attribute is used as parameter value in this case.

- If " $\$$ " is preceding a integer value, the value is a indexing a draw:modifiers attribute. The corresponding modifier value is used as parameter value then. 
A example of the draw:text-areas attribute that defines two text areas, including modifier and equation usage, would be: draw: text-areas="0 $0100 \quad 100$ ?Formula1 \$1 200 200"

\section{Glue Points}

The draw: glue-points attribute specifies a list of object defined glue points. In contradiction to the user defined glue points which are defined by the <draw: glue-point $>$ sub element, the object defined glue point can make use of equations and modifiers.

The first parameter specifies the horizontal position of the glue point.

The second parameter specifies the vertical position of the glue point.

Each parameter can be a float, or it can also have one of the following enhancements:

- A "?" is used to mark the beginning of a formula name. The result of the element's draw: formula attribute is used as parameter value in this case.

- If " $\$$ " is preceding a integer value, the value is a indexing a draw:modifiers attribute. The corresponding modifier value is used as parameter value then.

A example of the draw:glue-points attribute that defines two glue points, including modifier and equation usage, would be: draw:glue-points="0 ?Formula1 $100 \$ 1 "$

\section{Glue Point Type}

The draw:glue-point-type attribute specifies the glue-point type. If the draw:gluepoints attribute is also available this attribute is ignored.

- none: there are no special object glue points.

- segments: a connector will connect with each point of the draw: enhanced-path attribute

- rectangle: the middle of each side of the shape bound rectangle specifies a object specific glue point 


\section{Glue Point Leaving Directions}

The draw: glue-point-leaving-directions attribute is containing a comma separated list of angles in grad. The angle can be a float value. The position in the list is the same as the to be referenced glue-point of the draw: glue-points attribute.

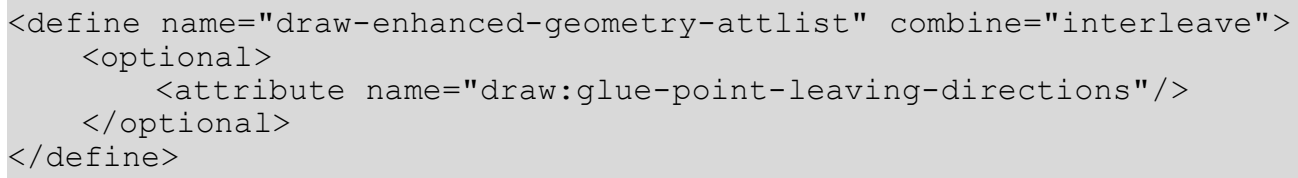

\subsubsection{Enhanced Geometry - Text Path Attributes}

\section{Text Path}

The draw: text-path attribute specifies if text is displayed on a text path.

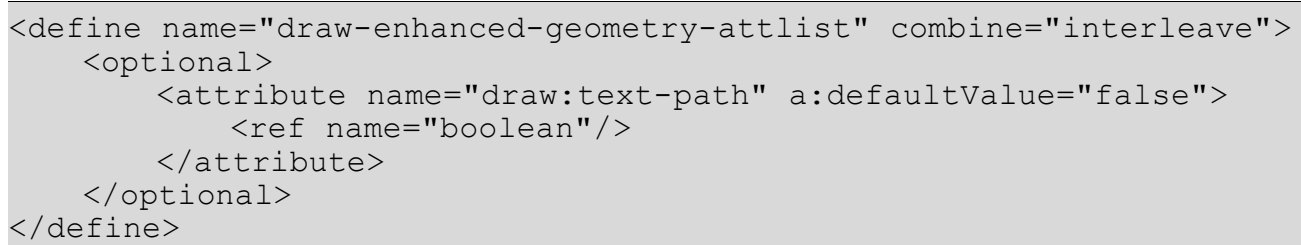

\section{Text Path Mode}

The draw:text-path-mode attribute specifies how the text is drawn.

- normal: the text is drawn along the path without scaling.

- path: the text is fit to the path.

- shape: the text is fit to the bounding box of the shape.

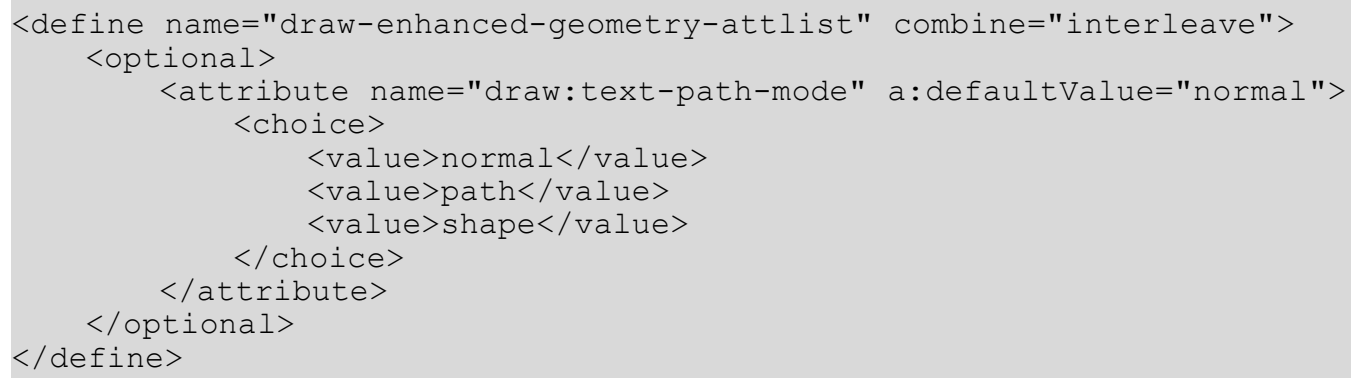

\section{Text Path Scale}

The draw: text-path-scale attribute specifies the scaling of the text path.

- path: The text scaling is determined by the length of the path from the draw: enhancedpath attribute.

- shape: The text scaling is determined by the width of a shape. 


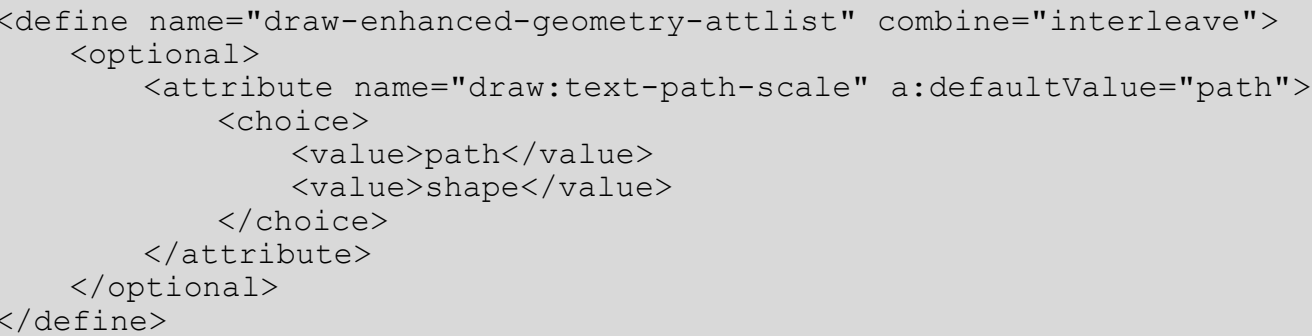

\section{Text Path Same Letter Heights}

The draw: text-path-same-letter-heights attribute specifies if all letters in the custom shape will have the same height.

<define name="draw-enhanced-geometry-attlist" combine="interleave">

\section{Modifiers}

The draw: modifiers attribute contains list of modifier values. The modifier can be a float value. In the majority of cases, the draw:modifiers attribute is being used by the draw: handle-position attribute to store the handle position.

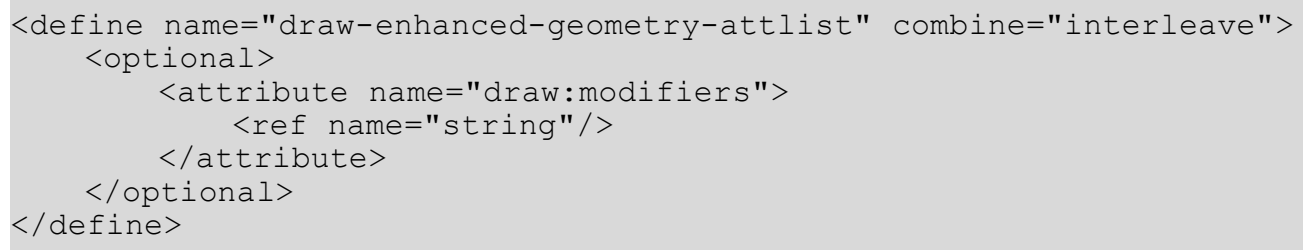

\subsubsection{Enhanced Geometry - Equation}

\section{Equation}

The <draw: equation $>$ element can be referenced by handles, text areas, glue points and enhanced paths to calculate values which are dependent to modifier values. Due to the fact that modifier values may changed by interaction it is a convenient way to integrate dynamic values into the shape geometry.

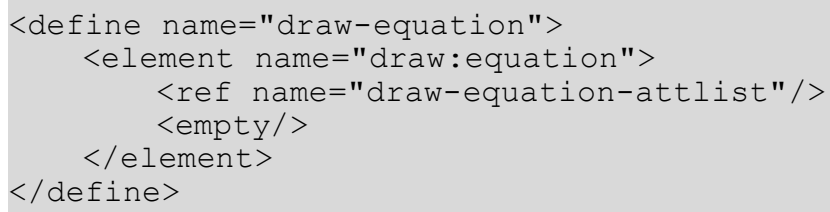

\section{Name}

The draw: name attribute specifies the name of the equation. The name is not allowed to include spaces. 


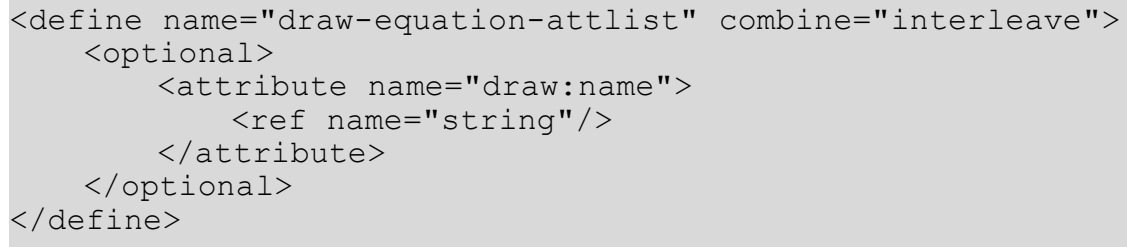

\section{Formula}

The draw: formula attribute specifies an equation that should be used to evaluate a value. A formula can make use of other formulas or modifier values by function and or modifier reference.

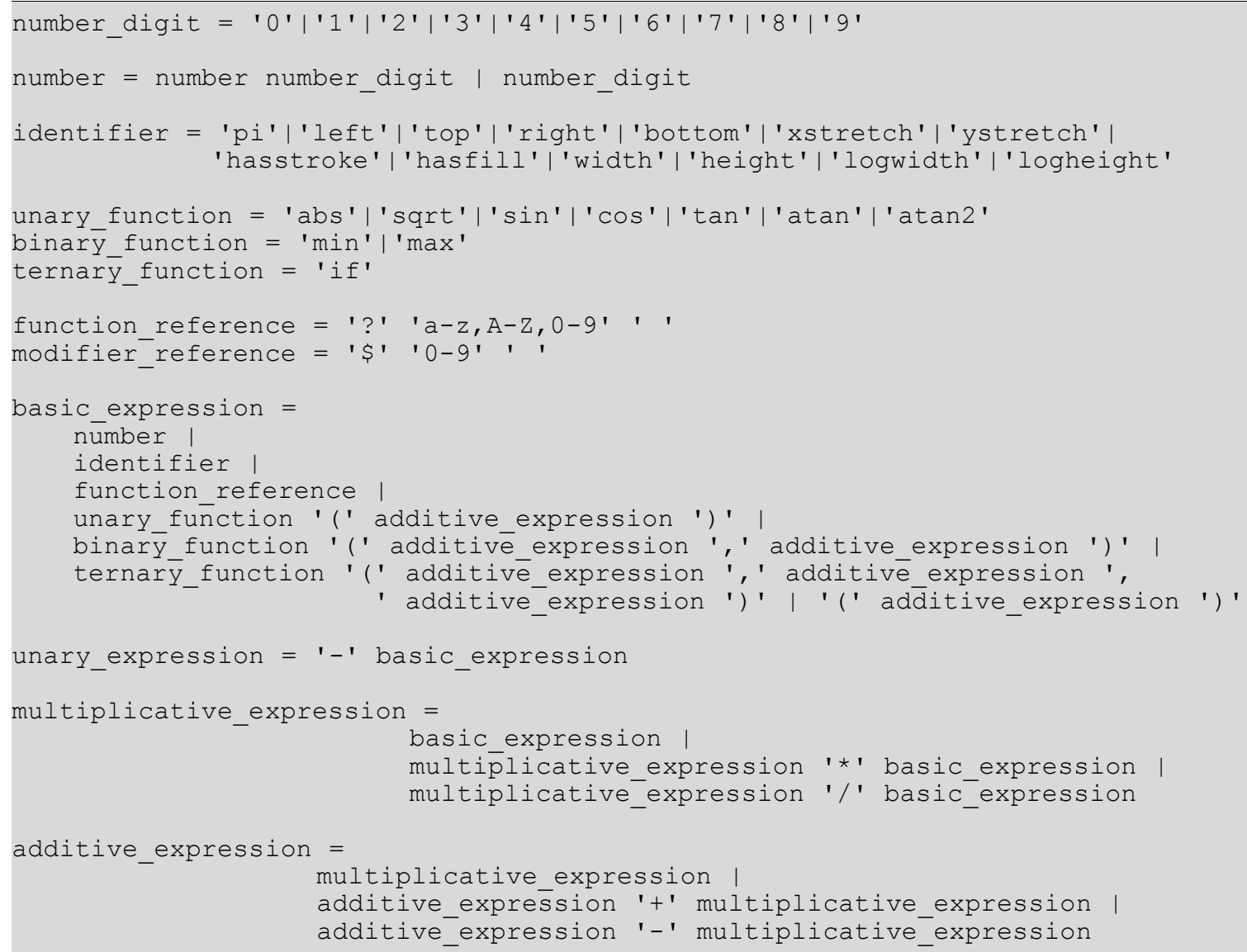

\begin{tabular}{|l|l|}
\hline identifier & \multicolumn{1}{|c|}{ Description } \\
\hline left & The left position of the $\mathrm{svg}: \mathrm{viewBox}$ attribute has to be used. \\
\hline top & The top position the $\mathrm{svg}: \mathrm{viewBox}$ attribute has to be used. \\
\hline right & The right position the $\mathrm{svg}: \mathrm{viewBox}$ attribute has to be used. \\
\hline bottom & $\begin{array}{l}\text { The bottom position the } \mathrm{svg}: \mathrm{viewB} \text { ( } \mathrm{x} \text { attribute has to be } \\
\text { used. }\end{array}$ \\
\hline
\end{tabular}




\begin{tabular}{|l|l|} 
xstretch & The value of draw:path-stretchpoint-x is used. \\
\hline ystretch & The value of draw:path-stretchpoint-y is used. \\
\hline hasstroke & If the shape has a line style, a value of 1 is used. \\
\hline hasfill & If the shape has a fill style, a value of 1 is used. \\
\hline width & The width of the svg:viewBox is used. \\
\hline height & The height of the svg:viewBox is used. \\
\hline logwidth & The width of the svg:viewBox in 1/100th $\mathrm{mm}$ is used. \\
\hline logheight & The height of the svg:viewBox in 1/100th $\mathrm{mm}$ is used. \\
\hline
\end{tabular}

A example for the draw: formula attribute would be: draw: formula="width+10- $\$ 0$ " If the value of the first modifier value is " 100 " and the width of the svg:viewbox is " 10000 ", then the result of the above formula would be $10000+10-100=9910$

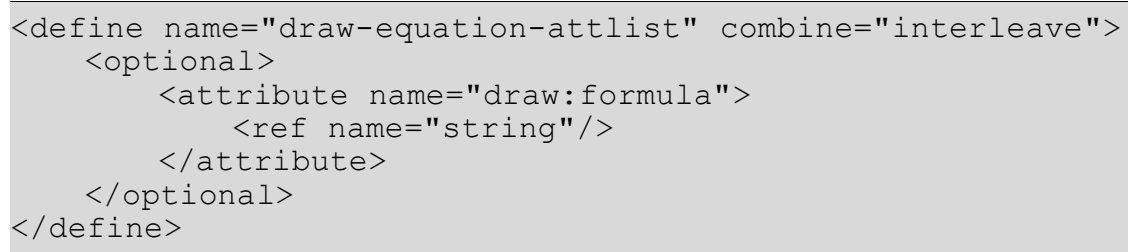

\subsubsection{Enhanced Geometry - Handle Attributes}

\section{Handle}

The <draw: handle $>$ element specifies a single interaction handle.

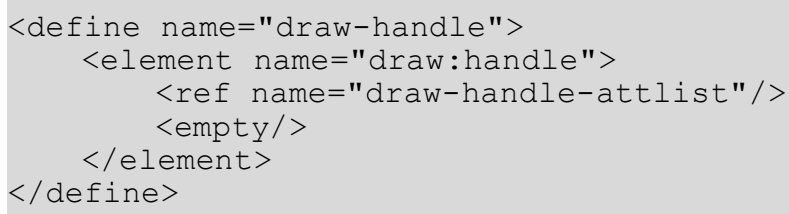

\section{Handle Mirror Vertical}

The draw: handle-mirror-vertical attribute specifies if the $\mathrm{x}$ position of the handle is mirrored.

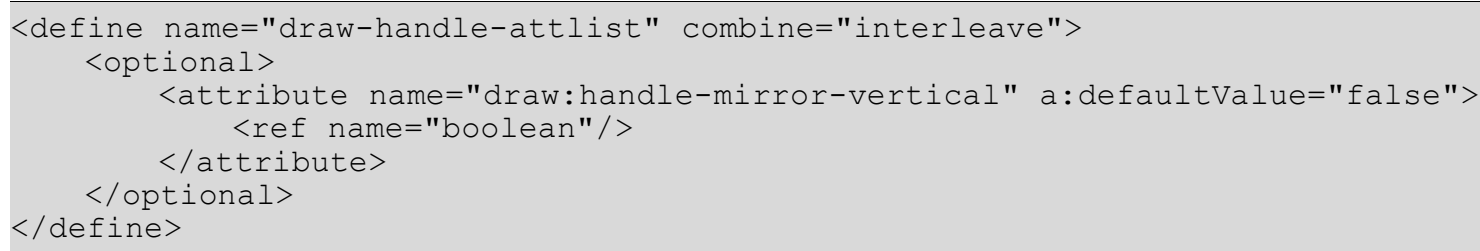




\section{Handle Mirror Horizontal}

The draw: handle-mirror-horizontal attribute specifies if the y position of the handle is mirrored.

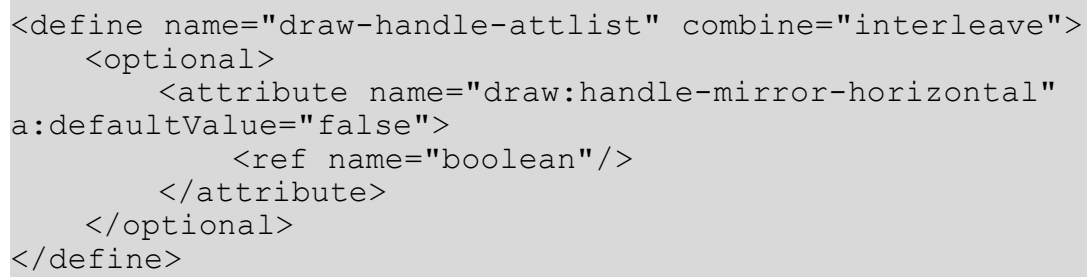

\section{Handle Switched}

The draw: handle-switched attribute specifies if the handle directions are swapped if the shape height is higher than the shape width.

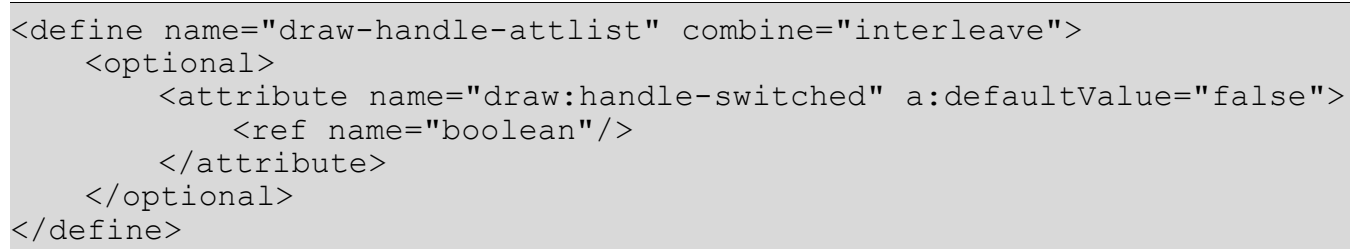

\section{Handle Position}

The draw: handle-position attribute specifies the position of the handle and consists of two parameters.

Each parameter can be a float or it can have one of the following enhancements:

- A "?" is used to mark the beginning of a formula name. The result of the element's draw: formula attribute is used as parameter value in this case.

- If " $\$$ " is preceding a integer value, the value is a indexing a draw:modifiers attribute. The corresponding modifier value is used as parameter value then.

- Instead of a number a parameter can also be one of the following constants:

\begin{tabular}{|l|l|}
\hline $\begin{array}{c}\text { Constan } \\
\boldsymbol{t}\end{array}$ & \multicolumn{1}{c|}{ Description } \\
\hline left & The value of the draw:coordinate-origin-x attribute has to be used. \\
\hline top & The value of the draw:coordinate-origin-y attribute has to be used. \\
\hline right & $\begin{array}{l}\text { The value of the draw:coordinate-origin-x attribute + the value of } \\
\text { the draw:coordinate-width has to be used. }\end{array}$ \\
\hline bottom & $\begin{array}{l}\text { The value of the draw:coordinate-origin-y attribute + the value of } \\
\text { the draw:coordinate-height has to be used. }\end{array}$ \\
\hline xstretch & The value of draw:path-stretchpoint-x is used. \\
\hline ystretch & The value of draw:path-stretchpoint-y is used. \\
\hline hasstroke & If the shape has a line style, a value of 1 is used. \\
\hline
\end{tabular}




\begin{tabular}{|l|l|}
\hline $\begin{array}{c}\text { Constan } \\
\boldsymbol{t}\end{array}$ & \multicolumn{1}{c|}{ Description } \\
\hline hasfill & If the shape has a fill style, a value of 1 is used. \\
\hline width & The width of the svg:viewBox is used. \\
\hline height & The height of the svg:viewBox is used. \\
\hline logwidth & The width of the svg:viewBox in $1 / 100$ th $\mathrm{mm}$ is used. \\
\hline logheight & The height of the svg:viewBox in $1 / 100$ th $\mathrm{mm}$ is used. \\
\hline
\end{tabular}

The draw: handle-position attribute specifies the position of the handle. If the draw: handle-polar attribute is not set, the first parameter of the draw: handle-position attribute specifies the horizontal handle position, the vertical handle position is described by the second parameter. If the draw: handle-polar attribute is set, then the handle is a polar handle and the first parameter of the draw:handle-position attribute specifies the angle in grad, the handle radius is specified by the second parameter. A example for the draw: handleposition attribute is: draw: handle-position = "left \$5"

\section{Handle Range X Maximum}

The draw: handle-range-x-maximum attribute specifies the horizontal maximum value of the range the handle can be moved within. The syntax for the attribute is the same as for the attribute draw: handle-range-x-minimum.

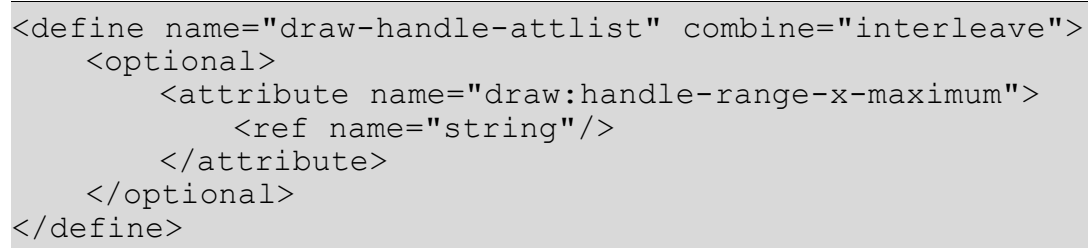




\section{Handle Range Y Minimum}

The draw: handle-range-y-minimum attribute specifies the vertical minimum value of the range the handle can be moved within. The syntax for the attribute is the same as for the attribute draw: handle-range-x-minimum.

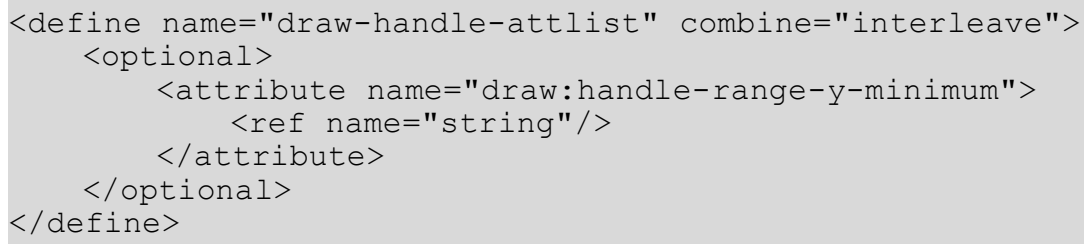

\section{Handle Range Y Maximum}

The draw: handle-range-y-maximum attribute specifies the vertical maximum value of the range the handle can be moved within. The syntax for the attribute is the same as for the attribute draw: handle-range-x-minimum.

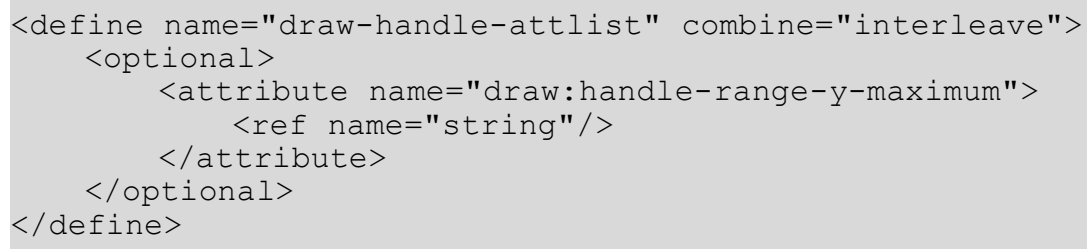

\section{Handle Polar}

The draw: handle-polar attribute specifies that the handle is a polar handle. The syntax for this attribute is the same as for the attribute draw: handle-position. The first parameter specifies the horizontal center position, the vertical center position is specified by the second parameter. If this attribute is set, the attributes draw: handle-range-x and draw: handlerange-y are ignored, instead the attributes draw: handle-radius-range-minumum and draw: handle-radius-range-maximum can be used.

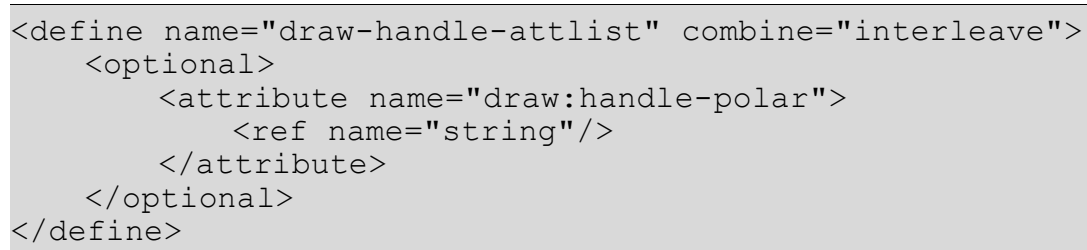

\section{Handle Radius Range Minimum}

If the attribute draw: handle-radius-range-minimum is set, it specifies the minimum radius range that can be used for a polar handle. The syntax is the same as for the attribute draw: handle-range-x-minimum.

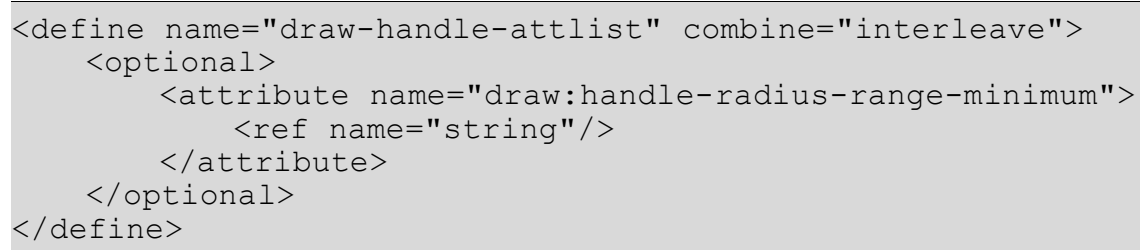




\section{Handle Radius Range Maximum}

If the attribute draw: handle-radius-range-maximum is set, it specifies the maximum radius range that can be used for a polar handle. The syntax is the same as for the attribute

draw: handle-range-x-minimum.

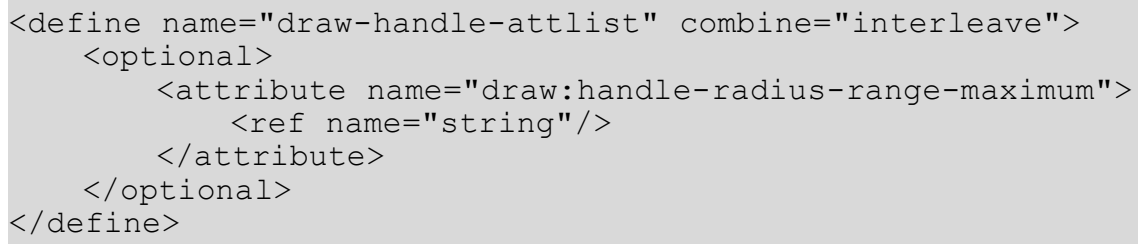

\subsection{Presentation Shapes}

Presentation shapes are special text box, image, object or thumbnail drawing shapes contained in a presentation. Presentation shapes use styles with a style family value of presentation, unlike drawing shapes which use styles with a style family value of graphic. Presentation shapes can be empty, acting only as placeholders. If a draw page's presentation layout (see section 14.15) is changed, all presentation shapes are adapted automatically.

Standard drawing shapes can also be used in presentations. The presentation:class attribute distinguishes presentation shapes from drawing shapes. Unlike presentation shapes, standard drawing shapes are not adapted if the presentation page layout is changed.

\subsubsection{Common Presentation Shape Attributes}

The attributes described in this section are common to all presentation shapes.

\section{Style}

Presentation shapes can have styles from the style family presentation assigned to them. A presentation shape can be distinguished from a drawing shape by checking whether it has a presentation:style-name attribute. A drawing shape uses a draw: style-name attribute with a style from the graphic family, while a presentation shape uses a

presentation:style-name attribute with a style from the presentation family. This name links to a <style:style $>$ element with the family presentation. The formatting properties in this style and its optional parent styles are used to format this shape. See also section 9.2.15.

\section{Class}

The presentation:class attribute classifies presentation shapes by their usage within a draw page (for instance as title or outline). The following classes are existing:

- title: Titles are standard text shapes.

- outline: Outlines are standard text shapes.

- subtitle: Subtitles are standard text shapes.

- text: Presentation texts are standard text shapes.

- graphic: Presentation graphics are standard graphic shapes

- object: Presentation objects are standard object shapes. 
- chart: Presentation charts are standard object shapes.

- table: Presentation tables are standard object shapes.

- orgchart: Presentation organization charts are standard object shapes.

- page: Presentation pages are used on notes pages.

- notes: Presentation notes are used on notes pages.

- handout: Presentation handouts are placeholder for the drawing page in an handout page.

The next four classes can be used only for drawing shapes that are contained in master pages. Depending on the settings of the page (see section 15.36), they are displayed automatically on drawing pages that use the master page.

- header: The drawing shape is used as a header. Header shapes are standard text shapes.

- footer: The drawing shape is used as a footer. Footer shapes are standard text shapes.

- date-time: The drawing shape is used as a date and/or time shape. Date and Time shapes are standard text shapes.

- page-number: The drawing shape is used as a page number shape. Page Number shapes are standard text shapes.

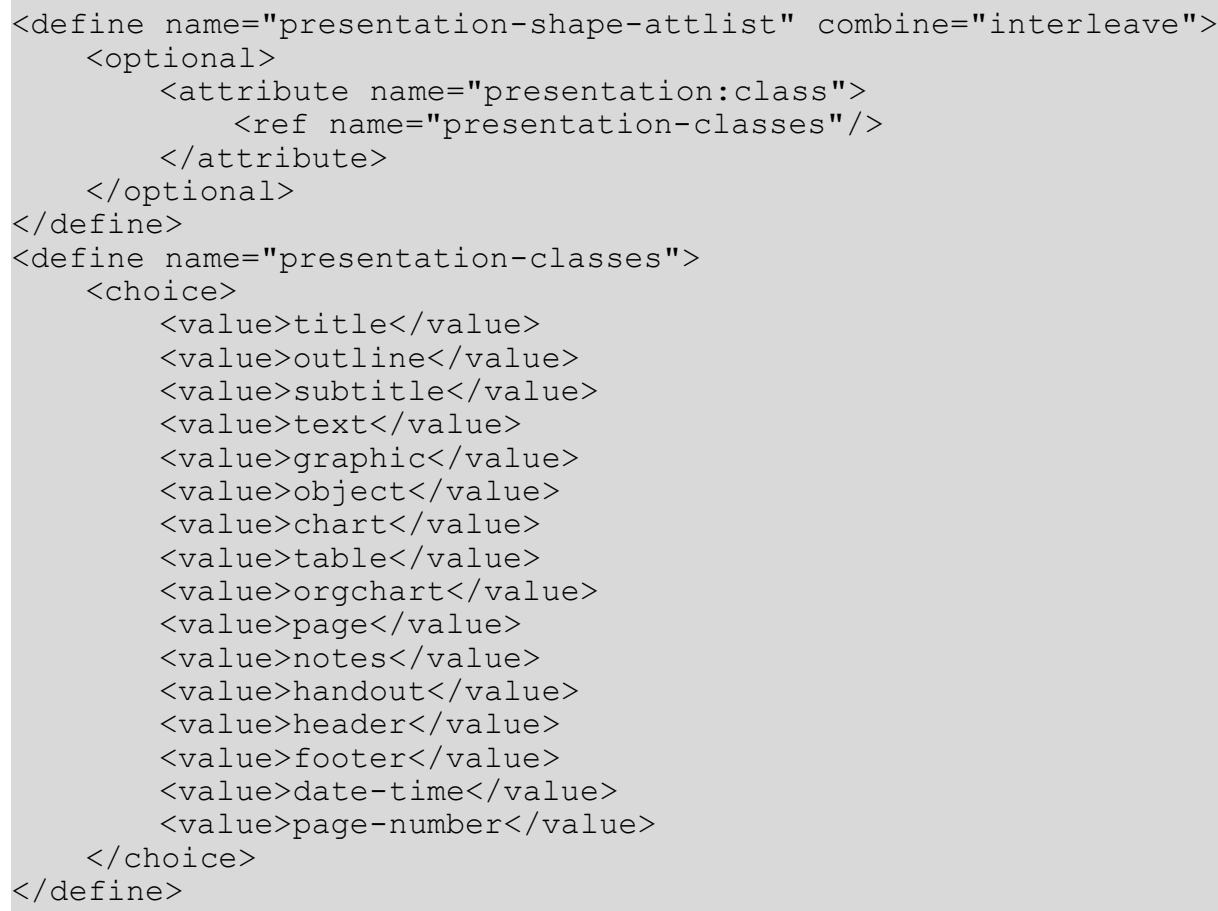

\section{Placeholder}

The presentation:placeholder attribute defines if a shape is a placeholder or a presentation object with actual content.

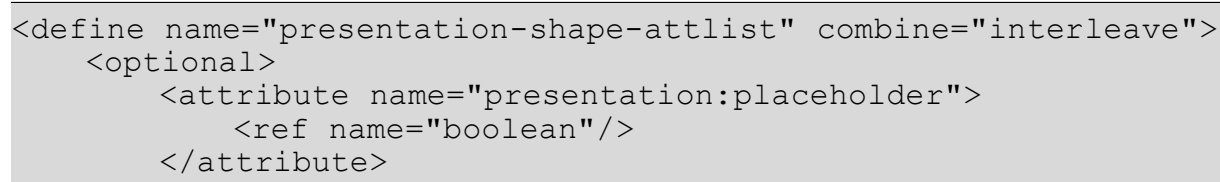




\section{User-Transform}

The presentation: user-transformed attribute specifies whether the size and position of the shape is set by the user or is set by the corresponding presentation shape on the master page.

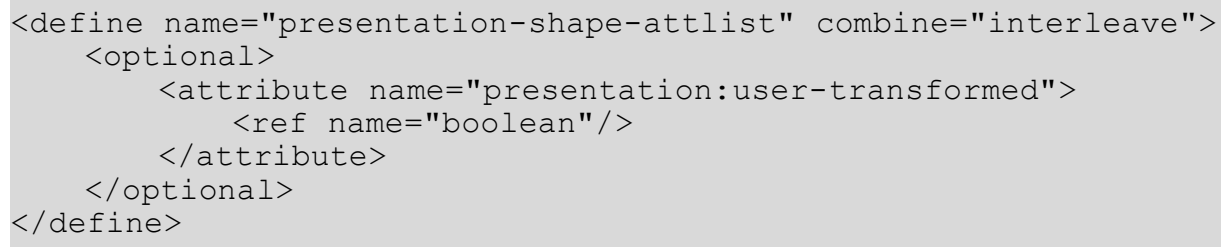

\subsection{Presentation Animations}

In a presentation document, shapes can be animated. Each presentation page can have an optional <presentation: animations > element, which is a container for animation effects. The animation is executed when the page is displayed during a presentation.

This specification allows multiple effects for one and the same shape within a page. Applications may have restrictions regarding the number and combination of effects applicable to a shape, for instance may support only one show and one hide effect per shape with an additional show and hide text and one dim and sound effect.

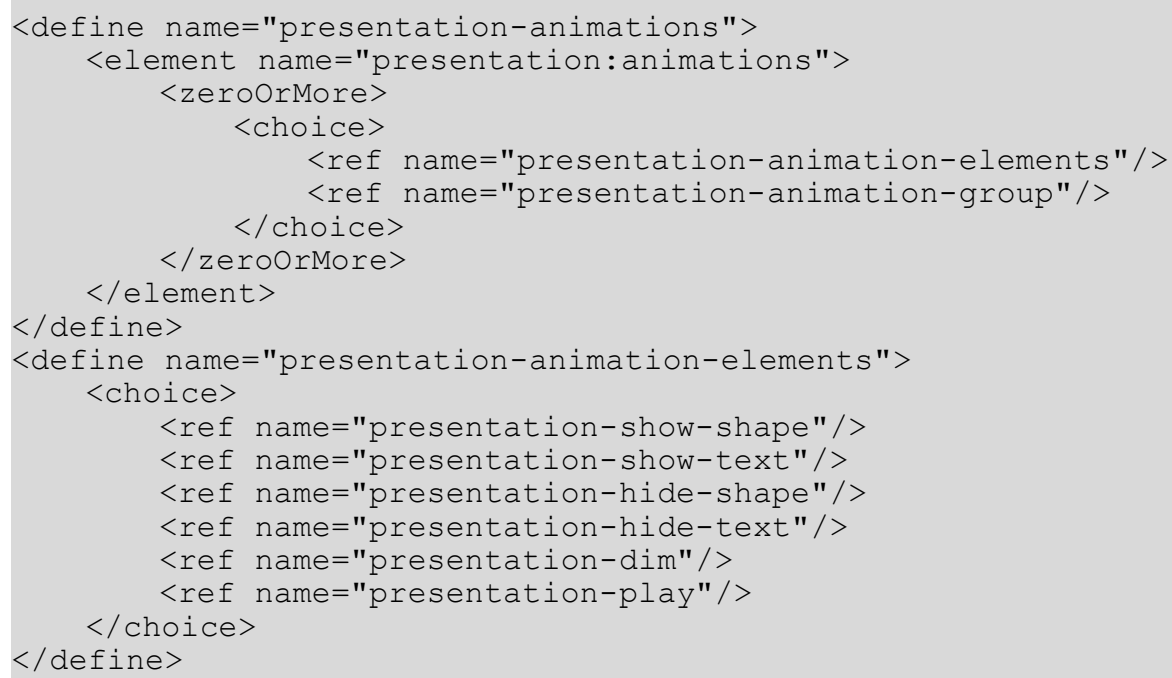

\subsubsection{Sound}

The element $<$ presentation: sound $>$ may be contained in all animation effect elements that support sounds. The sound file referenced by the XLink attributes is played when the effect is executed.

<define name="presentation-sound">

<element name="presentation:sound">

<ref name="presentation-sound-attlist"/>

<attribute name="xlink:href">

$<$ ref name="anyURI" />

$</$ attribute $>$ 


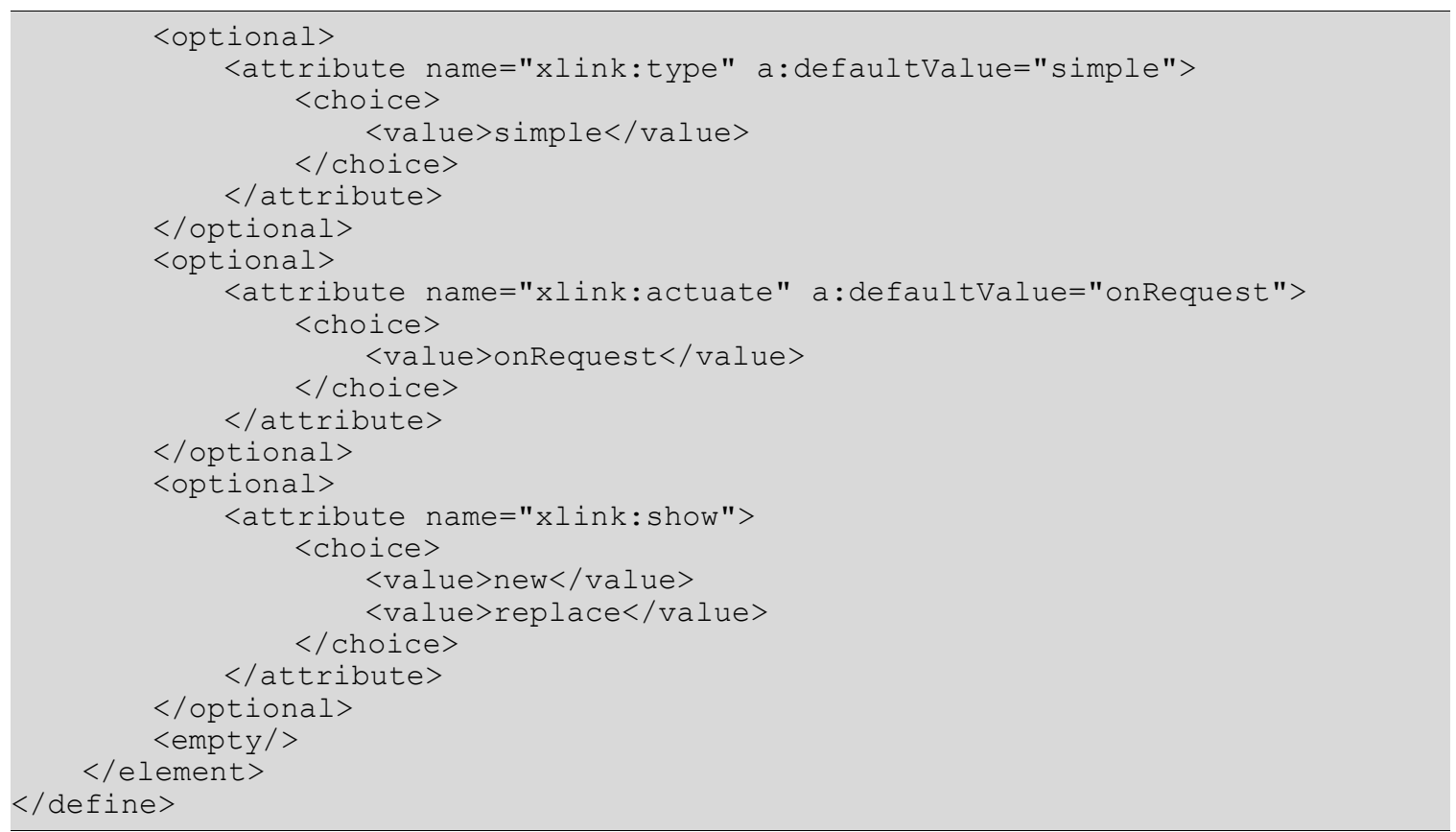

The attribute that may be associate with the <presentation: sound> element is:

- Play full

\section{Play Full}

If the value of the attribute presentation:play-full is true, the next effect starts after the sound is played. If the value of this attribute is false, the next effect starts when the current effect is finished.

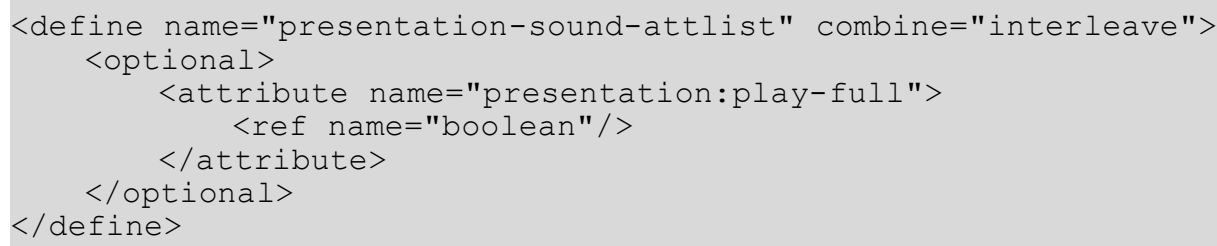

\subsubsection{Show Shape}

The element $<$ presentation:show-shape> makes a shape visible. If there is a <presentation:show-shape> element for one shape, this shape is automatically invisible before the effect is executed.

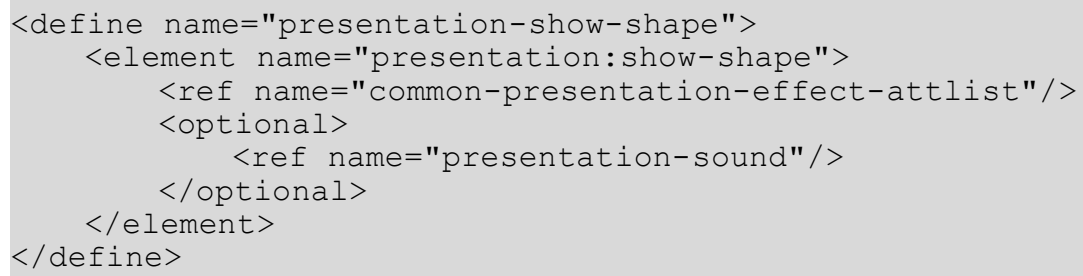

The attributes that may be associated with the <presentation: show-shape> element are:

- Shape 
- Effect

- Direction

- $\quad$ Speed

- Delay

- Start Scale

- Path

\section{Shape}

The attribute draw: shape-id specifies the shape of this effect using a shape ID.

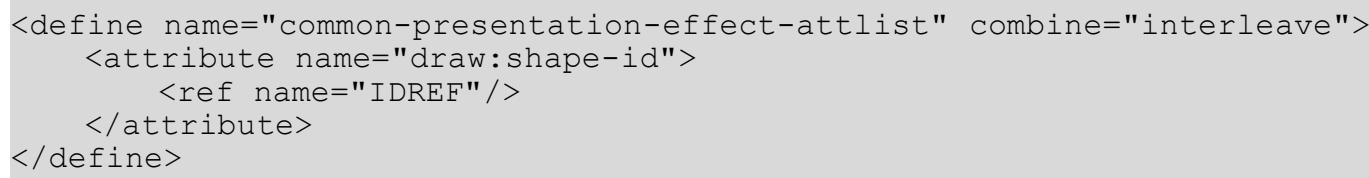

\section{Effect}

The attribute presentation: effect specifies the type of effect.

- none: no effect is used.

- fade: the shape fades from its visible or hidden state to a hidden or visible state.

- move: the shape moves from or to its final position.

- stripes: the shape is faded in or out by drawing or removing horizontal or vertical stripes that change their size.

- open: the shape is drawn or removed line by line, either horizontally or vertically, starting at the center of the shape.

- close: the shape is drawn or removed line by line, either horizontally or vertically, starting at the edge of the shape.

- dissolve: the shape is faded in or out by drawing or removing small blocks in a random fashion.

- wavyline: the shape is faded in our out by drawing or removing small blocks in a snake like fashion.

- random: an effect is chosen at random to fade the shape in or out.

- lines: the shape is faded in our out by drawing or removing line by line, either horizontally or vertically, in a random fashion.

- laser: this effect is only available for text shapes; the characters of the text are moved one by one from the top edge of the screen to their final position.

- appear: the shape is faded in by just switching its state from invisible to visible.

- hide: the shape is faded out by just switching its state from visible to invisible.

- move-short: like the move effect, but the moving shape is clipped to its final bounding rectangle during fade. 
- checkerboard: the shape is faded in or out by drawing or removing checkerboard like blocks that increase in size over time.

- rotate: the shape rotates horizontally or vertically for a short amount of time during this effect.

- stretch: the shape is faded in or out by changing its size during this effect.

\section{Direction}

The attribute presentation: direction specifies the direction of the effect. This is relevant for some effects only.

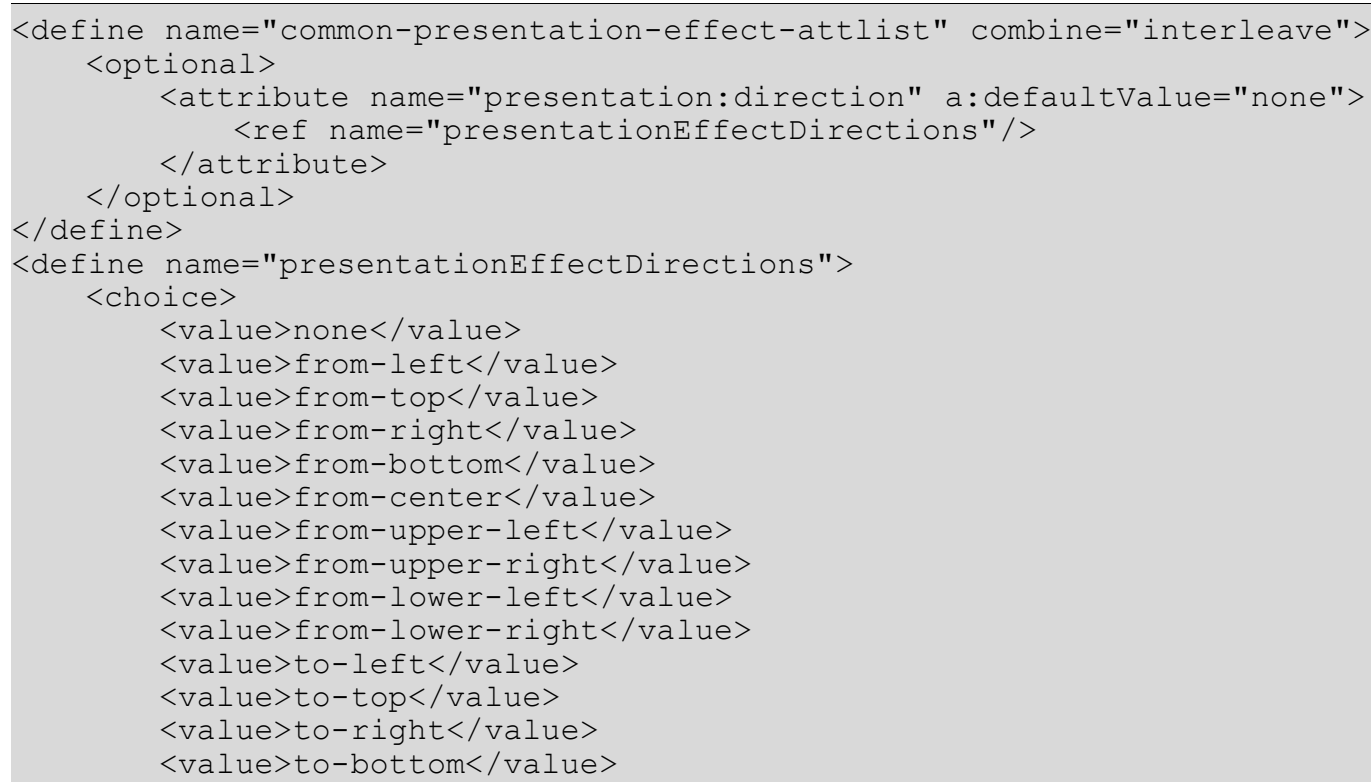




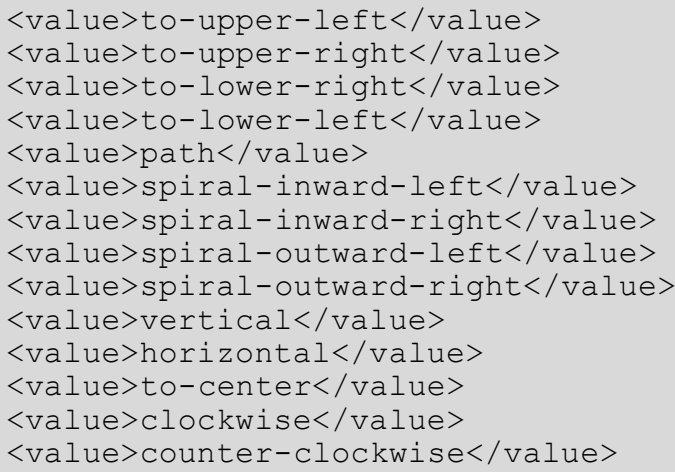

\section{Speed}

The attribute presentation:speed specifies the speed of the effect.

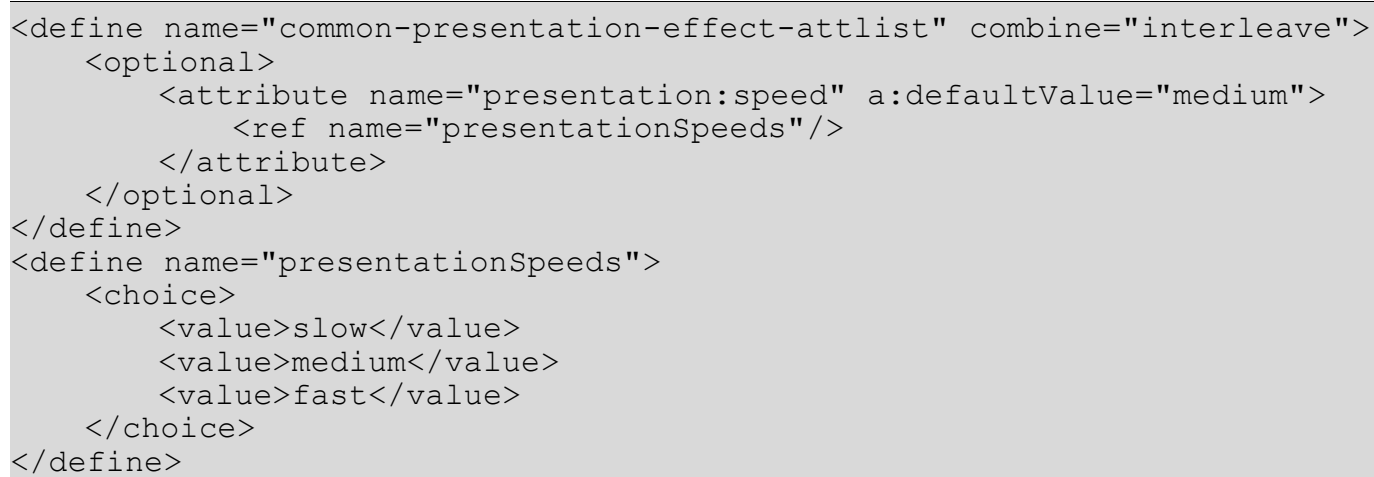

\section{Delay}

The attribute presentation: delay specifies the delay before a presentation effect starts after the previous one has been finished.

8001

8002

8003

8004

8005

8006

8007

\section{Start Scale}

Some effects scale a shape during execution of the effect. The attribute presentation: start-scale specifies the start size of the shape as a percentage of its original size. 


\section{Path}

The attribute presentation:path-id applies to move effects. The attribute specifies the shape-id of a polygon shape. The effect moves along the lines of the specified polygon. The referenced polygon is not visible during the presentation.

8015

8016

8017

8018

8019

\subsubsection{Show Text}

The element $<$ presentation: show-text $>$ makes the text of a shape visible. If there is a <show-text> element for one shape, the text of the shape is automatically invisible before the effect is executed.

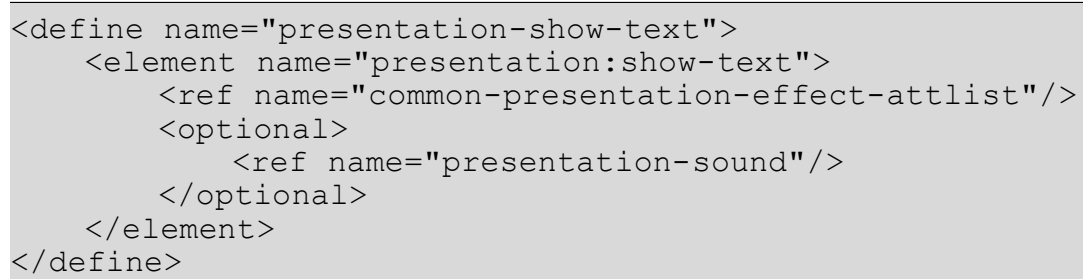

The attributes that may be associated with the <presentation: show-text> element are:

- $\quad$ Shape, Effect, Direction, Speed, Start Scale, Path - see section 9.7.2

\subsubsection{Hide Shape}

The element $<$ presentation: hide-shape $>$ makes a shape invisible.

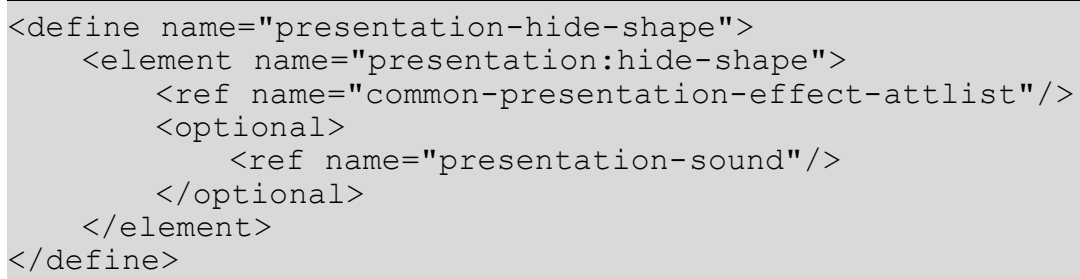

The attributes that may be associated with the <presentation:hide-shape> element are:

- Shape, Effect, Direction, Speed, Start Scale, Path - see section 9.7.2

\subsubsection{Hide Text}

The element $<$ presentation: hide-text $>$ makes the text of a shape invisible.

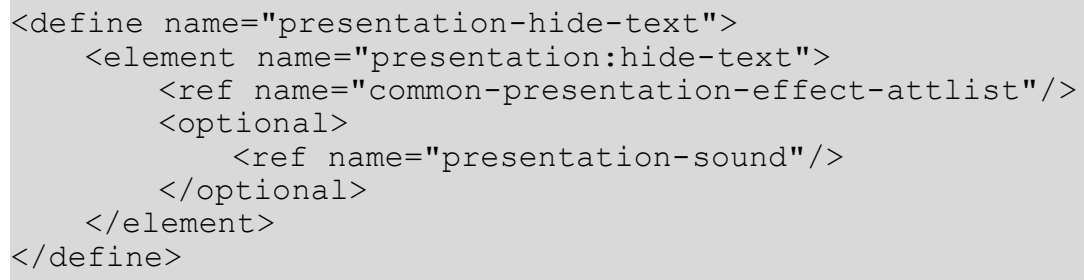

The attributes that may be associated with the <presentation: hide-text $>$ element are: 
- $\quad$ Shape, Effect, Direction, Speed, Start Scale, Path - see section 9.7.2

\subsubsection{Dim}

The element $<$ presentation: $\operatorname{dim}>$ fills a shape in a single color.

\section{4 \\ 8045 \\ 8046 \\ 8047 \\ 8048 \\ 8049 \\ 8050 \\ 8051}

\subsubsection{Play}

The element $<$ presentation:play $>$ starts the animation of a shape that supports animation.

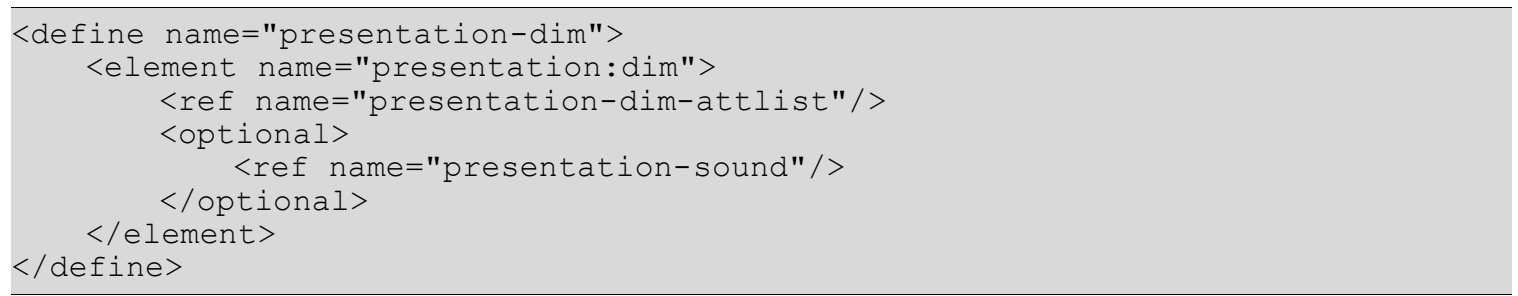

The attributes that may be associated with the <presentation: dim> element are:

- $\quad$ Shape - see section 9.7.2

\section{- Color}

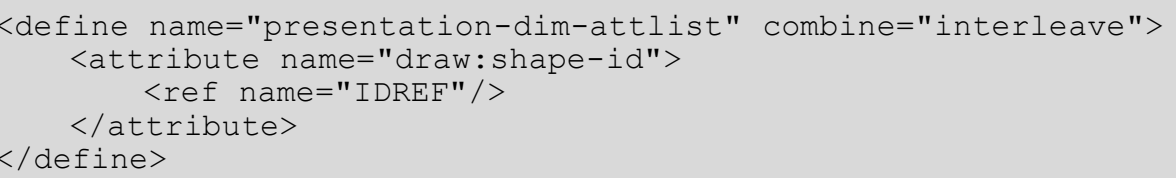

\section{Color}

The attribute draw: color specifies the color that is used to fill the shape when the shape is dimmed.

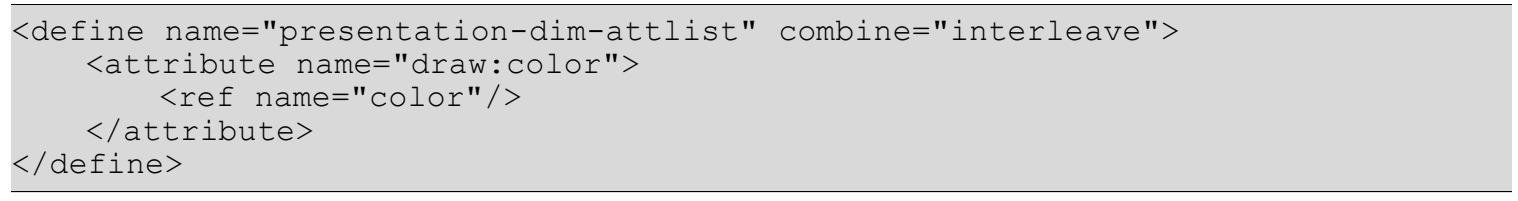

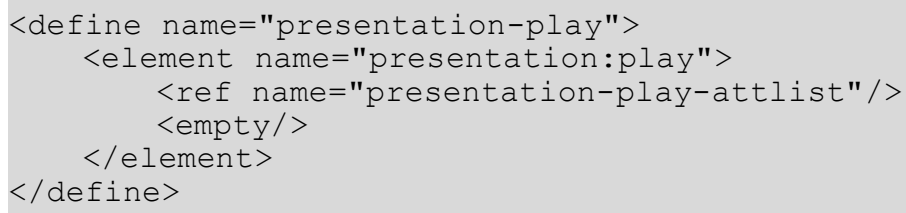

The attributes that may be associated with the <presentation:play> element are:

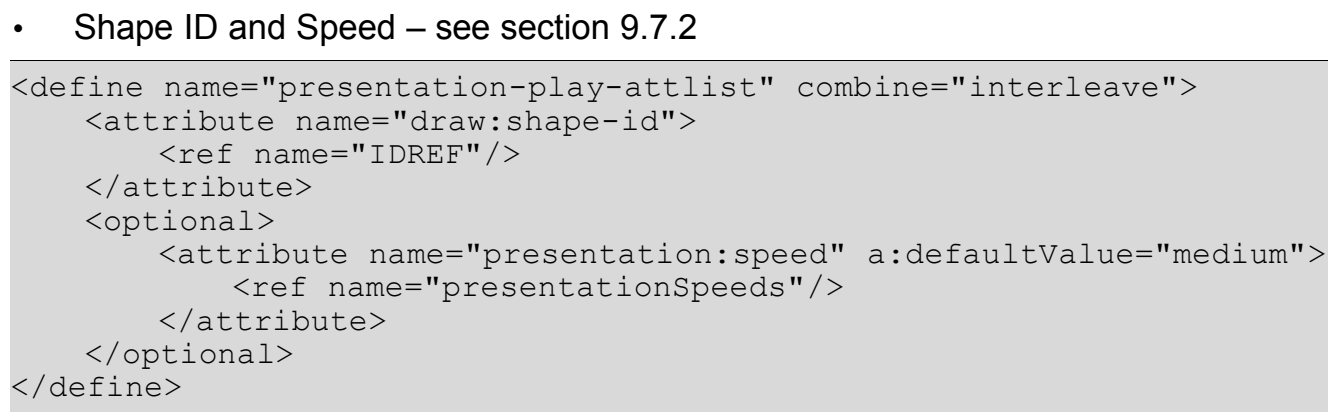




\subsubsection{Effect groups}

The element $<$ presentation:animation-group $>$ allows to specify that multiple effects should happen at the same time.

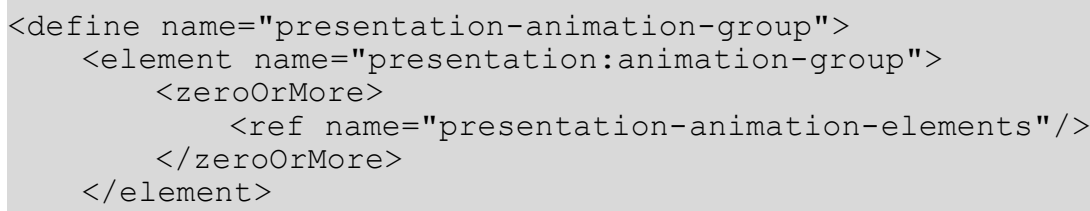

\subsection{SMIL Presentation Animations}

This chapter describes [SMIL20] based shape animations for presentation documents. This kind of animations can be used instead of the ones specified by the <presentation:animations> elements if one of the following items is required:

- Multiple animations per shape.

- A mixture of animations starting on user interaction and starting automatically per page.

- Multiple animations running at the same time.

- Additional effects 'programmed' in XML by combining basic animation elements.

- Document transformations to SVG including [SMIL20].

\subsubsection{Recommended Usage Of SMIL}

The following sections describe the usage of SMIL animation elements that enables an office application to present the animation elements in a simple and easy to use $\mathrm{UI}$ to the user. This UI may contain a single main sequence of effects, and in addition to this, multiple sequences of effects that are started as interactions on drawing shapes. An effect is a combination of one or more animation elements that animate a single shape and or a shape's paragraphs.

It is recommended, that in user interfaces, effects can be created by using presets that have localized and meaningful names. This way, the user will not work on a hierarchy of SMIL animation elements, but on one dimensional lists of effects, which are much easier to handle for the office application users.

\section{Slide Animation}

Each <draw: page > element may optionally have an <anim:par $>$ element that defines the animation of that page during a running slideshow. This $<$ anim: par $>$ element should contain one $<$ anim:seq $>$ element which is the main sequence for shape effects and zero or more $<$ anim:seq> elements that define interactive sequences for shapes that contain animation interactions. The animation elements are executed after the slide has executed its initial transition.

\section{Main Sequence}

The main sequence is a <anim:seq> element which contains the effects that should start after the slide has executed its initial transition. Since this is a sequential container, its child nodes are executed one after each other. If a child node's smil:begin attribute has the value 
indefinite, then the execution is stalled until the user advances the slideshow by a mouse or key interaction.

The first level of child nodes in the main sequence should be <anim:par> elements that group animation elements that are started with the same user interaction. The second level of child nodes should be <anim: par> elements that group animations elements that start at the same time. The third level of child nodes should be <anim:par> elements that group the animation elements for a single effect.

The following example shows a main sequence with the effects $A, B, C$ and $D$. Effect $A$ is started on user interaction, effect $B$ is started simultaneously with $A$. Effect $C$ is started 4 seconds after the effects $A$ and $B$. Effect $D$ is started on the next user interaction:

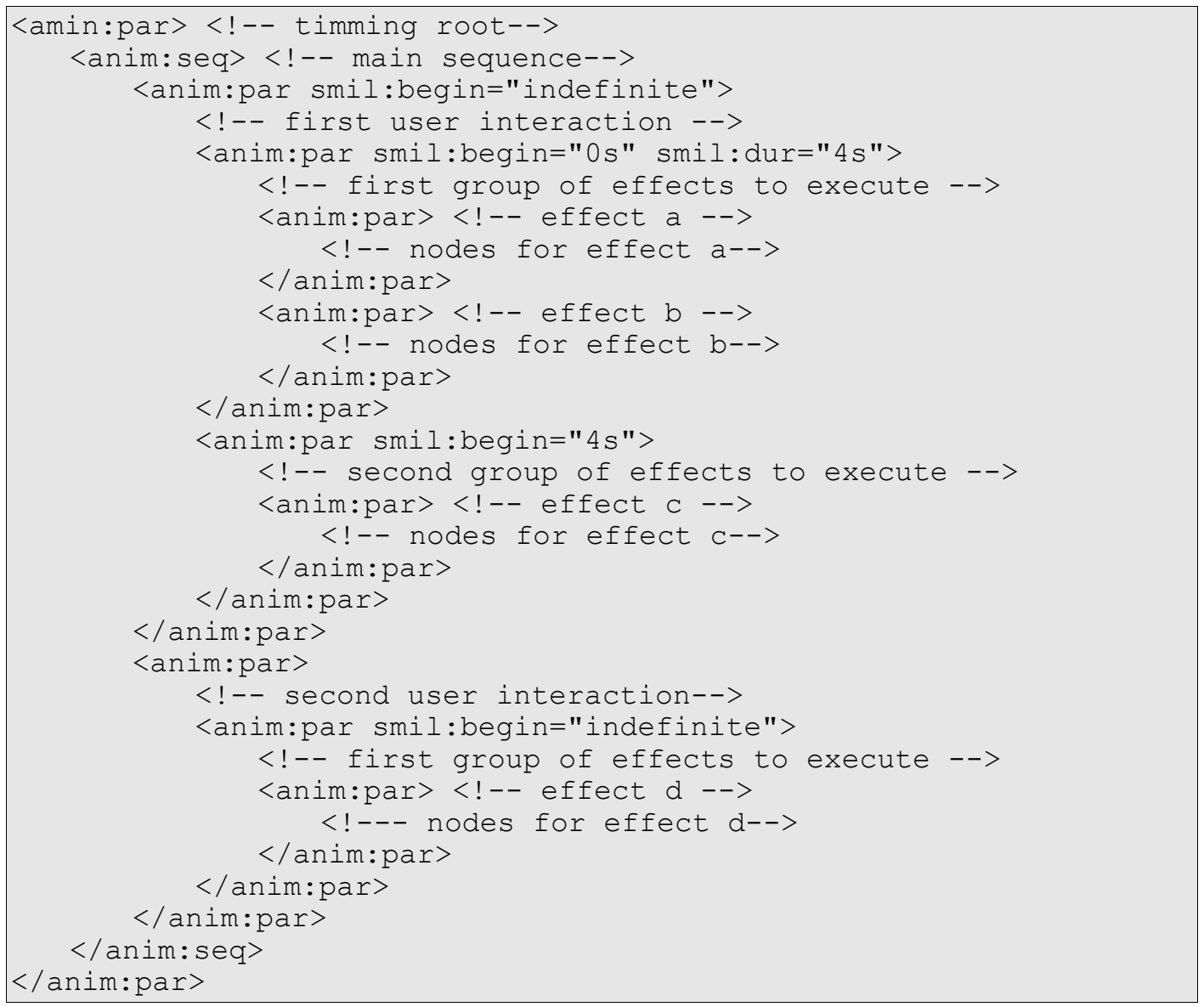

\section{Interactive Sequence}

An interactive sequence is a $<$ anim:seq $>$ element that should have the same structure as a main sequence. The only difference is that the <anim:par> element in the first level has a smil:begin attribute with a value like [shape-id].click, where [shape-id] identifies a drawing shapes by its draw:id attribute. These animation elements are triggered when the user interacts with the element defined by [shape-id].

\subsubsection{Document Dependent SMIL Animation Attribute Values}

This section describes the attribute values of the document type dependent attributes specified in section 13 if they are used within presentation documents. 


\section{Iteration Target Element}

For presentation documents, the smil:targetElement attribute of the <anim:iterate> element (see section 13.4.4) can reference drawing shape or paragraph elements. If the anim:sub-item attribute of $<$ anim: iterate $>$ has the value whole, the iteration includes the drawing shape's background and its text. If the anim:sub-item attribute's value is text, only the shape's text is iterated.

\section{Iteration Type}

For presentation documents, the anim:iterate-type attribute of the <anim:iterate> element (see section 13.4.4) can have the following values:

- by-paragraph: the target shape is iterated by paragraphs.

- by-word: the target shape or paragraph is iterated by words.

- by-letter: the target shape or paragraph is iterated by letters.

\section{Target Element}

For presentation documents, the smil: targetElement specified in section 13.3 .1 can reference drawing shapes by their draw: id attribute value and paragraphs by their text:id attribute value.

\section{Target Attribute}

For presentation documents, the smil:attributeName attribute specified in section 13.3.1 can have the following values:

- $\mathrm{x}$ : animates the elements $\mathrm{x}$ position, values are given in screen space where 0 is the left edge and 1 is the right edge.

- y animates the elements y position, values are given in screen space where 0 is the top and 1 is the bottom.

- width: animates the elements width, values are given in screen space where 0 is no width and 1 is the same width as the screen.

- height: animates the elements height, values are given in screen space where 0 is no height and 1 is the same height as the screen.

- color: animates the elements color, this animates both fill,line and char color. Values can be RGB or HSL

- rotate: animates the elements roation, this animates both the shapes and text animation.

- skewX: animates the elements horizontal skew.

- fillColor: animates the elements fill color.

- fillstyle: animates the elements fill style.

- lineColor: animates the elements line color.

- lineStyle: animates the elements line style.

- charColor: animates the elements char color. 
- charWeight: animates the elements text weight.

- charUnderline: animates the elements text underline.

- charFontName: animates the elements text font.

- charHeight: animates the elements text height.

- charPosture: animates the elements text posture.

- visibility: animates the elements visibility.

- opacity: animates the elements opacity.

\section{Target Element Sub Item}

For presentation documents, the anim: sub-item attribute specified in section 13.3.1 can have the following values:

- whole :animates both the shape and its text.

- background,:animates only the shapes background and not its text.

- Text: animates only the text.

\section{Formula}

For presentation documents, the anim: formula attribute specified in section 13.3.2 may contain the following additional identifiers:

- $e,:$ this is the Euler constant.

- $\mathrm{x}$ : this is the animated elements left edge in screen space where 0 is the left edge of the screen and 1 is the right edge.

- $\mathrm{y}$ : this is the animated elements top edge in screen space, where 0 is the top edge of the screen and 1 is the bottom edge.

- width: this is the animated elements width in screen space, where 0 is no width and 1 is the screens width.

- height: this is the animated elements height in screen space, where 0 is no height and 1 is the screens height.

\section{Command}

For presentation documents, The anim: command attribute of the <anim:command> element (see section 13.6.1) can have the following values:

- custom: the command is user defined.

- verb: the command targets an OLE2 shape. The parameter verb is the verb number that will be executed at the OLE2 shape.

- play: the command targets a media shape and starts its playback. The optional parameter media-time defines the playback start time in seconds. If this parameter is not set, playback starts at the last position. 
- toggle-pause: the command targets a media shape and toggles its playback state from play to paused or from paused to play.

- stop: the command targets a media shape and stops its playback.

- stop-audio: the command has no target and stops all running audio playback.

\subsubsection{SMIL Presentation Animation Attributes}

The attributes described in this section can be attached to the animation elements described in section 13.4, 13.5 and 13.6 if they are used inside presentation documents. They don't influence the actual animation behavior, but help office application user interfaces in presenting animation effect settings to the user.

\section{Node Type}

The presentation: node-type attribute specifies a node type for an animation element. This attribute does not alter the element's behavior but helps the application to quickly identify an elements purpose inside an animation element hierarchy. The value of this attribute can be:

- default: this animation element has no special meaning for the application. This is the default setting.

- on-click: this animation element is the root element of an effect that starts with a user click.

- with-previous: this animation element is the root element of an effect that starts with the previous effect.

- after-previous: this animation element is the root element of an effect that starts after the previous effect.

- timing-root: this animation element is the root element for the animation of a page.

- main-sequence: this animation element is the root element for the main sequence of effects of a page

- interactive-sequence: this animation element is the root element for a sequence of effects that are started when the user interactively clicks on a special element inside a page.

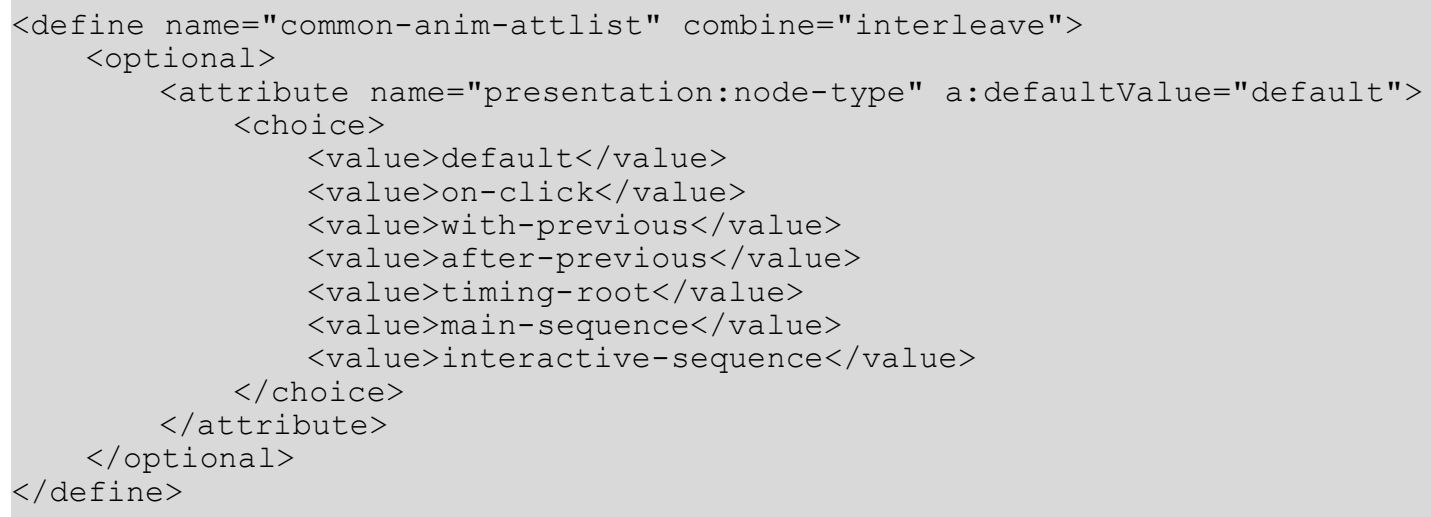




\section{Preset Id}

The presentation:preset-id attribute specifies the name of the preset that was used to create this animation element.

8100

8101

8102

8103

8104

8105

8106

8107

8108

8109

8110

8111

8112

8113

\section{Preset Class}

The presentation:preset-class attribute specifies the class of the preset that was used to create this animation element. The value of this attribute can be:

- custom: the preset was a user defined one. This is the default setting.

- entrance: the preset was an entrance effect.

- exit: the preset was an exit effect.

- emphasis: the preset was an emphasis effect.

- motion-path: the preset was a motion path.

- ole-action: the preset was an ole action.

- media-call: the preset was a media call.

8114

8115

8116

8117

8118

8119

8120

8121

8122

8123

8124

8125

8126

8127

8128

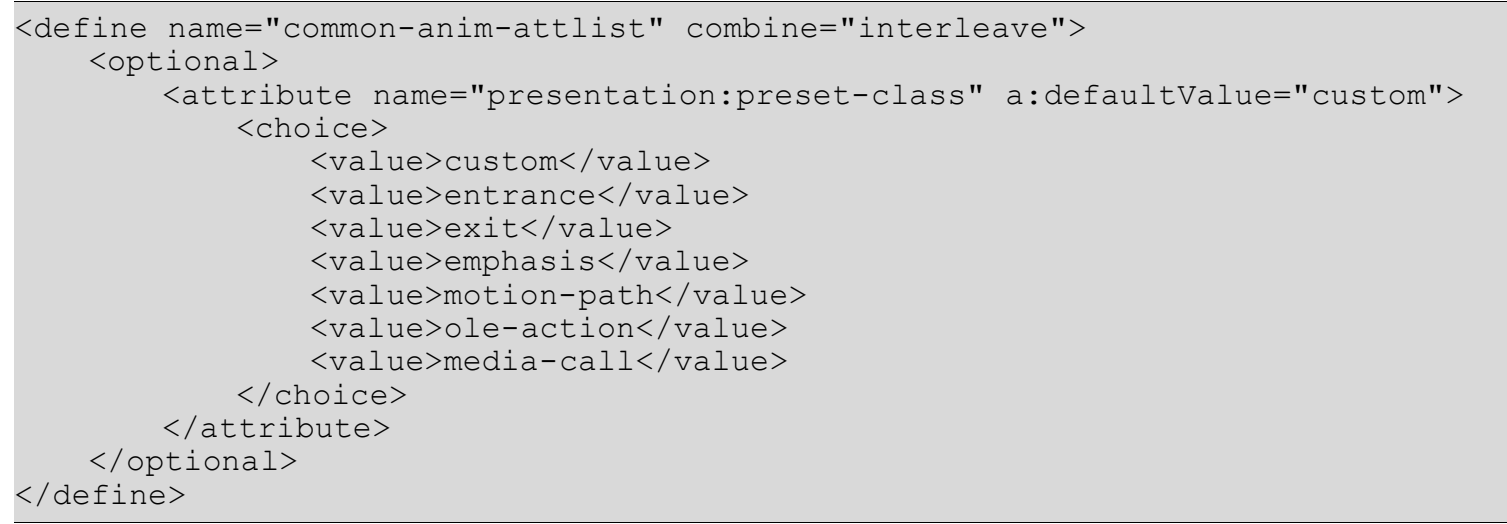




\section{Master Element}

The presentation:master-element attribute specifies the id of an animation element. Office application user interfaces may only display animation elements that don't have a presentation:master-element attribute, and may consider the ones that have a presentation:master-element to be a part of the animation element that is referenced.

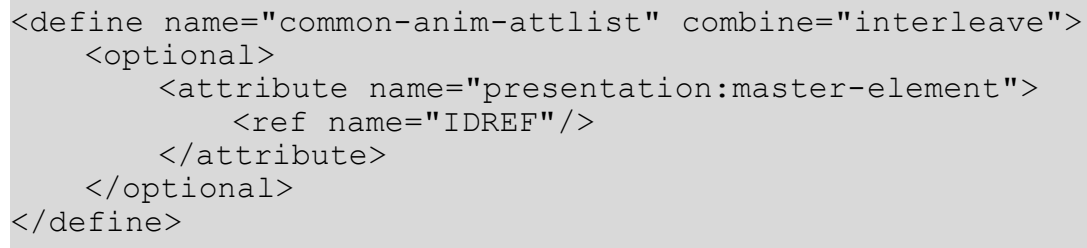

\section{Group Id}

The presentation: group-id attribute specifies a group id. This id can be used to group animation elements within the user interface, where a group consists of all animation elements that have the same group id. This can be used for instance to group the animation elements that animate the paragraphs of a single shape.

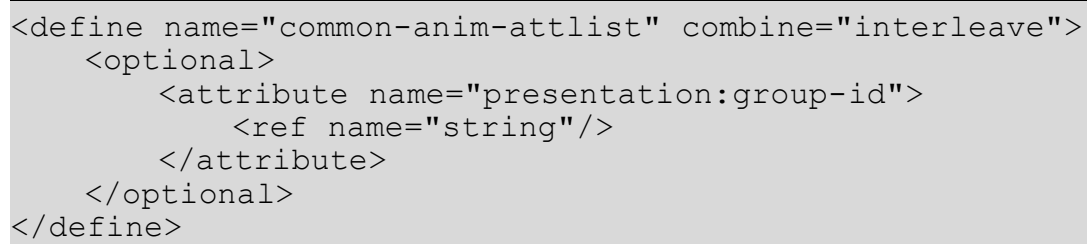

\subsection{Presentation Events}

Many objects inside a presentation document support special presentation events. For example, a user can advance the presentation one frame when he clicks on an object with a corresponding event. Presentation events are contained with a graphic object's event listener table. See section 9.2.20 for details.

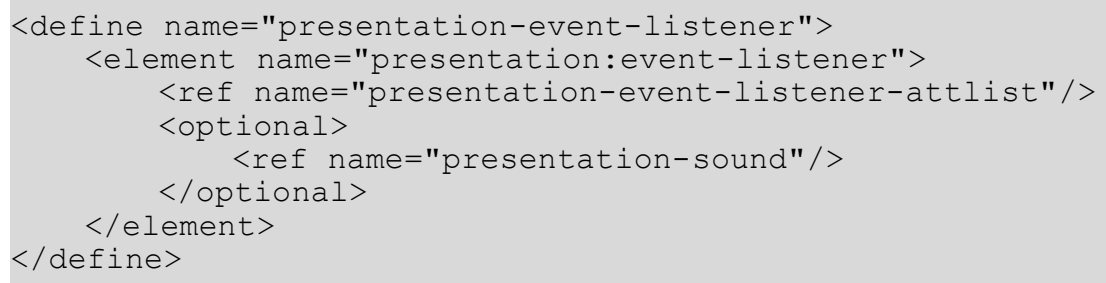

\section{Event Name}

The script: event-name attribute specifies the name of the event. See section 12.4 .1 for details.

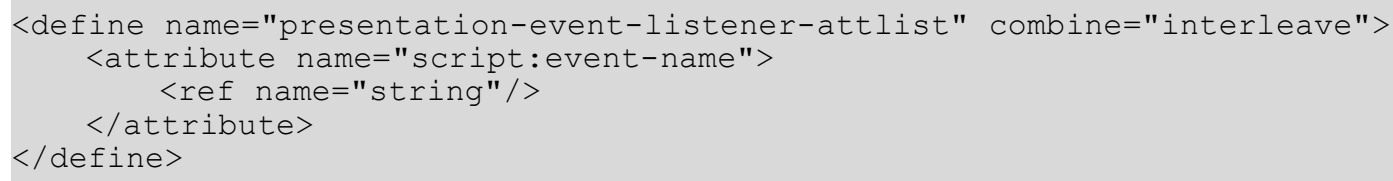




\section{Event Action}

The kind of action that is executed when the event is triggered can be selected with the presentation: action attribute. The following actions are available:

- none: no action is performed when this event is triggered.

- previous-page: the presentation jumps to the previous page.

- next-page: the presentation jumps to the next page.

- first-page: the presentation jumps to the first page of the current document.

- last-page: the presentation jumps to the last page of the current document.

- hide: the object that contains this event is hidden if the event is triggered.

- stop: if a slide show is active, it will be stopped.

- execute: another application is lunched when this event is triggered. The application can be set with an xlink.

- show: the target of a url is opened when this event is triggered. The url can be set with an xlink.

- verb: if the object that contains this event supports the execution of [OLE] verbs, the verb with the id set in the presentation:verb attribute is executed.

- fade-out: the object that contains this event is faded out when this event is triggered. The attributes presentation:effect, presentation:direction, presentation: speed and presentation:start-scale can be used to set the effect.

- sound: an audio effect is started when the effect is triggered. The audio effect is described by a <presentation: sound $>$ child element.

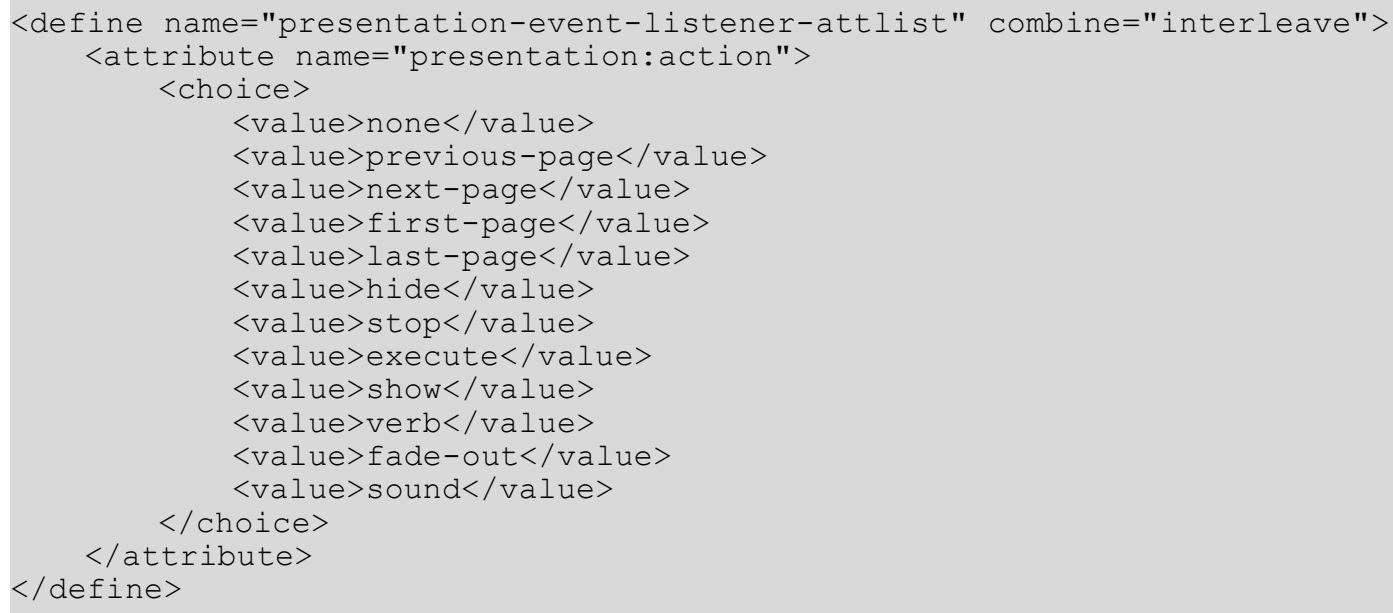

\section{Event Effect}

See presentation: effect attribute in section 9.7.2.

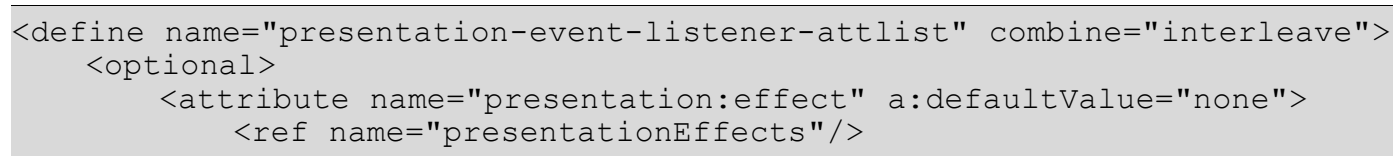




\section{Effect Direction}

See presentation: direction attribute in section 9.7.2.

\section{Effect Speed}

See presentation: speed attribute in section 9.7.2.

\section{Start Scale}

See presentation:start-scale attribute in section 9.7.2.

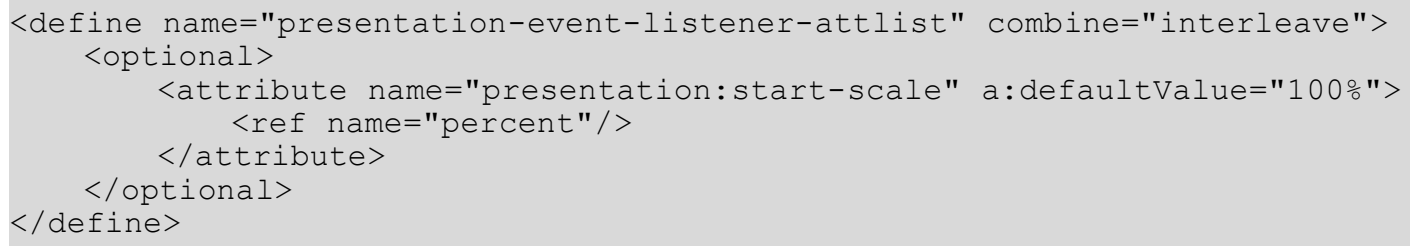

\section{Link}

Depending on the action selected by the presentation:action attribute, this xlink: href attribute either selects a document bookmark or an application. 


\section{Verb}

The [OLE] verb defined by the presentation: verb attribute is executed for event listeners of type verb at the object that contains this event.

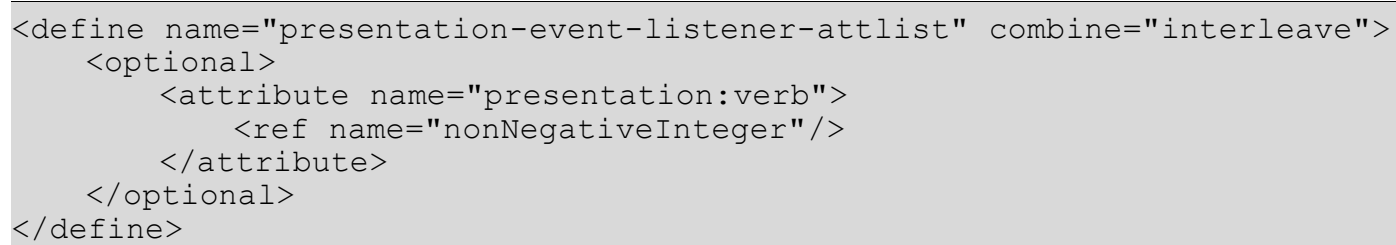

\subsection{Presentation Text Fields}

This section describes text fields that are specific to the text of drawing shapes that are contained presentations.

\subsubsection{Header Field}

Header fields display a header text specified in a header field declaration (see section 9.11.2). Which header field declaration is used is specified by the presentation: use-header-name attribute of the draw page where the field occurs. If the field is contained in a presentation shape inside a master page (see section 9.6.1), then the presentation: use-header-name attribute of the drawing page for which the drawing shape is displayed is used (see section 9.1.4).

This field is mainly used inside master pages. Since its value may differ for the individual drawing pages that make use of a master page, the current field value is not available.

\subsubsection{Footer Field}

Footer fields display a footer text specified in a footer field declaration (see section 9.11.3). Which footer field declaration is used is specified by the presentation: use-footer-name attribute of the draw page where the field occurs. If the field is contained in a presentation drawing shape inside a master page (see section 9.6.1), then the presentation: usefooter-name attribute of the drawing page for which the drawing shape is displayed is used (see section 9.1.4). 
This field is mainly used inside master pages. Since its value may differ for the individual drawing pages that make use of a master page, the current field value is not available.

\subsubsection{Header field declaration}

The <presentation:header-decl> element specifies the text of an header field. See section 9.10 .1 for details.

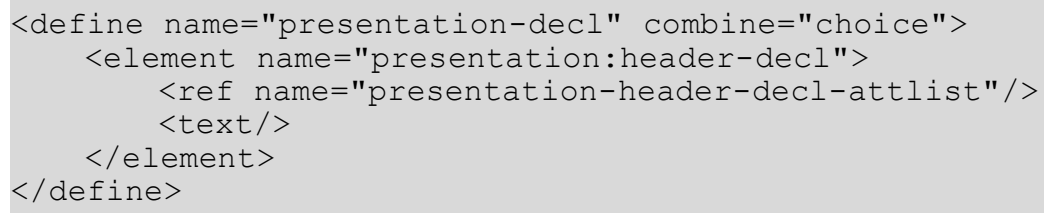

\section{Name}

The presentation: name attribute specifies the name of the header declaration. 


\section{Date and time formatting style}

The date style referenced by the style:data-style-name attribute is used to format the date and time of the presentation: date-time fields if the field is not fixed.

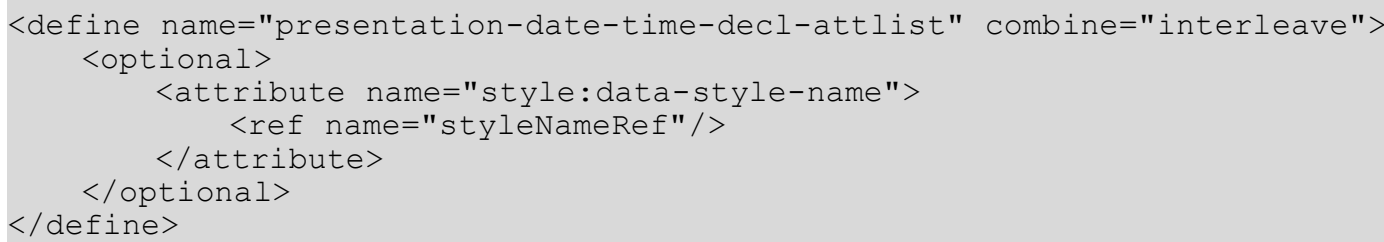

\section{$</ o p t i o n a l>$}

\subsubsection{Presentation Settings}

The settings for a presentation are stored in the element $\langle$ presentation: settings $>$ inside an <office:presentation> element. These settings affect the behavior if the document is displayed in a presentation.

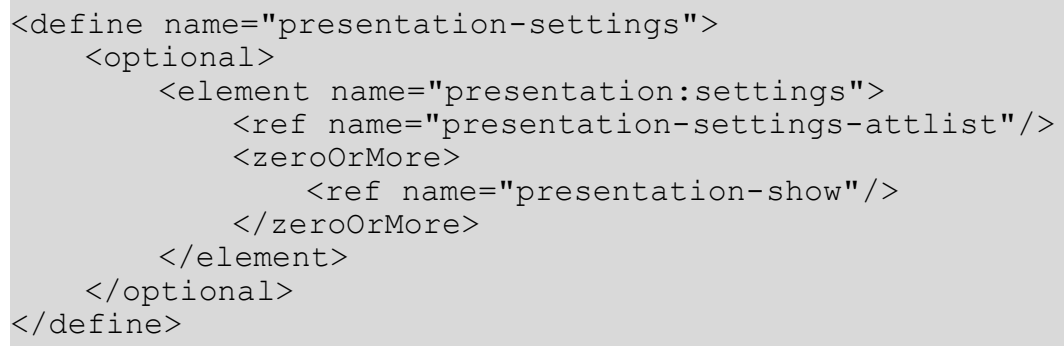

The attributes that may be associated with the <presentation: settings $>$ element are:

- $\quad$ Start page

- Show

- Full screen

- Endless

- Pause

- Show logo

- Force manual

- Mouse visible

- Mouse as pen

- Start with navigator

- Animation

- Transition on click

- Stay on top 


\section{Start page}

The attribute presentation:start-page specifies the name of the page on which the presentation starts. If this attribute is set, it overrides the presentation: show attribute.

8315

8316

8317

8318

8319

8320

8321

\section{Show}

The attribute presentation: show specifies the name of a show definition (see section 9.11.6) that is used for the presentation. If the presentation: start-page attribute is set, it overrides the value of this attribute.

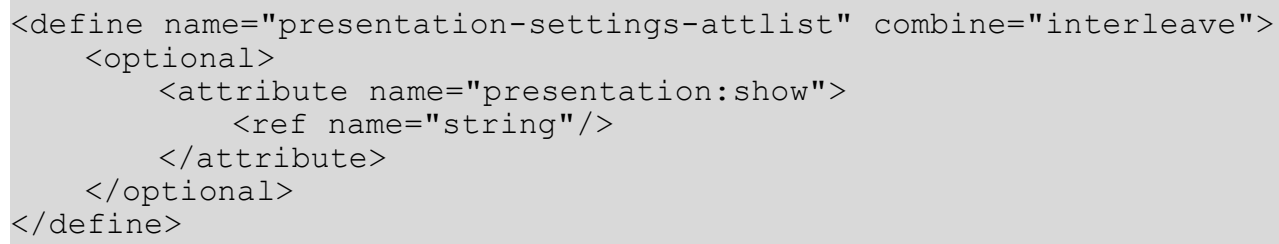

\section{Full Screen}

The attribute presentation:full-screen determines whether the presentation is displayed in full screen mode or in a window.

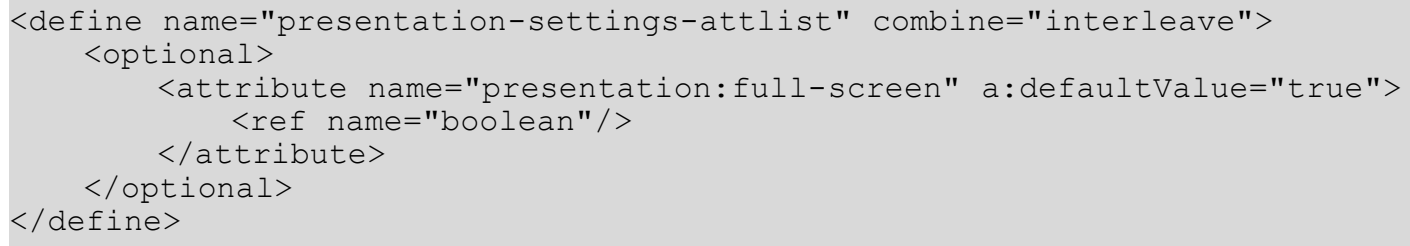

\section{Endless}

The attribute presentation: endless switches indefinite repetition of a presentation on and off.

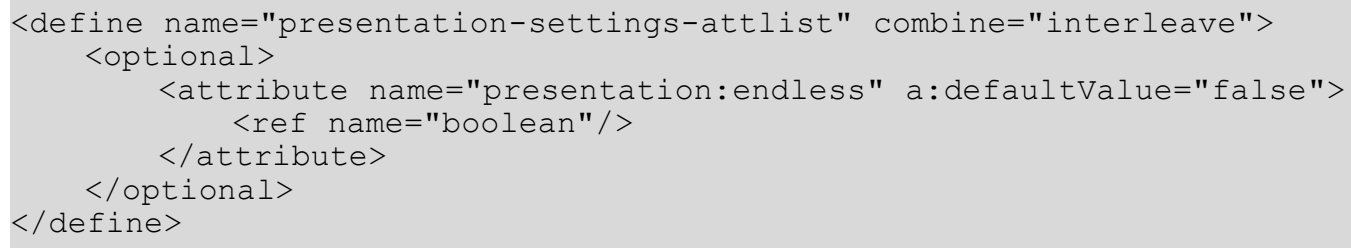

\section{Pause}

If a presentation is repeated indefinitely, the attribute presentation:pause specifies a time duration for displaying a pause screen before the presentation is played again. If this attribute is not set or has a value of 0 , a pause screen is not displayed in endless mode. The value of this attribute must conform to the time period format described in §3.2.6 of [xmlschema-2]. 


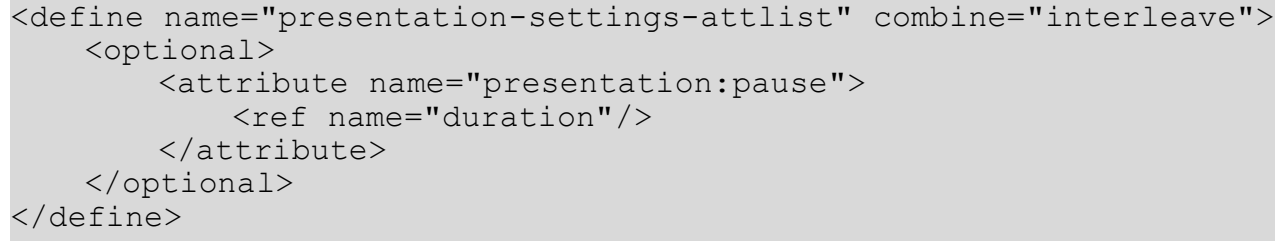

\section{Show Logo}

The attribute presentation:show-logo specifies whether or not a presentation application shows its logo on the pause screen.

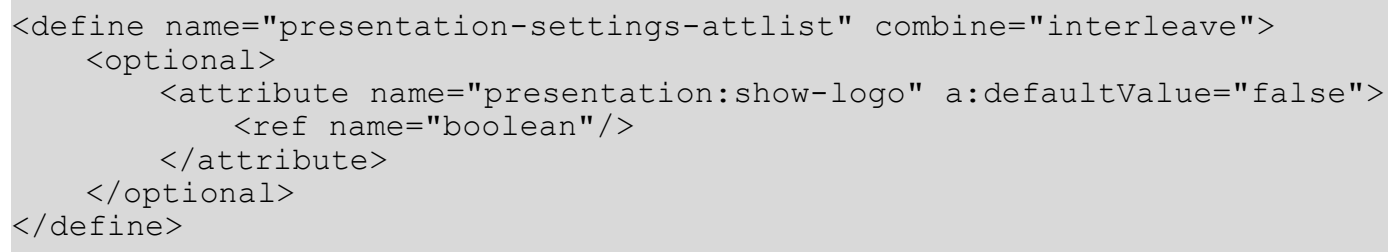

\section{Force Manual}

If set, the attribute presentation:force-manual overrides all presentation:transition-type properties that are specified within a presentation page (see section 15.36.1) and sets it to manual.

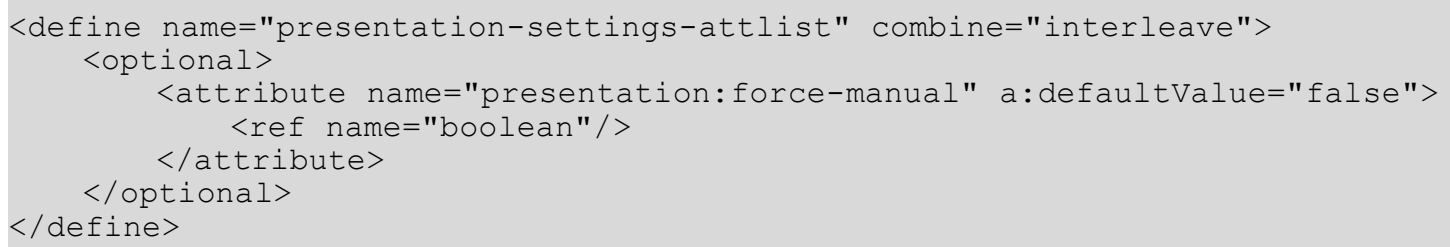

\section{Mouse Visible}

The attribute presentation:mouse-visible specifies whether or not the mouse pointer is visible during a presentation.

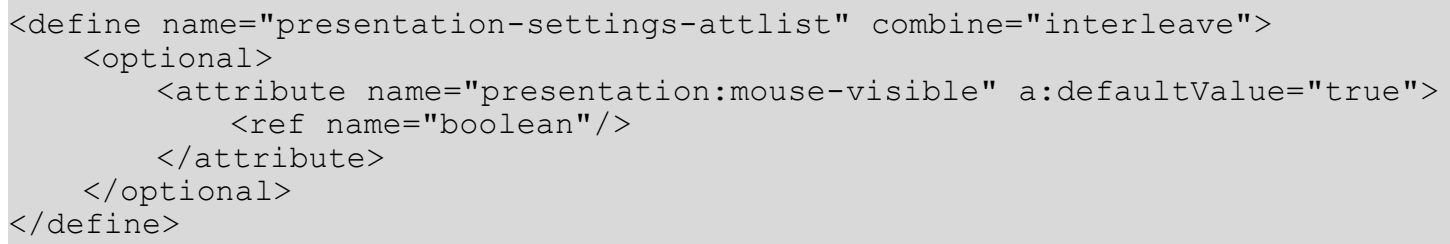

\section{Mouse As Pen}

The attribute presentation:mouse-as-pen specifies if the mouse pointer is displayed as a pen or a pointer. If the mouse is displayed as a pen the user can draw sketches on the pages during a presentation.

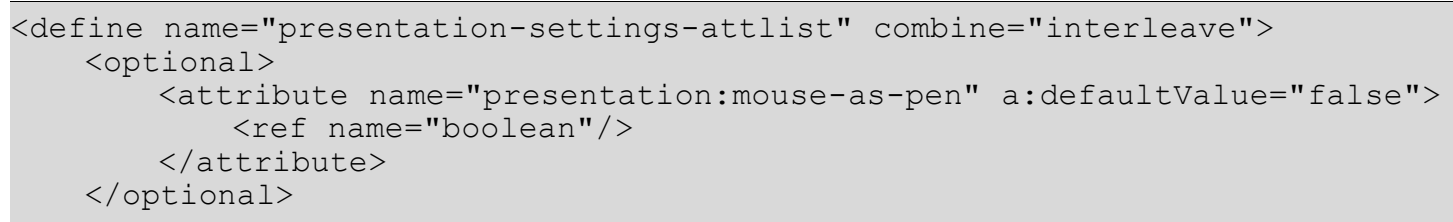




\section{Start With Navigator}

The attribute presentation:start-with-navigator specifies whether or not the navigator window is initially displayed during a presentation.

\section{Animations}

The attribute presentation:animations enables or disables the playback of bitmap animations during a presentation.
8386

8387

8388

8389

8390

8391

8392

8393

8394

8395

\section{Transition On Click}

The attribute presentation:transition-on-click enables or disables a manual transition by a mouse click on the slide during a presentation.

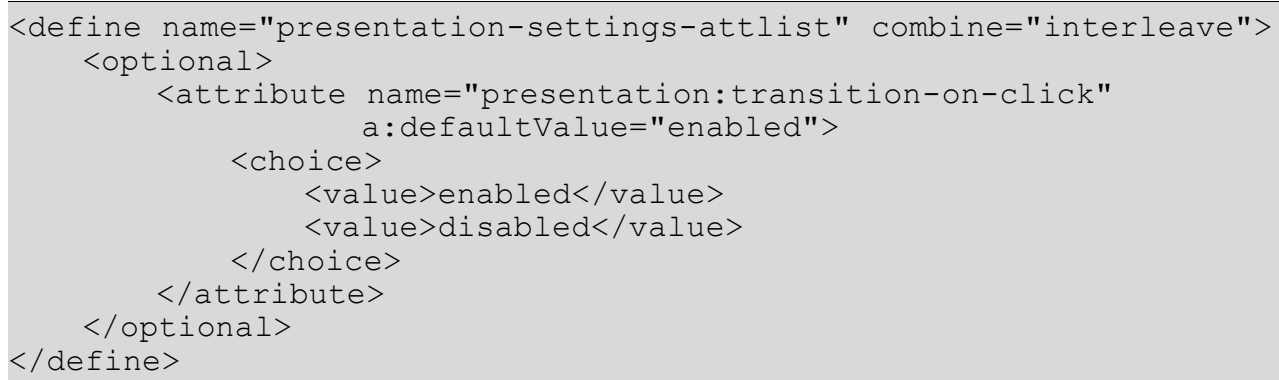

\section{Stay On Top}

If the attribute presentation:stay-on-top is set to true, the presentation window is displayed on top of other windows during a presentation.

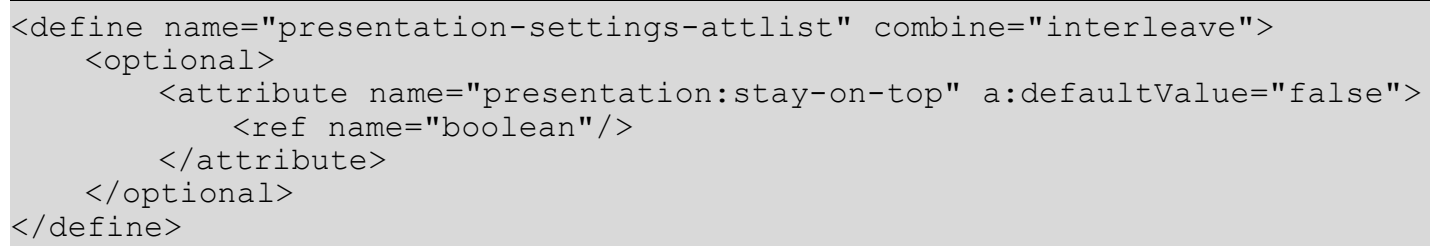




\subsubsection{Show Definitions}

A presentation document can contain one or more $<$ presentation: show $>$ elements. A $<$ presentation: show $>$ element customizes the order in which the pages are displayed during a presentation. It can be also used to omit pages from the presentation or to repeat pages during the presentation.

This element is optional.

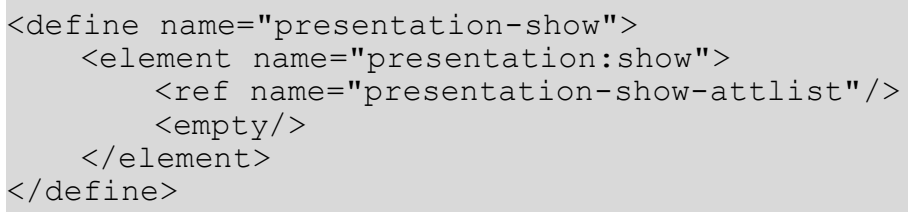

The attributes that may be associated with the $\langle$ presentation: show $>$ element are:

- Name

- Pages

\section{Name}

The attribute presentation: name uniquely identifies a $<$ presentation: show $>$ element.

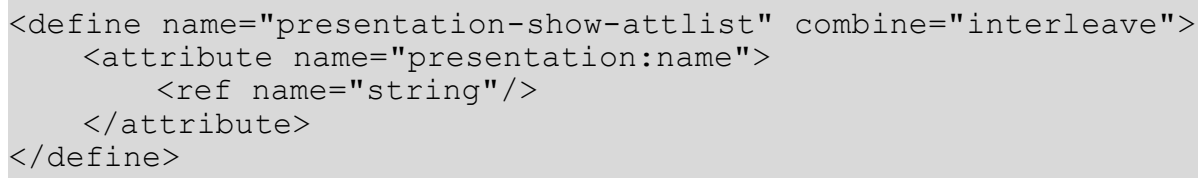

\section{Pages}

The attribute presentation:pages contains a comma separated list of page names. The pages are displayed in the order in which they are listed during a presentation that uses this show. Pages can be included more than once.

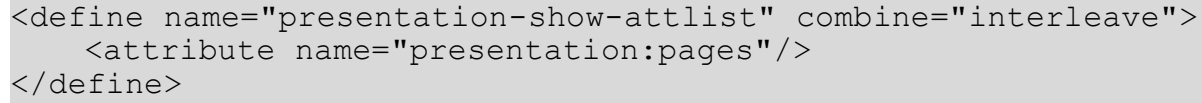




\section{Chart Content}

This chapter describes the XML representation of chart content. It contains the following sections:

- Introduction to Chart Documents

- Chart

- Title, Subtitle and Footer

- Legend

- Plot Area

- Wall

- Floor

- Axis

- Series

- Categories

- Data Point

- Mean Value

- Error Indicator

- Regression Curves

\subsection{Introduction to Chart Documents}

Chart documents are always contained within other XML documents. There are two types of chart container documents:

- Documents that do not provide data for the chart: The chart data is contained in a $<$ table: table> element inside the <chart: chart> element.

- Documents that provide data for the chart: The chart data may be contained in a $<$ table:table $>$ element in the parent document, for example, in a spreadsheet or text document.

The chart data is specified by the <chart:plot-area> element's table:cell-rangeaddress attribute. The <chart: plot-area> element represents the visualization container of all data series in the chart.

\subsection{Chart}

The <chart: chart $>$ element represents an entire chart, including titles, a legend, and the graphical object that visualizes the underlying data called the plot area. The data underlying the chart is represented by a table element. This element may also exist for embedded charts that get the data from the container document. In this case the chart can be rendered without getting the data from the container document. 


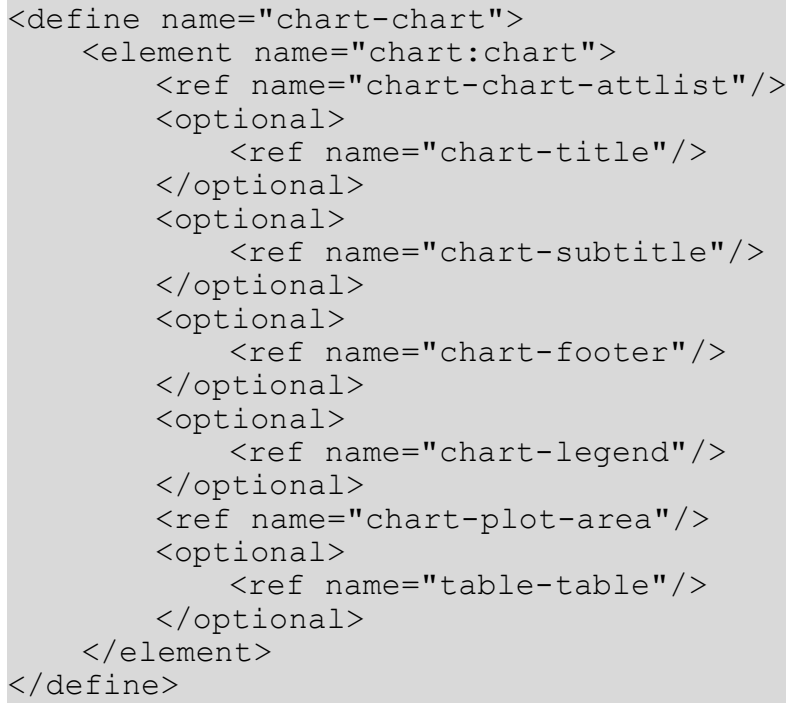

\section{Class}

The chart:class attribute specifies the chart type. The chart type is represented by a namespaced token, meaning an identifier prefixed by an XML namespace prefix, just like any attribute or element name in this specification. This specification defines a number of chart types in the chart namespace (URN: urn:oasis:names:tc:opendocument:xmlns:chart:1.0). Additional chart types may be supported by using a different namespace.

The chart type may be specified more precisely with formatting properties that may be attached to chart styles. For example, a 3D bar chart with horizontal bars is specified by setting the class attribute to chart: bar and an by adding the properties for three dimensional and horizontal arrangement in the corresponding style.

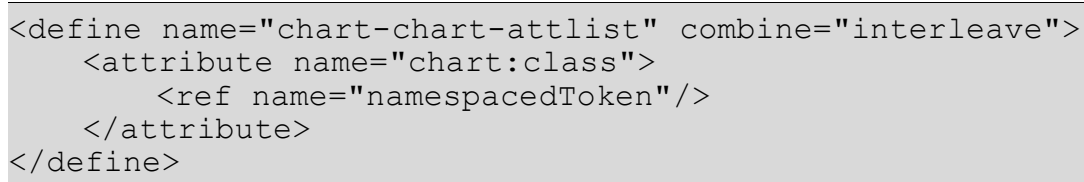

The pre-defined chart types are:

- line - the data points of each data series are connected through lines.

- area - the area below a data series is filled, and additional data series are stacked.

- circle - a circular chart is segmented according to the relative weights of the data points.

- ring - each data series is represented as a concentric rings, with each ring rendered as if it was part of a circle chart of the series.

- scatter - a pair of data series is used to determine $\mathrm{x}$ and $\mathrm{y}$ positions for each data point.

- radar - a radial plot of the data points, where the value of each point determines the distance from the chart origin. The data points of a series are connected, thus forming a closed line around the center.

- bar - each data point is depicted by a bar whose length is proportional to the data value.

- $\quad$ stock - four data series are interpreted as opening, minimum, maximum and closing stock values. 
- bubble - the first two of three data series are interpreted a positions as in a scatter chart, where the area of each data point is sized relative to the value in the third data series.

- surface - the data points are interpreted as tabular data, where each value defines a 'height' at a specific grid location. The graph may visualize these using colors for height intervals, creating color bands similar to geographical maps.

- gantt - a pair of data series is used to determine the start and end positions for horizontal bars

Example: The following table shows examples for the pre-defined chart types. Those charts that use one or two data series use two data series with the values $1 ; 2 ; 3 ; 4$ and $1 ; 4 ; 9 ; 16$ and the labels $a ; b ; c ; d$. Those chart types that use more than two data series (stock and bubble) use the data series 1;2;3;4 and multiples thereof. The radar chart uses two data series with five data points.

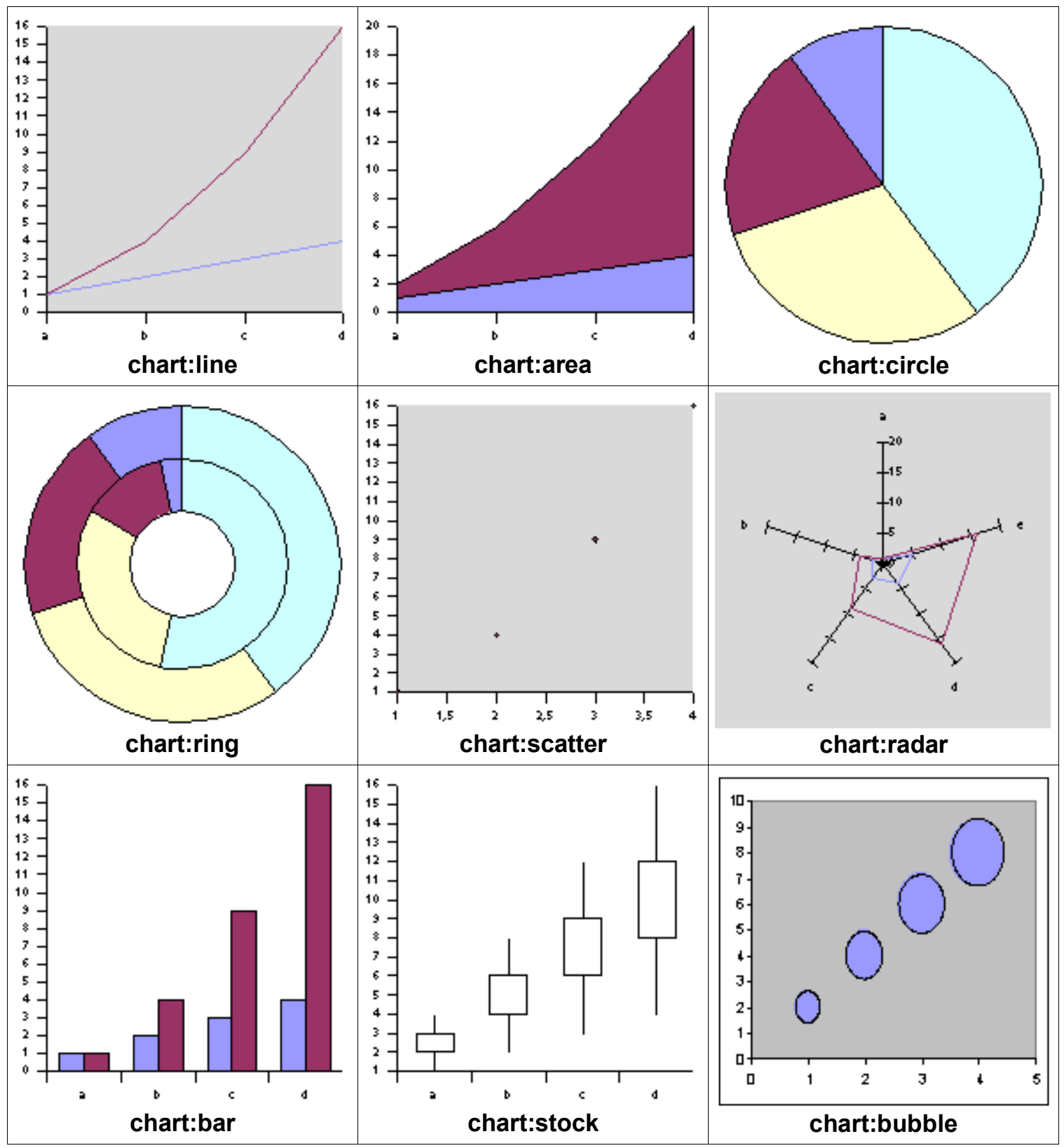




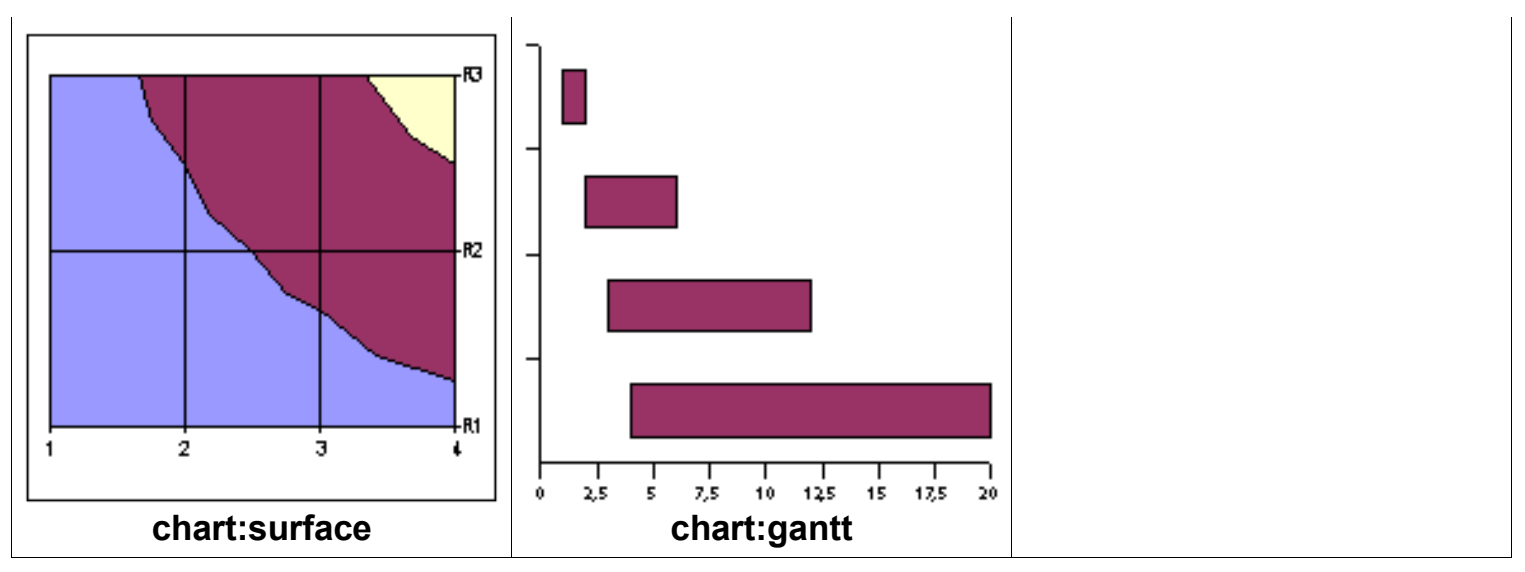

\section{Size}

The svg:width and svg: height (see section 9.2.15) attributes define the extent of the entire chart. If they are omitted, the size of the chart is determined by the size of the window in which the chart is displayed.

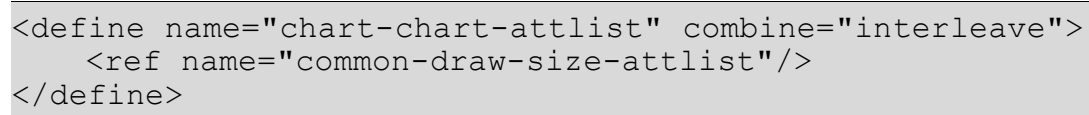

\section{Column and Row Mapping}

The chart:column-mapping and chart:row-mapping attributes contain, if provided, a list of indexes of series. The numbers define a reordering of data that comes from a container document that provides the data for the chart. The numbering begins with 1 . A list of ascending numbers beginning with 1 has no effect. To exchange two series, their numbers must be exchanged in the list. For example, 13324 exchanges the second and the third series.

The chart:column-mapping and chart:row-mapping attributes must not be used simultaneously.

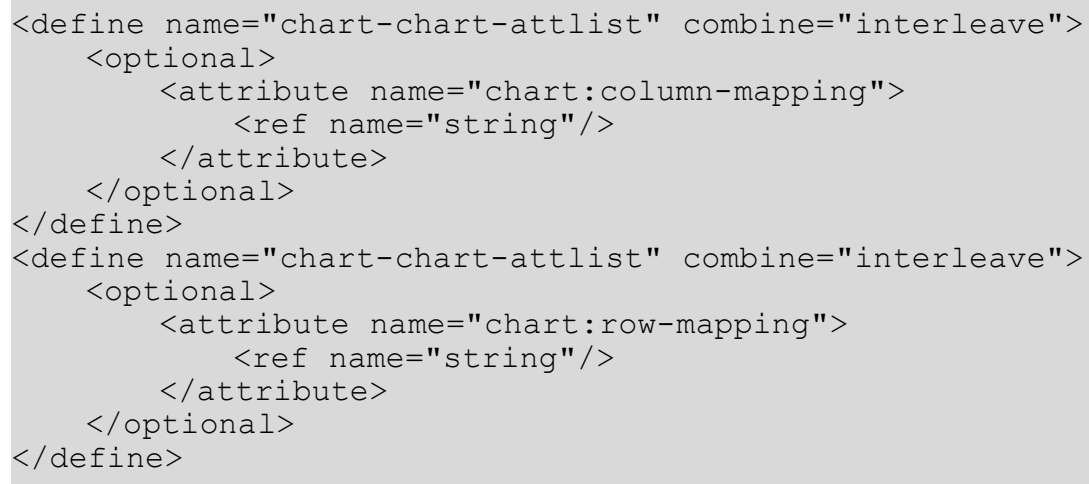

\section{Style Name}

The chart:style-name attribute references a chart style. See section 14.16 for details.

Within the style applied to the <chart: chart> element, fill properties (described in section 15.14) and the stroke properties (described in section 15.13) as well as the scale text property described in section 15.29.1 can be used. 


\subsection{Title, Subtitle and Footer}

\subsubsection{Title}

The <chart:title $>$ element represents a main title object in a chart document. This element can contain fixed text or it can contain a $<$ table: cell-address $>$ element pointing to the text that should be displayed as the title. This element can also be a sub-element of chart:axis, see section 10.8. In this case the title is displayed beside the axis object.

\section{Position and Size}

The common positioning attributes for drawing objects can be used on <chart: title> elements.

\section{Style Name}

The chart:style-name attribute specifies a chart style for the <chart:title> element. Within the referenced style, fill and stroke properties may be used. They are applied to the surrounding title box. See sections 15.14 and 15.13 for more information. In addition to this, text properties may be used. They are applied to the title text itself. See section 15.4 . 


\subsubsection{Subtitle}

The <chart: subtitle > element represents a subtitle which can be used for additional title information in a chart.

The structure of the <chart: subtitle> element is the same as that of the <chart:title> element. The attributes that may be associated with the <chart: subtitle $>$ element are the same as those that may be associated with the <chart:title> element. See section 10.3.1 for more information.

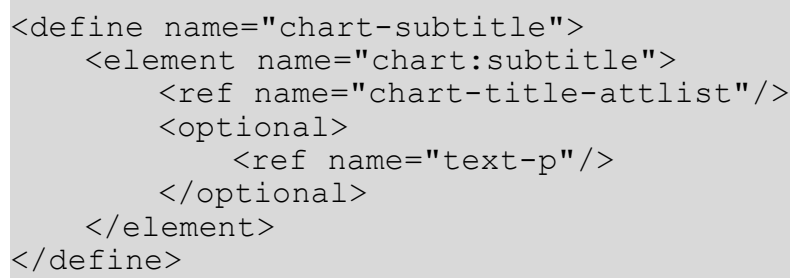

\subsubsection{Footer}

The <chart: footer $>$ element represents a footer below the chart's plot area.

The structure of the subtitle element is the same as that of the <chart:title $>$ title element. See section 10.3.1 for more information.

\subsection{Legend}

The <chart: legend $>$ element determines whether or not a legend is displayed in the chart. The legend's position may be specified either as a relative or as an absolute position. The size of the legend is calculated automatically and therefore cannot be set as attribute.

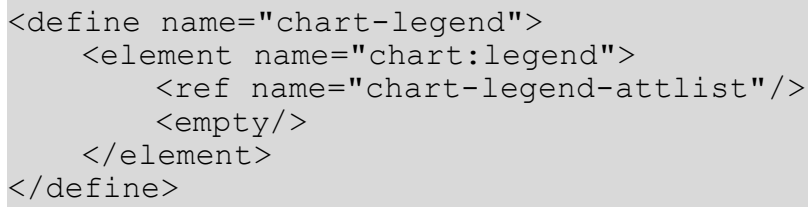

\section{Legend Placement}

The legend can be placed automatically, next to the plot area, or in one of the corners. This placement is determined by the chart: legend-position attribute, which may have the values start, end, top, bottom for legend positions next to the plot area and top-start, bottom-start, top-end or bottom-end for legend positions in the corners. If the legend is placed next to the plot area, in any of the four directions start, end, top bottom, an additional 
alignment attribute chart: legend-align determines which border (start, end) or axis (center) of the legend and the plot area are to be aligned.

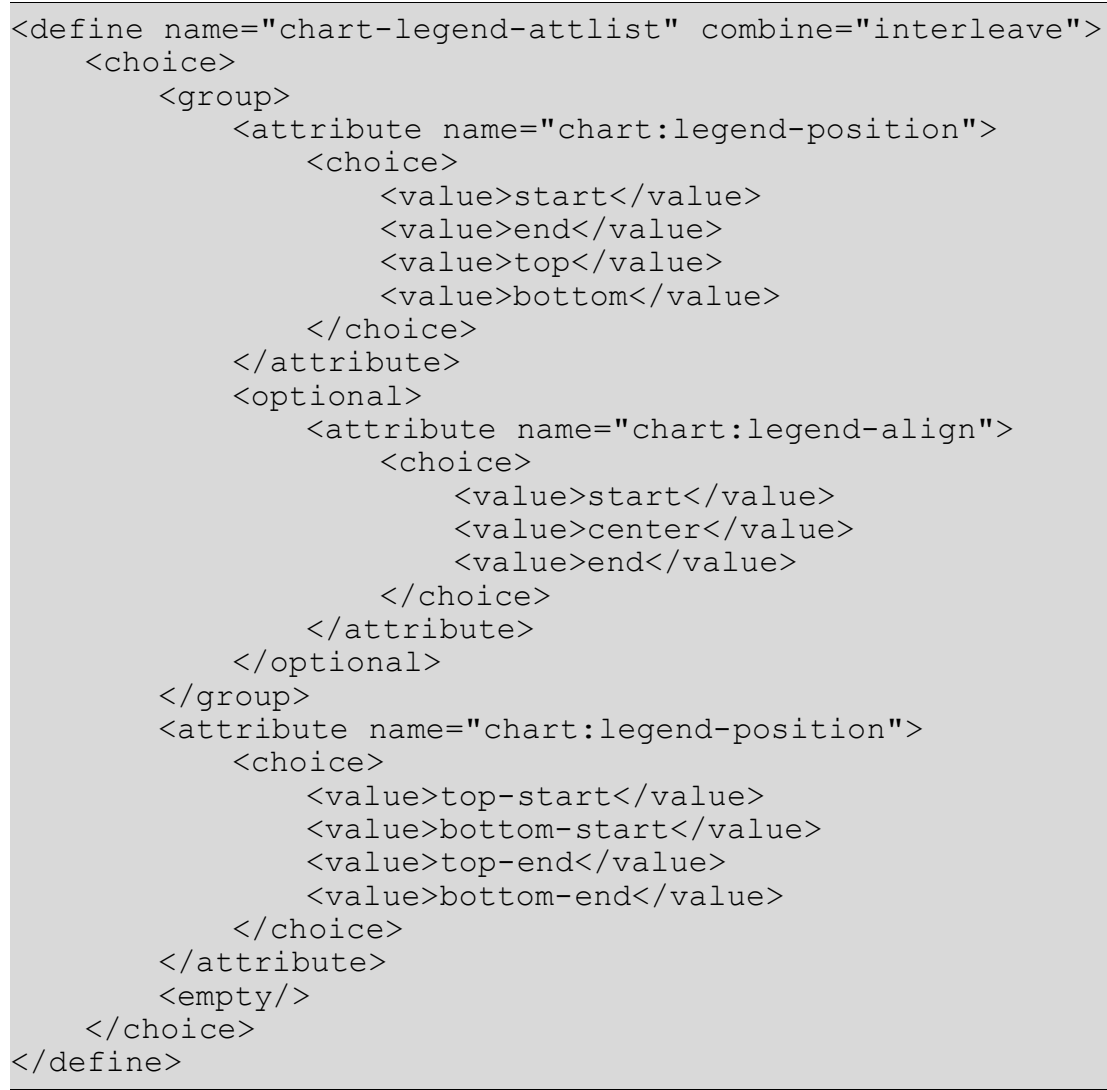

Example: If chart: legend-position="right", the legend will be positioned to the right of the chart's plot area. The chart: legend-align values of start, center, and end will yield legend positions as depicted by the green, red, and blue boxes, respectively.

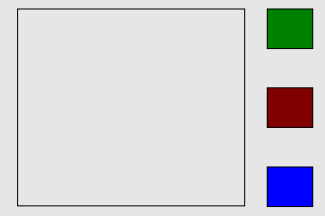

The legend position can also be given in absolute coordinates, as with any drawing object. If both a drawing position and legend placement options are available, the legend placement takes precedence and the position should reflect the automatic placement.

<define name="chart-legend-attlist" combine="interleave">

<ref name="common-draw-position-attlist"/>

\section{Legend Expansion}

The legend needs to be expanded to accommodate additional legend items. The style: legend-expansion attribute determines in which direction the legend expands. Legend expansion of wide and high causes the legend to be expanded horizontally and vertically. An expansion balanced causes expansion into both directions. An expansion value 


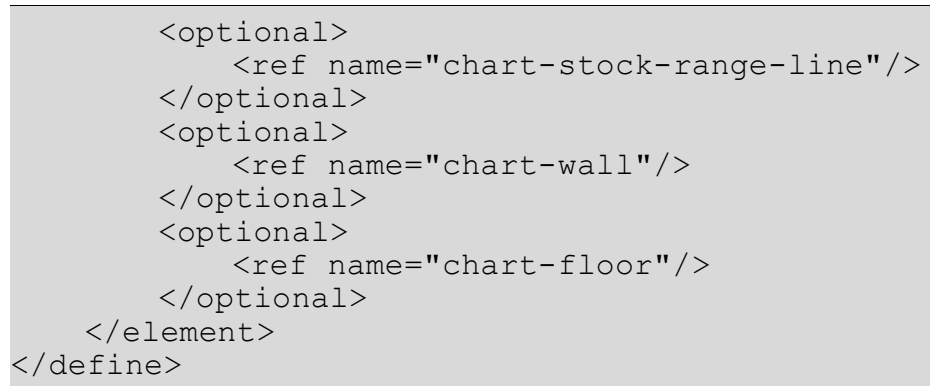

\section{Plot Area Positioning}

The plot area's position and size are determined the common positioning and sizing attributes for drawing objects. If the position and size attributes are not specified, the values are calculated by the render application.

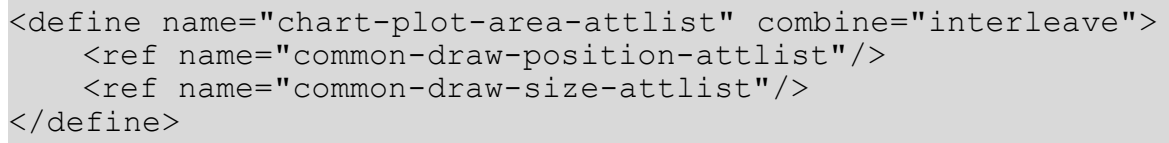

\section{Plot Area Style}

The chart:style-name attribute that is set for the <chart:plot-area> element is used for all data elements contained inside the plot area, unless extra styles are specified in one of those sub elements. These data elements can be <chart: series> and <chart: data-point> elements.

If the chart is three-dimensional, 3D scene properties may be applied to the plot area. See the section 15.22 - 15.26 for more information.

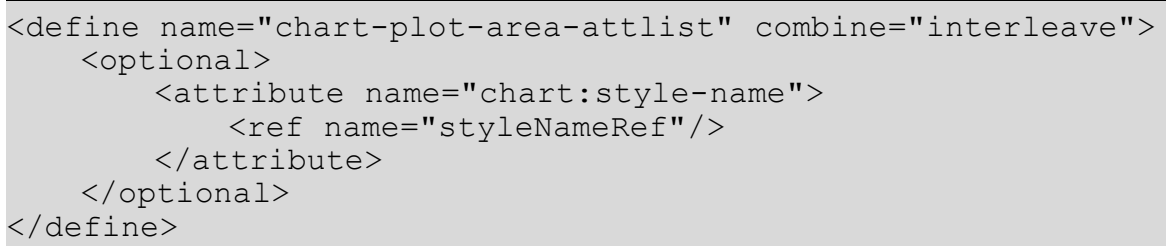

\section{Plot Area Data Attributes}

If a chart is embedded in a document that provides the data for the chart, the table: cellrange-address attribute reflects the ranges from which all the data for the chart comes. The range given here is interpreted by the chart as consecutive series.

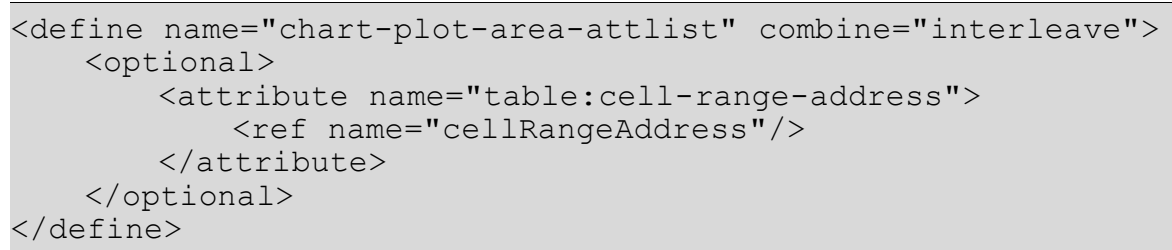

If the first row or column, or both contains labels, this is stated by the chart: data-sourcehas-labels attribute.

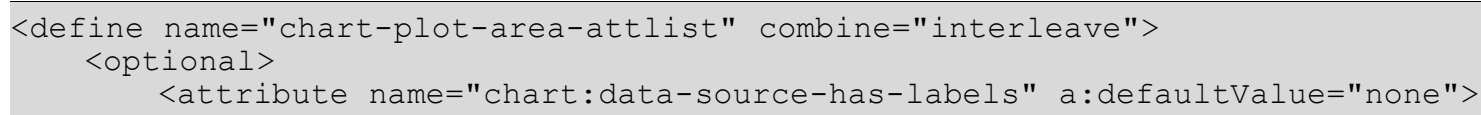




\section{Width}

The svg:width attributes specifies the width of the wall for three-dimensional charts.

\section{$</$ define $>$}

The chart:series-source formatting property specified in section 15.34 .1 determines whether the data table contains the data series in column-wise or row-wise fashion.

\subsubsection{D Plot Area}

The plot area may be displayed as an 3D scene as specified in section 9.4.1. All 3D attributes that can be applied to the $\langle\mathrm{dr} 3 \mathrm{~d}$ : scene $>$ element can be applied to the <chart:plot-area $>$ element, including the $\mathrm{dr} 3 \mathrm{~d}$ :transform attribute. It represents the rotation of a chart scene, that is the three-dimensional plot area. See section 9.4.1 for more information. In addition to this, the <chart: plot-area $>$ element may contain a $<\mathrm{dr} 3 \mathrm{~d}$ : light $>$ element as specified in section 9.4.2.

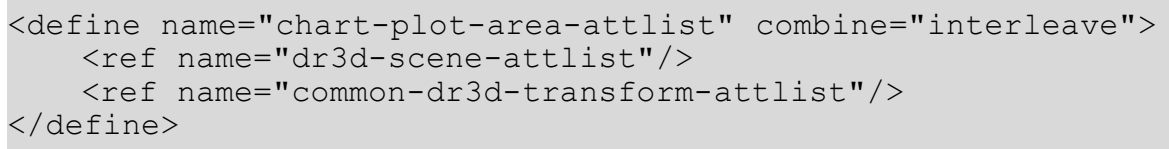

\subsection{Wall}

The <chart: wall > element can be contained in the <chart: plot-area $>$ element. It specifies a chart's wall. For two-dimensional charts, the wall spans the entire plot area. For threedimensional charts, the wall usually consists of two perpendicular rectangles.

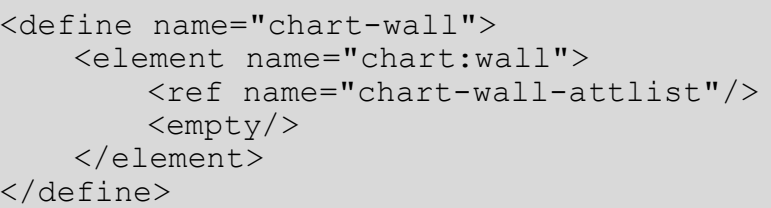

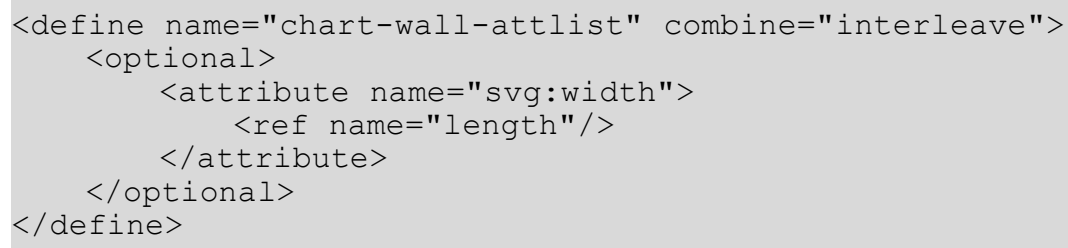

\section{Style}

The <chart: wall> element may have a chart: style-name attribute to specify further styling information. They style may contain fill and stroke properties. See sections 15.14 and 15.13 for more information. 


\section{Style}

The <chart:floor> element may have a chart:style-name attribute to specify further styling information. Fill and stroke properties can be applied to a floor. See sections 15.14 and 15.13 for more information.

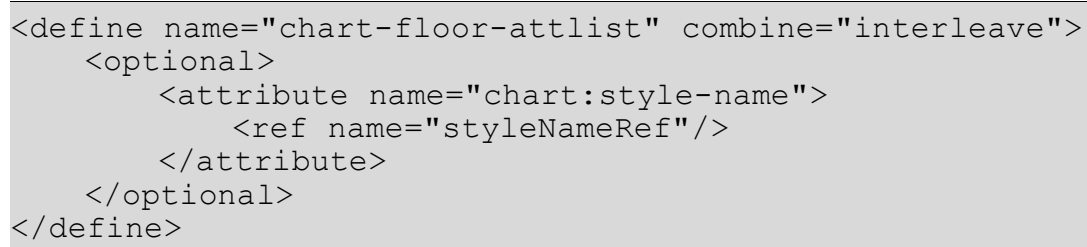

\subsection{Axis}

The <chart:axis > element mainly contains style information, in particular scaling information. Chart data is usually structured as follows:

- Several data series each consisting of a name, for example, the name of a company.

- Values, for example, the yield of the company in different years.

- One value in each series belongs to a category, for example, the year. 


\section{$</$ element $>$}

<element name="chart:axis">

<ref name="chart-axis-attlist"/>

<optional>

<ref name="chart-title"/>

$<$ /optional $>$

<optional>

<ref name="chart-categories"/>

$<$ /optional>

$<$ zeroorMore $>$

<ref name="chart-grid"/>

$</$ zeroorMore $>$

$</$ define $>$

\section{Dimension}

The chart:dimension attribute specifies along which physical axis on the chart the values of the current axis are displayed.

A chart may contain more than one axis with the same dimension. For example, it may have two axes with dimension y. Data series may be attached to either axis. This way, data may be grouped for different scaling. To attach a specific axis to a data series, the axis has to be referenced by the <chart:series> element's chart:axis-name attribute. If an axis is not references by a data series, it becomes a copy of an existing axis with the same dimension.

The position of an axis in a chart is determined by the rendering application and depends on the chart type. In a chart with horizontal bars, the rendering application usually paints the axis with dimension $x$ on the bottom of the plot area. If there are two axes with dimension $y$, a rendering application might paint the second axis at the top of the plot area.

8704

8705

8706

8707

8708

8709

8710

8711

8712

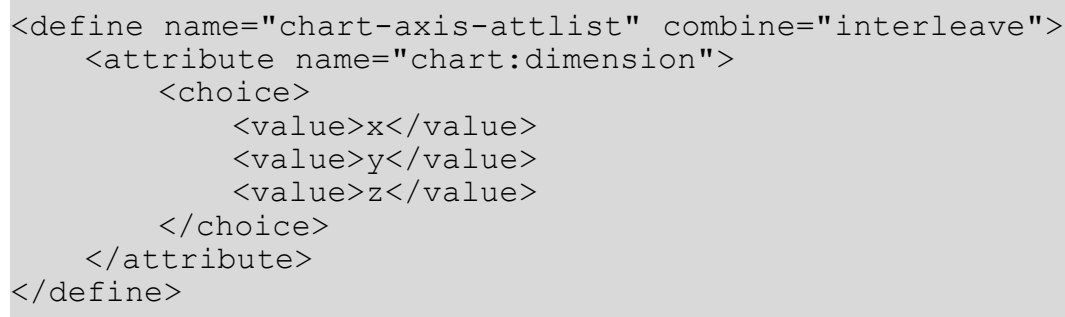

\section{Name}

The chart: name attribute can be used to assign a name to this axis, so it can be referenced from e.g. a data series.

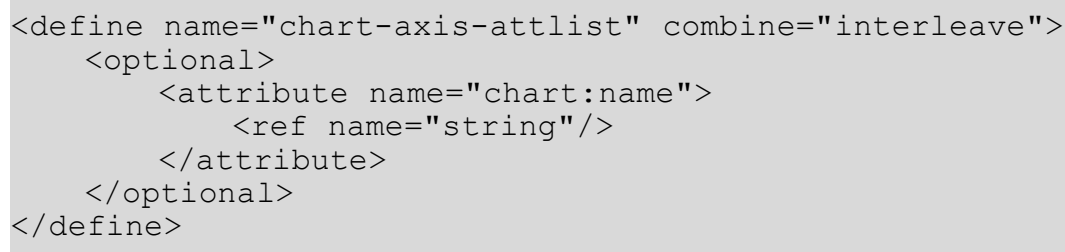

\section{Style}

A chart:style-name attribute can be associated with an axis. Stroke properties can be applied to axes; see section 15.13. These properties affect all lines of the axis object. Text properties can also be applied to axes; see section 15.4. These properties affect the appearance of all text objects. The axis properties described in section 15.31 can also be used. 
The chart style that is referenced by the chart: style-name attribute may specify a data style that is used to format the axis' labels. See section 14.1 for details.

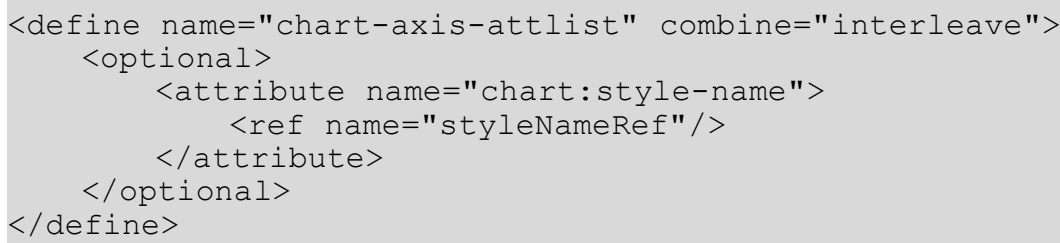

\section{$</ o p t i o n a l>$}

\section{Example: Bar chart}

In this example, there are two axes with dimension y. One of these axes has the name primary-value. A data series has been attached to that named axis. There is no data attached to the second axis, therefore an axis name has not been specified, and the axis is just a copy of the first one.

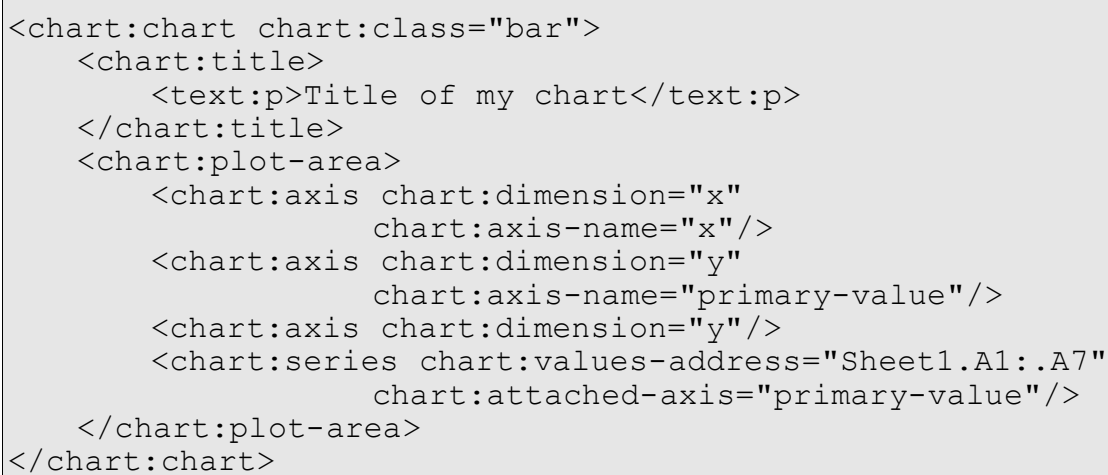

\subsubsection{Grid}

The <chart: grid> element can be contained in a <chart:axis> element. It adds a grids to the axis.

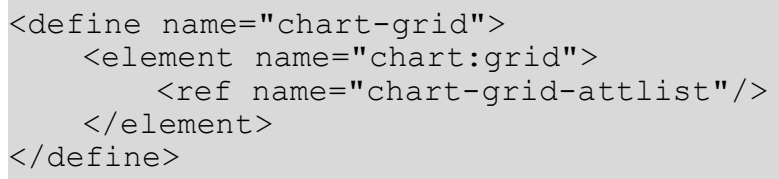

\section{Class}

The chart:class attribute specifies whether major or minor tickmarks are used. If a major grid is applied to an axis, the major tickmarks are extended to gridlines. If a grid is minor, any minor tickmarks assigned to the axis are used.

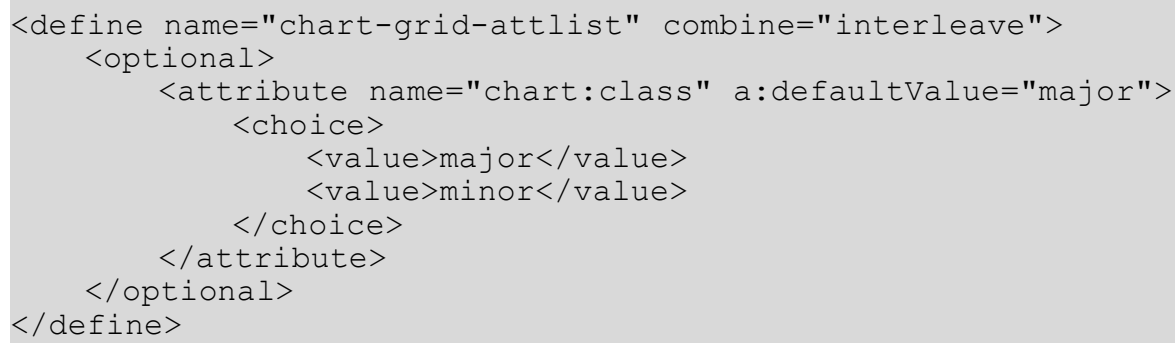




\section{Style Name}

The <chart: grid> element may have a chart: style-name attribute to specify further styling information. Stroke properties can be applied to grids, which affect the lines of the grid. See section 15.13 for information on these stroke properties.

\subsection{Series}

The <chart:series $>$ element represents a data series in a chart. If the chart requires more input data like scatter and bubble charts, <chart: domain $>$ sub-elements must be defined that mainly contain the cell-range-address of the corresponding data.

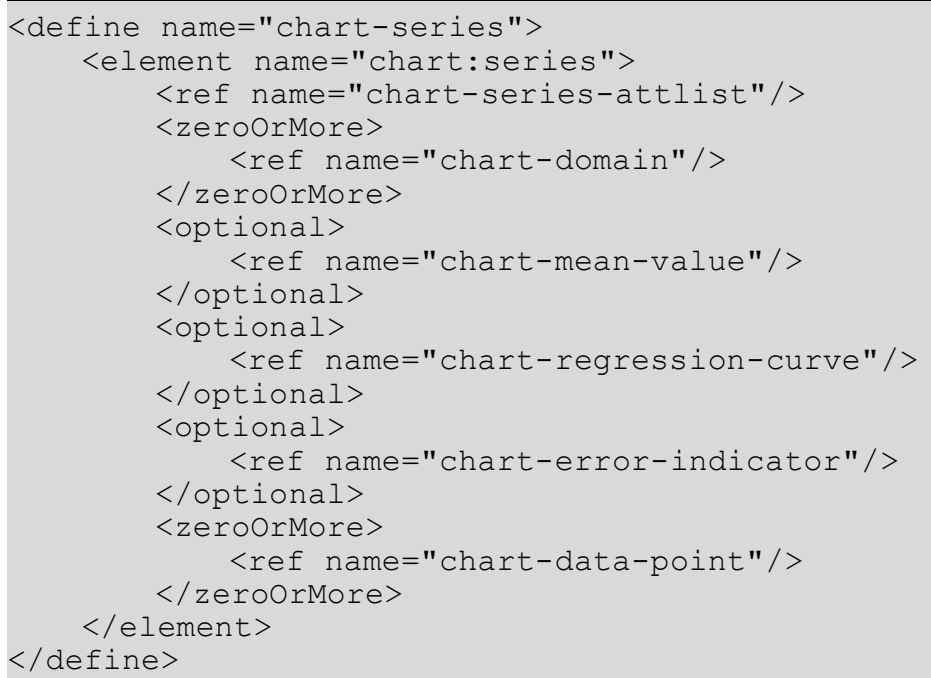

$</$ element $>$

\section{Cell Range}

The chart:values-cell-range-address attribute allows a range to be specified that contains the values that should be visualized by this data series.

\section{$</$ define $>$}

The chart:label-cell-address attribute allows a name to be provided for the series.

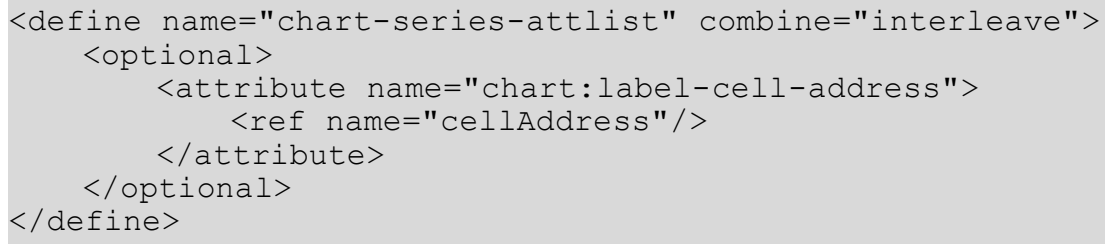




\section{Class}

The chart:class attribute can be used to assign a chart type to be used for rendering the data of this <chart: series> element. A chart: class attribute for a <chart: series $>$ element overrides the chart:class attribute for the entire chart. This allows the creation of charts with multiple sub-charts, e.g. a bar chart with one or more data series rendered as lines. For more information on the available chart classes, see section 10.2.

\section{Attached Axis}

The chart:attached-axis attribute can be used to assign the data series to a $<$ chart:axis> element.

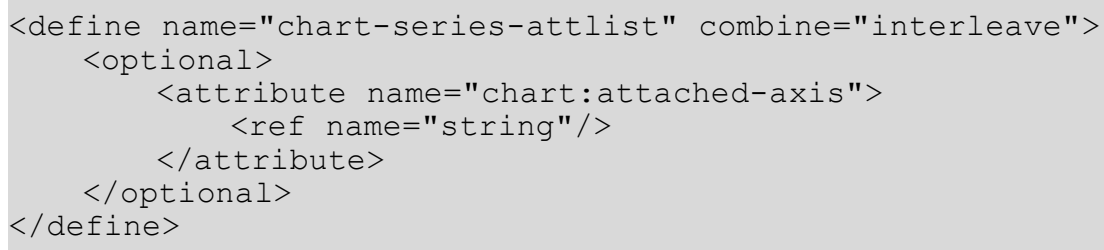

\section{Style Name}

Styling attributes for the data series can be assigned through the chart:style-name attribute. Fill and stroke properties may be applied for <chart: series> element, see sections 15.14 and 15.13 for information. Text properties can also be applied to the descriptive text underneath the series, see section 15.4 for information.

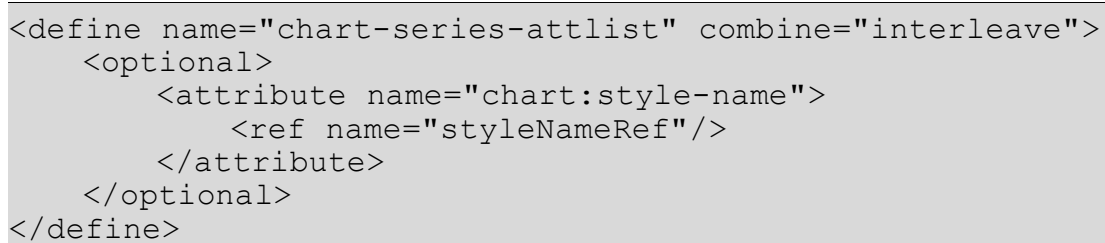

\subsubsection{Domain}

For scatter and bubble charts, one ore more <chart: domain> elements must be specified for the <chart: series > elements.

For scatter charts, one <chart: domain> element is required. Its cell-range-address attribute references the $\mathrm{x}$ coordinate values for the scatter chart.

For bubble charts, two <chart: domain> elements are required. Their cell-range-address attributes reference the $\mathrm{x}$ and $\mathrm{y}$ coordinate values for the bubble chart

For both chart types, there must be at least one <chart: series $>$ element with the necessary number of <chart: domain> sub-elements. All other <chart: series $>$ elements can omit these. In this case, the first domain that is specified is used. 


\section{Style}

The chart:style-name attribute referenced a chart style. Fill and stroke properties can be applied to each data point object, see sections 15.14 and 15.13. Text properties can also be applied to the descriptive text located underneath the data points, see section 15.4.

8842

\section{Style Name}

The chart:style-name attribute references a chart style that contains the formatting properties for the mean-value line.

\section{$</$ define $>$}

\subsection{Error Indicator}

The formatting properties of error-indicators are stored in the <chart:error-indicator $>$ elements which may be part of a series.

8856

8857

8858

8859

8860

8861

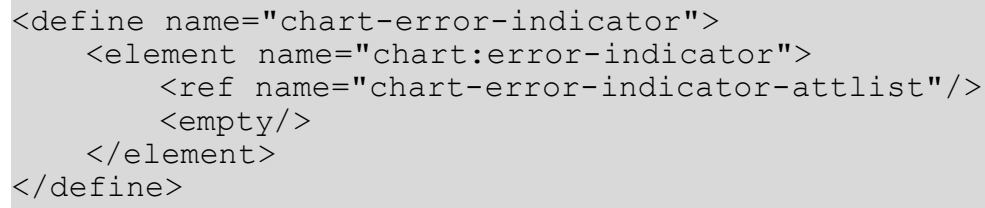




\section{Style Name}

The chart:style-name attribute references a chart style that contains the formatting properties for the error indicator.

8862

8863

8864

8865

8866

8867

8868

8869

8870

8871

8872

8873

8874

\subsubsection{Stock Chart Markers}

The properties of a stock chart, i.e. the different colors for filling the candlestick-bars or the linestyles of the lines pointing to the high and low values (the range-line), are stored in separate elements.

The candlestick-bars for stocks that have a higher close-value than open-value take their formatting from the <chart: stock-gain-marker> element's properties, whereas stocks which close value is lower than the open-value, use the properties stored in <chart: stock-

loss-marker>.

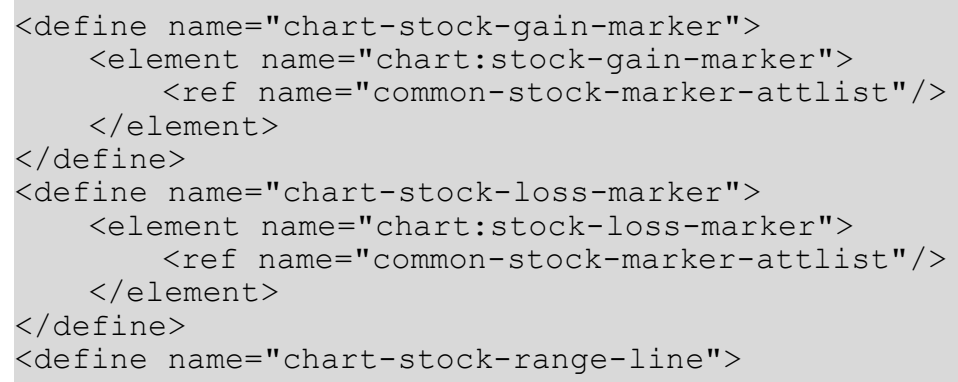


<element name="chart:stock-range-line"> $</$ element $>$

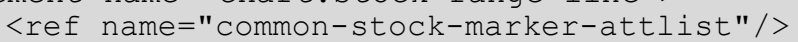

\section{Style Name}

The chart:style-name attribute referenced a chart style that contains the formatting properties for stock markers.

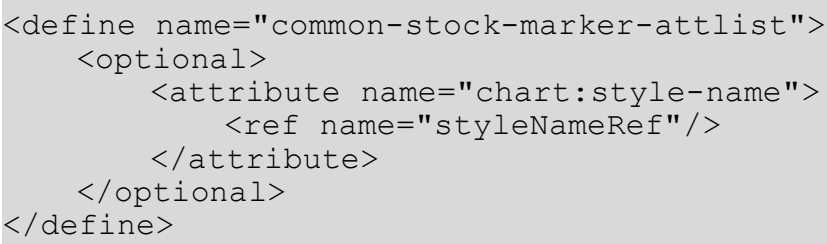




\section{Form Content}

A form is a container for user interface controls which a user interacts with. For example, buttons, text boxes, check boxes, and drop-down lists are user interface controls that can be contained in a form. In the XML file format, the following basic rules apply to user interface controls and forms:

- All controls must be located in a form.

- All controls that are not hidden have to be associated with an absolute or relative position. These visual aspects of the control are represented by drawing shapes that contain a reference to the control. See section 9.2.12 for details.

- Forms may be nested.

- Forms are not connected with the text flow and layout of a document. This does not apply to controls.

- Forms can be data-aware. The controls reflect the content of a database.

Forms define rules for the following form behavior:

- Submitting the form, which is similar to [HTML4].

Note: Form submission is only supported for non nested forms that contain only controls that can be converted to HTML.

- Connecting to a data source. When this happens, the controls in a form become data-aware.

- Submitting and binding according to the [XForms] data model.

Forms are contained in the <office: forms > section of an XML document. This element may contain an arbitrary sequence of $\langle$ form: form $>$ or $\langle$ xforms : model $>$ elements. Note that controls are always declared inside a <form: form $>$ element, while an <xforms:model $>$ element contains only the XForms data model. Thus, the <office: forms $>$ element may contain only <form: form> elements but no <xforms:model $>$ element, while an $<$ xforms:model > would typically be accompanied by an additional <form: form $>$ element.

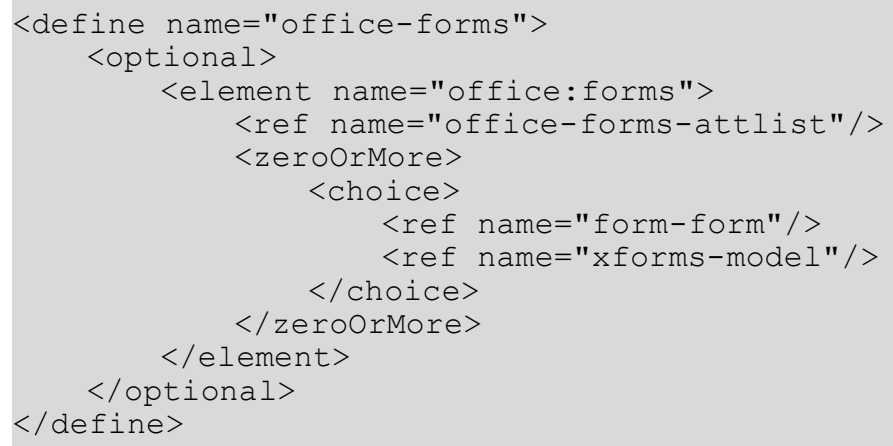

For ease of use when using (filling out) forms, applications may focus controls initially so that the user can immediately type into the first form control. To achieve this behavior, the form: automatic-focus flag may be set to true.

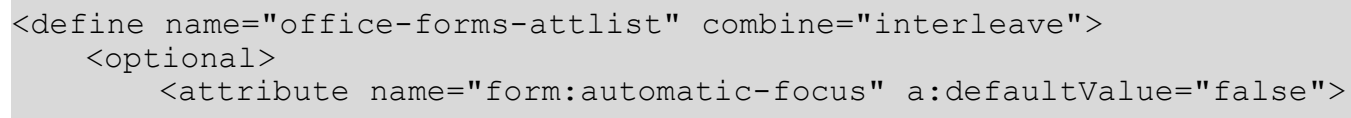




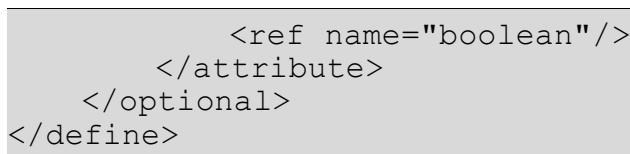

Application which support both creation and usage (filling out) of forms, the form: applydesign-mode flag determines whether the application is supposed to present the forms in this document in editable or fill-out state.

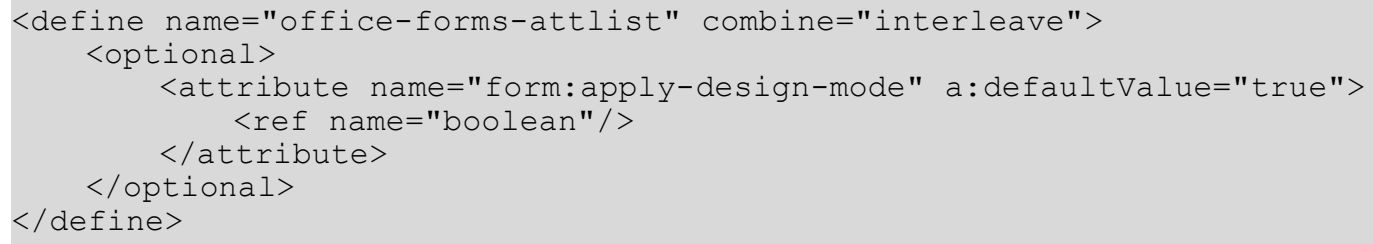

\subsection{Form}

The <form: form > element represents a user interface form and defines the contents and properties of the form.

This element is contained in either an <office: forms $>$ or a $<$ form: form $>$ element. It contains the controls and sub forms of the form, a $<$ form: properties $>$ element which defines the properties of the form, and an <office:events-listeners $>$ element that contains the events for the form.

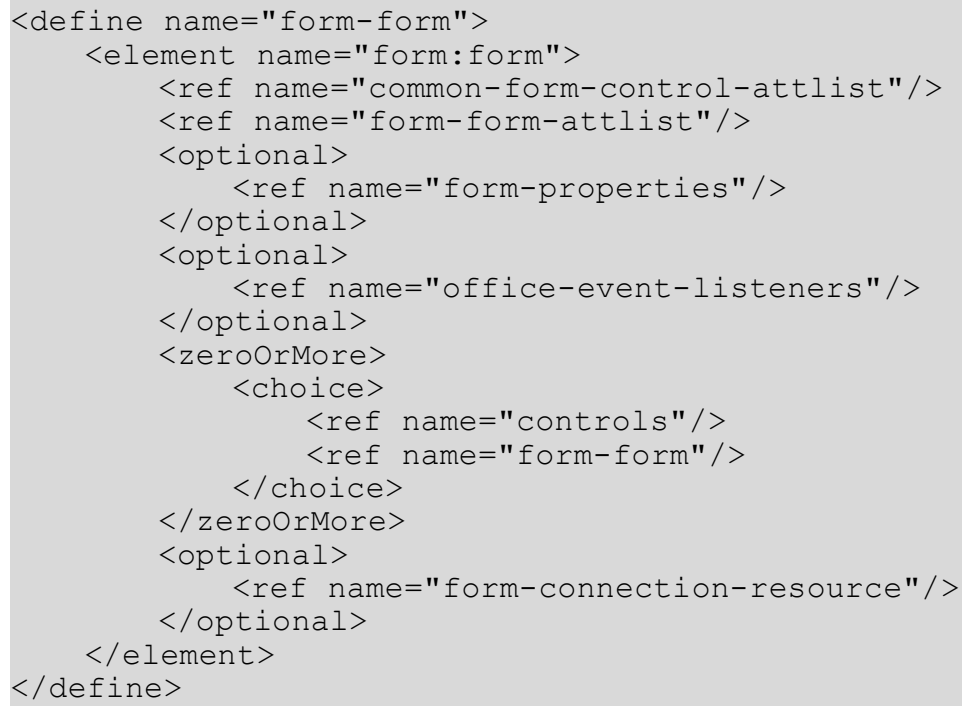

The attributes that may be associated with the <form: form $>$ are as follows:

- Name. See section 11.4.

- $\quad$ Service name. See section 11.4.

- Action

- Target frame

- Method

- Encoding Type

- Allow deletes 
- Allow inserts

- Allow updates

- Apply filter

- Command type

- Command

- Data source

- Master fields

- Detail fields

- Escape processing

- Filter

- Ignore result

- Navigation mode

- Order

- Tabbing cycle

\subsubsection{Action}

The $x l i n k$ : href attribute represents the URI of the processing agent for the form.

\subsubsection{Target Frame}

The office:target-frame attribute specifies the target frame of the form.

This attribute can have one of the following values:

- _ _self: The form replaces the content of the current frame.

- _blank: The form is displayed in a new frame.

- _ parent: The form is displayed in the parent frame of the current frame. 
- top: The form is displayed in the topmost frame, that is the frame that contains the current frame as a child or descendent but is not contained within another frame.

- A frame name: The form is displayed in the named frame. If the named frame does not exist, a new frame with that name is created.

\subsubsection{Encoding Type}

If the value of the form:method attribute is post, the form:enctype attribute specifies the content type used to submit the form to the server. The default value of this attribute is application/x-www-form-urlencoded. Other suitable MIME types are also acceptable.

See $\$ 17.3$ of [HTML4] for more information.

8987

8988

8989

8990

8991

8992

8993

8994

\subsubsection{Allow Deletes}

The form:allow-deletes attribute specifies whether or not data records can be deleted. It applies only if the form is data-aware.

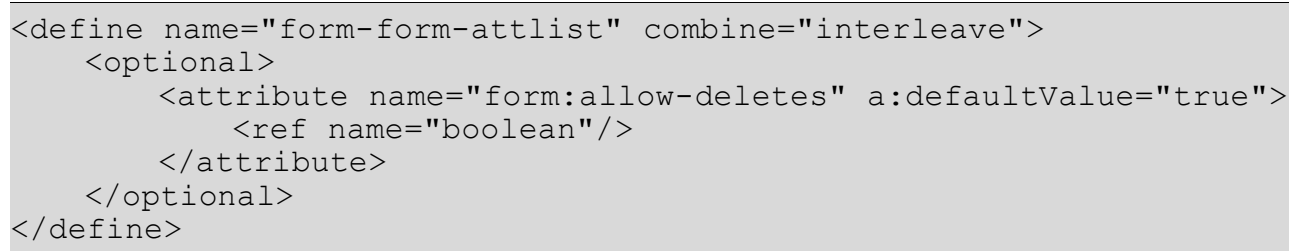




\subsubsection{Allow Inserts}

The form:allow-inserts attribute specifies whether or not new data records can be inserted. It applies only if the form is data-aware.

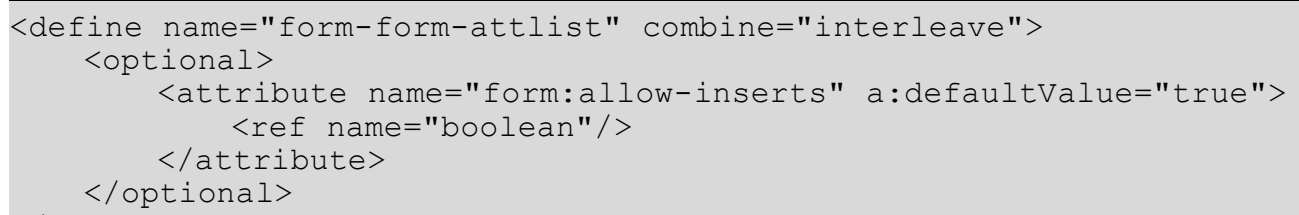

\subsubsection{Allow Updates}

The form:allow-updates attribute specifies whether or not data records can be updated.

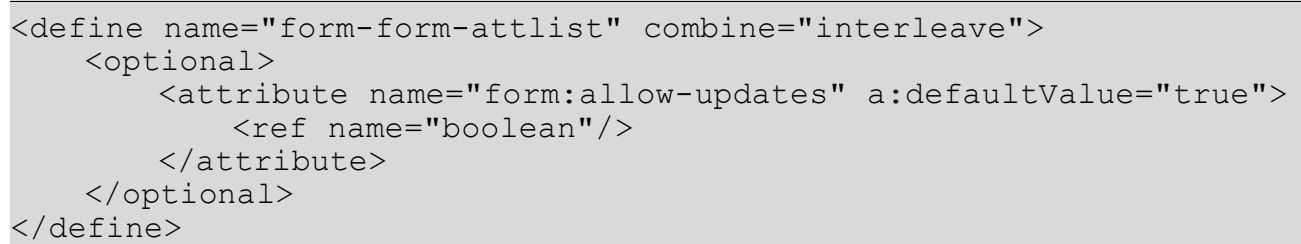

\subsubsection{Apply Filter}

The form:apply-filter attribute specifies whether or not filters should be applied to the form. See also the Filter attribute.

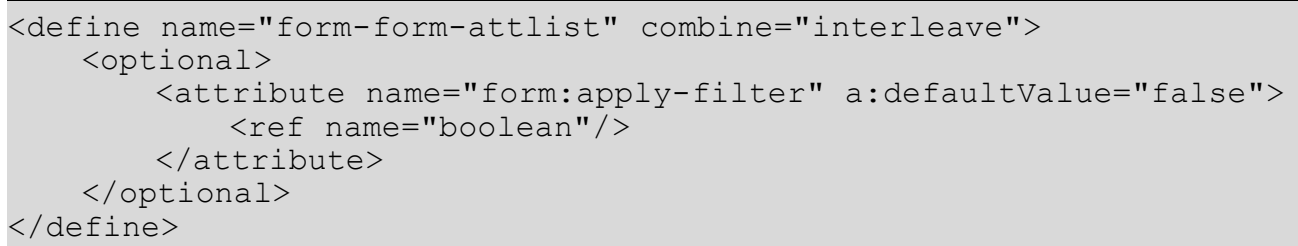

\subsubsection{Command Type}

The form: command-type attribute specifies the type of command to execute on the data source. The value of this attribute can be one of the following:

- table: The command contains a table name. The form retrieves all of the data in the table.

- query: The command contains the name of query. The form retrieves and executes the query.

- command: The command contains an SQL statement. The form executes the SQL statement.

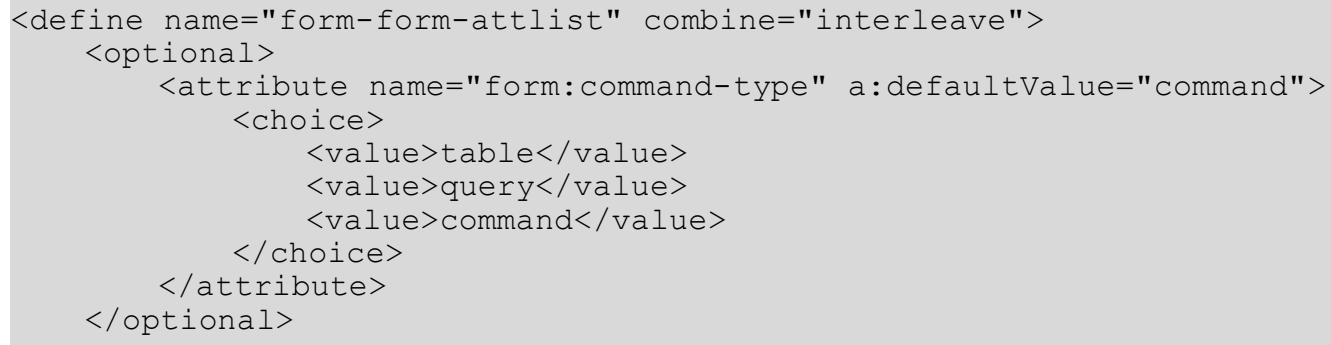




\subsubsection{Command}

The form: command attribute specifies the command to execute on the data source.

The value is interpreted differently, depending to the value of the Command Type attribute of the form. It can be the name of a database table, the name of a query object or an SQL statement.

\subsubsection{Master Fields}

The form:master-fields attribute is used for nested data-aware forms. It specifies the names of the columns in the result set represented by the parent form. Usually, they denote the foreign key fields of the parent form. The values of the columns are used to parameterize the data for the nested form. Each time the parent form changes the current row, the nested form queries the database again based on the values of the master fields.

The attribute contains a comma separated list of field names.

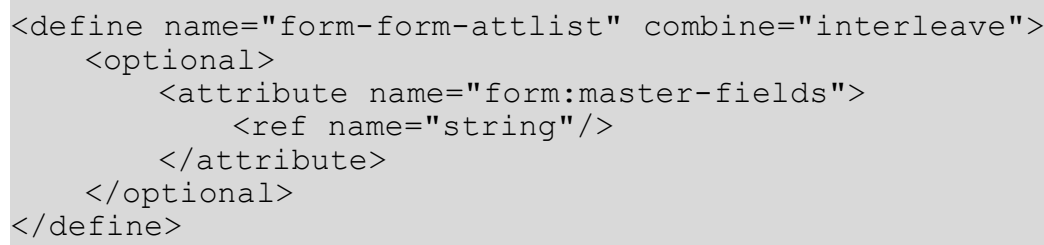

\subsubsection{Detail Fields}

The form: detail-fields attribute is used for nested database forms. It specifies the names of the columns in detail forms that are related to columns in the parent form. The columns are used as parameters in the command for the nested form to retrieve the details for a matching master form record. 
This attribute contains a comma separated list of field names.

\subsubsection{Filter}

The form: filter attribute specifies a filter for the command to base the form on. No matter whether the form is based on a query, a table, or an command, the filter is always conjunctively added to any possible existing filter. The filter usually forms a SQL "WHERE" clause, without the "WHERE" keyword.

The form:apply-filter attribute specifies whether or not the filter is actually applies to the command.

\subsubsection{Ignore Result}

The form:ignore-result attribute specifies whether or not to discard all results that are retrieved from the underlying data source. If true, a database-bound form will discard any data it queries from the database, and thus only inserting and editing of new records is available. Essentially, this allows a mode of operation where only new data can be inserted into a database.

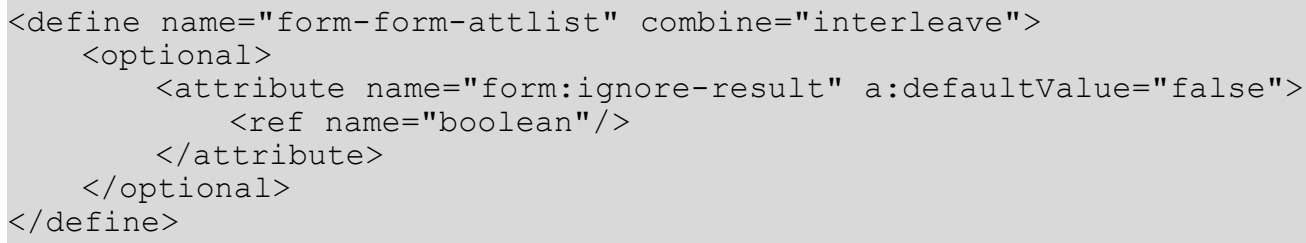




\subsubsection{Navigation Mode}

The form:navigation-mode attribute specifies how the records in a database form are navigated.

The value of this attribute can be one of the following:

- none: A dedicated navigation bar is not provided by the user interface. The form must be navigated using the TAB and SHIFT/TAB keys on the keyboard.

- current: A navigation bar is provided and the navigation is performed on the current form.

- parent: A navigation bar is provided and the navigation is performed on the parent form of the current form.

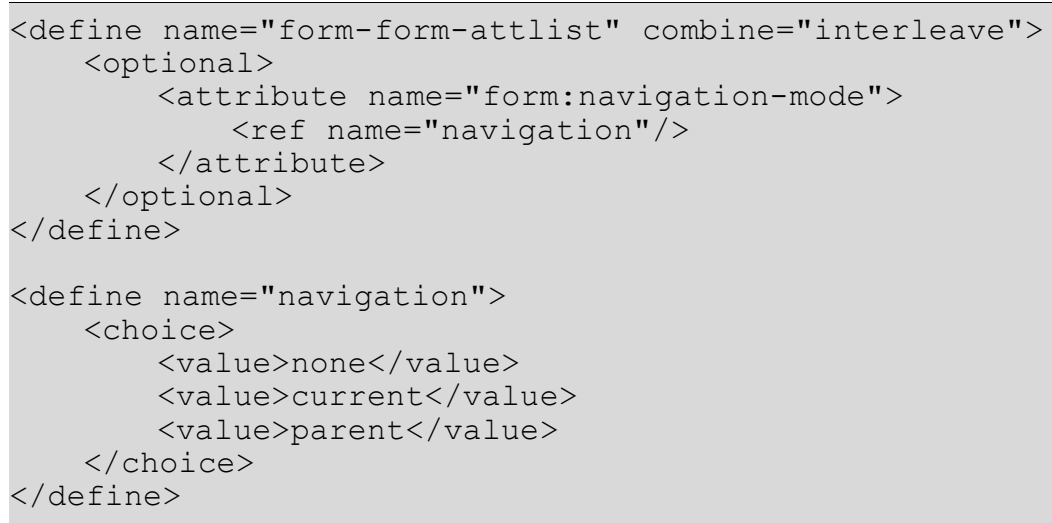

\subsubsection{Order}

The form: order attribute specifies a sort criteria for the command. No matter whether the form is based on a query, a table, or an command, the sorting is always conjunctively added to any possible existing sorting. The attribute value usually forms an SQL "ORDER BY" clause, without the "ORDER BY" keyword.

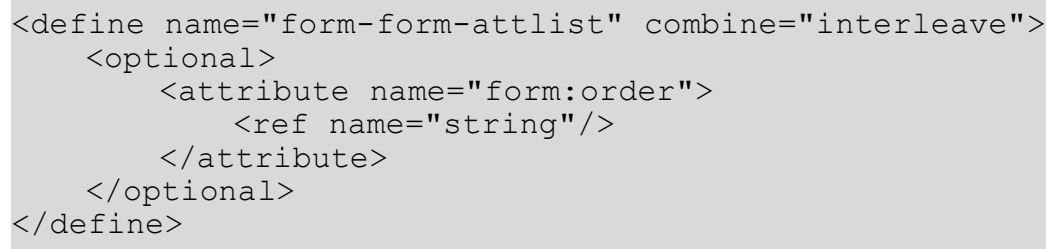

\subsubsection{Tabbing Cycle}

The form: tab-cycle attribute specifies how the application responds when the user presses the TAB key in the controls in a form. The behavior of the application depends on whether or not the form is bound to a data source.

The value of this attribute can be one of the following:

- records: If a user presses the TAB key in the last control of the form, the focus moves to the first control specified in the tab order of the same form, and moves the form to the next record.

- current: If a user presses the TAB key in the last control of the form, the focus moves to the first control specified in the tab order of the same form, while the record pointer of the form is not touched. 
- page: If a user presses the TAB key in the last control of a form, the focus moves to the first control specified in the tab order for the next form.

\subsection{XForms Model}

The form model described in the previous chapter implies a data model where each control defines a name-value-pair, with the name being determined by the control id and the value being editable through the control. No interaction between controls is possible (save for macro programming). For applications where this kind of form logic does not suffice, W3C has introduced XForms (see [XForms]), a standard for XML-based forms.

XForms is designed to be embedded in another XML format. It consists of two major parts, the XForms model which contains the form logic plus form data, and the XForms controls, which can be bound to a data model. In the OASIS Open Office 1.0 we embed the W3C XForms model as defined by the <xforms:model> element into the <office: forms > forms container. The controls (see 11.3) will be left as is, except that they receive an xforms : bind attribute, which allows to bind any OpenDocument control to a previously defined XForms model.

\subsubsection{XForms Model}

We import the XForms model defined in [XForms]. In order to avoid duplication of the XForms schema here, we only specify the XForms model element and allow arbitrary content. 


\subsection{Controls}

Controls are used to interact with forms. Each control in a form is identified by a name, though the names must not necessarily be unique.

Controls are connected to a the surrounding document (and its text flow, if applicable) by binding them to a shape that acts as a placeholder for the control. See section 9.2.12 for details.

In addition to the attributes defined in this file format, controls may have application-specific additional attributes. These attributes are stored in the <form:properties $>$ element in each control. Control events are specified in the <office: event-listeners> element.

When a user submits a form for processing, the names of some controls are paired with the current values of the controls and the pairs are submitted with the form. These controls are called successful controls. See section 17.13.2 of [HTML4]for more information.

The file format provides elements for the following standard controls:

- Text

- Text area

- Password

- File

- Formatted text

- Number

- Date

- $\quad$ Time

- Fixed text

- Combo box

- List box

- Button

- Image

- Check box

- Radio button

- Frame

- Image frame

- Hidden

- Grid

It is also possible to define application-specific controls. These controls are described by the <form: generic-control> element.

\subsubsection{Text}

The $<$ form: text $>$ element defines a control for displaying and inputting text. 


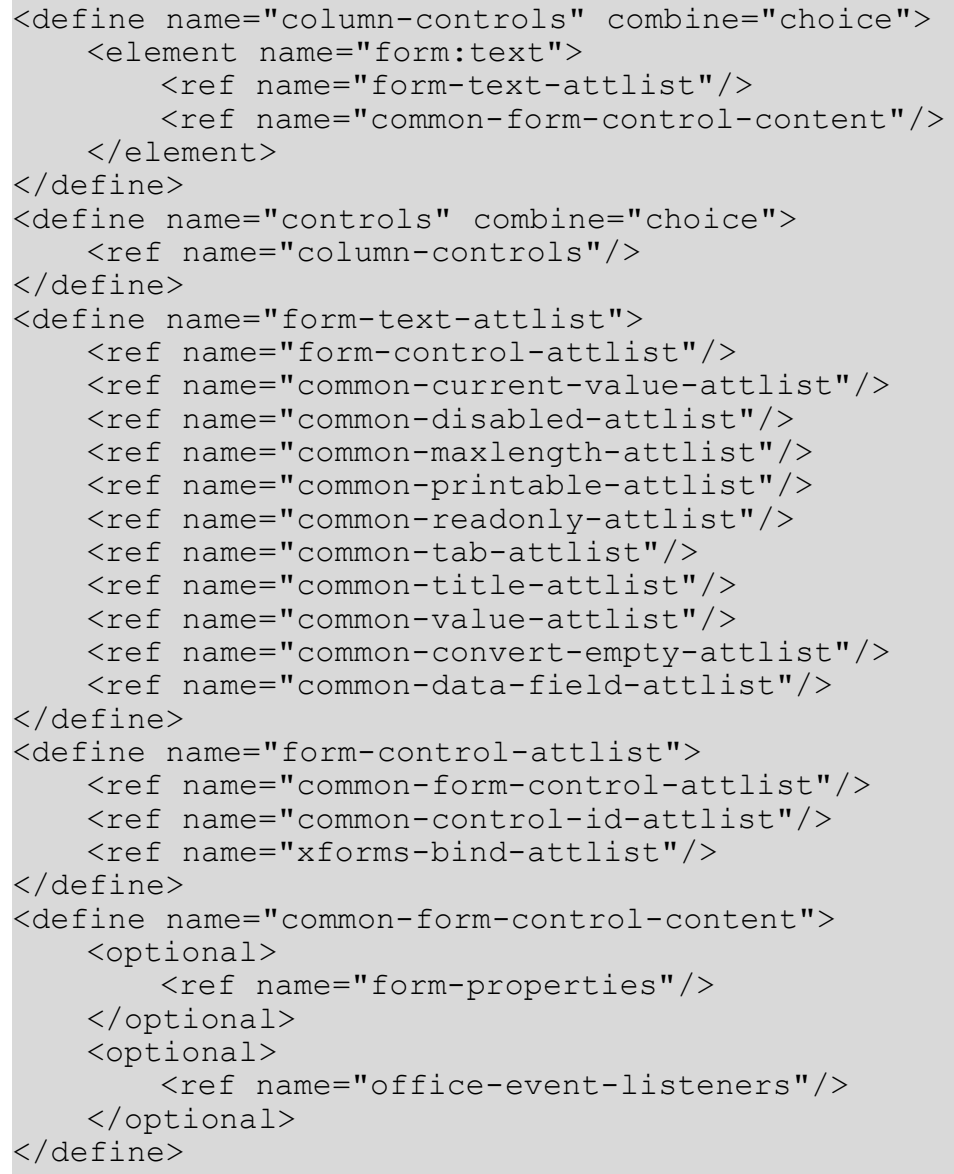

The attributes that may be associated with the <form: text $>$ element are:

- Name and Service Name. See section 11.4 for information about these attributes.

- Control ID, Current Value, Disabled, Maximum Length, Printable, Read only, Tab Index, Tab Stop, Title and Value. See section 11.5 for information about these attributes.

- Convert Empty and Data Field. See section 11.5.22 for information about these attributes.

\subsubsection{Text Area}

The <form: textarea $>$ element defines a control for displaying and inputting text on multiple lines.

The <form: textarea> element may be used with plain text values (specified by the form: current-value attribute) as well as with formatted text (specified as paragraph content). If both, the form: current-value and one or more <text:p> elements are present, it's up to the application reading the document to decide which information is used.

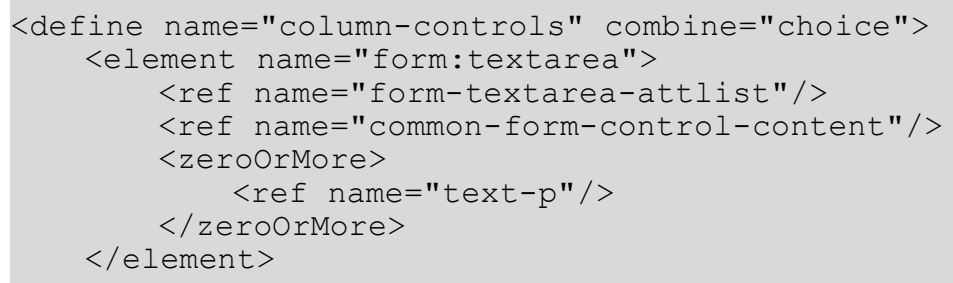




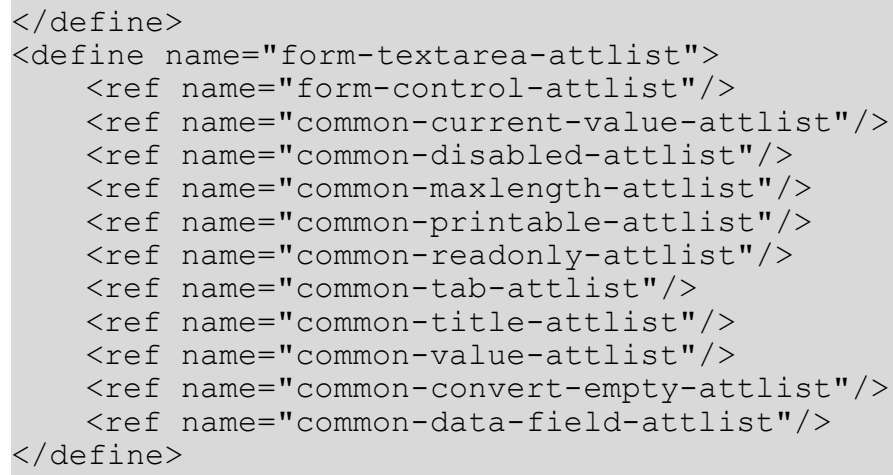

The attributes that may be associated with the <form: textarea $>$ element are:

- Name and Service Name. See section 11.4 for information about these attributes.

- Control ID, Current Value, Disabled, Maximum Length, Printable, Read only, Tab Index, Tab Stop, Title and Value. See section 11.5 for information about these attributes.

- Convert Empty and Data Field. See section 11.5.22 for information about these attributes.

\subsubsection{Password}

The <form:password $>$ element defines a control that hides the text that a user inputs using an echo character, for example, an asterisk. This type of control is usually used for inputting sensitive information such as a password.

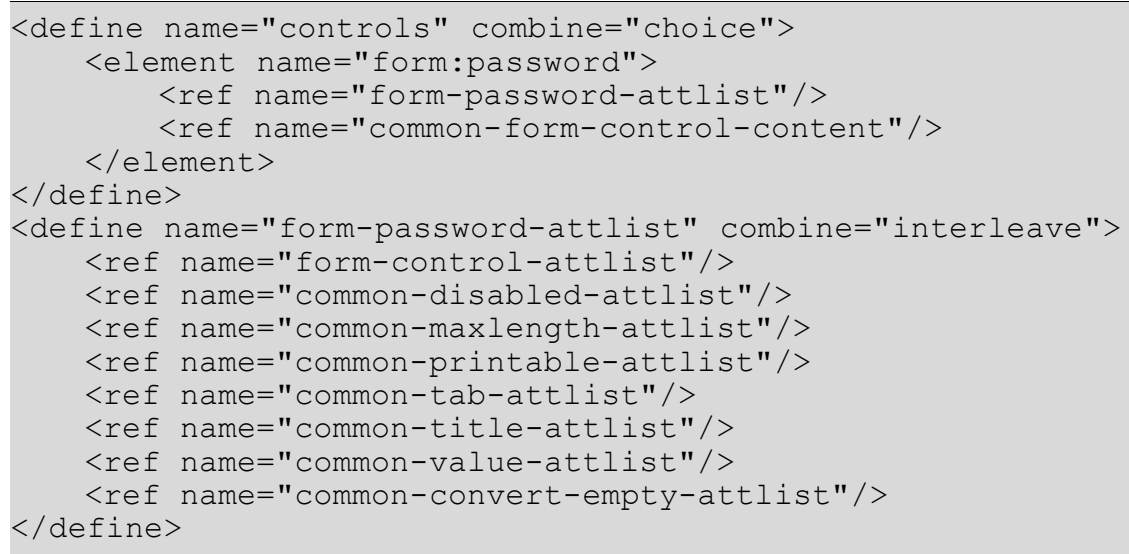

The attributes that may be associated with the <form:password $>$ element are:

- Name and Service Name. See section 11.4 for information about these attributes.

- Control ID, Disabled, Maximum Length, Printable, Tab Index, Tab Stop, Title and Value. See section 11.5 for information about these attributes.

- Echo Char

\section{Echo Char}

The form:echo-char attribute specifies the character that the form uses to mask the text which a user inputs in a password control. 


\subsubsection{File}

The $<$ form: file $>$ element defines a control for selecting a file.
The attributes that may be associated with the $<$ form: file $>$ element are:

- Name and Service Name. See section 11.4 for information about these attributes.

- Control ID, Current Value, Disabled, Printable, Read only, Tab Index, Tab Stop, Title and Value. See section 11.5 for information about these attributes.

\subsubsection{Formatted Text}

The <form: formatted-text> element defines a control for inputting formatted text, which follows a certain formatting in both input and display.

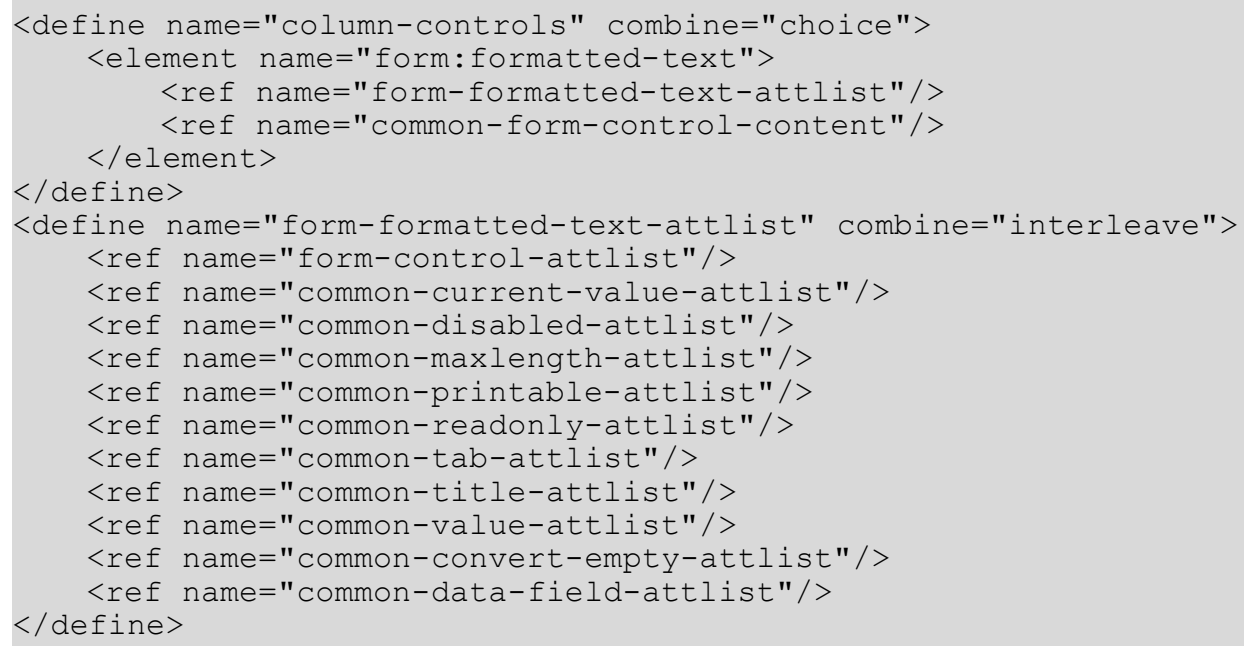

The attributes that may be associated with the $<$ form: formatted-text $>$ element are: 
- Name and Service Name. See section 11.4 for information about these attributes.

- Control ID, Current Value, Disabled, Maximum Length, Printable, Read only, Tab Index, Tab Stop, Title and Value. See section 11.5 for information about these attributes.

- Convert Empty and Data Field. See section 11.5.22 for information about these attributes.

- Maximum Value

- Minimum Value

- Validation

\section{Maximum Value}

The form:max-value attribute specifies the maximum value that a user can enter.

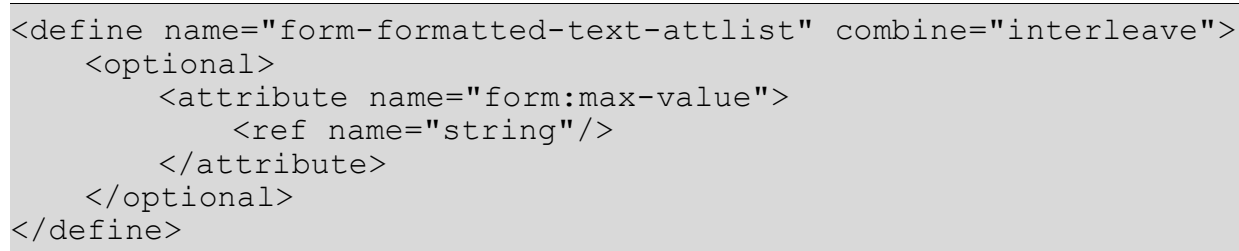

\section{Minimum Value}

The form:min-value attribute specifies the minimum value that a user can enter.

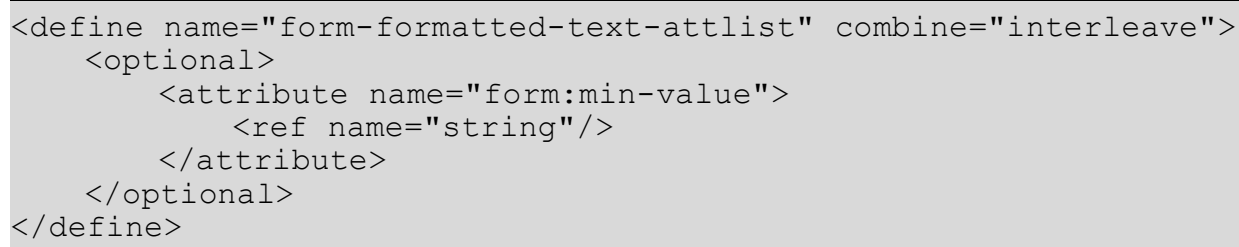

\section{Validation}

The form:validation attribute specifies whether or not the text that the user enters is validated during input.

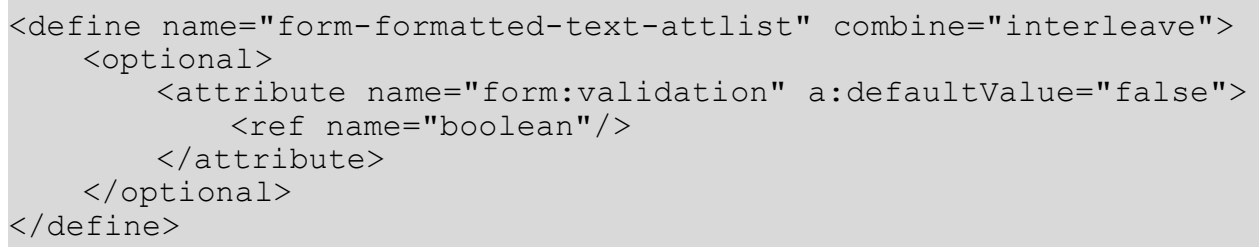

\subsubsection{Number}

The $<$ form: number $>$ element describes a control which allows the user to enter a floating point number. The attributes that may be associated on this control are similar to those of the <form: formatted-text>, except that the data type is fixed to numeric data.

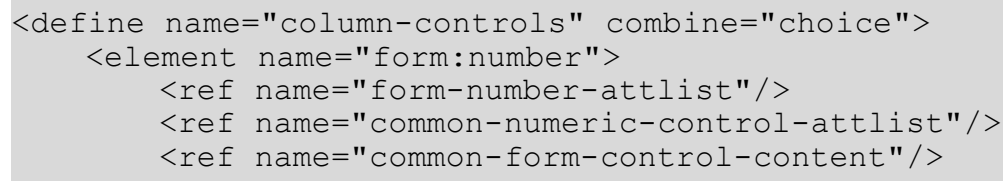




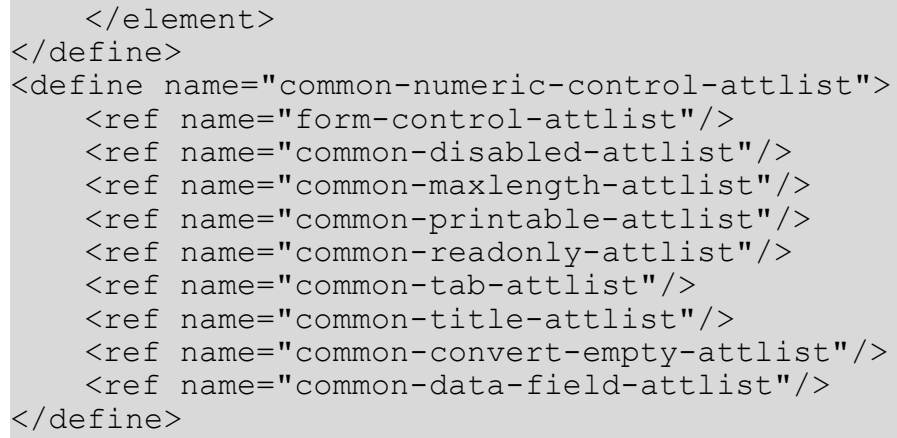

The attributes that may be associated with the $<$ form: number $>$ element are:

- Name and Service Name. See section 11.4 for information about these attributes.

- Control ID, Disabled, Maximum Length, Printable, Read only, Tab Index, Tab Stop, Title and Value. See section 11.5 for information about these attributes.

- Convert Empty and Data Field. See section 11.5.22 for information about these attributes.

- Value and Current Value

- Minimum and Maximum Value

\section{Value}

The attributes for value and current value are the same as those for other fields, except that they can contain only floating point data.

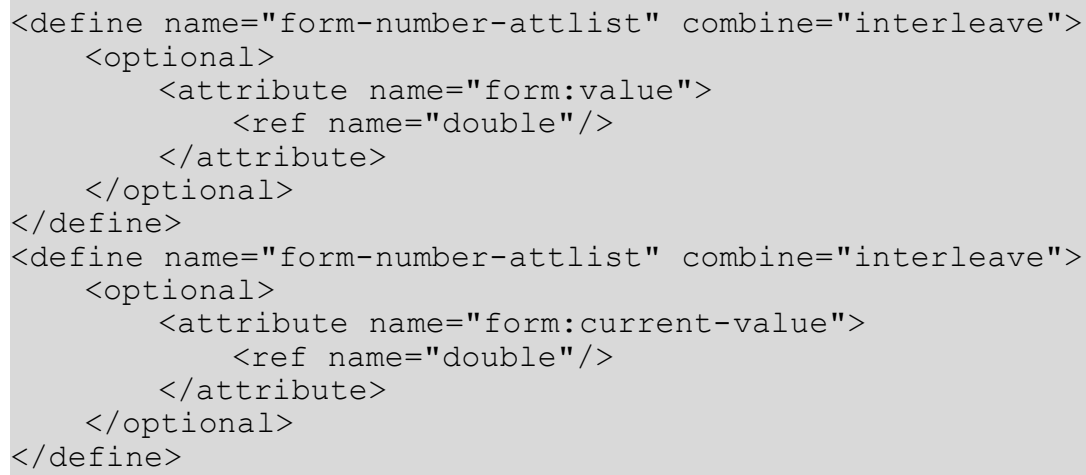

\section{Minimum and Maximum}

The attributes for minimum and maximum value define the smallest and largest numerical values that are acceptable for this control.

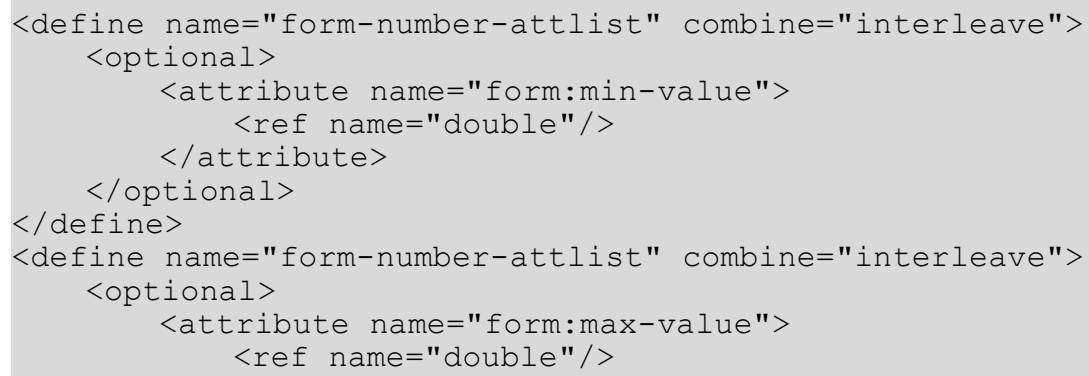




\subsubsection{Date And Time}

The controls for date and time are the same as those for number values, except that they accept date and time values, respectively. They support the same attributes as the numerical field, except for the different data types of their value attributes.

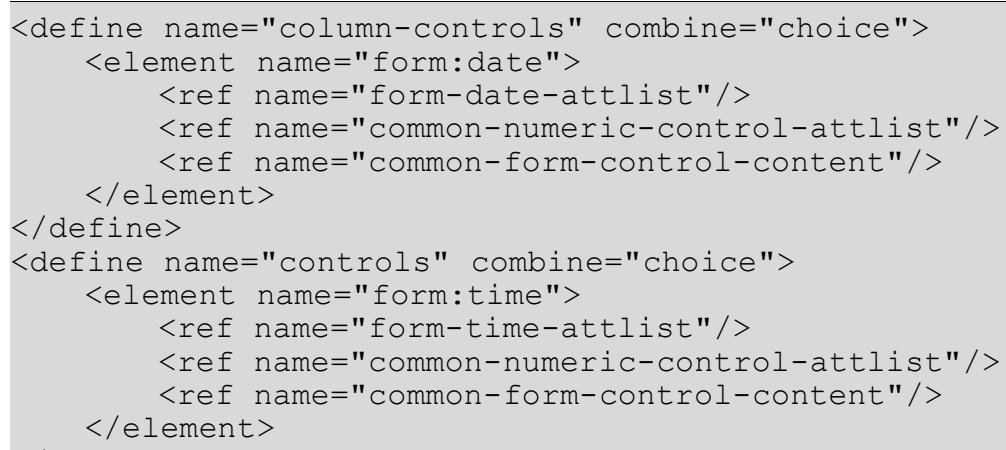

The attributes that may be associated with the $<$ form: date $>$ and $<$ form: time $>$ elements are:

- Name and Service Name. See section 11.4 for information about these attributes.

- Control ID, Disabled, Maximum Length, Printable, Read only, Tab Index, Tab Stop, Title and Value. See section 11.5 for information about these attributes.

- Convert Empty and Data Field. See section 11.5.22 for information about these attributes.

- Value and Current Value

- Minimum and Maximum Value

\section{Value}

The attributes for value and current value are the same as those for $\langle$ form: number $\rangle$, except that they can contain only date or time data, respectively.

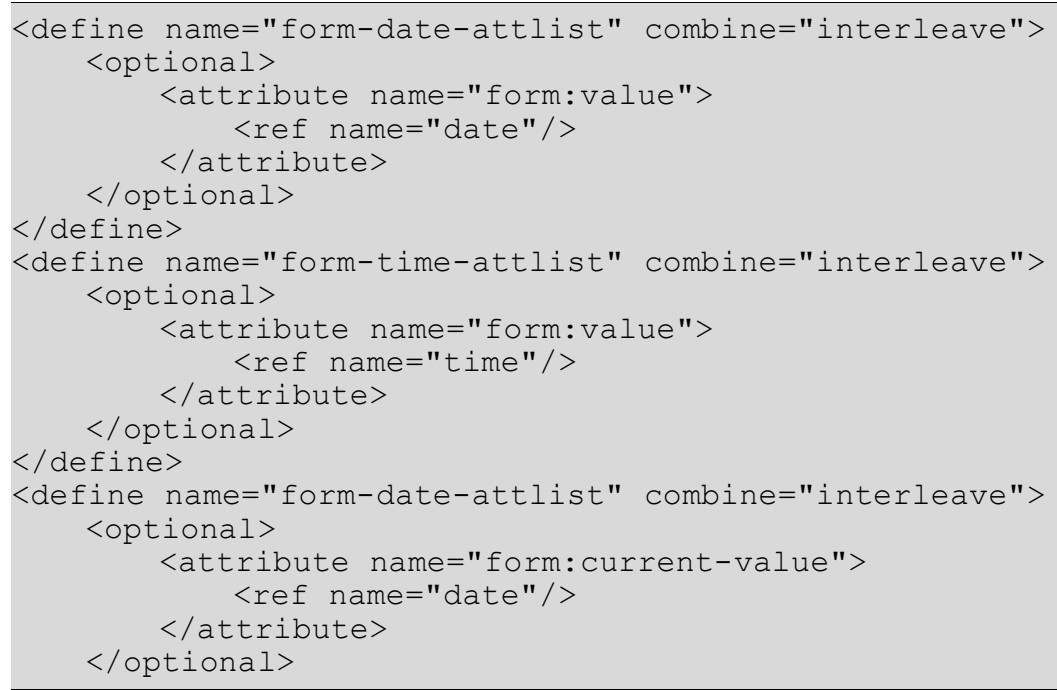




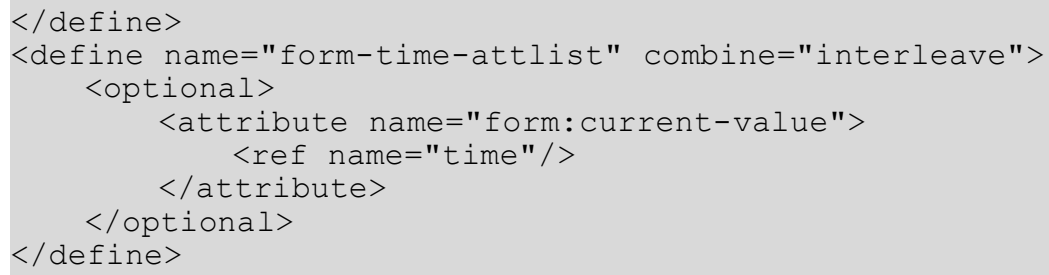

\section{Minimum and Maximum}

The attributes for minimum and maximum value define the smallest and largest dates (or times) that are acceptable for this control.

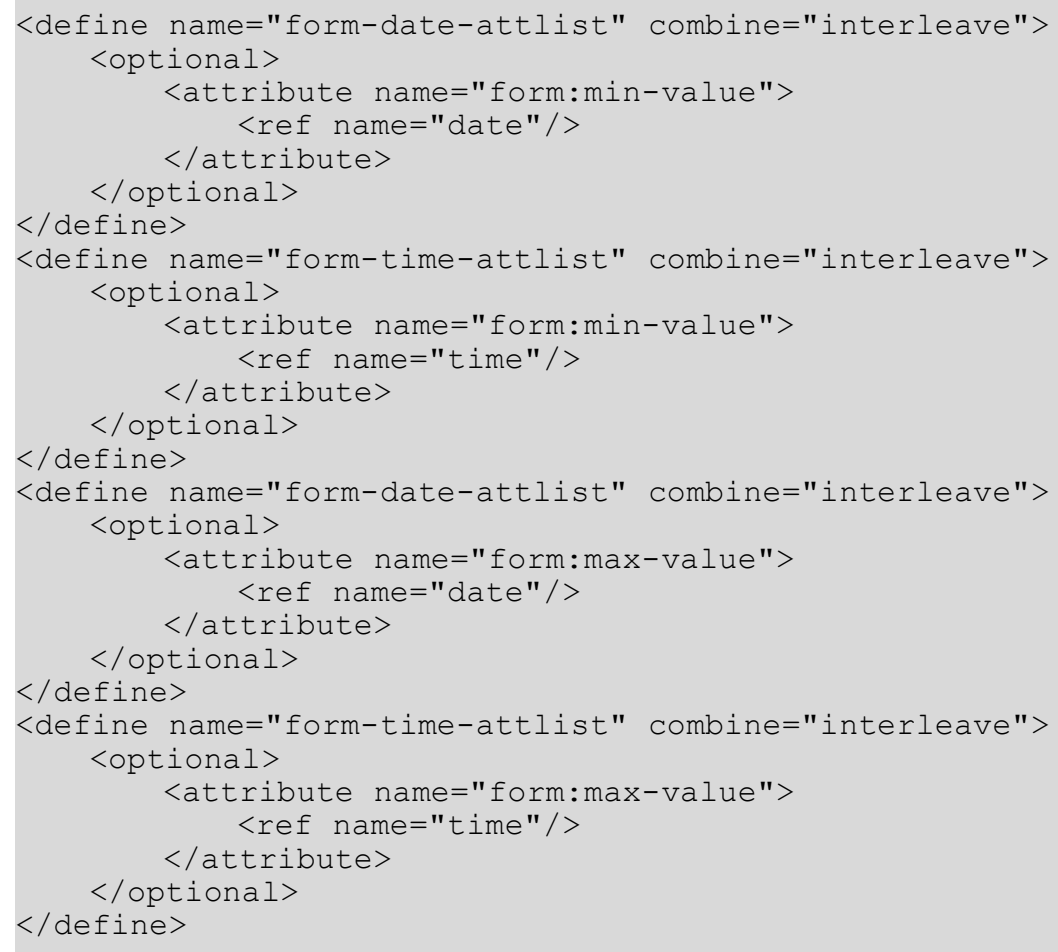

\subsubsection{Fixed Text}

The <form: fixed-text $>$ element describes a control which attaches additional information to controls, or merely displays information in the application. Relations between a labeling and a labeled control can be established by specifying the form: for attribute of the label. Only one label may be associated with the same control.

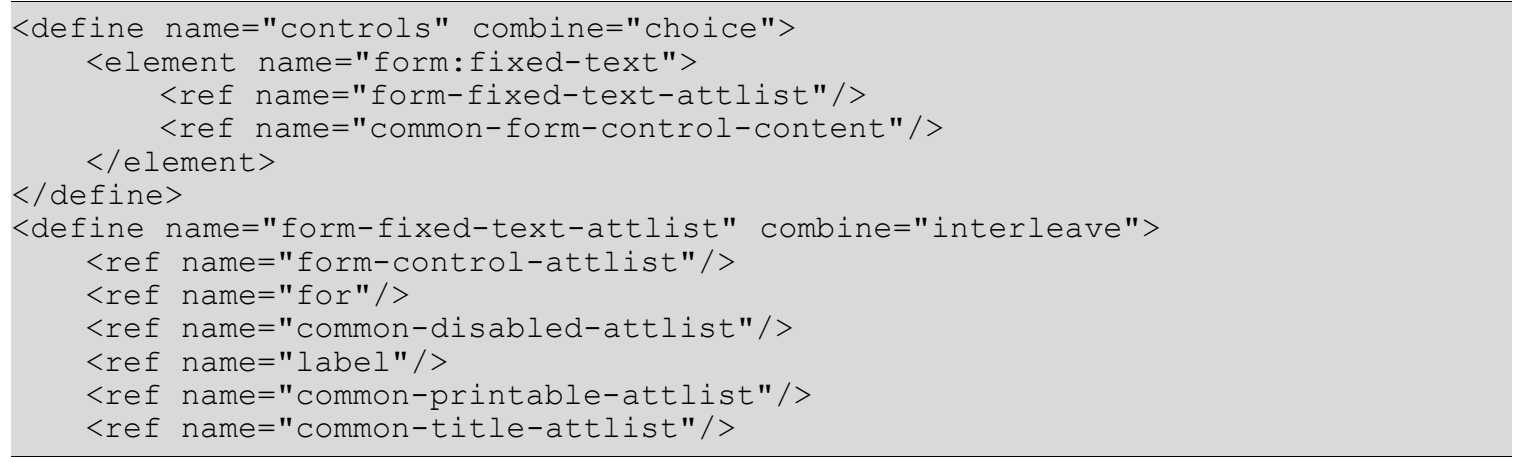


The attributes that may be associated with the <form: fixed-text> element are:

- Name and Service Name. See section 11.4 for information about these attributes.

- Control ID, Disabled, For, Label, Printable, and Title. See section 11.5 for information about these attributes.

- Multi-Line

\section{Multi-Line}

The form:multi-line attribute specifies whether or not the label is displayed on multiple lines.

9400

9401

9402

9403

9404

9405

9406

\subsubsection{Combo Box}

The $<$ form: combobox $>$ element defines a control which allows displaying and editing of text, and containing a list of possible values for this text.

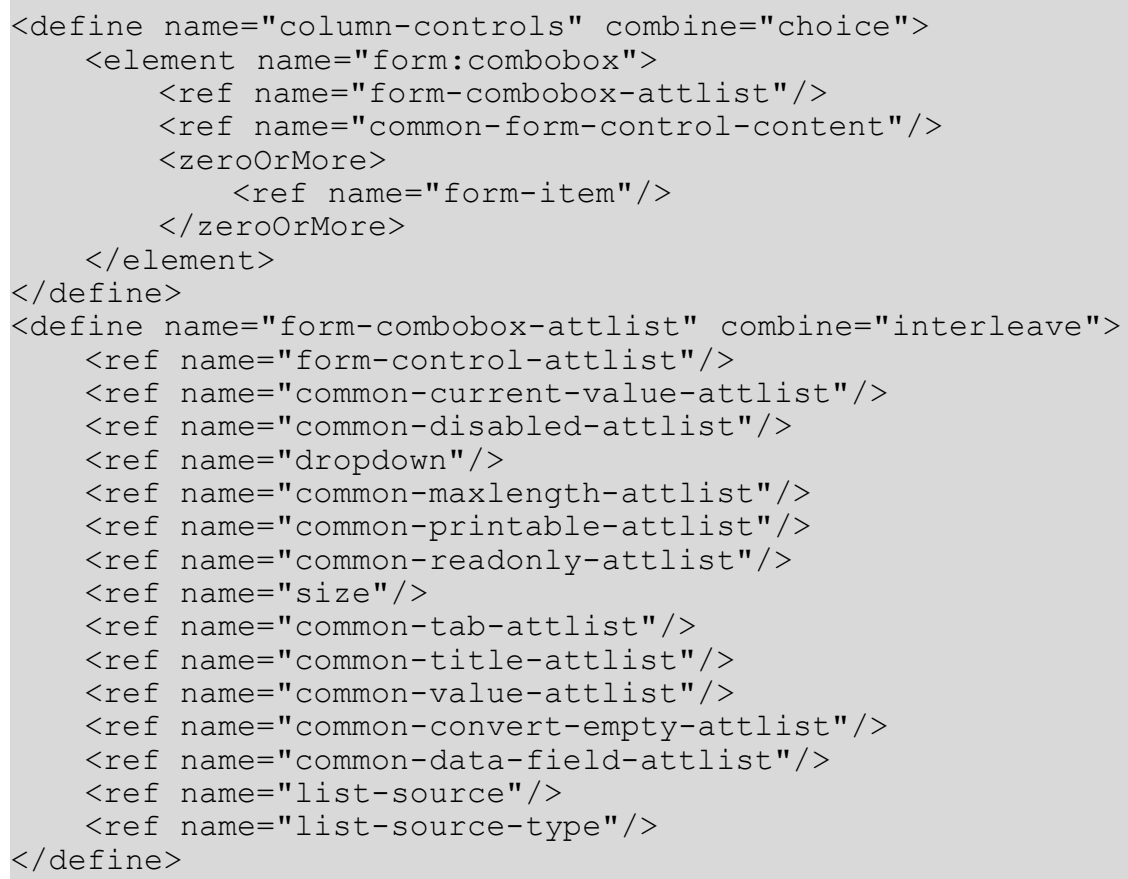

The attributes that may be associated with the <form: combobox $>$ element are:

- Name and Service Name. See section 11.4 for information about these attributes.

- Control ID, Current Value, Disabled, Dropdown, Max Length, Printable, Read only, Size, Tab Index, Tab Stop, Title, and Value. See section 11.5 for information about these attributes. 
- Convert Empty, Data Field, List Source, and List Source Type. See section 11.5.22 for information about these attributes.

- Automatic Completion

\section{Automatic Completion}

The form: auto-complete attribute specifies whether, when the user enters text in the combobox that matches one of the list items in the combobox, the application automatically completes the text for the user.

\section{Item}

The $<$ form: item> element defines a list item for a combobox control.

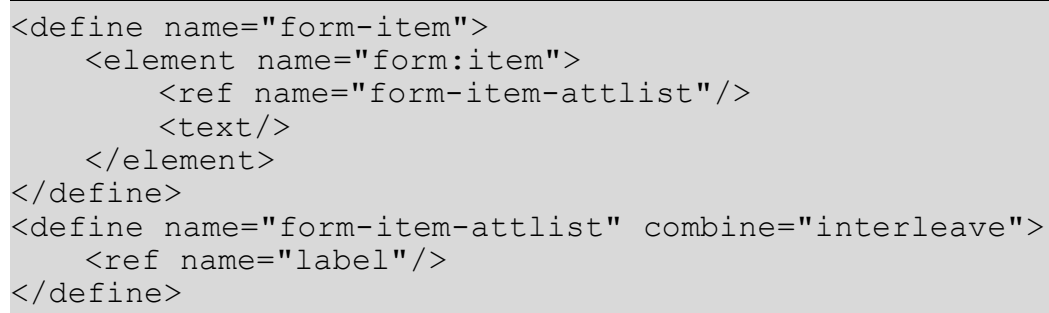

The attribute that may be associated associate with the $<$ form: item $>$ element is:

- Label. See section 11.5 for information about this attribute.

\subsubsection{List Box}

The <form: listbox $>$ element defines an input control that allows a user to select one or more items from a list. It is an alternative representation for a group of radio buttons.

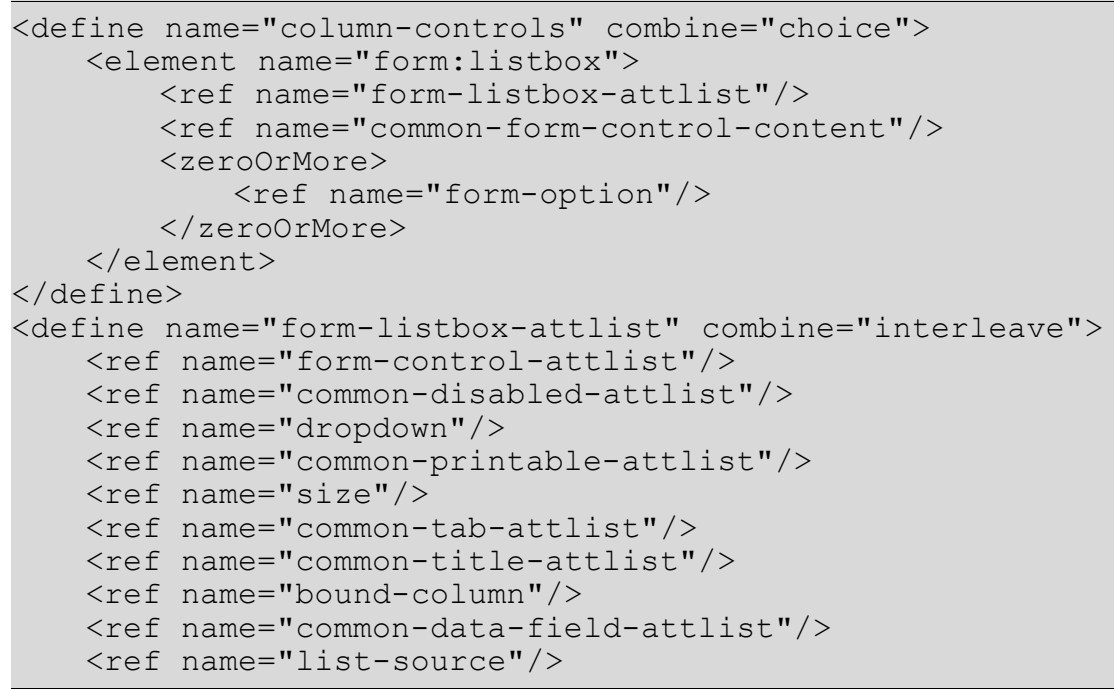


The attributes that may be associated with the $<$ form: listbox $>$ element are:

- Name and Service Name. See section 11.4 for information about these attributes.

- Control ID, Disabled, Dropdown, Printable, Read only, Size, Tab Index, Tab Stop, and Title. See section 11.5 for information about these attributes.

- Bound Column, Data Field, List Source, and List Source Type. See section 11.5.22 for information about these attributes.

- Multiple

- XForms source

\section{Multiple}

The form:multiple attribute determines whether or not a user can select multiple items from a list box.

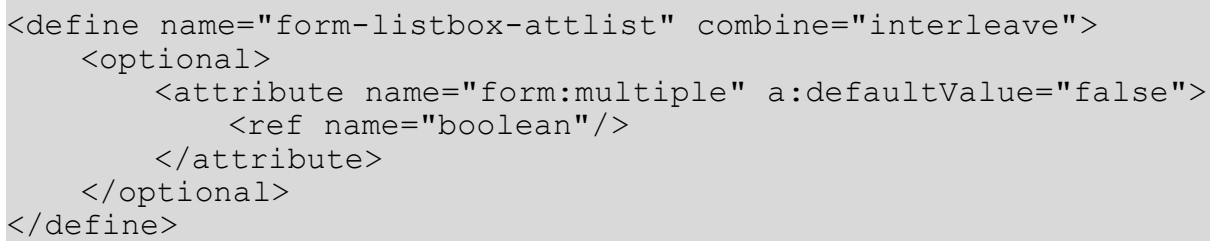

\section{XForms source}

The form:xforms-list-source allows to dynamically create the list of choices by binding the list content to XForms (see section 11.2, as well as [XForms]). The attribute references an <xforms:bind> element, and creates a list entry for each node in the nodeset defined by that attribute.

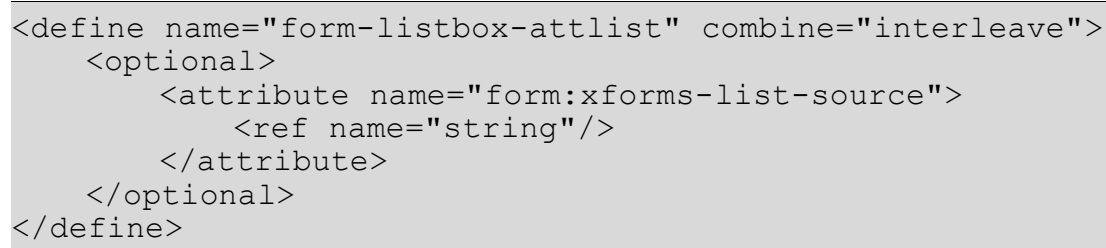

\section{Option}

The <form: option> element defines the list items for a list box control. An item can be preselected and can contain a related value.

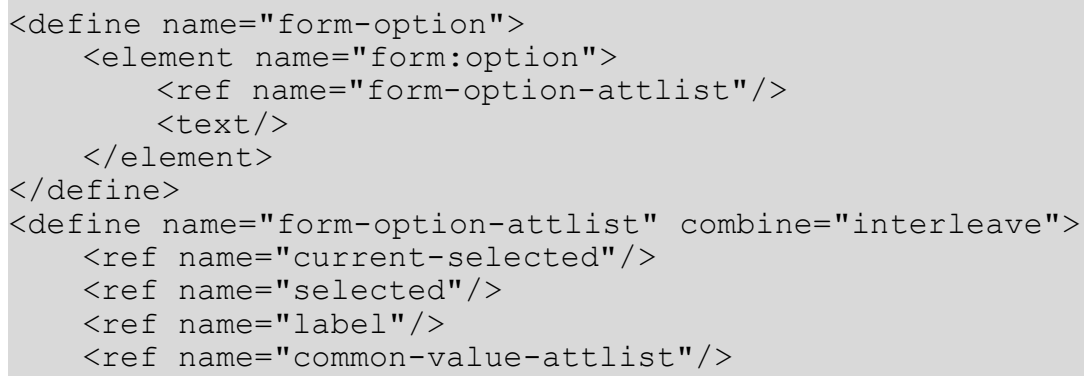


The attributes that may be associated with the <form: option $>$ element are:

- Current Selected, Selected, Label, and Value. See section 11.5 for information about these attributes.

\subsubsection{Button}

The <form: button> element defines a button. When pressed, a button usually triggers an action.

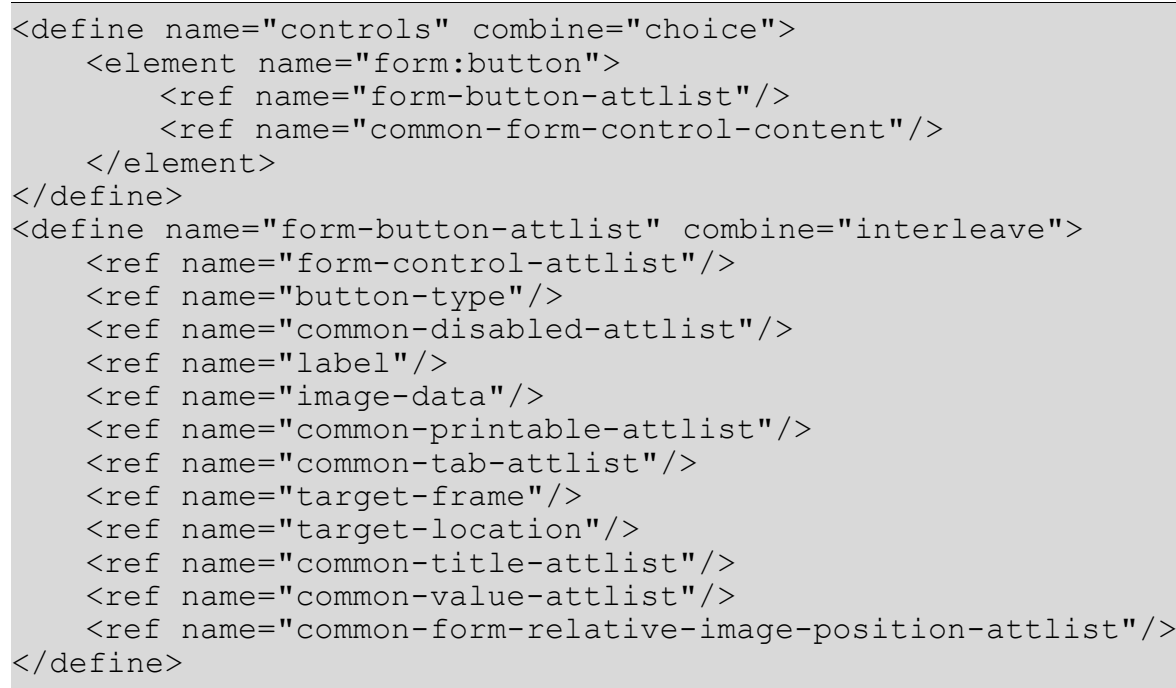

The attributes that may be associated with the $<$ form: button $>$ element are:

- Name and Service Name. See section 11.4 for information about these attributes.

- Button Type, Control ID, Disabled, Image Data, Printable, Tab Index, Tab Stop, Target Frame, Target Location, Title, Value and relative image position. See section 11.5 for information about these attributes.

- Default Button

- Toggle

- Focus on Click

- XForms Submission

\section{Default Button}

The form: default-button attribute determines whether or not the button is the default button on the form. If a user clicks the default button or presses Return while an input control is focused, the application takes the same action.

If a form contains more than one default button, the behavior of the application is undefined.

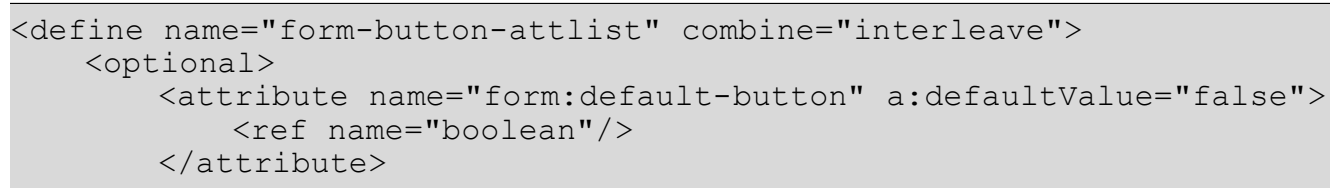




\section{Toggle}

The form: toggle attribute specifies whether a form button control, when it is operated (via mouse or keyboard), should be toggled between a "pressed" and a "not pressed" state. If this attribute is set to false, the button controls behaves like an usual push button.

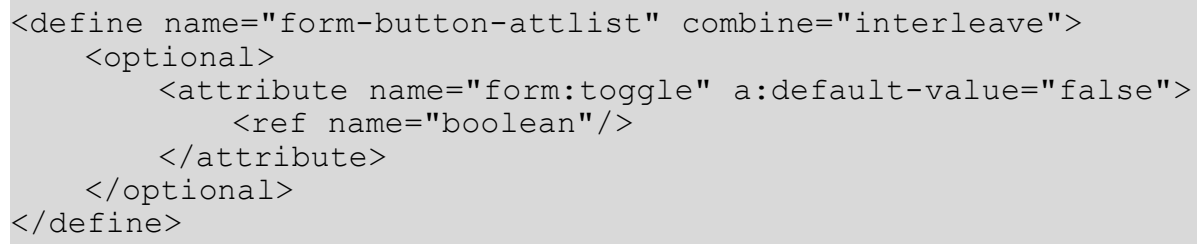

\section{Focus on click}

The form: focus-on-click attribute specifies whether a form button control should grab the focus when it is clicked with the mouse.

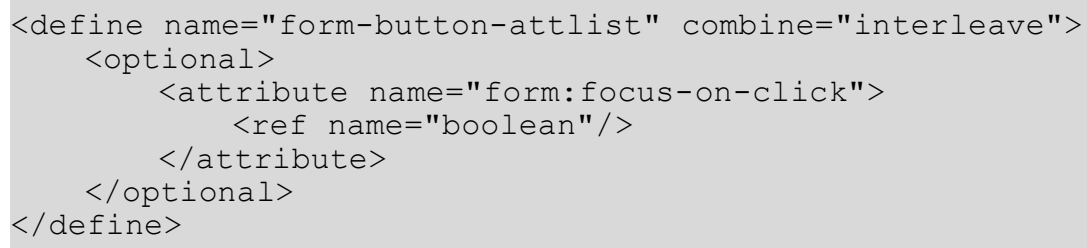

\section{XForms Submission}

Buttons may be used to trigger an XForms submission by adding an form:xformssubmission attribute. If such a button is triggered, a previously declared XForms submission with the given name is executed.

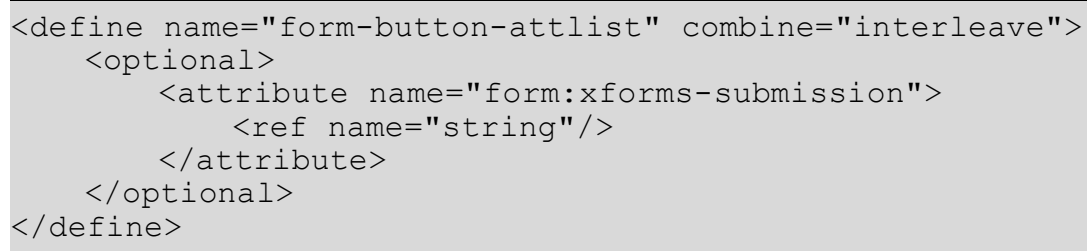

\subsubsection{Image}

The <form: image $>$ element defines a graphical button control. This element corresponds to the input element of type image in HTML 4.01. Note: HTML 4.01 only allows the button type to be submit for an image button. In office application file format, an image button can be of any type.

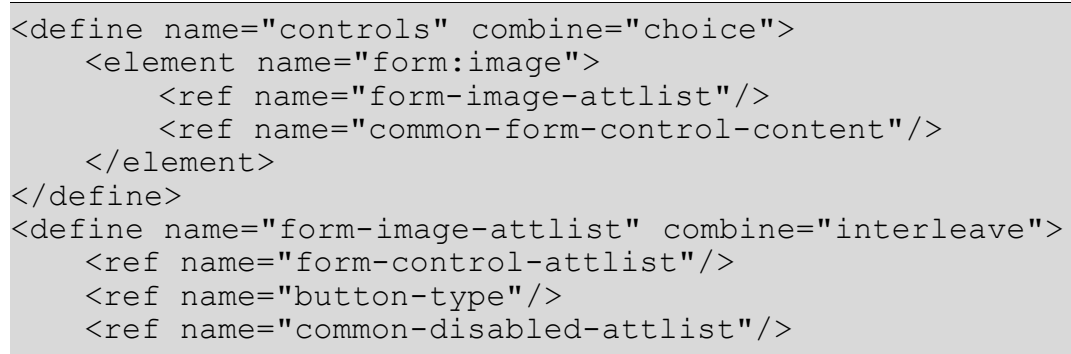


<ref name="image-data" / >

<ref name="common-printable-attlist" / >

<ref name="common-tab-attlist" / >

<ref name="target-frame"/>

<ref name="target-location"/>

<ref name="common-title-attlist"/>

<ref name="common-value-attlist"/>

$</$ define $>$

The attributes that may be associated with the <form:image> element are:

- Name and Service Name. See section 11.4 for information about these attributes.

- Button Type, Control ID, Disabled, Image Data, Printable, Tab Index, Tab Stop, Target Frame, Target Location, Title, and Value. See section 11.5 for information about these attributes.

\subsubsection{Check Box}

The $<$ form: checkbox $>$ element defines an on/off control which a user can toggle. The control is on when the value of the form: current-state attribute associated with the control element is checked. When a user submits a form, only the controls whose current state is checked are successful.

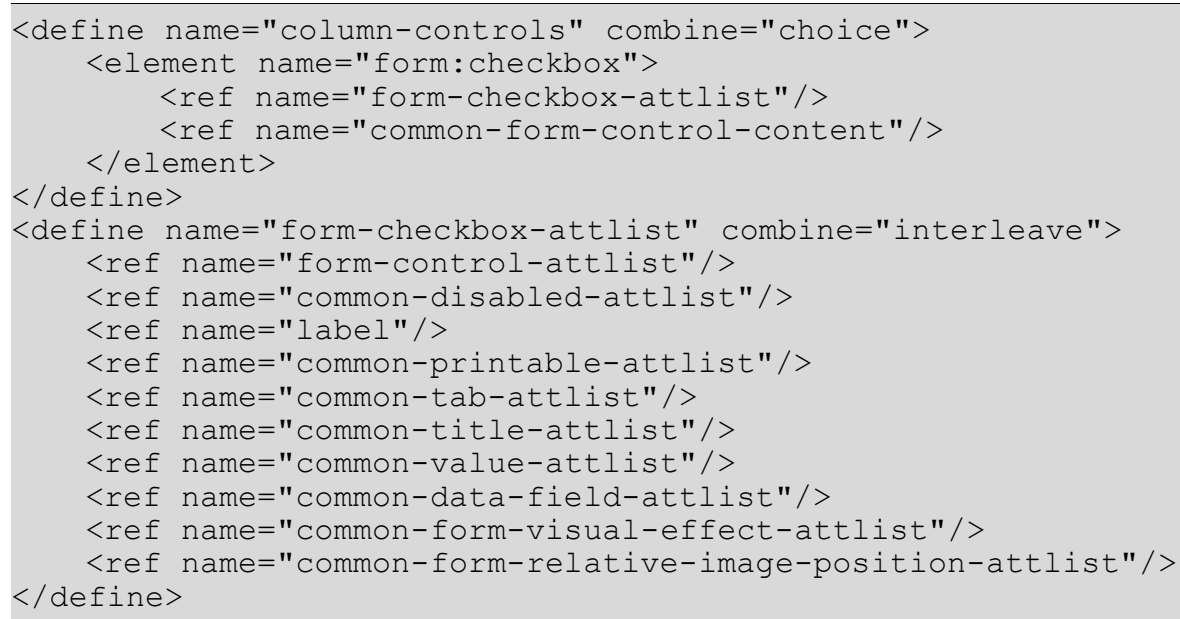

The attributes that may be associated with the <form: checkbox > element are:

- Name and Service Name. See section 11.4 for information about these attributes.

- Control ID, Disabled, Label, Printable, Tab Index, Tab Stop, Title, Value, Visual Effect and Relative Image Position. See section 11.5 for information about these attributes.

- Data Field. See section 11.5.22 for information about this attribute.

- Current State

- Is Tristate

- State

\section{Current State}

The form: current-state attribute specifies the current state of the check box control. 
The value of this attribute can be one of the following:

- unchecked: The check box is not checked.

- checked: The check box is checked. The value of the control is submitted with the form.

- unknown: This value is only available when the control is in tristate mode (See the "ls Tristate" attribute) . This value may, for instance, be used in connection with a database field binding to indicate that the value is NULL.

9581

9582

9583

9584

9585

9586

9587

9588

9589

9590

9591

9592

9593

9594

\section{Is Tristate}

The form: is-tristate attribute specifies that the check box can have three states instead of the common two states.

9595

9596

9597

9598

9599

9600

9601

\section{State}

The form: state attribute specifies the default state of the check box control. This state is used to initialize the control.

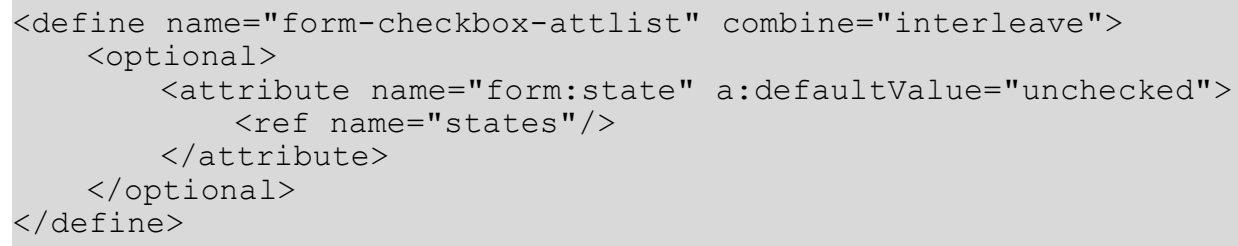

\subsubsection{Radio Button}

The <form:radio $>$ element describes controls which act like check boxes except that when several radio buttons share the same control name they are mutually exclusive. When one button is on, all of the other buttons with the same name are off. If no radio button is initially on, the way in which the application chooses which button to turn on initially is undefined.

If a group of radio buttons is bound to one database field, the reference value of the selected radio button is written into the database field. 


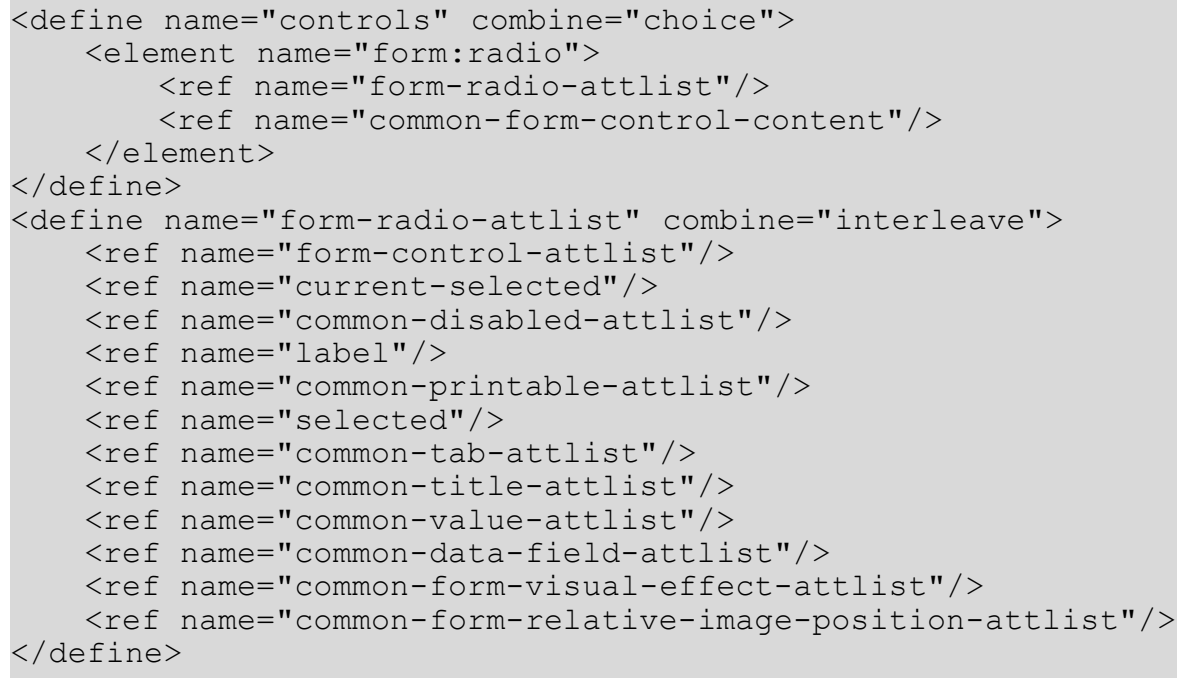

The attributes that may be associated with the $<$ form: radio $>$ element are:

- Name and Service Name. See section 11.4 for information about these attributes.

- Control ID, Current Selected, Disabled, Label, Printable, Selected, Tab Index, Tab Stop, Title, Value, Visual Effect and Relative Image Position. See section 11.5 for information about these attributes.

- Data Field. See section 11.5.22 for information about this attribute.

\subsubsection{Frame}

The <form: frame> element defines a frame, which may be used to arrange controls visually. This element does not have a value and it does not allow any user input.

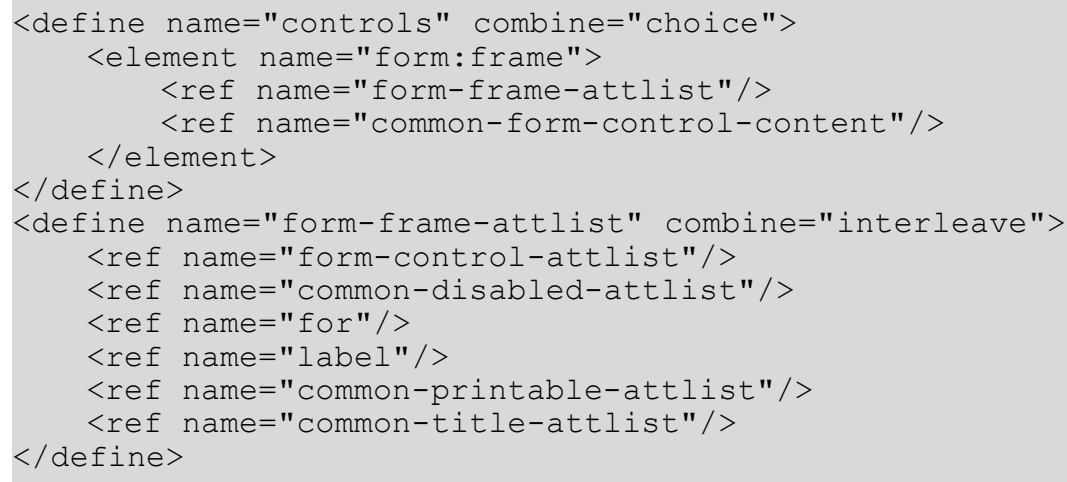

The attributes that may be associated with the <form: frame> element are:

- Name and Service Name. See section 11.4 for information about these attributes.

- Control ID, Disabled, For, Label, Printable, and Title. See section 11.5 for information about these attributes.

\subsubsection{Image Frame}

The <form: image-frame> element defines a graphical control. The control displays an image, whose location is described in the control. 


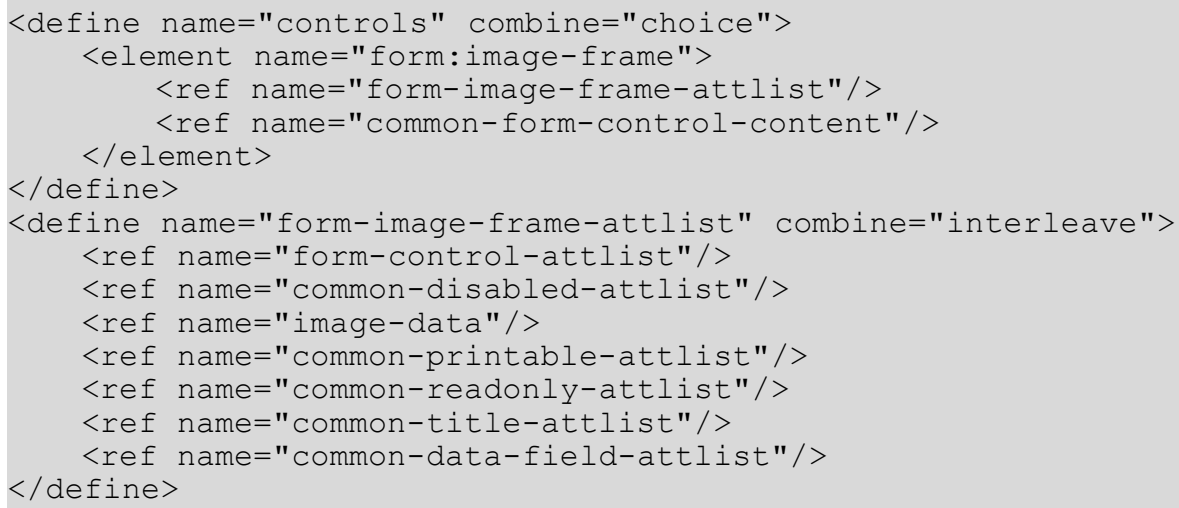

The attributes that may be associated with the $<$ form: image-frame> element are:

- Name and Service Name. See section 11.4 for information about these attributes.

- Control ID, Disabled, Image Data, Printable, Read only, and Title. See section 11.5 for information about these attributes.

- Data Field. See section 11.5.22 for information about this attribute.

\subsubsection{Hidden}

The <form: hidden> element defines a control that does not have a visual representation. This element is usually used as a container for information.

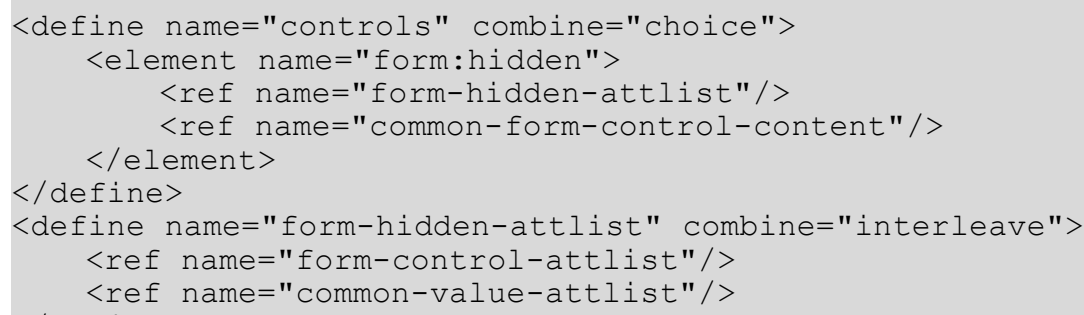

The attributes that may be associated with the <form: hidden $>$ element are:

- Name and Service Name. See section 11.4 for information about these attributes.

- Value. See section 11.5 for information about this attribute.

\subsubsection{Grid}

The <form:grid> element defines a control that displays table data. This control is data-aware and is bound to a form which retrieves data from a data source. The actual data to display in a grid control is determined by the parent form, which is data-aware and thus based on a certain row set. The rows in the grid contain these data rows.

Each column in the grid is specified by a $<$ form: column> element. Each column is bound to a field in the form's row set. 


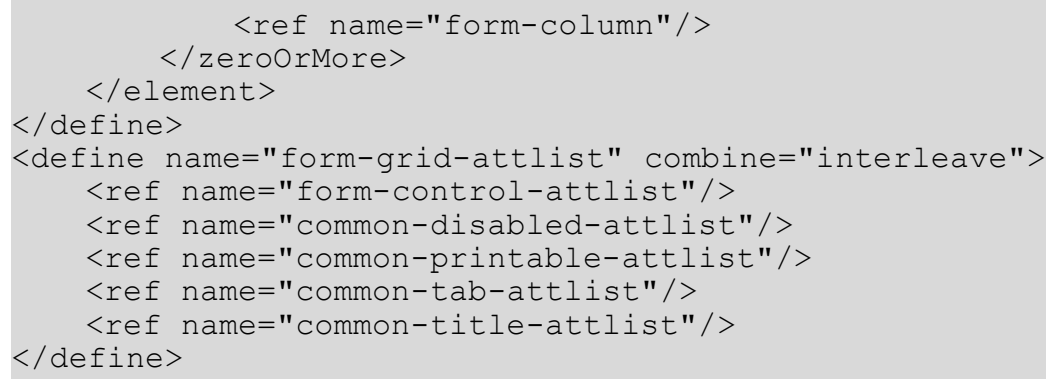

$$
</ \text { element }>
$$

The attributes that may be associated with the <form: grid> element are:

- Name and Service Name. See section 11.4 for information about these attributes.

- Control ID, Disabled, Printable, Tab Index, Tab Stop, and Title. See section 11.5 for information about these attributes.

\section{Column}

The <form: column> element defines a column in a grid control. The column contains a control that displays the grid data for the column.

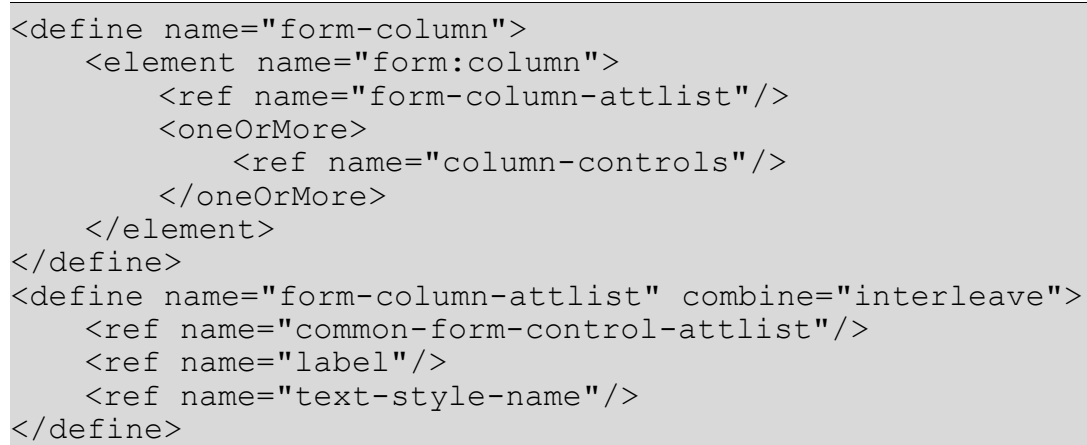

The attributes that may be associated with the <form: column> element are:

- Name and Service Name. See section 11.4 for information about these attributes.

- Label. See section 11.5 for information about this attribute.

- Column Style

\section{Column Style}

The form: text-style-name attribute specifies paragraph style that is applied to all controls with the column. See also section 9.2.12. Unlike other paragraph styles, this style may reference a data style.

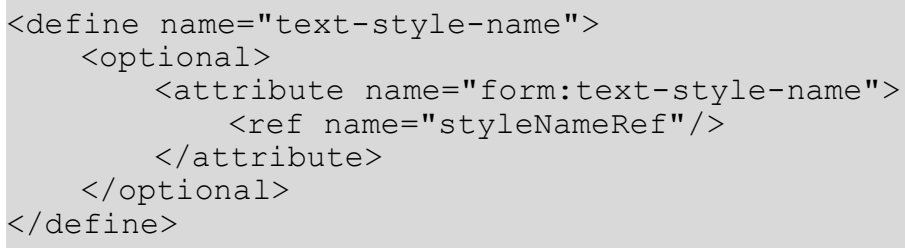




\subsubsection{Value Range}

The new <form:value-range> element defines a control which allows the user to select a value from a continuous number range. Possible representations include scroll bars and spin buttons.

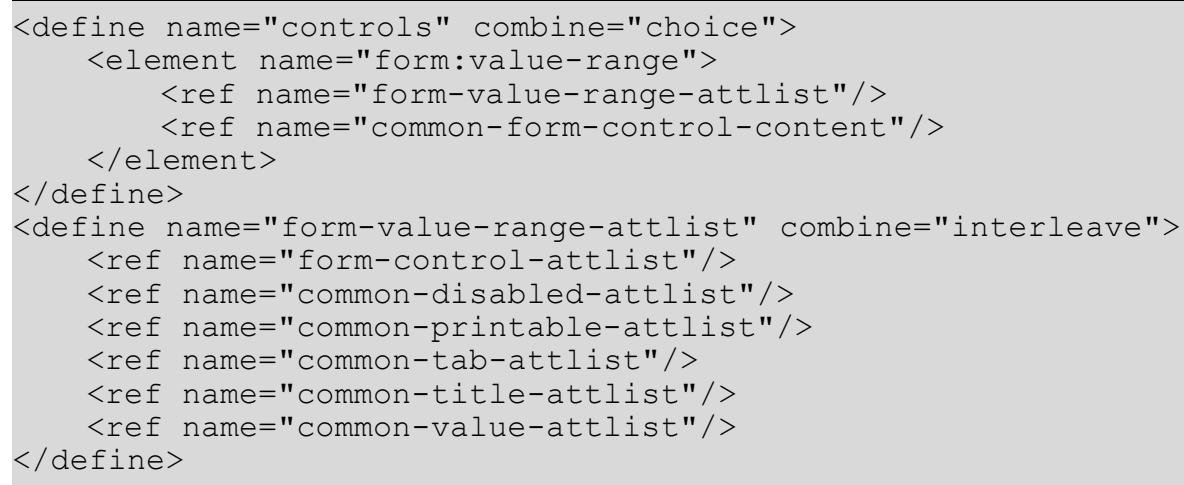

The attributes that may be associated with a <form:value-range> element are:

- Name and Service Name. See section 11.4 for information about these attributes.

- Control ID, Current Value, Disabled, Printable, Read only, Tab Index, Tab Stop, Title and Value. See section 11.5 for information about these attributes.

- Maximum Value

- Minumum Value

- Step Size

- $\quad$ Page Step Size

- Repeat Delay

- Orientation

\section{Maximum Value}

The form:max-value attribute specifies the maximum value that a user can enter.

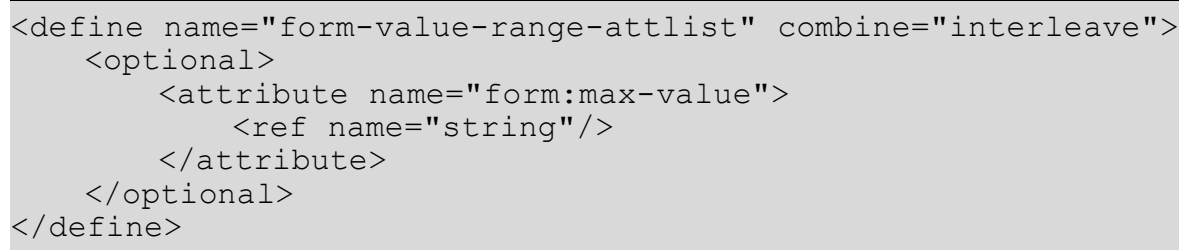

\section{Minimum Value}

The form:min-value attribute specifies the minimum value that a user can enter.

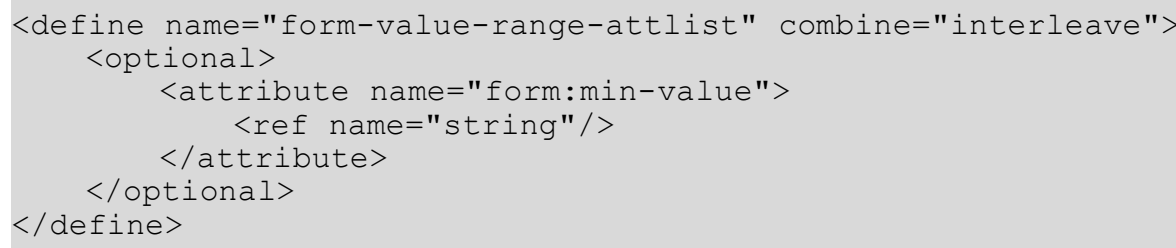




\section{Step Size}

The form:step-size attribute specifies the increment to be used for a control representing a value.

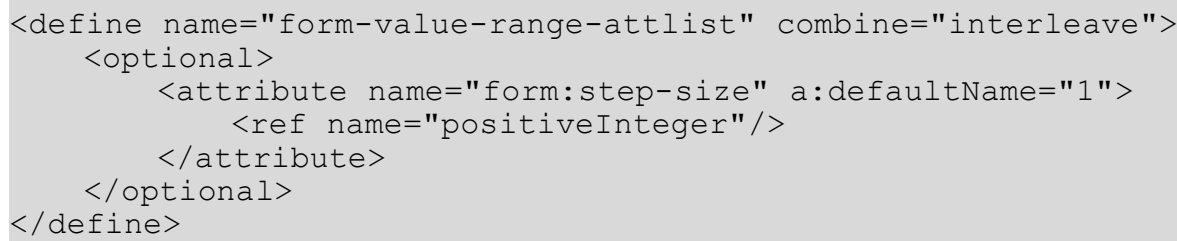

\section{Page Step Size}

The form:page-step-size attribute specifies a second-level increment to be used for a control representing a value. In the user interface, this is usually associated with the user pressing the "Page Up" or "Page Down" key.

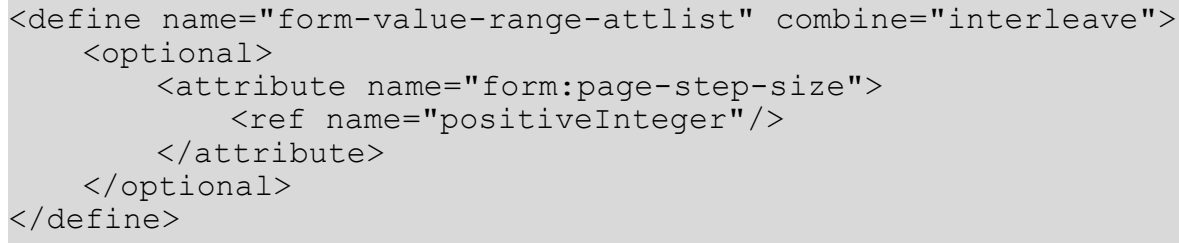

\section{Repeat Delay}

The form: delay-for-repeat attribute specifies a time-out to be used before a pressed mouse button results in repeating an action.

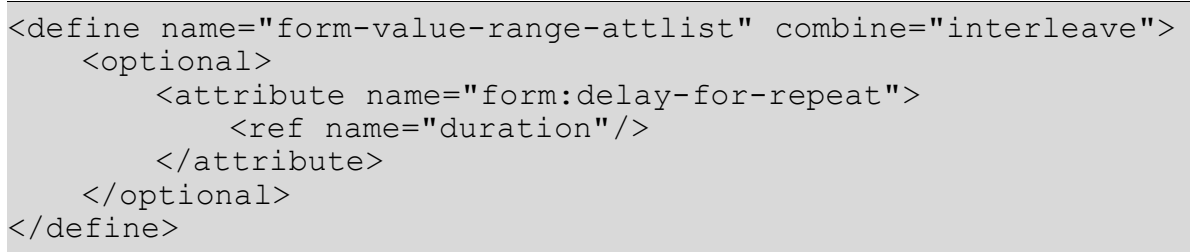

\section{Orientation}

The form: orientation attribute specifies the orientation of the control, which could be either horizontal or vertical.

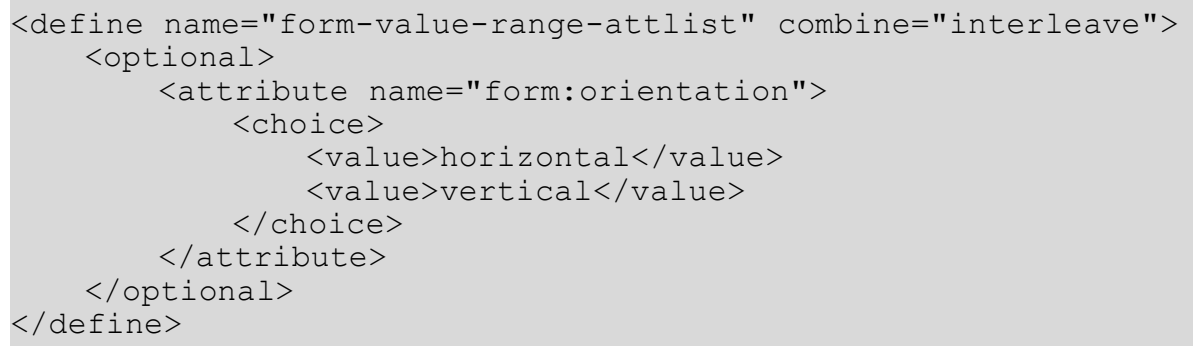




\subsubsection{Generic Control}

The <form: generic-control> element defines a placeholder for a generic control. The generic control can contain any properties and any events. The application detects the type of the control and instantiates the correct control.

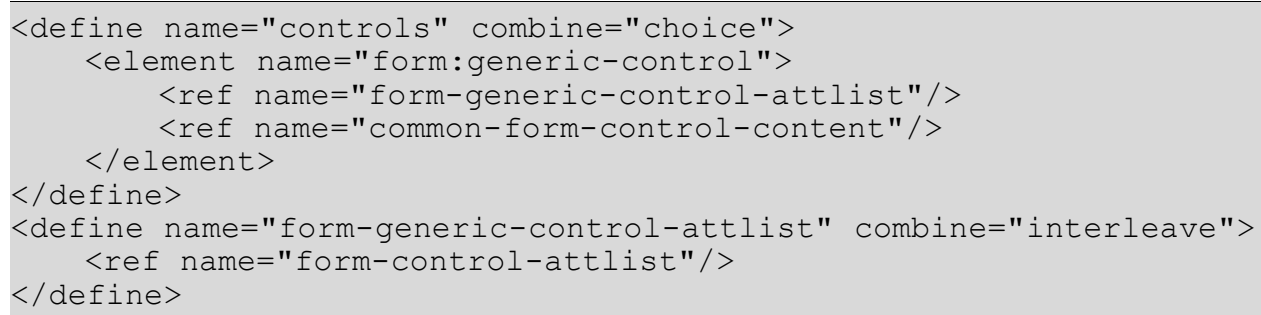

The attributes that may be associated with the <form:generic-control> element are:

- Name and Service Name. See section 11.4 for information about these attributes.

\subsection{Common Form and Control Attributes}

\subsubsection{Name}

The form: name attribute specifies the name of the form or control element. This may be used to give a form or control element an identity, which is important for scripting and for submitting the content of controls.

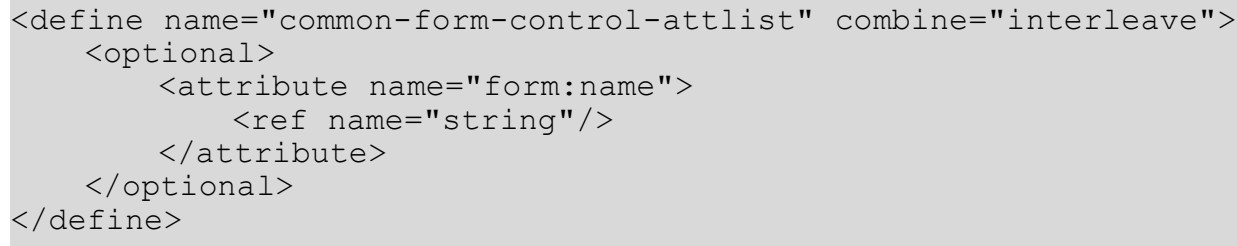

\subsubsection{Control Implementation}

A control may be given a control type attribute, which determines which concrete rendition or implementation the user agent should instantiate. For easy extensibility, the value of this attribute is a namespaced token, i.e. it is token using a namespace prefix, much like attributes in $\mathrm{XML}$.

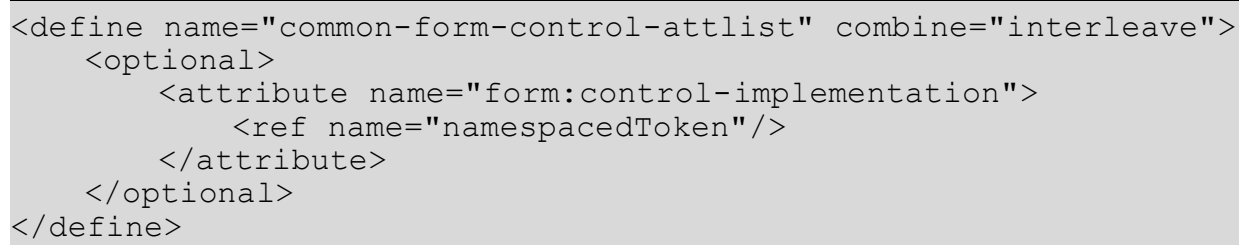

\subsubsection{Bind to XForms}

Any control can be bound to an XForms form (see section 11.2, as well as [XForms]) by using the $x$ forms:bind attribute. With buttons the bind attribute refers to an $<x$ forms:submission> element with the given ID. Pushing the button causes the appropriate XForms submission action to be performed. For all other control types, the xforms: bind 
attribute refers to an <xforms:bind> element with the given ID. Any such bound control reads and writes its data as determined by the appropriate bind element.

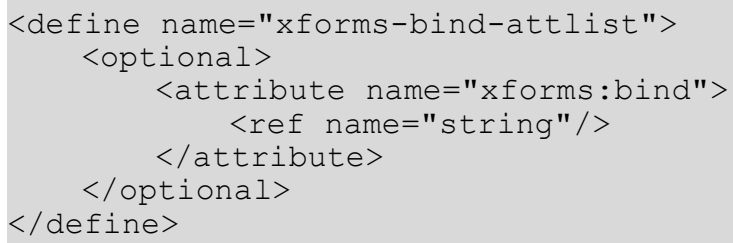

\subsection{Common Control Attributes}

\subsubsection{Button Type}

The form: button-type attribute specifies the type of a button. This attribute is supported for the following elements:

- <form:button>

- <form:image>

The value of this attribute can be one of the following:

- submit: Pressing the button submits the form.

- reset: Pressing the button resets every control in the form to its default value.

- push: Pressing the button does not perform any action by default. The use then can add scripts to the button. and the script is run when the button is pressed.

- url: Pressing the button loads the URL that is specified in the form:target-url attribute.

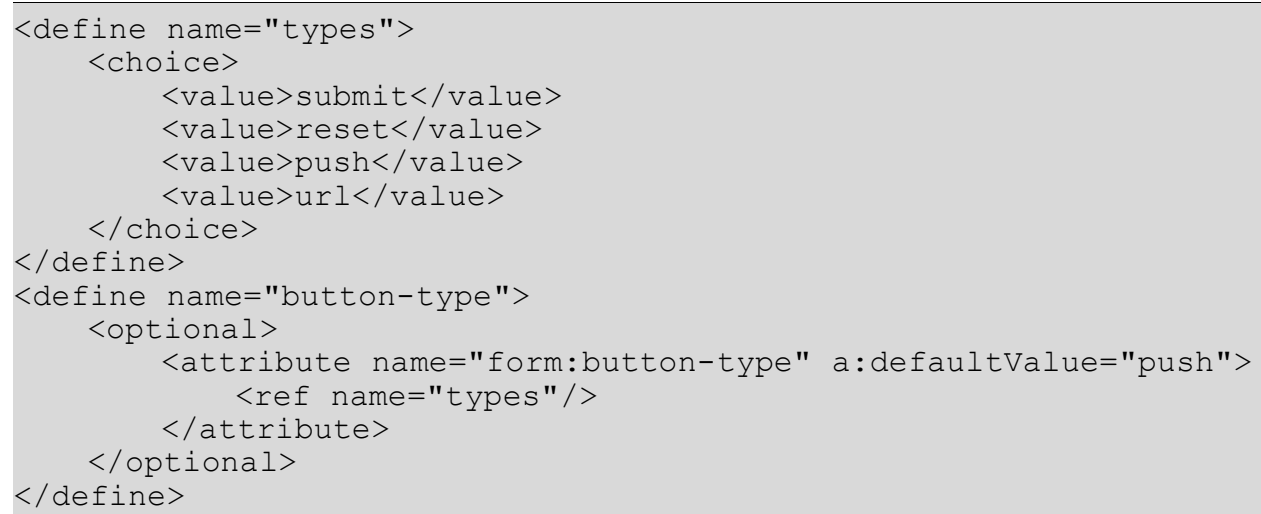

\subsubsection{Control ID}

All controls except Hidden Controls have a visual representation in the host document. Thus, they need an absolute or relative position, describing the location in the document. The position is represented by a shape that contains a reference to the control element within the form element.

The form: id attribute is used to uniquely identify a control element. Every control that is not hidden must have such an attribute associated with it, which in turn can be used to reference the control. 
This attribute is supported for the following elements:

- <form:text>

- <form:textarea>

- <form:password>

- <form:file>

- <form: formatted-text>

- <form:fixed-text>

- <form: combobox>

- <form:listbox>

- <form:button>

- <form:image>

- <form: checkbox>

- <form:radio>

- <form:frame>

- <form:image-frame>

- <form:grid>

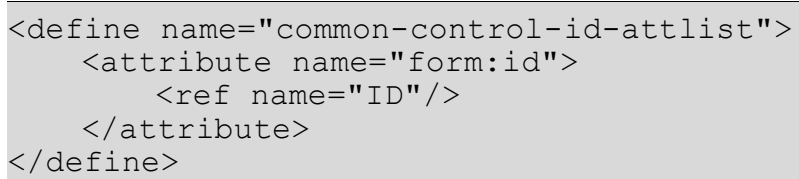

\subsubsection{Current Selected}

The form: current-selected attribute determines the current state of a radio button or option element.

This attribute is supported for the following elements:

- <form:option>

- <form:radio>

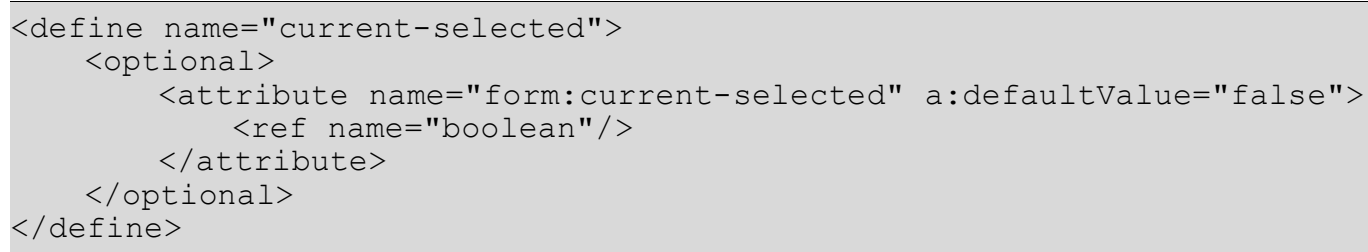

\subsubsection{Value and Current Value}

Every control has a default value and a current value. The current value changes with user interaction; the default value of a control does not. In general, the default value is specified in a form: value attribute. 
The default value is used during special events, such as resetting the form, which transfers the default value of every control to it's current value. If a control does not have an default value, the result of resetting the form is undefined.

Besides storing the current value together with the control, it is also possible to bind controls to other value providers, which act as value sink and source, such as database fields (in dataaware forms) or e.g. cells in a spreadsheet document the controls live in. In this case, the current value is not stored with the control itself, but in the external instance, which may or may not store it together with the document. See section 11.5.22 for more details on database properties.

\section{Default Value}

The form:value attribute specifies the default value of an input control. This attribute is supported for the following elements:

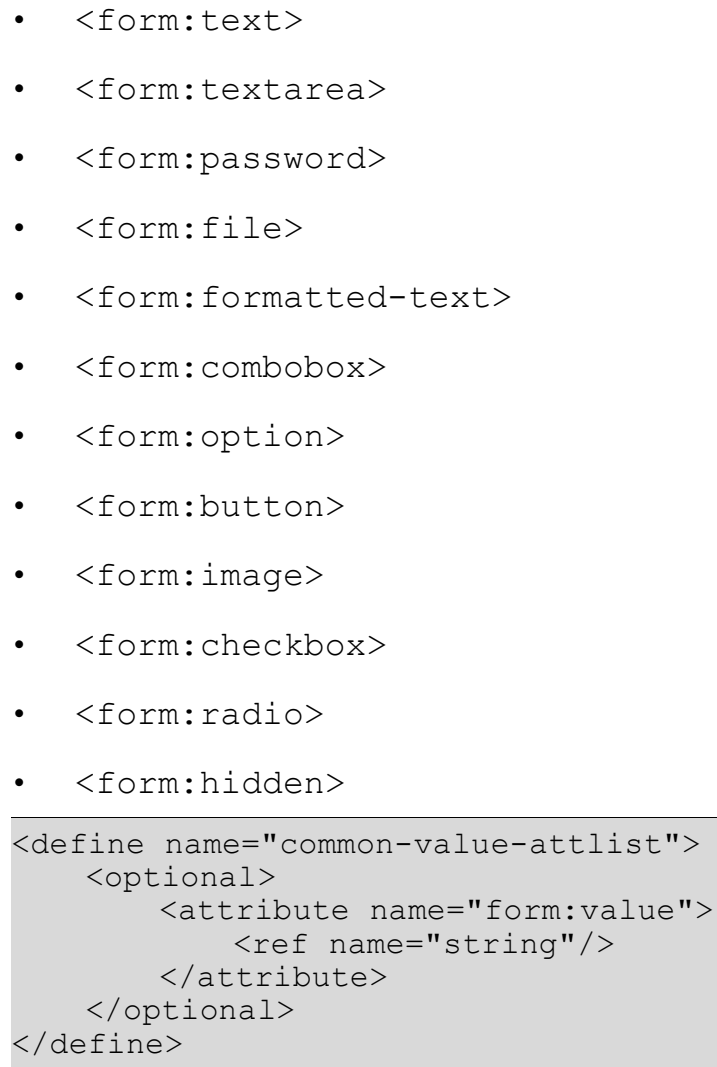

\section{Current Value}

The form: current-value attribute specifies the current status of an input control. It overrides the value of a form: value attribute, if one is present.

This attribute is supported for the following elements:

- <form:text>

- <form:textarea>

- <form:file>

- <form: formatted-text> 
- $\quad$ fform: combobox >

\subsubsection{Disabled}

The form: disabled attribute specifies whether or not a control can accept user input. This attribute is supported for the following elements:

- $\quad$ form:text>

- <form:textarea>

- <form:password>

- <form:file>

- <form: formatted-text>

- $\quad$ fform:fixed-text>

- <form: combobox>

- <form:listbox>

- $\quad<$ form: button>

- <form:image>

- <form: checkbox>

- <form:radio>

- <form: frame>

- <form:image-frame>

- <form:grid>

Controls that are disabled are not included in the tabbing navigation sequence and can not be focused.

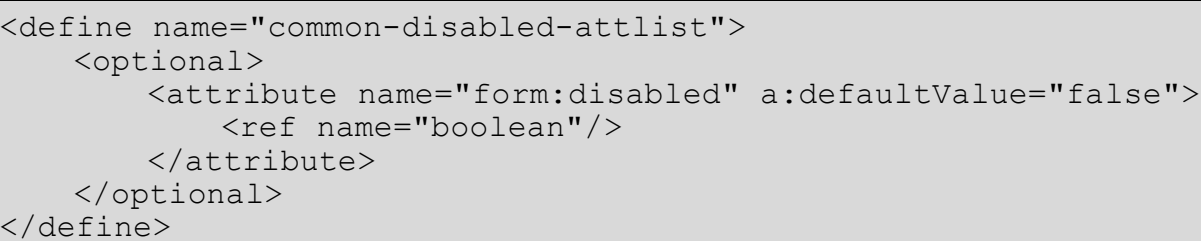

\subsubsection{Dropdown}

The form: dropdown attribute specifies whether the list in a combo box or list box is always visible or is only visible when the user clicks the drop-down button. This attribute is supported for the following elements: 
- <form:combobox >

- <form:listbox>

If the value is true, the list is always visible. If the value is false, the list is only visible when the user clicks the drop-down button.

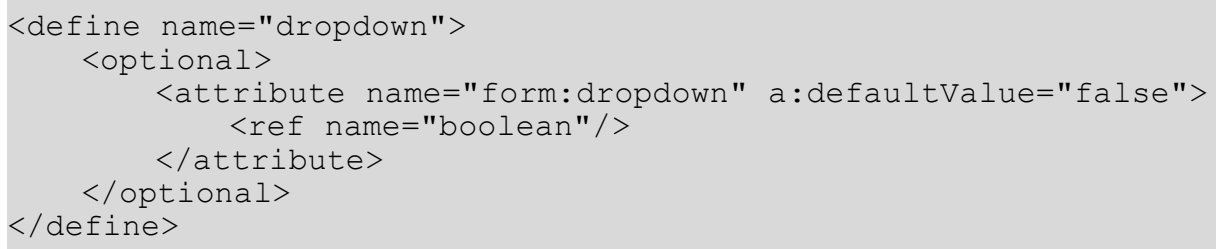

\subsubsection{For}

The form: for attribute specifies the IDs of the controls with which control element is labeling. This attribute is supported for the following elements:

- <form:fixed-text>

- <form:frame>

This attribute contains a comma separated list of control IDs.

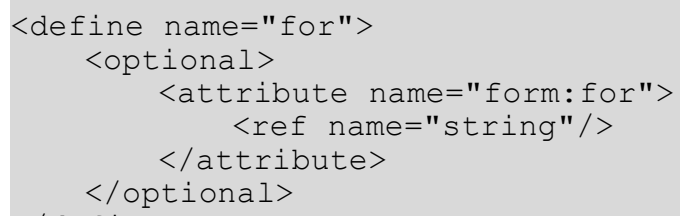

\subsubsection{Image Data}

The form: image-data attribute links the control to an external file containing image data. This attribute is supported for the following elements:

- <form:button>

- <form:image>

- <form:image-frame>

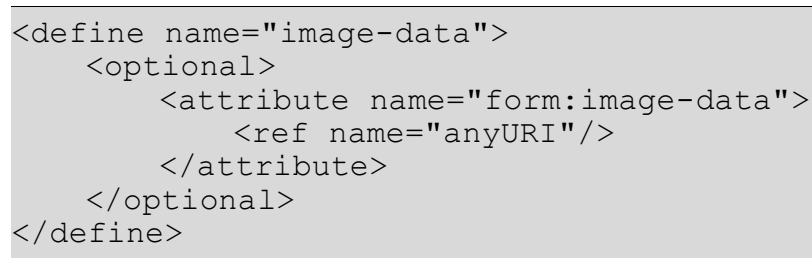

\subsubsection{Label}

The form: label attribute contains a label for a control such as a radio button or check box. This attribute is supported for the following elements:

- <form:fixed-text>

- <form:item> 
- <form:option>

- <form: checkbox>

- <form:radio>

- <form:frame>

- <form:column>

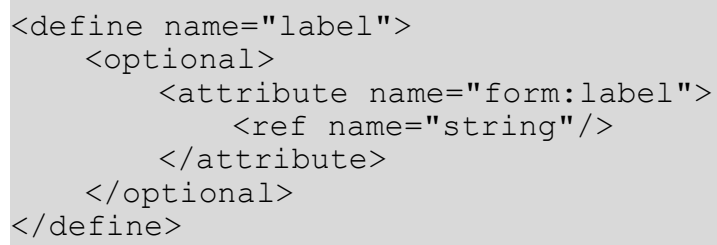

\subsubsection{Maximum Length}

The form:max-length attribute specifies the maximum number of characters that a user can enter in an input control. This attribute is supported for the following elements:

- <form:text>

- <form:textarea>

- <form:password>

- <form: formatted-text>

- $\quad$ <form: combobox>

The default value of this attribute is unlimited, which allows a user to enter an unlimited number of characters.

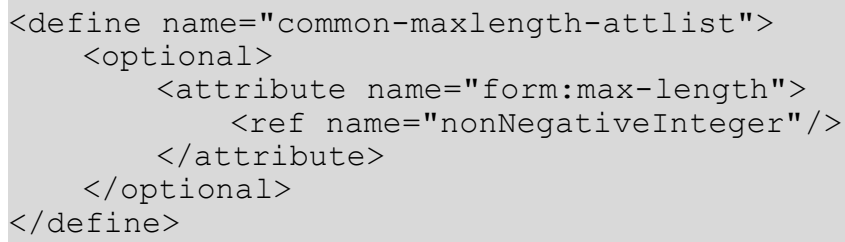

\subsubsection{Printable}

The form:printable attribute specifies whether or not a control is printed when a user prints the document in which the control is contained. This attribute is supported for the following elements:

- <form:text>

- <form:textarea>

- <form:password>

- <form:file>

- <form: formatted-text>

- <form:fixed-text> 
- <form: combobox>

- <form:listbox>

- <form:button>

- <form:image>

- <form: checkbox>

- <form:radio>

- <form:frame>

- <form:image-frame>

- <form:grid>

<define name="common-printable-attlist"> <optional>

<attribute name="form:printable" a:defaultValue="true"> $<$ ref name="boolean"/>

$</$ optional $>$ $</$ attribute $>$

$</$ define $>$

\subsubsection{Read only}

The form:readonly attribute specifies whether or not a user can modify the value of a control. This attribute is supported for the following elements:

- $\quad$ form:text>

- <form:textarea>

- <form:file>

- <form: formatted-text>

- <form: combobox>

- <form:listbox>

- <form:image-frame>

Read-only controls are included in the tabbing navigation sequence.

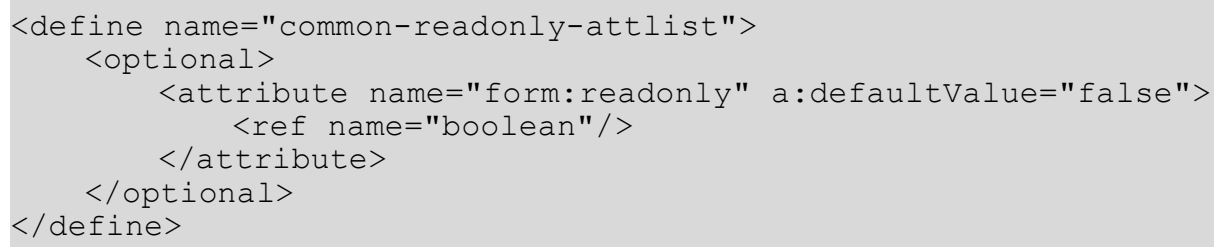

\subsubsection{Selected}

The form: selected attribute specifies the default state of a radio button or option. When the control is initialized, it is in the default state specified by this attribute. This attribute is supported for the following elements:

- <form:option> 
- <form:radio>

In a group of radio buttons that share the same name, only one radio button can have this attribute set to true.

\subsubsection{Size}

The form:size attribute specifies the number of rows that are visible at a time in a combo box list or a list box list. This attribute is supported for the following elements:

- <form: combobox>

- <form:listbox>

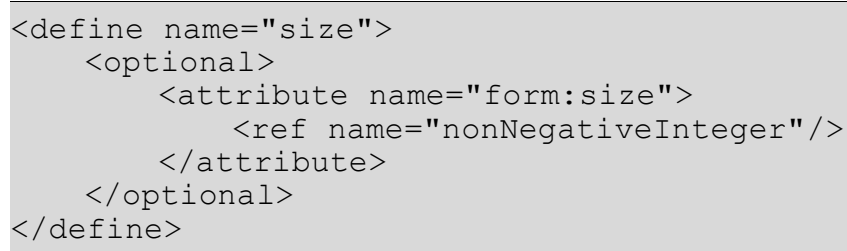

\subsubsection{Tab Index}

The form:tab-index attribute specifies the tabbing navigation order of a control within a form. The tabbing order is the order in which controls are given focus when a user navigates through the form using the TAB key on the keyboard. The tabbing order can include elements that are nested in other elements. This attribute is supported for the following elements:

- <form:text>

- <form:textarea>

- <form:password>

- <form:file>

- <form: formatted-text>

- <form:combobox>

- <form:listbox>

- <form:button>

- <form:image>

- <form: checkbox>

- <form:radio>

- <form:grid>

The rules for tabbing are similar to the tabbing rules used in HTML 4.0. 
Controls that can be given focus are navigated in the order described in the following rules:

1. The controls that have a positive value for the form:tab-index attribute are navigated first.

2. The navigation starts at the control with lowest form:tab-index value and ends at the control with the highest value. Values do not have to be sequential and they do not have to begin with a particular value.

3. Controls that have the same values for the form:tab-index attribute are navigated according their position in the form.

4. Controls that do not contain the form:tab-index attribute or contain the attribute with a value of 0 are navigated next. These controls are navigated according to their position in the form.

5. Controls that have the form: disabled attribute set to true are not included in the navigation, independent on their form:tab-index value.

\subsubsection{Tab Stop}

The form: tab-stop attribute specifies whether or not a control is included in the tabbing navigation order. This attribute is supported for the following elements:

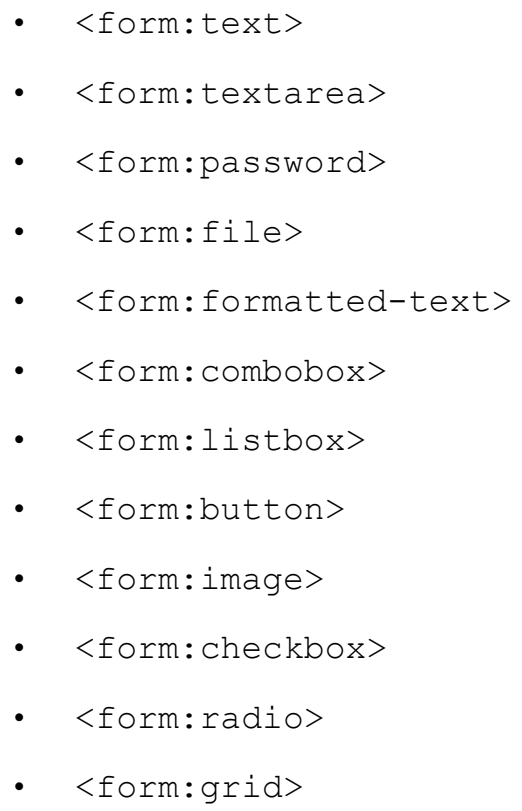

If the value is false, the control is not included in the tabbing navigation.

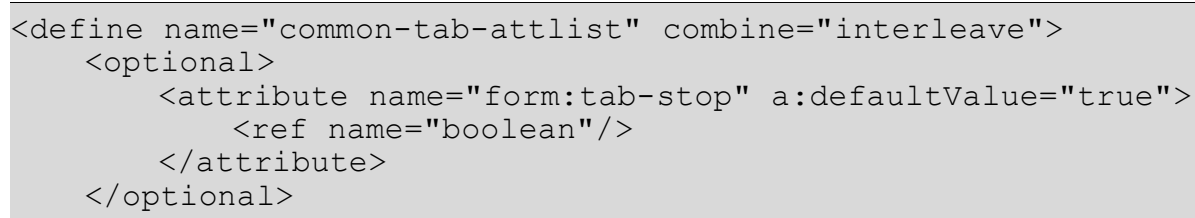




\subsubsection{Target Frame}

The office:target-frame attribute specifies the link target frame of the area. This attribute is supported for the following elements:

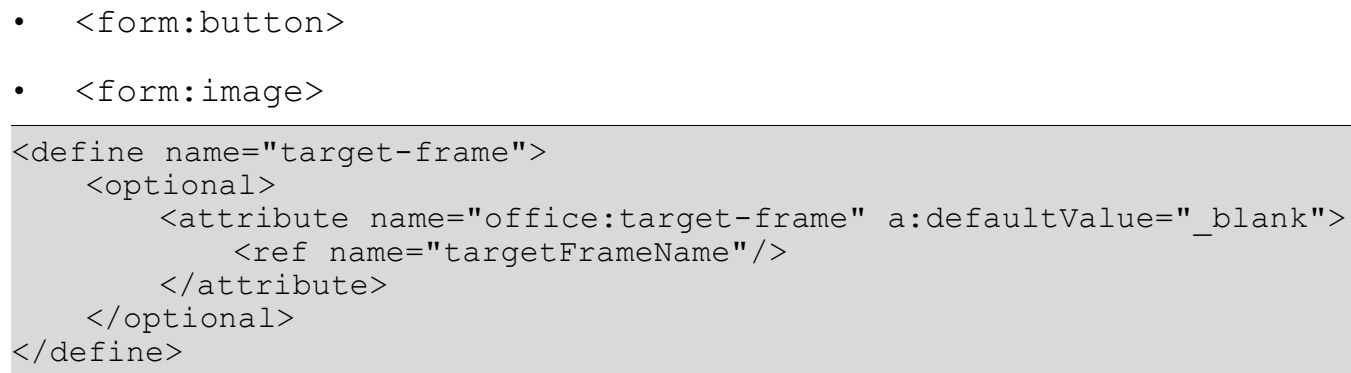

\subsubsection{Target Location}

An xlink: href attribute specifies the URL that is loaded if a button is clicked. This attribute is supported for the following elements:

- <form:button>

- <form:image>

This attribute is only evaluated if the value of the form:button-type attribute is location.

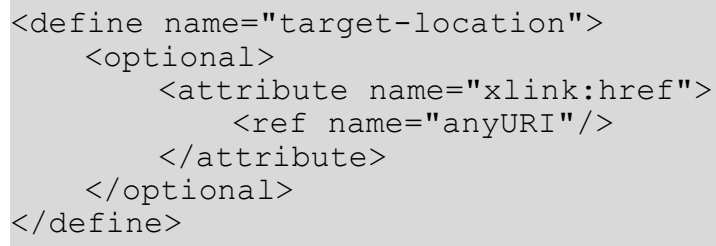

\subsubsection{Title}

The form: title attribute contains additional information about a control. The value of the attribute can be used as a tool tip. This attribute is supported for the following elements:

- <form:text>

- <form:textarea>

- <form:password>

- <form:file>

- <form: formatted-text>

- <form:fixed-text>

- <form: combobox>

- <form:listbox>

- <form:button> 
- <form:image>

- <form: checkbox>

- <form:radio>

- <form:image>

- <form:image-frame>

- <form:grid>

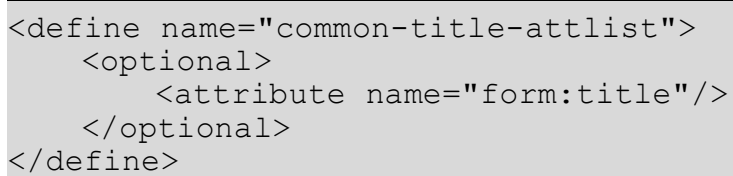

\subsubsection{Visual Effect}

The form:visual-effect attributes specifies a visual affect to apply to a control. The attribute values can be flat for a flat visual effect and $3 d$ for a 3D effect. This attribute is supported for the following elements:

- <form: checkbox>

- <form:radio>

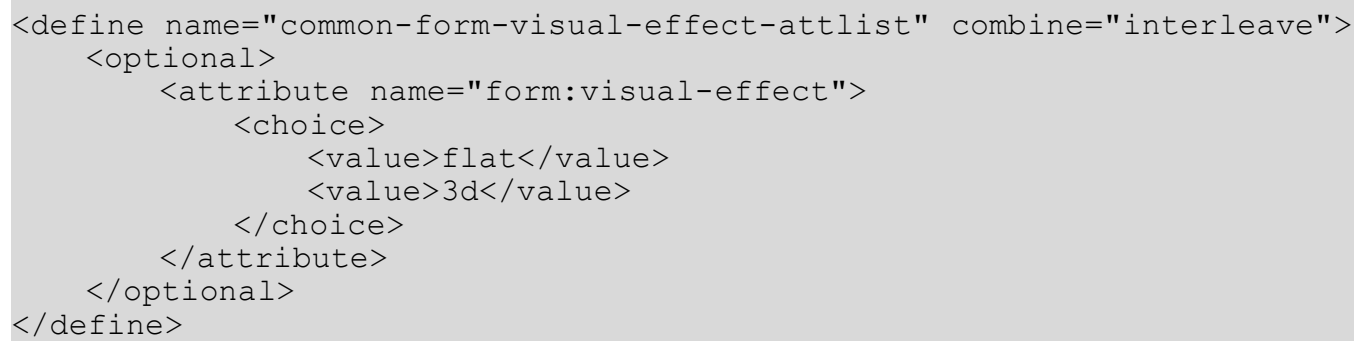

\subsubsection{Relative Image Position}

The form: image-position and form: image-align together specify the position of an image to be displayed in a form control, relative to the label text.

If the form: image-position attribute has the value center, the image shown in a control should be centered relative to the control's text.

If the form:image-position attribute has one of the values start, end, top, bottom, the image is to be placed before, after, above, or below the text. In this case, the form: imagealign attribute specifies which border (start, end) or axis (center) of the image and the text are to be aligned. If the form:image-position attribute is not present, it is assumed to be center. The form:image-position and form: image-align attributes are supported for the following elements:

- <form:button>

- $\quad$ fform: checkbox>

- <form:radio> 


\subsubsection{Database Binding Attributes}

A control may be bound to a database fields. In this case, the controls becomes data-aware. The control acquires the values of a database field by going through a result set that is provided by the form. Each time there is a row change in the form, the value of the control may change. The value changes are stored in the associated database field.

\section{Bound Column}

The form:bound-column attribute specifies the column values of the list source result set that are used to fill the data field values. This attribute is supported for the $<$ form: listbox $>$ element.

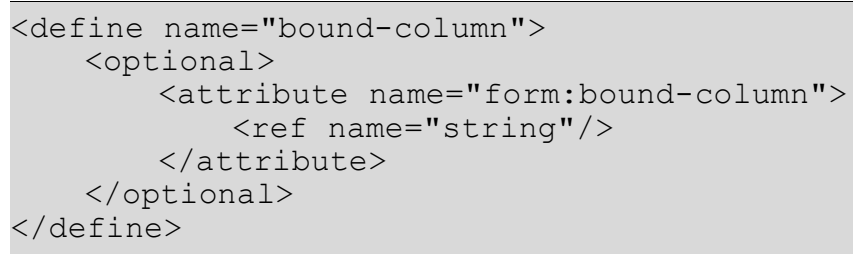

\section{Convert Empty To Null}

The form:convert-empty-to-null attribute specifies whether or not empty current values are regarded as NULL This attribute is important for data-aware controls to determine which values to store for the bound database field. This attribute is supported for the following elements:

- <form:text>

- <form:textarea> 
- <form:formatted-text>

- <form: combobox>

If the value of the attribute is true, an empty string in the control is regarded as the dedicated NULL value. If the value of the attribute is false, an empty string in the control is regarded as an empty string.

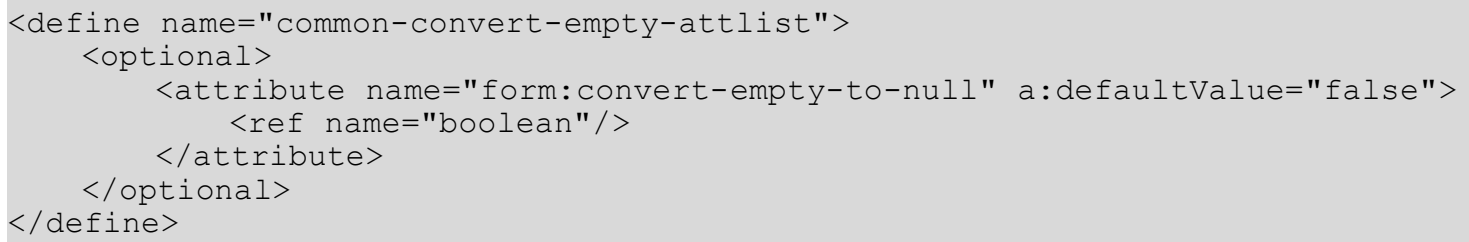

\section{Data Field}

The form: data-field attribute specifies the name of a result set column. The result set is determined by the form which the control belongs to. This attribute is supported for the following elements:

- <form:text>

- <form:textarea>

- <form: formatted-text>

- <form: combobox>

- $\quad<$ form:listbox $>$

- <form:checkbox>

- <form:radio>

- <form:image-frame>

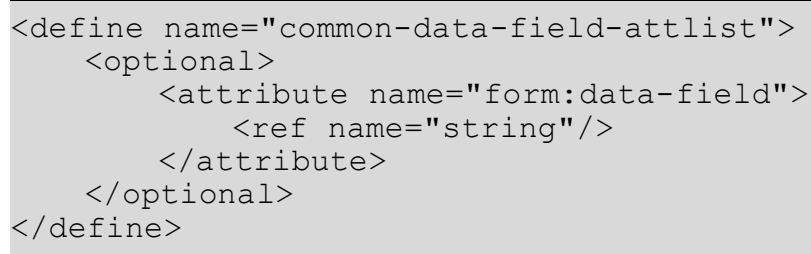

\section{List Source}

The form: list-source attribute specifies the source used to populate the list in a list box or combo box. The first column of the list source result set populates the list. This attribute is supported for the following elements:

- <form: combobox>

- <form:listbox>

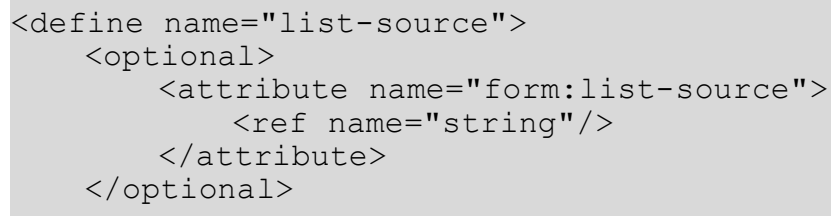




\section{List Source Type}

The form:list-source-type attribute specifies the type of data source that is used to populates the list data in a list box or combo box. This attribute is supported for the following elements:

- <form: combobox>

- $\quad<$ form:listbox>

The value of this attribute can be one of the following:

- table: The list is populated using the content of a database table.

- query: The list is populated by executing a query.

- $\quad$ sql: The list is populated by executing an SQL statement.

- sql-pass-through: The list is populated by executing any type of statement that is passed directly to a database driver, without being interpreted by the application.

- value-list: The list is populated with values specified by the user using the form:value attribute in the <form: option> element. This setting is only applicable to list boxes.

- table-fields: The list is populated using the field names in a database table.

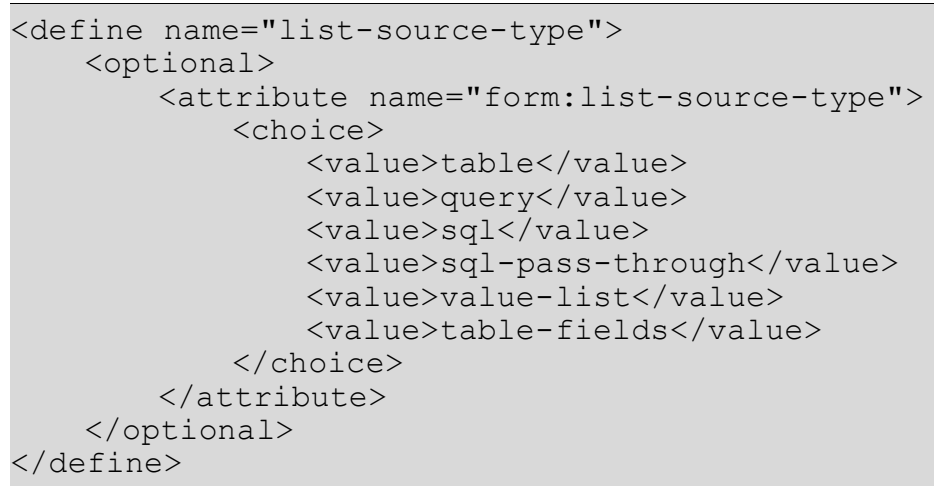

\subsection{Events}

HTML defines a list of standard events for controls. These events are represented by attributes, which are associated with the control elements. In the office application XML file format, these events and any additional events defined by the application component are stored as elements in an <office:event-listeners> element.

For a single event element, the script:event-name attribute specifies the type of event and other attributes specify the language and the event handler.

\subsubsection{Events with an Equivalent HTML Event Type}

The following table describes the XML events that have an equivalent event in HTML. Their names are contained in the namespace "http://www.w3.org/2001/xml-events". The namespace prefix used in this specification is DOM. See also 12.4.1. 


\begin{tabular}{|c|c|c|}
\hline $\begin{array}{l}\text { Value of script:event- } \\
\text { name Attribute }\end{array}$ & $\begin{array}{l}\text { Equivalent } \\
\text { HTML Event }\end{array}$ & Description of Event \\
\hline dom: change & onchange & $\begin{array}{l}\text { Occurs when a control is no longer focussed } \\
\text { and the value of the control was modified } \\
\text { since it was given focus. }\end{array}$ \\
\hline dom:DOMFocus In & onfocus & $\begin{array}{l}\text { Occurs when a control is given focus using the } \\
\text { mouse or the TAB key. }\end{array}$ \\
\hline dom: DOMFocusOut & onblur & $\begin{array}{l}\text { Occurs when a control is no longer focussed } \\
\text { as a result of moving the mouse or by tabbing } \\
\text { navigation. It may be used with the same } \\
\text { elements as form:on-focus. }\end{array}$ \\
\hline dom: keydown & onkeydown & Occurs when a key is pressed on a control. \\
\hline dom: keyup & on keyup & Occurs when a key is released on a control. \\
\hline dom:mouseover & onmouseover & $\begin{array}{l}\text { Occurs when the mouse pointer is moved over } \\
\text { the control. }\end{array}$ \\
\hline dom:mousemove & onmousemove & $\begin{array}{l}\text { Occurs when the mouse pointer is moved onto } \\
\text { a control. }\end{array}$ \\
\hline dom:mousedown & onmousedown & $\begin{array}{l}\text { Occurs when a mouse button is pressed on a } \\
\text { control. }\end{array}$ \\
\hline dom:mouseup & onmouseup & $\begin{array}{l}\text { Occurs when a mouse button is released on a } \\
\text { control. }\end{array}$ \\
\hline on-mouseout & onmouseout & $\begin{array}{l}\text { Occurs when the mouse pointer is moved } \\
\text { away from a control. }\end{array}$ \\
\hline dom: reset & onreset & Occurs when a form is reset. \\
\hline dom: submit & onsubmit & Occurs when a form is submitted. \\
\hline
\end{tabular}

\subsubsection{Event Types}

In addition to the HTML event types, the XML file format for office applications allows additional events to be handled at run time.

\begin{tabular}{|l|l|l|}
\hline $\begin{array}{c}\text { Value of script:event- } \\
\text { name Attribute }\end{array}$ & \multicolumn{1}{|c|}{ Applies To } & \multicolumn{1}{c|}{ Description of Event } \\
\hline form: approveaction & Button or image. & $\begin{array}{l}\text { Occurs before the on-performaction event } \\
\text { takes place. Allows the user to veto the action. }\end{array}$ \\
\hline form: performaction & Button or image. & $\begin{array}{l}\text { Occurs when the control action is to be } \\
\text { performed. The common interpretation of this } \\
\text { event is "pressing the button". }\end{array}$ \\
\hline form: textchange & $\begin{array}{l}\text { All controls that } \\
\text { allow text input. }\end{array}$ & $\begin{array}{l}\text { Occurs when a user changes the text in a } \\
\text { control. }\end{array}$ \\
\hline form: itemstatechange & $\begin{array}{l}\text { Check box or } \\
\text { radio button. }\end{array}$ & $\begin{array}{l}\text { Occurs when the state of a check box or radio } \\
\text { button changes. }\end{array}$ \\
\hline
\end{tabular}




\begin{tabular}{|c|c|c|}
\hline $\begin{array}{l}\text { Value of script:event- } \\
\text { name Attribute }\end{array}$ & Applies To & Description of Event \\
\hline form:mousedrag & All controls. & $\begin{array}{l}\text { Occurs when a user presses and holds one of } \\
\text { the mouse buttons and moves the mouse } \\
\text { pointer onto a control. }\end{array}$ \\
\hline form:approvereset & $\begin{array}{l}\text { same objects as } \\
\text { for form:on-reset }\end{array}$ & $\begin{array}{l}\text { Occurs before the on-reset event takes place. } \\
\text { Allows the user to veto the reset event. }\end{array}$ \\
\hline form:approveupdate & $\begin{array}{l}\text { All controls that } \\
\text { can be bound to a } \\
\text { database field, } \\
\text { that is controls } \\
\text { that contain the } \\
\text { data-field } \\
\text { attribute. }\end{array}$ & $\begin{array}{l}\text { Occurs before the on-update event takes } \\
\text { place. Allows the user to veto the update. }\end{array}$ \\
\hline form:update & $\begin{array}{l}\text { All controls that } \\
\text { can be bound to a } \\
\text { database field, } \\
\text { that is controls } \\
\text { that contain the } \\
\text { data-field } \\
\text { attribute. }\end{array}$ & $\begin{array}{l}\text { Occurs when the content of a control that is } \\
\text { bound to a database field is committed. }\end{array}$ \\
\hline form:load & Forms. & $\begin{array}{l}\text { Occurs when the form establishes a } \\
\text { connection to the data source. }\end{array}$ \\
\hline form:startrealod & Forms. & $\begin{array}{l}\text { Occurs when the form is about to refresh a } \\
\text { data source connection. }\end{array}$ \\
\hline form:reload & Forms. & $\begin{array}{l}\text { Occurs when the form has refreshed a data } \\
\text { source connection. }\end{array}$ \\
\hline form:startunload & Forms. & $\begin{array}{l}\text { Occurs when the form is about to drop a data } \\
\text { source connection. }\end{array}$ \\
\hline form:unload & Forms. & $\begin{array}{l}\text { Occurs when the form has dropped a data } \\
\text { source connection. }\end{array}$ \\
\hline form:confirmdelete & Forms. & $\begin{array}{l}\text { Occurs when the user is about to delete a } \\
\text { record. }\end{array}$ \\
\hline $\begin{array}{l}\text { form: approverowchang } \\
\text { e }\end{array}$ & Forms. & $\begin{array}{l}\text { Occurs before the on-rowchange event takes } \\
\text { place. Allows the user to veto the change. }\end{array}$ \\
\hline form:rowchange & Forms. & $\begin{array}{l}\text { Occurs after changes to a row are complete, } \\
\text { such as deletions, updates, and insertions. }\end{array}$ \\
\hline $\begin{array}{l}\text { form: approvecursormo } \\
\text { ve }\end{array}$ & Forms. & $\begin{array}{l}\text { Occurs before the form is moved to another } \\
\text { row. Allows the user to veto the move. }\end{array}$ \\
\hline form: cursormove & Forms. & $\begin{array}{l}\text { Occurs after the form is moved to another } \\
\text { row. }\end{array}$ \\
\hline form:supplyparameter & Forms. & $\begin{array}{l}\text { Occurs when the form needs to fill parameters } \\
\text { to connect to a data source. }\end{array}$ \\
\hline
\end{tabular}




\begin{tabular}{|l|l|l|}
\hline $\begin{array}{c}\text { Value of script:event- } \\
\text { name Attribute }\end{array}$ & \multicolumn{1}{|c|}{ Applies To } & \multicolumn{1}{c|}{ Description of Event } \\
\hline form: error & $\begin{array}{l}\text { Forms, combo } \\
\text { boxes and list } \\
\text { boxes. }\end{array}$ & Occurs when a database-related error occurs. \\
\hline form: adjust & Value Range & $\begin{array}{l}\text { Occurs when the value of a Value Range } \\
\text { element has been adjusted. }\end{array}$ \\
\hline
\end{tabular}

\subsection{Properties}

The <form:properties $>$ element may be used to store the following settings for controls and forms:

- Settings that are not known by the document format.

- Settings that are provided by external vendors.

- Settings that are specific to the application.

Properties consist of a name/value pair. The name identifies the property. The value can be given in a fundamental data type or as a list of fundamental data types.

\subsubsection{Property Set}

The <form:properties $>$ element contains the property elements. Properties are encoded using the form:property element, except for list properties, which make use of the form:list-property element.

\section{Property Name}

The form:property-name attribute specifies the name of a property element.

10031

10032

10033

10034 


\section{Property Value and Type}

The value and type of form properties are represented through the common office:valuetype and suitable value attributes. See section 6.7 .1 for more information on these attributes.

In addition to these value types, form properties can also be empty. This is represented by the special value type void. Such properties have no value attribute.

\section{List Value}

The list value element contains value attributes for the value type given in the containing $<$ form: list-property > element. 


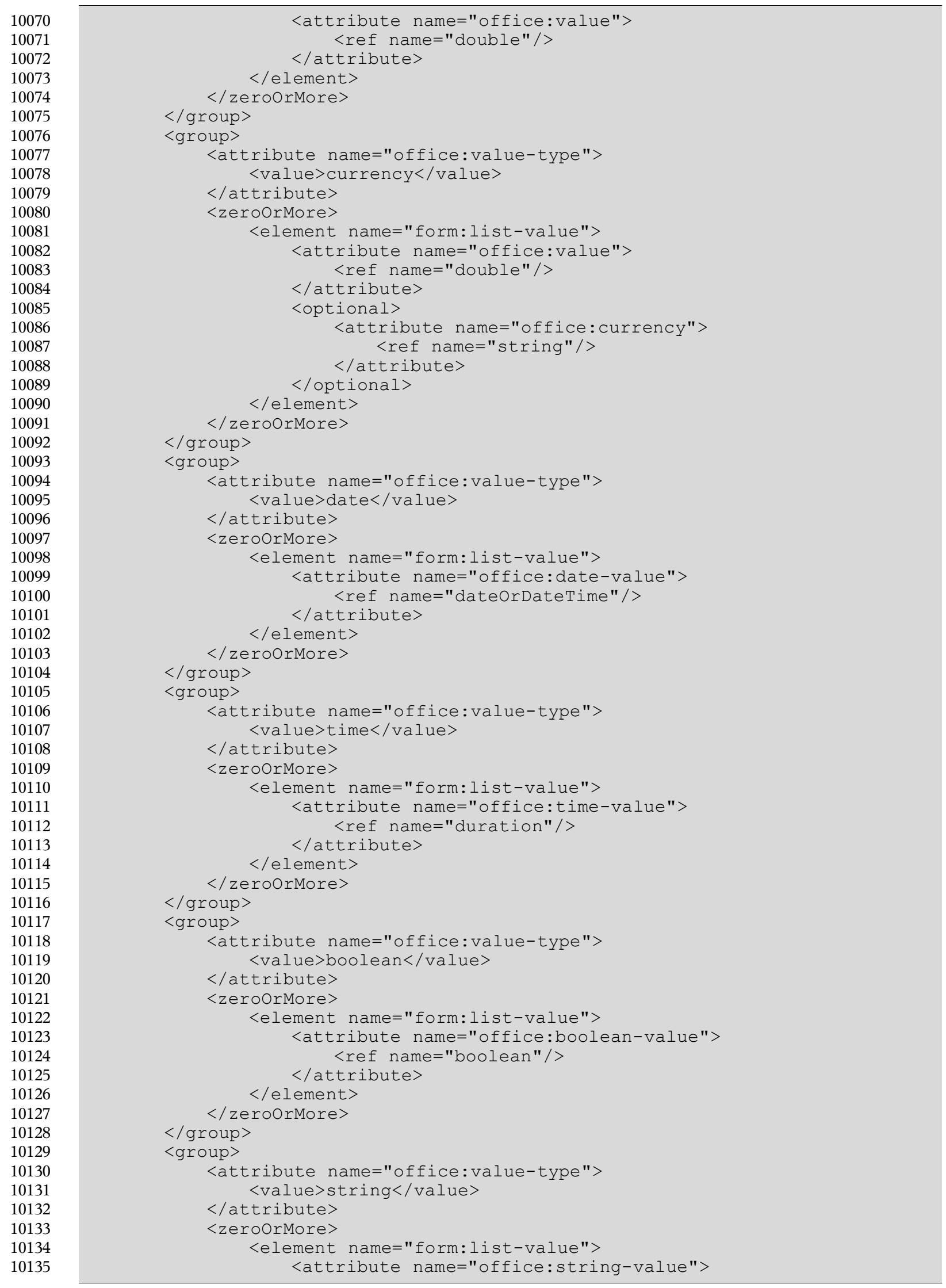




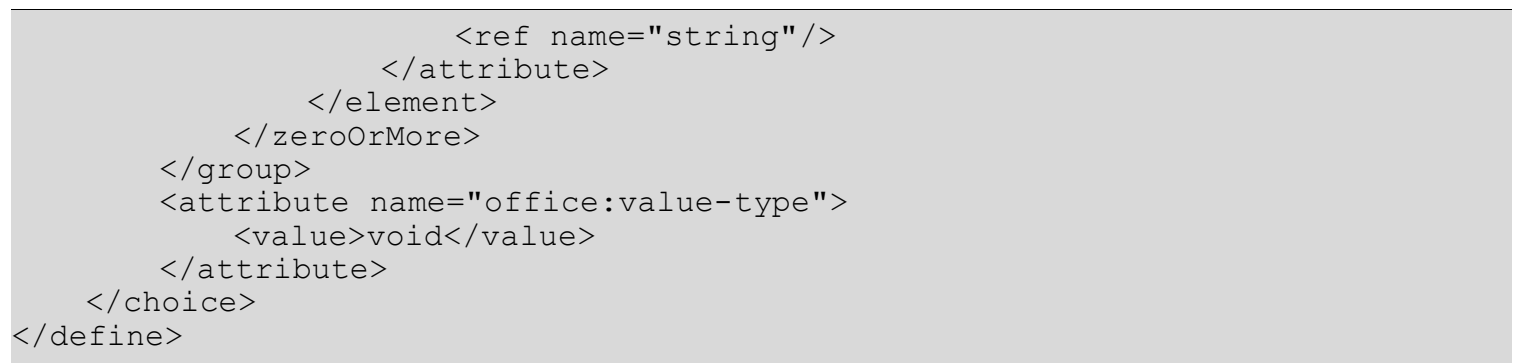

\section{Example: Form properties}

The following contains a string property "Name" with value "Name 1", and a string list property "Items" containing the strings "Item 1", "Item 2", "Item 3".

$<$ form:properties>

<form:property form:property-name="Name" office:value-type="string" office:string-value="Name 1">

<form:list-property form:property-name="Items" office:value-type="string" > <form:list-value office:string-value="Item $1 " />$ <form:list-value office:string-value="Item 2"/> <form:list-value office:string-value="Item $3 " />$ $</$ form:list-property $>$

$</$ form:properties $>$ 


\section{Common Content}

\subsection{Annotation}

The <office: annotation> element specifies an OpenDocument annotation. The annotation's text is contained in <text: $p>$ and $<$ text: list $>$ elements.

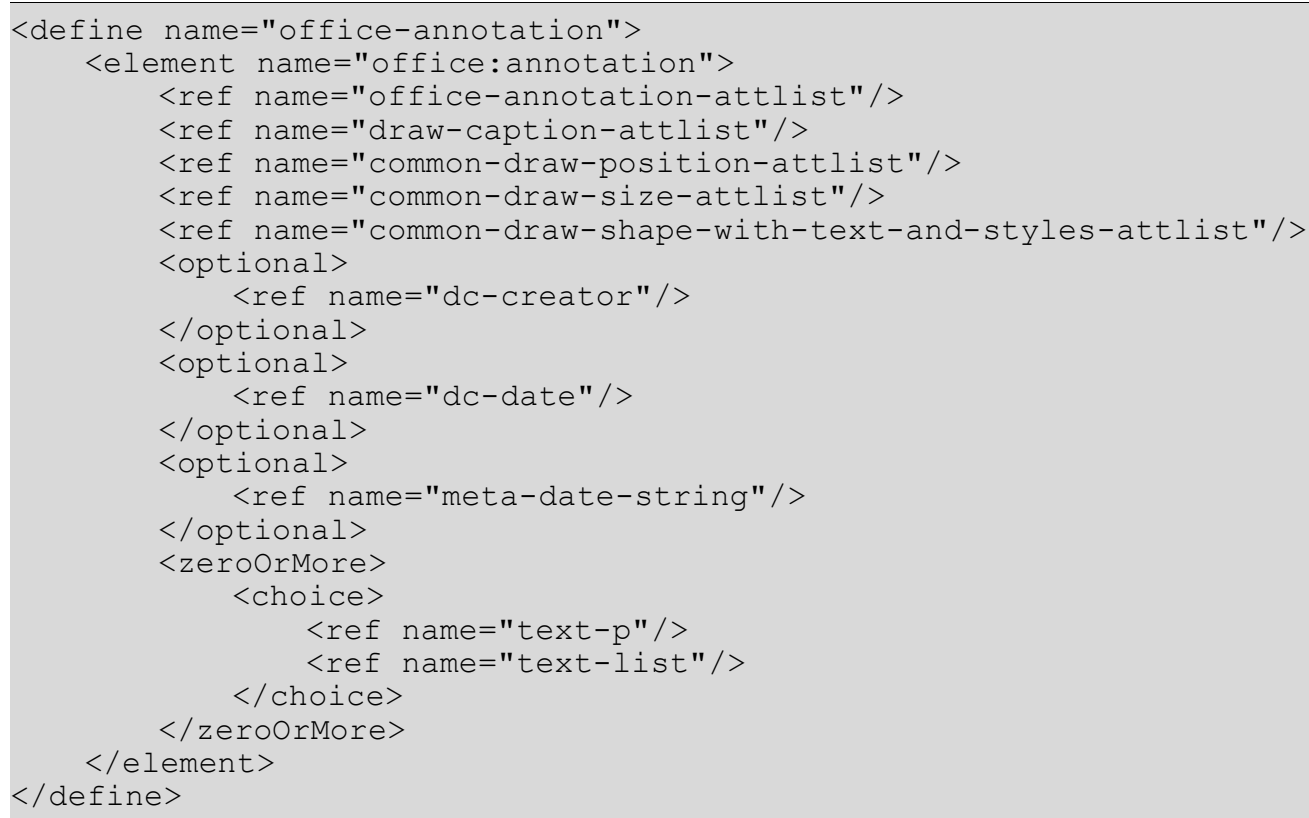

The attributes associated with the <office: annotation> element are:

- Display

- $\quad$ Position, size, style, layer, z-index, id, and transformation (see section 9.2.15)

- Text anchor, table background, draw end position (see section 9.2.16)

- Caption point, round corners (see section 9.2.10)

\section{Display}

The office: display attribute specifies whether or not the annotation is visible.

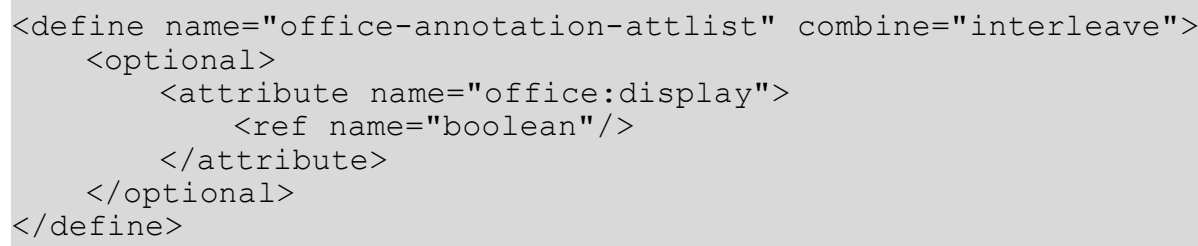

\section{Caption Attributes}

The following attributes can be attached to the <office: annotation> element to influence how it is displayed: $s v g: x, s v g: y, s v g: w i d t h, s v g:$ height, draw:caption-point-x, 
draw: caption-point-y, draw: corner-radius, table:end-cell-address,

table:end-x, table:end-y, text:anchor-type, text:anchor-page-number, draw:layer, draw:style-name, draw:text-style-name, draw:transform, draw: name, draw:z-index and draw:id. Their meaning is the same as if they are applied to $\mathrm{a}<\mathrm{draw}$ : caption $>$ element (see section 9.2.10). The use of these attributes is optional.

\subsubsection{Creator}

The optional $<\mathrm{dc}$ : creator $>$ element described in section 3.1.7 specifies the author of the annotation.

\subsubsection{Creation Date and Time}

The optional $<\mathrm{dc}$ : date $>$ element described in section 3.1.9 specifies the creation date and time of the annotation.

\subsubsection{Creation Date and Time String}

If the application only has a date string and cannot parse this string, it may write the string into the $<$ meta: date-string $>$ element.

10177

10178

10179

10180

10181

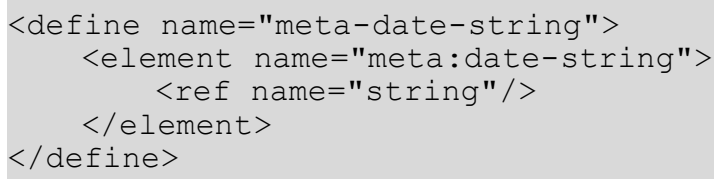

\subsection{Number Format}

The OpenDocument number format consists of three parts:

- Prefix - the text that is displayed before the number

- Display format specification, for example, A, B, C, or 1, 2, 3

- Suffix - the text that is displayed after the number

\subsubsection{Prefix and Suffix}

The style:num-prefix and style:num-suffix attributes specify what to display before and after the number.

If the prefix and suffix do not contain alphanumeric characters, an [XSLT] format attribute can be created from the OpenDocument attributes by concatenating the values of the style:numprefix, style:num-format, and style:num-suffix attributes.

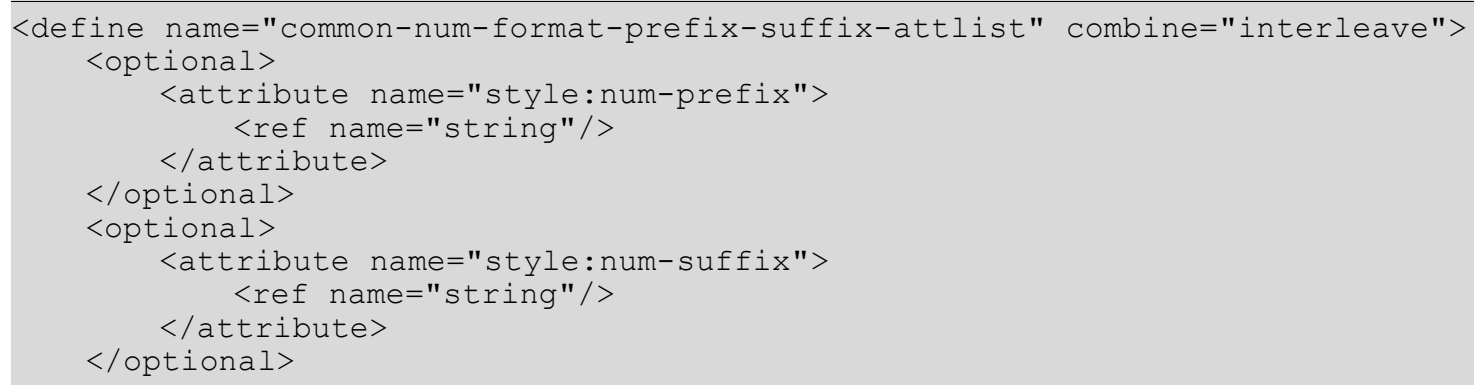




\subsubsection{Format Specification}

The style:num-format attribute specifies the format of the number in the same way as the [XSLT] format attribute. The number styles supported are as follows:

- $\quad$ Numeric: $1,2,3, \ldots$

- Alphabetic: $a, b, c, \ldots$ or $A, B, C, \ldots$

- Roman: i, ii, iii, iv, ... or I, II, III, IV, ...

The value of this attribute can be "1", "a", "A", "i", or "I". For some elements, the attribute value also can be empty. In this case, no number is displayed.

10194

10195

10196

10197

10198

10199

10200

10201

10202

10203

10204

10205

10206

10207

10208

10209

10210

10211

10212

10213

10214

10215

10216

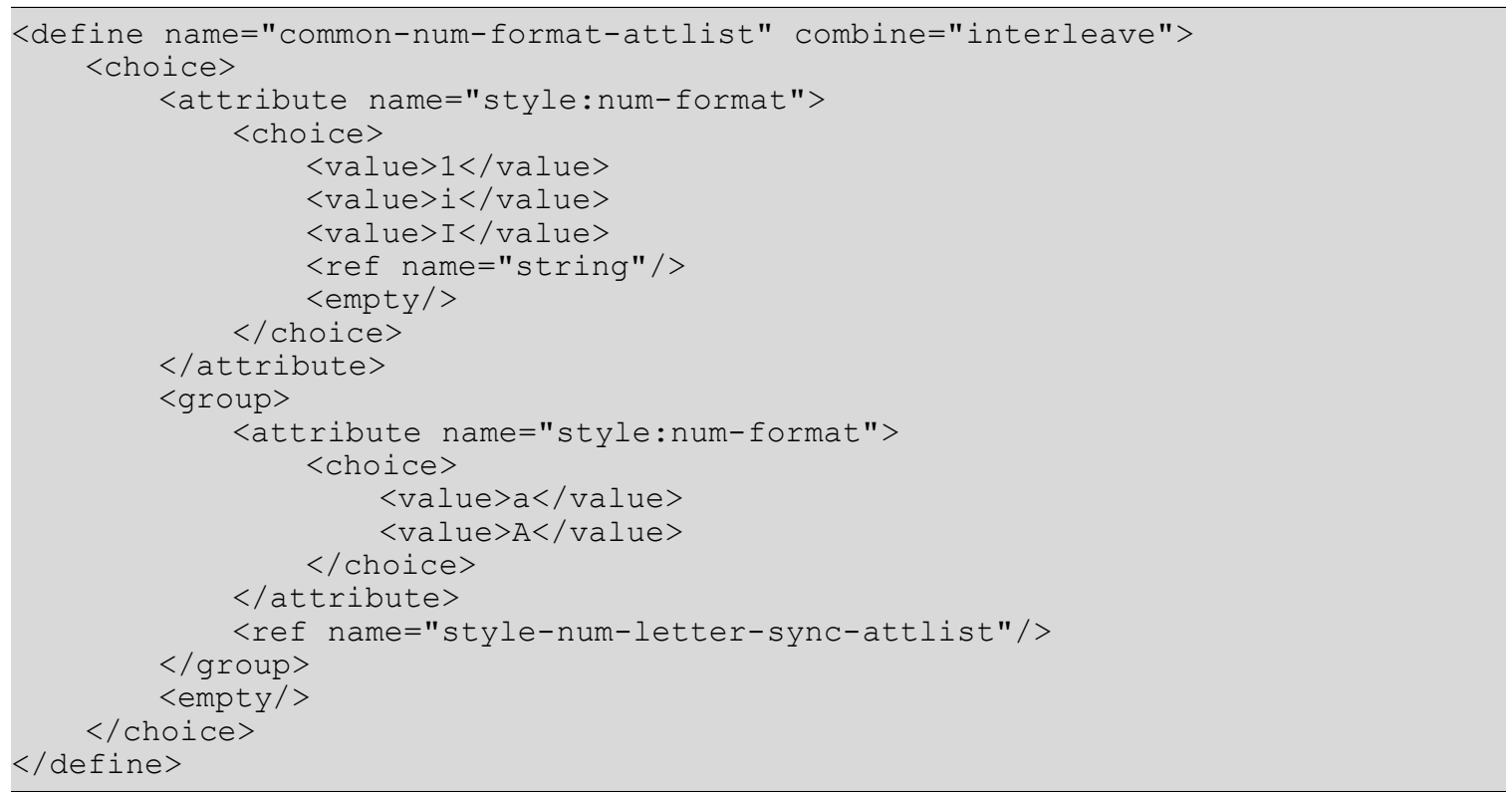

\subsubsection{Letter Synchronization in Number Formats}

If letters are used in alphabetical order for numbering, there are two ways to process overflows within a digit, as follows:

- A new digit is inserted. Its start value is $A$, and it is incremented every time an overflow occurs in the following digit. The numbering sequence in this case is something like a,b,c, ..., $\mathrm{z}$, aa, ab, ac, ...,az, ba, ..., and so on.

- A new digit is inserted that always has the same value as the following digit. The numbering sequence in this case is something like $a, b, c, \ldots, z$, aa, bb, cc, .., zz, aaa, ..., and so on. This is called letter synchronization.

The style:num-letter-sync specifies whether letter synchronization shall take place.

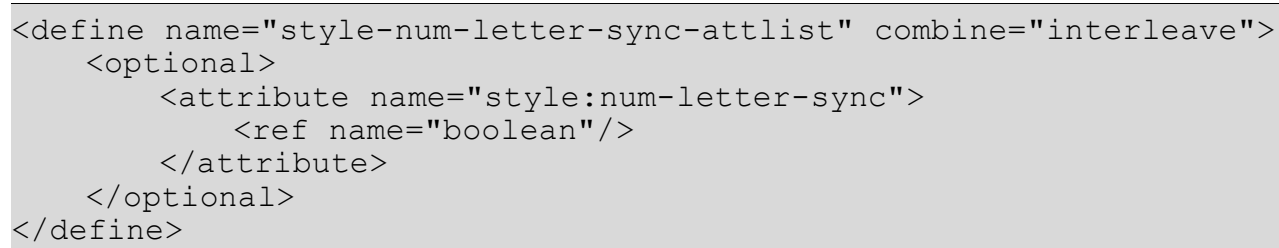




\subsection{Change Tracking Metadata}

Meta-data for change tracking is contained inside an <office: change-info > element. It contains the author and creation date of a tracked change, as well as an optional comment.

\section{Creator}

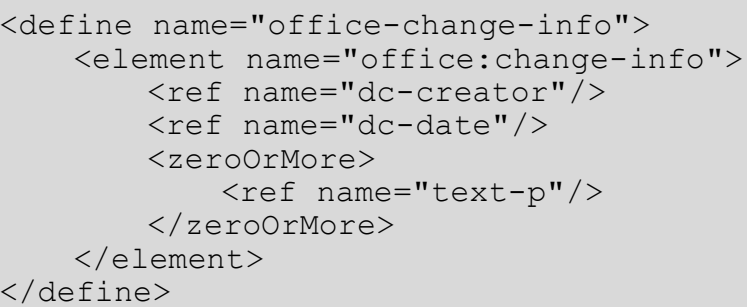

The $<\mathrm{dc}$ : creator $>$ element as described in section 3.1.7 specifies the name of the author who changed the document.

\section{Date and Time}

The $<\mathrm{dc}$ : date $>$ element as described in section 3.1.9 specifies the date and time when the change took place.

\section{Comment}

An additional comment may be included as $<$ text $: p>$ elements.

\subsection{Event Listener Tables}

Many objects such as controls, images, text boxes, or an entire document support events. An event binds the occurrence of a particular condition to an action that is executed if the condition arises. For example, if a user places the cursor over a graphic, this condition triggers an action that is supported by the office application. This event, called "on-mouse-over", can be associated with an macro that is executed whenever the condition occurs, that is, whenever a user places the cursor over a graphic.

The XML representation of events and event tables is structured as follows:

- All of the event elements that are associated with an object are located in a container element called <office: event-listeners>.

- Each event-to-action association is recorded in one <script:event-listener $>$ element.

- Depending on the type of action that the event triggers, the following elements are used:

- The <script:event-listener> element represents events that are bound to a macro or script.

- The <presentation: event-listener> element represents events that are bound to an action that is specific to a presentation, for example, go to the next page. Presentation events are described in section .

The <office:event-listeners> element specifies the table of events that are associated with an object. 


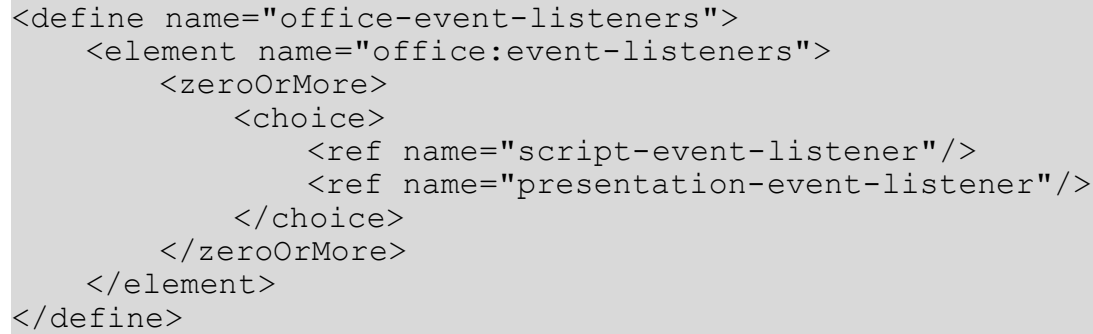

\section{$</$ define $>$}

\subsubsection{Event Listener}

The <script:event-listener $>$ element binds an event to a macro.

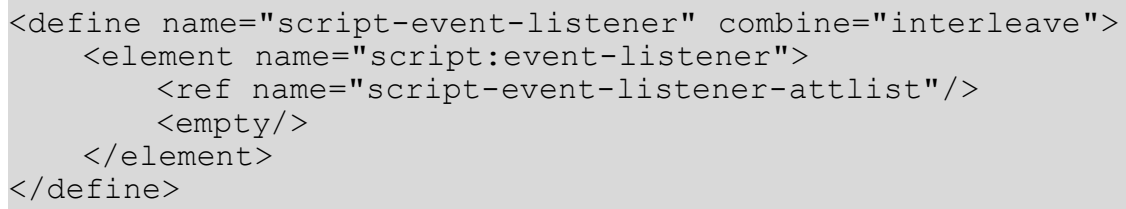

The attributes that may be associated with the <script: event-listener $>$ element are:

- Event name

- Script language

- Macro Name and Location

\section{Event Name}

The script:event-name attribute specifies the name of the event. Since the available events, their names and their meanings are application and script language dependent, the name should be preceded by a namespace prefix, so that the corresponding namespace together with the event name can be used to identify the semantic of the event. For events that are specified in the DOM event model, it is recommended to use the event names described in $\S 1.4 .2$ of [DOMEvents]. The corresponding namespace is "http://www.w3.org/2001/xml-events" .

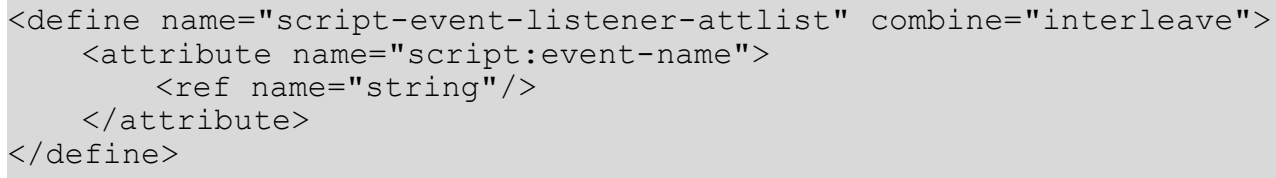

\section{Script Language}

The script: language attribute specifies the scripting language in which the macro or script which is associated with the event is written. See also section 2.5.1.

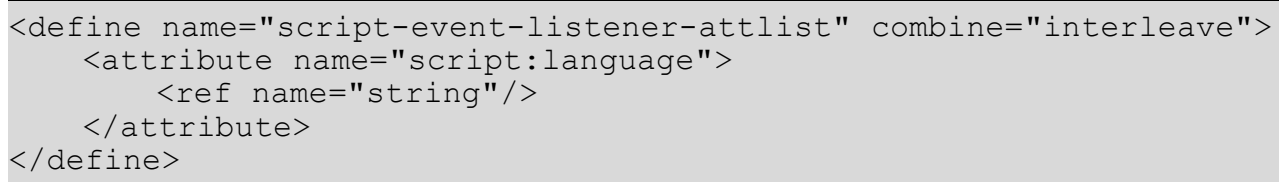

\section{Macro Name and Location}

The macro code that should be called for the event can be either specified by an URI in [XLink] notation, or a simple name specified by a script:macro-name attribute. If an XLink is used, 
the URI may have an arbitrary protocol, for instance one that encodes the name of a macro library name together with macro name defined in this library. Both, the XLink URI as well as a simple name, are script language dependent.

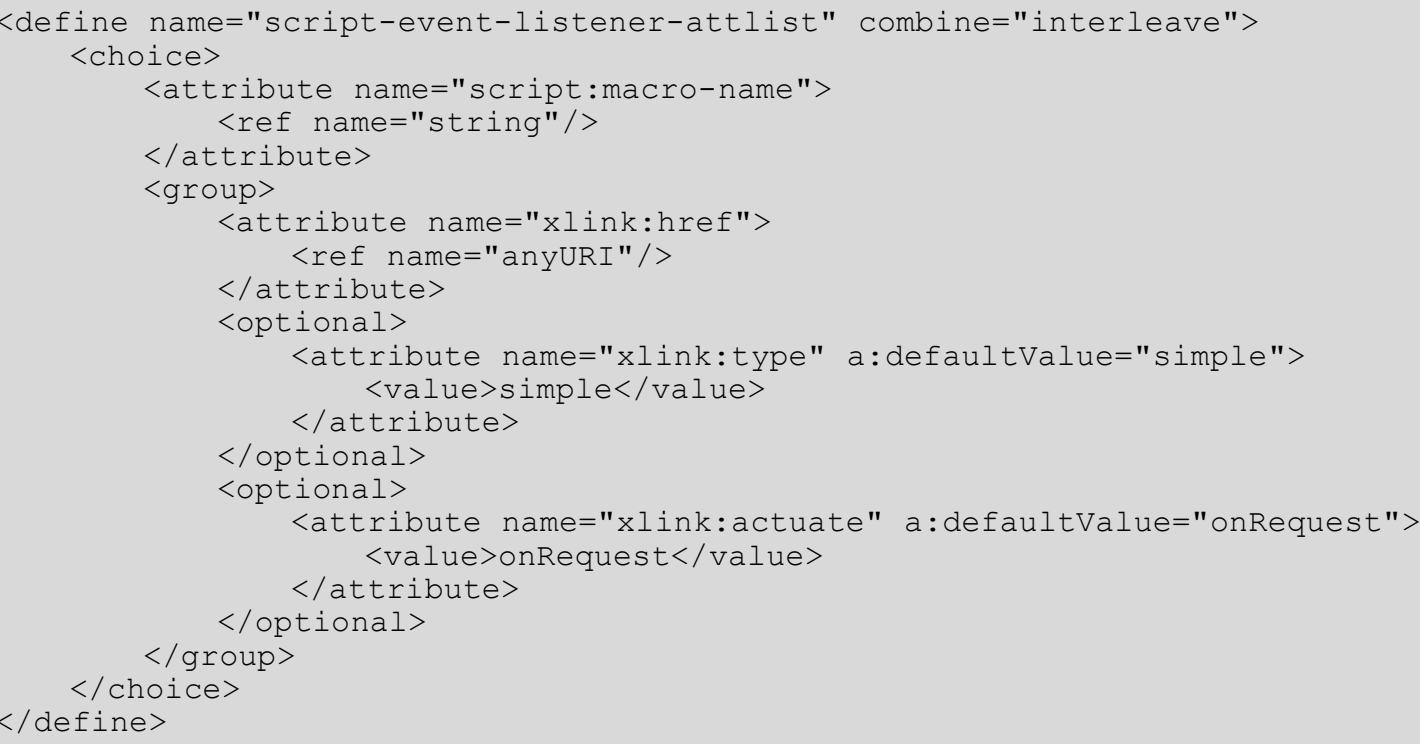

\subsection{Mathematical Content}

Mathematical content is represented by MathML 2.0 (see [MathML])

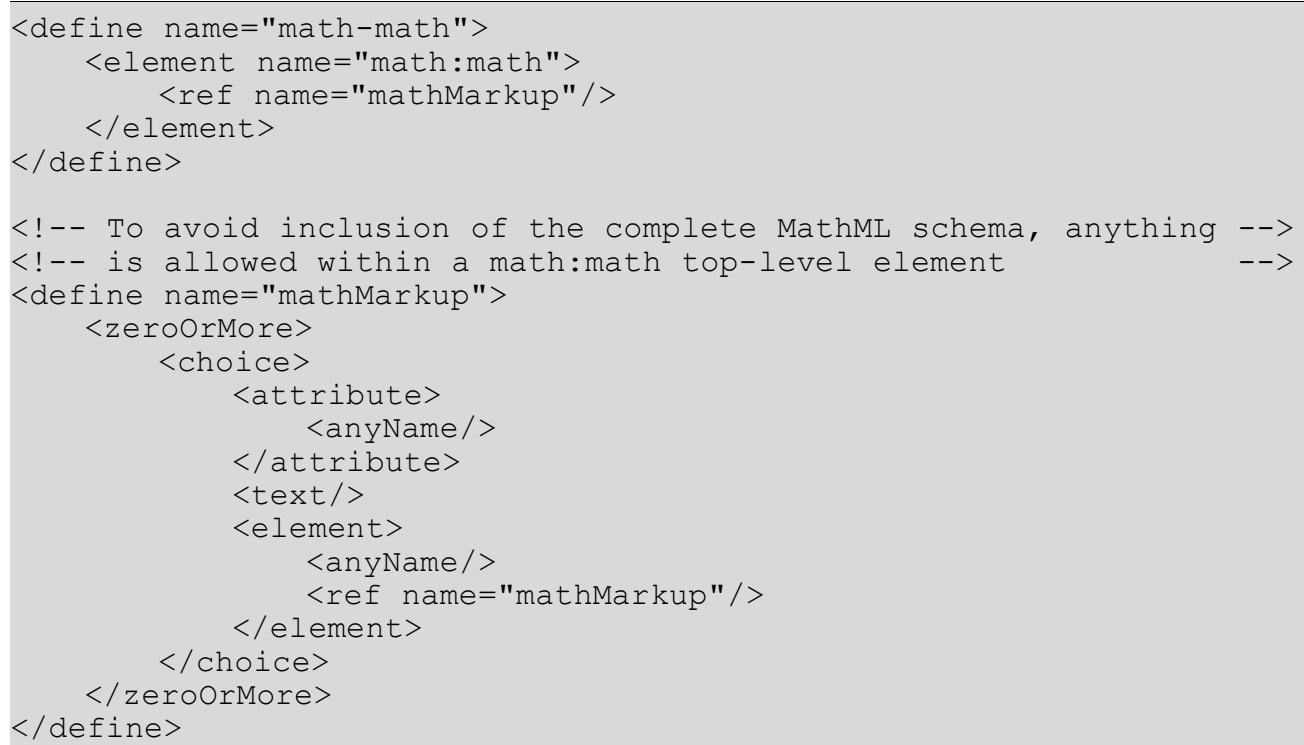

\subsection{DDE Connections}

A Dynamic Data Exchange (DDE) connection consists of the parameters for the DDE target application, a file name, and a command string. A DDE connection also takes a parameter that specifies whether it will be updated automatically or only on the user's request. Every DDE connection must be named.

All elements making use of DDE connections must contain their content (or its presentation), so that documents using DDE can still be properly displayed on machines which do not support the 
DDE mechanism, or where the DDE target is not available. Applications should preserve the DDE connection information even if they cannot make use of it, so that other applications can make use the DDE facilities.

\subsubsection{Container for DDE Connection Declarations}

Within text and spreadsheet documents, DDE connection declarations are contained in one declaration element. For text documents, the element is $\langle$ text:dde-connection-decls $>$ as described in section 4.7. For spreadsheet documents, it is $\langle$ table: dde-links $>$ as described in section 8.10 .

\subsubsection{Declaring DDE Connections for Text Fields}

Every DDE connection used by a text field is declared using a declaration element. Multiple DDE fields can refer to one DDE connection by using the same name. The declaration element has no content.

10303

10304

10305

10306

10307

10308

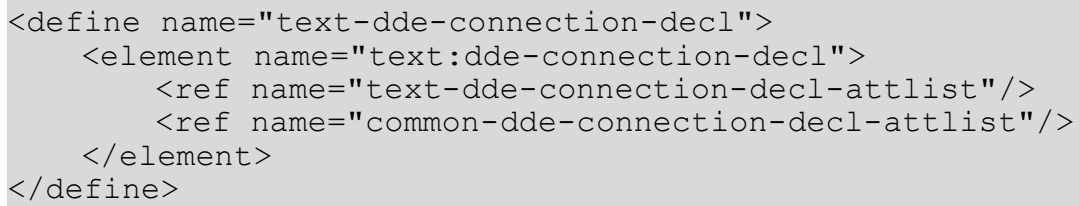

The attributes that may be associated with the <text: dde-connection-decl> element are:

- Connection name

- DDE target application

- DDE target topic

- DDE target item

- Automatic update flag

\section{Connection Name}

The office: name attribute specifies the name by which the connection will be referred.

\section{Target Application}

The office:dde-application attribute specifies the name of the target application to use for the DDE connection.

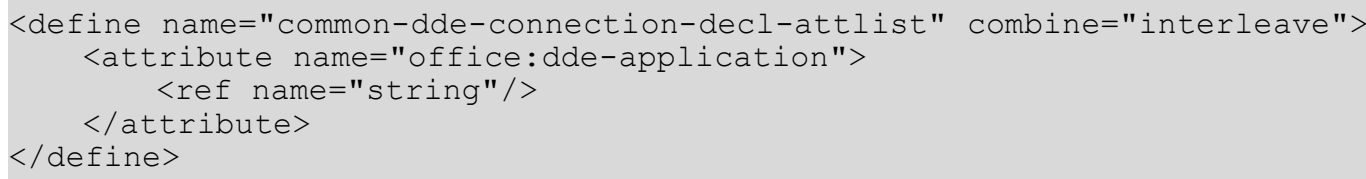


Example: The target name for the OpenOffice.org software is soffice.

Therefore, internal DDE links have the attribute text: dde-

application="soffice".

\section{Target Topic}

The office:dde-topic attribute specifies the name of the topic to use for the DDE connection.

10319

10320

10321

10322

10323

10324

10325

10326

10327

10328

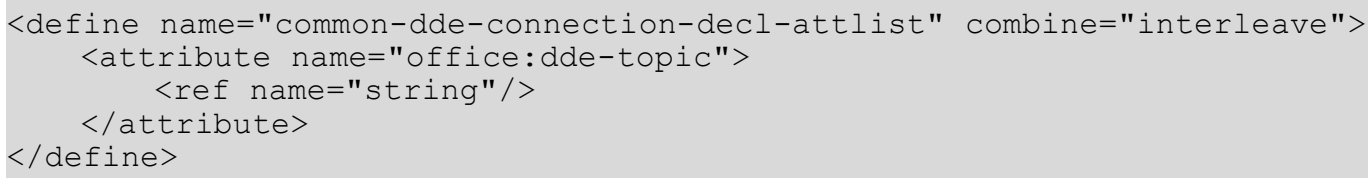

Example: The OpenOffice.org software interprets the DDE topic as the name of the file.

\section{Target Item}

The office: dde-item attribute specifies which information the target application should deliver.

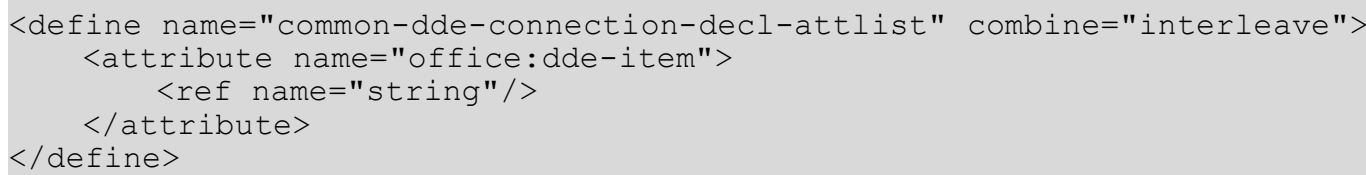

Example: If the target application for the DDE connection is the OpenOffice.org Writer software, the item represents the name of a bookmark. OpenOffice.org delivers the current text content to the requesting application.

\section{Automatic Update}

Office applications by default automatically update DDE links. If a manual update of the link is preferred, the text:automatic-update attribute my be used to specify that the DDE connection links should only be updated the request of the user.

If the value of this attribute is true, then the application is expected to automatically update the DDE links. If this value of this attribute is false, the DDE links are updated on user request only.

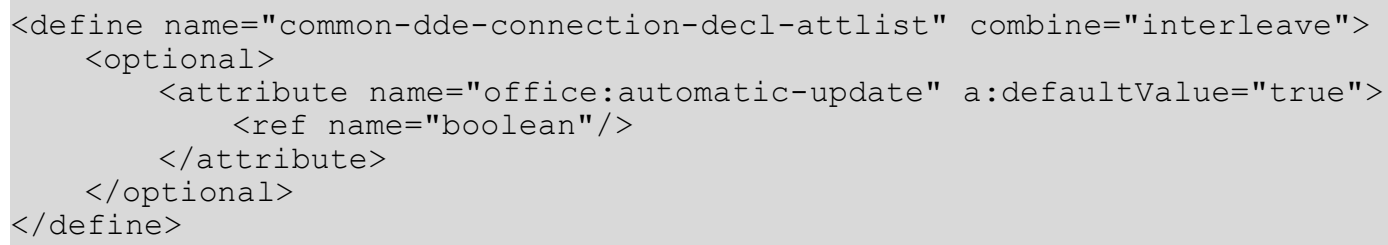

\subsubsection{Declaring DDE Connections for Tables}

The DDE connection data of tables is contained in an <office: dde-source> element. The usage of this element differs between spreadsheet and text document tables. For text document tables, the element is contained within the table's $\langle$ table: table $\rangle$ element directly. For 
spreadsheet documents, it is contained in a $<$ table: dde-link> element, that describes a single DDE connection.

The $<$ table: dde-link> element contains the DDE source data in the <office:ddesource> element and a simple table element that might be used to cache the data of the DDE source. The table does not need a name and does not contain style information. Only the data contained in the cell attributes is used. The cells themselves remain empty.

10336

10337

10338

10339

10340

10341

10342

10343

10344

10345

10346

10347

\section{Connection Name}

The office: name attribute specifies the name by which the connection can be referred.

10348

10349

10350

10351

10352

10353

10354

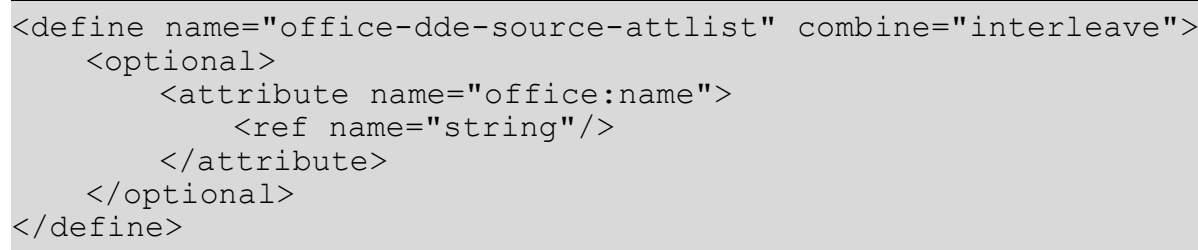

\section{Conversion Mode}

The office:conversion-mode attribute specifies the method by which the DDE server converts its data into numbers. There are three possible values:

- into-default-style-data-style: Numbers are converted into the data style which is set on the default style.

- into-english-number: numbers are converted into the English default format.

- keep-text: Numbers are not converted. They are treated as text. 
10363

10364

10365

10366

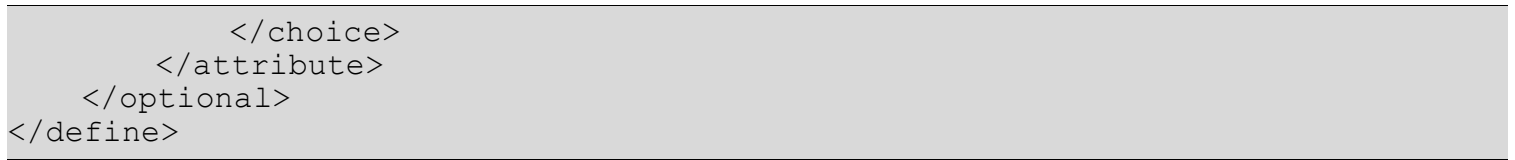




\section{SMIL Animations}

This section describes [SMIL20] based elements and attribute that can be used within the OpenDocument format for animation effects.

\subsection{Basic Animation Elements}

The basic animation elements are directly derived from basic animation elements specified §3.5 and $\S 12.5$ of [SMIL20], and in section §19.2 of [SVG].

\subsubsection{Animate}

The <anim:animate> element behaves like the [SMIL20] <smil:animate> element. See $\S 3.5 .1$ of [SMIL20] for details.

\subsubsection{Animate Motion}

The <anim:animateMotion> element behaves as the [SVG] <svg:animateMotion> element. See $\S 19.2 .12$ of [SVG] and $\S 3.5 .3$ of [SMIL20] for details.

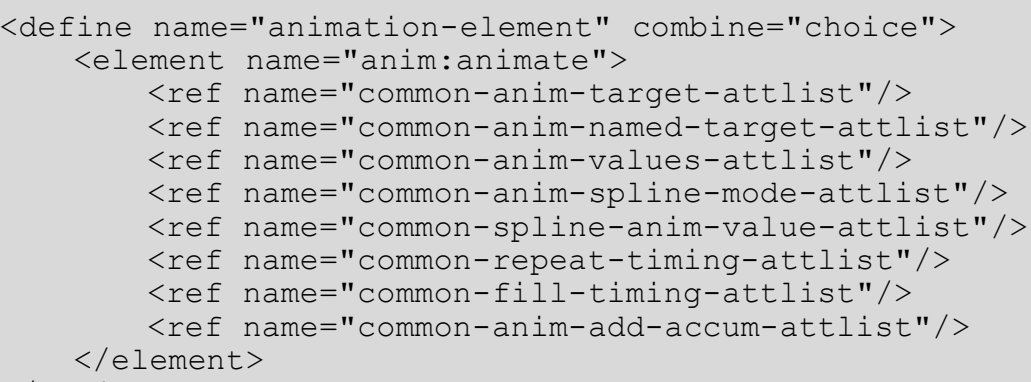

\subsubsection{Set}

The <anim: set> element behaves like the [SMIL20] <smil : set> element. See §3.5.2 of [SMIL20] for details.

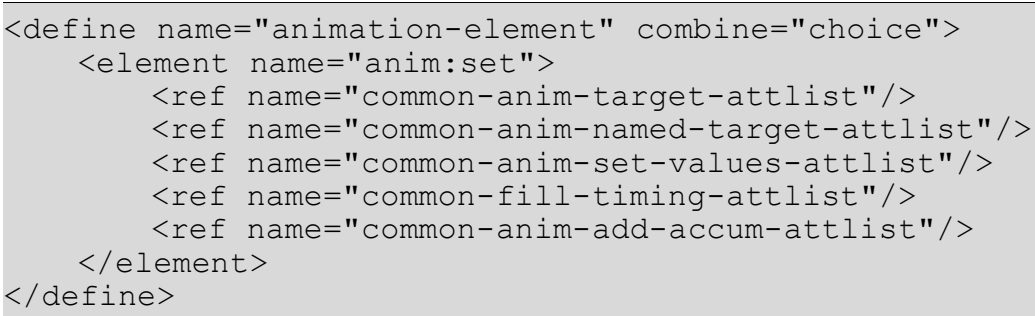

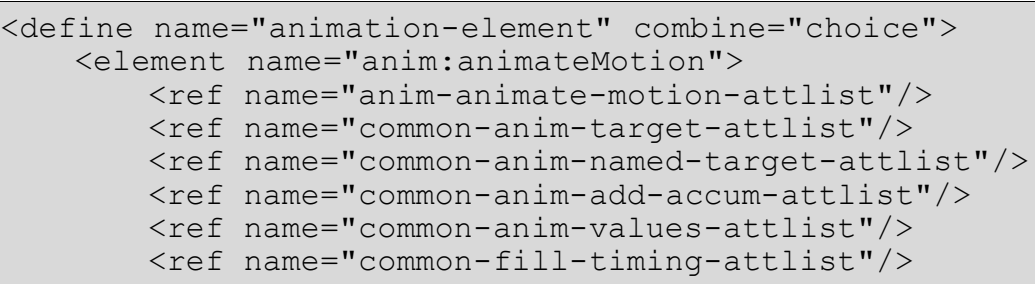




\section{The Motion Path}

The [SVG] svg:path attribute can be used to specify a path along which the element is animated. See $\S 19.2 .12$ of [SVG] for details.

\section{Origin}

The [SVG] svg:origin attribute can be used to specify an origin. See §19.2.12 of [SVG] for details.

\section{$</$ define $>$}

\section{Calc Mode}

The [SMIL20] smil: calcMode attribute is used to specify the interpolation mode of the animation. See $\$ 19.2 .12$ of [SVG] for details.

10413

10414

10415

10416

10417

10418

10419

10420

10421

10422

10423

10424

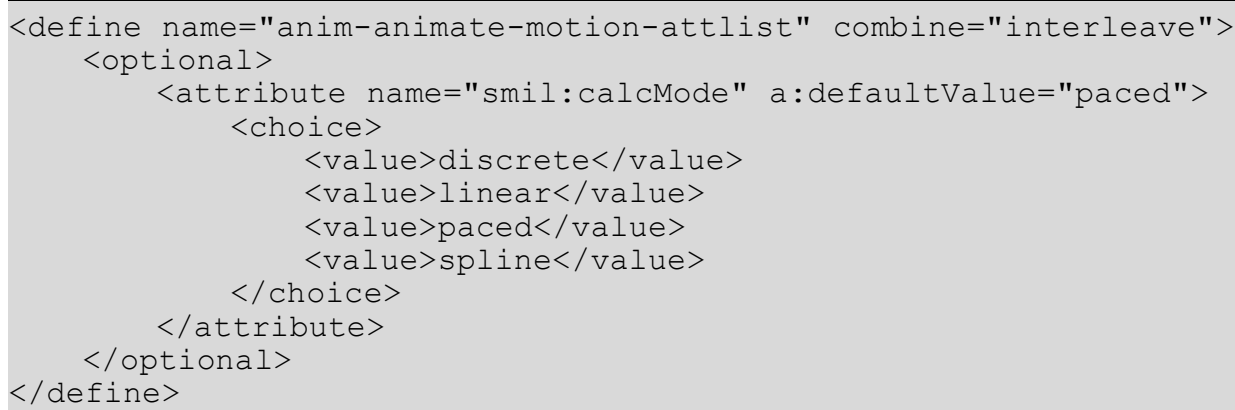

\subsubsection{Animate Color}

The <anim: animateColor > element behaves like the [SMIL20]<smil:animateColor > element. See $§ 3.5 .4$ of [SMIL20] for details. 


\section{Color Interpolation}

The anim:color-interpolation attribute specifies the color space that is used for color interpolation.

\subsubsection{Animate Transform}

The <anim:animateTransform> element is based on the [SVG] <svg:animateTransform> element. See \$19.2.14 of [SVG] for details.

10458

10459

10460

10461

10462

10463

10464

10465

10466

10467

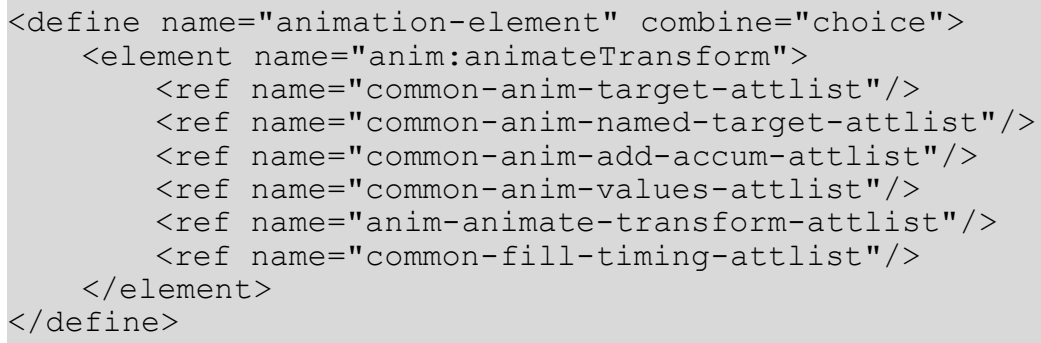

\section{Transformation Type}

The [SVG] svg: type attribute is used to specify the transformation type. See §19.2.14 of [SVG] for details. <attribute name="svg:type"> 


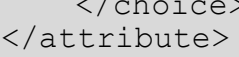

\section{Fade Color}

The [SMIL20] smil: fadeColor attribute can be used to specify the transition fade color for transitions that makes use of a start or end color. See \$12.5.1 of [SMIL20] for details.

10511

10512

10513

10514

10515

10516

10517

10518

10519

10520

\section{The Transition Mode}

The [SMIL20] smil:mode attribute is used to specify if the animated element will be transition in or out. See §12.5.1 of [SMIL20] for details.

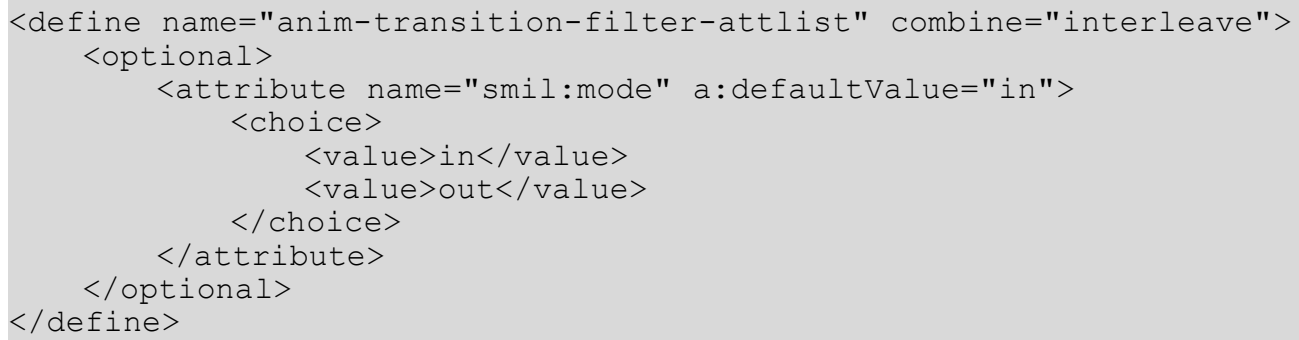

\subsection{Animation Model Attributes}

The animation model uses the same concepts and syntax as specified in $\S 3$ of [SMIL20].

\subsection{Common Animation Attributes}

\section{Element Id}

The anim:id attribute defines an ID that is used to identify the element inside a document.

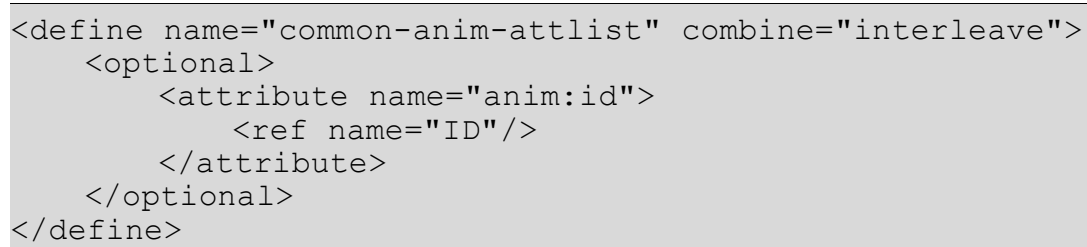




\subsubsection{Animation Target Attributes}

\section{Target Element}

The [SMIL20] smil: targetElement attribute is used to specify the target element to be animated. See $\S 3.4 .1$ of [SMIL20] for details. See section 9.8 .2 for details about the usage of this attribute in presentation documents.

10538

10539

10540

10541

10542

10543

10544

10545

10546

10547

10548

10549

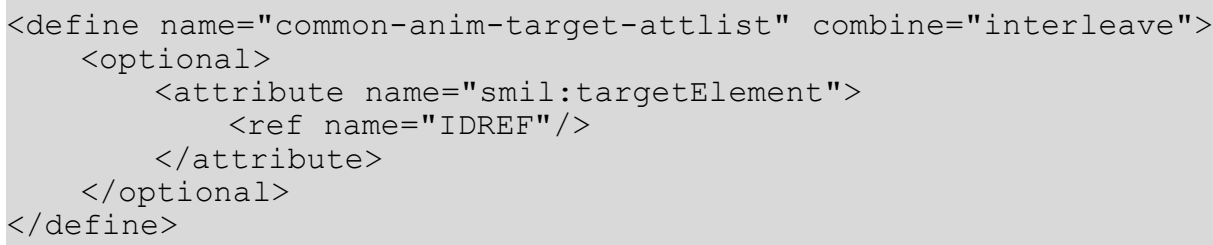

\section{Target Attribute}

The [SMIL20] smil: attributename attribute is used to specify a target attribute by name. See $\S 3.4 .1$ of [SMIL20] for details. See section 9.8.2 for details about the usage of this attribute in presentation documents.

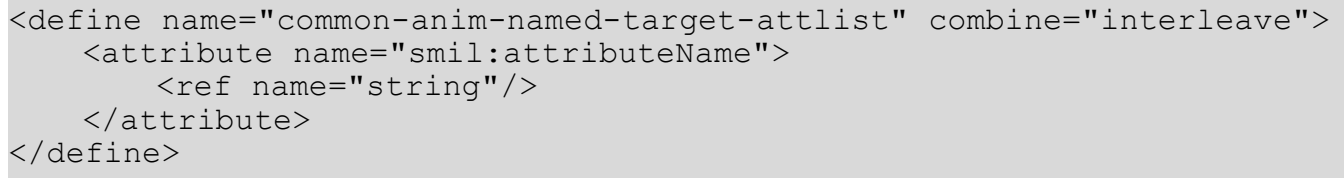

\section{Target Element Sub Item}

The anim:sub-item attribute specifies an optional sub item of the target element. Possible values for this element depend on the document type and the target element type. See section 9.8.2 for details about the usage of this attribute in presentation documents.

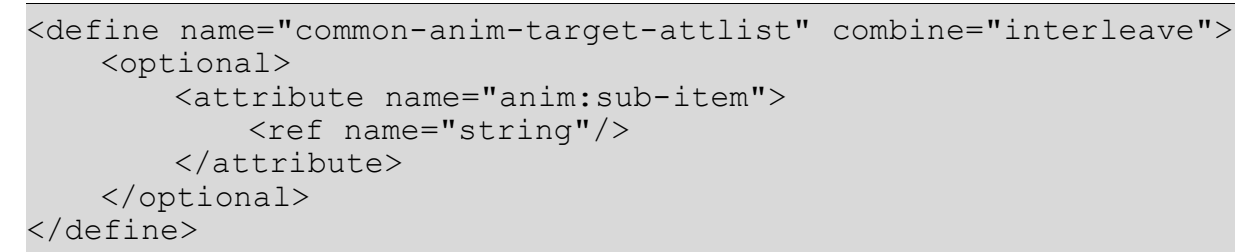

\subsubsection{Animation Function Attributes}

\section{Value List}

The [SMIL20] smil:values attribute specifies the values used to animate the target element. See $\$ 3.4 .2$ of [SMIL20] for details.

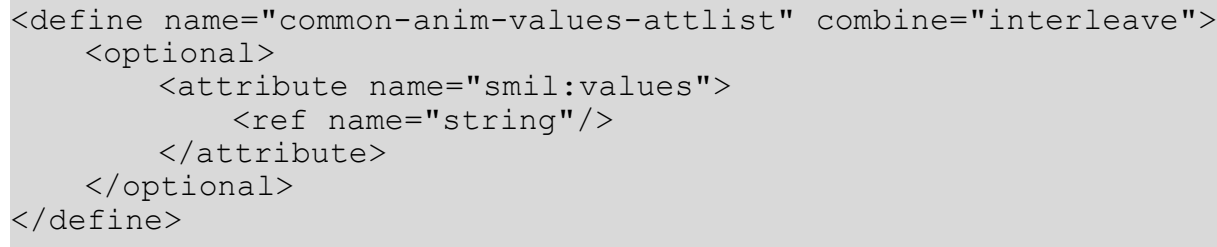




\section{Calc Mode}

The [SMIL20] smil: calcMode attribute is used to specify the interpolation mode of the animation function. See $\$ 3.4 .2$ of [SMIL20] for details.

10564

10565

10566

10567

10568

10569

10570

10571

10572

10573

10574

10575

\section{Key Times}

The [SMIL20] smil: keyTimes attribute specifies the pacing of the animation. See \$3.7.1 of [SMIL20] for details.

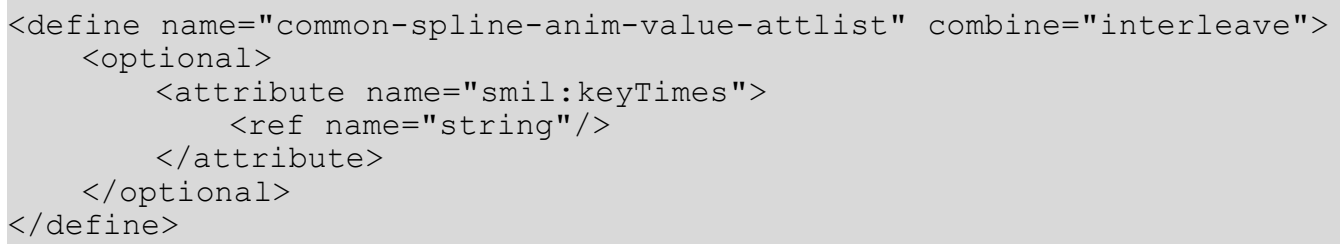

\section{Key Splines}

The [SMIL20] smil: keySplines attribute specifies a cubic Bezier function that controls interval pacing. See $\$ 3.7 .1$ of [SMIL20] for details.

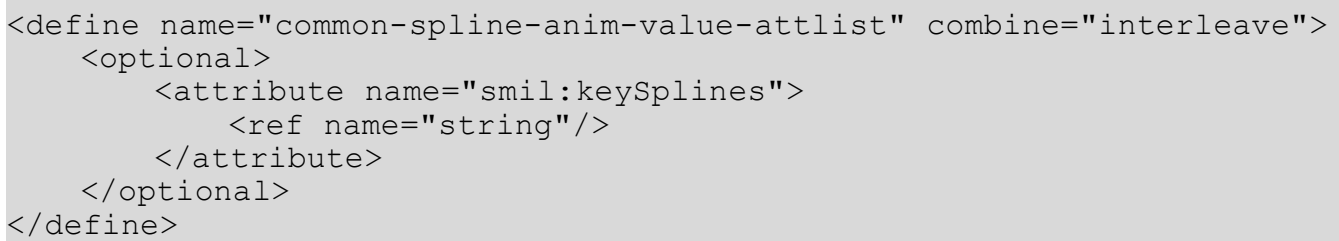

\section{Accumulation}

The [SMIL20] smil: accumulate attribute specifies the accumulation of the animation function. See $\$ 3.4 .3$ of [SMIL20] for details.

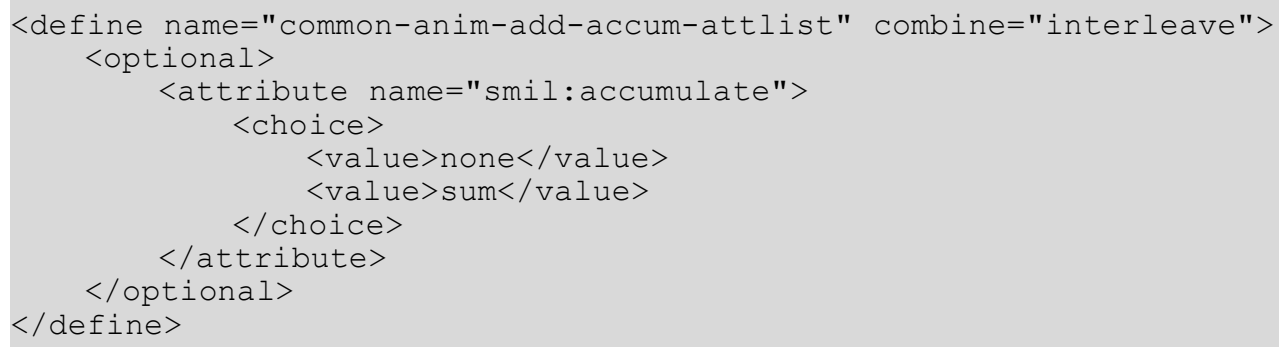




\section{Additive}

The [SMIL20] smil:additive attribute specifies if the additive of the animation function. See $\$ 3.4 .3$ of [SMIL20] for details.

10600

10601

10602

10603

10604

10605

10606

10607

10608

10609

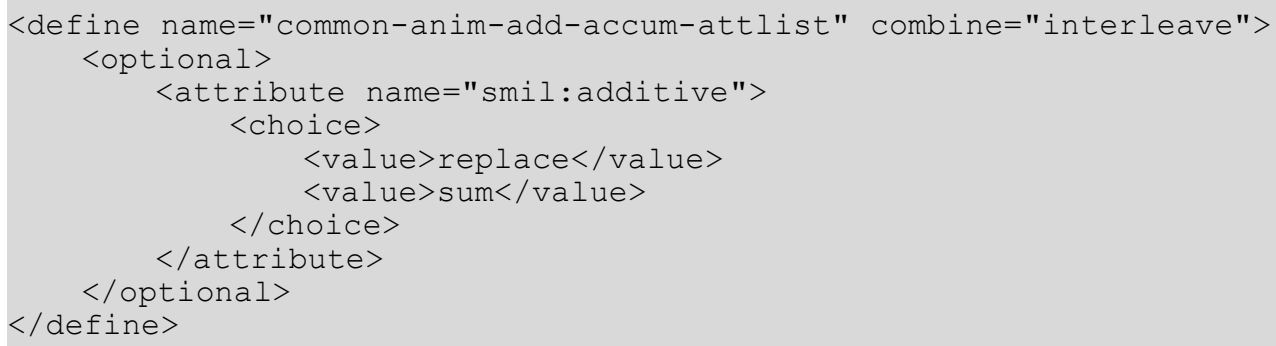

\section{Formula}

The anim: formula attribute specifies a formula that is used as the animation function. The identifier ' $\$$ ' will be replaced by a value between 0 and 1 (inclusive) that represents the proportional offset into the animation element's duration. For specific document types, additional identifiers may exist. The following is the minimum supported grammar:

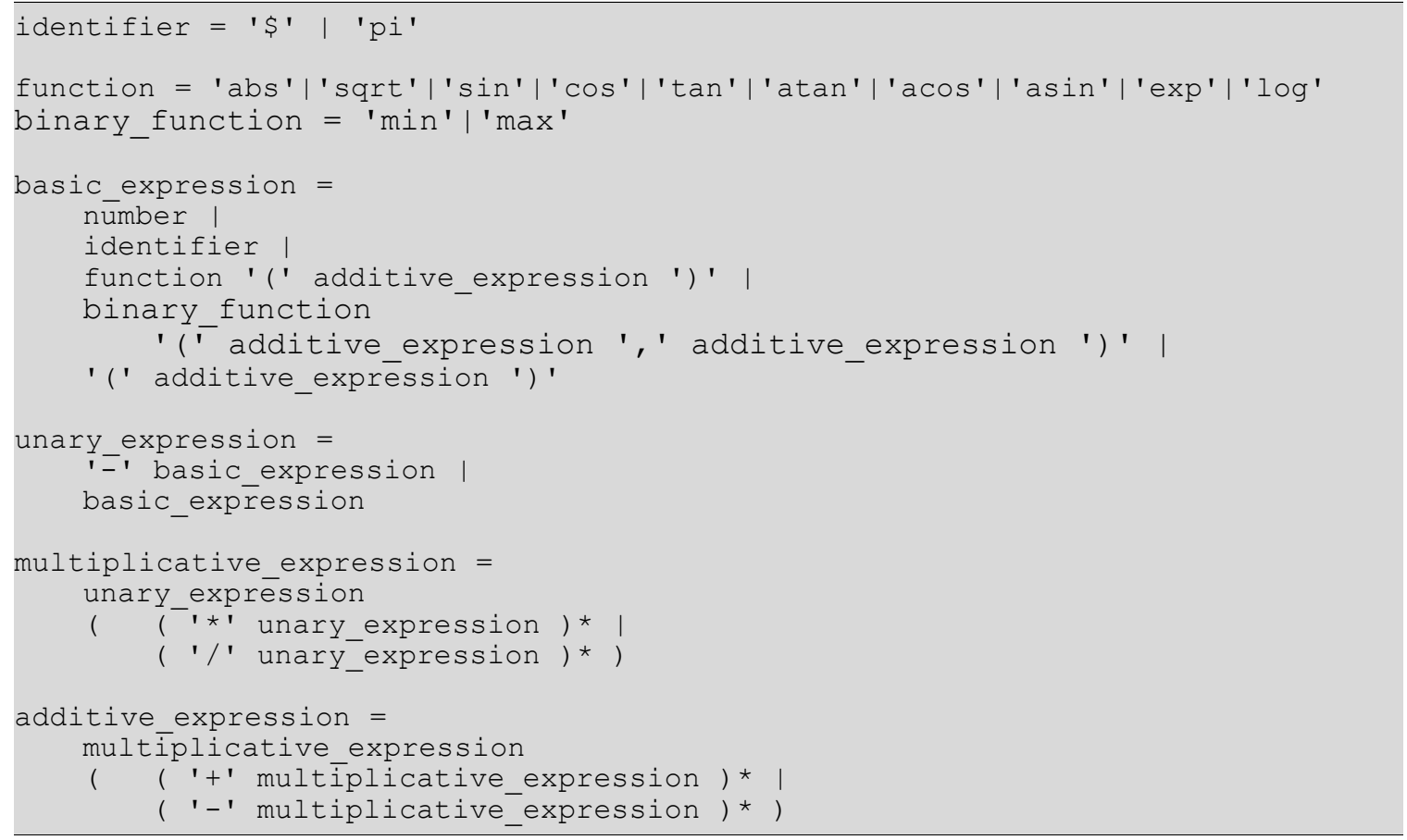

See section 9.8.2 for details about additional identifiers for presentation documents.

If a anim:formula attribute is given, it overrides the smil:values, smil:to, smil:from and smil:by attributes as specified in the next section.

10610

10611

10612

10613

10614

10615

10616

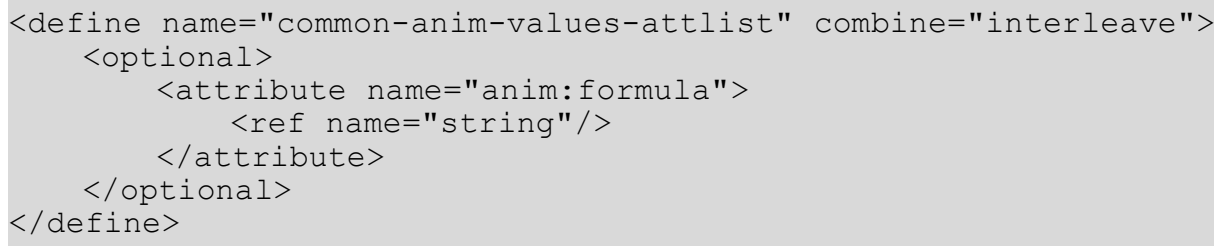




\section{Simple Animation Functions}

In addition to describing an animation with a list of values, a simplified version using the [SMIL20] smil: from, smil: to and smil: by attributes can be used. See §3.4.4 of [SMIL20] for details.

10617

10618

10619

10620

10621

10622

10623

10624
10625

10626

10627

10628

10629

10630

10631

10632

10633

10634

10635

10636

10637

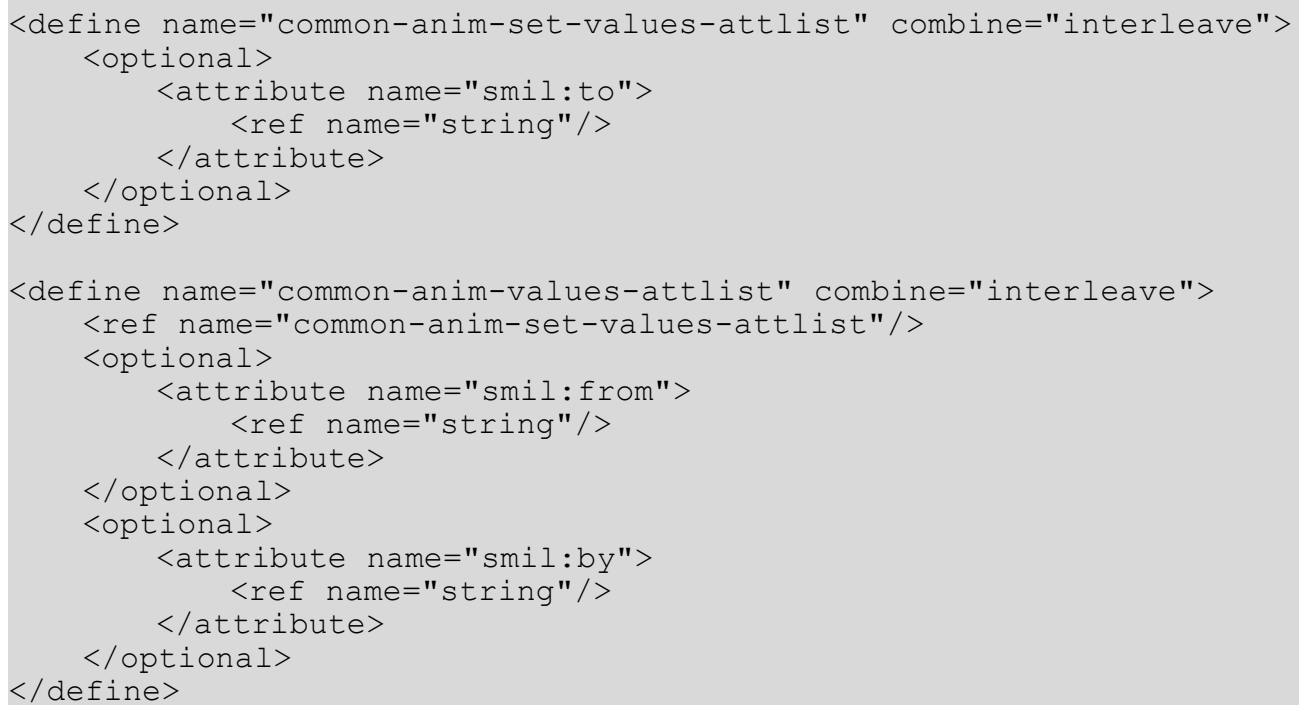

\subsection{Animation Timing}

The animation timing uses the same concepts and syntax as specified in $\S 10$ and $\S 11$ of [SMIL20] chapters.

\subsubsection{Animation Timing Attributes}

\section{Element Start}

The [SMIL20] smil:begin attribute can be used to specify the begin time of an element. See $\S 10.3 .1$ of [SMIL20] for details.

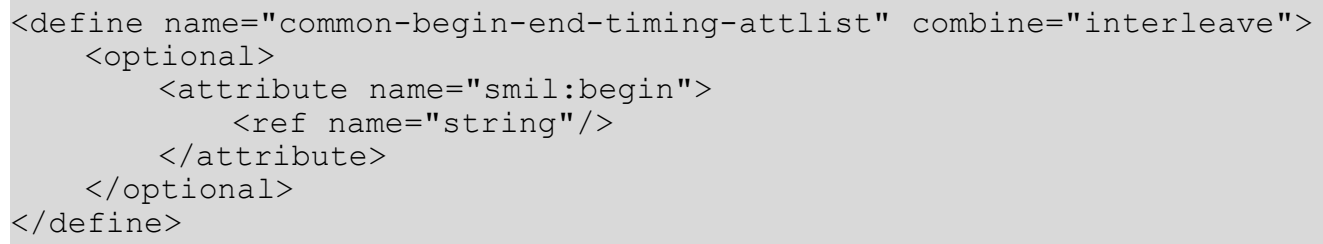

\section{Element End}

The [SMIL20] smil: end attribute can be used to specify the end time of an element. See $\S 10.3 .1$ of [SMIL20] for details.

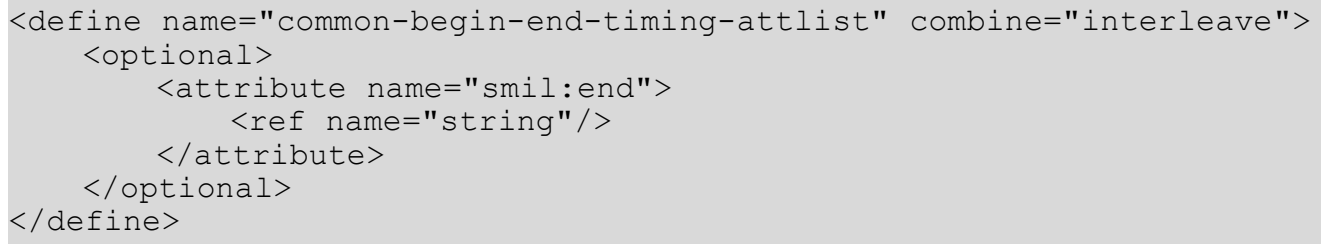




\section{Element Duration}

The [SMIL20] smil : dur attribute can be used to specify the duration of an element. See $\S 10.3 .1$ of [SMIL20] for details.

10652

10653

10654

10655

10656

10657

10658

10660

10661

10662

10663

10664

10665

10666

10667

10668

10669

10670

\section{Repeating Elements}

The [SMIL20] smil: repeatCount and smil: repeatDur attributes specifies the behavior of repeated animations. See $\S 10.3 .1$ of [SMIL20] for details.

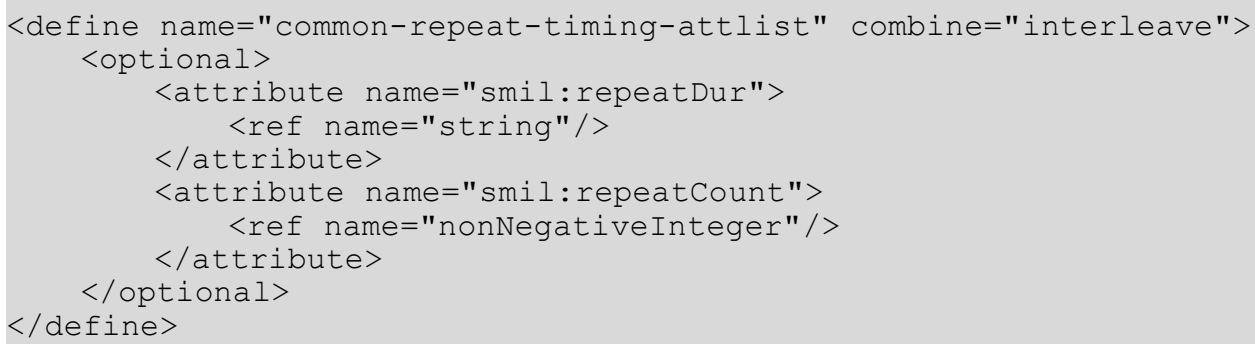

\section{Fill}

The [SMIL20] smil: fill attribute specifies the behavior of an element after an animation is finished. See $\$ 10.3 .1$ of [SMIL20] for details. 


\section{Fill Default}

The [SMIL20] smil:fillDefault attribute specifies the default behavior for the smil:fill attribute. See $\S 10.3 .1$ of [SMIL20] for details.

10695

10696

10697

10698

10699

10700

10701

10702

10703

10704

10705

10706

10707

10708

\section{Restart}

The [SMIL20] smil:restart attribute can be used to specify the restart behavior of an element. See $\S 10.3 .1$ of [SMIL20] for details.

10709

10710

10711

10712

10713

10714

10715

10716

10717

10718

10719

10720

\section{Restart Default}

The [SMIL20] smil: restartDefault attribute can be used to specify the default restart behavior of an element. See $\$ 10.3 .1$ of [SMIL20] for details. 


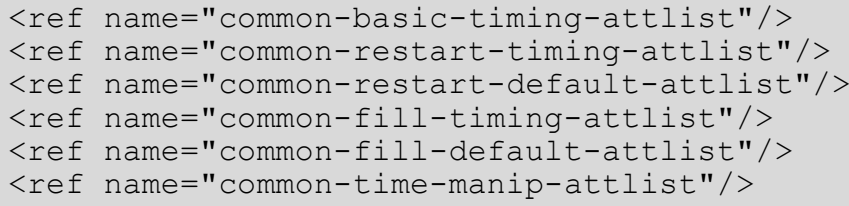

\subsubsection{Sequential Animations}

The <anim: seq > element is based on the [SMIL20] <smil : seq > element and defines a sequential time container. See $\$ 10.3 .2$ of [SMIL20] for details.

10779

10780

10781

10782

10783

10784

10785

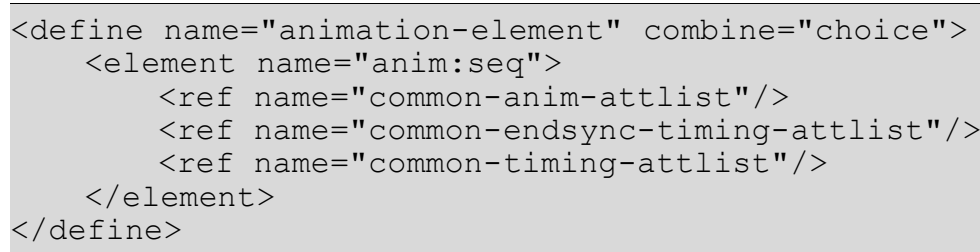

\subsubsection{Iterative Animations}

The <anim: iterate> element defines a parallel time container. The difference to a <anim:par> element is that the <anim: iterate> element does not specify effects for its target element itself. Instead of this, it iterates over possible child elements of the target element and executes all its child effects with the children of the target element as target.

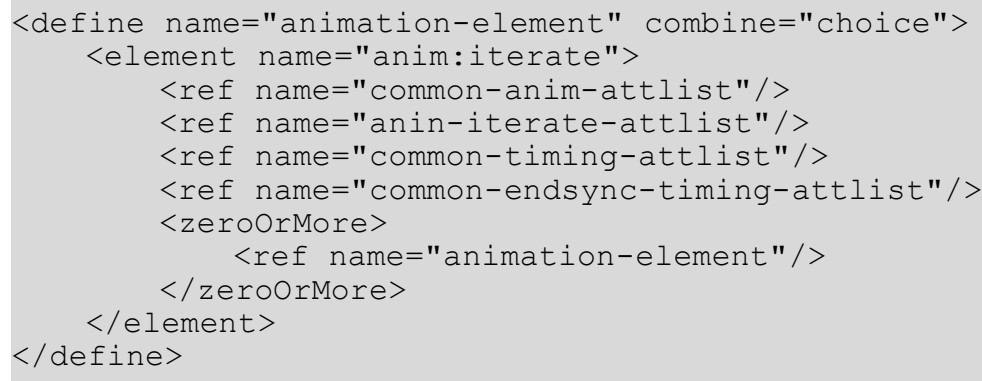

\section{The Target Element}

The [SMIL20] smil: targetElement attribute specifies the target element to that's children the effects should be applied. See section 9.8 .2 for details about the attribute's usage in presentation documents.

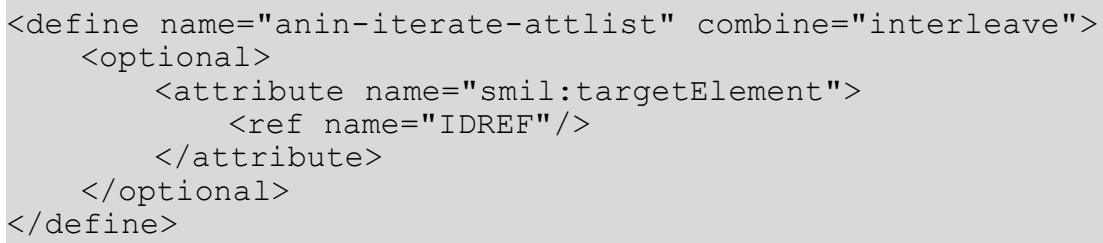

\section{The Iterate Type}

The anim:iterate-type attribute specifies how the iteration targets child elements are iterated. Possible values depends on the document type and the target element type. See section 9.8.2 for details about the attribute's usage in presentation documents. 


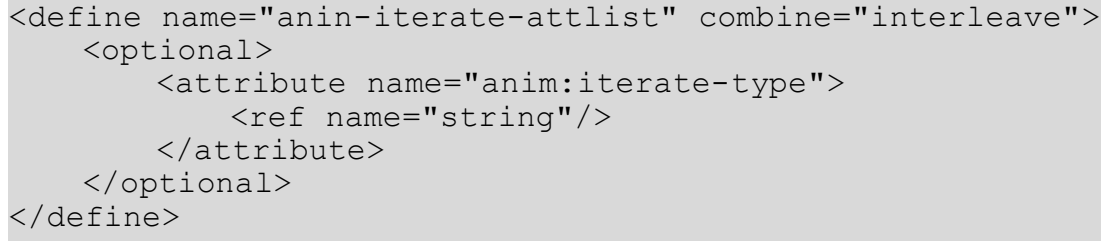

\section{The Iterate Interval}

The anim:iterate-interval attribute specifies the delay between the execution of the child effects of this element. The effects of the next iterated child of the target element are started when the given time is elapsed since the effects for the previous child has been started. An iterate interval of zero seconds would have the same behavior as using a <anim:par $>$ element.

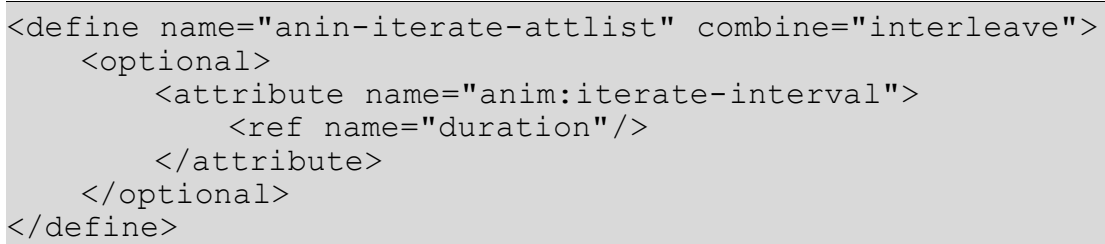

\subsection{Media Elements}

\subsubsection{Audio}

The <anim:audio> element is based on the [SMIL20] <smil:audio> element. It allows the playback of audio streams during an animation. See §7.3.1 of [SMIL20] for details.

10818

10819

10820

10821

10822

10823

10824

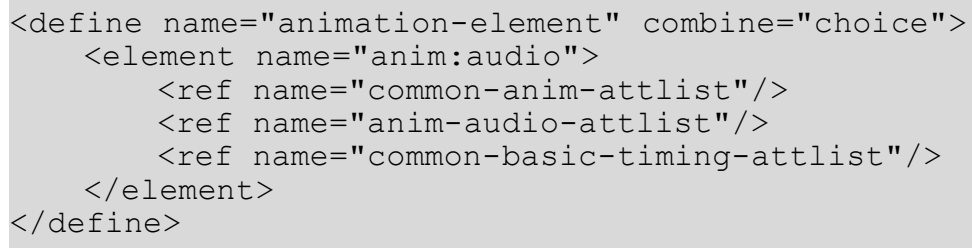

\section{Source}

The xlink: href attribute specifies the URI of the audio stream.

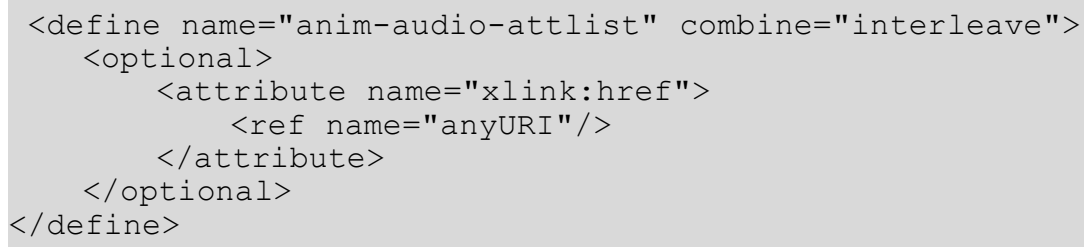

\section{Audio Level}

The anim:audio-level attribute specifies the volume during playback. Its value is a number in the range 0 (inaudible) to 1 (the system volume).

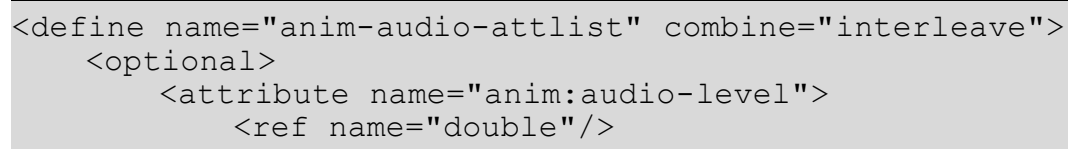




\subsection{Special Elements}

\subsubsection{Command}

The <anim: command> element is used to send generic commands to the application during an animation. The available command types and its parameters depend on the document type and the type of the target element. See section 9.8.2 for details about the element's usage in presentation documents.

\section{9 \\ 10840 \\ 10841 \\ 10842 \\ 10843 \\ 10844 \\ 10845 \\ 10846 \\ 10847 \\ 10848 \\ 10849 \\ 10850 \\ 10851 \\ 10852}

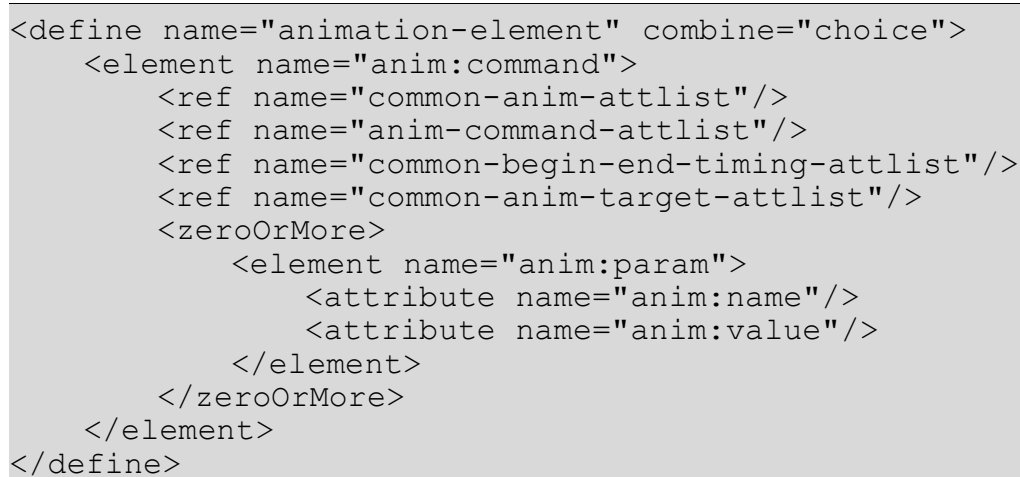

\section{Command}

The anim: command attribute specifies the command that will be executed at the application when this animation element is started.

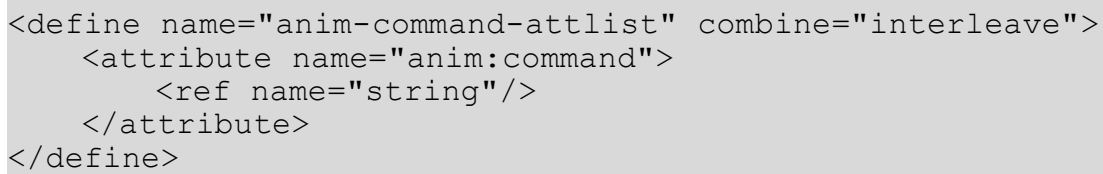




\section{Styles}

Many objects in an office document have formatting properties. A formatting property influences the visual representation of an object but it does not contribute to the content or structure of the document. Examples of formatting properties are:

- Font family

- Font size

- Font color

- Page margins

In the OpenDocument format, formatting properties are only stored within styles. This differs to the user interface of typical office applications, where formatting properties may be assigned to an object directly, or indirectly by applying a style to the object. Assigning formatting properties to an object directly has the same effect as assigning an unnamed style with the same properties to that object. Therefore, user interface styles remain unchanged conceptually in the OpenDocument file format, while formatting properties assigned directly to an object are assumed to be unnamed styles. In order to use unnamed styles, they are assigned a name and therefore become automatic styles.

There are two main reasons for using styles to store formatting properties:

1. The format and layout of the document get separated from the document content.

2. If two or more objects have the same formatting properties and styles assigned, the formatting properties that are assigned to the objects directly can be represented by a single automatic style for all objects. This saves disk space and allows styles to integrate seamlessly into the overall document style.

Within this chapter, the various style types are explained.

\subsection{Style Element}

Some style families are very similar in structure and can be represented by the same element. For example, the <style:style> element can represent paragraph, text, and graphic styles.

The individual style families that make use of these element are described separately. Within this section, the common attributes of the style element are described.

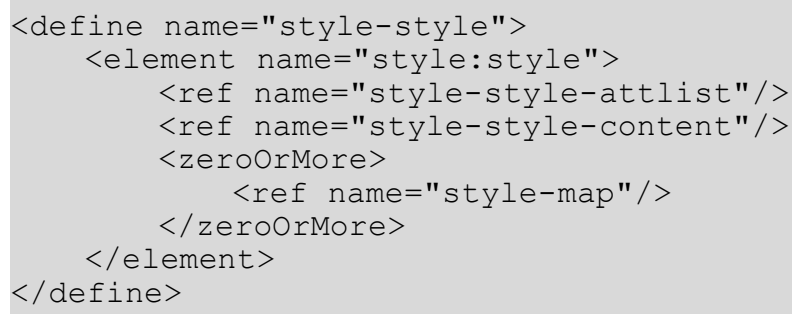

The attributes that may be associated with the <style:style $>$ element are:

- Style name

- Display name 
- Style family

- Parent style

- Next style

- List style

- Master page name

- Automatically update

- Data style name

- Class

- Outline numbering level

\section{Style Name}

The style: name attribute identifies the name of the style. This attribute, combined with the style:family attribute, uniquely identifies a style. The <office:styles <office:automatic-styles> and <office:master-styles> elements each must not contain two styles with the same family and the same name.

For automatic styles, a name is generated during document export. If the document is exported several times, it cannot be assumed that the same name is generated each time.

In an XML document, the name of each style is a unique name that may be independent of the language selected for an office applications user interface. Usually these names are the ones used for the English version of the user interface.

10867

10868

10869

10870

10871

\section{Display Name}

The style:display-name attribute specifies the name of the style as it should appear in the user interface. In contrast to the style name itself, this name may contain arbitrary characters. If this attribute is not present, the display name equals the style name.

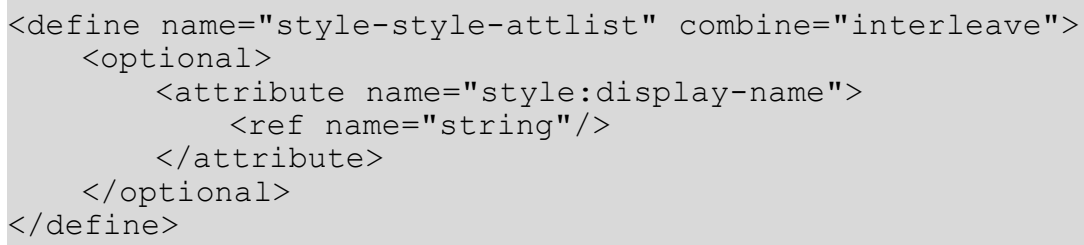

\section{Style Family}

The style:family attribute identifies the family of the style, for example, paragraph, text, or frame. It might have one of the following values: paragraph, text, section, table, tablecolumn, table-row, table-cell, table-page, chart, default, drawing-page, graphic, presentation, control and ruby. 


\section{Parent Style}

The style:parent-style-name attribute specifies the name of the parent style. If a parent style is not specified, a default parent style defined by the application is used. The parent style cannot be an automatic style and has to exist.

10879

10880

10881

10882

10883

10884

10885

10886

10887

10888

10889

10890

10891

10892

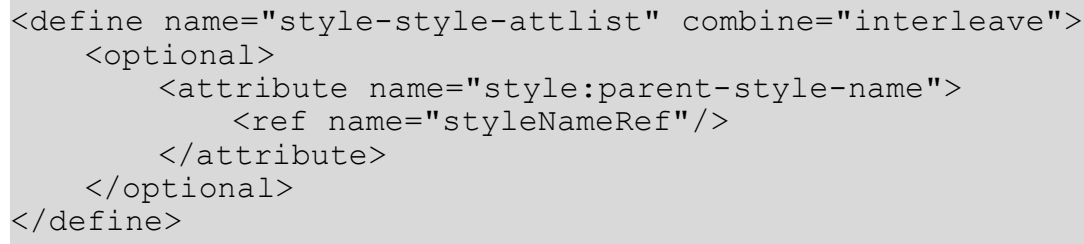

\section{Next Style}

The style:next-style-name attribute specifies the style to used for the next paragraph if a paragraph break is inserted in the user interface. By default, the current style is used as the next style.

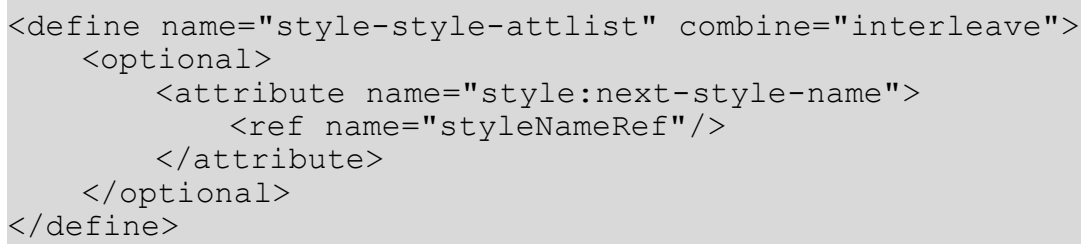

\section{List Style}

A paragraph style and styles of other families that may contain paragraph properties (for instance graphic styles) can have an associated list style. This applies to automatic and common styles.

The list style specified by the style:list-style-name attribute is only applied to headings and to paragraphs that are contained in a list, where the list does not specify a list style itself, and the list has no list style specification for any of its parents.

The style:list-style-name attribute's value can be empty. In this case, an association with a list style that is inherited from a parent style will be removed.

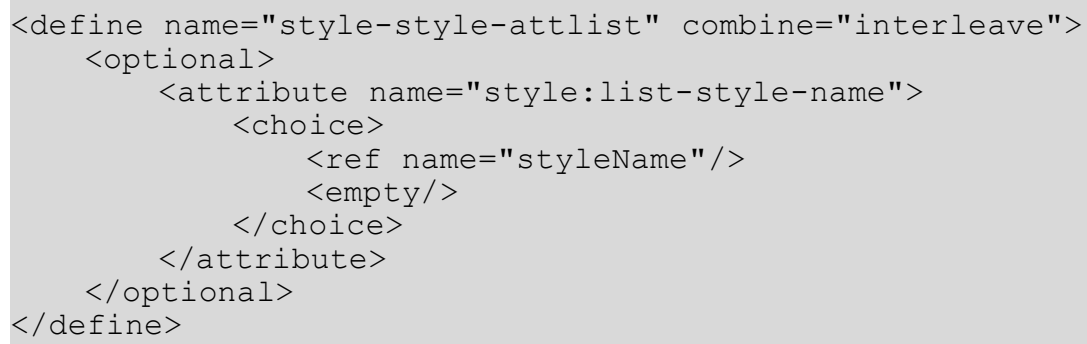

\section{Master Page Name}

A paragraph or table style can have an associated style:master-page-name attribute. This applies to automatic and common styles. If this attribute is associated with a style, a page break is inserted when the style is applied and the specified master page is applied to the preceding page. 


\section{Outline Numbering Level}

For style with family paragraph, the style: default-outline-level attribute specifies a default outline level. It takes a number like the text:outline-level attribute of the heading element <text:h>. If this attribute is existing for a paragraph style, and if the paragraph style is assigned to a paragraph by an user interface action, then office applications should convert the paragraph into a heading of the given level. However, the attribute has no effect to the differentiation of headings and paragraphs in the file format itself. The differentiation between headings and paragraphs still takes place by using either $a<t e x t: h>$ or $a<t e x t: p>$ element. If $a<t e x t: p>$ element references a paragraph style that has a style:default-outlinelevel attribute, the paragraph remains a paragraph and will not become a heading.

\section{Formatting Properties}

If a style has formatting attributes assigned, the style element contains one ore more formatting property container elements. See section 15 for detailed information about these element.

\section{Sample Style}

Example: OpenDocument representation of the "Text body" paragraph style

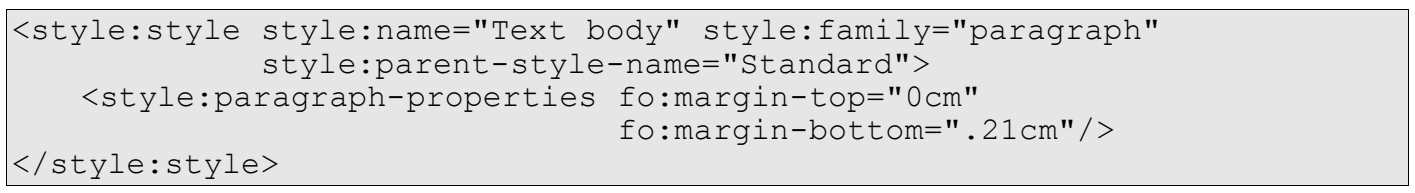

\subsubsection{Style Mappings}

The <style:map > element specifies the mapping to another style, if certain conditions exist. If a style contains such mappings, it is called an conditional style. There is one element for every condition that the style uses.

Conditional styles usually are supported by paragraph styles contained in text documents and table cell styles contained in spreadsheets only. Conditional styles are also supported by data styles.

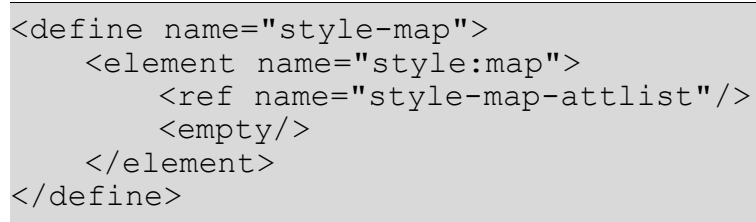

The attributes that may be associated with the $\langle$ style:map $>$ element are:

- Condition

- Applied style

- Base cell address 


\section{Condition}

The style:condition attribute specifies the condition in which a style map should be applied.

The value of this attribute is a Boolean expression. The syntax of the expression is similar to the $X P a t h$ syntax. If an application detects a condition that it does not recognize, it must ignore the entire <style:map> element.

The following conditions are valid for paragraph styles:

- $\quad$ list-level ()$=n$, where $n$ is a number between 1 and 10

- outline-level ()$=n$, where $n$ is a number between 1 and 10

- table() and table-header()

- $\operatorname{section}()$

- header() and footer()

- footnote() and endnote()

The following conditions are valid for paragraph styles:

- is-true-formula (formula)

- cell-content-is-between(value, value)

- cell-content-is-not-between(value, value)

- cell-content() operator value, where operator is one of; '<', '> ', '<=', ' $>=$ ', '=' or ' !=', and value is a numbervalue, a string or a formula.

- A numberValue is a whole or decimal number. The number cannot contain comma separators for numbers of 1000 or greater.

- A string comprises one or more characters surrounded by quotation marks.

- A formula is a formula (see 8.1.3) without the equals (=) sign at the beginning.

The following conditions are valid for data styles:

- value () op $n$, where op is a relational operator and $n$ is a number.

- For Boolean styles the condition value must be true and false.

The conditions that apply for different types of styles may differ.

\section{Applied Style}

The style:apply-style-name attribute specifies the style to apply when the condition specified by the style:condition attribute is true. If the referenced style is undefined or is an automatic style, an error occurs.

<define name="style-map-attlist" combine="interleave"> <attribute name="style:apply-style-name"> 


\section{Base Cell Address}

For table cell styles, the style:base-cell-address attribute specifies the base cell for relative addresses in formulas. This attribute only applies to cell styles where the condition contains a formula. The value of this attribute must be an absolute cell address with a table name.

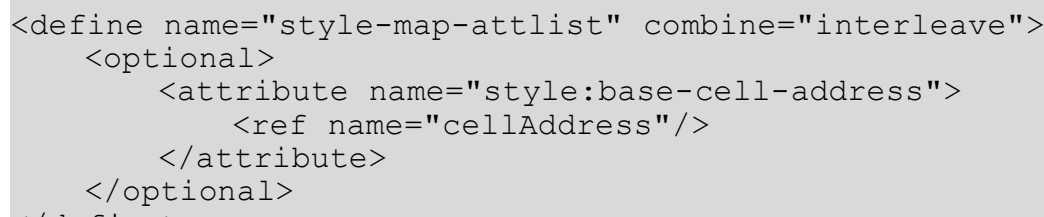

Example: Style mapping

\begin{tabular}{|} 
<style:style style:name="Text body" style:family="paragraph" \\
style:parent-style-name="Standard" \\
style:next-style-name="Text body"> \\
<style:paragraph-properties fo:margin-top="0cm" \\
fo:margin-bottom=".21cm"/> \\
<style:map style:condition="footnote" \\
style:apply-style-name="footnote"/> \\
<style:map style:condition="heading (1)" \\
style:apply-style-name="Heading $1 " />$ \\
<style:map style:condition="heading(2)" \\
style:apply-style-name="Heading $2 " />$ \\
$<$ style:style>
\end{tabular}

\subsection{Default Styles}

A default style specifies default formatting properties for a certain style family. These defaults are used if a formatting property is neither specified by an automatic nor a common style. Default styles exist for all style families that are represented by the $\langle$ style:style $\rangle$ element specified in section 14.1.

Default styles are represented by the $\langle$ style: default-style $>$ element. The only attribute supported by this element is style:family. Its meaning equals the one of the same attribute for the <style:style $>$ element, and the same properties child elements are supported depending on the style family.

10961

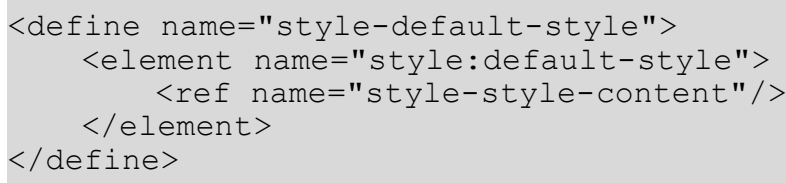

\subsection{Page Layout}

The <style:page-layout $>$ element specifies the physical properties of a page. This element contains a <style:page-layout-properties $>$ element which specifies the formatting properties of the page and two optional elements that specify the properties of headers and footers.

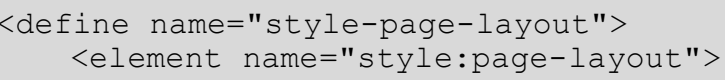




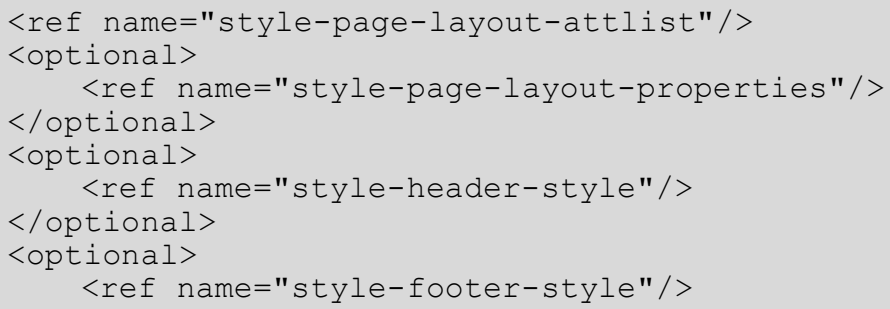

The attributes that may be associated with the <style:page-layout> element are:

- Name

- Page usage

\section{Name}

The style:name attribute specifies the name of the page layout.

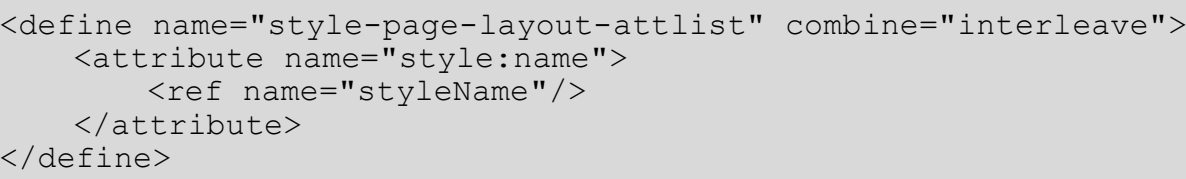

\section{Page Usage}

The style:page-usage attribute specifies the type of pages that the page master should generate.

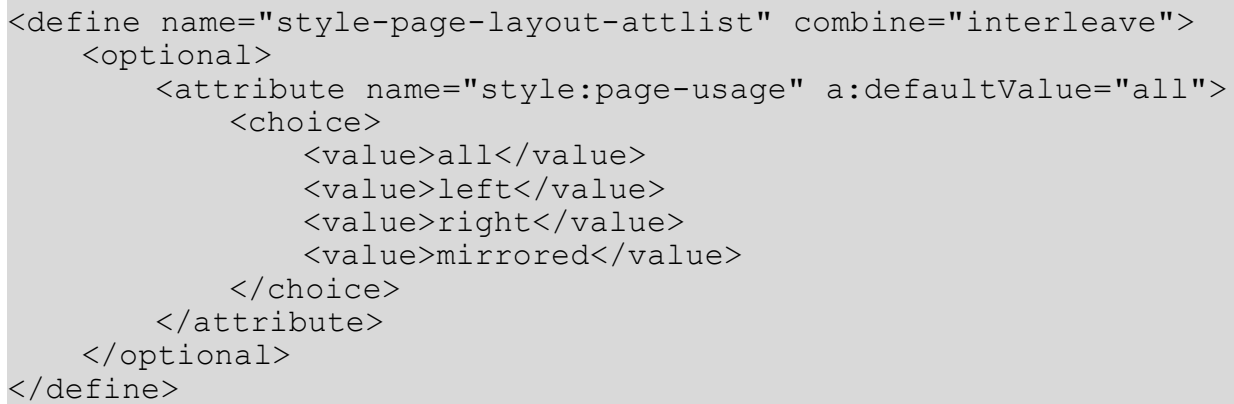

\subsubsection{Header and Footer Styles}

The header and footer style elements <style: header-style $>$ and <style:footer-style $>$ specify the formatting properties for headers and footers on a page. These elements must be contained within a page layout element. The contain a $<$ style: header-footerproperties $>$ element that contains the formatting properties of the header or footer.

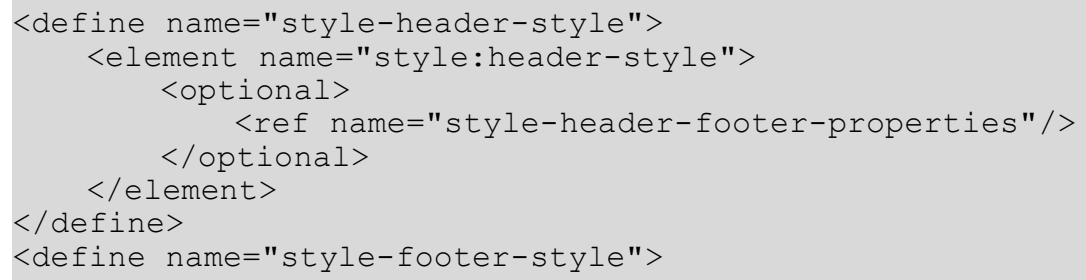




\subsection{Master Pages}

In text and spreadsheet documents, the <style:master-page $>$ element contains the content of headers and footers. In these applications, a sequence of pages is generated by making use of a single master page or a set of master pages.

In drawing and presentation documents, the <style:master-page $>$ element is used to define master pages as common backgrounds for drawing pages. Each drawing page here is directly linked to one master page, which is specified by the draw:master-page-name attribute of the drawing pages style.

Master pages are contained in the <office:master-styles> element. See also section 2.8.

All document must contain at least one master page element.

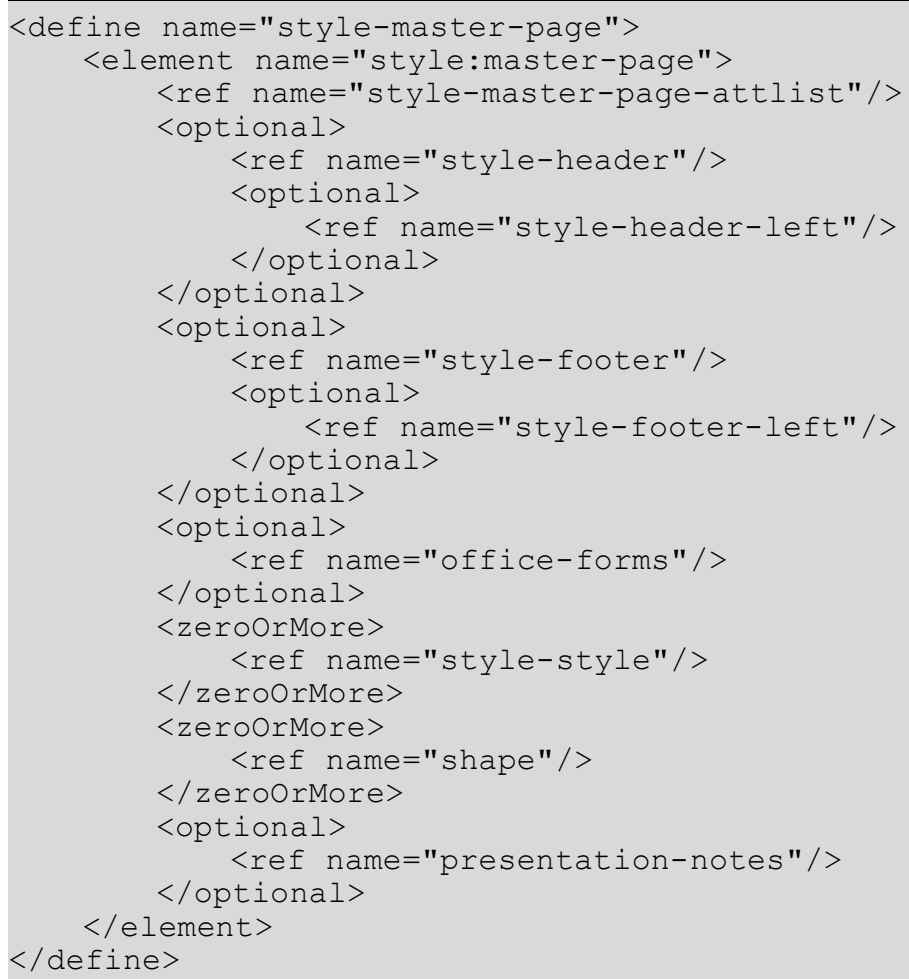

The attributes that may be associated with the <style:master-page $>$ element are:

- Page name

- Display name

- Page layout

- Page style

- Next style name 
The elements that my be included in the <style:master-page> element are:

- Headers and Footers

- Forms

- Styles

- Shapes

- Presentation notes

\section{Page Name}

The style: name attribute specifies the name of a master page. Each master page is referenced using the page name. This attribute is required and the name specified must be unique.

11040

11041

11042

11043

11044

11045

11046

11047

11048

11049

11050

11051

11052

11053

11054

11055

11056

\section{Page Style}

In graphic applications, additional drawing page attributes my be assigned to a drawing page using the draw:style-name attribute. This attribute is optional. The fixed family for page styles is drawing-page. This is used to define an optional background filling.

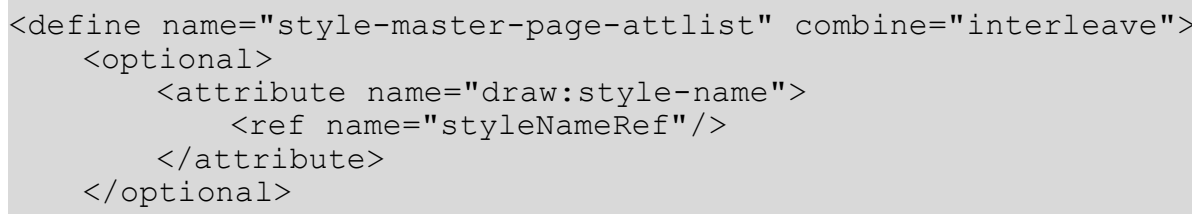




\section{Next Style Name}

For text and spreadsheet documents, the style:next-style-name attribute identifies the master page that is used for the next page if the current page is entirely filled. This attribute is optional. If the next style name is not specified, the current master page is used for the next page. The value of this attribute must be the name of another style:master-page element.

\subsubsection{Headers and Footers}

The header and footer elements specify the content of headers and footers. They are contained within a master page element. The <style: header $>$ and $\langle$ style: footer $\rangle$ elements contain the content of headers and footers. The two additional elements, <style: header-left $>$ and <style:footer-left>, can be used to specify different content for left pages, if appropriate. If the latter two elements are missing, the content of the headers and footers on left and right pages is the same.

If the style:page-usage attribute associated with the page layout has a value of all or mirrored and there are no <style: header-left> or <style: footer-left> elements, the header and footer content is the same for left and right pages.

If the style:page-usage attribute has a value of left or right, the <style:headerleft> or <style:footer-left> elements are ignored.

The content of headers and footers is either:

- Standard text content, for example paragraphs, tables, or lists. Such headers and footers usually are supported by text documents.

- A sequence of any of the following elements; <style:region-left $>$, <style:regioncenter $>$ and $<$ style:region-reight $>$. These elements usually are supported by spreadsheet documents.

- Empty, which switches off the display of all headers or footers. It is not possible to switch off the display of headers or footers for left pages only.

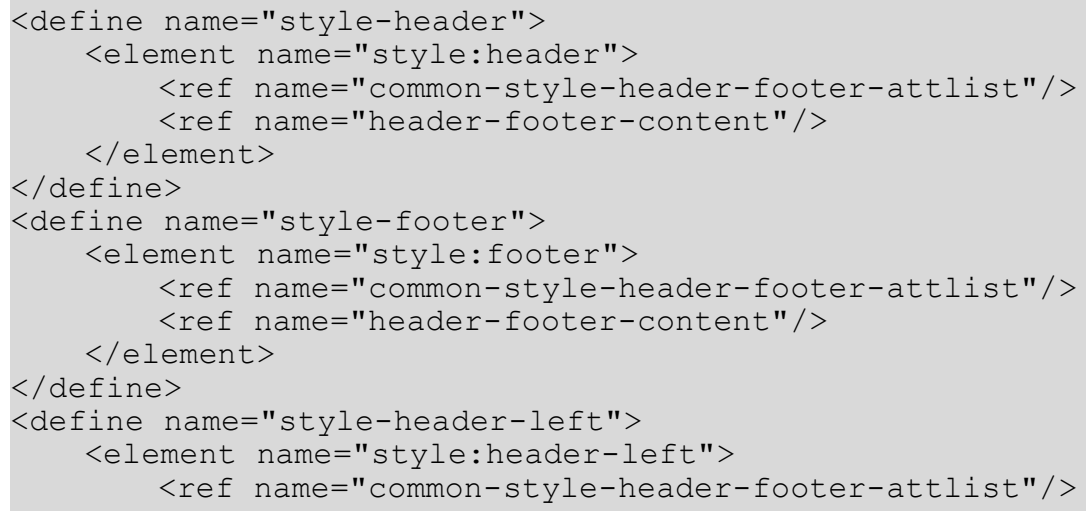




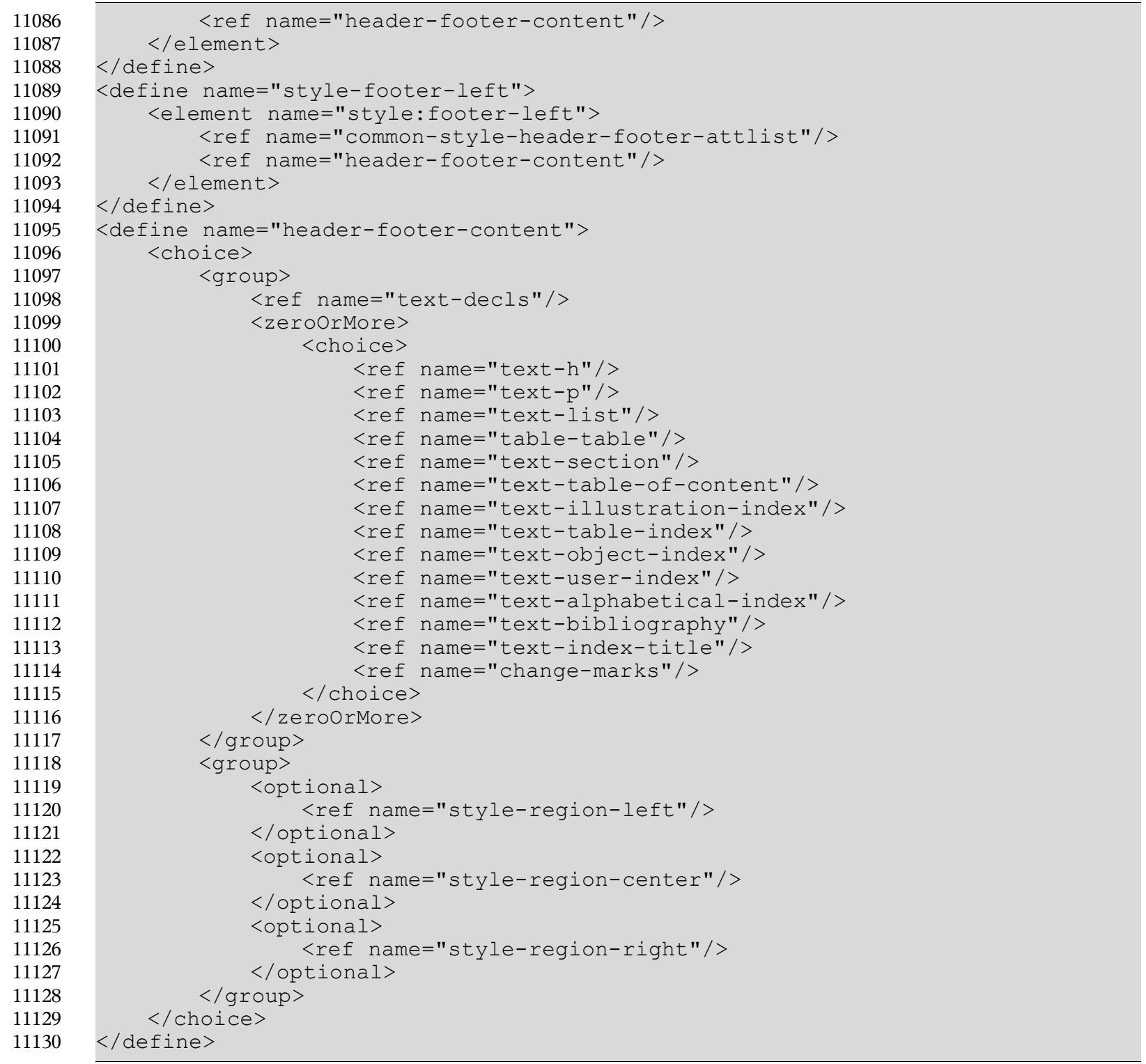

\section{Display}

The style:display attribute specifies whether the header or footer is displayed or not.

11131

11132

11133

11134

11135

11136

11137

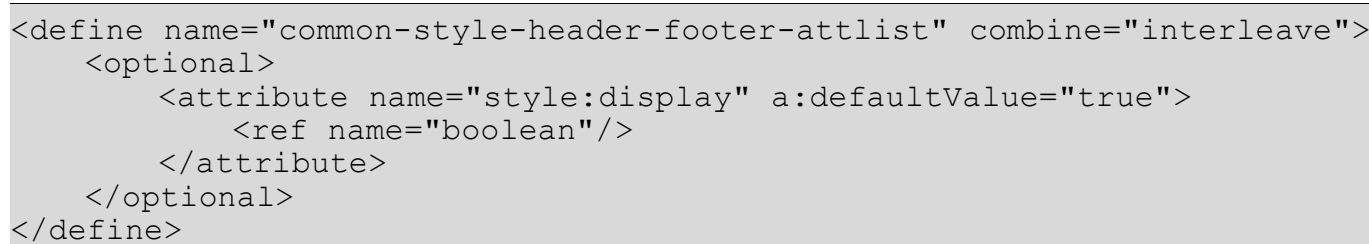

\section{Regions}

The region elements <style:region-left>, <style:region-center> and <style:region-right> specify three regions of a header or footer that are displayed left aligned, centered or right aligned. Each of these regions can contain a sequence of paragraphs. 


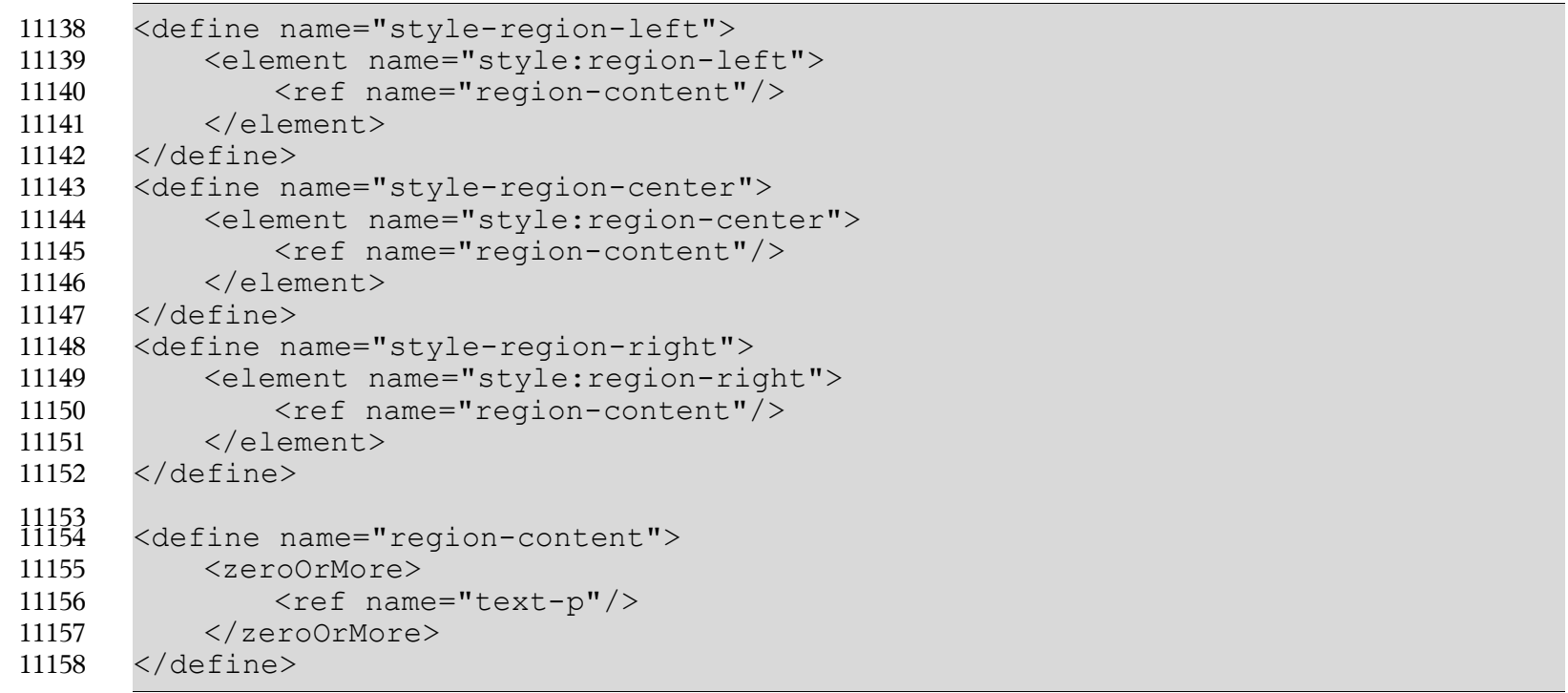

\subsubsection{Presentation Notes}

The <presentation: notes $>$ element is usually supported only by presentation applications, where each master page as well as each drawing page in a presentation can have an additional presentation notes page. The presentation notes page contains:

- A preview of the drawing page.

- Additional graphic shapes as contained in the <presentation: notes $>$ element. While the $<$ presentation: notes $>$ may contain any kind of shapes, the only shape type that should be supported by presentation applications are text boxes (i.e. <draw: text-box > contained in a <draw: frame>).

\section{Page Layout}

The style:page-layout-name attribute specifies a page layout which contains the sizes, border and orientation of the notes page. See section 14.3 for details on page layouts. 


\section{Page Style}

The attribute draw:style-name assigns an additional formatting attributes to a notes page by assigning a drawing page style. This attribute is optional. The fixed family for page styles is drawing-page.

\section{Header Declaration}

The presentation: use-header-name attribute specifies the name of the header field declaration (see section 9.11.2) that is used for all header fields (see section 9.10.1) that are displayed on the notes page. See also section 9.1.4.

\section{Footer Declaration}

The presentation: use-footer-name attribute specifies the name of the footer field declaration (see section 9.11.3) that is used for all footer fields (see section 9.10.2) that are displayed on the notes page. See also section 9.1.4.

\section{Date and Time Declaration}

The presentation: use-date-time-name attribute specifies the name of the date-time field declaration (see section 9.11.4) that is used for all date-time fields (see section 9.10.3) that are displayed on the notes page. See also section 9.1.4.

Example: Master page containing presentation notes.

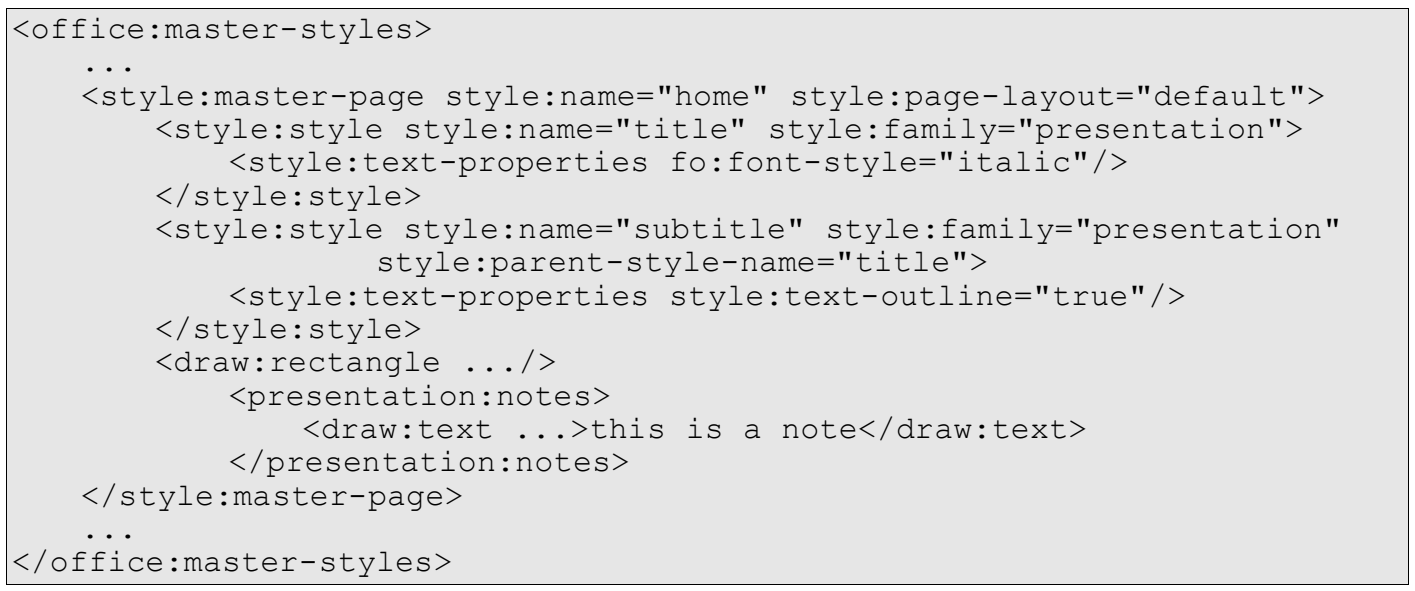

\subsection{Table Templates}

A table template is a set formatting properties, like borders, background color, and text properties that can be applied to a table when creating it. In contrast to other styles, it is not referenced by a table, but if a table is created, a set of table-cell styles is created from the table template. To change the formatting properties of a table, the cell styles and other styles 
themselves have to be changed. Table are contained in the <style:master-styles $>$ element.

11182
11183
11184
11185
11186
11187
11188
11189
11190
11191
11192
11193
11194
11195
11196
11197
11198
11199
11200
11201
11202
11203
11204
11205
11206
11207
11208
11209

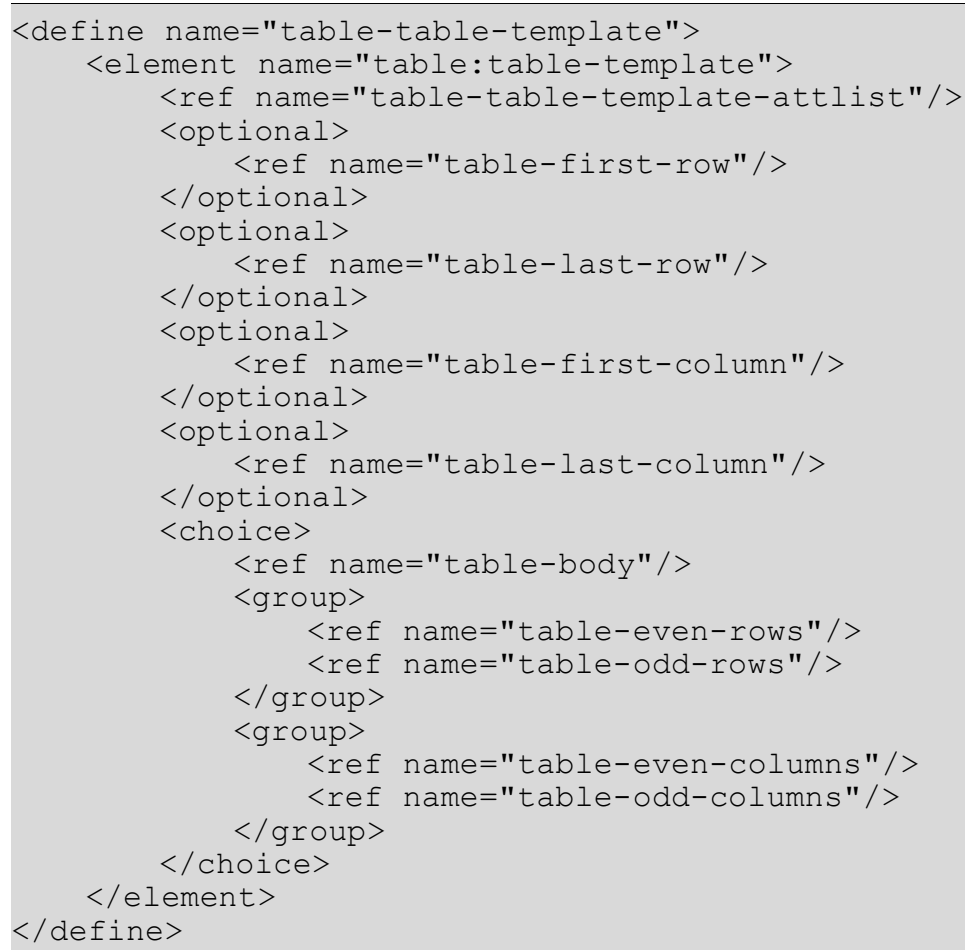

\section{Style Name}

The table:name attribute specifies the name of the table template.

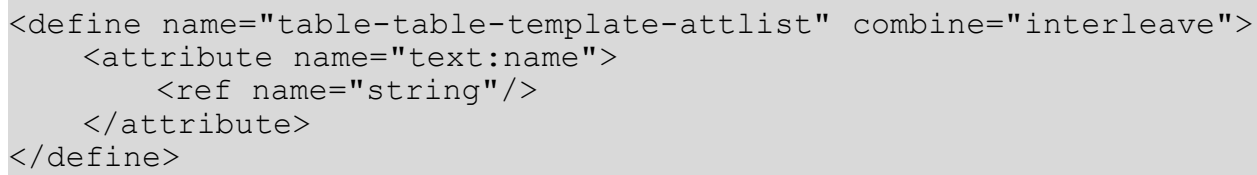

\section{Corner Styles}

The attributes table:first-row-start-column, table:first-row-end-column, table: last-row-start-column and table:last-row-end-column specify whether the cells in the four corners of the table should get the style from the row they are in or from the column. The possible values of these attributes are row and column.

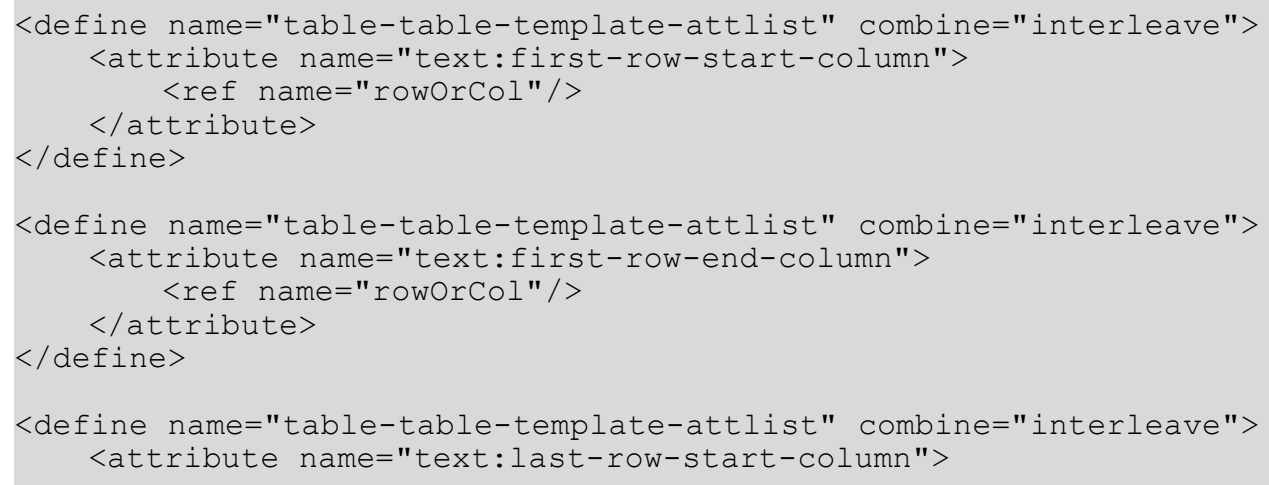




\subsubsection{Row and Column Styles}

The elements $<$ table: first-row $>$ and $<$ table: last-row $>$ specify the cell styles that shall be applied to the first and last row of a table. They have a table:style-name attribute that references these styles.

The elements $<t a b l e: f i r s t-c o l>$ and $<t a b l e: l a s t-c o l>$ do the same for the first and last table column.

For the remaining cells, the cells styles can either be specified by the $<$ table:body $>$ element, or by the $\langle$ table: even-rows $>/<$ table: odd-rows $>$ or $\langle$ table: evencolumns $>\mid<$ table:odd-columns $>$ element pairs if different cell styles should be applied to even and odd rows or columns.

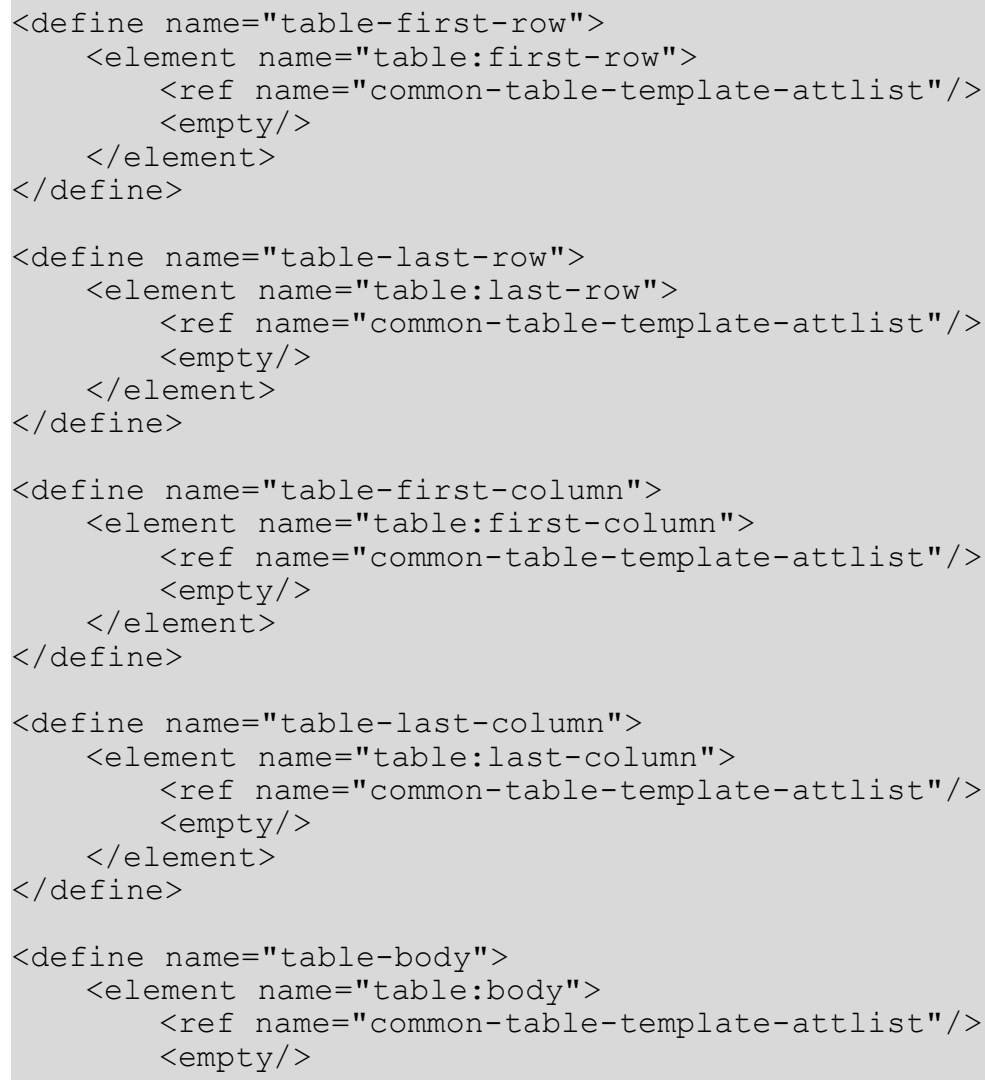




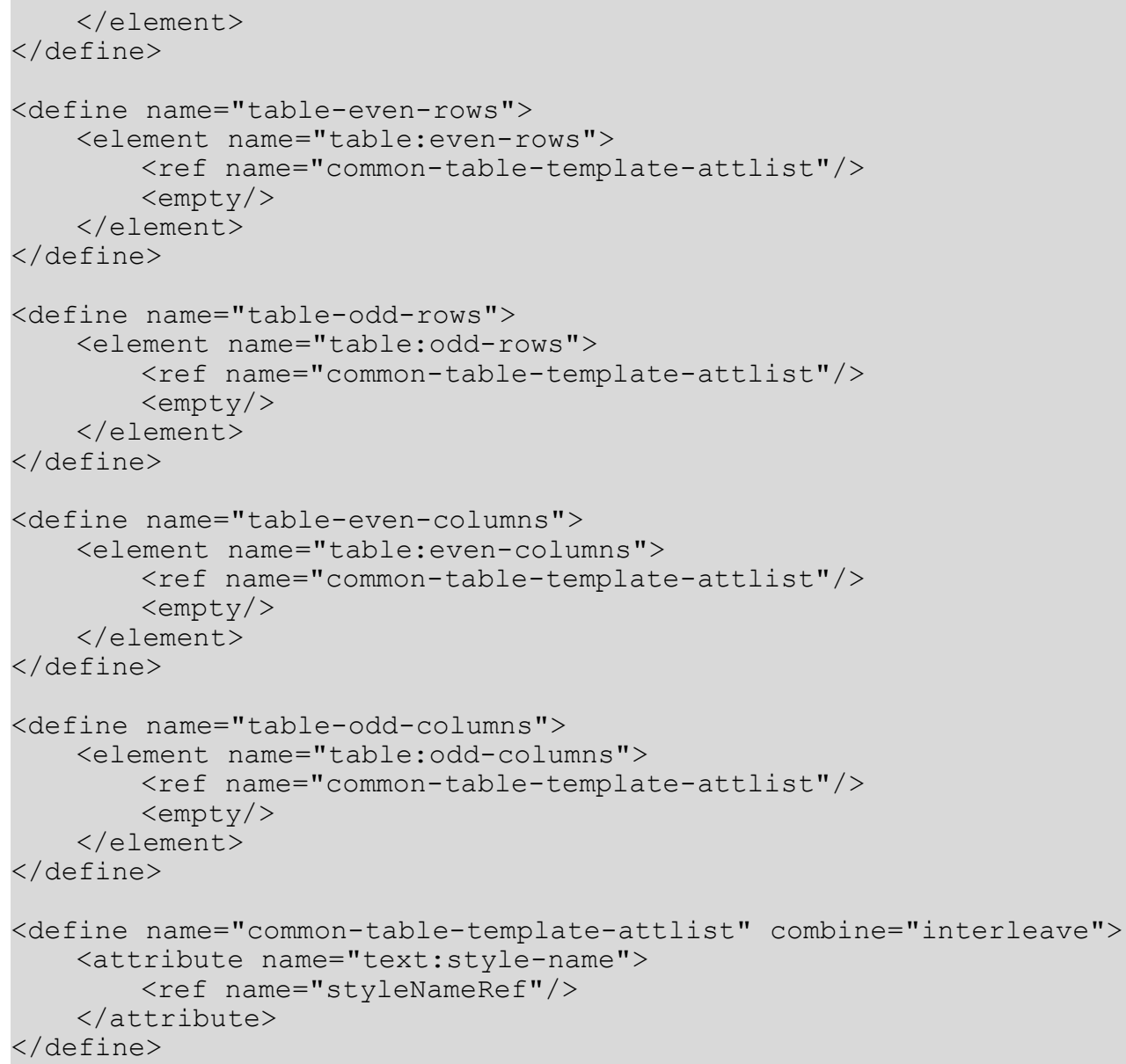

\subsection{Font Face Declaration}

OpenDocument font face declarations directly correspond to the a font-face font description of [CSS2] (see §15.3.1) and the <font-face> element of [SVG] (see §20.8.3), but have the following two extensions:

- OpenDocument font face declarations optionally may have an unique name This name can be used inside styles (i.e. as attribute of <stye: text-properties $>$ element) as value of the style: font-name attribute to immediately select a font face declaration. If a font face declaration is referenced this way, the steps described in §15.5 the [CSS2] font matching algorithms for selecting a font declaration based on the font-family, font-style, font-variant, font-weight and font-size descriptors will not take place, but the referenced font face declaration is used directly.

- Some additional font descriptor attributes exist. The are described below.

With the exception mentioned above, conforming applications should implement the CSS2 font matching algorithm as described in described in \$15.5 the [CSS2], but they may also implement variants of it. They are especially allowed to implement a font matching based only on the font face declarations, that is, a font matching that is not applied to every character independently but only once for each font face declaration. This is useful for editing applications, where a font matching based on characters might be to expensive. 
11315

11316

11317

11318

11319

11320

11321

11322

11323

\section{$</$ define $>$}

\subsubsection{CSS2/SVG Font Descriptors}

Font face declarations support the font descriptor attributes and elements described in $\S 20.8 .3$ of [SVG].

11324

11325

11326

11327

11328

11329

11330

11331

11332

11333

11334

11335

11336

11337

11338

11339

11340

11341

11342

11343

11344

11345

11346

11347

11348

11349

11350

11351

11352

11353

11354

11355

11356

11357

11358

11359

11360

11361

11362

11363

11364

11365

11366

11367

11368

11369

11370

11371

11372

11373

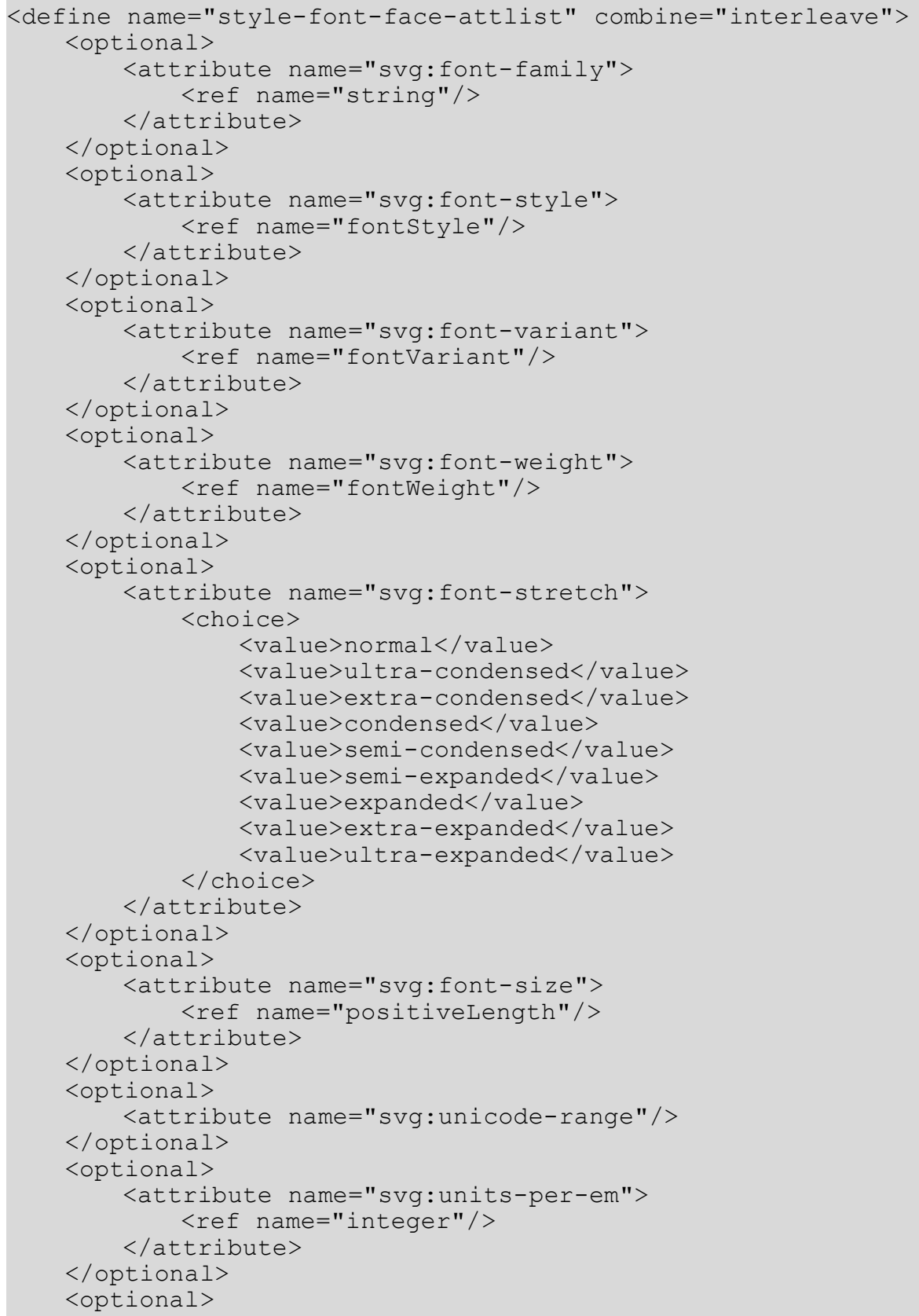




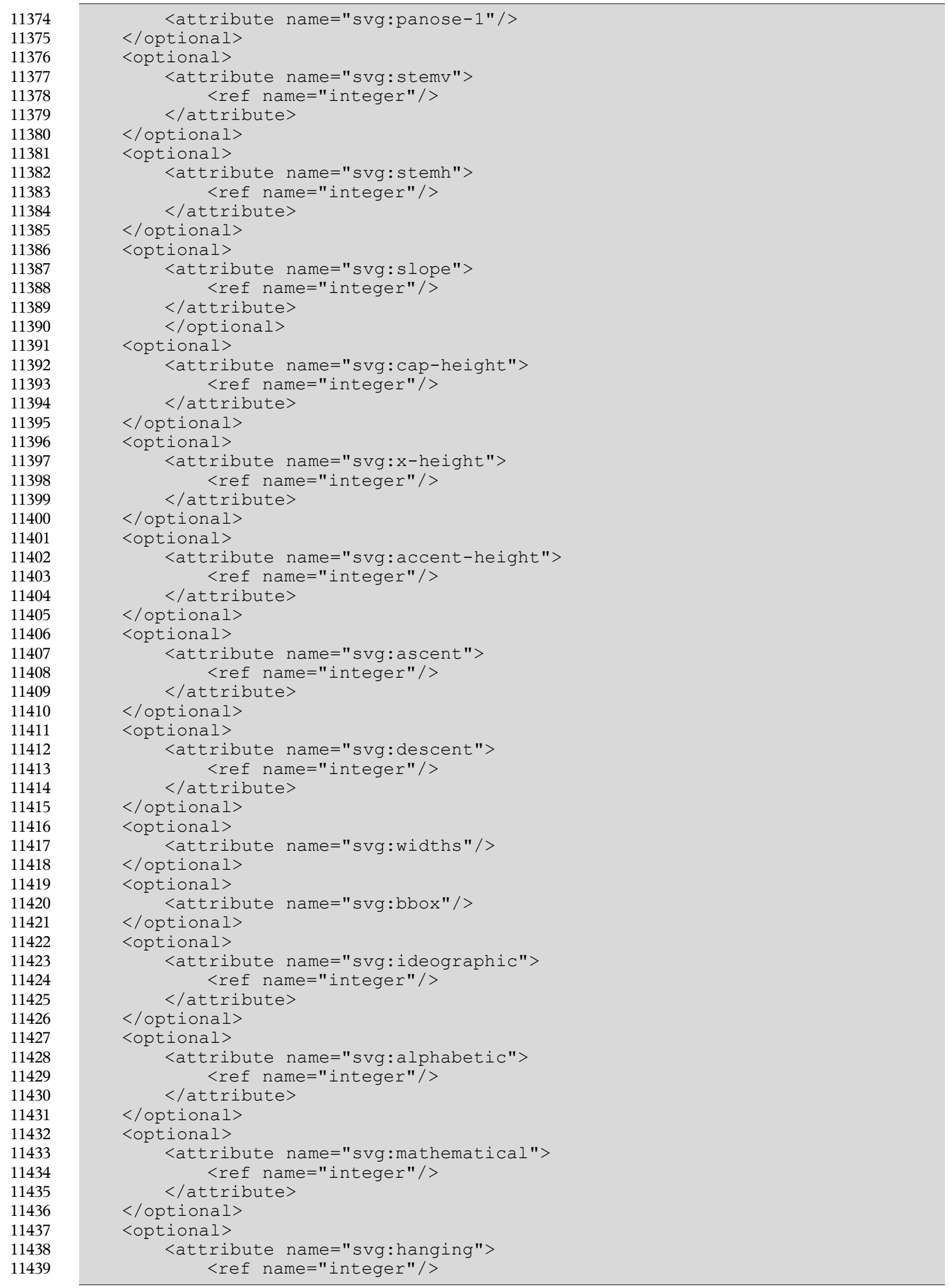




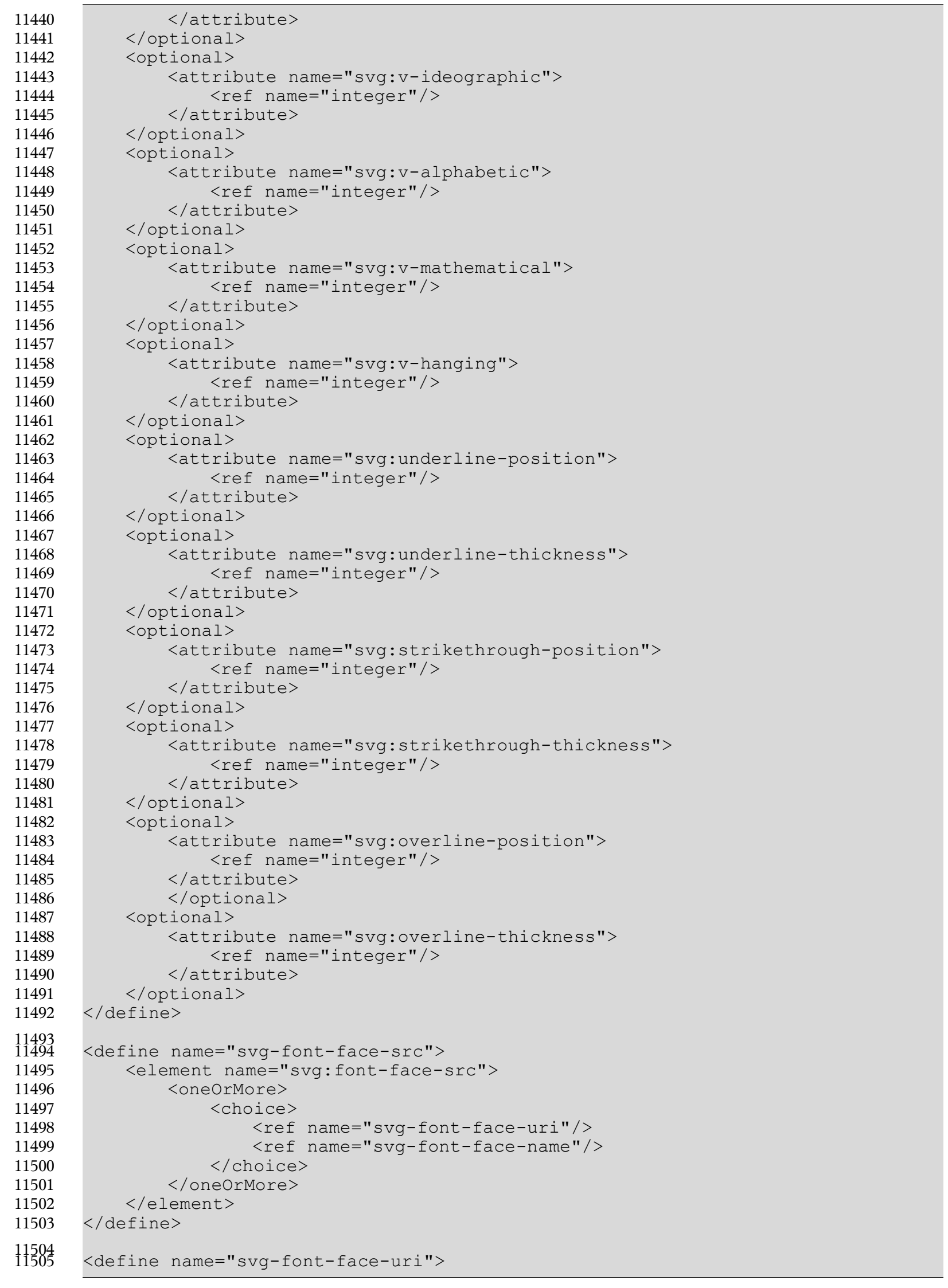




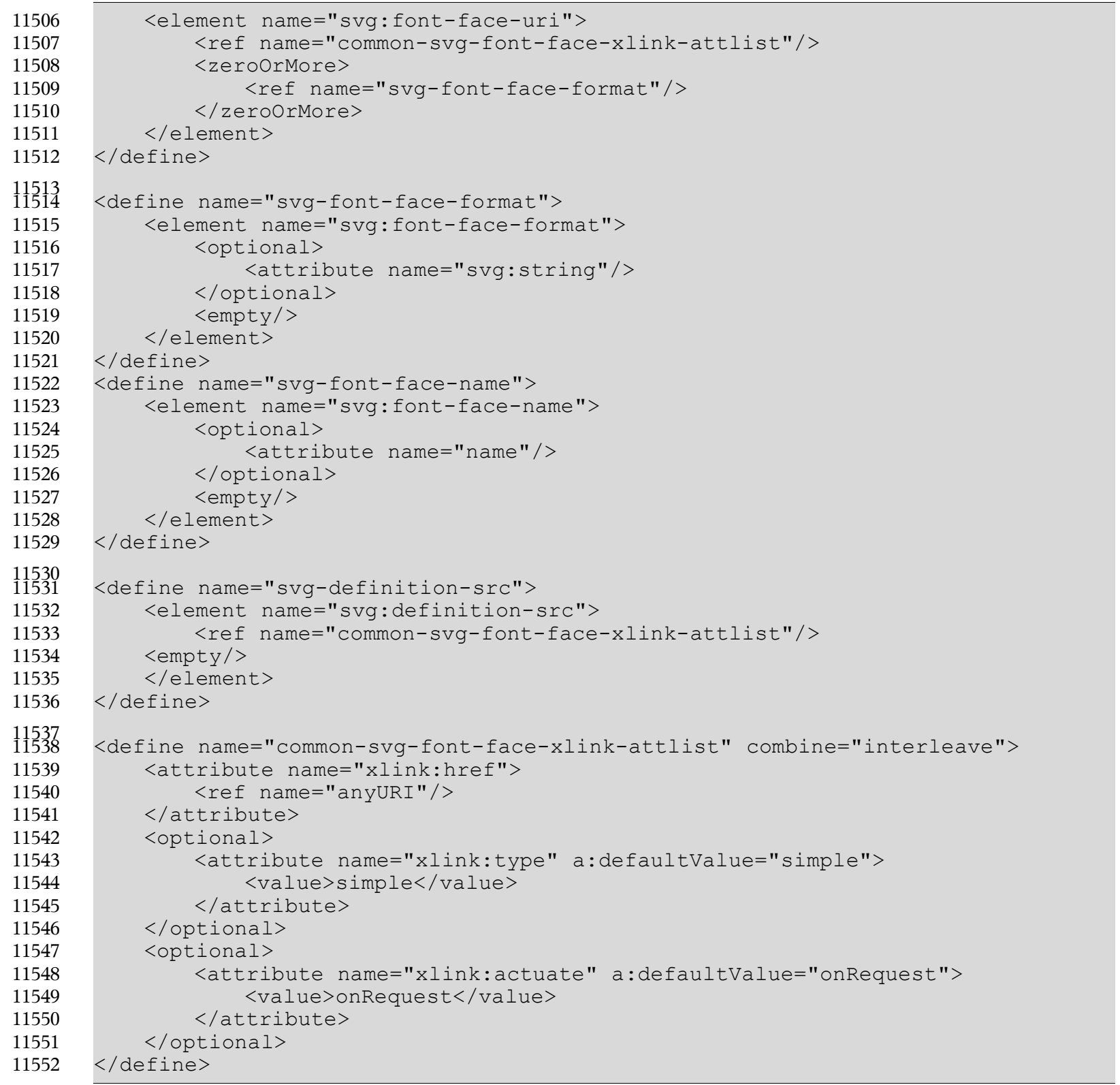

\subsubsection{Name}

The style: name attribute specifies the unique name of the font declaration. This name can be used inside styles (i.e. as attribute of <stye: text-properties $>$ element) as value of the style: font-name attribute to immediately select a font face declaration

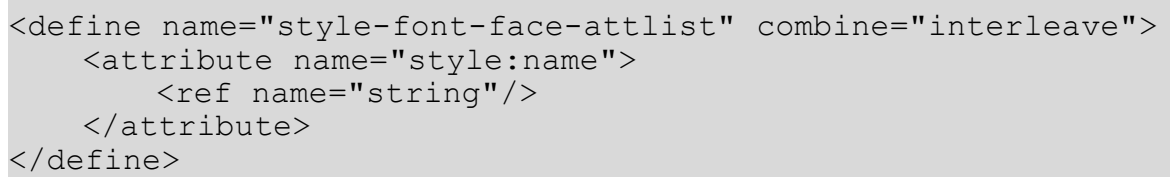




\subsubsection{Adornments}

The style: font-adornments attributes specifies adornments, like bold or italic that can be used to locate a font in addition to the family name.

\subsubsection{Font Character Set}

The style: font-charset attribute specifies the character set of a font. See section 15.4.18 for details.

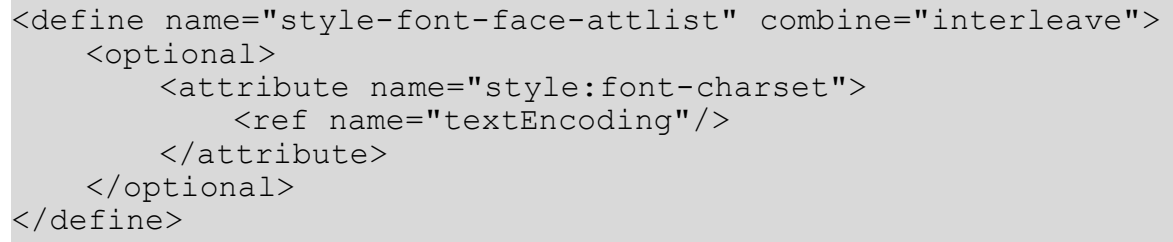

\subsection{Data Styles}

Data styles describe how to display different types of data, for example, a number or a date. The elements and attributes that are used to represent data styles are contained in the namespace urn:oasis:names:tc:opendocument:xmlns:datastyle:1.0. The prefix number denotes the data styles namespace. 
This section describes the OpenDocument representation of the following data styles:

- Number style

- Currency style

- Percentage style

- Date style

- Boolean style

- Text style

\subsubsection{Number Style}

The $<$ number: number-style $>$ element describes the style for decimal numbers.

This element can contain one of the following elements:

- <number: number>

- <number:scientific-number>

- <number:fraction>

These elements describe the display format of the number. The elements can be preceded or followed by $<$ number: text $>$ elements, which contain any additional text to be displayed before or after the number.

In addition, this element can contain a $<$ style: text-properties $>$ element and a $<$ style:map > element.

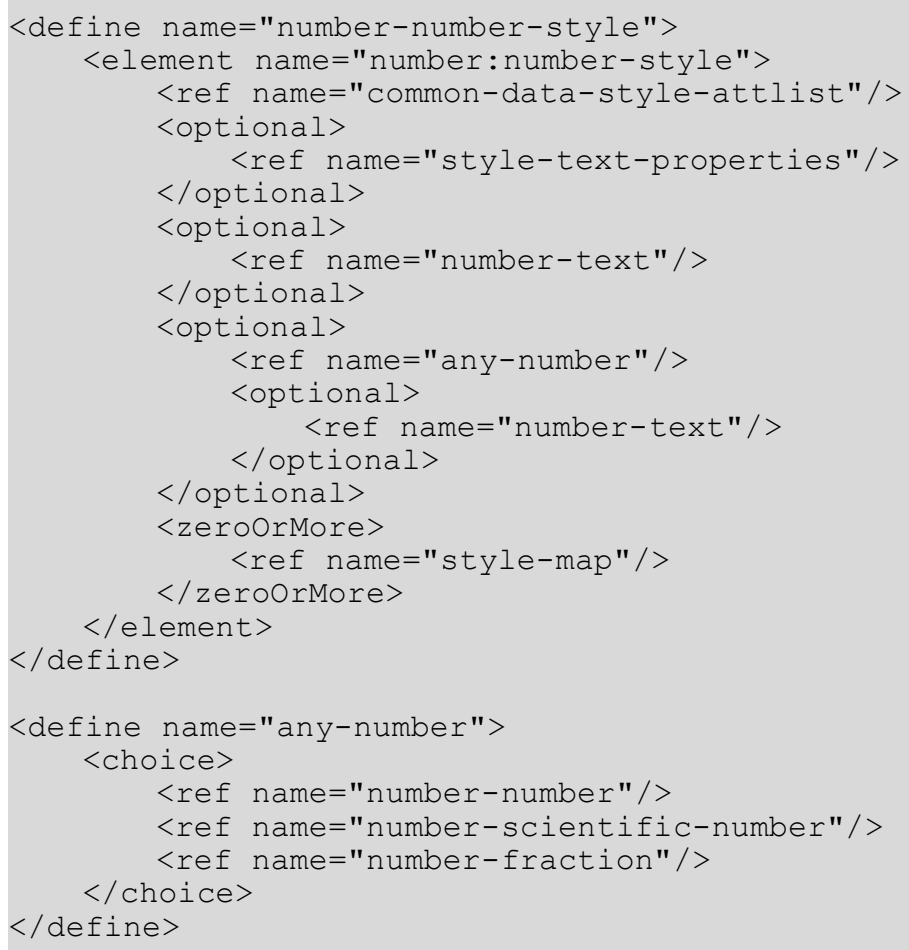

See section 14.7.9 for information about the attributes that may be associated with the number style elements. 
The following elements may be contained in the <number: number-style> element:

- Number

- Scientific number

- Fraction

\section{Number}

The $<$ number: number $>$ element specifies the display properties for a decimal number.

This element is contained in the $<$ number: number-style $>$ element. The $<$ number: number $>$ element can contain multiple $<$ number : embedded-text $>$ elements.

The number:decimal-replacement and number:display-factor attributes may be used with this element. See also section 14.7.11 for information about additional attributes that may be associate with the <number: number $>$ element.

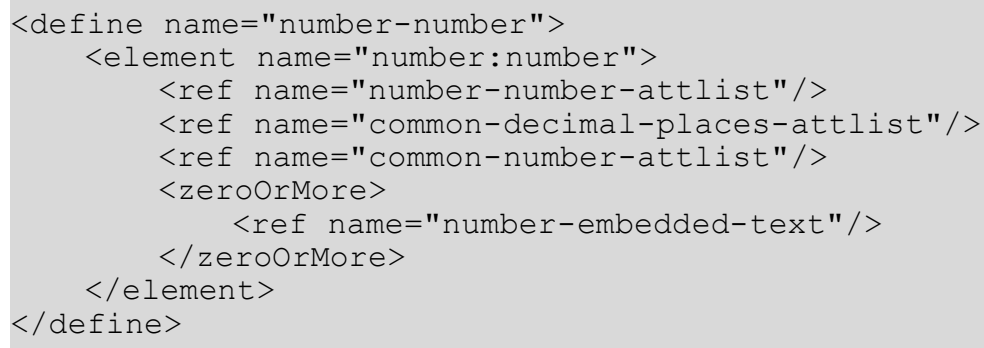

\section{Decimal Replacement}

If a number style specifies that decimal places are used but the number displayed is an integer, a replacement text may be displayed instead of the decimal places. The number: decimalreplacement attribute specifies the replacement text.

Some applications may supports replacement text only that consists of the same number of "-" characters as decimal places.

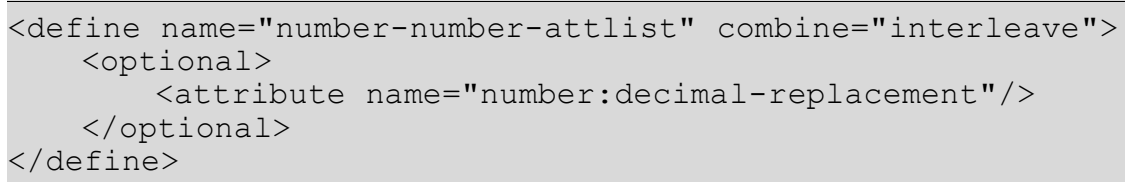

\section{Display Factor}

The number: display-factor attribute specifies a factor by which each number is scaled (divided) before displaying. A factor of 1000 , for example, causes numbers to be displayed in thousands.

Some applications may only support display factors of 1000 to the power of a non-negative integer number, that is $1,1000,1000000,1000000000$, etc.

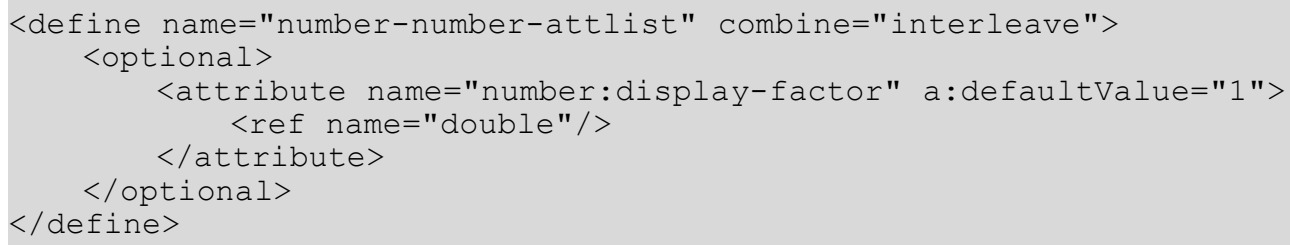




\section{Embedded Text}

The $<$ number: embedded-text $>$ element specifies text that is displayed at one specific position within a number. This element is different to a grouping separator, which appears several times within a number.

This element is contained in the <number: number $>$ element. The <number: number $>$ element can contain multiple occurrences of the $<$ number: embedded-text $>$ element to describe text at different positions in the number. 


\section{Fraction}

The <number: fraction> element specifies the display properties for a number style that should be displayed as a fraction.

This element is contained in the $<$ number: number-style $>$ element.

The number:min-numerator-digits and number:min-denominator-digits attributes may be used with this element. See section 14.7 .11 for information on the attributes that may be associated with the <number: fraction $>$ elements.

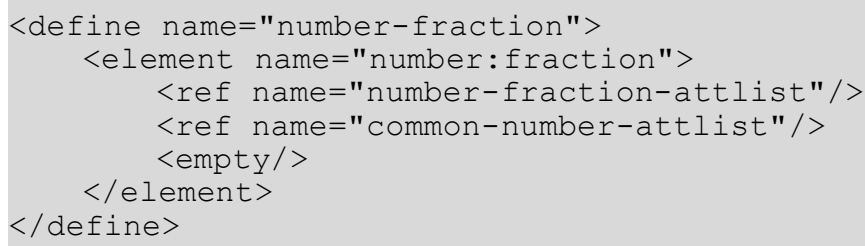

\section{Minimum Numerator Digits}

The number:min-numerator-digits attribute specifies the minimum number of digits to use to display the numerator in a fraction.

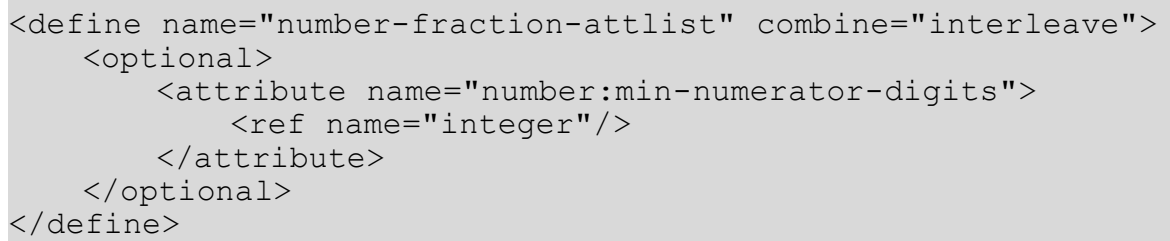

\section{Minimum Denominator Digits}

The number:min-denominator-digits attribute specifies the minimum number of digits to use to display the denominator of a fraction.

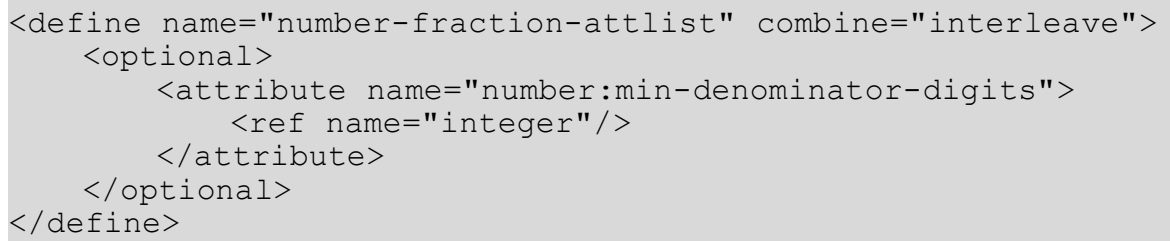

\section{Denominator Value}

The number:denominator-value attribute specifies an integer value that is used as denominator of a fraction. If this attribute is not present, the application may choose an arbitrary denominator value.

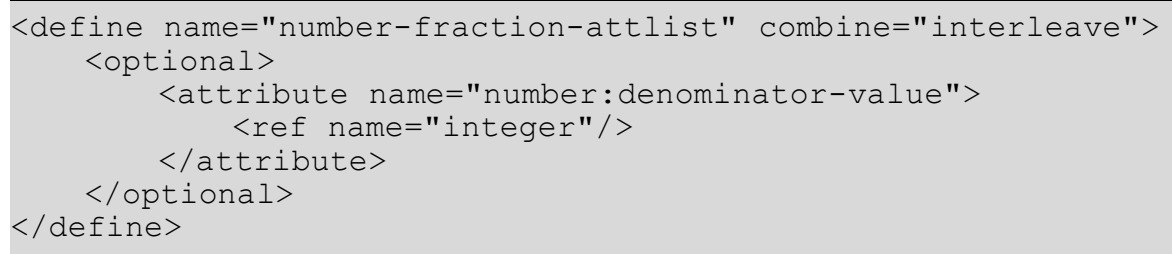




\subsubsection{Currency Style}

The <number: currency-style> element describes the style for currency values.

This element can contain one <number: number $>$ element and one <number: currencysymbol> element. It can also contain <number: text> elements, which display additional text, but it cannot contain two of these elements consecutively.

In addition, this element can contain a $<$ style:text-properties $>$ element and a $<$ style:map> element.

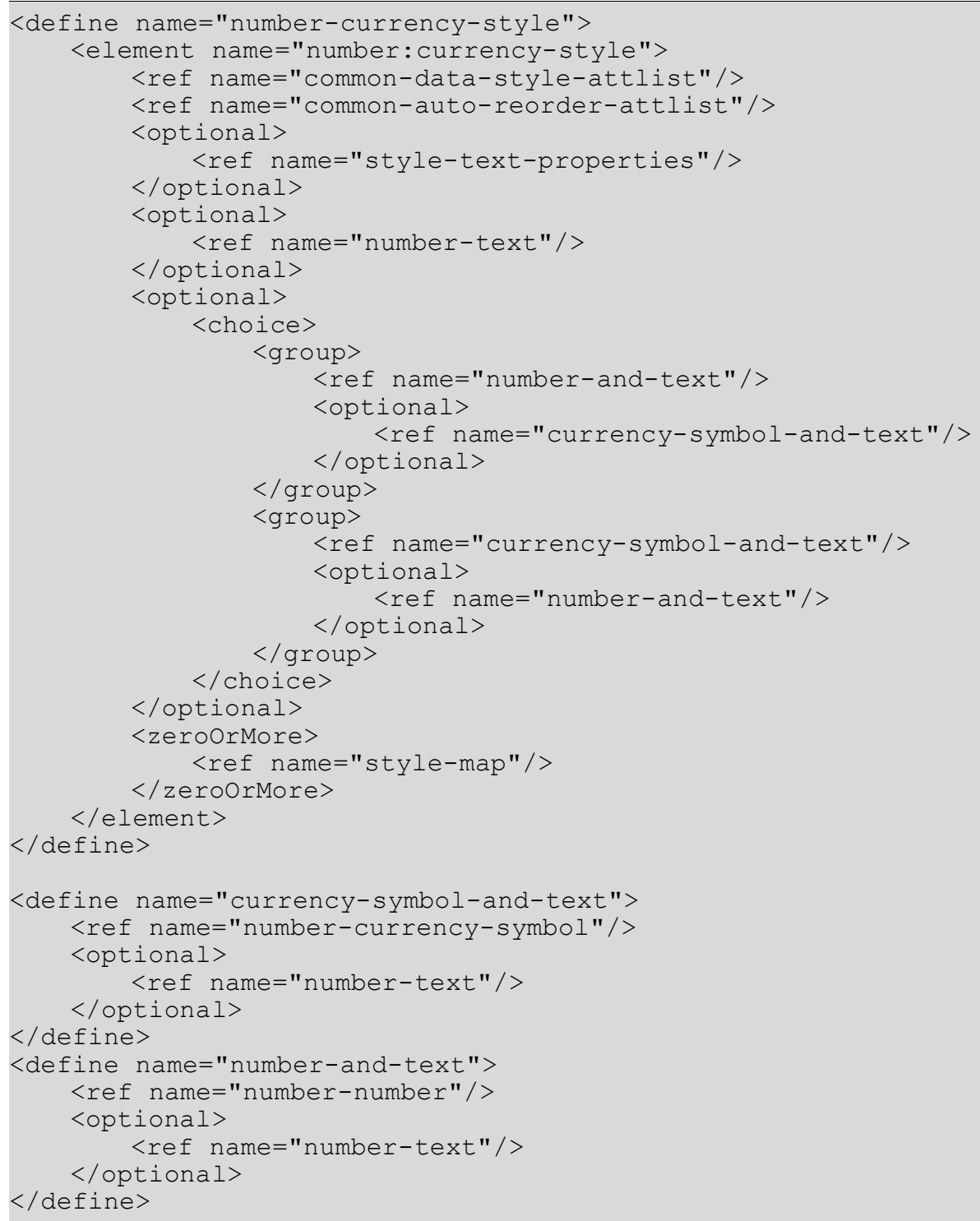

See section 14.7.9 for information about the attributes that may be associated with the number style elements.

The following elements may be contained in the <number: currency-style> element:

- $\quad$ Number, see section 14.7.1.

- Currency symbol 


\section{Currency Symbol}

The <number: currency-symbol> element determines whether or not a currency symbol is displayed in a currency style.

The content of this element is the text that is displayed as the currency symbol. If the element is empty or contains white space characters only, the default currency symbol for the currency style or the language and country of the currency style is displayed.

This element is contained in the <number: currency-style> element.

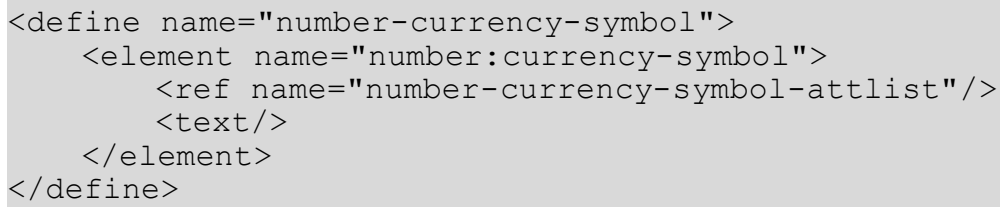

The number: language and number: country attributes may be used to specify the language and country of the currency symbol. See section 14.7 .11 for information on the other attributes that may be associated with the currency style elements.

\section{Currency Language and Country Attributes}

If the currency symbol contained in a currency style belongs to a different language or country than the currency style itself, then the number: language and number:country attributes may be used to specify the language and country of the currency symbol.

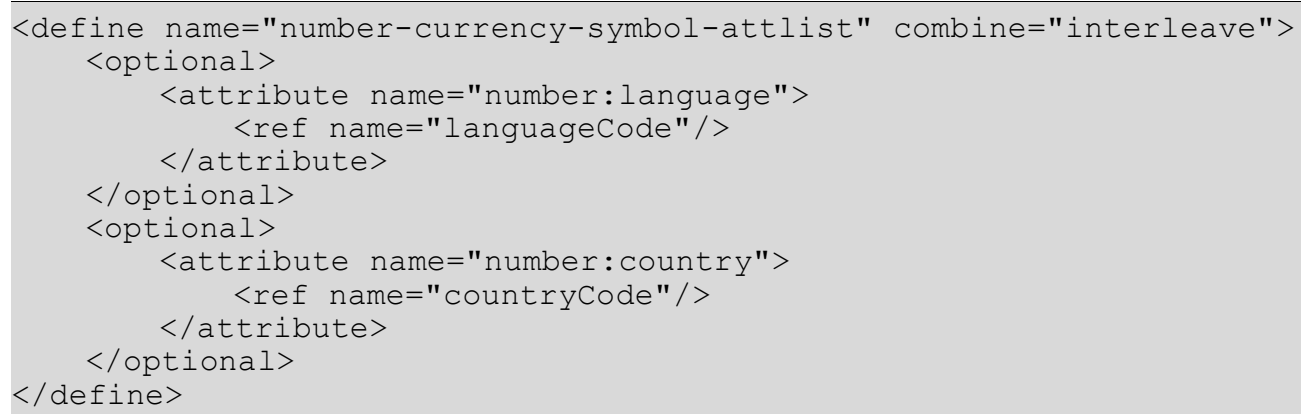

\subsubsection{Percentage Style}

The <number: percentage-style $>$ element describes the style for percentage values.

This element can contain one $<$ number : number $>$ element, which describes the display format for the percentage. The element can be preceded or followed by <number: text $>$ elements, which contain any additional text to display before or after the percentage. Some applications require that at least one $<$ number : text > element exist and that its text must contain a "\%" character.

In addition, the <number: percentage-style $>$ element can contain a $<$ style: textproperties $>$ element and a $<$ style:map $>$ element.

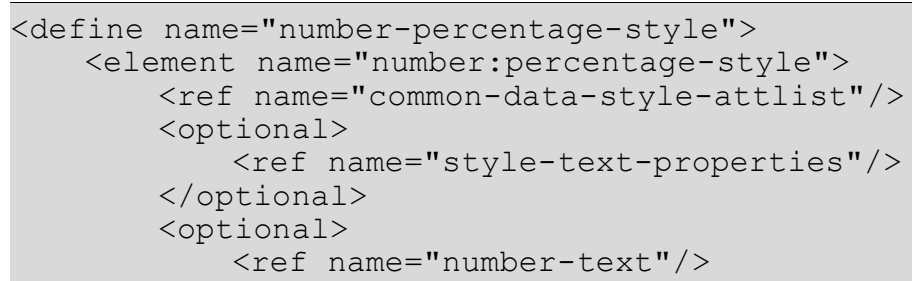


11761

11762

11763

11764

11765

11766

11767

11768

11769

11771

11772

11773

11774

11775

11776

11777

11778

11779

11780

11781

11782

11783

11784

11785

11786

11787

11788

11789

11790

11791

11792

11793

11794

11795
11796

11797

11798

11799

11800

11801

11802

11803

11804

11805

11806

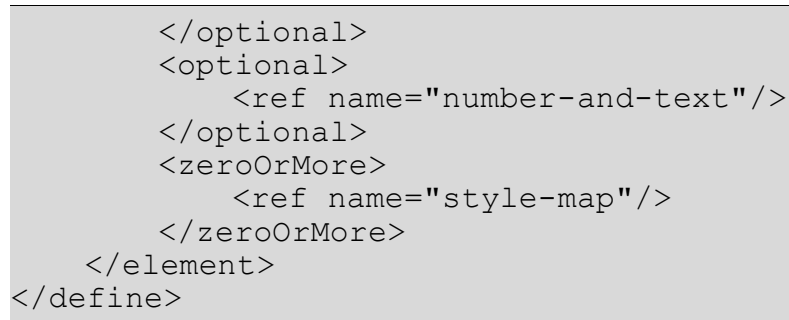

See section 14.7.9 for information on the attributes that may be associated with the percentage style element.

\subsubsection{Date Style}

The $<$ number: date-style $>$ element describes the style for date values.

This element can contain one instance of each of the following elements: $<$ number: day $>$, $<$ number:month>, <number:year>, <number:era>, <number:day-of-week $>$, $<$ number: week-of-year>, <number: quarter>, <number:hours $>$, <number:minutes $>$, $<$ number: seconds $>$, and $<$ number: am-pm $>$.

The <number: date-style $>$ element can also contain <number: text $>$ elements, which display additional text, but it cannot contain two of these elements consecutively. In addition, it can contain a <style:text-properties $>$ element and a $\langle$ style:map $>$ element.

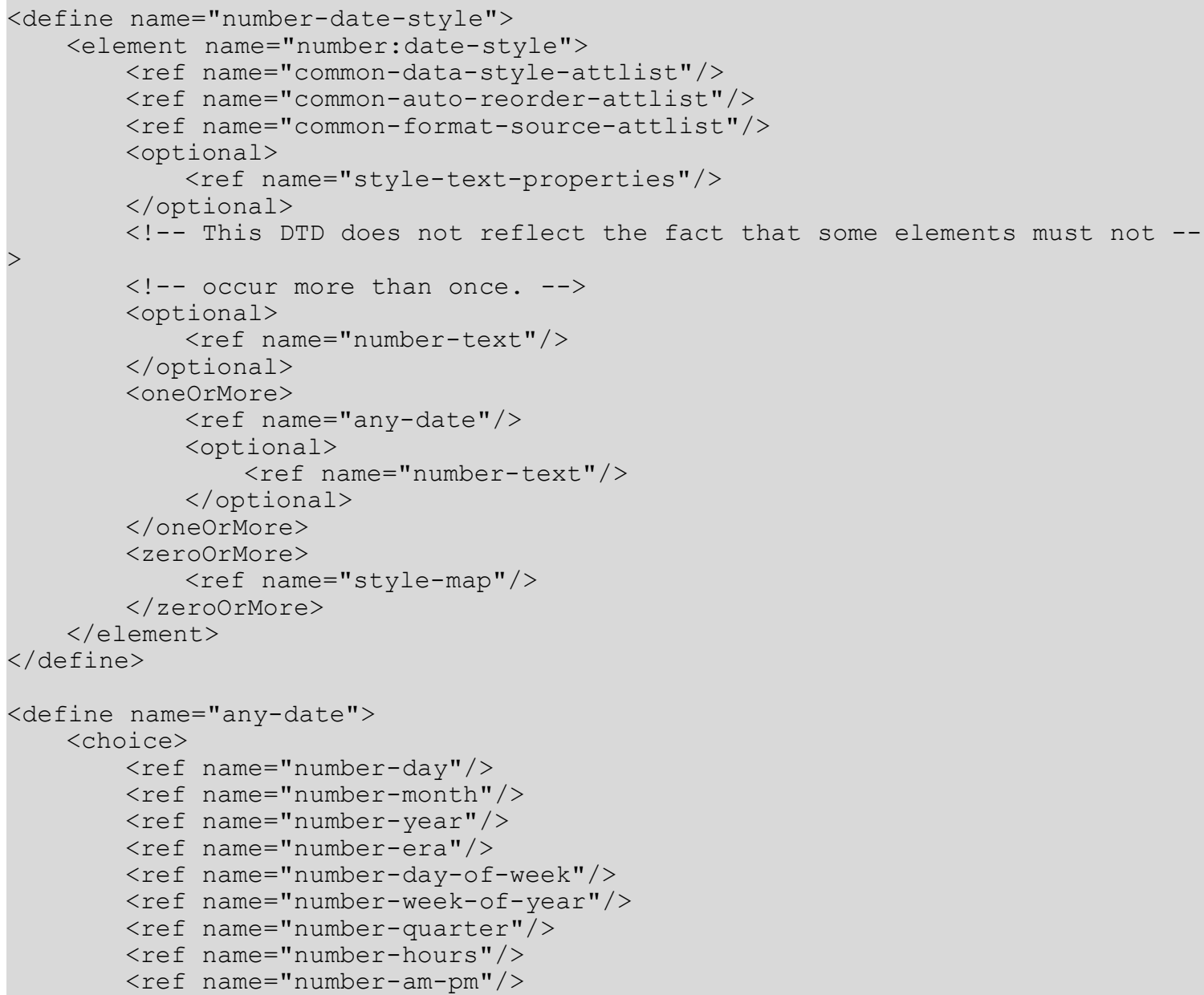




$$
</ \text { choice }>
$$

$</$ define $>$

See section 14.7 .9 for information on the attributes that may be associated with the date style elements.

The <number: date-style> element can contain the following elements:

- <number:day $>$ - day of month

- <number:month> - month

- <number:year> - year

- <number:era $>$ - era

- <number:day-of-week>- day of week

- <number:week-of-year> - week of year

- <number:quarter> - quarter

\section{Day of Month}

The <number: day $>$ element specifies the day of the month in a date.

If this element is used, it should be contained in the <number: date-style> element.

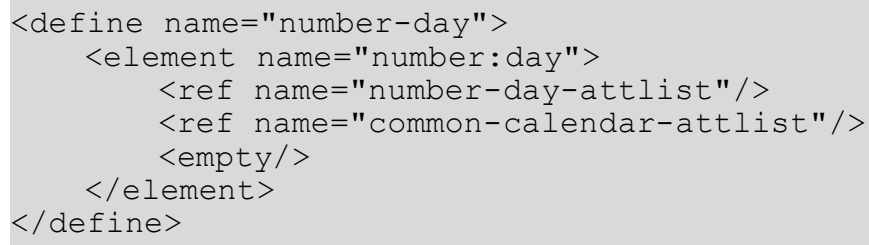

The number:style attribute may be used with this element. See section 14.7 .11 for information on the other attributes that may be associated with the element.

\section{Format Attribute}

The number:style attribute specifies whether the day of month element is displayed in short or long format. The value of this attribute can be short or long. The meaning of these values depends on the value of the number: format-source attribute that is attached to the date style.

For days, if the value of the number:format-source attribute is fixed:

- $\quad$ short means that the day of the month is displayed using one or two digits

- long means that the day of the month is displayed using two digits

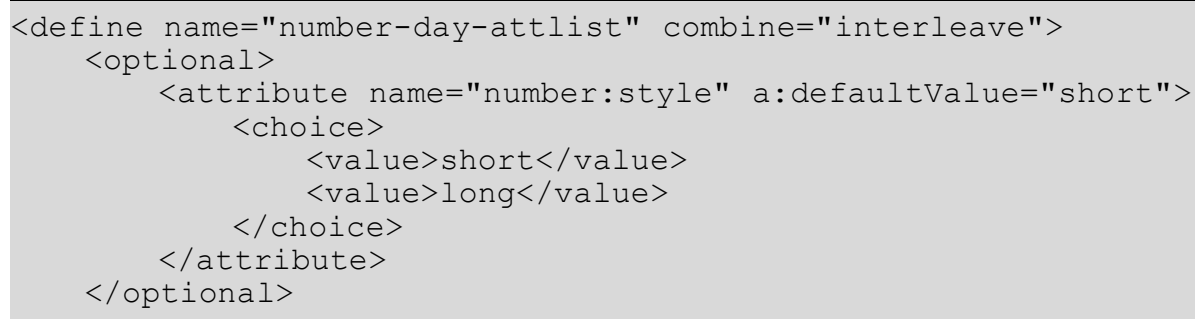




\section{Month}

The <number:month $>$ element specifies the month in a date.

If used, this element must be contained in the <number: date-style > element.

11828

11829

11830

11831

11832

11833

11834

/define $>$

The number: textual and number:style attributes may be used with this element. See section 14.7 .11 for information on the other attributes that may be associated with the element.

\section{Textual Representation Attribute}

The number: textual attribute determines whether the name or number of a month is displayed in the month element of a date. If the value of this attribute value is true, the name of the month is displayed. If the attribute value is false, the number of the month is displayed.

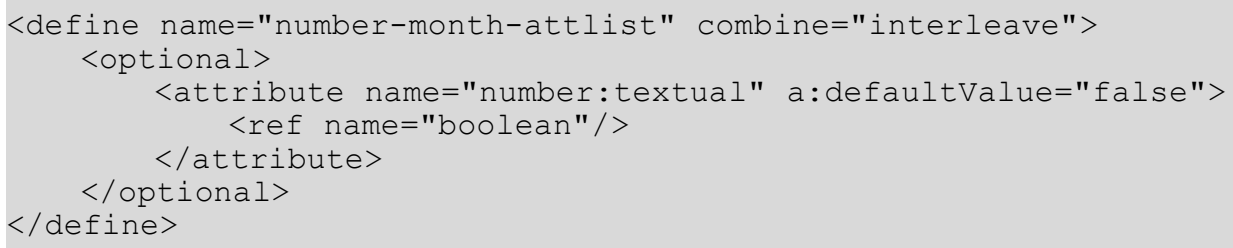

\section{Possessive Form Attribute}

The number:possessive-gorm attribute determines whether the month is displayed as is (e.g. as in "17 January 2004") or using the possessive form (e.g. as in "17th day of January"). If the value of this attribute value is true, the name of the month is displayed in possessive form. If the attribute value is false, the number of the month is displayed as is.

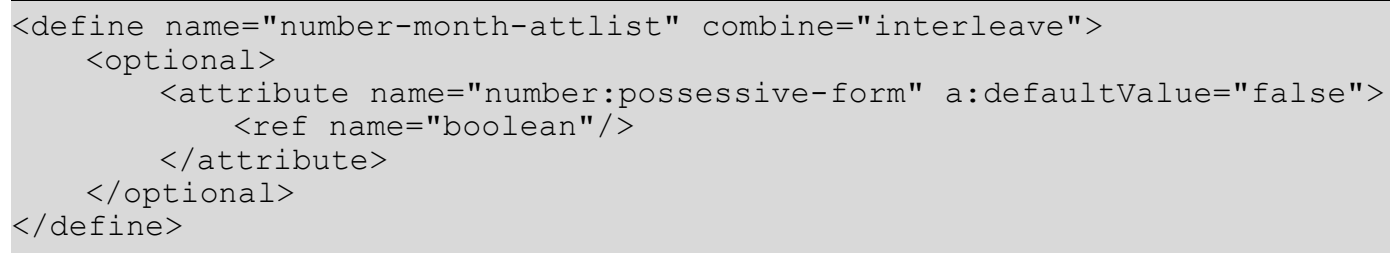

\section{$</$ define $>$}

\section{Format Attribute}

The number:style attribute specifies whether the month element is displayed in short or long format. The value of this attribute can be short or long. The meaning of these values depends on the value of the number:format-source attribute that is attached to the date style.

For months, if the value of the number: format-source attribute is fixed:

- short means that the abbreviated name of the month is displayed or the month is displayed using one or two digits

- long means that the full name of the month is displayed or the month is displayed using two digits 
$<$ /optional>

\section{Year}

The <number: year > element specifies the year in the date.

If used, this element must be contained in the <number: date-style $>$ element.

11859

11860

11861

11862

11863

11864

11865

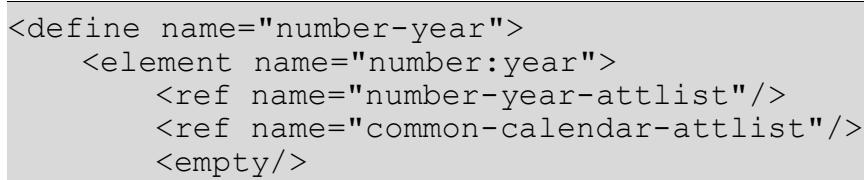

The number: style attribute may be used with this element. See section 14.7 .11 for information on the other attributes that may be associated with the element.

\section{Format Attribute}

The number:style attribute specifies whether the year element is displayed in short or long format. The value of this attribute can be short or long. The meaning of these values depends on the value of the number: format-source attribute that is attached to the date style.

For years, if the value of the number: format-source attribute is fixed:

- $\quad$ short means that the year is displayed using two digits

- long means that the year is displayed using four digits

11866

11867

11868

11869

11870

11871

11872

11873

11874

11875

\section{Era}

The $<$ number:era $>$ element specifies the era in which the year is counted.

If used, this element must be contained in the <number: date-style > element.

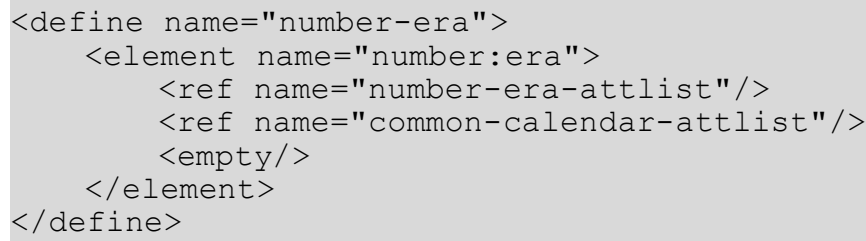


The number:style attribute may be used with this element. See section 14.7 .11 for information on the other attributes that may be associated with the element.

\section{Format Attribute}

The number:style attribute specifies whether the era element is displayed in short or long format. The value of this attribute can be short or long. The meaning of these values depends on the value of the number:format-source attribute that is attached to the date style.

For eras, if the value of the number:format-source attribute is fixed:

- short means that the abbreviated era name is used

- long means that the full era name is used

\section{Day Of Week}

The <number:day-of-week> element specifies the day of the week in a date.

If used, this element must be contained in the <number: date-style> element.

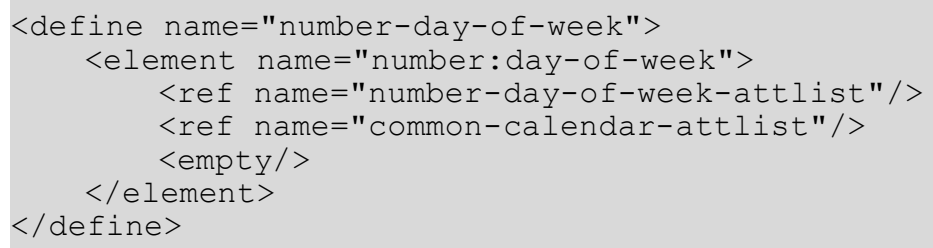

The number:style attribute may be used with this element. See section 14.7 .11 for information on the other attributes that may be associated with the element.

\section{Format Attribute}

The number:style attribute specifies whether the day of week element is displayed in short or long format.

The value of this attribute can be short or long. The meaning of these values depends on the value of the number: format-source attribute that is attached to the date style.

For days of the week, the value of the number:format-source attribute is fixed:

- short means that the abbreviated name of the day is displayed

- long means that the full name of the day is displayed 


\section{Week Of Year}

The <number: week-of-year> element specifies the week of the year in the date.

If used, this element must be contained in the <number: date-style $>$ element.

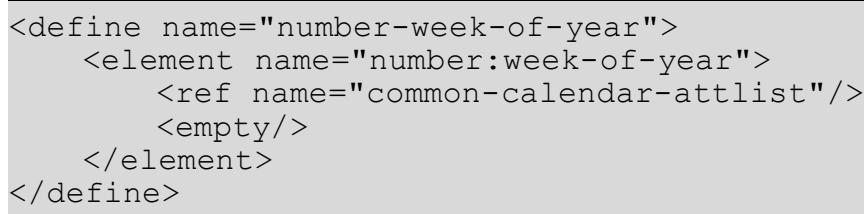

See section 14.7.11 for information on the the attributes that may be associated with the element.

\section{Quarter}

The <number:quarter $>$ element specifies the quarter of the year in the date.

If used, this element must be contained in the <number: date-style> element.

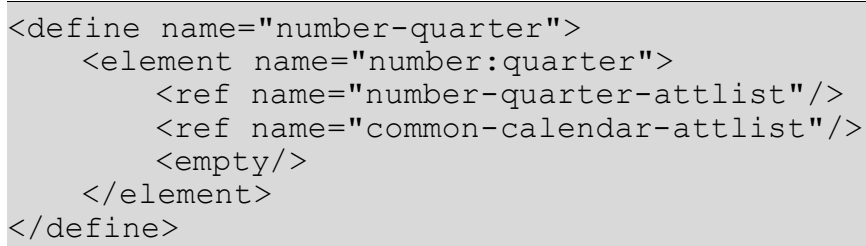

The number: style attribute may be used with this element. See section 14.7 .11 for information on the other attributes that may be associated with the element.

\section{Format Attribute}

The number:style attribute specifies whether the quarter element is displayed in short or long format.

The value of this attribute can be short or long. The meaning of these values depends on the value of the number: format-source attribute that is attached to the date style.

For quarters, if the value of the number:format-source attribute is fixed:

- short means that the abbreviated name of the quarter is displayed, for example, Q1

- long means that the full name of the quarter is displayed, for example, Quarter 1

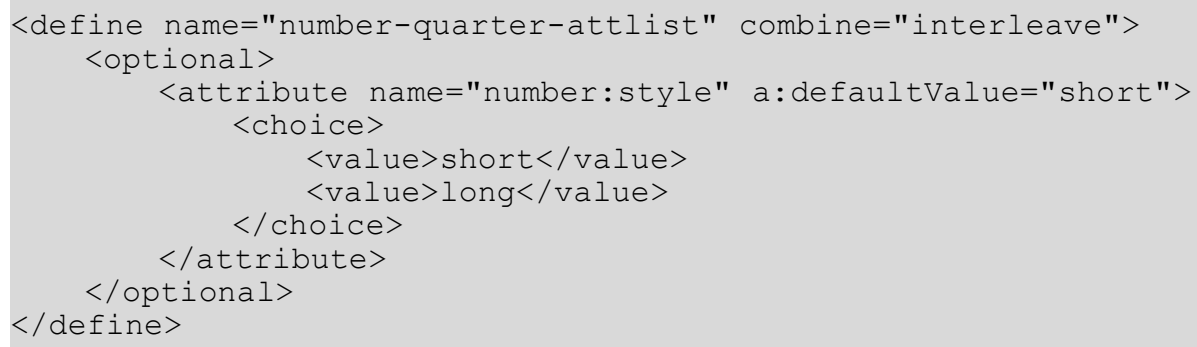




\subsubsection{Time Style}

The <number:time-style> element describes the style for time values.

This element can contain one instance of any of the following elements: <number: hours $>$, $<$ number:minutes $>$, <number: seconds $>$ and $<$ number:am-pm $>$.

The <number: time-style> element can also contain <number: text> elements, which display additional text, but it cannot contain two of these elements consecutively. In addition, it can contain a $<$ style: text-properties $>$ element and a $<$ style:map $>$ element.

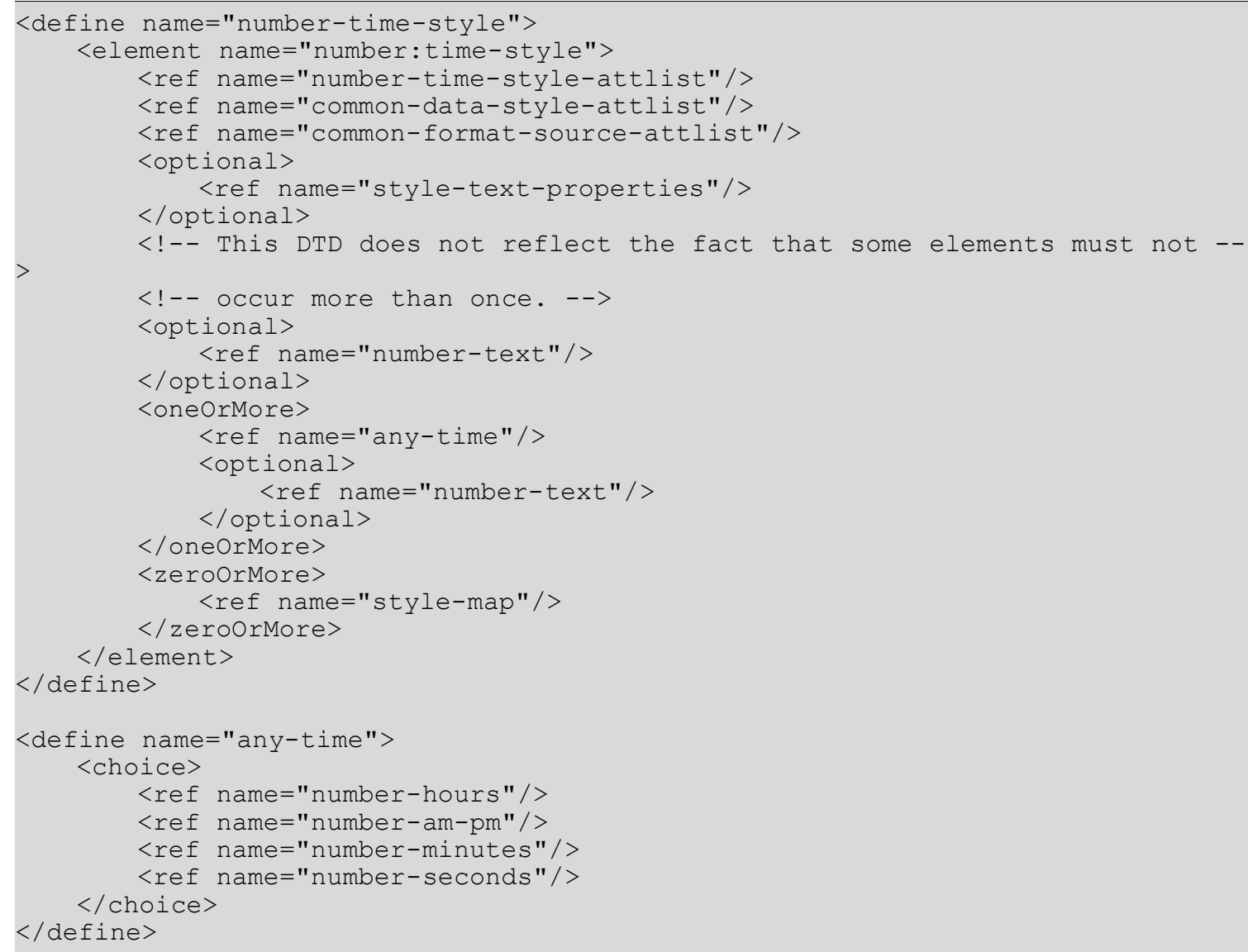

See section 14.7 .9 for information on the attributes that may be associated with the time style elements.

The following elements can be contained in the <number:time-style > element:

- $\quad<$ number:hours $>$ - hours

- $\quad<$ number:minutes $>$ - minutes

- $\quad$ number:seconds $>$ - seconds

- $\quad$ number:am-pm>-am/pm

\section{Time Value Truncation}

If a time or duration is too large to be displayed using the default value range for a time component, ( 0 to 23 for <number:hours $>$ ), the number:truncate-on-overflow attribute 
may be used to specify whether the time or duration value should be truncated or whether the value range becomes extended.

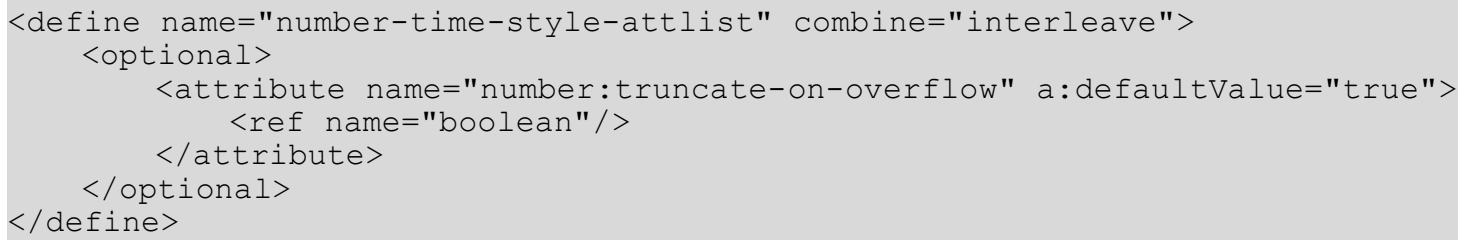

\section{Hours}

The <number: hours $>$ element specifies if hours are displayed as part of a date or time.

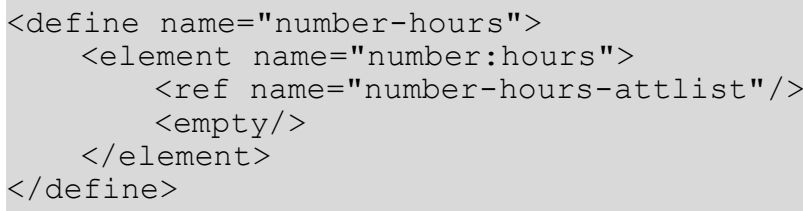

\section{Format Attribute}

The number:style attribute specifies whether the hours element is displayed in short or long format.

The value of this attribute can be short or long. The meaning of these values depends on the value of the number: format-source attribute that is attached to the time style.

For hours, if the value of the number: format-source attribute is fixed:

- $\quad$ short means that the hours are displayed using at least one digit

- $\quad$ long means that the hours are displayed using at least two digits

\section{Minutes}

The <number:minutes $>$ element specifies if minutes are displayed as part of a date or time.

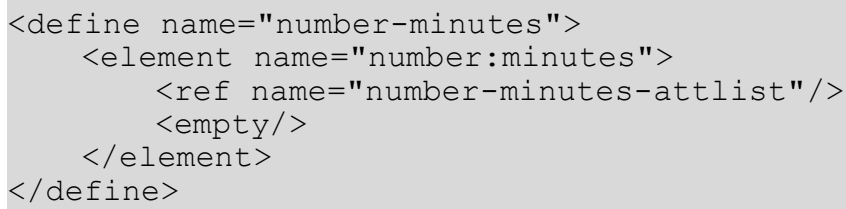

\section{Format Attribute}

The number:style attribute specifies whether the minutes element is displayed in short or long format. 
The value of this attribute can be short or long. The meaning of these values depends on the value of the number: format-source attribute that is attached to the time style.

For minutes, if the value of the number: format-source attribute is fixed:

- short means that the minutes are displayed using at least one digit

- long means that the minutes are displayed using at least two digits

11996

11997

11998

11999

12000

12001

12002

12003

12004

12005

\section{Seconds}

The <number: seconds $>$ element specifies if seconds are displayed as part of a date or time.

\section{Decimal Places Attribute}

The number:decimal-places attribute determines the number of decimal places to use when displaying fractions.

If this attribute is not present or if the value of the attribute is 0 , fractions are not displayed. 


\subsubsection{Text Style}

The <number: text-style $>$ element describes the style for displaying text.

This element can contain any number of <number: text-content $>$ elements. It can also contain <number: text > elements, which display additional text, but it cannot contain two of these elements consecutively. In addition, it can contain a <style:text-properties $>$ element and a $<$ style: map $>$ element. The $<$ number: text-content $>$ elements represent the variable text content to display, while the $<$ number : text $>$ elements contain any additional fixed text to display.

\section{Text Content}

The <number: text-content $>$ element contains the variable text content of a text style.

\subsubsection{Common Data Style Elements}

The following common style elements may be contained within data style elements: 
- Text formatting properties

- Style mappings

\section{Formatting Properties}

The <style:text-properties $>$ element specifies the text formatting properties to apply to any text displayed in the data style. See section 15.4 for information on the formatting properties element.

The purpose of specifying text formatting properties within data styles is mainly to highlight certain values (for instance negative ones) by using style mappings. For this reason, data styles usually support only very few text formatting properties, for instance a text color. There may be also restrictions for the values of text formatting properties. For instance, the only value allowed for the text color might be read.

\section{Style Mappings}

The <style:map $>$ element specifies an alternative data style to map to if a certain condition exists. See section 14.1.1 for information on the <style: map > element.

The following rules exist for using style maps element with data style elements:

- The style referenced by the style:apply-style attribute must be of the same type as the style containing the map.

- The condition must be in the format value() op $n$, where op is a relational operator and $n$ is a number. For Boolean styles the condition value must be true and false.

\subsubsection{Common Data Style Attributes}

Many of the data style attributes are applicable to more than one data style element. The following data style attributes are common to many of the data style elements:

- Name

- Language

- Country

- Title

- Volatility

- Automatic Order

- Format Source

- Transliteration

\section{Name}

The style:name attribute specifies the name of the data style. It can be used with all data style elements.

<define name="common-data-style-attlist" combine="interleave">

<attribute name="style:name">

<ref name="styleName"/> 


\section{Display Name}

The style:display-name attribute specifies the name of the style as it should appear in the user interface. In contrast to the style name itself, this name may contain arbitrary characters. If this attribute is not present, the display name equals the style name.

The style:display-name attribute can be used with all data style elements.

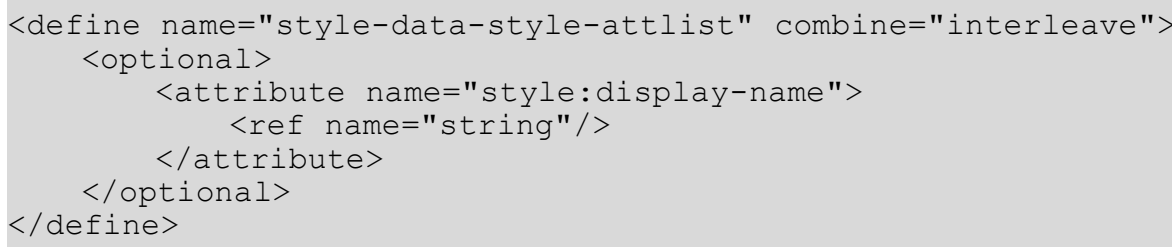

\section{Language}

The number: language attribute specifies the language of the style. The value of the attribute is a language code in conformance with [RFC3066]. The language code is used to retrieve information about any display properties that are language-dependent. The language attribute can be used with all data style elements.

If a language code is not specified, either the system settings or the setting for the system's language are used, depending on the property whose value should be retrieved.

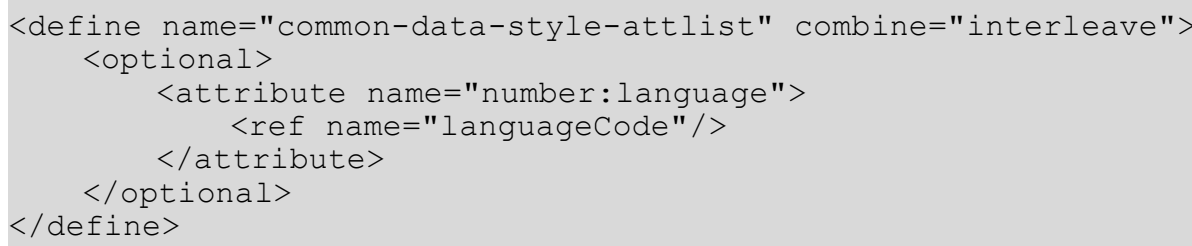

\section{Country}

The number: country attribute specifies the country of the style. The value of the attribute is a country code in conformance with [RFC3066]. The country code is used to retrieve information about any display properties that are country-dependent. The language attribute can be used with all data style elements.

If a country is not specified, either the system settings or the setting for the system's country are used, depending on the property whose value should be retrieved.

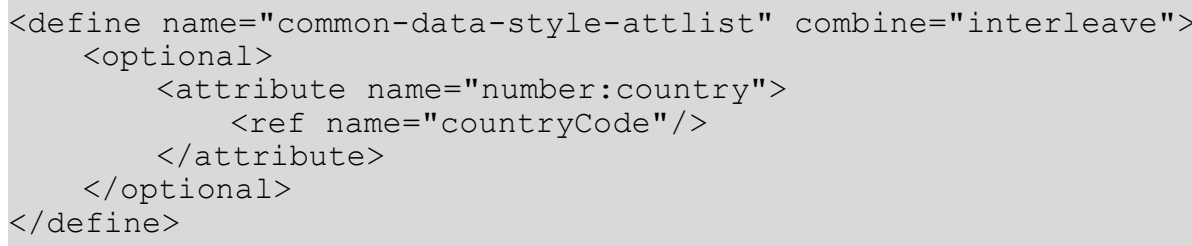

\section{Title}

The number:title attribute specifies the title of the data style. It can be used with all data style elements. 


\section{Automatic Order}

The number:automatic-order attribute can be used to automatically order data to match the default order for the language and country of the data style. This attribute is used with the following elements:

- <number: currency-style>, where number and the currency symbols are reordered

- <number:date-style>, where the <number: date-style $>$ child elements that are not $<$ number:text $>$ or $<$ style:text-properties $>$ elements are reordered

The attribute value can be true or false.

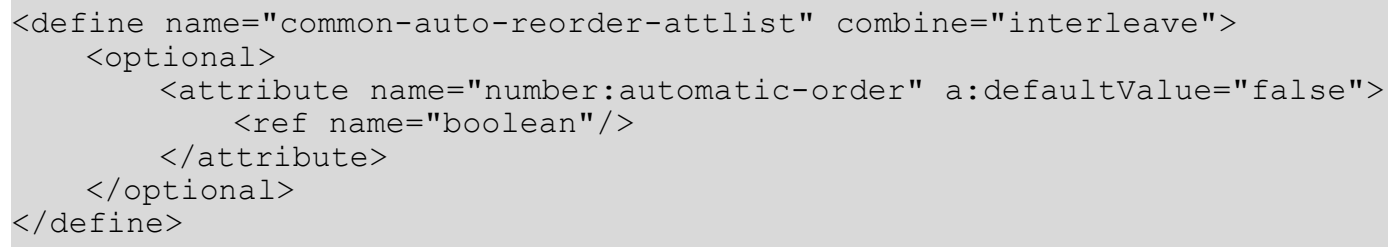

\section{Format Source}

The number:format-source attribute specifies the source of the short and long display formats. It is used with the following elements:

- $\quad$ number:date-style>

- <number:time-style>

The value of this attribute can be fixed or language.

If the value is fixed, the meaning of the values number: style attribute's values short and long is as described in this specification. 
If the value of the number:format-source attribute is language, the meaning of short and long depends on the language and country of the date style, or, if neither of these are specified, applications should use the system settings for short and long date and time formats.

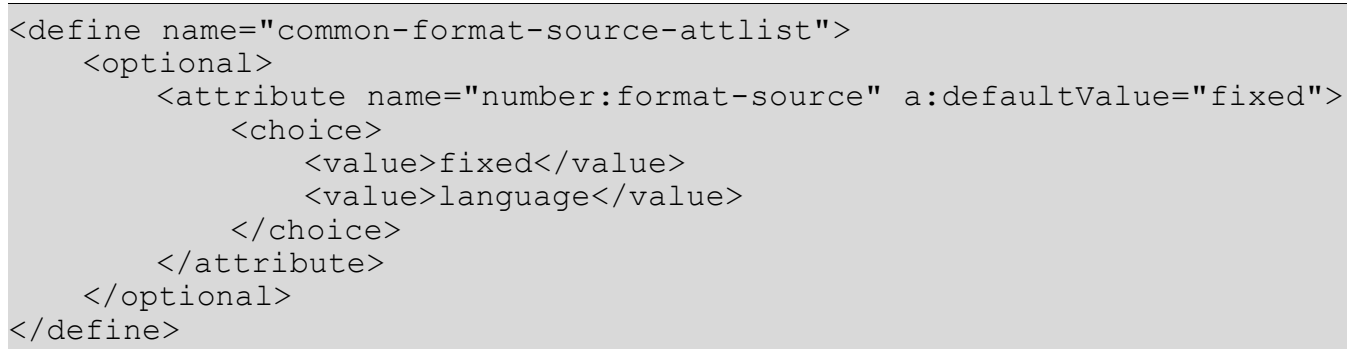

\subsubsection{Transliteration}

The various number:transliteration $-*$ attributes specify the native number system of the style to display the number using, for example, CJK number characters. The notation is inspired by the W3C XSLT $2.0 \mathrm{draft}$, see $\S 12.3$ of [XSLT2]. However, to be able to fully distinguish between all possible native number systems additional attributes are needed in combination. For example, Korean uses 11 different systems where the digits are not always different but short and long and formal and informal forms exist.

The transliteration attributes can be used with all data style elements.

\section{Transliteration Format}

The number:transliteration-format attribute specifies which number characters to use. The value of the attribute is the digit "1" expressed as a native number.

If no format is specified the default ASCII representation of Arabic digits is used, other transliteration attributes present in this case are ignored.

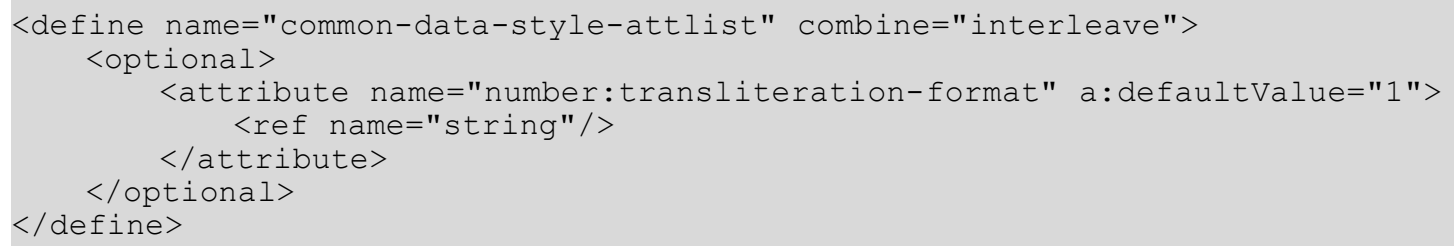

\section{Transliteration Language}

The number:transliteration-language attribute specifies which language the native number system belongs to. The value of the attribute is a language code in conformance with [RFC3066].

If no language/country (locale) combination is specified the locale of the data style is used.

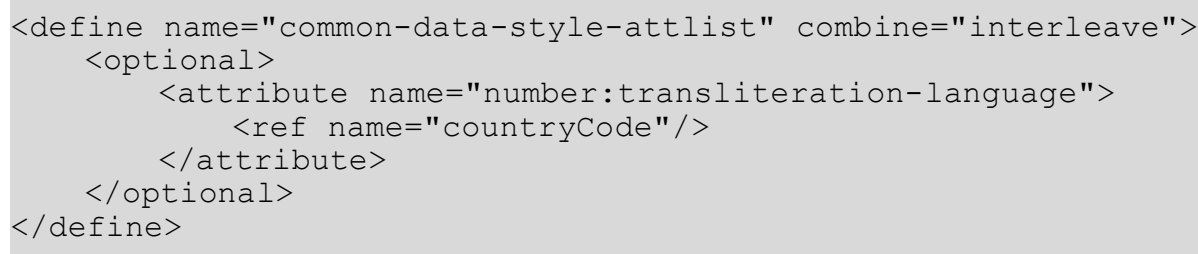




\section{Transliteration Country}

The number:transliteration-country attribute specifies which country the native number system belongs to. The value of the attribute is a country code in conformance with [RFC3066].

If no language/country (locale) combination is specified the locale of the data style is used.

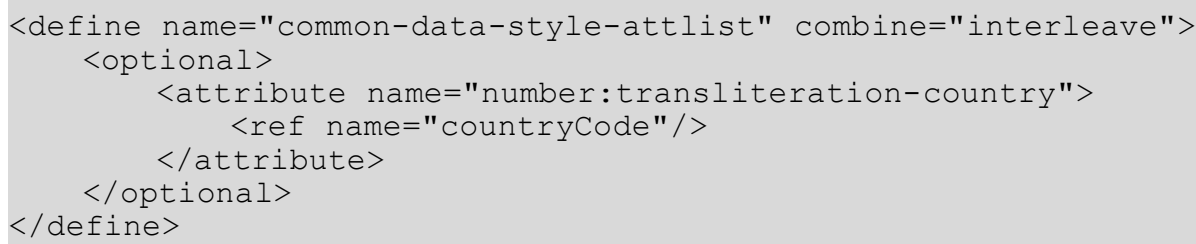

\section{Transliteration Style}

The number:transliteration-style attribute specifies which style the native number system belongs to. If more than one native number system matches the transliteration-format this attribute selects one. A short style should result in a one to one mapping of Arabic digits to native number digits if possible.

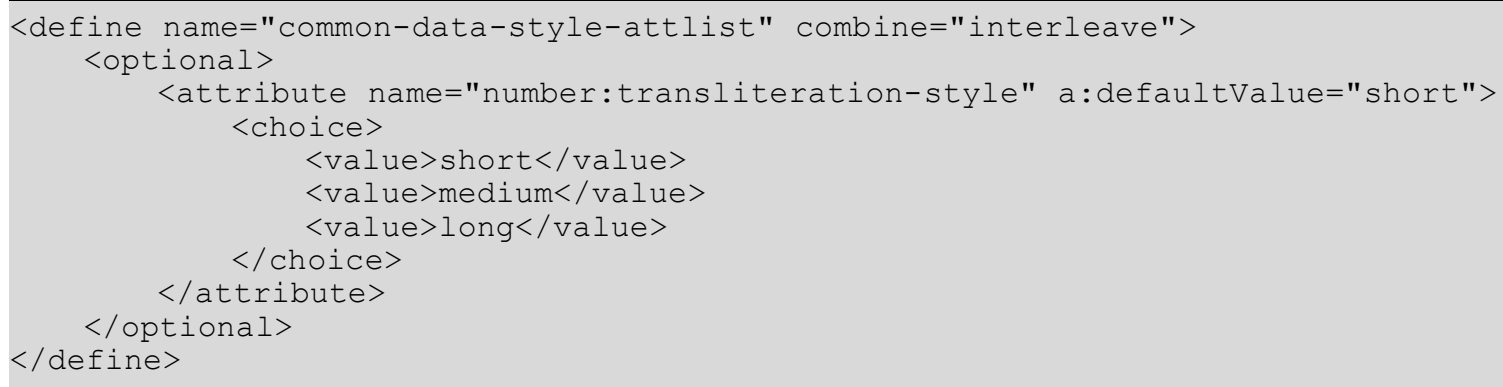

\subsubsection{Common Data Style Child Element Attributes}

Many of the number style attributes are applicable to more than one number style element. The following attributes are common to many of the number style elements:

- Decimal places

- Minimum integer digits

- Grouping separator

- Decimal replacement

- Minimum exponent digits

- Minimum numerator digits

- Minimum denominator digits

- Calendar system

\section{Decimal Places}

The number:decimal-places attribute specifies the number of decimal places to display. This attribute is supported for the following elements:

- <number: number> 
- <number:scientific-number>

If this attribute is not specified, a default number of decimal places is used.

\section{Minimum Integer Digits}

The number:min-integer-digits attribute specifies the minimum number of integer digits to display in a number, a scientific number, or a fraction. This attribute is supported for the following elements:

- <number: number >

- <number:scientific-number>

- <number:fraction>

If this attribute is not specified, a default number of integer digits is used.

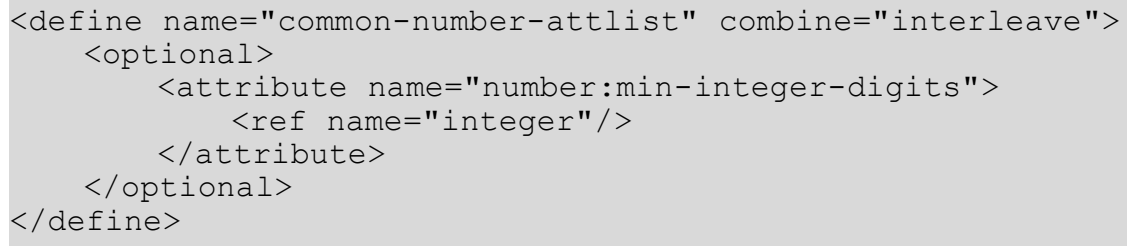

\section{Grouping Separator}

The number:grouping attribute specifies whether or not the integer digits of a number should be grouped using a separator character. This attribute is supported for the following elements:

- <number: number>

- <number:scientific-number>

- <number:fraction>

The grouping character that is used and the number of digits that are grouped together depends on the language and country of the style.

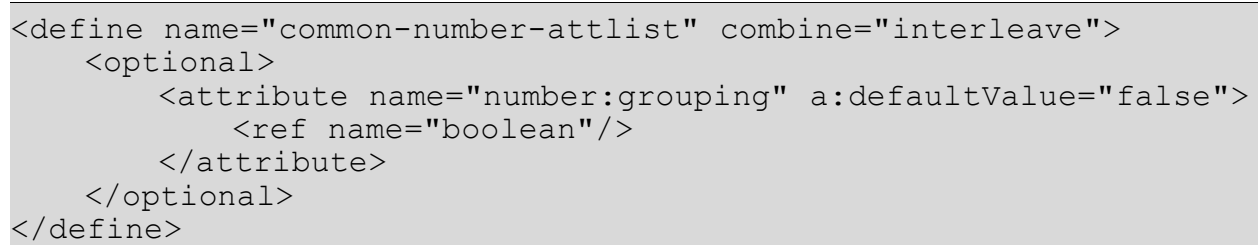

\section{Calendar System}

The number: calendar attribute specifies the calendar system used to extract parts of a date. This attribute is supported for the following elements:

- <number: day $>$ 
- $\quad<$ number:month>

- <number:year>

- <number:era>

- <number:day-of-week>

- $\quad$ <number:week-of-year>

- <number:quarter>

The attribute may have the values gregorian, gengou, RoC, hanja_yoil, hanja, hijri, jewish, buddhist or an arbitrary string value. If this attribute is not specified, the default calendar system is used.

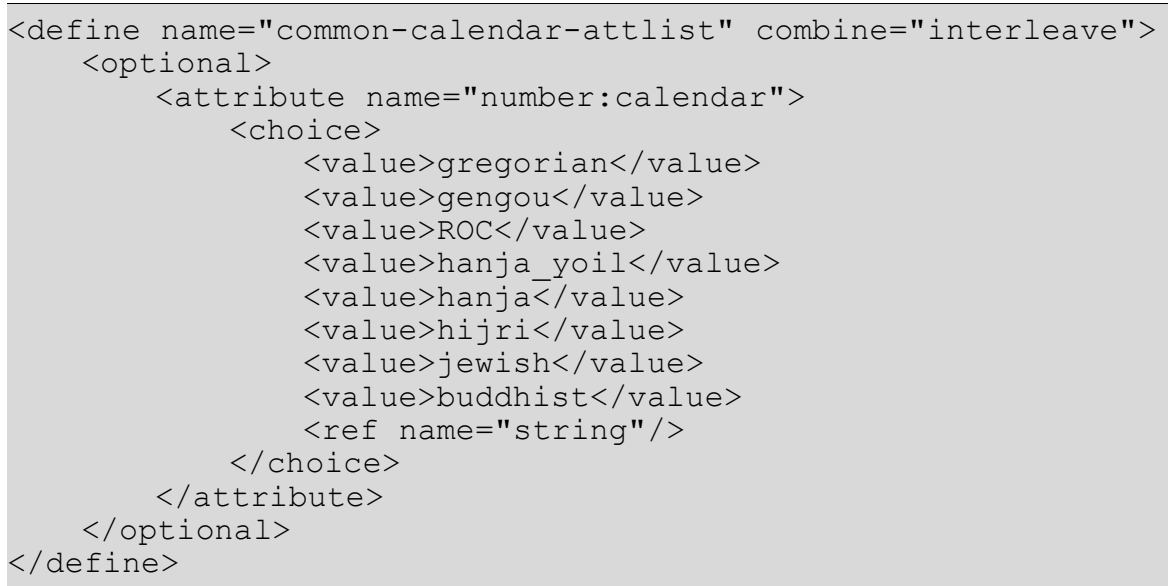

\subsection{Text Styles}

\subsubsection{Text Styles}

Text styles are <style:style $>$ elements that have the family text. They can be used within all kind of applications to specify formatting properties for piece of text. They support the text properties as described in section 15.4.

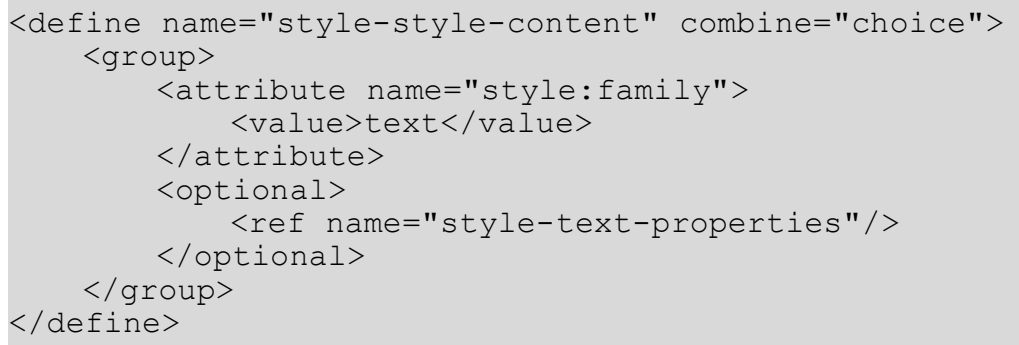

\subsubsection{Paragraph Styles}

Paragraph styles are <style:style $>$ elements that have the family paragraph. They can be used within all kind of applications to specify formatting properties for paragraphs and headings. They support the paragraph properties described in section 15.5 as well as the text properties described in section 15.4 .

<define name="style-style-content" combine="choice"> 
$</$ group $>$

\subsubsection{Section Styles}

Section styles are <style:style $>$ elements that have the family section. They can be used within text documents to specify formatting properties for a text section. They support the section properties as described in section 15.7.

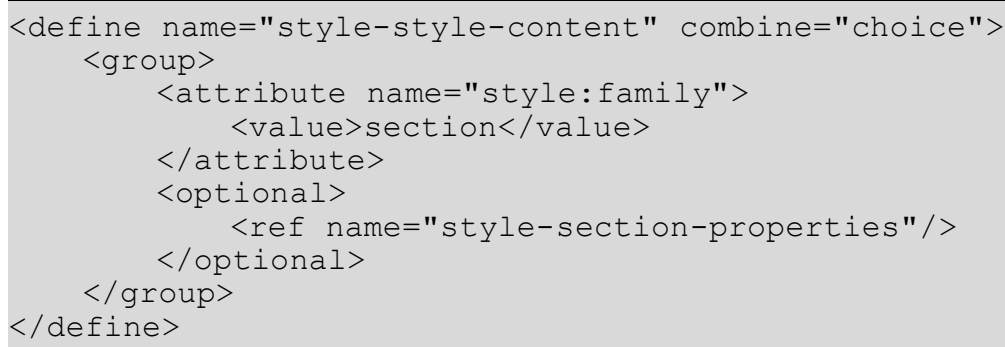

$</$ group $>$

\subsubsection{Ruby Style}

A ruby style specifies how the ruby text is displayed relative to the base text. It is represented by a <style:style $>$ element those family is ruby. The ruby style is assigned to the ruby element using a text: style-name attribute. Ruby styles support the formatting properties described in section 15.6 .

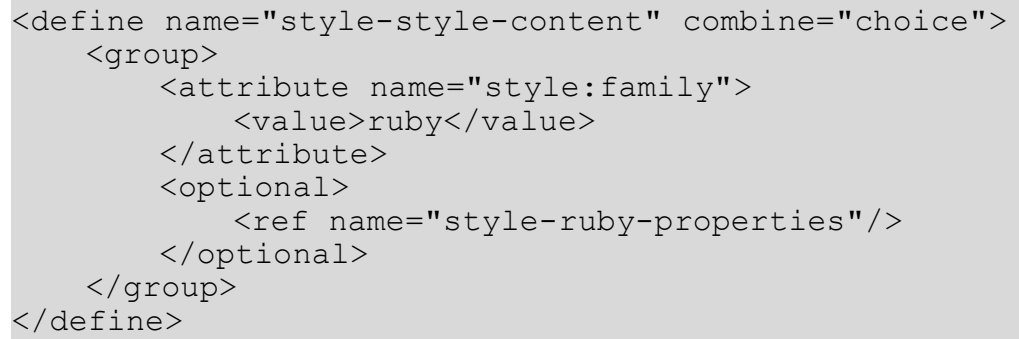

\subsection{Enhanced Text Styles}

\subsubsection{Line Numbering Configuration}

A document can contain none or one line numbering configuration element <text: linenumbering-configuration> within the <office:styles> element. If the element is not present, a default line numbering configuration is used. The default line numbering may vary on the office application software, but every document saved by an application that supports line numbering should contain a line numbering configuration element. 


\section{$</$ define $>$}

<element name="text:linenumbering-configuration">

<ref name="text-linenumbering-configuration-attlist"/>

<optional>

<ref name="text-linenumbering-separator"/> $</ o p t i o n a l>$

The attributes that may be associated with the <text:linenumbering-configuration> element are:

- Line numbering enable

- Number format

- Text style

- Increment

- Position

- Offset

- Count empty lines

- Count line in text boxes

- Restart numbering on every page

The following element may be included in the <text: linenumbering-seperator $>$ element:

- Separator

\section{Line Numbering Enable}

The text:number-lines attribute controls whether or not lines are numbered.

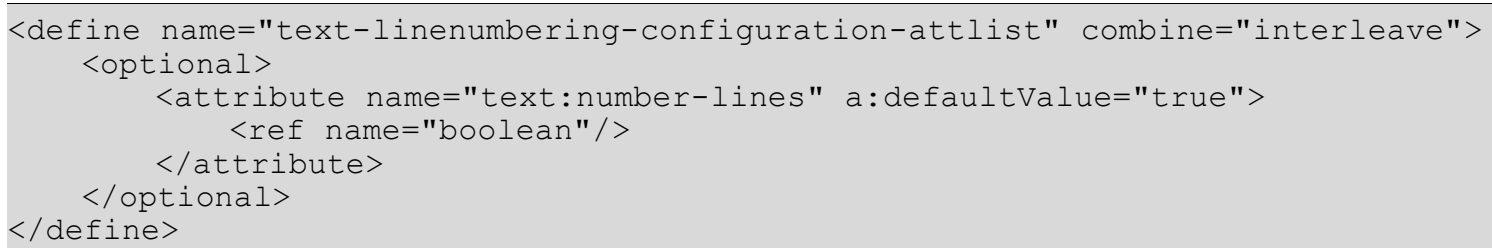

\section{Number Format}

See section 12.2 for detailed information on number format attributes. The attributes described in section 12.2 can also be associated with the <text: linenumbering-configuration> element.

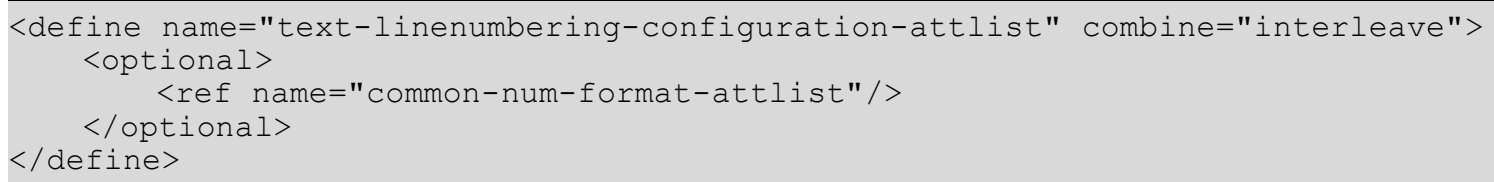

\section{Text Style}

The text:style-name attribute specifies the text style for all line numbers. The value of this attribute is the name of the text style that is applied to all line numbers. 


\section{Increment} numbered.

\section{Position}

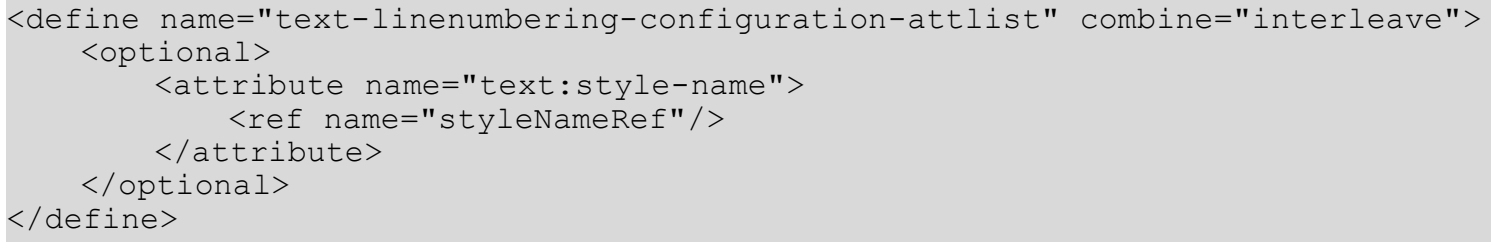

The text: increment attribute causes line numbers that are a multiple of the given increment to be numbered. For example, if the increment is 5 , only lines number $5,10,15$, and so on are

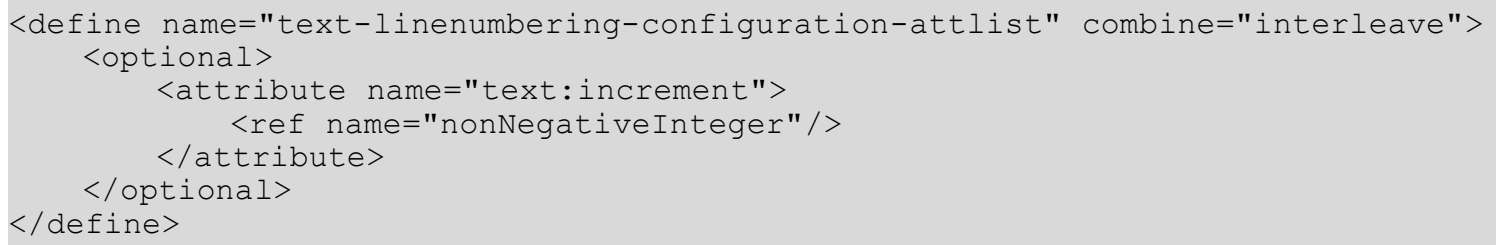

The text:position attribute determines whether the line numbers are printed on the left , right, inner, or outer margins.

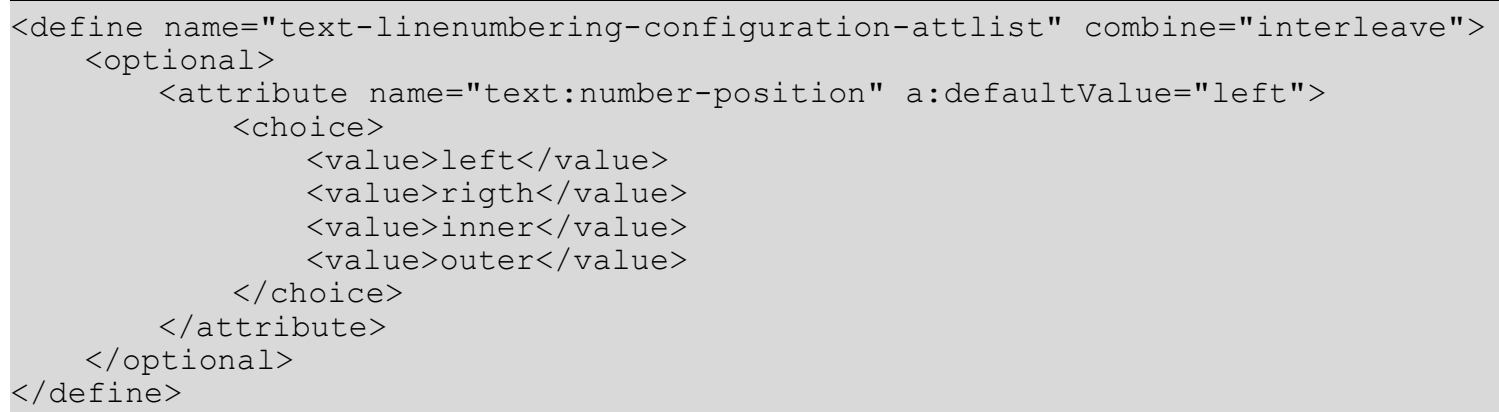

$</ o p t i o n a l>$

\section{Offset}

The text: offset attribute determines the distance between the line number and the margin.

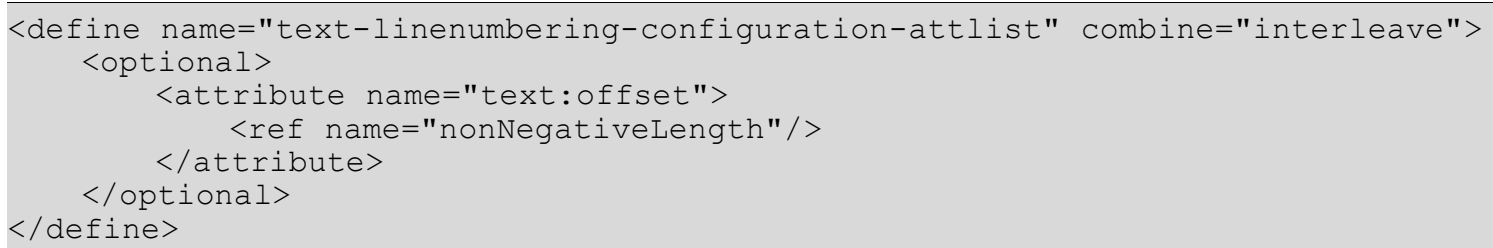

\section{Count Empty Lines}

The text: count-empty-lines attribute determines whether or not empty lines are included in the line count. If the value of this attribute is true, empty lines are included in the line count. 


\section{Count Lines in Text Boxes}

The text:count-in-text-boxes attribute determines whether or not text in text boxes is included in the line count. If the value of this attribute is true, text within text boxes is included in the line count.

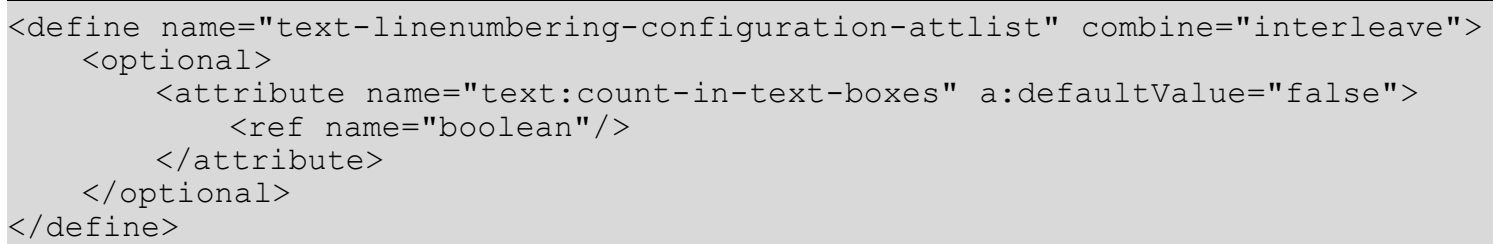

\section{Restart Numbering on Every Page}

The text:restart-on-page attribute determines whether or not the line count is reset to 1 at the start of every page.

If the value of this attribute is true, the line count is reset to 1 at the beginning of every page, resulting in page -specific numbering of lines. The default value of this attribute is false, resulting in document-specific numbering of lines.

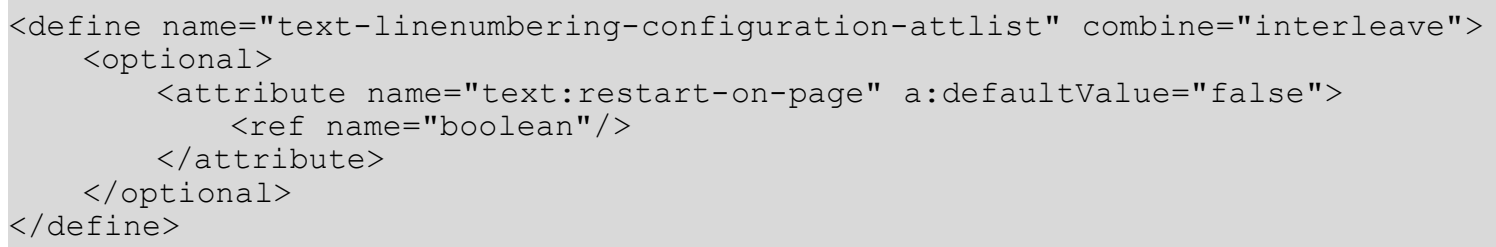

\section{Separator}

The <text: linenumbering-seperator $>$ element contains the text that is displayed as a separator. A separator is text that is displayed instead of a line number for lines where no number is displayed.

This element is contained in the line numbering configuration element. If the element is not present, no separator is displayed.

The element's text: increment attribute causes the separator to appear on lines that are a multiple of the given increment. For example, if the increment is 2 , only lines $2,4,6$, and so on get a separator, provided that no number is displayed already.

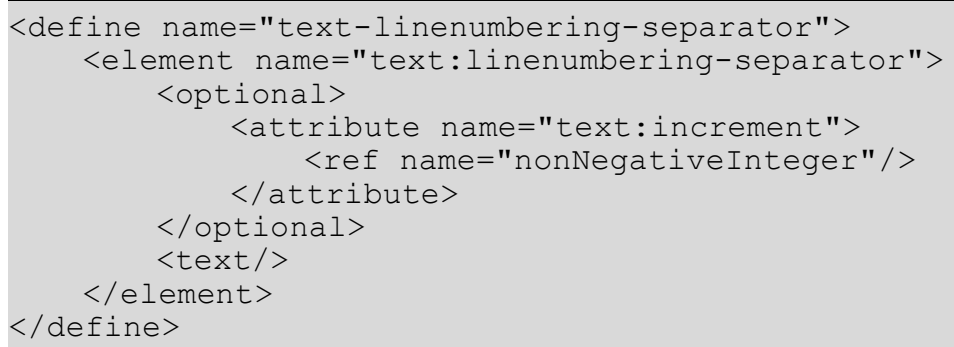




\subsubsection{Notes Configuration Element}

A document in OpenDocument format contains at most one notes configuration element for every notes class used in the document. If there is no note configuration element, a default note configuration is used.

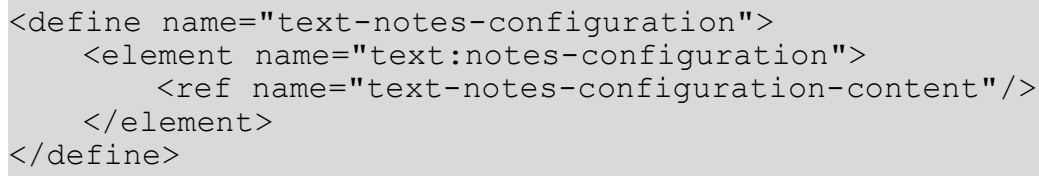

The attributes that may be associated with the <text: notes-configuration> element are:

- Note class

- Citation text style

- Citation body text style

- Default footnote paragraph style

- Master page

- Start value

- Number format

- Numbering scheme

- Footnote position

The following element may be included in the <text: footnotes-configuration> element:

- Footnote continuation notice (forward and backward)

\section{Note class}

The note class attribute determines which note elements this notes configuration applies to.

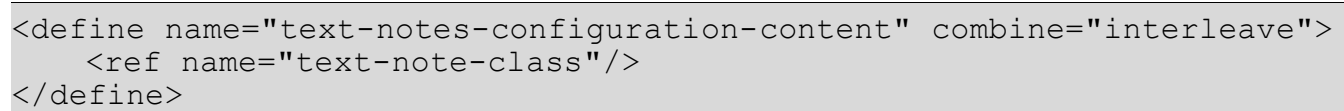

\section{Citation Text Style}

The text:citation-style attribute specifies the text style to use for the footnote citation within the footnote.

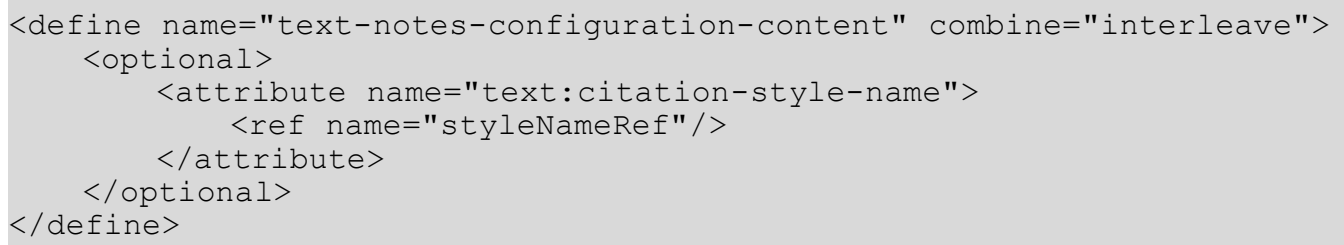

\section{Citation Body Text Style}

The text:citation-body-style-name attribute specifies the text style to use for the footnote citation in the text flow. 


\section{Start Value}

The start:value attribute specifies the value at which the footnote numbering starts.

\section{$</$ define $>$}

\section{Number Format}

See section 12.2 for information on the number format for footnotes.

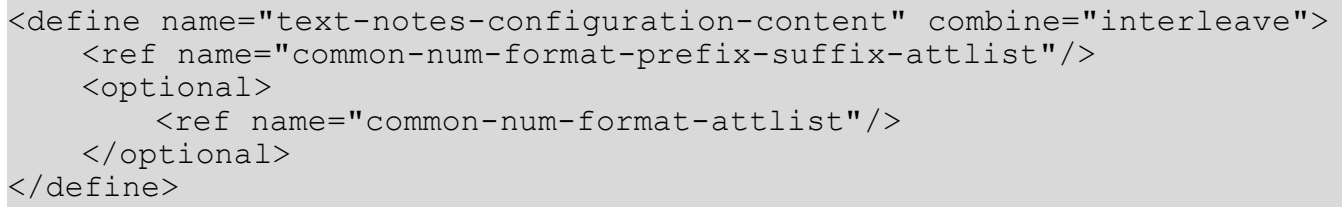




\section{Numbering Scheme}

The text:start-numbering-at attribute specifies if footnote numbers start with a new number at the beginning of the document or at the beginning of each chapter or page.

Note: [XSLT] does not have the capability to start with new footnote numbers on every page.

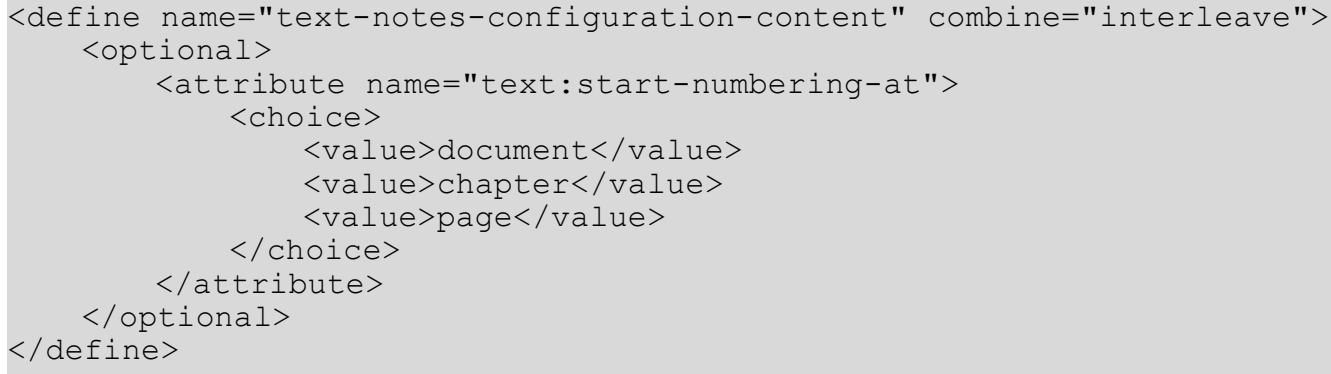

\section{Footnotes Position}

The text: footnotes-position attribute specifies one of the following positions for footnotes:

- text: At the page where the footnote citation is located, immediately below the page's text.

- page: The bottom of the page where the footnote citation is located.

- section: The end of the section

- document: The end of the document.

Note: $[\mathrm{XSL}]$ does not have the capability to display footnotes at the end of the document. However, an [XSLT] stylesheet may generate some other flow objects to display such footnotes.

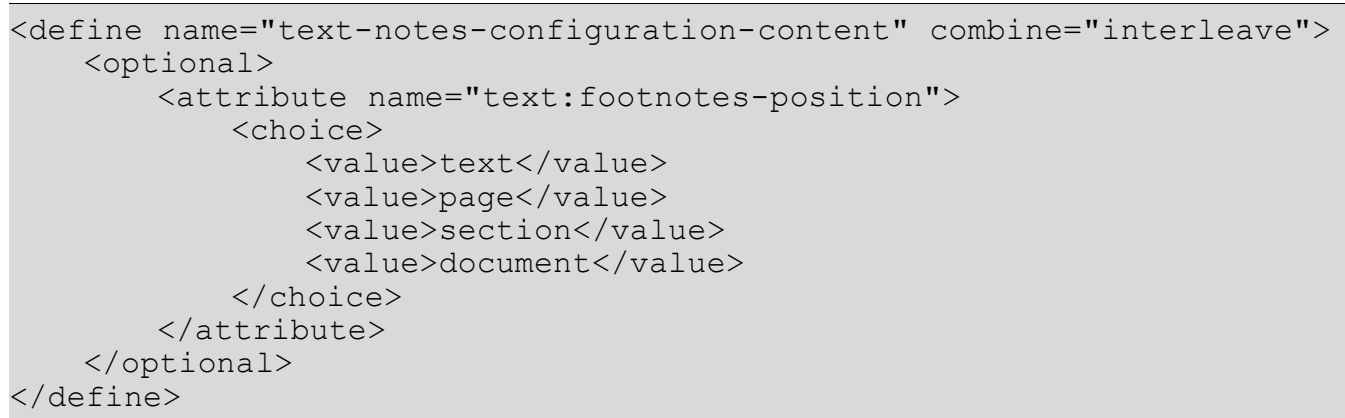

\section{Footnote Continuation}

The footnote continuation elements specify:

- Text displayed at the end of a footnote that is continued on the next page

- Text displayed before the continued text

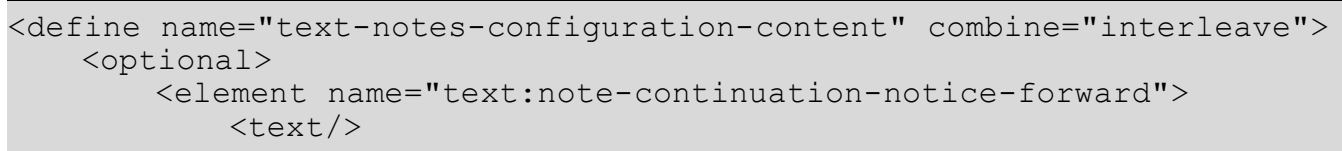




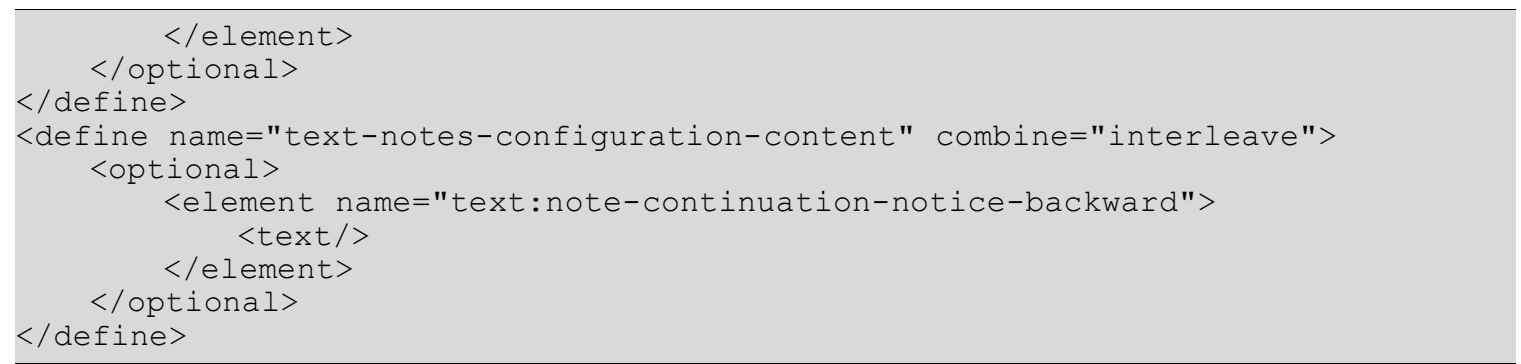

Example: Footnote configuration

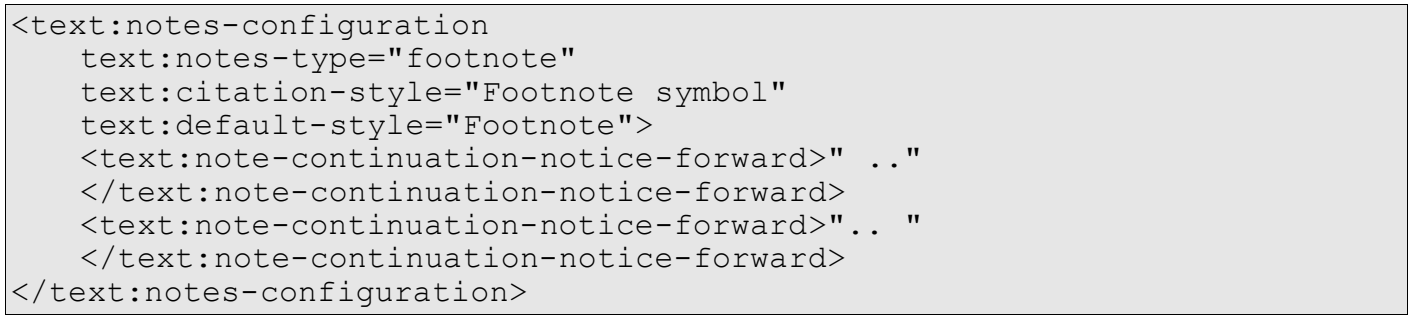

\subsubsection{Bibliography Configuration}

The bibliography configuration element <text:bibliography-configuration> is contained in the document's style section. It contains information how bibliography entries are displayed inline, and how they are displayed in the bibliography index.

\section{Prefix and Suffix}

The text:prefix and text:suffix attributes contain a string that is displayed before and after an bibliography entry's short name or number if it occurs in the document body.

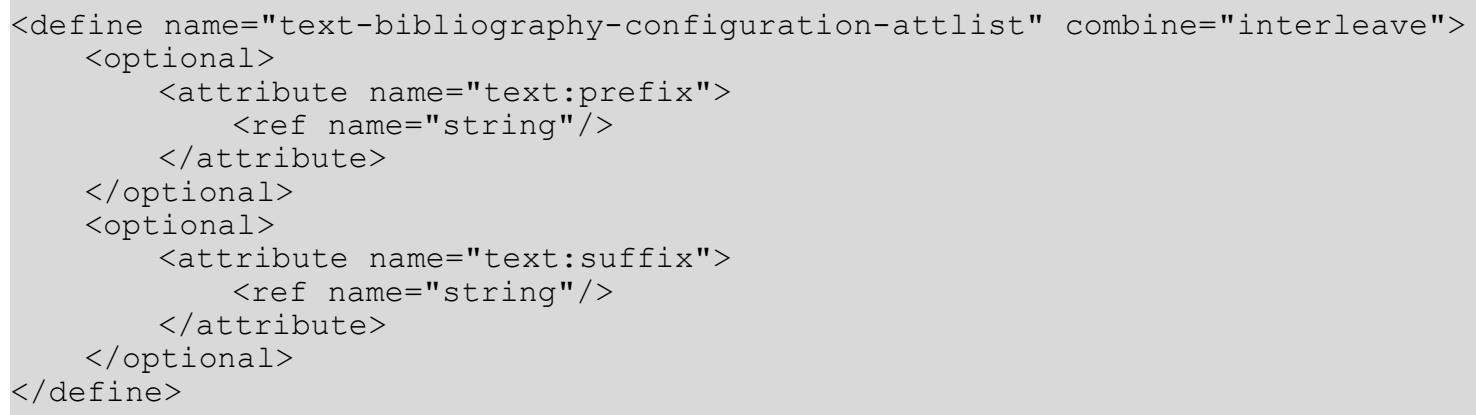

\section{Numbered Entries}

The text: numbered-entry attribute specifies whether a number is displayed for bibliography entries instead of their short name. 
Example: With prefix and suffix "[" and "]" a bibliography entry with short name "Abc123" would be displayed as "[Abc123]" in the document body if text: numbered-entry has the value false, and for instance as "[5]", if it has the value true.

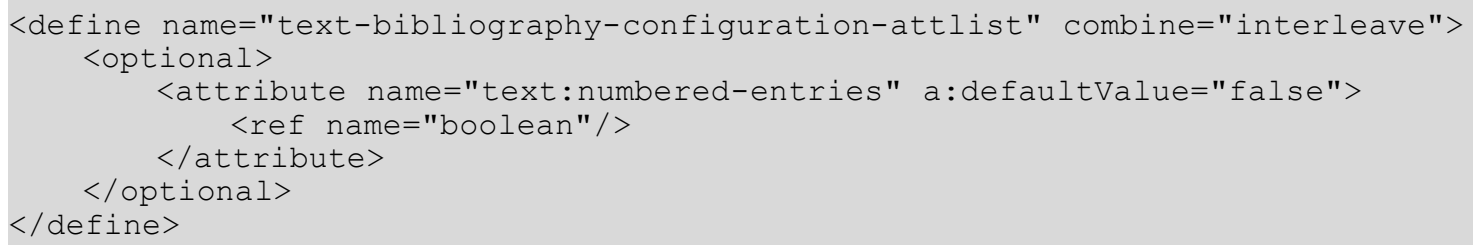

\section{Sorting}

The text:sort-by-position attribute specifies whether bibliography entries are displayed in the order of their positions in the document, or by an arbitrary selection of entry fields, e.g. author name or publication date. In the later case, the collating order for entries is determined by the triplet language/country/sort-algorithm as specified in the attributes fo: language, fo: country and text: sort-algorithm. See also section 7.8.

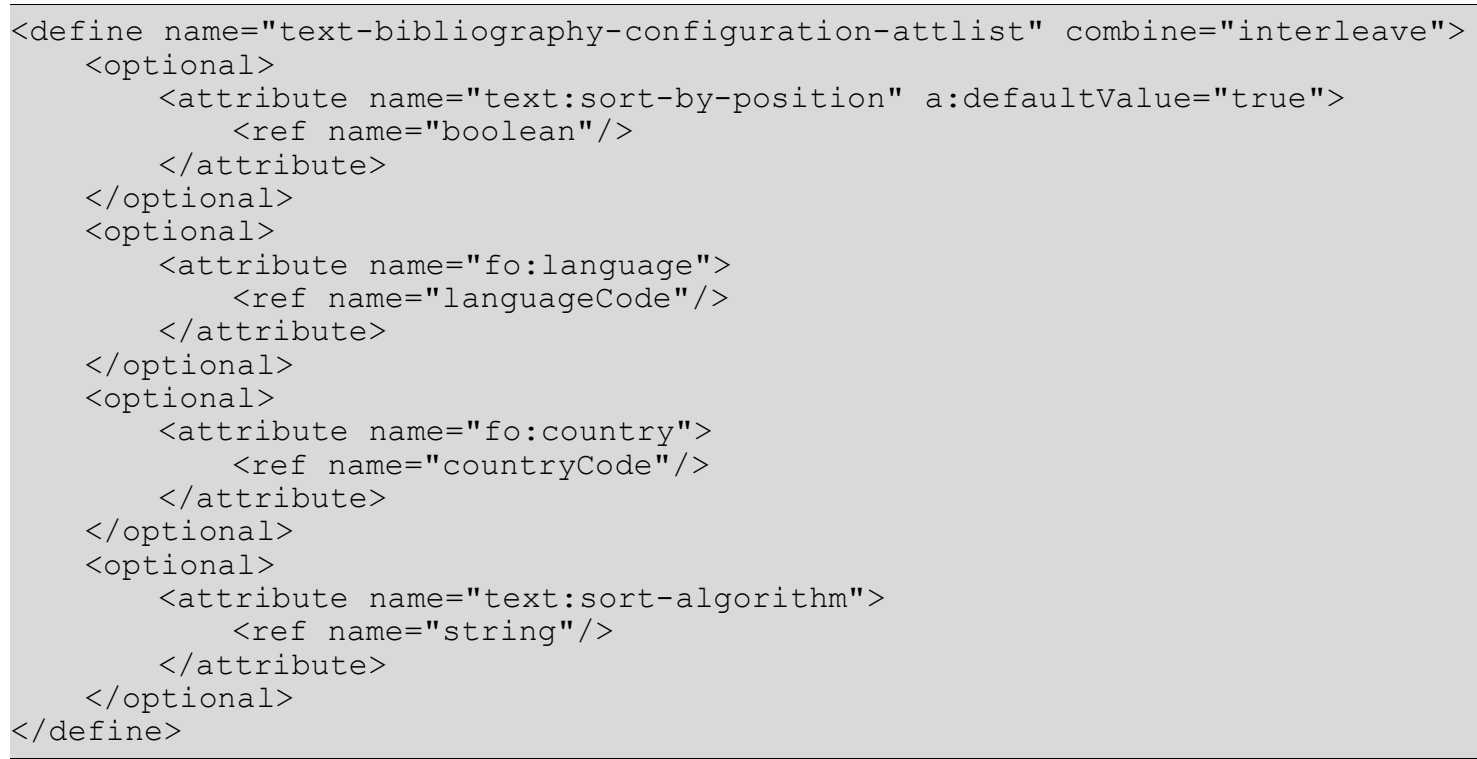

\section{Sort Keys}

The <text: sort-key> element specifies a single sort key if bibliography entries are not displayed in document order. It has an attribute text: key, that contains the type of index entry data that should be used for sorting (see also section 7.1.4) and an attribute text: sortascending that specifies whether sorting takes pace in ascending or descending order.

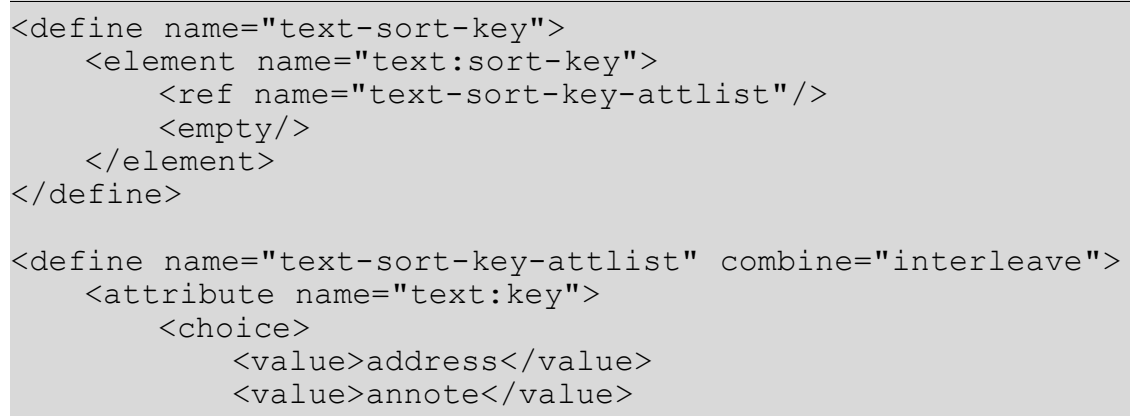




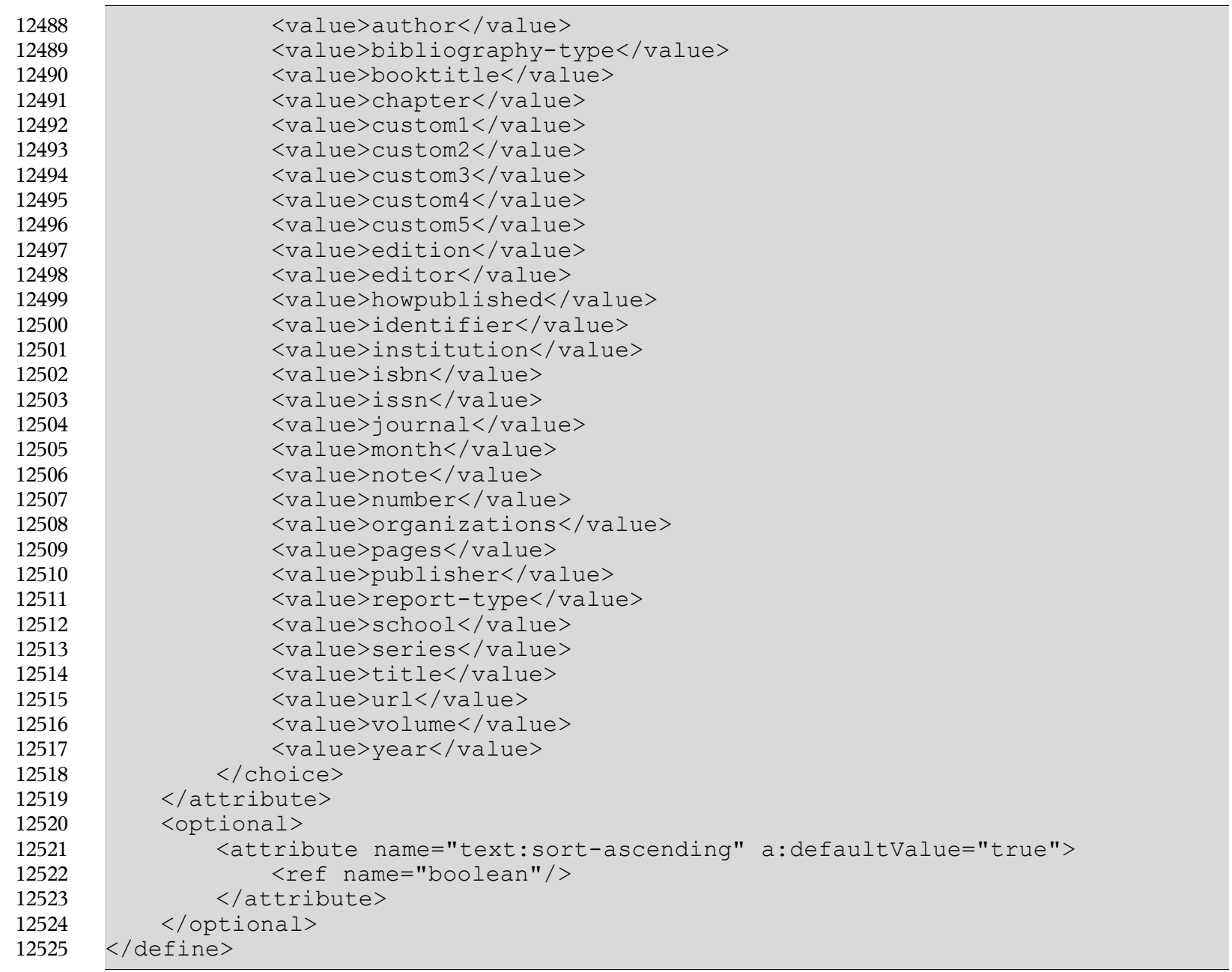

\subsection{List Style}

List styles specify the formatting properties for lists. A <text: list-style $>$ element contains a set of style elements for each list level, which are called list level styles. There are three different list level style elements, depending on whether this particular list level is to have a list label containing the list numbering, a bullet, or an image.

If a list style is applied to a list but does not contain a list level specification for the suitable level, the list level style of the next lower level is used. If no suitable list level style does exist, a default style is used.

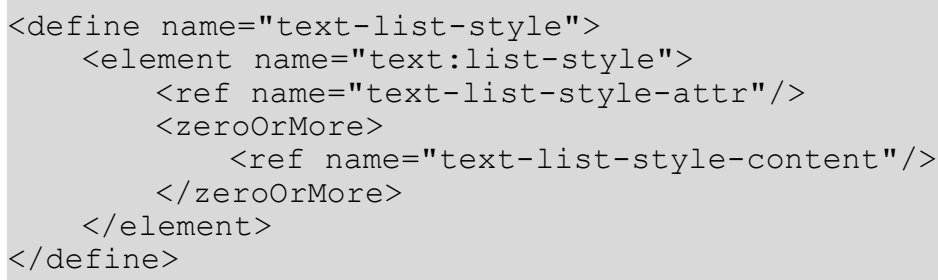

Note: List styles contain different properties than paragraph or text styles. This is why they are represented by a different element.

The attributes that may be associated with the <text: list-style $>$ element are: 
- Name

- Display name

- Consecutive numbering

\section{Name}

The style: name attribute specifies the name of the list style.

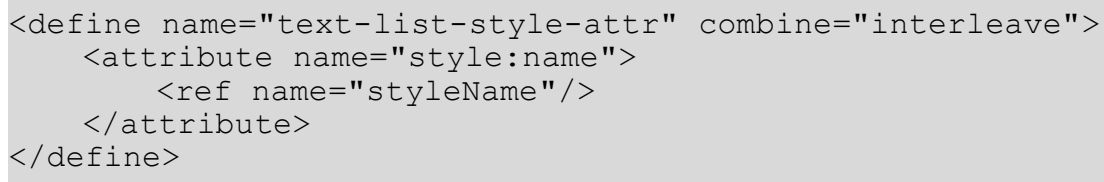

\section{Display Name}

The style:display-name attribute specifies the name of the list style as it should appear in the user interface. In contrast to the style name itself, this name may contain arbitrary characters. If this attribute is not present, the display name equals the style name.

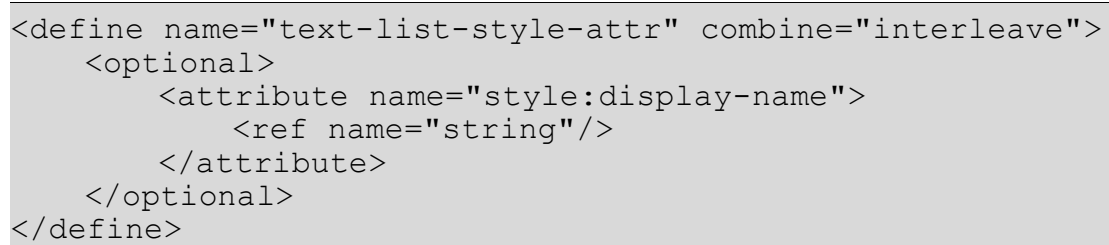

\section{Consecutive Numbering}

The text:consecutive-numbering attribute specifies whether or not the list style uses consecutive numbering for all list levels or whether each list level restarts the numbering.

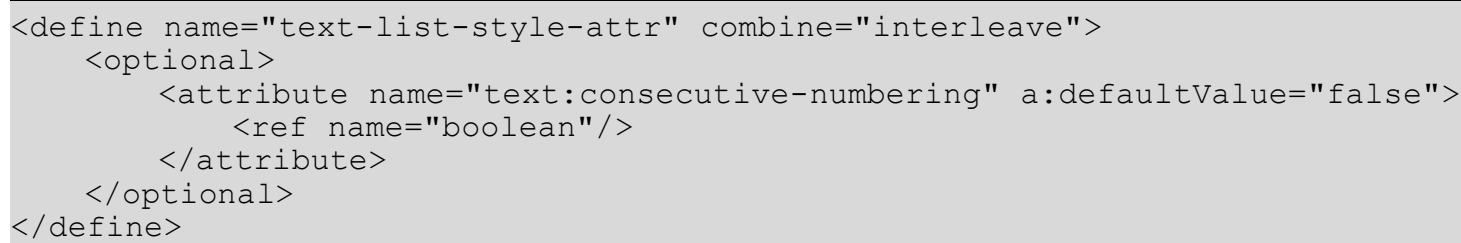

\subsubsection{Common List-Level Style Attributes}

The following attributes can be used on all list-level styles:

\section{Level}

The text: level attribute specifies the level of the number list style.

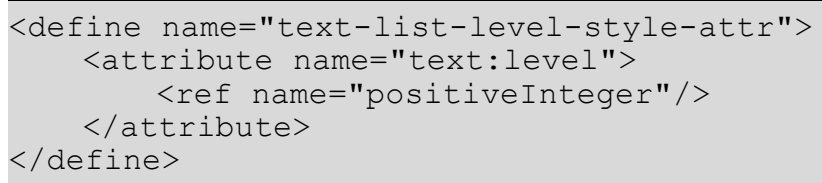




\subsubsection{Number Level Style}

A number level style specifies a list style where the list items are preceded by numbers.

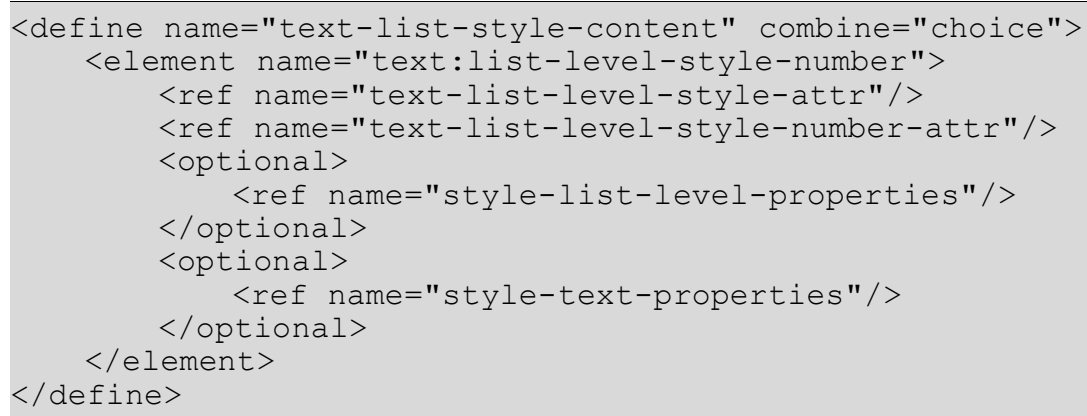

The attributes that may be associated with the <text: list-level-style-number $>$ element are:

- Level (see section 14.10.1)

- Text style

- Number format

- Display levels

- Start value

Additional formatting properties may be contained in the <style:list-level-properties $>$ and <style: text-properties> elements. See sections 15.12 and 15.4 for details.

\section{Text Style}

The text:style-name attribute specifies the name of the character style to use to format the number of the list.

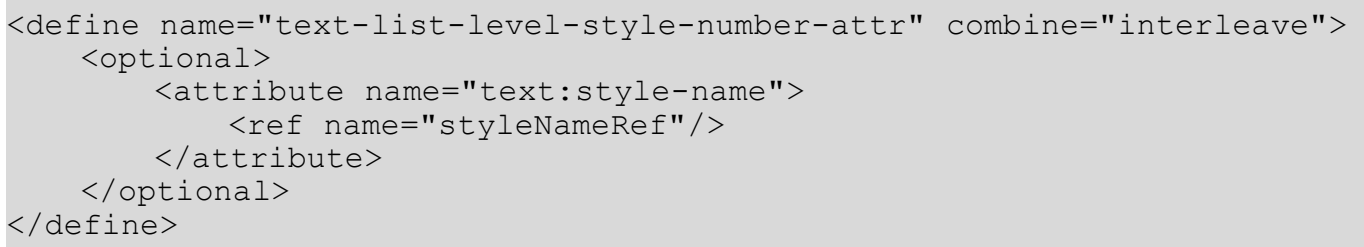

\section{Number Format}

See section 12.2 for detailed information on number format attributes. The attributes described in section 12.2 can also be associated with the <text: list-level-style-number > element. The style:num-format attribute can be empty. In this case, no number is displayed.

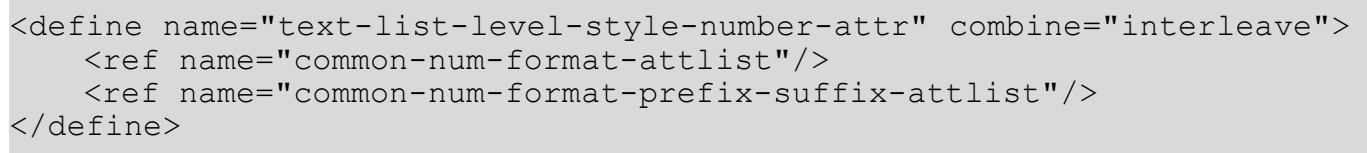

\section{Display Levels}

The text:display-levels attribute specifies the number of levels whose numbers are displayed at the current level. 


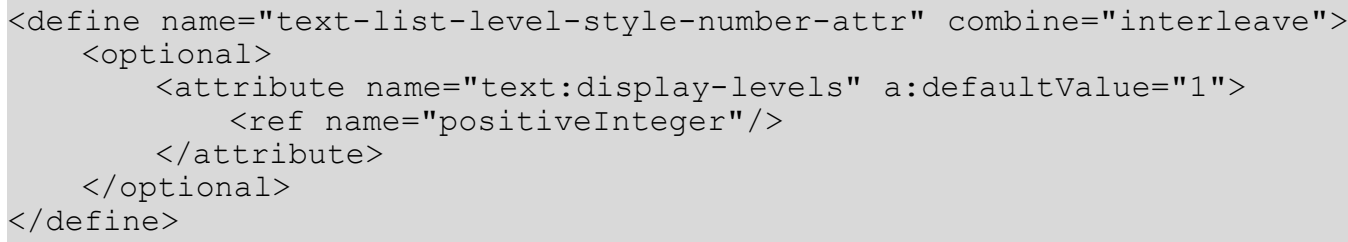

$</$ define $>$

Example: Given a third-level chapter number 1.2.3. Values of text:display-number from 1 to three would achieve the following results:

\begin{tabular}{|c|c|}
\hline text:display-number & display \\
\hline 1 & 1 \\
\hline 2 & 1.2 \\
\hline 3 & 1.2 .3 \\
\hline
\end{tabular}

\section{Start Value}

The text:start-value attribute specifies the first number of an list item of the current level.

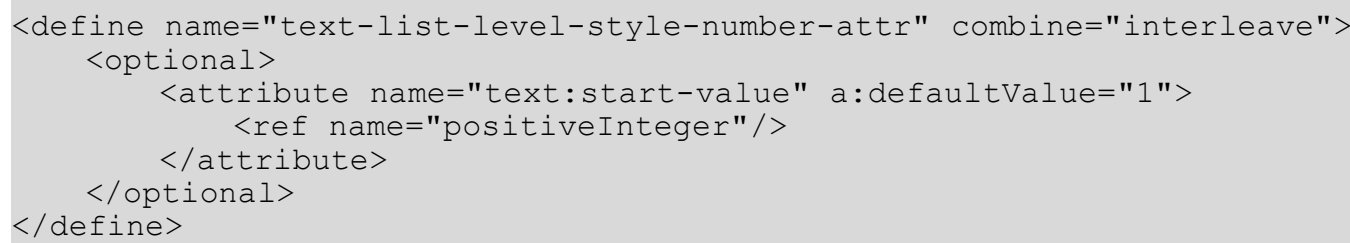

\subsubsection{Bullet Level Style}

A bullet level style element specifies a list style where the list items are preceded by bullets.

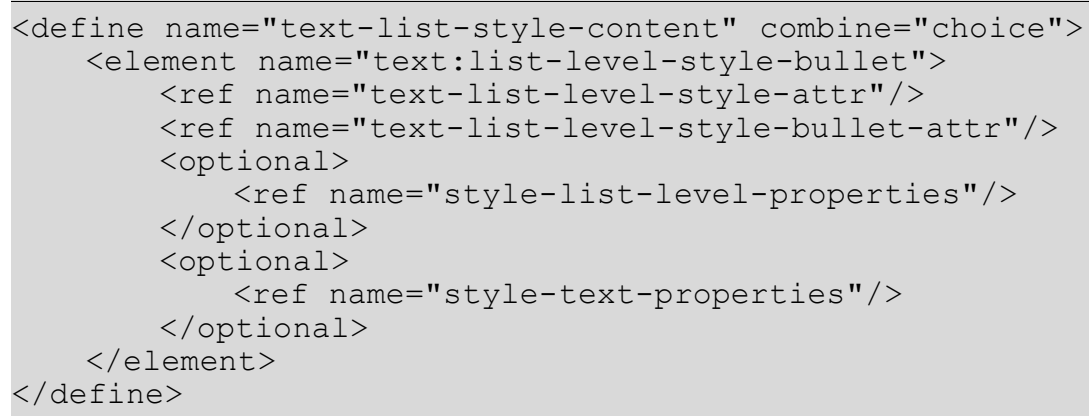

The attributes that may be associated with the <text: list-level-style-bullet> element are:

- Level (see section 14.10.1)

- Text style

- Bullet character

- Prefix and suffix

- Bullet relative size 
Additional formatting properties may be contained in the <style:list-level-properties> and <style: text-properties> elements. See sections 15.12 and 15.4 for details.

\section{Text Style}

The text:style-name attribute specifies the name of the character style to use to format the list bullet.

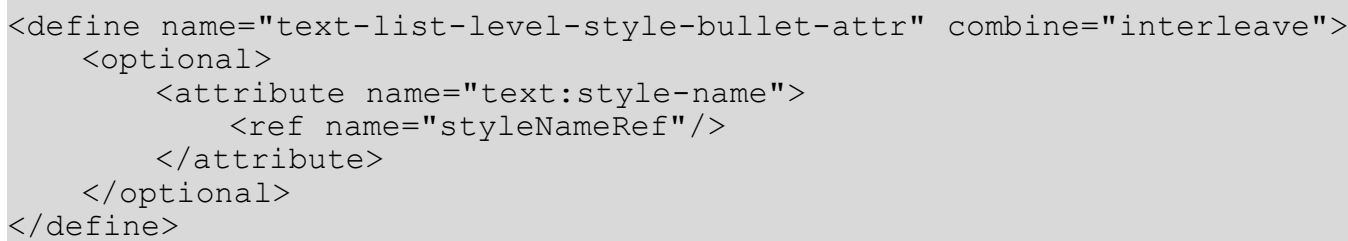

\section{Bullet Character}

The bullet character attribute specifies the UNICODE character to use as the bullet in a bullet level style.

Typical bullet characters are:

- $\mathrm{U}+2022$

- $\mathrm{U}+25 \mathrm{CF}$

$\rightarrow \mathrm{U}+2794$

$\mathrm{U}+27 \mathrm{~A} 2$

$x \quad \mathrm{U}+2717$

$\checkmark \mathrm{U}+2714$

These characters might not be available within some fonts.

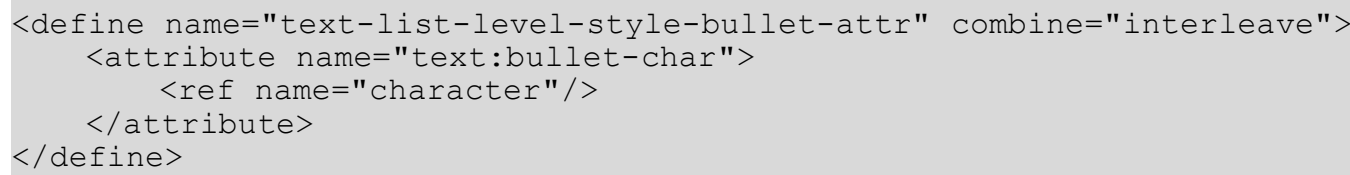

\section{Prefix and Suffix}

The attributes style:num-format-prefix and style:num-format-suffix specified in section 12.2 can be used to add characters before or behind the bullet character.

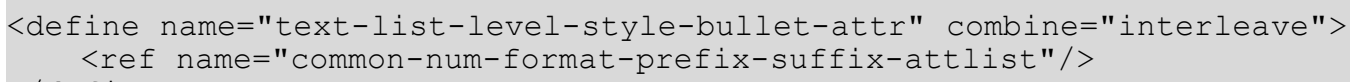

\section{Bullet Relative Size}

The text:bullet-relative-size attribute specifies a percentage value for the bullet size relative to the font size of the paragraphs in the bullet list. For example, if the value of the text:bullet-relative-size attribute is 75 , the bullet used in the list is $75 \%$ of the font size for the paragraph. 
<optional>

<attribute name="text:bullet-relative-size">

<ref name="percent" />

$</$ attribute $>$

$</$ optional $>$

$</$ define $>$

\subsubsection{Image Level Style}

An image level style element specifies a list style where the list items are preceded by images.

This element can be an [XLink] and can only be contained in list style elements.

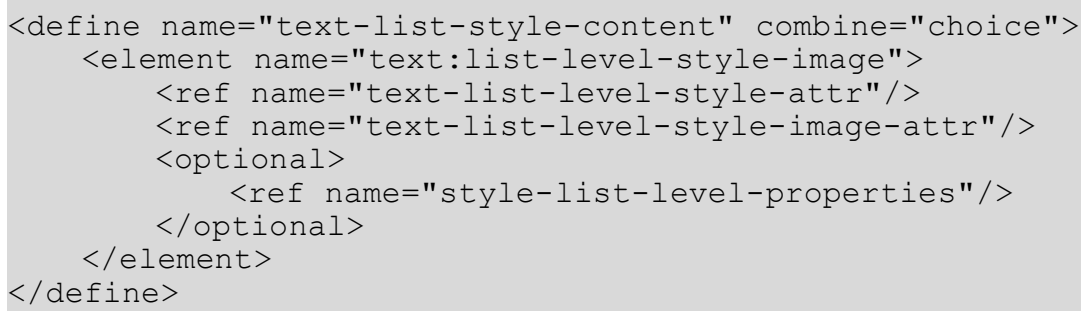

The following elements and attributes may be associated with the <text:list-level-styleimage> element are:

- Level (see section 14.10.1)

- Image location

Additional formatting properties may be contained in the <style:list-level-properties $>$ element. See section 15.12 for details.

\section{Image Location}

The image data can be stored in one of the following ways (see also section 9.3.2):

- The image data is located in an external file. Use the xlink: href attribute described below to specify the location of the file.

- The image data is contained in the <text:list-level-style-image $>$ element. The <text: list-level-style-image> element must contain an <office:binary-data> element that contains the image data in BASE64 encoding. In this situation, the xlink: href attribute is not required.

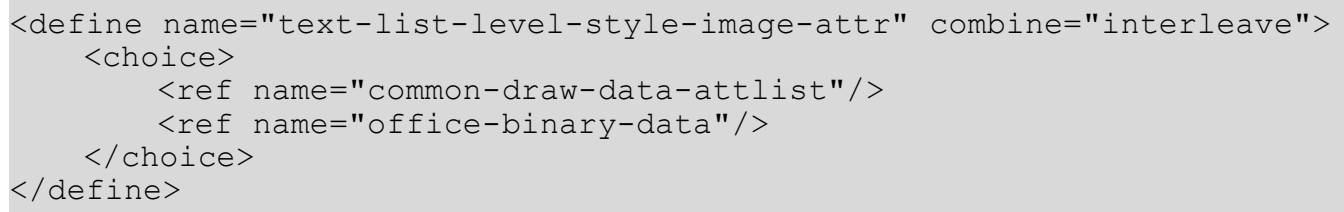

\subsubsection{List Level Style Example}

\section{Example: List level style}

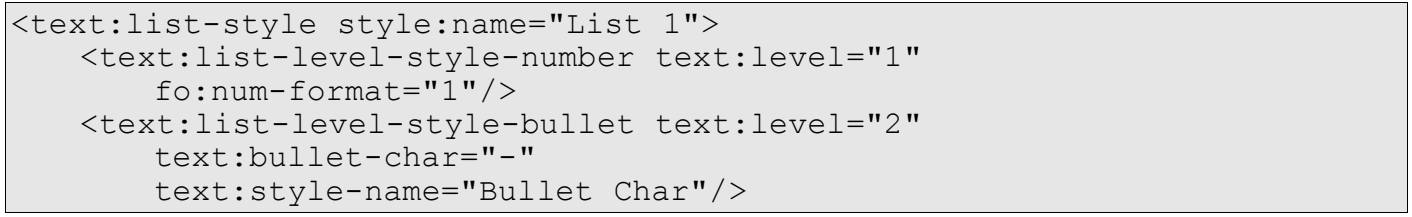




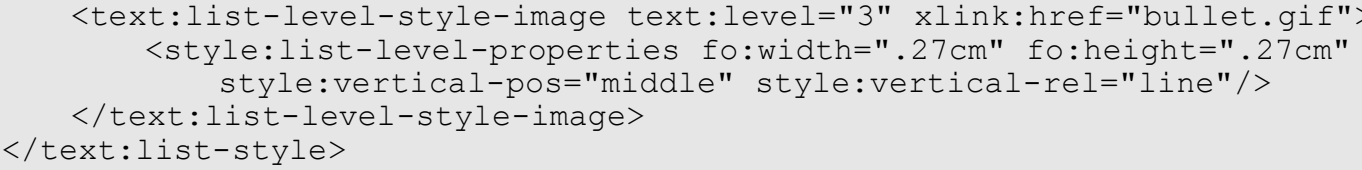

The following is the output from the above example:

1. This is the first list item.

This is a continuation of the first list item.

2. This is the second list item. It contains an unordered sub list.

- This is a sub list item.

- This is a sub list item.

- This is a sub list item.

This is a sub sub list item.

This is a sub sub list item.

3. This is the third list item.

\subsection{Outline Style}

The outline style is a list style that is applied to all headings within a text document where the the heading's paragraph style does not define a list style to use itself.

The way in which the OpenDocument format represents outline numbering styles is very similar to the way it represents list styles. The <text: outline-style> element contains elements that specify the style of each outline level. It can be contained within the <office:styles $>$ element only.

12661

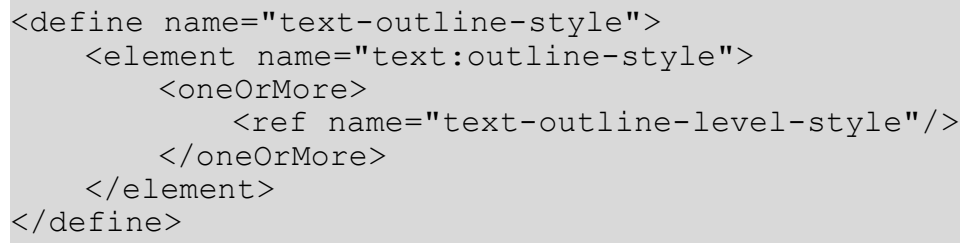

\subsubsection{Outline Level Style}

The <text: outline-level-style> element specifies the style for each outline level. This element is contained in <text:outline-style> elements only.

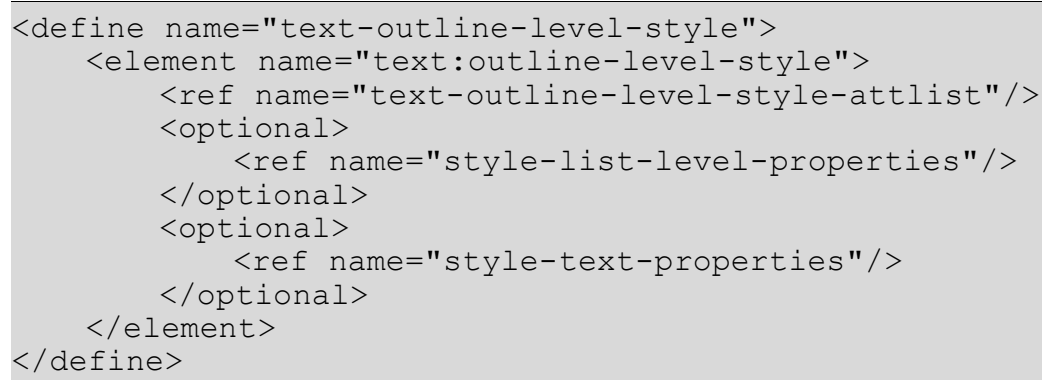

The attributes that may be associated with the <text: outline-level-style $>$ element are: 
- Level

- Text style

- Number format

- Display levels

- $\quad$ Start value

Additional formatting properties may be contained in the <style: list-level-properties> and <style: text-properties> element. See sections 15.12 and 15.4 for details.

\section{Level}

The text: level attribute specifies the level of the outline style.

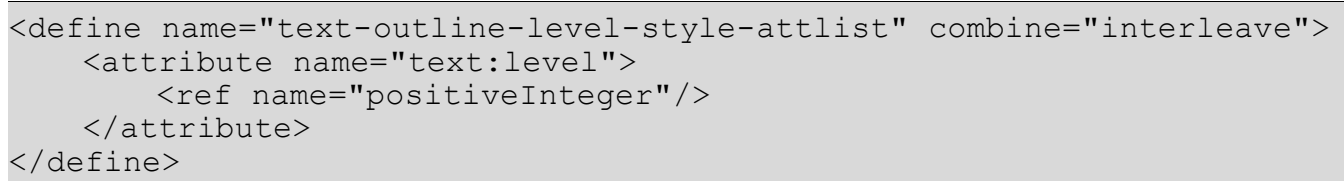

\section{Text Style}

The text:style-name attribute specifies the name of the character style to use to format the number of the heading.

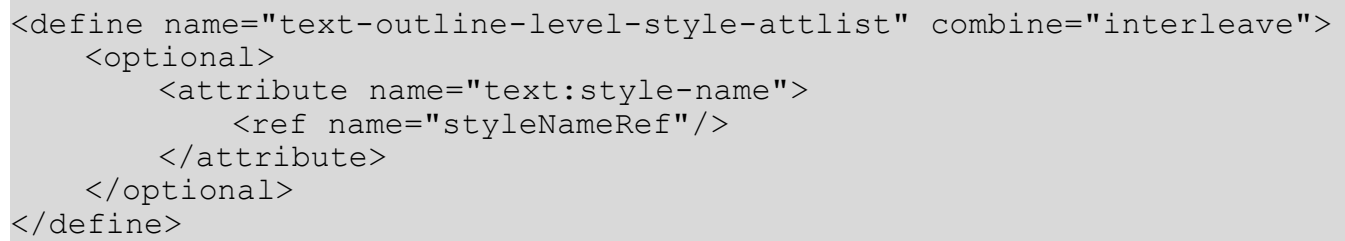

\section{Number Format}

See section 14.10.2 for information on the number format attributes.

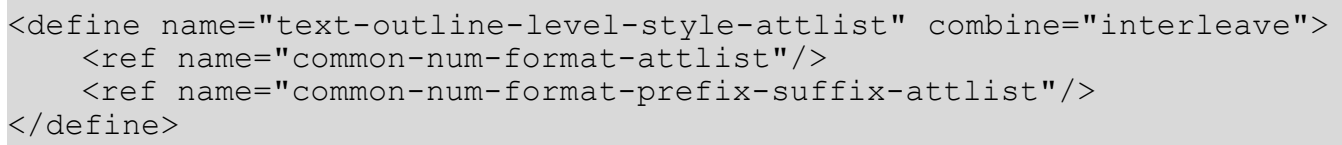

\section{Display Levels}

The text:display-levels attribute specifies the number of levels whose numbers are displayed at the current level. See also section 14.10.2.

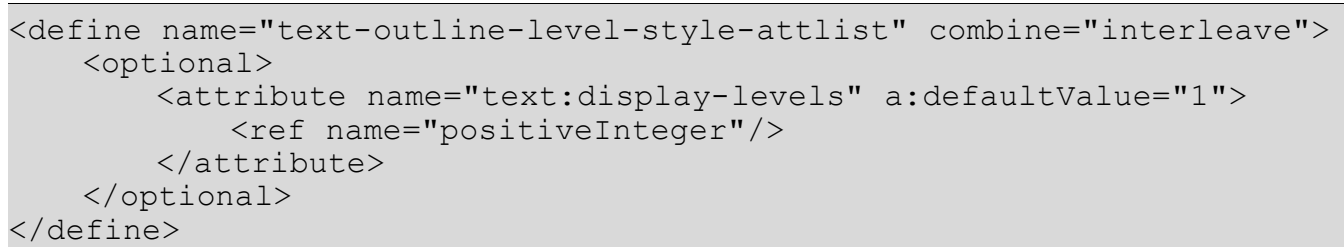




\section{Start Value}

The text:start-value attribute specifies the first number of an heading of the current level.

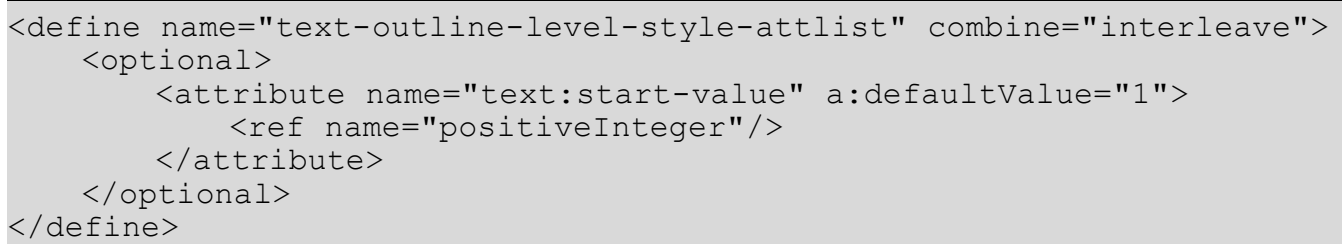

\subsection{Table Styles}

\subsubsection{Table Styles}

Table styles are <style:style> elements that have the family table. They can be used within all kind of applications to specify formatting properties for tables. They support the table properties as described in section 15.8.

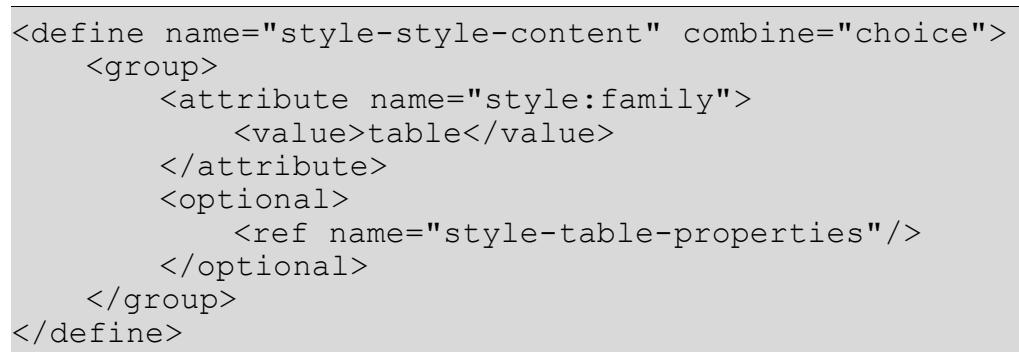

\subsubsection{Table Column Styles}

Table column styles are <style:style> elements that have the family table-column. They can be used within all kind of applications to specify formatting properties for table columns. They support the table column properties as described in section 15.9.

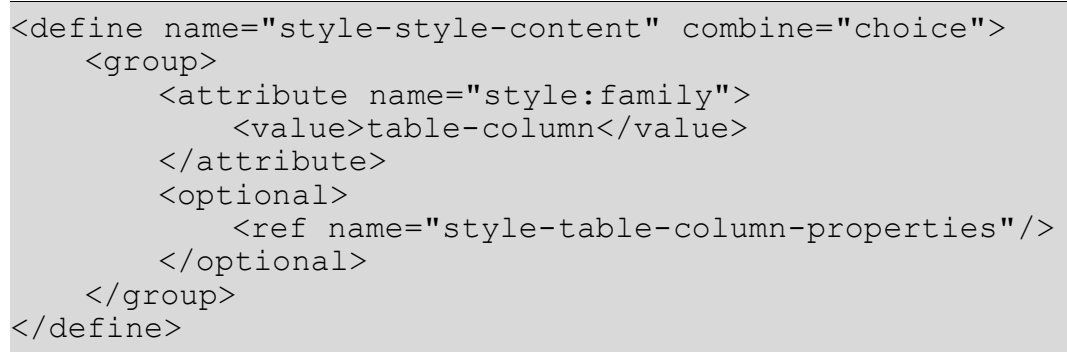

\subsubsection{Table Row Styles}

Table row styles are <style:style> elements that have the family table-row. They can be used within all kind of applications to specify formatting properties for table rows. They support the table properties as described in section 15.10.

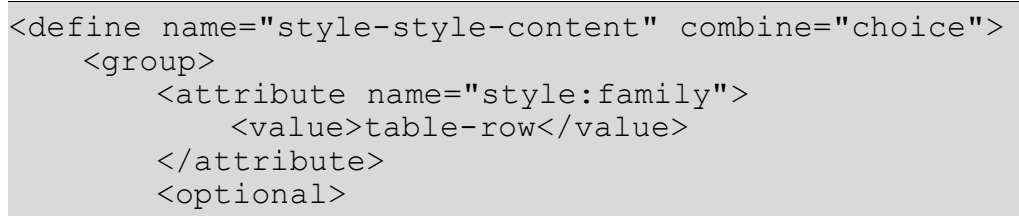




\subsubsection{Table Cell Styles}

Table styles are <style:style> elements that have the family table-cell. They can be used within all kind of applications to specify formatting properties for table cells. They support the table properties as described in section 15.11 as well as the paragraph and text properties as described in sections 15.5 and 15.4 .

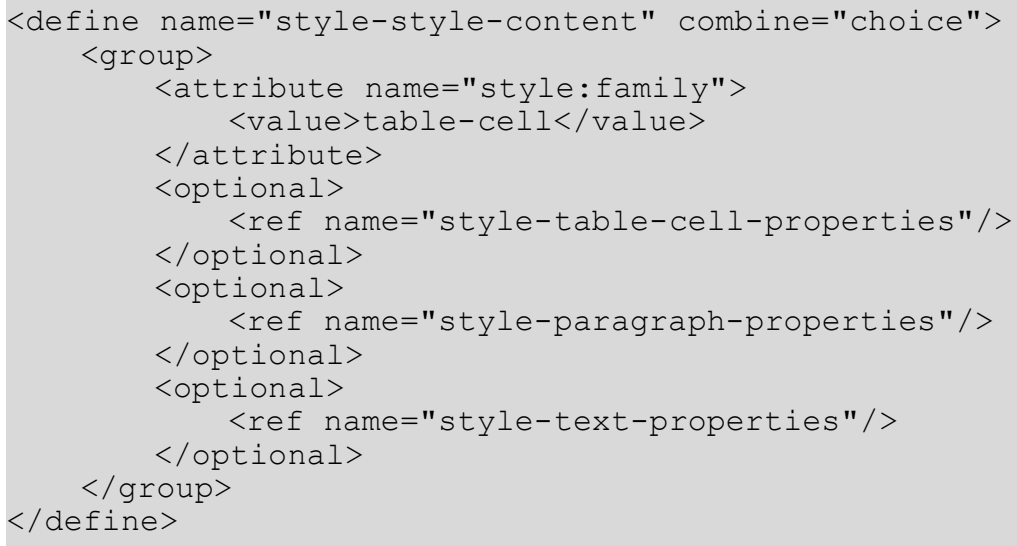

\subsection{Graphic Styles}

\subsubsection{Graphic and Presentation Styles}

Graphic and presentation styles are <style:style> elements that have either the family graphic or presentation. Graphic styles with family graphic may occur within all kinds of applications, graphic styles with family presentation may occur only within presentation documents. Both kind of styles support the graphic properties described in section 15.17. They may also contain paragraph and text properties as described in sections 15.5 and .

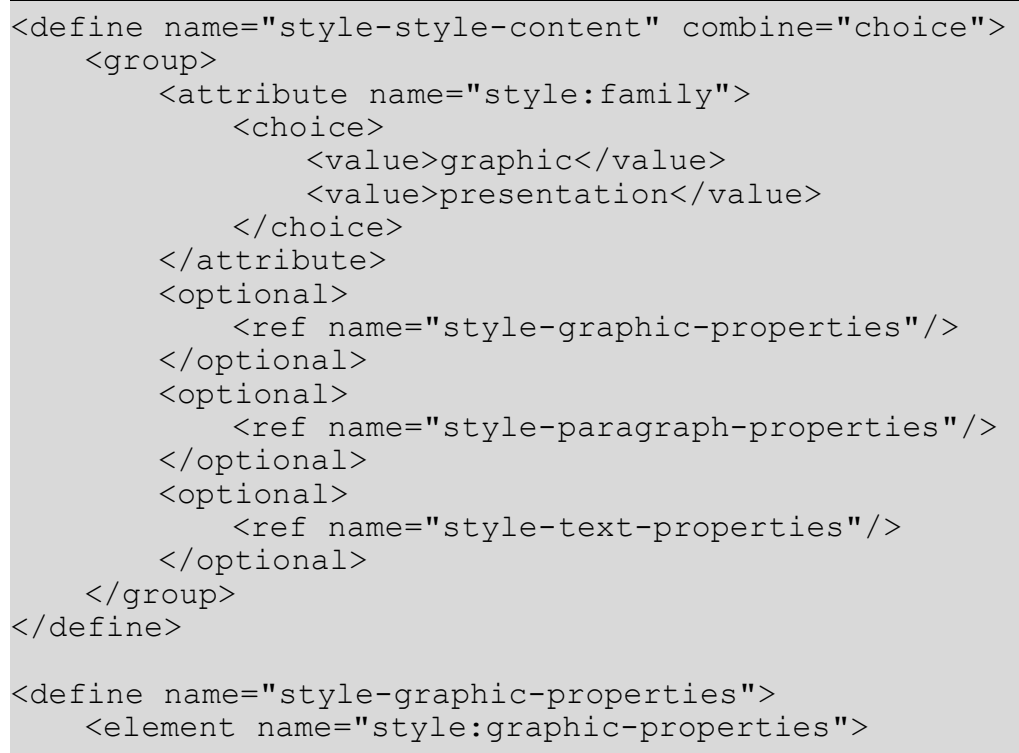




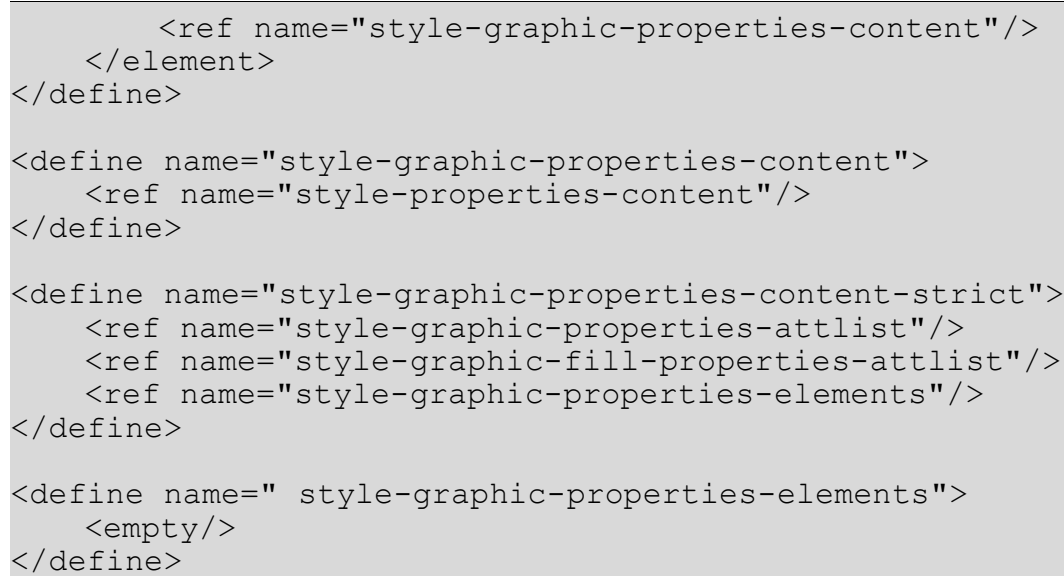

\subsubsection{Drawing Page Style}

A drawing page style is a <style:style> element with family drawing-page. Within graphical applications, drawing page styles can be used to change the background of draw page. If a background is set with the help of a drawing page style, then it overrides the background of the master page that is assigned to the draw page, but not the shapes that are on the master page.

Within presentation applications, the draw page style additionally may contain presentation properties, for example, the duration for which a page is displayed or fade effects.

The properties that can be used in a draw page style to change the background are the ones described in section 15.14 .

The presentation properties that can be used in a draw page style are described in section 15.36.

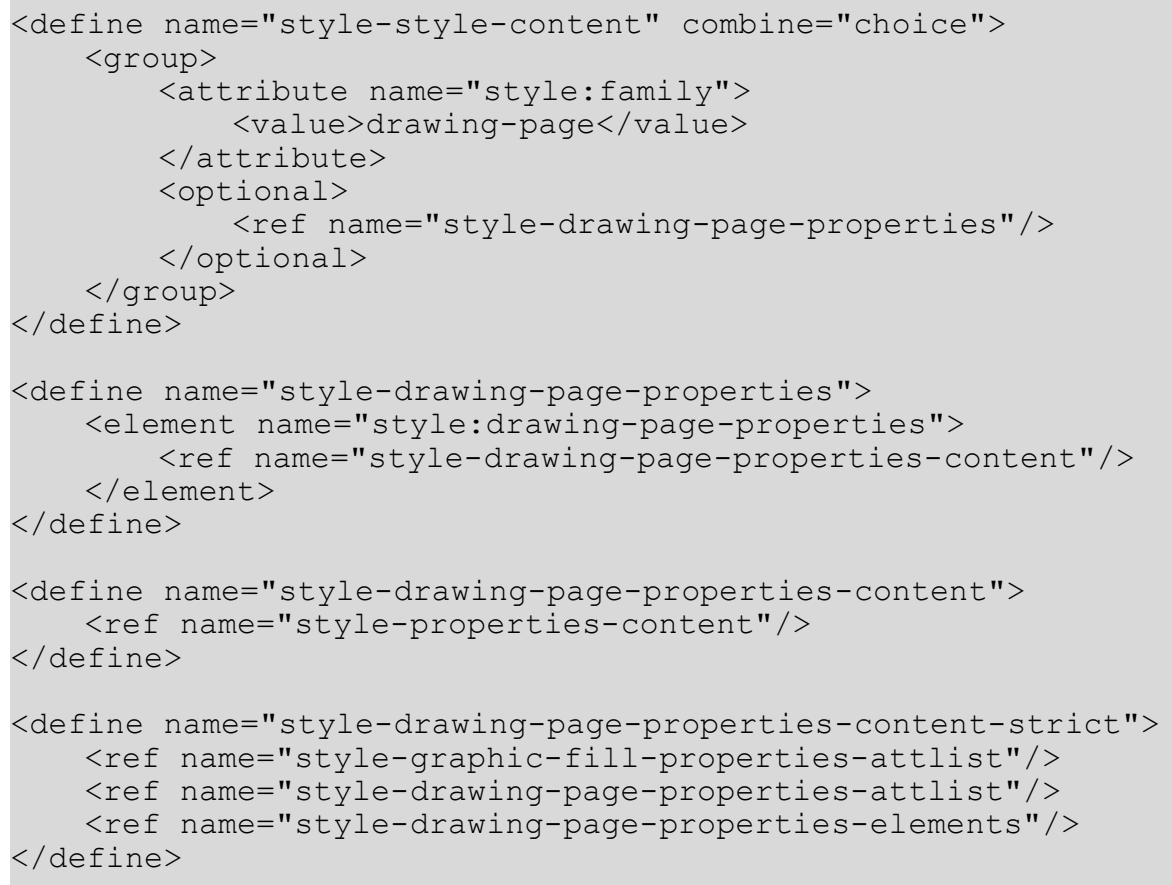




\subsection{Enhanced Graphic Style Elements}

The elements described in this section are enhanced graphic style. They cannot be used as automatic styles, that is, they have to be located in the <office:styles $>$ section of a document. Like all other style elements, they are referenced to by a unique name. The following styles for filling graphic objects are available:

- Gradient

- SVG Gradient

- Hatch

- Image

- Opacity Gradient

- Marker

- Dash

- Presentation Page Layout

\subsubsection{Gradient}

The element <draw: gradient> defines a gradient for filling a drawing object. Gradients are not available as automatic styles.

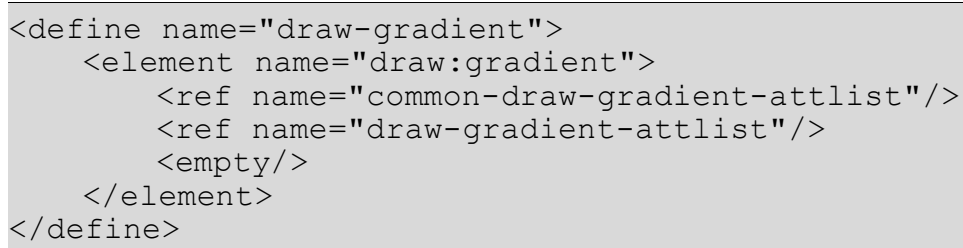

The attributes that may be associated with the gradient element are:

- Name

- Display name

- Gradient style

- Gradient center

- Colors

- Intensity

- Angle

- Border

\section{Name}

The attribute draw: name uniquely identifies a gradient inside an <office:styles $>$ element. 


\section{Display Name}

The draw: display-name attribute specifies the name of the gradient as it should appear in the user interface. In contrast to the style name itself, this name may contain arbitrary characters. If this attribute is not present, the display name equals the style name.

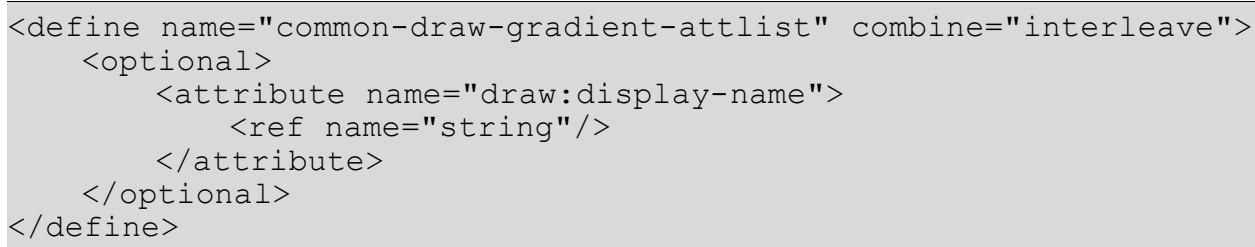

\section{Gradient Style}

The attribute draw: style specifies the style of the gradient. The gradient styles that an office application should support are linear, axial, radial, ellipsoid, square, and rectangular.

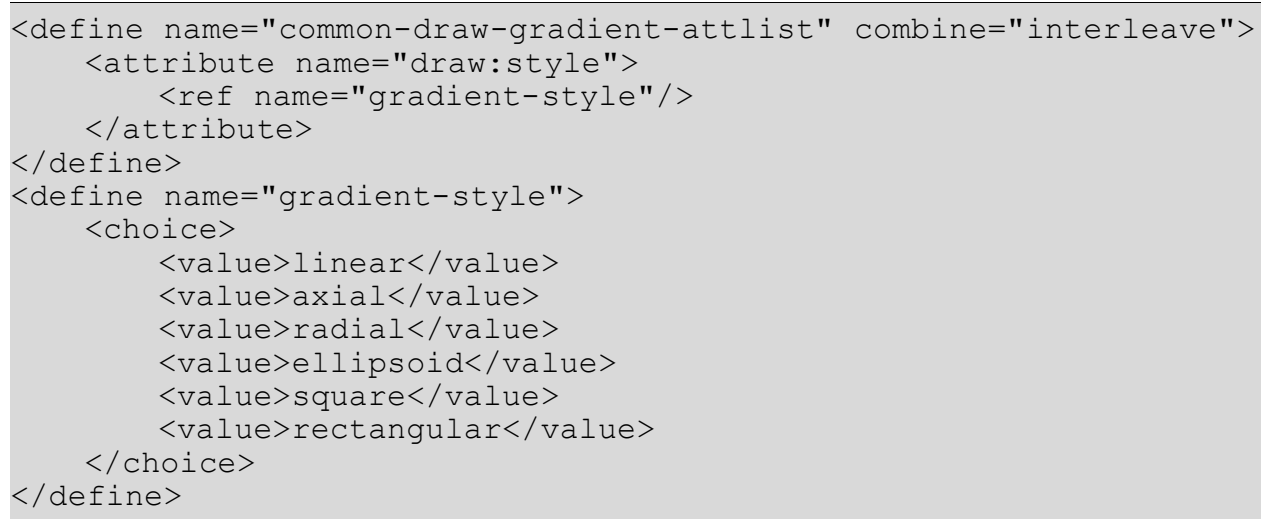

\section{Gradient Center}

If the gradient style is radial, ellipsoid, square, or rectangular, the gradient center attributes draw: cx and draw: cy specifies the center of the geometry that is used for the gradient. The values of these attributes are always percentage values.

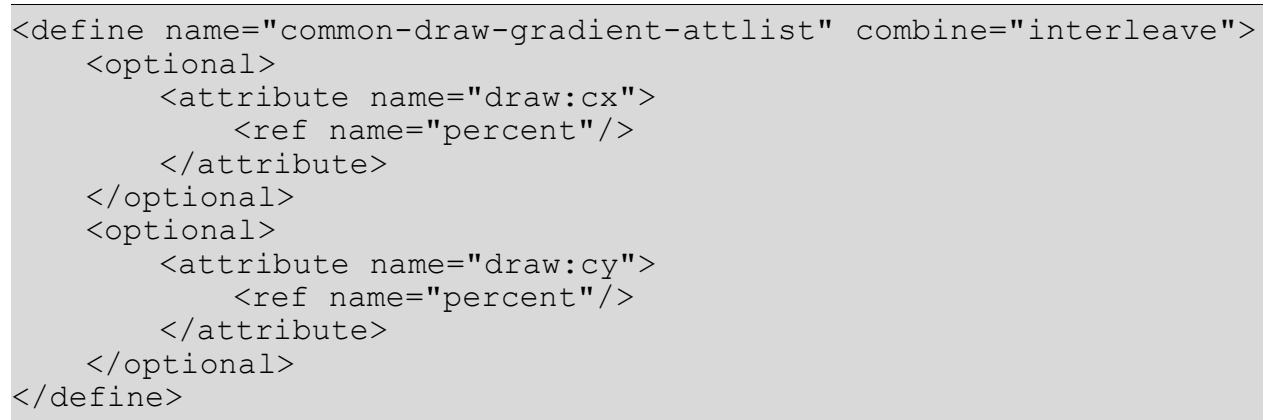




\section{Colors}

The gradient interpolates between a start color and an end color, which are specified using the attributes draw: start-color and draw:end-color.

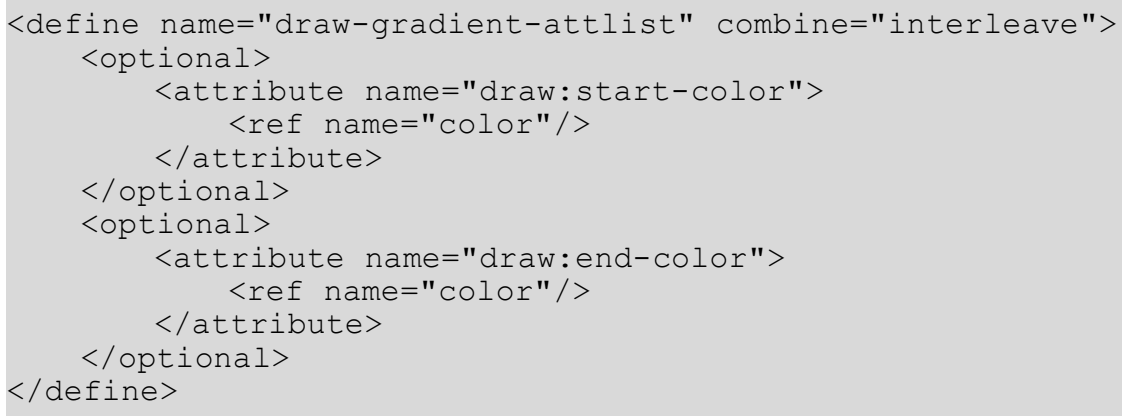

\section{Intensity}

The attributes draw:start-intensity and draw:end-intensity specify the intensity of the gradient's start and end color as percentage values. These attributes are optional. If the attributes are not specified, the colors are used as they are, that is at $100 \%$ intensity.

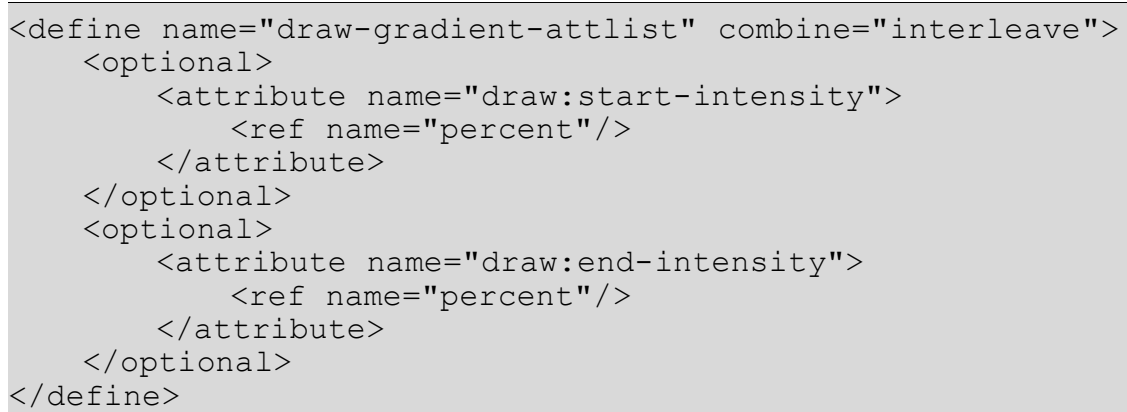

$</$ optional $>$

\section{Angle}

The draw: angle attribute specifies an angle that rotates the axis at which the gradient values are interpolated. This attribute is ignored for radial style gradients.

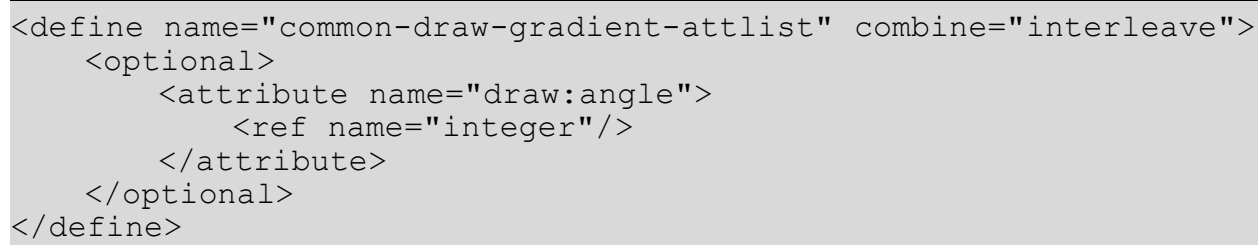

\section{Border}

Depending on the style of the gradient, the draw:border attribute specifies a percentage value which is used to scale a border which is filled by the start or end color only.

For example, a border of $10 \%$ means that the first $10 \%$ of the gradient is colored completely in the start color and the remaining $90 \%$ are an interpolation between start and end color.

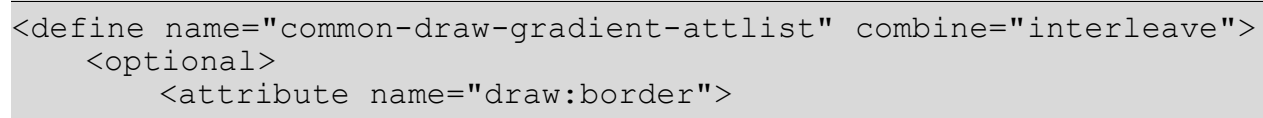




\subsubsection{SVG Gradients}

In addition to the gradients specified in section 14.14.1, gradient may be defined by the SVG gradient elements <linarGradient> and <radialGradient> as specified in §13.2 of [SVG]. The following rules apply to SVG gradients if they are used in documents in OpenDocument format:

- The gradients must get a name. It is specified by the draw: name attribute.

- For <linarGradient>, only the attributes gradientTransform, x1, y1, x2, y2 and spreadMethod will be evaluated.

- For <radialGradient>, only the attributes gradientTransform, cx, cy, r, fx, fy and spreadMethod will be evaluated.

- The gradient will be calculated like having a gradientUnits of objectBoundingBox, regardless what the actual value of the attribute is.

- The only child element that is evaluated is $\langle$ stop $\rangle$.

- For $\langle$ stop $\rangle$, only the attributes offset, stop-color and stop-opacity will be evaluated.

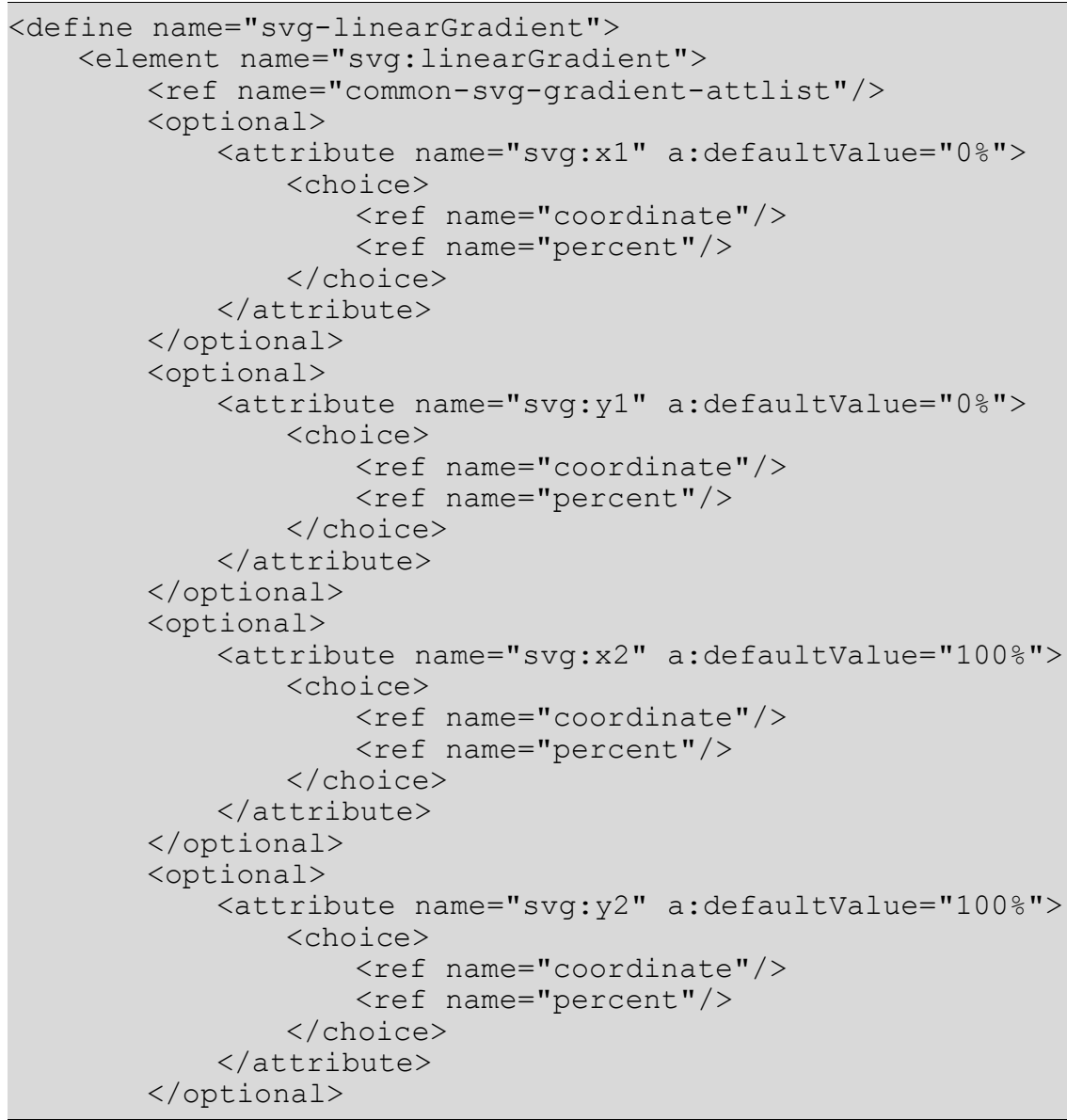




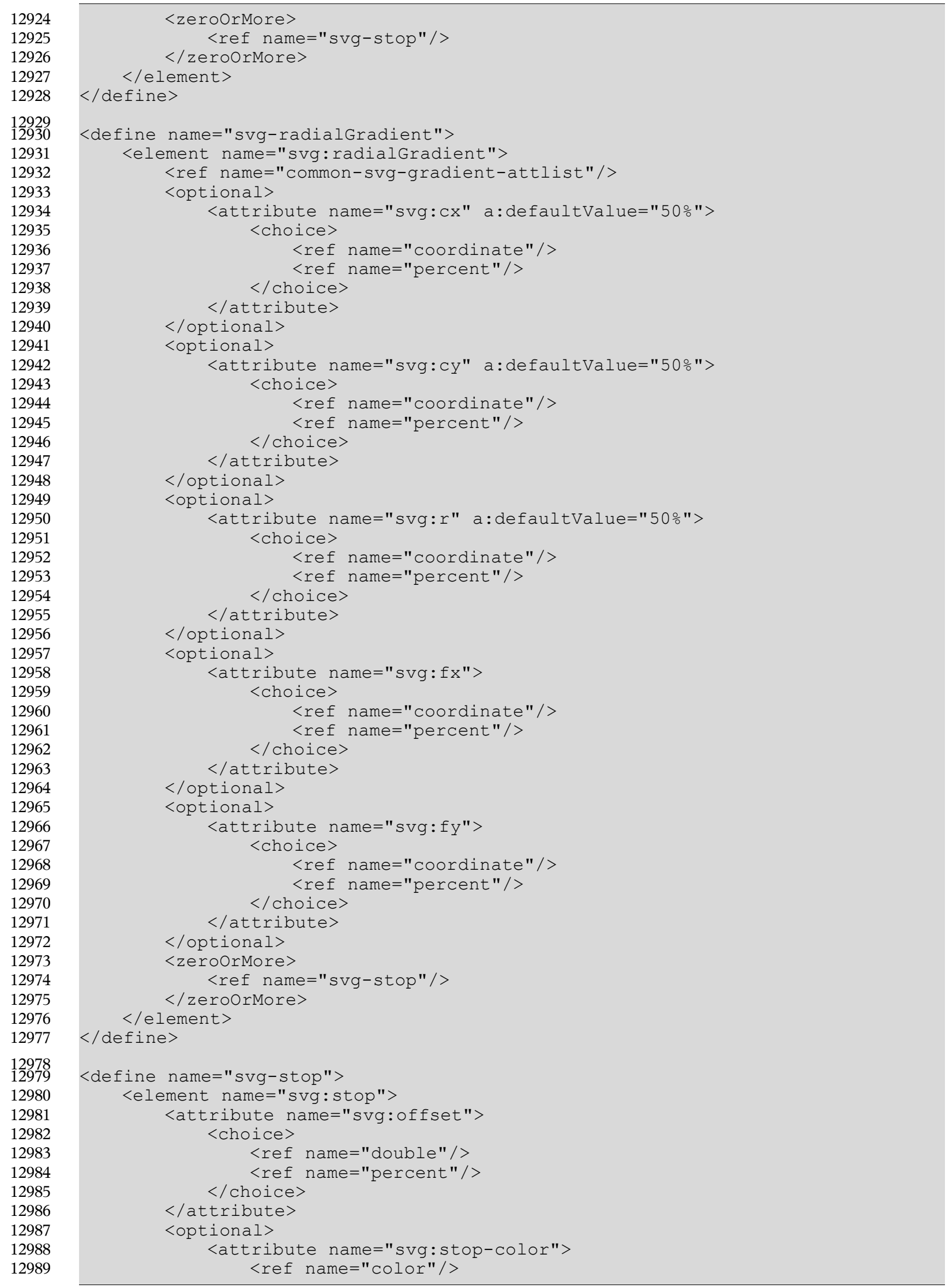




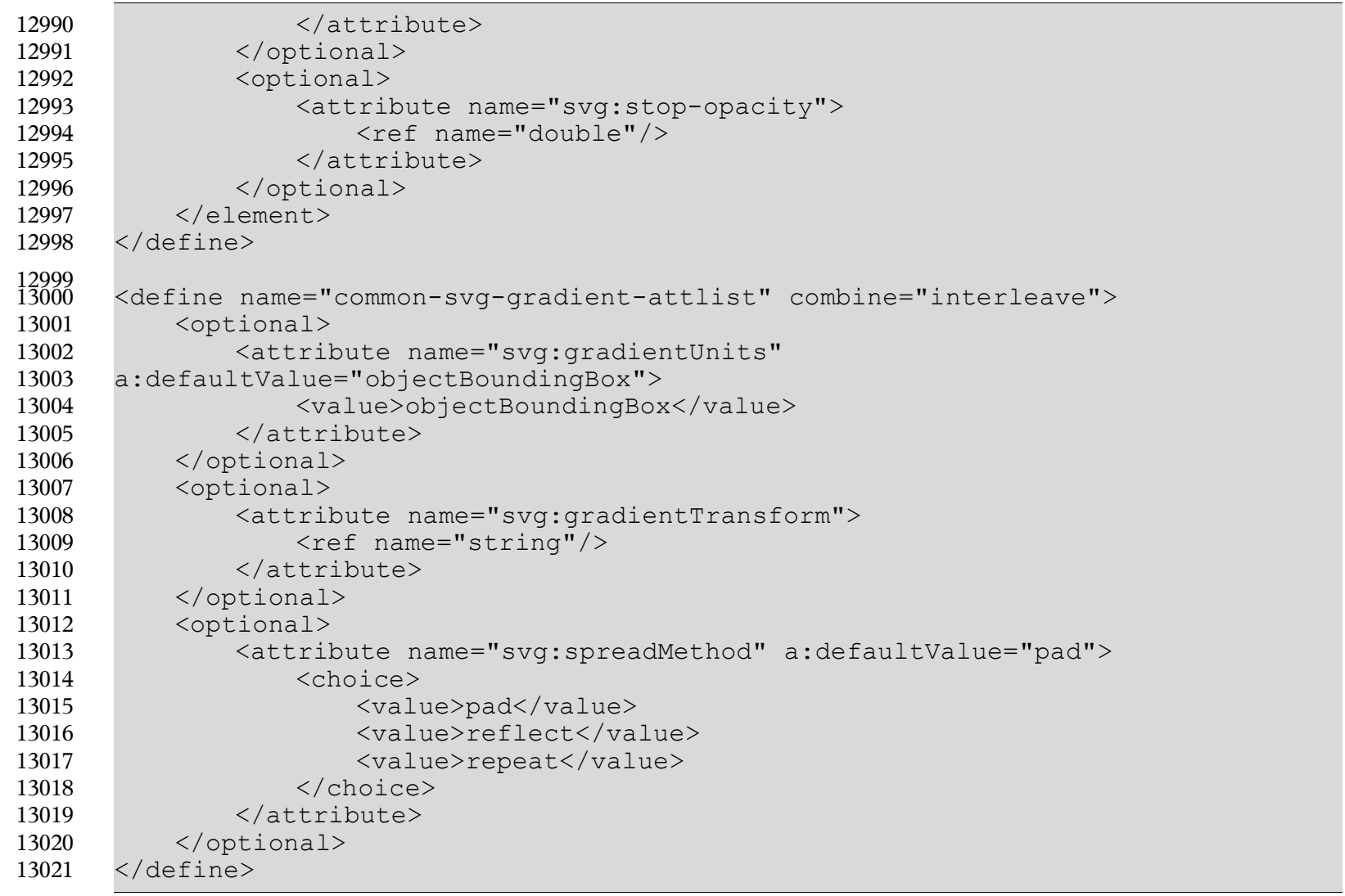

\section{Name}

The attribute draw: name uniquely identifies a gradient inside an <office:styles $>$ element. Like <draw: gradient> elements, SVG gradients are referenced by this name using the draw:fill-gradient-name attribute within a graphic style. SVG gradients cannot be referenced by a draw: opacity-name attribute. The result of referencing a SVG gradient with draw:fillgradient-name attribute and an opacity gradient with a draw:opacity-name attribute at the same time is unspecified.

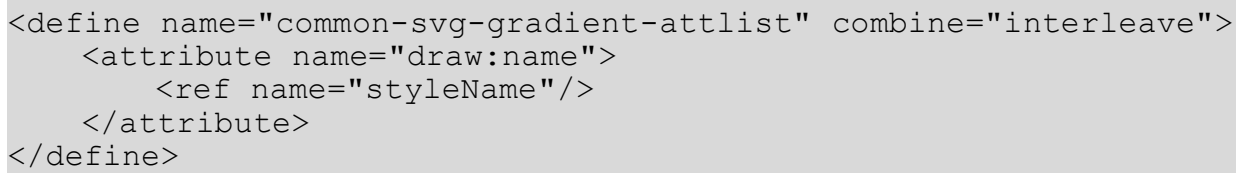

\section{Display Name}

The draw: display-name attribute specifies the name of the gradient as it should appear in the user interface. In contrast to the style name itself, this name may contain arbitrary characters. If this attribute is not present, the display name equals the style name.

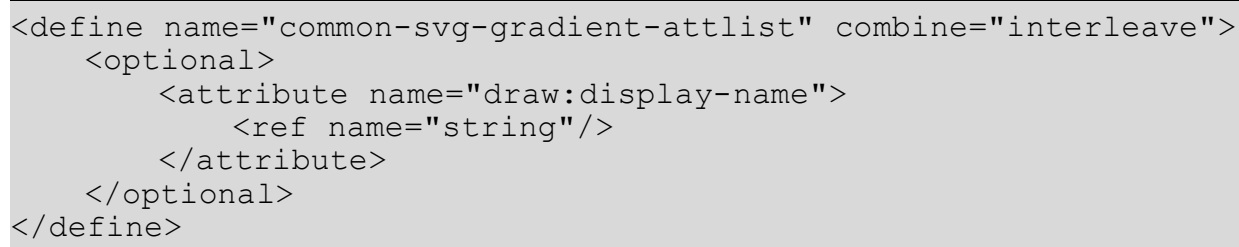




\subsubsection{Hatch}

The <draw: hatch> element defines a hatch for filling graphic objects. A hatch is a simple pattern of straight lines that is repeated in the fill area. Hatches are not available as automatic styles.

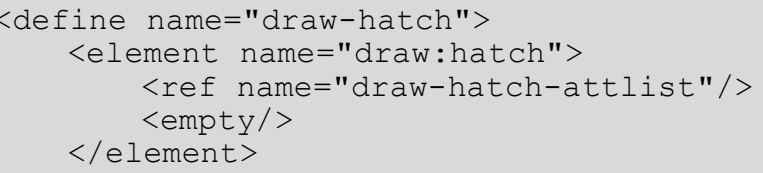

The attributes that may be associated with the hatch element are:

- Name

- Display name

- Style

- Color

- Distance

- Angle

- Background

\section{Name}

The draw: name attribute uniquely identifies a hatch inside an <office:styles $>$ element.

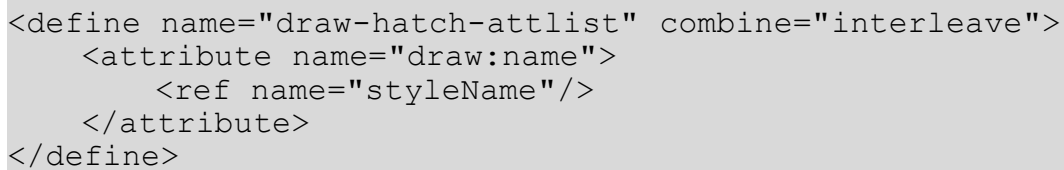

\section{Display Name}

The draw: display-name attribute specifies the name of the hatch style as it should appear in the user interface. In contrast to the style name itself, this name may contain arbitrary characters. If this attribute is not present, the display name equals the style name.

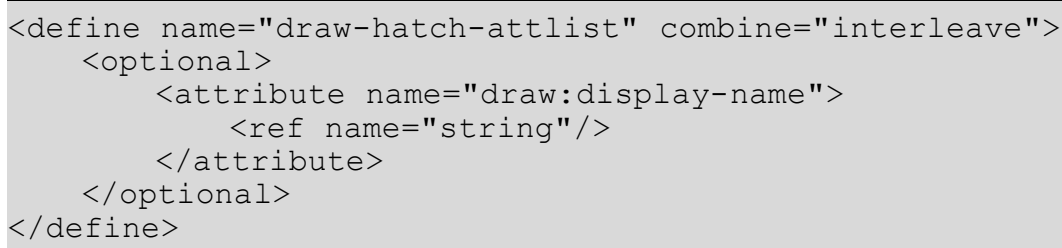

\section{Style}

The draw:style attribute specifies the style of the hatch.

The hatch can have one of three styles: single, double, or triple. 


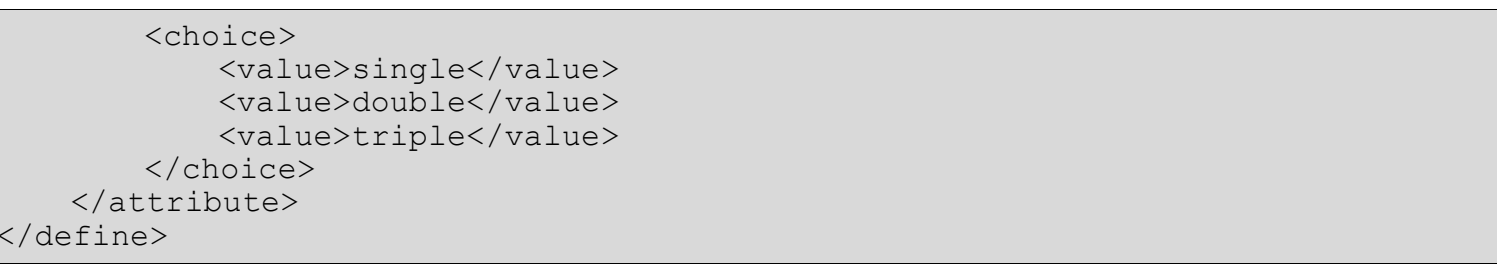

\section{Color}

The draw: color attribute specifies the color of the hatch lines.

13061

13062

13063

13064

13065

13066

13067

13068

13069

13070

13071

13072

13073

13074

\section{Angle}

The draw: rotation attribute specified the rotation angle of the hatch lines.

13075

13076

13077

13078

13079

13080

13081

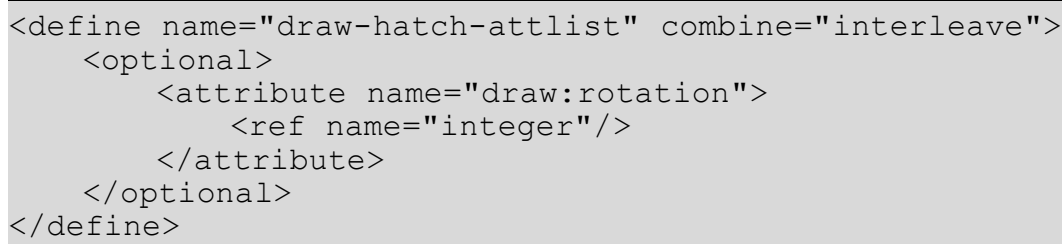

\subsubsection{Fill Image}

The <draw: fill-image> element specifies a link to a bitmap resource, for example, a .PNG file. This element follows the XLink specification. Fill image are not available as automatic styles.

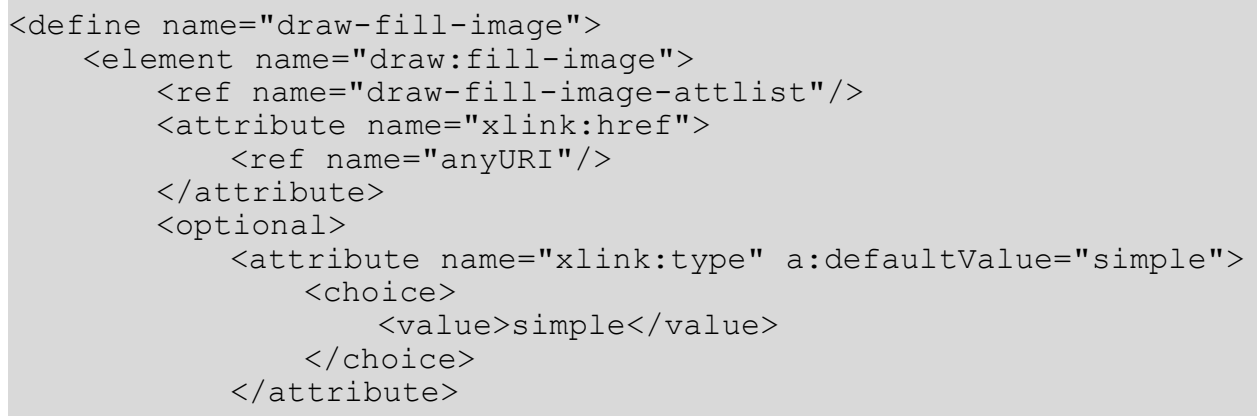


$</$ define $>$

$</ o p t i o n a l>$

<optional>

<attribute name="xlink:show" a:defaultValue="embed"> $<$ choice>

<value>embed</value>

$</$ choice $>$

$</ a t t r i b u t e>$

$</ o p t i o n a l>$

<optional>

<attribute name="xlink:actuate" a:defaultValue="onLoad">

$<$ choice>

$<$ value $>$ onLoad $</$ value $>$

$</$ choice $>$

$</ a t t r i b u t e>$

$</$ optional $>$

$<$ empty/>

$</$ element $>$

The attributes that may be associated with the fill image element are:

- Name

- Display name

- $\quad$ Size

\section{Name}

The draw: name attribute uniquely identifies a fill image inside an <office:styles $>$ element.

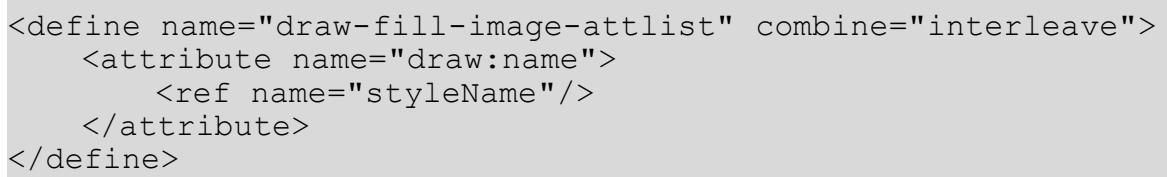

\section{Display Name}

The draw: display-name attribute specifies the name of the fill image as it should appear in the user interface. In contrast to the style name itself, this name may contain arbitrary characters. If this attribute is not present, the display name equals the style name.

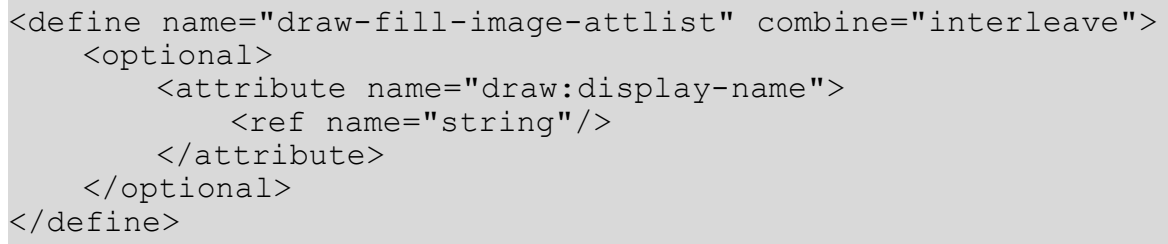

\section{Size}

The optional attributes svg:width and svg:height specify the size of the linked image. These values are optional and are overridden by the physical size of the linked image resource. They can be used to get the size of an image before it is loaded.

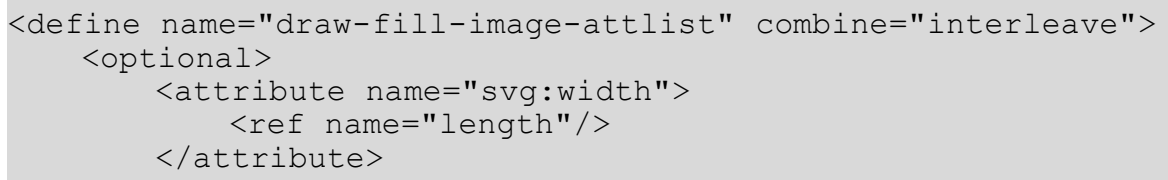




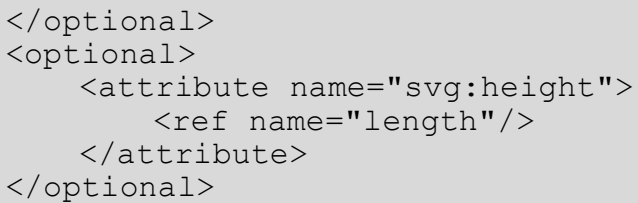

\subsubsection{Opacity Gradient}

The <draw: opacity > element specifies an opacity gradient for a graphic object. An opacity gradient works like a gradient, except that the opacity is interpolated instead of the color. Opacity gradients are not available as automatic styles.

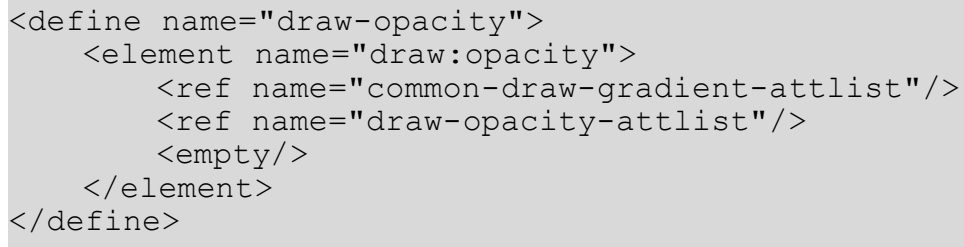

The attributes that may be associated with the $\langle$ draw: opacity $>$ element are:

- $\quad$ Name, Display name, Style, Opacity center, Angle, Border - see section 14.14.1.

- Opacity

\section{Opacity}

The opacity interpolates between a start and an end value.

The values of the attributes draw: start and draw:end are percentages where $0 \%$ is fully transparent and $100 \%$ is fully opaque.

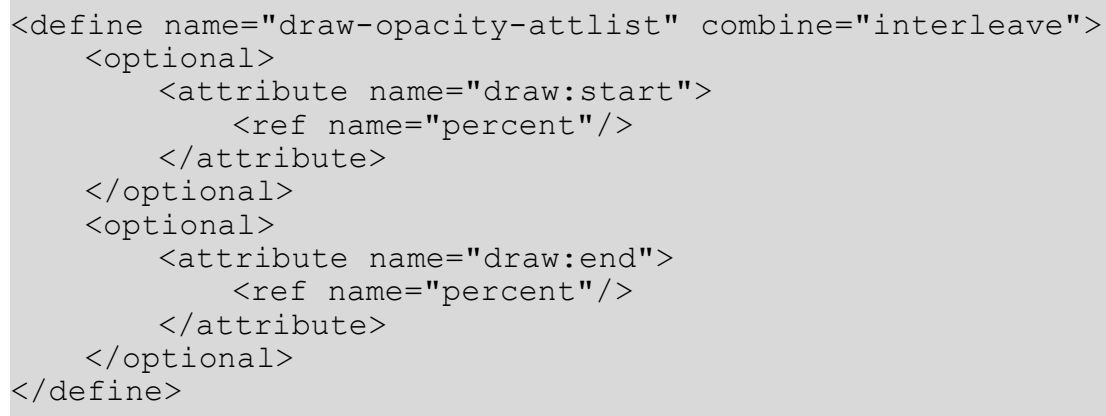

\subsubsection{Marker}

The element $<$ draw: marker $>$ represents a marker, which is used to draw polygons at the start and end points of strokes. Markers are not available as automatic styles.

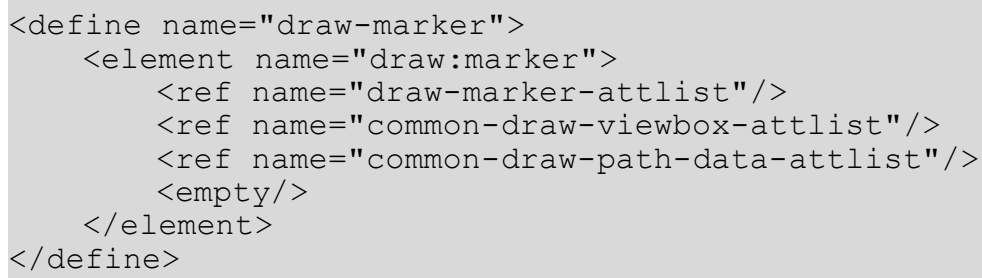


See sections 9.2.4 and 9.2.15 for information on the path data and viewbox attributes that may be associated with the <draw:marker> element.

\section{Name}

The draw: name attribute uniquely identifies a fill image inside an <office:styles $>$ element.

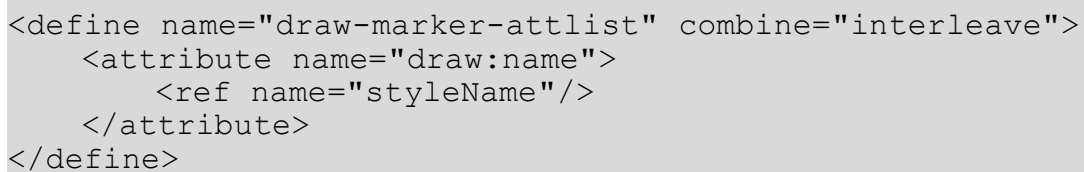

\section{Display Name}

The draw: display-name attribute specifies the name of the marker as it should appear in the user interface. In contrast to the style name itself, this name may contain arbitrary characters. If this attribute is not present, the display name equals the style name.

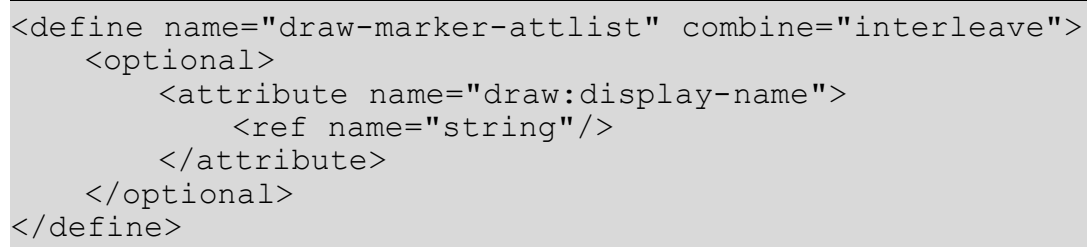

\subsubsection{Stroke Dash}

The dash element $<$ draw : stroke-dash $>$ represents a dash style that can be used to render strokes of shapes. Stroke dashes are not available as automatic styles.

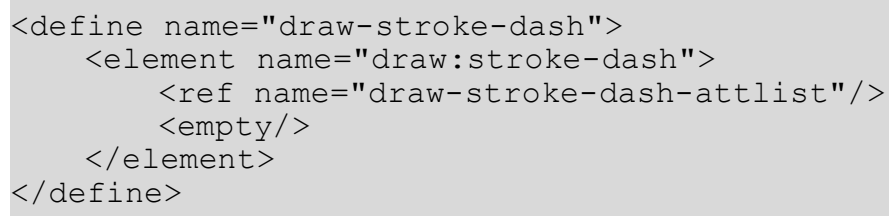

The attributes that may be associated with the <draw: stroke-dash> element are:

- Name

- Display name

- Style

- Dots

- Distance

\section{Name}

The attribute draw: name uniquely identifies a dash inside an <office:styles > element.

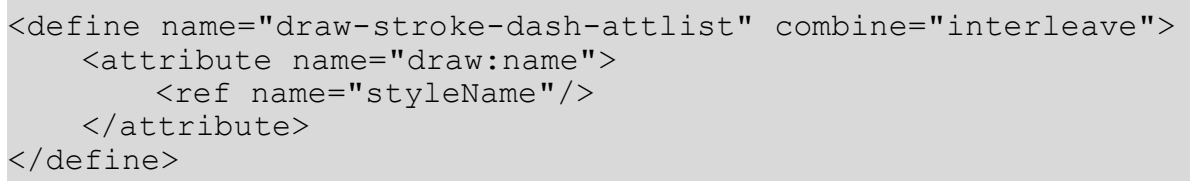




\section{Display Name}

The draw: display-name attribute specifies the name of the dash as it should appear in the user interface. In contrast to the style name itself, this name may contain arbitrary characters. If this attribute is not present, the display name equals the style name.

13186

13187

13188

13189

13190

13191

13192

\section{Style}

The attribute draw:style specifies whether the points of a dash are round or rectangular.

13193

13194

13195

13196

13197

13198

13199

13200

13201

13202

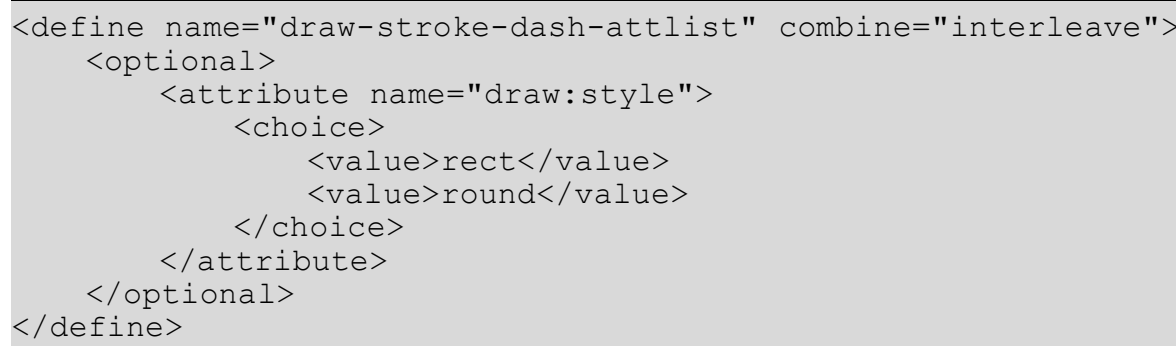

\section{Dots}

The attribute pairs draw: dots1, draw: dots1-length and draw:dots2, draw:dots2length each define a repeating sequence of dots that are used to render a dash. Both sequences are used alternating. The draw: dots 1 and draw: dots 2 attributes specify the number of dots to draw for both sequences, and the draw:dots1-length and draw:dots2length attributes specify the length of each dot.

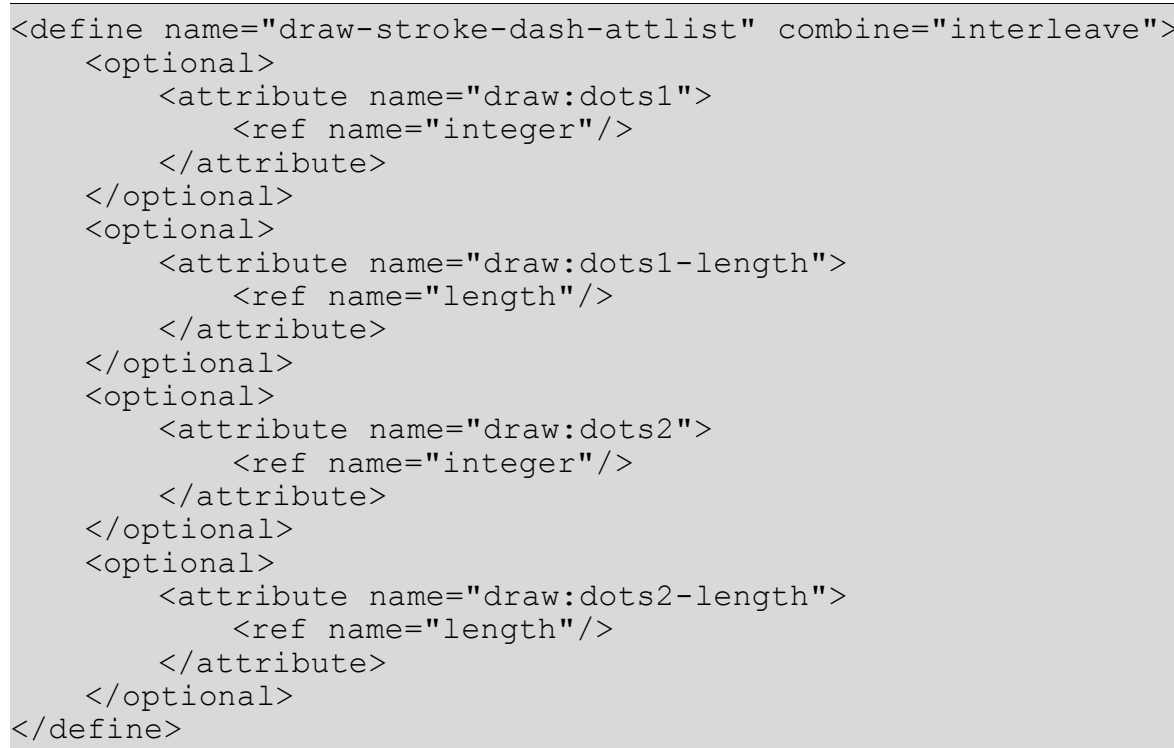




\section{Distance}

The draw: distance attribute specifies the distance between the dots of a dash.

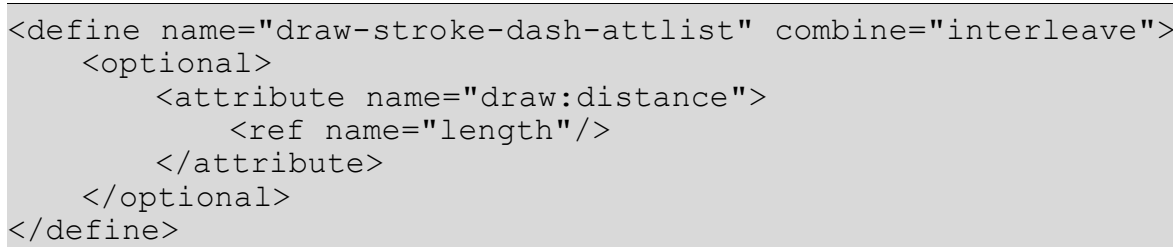

\subsection{Presentation Page Layouts}

The element <style:presentation-page-layout> is a container for placeholders, which define a set of empty presentation objects, for example, a title or outline. These placeholders are used as templates for creating new presentation objects and to mark the size and position of an object if the presentation page layout of a drawing page is changed.

The <style:presentation-page-layout> element has an attribute style: name. It defines the name of the page layout. If a drawing page has been created using a presentation page layout, the name of the layout is contained in the draw page's presentation:presentation-page-layout-name attribute. The optional style:display-name attribute specifies the name of the presentation page layout as it should appear in the user interface.

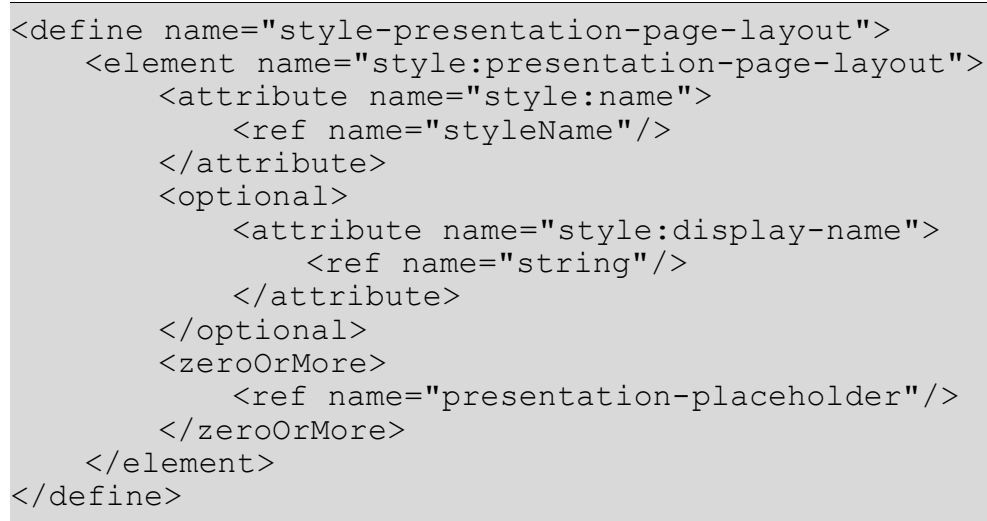

\subsubsection{Presentation Placeholder}

The element $<$ presentation:placeholder $>$ specifies a placeholder for presentation objects, for example, a title or outline.

The element has the following attributes:

- object: Specifies the kind of object the element is a placeholder for. The value equals the one of the presentation:class attribute for presentation shapes. See section 9.6.

- $\quad \mathrm{svg}: \mathrm{x}, \mathrm{svg}: \mathrm{y}, \mathrm{svg}:$ width, svg:height: position and size attributes as specified in section 9.2.15, with the exception that percentage values are allowed for placeholders.

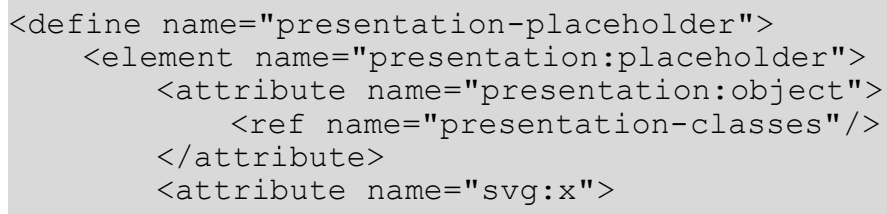




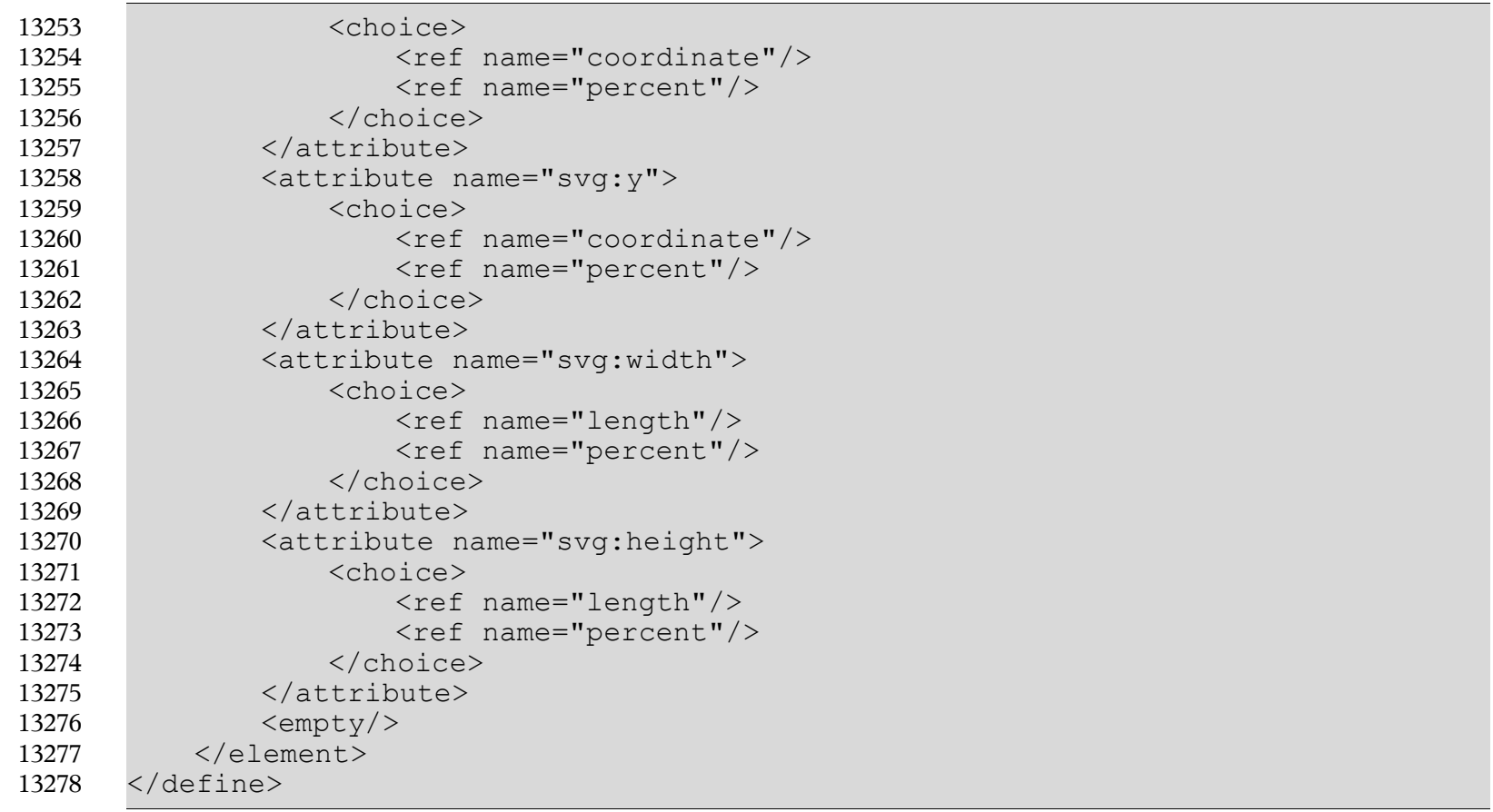

\subsection{Chart Styles}

Chart styles are <style:style> elements that have the family chart. They can be used within chart documents to specify formatting properties for the chart, but also for certain objects within a chart. They support the chart properties described in section 15.29, but also graphic, paragraph and text properties as described in sections $15.17,15.5$ and 15.4.

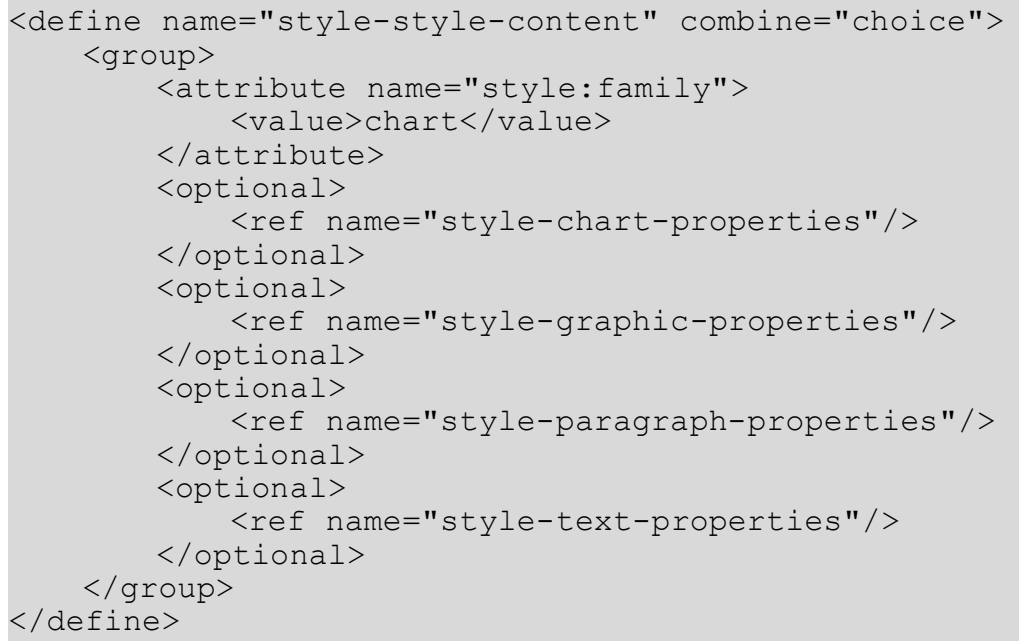




\section{Formatting Properties}

A document can contain several style elements. To acquire a common set of formatting properties, all formatting properties are contained in formatting property elements which are included as a child elements of any style element. This container elements offers two important advantages, as follows:

- Formatting properties can be addressed by [CSS2] or [XSLT] stylesheets regardless of the style type.

- Styles contain additional information that is not a formatting property, for example, the style name and parent style. It is good practice to separate this type of information.

The following formatting property elements do exist:

- <style:page-layout-properties> for page layout properties

- <style:header-footer-properties> for page header and footer properties

- <style:text-properties> for text properties

- <style:paragraph-properties> for paragraph properties.

- <style:section-properties> for text section properties.

- <style:ruby-properties> for ruby section properties.

- <style:list-level-properties> for list properties.

- <style:table-properties> for table properties.

- <style:table-column-properties> for table column properties.

- <style:table-row-properties> for table row properties.

- <style:table-cell-properties> for table cell properties.

- <style:graphic-properties> for drawing object properties.

\subsection{Simple and Complex Formatting Properties}

\subsubsection{Simple Formatting Properties}

Most formatting properties are simple and can be represented as attributes of the formatting property elements. Where possible, [XSL] attributes or attributes from other specifications are used to represent formatting properties. In this specification, the namespace prefix $f \circ$ is used for $\mathrm{XSL}$ properties, that is properties that are part of the XSL namespace.

In office application, there are very often formatting properties that cannot be specified independent of other formatting properties. If this is the case, and if some of the required properties are missing, the application assumes reasonable default values.

Example: Simple style properties

This example shows a formatting property container that specifies an upper paragraph margin of $1 \mathrm{~cm}$ as well as a lower margin of $0.5 \mathrm{~cm}$ : 


\subsubsection{Complex Formatting Properties}

If a formatting property is too complex to be represented by XML attributes, it is represented by an XML element. Each such property is represented by an element type of its own.

Example: Complex formatting properties

This is an example of a formatting property container that specifies upper and lower margins as well as tab stop position at 2 and $4 \mathrm{~cm}$.

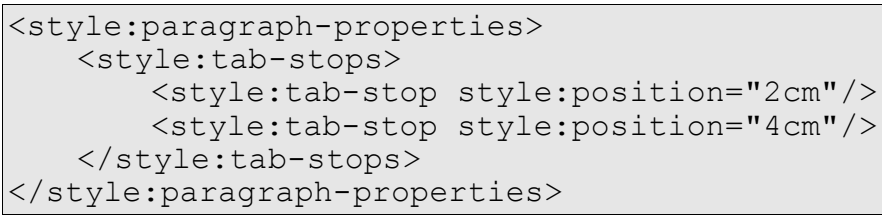

\subsubsection{Processing Rules for Formatting Properties}

In the OpenDocument schema the various <style: ${ }^{\star}$-properties $>$ elements may contain pre-defined formatting attributes and elements as well as custom formatting attributes and elements. The pre-defined attributes and elements have defined semantics, and are described within this chapter.

Custom formatting attributes and elements are arbitrary attributes and elements inside <style: *-properties> elements. Their semantics are not defined in this specification,

Conforming applications in general SHOULD preserve both, pre-defined and custom formatting attributes and elements when editing the document.

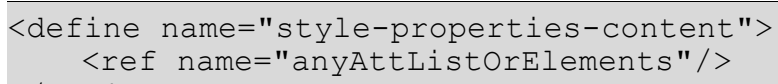

\subsection{Page Layout Formatting Properties}

The properties described in this section can be contained within style page layouts (see section 14.3) They are contained in a <style:page-layout-properties> element.

- Page size

- Page number format

- Paper tray

- Print orientation

- Margins

- Border

- Border line width

- Padding

- Shadow

- Background 
- Columns

- Register-truth

- Print

- Print page order

- First page number

- Scale

- Table centering

- Maximum footnote height

- Footnote separator

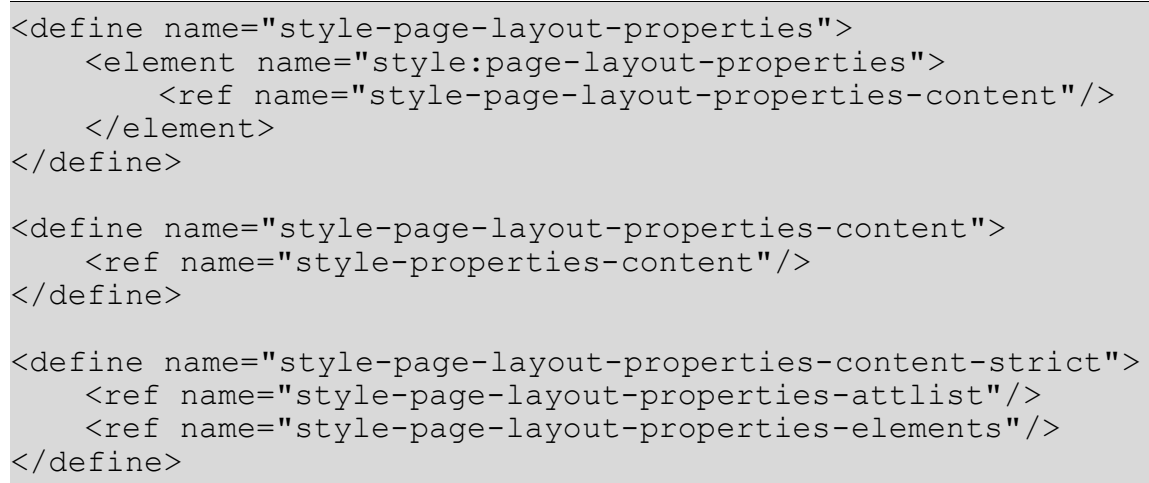

\subsubsection{Page Size}

The fo:page-width and fo:page-height attributes specify the physical size of the page.

The fo:page-width attribute must correspond to the orientation of the page. For example, if a page is printed in portrait, the fo:page-width attribute specifies the width of the shorter page side. If the page is printed in landscape, the fo:page-width attribute specifies the width of the longer page side.

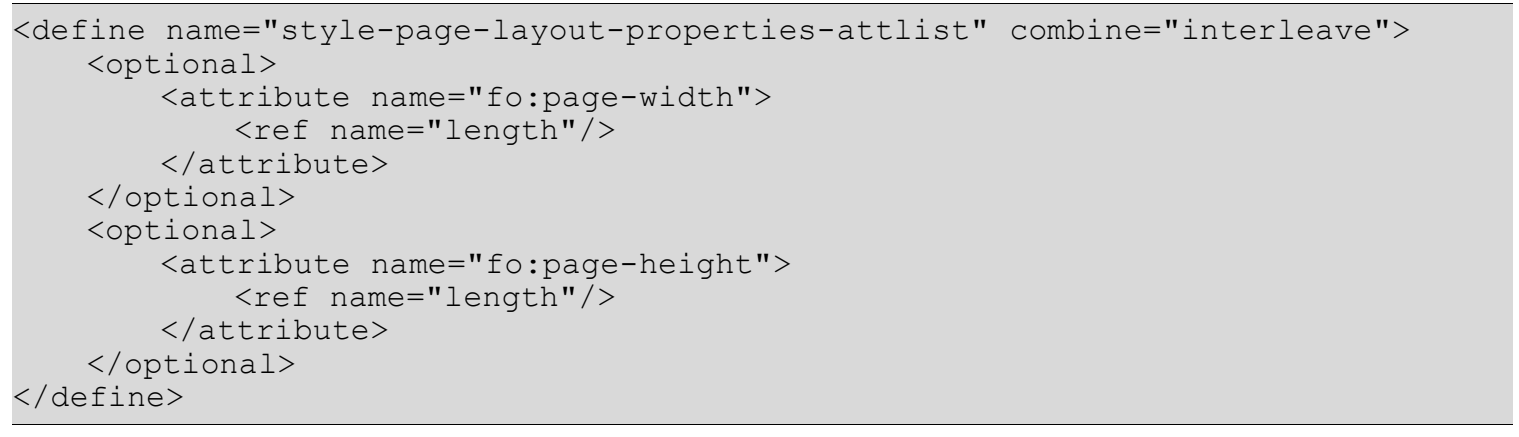

\subsubsection{Page Number Format}

The style:num-format, style:num-prefix and style:num-suffix attributes specify a default number format for page styles, which is used to display page numbers within headers and footers. See section 12.2 for detailed information on number format attributes. 
The style:num-format attribute can be empty. In this case, no page number will be displayed by default.

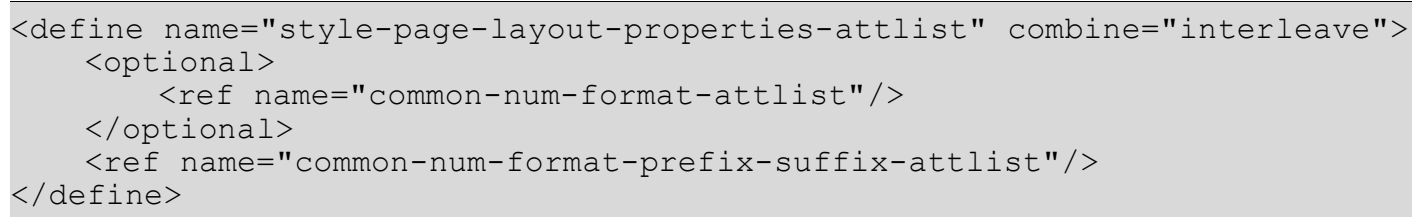

\subsubsection{Paper Tray}

The style:paper-tray-name attribute specifies the paper tray to use when printing the document. The names assigned to the printer trays depend on the printer. If the value of this attribute is default, the default tray specified in the printer configuration settings is used.

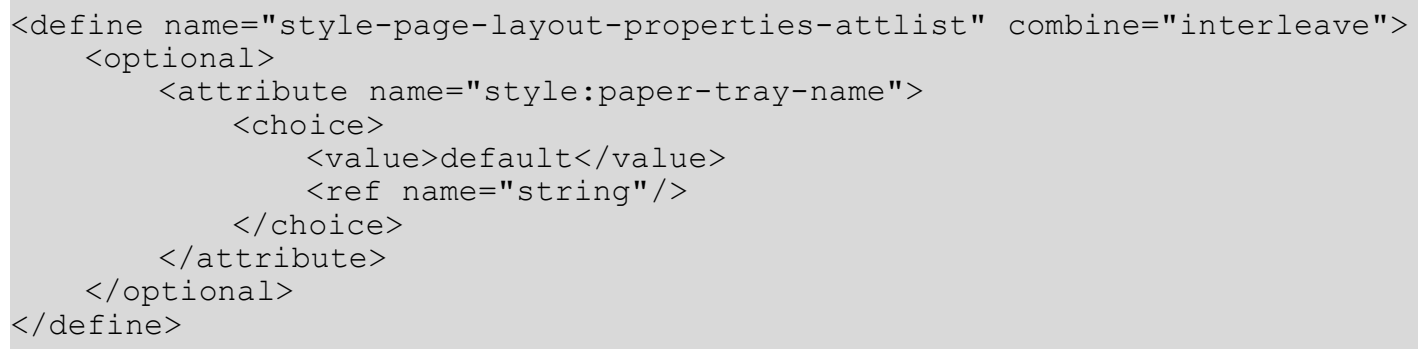

\subsubsection{Print Orientation}

The style:print-orientation attribute specifies the orientation of the printed page. The value of this attribute can be portrait or landscape.

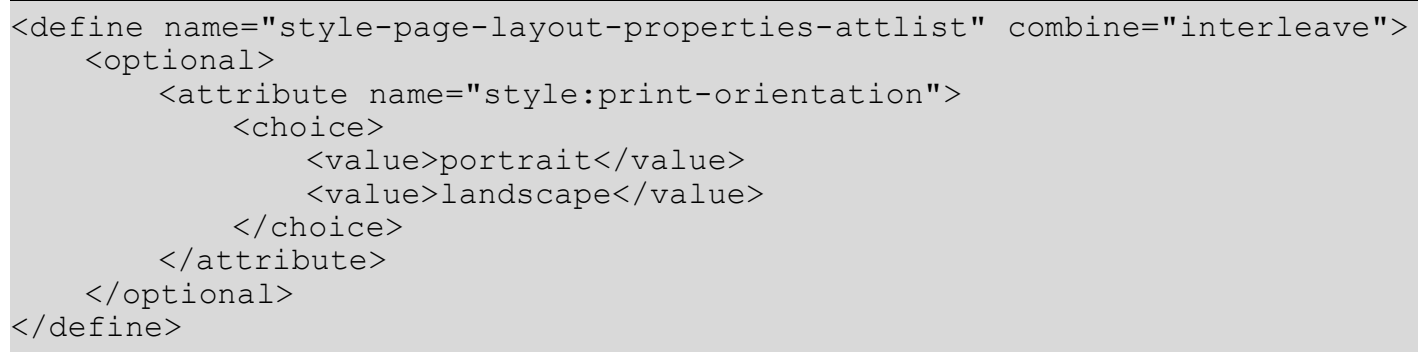

\subsubsection{Margins}

The margins attributes fo:margin, fo:margin-top, fo:margin-bottom, fo:marginleft and fo:margin-right specify the size of the page margins. See sections 15.5.17, 15.5.20 and 15.5.21 for detailed information on these attributes. Percentage values are not supported.

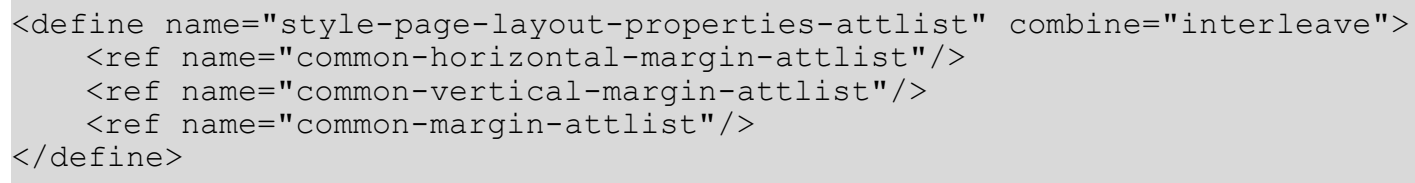




\subsubsection{Border}

The border attributes fo:border, fo:border-top, fo:border-bottom, fo:border-left and $f \circ$ :border-right specify the border properties of the page. See section 15.5.25 for detailed information on these attributes.

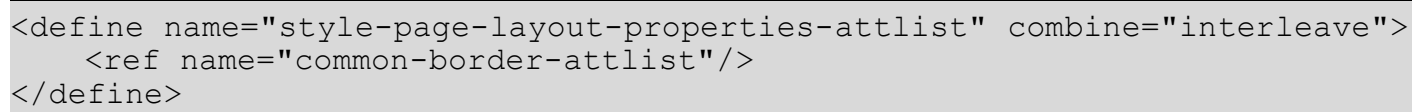

\subsubsection{Border Line Width}

If a page contains borders, the border line width attributes style:border-line-width, style:border-line-width-top, style:border-line-width-bottom, style:borderline-width-left and style:border-line-width-right specify the properties of the border lines of the page. See section 15.5.26 for detailed information on these attributes.

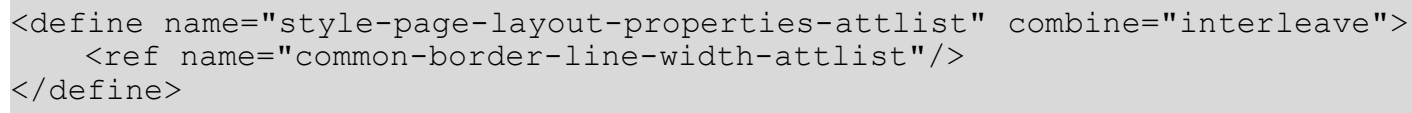

\subsubsection{Padding}

The padding attributes fo:padding, fo:padding-top, fo:padding-bottom, fo:paddingleft and fo:padding-right specify the padding properties of the page. See section 15.5.27 for detailed information on these attributes.

\subsubsection{Shadow}

The shadow attribute style: shadow specifies the shadow of the page. See section 15.5.28 for detailed information on this attribute.

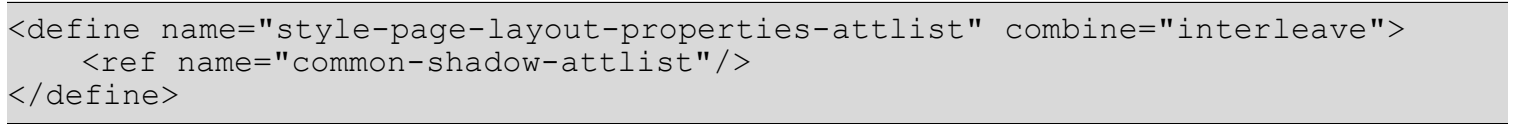

\subsubsection{Background}

The background attribute fo:background-color and the background element <style:background-image> specify the background properties of the page. See sections 15.5.23 and 15.5.24 for detailed information on this attribute and element.

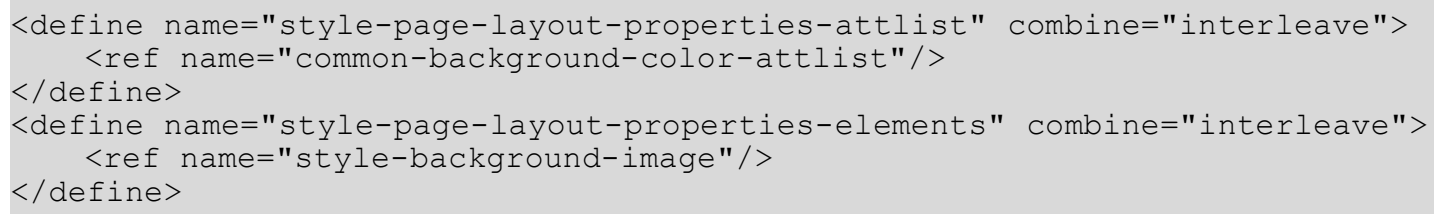




\subsubsection{Columns}

The <style:columns $>$ element specifies if the page contains columns. See section 15.7.3 for detailed information on this element.

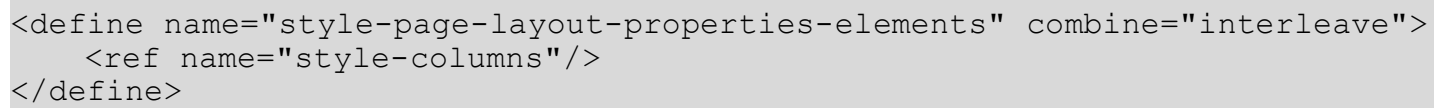

\subsubsection{Register-truth}

The style:register-truth-ref-style-name attribute references a paragraph style. The line distance specified of the paragraph style is used as the reference line distance for all paragraphs that have the register-truth feature enabled.

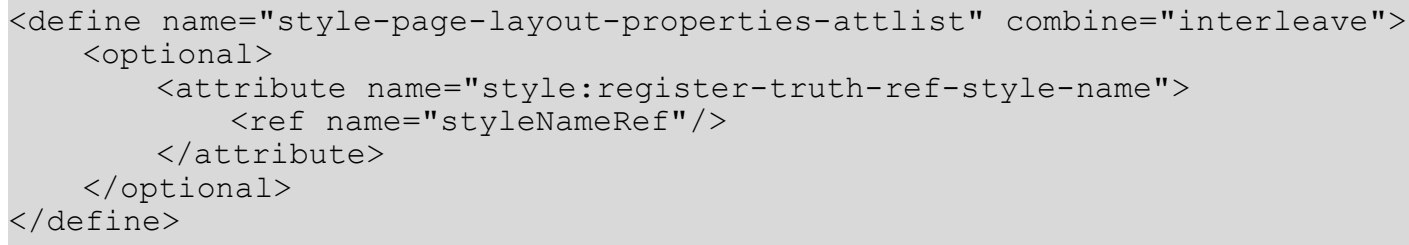

\subsubsection{Print}

The style:print attribute specifies which components in a spreadsheet document to print.

The value of this attribute is a list of the following values separated by blanks:

- headers

- $\operatorname{grid}$

- annotations

- objects (including graphics)

- charts

- drawings

- formulas

- zero-values

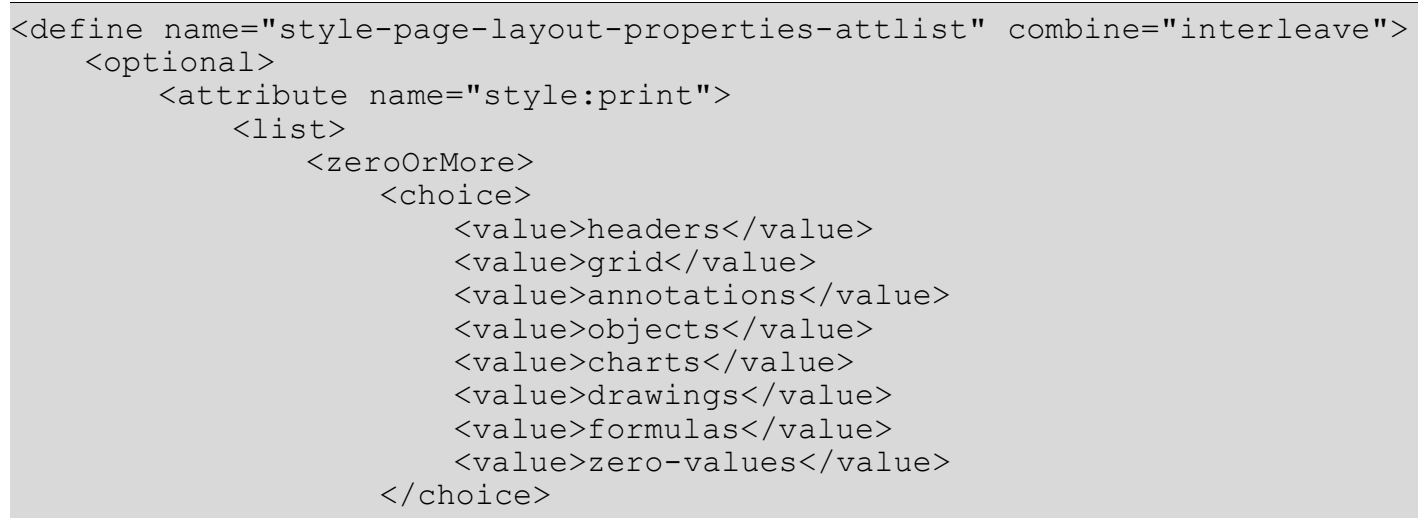




\subsubsection{Print Page Order}

The style:print-page-order attribute specifies the order in which data in a spreadsheet is numbered and printed when the data does not fit on one printed page.

The value of this attribute can be $t t b$ or $l t r$. Use $t t b$ to print the data vertically from the left column to the bottom row of the sheet. Use 1 tr to print the data horizontally from the top row to the right column of the sheet.

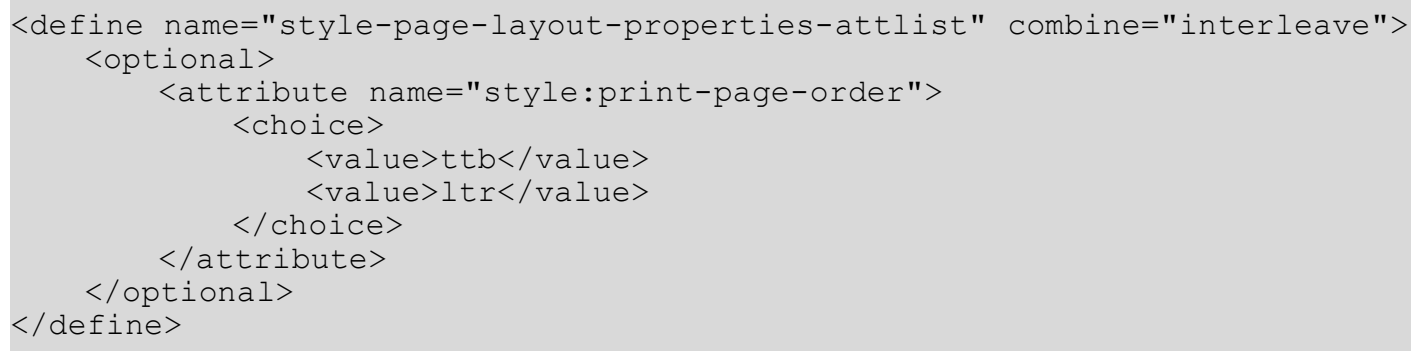

\subsubsection{First Page Number}

The style:first-page-number specifies the number of the first page of a text or graphical document, or for the first page of a table within a spreadsheet document.

The value of this attribute can be an integer or continue. If the value is continue, the page number is the last page number incremented by 1 . The default first page number is 1 .

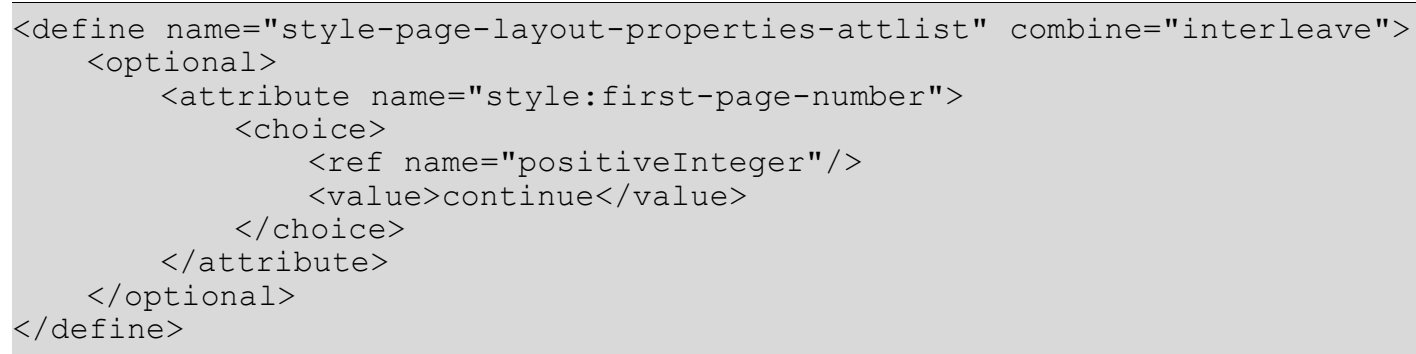

\subsubsection{Scale}

The style:scale-to and style:scale-to-pages attributes specify how the application should scale spreadsheet documents for printing.

The style:scale-to attribute specifies that the document is scaled to a percentage value, where $100 \%$ equals no scaling. When using this attribute, all pages are enlarged or reduced in size while printing.

The style:scale-to-pages attribute specifies the number of pages on which the the document should be printed. The document is then scaled to fit the defined number of pages. 
If none of these attributes are present, the document is not scaled.

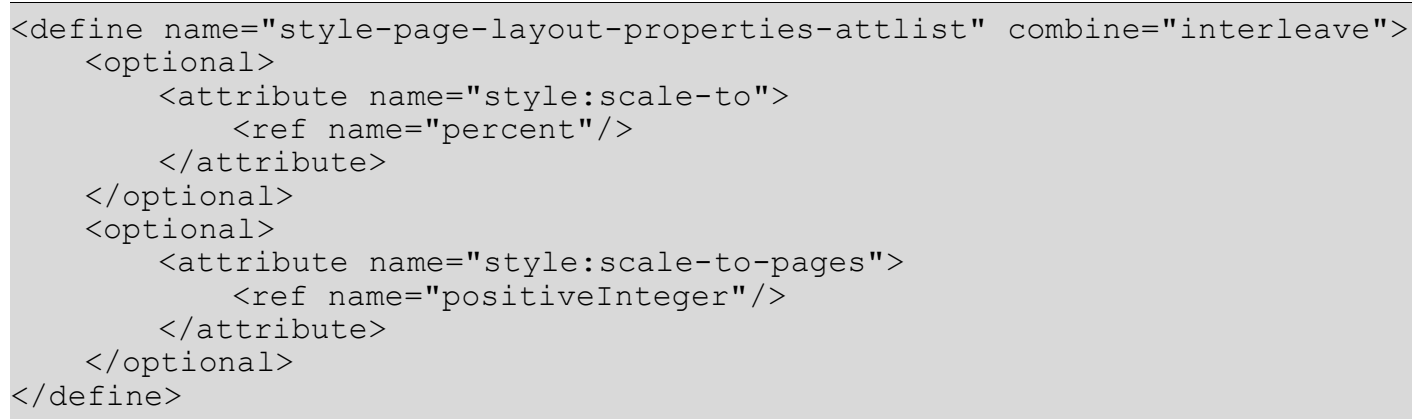

\subsubsection{Table Centering}

The style:table-centering attribute specifies how the application should center tables on the page. This attribute only applies to spreadsheet documents.

The value of this attribute can be horizontal, vertical, both, or none. If this attribute is not present, the table is not centered.

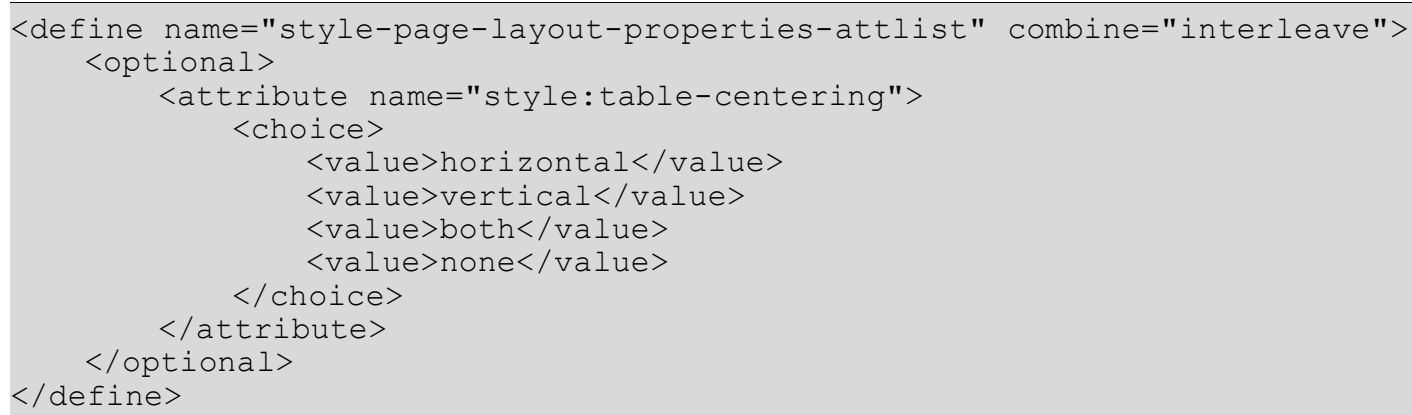

\subsubsection{Maximum Footnote Height}

The style:footnote-max-height attribute specifies the maximum amount of space on the page that a footnote can occupy. The value of the attribute is a length, which determines the maximum height of the footnote area.

If the value of this attribute is set to $0 \mathrm{in}$, there is no limit to the amount of space that the footnote can occupy.

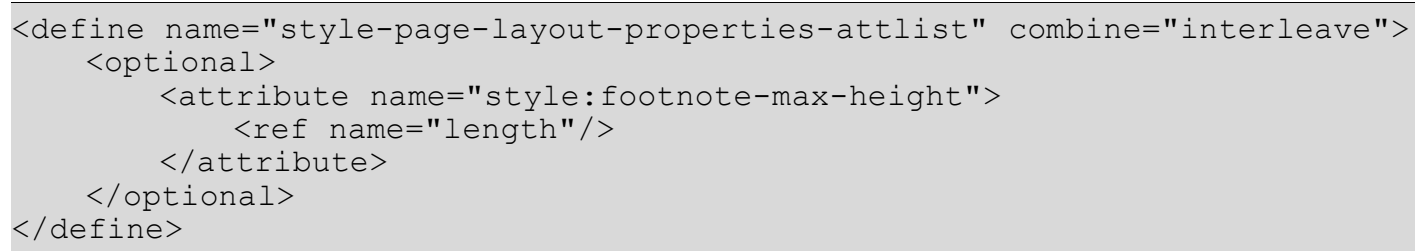

\subsubsection{Writing Mode}

The style:writing mode attribute specifies the writing mode that should is used by all paragraphs that appear on the page. See section 15.5 .36 for details. The value page is not allowed within page layouts.

<define name="style-page-layout-properties-attlist" combine="interleave"> 


\subsubsection{Footnote Separator}

The <style:footnote-sep> element describes the line that separates the footnote area from the body text area on a page.

The <style: footnote-sep> element supports the following attributes:

- style:width - specifies the width or thickness of the line.

- style:rel-width - specifies the length of the line as a percentage of the body text area.

- style:color - specifies the color of the line.

- style:adjustment - specifies how the line is aligned on the page, that is left, right, or center.

- style:distance-before-sep - specifies the space between the body text area and the footnote line.

- style:distance-after-sep-specifies the space between the footnote line and the footnote text.

- style:line-style-specifies the style of the line.

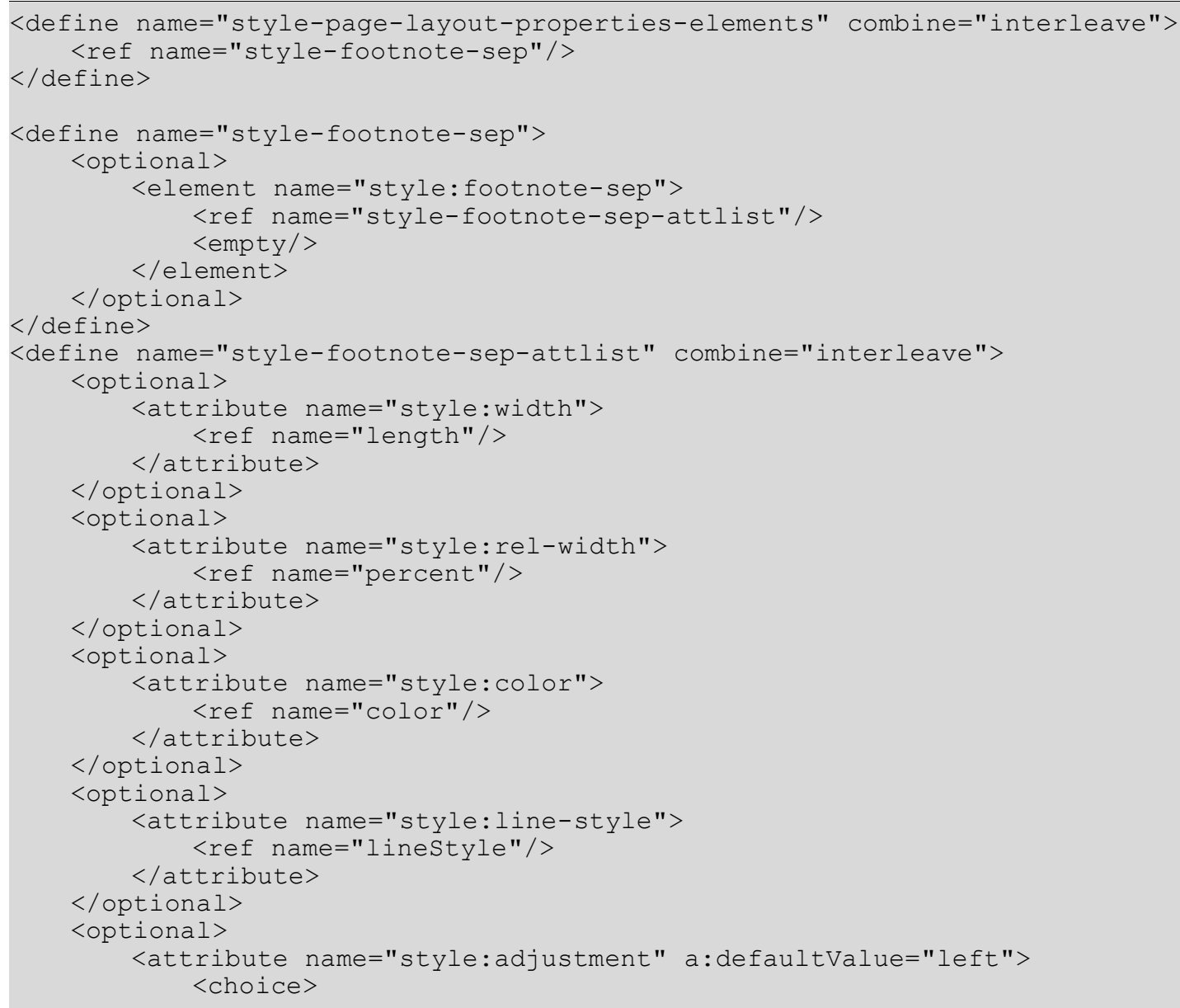




\section{$</$ optional $>$}

$</$ choice $>$

optional>

<optional>

<attribute name="style:distance-before-sep">

<ref name="length"/>

$</$ attribute $>$

$</$ optional $>$

$<$ optional>

<attribute name="style:distance-after-sep">

<ref name="length"/>

$</$ define $>$

\subsubsection{Layout Grid}

The style:layout-grid-mode property enables Asian layout grids. It has the following values:

- none: Disables the layout grid.

- lines: Enables a line layout, this is, the page is divided in a fixed number of lines. The exact number of lines depends on the other grid layout properties described below. There is no space between the layout grid lines. The layout grid itself is centered on the page.

- both: Like lines, except that the lines are divided into square cells. The number of cells per line depends on the line height, where the line height is the sum of the base height and the ruby height as specified below. Within a layout cell, nor more than one Asian UNICODE character is displayed. Asian characters that do not fit into a single cell are displayed centered into as many cells as required. Non Asian text is centered within as many cells as required.

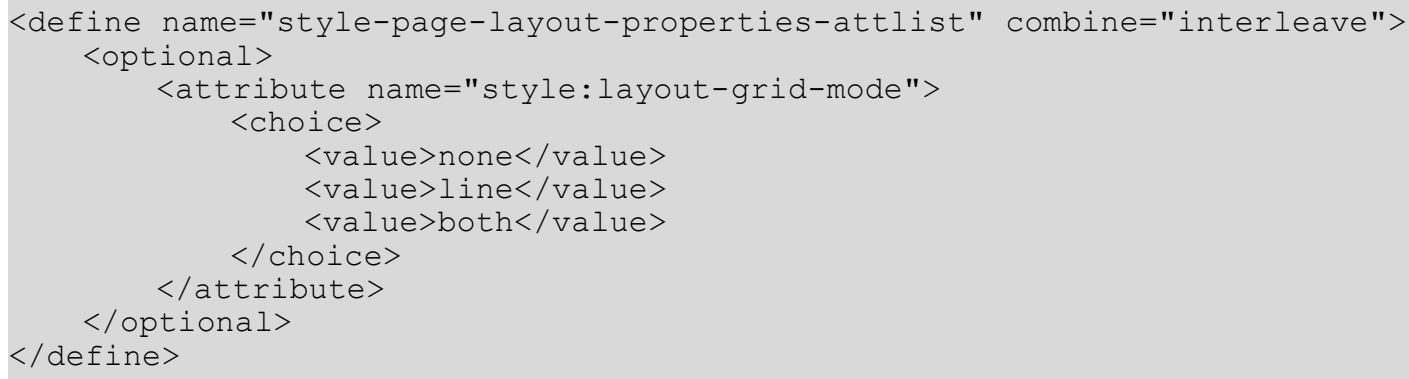

\subsubsection{Layout Grid Base Height}

The style:layout-grid-base-height attribute specifies the height reserved in the layout grid lines for non ruby text.

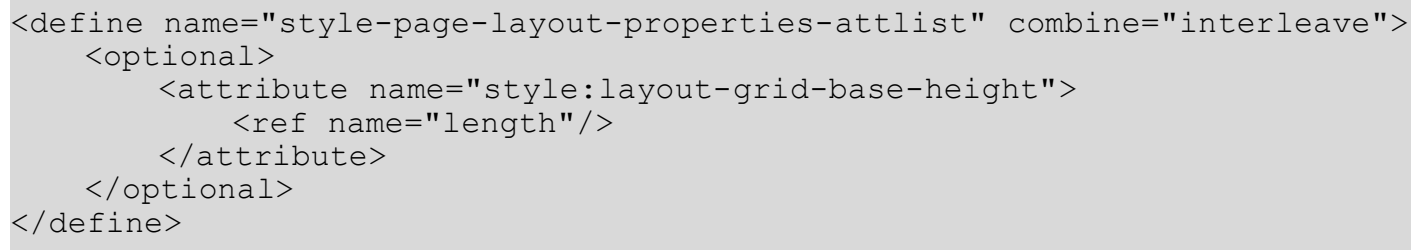




\subsubsection{Layout Grid Ruby Height}

The style:layout-grid-ruby-height attribute specifies the height reserved in the layout grid lines for ruby text.

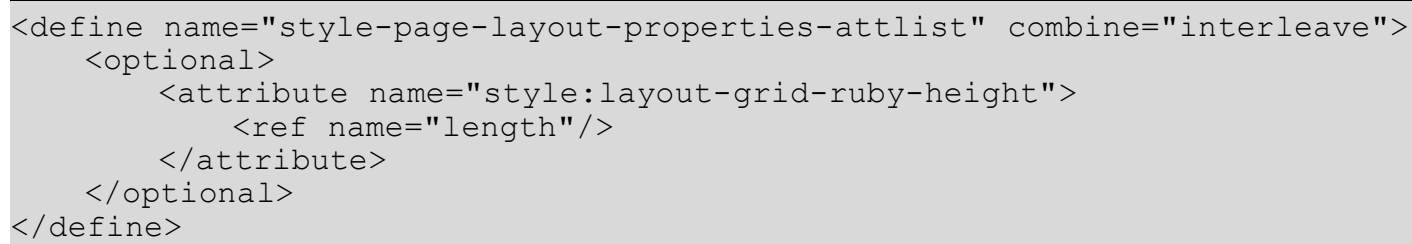

\subsubsection{Layout Grid Lines}

The style:layout-grid-lines attribute specifies the number of layout grid lines per page. The number of lines actually displayed may be smaller than specified if the page has not enough space to display the specified number of lines with the specified line height (i.e. the sum of the base and ruby height).

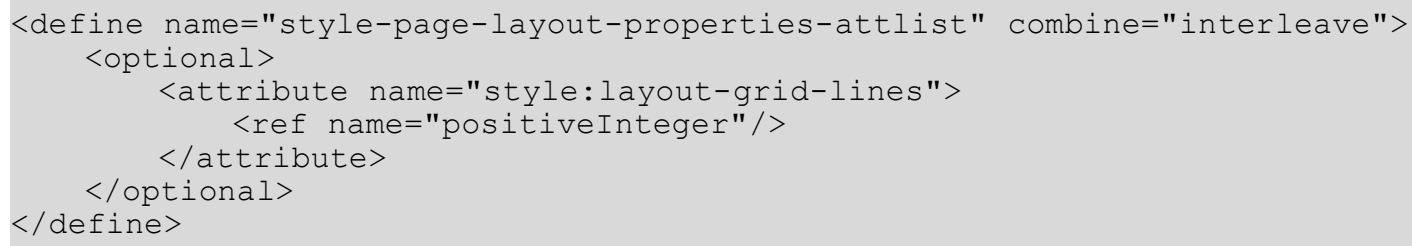

\subsubsection{Layout Grid Color}

The style:layout-grid-color attribute specifies the color of the layout grid border lines.

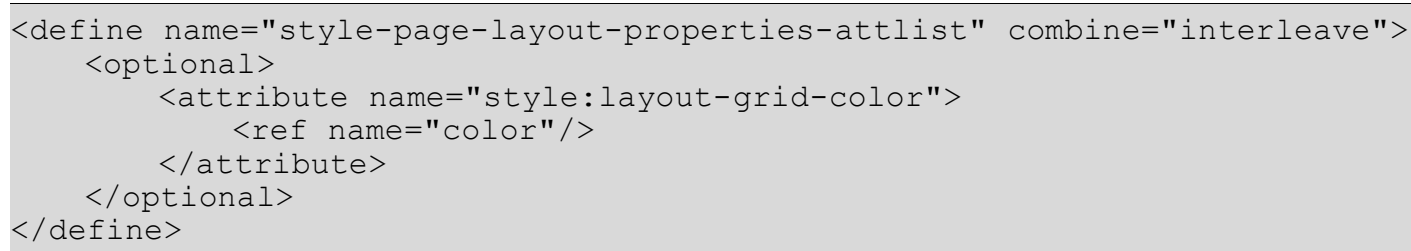

\subsubsection{Layout Grid Ruby Below}

The style:layout-grid-ruby-below attribute specifies whether ruby text is displayed above or below the base text.

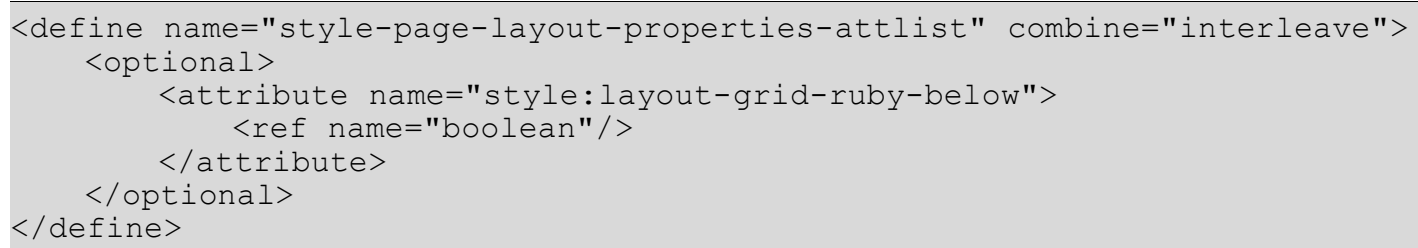

\subsubsection{Layout Grid Print}

The style:layout-grid-ruby-print attribute specifies whether the layout grid border lines are printed.

<define name="style-page-layout-properties-attlist" combine="interleave"> <optional> 


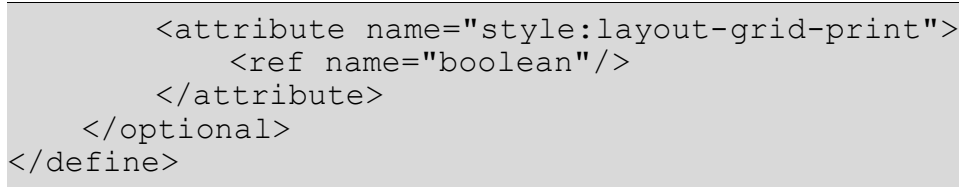

\subsubsection{Layout Grid Display}

The style:layout-grid-ruby-print attribute specifies whether the layout grid border lines are displayed.

<define name="style-page-layout-properties-attlist" combine="interleave"> <optional>

<attribute name="style:layout-grid-display">

$<$ ref name="boolean"/>

13569

13570

13571

13572

$</$ attribute $>$

$</$ optional $>$

$</$ define $>$

\subsection{Header Footer Formatting Properties}

The properties described in this section can be contained within the header and footer style elements contained in page layouts (see section 14.3) They are contained in a

<style:header-footer-properties> element.

These attributes are:

- $\quad$ Fixed and minimum heights - see section 15.27

- Left and right margins - see section 15.5.17

- Bottom (for headers only) and top (for footers only) margins - see section 15.5.20.

- $\quad$ Borders - see section 15.5.25 and 15.5.26

- Shadows - see section 15.5 .28

- Backgrounds - see section 15.5.23 and 15.5.24.

- Dynamic-Spacing

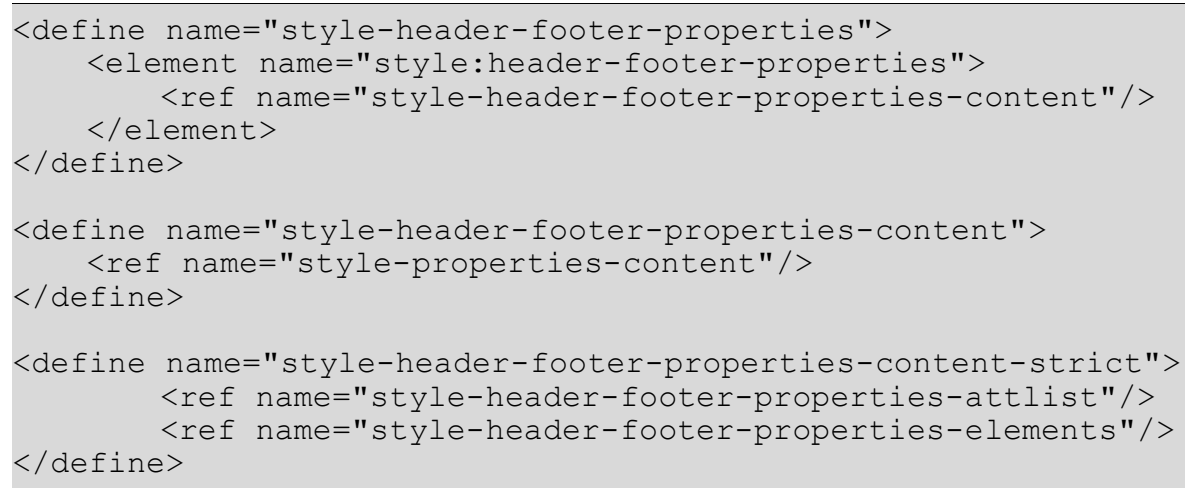

\subsubsection{Fixed and Minimum heights}

The attributes svg: height and fo:min-height properties specify a fixed or a minimum height for the header or footer. 


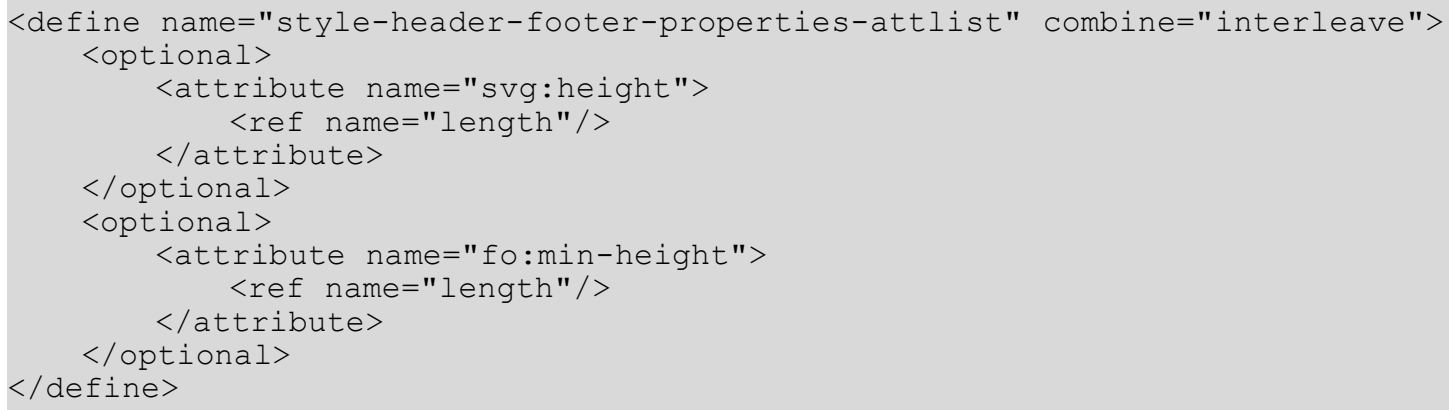

\subsubsection{Margins}

The margins attributes fo:margin, fo:margin-top, fo:margin-bottom, fo:marginleft and fo:margin-right specify the size of the header and footer margins. See sections 15.5.17, 15.5.20 and 15.5.21 for detailed information on these attributes. Percentage values are not supported. Bottom margins are only supported for headers, top margins only for footers.

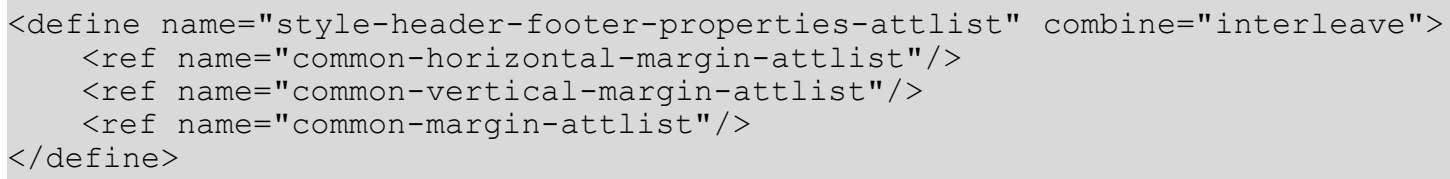

\subsubsection{Border}

The border attributes fo:border, fo:border-top, fo:border-bottom, fo:border-left and $f \circ:$ border-right specify the border properties of the headers and footers. See section 15.5.25 for detailed information on these attributes.

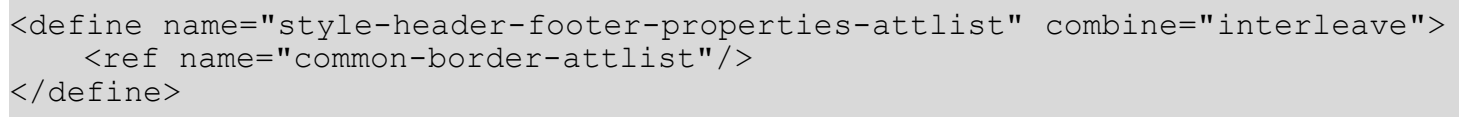

\subsubsection{Border Line Width}

If a page contains borders, the border line width attributes style:border-line-width, style:border-line-width-top, style:border-line-width-bottom, style:borderline-width-left and style:border-line-width-right specify the properties of the border lines of the headers and footers. See section 15.5.26 for detailed information on these attributes.

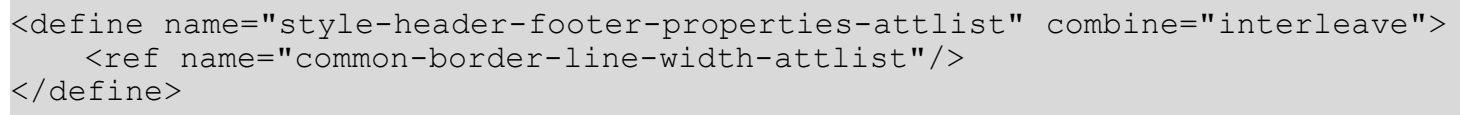

\subsubsection{Padding}

The padding attributes fo:padding, fo:padding-top, fo:padding-bottom, fo:paddingleft and fo:padding-right specify the padding properties of the headers and footers. See section 15.5.27 for detailed information on these attributes. 


\subsection{Text Formatting Properties}

The properties described in this section can be contained within text styles (see section 14.8.1), but also within other styles, like paragraph styles (see section 14.8.2) or cell styles (see section 14.12.4) They are contained in a <style: text-properties $>$ element.

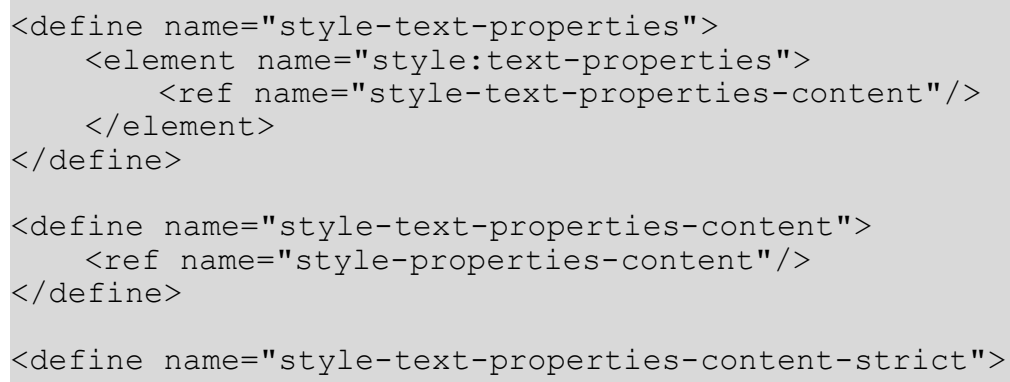




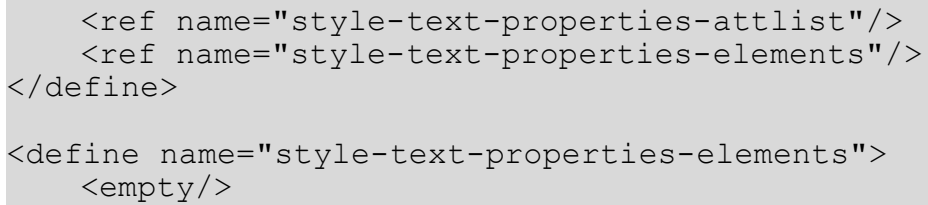

\subsubsection{Font Variant}

Use the fo: font-variant property to switch the option to display text as small capitalized letters on or off. See $\S 7.8 .8$ of [XSL] for details.

For some implementations, the fo:font-variant and fo:text-transform properties are mutually exclusive. If both properties are attached used simultaneously, the result is undefined except that the fo:text-transform value is none and the fo:font-variant value is normal.

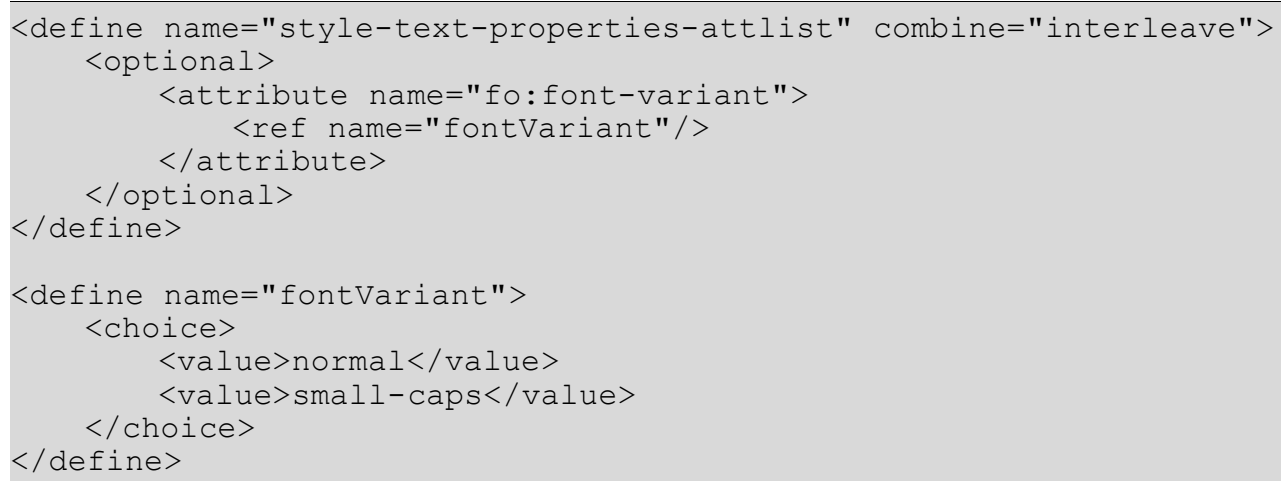

\subsubsection{Text Transformations}

Use the fo:text-transform property to describe text transformations to uppercase, lowercase, and capitalization. See $§ 7.16 .6$ of [XSL] for details.

For some implementations, the fo:font-variant and fo:text-transform properties are mutually exclusive. If both properties are attached used simultaneously, the result is undefined except that the fo:text-transform value is none and the fo:font-variant value is normal.

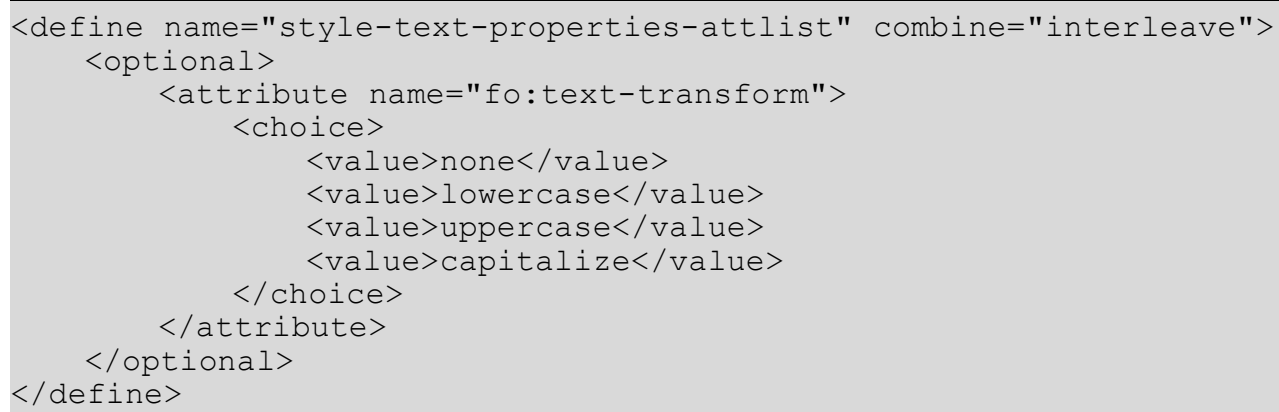

\subsubsection{Color}

Use the fo: color property to specify the foreground color of text. See $\S 7.17 .1$ of $[X S L]$ for details. 


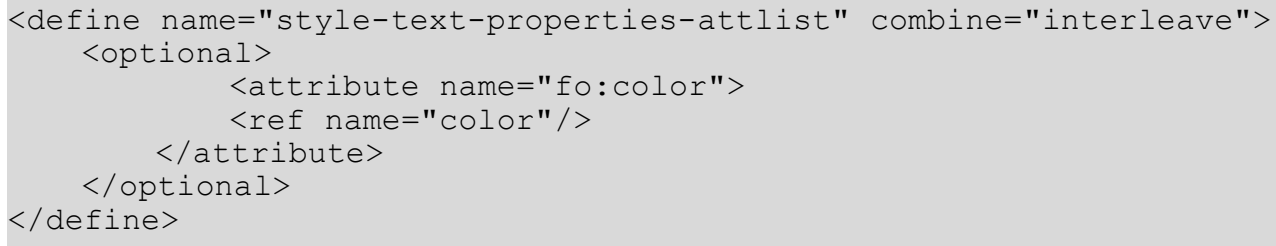

\subsubsection{Window Font Color}

Use the style:use-window-font-color property to specify whether or not the window foreground color should be as used as the foreground color for a light background color and white for a dark background color.

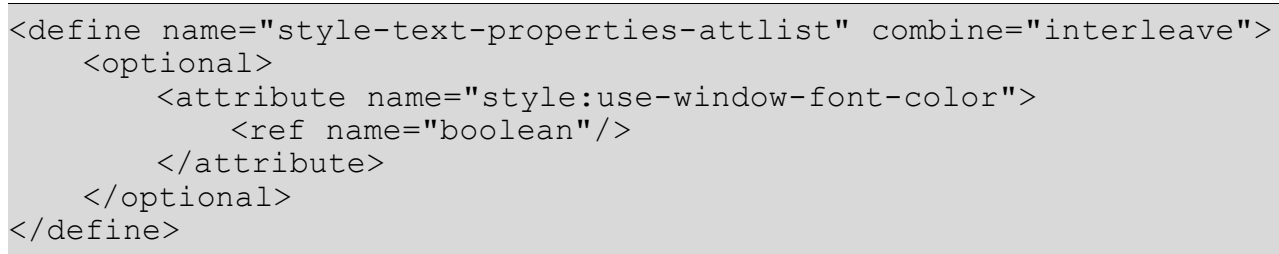

\subsubsection{Text Outline}

Use the style:text-outline property to specify whether to display an outline of text or the text itself. This attribute can have a value of true or false.

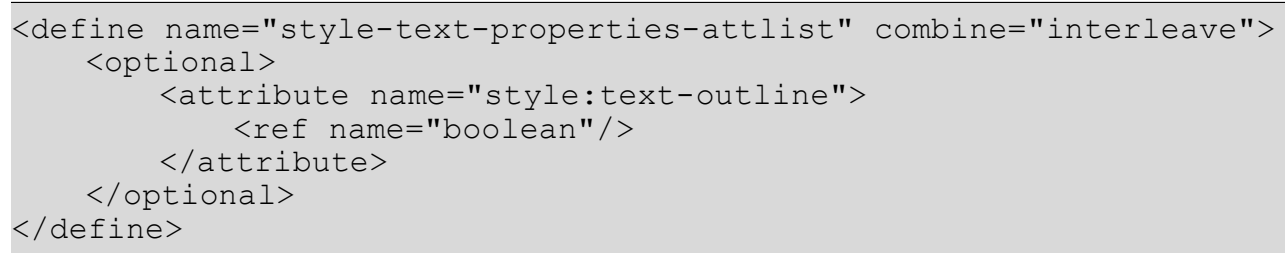

\subsubsection{Line Through Type}

Use the style:text-line-through-type property to specify whether text is lined through, and if so, whether a single or double line will be used. See section 15.4.28 for details.

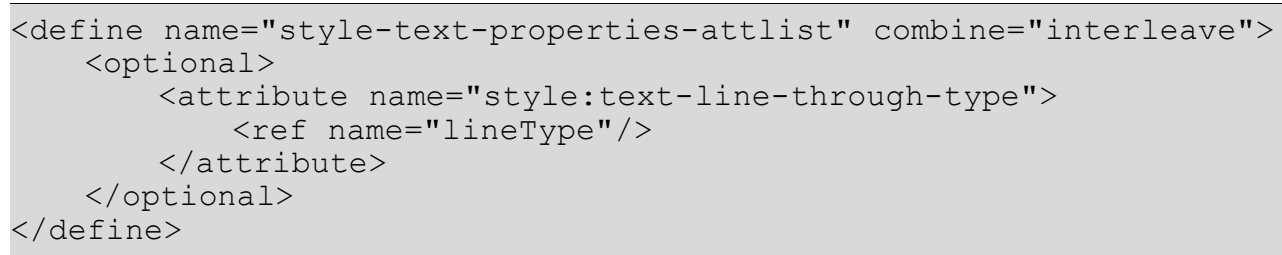

\subsubsection{Line Through Style}

Use the style:text-line-through-style property to specify if and how text is lined through. This property is similar to the [CSS3Text] text-line-style property, except that it has the additional value long-dash and that it does not have the value double. Instead of this, the attribute style:text: line-through-type can be used to turn each line style into a double line. See $\S 9.2$ of [CSS3Text] for details. See also section 15.4.29.

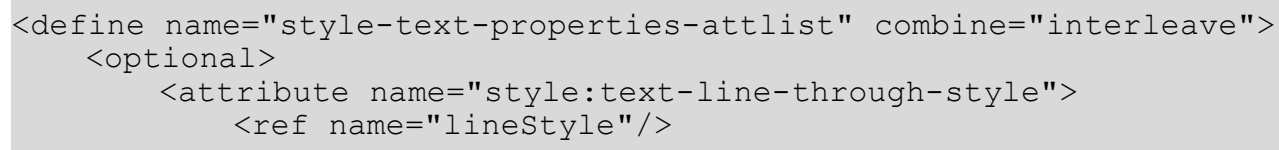




\subsubsection{Line Through Width}

Use the style:text-line-through-width property to specifies the width of a line through line. This property is very similar to the [CSS3Text] text-line-through-width property, except that it has an additional value bold. bold specifies a line width that is calculated from the font sizes like an auto width, but is wider than an auto width. See $\S 9.3$ of [CSS3Text] for details. See also section 15.4.30.

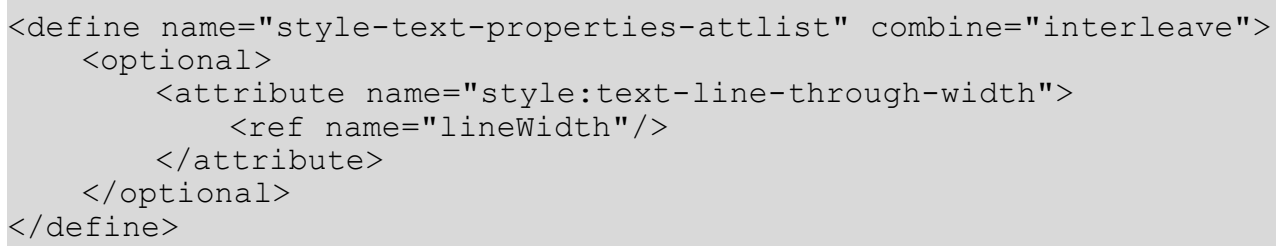

\subsubsection{Line Through Color}

Use the style:text-line-through-color property to specify the color that is used to line through text. The value of this property is either font-color or a color. If the value is fontcolor, the current text color is used for underlining.

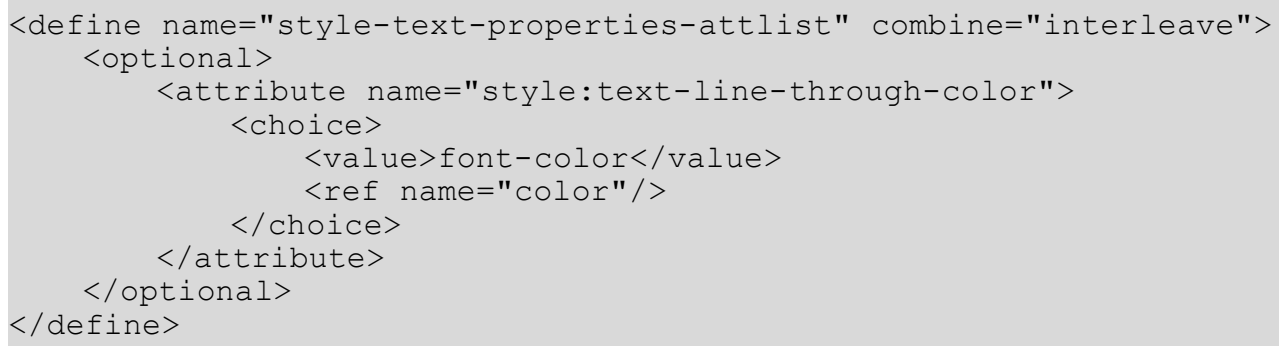

\subsubsection{Line Through Text}

The style:text-line-through-text attribute is evaluated only if the value of style:text-line-through-style attribute is different than none. If the attribute value is not empty, the attribute value string is used for line-through instead of the line that has been specified, provided that the application supports line-through with text. If the application does not support line-through with text, the attribute is ignored, this means, style:text-linethrough-style will be evaluated only. If the application supports line-trough with single characters only, and the text-line-through-text has more than one character, the first character of the line-through-text should be used only. If the applications supports line-through with with certain characters only (like "x" or "/"), the application should use one of these characters if the text-line-through-text specifies characters that are not supported. In other words: line-through with text has an higher priority than line-through with lines, even if the line through text that is specified has to be adapted to be usable by the application.

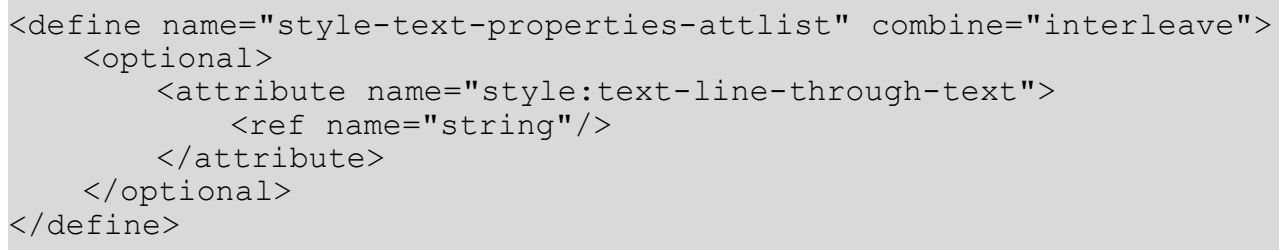




\subsubsection{Line Through Text Style}

The style:text-line-through-text-style specifies a text style that is applied to the text-line-through characters. It is not applied to line through lines. If the attribute appears in an automatic style, it may reference either an automatic text style or an common style. If the attribute appears in an common style, it may reference a common style only.

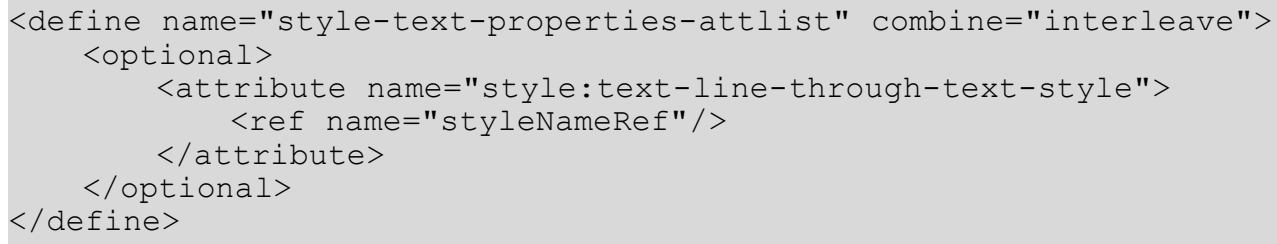

\subsubsection{Text Position}

Use the style:text-position formatting property to specify whether text is positioned above or below the baseline and to specify the relative font height that is used for this text.

This attribute can have one or two values.

The first value must be present and specifies the vertical text position as a percentage that relates to the current font height or it takes one of the values sub or super. Negative percentages or the sub value place the text below the baseline. Positive percentages or the super value place the text above the baseline. If sub or super is specified, the application can choose an appropriate text position.

The second value is optional and specifies the font height as a percentage that relates to the current font-height. If this value is not specified, an appropriate font height is used. Although this value may change the font height that is displayed, it never changes the current font height that is used for additional calculations.

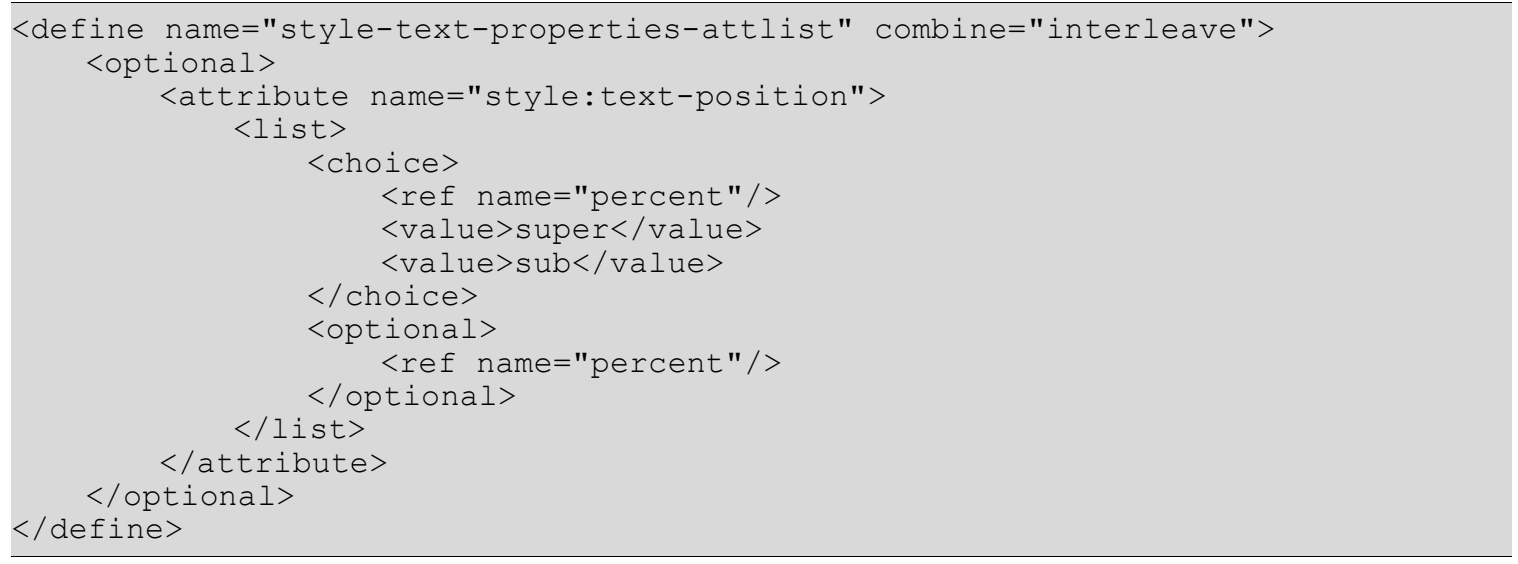

\subsubsection{Font Name}

Use the style: font-name, style:font-name-asian and style:font-name-complex properties to assign a font to the text.

The values of these attributes form the name of a font that is declared by a $<$ style: fontface> element within the <office: font-face-decls> element.

The style:font-name-asian attribute is evaluated for UNICODE characters that are CJK characters. 
The style:font-name-complex attribute is evaluated for UNICODE characters that are complex text layout (CTL) characters.

The style:font-name attribute is evaluated for any other UNICODE character.

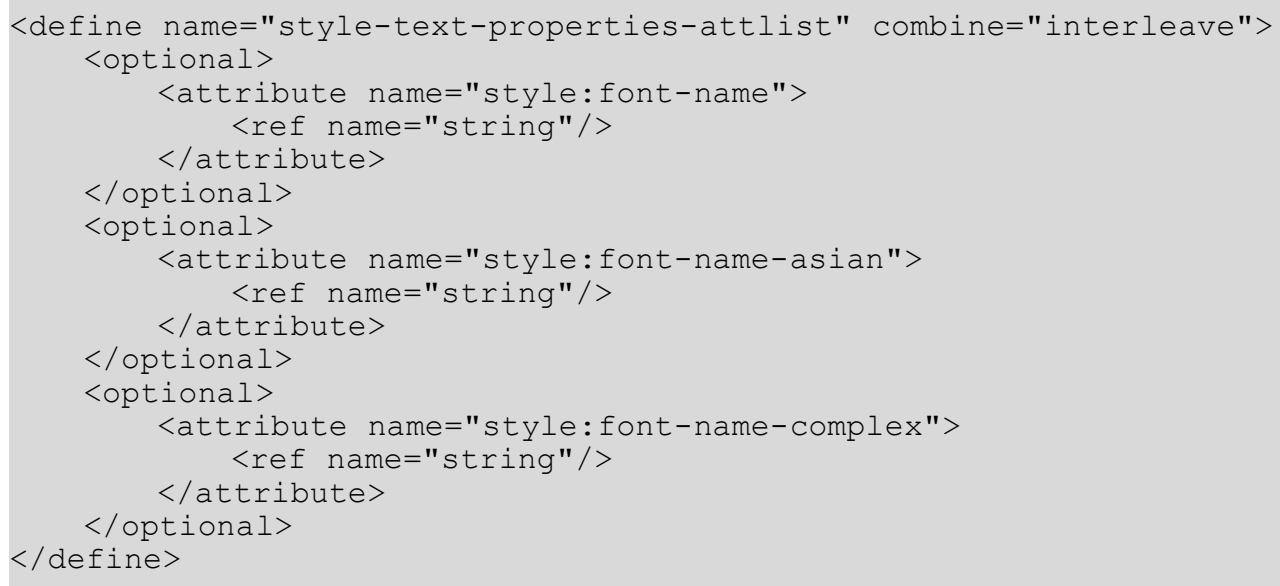

\subsubsection{Font Family}

Use the fo:font-family, style:font-family-asian and style:font-familycomplex properties to specify the font family for the text.

These attributes may be used instead of the font name attributes to specify the properties of a font individually. However, it is advisable to use the style: font-name attributes instead. See section 15.4.13 for information about when Asian and complex variants of the attribute are evaluated. See also $§ 7.8 .2$ of [XSL].

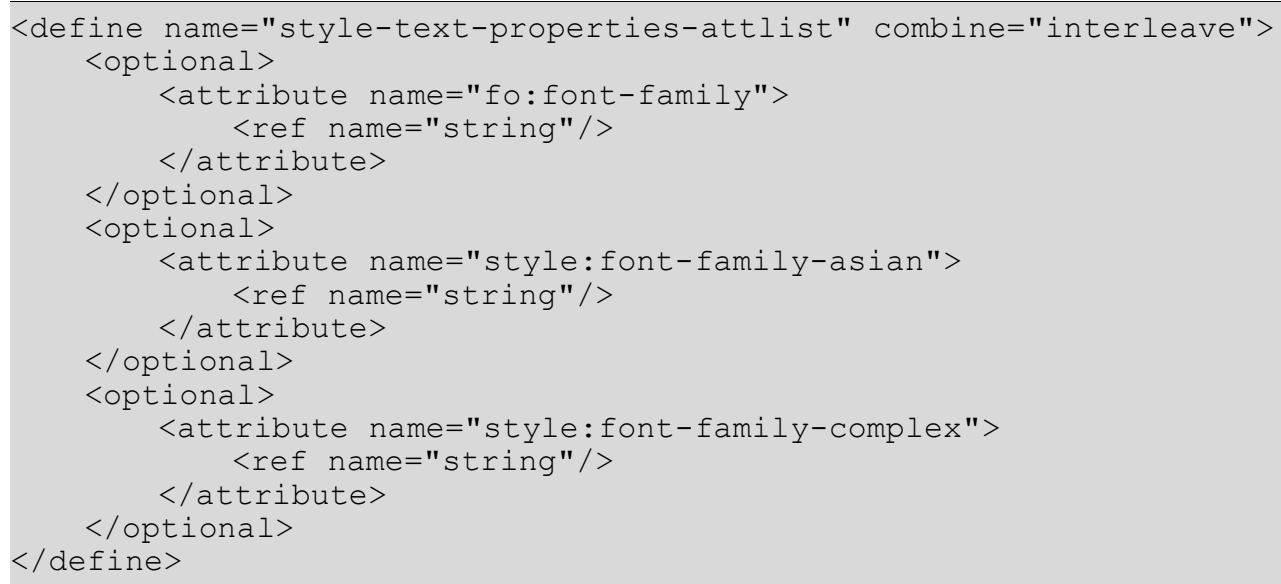

\subsubsection{Font Family Generic}

Use the style:font-family-generic, style:font-family-generic-asian and style:font-family-generic-complex properties to specify a generic font family name.

These properties are ignored if there is no corresponding $f o: f o n t-f a m i l y$ property attached to the same properties element.

Although it is recommended to use the font name attributes (see section 15.4.13), these properties may be used instead of them to specify the properties of a font. 
See section 15.4.13 for information about when the Asian and complex variants of the attribute are evaluated.

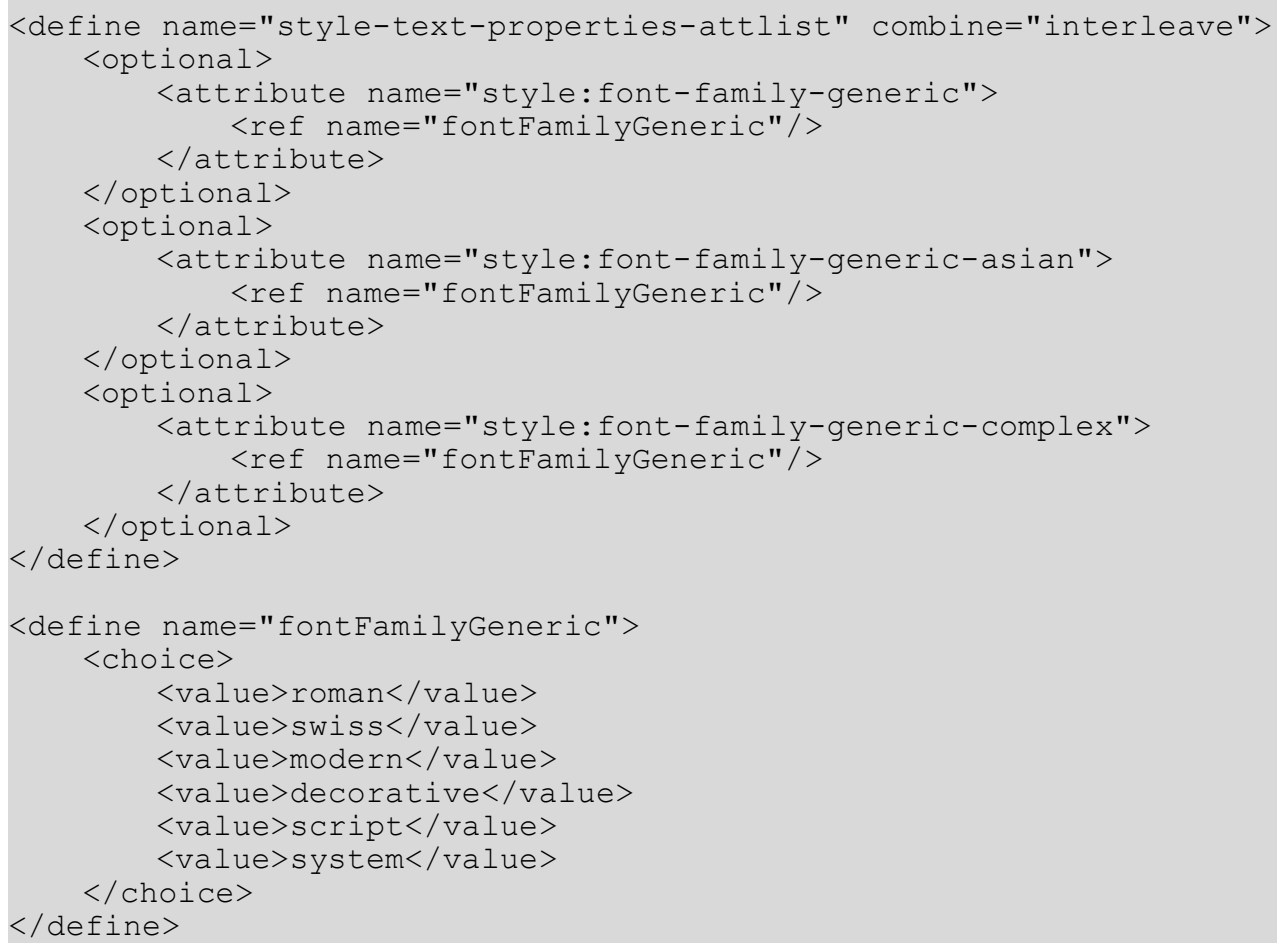

\subsubsection{Font Style}

Use the style:font-style-name, style:font-style-name-asian and style:fontstyle-name-complex properties to specify a font style name.

These properties are ignored if there is no corresponding $f \circ: f o n t-f a m i l y$ property attached to the same properties element.

Although it is recommended to use the font name attributes (see section 15.4.13), these properties may be used instead of them to specify the properties of a font.

See section 15.4 .13 for information about when the Asian and complex variants of the attribute are evaluated.

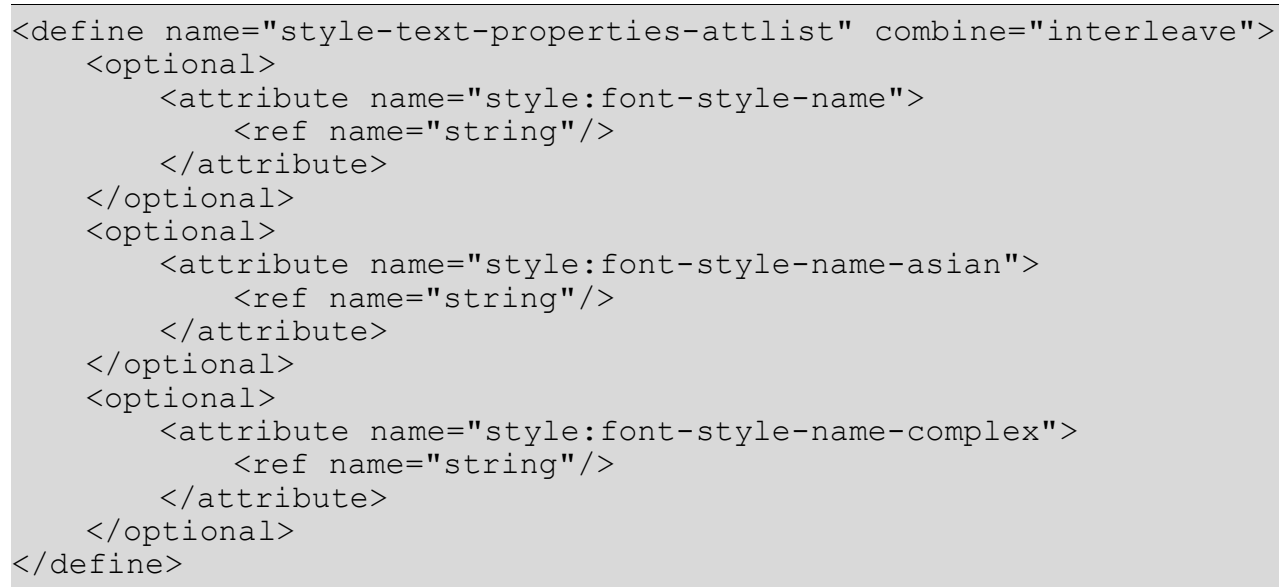




\subsubsection{Font Pitch}

Use the style:font-pitch, style: font-pitch and style:font-pitch-complex properties to specify whether a font has a fixed or variable width.

These properties are ignored if there is no corresponding fo:font-family property attached to the same properties element.

Although it is recommended to use the font name attributes (see section 15.4.13), these properties may be used instead of them to specify the properties of a font.

See section 15.4 .13 for information about when the Asian and complex variants of the attribute are evaluated.

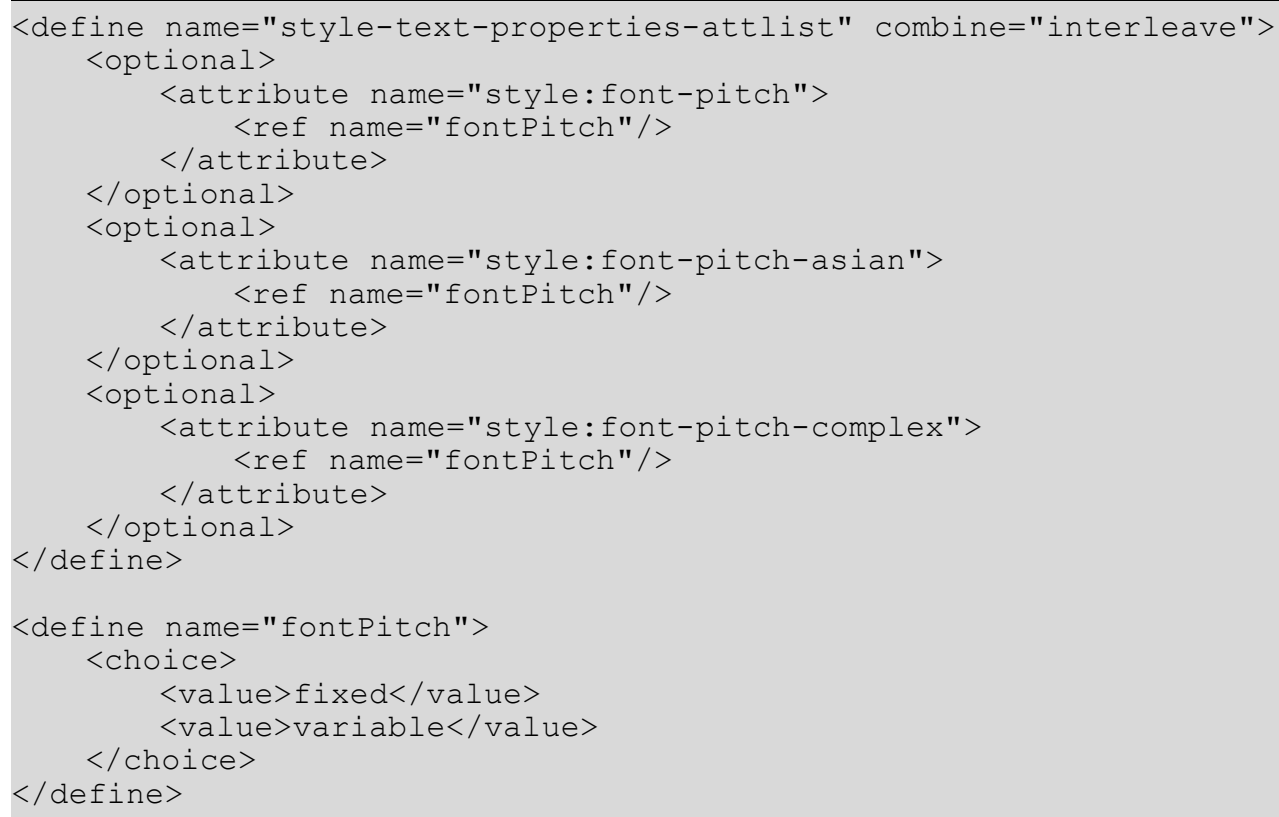

\subsubsection{Font Character Set}

Use the style:font-charset, style:font-charset-asian and style:font-charsetcomplex properties to specify the character set of a font.

The value of these attributes can be $x$-symbol or the character encoding in the notation described in the $\S 4.3 .3$ of [XML1.0]. If the value is $x-$ symbol, all characters that are displayed using this font must be contained in the UNICODE character range 0xf000 to 0xf0ff.

These properties are ignored if there is no corresponding fo:font-family property attached to the same properties element.

Although it is recommended to use the font name attributes (see section 15.4.13), these properties may be used instead of them to specify the properties of a font.

See section 15.4.13 for information about when the Asian and complex variants of the attribute are evaluated.

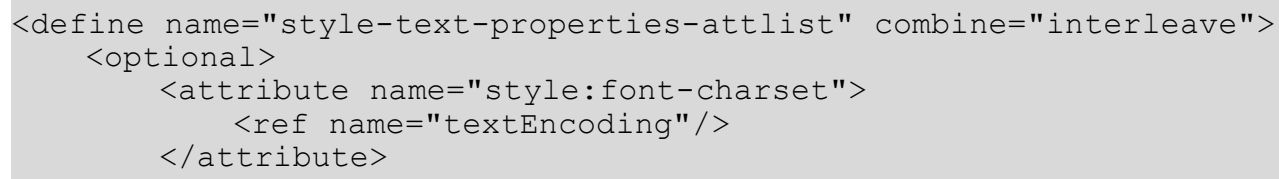




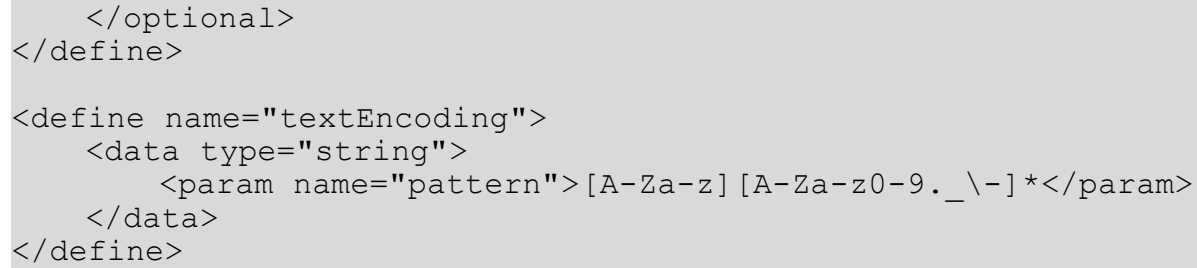

\subsubsection{Font Size}

Use the fo:font-size, style:font-size-asian and style:font-size-complex properties to specify the size of font.

The value of these property is either an absolute length or a percentage as described in §8.8.4 of [XSL]. In contrast to XSL, percentage values can be used within common styles only and relates to the font height of the parent style rather than to the font height of the attributes neighborhood. Absolute font heights such as medium, large, $x$-large, and so on, and relative font heights such as smaller, and larger are not supported.

See section 15.4.13 for information about when the Asian and complex variants of the attribute are evaluated.

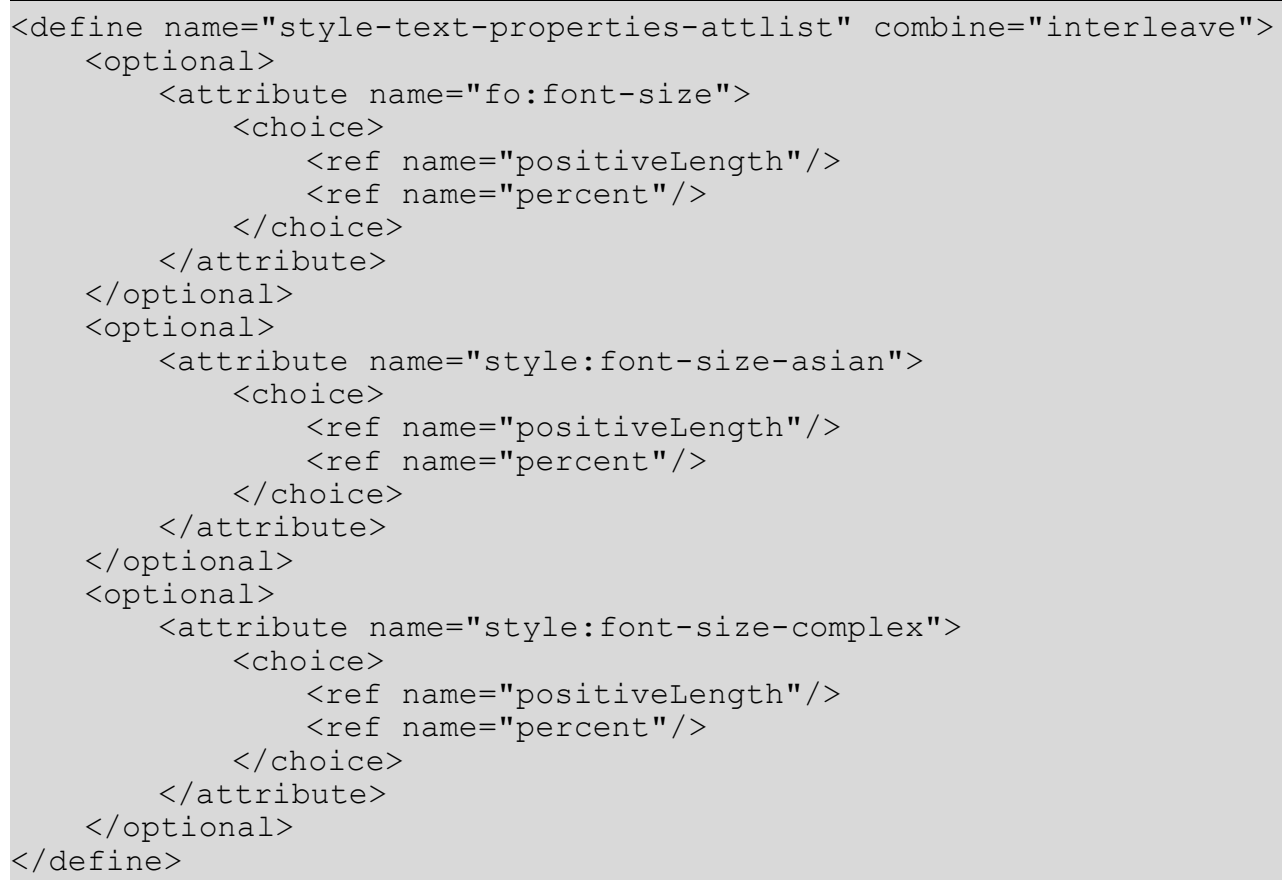

\subsubsection{Relative Font Size}

Use the style: font-size-rel, style: font-size-rel-asian and style:font-sizerel-complex properties to specify a relative font size change.

These properties specify a relative font size change as a length such as $+1 p t,-3 p t$. It cannot be used within automatic styles. The size changes relates to the font size setting that applies to the parent style of the style.

See section 15.4 .13 for information about when the Asian and complex variants of the attribute are evaluated. 


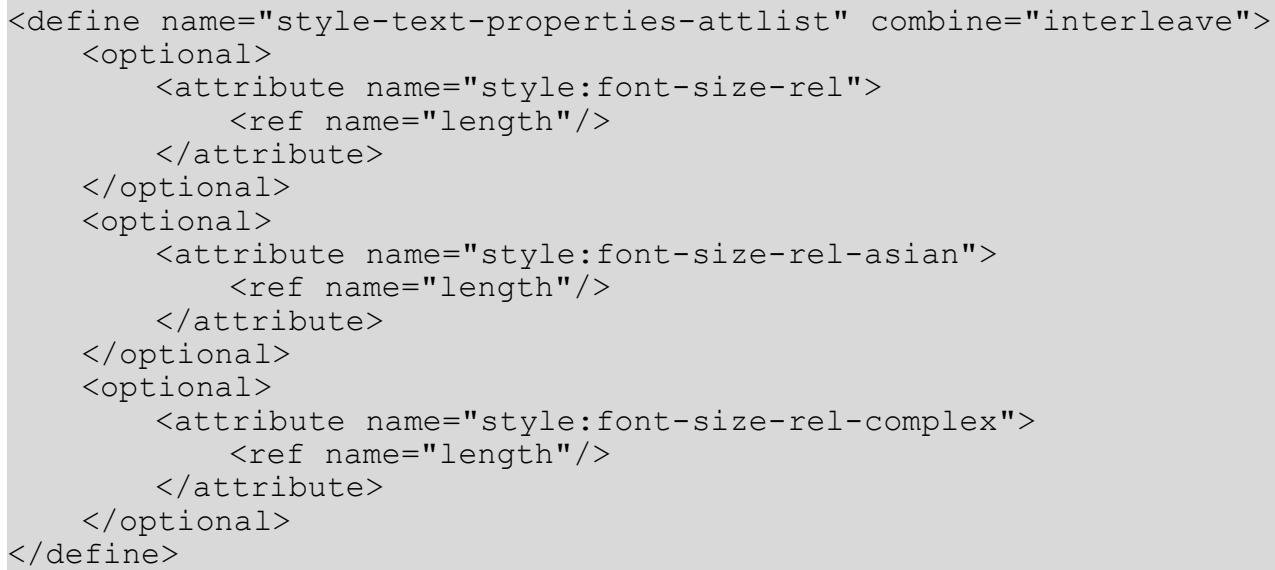

\subsubsection{Script Type}

The style:script-type property may be used to specify which script dependent attributes (like fo: font-family, style: font-family-asian, style:font-family-complex) are currently active for some text. The attribute should be evaluated by applications that do not support script types to select the correct script dependent properties. Application that support script types may also evaluate the attribute and overwrite the script type they would evaluate for a certain character, but they don't have to.

The usage of this property simplifies for instance transformations from and to [CSS2]/[XSL] and other formats that don't have script-dependent attributes, and also can be used to assign scripttypes to weak UNICODE characters, where application may choose different script types.

The values of this property are latin, asian, complex and ignore. The value ignore can be used only within default styles. If it is set, all script-dependent attributes are applied to all script types. This would mean for example that a fo:font-family would be applied to all script types as well as a style:font-family-asian or style:font-family-complex. This simplifies saving documents with application that do not support a script type.

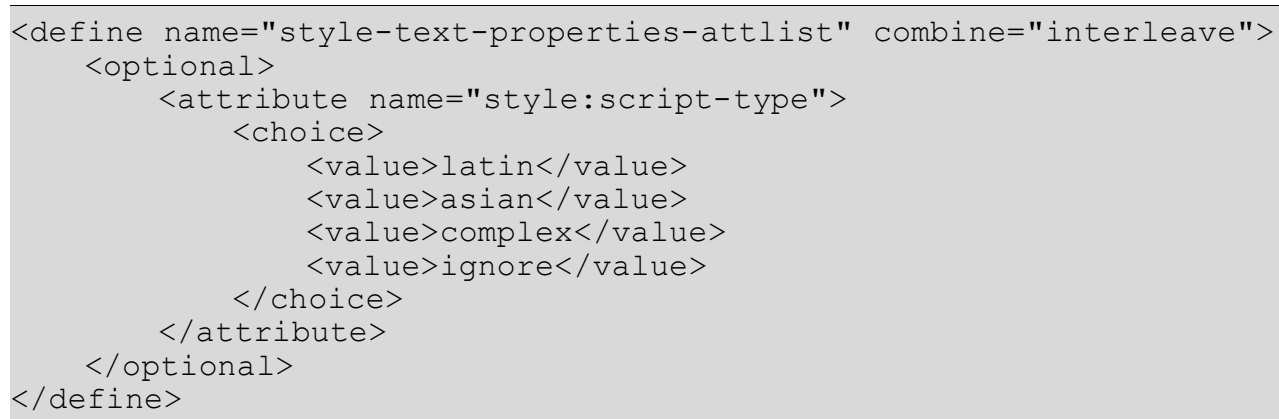

\subsubsection{Letter Spacing}

Use the fo:letter-spacing property to specify the amount of space between letters. The value of this property can be normal or it can specify a length. See $\$ 7.16 .2$ of [XSL] for details.

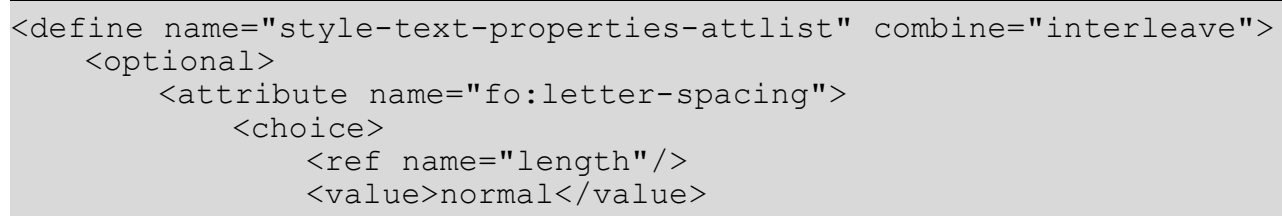




\subsubsection{Language}

Use the fo: language, fo: language-asian and fo:language-complex properties to specify the language of the text. See $\S 7.9 .2$ of $[X S L]$ for details.

Some applications ignore these properties if they are not specified together with the corresponding fo: country property.

See section 15.4.13 for information about when the Asian and complex variants of the attribute are evaluated.

fo: language, fo:language-asian and fo: language-complex

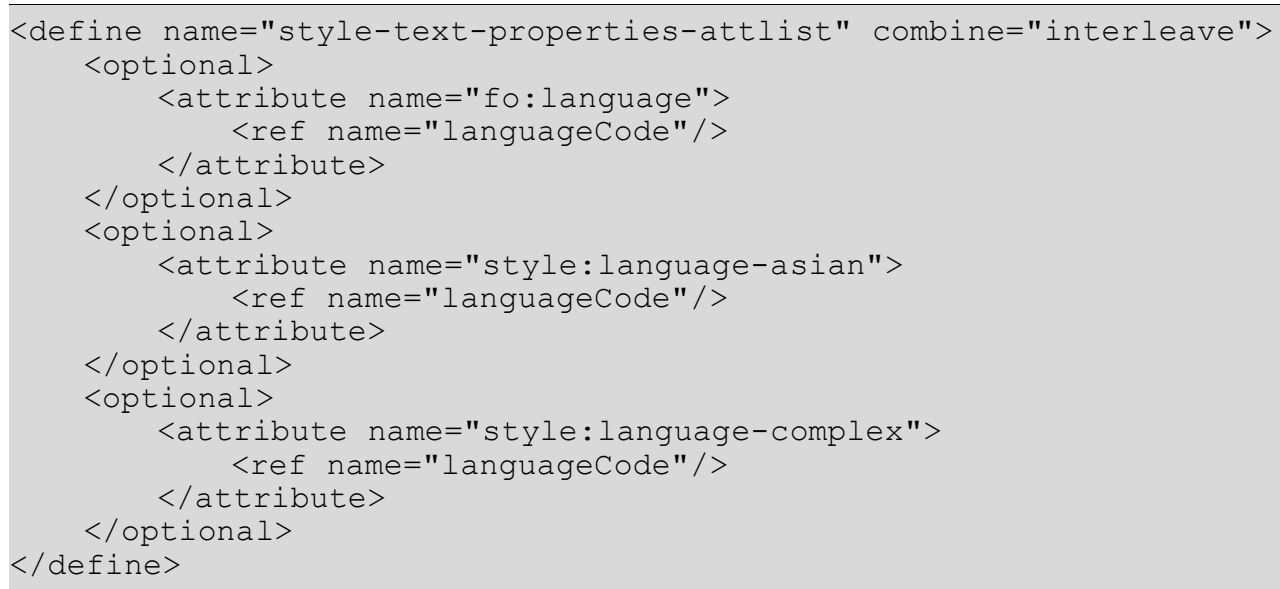

\subsubsection{Country}

Use the fo:country, style:country-asian and style: country-complex properties to specify the country of the text. See $\S 7.9 .1$ of [XSL] for details.

Some application ignore these properties if they are not specified together with the corresponding fo: language property.

See section 15.4.13 for information about when the Asian and complex variants of the attribute are evaluated.

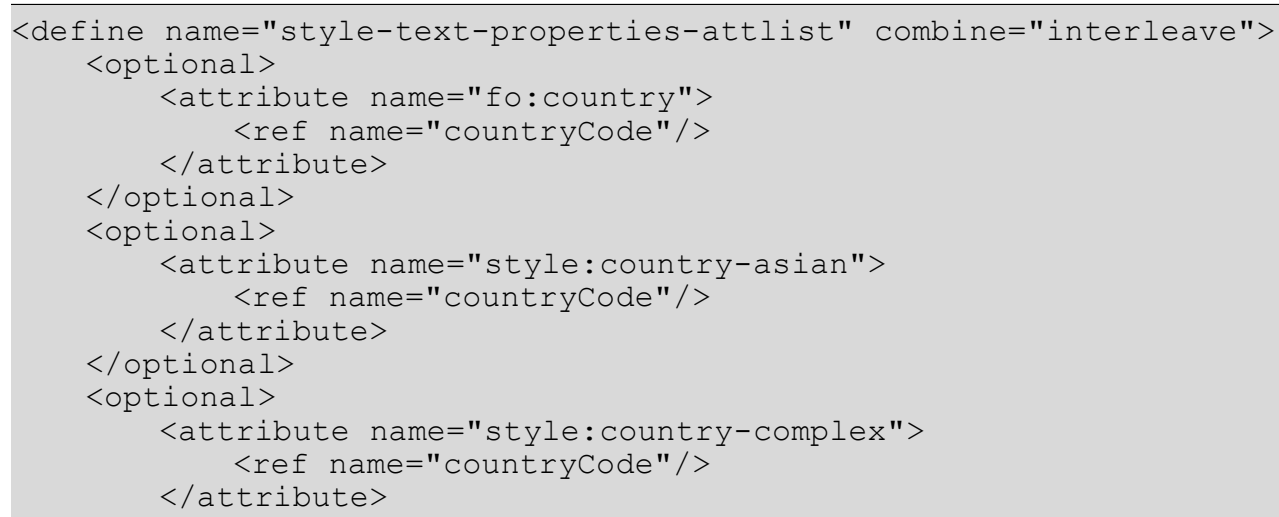




\subsubsection{Font Style}

Use the fo:font-style, style:font-style-asian and style:font-style-complex properties to specify whether to use normal or italic font face. See $\S 7.8 .7$ of [XSL] for details.

See section 15.4.13 for information about when the Asian and complex variants of the attribute are evaluated.

13970

13971

13972

13973

13974

13975

13976

13977

13978

13979

13980

13981

13982

13983

13984

13985

13986

13987

13988

13990

13991

13992

13993

13994

\subsubsection{Font Relief}

Use the style:font-relief property to specify whether the font should be embossed, engraved, or neither.

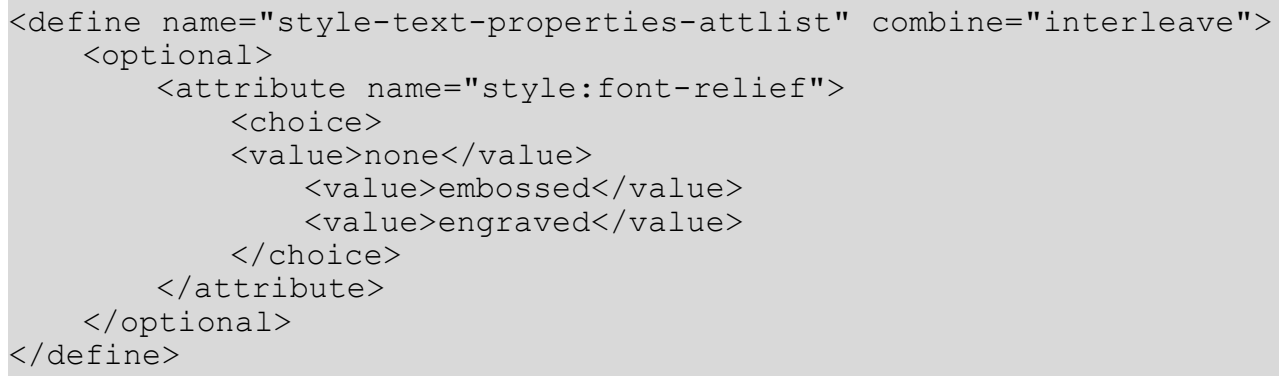

\subsubsection{Text Shadow}

Use the fo:text-shadow property to specify the text shadow style to use. See $§ 7.16 .5$ of [XSL] for details.

Some applications may only supports a limited number of shadow effects, for instance a default text shadow style only. 


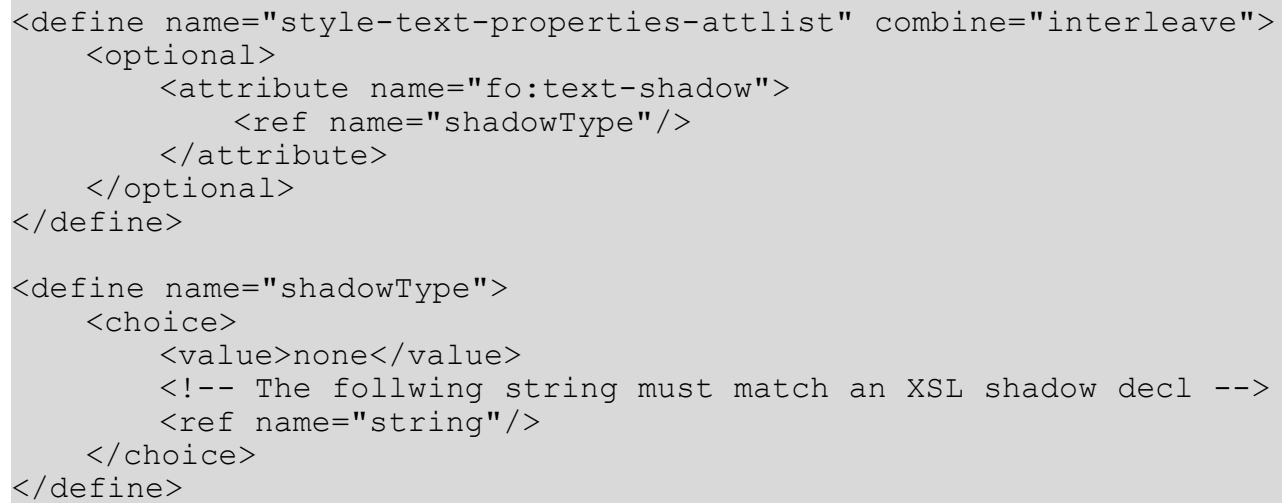

\subsubsection{Underlining Type}

Use the style:text-underline-type property to specify whether text is underlined, and if so, whether a single or double line will be used for underlining.

14021

14022

14023

14024

14025

14026

14027

14028

14030

14031

14032

14033

14034

14035

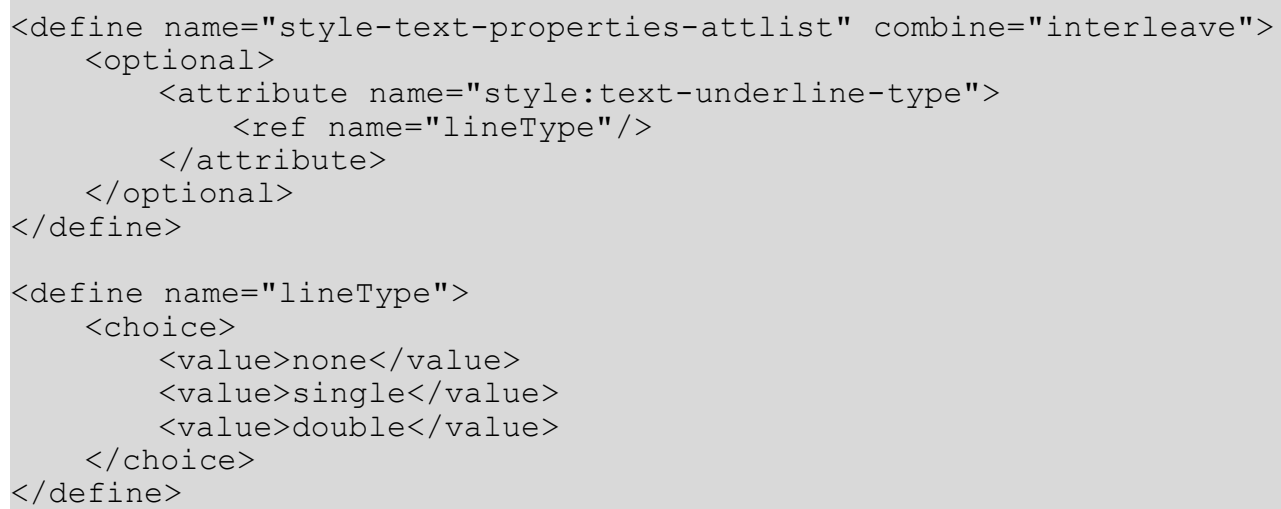

\subsubsection{Underlining Style}

Use the style:text-underline-style property to specify if and how text is underlined. The value of this property is the underlining style for the text, for example, single, dotted, dash. This property is similar to the [CSS3Text] text-underline-style property, except that has the additional value long-dash and that it does not have the value double. Instead of this, the attribute style:text: underline-type can be used to turn each line style into a double line. See $\S 9.2$ of [CSS3Text] for details.

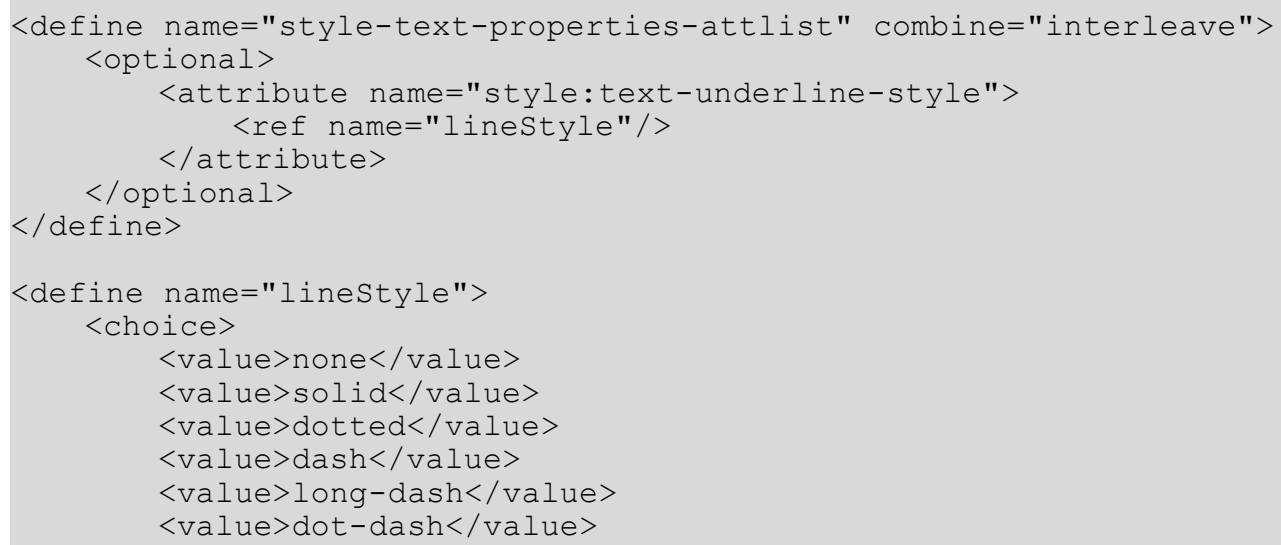




\subsubsection{Underling Width}

Use the style:text-underline-width property specifies the width of an underline. This property is very similar to the [CSS3Text] text-underline-width property, except that it has an additional value bold. bold specifies a line width that is calculated from the font sizes like an auto width, but is wider than an auto width. See $\S 9.3$ of [CSS3Text] for details.

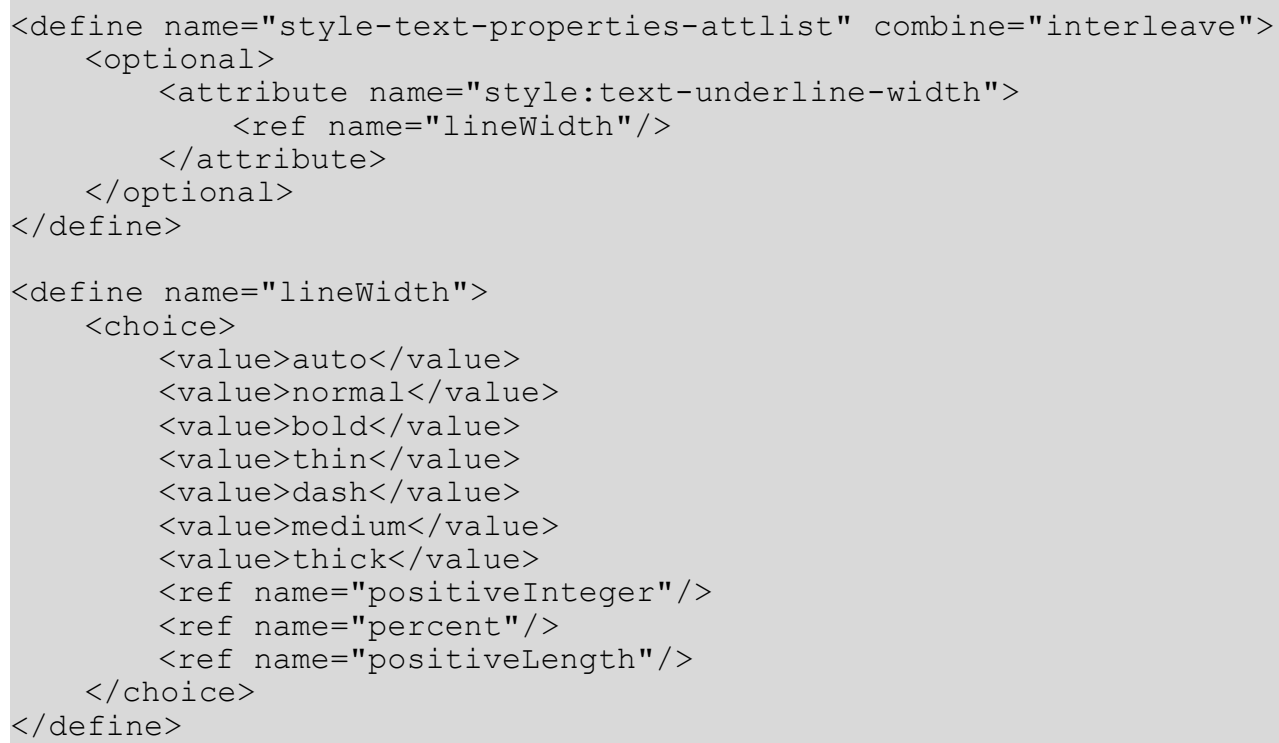

\subsubsection{Underline Color}

Use the style:text-underline-color property to specify the color that is used to underline text. The value of this property is either font-color or a color. If the value is font-color, the current text color is used for underlining.

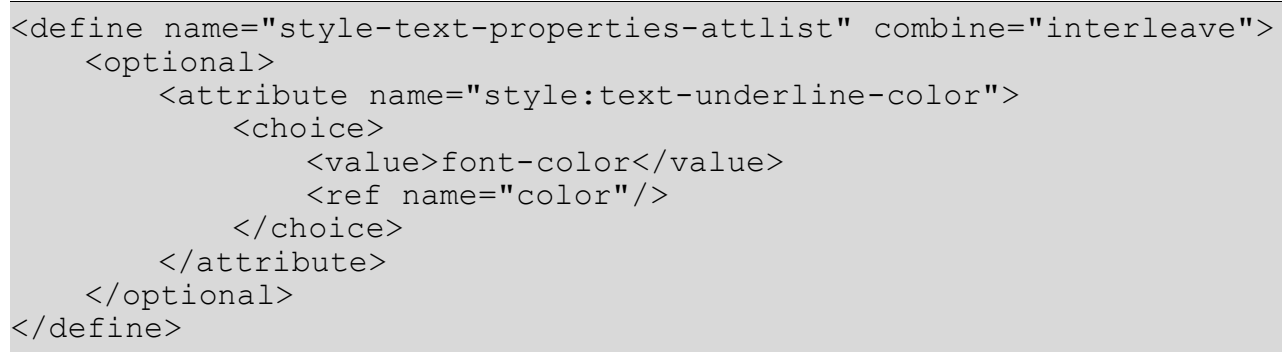

\subsubsection{Font Weight}

Use the fo: font-weight, style: font-weight-asian and style: font-weightcomplex properties to specify the weight of the font. See $§ 7.8 .9$ of [XSL] for details.

The relative values lighter or bolder are not supported and only a few distinct numerical values are supported. Unsupported numerical values are rounded off to the next supported value. 
See section 15.4.13 for information about when the Asian and complex variants of the attribute are evaluated.

14088

14089

14090

14091

14092

14093

14094

14095

14096

14097

14098

14099

14100

14101

14102

14103

14104

14105

14107

14108

14109

14110

14111

14112

14113

14114

14115

14116

14117

14118

14119

14120

14122

14123

14124

14125

14126

14127

14128
14129

14130

14131

14132

14133

14134

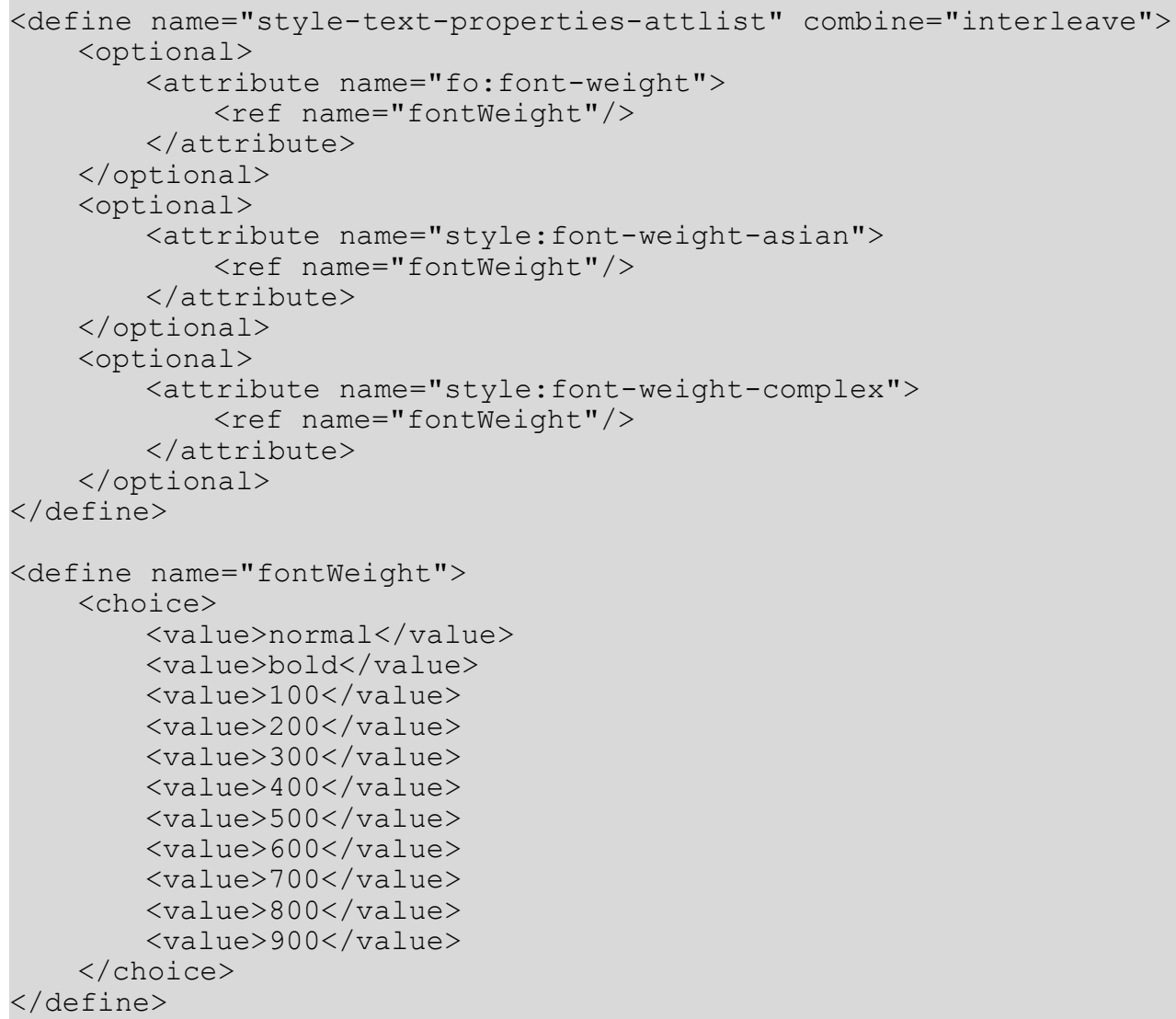

\subsubsection{Text Underline Word Mode}

Use the style:text-underline-mode property to specify whether underlining is applied to words only or to portions of text. If underlining is applied to text portions, the spaces between words and the words are underlined. See $\S 9.5$ of [CSS3Text] for details.

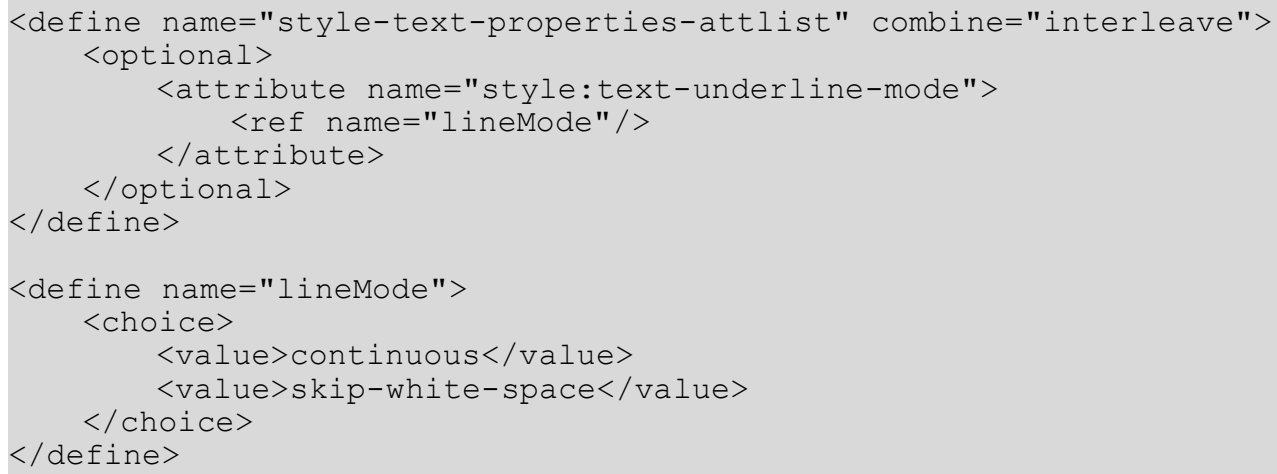

\subsubsection{Text Line Through Word Mode}

Use the style:text-line-through-mode property to specify whether lining through is applied to words only or to portions of text. If lining through is applied to text portions, the spaces between words and the words are line-through. See $\S 9.5$ of [CSS3Text] for details. 


\subsubsection{Text Combine}

Use the style:text-combine property to combine characters so that they are displayed within two lines.

The value of this attribute can be none, letters or lines.

If the value is lines, all characters with this attribute value that immediately follow each other are displayed within two lines of approximately the same length. There can be a line break between any two characters to meet this constraint.

If the value of the attribute is letters, up to 5 characters are combined within two lines. Any additional character is displayed as normal text. 
14162

14163

14164

14165

14166

14167

14168

14169

14171

14172

14173

14174

14175

14176

14177

14178

14179

14180

14181

<choice $>$
$\quad<$ value $>$ none $</$ value $>$
$\quad<$ value $>$ letters $</$ value $>$
$\quad<$ value $>$ lines $</$ value $>$
$</$ choice $>$
$</$ attribute $>$
$</$ optional $>$

\subsubsection{Text Combine Start and End Characters}

Use the two properties style:text-combine-start-char and style:text-combineend-char to specify a start and end character that is displayed before and after a portion of text whose style:text-combine property has a value of lines.

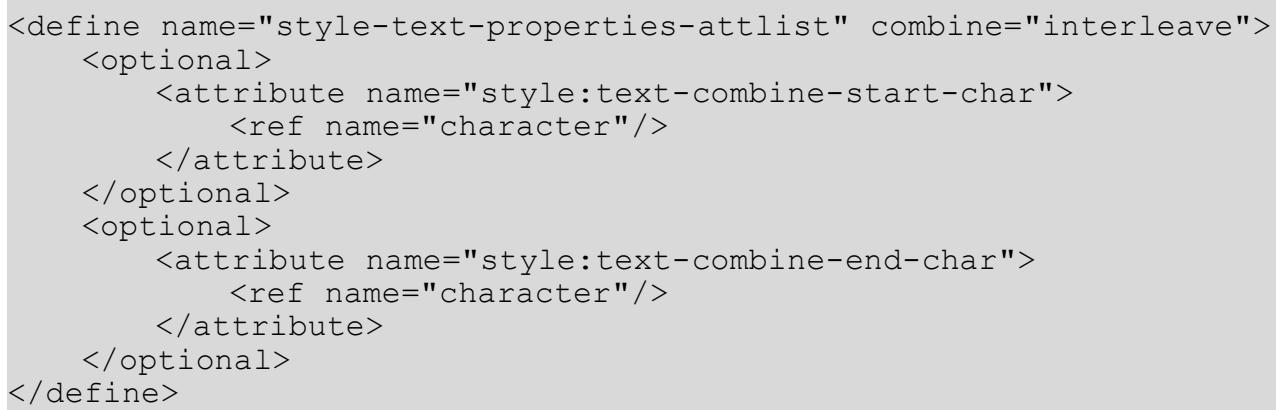

\subsubsection{Text Emphasis}

Use the style:text-emphasize property to emphasize text in Asian documents.

The value of this attribute consists of two space-separated values.

The first value represents the style to use for emphasis and it can be none, accent, dot, circle, or disc.

The second value represents the position of the emphasis and it can be above or below. If the first value is none, this value can be omitted.

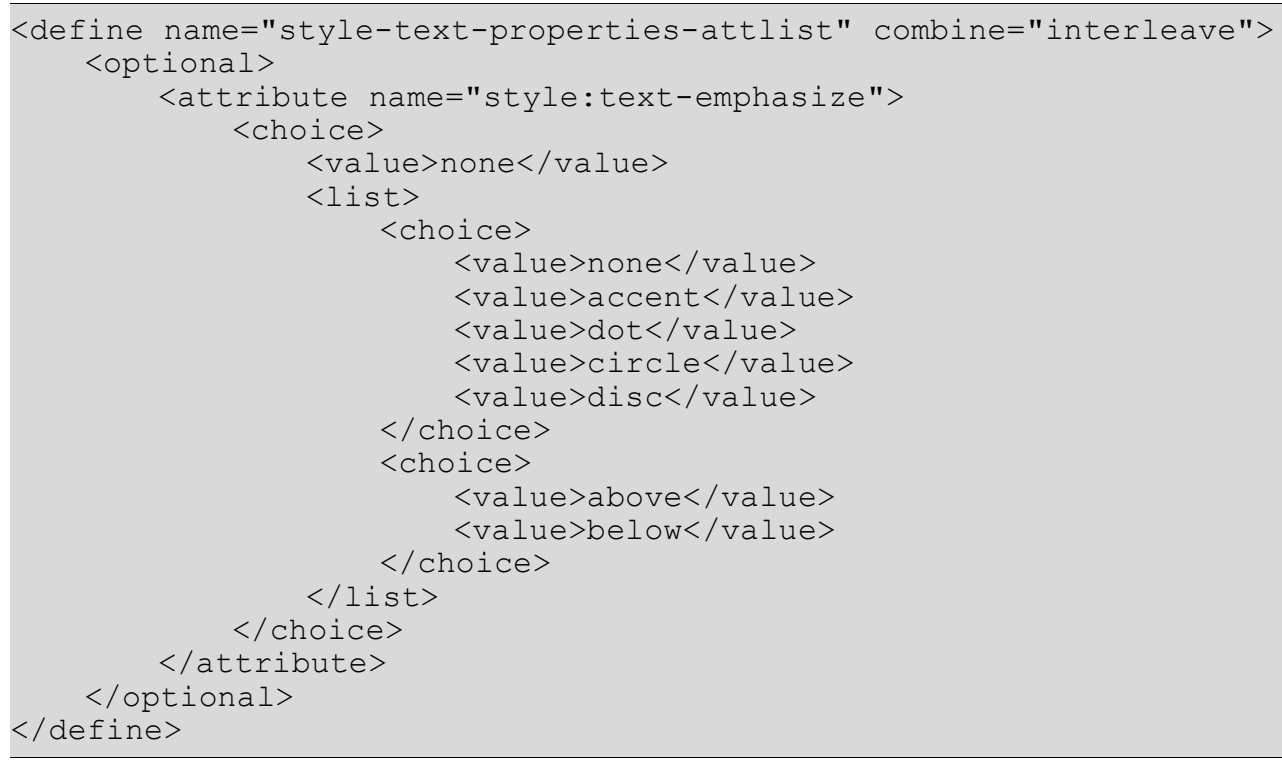




\subsubsection{Text Scale}

Use the style:text-scale property to decrease or increase the width of the text by scaling the font width.

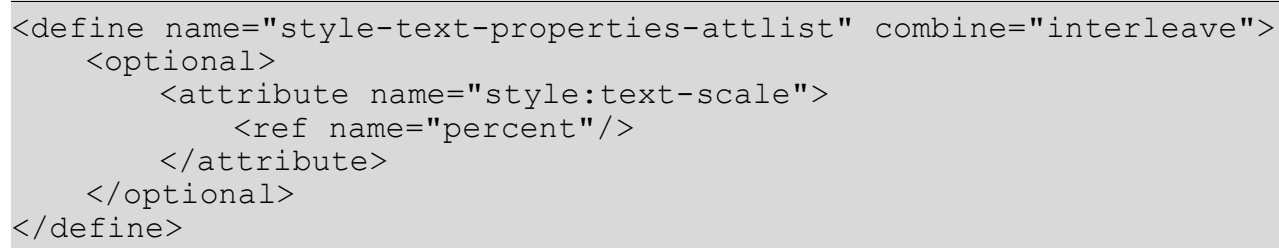

\subsubsection{Text Rotation Angle}

The style:text-rotation-angle property specifies an angle to which text is rotated. The value of this attribute can be 0,90 , or 270 . For any angle greater than 359 the remainder of a division by 360 is used. Any angle other than 0,90 or 270 is rounded to the nearest possible value.

If this attribute is specified for more than one character, all text containing these characters is rotated.

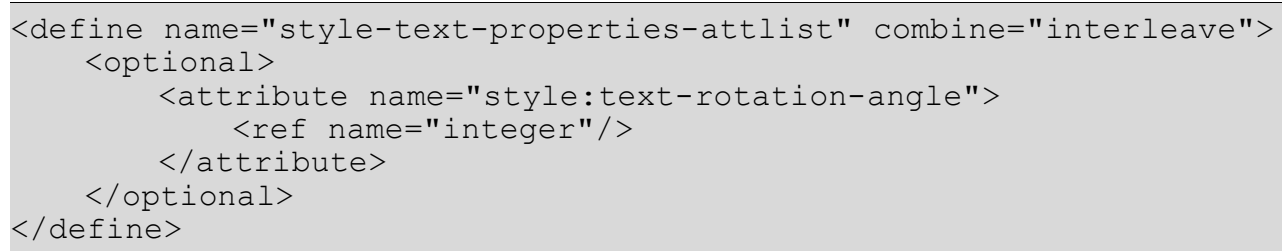

\subsubsection{Text Rotation Scale}

If text is rotated, the style:text-rotation-scale property specifies whether the width of the text should be scaled to fit into the current line height or the width of the text should remain fixed, therefore changing the current line height.

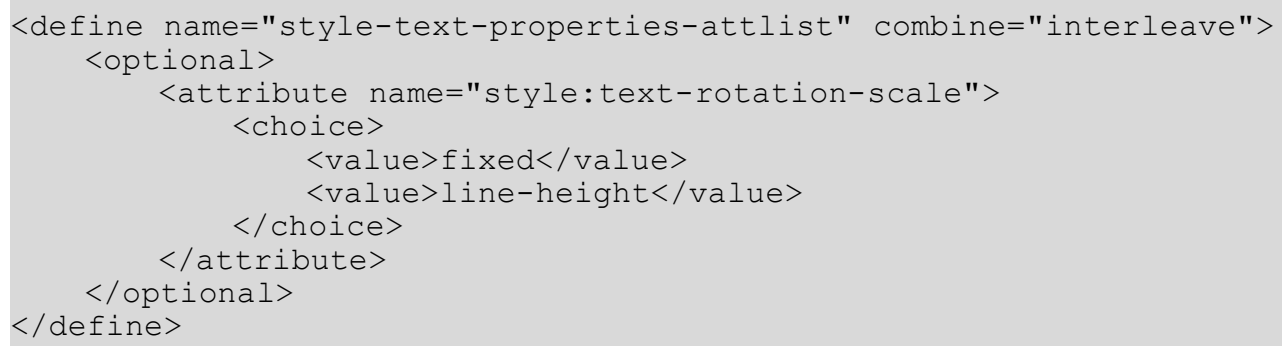

\subsubsection{Hyphenation}

Use the fo: hyphenate property to enable or disable automatic hyphenation. See $§ 7.9 .4$ of [XSL] for details.

Some application might not support setting the properties fo:hyphenate, fo: hyphenationkeep, fo:hyphenation-remain-char-count, fo:hyphenation-push-char-count and fo: hyphenation-ladder-count independent of each other within a style. A reasonable default for $f \circ:$ hyphenate in this case is false.

<define name="style-text-properties-attlist" combine="interleave"> <optional> 


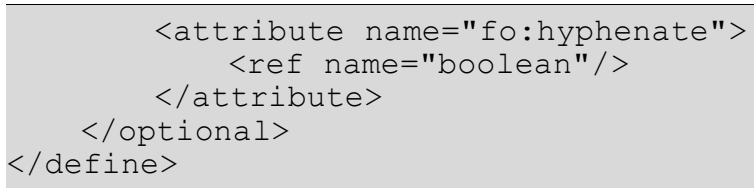

\subsubsection{Hyphenation Remain Char Count}

Use the fo:hyphenation-remain-char-count property to specify the number of characters that must be present before a hyphenation character. See $§ 7.9 .7$ of [XSL] for details.

Some application might not support setting the properties fo:hyphenate, fo:hyphenationkeep, fo:hyphenation-remain-char-count, fo:hyphenation-push-char-count and fo: hyphenation-ladder-count independent of each other within a style. A reasonable default for fo:hyphenation-remain-char-count in this case is 0 .

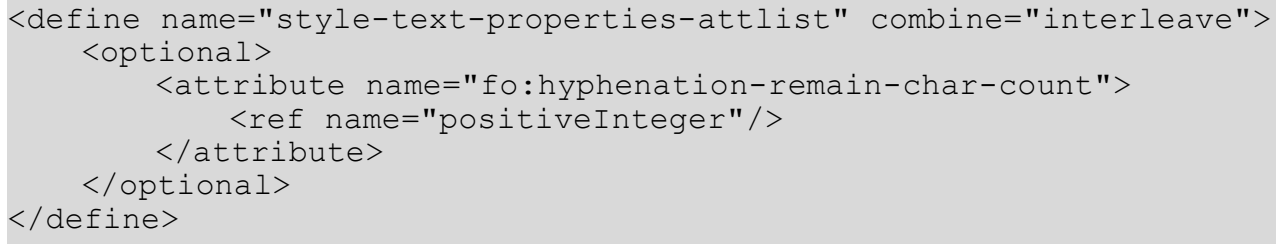

\subsubsection{Hyphenation Push Char Count}

Use the fo:hyphenation-push-char-count property to specify the minimum number of characters that are moved to the next line. See $§ 7.9 .6$ of [XSL] for details.

Some application might not support setting the properties fo:hyphenate, fo:hyphenationkeep, fo:hyphenation-remain-char-count, fo:hyphenation-push-char-count and fo: hyphenation-ladder-count independent of each other within a style. A reasonable default for fo:hyphenation-push-char-count in this case is 0 .

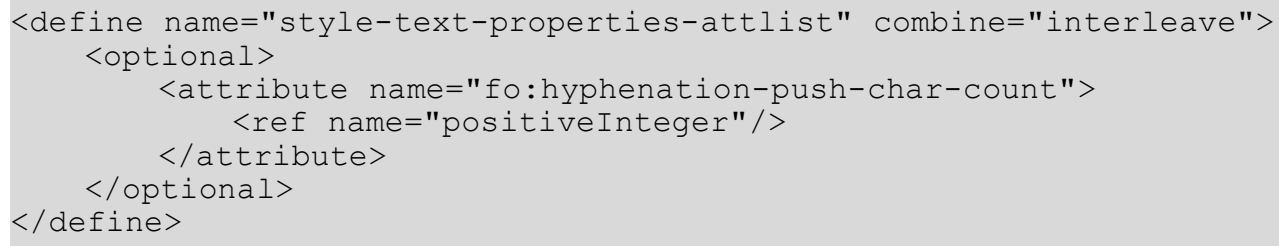

\subsubsection{Hidden or Conditional Text}

The text: display property allows text to be hidden. This can be made dependent on a condition as well. This attributes and its values are the same as for text:display attribute on text sections (see also section 4.4). The values of this attribute may be any of:

- true - the text will be displayed normally. This is the default.

- none - the text will be hidden.

- condition - a condition determines whether the text will be displayed or hidden. In this case, a text:condition attribute must be present specifying the condition.

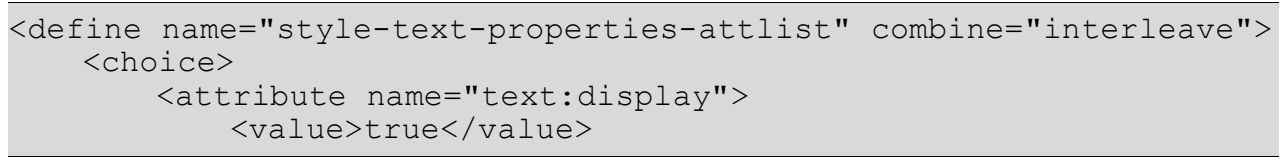




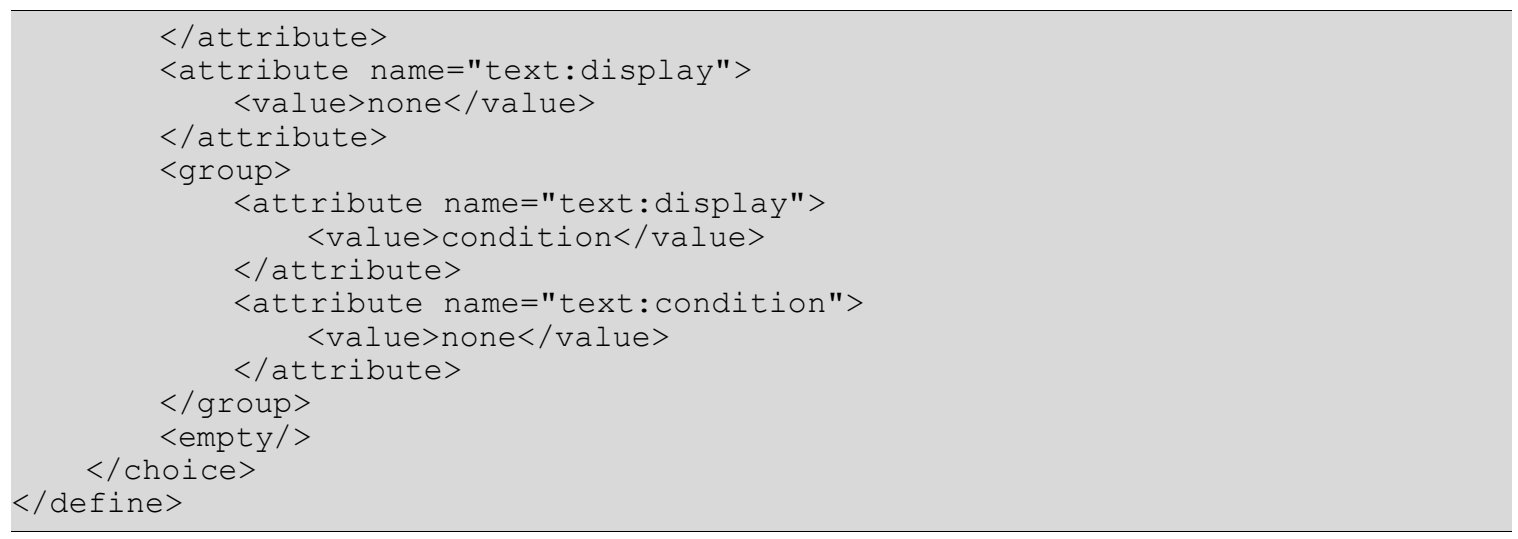

\subsection{Paragraph Formatting Properties}

The properties described in this section can be contained within paragraph styles (see section 14.8.2), but also within other styles, like cell styles (see section 14.12.4) They are contained in a $<$ style:paragraph-properties> element.

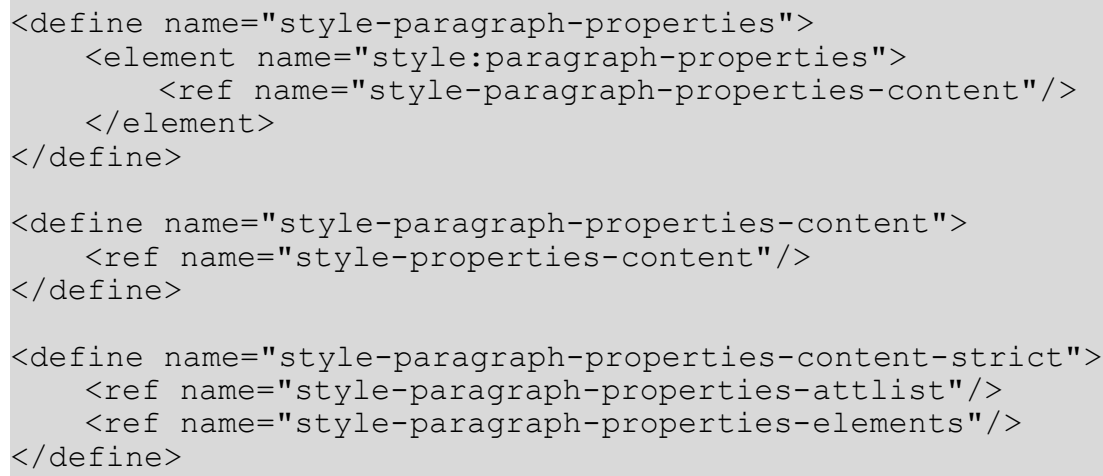

\subsubsection{Fixed Line Height}

Use the fo:line-height property to specify a fixed line height either as a length or a percentage that relates to the highest character in a line. A special value of normal activates the default line height calculation. It is also used to deactivate the effects of the style: lineheight-at-least and style:line-spacing properties. The value of this property can be a length, a percentage, or a value of normal. See $\S 7.15 .4$ of $[X S L]$ for details.

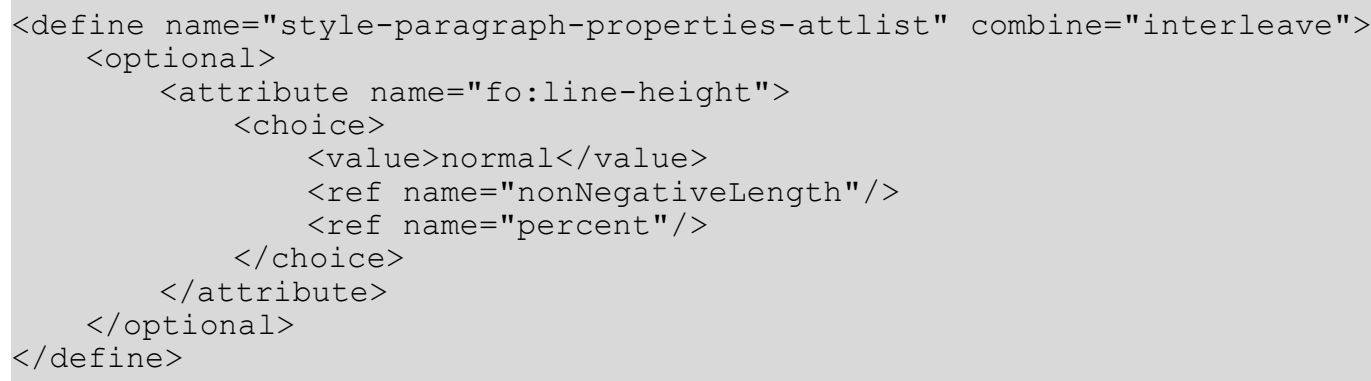

\subsubsection{Minimum Line Height}

Use the style:line-height-at-least property to specify a minimum line height. The value of this property is a length. There is no normal value for the property. 


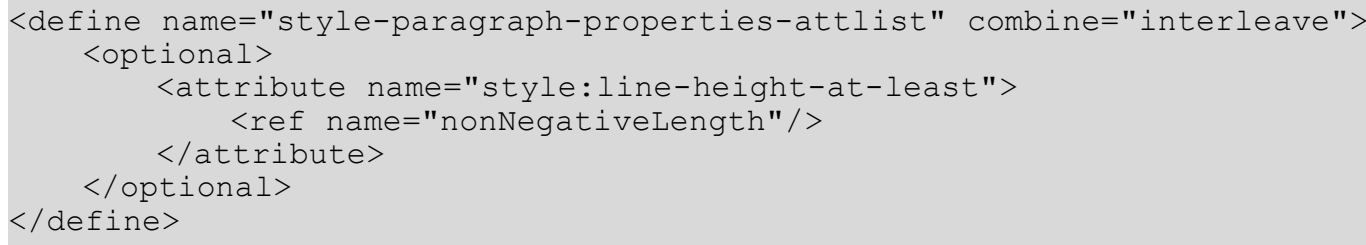

\subsubsection{Line Distance}

Use the style:line-spacing property to specify a fixed distance between two lines. There is no normal value for this property.

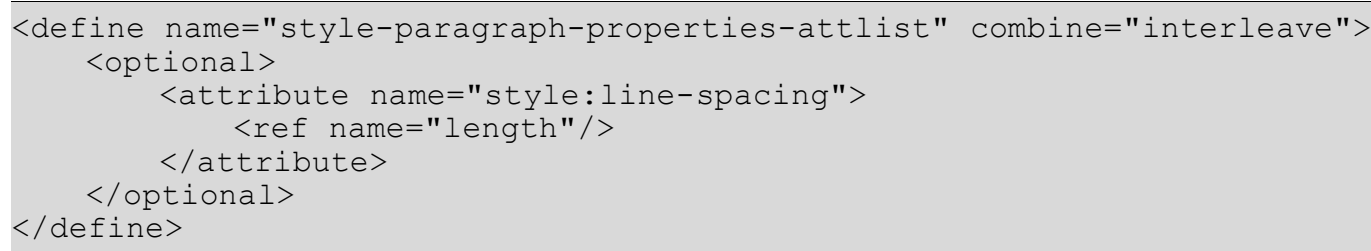

\subsubsection{Font-Independent Line Spacing}

The style:font-independent-line-spacing property specifies if font independent line spacing is used. If the attribute's value is true, then the line height is calculated only from the font height as specified by the font size attributes fo:font-size, style: font-size-asian and style:font-size-complex. If the value is false, the font metric of the actual font is taken into account.

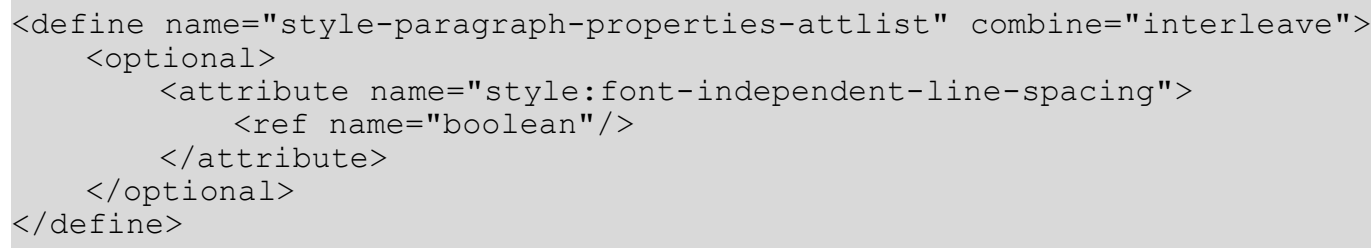

\subsubsection{Text Align}

Use the fo:text-align property to specify how to align text in paragraphs.

The value of this property can be start, end, left, right, center, or justify. See $§ 7.15 .9$ of [XSL] for details. The values inside and outside are not supported.

If there are no values specified for the fo:text-align-last and style:justify-singleword properties within the same item set element, the values of these properties are set to start and false respectively.

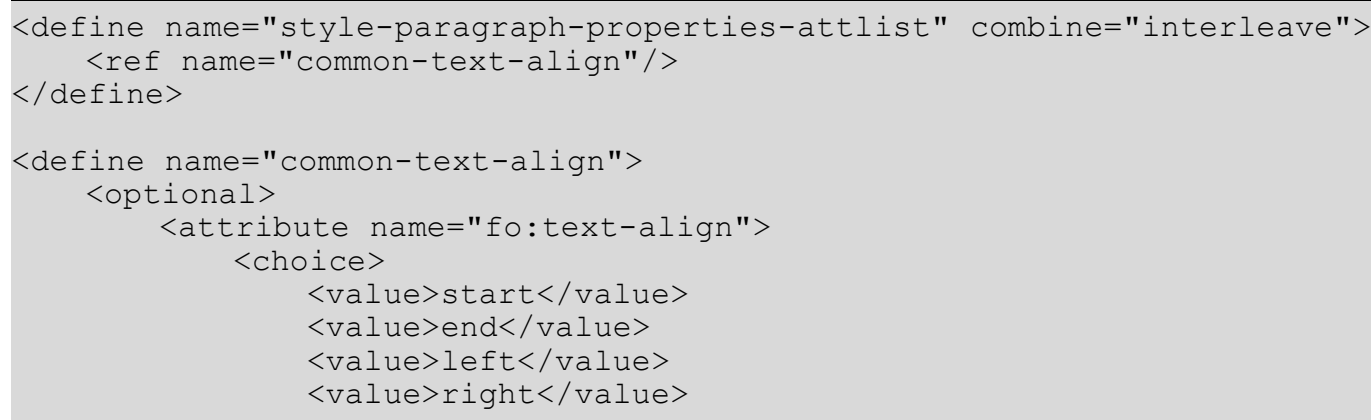




\subsubsection{Text Align of Last Line}

Use the fo:text-align-last property to specify how to align the last line of a justified paragraph. See $\S 7.15 .9$ of [XSL] for details. The only values of this property that are supported are start, center, or justify.

This property is ignored if it not accompanied by an fo:text-align property.

If there are no values specified for the fo:text-align and style:justify-single-word properties, these values of these properties is set to start and false respectively.

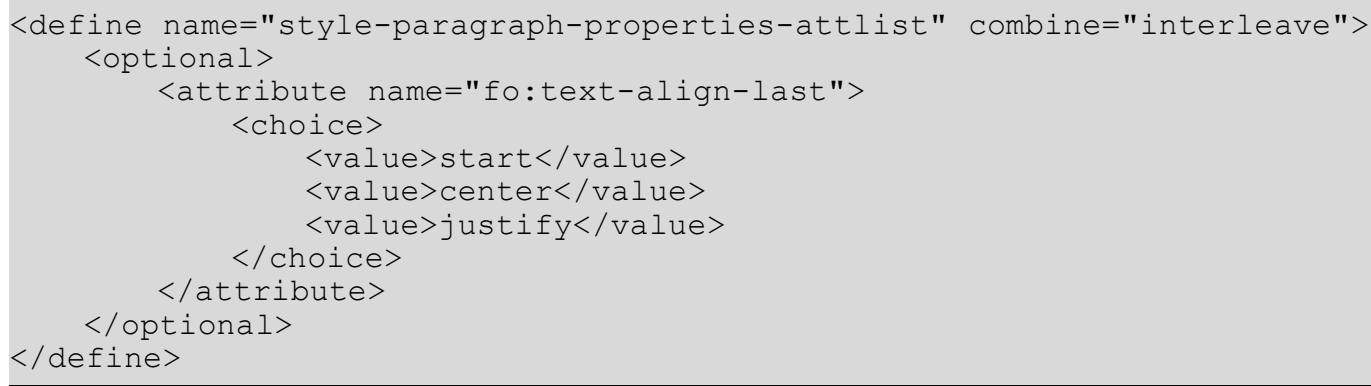

\subsubsection{Justify Single Word}

If the last line in a paragraph is justified, use the style: justify-single-word property to specify whether or not a single word should be justified.

If there are no values specified for the fo:text-align and fo:text-align-last properties, the values of these properties are set to start. This means that specifying a style:justifysingle-word property without specifying a fo:text-align and fo:text-align-last property has no effect.

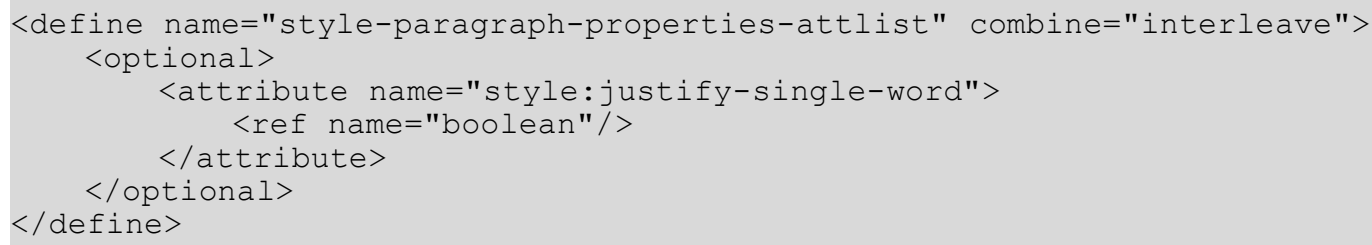

\subsubsection{Keep Together}

Use the fo:keep-together property to control whether the lines of a paragraph should be kept together on the same page or column (if the value is always), or whether breaks are allowed within the paragraph (if the value is auto). See $\S 7.19 .3$ of [XSL] for details.

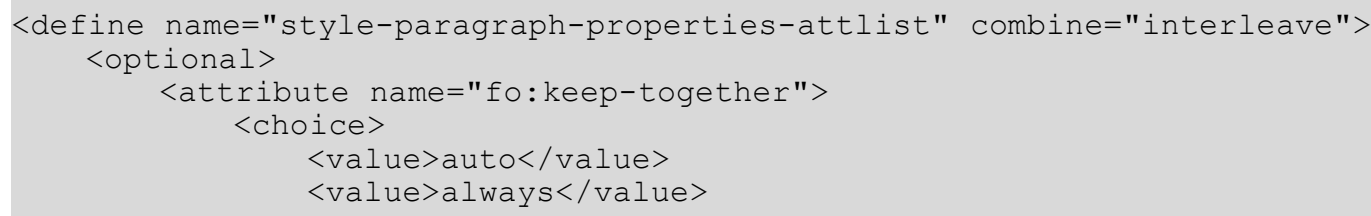




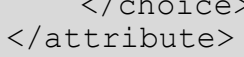

\subsubsection{Widows}

Use the $f \circ$ :widows property to specify the minimum number of lines allowed at the top of a page to avoid paragraph widows. See $\S 7.19 .7$ of [XSL] for details.

14360

14361

14362

14363

14364

14365

14366

\subsubsection{Tab Stops}

Use the tab stop element $<$ style:tab-stops $>$ to specify tab stop definitions.

Every tab stop position is represented by a single $<$ style:tab-stop $>$ element that is contained in the <style:tab-stops $>$ element.

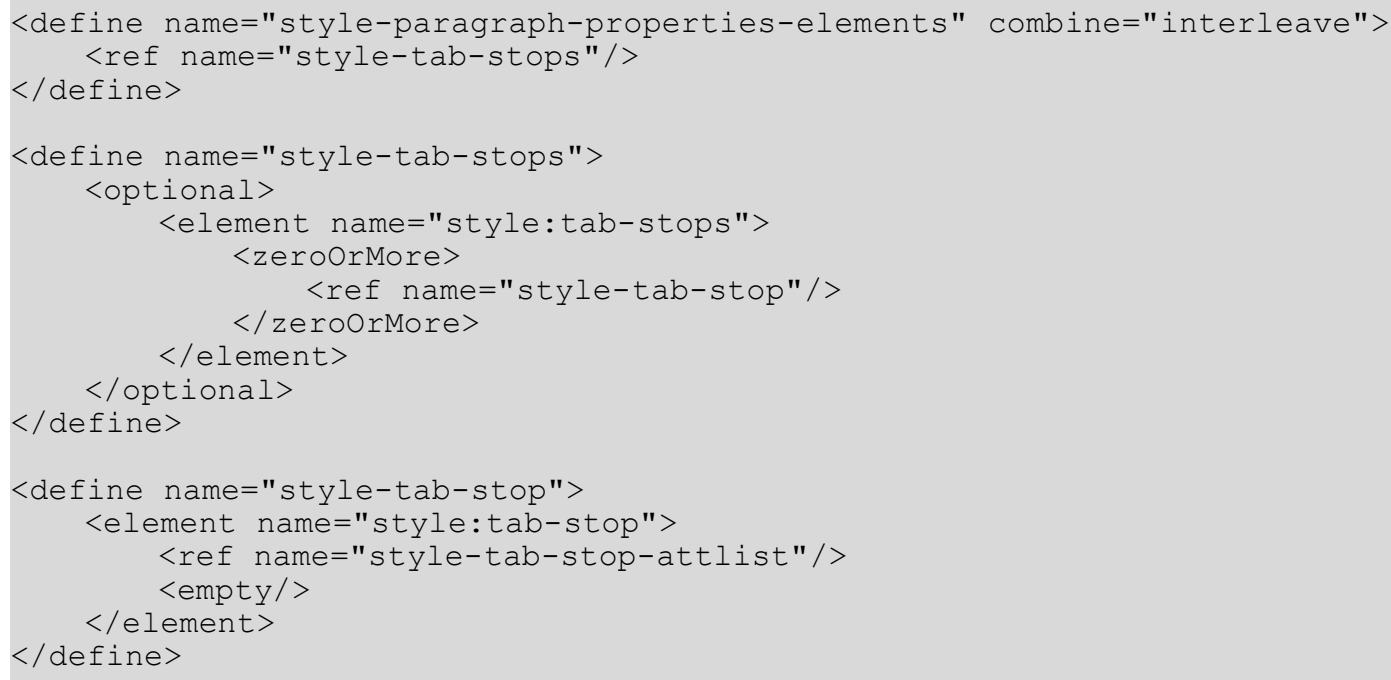

The attributes that may be associated with the <style:tab-stop $>$ elements are:

- Tab position 
- Tab type

- Delimiter character

- Leader type

- Leader style

- Leader width

- Leader color

- Leader text

- Leader text style

\section{Tab Position}

The style:position attribute specifies the position of a tab stop.

This attribute is associated with the <style:tab-stop $>$ element and its value is a length.

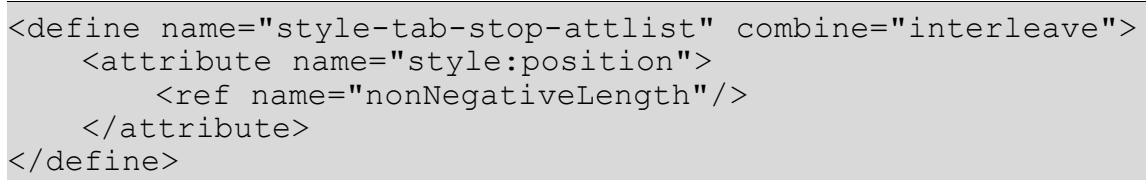

\section{Tab Type}

The style:type attribute specifies the type of tab stop.

This attribute is associated with the <style:tab-stop> element and its value can be left, center, right or char.

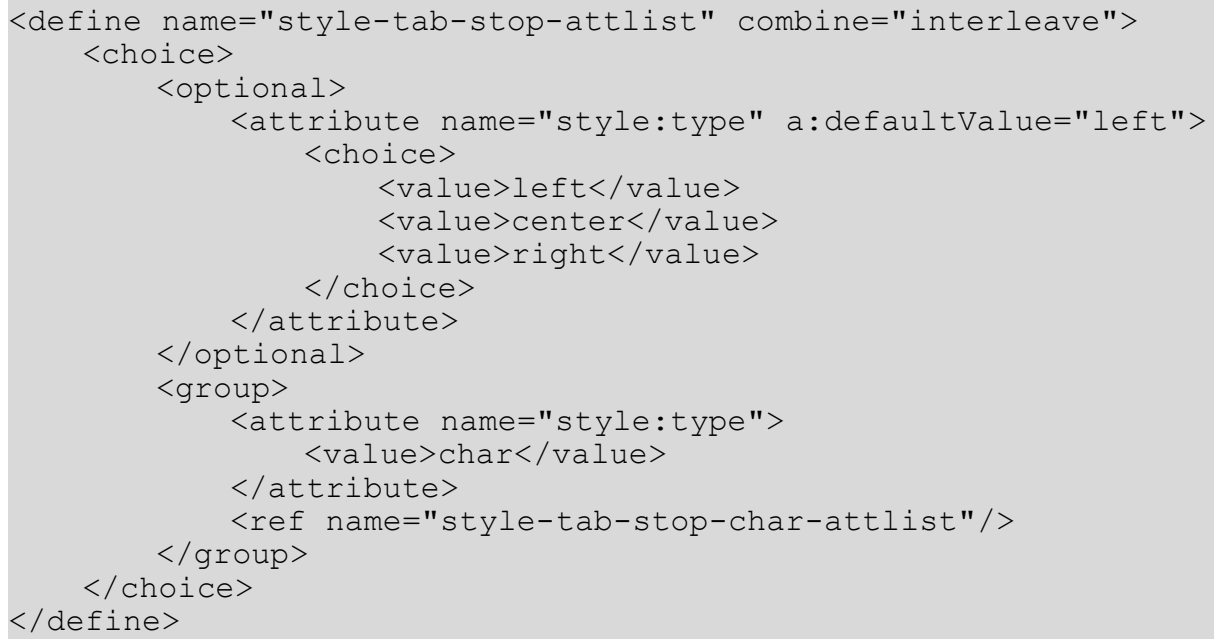

\section{Delimiter Character}

The style:char attribute specifies the delimiter character for tab stops of type char.

This attribute is associated with the <style:tab-stop $>$ element and it must be present if the value of the style:type attribute is char. If the value of style:type attribute is not char, it is ignored. 
The value of the attribute must be a single UNICODE character.

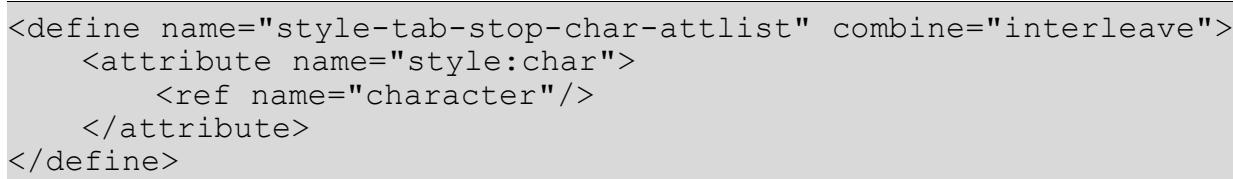

\section{Leader Type}

Use the style: leader-type attribute to specify whether a leader line should be drawn, and if so, whether a single or double line will be used. See also section 15.4.28.

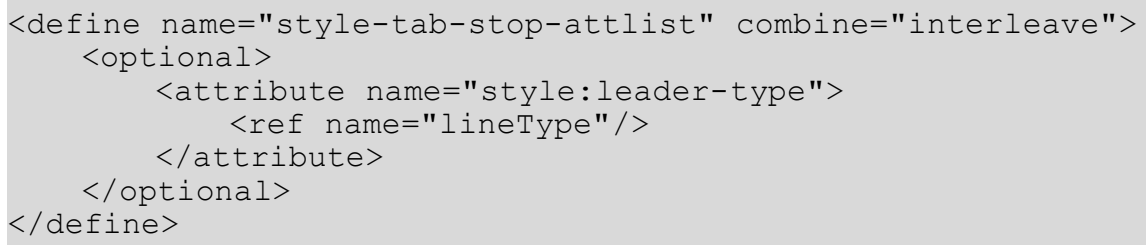

\section{Leader Style}

Use the style: leader-style property to specify if and how a leader line is drawn. The line styles that can be used are described in section 15.4.29.

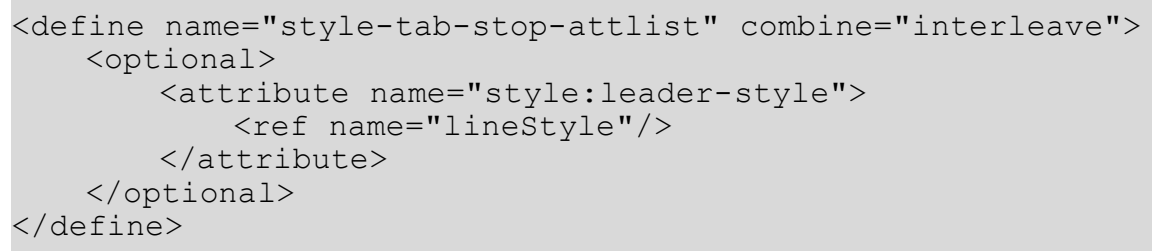

\section{Leader Width}

Use the style: leader-width property to specifies the width of a leader line. See section 15.4.30 for the values of this attribute.

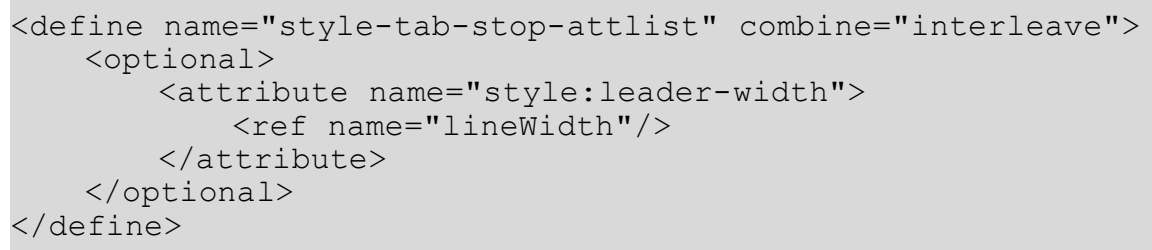

\section{Leader Color}

Use the style:leader-color property to specify the color that is for the leader line. The value of this property is either font-color or a color. If the value is font-color, the current text color is used for the leader line.

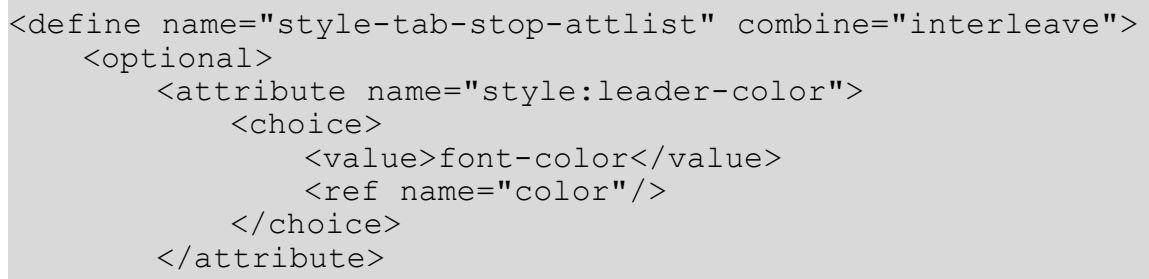




\section{Leader Text}

The style: leader-text attribute specifies the leader text to use for tab stops. If the attribute value is not empty, the attribute value string is used as leader instead of the line that has been specified, provided that the application supports textual leaders. If the application does not support textual, the attribute is ignored, this means, style: leader-style will be evaluated only. If the application supports textual consisting of a single characters only, and the leader text has more than one character, the first character of the leader text should be used only. If the applications supports textual leaders with with certain characters only (like "." or "_"), the application should use one of these characters if the leader-text specifies characters that are not supported. In other words: textual leaders have an higher priority than line leaders, even if the leader text that is specified has to be adapted to be usable by the application.

This attribute is associated with the $\langle$ style:tab-stop $>$ element and its value must be a single UNICODE character.

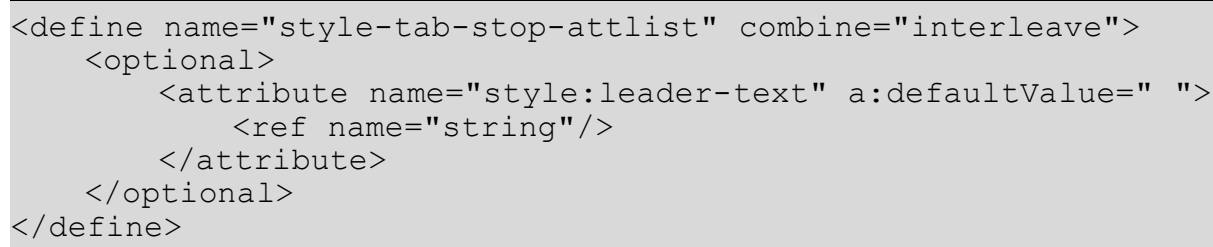

\section{Leader Text Style}

The style:leader-text-style specifies a text style that is applied to a textual leader. It is not applied to leader lines. If the attribute appears in an automatic style, it may reference either an automatic text style or an common style. If the attribute appears in an common style, it may reference a common style only.

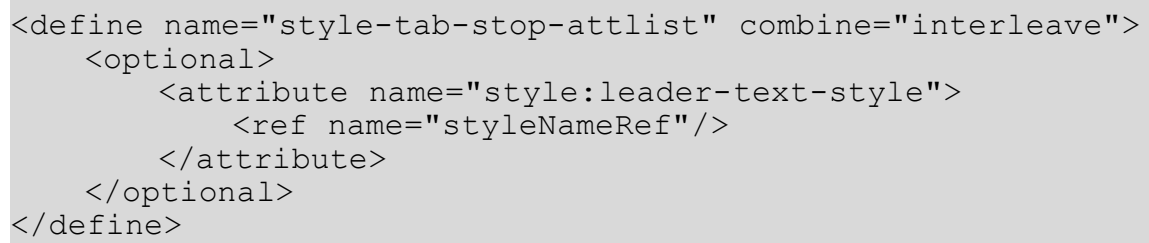

\subsubsection{Tab Stop Distance}

The attribute style:tab-stop-distance specifies the distance between default tab stops. A default tab stop is repeated automatically after the specified distance. Default tab stops usually are only evaluated if they are specified within a default style (see section 14.2).

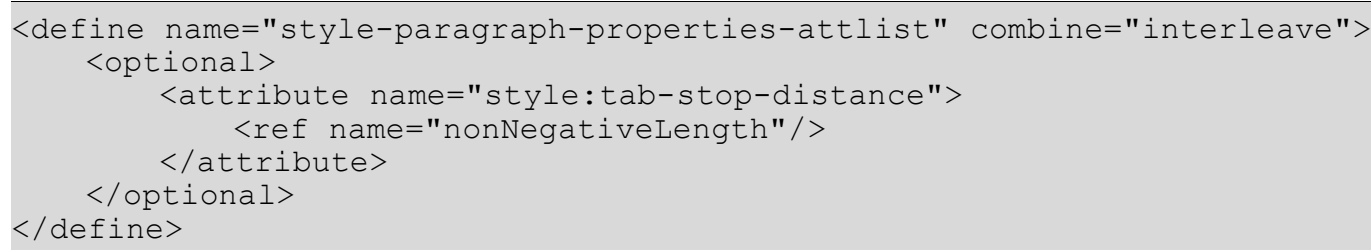




\subsubsection{Hyphenation Keep}

Use the fo:hyphenation-keep property to enable or disable the hyphenation of the last word on a page. See $\S 7.15 .1$ of [XSL] for details.

Some application might not support setting the properties fo:hyphenate, fo:hyphenationkeep, fo:hyphenation-remain-char-count, fo:hyphenation-push-char-count and fo: hyphenation-ladder-count independent of each other within a style. A reasonable default for fo:hyphenation-keep in this case is auto.

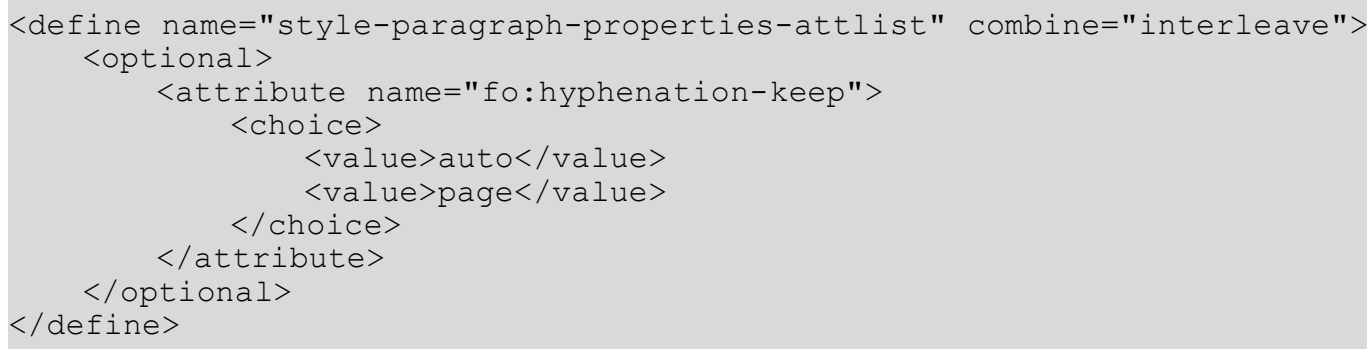

\subsubsection{Maximum Hyphens}

Use the fo: hyphenation-ladder-count property to specify the maximum number of successive lines that can contain a hyphenated word. See $§ 7.15 .2$ of [XSL] for details.

Some application might not support setting the properties fo: hyphenate, fo: hyphenationkeep, fo:hyphenation-remain-char-count, fo:hyphenation-push-char-count and fo: hyphenation-ladder-count independent of each other within a style. A reasonable default for fo:hyphenation-push-char-count in this case is no-limit.

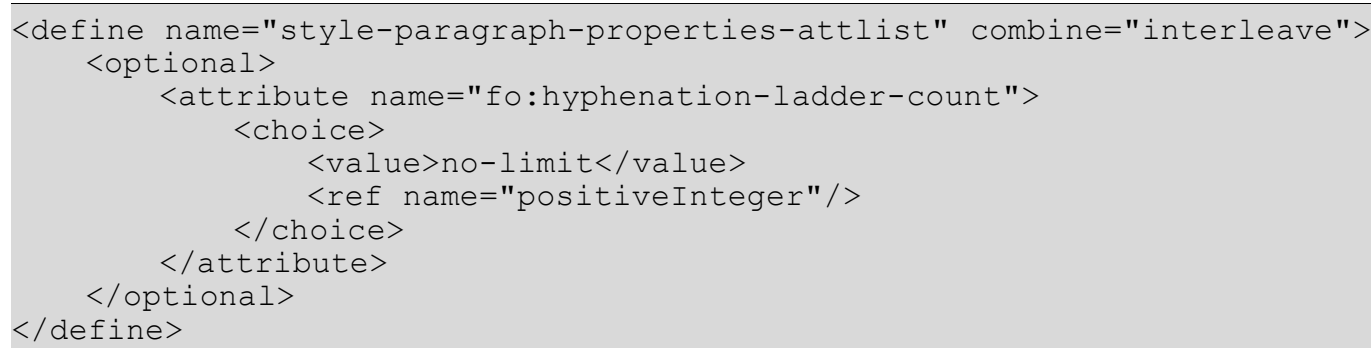

\subsubsection{Drop Caps}

Use the <style: drop-cap> element to specify if the first character or more of a paragraph is displayed in a larger font. This element can be contained in a <style:paragraphproperties> element.

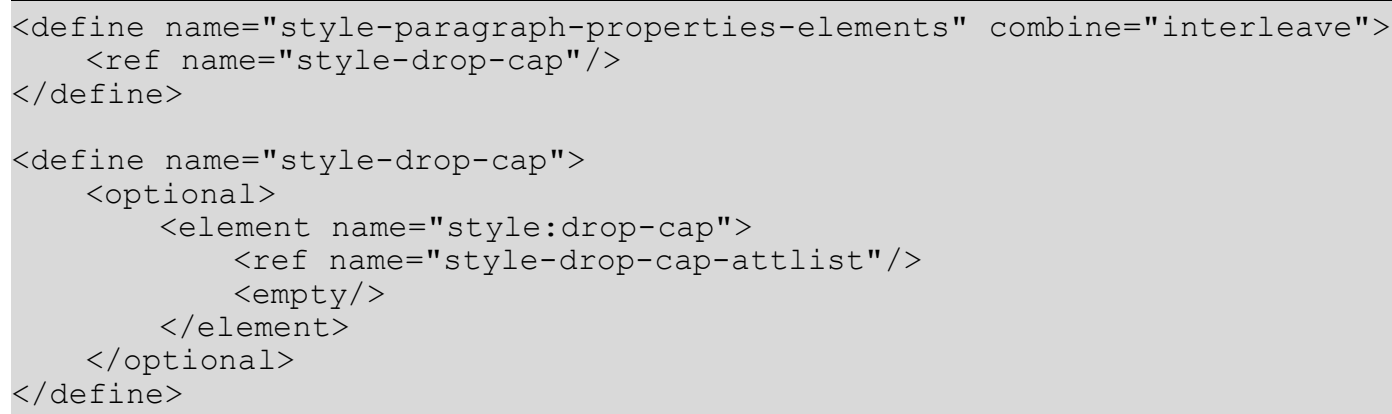




\subsubsection{Register True}

The style:register-true property specifies whether the lines on both sides of a printed page match when a document is printed using two-sided printing, It also ensures that the text in page columns or text box columns is arranged in such a way that the text baselines seem to run from one column to another. See also section 15.2.12.

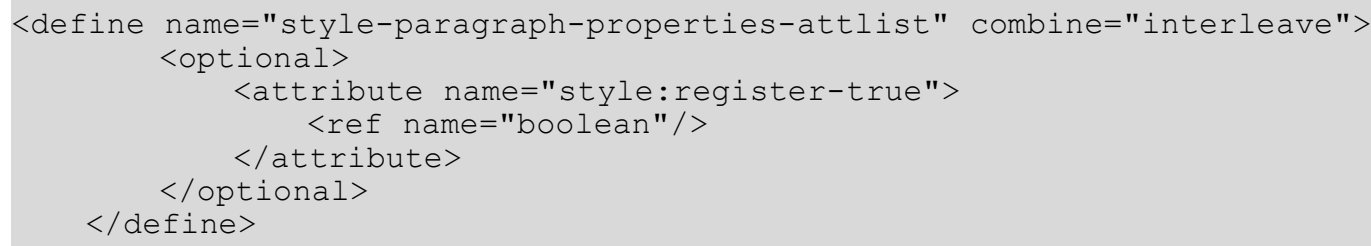

\subsubsection{Left and Right Margins}

Use the fo:margin-left and fo:margin-right properties to specify the left and right margins for a paragraph. See $\S 7.10 .3$ and $\S 7.10 .4$ of [XSL] for details. The value auto is not supported. Percentage values are only supported in common styles. They here relate to the corresponding margin of the parent style.

For some applications. these two properties must be used simultaneously and also together with the fo:text-indent property. If any of the properties is missing, its value is assumed to be $0 \mathrm{~cm}$.

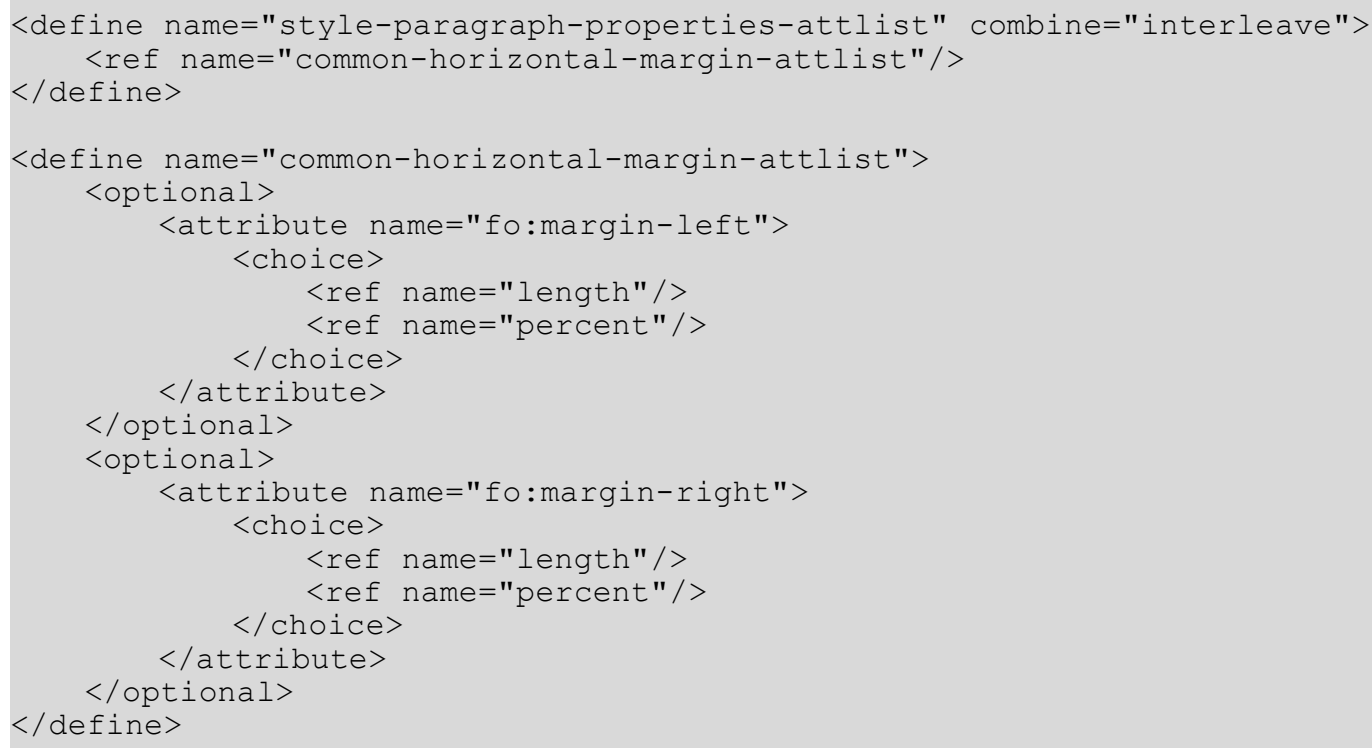

\subsubsection{Text Indent}

Use the fo:text-indent property to specify a positive or negative indent for the first line of a paragraph. See $\S 7.15 .11$ of [XSL] for details. Percentage values are only supported in common styles. They here relate to the corresponding margin of the parent style. 
For some applications. the fo:text-indent property must be used together with the fo:margin-left and fo:margin-right properties. If any of these properties is missing, its value is assumed to be $0 \mathrm{~cm}$.

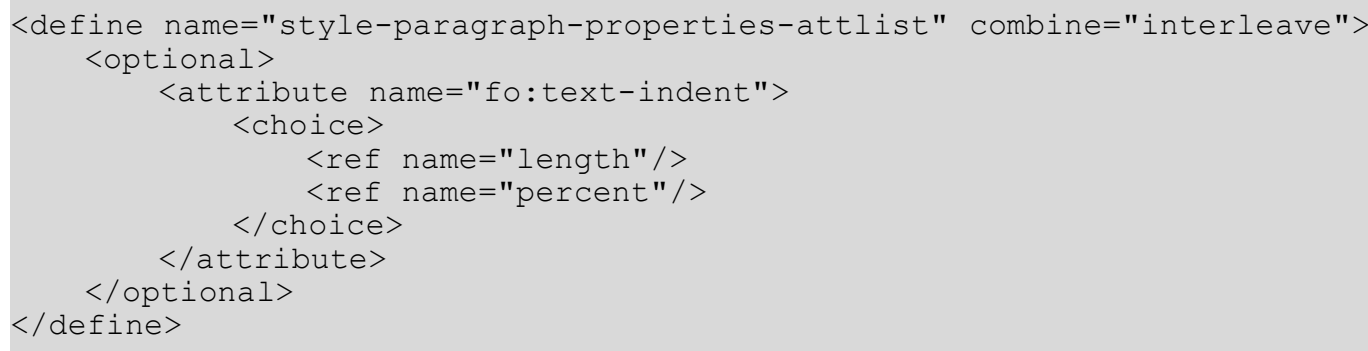

\subsubsection{Automatic Text Indent}

Use the style:auto-text-indent property to specify that the first line of a paragraph is indented by a value that is based on the current font size.

For some applications. the style:auto-text-indent property must be used together with the fo:margin-left and fo:margin-right properties. If any of these properties is missing, its value is assumed to be $0 \mathrm{~cm}$.

If this property has a value of true and is used together with a fo:text-indent property, then the fo:text-indent property is ignored.

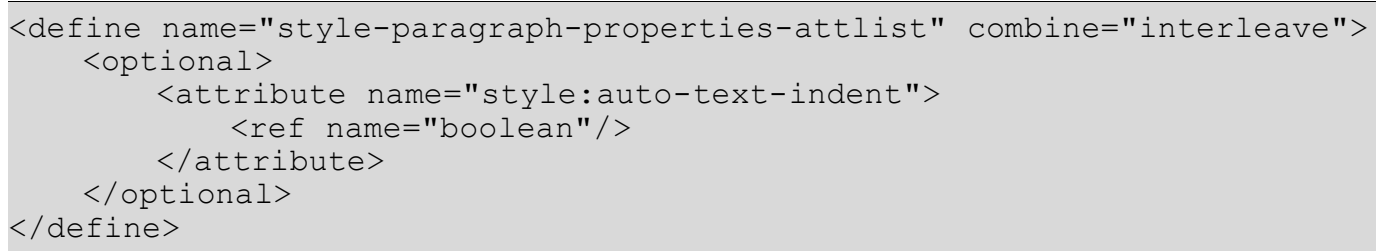

\subsubsection{Top and Bottom Margins}

Use the fo:margin-top and fo:margin-bottom properties to specify the top and bottom margins for paragraphs. See $\S 7.10 .1$ and $\S 7.10 .2$ of [XSL] for details. The value auto is not supported. Percentage values are only supported in common styles. They here relate to the corresponding margin of the parent style.

For some applications. these two properties must be used simultaneously. If any of the properties is missing, its value is assumed to be $0 \mathrm{~cm}$.

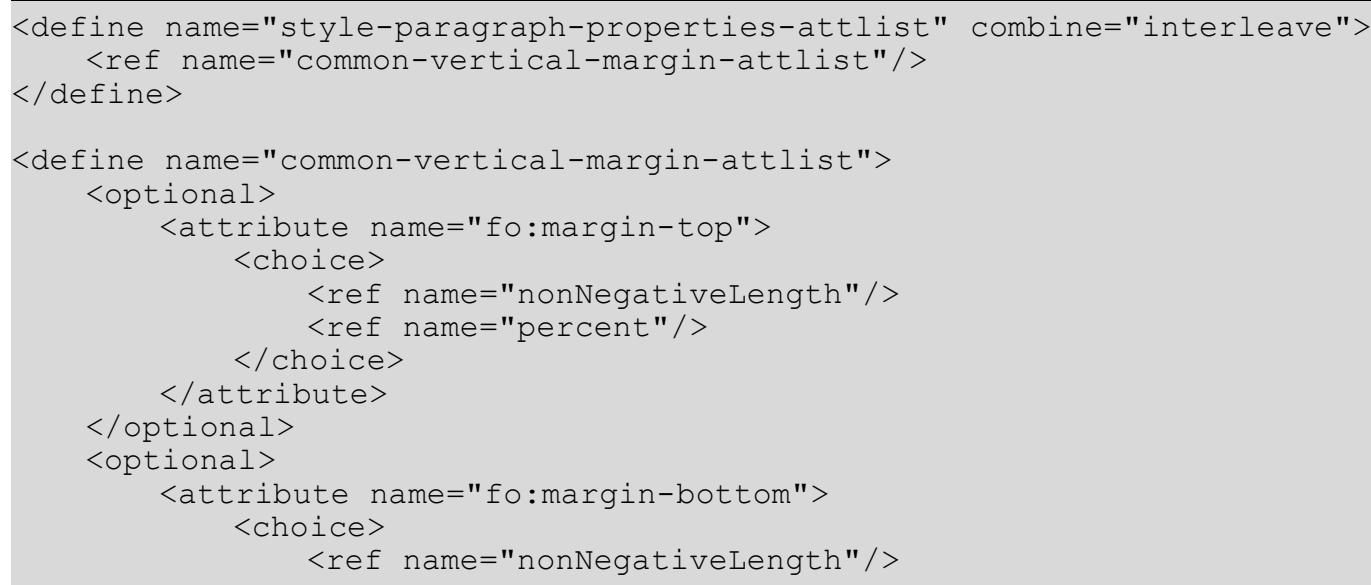




<ref name="percent"/>
$</$ choice $>$
$</$ attribute $>$
$</$ optional $>$
$</$ define $>$

\subsubsection{Margins}

Use the fo:margin property to specify the top, bottom, left and right margins for paragraphs simultaneously. See §7.29.4 of [XSL] and sections 15.5.17 and 15.5.20 for details.

14606

14607

14608

14609
14610

14611

14612

14613

14614

14615

14616

14617

14618

14619

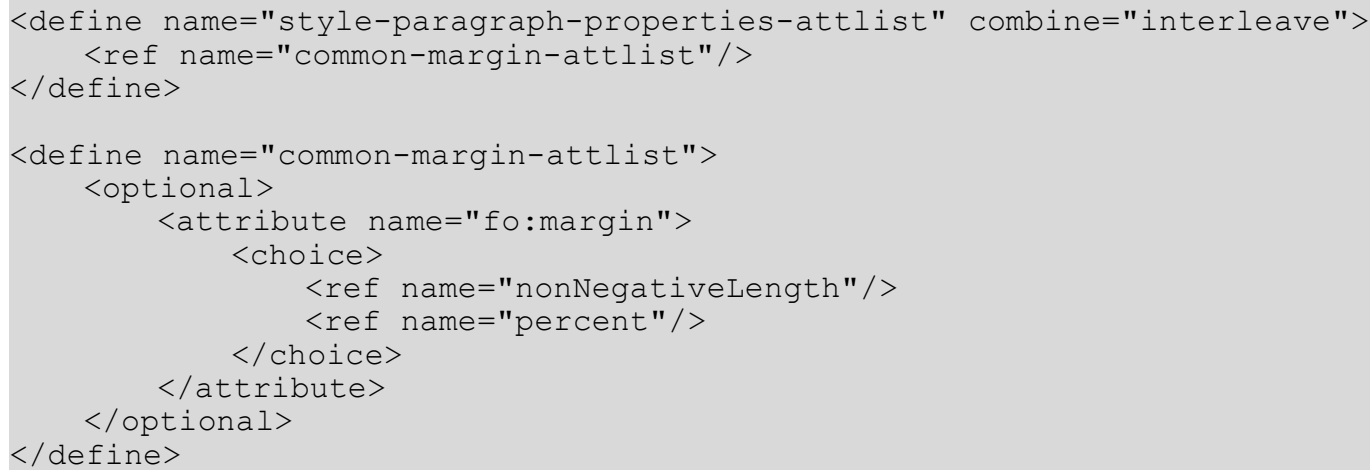

\subsubsection{Break Before and Break After}

Use the fo:break-before and fo:break-after properties to insert a page or column break before or after a paragraph. See $\S 7.19 .1$ and $§ 7.19 .2$ of [XSL] for details. The values odd-page and even-page are not supported.

These two properties are mutually exclusive. If they are used simultaneously, the result is undefined.

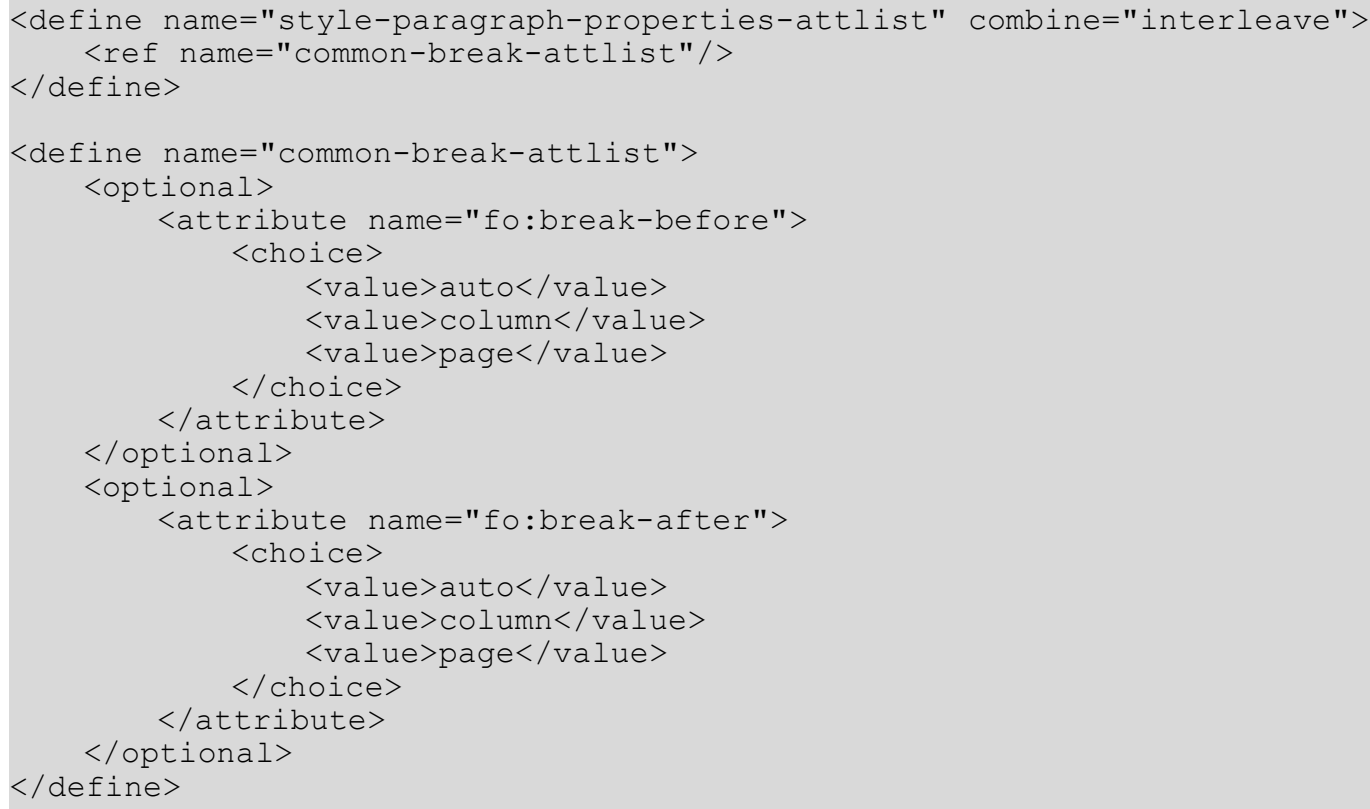




\subsubsection{Paragraph Background Color}

Use the fo:background-color property to specify the background color of a paragraph. See $\S 7.7 .2$ of $[\mathrm{XSL}]$ for details.

The value of this attribute can be either transparent or it can be a color. If the value is transparent, it switches off any background image that is specified by a

<style:background-image> element simultaneously.

14644

14645

14646

14647

14649

14650

14651

14652

14653

14654

14655

14656

14657

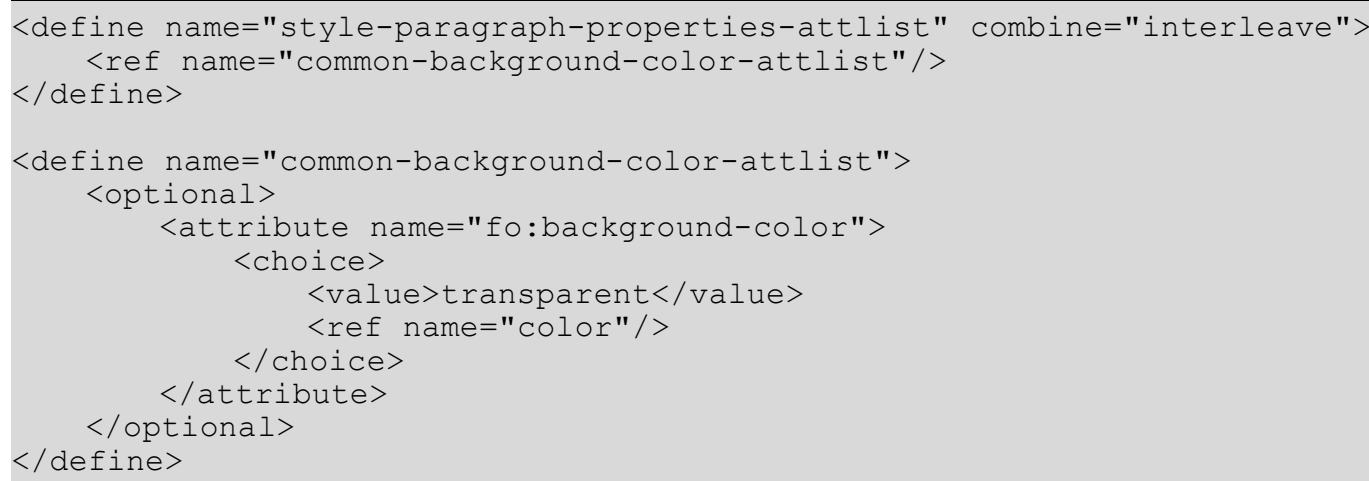

\subsubsection{Paragraph Background Image}

Use the <style:background-image> element to specify a background image for a paragraph.

The background image can be stored in one of the following ways (see also section 9.3.2):

- The image data is stored in an external file. Use the [XLink] attributes to specify the location of the image.

- The image data is contained in an <office:binary-data> sub element in BASE64 encoding.

If the <style:background-image> element is empty and if there is no color specified by an fo:background-color element in the same properties element, the background color is set to transparent.

14658

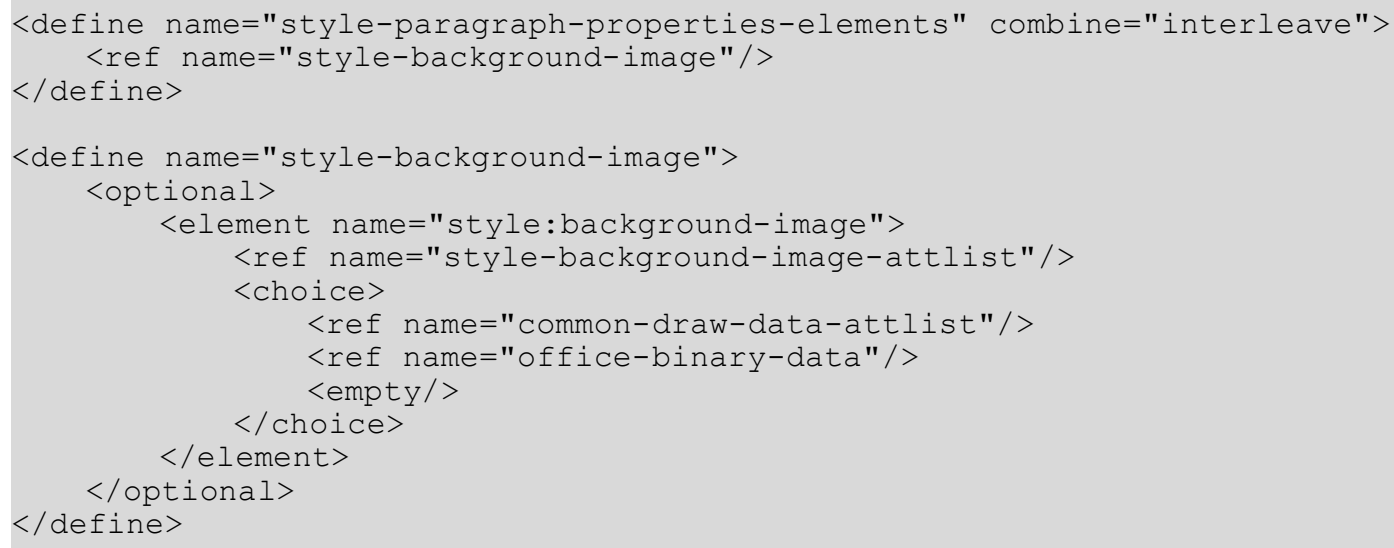

The attributes that may be associated with the <style:background-image> element are:

- Repetition

- Position 
- Filter

- Opacity

\section{Repetition}

The style:repeat attribute specifies whether a background image is repeated or stretched in a paragraph.

This attribute is attached to the <style:background-image $>$ element and its value can be no-repeat, repeat, or stretch.

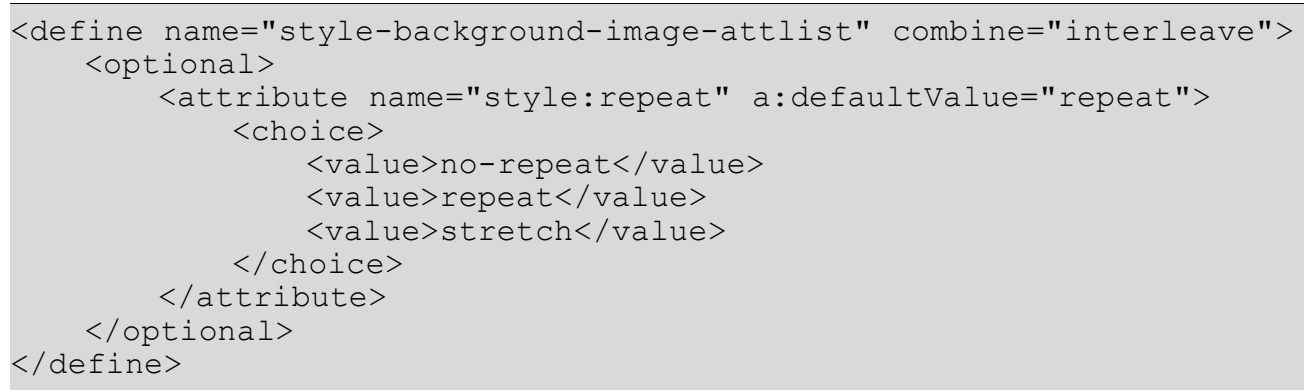

\section{Position}

The style:position attribute specifies where to position a background image in a paragraph.

This attribute is attached to the <style:background-image> element and its value can be a space separated combination of top, center or bottom for the vertical position and left, center or right for the horizontal position. The vertical and horizontal positions can be specified in any order. If one position is specified, the other position defaults to center.

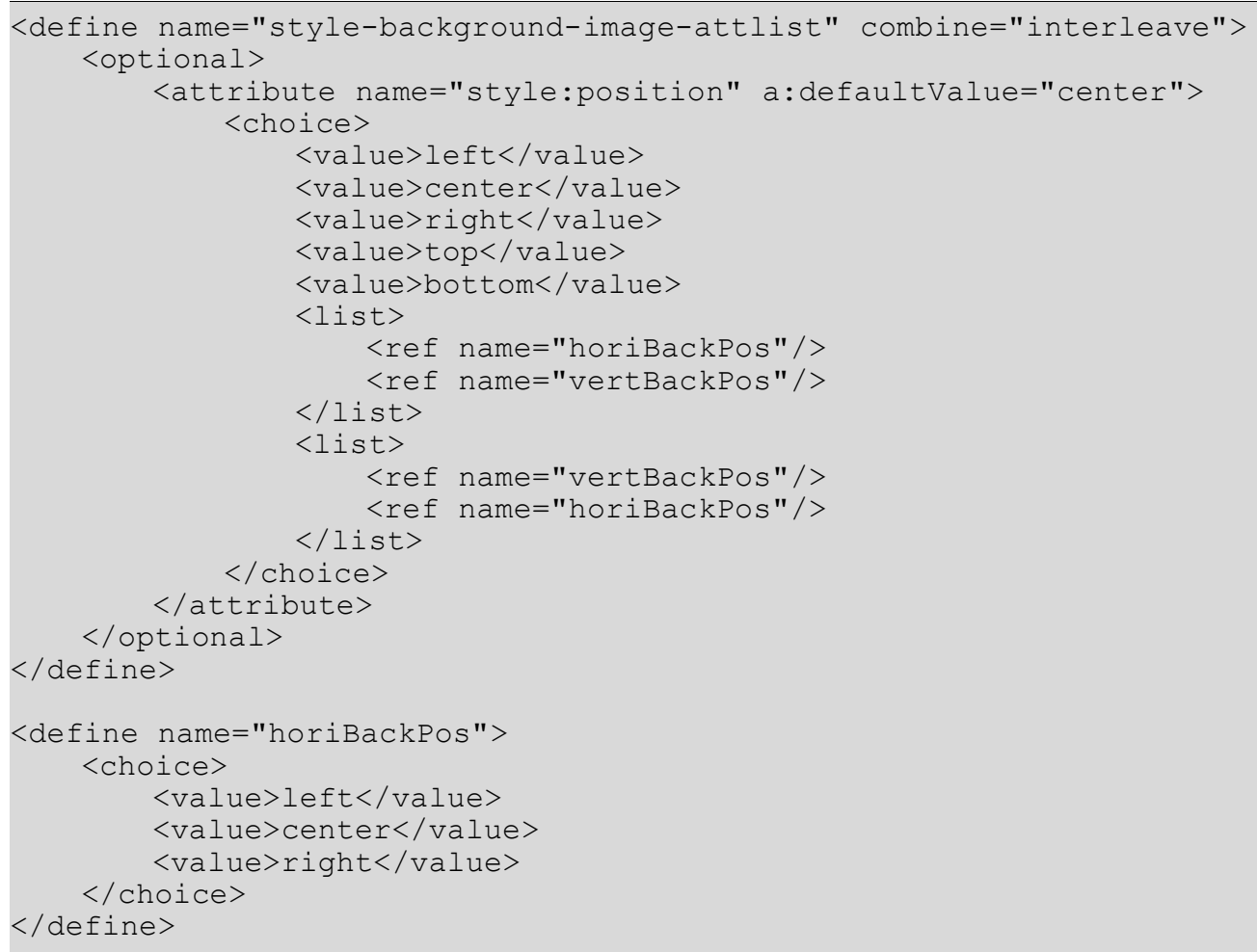




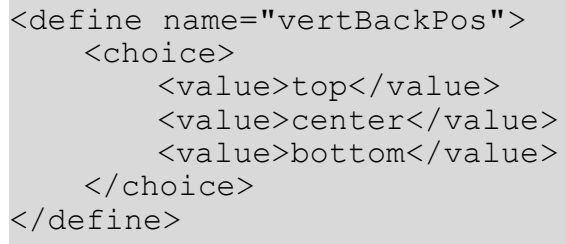

\section{Filter}

The style:filter-name attribute specifies the application specific filter name that is used to load the image into the document.

This attribute is attached to the <style:background-image> element.

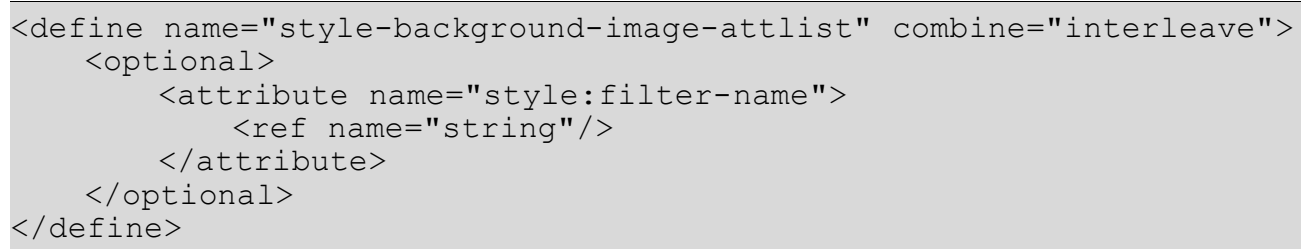

\section{Opacity}

The draw: opacity attribute specifies the opacity of the background image. The value is a percentage, where $0 \%$ is fully transparent and $100 \%$ is fully opaque.

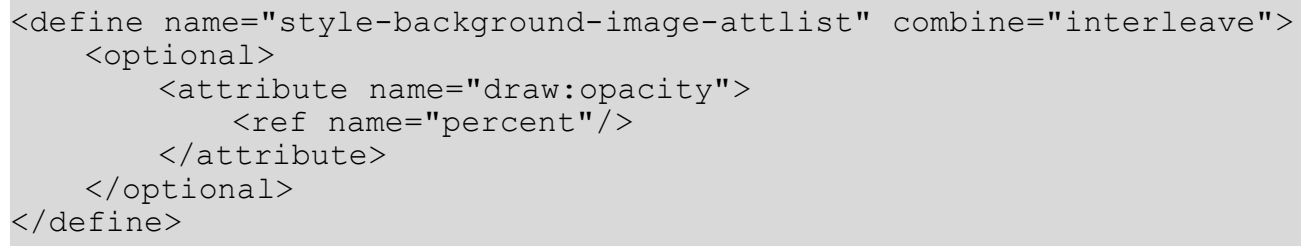

\subsubsection{Border}

Use the border properties fo:border, fo:border-top, fo:border-bottom, fo:borderleft and fo:border-right to specify the border properties for paragraphs. See $§ 7.29 .3$ $\S 7.29 .7$ of $[\mathrm{XSL}]$ for details.

The fo:border property applies to all four sides of a paragraph while the other properties apply to one side only.

For some applications, all four borders must be set simultaneously by using either the fo:border property or by attaching all four of the other border properties to a properties element. In the latter case, if one or more of the properties is missing their values are assumed to be none.

There may be also restriction regarding the border styles and widths that are supported. In addition to this, some applications may add a default padding for sides that have a border.

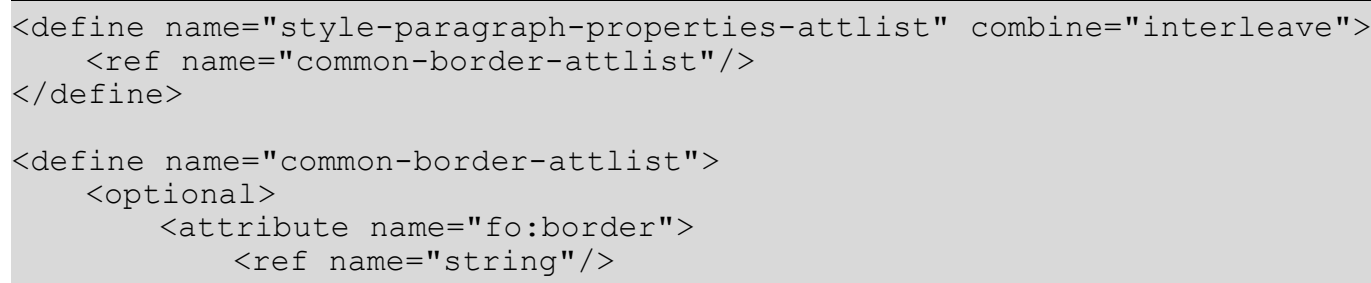




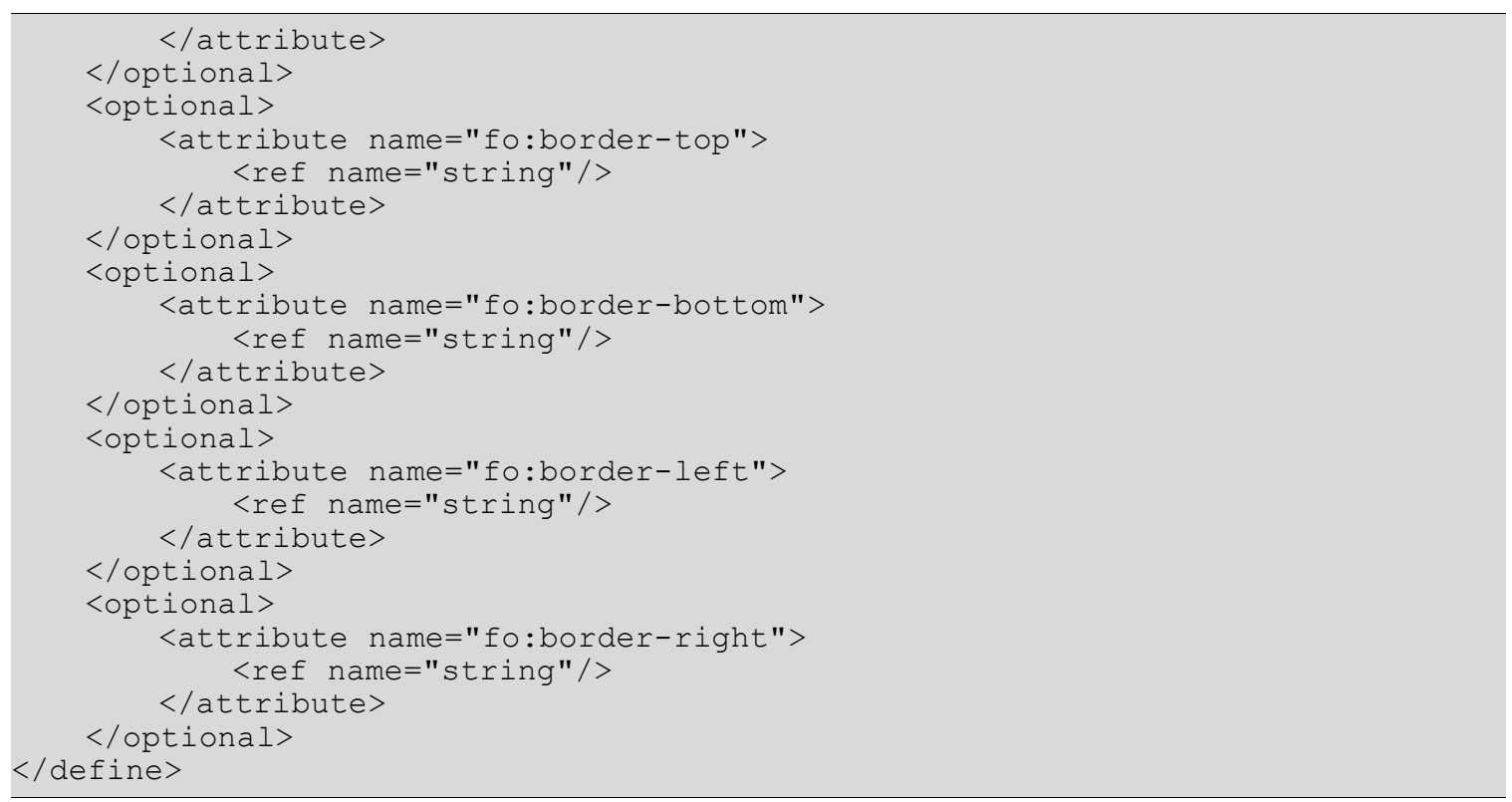

\subsubsection{Border Line Width}

If the line style for a border is double, use the border line properties style:border-linewidth, style:border-line-width-top, style:border-line-width-bottom, style:border-line-width-left and style:border-line-width-right to individually specify the width of the inner and outer lines and the distance between them.

The style:border-line-width specifies the line widths of all four sides, while the other attributes specify the line widths of one side only.

The value of the attributes can be a list of three space-separated lengths, as follows:

- The first value specifies the width of the inner line

- $\quad$ The second value specified the distance between the two lines

- The third value specifies the width of the outer line

The result of specifying a border line width without specifying a border width style of double for the same border is undefined.

14766

14767

14768

14769

14771

14772

14773

14774

14775

14776

14777

14778

14779

14780

14781

14782

14783

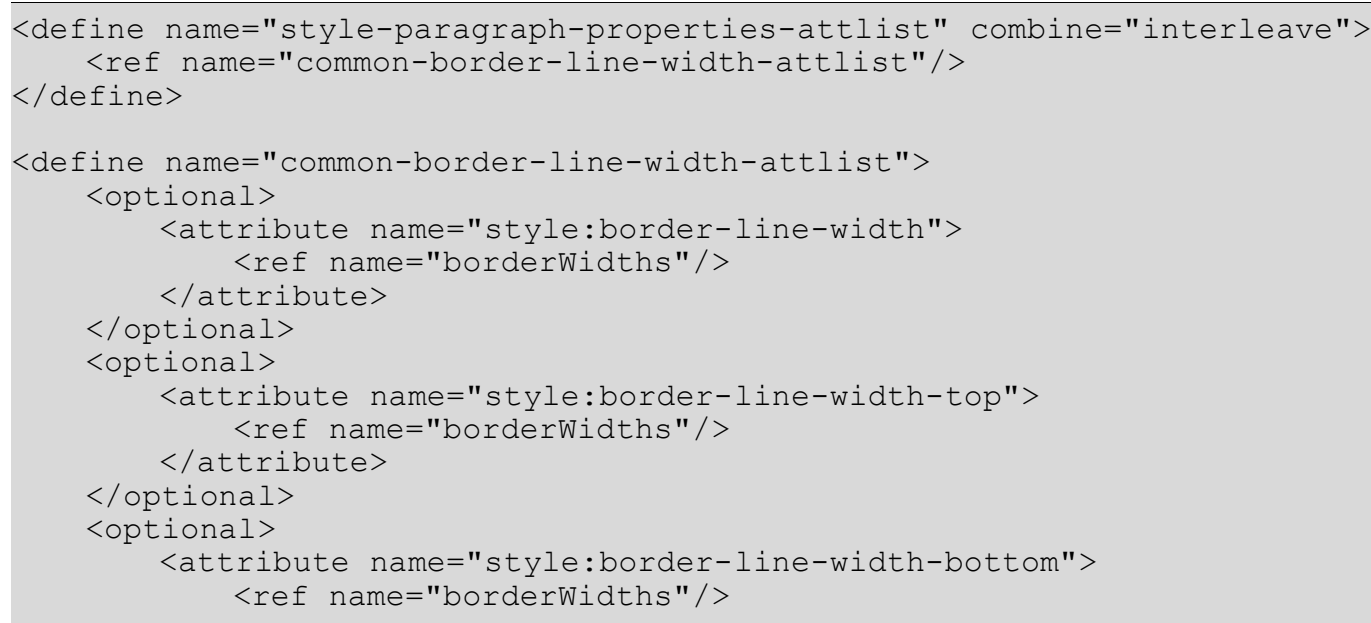




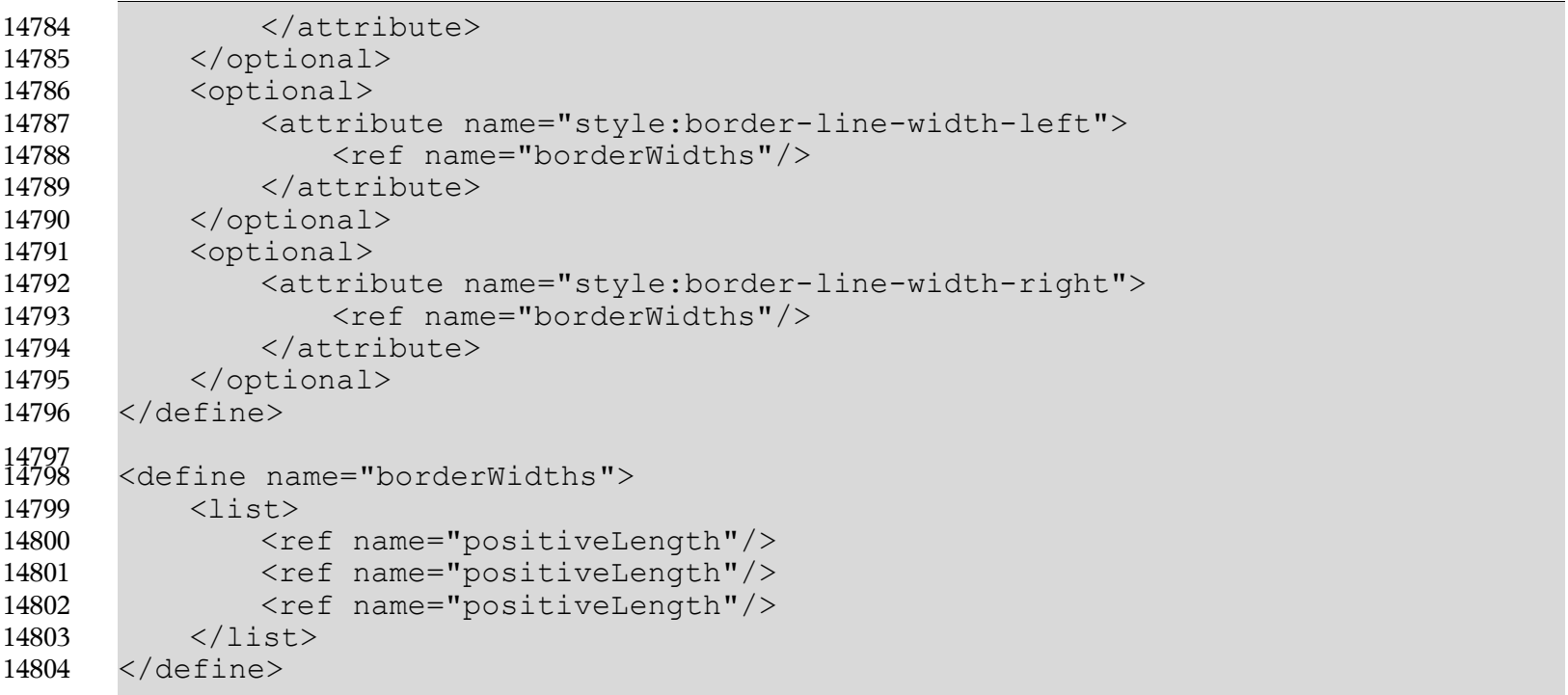

\subsubsection{Padding}

Use the padding properties fo:padding, fo:padding-top, fo:padding-bottom, fo:padding-left and fo:padding-right to specify the spacing around a paragraph. See $\S 7.29 .15$ and $\S 7.7 .35-\S 7.7 .38$ of $[X S L]$ for details.

For some application, the value of these properties can be a non-zero value only if there is a border at the same side and the border is specified within the same properties element. If a properties element contains a padding specification for one but not all four sides, some applications may also assign a zero or a default padding to these sides depending on whether or not there is a border at that side. There might be also other restriction regarding the combination of borders and paddings.

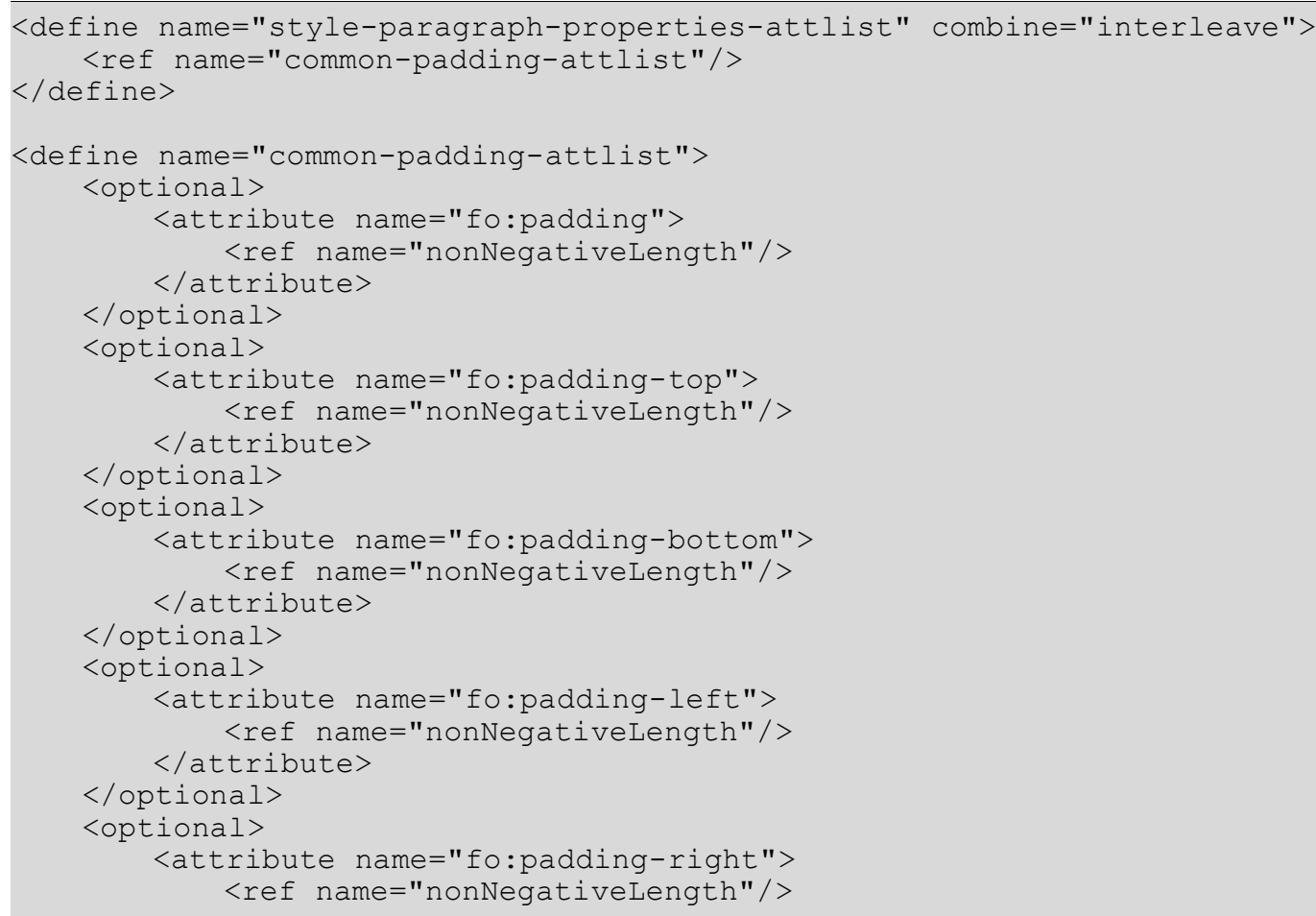




\subsubsection{Shadow}

Use the style:shadow property to specify a shadow effect for the paragraph.

The valid values for this attribute are the same as the values for the fo:text-shadow property. See section 15.4.27 for information.

Some applications may only supports a limited number of shadow effects, for instance only one effect where the the horizontal and vertical positions have the same value.

14836

14837

14838

14839

14841

14842

14843

14844

14845

14846

\section{7}

14848

14849

14850

14852

14853

14854

14855

14856

14857

14858

14859

14860

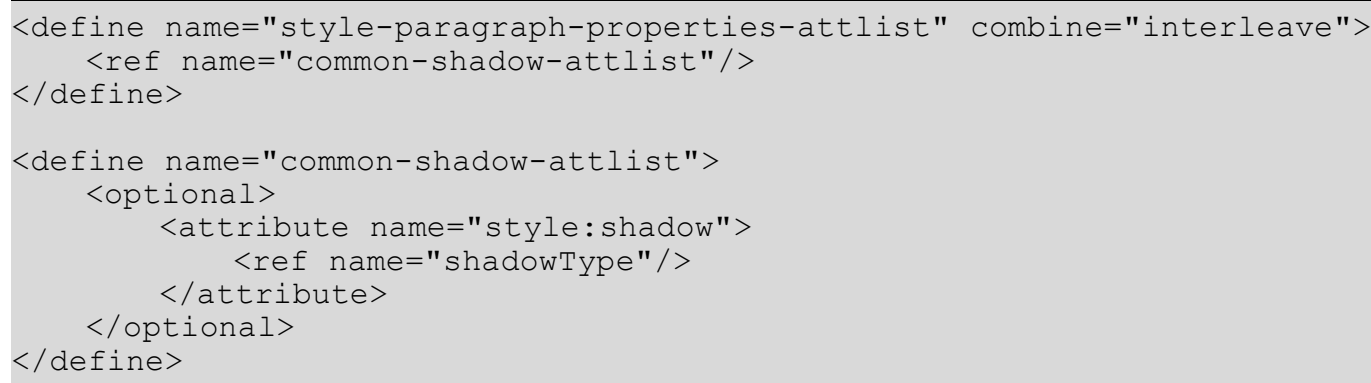

\subsubsection{Keep with Next}

Use the fo:keep-with-next property to specify whether or not to keep the current paragraph and the next paragraph together on a page or in a column after a break is inserted. See \$7.9.14 of [XSL] for details. The only supported values are auto and a lways.

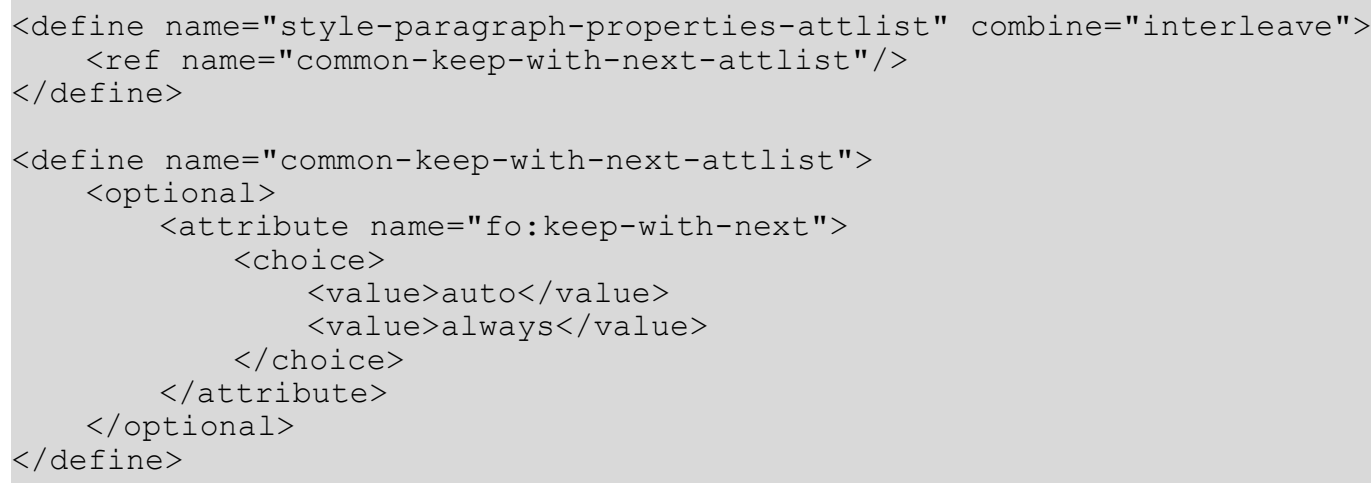

\subsubsection{Line Numbering}

The text: number-lines attribute controls whether or not lines are numbered.

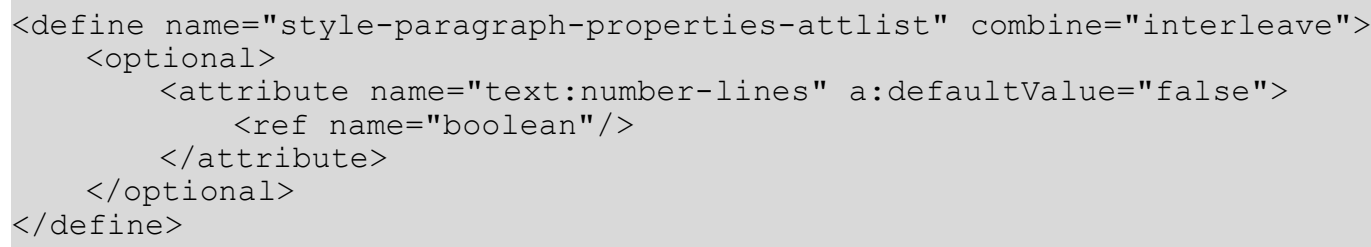




\subsubsection{Line Number Start Value}

The text:line-number property specifies a new start value for line numbering. The attribute is only recognized if there is also a text:number-lines attribute with a value of true in the same properties element.

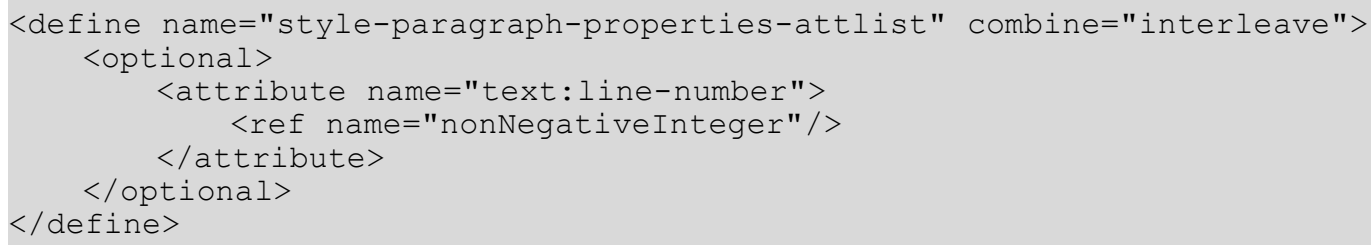

\subsubsection{Text Autospace}

Use the style:text-autospace property to specify whether to add space between Asian, western, and complex text.

The possible values are none and ideograph-alpha.

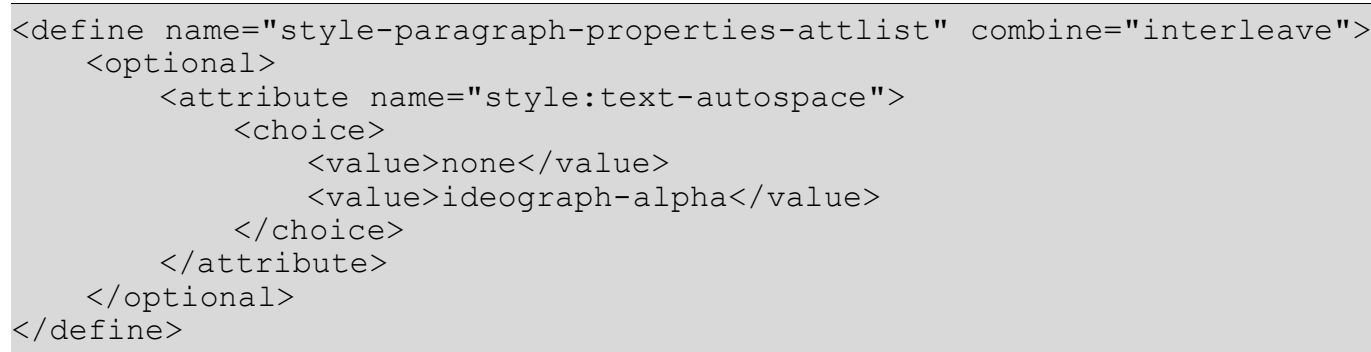

\subsubsection{Punctuation Wrap}

Use the style:punctuation-wrap property to determine whether or not a punctuation mark, if one is present, can be hanging, that is, whether it can placed in the margin area at the end of a full line of text. This is a common setting in East Asian typography.

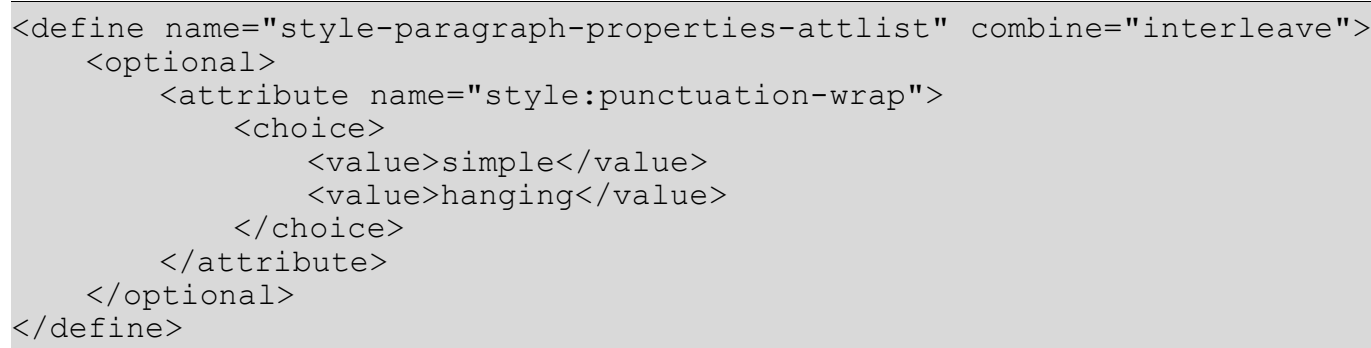

\subsubsection{Line Break}

Use the style: line-break property to select the set of line breaking rules to use for text. If the value is strict, line breaks are forbidden between certain user and application configurable characters. If the value is normal, line breaks may occur between arbitrary characters.

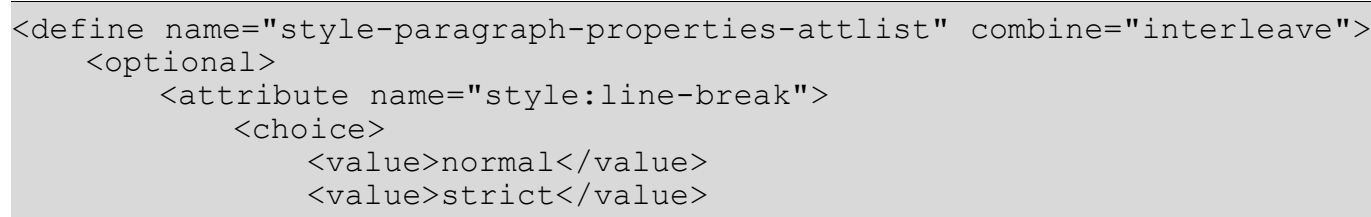




\subsubsection{Vertical Alignment}

The style:vertical-align property specifies the vertical position of a character. By default characters are aligned according to their baseline, which is the default for most European languages. This is also the alignment used in this specification. Alternatively, characters may be vertically aligned as follows:

- bottom - To the bottom of the line.

- top -To the top of the line.

- middle -To the center of the line.

- auto - Automatically, which sets the vertical alignment to suit the text rotation. Text that is rotated 0 or 90 degrees is aligned to the baseline, while text that is rotated 270 degrees is aligned to the center of the line.

The following graphic illustrates the effect of the vertical alignment property when it is set to baseline, top, bottom, and center respectively.

\section{mgk mgk mgk mgk mgk mgk mgk mg]}

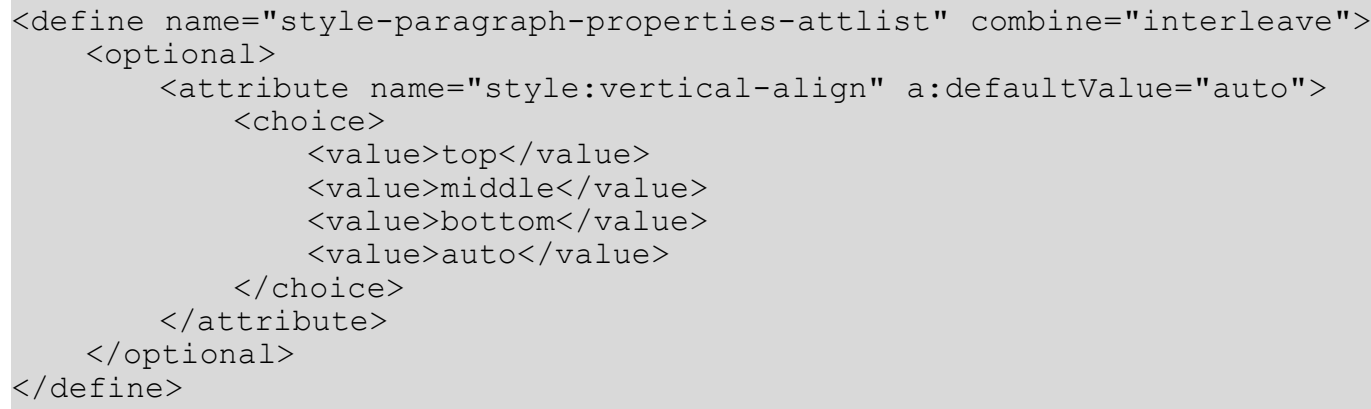

\subsubsection{Writing Mode}

The style:writing mode attribute specifies the writing mode of a paragraph. The attribute is similar to the writing-mode attribute specified in §7.27.7 of [XSL], except hat it has the additional value page. This value specifies that the writing mode is inherited from the page that contains the paragraph.

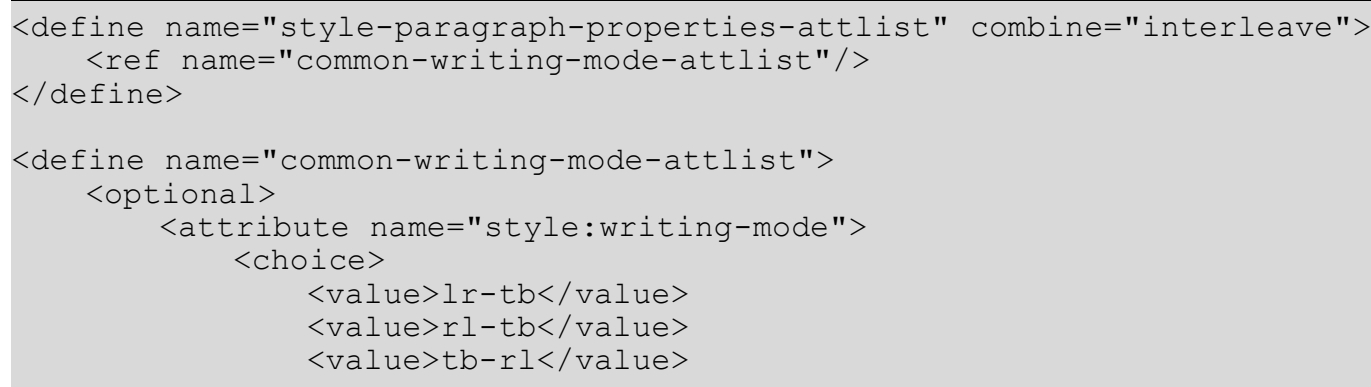




\subsubsection{Automatic Writing Mode}

If the style:writing-mode-automatic attribute is given for a paragraph and if its value is true, then an application is allowed to recalculate the writing mode of the paragraph based on it's content whenever the content changes. The actual value for the writing-mode should be contained in style:writing-mode attribute, so that applications that do not support an automatic writing mode calculation or use a different algorithm always know the actual value.

By specifying a fo:text-align='start' attribute additionally, the text alignment can be adapted to the writing mode simultaneously.

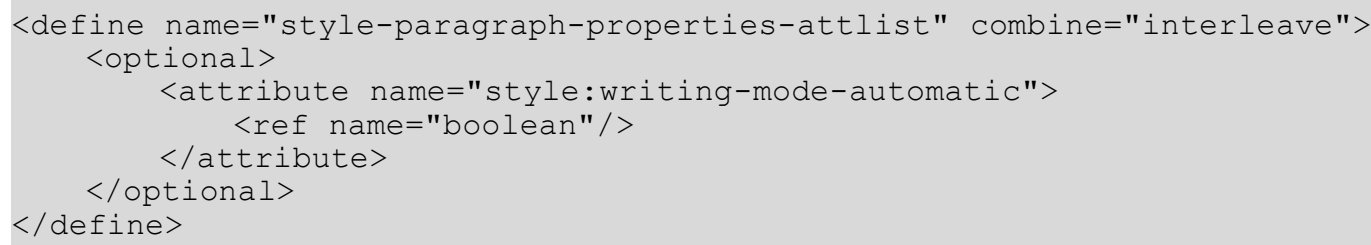

\subsubsection{Snap To Layout}

The style:snap-to layout-grid attribute specifies whether the paragraph should consider the layout grid settings of the page. See section 15.2.21.

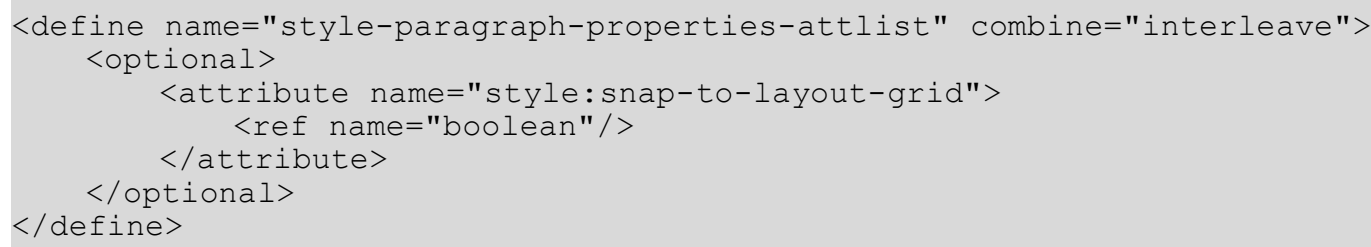

\subsubsection{Page Number}

If a paragraph style specifies a master page that should be applied beginning from the start of the paragraph, the style:page-number attribute specifies the page number that should be used for new page.

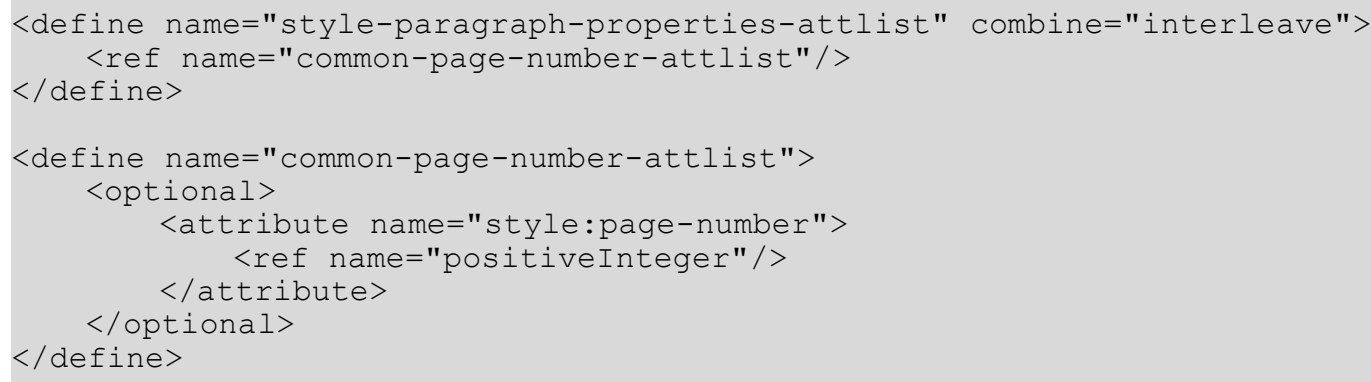




\subsubsection{Background Transparency}

The properties described in this section can be used within ruby styes (see section 14.8.4 for details). They are contained in a <style:ruby-properties $>$ element.

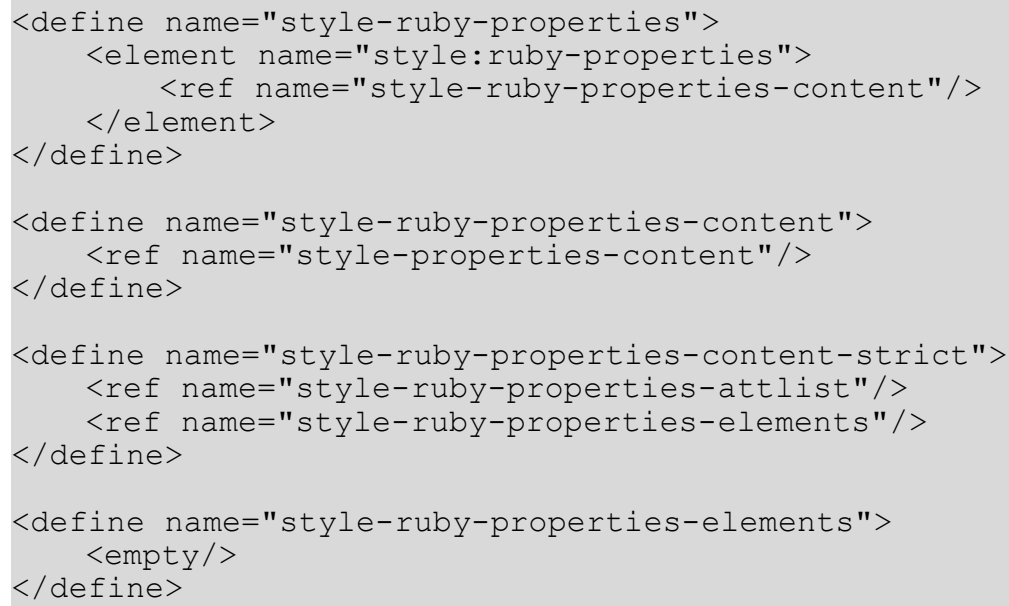

\subsubsection{Ruby Position}

This property specifies the position of the ruby text relative to the ruby base.

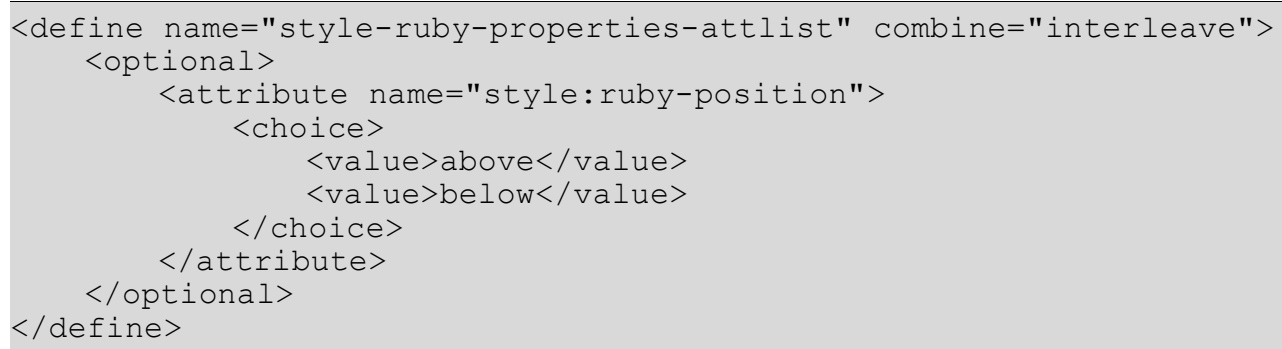

$</$ optional $>$

\subsubsection{Ruby Alignment}

This property specifies the alignment of the ruby text relative to the ruby base.

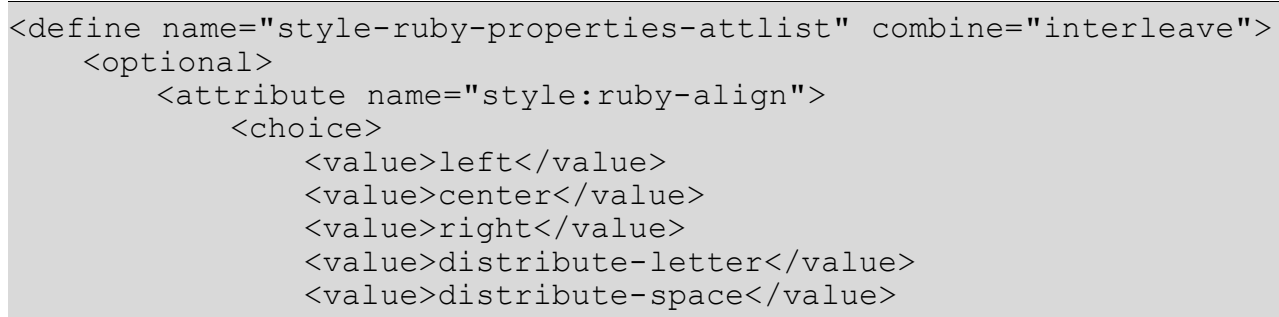




\subsection{Section Formatting Properties}

The properties described in this section can be used within section styes (see section 14.8.3 for details). They are contained in a <style: section-properties $>$ element.

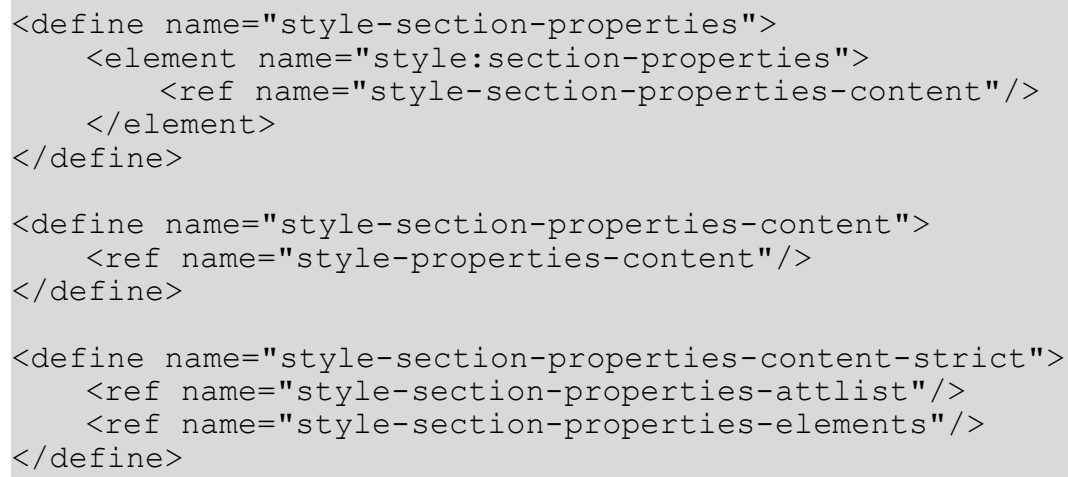

\subsubsection{Section Background}

The background attribute $f \circ$ :background-color and the background element <style:background-image> specify the background properties of the section. See sections 15.5.23 and 15.5.24 for detailed information on this attribute and element.

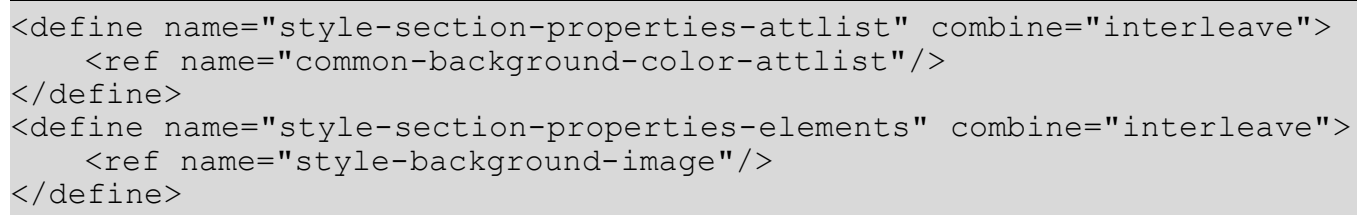

\subsubsection{Margins}

The margins attributes fo:margin-left and fo:margin-right specify the size of the section margins. See sections 15.5 .17 for detailed information on these attributes. Percentage values are not supported.

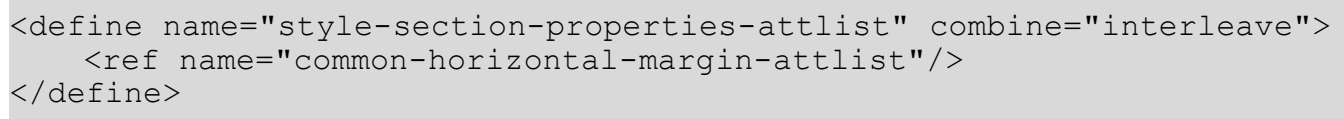

\subsubsection{Columns}

The <style:columns > element contains <style:column> elements that specify each column individually (see section 15.7.4). If these elements are not present, all columns are assigned the same width.

The <style: columns > can contain a <style:column-sep> element that describes the separator line between columns. See section 15.7 .5 for information on this element. 
$</$ define $>$

<ref name="style-columns"/>

$</$ define $>$

<define name="style-columns">

<optional>

<element name="style:columns">

<ref name="style-columns-attlist"/>

<optional>

<ref name="style-column-sep"/>

$<$ /optional>

$<$ zeroorMore>

<ref name="style-column"/>

$</ e l e m e n t>$

$</$ optional $>$

The attributes that may be associated with the <style:columns $>$ element are:

- Column count

- Column gap

\section{Column Count}

The fo: columns-count attribute specifies the number of columns in a section.

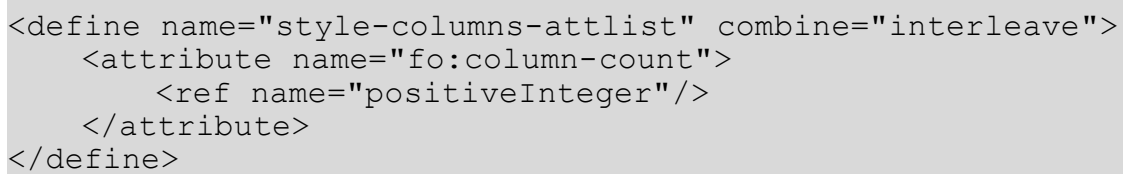

Note: This attribute has the same name as an [XSL] property but it is attached to a different element.

\section{Column Gap}

If the <style:columns $>$ element does not contain individual $<$ style:column $>$ elements, then the gap between columns may be specified by the fo:column-gap attribute. If there are individual column elements, this attribute is ignored.

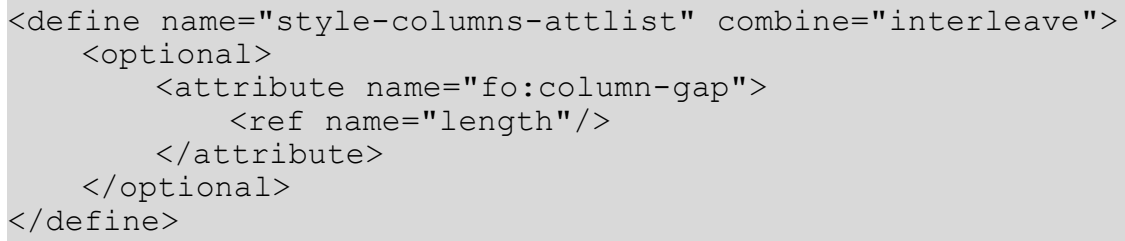

Note: This attribute has the same name as an [XSL] property but it is attached to a different element.

\subsubsection{Column Specification}

The <style:column $>$ element can be contained in a $\langle$ style:columns $>$ element, to specify details of an individual column. This element is contained in the $\langle$ styles : columns $>$ element. There can be either no column elements or there can be the same number of column elements as specified by the fo: column-count attribute. 


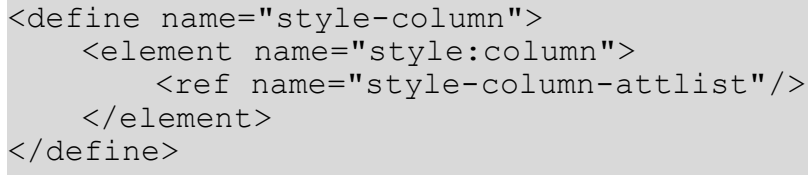

Note: In [XSL], it is not possible to specify columns individually.

The attributes that may be associated with the $\langle$ style: column $>$ element are:

- Column width

- Column left, right, upper, and lower space

\section{Column Width}

Use the style:rel-width attribute to specify the width of a column. The column widths are specified as number values instead of lengths. To get the absolute column width, the space that is available for a columned area is distributed among the columns proportional to these numbers.

The column width is not specified in a percentage length, but rather in terms of relative weights, that is, a number followed by $a^{\prime * \prime}$ character. The total space available for the entire table is distributed among its columns according to its relative widths. For example, if three columns are assigned the relative widths 1,2 and 3 , then the first column will take up $1 / 6$ of the available width, the second will take up $1 / 3$, and the last column will take up $1 / 2$ of the available space. To achieve these figures, all given relative widths must be summed up (six in the example), and then each column will get as much space as the proportion of its own relative width to the sum of all relative widths indicates $(3 / 6=1 / 2$ for the last column in the example).

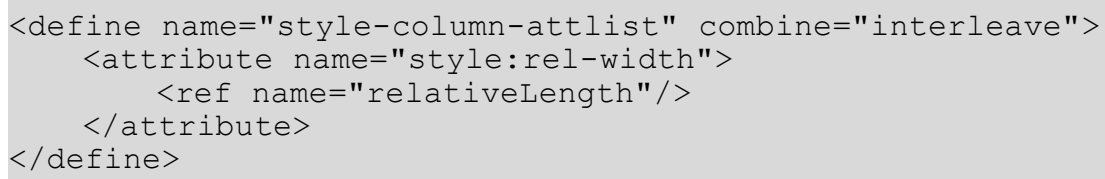

\section{Column Left, Right, Upper, and Lower Space}

For each column, its left, right, upper, and lower space may be specified. The right space of a column together with the left space of the next column corresponds to the gap between two columns. If a columned area contains a separator line between columns, the space that is occupied by the line is contained within the left and right spaces and therefore is not added to them.

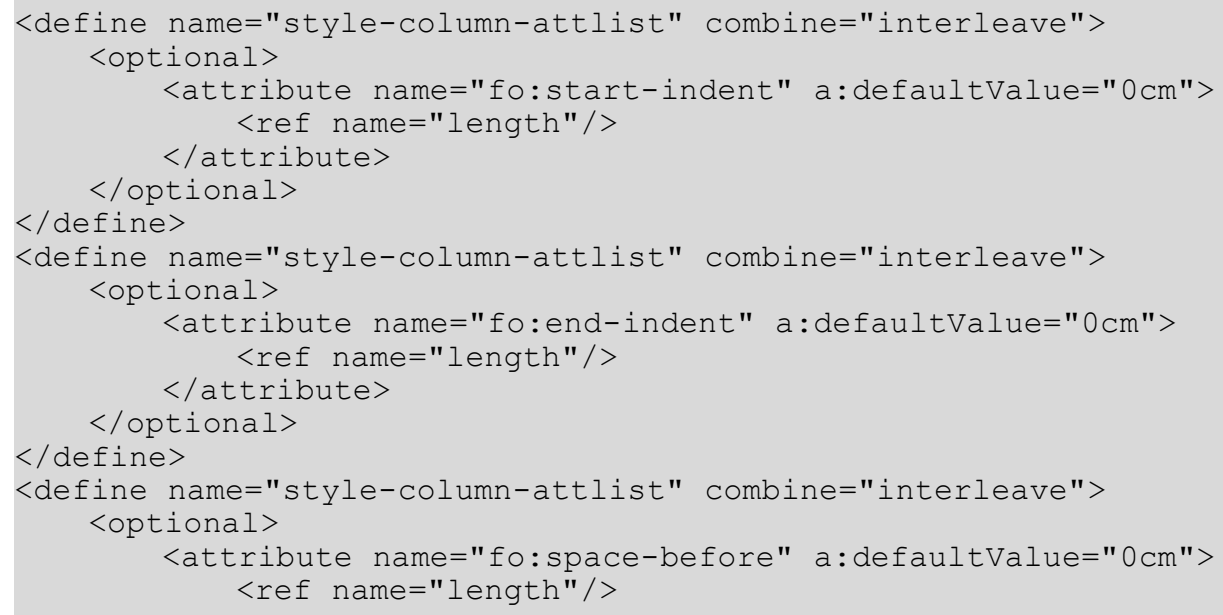




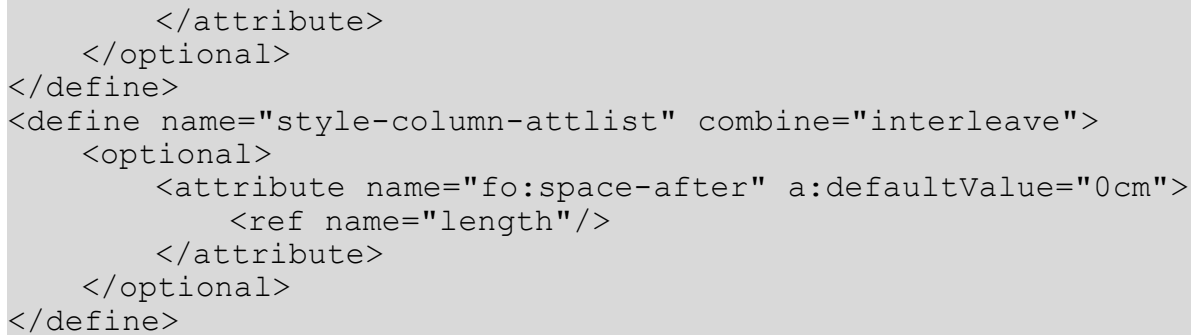

\subsubsection{Column Separator}

The <style:column-sep> element specifies the separator line to use between columns. This element can be contained in a $<$ style:columns $>$ element to specify the type of separator line to use between columns.

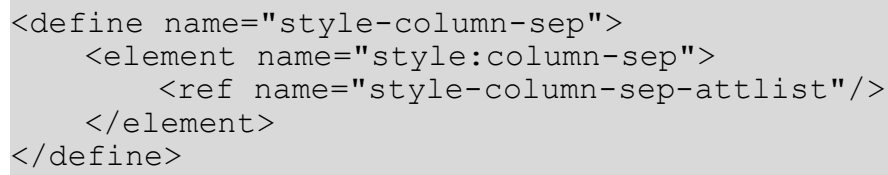

Note: $[X S L]$ does not support column separators.

The attributes that may be associated with the $\langle$ style:column-sep $\rangle$ element are:

- Line style

- $\quad$ Line width

- $\quad$ Line height

- Vertical line alignment

- Line color

\section{Line Style}

Use the style:style attribute to specify the line style of the column separator line.

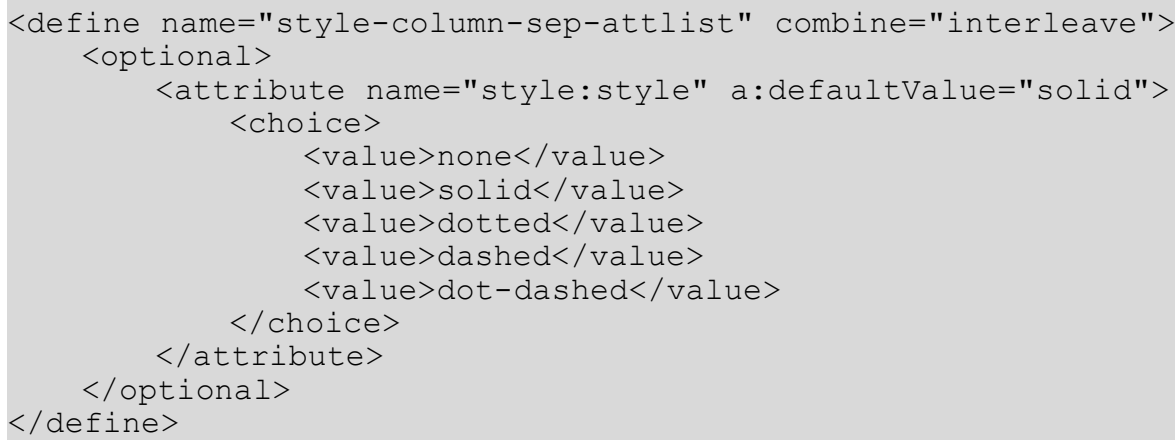

\section{Line Width}

Use the style:width attribute to specify the width of the column separator line. 


\section{Line Height}

Use the style: height to specify the height of the column separator line. The value of this attribute is a percentage that relates to the height of the columned area.

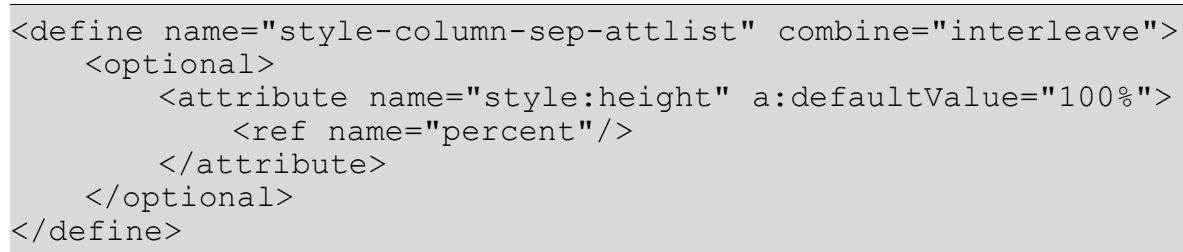

\section{Vertical Line Alignment}

Use the style:vertical-align attribute to specify how to vertically align a line that is less than $100 \%$ of its height within the columned area. The value of this attribute can be either top, middle, or bottom.

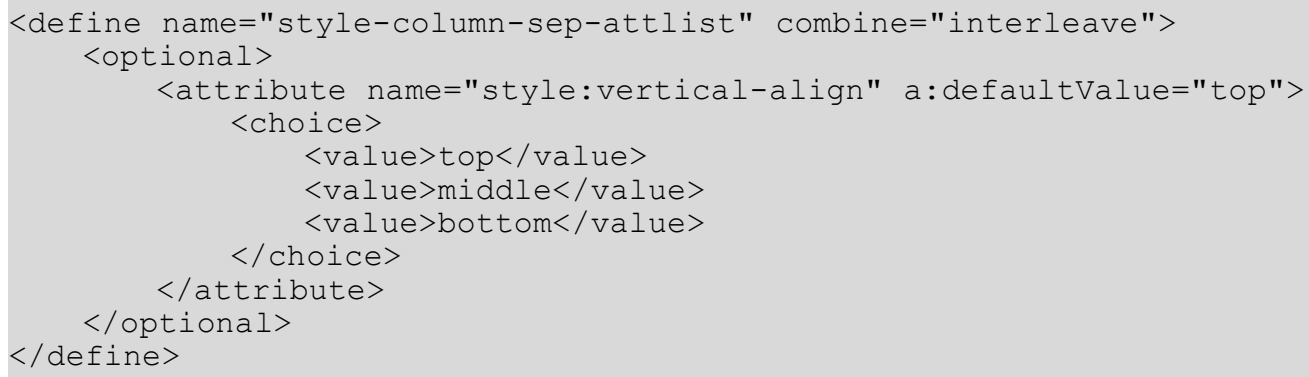

\section{Line Color}

Use the style:color attribute to specify the color of the column separator line.

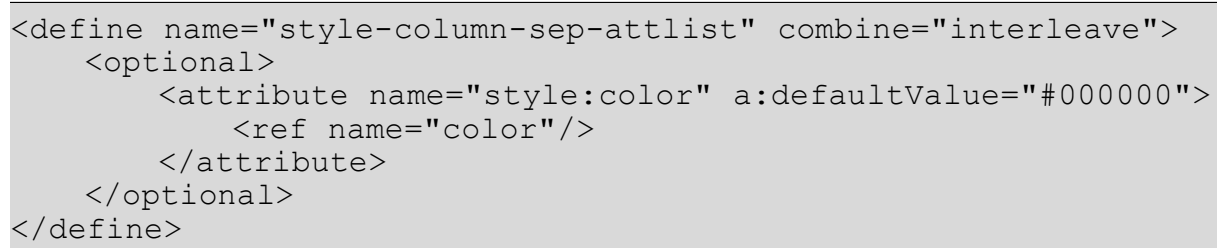

\subsubsection{Protect}

Sections marked with the style: protect attribute should not be changed. The user interface should prevent the user from manually making any changes. The style:protect attribute should be set by default for linked sections or indexes. Removing the protection makes these sections accessible to the user, but updating the links or the index will not preserve the changes.

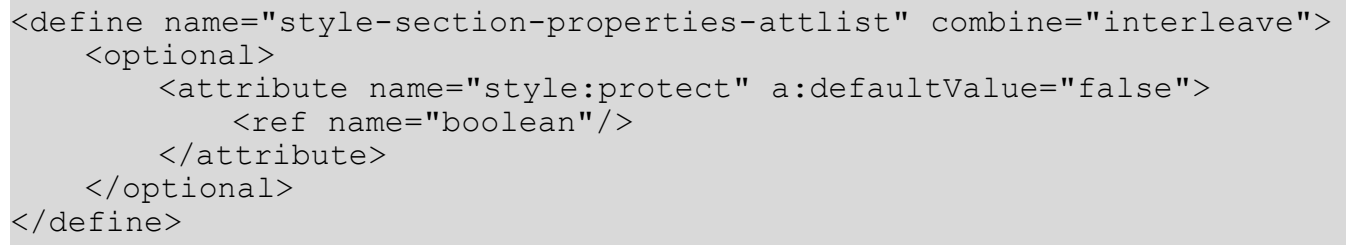




\subsubsection{Don't Balance Text Columns}

The text:dont-balance-text-columns attribute specifies whether the text column content should be evenly distributed over all text columns or not.

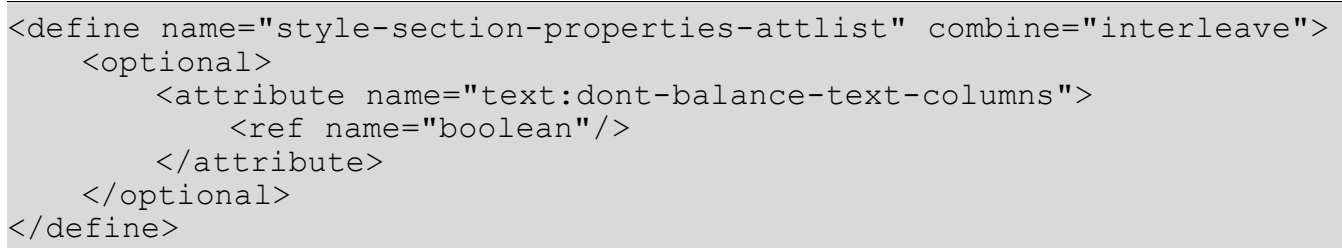

\subsubsection{Writing Mode}

The style:writing-mode attribute specifies the writing mode that should be used for the section. See section 15.5 .36 for details.

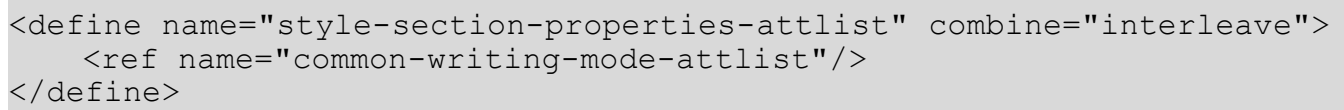

\subsubsection{Notes Configuration}

A section style may contain have its own notes configurations (see section 14.9.2). If this is the case, notes of the corresponding notes type are displayed at the end of the columns of the section or the section itself instead of the end of the page's columns or the end of the document.

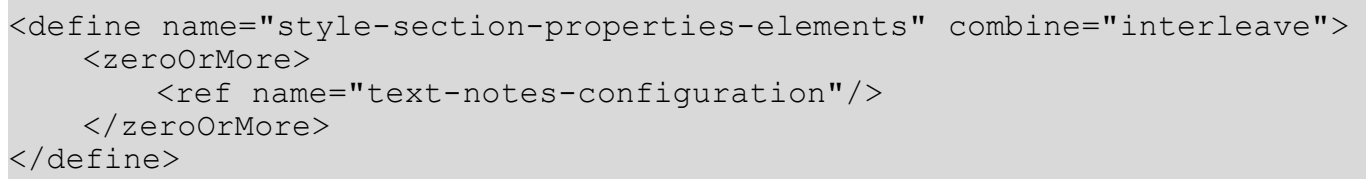

\subsection{Table Formatting Properties}

The properties described in this section can be contained within table styles (see section 14.12.1) They are contained in a $<$ style:table-properties $>$ element.

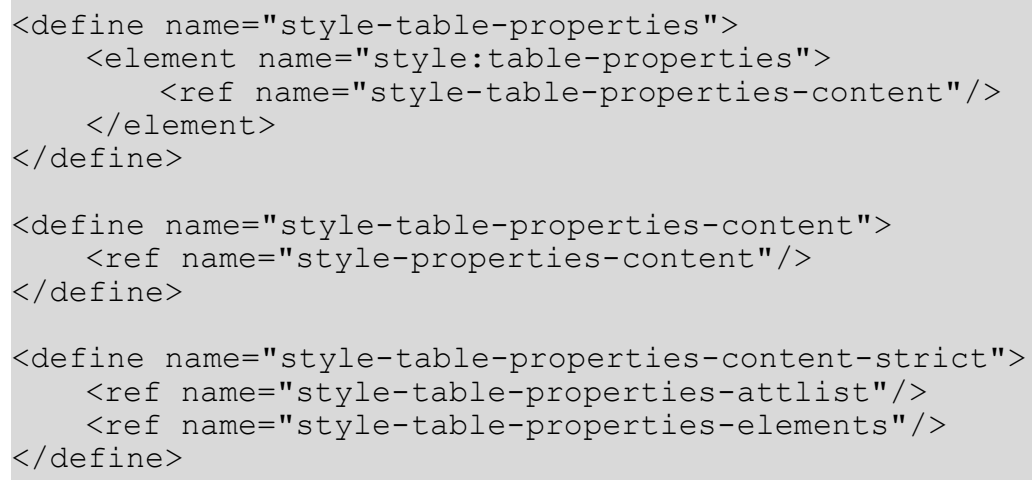

\subsubsection{Table Width}

Every table must have a fixed width. This width is specified by the style:width attribute.

The width of a table may be also specified relative to the width of the area that the table is in. In this case, the width is specified as a percentage using the style:rel-width attribute. User 
agents that support specifying the relative width of a table can specify widths in this way, but it is not essential.

The reasons why every table must have a fixed width and relative widths are only an option are as follows:

- Specifying the width of a table by a percentage is useful for current web browsers and other applications where the percentage is relative to the width of a window. But it may cause problems if the percentage relates to a fixed paper width.

- Relative widths can also cause problems for applications such as spreadsheet applications, where there is no requirement for a table to fit on a page.

However, if an application supports relative widths, it is relatively easy to program the application to calculate a fixed table width, based on a percentage.

\subsubsection{Table Left and Right Margin}

The fo:margin-left and fo:margin-right properties specify the distance of the table from the left and right margins. See section 15.5.17 for a full explanation of left and right margin properties. An application may recognize table margins, but this is not essential. 
Tables that align to the left or to the center ignore right margins, and tables align to the right or to the center ignore left margins.

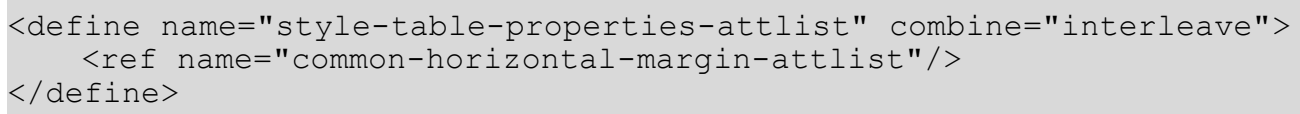

\subsubsection{Table Top and Bottom Margin}

The fo:margin-top and fo:margin-bottom properties specify the distance of the table from the top and bottom. See section 15.5.20 for a full explanation of top and bottom margin properties.

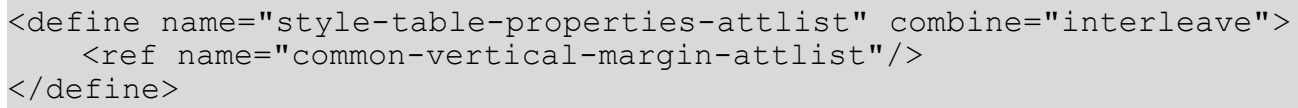

\subsubsection{Table Margins}

The fo:margin property specifies the distance of the table from the left, right, top and bottom.

See section 15.5.21 for a full explanation of this property.

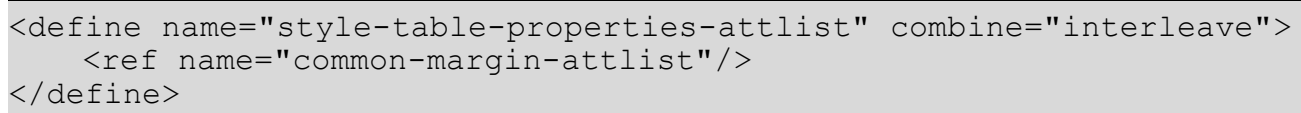

\subsubsection{Page Number}

If the table style specifies a master page that should be applied beginning from the start of the table, the style:page-number attribute specifies the page number that should be used for the first page of the table. See also section 15.5.39.

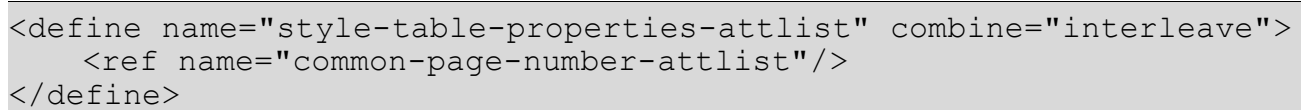

\subsubsection{Break Before and Break After}

The fo:break-before and fo:break-after properties insert a page or column break before or after a table. See section 15.5.22 for a full explanation of these properties.

\subsubsection{Table Background and Background Image}

The background attribute fo:background-color and the background element <style:background-image> specify the background properties of the table. See sections 15.5.23 and 15.5.24 for detailed information on this attribute and element.

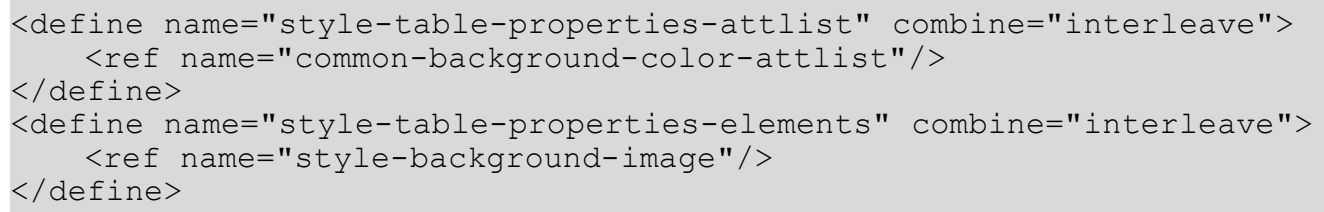




\subsubsection{Table Shadow}

The style:shadow property specifies that a shadow visual effect appears on a table. See section 15.5 .28 for a full explanation of this property.

\subsubsection{Border Model Property}

The table:border-model property specifies what border model to use when creating a table with a border. There are two types of border model, as follows:

\section{- Collapsing border model}

When two adjacent cells have different borders, the wider border appears as the border between the cells. Each cell receives half of the width of the border.

\section{- Separating border model}

Borders appear within the cell that specifies the border.

Both border models are very similar to the collapsing and separating border models of [XSL] and [CSS2]. They differ in how border widths relate to row and column widths.

In OpenDocument, a row height or column width includes any space required to display borders or padding. This means that, while the width and height of the content area is less than the column width and row height, the sum of the widths of all columns is equal to the total width of the table.

In XSL and CSS2, a column width or row height specifies the width or height of the content area of a cell. This means that the sum of the widths of all columns is less than the width of the table. 


\subsubsection{Writing Mode}

The style:writing-mode attribute specifies the writing mode that should is used for the table. See section 15.5 .36 for details.

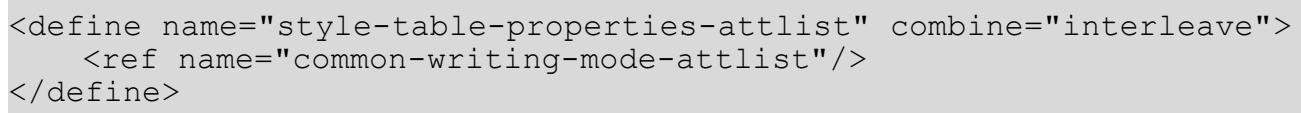

\subsubsection{Display}

The table:display attribute specifies whether or not a table is displayed.

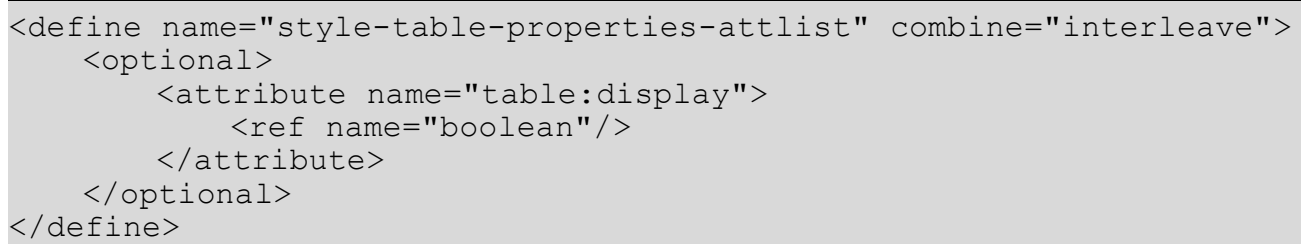

\subsection{Column Formatting Properties}

The properties described in this section can be contained within table column styles (see section 14.12.2) They are contained in a <style:table-column-properties $>$ element.

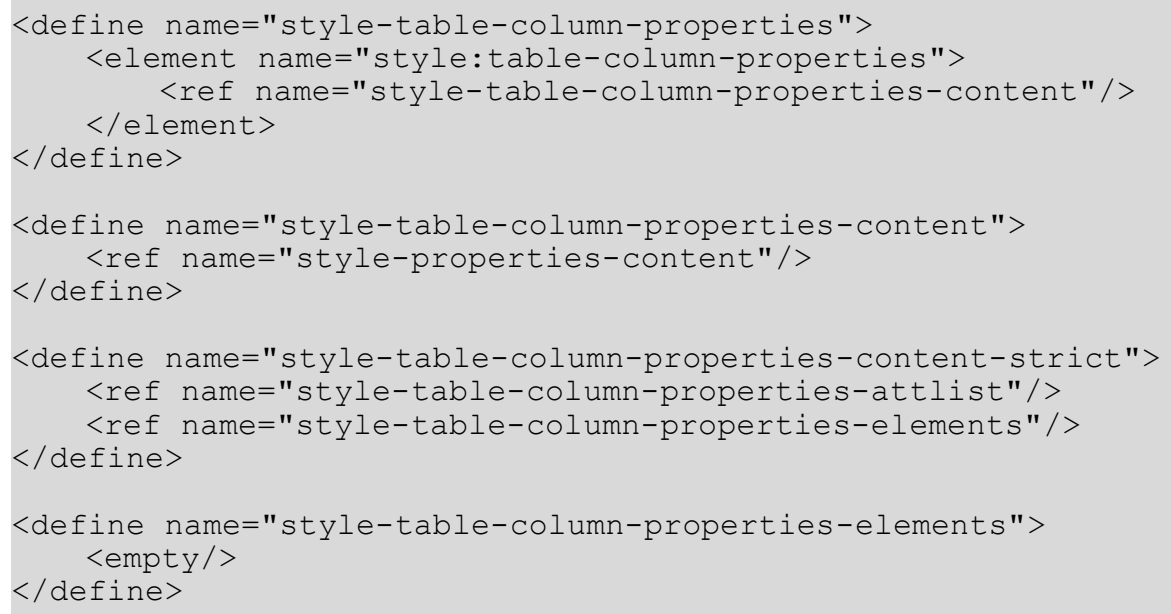

\subsubsection{Column Width}

Every table column must have a fixed width. This width is specified by the style: columnwidth attribute.

The width of a column may be also specified relative to the other column widths. Applications that support specifying the relative width of a column may specify widths in this way, but it is not essential. 
A relative width is specified by the style:rel-column-width property that takes a number value, followed by a ${ }^{1 * 1}$ character. If $r_{c}$ is the relative with of the column, $r_{s}$ the sum of all relative columns widths, and $w_{s}$ the absolute width that is available for these columns, then the absolute with $w_{c}$ of the column is $w_{c}=r_{c} w_{s} / r_{s}$.

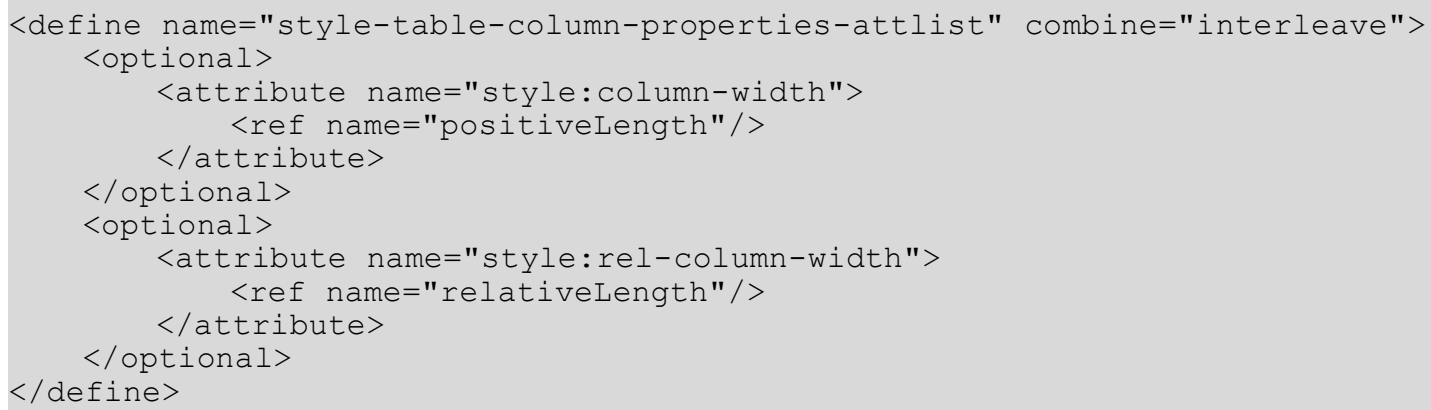

\subsubsection{Optimal Table Column Width}

The style: use-optimal-column-width attribute specifies that the column width should be recalculated automatically if some content in the column changes.

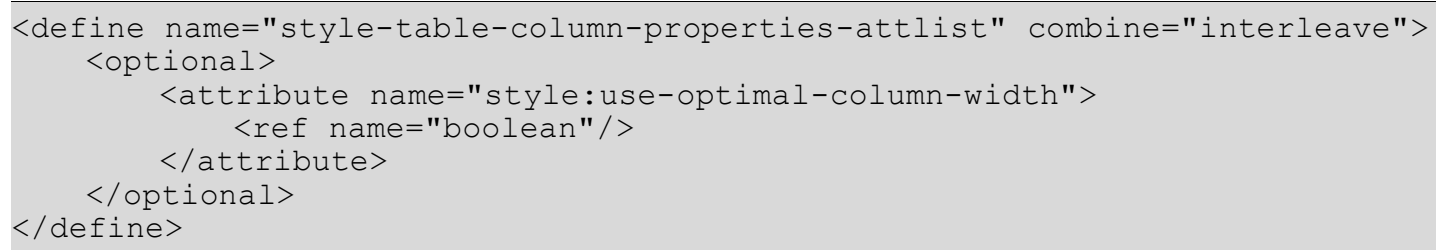

\subsubsection{Break Before and Break After}

The fo:break-before and fo:break-after properties insert a page or column break before or after a table column. See section 15.5.22 for a full explanation of these properties.

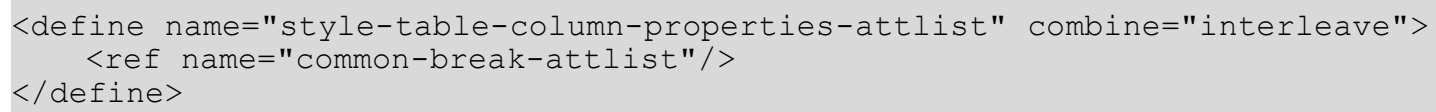

\subsection{Table Row Formatting Properties}

The properties described in this section can be contained within table column styles (see section 14.12.3) They are contained in a <style:table-column-properties> element.

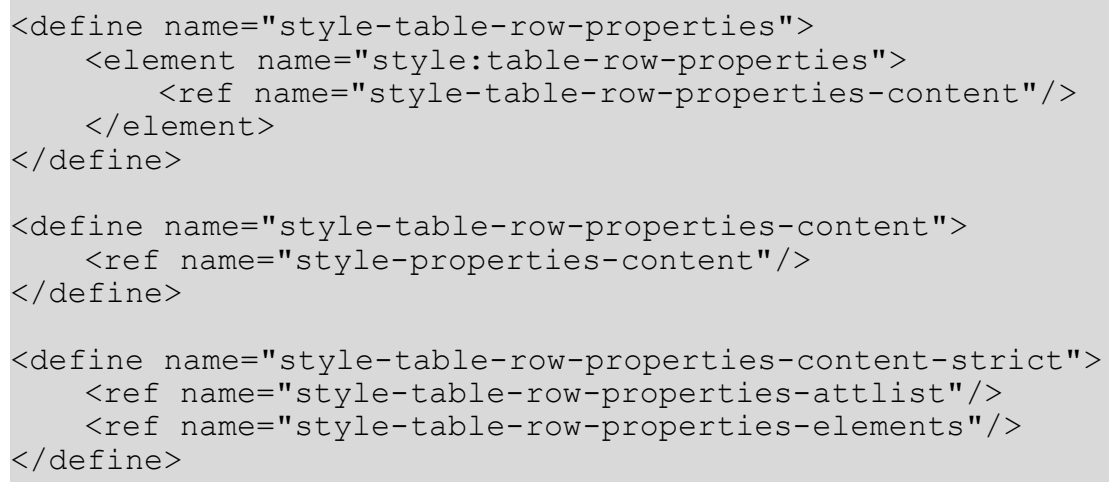




\subsubsection{Row Height}

The style:row-height and style:min-row-height properties specifies the height of a table row. By default, the row height is the height of the tallest item in the row.

The style:row-height property specifies a fixed row height, while the style:min-rowheight property specifies a fixed height.

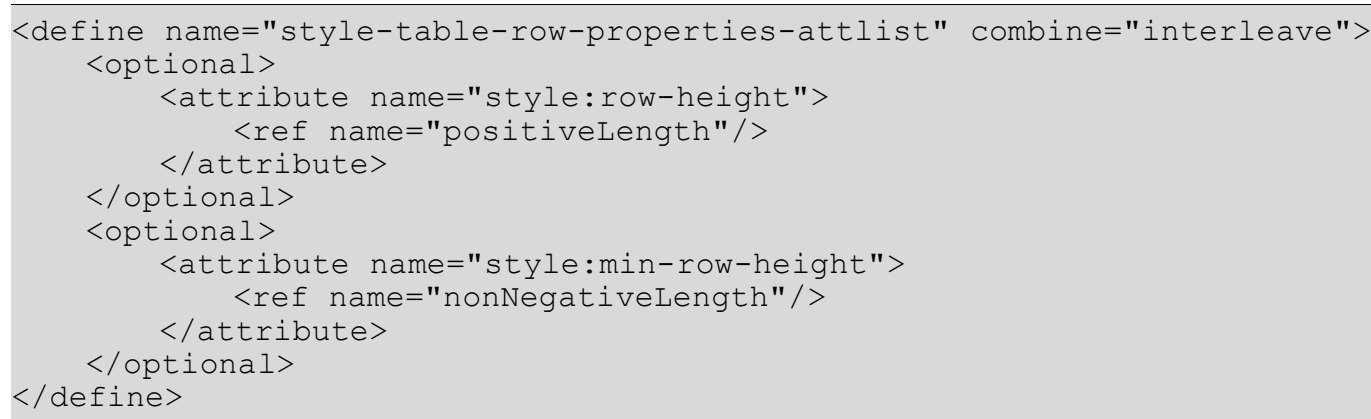

\subsubsection{Optimal Table Row Height}

The style: use-optimal-row-height attribute specifies that the row height should be recalculated automatically if some content in the row changes.

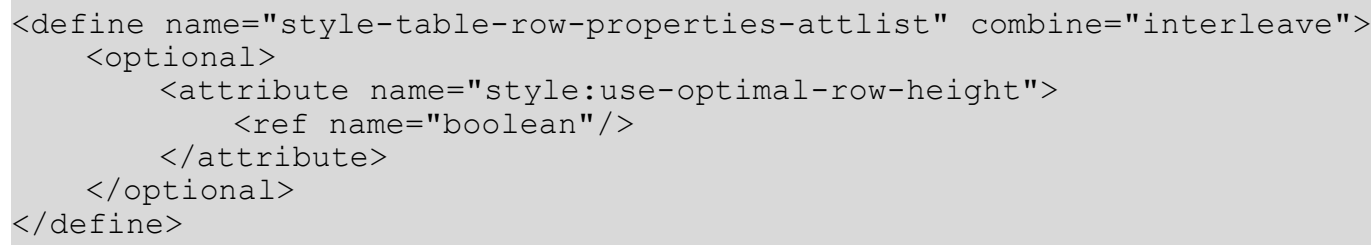

\subsubsection{Row Background}

The background attribute fo:background-color and the background element <style:background-image> specify the background properties of the table. See sections 15.5.23 and 15.5.24 for detailed information on this attribute and element.

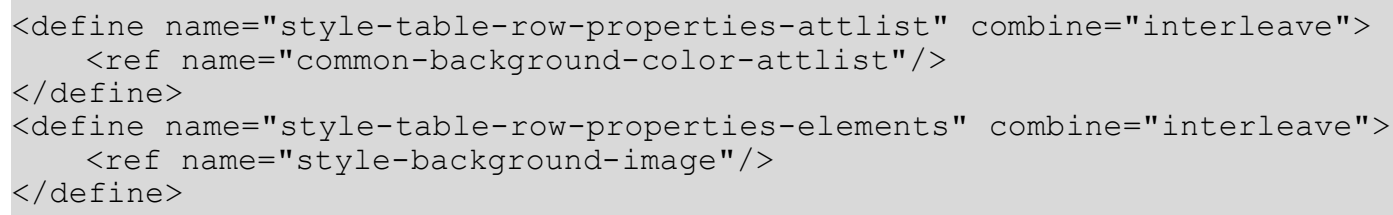

\subsubsection{Break Before and Break After}

The fo:break-before and fo:break-after properties insert a page or column break before or after a table column. See section 15.5.22 for a full explanation of these properties.

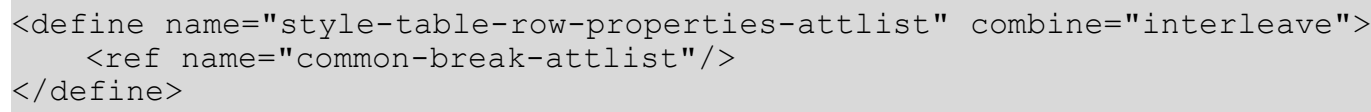




\subsubsection{Keep Together}

Use the fo:keep-together property to control whether the contents of a table cell should be kept together on the same page or column (if the value is always), or whether breaks are allowed within the cell (if the value is auto). See $\S 7.19 .3$ of [XSL] for details.

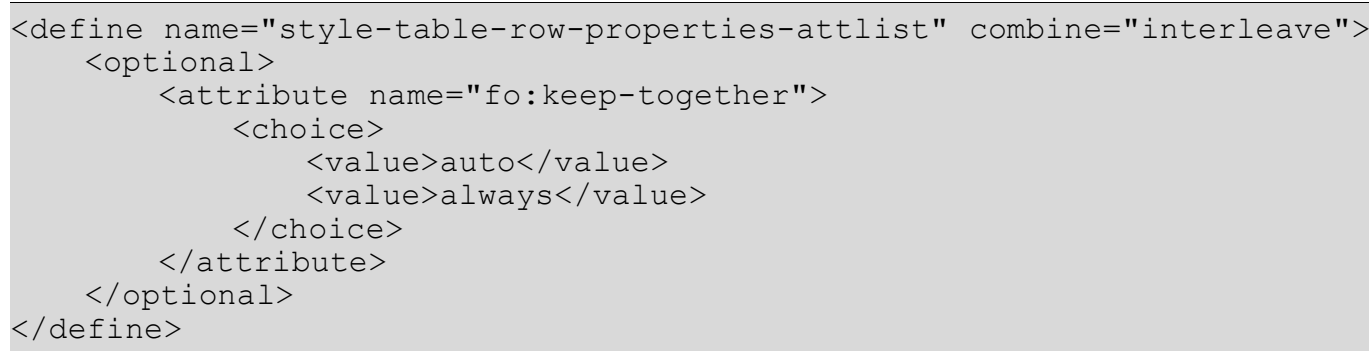

\subsection{Table Cell Formatting Properties}

The properties described in this section can be contained within table cell styles (see section 14.12.4) They are contained in a <style:table-column-properties $>$ element.

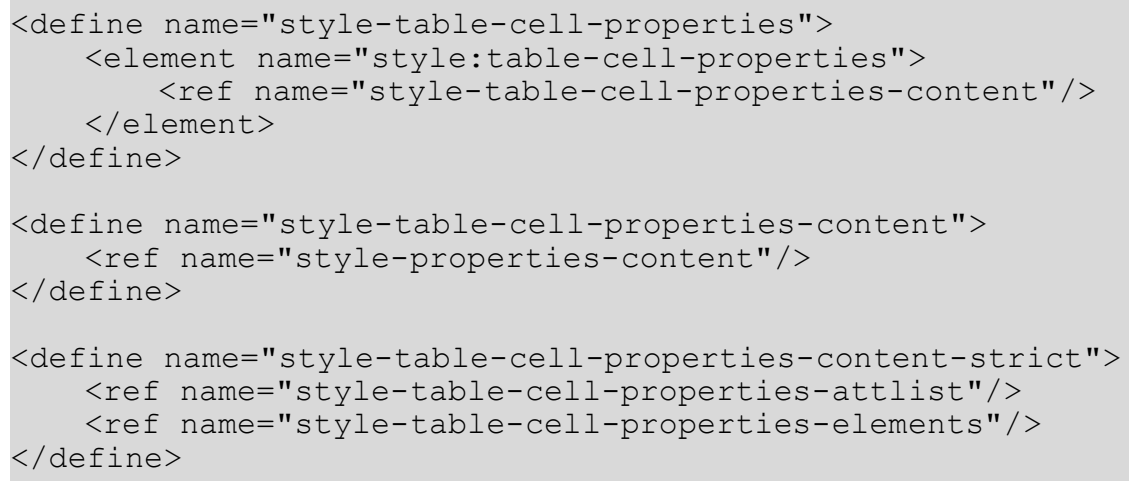

\subsubsection{Vertical Alignment}

The vertical alignment property style:vertical-align is used to specify the vertical alignment of text in a table cell.

The options for the vertical alignment property are as follows:

- top - Aligns text vertically with the top of the cell.

- middle - Aligns text vertically with the middle of the cell.

- bottom - Aligns text vertically with the bottom of the cell.

- automatic - The application decide how to align the text.

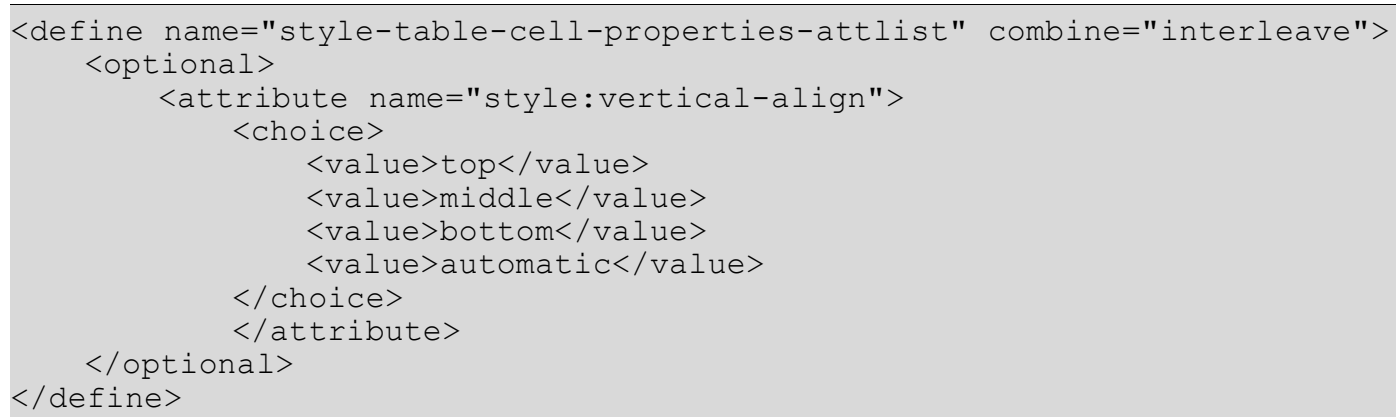




\subsubsection{Text Align Source}

The style:text-align-source property specifies the source of the text-align property. If the value of this attribute is $f i x$, the value of the $f o$ :text-align property is used. If the value is value-type, the text alignment depends on the value-type of the cell.

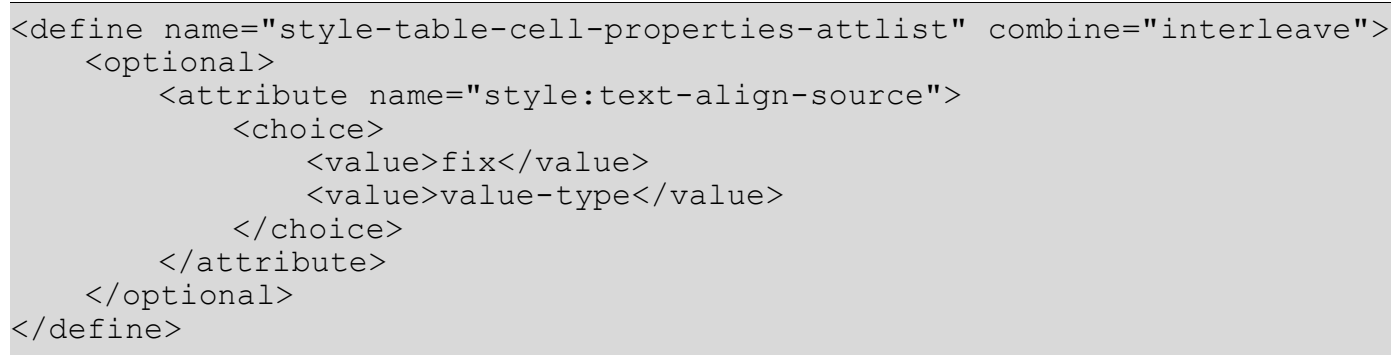

\subsubsection{Direction}

The style: direction property specifies the direction of characters in a cell. The most common direction is left to right ( $l t r)$. The other direction is top to bottom ( $\left.t t_{b}\right)$, where the characters in the cell are stacked but not rotated.

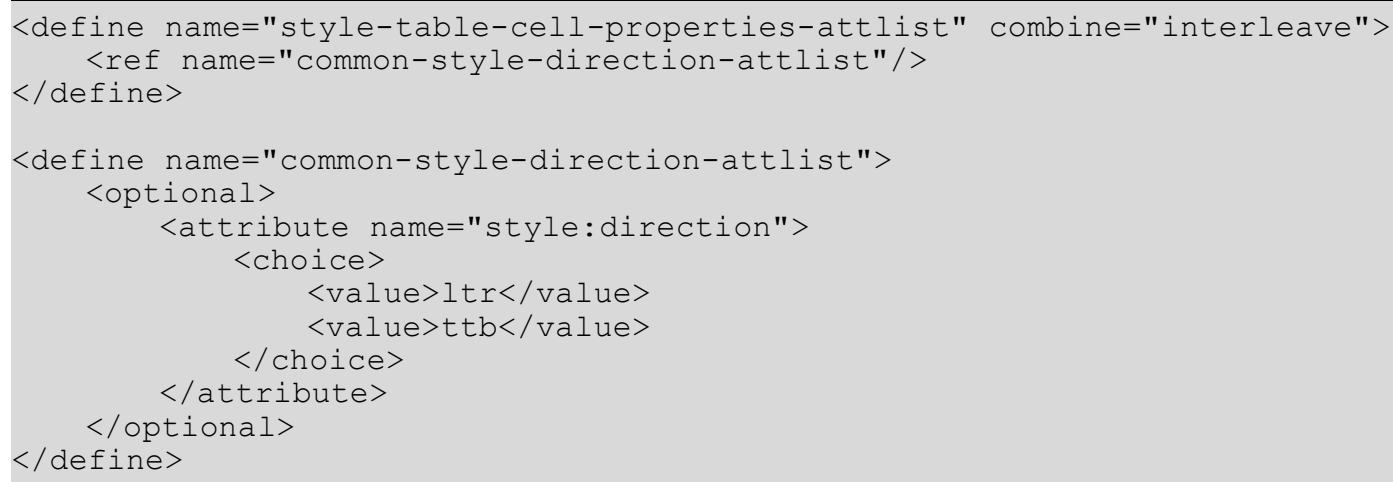

\subsubsection{Vertical Glyph Orientation}

The style:glyph-orientation-vertical property specifies the vertical glyph orientation. The property specifies an angle or automatic mode. The only possible angle is 0 , which disables this feature.

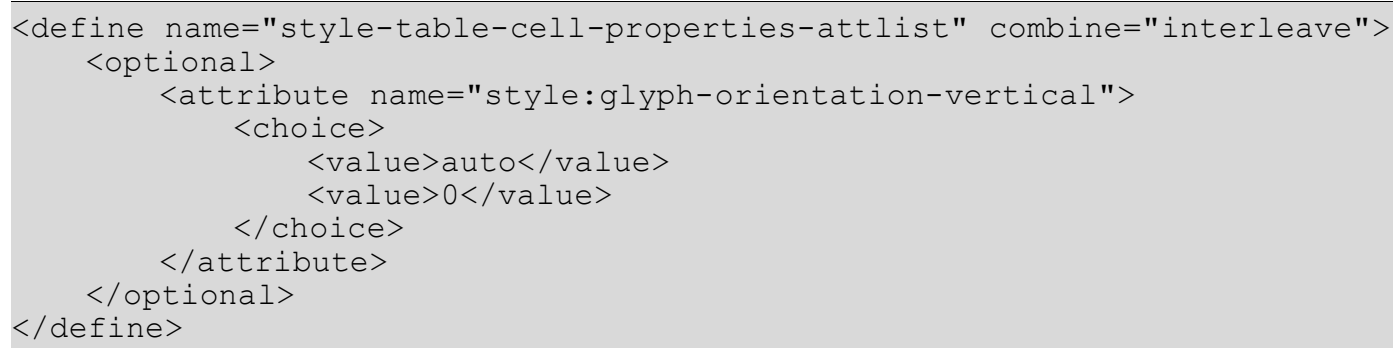

\subsubsection{Cell Shadow}

The style: shadow property specifies that a shadow visual effect appears on a table cell. See section 15.5 .28 for a full explanation of this property. 


\subsubsection{Cell Background}

The background attribute fo:background-color and the background element <style:background-image> specify the background properties of the table cell. See sections 15.5.23 and 15.5.24 for detailed information on this attribute and element.

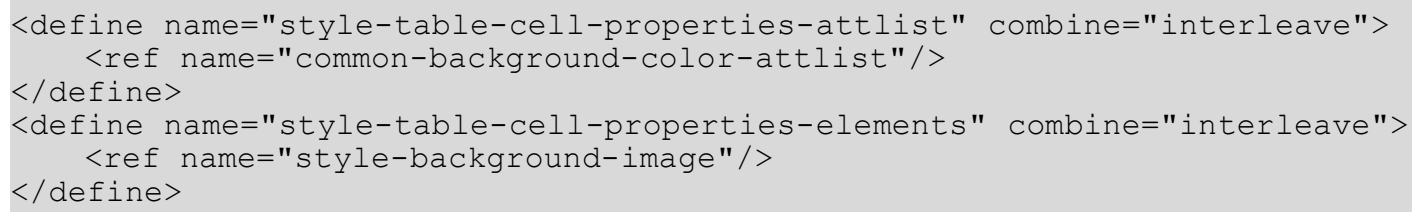

\subsubsection{Cell Border}

The border attributes fo:border, fo:border-top, fo:border-bottom, fo:border-left and $f \circ$ :border-right specify the border properties of the table cell. See section 15.5 .25 for detailed information on these attributes.

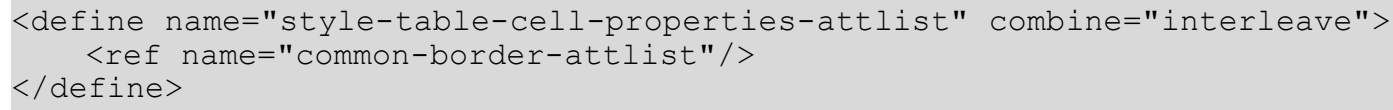

\subsubsection{Diagonal Lines}

Spreadsheet cells can also have diagonal lines, which follow the same specification as borders.

style:diagonal-tl-br defines the style of "border" to use for the topleft-bottomright diagonal (see section 15.5.25 for detailed information). In case of a double line, style:diagonal-bl-tr-widths allows to specify the width of the inner and outer lines and the distance between them (see section 15.5.26 for detailed information).

style:diagonal-bl-tr and style:diagonal-tl-br-widths define the same properties for the bottomleft-topright diagonal.

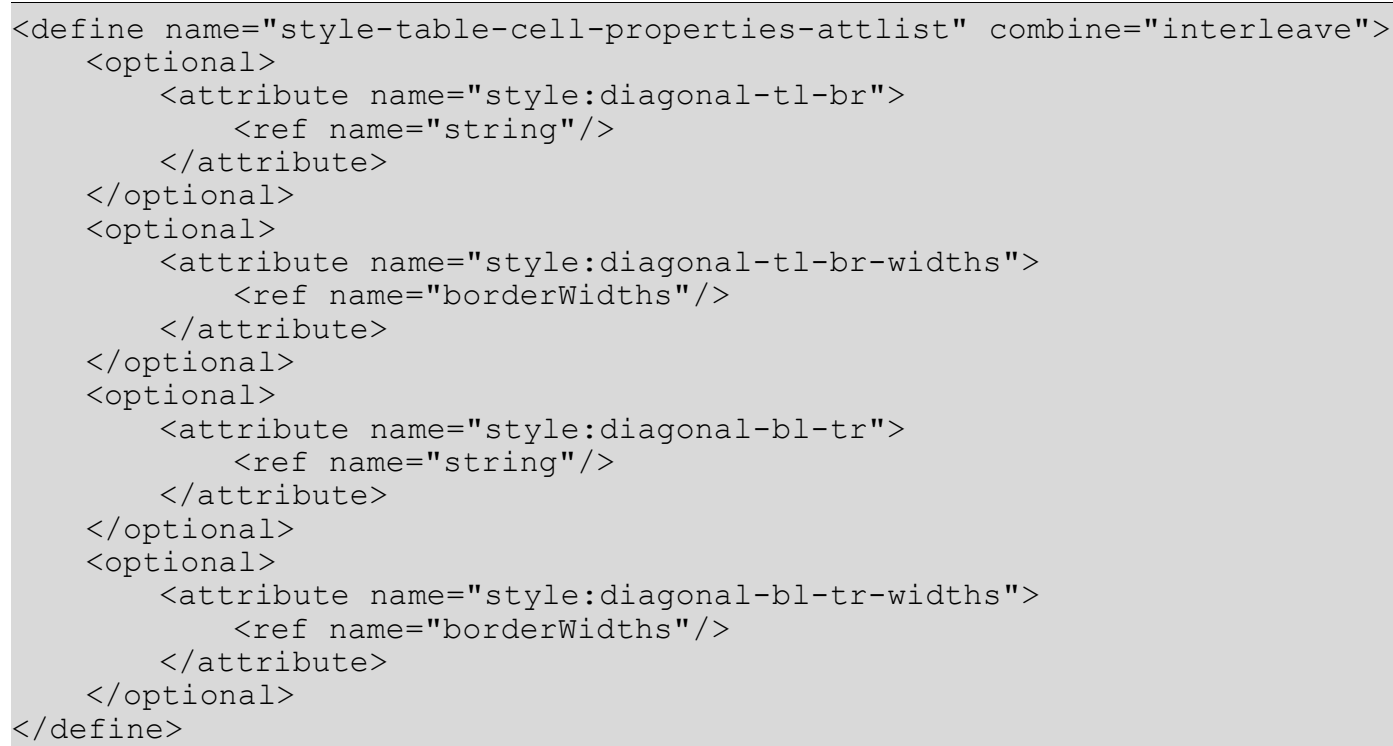




\subsubsection{Border Line Width}

The border line width attributes style:border-line-width, style:border-line-widthtop, style:border-line-width-bottom, style:border-line-width-left and style:border-line-width-right specify the properties of the border lines of the page.

See section 15.5.26 for detailed information on these attributes.

<define name="style-table-cell-properties-attlist" combine="interleave">

<ref name="common-border-line-width-attlist"/>

$</$ define $>$

\subsubsection{Padding}

The padding attributes fo:padding, fo:padding-top, fo:padding-bottom, fo:paddingleft and fo:padding-right specify the padding properties of the table cell. See section 15.5.27 for detailed information on these attributes.

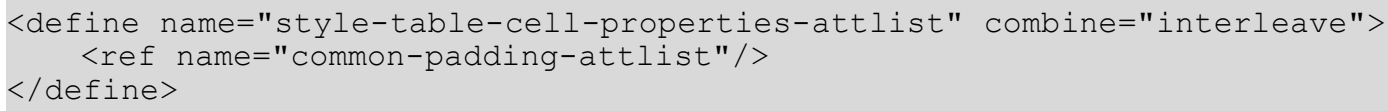

\subsubsection{Wrap Option}

The fo:wrap-option property specifies whether text wraps within a table cell. See $§ 7.5 .13$ of [XSL] for details. If wrapping is disabled, the application determines whether the clipped text is visible or hidden. If the text is hidden applications may support a scrolling mechanism to access the text. This is similar to setting a fo: overflow property to a value of auto. See also $§ 7.20 .2$ of [XSL].

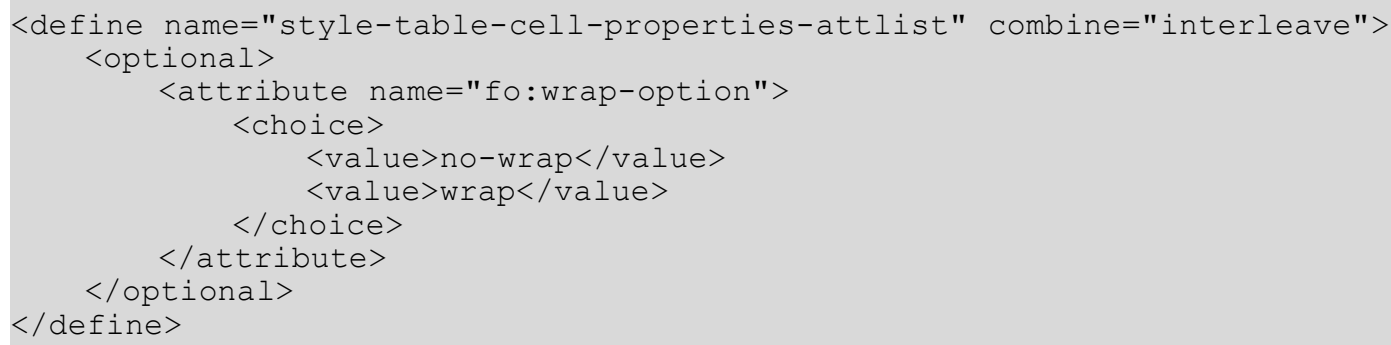

\subsubsection{Rotation Angle}

The style:rotation-angle property specifies the rotation angle of the cell content in degrees.

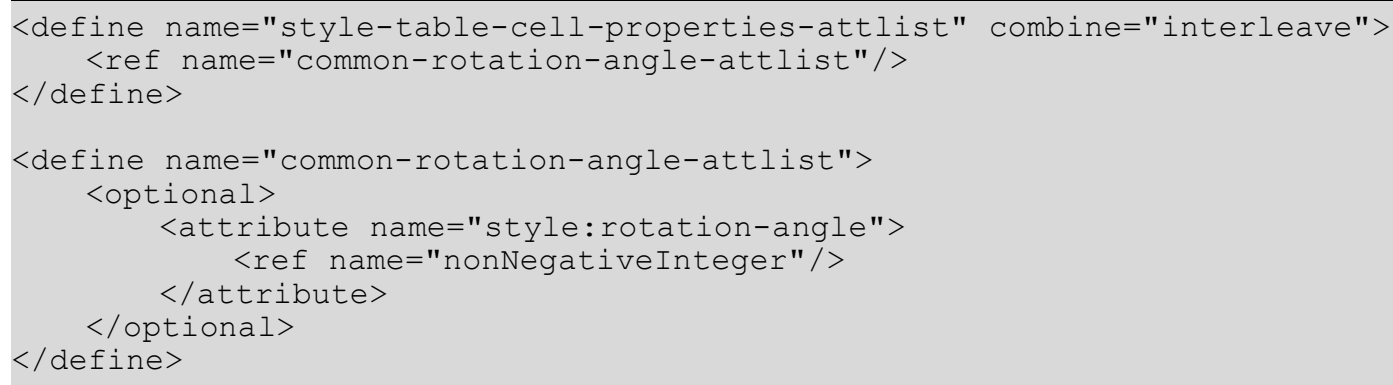




\subsubsection{Rotation Align}

The style:rotation-align property specifies how the edge of the text in a cell is aligned after a rotation. There are four alignment options: "none", "bottom", "top", or "center".

\begin{tabular}{|l|l|l|}
\hline \multicolumn{1}{|c|}{ Alignment } & \multicolumn{1}{|c|}{ Text is... } & \multicolumn{1}{c|}{ Borders and background are... } \\
\hline None. & Rotated. & Unchanged. \\
\hline Bottom of the cell. & $\begin{array}{l}\text { Rotated and may overlap with } \\
\text { other cells if the text is longer } \\
\text { than the length of the cell. }\end{array}$ & $\begin{array}{l}\text { Positioned parallel to the text, whereby } \\
\text { the upper or lower edge is drawn at the } \\
\text { original position of the cell. }\end{array}$ \\
\hline Top of the cell. & & \\
\hline Center of the cell. & & \\
\hline
\end{tabular}

\subsubsection{Print Content}

The style:print-content property specifies whether or not the cell content is printed.

\subsubsection{Cell Protect}

The style:cell-protect property specifies how a cell is protected.

This attribute is only evaluated if the current table is protected (see section 8.1.1). The value of the attribute can be "none", "hidden-and-protected", or a space-separated list containing the values "protected" or "formula-hidden".

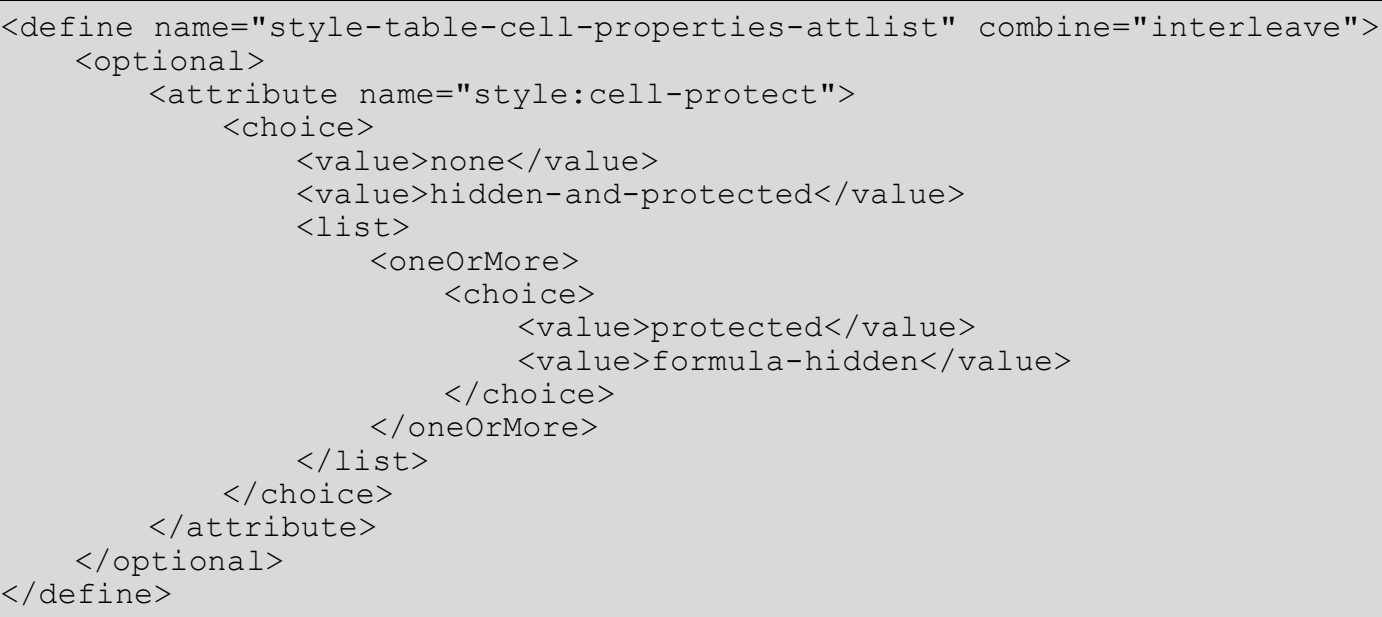


<optional>

<attribute name="style:print-content">

<ref name="boolean"/>

$</$ attribute $>$

$</$ optional $>$

\subsubsection{Decimal places}

The style:decimal-places attribute specifies the maximum number of decimal places that are displayed if numbers are formatted by a data style that has no setting for number of decimal places itself. See also section 14.7.9.

This property is usually only evaluated if it is contained in a default style (see section 14.2).

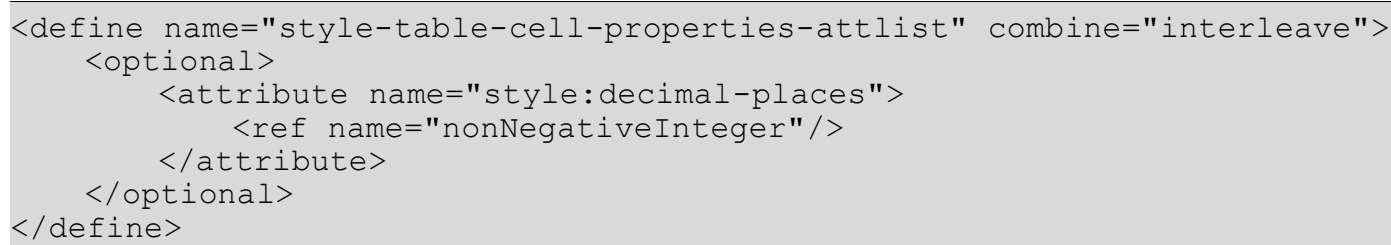

\subsubsection{Repeat Content}

The style:repeat-content property specifies whether the content of a cell is displayed as many times as there is space left in the cell's writing direction. Only full instances of the text are displayed. The property has no effect for cell content that contains a line break. This property is for instance used to "fill" a table cell with "-" or "x" characters so that no other data can be entered.

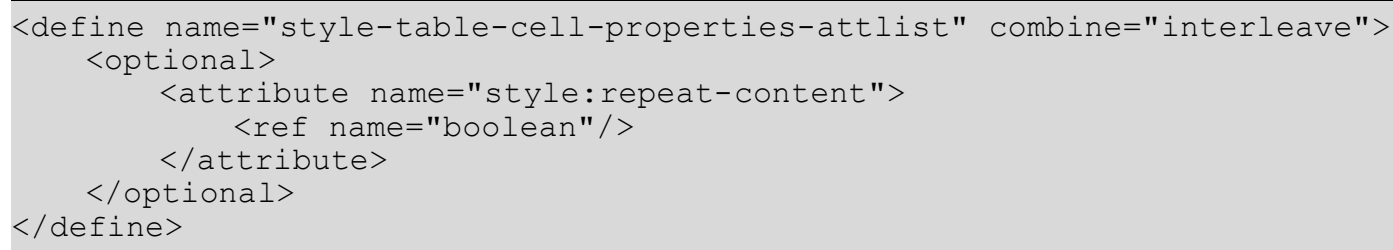

\subsubsection{Shrink To Fit}

The style:shrink-to-fit property specifies whether the content of a cell, if necessary, gets shrunk to fit into the cell. Shrinking does mean that the cell's font size is decreased, so that the complete text fits into the cell. The property has no effect on cells where the cell content fits already into the cell.

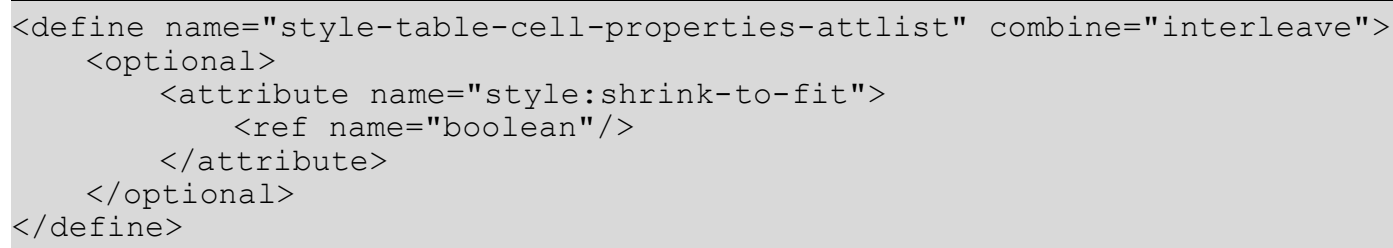

\subsection{List-Level Style Properties}

The properties described in this section can be contained within the various list style level elements (see section 14.10). They are contained in a <style: list-level-properties> element. 


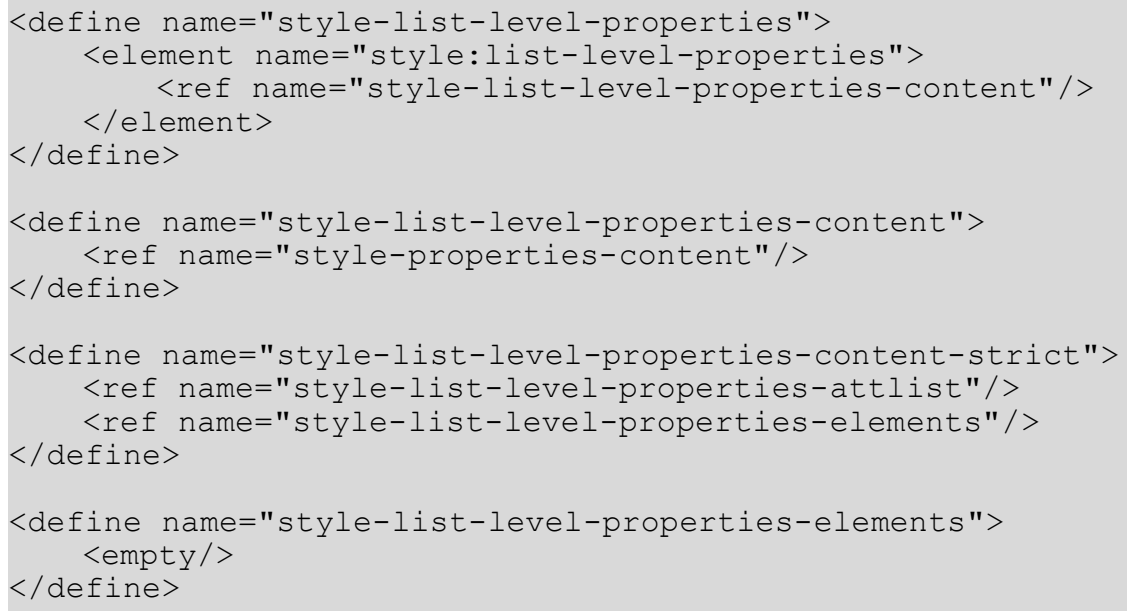

\section{Label Alignment}

The fo:text-align attribute specifies the horizontal alignment of a label (number) within the width specified by the text:min-label-width attribute. See also section 15.5.5,

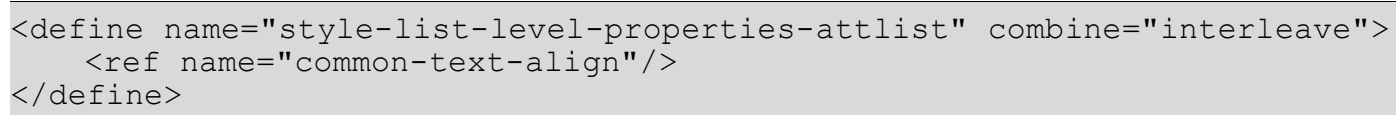

\section{Start Indent}

The text:space-before attribute specifies the space to include before the number for all paragraphs at this level. If a paragraph has a left margin that is greater than 0 , the actual position of the list label box is the left margin width plus the start indent value.

This attribute can be associated with an item set element that is contained in a <text: listlevel-style-*> element.

The value of the attribute is an absolute value. This means that when the position of a label is calculated the start indent value of the current level is only considered. The start indent values for lower levels do not affect the label position.

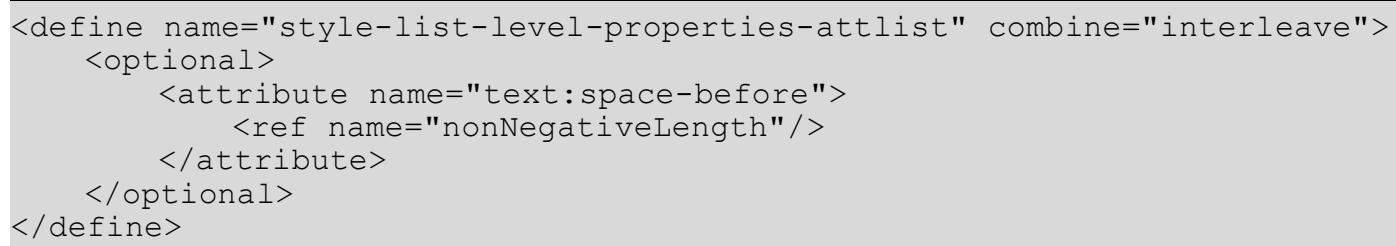

\section{Minimum Label Width}

The text:min-label-width attribute specifies the minimum width of a number.

This attribute can be associated with an item set element that is contained in a $<$ text: 1 istlevel-style-*> element.

The label can be aligned horizontally with the width using an fo:text-align property. See the Label Alignment attribute below for more information.

<define name="style-list-level-properties-attlist" combine="interleave"> <optional> 


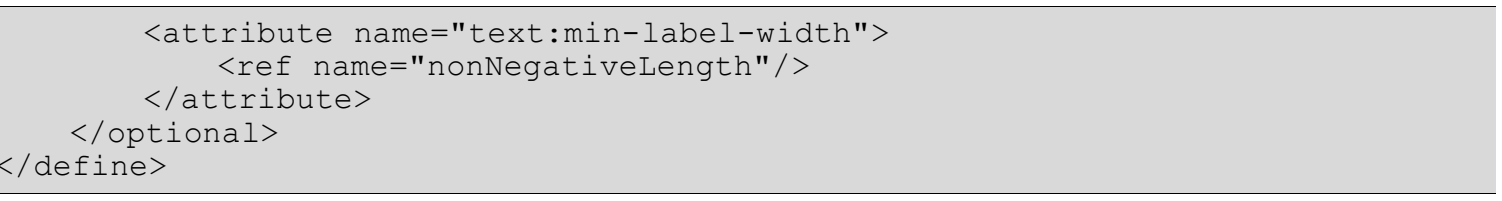

\section{Minimum Label Distance}

The text:min-label-distance attribute specifies the minimum distance between the number and the text of the list item.

This attribute can be associated with an item set element that is contained in a $<$ text: listlevel-style-*> element.

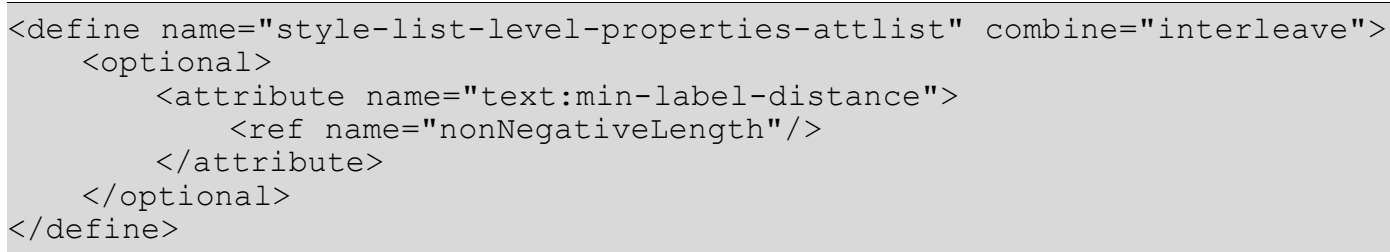

\section{Font Name}

The style:font-name attribute species the name of a font that is used to display a bullet character. See also section 15.4.13.

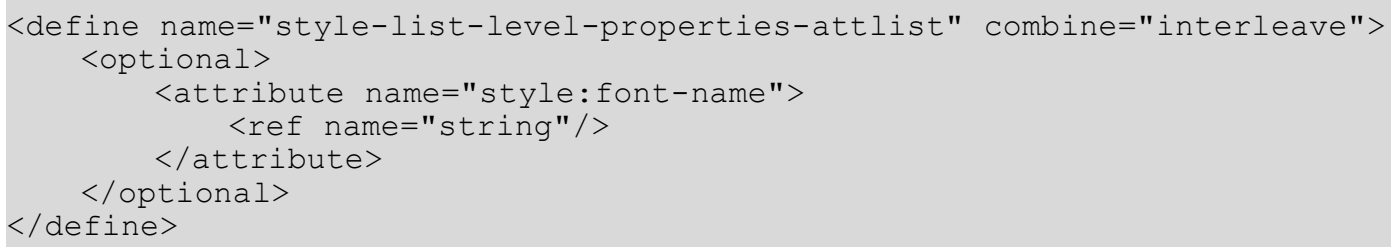

\section{Image Size}

The size of the image is specified by the following attributes:

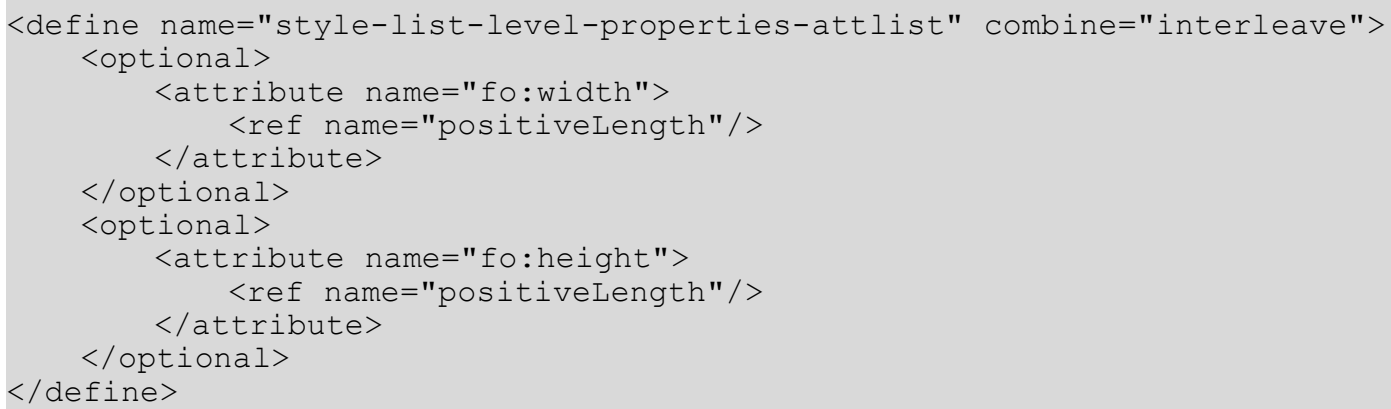

\section{Vertical Alignment}

The vertical alignment of the image is specified by the style:vertical-pos and style:vertical-rel properties. See sections 15.27.11 and 15.27.12 for details.

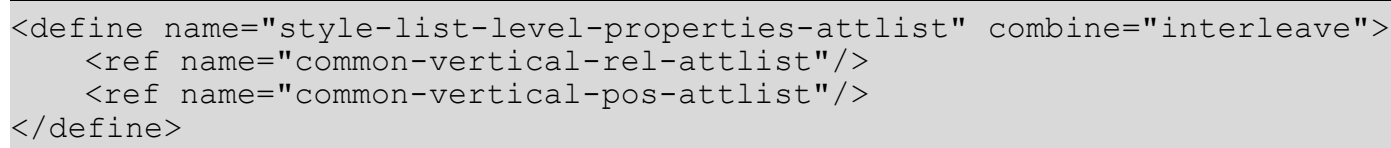




\subsection{Stroke Properties}

The following stroke properties are used to define drawing object line characteristics. They are available for drawing objects contained in all kinds of applications.

- Style

- Dash

- Width

- Color

- Start marker

- End marker

- Start marker width

- End marker width

- Start marker center

- End marker center

- Opacity

- Joint

The properties described in this section can be contained within style elements $\langle$ style:style $\rangle$ whose family is either graphic or presentation. They are contained in a <style:graphicproperties> element.

\subsubsection{Stroke Style}

The attribute draw: stroke specifies the style of the stroke on the current object. The value none means that no stroke is drawn, and the value solid means that a solid stroke is drawn. If the value is dash, the stroke referenced by the draw:stroke-dash property is drawn.

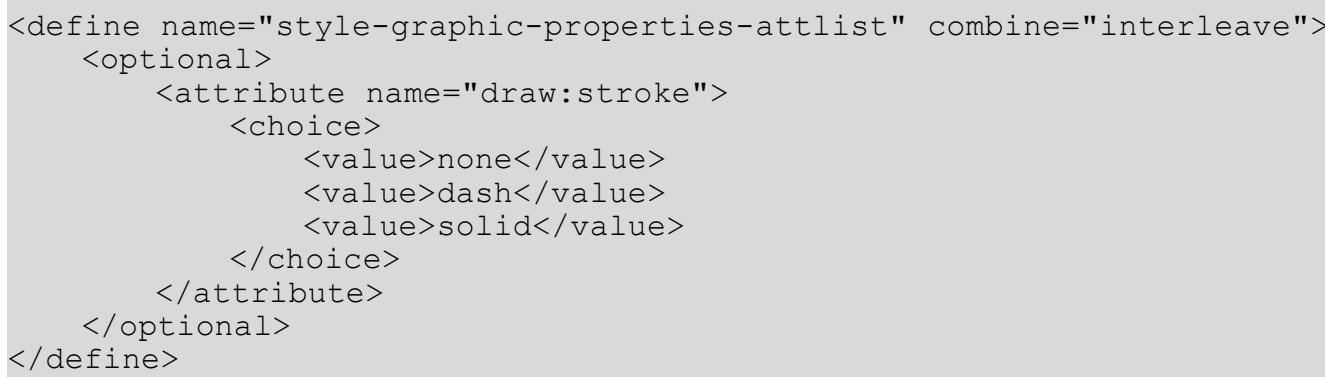

\subsubsection{Dash}

The attribute draw:stroke-dash specifies the dash style that is used for the stroke. See section 14.14.7 for dash styles.

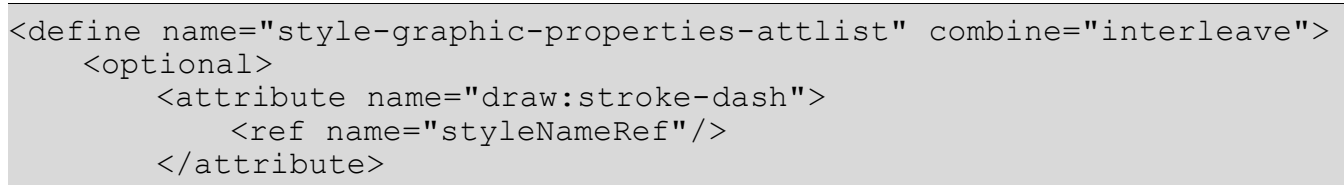




\subsubsection{Multiple Dashes}

The attribute draw: stroke-dash-names specifies a list of dash styles that are used for the stroke in addition to the dash specified by the draw: stroke-dash attribute. See section 15.13.2 for the draw: stroke-dash attribute and section 14.14.7 for dash styles.

\subsubsection{Color}

The attribute svg:stroke-color specifies the color of the stroke on the current object.

\subsubsection{End Marker}

The attribute draw: marker-end specifies a stroke end marker, which is a path that can be connected to the end of a stroke. See section 14.14.6 for markers. 


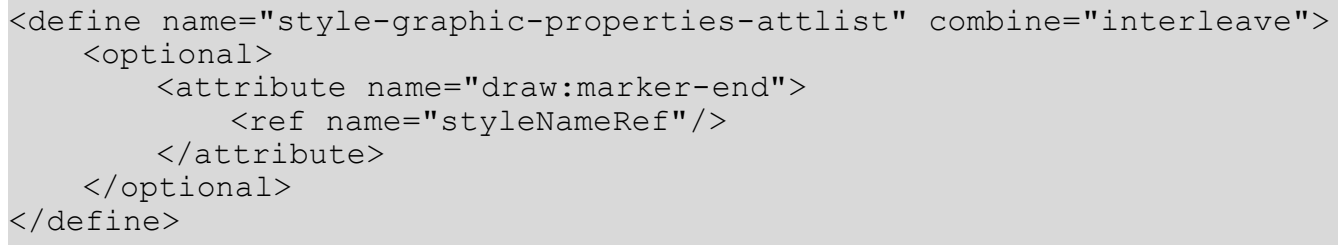

\subsubsection{Start Marker Width}

The attribute draw:marker-start-width specifies the width of the marker at the start of the stroke.

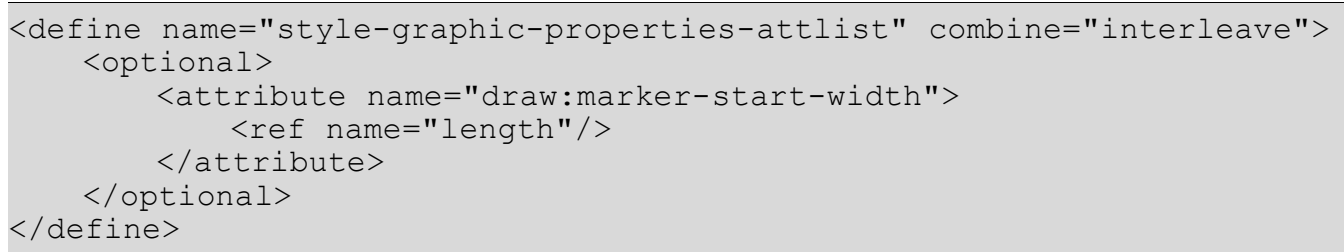

\subsubsection{End Marker Width}

The attribute draw: marker-end-width specifies the width of the marker at the end of the stroke.

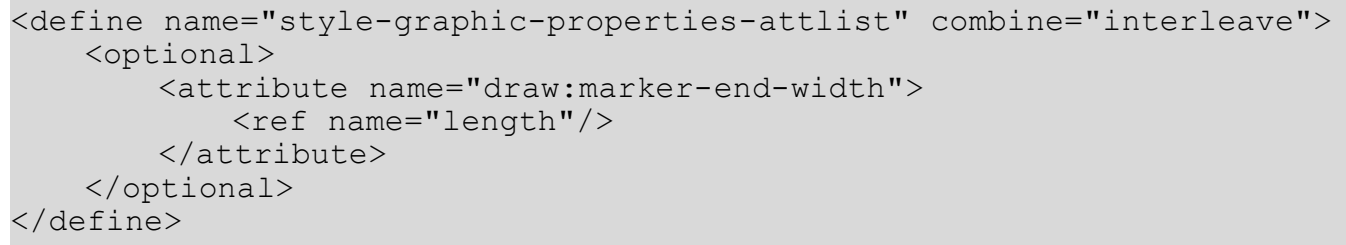

\subsubsection{Start Marker Center}

The attribute draw:marker-start-center specifies whether or not a start marker is centered at the start of a stroke.

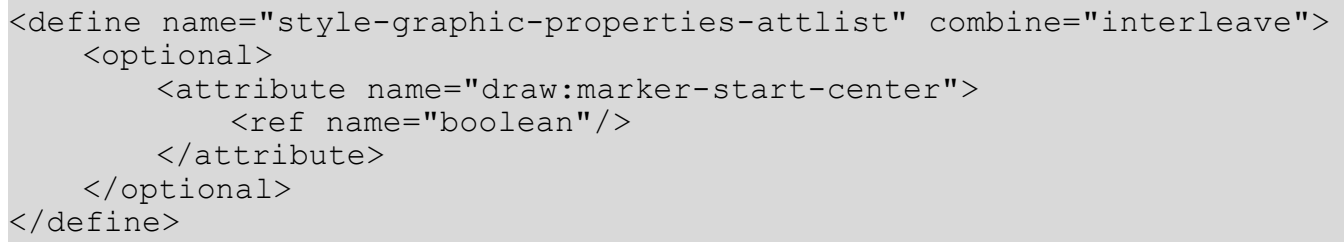

\subsubsection{End Marker Center}

The attribute draw:marker-end-center specifies whether or not an end marker is centered at the end of a stroke.

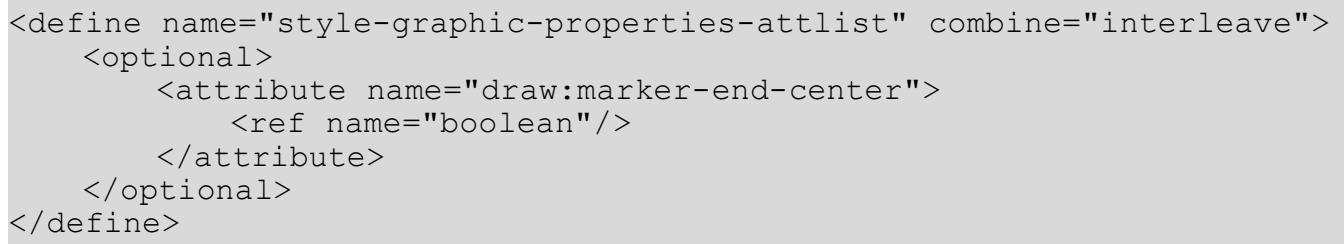




\subsubsection{Opacity}

The attribute svg:stroke-opacity specifies the opacity of a stroke. The value of this attribute can be a number between 0 (fully transparent) and 1 (fully opaque) or a percentage.

15679

15680

15681

15682

15683

15684

15685

15686

15687

15688

15689

15690

15691

\subsubsection{Line Join}

The attribute draw: stroke-linejoin specifies the shape at the corners of paths or other vector shapes, when they are stroked. The values are the same as for [SVG]'s strokelinejoin attribute, except that the attribute in addition to the values supported by SVG may have the value middle, which means that the mean value between the joints is used.

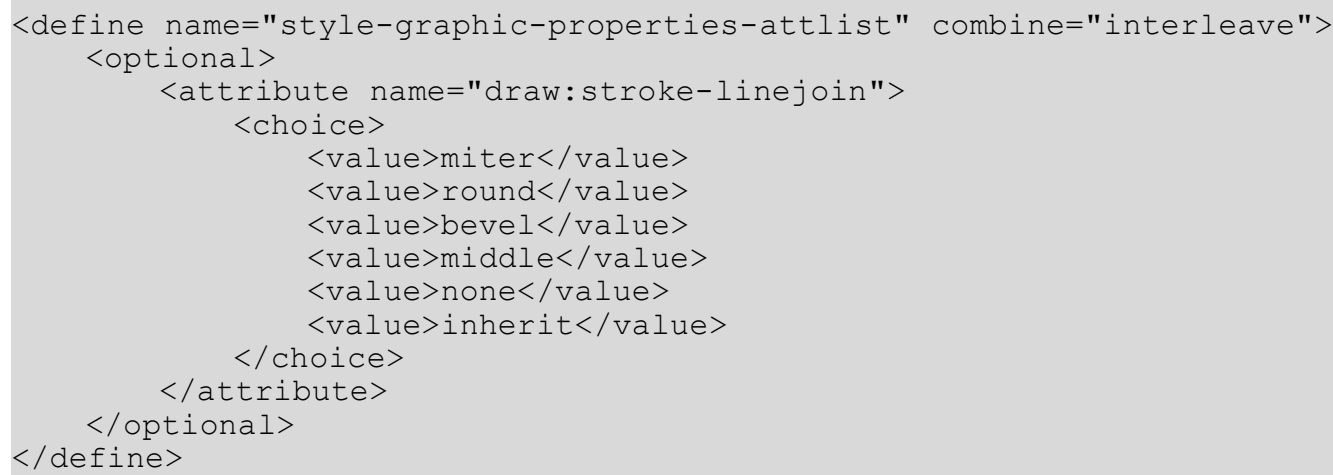

\subsection{Fill Properties}

The following fill properties are used to define drawing object fill characteristics. They are available for drawing objects contained in all kinds of applications.

- Style

- Color

- Gradient

- Gradient step count

- Hatch

- Solid hatch

- Bitmap

- Opacity 
- Fill rule

\subsubsection{Fill Style}

The attribute draw: fill specifies the fill style for a graphic object. Graphic objects that are not closed, such as a path without a closepath at the end, will not be filled. The fill operation does not automatically close all open subpaths by connecting the last point of the subpath with the first point of the subpath before painting the fill. The attribute has the following values:

- none: the drawing object is not filled.

- solid: the drawing object is filled with color specified by the draw:fill-color attribute.

- bitmap: the drawing object is filled with the bitmap specified by the draw: fill-imagename attribute.

- gradient: the drawing object is filled with the gradient specified by the draw: fillgradient-name attribute.

- hatch: the drawing object is filled with the hatch specified by the draw: fill-hatch-name attribute.

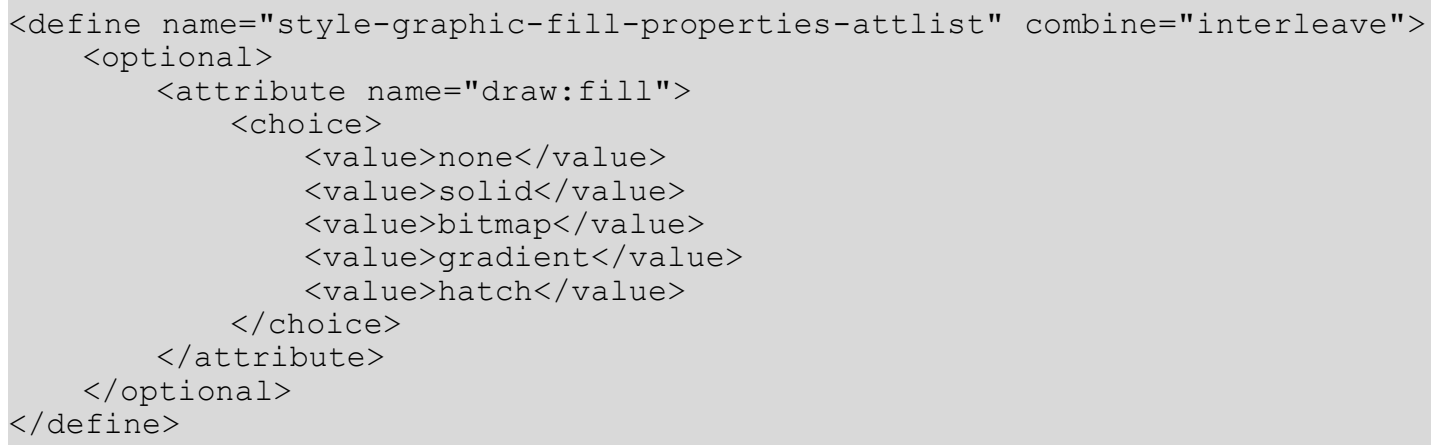

\subsubsection{Color}

The attribute draw: fill-color specifies the color of the fill for a graphic object. It is used only if the draw: fill attribute has the value solid.

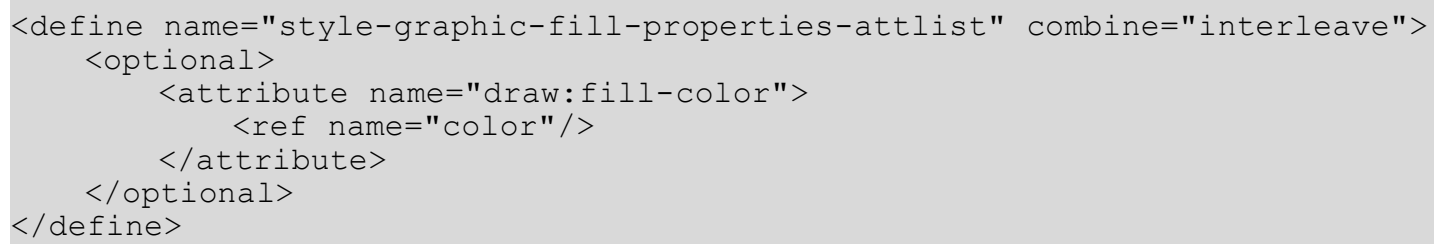

\subsubsection{Secondary Fill Color}

The draw: secondary-fill-color attribute specifies the secondary fill color. It may be used as fill color for the extrusion.

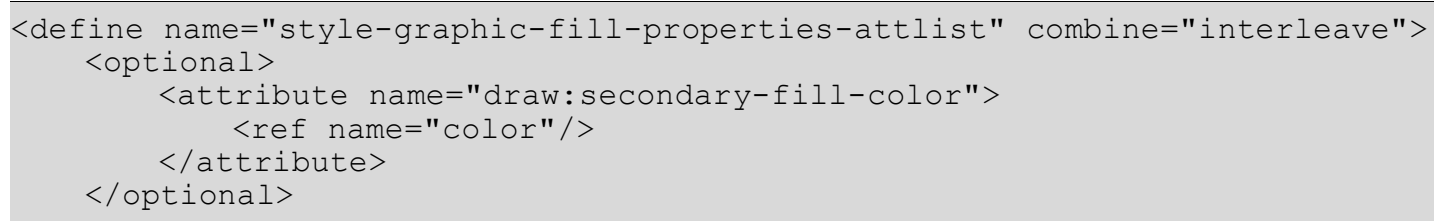




\subsubsection{Gradient}

The attribute draw:fill-gradient-name specifies a gradient style that is used for filling graphic objects. It is used only if the draw: fill attribute has the value gradient. See section 14.14.1 and 14.14.2 for gradients.

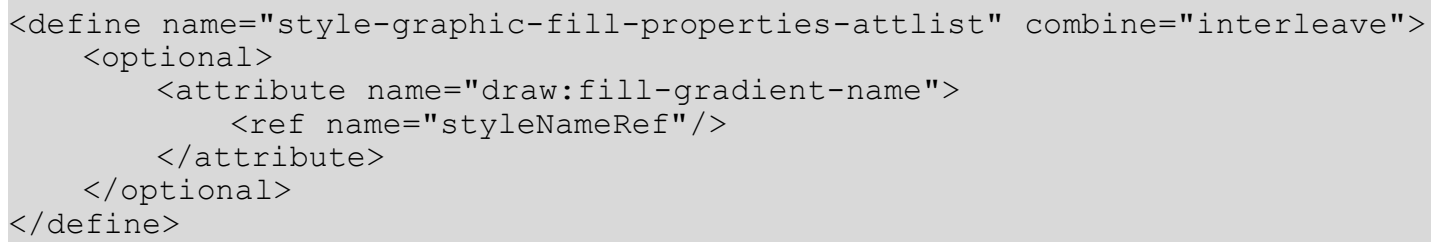

\subsubsection{Gradient Step Count}

If a gradient is used for filling, the attribute draw: gradient-step-count can be used to set the gradient step count of the color interpolation to be a fixed value. By default, the step count is automatically calculated based on the size and resolution of the filled area.

A step count less than 3 is not valid as there would be no interpolation possible. Values above 256 may not be supported or may result in performance issues.

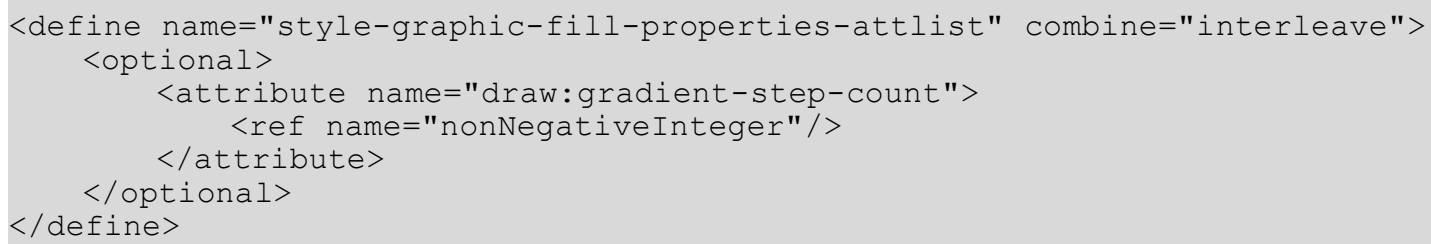

\subsubsection{Hatch}

The attribute draw: fill-hatch-name specifies a hatch style that is used for filling. It is used only if the draw: fill attribute has the value hatch. See section 14.14.3 for hatches.

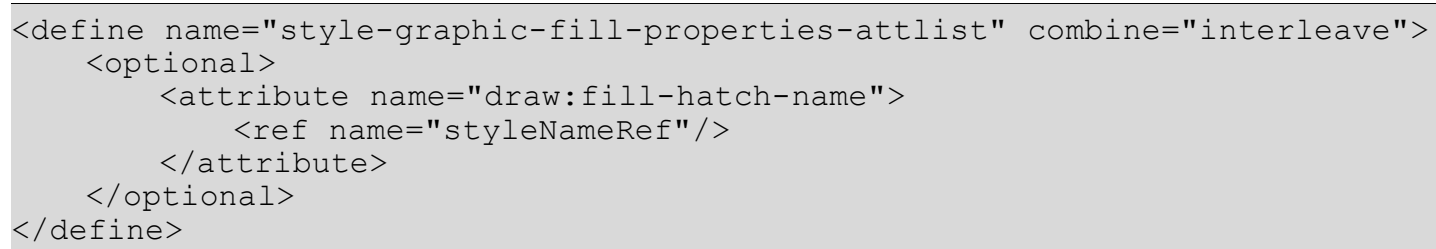

\subsubsection{Solid Hatch}

The attribute draw:fill-hatch-solid specifies whether the background of a hatch filling is solid or transparent.

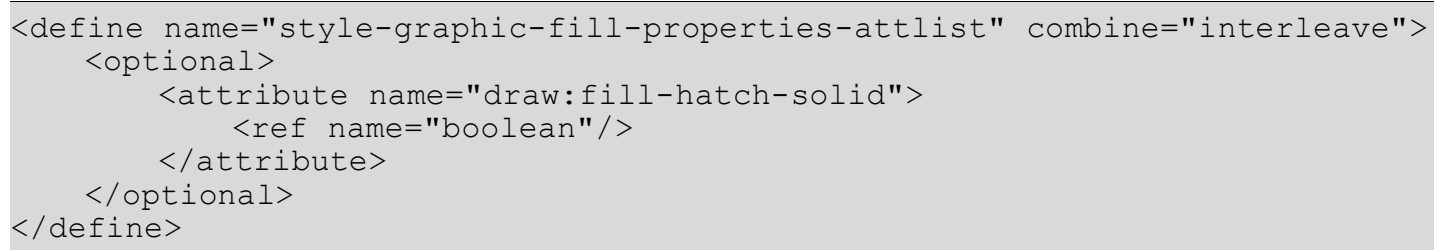




\subsubsection{Fill Image}

The attribute draw:fill-image-name specifies a fill image that is used for filling. It is used only if the draw: fill attribute has the value bitmap. See section 14.14.4 for fill images.

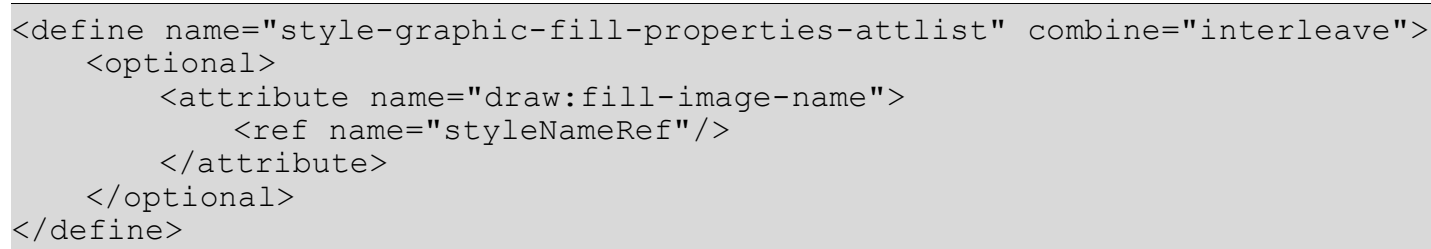

\subsubsection{Fill Image Rendering Style}

If an image is used for filling, the bitmap image can either be rendered in the given size, stretched to the filled area, or tiled over the area. The attribute style: repeat specifies how the bitmap image should be treated.

The value of the attribute can be no-repeat, repeat, or stretch.

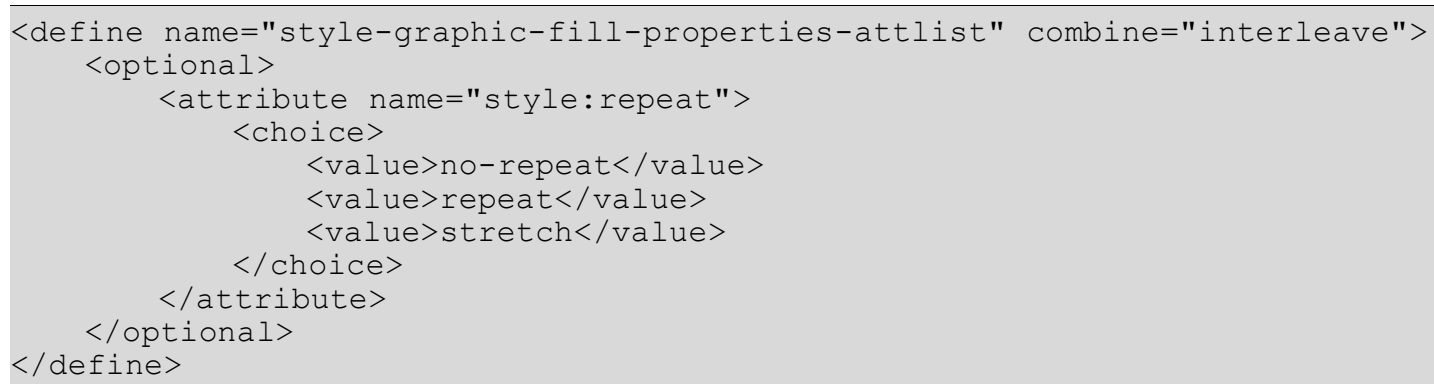

\subsubsection{Fill Image Size}

If an image is used for filling, the optional attributes draw: fill-image-width and draw: fill-image-height can be used to override the logical size of the source image data. If the value of the style:repeat attribute is stretch, these attributes are ignored.

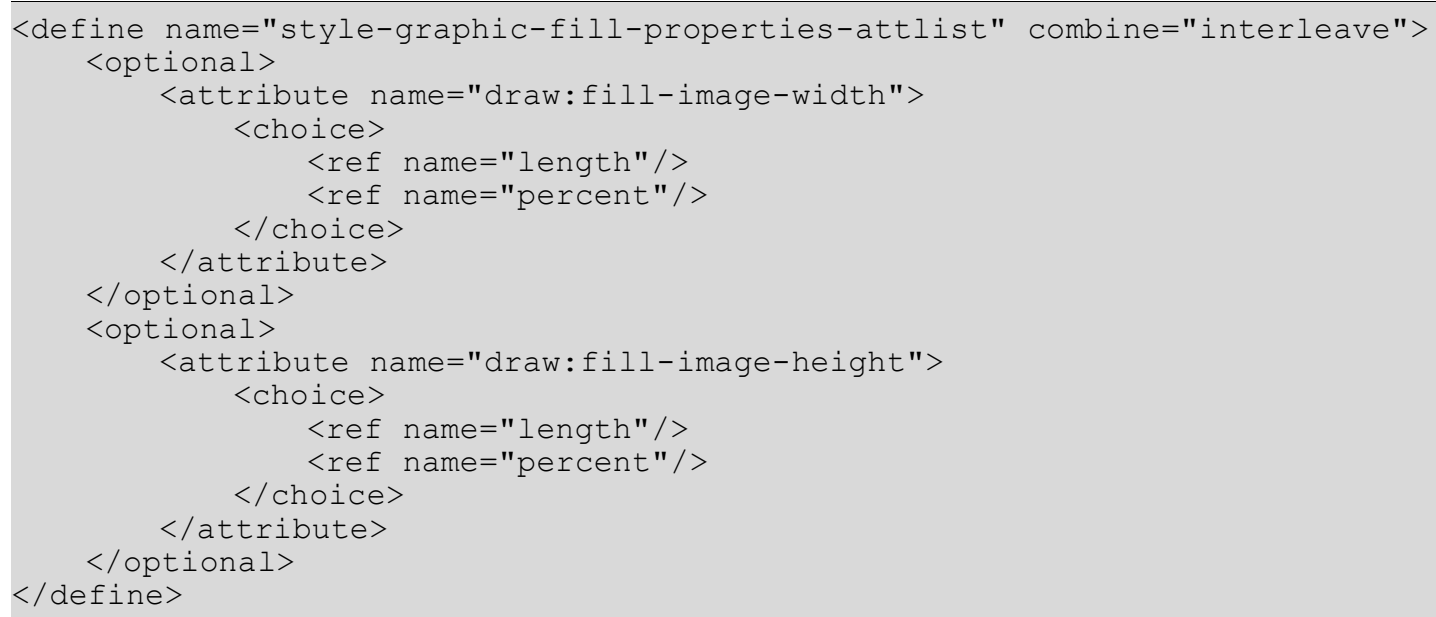




\subsubsection{Fill Image Tile Reference Point}

If an image is used for filling, the attributes draw: fill-image-ref-point, draw:fillimage-ref-point-x and draw:fill-image-ref-point-y specify the reference position of the image. The draw: fill-image-ref-point attribute specifies the position as an alignment of the image within the filling area, while the draw:fill-image-ref-point-x and draw:fill-image-ref-point-y attributes specify an horizontal and vertical movement as percentage values, where the percentage value relates to the image width and height. If an alignment and a movement is specified at the same time, the image first is aligned and afterwards moved.

These attributes are only interpreted if the value of the current style: repeat attribute is repeat.

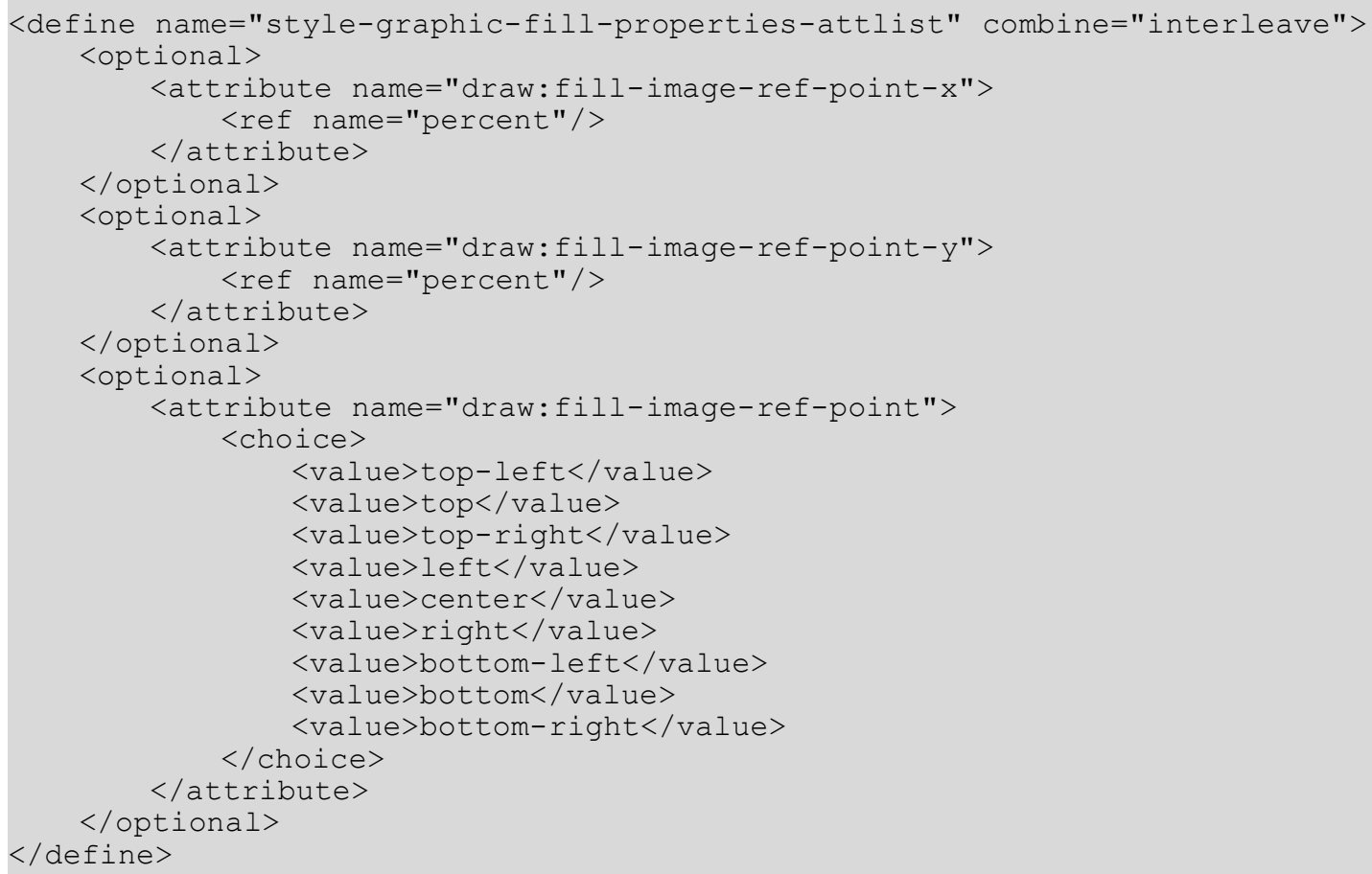

\subsubsection{Fill Image Tile Translation}

If an image is used for filling, the attribute draw:tile-repeat-offset defines the translation of each tile in relation to the previous tile. This attribute is only interpreted if the value of the current style: repeat attribute is tiled. The value of this attribute is a percentage value representing the tiles repeat offset relative to the tiles height or width, followed by either the word horizontal or vertical.

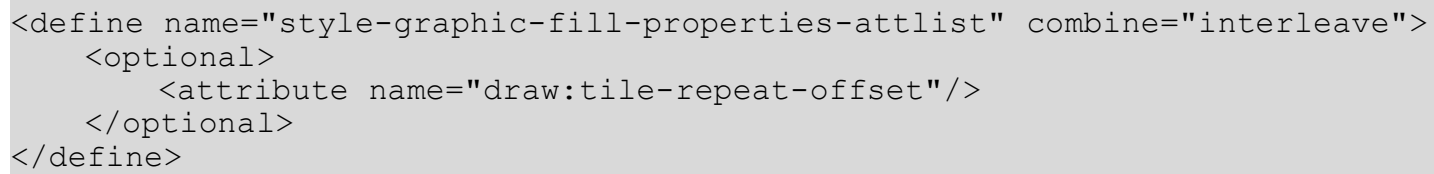

Example: Tile translation 


\subsubsection{None and Linear Opacity}

The fill area of a graphic object can either have a full, a linear, or gradient opacity. Full and linear opacity is selected using the draw: opacity attribute, while gradient opacity is selected using the draw: opacity-name attribute.

The draw: opacity attribute disables any transparency effect or sets a linear opacity for the fill area of a graphic object.

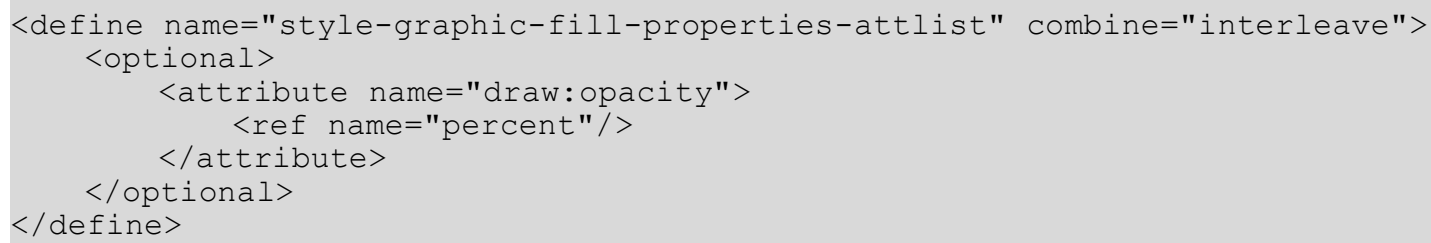

\subsubsection{Gradient Opacity}

The draw: opacity-name attribute specifies an opacity gradient that defines the opacity for the fill area of a graphic object. When applying an opacity gradient, the opacity is interpolated as defined in the referenced opacity gradient style. This fill style is rendered independently from other fill styles like gradient, image, and hatch. See section 14.14 .5 for opacity gradients.

The value of this attribute overrides the draw: opacity attribute.

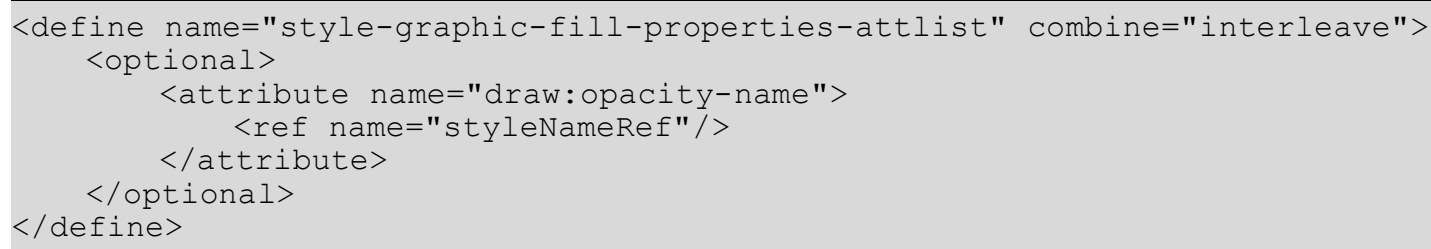

\subsubsection{Fill Rule}

The svg: $f i l l-r u l e$ specifies the algorithm which is to be used to determine what parts of the canvas are included inside the shape. See $\S 11.3$ of [SVG] for more details.

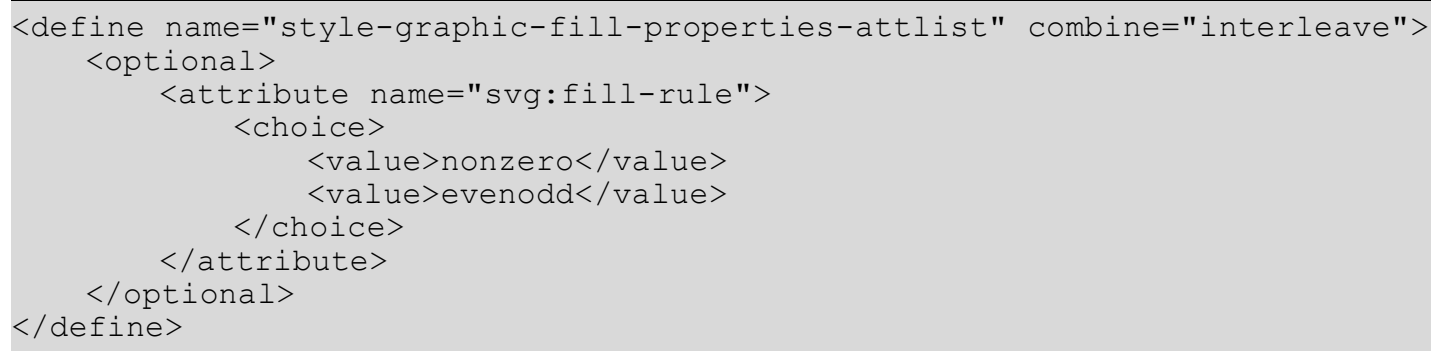

\subsubsection{Symbol color}

The draw:symbol-color attribute defines the color to be used to draw symbols contained on the drawing object. This could be for instance arrows displayed within a control.

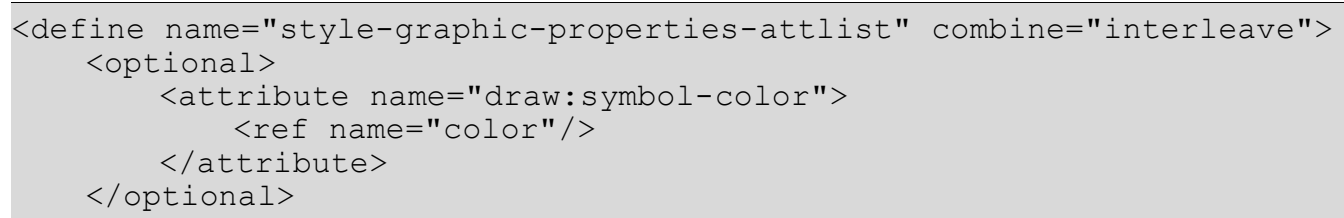




\subsection{Text Animation Properties}

Drawing objects that contain text and text boxes can have optional text animation properties. These properties always animate the complete text of a drawing object or text frame. The following attributes define the text animation:

- Animation

- Animation direction

- Animation start inside

- Animation stop inside

- Animation repeat

- Animation delay

- Animation setps

These properties are available for drawing objects contained in all kinds of applications.

\subsubsection{Animation}

The attribute text:animation specifies the type of animation that is used for the text.

The value of this attribute can be one of the following:

- none, disables the text animation.

- scroll, scrolls the text from one side to another.

- alternate, scrolls the text from one side to another and back.

- slide, scrolls the text from one side to the original text position and stops there.

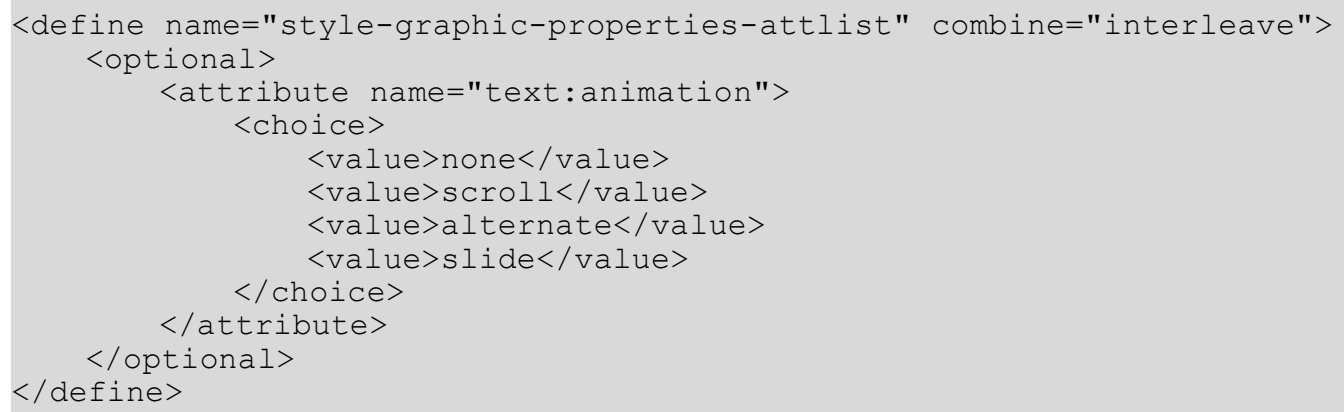

\subsubsection{Animation Direction}

The attribute text:animation-direction specifies the scroll direction of animated text.

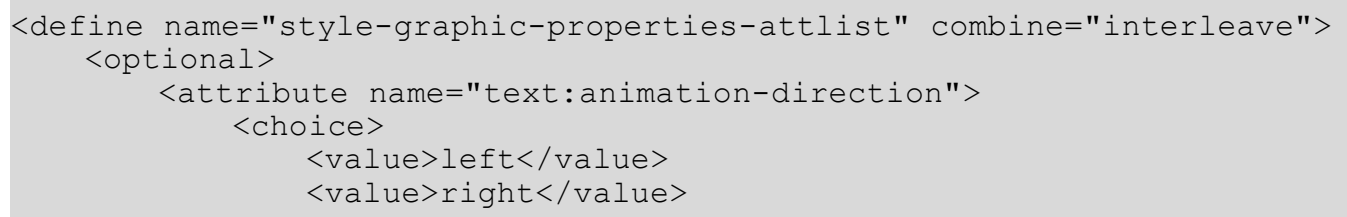




\subsubsection{Animation Start Inside}

If this attribute text:animation-start-inside is true, the text starts its animation inside the shape. If its false, the text starts its animation just outside the shapes bounding rectangle.

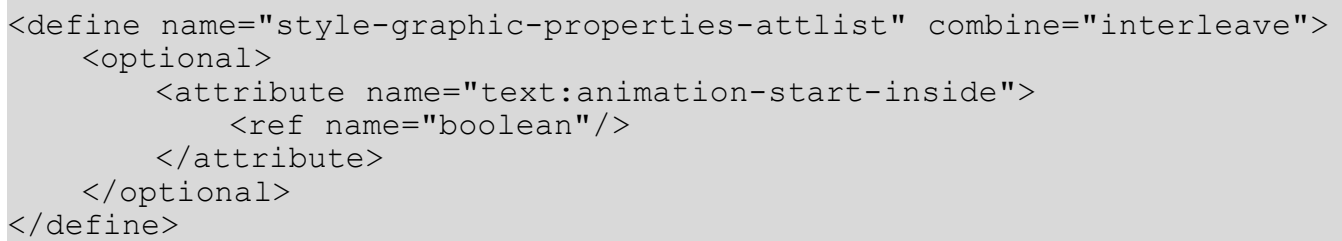

\subsubsection{Animation Stop Inside}

If this attribute text:animation-stop-inside is true, the text stops when it is inside the the shape. If its false, the text stops its animation just outside the shapes bounding rectangle.

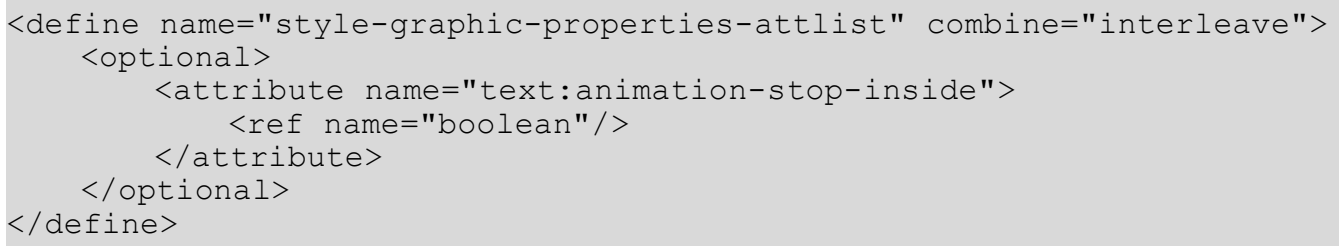

\subsubsection{Animation Repeat}

The attribute text:animation-repeat specifies the number of times the animation is repeated. If the value of the attribute is 0 , the animation is repeated indefinitely.

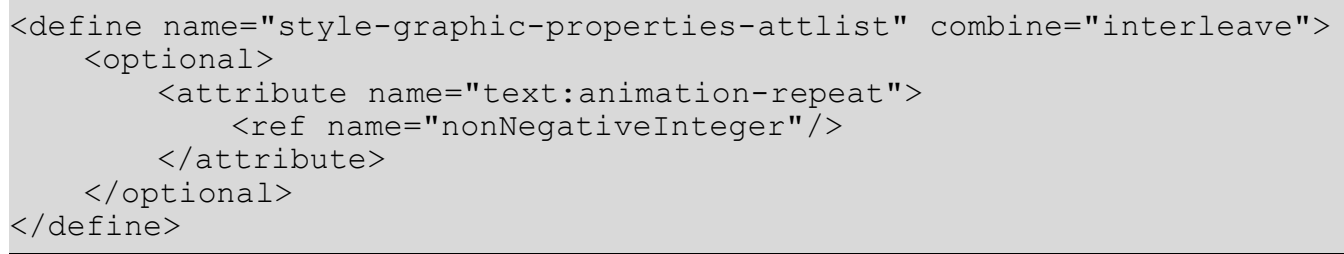

\subsubsection{Animation Delay}

The attribute text:animation-delay specifies a delay before the animation is started. The value of this attribute must conform to the time period format described in §3.2.6 of [xmlschema2].

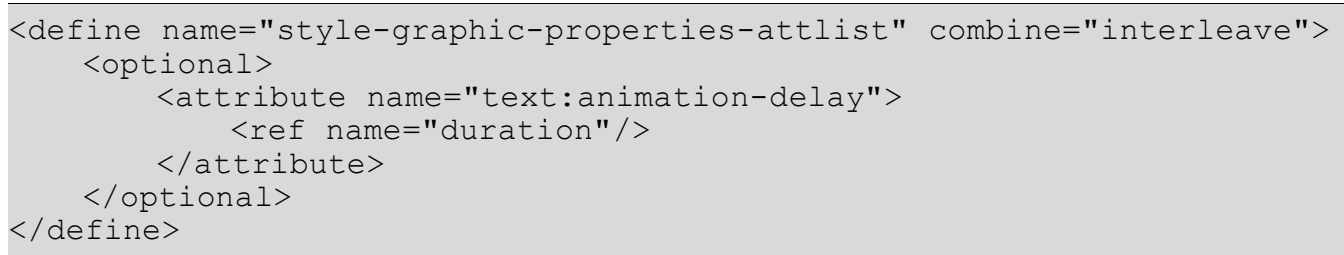




\subsubsection{Animation Steps}

The attribute text:animation-steps specifies the distance by which text is moved within each scrolling step.

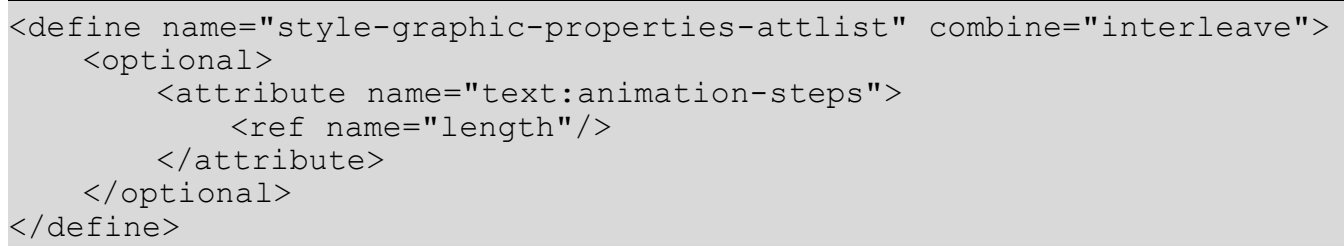

\subsection{Text and Text Alignment Properties}

Drawing objects that contain text and text boxes can have optional properties that specify how the text is aligned within the drawing object. These properties are available for drawing objects contained in all kinds of applications.

\subsubsection{Auto Grow Width and Height}

The attributes draw:auto-grow-width and draw:auto-grow-height specify whether or not to automatically increase the width and height of the drawing object if text is added to the drawing object. These attributes usually are evaluated only for text boxes.

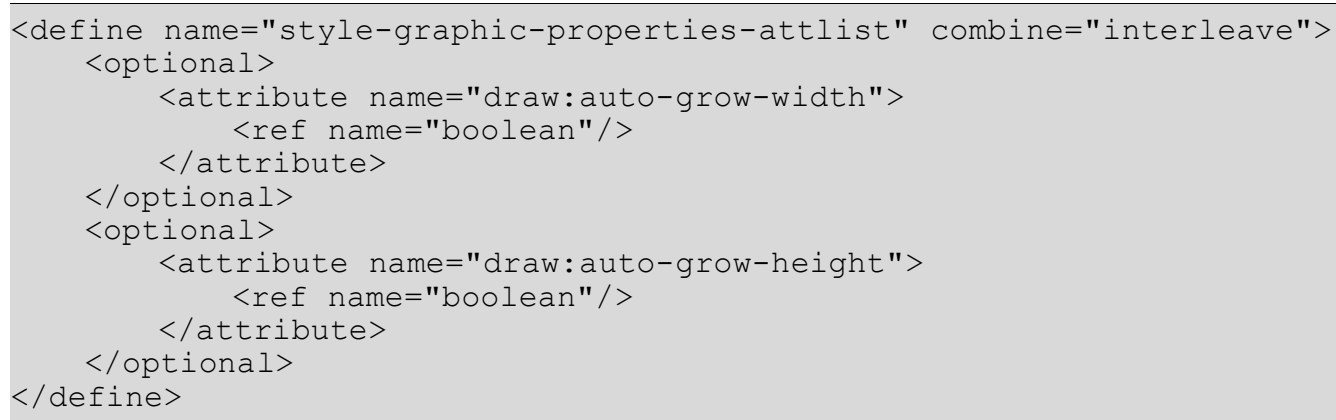

\subsubsection{Fit To Size}

The attribute draw: fit-to-size specifies whether or not to stretch the text content of a drawing object to fill the entire object. If the value of the attribute is true, the text content is stretched.

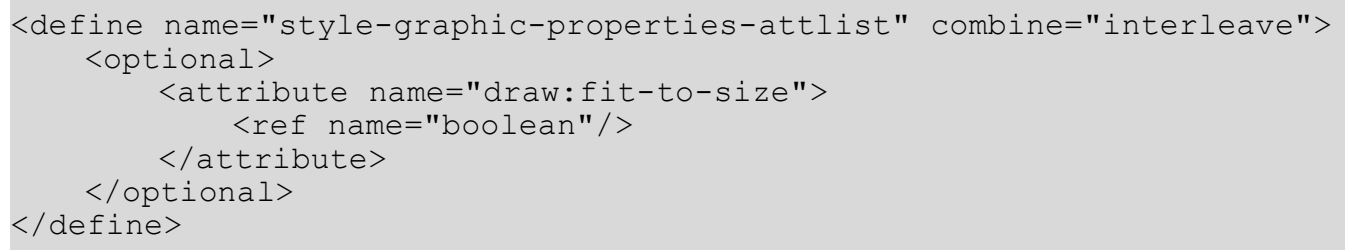

\subsubsection{Fit To Contour}

The attribute draw: fit-to-contour specifies whether or not to stretch the text content of a drawing object to fill the contour of the object. If the value of the attribute is true, the text content is stretched.

<define name="style-graphic-properties-attlist" combine="interleave"> 


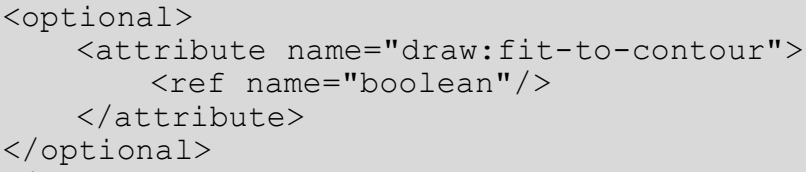

15.16.4 Text Area Vertical Align

The attribute draw:textarea-vertical-align specifies the vertical alignment of the text area inside a shape.

\subsubsection{Text Area Horizontal Align}

The attribute draw:textarea-horizontal-align specifies the horizontal alignment of the text area inside a shape.

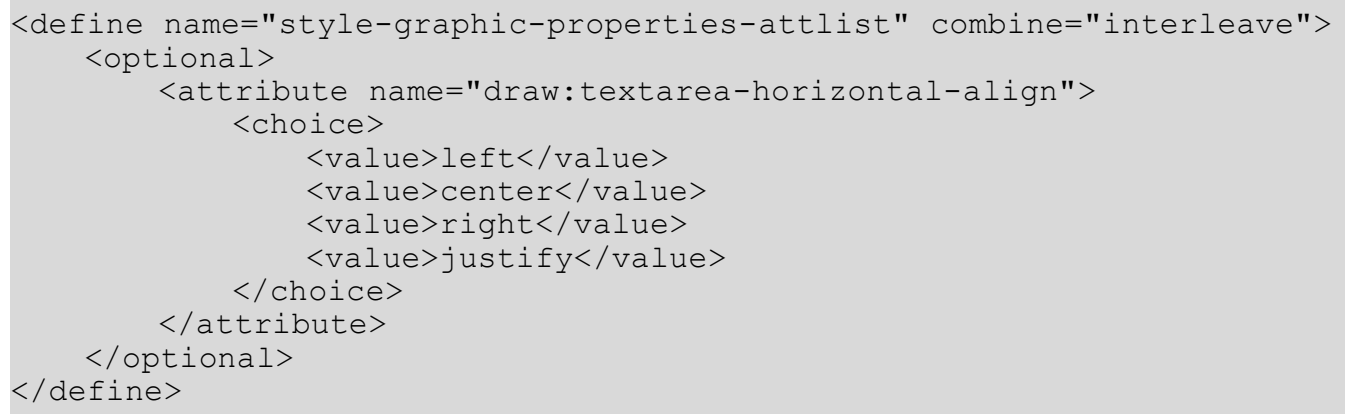

\subsubsection{Word Wrap}

The fo:wrap-option attribute specifies if text is word wrapped in a shape.

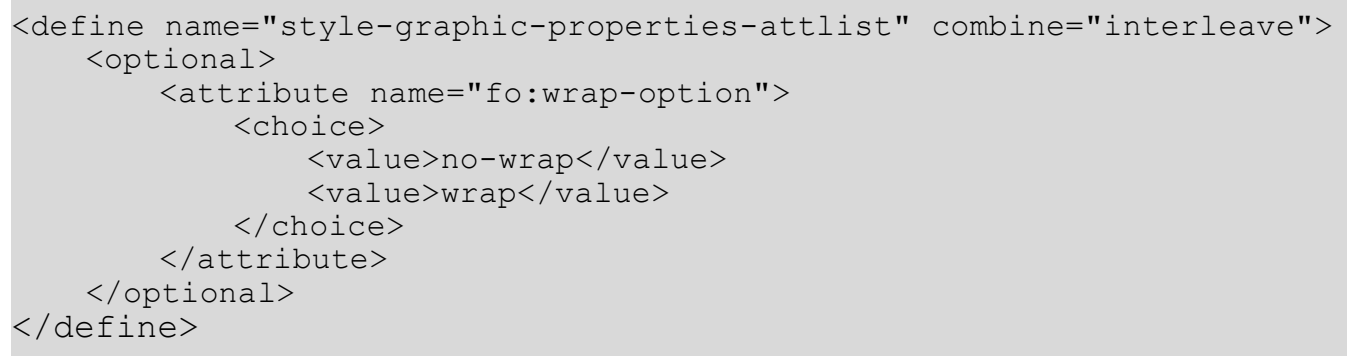




\subsubsection{List Styles}

The <text: list-style> element as described in section 14.10 specifies a list style that is applied to the paragraphs contained in a text box. Although the list style has a name, it is not displayed in the user interface, even if the graphic style that contains it is a common style.

Including a list style element into a graphic style has the same semantics as adding a style: list-style-name attribute (see section 14.1) to the style that references a list style that is declared outside a graphic style. The inclusion of a list style element is required in cases where a common graphic style should be associated with an automatic list style.

List styles contained in a graphic style can be referenced by other graphic styles using the style:list-style-name attribute.

\subsection{Color Properties}

Drawing objects that display a bitmap graphic can have optional properties that adjust the colors of the bitmap. These properties are available for drawing objects contained in all kinds of applications.

\subsubsection{Color Mode}

The attribute draw: color-mode affects the output of colors from a source bitmap or raster graphic.

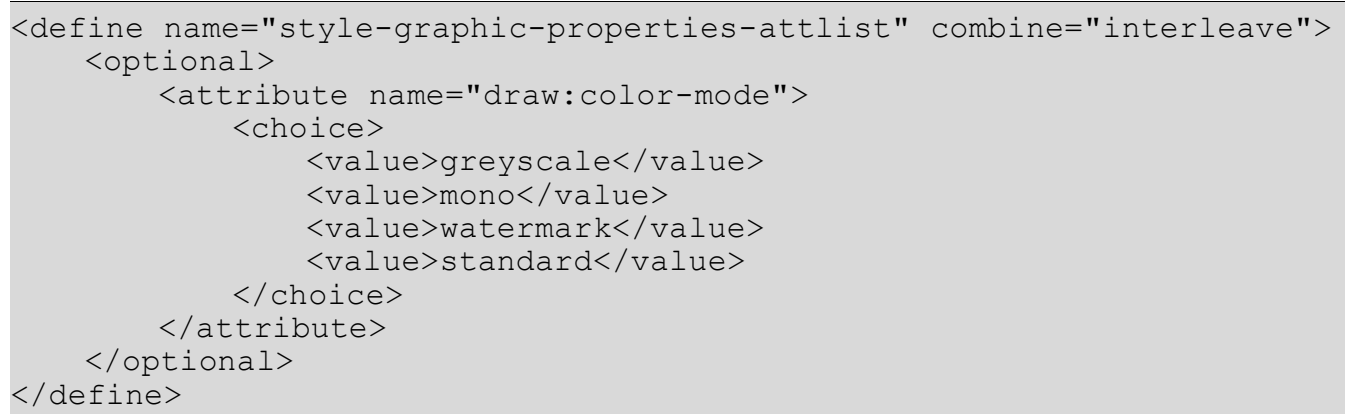

\subsubsection{Color Inversion}

The attribute draw:color-inversion specifies whether or not the colors in the graphic shape should be inverted.

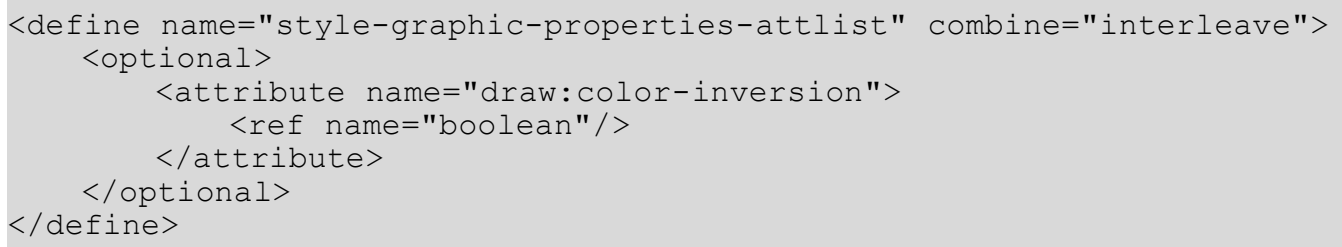




\subsubsection{Adjust Luminance}

The attribute draw: Iuminance specifies a signed percentage value that affects the output luminance of a bitmap or raster graphic.

16003

16004

16005

16006

16007

16008

16009

16010

16011

16012

16013

16014

16015

16016

16017

16018

16019

16020

16021

16022

16023

\subsubsection{Adjust Red}

The attribute draw:red specifies a signed percentage value that affects the output of the red color space of a bitmap or raster graphic.
16024

16025

16026

16027

16028

16029

16030

\subsubsection{Adjust Green}

The attribute draw: green specifies a signed percentage value that affects the output of the green color space of a bitmap or raster graphic. 


\subsubsection{Adjust Blue}

The attribute draw:blue specifies a signed percentage value that affects the output of the blue color space of a bitmap or raster graphic.

\subsection{Shadow Properties}

Most drawing objects can have a shadow. The following attributes specify how the shadow is rendered. These properties are available for drawing objects contained in all kinds of applications.

\subsubsection{Shadow}

The attribute draw: shadow enables or disables the visibility of a shadow.

16052

16053

16054

16055

16056

16057

16058

16059

16060

16061

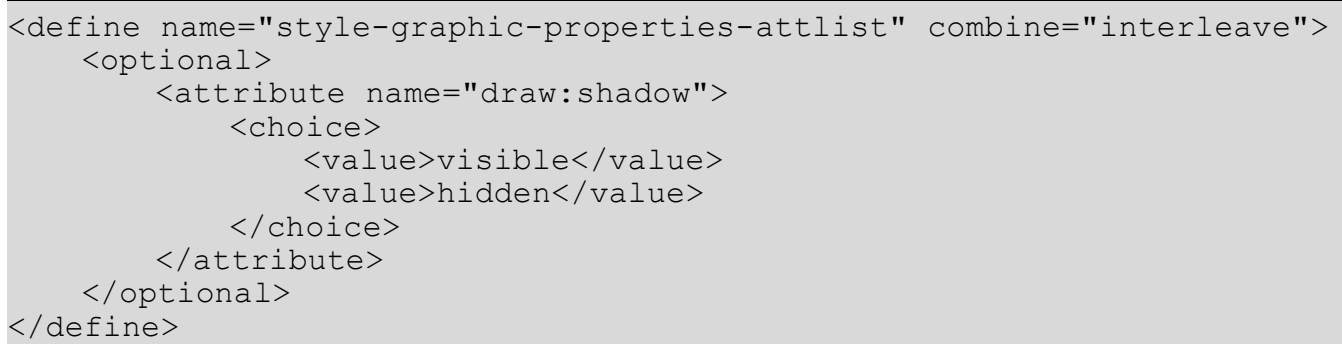

\subsubsection{Offset}

The attributes draw: shadow-offset-x and draw: shadow-offset-y are used to render a shadow. A copy of the shape is rendered in the single shadow color (specified by draw: shadow-color) behind the shape. The offset attributes specify the offset between the top left edge of the shape and the top left edge of the border 


\subsubsection{Color}

The attribute draw:shadow-color specifies the color in which the shadow is rendered.

16074

16075

16076

16077

16078

16079

16080

16081

16082

16083

16084

16085

16086

16087

\subsection{Connector Properties}

The properties described in this section are specific to connector drawing objects. These properties are available for connector drawing objects contained in all kinds of applications.

\subsubsection{Start Line Spacing}

For standard connectors, the attributes draw: start-line-spacing-horizontal and draw: start-line-spacing-vertical increment the length of the escape line from the start shape for standard connectors. For lines connectors, these attributes specify the absolute length of the escape line from the start shape. For other connector types, they are ignored.

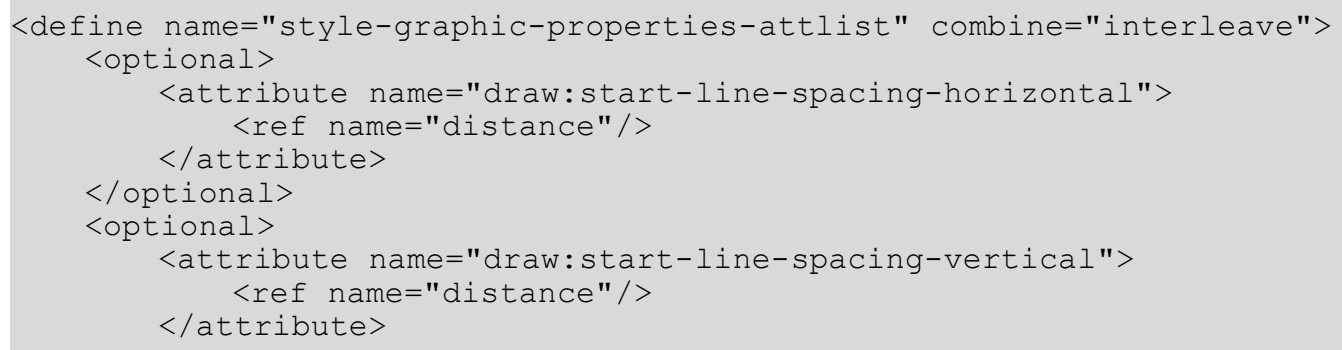




\subsubsection{End Line Spacing}

For standard connectors, the attributes draw:end-line-spacing-horizontal and draw: end-line-spacing-vertical increment the length of the escape line from the end shape. For lines connectors, they specify the absolute length of the escape line from the end shape. For other connector types, they are ignored.

16100

16101

16102

16103

16104

16105

16106

16107

16108

16109

16110

16111

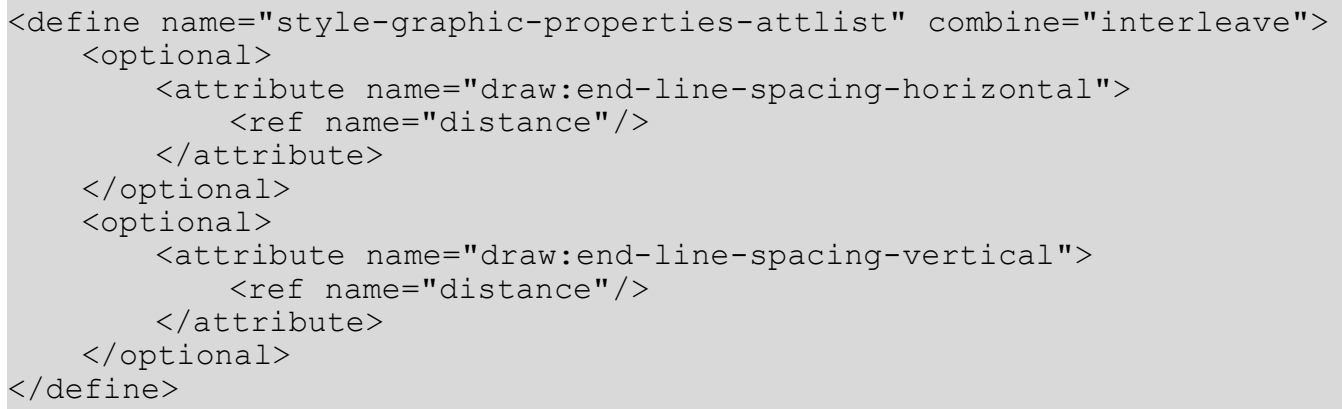

\subsection{Measure Properties}

The properties described in this section are specific to measure drawing objects. These properties are available for measure drawing objects contained in all kinds of applications.

\subsubsection{Line Distance}

The attribute draw: line-distance specifies the distance from the reference points to the measure line.

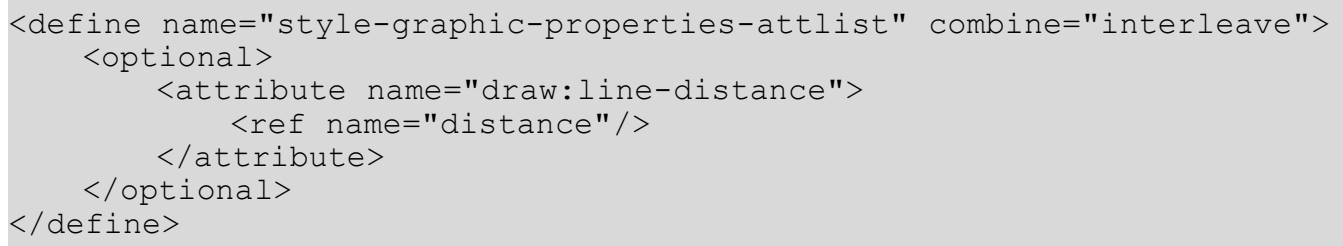

\subsubsection{Guide Overhang}

The guides are the two lines from the reference points to the measure line. The attribute draw: guide-overhang specifies the length that the guides are drawn after they cross the measure line.

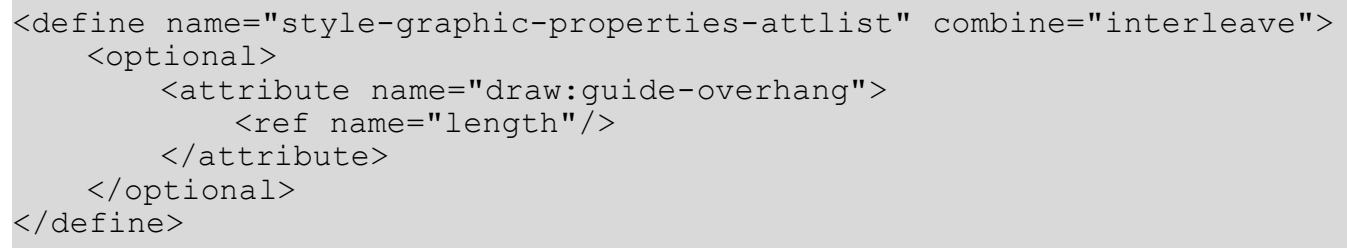




\subsubsection{Guide Distance}

The attribute draw: guide-distance specifies the distance between the reference points and the start point of the guide lines. This distance does not take the attributes draw:start-guide and draw: end-guide into account, that is, the distance specified in draw: guide-distance equals the distance that is actually drawn only if draw:start-guide and draw:end-guide both are 0 .

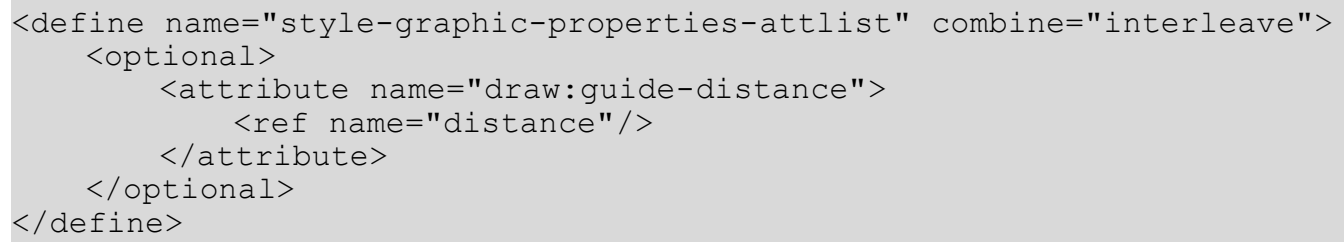

\subsubsection{Start Guide}

The draw: start-guide attribute specifies a length that is added to the length of the guide from the first reference point to the measure line. The guide is extended by this length at the end that points towards the reference points.

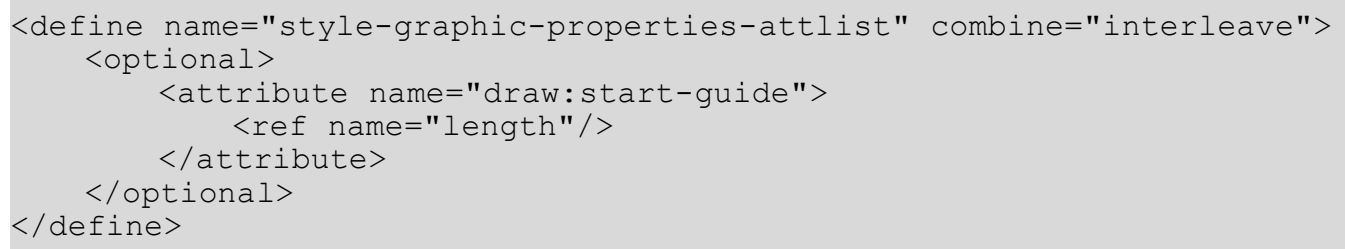

\subsubsection{End Guide}

The draw: end-guide attribute specifies a length that is added to the length of the guide from the second reference point to the measure line. The guide is extended by this length at the end that points towards the reference points.

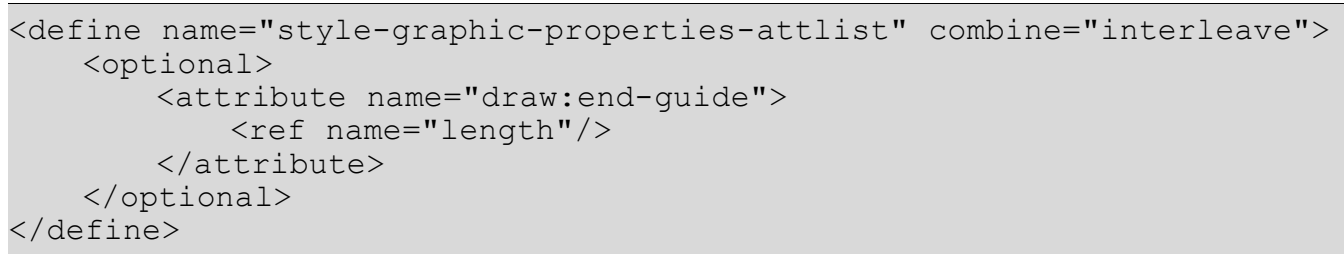

\subsubsection{Placing}

The attribute draw:placing specifies whether the measure line is rendered below or above the edge defined by the two reference points. The value of this attribute can be below or above.

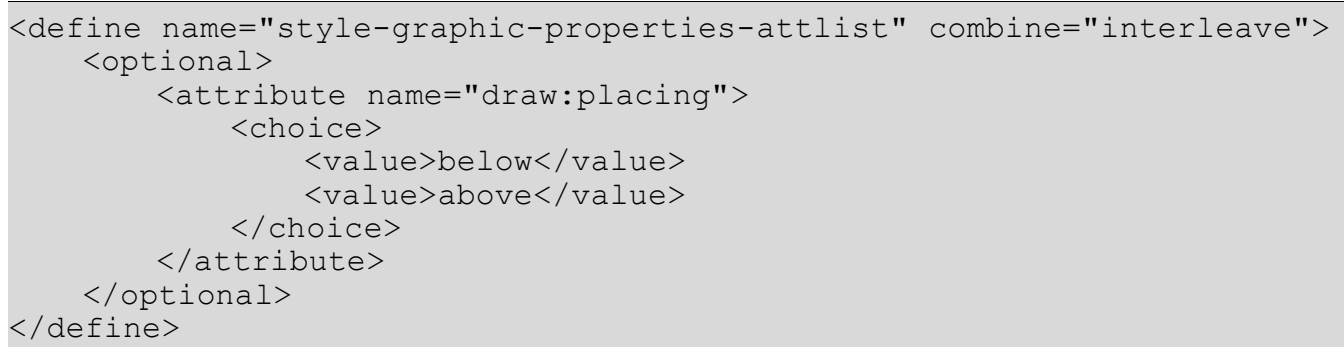




\subsubsection{Show Unit}

The attribute draw:show-unit toggles the display of the unit in the textual presentation of a measure shape.

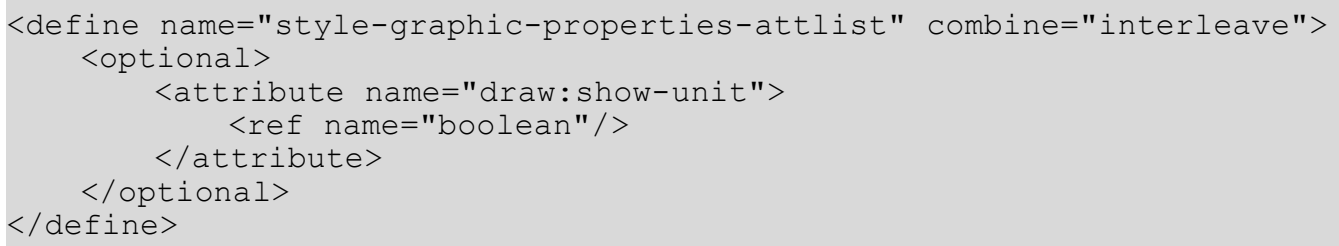

\subsubsection{Decimal Places}

The attribute draw: decimal-places specifies the number of decimal places that are used for the measure text.

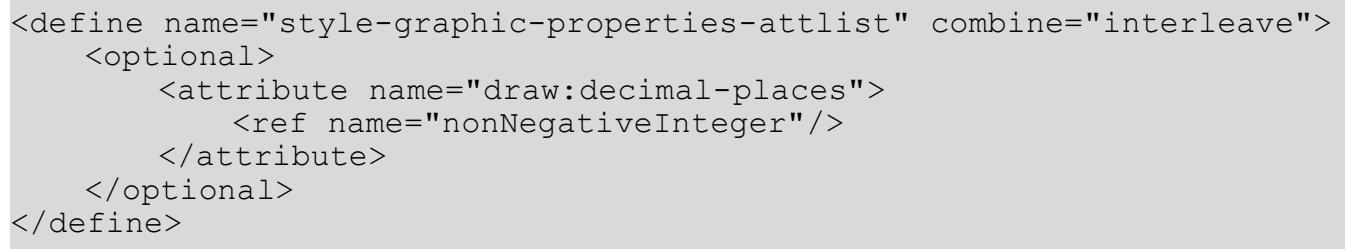

\subsection{Caption Properties}

The following attributes can be used in the styles for caption shapes. These properties are available for caption objects contained in all kinds of applications.

- Type

- Angle type

- Angle

- Gap

- Escape direction

- Escape

- $\quad$ Line length

- Fit line length

\subsubsection{Type}

The attribute draw: caption-type specifies the geometry of the line of a caption.

- straight-line: a straight perpendicular line is drawn to the caption point.

- angled-line: a straight line is drawn to the caption point.

- angled-connector-line: a straight perpendicular line, followed by a straight line is drawn to the caption point. 


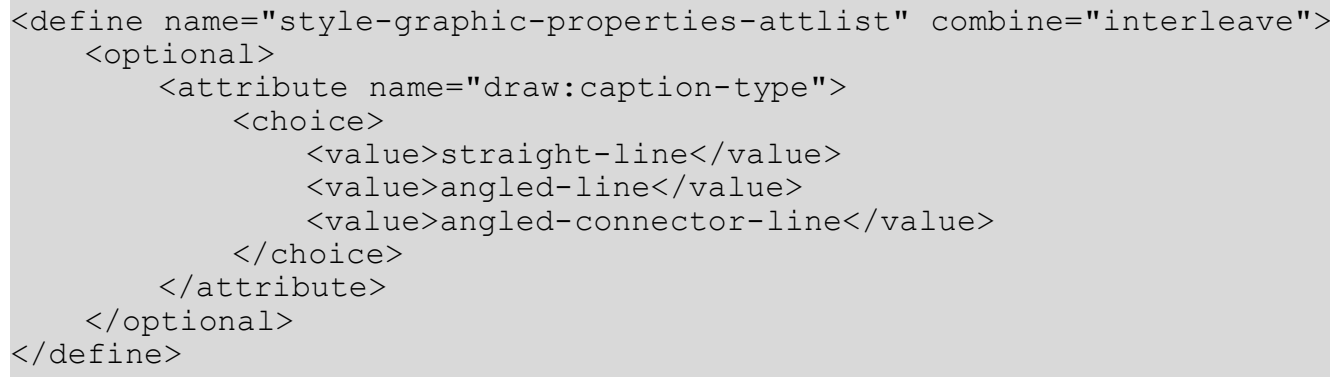

\subsubsection{Angle Type}

The attribute draw: caption-angle-type specifies if the escape angle of the line of a caption is fixed or free. If this is set to free the application can choose the best possible angle.

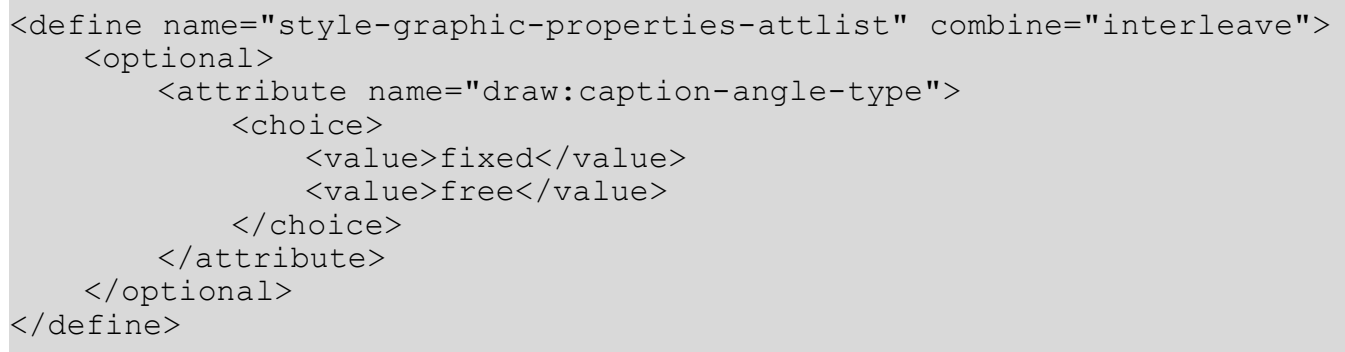

\subsubsection{Angle}

The attribute draw: caption-angle specifies the escape angle of the line of a caption. It is evaluated only if draw: caption-angle-type has the value fixed.

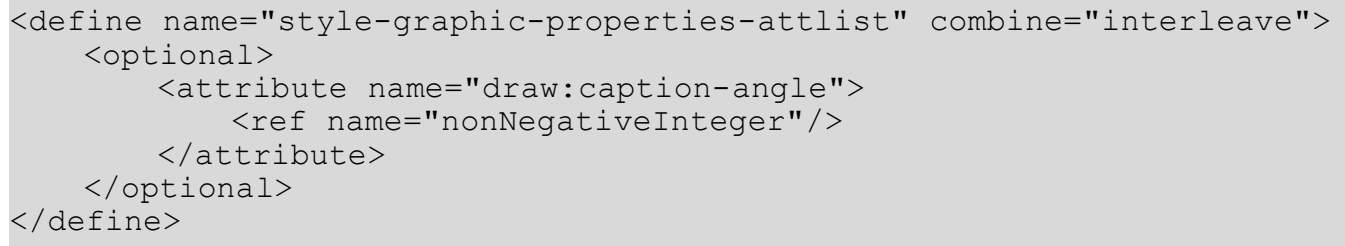

\subsubsection{Gap}

The attribute draw: caption-gap specifies the distance between the text area of the caption and the start of the line.

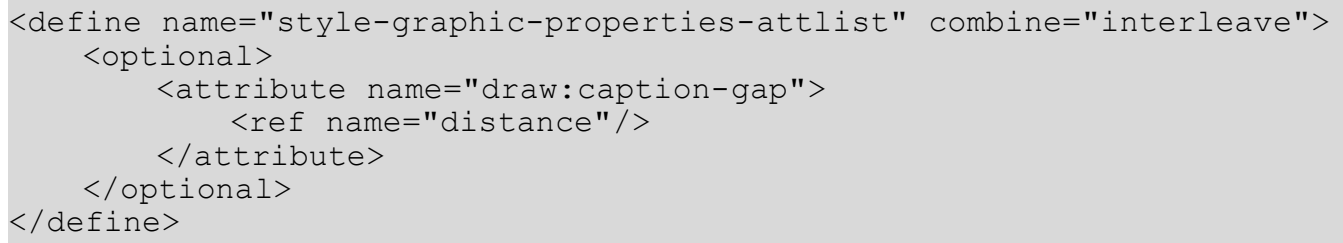

\subsubsection{Escape Direction}

The attribute draw: caption-escape-direction specifies the escape direction for the line of a caption. If this is set to auto the application can choose the best direction. 
$</$ define $>$

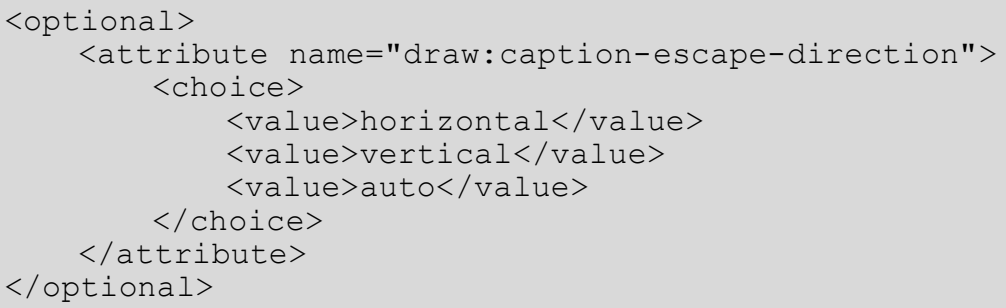

\subsubsection{Escape}

The attribute draw: caption-escape specifies the escape point of the caption line measured from the top left corner of the text area. The value can be an absolute length or an percentage.

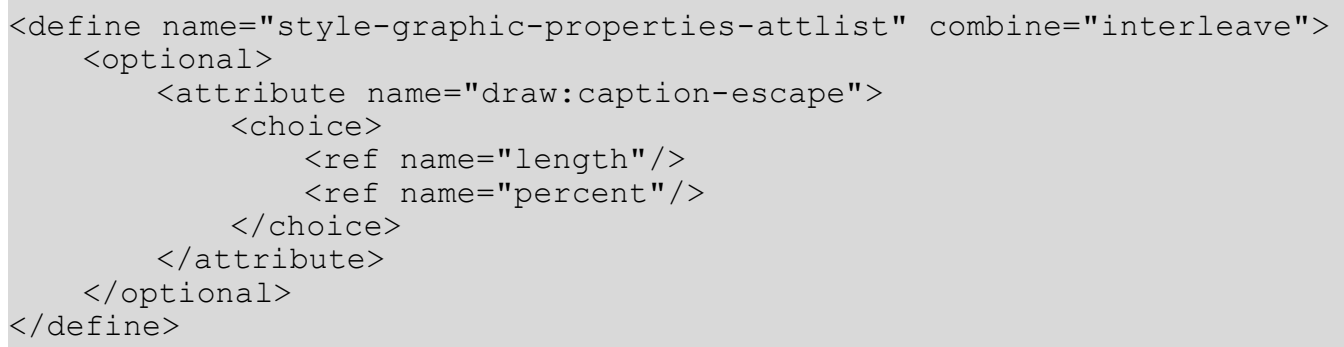

\subsubsection{Line Length}

The attribute draw: caption-line-length specifies the length of the first caption line (i.e. the one that starts at the caption's text area). The attribute is only evaluated if draw: captionfit-line-length has the value false.

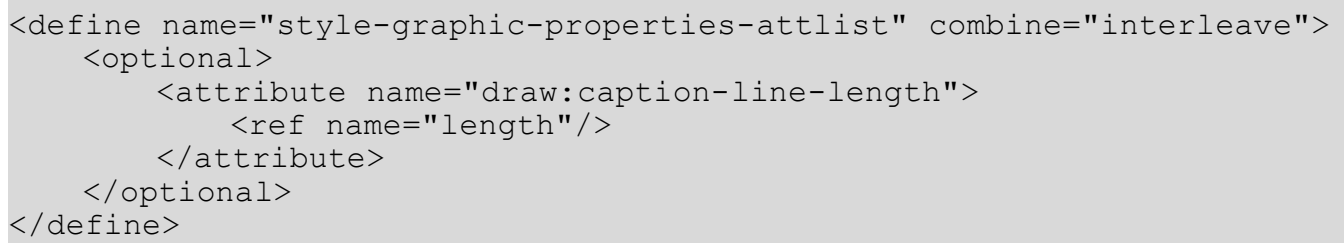

\subsubsection{Fit Line Length}

If the attribute draw: caption-fit-line-length is true, the application determines the best possible length for the caption line.

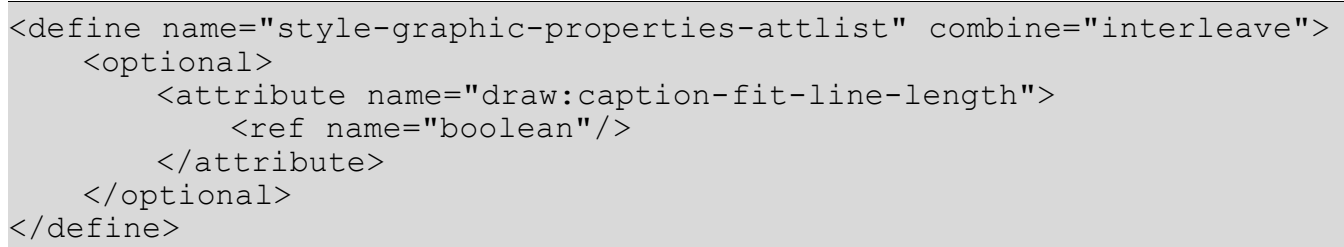

\subsection{D Geometry Properties}

The 3D geometry properties described in this section are applicable to 3D drawing objects. These properties are available for 3D drawing objects contained in all kinds of applications. 


\subsubsection{Horizontal Segments}

If the geometry of a $3 D$ object is generated during run-time, the $d r 3 d$ :horizontal-segments attribute is used to specify the number of horizontal segments that are used to generate the geometry. Typical applications support values between 2 and 256.

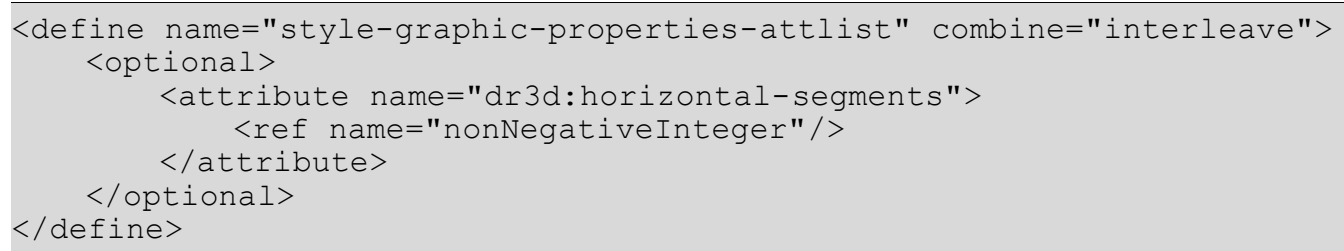

\subsubsection{Vertical Segments}

If the geometry of a 3D object is generated during run-time, the $\mathrm{dr} 3 \mathrm{~d}$ :vertical-segments attribute is used to specify the number of vertical segments that are used to generate the geometry. Typical applications support values between 2 and 256 .

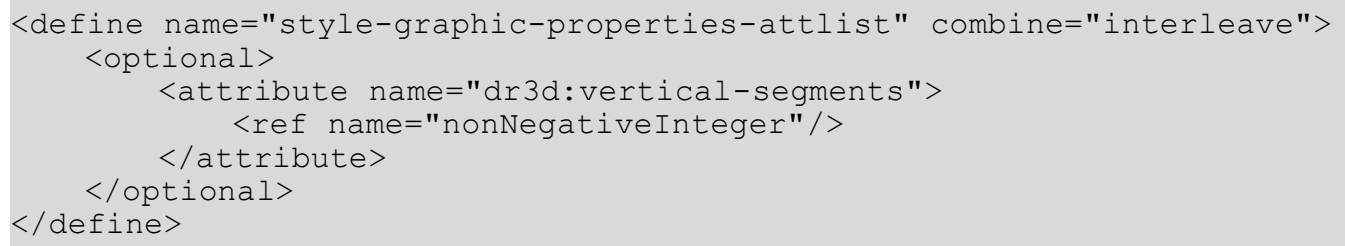

\subsubsection{Edge Rounding}

If the geometry of a 3D object is generated during run-time, the $\mathrm{dr} 3 \mathrm{~d}$ :edge-rounding attribute is used to specify the size of an area at the edges of the geometry that is used for rounding the edges.

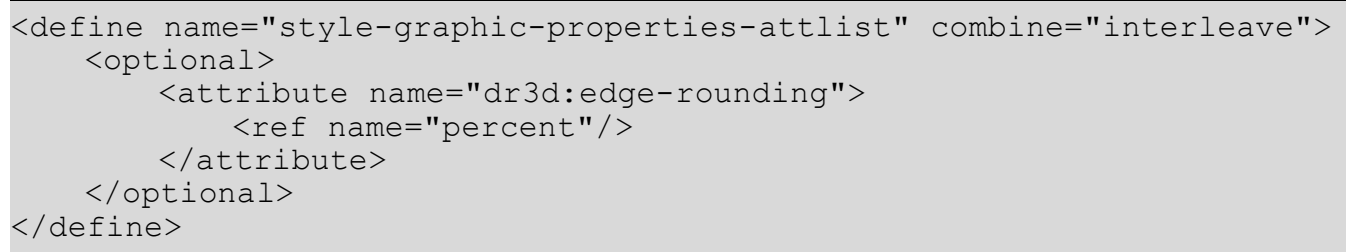

\subsubsection{Edge Rounding Mode}

The attribute $d r 3 d$ :edge-rounding-mode specifies how to generate rounded edges.

The value of this attribute can be correct or attractive. If the value is correct, the mathematically correct method is used. If the value is attractive, a method which preserves the visual appearance of the text is used.

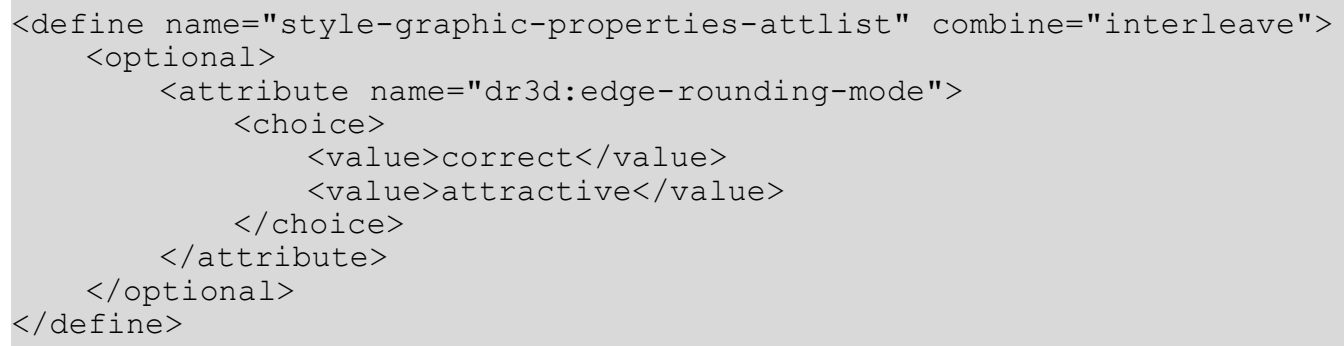




\subsubsection{Back Scale}

The attribute $d r 3 d:$ back-scale specifies the proportion of the background geometry for lathe and extrude objects.

For example, with a back scale of $50 \%$, the background plane of an extrude object is half the size of the foreground plane.

\subsubsection{Backface Culling}

The $d r 3 d$ :backface-culling attribute enables or disables backface culling.

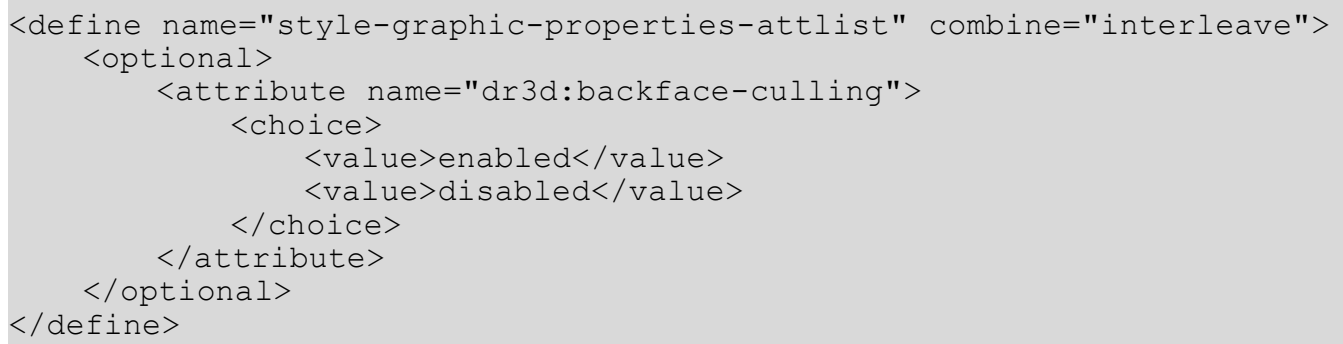

\subsubsection{End Angle}

The attribute $\mathrm{dr} 3 \mathrm{~d}$ : end-angle specifies the rotation angle for 3D lathe objects. If it's the default $\left(360^{\circ}\right)$, the lathe object is closed and completely rotated. With smaller values it is possible to define opened lathe objects (segments). The then visible sides are closed and take into account the $\mathrm{dr} 3 \mathrm{~d}$ : back-scale and $\mathrm{dr} 3 \mathrm{~d}$ : edge-rounding attributes. With bigger values it is possible to create lathe objects with more than one rotation. This will only have a visible effect when e.g. dr $3 d$ : back-scale is used.

For example, with a end angle of $270^{\circ}$, the lathe object will be opened by $90^{\circ}$.

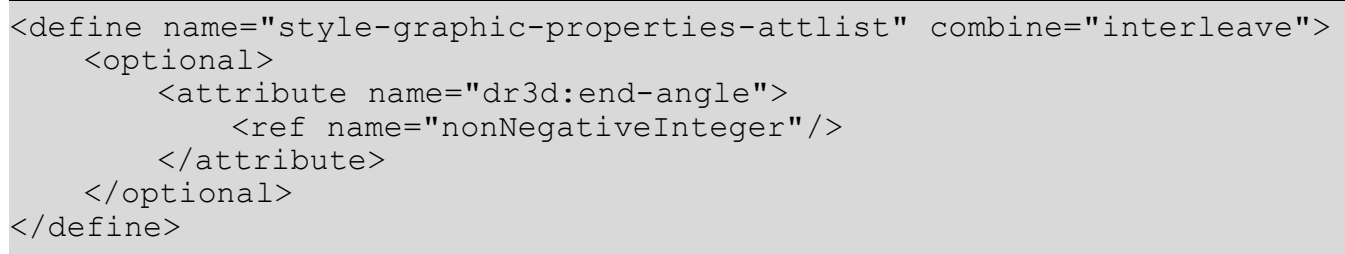




\subsubsection{Close Front}

The $d r 3 d: c l o s e-f r o n t$ property specifies whether a front plane shall be generated. E.g. if an ellipse is extruded, and this attribute is set, the ellipse will have an open front. The attribute can be used with extrudes and lathe objects.

16350

16351

16352

16353

16354

16355

16356

\subsection{D Lighting Properties}

The 3D lightning properties described in this section are applicable to 3D drawing objects. These properties are available for $3 \mathrm{D}$ drawing objects contained in all kinds of applications.

\subsubsection{Mode}

The attribute $d r 3 d$ :lighting-mode determines the lighting algorithm used to render the corresponding $3 \mathrm{D}$ object.

The value of this attribute can be standard or double-sided. If the value is double-sided, the reverse sides of the objects are also lighted.

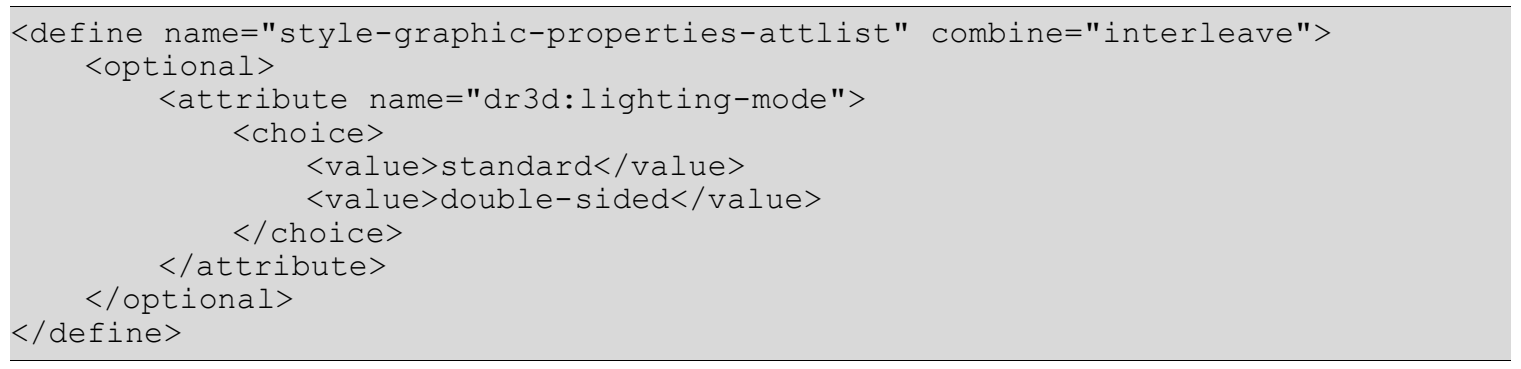

\subsubsection{Normals Kind}

The attribute $\mathrm{dr} 3 \mathrm{~d}$ : normals-kind specifies how the normal settings for the generated lighting.

- object: does not produce standard normals, but leaves the object-specific ones untouched.

- flat: forces one normal per flat part 
- sphere: forces normals to behave as the object would be a sphere.

\subsection{D Texture Properties}

The 3D texture properties described in this section are applicable to 3D drawing objects. These properties are available for 3D drawing objects contained in all kinds of applications.

\subsubsection{Generation Mode}

The attributes $d r 3 d$ :texture-generation-mode-x and $d r 3 d$ :texture-generationmode-y specify how the texture coordinates are generated.

- object: This value specifies that the standard object projection method is used

- parallel: This value specifies a flat parallel projection in the specified degree of freedom (X or $\mathrm{Y})$.

- sphere: This value forces projection to wrapping in $X$ and/or $Y$ direction 


\subsubsection{Kind}

The attribute $d r 3 d$ : texture-kind is used to select whether the texture changes the luminance, intensity, or color of the shape.

16415

16416

16417

16418

16419

16420

16421

16422

16423

16424

16425

\subsubsection{Filter}

The attribute $d r 3 d$ :texture-filter is used to enable or disable texture filtering.

16426

16427

16428

16429

16430

16431

16432

16433

16434

16435

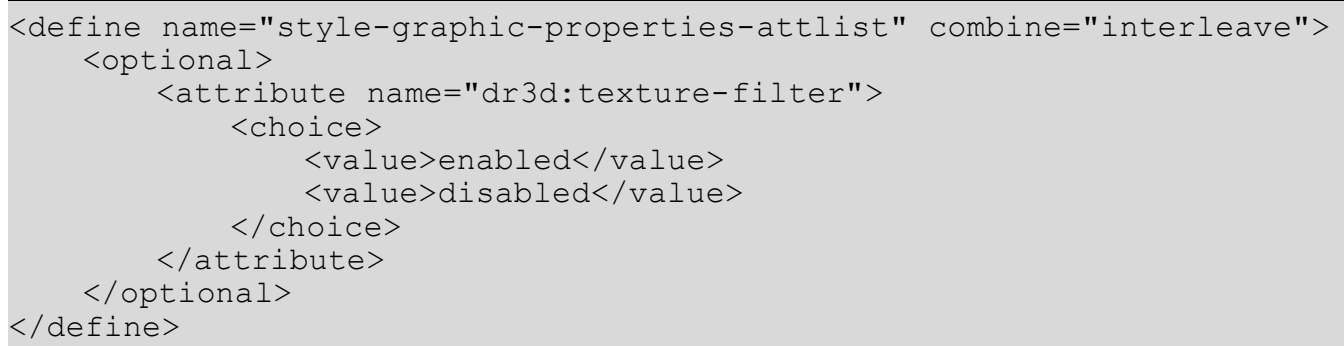

\subsubsection{Mode}

The attribute $d r 3 d$ :normals-direction is used to specify how the texture is modulated.

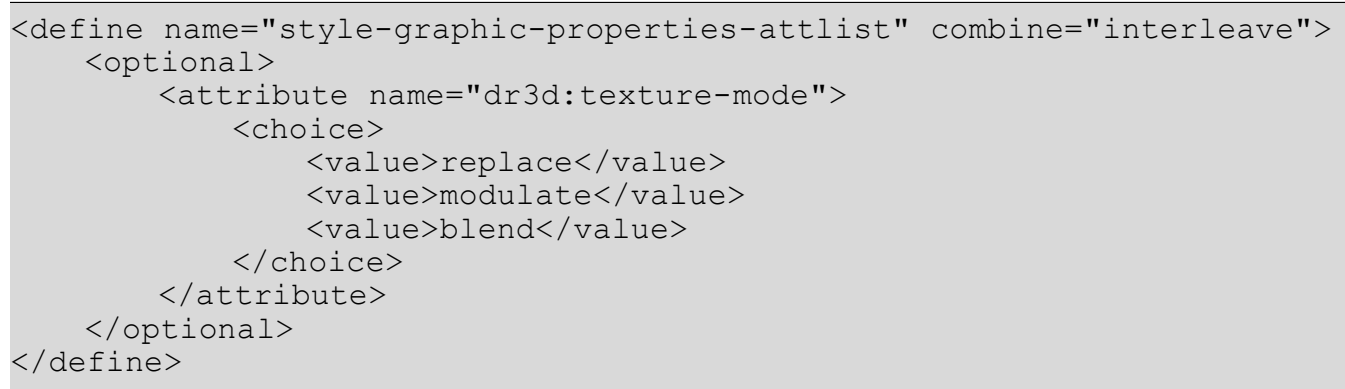

\subsection{D Material Properties}

The 3D texture properties described in this section are applicable to 3D drawing objects. These properties are available for 3D drawing objects contained in all kinds of applications. 


\subsubsection{Colors}

The attributes $d r 3 d$ :ambient-color, $d r 3 d$ :emissive-color, $d r 3 d$ :specular-color and dr3d:diffuse-color specify the four colors that define a material.

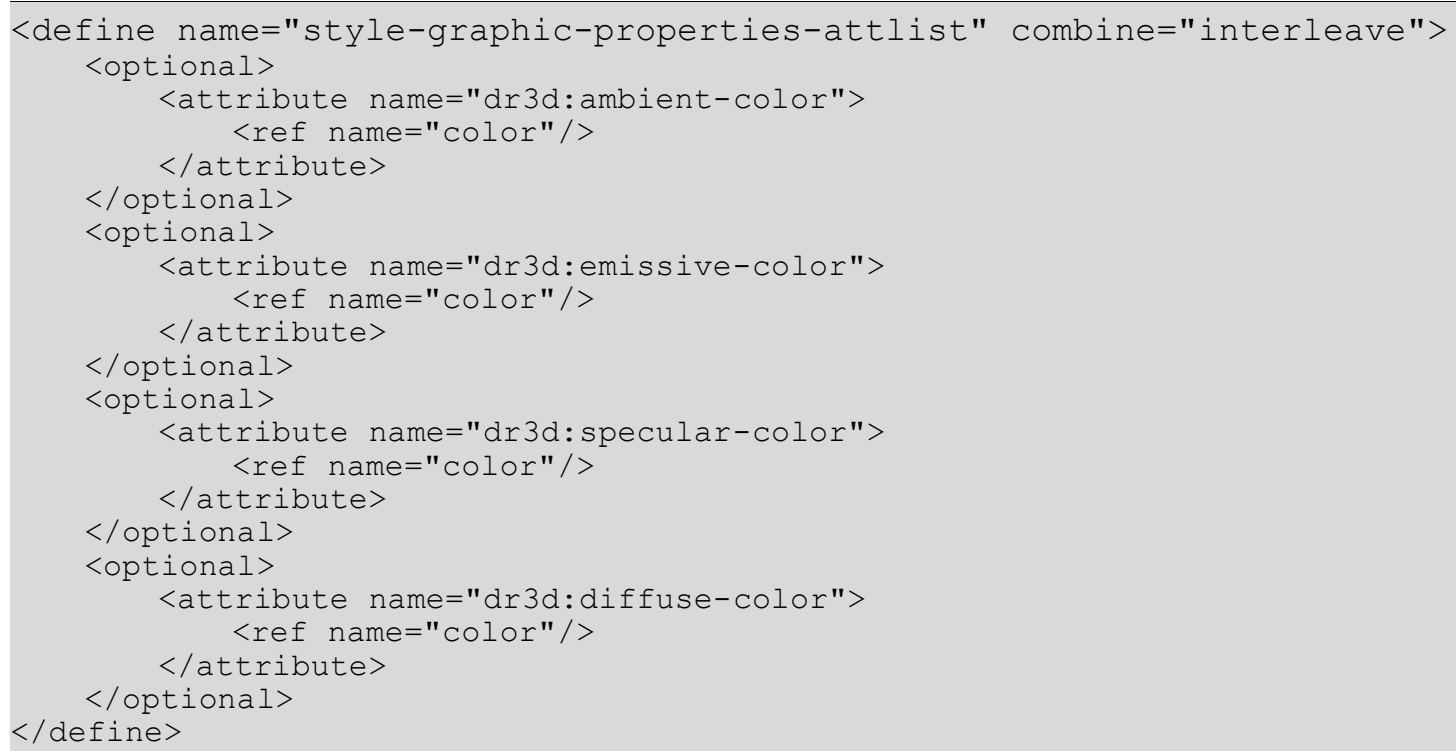

\subsubsection{Shininess}

The attribute $d r 3 d$ :shininess specifies the shine of the used material.

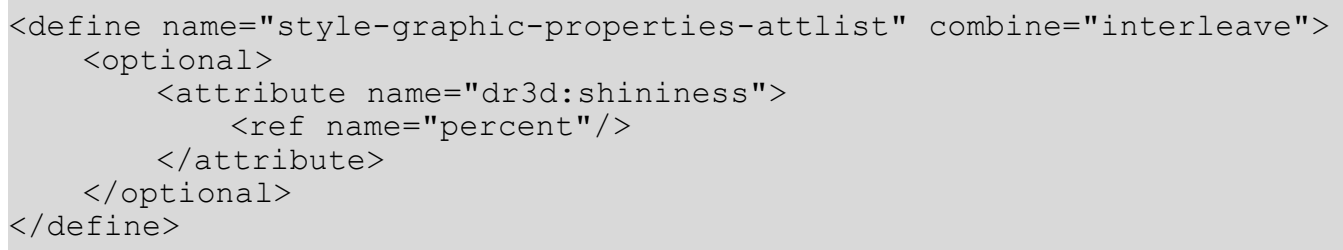

\subsection{D Shadow Properties}

The 3D shadow properties described in this section are applicable to 3D drawing objects. These properties are available for 3D drawing objects contained in all kinds of applications.

\subsubsection{Shadow}

The attribute $\mathrm{dr} 3 \mathrm{~d}$ : shadow enables or disables a three-dimensional shadow for a threedimensional object.

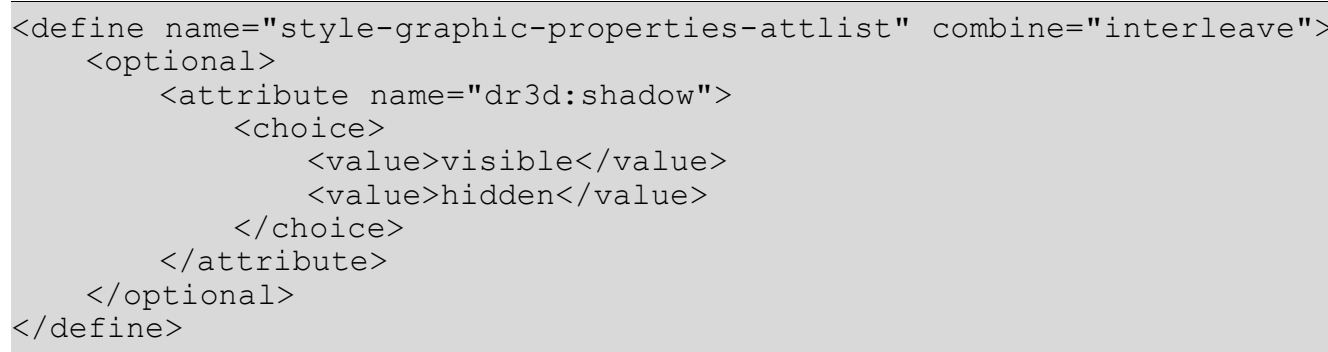




\subsection{Frame Formatting Properties}

The properties described in this section apply to draw frames (see section 9.3). They can be used within graphic styles (see section 14.13.1) and they are contained in a <style: graphicproperties $>$ element.

\subsubsection{Frame Widths}

There are three types of frame widths; fixed widths, minimum widths and relative widths. Fixed widths are specified using the svg:width attribute, minimum widths are specified using the fo:min-width attribute and relative widths are specified using the style:rel-width attribute. The meaning of these attributes is the same as described in section 9.3, except that the attributes specify the default width for new created frames only. The style:rel-width attribute will be evaluated only for graphic styles that are applied to text boxes.

\subsubsection{Frame Heights}

There are three types of frame heights; fixed heights, minimum heights and relative heights. Fixed heights are specified using the svg: height attribute, minimum heights are specified using the fo:min-height attribute and relative heights are specified using the style:relheight attribute. The meaning of these attributes is the same as described in section 9.3, except that the attributes specify the default height for new created frames only. The style:rel-height attribute will be evaluated only for graphic styles that are applied to text boxes. See also section 15.27.1.

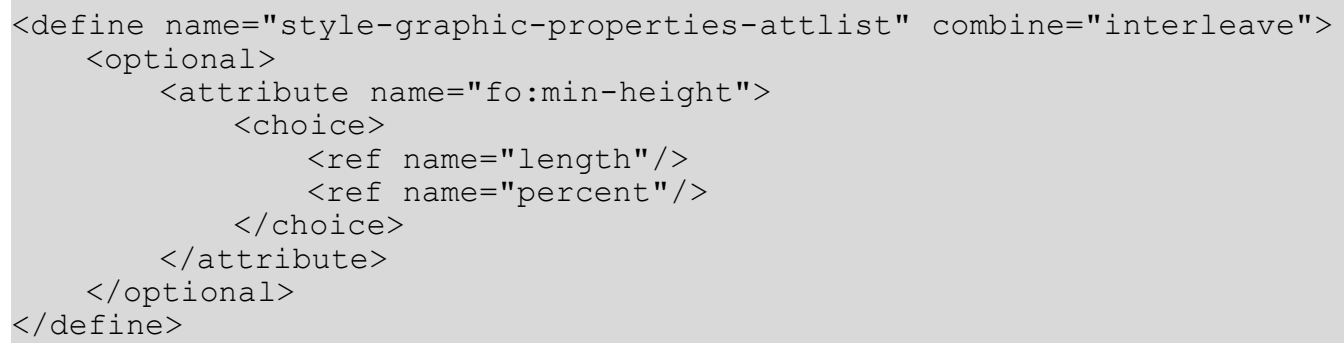

\subsubsection{Maximum Width and Height}

Text boxes can increase in size automatically when content is added. The fo:max-width and fo:max-height attributes specify a maximum width and height for the frame. When the maximum values are reached, the frame stops increasing in size. The attributes' value can be either a length or a percentage. If the anchor for the text box is in a table cell, the percentage value relates to the surrounding table box. If the anchor for the text box is in a text box, the percentage value relates to the surrounding text box. In other cases, the percentage values relate to the height of the page or window. 


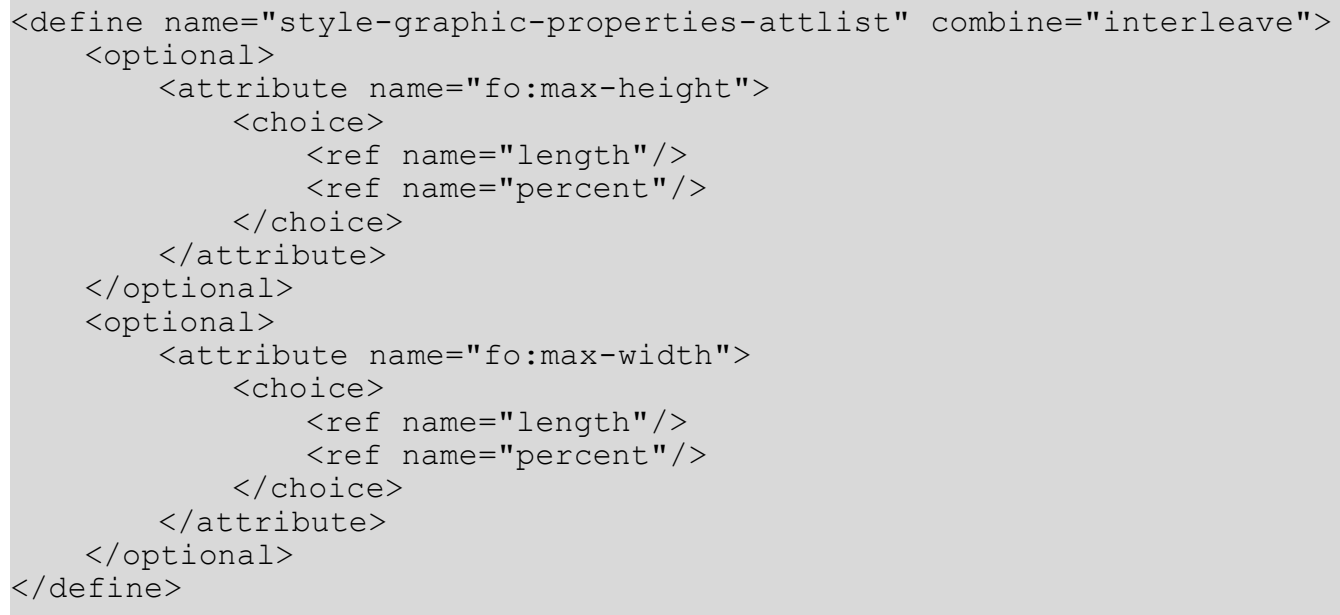

\subsubsection{Left and Right Margins}

The fo:margin-left and fo:margin-right properties determine the left and right margins to set around a frame. See sections 15.5.17 for detailed information on these attributes.

Percentage values are not supported.

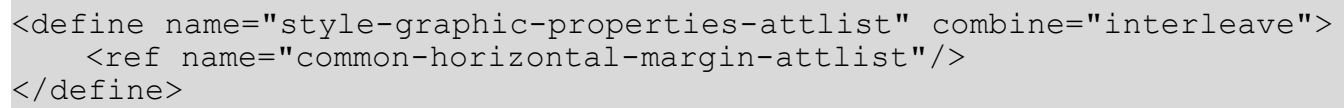

\subsubsection{Top and Bottom Margins}

The fo:margin-top and fo:margin-bottom properties determine the top and bottom margins to set around a frame. See sections 15.5 .20 for detailed information on these attributes. Percentage values are not supported.

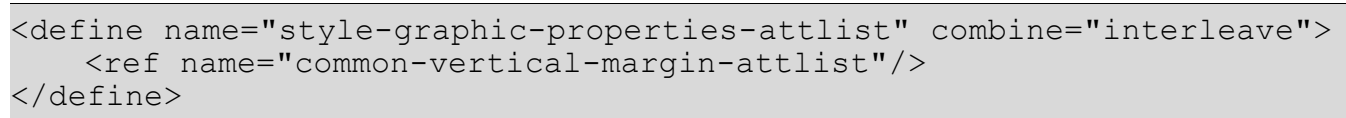

\subsubsection{Margins}

The fo:margin property specifies the the margin for all four edges of a frame. See section 15.5.21 for a full explanation of this property.

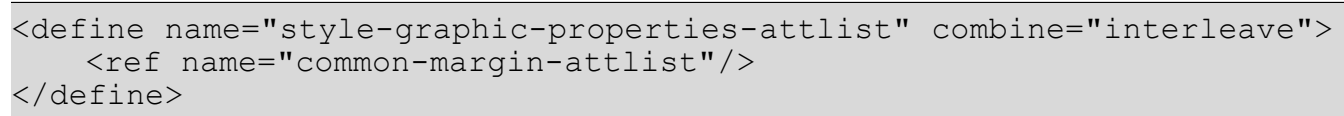

\subsubsection{Print Content}

The style:print-content property specifies whether or not the content of a frame is printed.

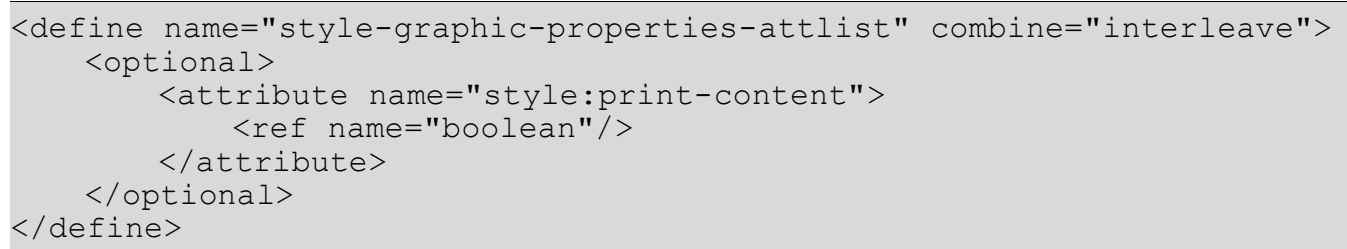




\subsubsection{Protect}

The style:protect property specifies whether the content, size, or position of a frame is protected. The value of this property can be either none or a space separated list that consists of any of the values content, position, or size.

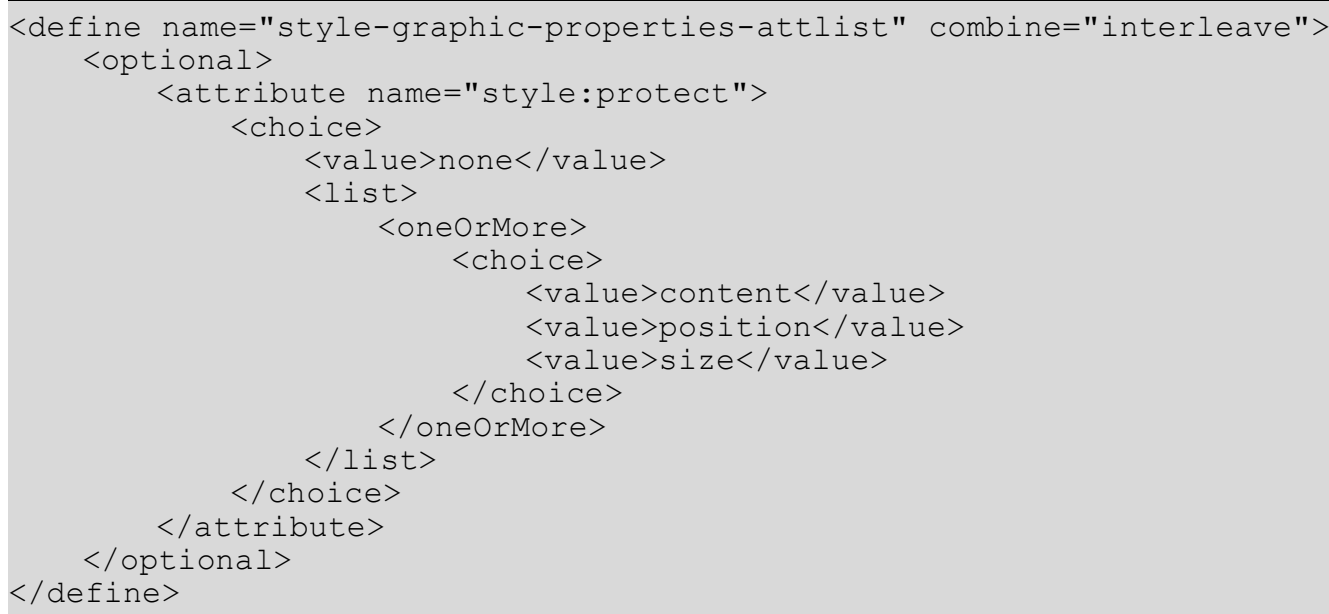

\subsubsection{Horizontal Position}

Within text documents, the style: horizontal-pos property specifies the horizontal alignment of the frame in relation to the specific area.

The value of this property can be one of the following: from-left, left, center, right, from-inside, inside, or outside. The area that the position relates to is specified by the style:horizontal-rel property. The values from-inside, inside and outside correspond to the values from-left, left, and right on pages that have an odd page number and to the opposite values on pages that have an even page number.

If the property value is $f r o m-l e f t$ or $f r o m-i n s i d e$, the $s v g: x$ attribute associated with the frame element specifies the horizontal position of the frame. Otherwise the $s v g: x$ attribute is ignored for text documents.

It is also possible to use an svg:x attribute within a graphic style. If this is the case, then the attribute specifies a default position for new frames that are created using this style.

Some values may be used in connection with certain frame anchor and relation types only.

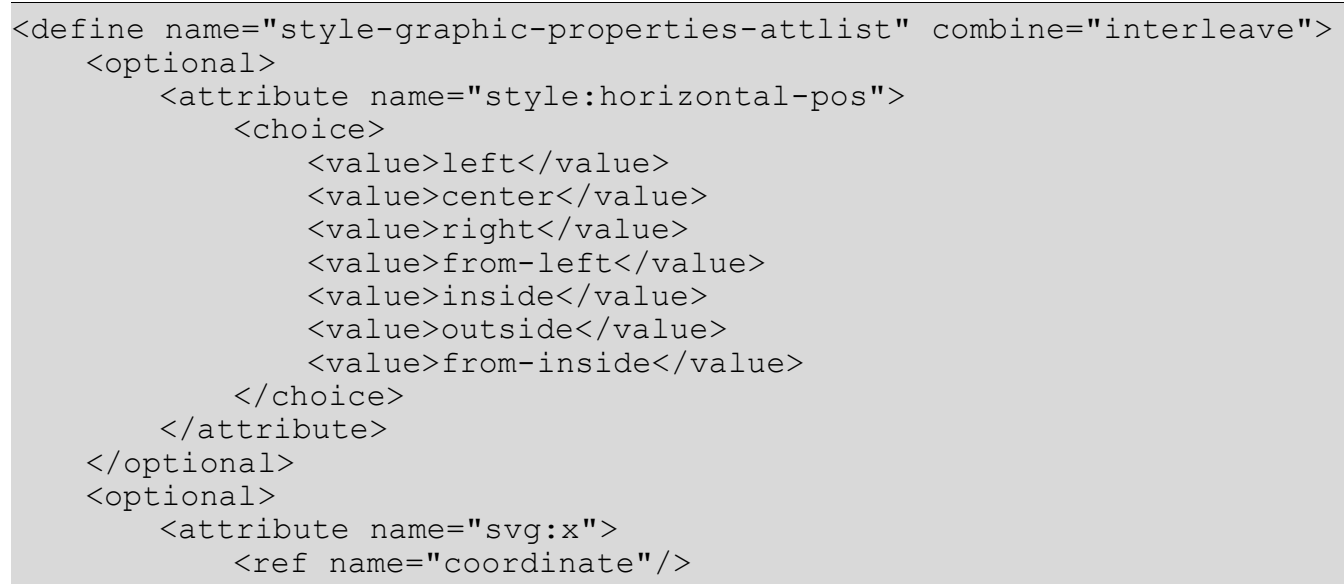


The following tables display the possible values of the attributes style:horizontal-pos and style:horizontal-rel. The possible values of these alignment attributes are listed in the first column on the left, and an alignment attribute value/anchor type value match is indicated by an X.

\begin{tabular}{|l|l|l|l|l|l|}
\hline \multirow{2}{*}{$\begin{array}{c}\text { Value of } \\
\text { style:horizon } \\
\text { tal-pos }\end{array}$} & \multicolumn{5}{|c|}{ Value of text:anchor-type } \\
\cline { 2 - 7 } & page & frame & paragraph & char & as-char \\
\hline any & $\mathrm{X}$ & $\mathrm{X}$ & $\mathrm{X}$ & $\mathrm{X}$ & \\
\hline
\end{tabular}

\begin{tabular}{|l|l|l|l|l|l|}
\hline \multirow{2}{*}{$\begin{array}{c}\text { Value of } \\
\text { style:horizon } \\
\text { tal-rel }\end{array}$} & \multicolumn{5}{|c|}{ Value of text:anchor-type } \\
\cline { 2 - 6 } page & page & frame & paragraph & char & as-char \\
\hline page-content & $\mathrm{X}$ & & $\mathrm{X}$ & $\mathrm{X}$ & \\
\hline page-start-margin & $\mathrm{X}$ & & $\mathrm{X}$ & $\mathrm{X}$ & \\
\hline page-end-margin & $\mathrm{X}$ & $\mathrm{X}$ & $\mathrm{X}$ & \\
\hline frame & & $\mathrm{X}$ & $\mathrm{X}$ & $\mathrm{X}$ & \\
\hline frame-content & & $\mathrm{X}$ & & & \\
\hline frame-start-margin & & $\mathrm{X}$ & & & \\
\hline frame-end-margin & & $\mathrm{X}$ & & $\mathrm{X}$ & \\
\hline paragraph & & & $\mathrm{X}$ & $\mathrm{X}$ & \\
\hline paragraph-content & & & $\mathrm{X}$ & $\mathrm{X}$ & \\
\hline $\begin{array}{l}\text { paragraph-start- } \\
\text { margin }\end{array}$ & & & $\mathrm{X}$ & $\mathrm{X}$ & \\
\hline $\begin{array}{l}\text { paragraph-end- } \\
\text { margin }\end{array}$ & & & $\mathrm{X}$ & $\mathrm{X}$ & \\
\hline char & & & & $\mathrm{X}$ & \\
\hline
\end{tabular}

\subsubsection{Horizontal Relation}

The style:horizontal-rel property specifies the area to which the horizontal position of a frame relates. See the previous section for information on the style:horizontal-pos property.

The value of this property can be one of the following: page, page-content, page-startmargin, page-end-margin, frame, frame-content, frame-start-margin, frame-endmargin, paragraph, paragraph-content, paragraph-start-margin, paragraph-endmargin, or char. 
Some values can be used with only certain frame anchor types.

The value start-margin determines the left margin, except when the horizontal position is from-inside, inside or outside and the anchor for the frame is on a page with an even page number, in which case it determines the right margin. The value end-margin determines the opposite margin to the start-margin values.

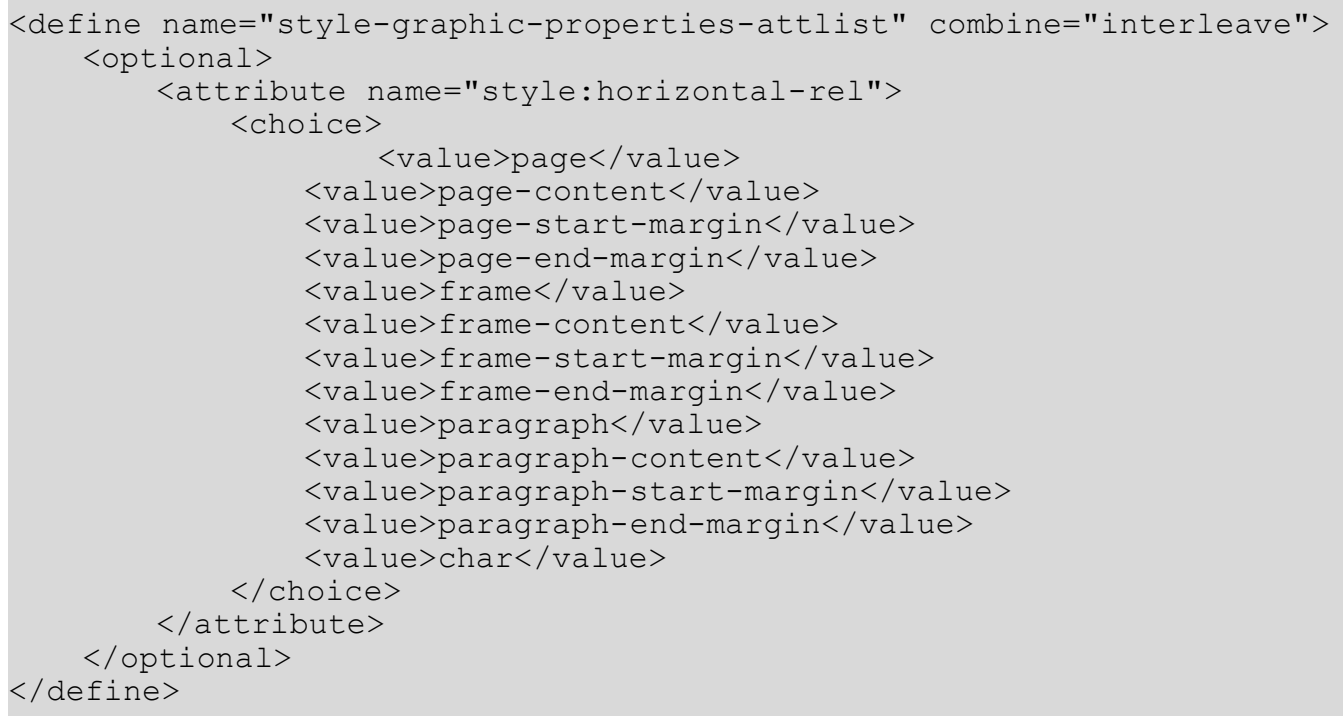

\subsubsection{Vertical Position}

The style:vertical-pos property specifies the vertical alignment of the frame in relation to a specific area.

The value of this property can be one of the following: from-top, top, middle, below or bottom. The area that the position relates to is specified by the style:vertical-rel property. top, middle and bottom specify the the given corners of the frame and the reference area get aligned. below specifies that the top corner of frame is positioned below the reference area.

If the value of this property is $f r o m-t o p$, the $s v g: y$ attribute associated with the frame element specifies the vertical position of the frame. Otherwise, the svg:y attribute is ignored for text documents.

It is also possible to use an $\mathrm{svg}: \mathrm{y}$ attribute within a graphic style. If this is the case, the attribute specifies a default position for new frames that are created using this style.

Some values may be used in connection with certain frame anchor and relation types only.

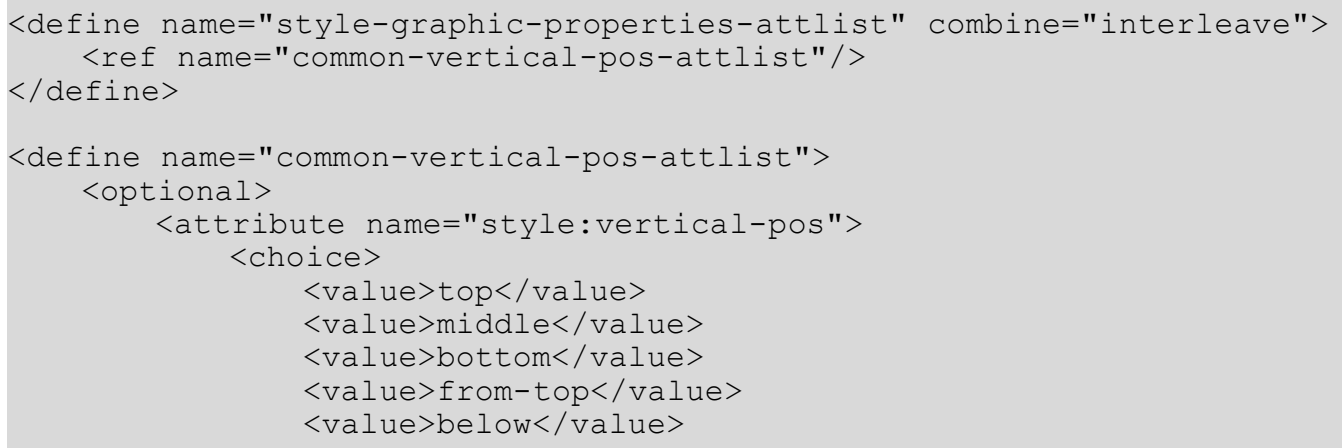




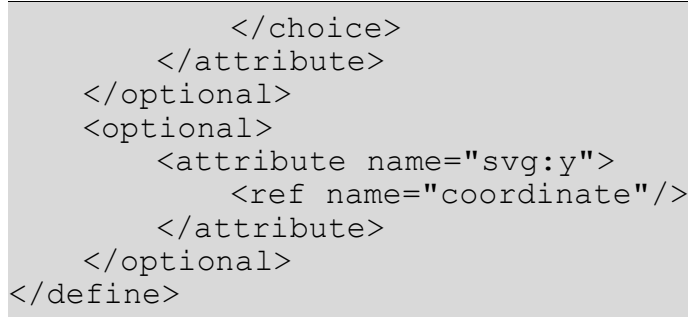

The following tables display the possible values of the attributes style:vertical-pos and style:vertical-rel. The possible values of these alignment attributes are listed in the first column on the left, and an alignment attribute value/anchor type value match is indicated by an $\mathrm{X}$.

\begin{tabular}{|c|c|c|c|c|c|}
\hline \multirow{2}{*}{$\begin{array}{c}\text { Value of } \\
\text { style: vertical } \\
\text {-pos }\end{array}$} & \multicolumn{6}{|c|}{ Value of text:anchor-type } \\
\cline { 2 - 7 } any & page & frame & paragraph & char & as-char \\
\hline
\end{tabular}

\begin{tabular}{|l|l|l|l|l|l|}
\hline \multirow{2}{*}{$\begin{array}{c}\text { Value of } \\
\text { style:vertical } \\
- \text {-rel }\end{array}$} & \multicolumn{5}{|c|}{ Value of text:anchor-type } \\
\cline { 2 - 6 } page & $\mathrm{X}$ & frame & paragraph & char & as-char \\
\hline page-content & $\mathrm{X}$ & & & & \\
\hline frame & & $\mathrm{X}$ & & & \\
\hline frame-content & & $\mathrm{X}$ & & & \\
\hline paragraph & & & $\mathrm{X}$ & $\mathrm{X}$ & \\
\hline paragraph-content & & & $\mathrm{X}$ & $\mathrm{X}$ & \\
\hline char & & & & $\mathrm{X}$ & $\mathrm{X}$ \\
\hline line & & & & & $\mathrm{X}$ \\
\hline baseline & & & & & $\mathrm{X}$ \\
\hline text & & & & & $\mathrm{X}$ \\
\hline
\end{tabular}

\subsubsection{Vertical Relation}

The style:vertical-rel property specifies the area to which the vertical position of a frame relates. See the previous section for information on the style:vertical-pos property.

The value of this property can be one of the following: page, page-content, frame, framecontent, paragraph, paragraph-content, line, baseline, text or char.

Some values can be used with only certain frame anchor types.

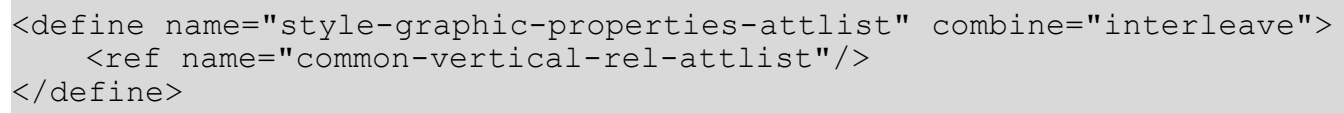




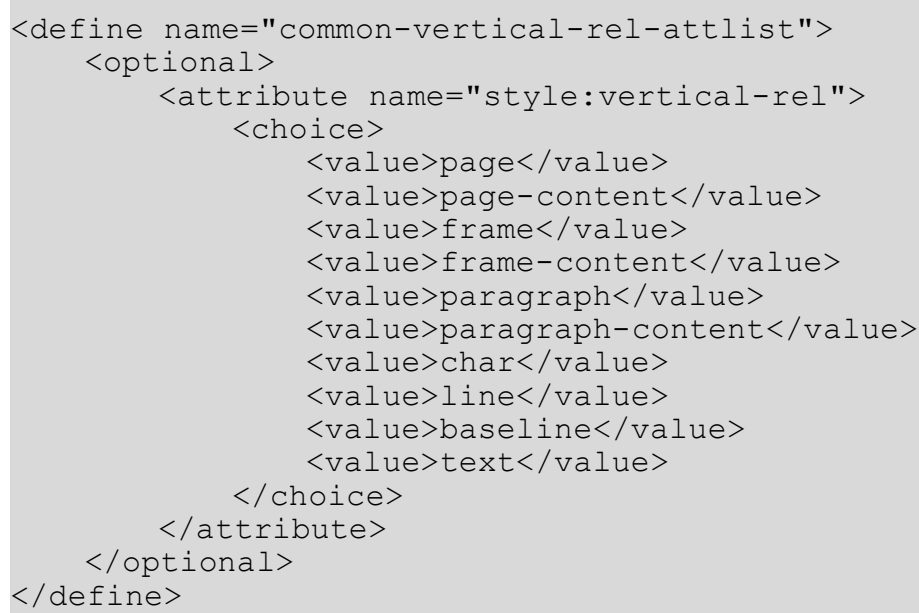

\subsubsection{Frame Anchor}

The text:anchor-type and text:anchor-page-number specify the default anchor for new frames and drawing objects. See section 9.2.16 for details.

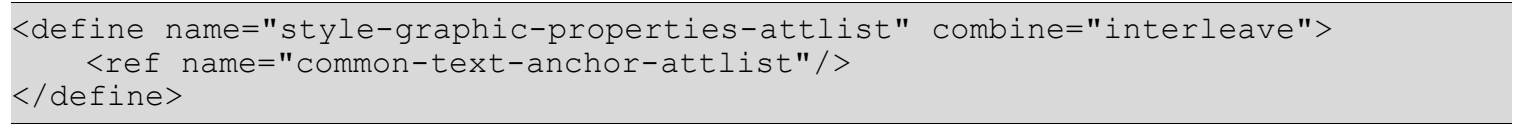

\subsubsection{Border}

The border attributes fo:border, fo:border-top, fo:border-bottom, fo:border-left and fo:border-right specify the border properties of the frame. See section 15.5.25 for detailed information on these attributes.

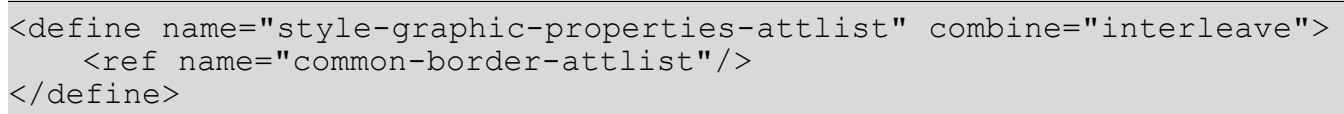

\subsubsection{Border Line Width}

If a frame has borders, the border line width attributes style:border-line-width, style:border-line-width-top, style:border-line-width-bottom, style:borderline-width-left and style:border-line-width-right specify the properties of the border lines of the frame. See section 15.5.26 for detailed information on these attributes.

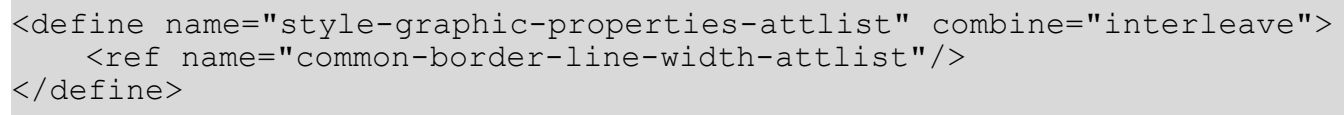

\subsubsection{Padding}

The padding attributes fo:padding, fo:padding-top, fo:padding-bottom, fo:paddingleft and fo:padding-right specify the padding properties of the frame. See section 15.5.27 for detailed information on these attributes. 


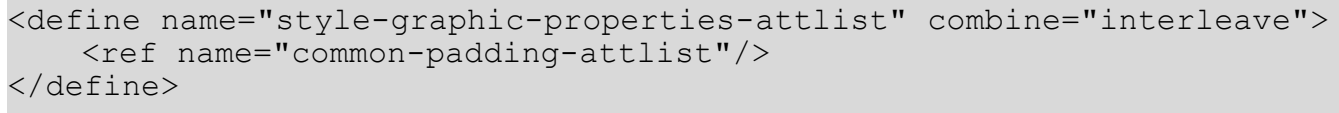

\subsubsection{Shadow}

The shadow attribute style: shadow specifies the shadow of the frame. See section 15.5 .28 for detailed information on this attribute.

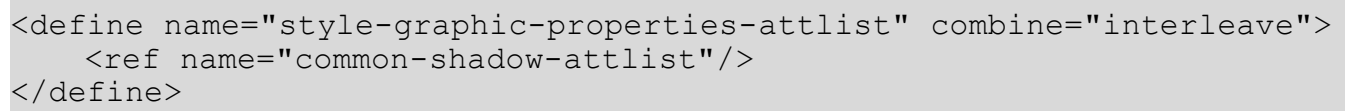

\subsubsection{Background}

The background attribute $\mathrm{fo}$ :background-color and the background element <style:background-image> specify the background properties of the frame. See sections 15.5.23 and 15.5.24 for detailed information on this attribute and element.

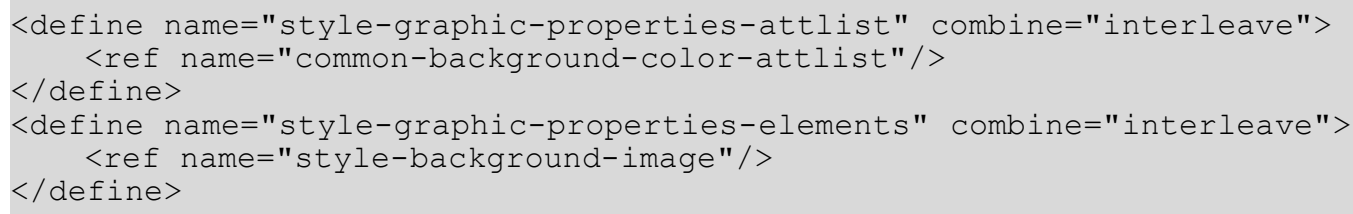

\subsubsection{Columns}

The <style:columns > element specifies if a text box contains columns. See section 15.7 .3 for detailed information on this element.

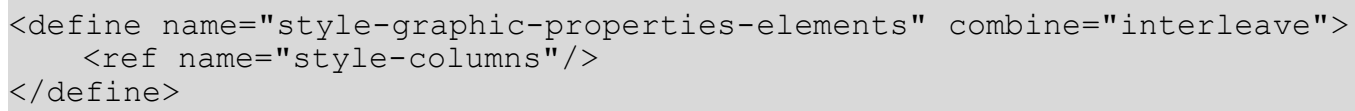

\subsubsection{Editable}

Within text documents, a text box can be editable even if the document in which it is contained is a read-only document. The style: editable property specifies if a text box can be edited.

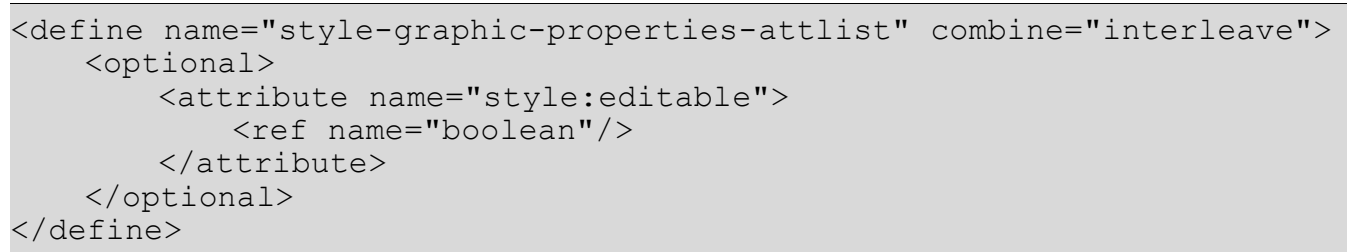

\subsubsection{Wrapping}

Within text documents, the style: wrap property specifies how text around a frame or graphic object is treated. For example, text can run around the left side of the frame, around the right side of the frame, or through the frame. The possible values are: 
- none: no text wraps around the drawing shape.

- left: Text may wrap around the left side of the drawing shape.

- right: Text may wrap around the left side of the drawing shape.

- parallel: Text may wrap around both sides of the drawing shape.

- dynamic: Text may wrap around both sides of the drawing shape, provided that there is sufficient space left.

- biggest: Text may wraps around the object border where the difference to the left or right page or column border is largest.

- run-through: Text runs through the drawing object.

16675

16676

16677

16678

16679

16680

16681

16682

16683

16684

16685

16686

16687

16688

16689

16690

16691

16692

16693

16694

16695

16696

\subsubsection{Paragraph-only Wrapping}

If the anchor position of a frame or drawing shape is a paragraph or a character, and the wrap mode specified by the style:wrap property is left, right, parallel, or dynamic, the number of paragraphs that wrap around the frame can be specified using a style: numberwrapped-paragraphs attribute.

This property is only recognized by frames or styles that have a style:wrap property attached with a value of left, right, parallel, or dynamic.

If the value is no-limit, there is no limit on the number of paragraphs that are allowed to wrap around a frame.

$$
\text { <optional> }
$$




\subsubsection{Contour Wrapping}

Within text documents, the style:wrap-contour attribute specifies for some frame types that the text should wrap around the shape of the object in the frame rather than around the frame itself . This is called contour wrapping.

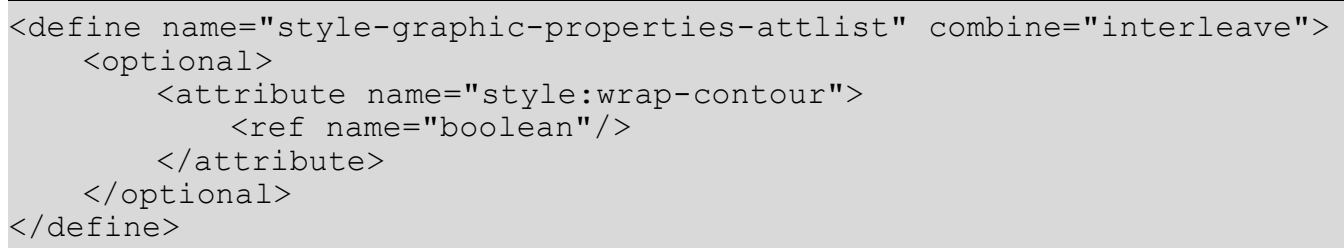

\subsubsection{Contour Wrapping Mode}

The style:wrap-contour-mode attribute is used to further specify how the text should wrap around the contour.

This attribute is recognized only by frames/drawing shapes or styles that already have the style:wrap and style:wrap-contour attributes attached.

The value of the attribute can be outside or full. If the value of the attribute is outside, the text wraps around the general area to the left and right of the shape. If the value of the attribute is full, the text wraps around the shape and fills any possible spaces and indentations in the shape.

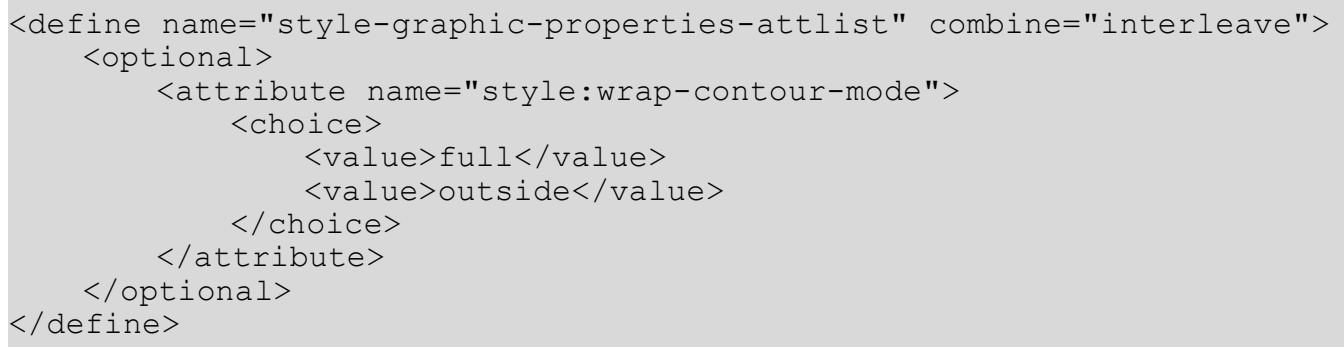

\subsubsection{Run Through}

If the value of the style:wrap attribute is run-through, it can be further specified whether the content of the frame should be displayed in the background or in the foreground. The style: run-through attribute is usually used for transparent objects.

The value of this attribute can be foreground or background. If the value is foreground, the frame content is displayed in front of the text. If the value is background, the frame content is displayed behind the text.

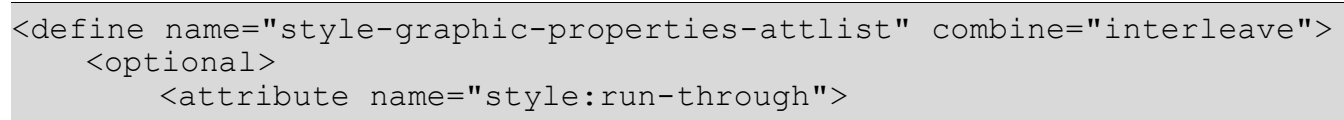




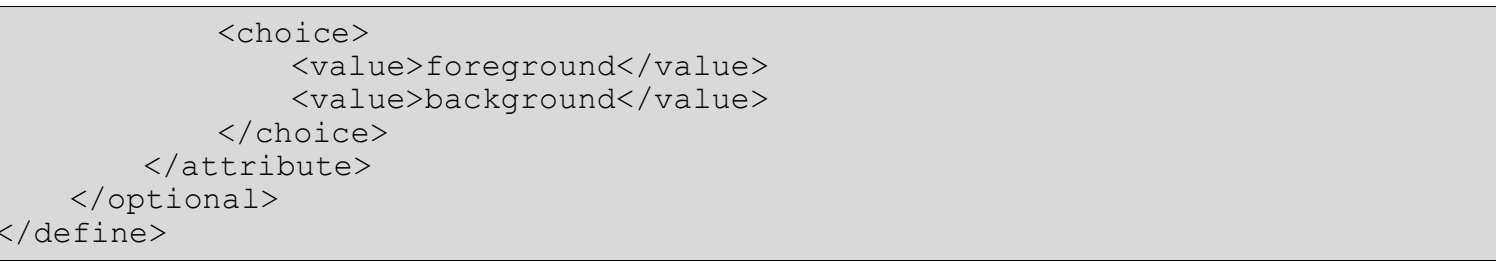

\subsubsection{Flow with Text}

The style:flow-with-text attribute specifies the behavior of drawing shapes that are positioned at a certain distance below an anchor and do not fit on the page where the anchor is. If the value of the property is true, such drawing objects follow the text flow, that is, they a displayed on the next page. If the attribute value is false, such drawing objects are displayed outside the page's text area.

Example: A graphic is to be positioned $10 \mathrm{~cm}$ below its anchor. It is followed by only $8 \mathrm{~cm}$ of text before the next page break. With style:flow-with-text='false' the graphics would then be positioned $2 \mathrm{~cm}$ below the text area (somewhere in the footer); with style: flow-withtext=' true' it would positioned $2 \mathrm{~cm}$ into the text flow of the following page.

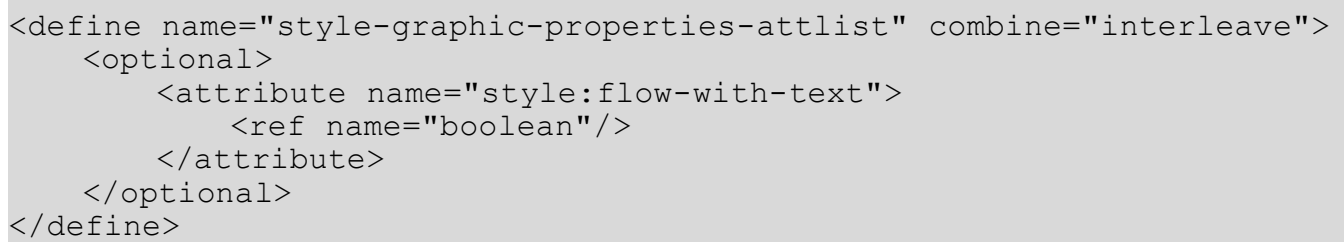

\subsubsection{Overflow behavior}

For text boxes contained within text document, the style:overflow-behavior property specifies the behavior of text boxes where the containing text does not fit into the text box. If the attribute's value is clip, the text that does not fit into the text box is not displayed. If the attribute value is auto-create-new-frame, a new frame will be created on the next page, with the same position and dimensions of the original frame.

If the style:overflow-behavior property's value is auto-create-new-frame and the text box has a minimum width or height specified, then the text box will grow until the page bounds are reached before a new frame is created.

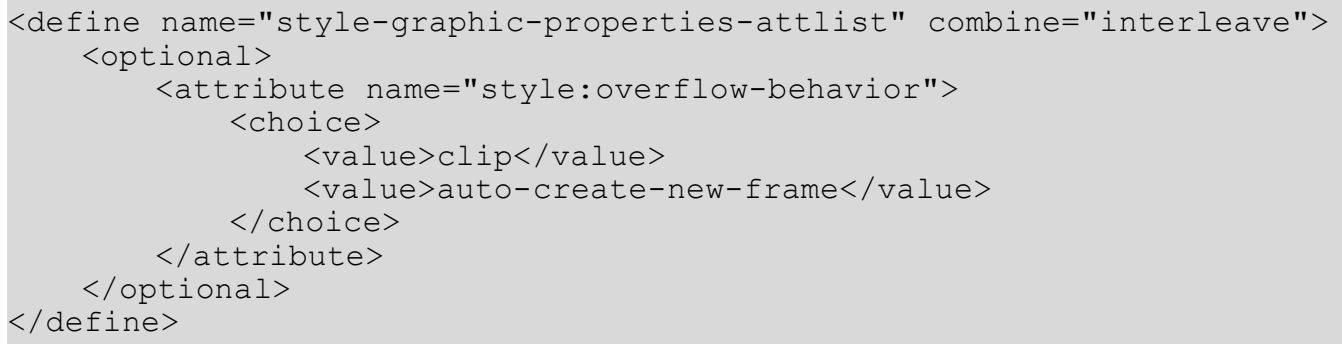

\subsubsection{Mirroring}

The style:mirror property specifies whether or not an image is mirrored before it is displayed. The mirroring can be vertical or horizontal. Horizontal mirroring can be restricted to images that are only located on either odd or even pages. 
The value of this attribute can be none, vertical, horizontal, horizontal-on-odd, or horizontal-on-even. The value vertical and the various horizontal values can be specified together, separating them by a white space.

\subsubsection{Clipping}

The fo:clip property specifies whether to display:

- A rectangular section of an image, or

- the entire image.

See $§ 7.20 .1$ of $[X S L]$ for details.

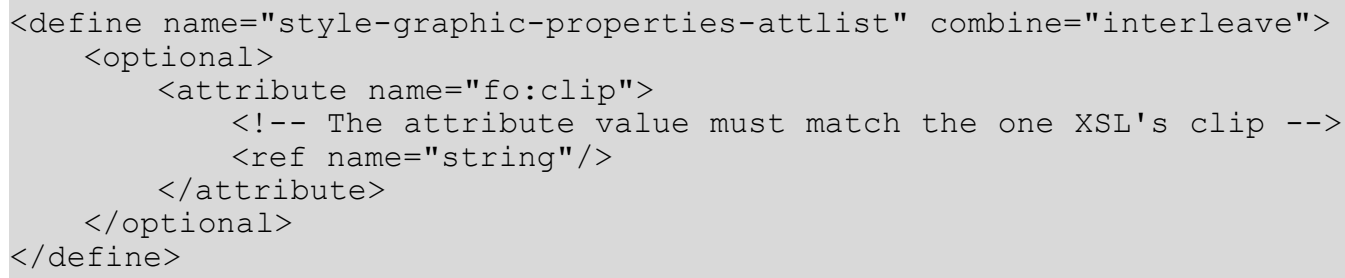

\subsubsection{Wrap Influence on Position}

This attribute details how the wrapping mode (see the style:wrap attribute) influences the positioning of a frame. It is intended as a hint to the layout algorithm to help decide on the placement of frames in certain cases where several correct placements could be used. All three options describe different, correct interpretations of the layout constraints already in the format. The new hint would allow to disambiguate between these situations. 


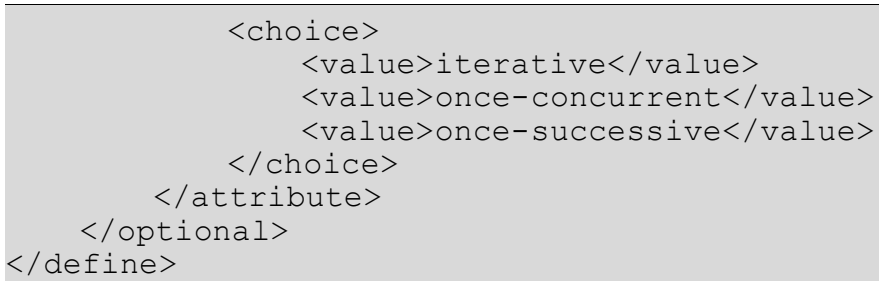

The situation in which this attribute makes a difference is when the anchor, position and wrapping mode of a frame are such that they influence each other. For example, consider a paragraph of text with two images positioned somewhat above the anchor. Without wrapping, the images overly the text and can simply be placed at the given offset from the anchor.

If wrap-around is enabled, the text hidden behind the images now needs to flow around the images, making the first paragraph use more space than previously. This moves the anchor position further down. If one does the placement only once and concurrently for all objects, this is the final result. This corresponds to the object once-concurrently.

If one proceeds as above, but does the process one image at a time, one arrives at the positions given to the right. This corresponds to the option oncesuccessive.

If one places the images iteratively, until a position is found which corresponds to the given offset from the anchor, one can often achieve a placement that fully satisfy all the given layout properties (at a certain price in implementation cost). This corresponds to the option iterative.

\subsection{Floating Frame Formatting Properties}

The attributes described in this section can be assigned to a graphic style that is assigned to floating frames.

\subsubsection{Display Scrollbar}

The draw: display-scrollbar attribute specifies whether or not vertical and horizontal scrollbars are displayed. This attribute can be assigned to automatic styles only.

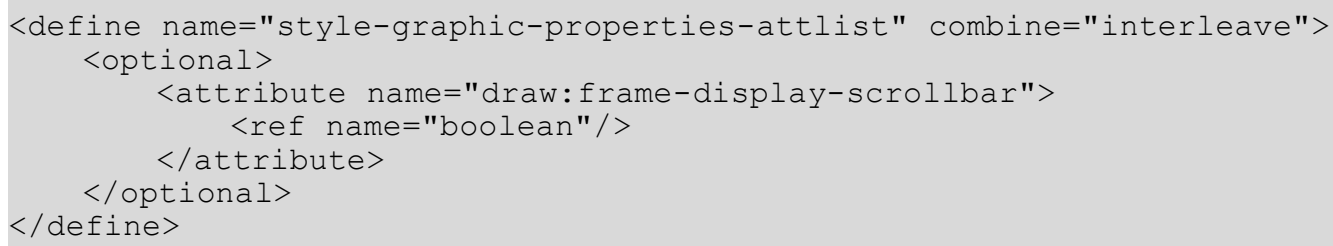

\subsubsection{Display Border}

The draw: display-border attribute specifies whether or not a border is displayed on the floating frame. This attribute can be assigned to automatic styles only. 


\subsubsection{Object Formatting Properties}

The attributes described in this section can be assigned to a graphic style that is assigned to objects.

\subsubsection{Visible Area}

The visible area of an object is the rectangular area of the object that is currently visible. The attributes draw:visible-area-left, draw:visible-area-top, draw:visible-areawidth and draw:visible-area-height specify a default visible area that the object has the option to use.

When the entire object is visible, the values of the draw:visible-area-left and draw:visible-area-top attributes are 0 and the draw:visible-area-width and draw:visible-area-height attributes specify the size of the object. These attributes can be assigned to automatic styles only.

Not all objects support these attributes. Some objects, may store and load their own visible area.

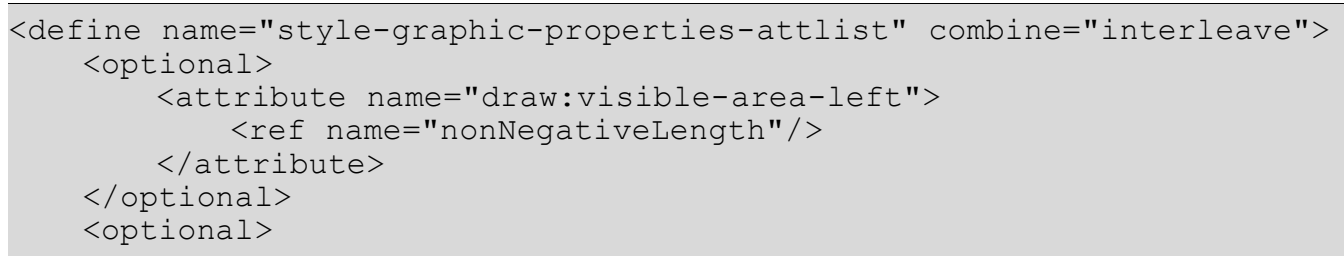




\subsubsection{Draw Aspect}

The draw: ole-draw-aspect attribute specifies the draw aspect that is used to display embedded OLE objects (see [OLE]). The draw aspect controls whether the object is displayed as a normal sub document, or whether the object is for instance displayed as an icon only. Within the [OLE] API, the draw aspect is an unsigned integer value that the host application passes to the object when it requests its presentation.

The draw: ole-draw-aspect attribute takes a non negative integer value and has only a meaning for objects that are embedded using the [OLE] API. In this case, it's value specifies a default value for method calls that require a draw aspect. The interpretation of this integer value is left to the OLE object's discretion and not part of this specification.

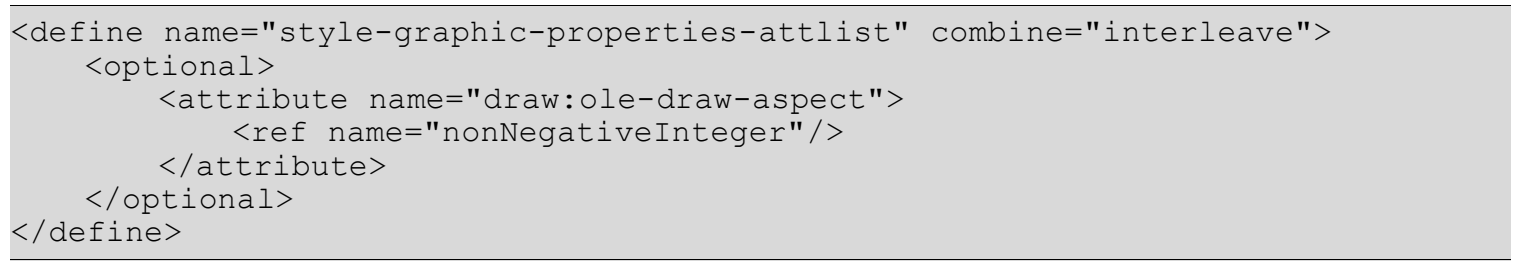

\subsection{Chart Formatting Properties}

The properties described in this section can be applied to all charts. They can be used within chart styles (see section 14.16) and are contained in a $<$ style: chart-properties $>$ element.

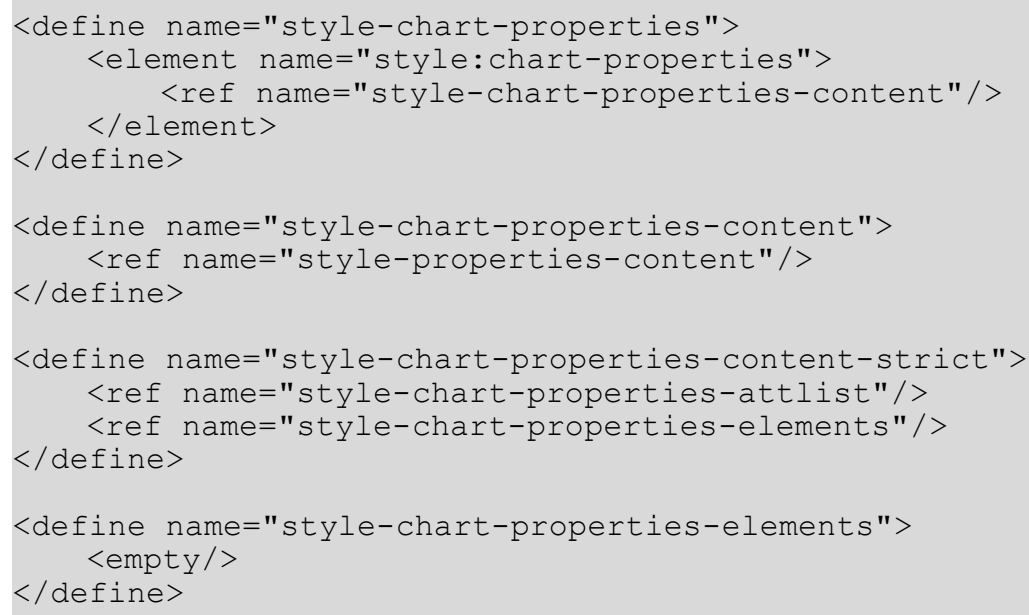




\subsubsection{Scale Text}

The chart:scale-text property is used to specify that all text objects in the chart should be scaled whenever the size of the chart changes. To enable scaling, set the value of this property to true.

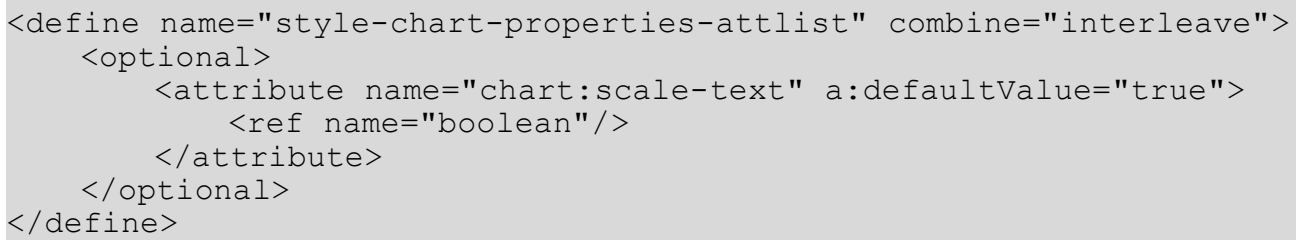

\section{$</$ optional $>$}

\subsection{Chart Subtype Properties}

The properties described in this section can be used to customize the basic chart type set in the $<$ chart: chart> element. They can be used within chart styles (see section 14.16) and are contained in a <style: chart-properties $>$ element.

\subsubsection{Three-dimensional Charts}

The chart:three-dimensional property specifies whether chart is displayed as a 3D scene.

16884

16885

16886

16887

16888

16889

16890

\subsubsection{Chart Depth}

The chart: deep property is only relevant with the chart:three-dimensional property. It specifies that the data series are displayed back-to-back rather than side by side.

16891

16892

16893

16894

16895

16896

16897

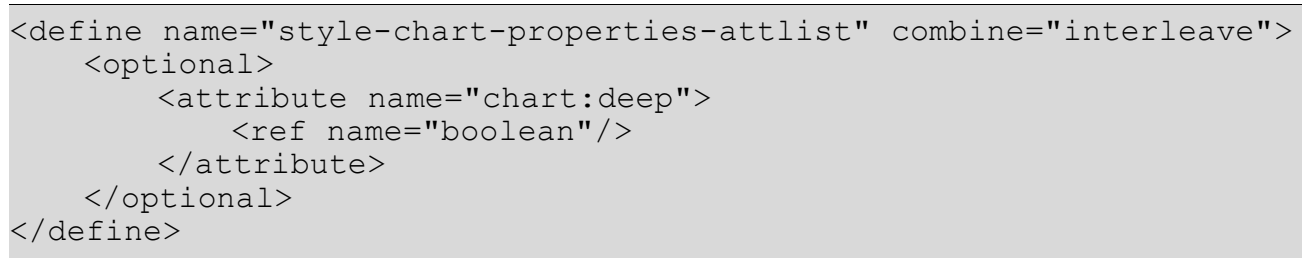

\subsubsection{Chart Symbol}

For some chart types, the data points can be denoted by symbols. The chart:symbol-type attribute determines whether a symbol is used, and whether it's a pre-defined symbol type, and image, or whether the application is free to automatically choose a type out of the set of predefined symbol types, e.g. choose one symbol per series in round-robin fashion.

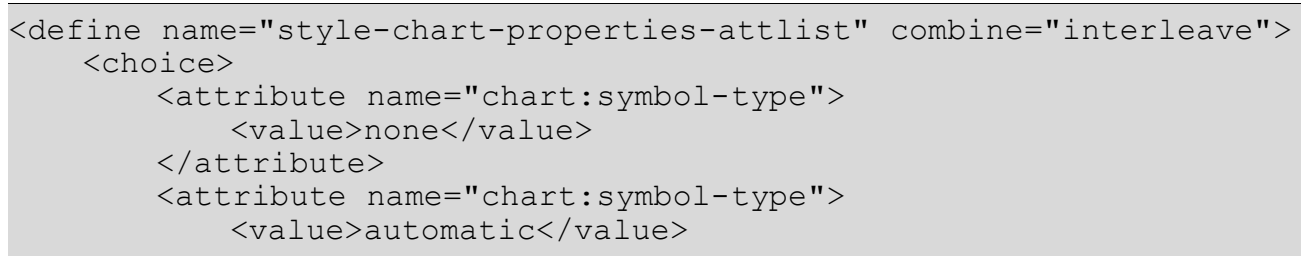




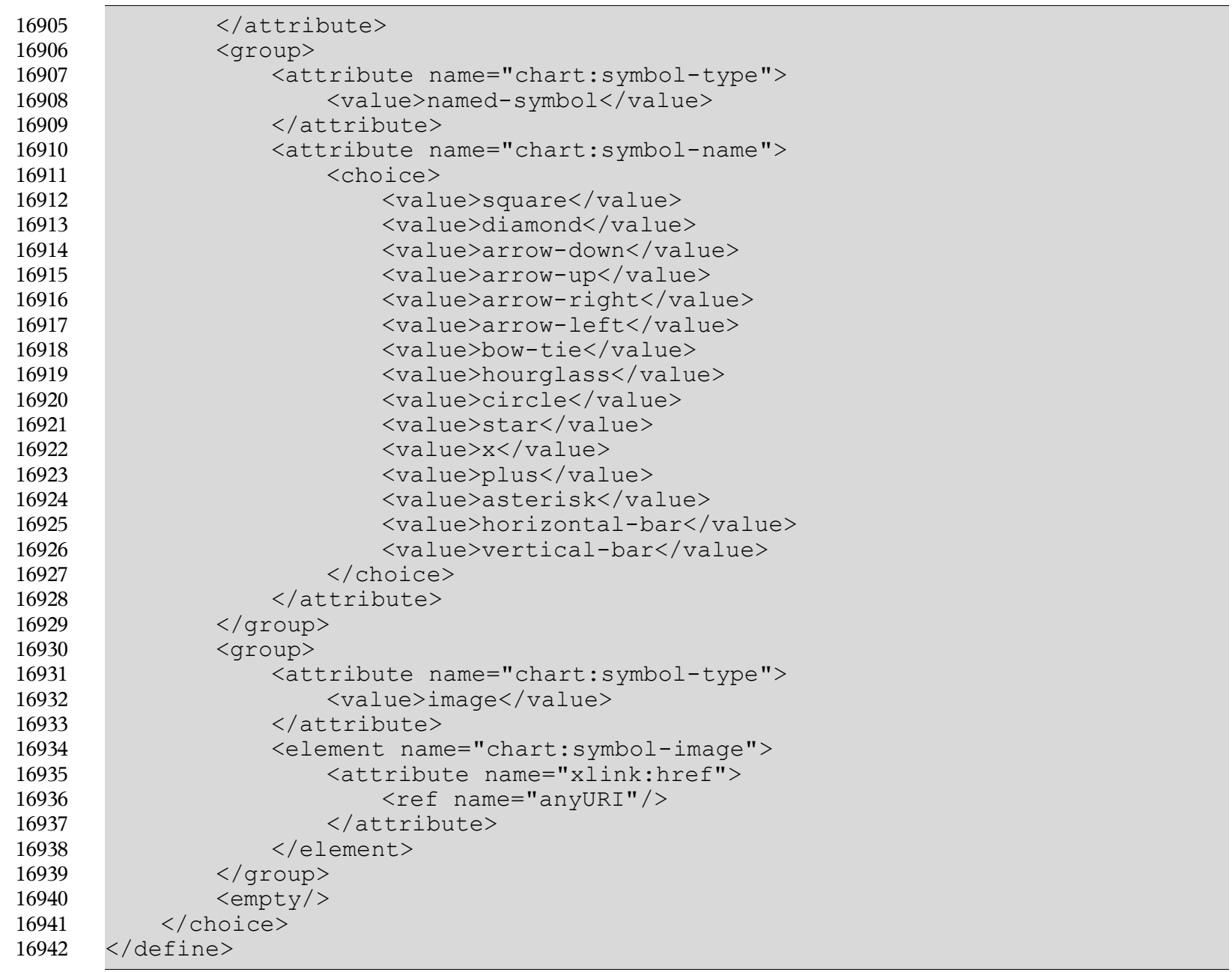

\subsubsection{Chart Symbol Size}

The width and height of each symbol can be set using the attribute chart:symbol-width and chart: symbol-length.

16943

16944

16945

16946

16947

16948

16949

16950

16951

16952

16953

16954

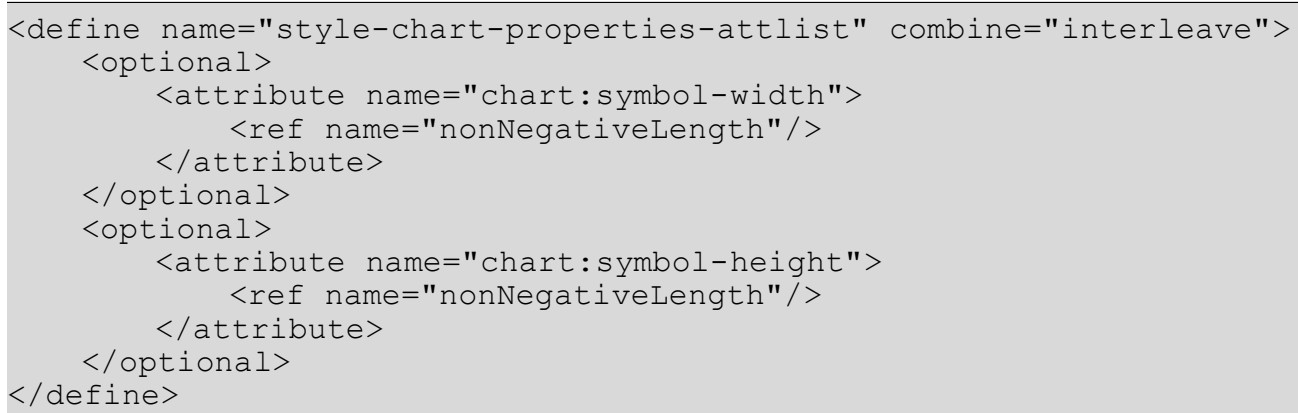

\subsubsection{Bar Chart Properties}

The chart:vertical and chart: connect-bars properties are for bar charts only. chart:vertical determines whether the bars will be oriented horizontally or vertically. If chart: connect-bars is set to true, the data points (the top of the bars) are additionally connected by lines. 


\subsubsection{Stock Chart Properties}

These attributes are only effective for stock charts.

Stock charts display a span from minimum to maximum values as a straight line. Opening and closing courses can be displayed either as left and right tick-lines, respectively, or as colored bars, with their color depending on whether the opening value is larger than the closing value. The chart:japanese-candle-stick attribute distinguish between those two representations.

Example: A stock chart in Japanese-candle-stick fashion (left), and as default (right).
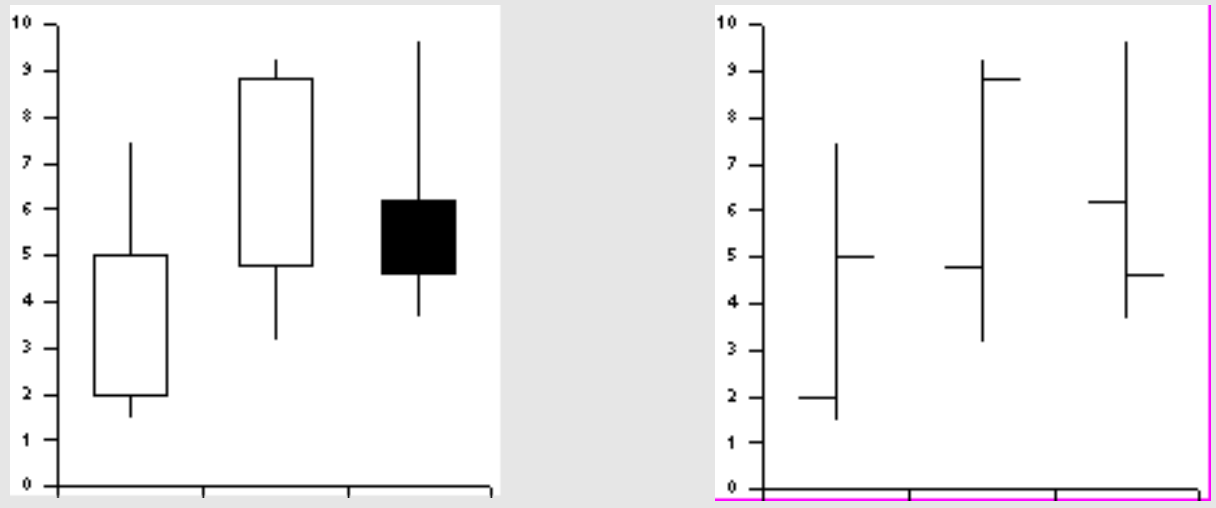


\subsubsection{Line Chart Properties}

For line chart-types, the attribute chart: interplation can be set to one of the following values:

- none -Straight lines - don't use spline interpolation

- cubic-spline-Cubic Splines (chart:spline-resolution determines the number of interpolated points between two data points)

- b-spline-B-Splines (chart:spline-order determines the order of the polygons used for calculation. The chart:spline-resolution is also taken into account.)

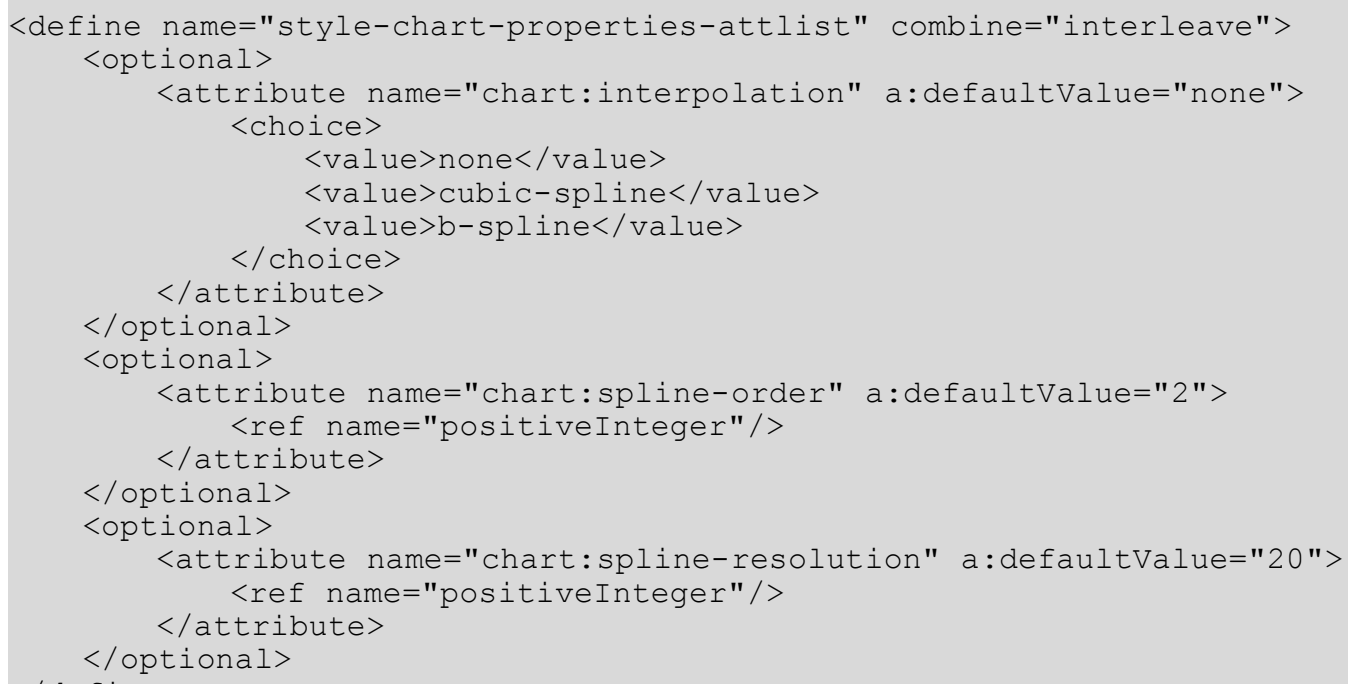

\subsubsection{Pie Chart Properties}

The chart:pie-offset attribute is only interpreted by pie charts. It determines the offset the tip of a 'pie' in a pie chart (or circle chart) has from the center of the circle.

17010 


\subsubsection{Solid Charts Bars}

The chart:solid-type attribute determines how the bars in three-three-dimensional bar charts should be rendered.

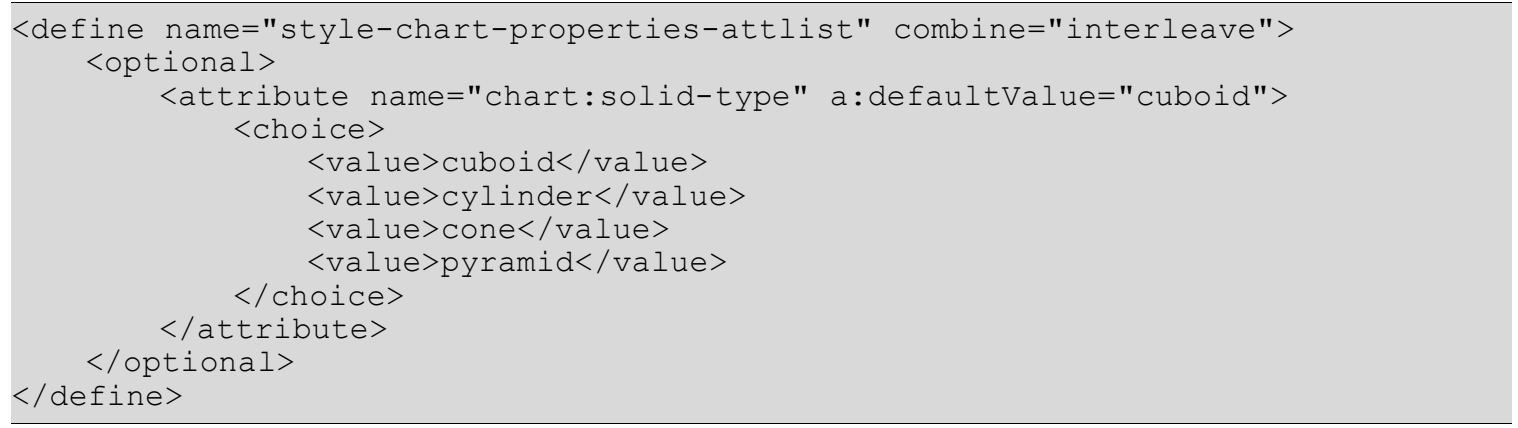

\subsubsection{Stacked Chart Bars}

The attribute chart:stacked attribute causes bars in bar charts to be stacked on top of each other, instead of next to each other. If chart:percentage is set to true, the stacked bars will all be scaled to the full height of the plot area, so that the bar segments represent the percentage of their respective data point in the total bar stack.

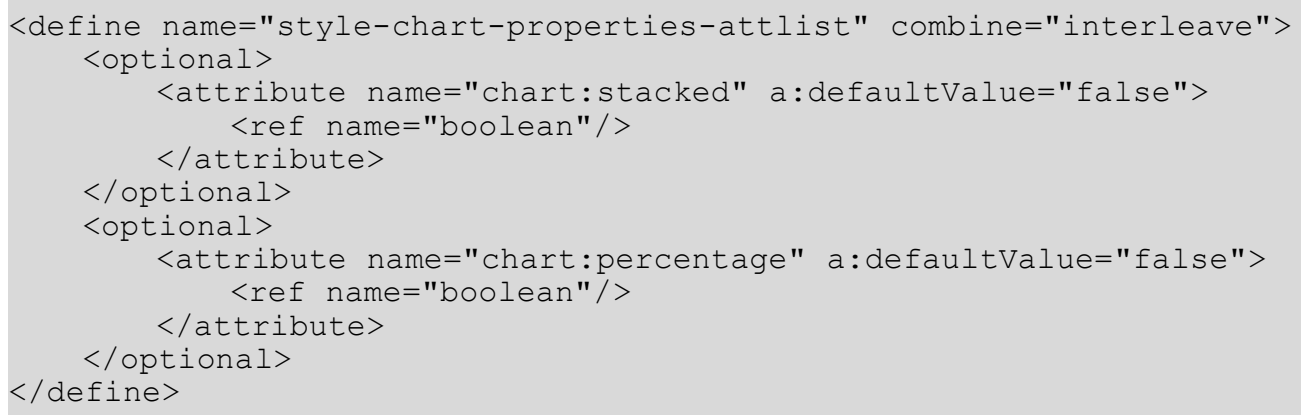

\subsection{Chart Axes Properties}

The properties described in this section can be applied to chart axis elements (see section 10.8). They can be used within chart styles (see section 14.16) and are contained in a <style:chart-properties> element.

\subsubsection{Linked Data Formats}

The chart:link-data-style-to-source attribute can only be used in chart documents that reside in a document that provides the data for the chart. If the value of the attribute is true, the number format used for rendering the axis is the format that the container document suggests based on the selected cell range. For example, if a cell range contains currencies all formatted in $€$, then this format will also be used at this axis. 


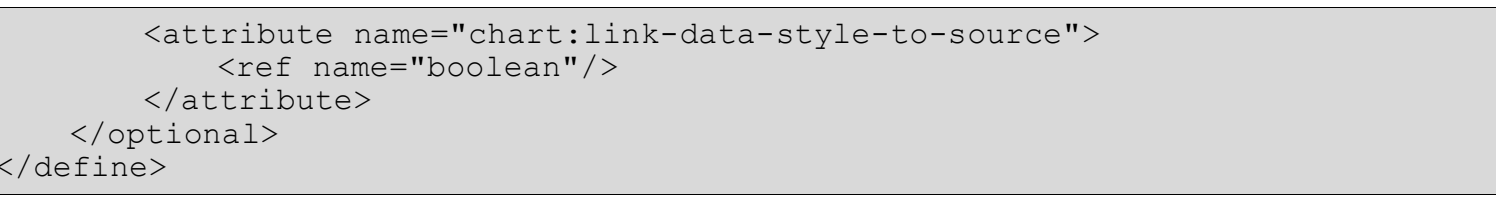

\subsubsection{Visibility}

To determine whether or not an axis object is visible, use the chart:axis-visible style property. This way, a chart with scaling information can be provided without displaying the axis object.

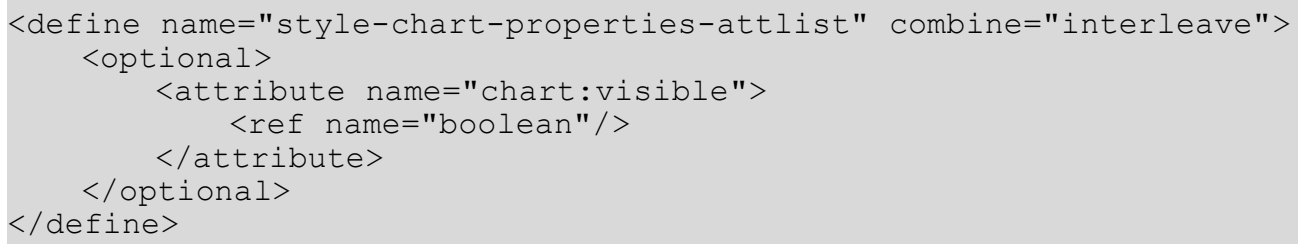

\subsubsection{Scaling}

If a scaling attribute is omitted, the axis is set to adaptation mode. This means that the value is not set to a fixed value but may be changed by the render application if data changes. However, the chart:axis-logarithmic attribute is set to false.

The optional chart:axis-logarithmic attribute can be used to cause logarithmic scaling on an axis. By default, proportional scaling is used.

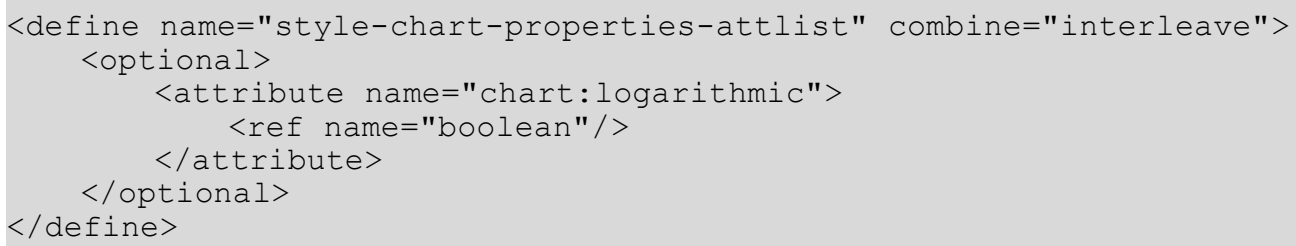

The following set of optional attributes further details the scaling of an axis. The properties have the following uses:

chart:minimum, chart:maximum - set minimal and maximal scaling values of an axis

chart:origin - determine the origin of the chart axis

chart: interval-major, chart:interval-minor-divisor - set major and minor interval for ticks or markings on the axis. The chart:interval-major defines the interval value. The minor interval is determined by dividing the chart:interval-major value by the

chart:interval-minor-divisor.

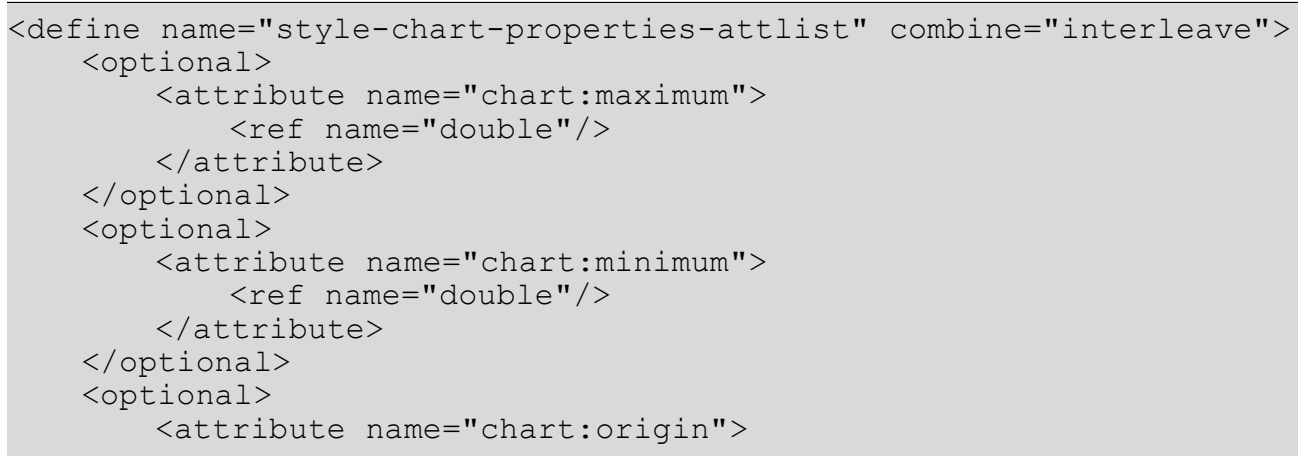


</optional>

\subsubsection{Tickmarks}

The tickmark properties are used to specify the existence of tickmarks at an axis. The major marks are drawn with respect to the major interval that may be specified by the chart:axisinterval-major attribute. The minor tick marks refer to the chart:axis-interval-minor attribute. Inner marks are drawn towards the inside of the plot area, that is to the right for an axis displayed on the left hand side of the plot area, and to the left for an axis displayed on the right hand side of the plot area. Outer marks point in the opposite direction. If both properties are specified, one tick mark is drawn that crosses the axis.

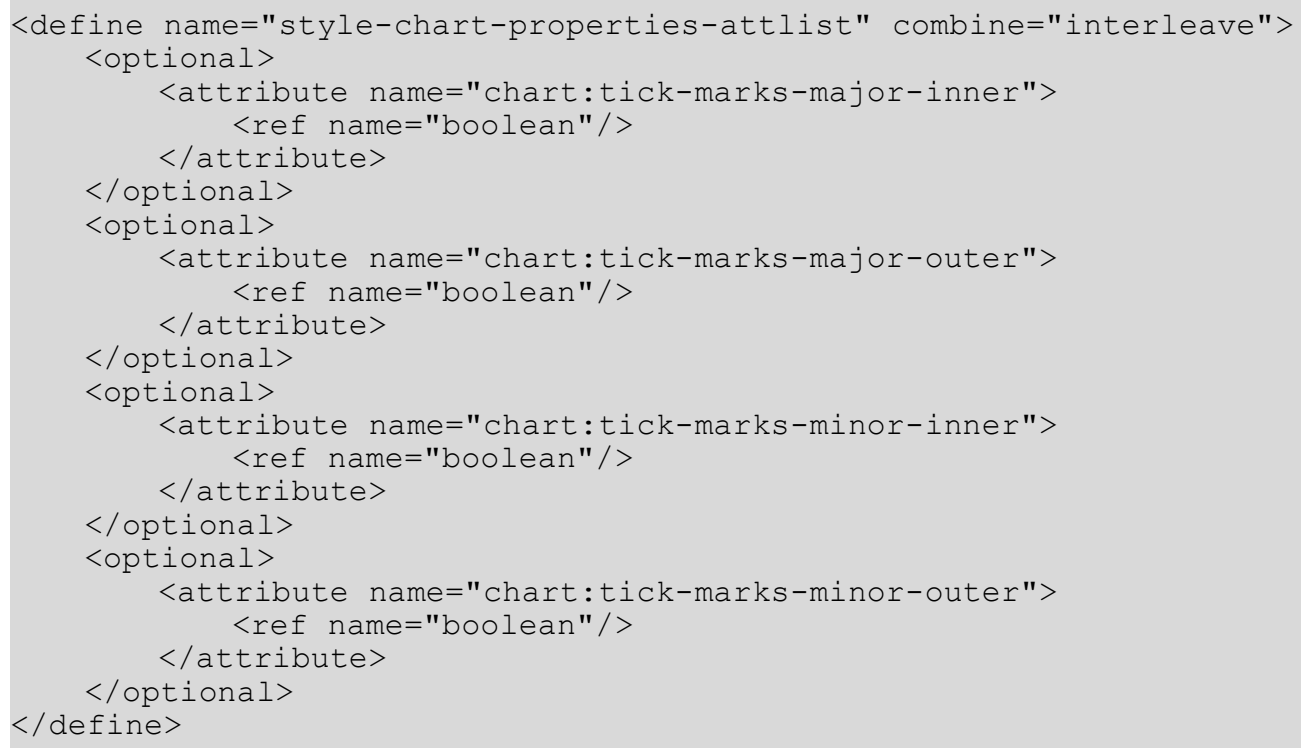

\subsubsection{Labels}

The following set of properties describes how axis labels are being represented. chart: display-label determines whether labels will be displayed at all. If chart: textoverlap is set true, labels may overlap. text: line-break determines whether label lines may be broken into multiple lines.

The chart:label-arrangement property allows labels to be arranged either side-by-side (i.e. all labels start on one line), or staggered (i.e., labels are distributed to two lines, with every other label starting on the same line). In case of staggered labels, one can choose between even or odd staggering, i.e. one can choose whether even or odd labels are aligned on the line that would be used for side-by-side arrangement. 


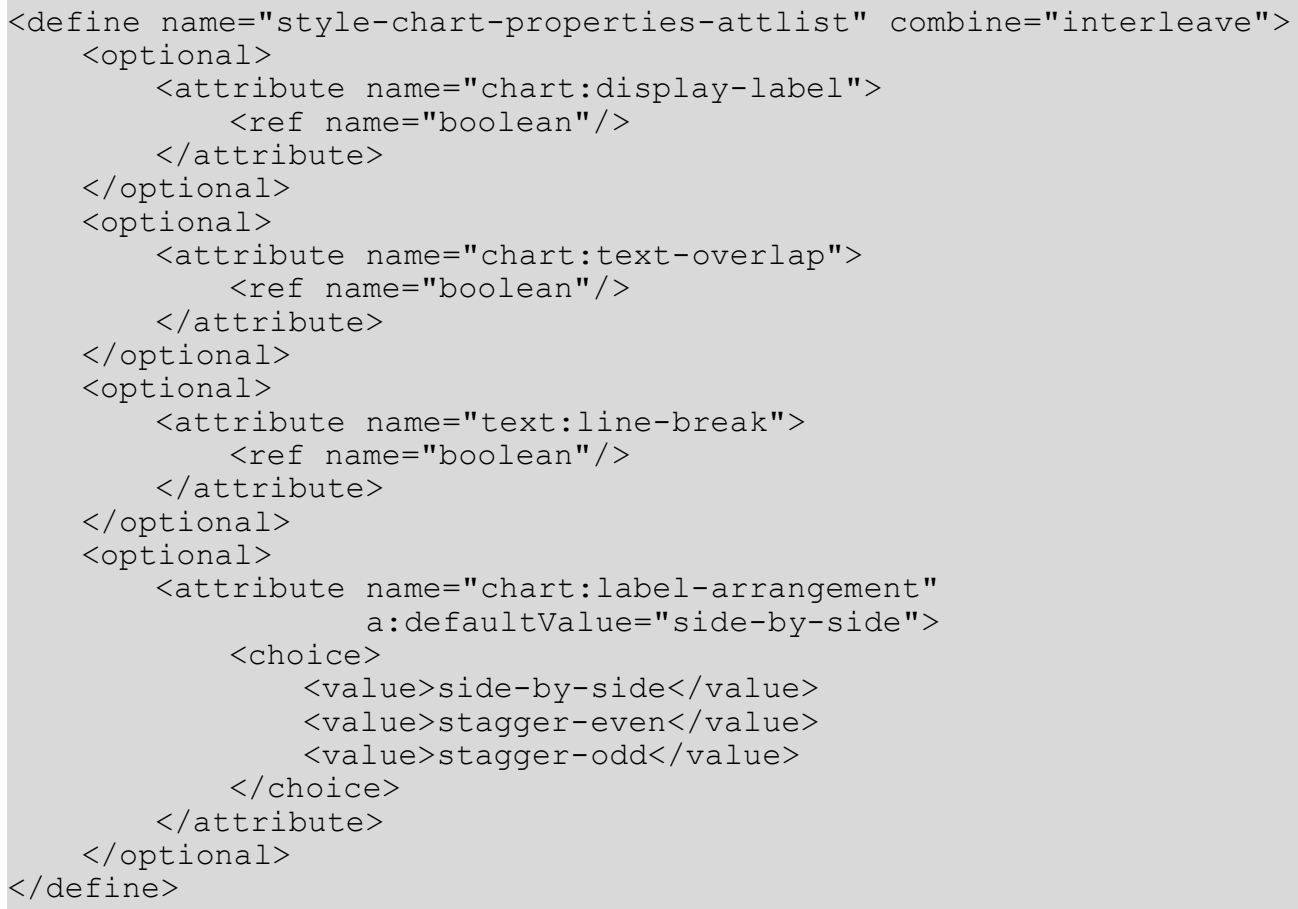

\subsection{Common Chart Properties}

The properties described in this section apply to all types of data representation objects, including the elements <chart: plot-area>, <chart: series>, and <chart: data-point>. They can be used within chart styles (see section 14.16) and are contained in a <style:chart-properties> element.

Properties are applied in a hierarchical manner. If a property is set in the <chart: chart> element, it applies to all data points contained in the chart. If the same property is set in a $<$ chart:series > element, it only applies to the data points contained in that specific series. To set a formatting property for one data point only, set the property in the <chart: data-point $>$ element.

\subsubsection{Stacked Text}

The property style:direction determines whether or not text is displayed vertically without rotating the letters. It can be applied to several text objects.

The value of this property can be $1 t r$ if text goes from left to right or $t t b$ if the text is stacked, that is goes from top to bottom. It can be applied to several text objects. See section 15.11 .3 for details.

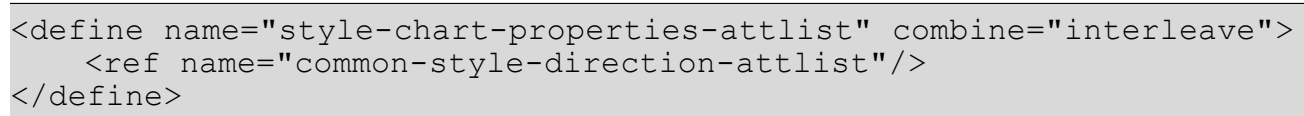

\subsubsection{Rotation Angle}

The style:rotation-angle property specifies the value of a rotation angle in degrees. See section 15.11 .12 for information on using this property. 


\subsubsection{Data Labels}

Data labels can be applied to data series and data points as well as to an entire chart. In the latter case, labels are shown for all data points. Data labels can consist of the following three parts:

- The value, which can be displayed as a percentage or the value itself.

- The label of the corresponding series.

- The legend symbol.

\section{Value}

The chart:data-label-number attribute represents the value of the data label.

\section{Label}

The chart:data-label-text attribute determines whether or not to display the label of the corresponding series.

The value of this attribute can be true or false.

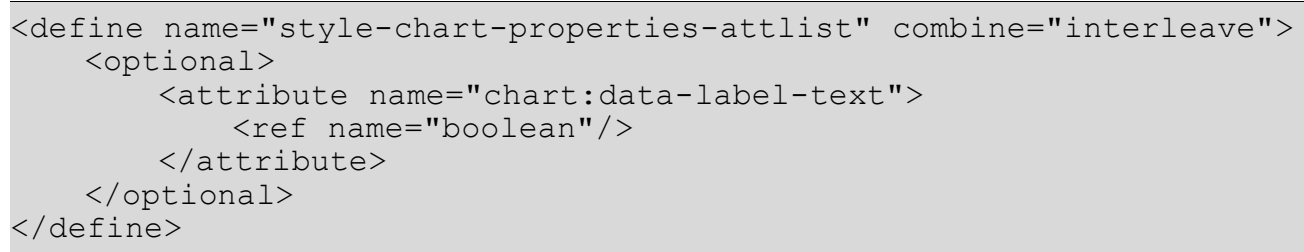

\section{Legend Symbol}

The chart:data-label-symbol attribute determines whether or not to display the legend symbol. The value of this attribute can be true or false.

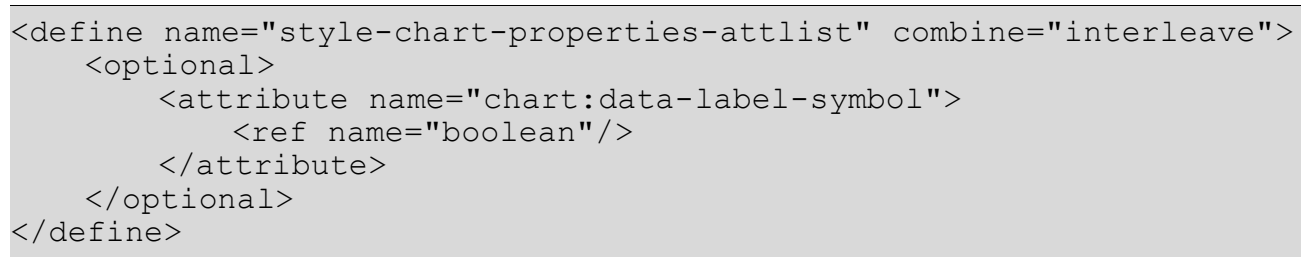




\subsection{Statistical Properties}

Statistical properties can be applied to data series or to an entire chart. In the latter case, the properties apply to all series in the chart. They can be used within chart styles (see section 14.16) and are contained in a <style: chart-properties $>$ element.

\subsubsection{Mean Value}

The chart:mean-value attribute determines whether or not to display a line that represents the statistical mean value of all data points of a series. The value of this attribute can be true or false.

\section{Error Percentage}

The chart:error-percentage attribute determines the percentage that is used to display error indicators for each data point of a series.

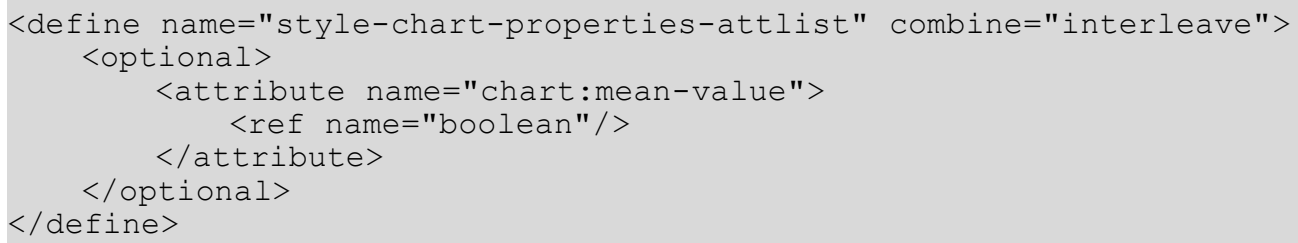

\subsubsection{Error Category}

The chart:error-category attribute is used to determine which function is used to display error indicators at data points. The following functions are available:

- Variance of the values of a series assuming an equal distribution.

- Standard-deviation of the values of a series assuming an equal distribution.

- Use a fixed percentage of each value

- Use a fixed percentage of the biggest value - this is called error-margin.

- Use fixed absolute values for both directions: positive and negative

If this attribute is set to any value other than none, error indicators are shown. To determine in which direction the indicators are pointing see the attributes chart:error-upper-indicator and chart:error-lower-indicator.

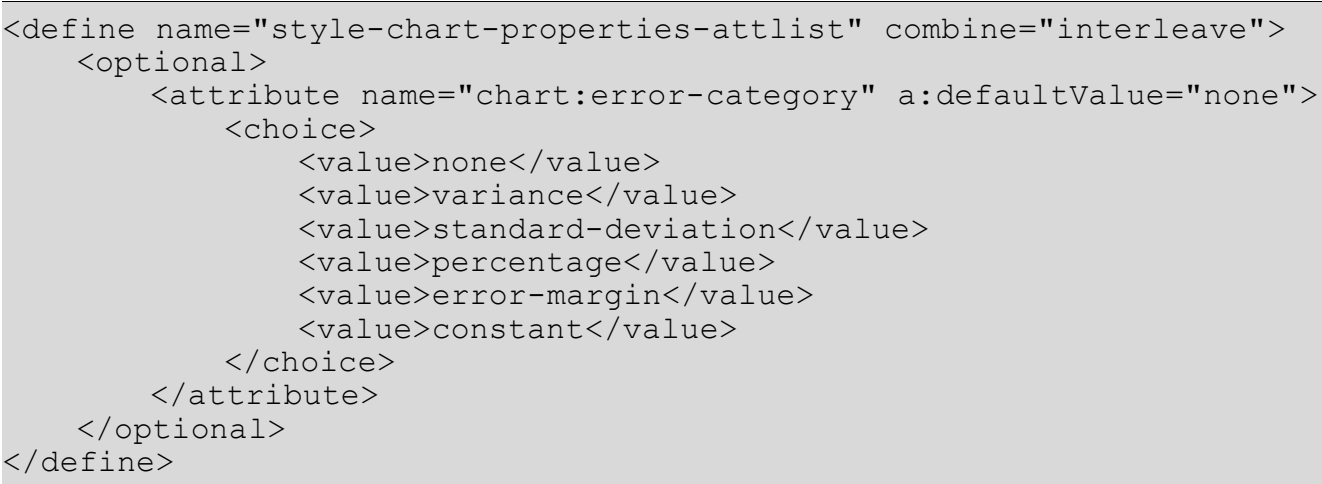

<define name="style-chart-properties-attlist" combine="interleave"> 


\section{Error Margin}

The chart:error-margin attribute determines the percentage that is used to display error indicators for the biggest value in a series.

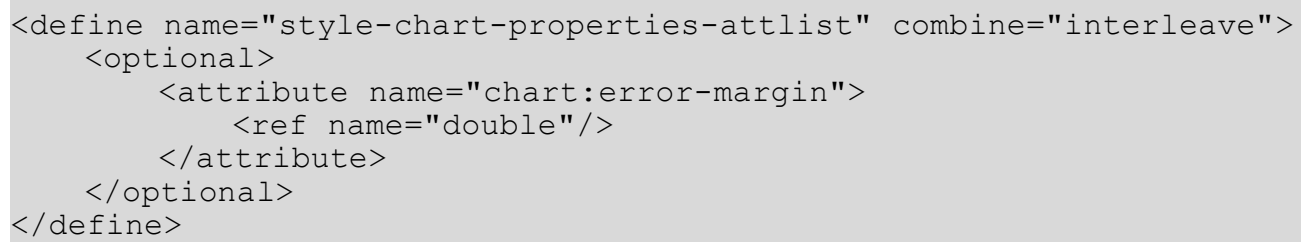

\section{Constant Error Lower and Upper Limit}

If the error category is set to constant, the chart:error-lower-limit and chart:error-upper-limmit attributes determine the absolute values in a positive and negative direction that are used to display the error indicators.

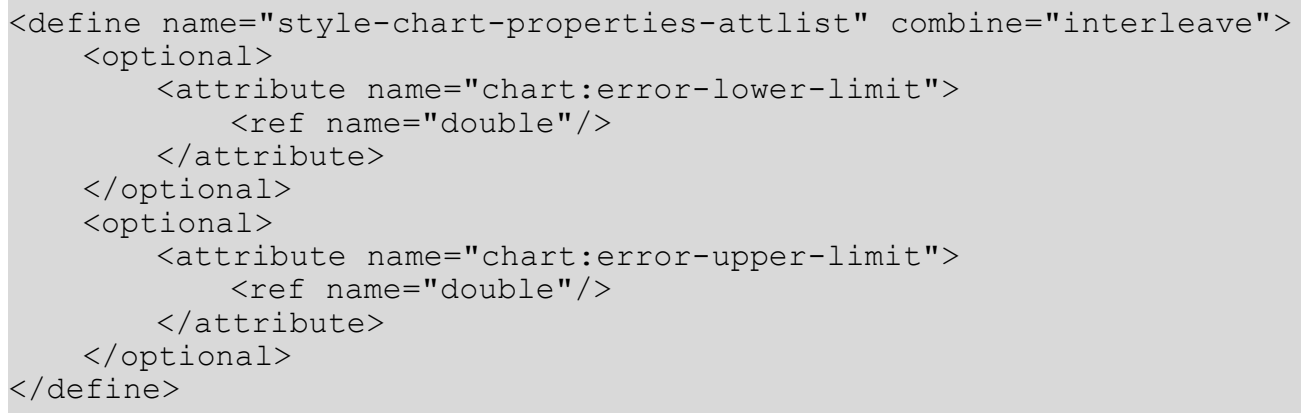

\section{Error Indicators}

The chart:error-lower-indicator and chart:error-upper-indicator attributes determine in which direction indicators should be drawn.

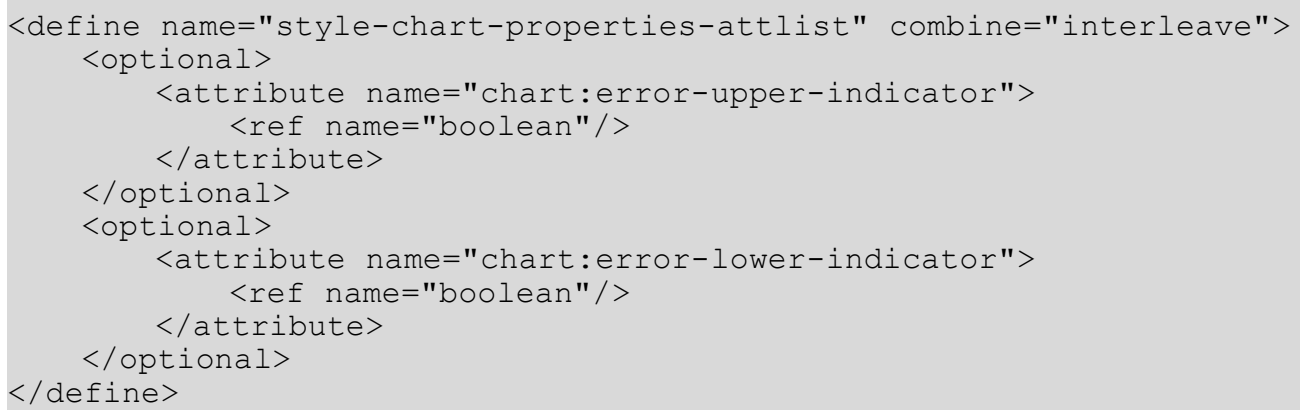




\subsection{Plot Area Properties}

The properties described in this section can be applied to chart plot area elements (see section 10.5). They can be used within chart styles (see section 14.16) and are contained in a <style: chart-properties> element.

\subsubsection{Series Source}

The chart:series-source attribute determines whether the data table contains the data series in column-wise or row-wise fashion.

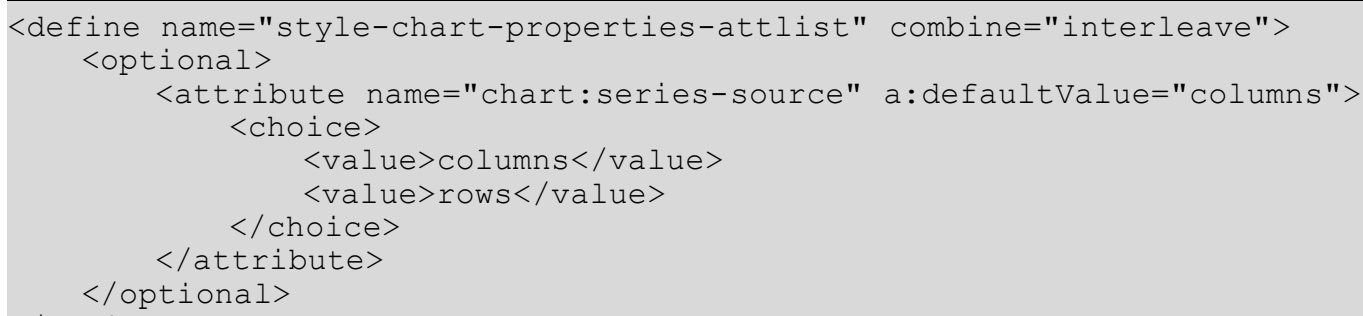

\subsection{Regression Curve Properties}

The properties described in this section can be applied to chart regression curves elements (see section 10.14). They can be used within chart styles (see section 14.16) and are contained in a <style: chart-properties> element.

\subsubsection{Regression Type}

Use the chart: regression-type attribute to display a regression for a series. A regression can be used to approximate the data points in a series by a mathematical function. The following models for approximation are available:

- Linear regression - approximate the values of the series using the model: $y=A \cdot x+B$.

- Logarithmic regression - approximate the values of the series using the model: $y=A \cdot \log (x)$ $+B$.

- Exponential regression - approximate the values of the series using the model: $y=A \cdot e^{B \cdot x}$.

- Regression with a power function - approximate the values of the series using the model: $y$ $=A \cdot x^{B}$.

This property is only relevant in scatter charts, because regression needs both $x$ and $y$ values for calculation

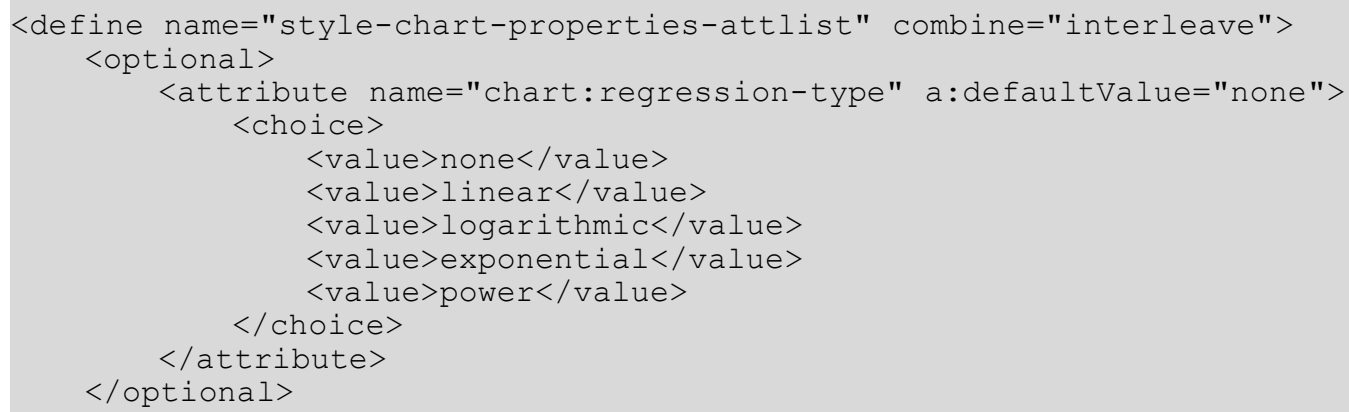




\subsection{Presentation Page Attributes}

The properties described in this section can be contained within style elements <style:style> whose family is drawing-page. They are contained in a style:style-drawpageproperties > element.

The following presentation properties do exist:

- Transition Type

- Transition Style

- Transition Speed

- Page Duration

- Page Visibility

- Sound

- Background Size

- Background Objects Visible

- Background Visible

- Display Header

- Display Footer

- Display Page Number

- Display Date and Time

\subsubsection{Transition Type}

The mode of transition, for example manual, can be set using the attribute presentation:transition-type.

- manual : slide transition and shape effects must be started separately by the user.

- automatic: slide transition and shape effects start automatically.

- semi-automatic: slide transition starts automatically, shape effects must be started by the user.

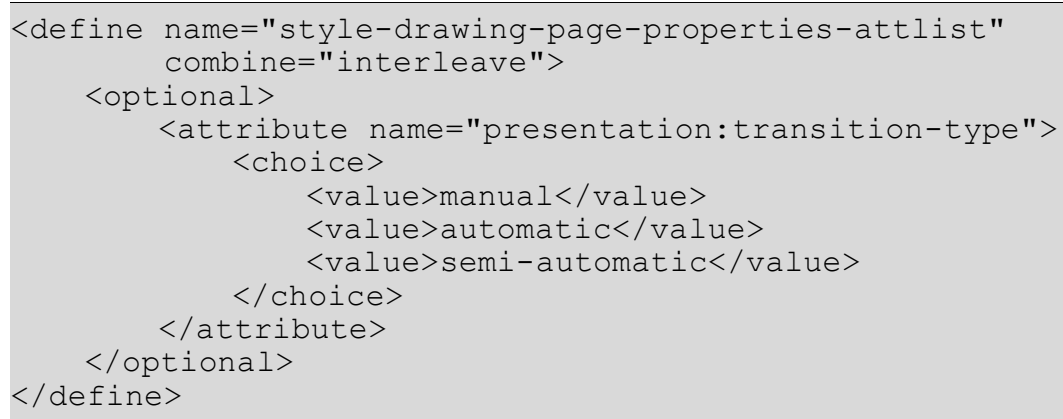




\subsubsection{Transition Style}

The attribute presentation:transition-style specifies the way that each presentation page replaces the previous presentation page, for example left-to-right replacement, or fading.

- none: no effect is used.

- fade-* : the pages fades from a visible or hidden state to a hidden or visible state in the specified direction.

- move-* : the page moves in the specified direction to its final position.

- uncover-* : the page get uncovered in the specified direction.

- *-stripes: the page is uncovered by drawing horizontal or vertical stripes that change their size during this effect.

- clockwise: the page is uncovered by the hand of a watch, moving clockwise.

- counterclockwise: the page is uncovered by the hand of a watch, moving counterclockwise.

- open-* : the page is uncovered by drawing it line by line, either horizontally or vertically, starting at the center of the page.

- close-* : the page is uncovered by drawing it line by line, either horizontally or vertically, starting at the edge of the page.

- wavyline-* : the page is uncovered by drawing small blocks in a snake like fashion.

- spiralin-* : the page is uncovered by drawing blocks in a spiral fashion, starting from the edge of the page.

- spiralout-* : the page is uncovered by drawing blocks in a spiral fashion, starting from the center of the page.

- roll-* : the pages moves in the specified direction to its final position, pushing the old page out.

- stretch-* : the page is uncovered by changing its size during this effect.

- $*-l$ ines: the page is uncovered by drawing it line by line, either horizontally or vertically in a random fashion.

- dissolve: the page is faded in by drawing small blocks in a random fashion.

- random: an effect is chosen at random to uncover the page.

- *-checkerboard: the page is uncovered by drawing checkerboard like blocks that increase in size horizontally or vertically.

- interlocking-horizontal-* : the new page appears in 4 horizontal stripes (i.e. the height is divided in 4 , a bit like in the horizontal-stripes effect) but those stripes come from left, right, left, and right, and cross each other in the middle of the screen.

- interlocking-vertical-* : similar effect with vertical stripes crossing each other.

- $f l y-a w a y:$ the page first reduces itself to a smaller size (while remaining centered in the screen), and then "flies away" (turns around a bit and moves to the bottom-right corner of the screen). The next slide appears under it meanwhile. 
- open: Combination of open-horizontal and open-vertical, i.e. a sort of plus sign opening.

- close: Combination of close-horizontal and close-vertical, i.e. a sort of plus sign closing.

- melt: Small vertical stripes move down at random speed, which gives the effect of the current page "melting down".

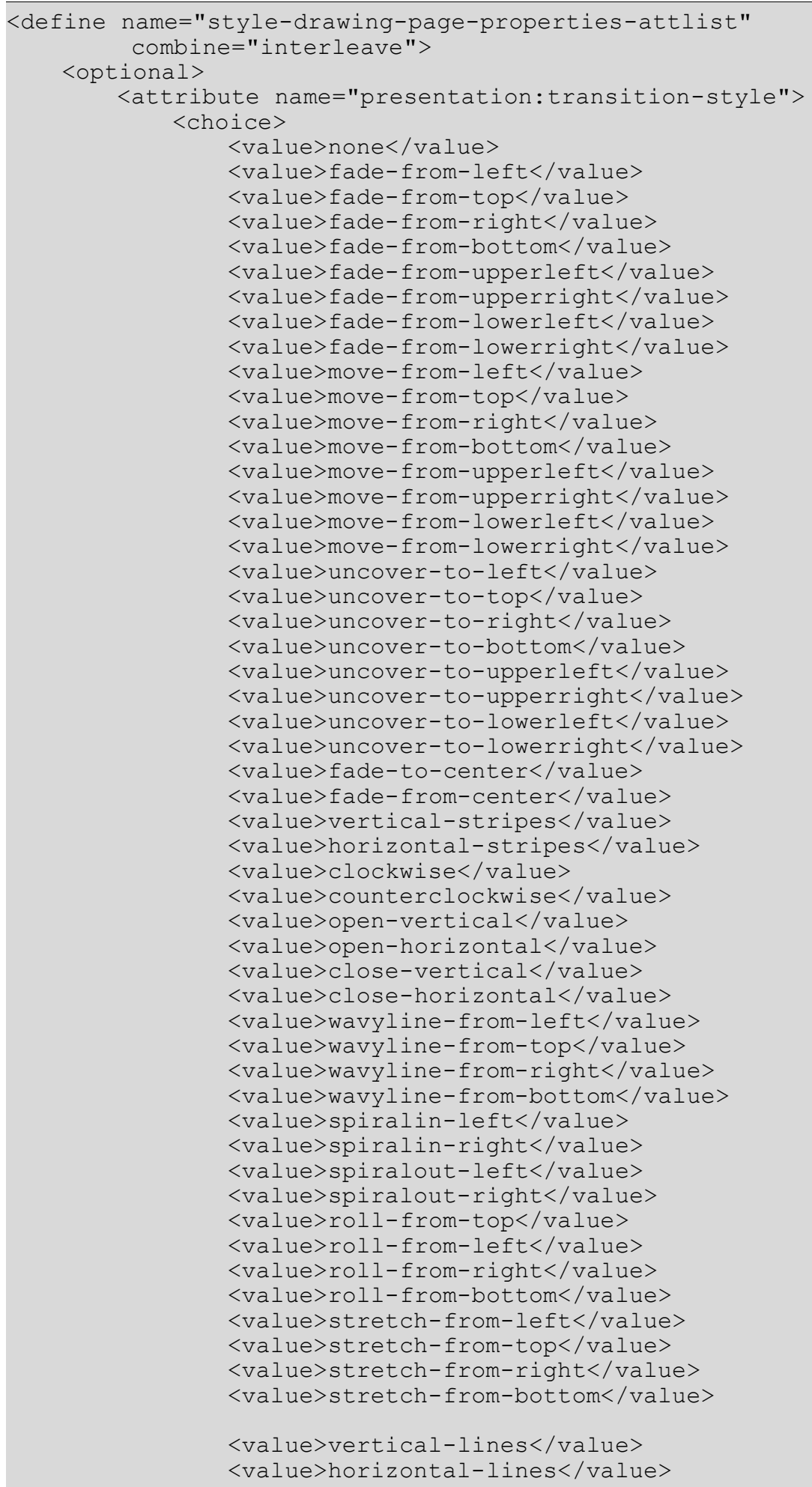




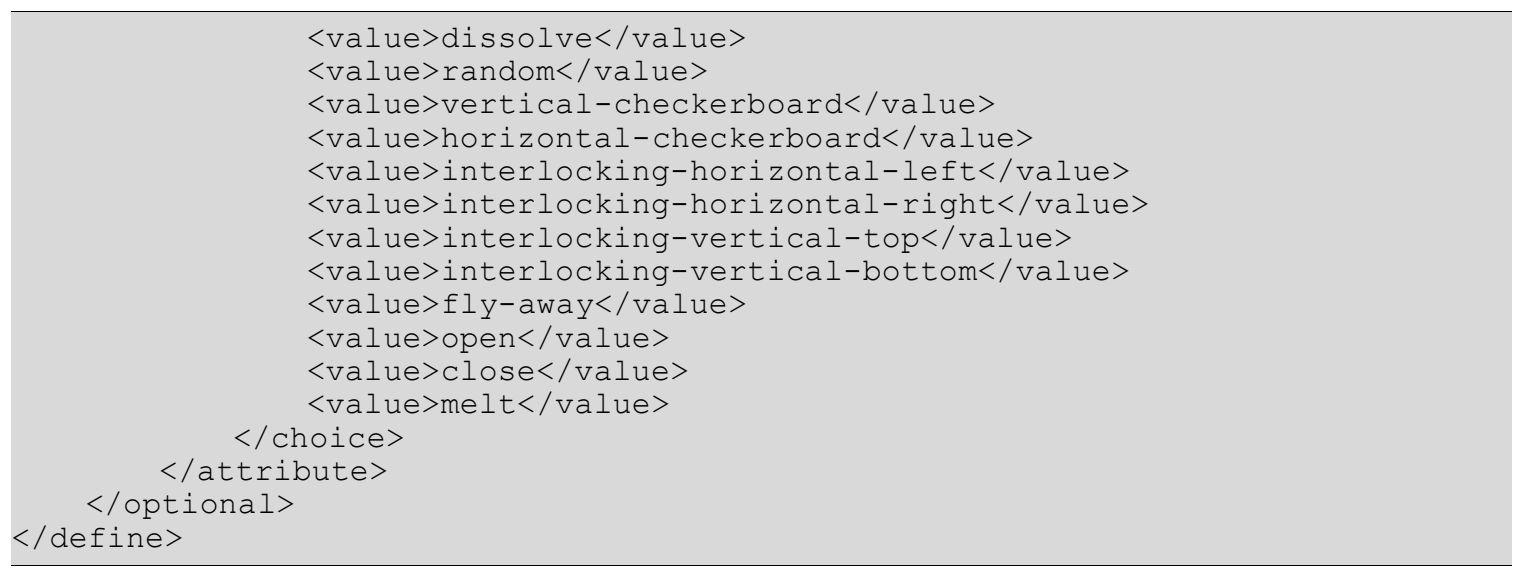

\subsubsection{Transition Speed}

The attribute transition-speed controls the speed at which a presentation page is removed from display, and replaced by a new presentation page. See

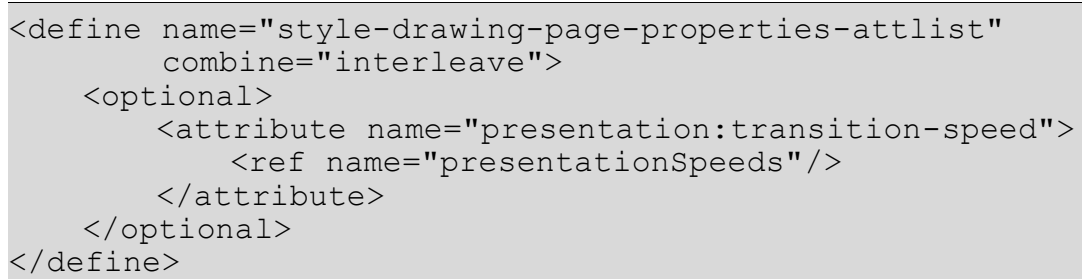

\subsubsection{Transition Type or Family}

The [SMIL20] smil: type attribute is used to specify the transition type or family. See $§ 12.4 .1$ of [SMIL20] for details. See $\S 12.8$ of [SMIL20] for a list of supported types.

If this attribute is present, the attributes presentation:transition-type and presentation:transition-style attributes should be ignored.

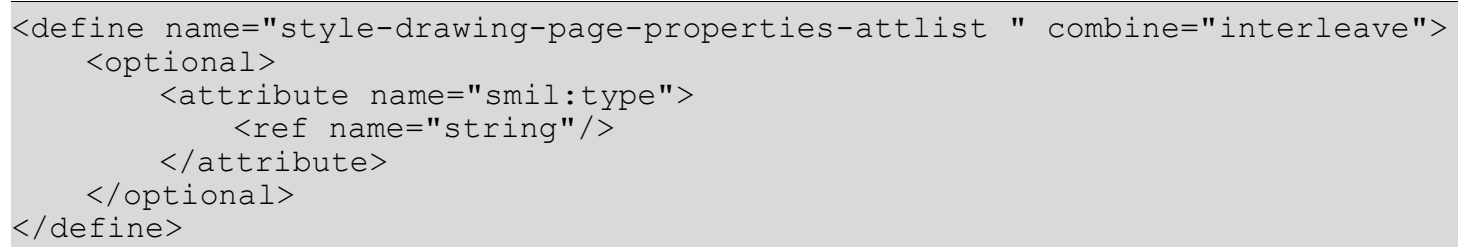

\subsubsection{Transition Subtype}

The [SMIL20] smil : subtype attribute is used to specify the transition subtype. See $\S 12.4 .1$ of [SMIL20] for details. See $\S 12.8$ of [SMIL20] for a list of supported subtypes.

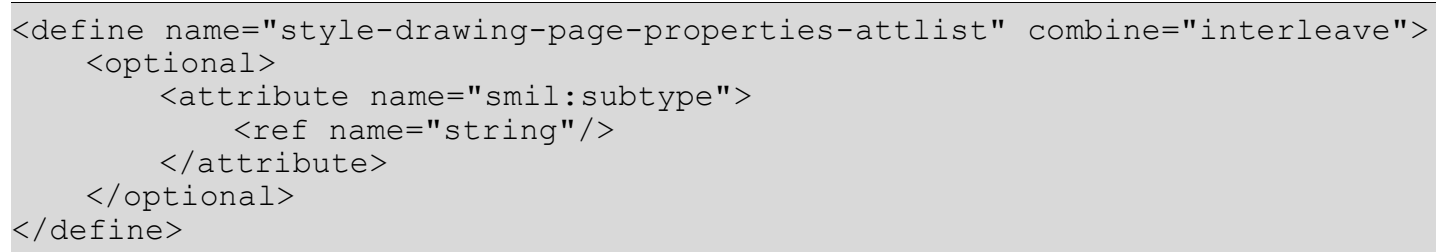




\subsubsection{Transition Direction}

The [SMIL20] smil: direction attribute is used to specify the transition direction. See $\$ 12.4 .1$ of [SMIL20] for details.

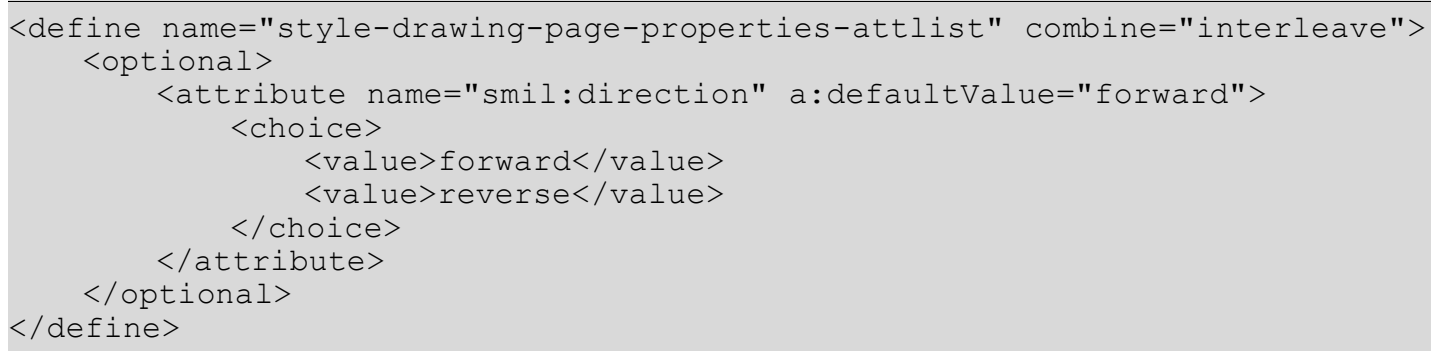

\subsubsection{Fade Color}

The [SMIL20] smil: fadeColor attribute is used to specify the transition fade color for transitions that make use of a start or end color. See §12.4.1 of [SMIL20] for details.

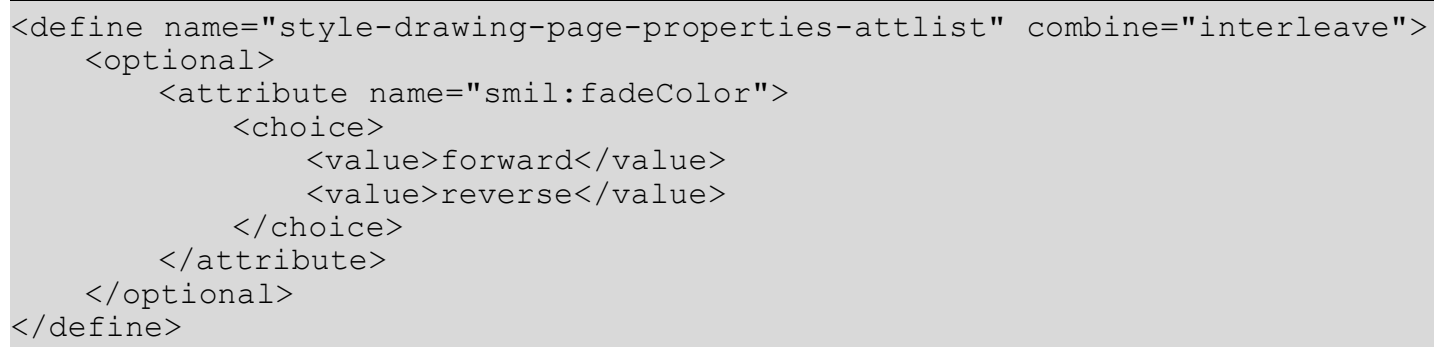

\subsubsection{Page Duration}

The attribute presentation:page-duration controls the amount of time that the presentation page is displayed. The value of this attribute must conform to the time period format described in §3.2.6 of [xmlschema-2].

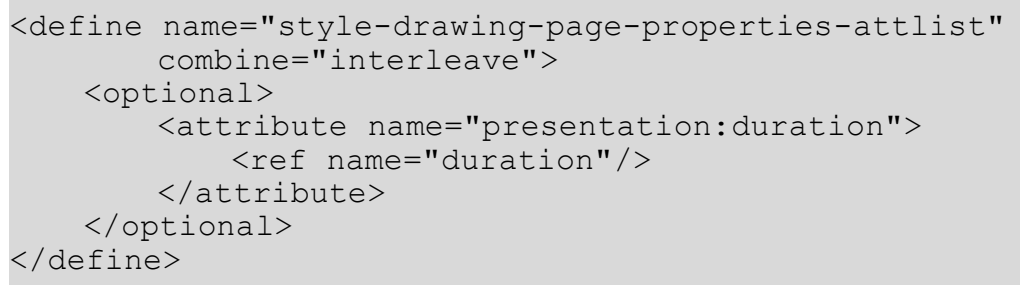

\subsubsection{Page Visibility}

A drawing page can be marked as hidden during a presentation by using the attribute presentation:visibility. A page marked with this attribute is only shown while editing the document but not during the presentation.

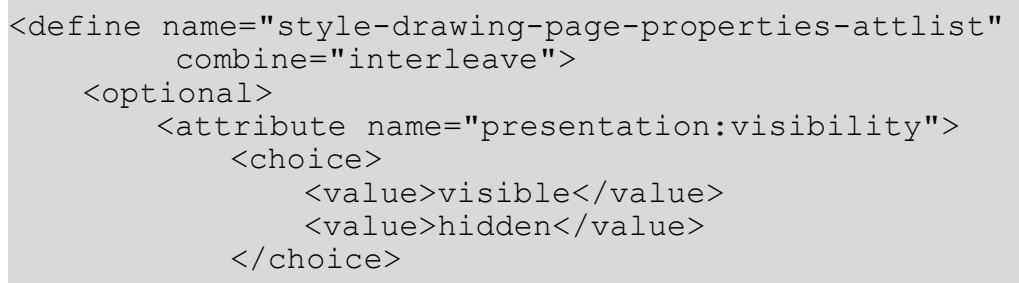




\subsubsection{Sound}

Sound effects can be added to your presentation pages using the element presentation: sound. It must be included in the <style:presentation-properties > element.

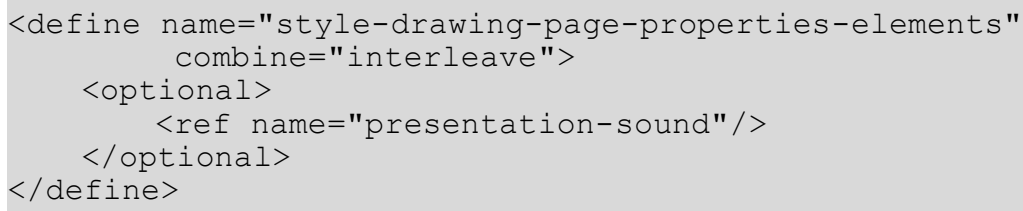

\subsubsection{Background Size}

The attribute draw:background-size specifies whether the background of a page is rendered on the full page or only inside the borders of the page.

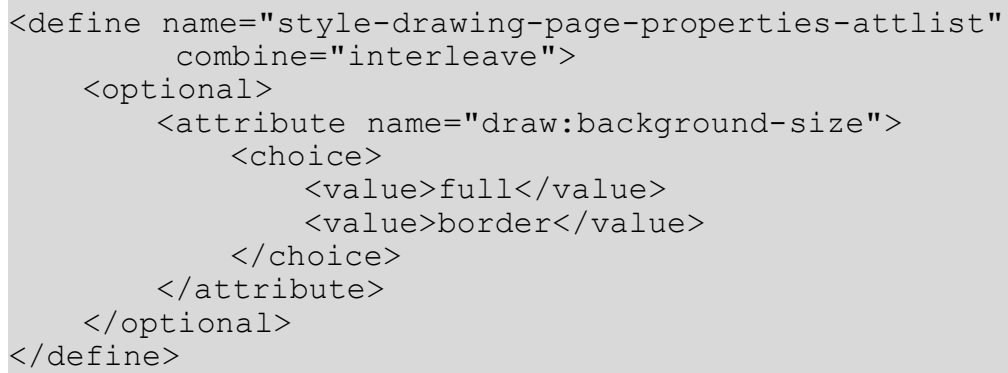

\subsubsection{Background Objects Visible}

The attribute presentation:background-objects-visible specifies whether or not to hide objects on the background of the master page when displaying the presentation page.

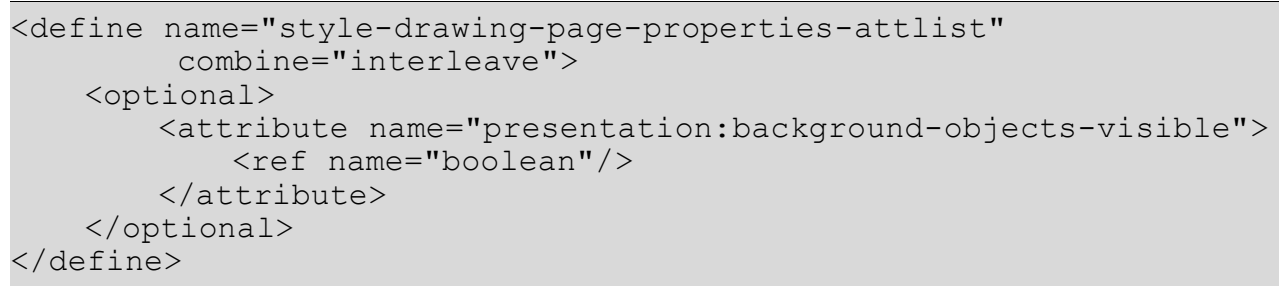

\subsubsection{Background Visible}

The attribute presentation:background-visible specifies whether or not to hide the background of the master page when displaying the presentation page.

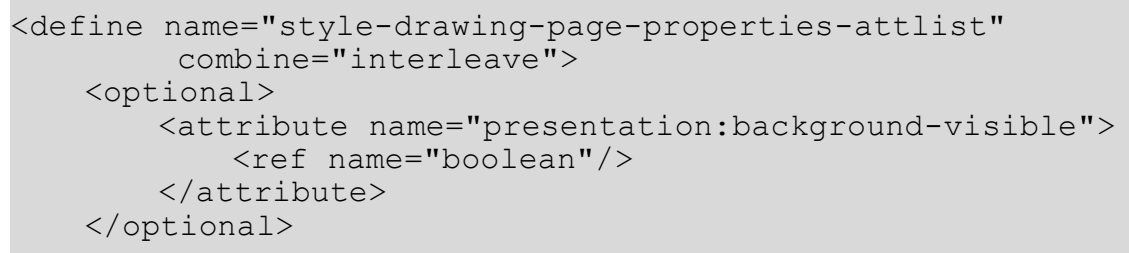




\subsubsection{Display Header}

The presentation: display-header attribute sets the visibility of presentation shapes from the master page with the presentation class header (see section 9.6.1).

17466

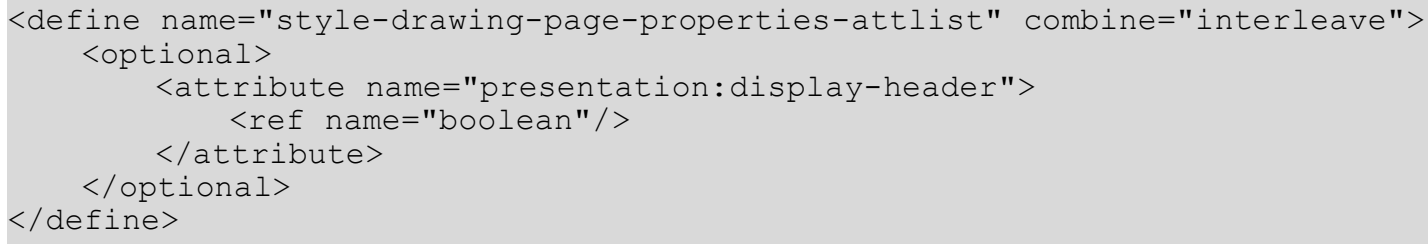

\subsubsection{Display Footer}

The presentation: display-footer attribute sets the visibility of presentation shapes from the master page with the presentation class footer (see section 9.6.1).

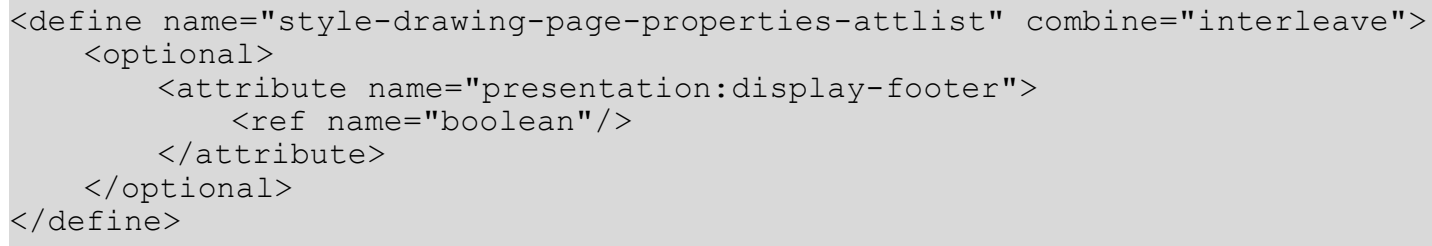

\subsubsection{Display Page Number}

The presentation:display-page-number attribute sets the visibility of presentation shapes from the master page with the presentation class page-number (see section 9.6.1).

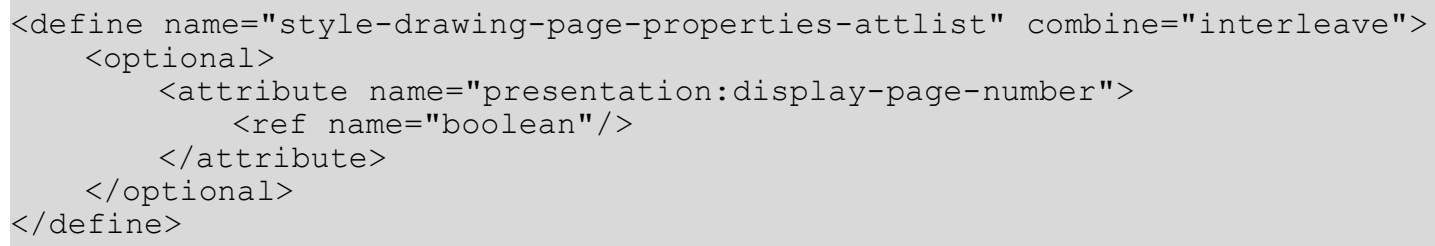

\subsubsection{Display Date And Time}

The presentation:display-date-time attribute sets the visibility of presentation shapes from the master page with the presentation class date-time (see section 9.6.1).

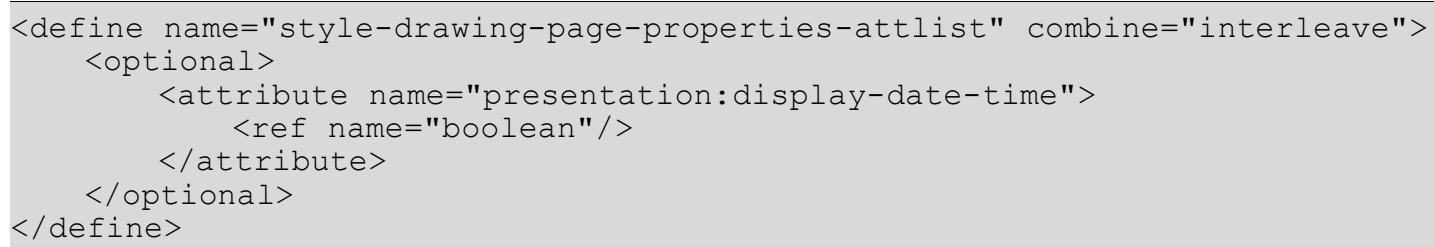




\section{Data Types and Schema Definitions}

\subsection{Data Types}

The following data types are used within this specification:

- W3C Schema data types as defined in [xmlschema-2] (referenced by $<$ ref $>$ elements named the same as the corresponding data types)

$$
\begin{aligned}
& \text { - string } \\
& \text { - date } \\
& \text { - time } \\
& \text { - dateTime } \\
& \text { - duration } \\
& \text { - integer } \\
& \text { - nonNegativelnteger } \\
& \text { - positivelnteger } \\
& \text { - double } \\
& \text { - anyURI } \\
& \text { - base64Binary } \\
& \text { - ID } \\
& \text { - IDREF }
\end{aligned}
$$

Relax-NG definitions for the W3C schema data types:

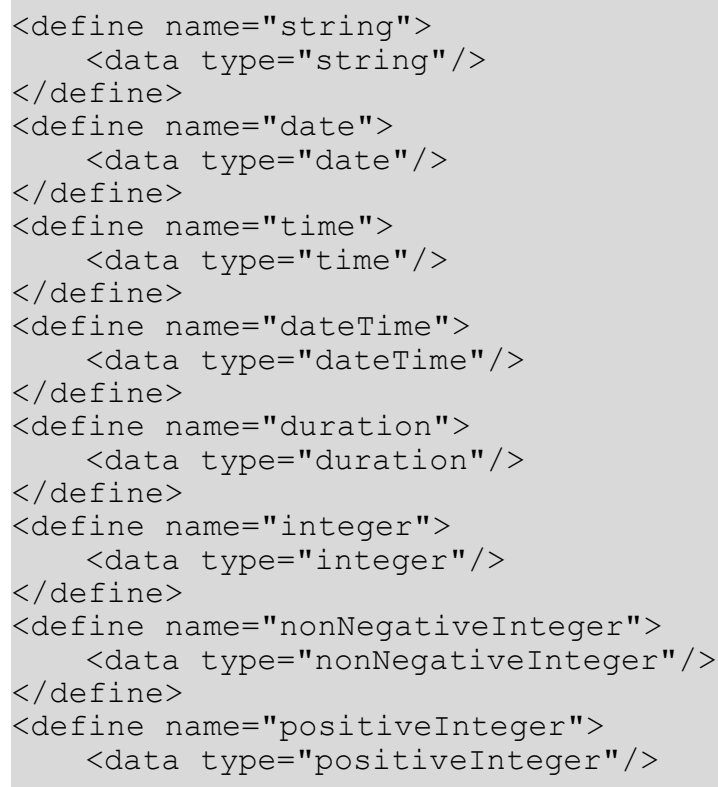




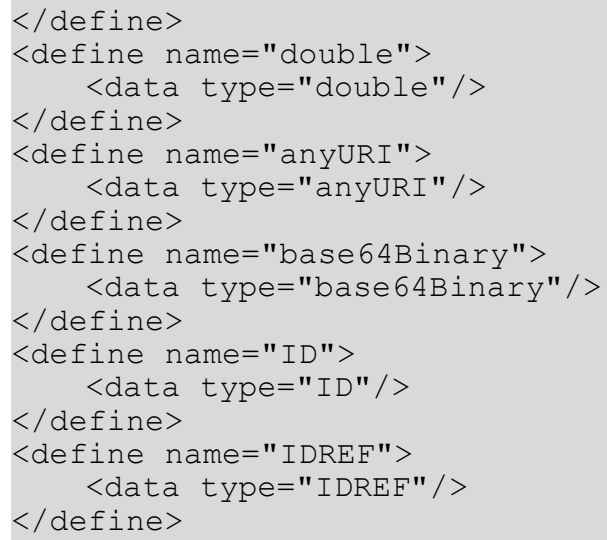

- custom data types (usually specializations of W3C Schema data types)

- boolean

A Boolean value may have either of the values true or false.

- dateOrDateTime

A dateOrDateTime value is essentially an [xmlschema-2] date and time value with an optional time component. In other words, it may contain either a date, or a date and time value.

- timeOrDateTime

A timeOrDateTime value is essentially an [xmlschema-2] date and time value with an optional date component. In other words, it may contain either a time, or a date and time value.

- language

A language is a the same as a W3C schema language data type, except that the values a described by [RFC3066] rather than [RFC1766].

- countryCode

A countryCode is a country code in conformance with [RFC3066], as specified in [XSL].

- languageCode

A languageCode is a language code in conformance with [RFC3066], as specified in [XSL].

- character

A character value is a string with only one character.

- length

A (positive or negative) physical length, consisting of magnitude and unit, in conformance with $\S 5.9 .11$ of [XSL]. Valid lengths would be " $2.54 \mathrm{~cm}$ " or " 1 inch". A number without unit is not a valid length, e.g. "3.2". The support of pixel values is optional.

- nonNegativeLength

Like length, except that the value must be zero or positive. 
- positiveLength

Like length, except that the value must be positive.

- percent

(Positive or negative) percentage values in conformance with $\S 5.9 .11$ of [XSL], e.g. "40\%".

- relativeLength

A relative length is a positive integer, followed by $a^{\prime * 1}$ character.

- coordinate

Like a length, except that the physical length denotes a certain point.

- distance

Like a length, except that the physical length measures the distance between to points.

- color

A RGB color in conformance with §5.9.11 of [XSL], that is a RGB color in notation "\#rrggbb", where rr, gg and bb are hexadecimal digits.

- styleName

A NCName as specified in [xmlschema-2] that is the name of a style.

- StyleNameRef

A NCName as specified in [xmlschema-2] that is the name of a referenced style, or an empty value.

- StyleNames

A whitespace separated list of NCNames as specified in [xmlschema-2] that are the names of a styles.

- VariableName

A string specifying the name of a variable

- formula

A string containing a formula. Formulas don't have a predefined syntax, but should start with a namespace prefix that specifies the syntax used within the formula.

- valueType

A list of value types supported for certain generic values, such as "string" or "date".

- targetFrameName

The name of a target frame in conformance with $\S 6.16$ of [HTML4].

- points

A sequence of points. The points are two integer coordinates separated by a comma. The points are separated by white space.

- pathData

Path data as described in §8 of [SVG]. 
- vector3D

A 3-element vector that is represented by floating point $x, y, z$ coordinates. The coordinates are encapsulated between parentheses and the coordinates are noted in the order $\mathrm{x}, \mathrm{y}$ and $\mathrm{z}$, separated by whitespaces. If this value represents a normal, then it should be normalized.

Example: A directional vector with the coordinates $x=0.5, y=0$ and $z=1$ looks like "(0.5 0 1)".

- namespacedToken

A namespaced token is a token id that makes use of the XML namespace mechanism for modularization purposes.

Example: The predefined chart types make use of the chart namespace

urn:oasis:names:tc:opendocument:xmlns: chart: 1.0 . Assuming a namespace declaration of

xmlns: chart="urn:oasis: names: tc : opendocument: xmlns: chart: 1.0 ", a bar chart would be identified as chart:bar.

Relax-NG definitions for custom data types:

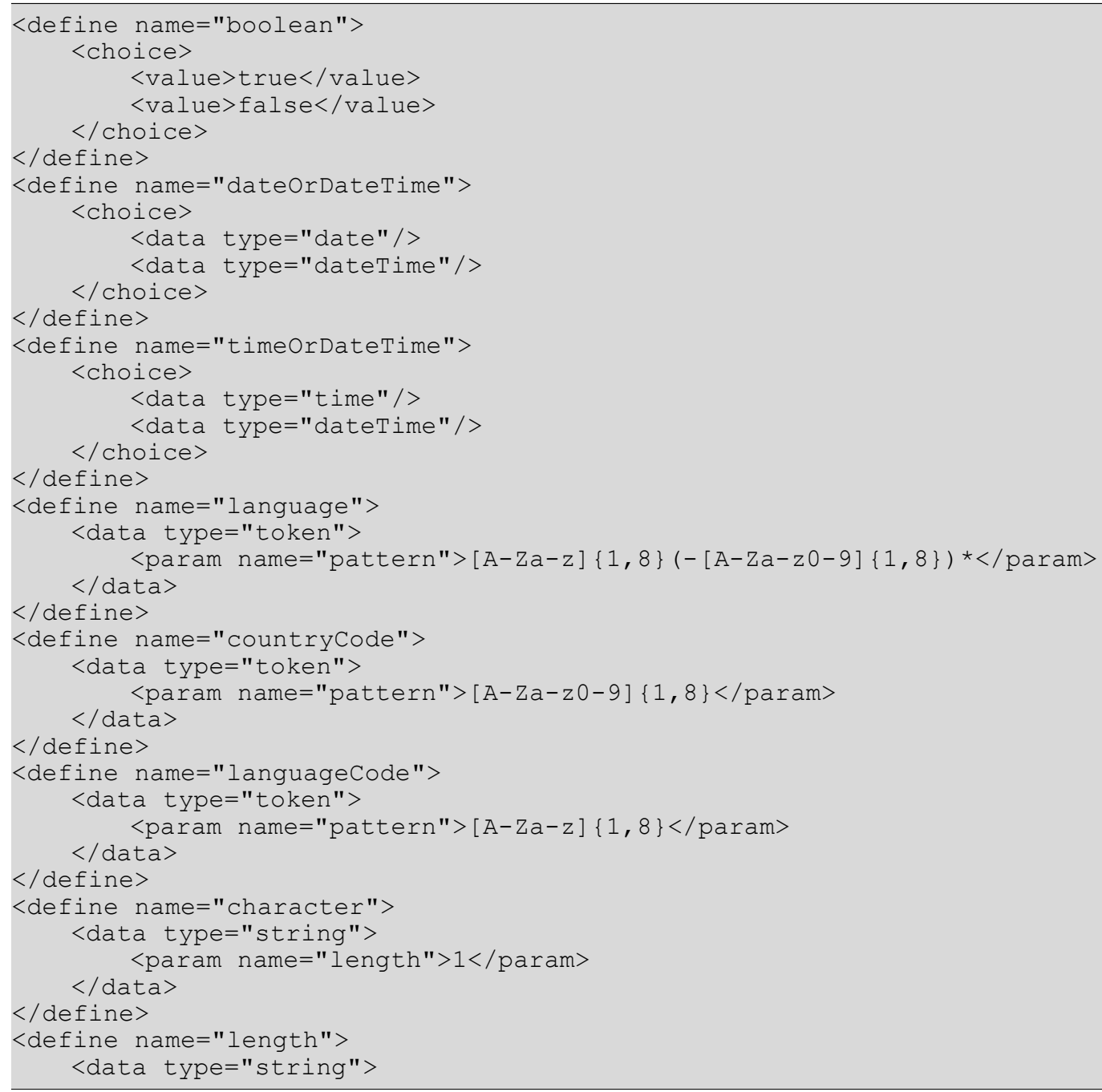




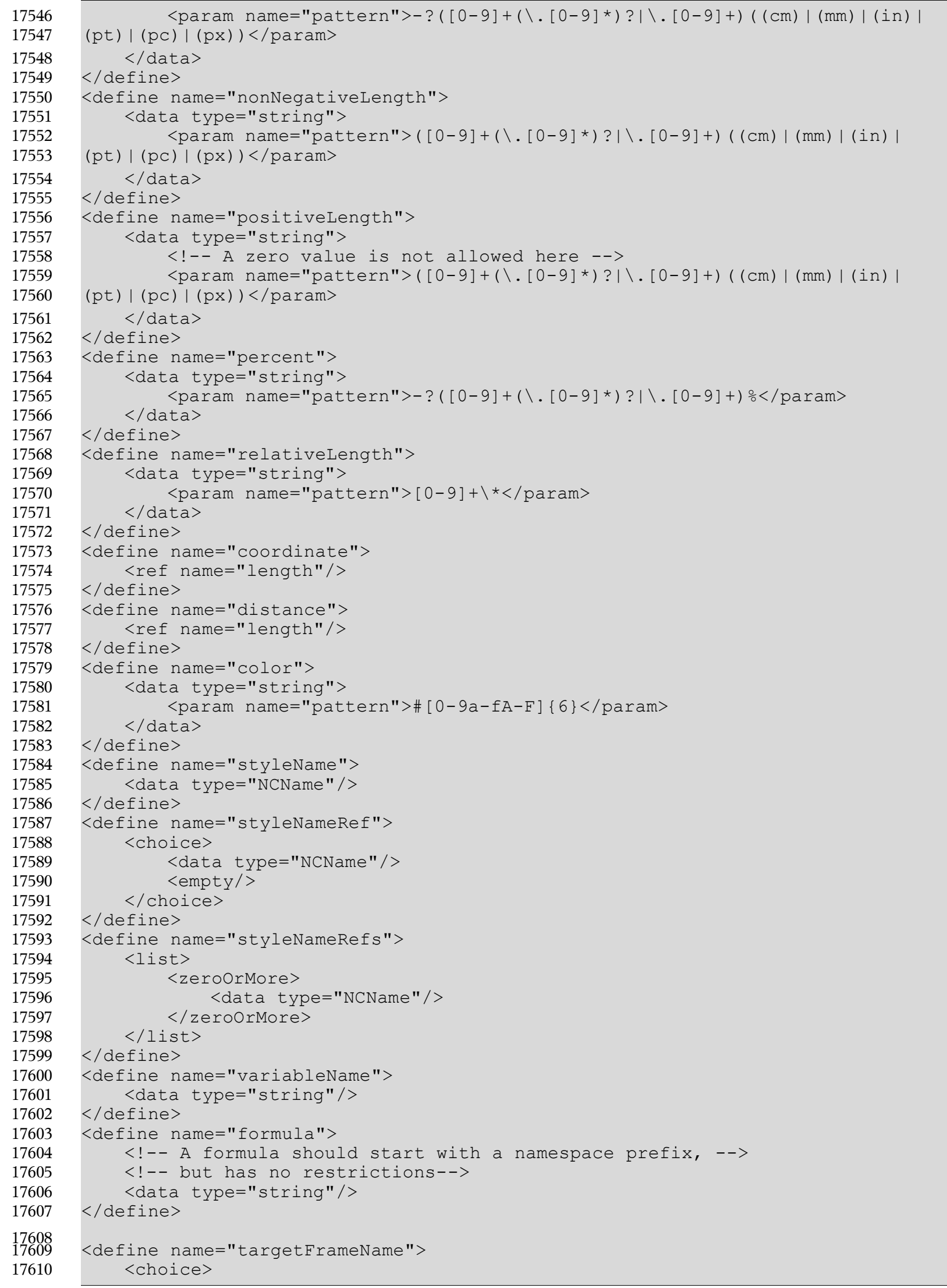




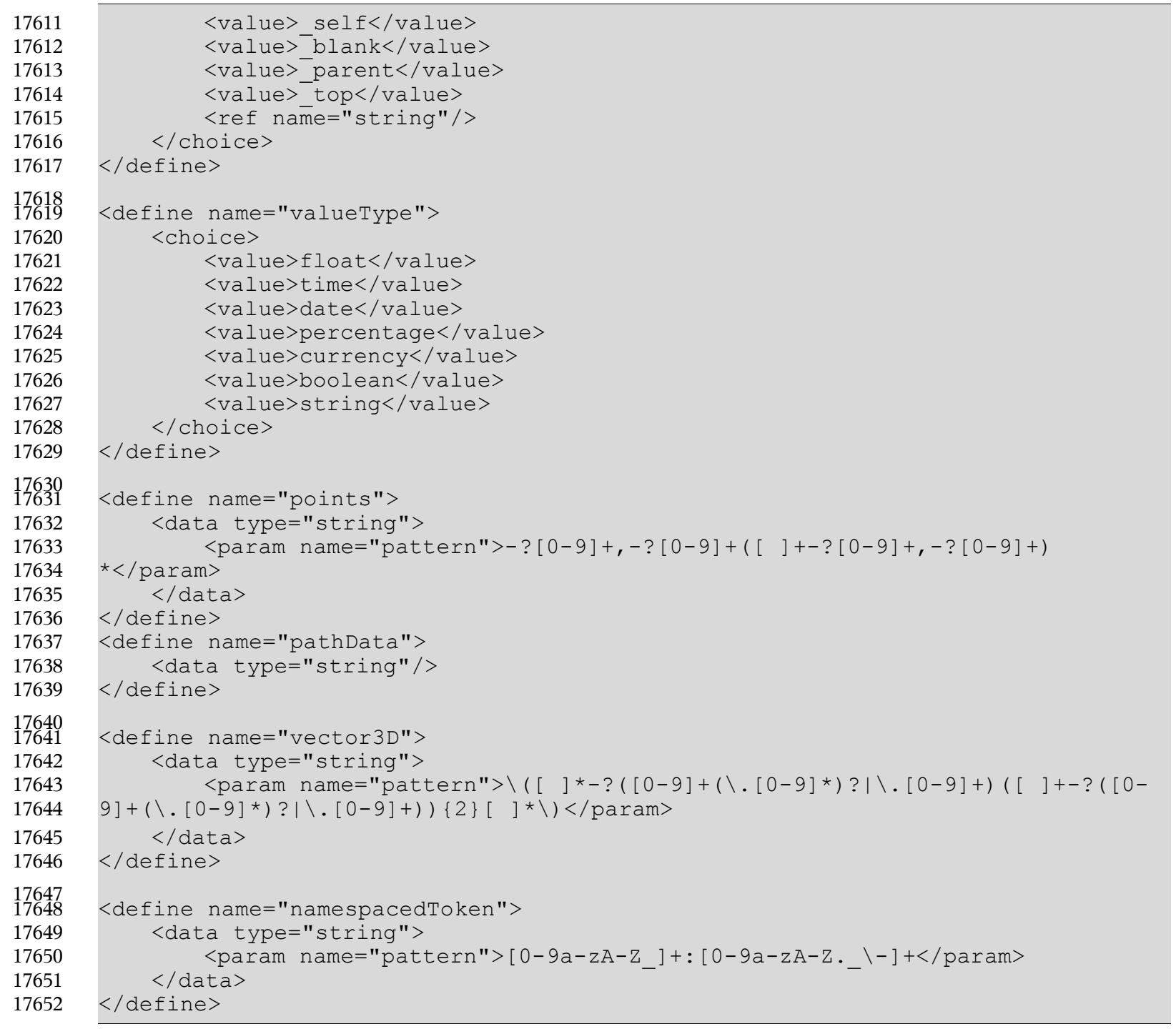

\subsection{Other Definitions}

To provide for extensibility of the format, inclusion of custom content is allowed on several occasions. The following definitions allow for inclusion of arbitrary attributes or elements (with arbitrary content models).

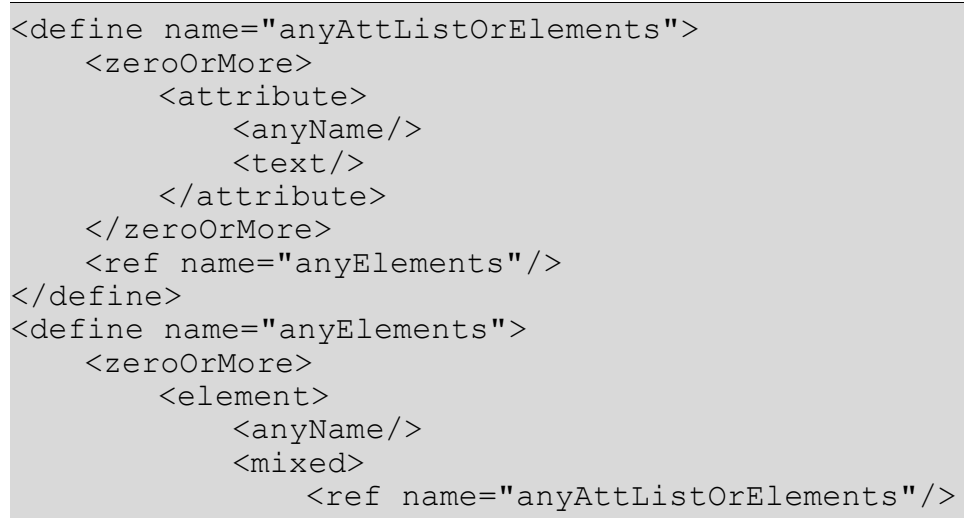




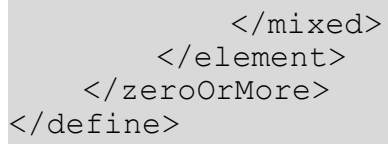

\subsection{Relax-NG Schema Suffix}

Suffix for the normative Relax-NG schema: 


\section{Packages}

This chapter describes the package format that optionally can be used in OpenDocument. It contains the following sections:

- Introduction

- Zip File Structure

- Encryption

- Preview Image

- Manifest File

\subsection{Introduction}

As XML has no native support for binary objects such as images, [OLE] objects, or other media types, and because uncompressed XML files can get very large, OpenDocument uses a package file to store the XML content of a document together with its associated binary data, and to optionally compress the XML content. This package is a standard Zip file, whose structure is discussed below.

Information about the files contained in the package is stored in an XML file called the manifest file. The manifest file is always stored at the pathname META-INF/manifest.xml. The main pieces of information stored in the manifest are as follows:

- A list of all of the files in the package.

- The media type of each file in the package.

- If a file stored in the package is encrypted, the information required to decrypt the file is stored in the manifest.

\subsection{Zip File Structure}

A Zip file starts with a sequence of files, each of which can be compressed or stored in raw format. Each file has a local header immediately before its data, which contains most of the information about the file, including time-stamps, compression method and file name. The compressed file contents immediately follow, and are terminated by an optional data descriptor. The data descriptor contains the CRC and compressed size of the file, which are frequently not available when writing the local file header. If these details were included, the data descriptor can be skipped. 
Each file in the archive is laid down sequentially in this format, followed by a central directory at the end of the Zip archive. The central directory is a contiguous set of directory entries, each of which contains all the information in the local file header, plus extras such as file comments and attributes. Most importantly, the central directory contains pointers to the position of each file in the archive, which makes navigation of the Zip file quick and easy.

For more details about the Zip file format, see [ZIP].

\subsection{Encryption}

The encryption process takes place in the following multiple stages:

1. A 20-byte SHA1 digest of the user entered password is created and passed to the package component.

2. The package component initializes a random number generator with the current time.

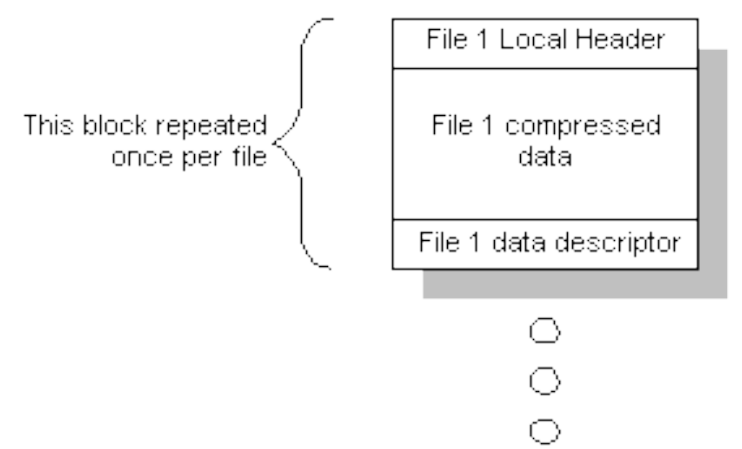

File N Local Header

File w cormpressed data

File N data descriptor

Directory Entry 1

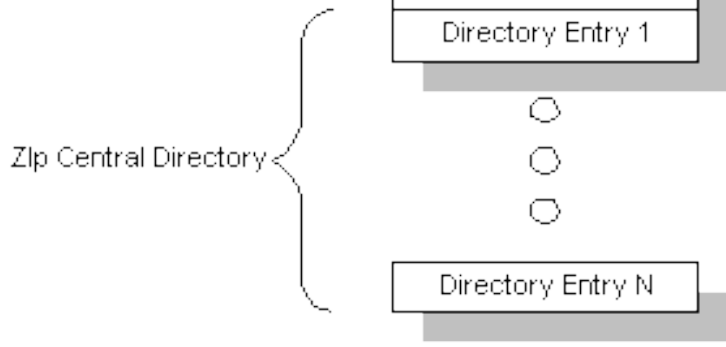

3. The random number generator is used to generate a random 8-byte initialization vector and 16-byte salt for each file.

4. This salt is used together with the 20-byte SHA1 digest of the password to derive a unique 128-bit key for each file. The algorithm used to derive the key is PBKDF2 using HMAC-SHA1 (see [RFC2898]) with an iteration count of 1024.

5. The derived key is used together with the initialization vector to encrypt the file using the Blowfish algorithm in cipher-feedback (CFB) mode.

Each file that is encrypted is compressed before being encrypted. To allow the contents of the package file to be verified, it is necessary that encrypted files are flagged as 'STORED' rather than 'DEFLATED'. As entries which are 'STORED' must have their size equal to the compressed size, it is necessary to store the uncompressed size in the manifest. The compressed size is stored in both the local file header and central directory record of the Zip file.

\subsection{MIME Type Stream}

If a MIME type for a document that makes use of packages is existing, then the package SHOULD contain a stream called "mimetype". This stream SHOULD be first stream of the package's zip file, it MUST NOT be compressed, and it MUST NOT use an 'extra field' in its header (see [ZIP]).

The purpose is to allow packaged files to be identified through 'magic number' mechanisms, such as Unix's file/magic utility. If a ZIP file contains a stream at the beginning of the file that is uncompressed, and has no extra data in the header, then the stream name and the stream content can be found at fixed positions. More specifically, one will find:

- a string 'PK' at position 0 of all zip files 
- a string 'mimetype' at position 30 of all such package files

- the mimetype itself at position 38 of such a package.

\subsection{Usage of URIs Within Packages}

Within a file that is contained in a package, relative URIs are used to reference other sub files of the package, but can also be used to reference files within the file system.

The following restrictions exist for URIs that are used within a package:

- only sub files within the same package can be referenced.

- URIs that reference a sub file of a package MUST be relative, and they MUST NOT contain paths that are not within the package. This especially means that sub files of a package MUST NOT be referenced by an absolute URI.

- sub file of a package can not be referenced from outside the package, for instance from the file system or another package.

A relative-path reference (as described in $\S 5$ of [RFC2396]) that occurs in a file that is contained in a package has to be resolved exactly as it would be resolved if the whole package gets unzipped into a directory at its current location. The base URI for resolving relative-path references is the one that has to be used to retrieve the (unzipped) file that contains the relativepath reference.

All other kinds of URI references, namely the ones that start with a protocol (like http:), an authority (i.e. //) or an absolute-path (i.e. /) do not need any special processing. This especially means that absolute-paths do not reference files inside the package, but within the hierarchy the package is contained in, for instance the file system. URI references inside a package may leave the package, but once they have left the package, they never can return into the package or another one.

\subsection{Preview Image}

A thumbnail representation of a document should be generated by default when the file is saved. It should be a representation of the first page, first sheet, etc. of the document. For maximum reusability of the thumbnails they have to be generated without any effects, surrounding frames, or borders. Such effects might interfere with effects added to the thumbnails by the different file system explorers or may not be desired at all for certain use cases.

The thumbnail must be saved as "thumbnail.png" in a separate folder named "Thumbnails".

The "Thumbnails" folder must not get a media type in the manifest.xml file, since it is not actually part of the document.

Encrypted files are intended to be unreadable for unauthorized users that's why a thumbnail for such files must not be generated. Instead of saving a thumbnail of the first page a replacement representation that doesn't depend on the contents of the document is saved for encrypted files which makes obvious that the corresponding file is encrypted.

In order to conform to the Thumbnail Managing Standard (TMS) at www.freedesktop.org, thumbnails must be saved as 24bit, non-interlaced PNG image with full alpha transparency. The required size for the thumbnails is $128 \times 128$ pixel. 


\subsection{Manifest File}

The elements and attributes in the manifest file are in the namespace: urn:oasis:names:tc:opendocument:xmlns:manifest:1.0.

\subsubsection{Relax-NG Schema}

The normative XML Schema for OpenDocument Manifest files is embedded within this specification. It can be obtained from the specification document by concatenating all schema fragments contained in this chapters. All schema fragments have a gray background color and line numbers.

The schema language used within this specification is Relax-NG (see [RNG]).

Prefix for the normative Relax-NG Manifest schema:

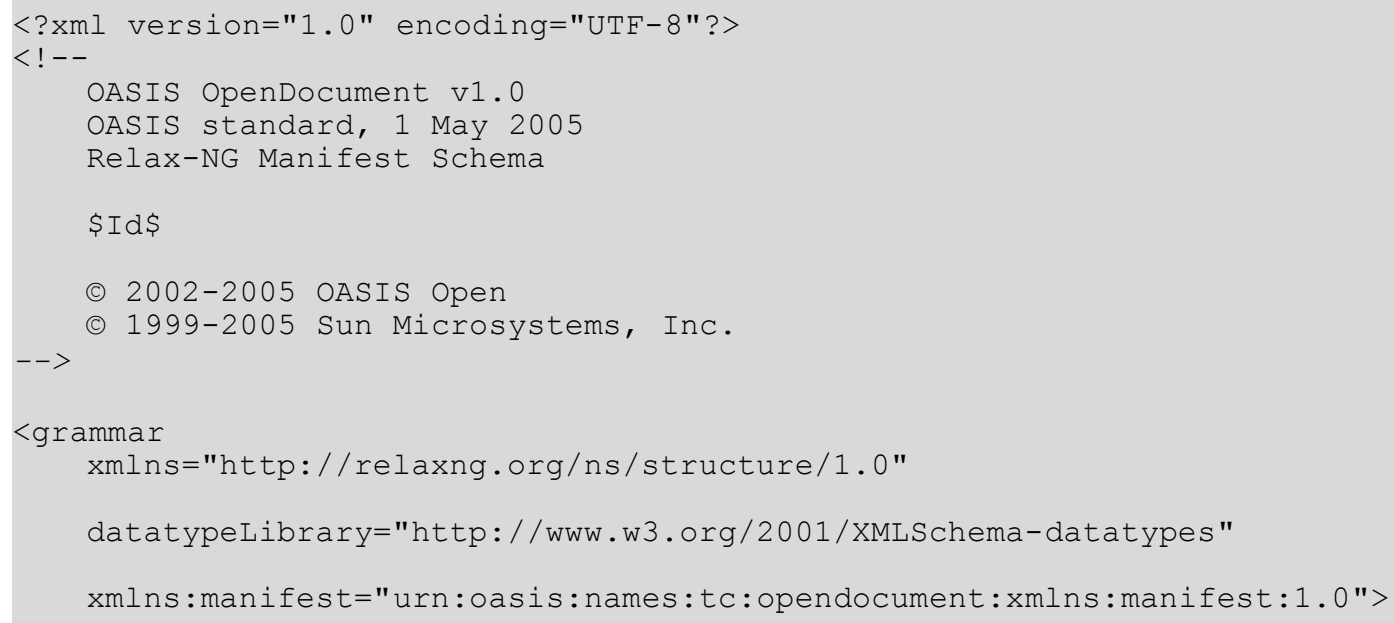

\subsubsection{Manifest Root Element}

The root element is called manifest. The root element contains one fixed attribute which specifies the namespace as described above and multiple <manifest:file-entry> elements, each of which describes a single file in the package.

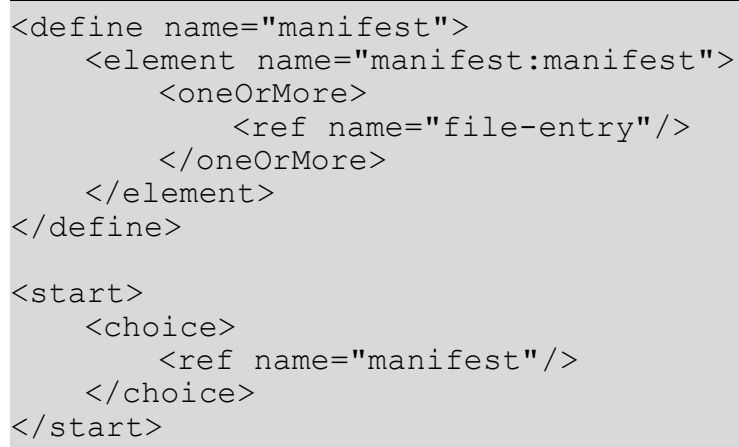

\subsubsection{File Entry}

The <manifest:file-entry> element represents a single file within the package, and stores the files location in the package, the mime-type of the file and optionally the data required to decrypt this file. 
Directories only receive <manifest: file-entry> entries if they have inherent semantics. For example, a directory that constitutes a sub-document referenced as an object from within the main document would contain a <manifest: file-entry> with a suitable media type. A directory for administrative or convenience purposes, such as a directory that contains various image files, would not receive an entry in the manifest file.

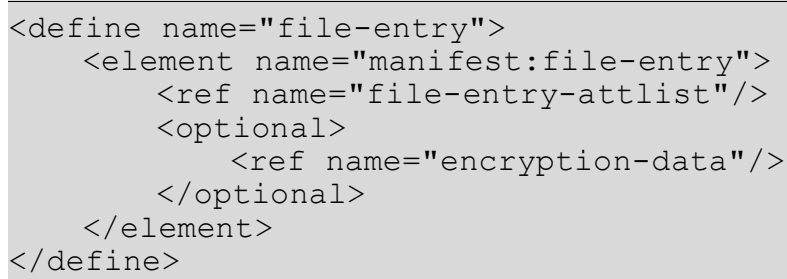

The attributes associated with a <manifest: file-entry $>$ are as follows:

- Full path

- Size

- Media type

\section{Full Path}

The manifest:full-path attribute describes the location of the file within the package.

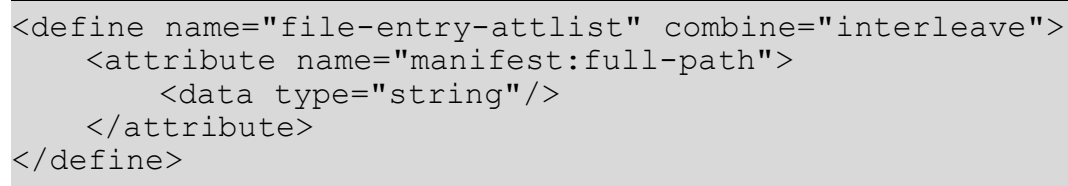

\section{Size}

The manifest:size attribute is only present if the file is stored in an encrypted format. The reason why this attribute is required is explained in section 17.3. This attribute is only used for encrypted files.

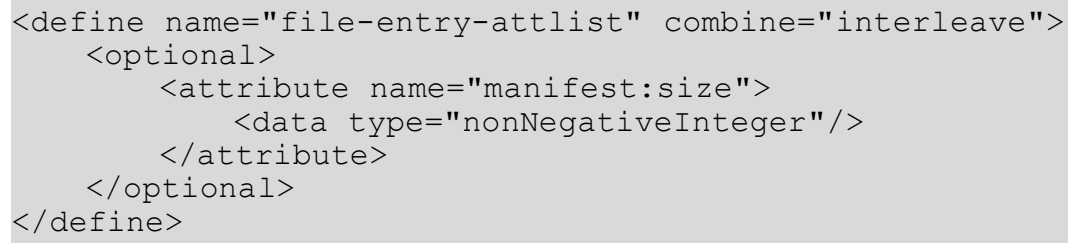

\section{Media Type}

The manifest:media-type attribute specifies the mime type of the specified file. For a full list of mime types see http://www.isi.edu/in-notes/iana/assignments/media-types/media-types. As an example, all XML streams have the media type "text/xml".

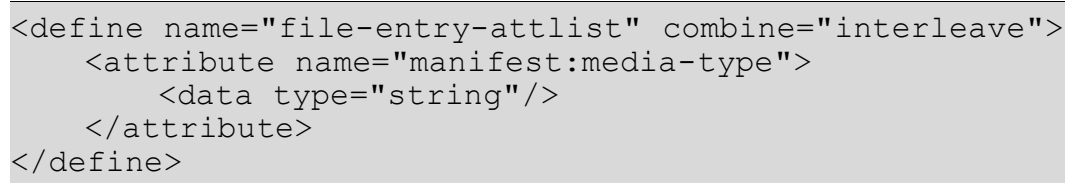




\subsubsection{Encryption Data}

The <manifest:encryption-data> element contains all of the information required to decrypt the file.

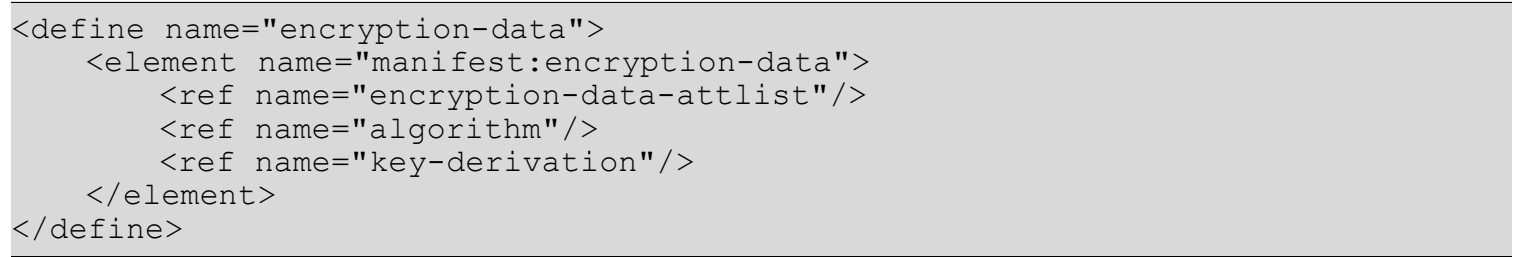

The <encryption-data> element contains the following elements:

- Algorithm

- Key Derivation

\section{Checksum Type}

The manifest: checksum-type attribute specifies the name of digest algorithm that can be used to check password correctness. Currently, the only supported digest algorithm is SHA1.

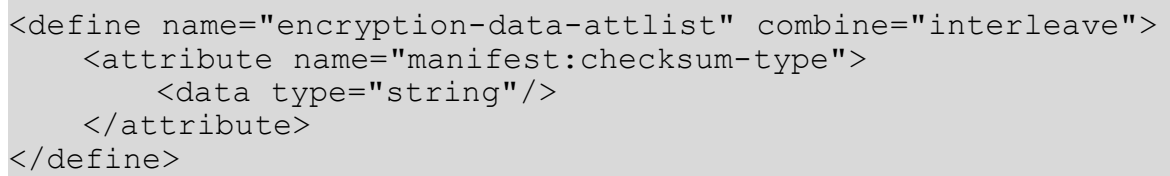

\section{Checksum}

The manifest: checksum attribute specifies the base64-encoded digest that can be used to detect password correctness as specified within manifest: checksum-type attribute.

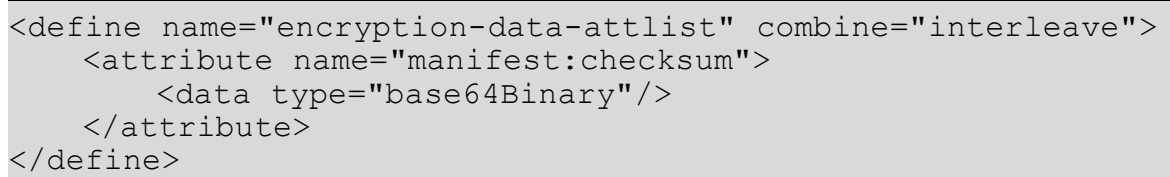

\subsubsection{Algorithm}

The <manifest:algorithm> element contains information about the algorithm used to encrypt the data.

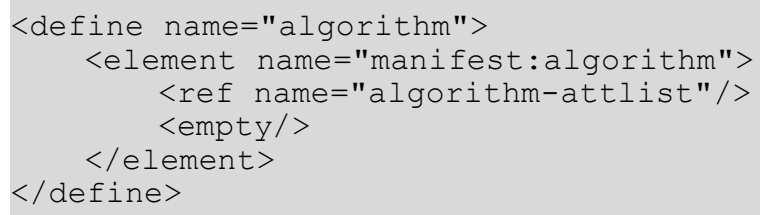

The attributes associated with <manifest:algorithm> are as follows:

- Algorithm name

- Initialization vector 


\section{Algorithm Name}

The manifest:algorithm-name attribute specifies the name of the algorithm used to encrypt the file, and also specifies in which mode this algorithm was used. Currently, the only supports algorithm is the Blowfish algorithm in CFB mode.

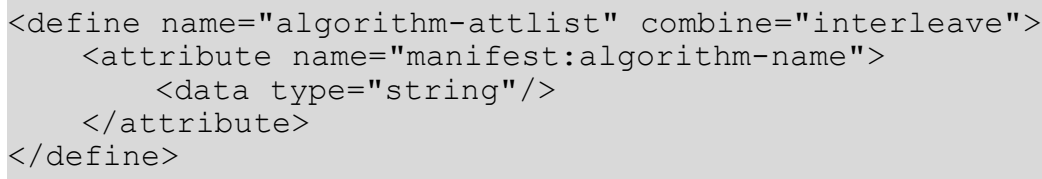

\section{Initialization Vector}

The manifest:initialisation-vector attribute specifies the 8 bytes used as an initialization vector to the stream cipher. The initialization vector is an 8 byte binary sequence, and so is encoded in base64 when written to the manifest file.

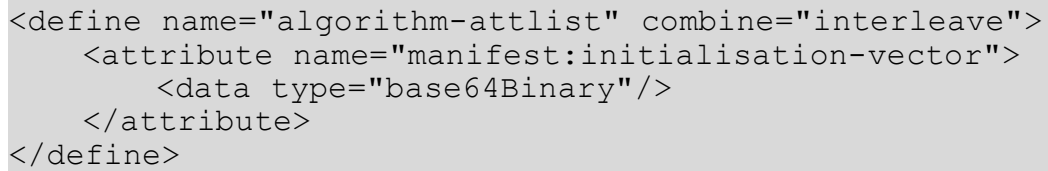

\subsubsection{Key Derivation}

The <manifest: key-derivation> element contains the information that was used to derive the encryption key for this file from the user specified password.

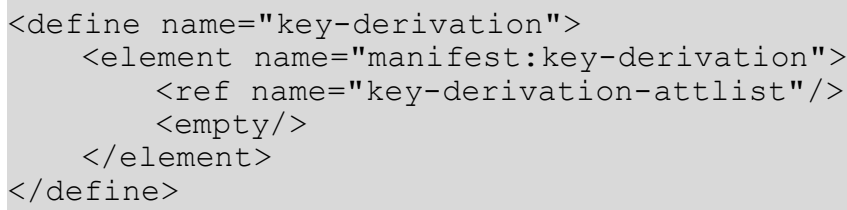

The attributes associated with the <manifest: key-derivation> element are as follows:

- Key derivation name

- Salt

- Iteration count

\section{Key Derivation Name}

The manifest:key-derivation-name attribute specifies the name of the algorithm used to derive the name. At this time, the packages only supports the use of the PBKDF2 key derivation method. For further details see [RFC2898].

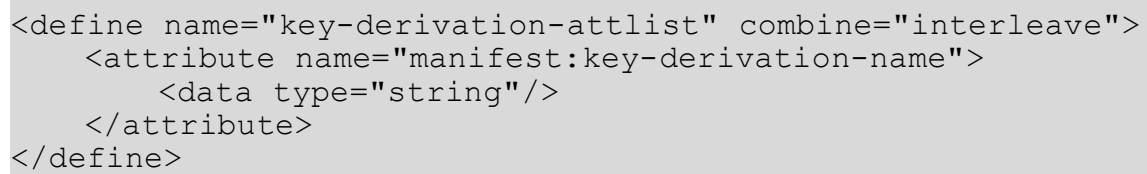




\section{Salt}

The manifest:salt attribute specifies the 16-byte sequence used as the 'salt' by the key derivation algorithm. The salt is a 16 byte binary sequence, and thus is encoded in base64 before being written to the manifest file.

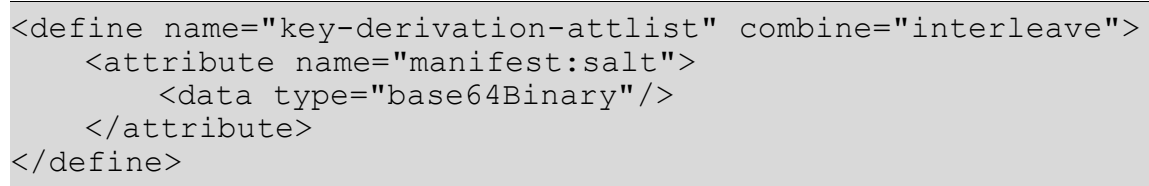

\section{Iteration Count}

The manifest:iteration-count attribute specifies the number of iterations used by the key derivation algorithm to derive the key.

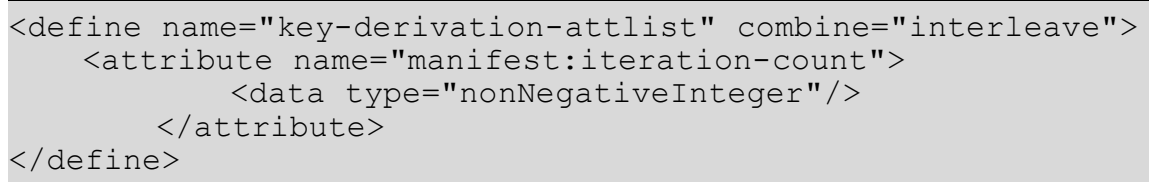

\section{Sample Manifest}

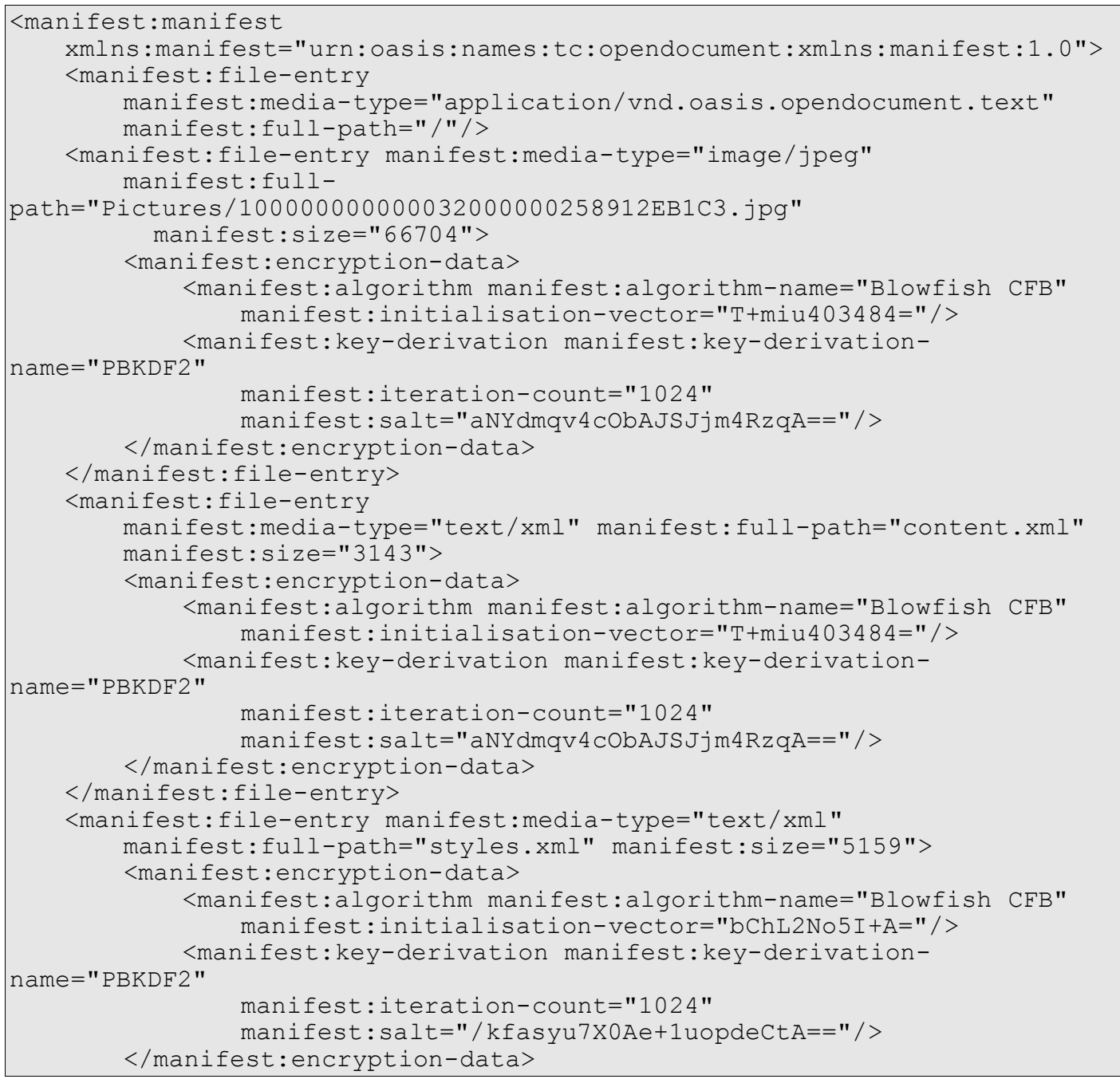




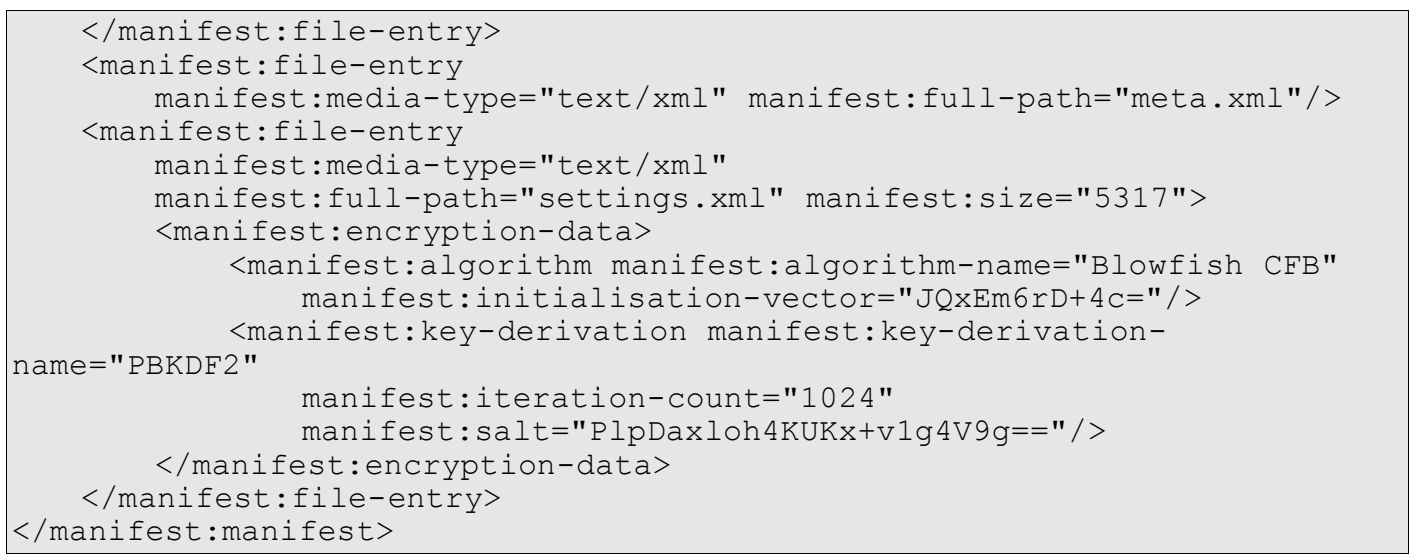

\subsubsection{Relax-NG Schema Suffix}

Suffix for the normative Relax-NG Manifest schema: 


\title{
Appendix A.Strict Relax NG Schema
}

\author{
The Relax-NG (see [RNG])schema provided in this appendix equals the schema defined in \\ chapters 1 to 16 of this specification, but restricts the content of meta information elements and \\ formatting properties elements to the attributes and elements defined in this specification. See \\ also section 1.5 .
}

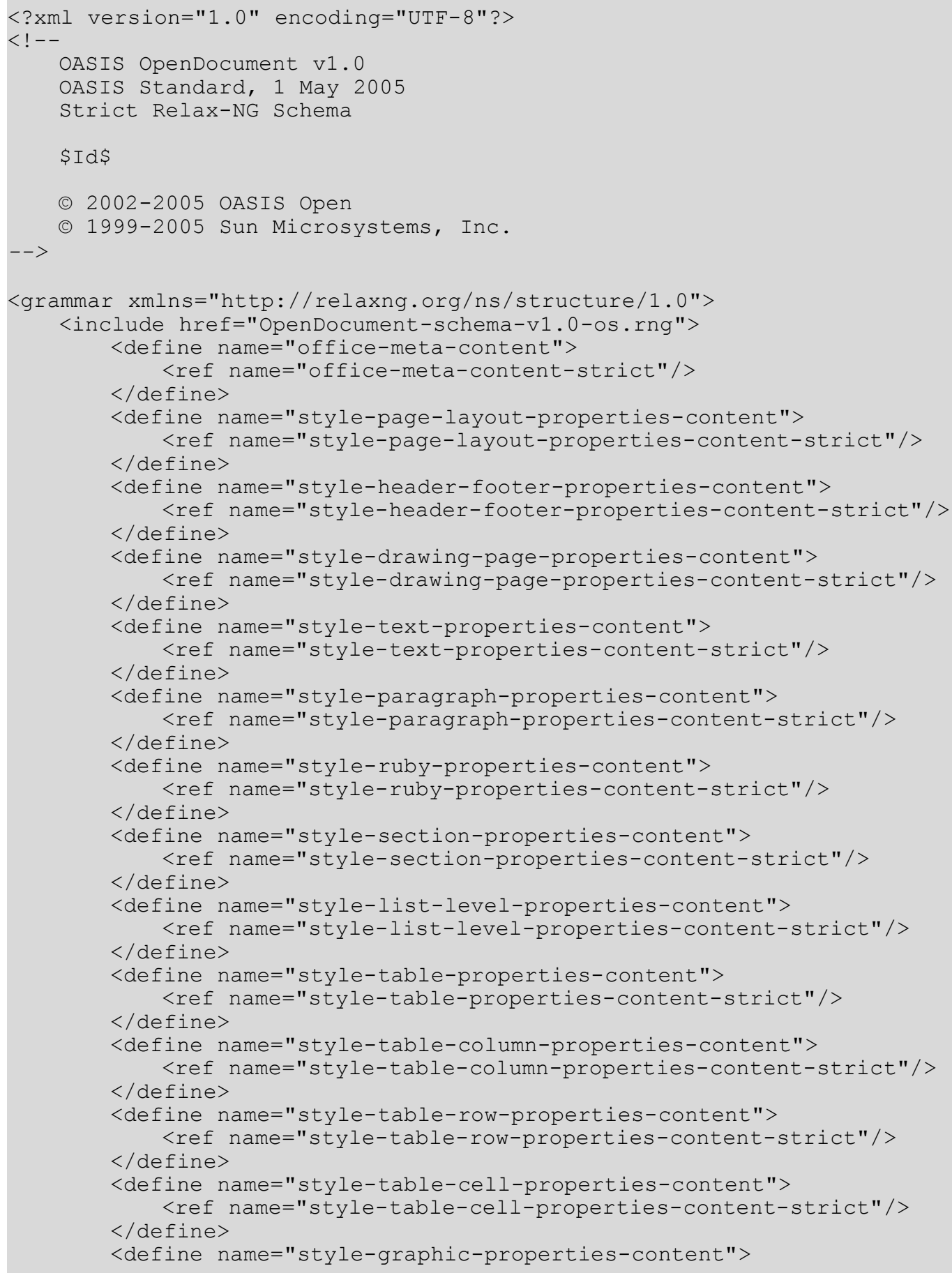




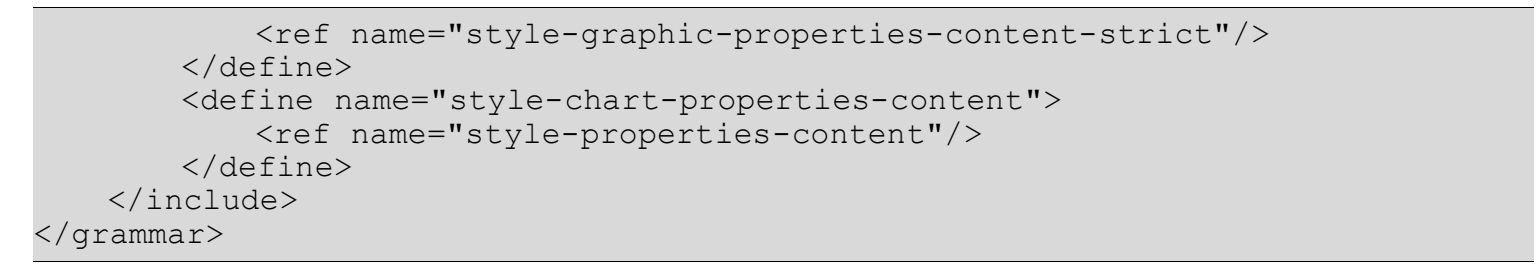




\section{Appendix B.References}

[CSS2] Bert Bos, Håkon Wium Lie, Chris Lilley, lan Jacobs, Cascading Style Sheets, level 2 , http://www.w3.org/TR/1998/REC-CSS2-19980512, W3C, 1998.

[CSS3Text] Michel Suignard, CSS3 Text Module, CSS3 Text Module, W3C, 2003.

[DCMI] -, Dublin Core Metadata Element Set, Version 1.1: Reference Description, http://www.dublincore.org/documents/dces/, Dublin Core Metadata Initiative, 2003.

[DOMEvents] Philippe Le Hégaret, Tom Pixley, Document Object Model (DOM) Level 3 Events Specification, http://www.w3.org/TR/2003/WD-DOM-Level-3-Events-20030331, W3C, 2003.

[HTML4] Dave Raggett, Arnoud Le Hors, lan Jacobs, HTML 4.01 Specification, http://www.w3.org/TR/1999/REC-html401-19991224, W3C, 1999.

[JDBC] Jon Ellis, Linda Ho, Maydene Fisher, JDBC 3.0 Specification, http://java.sun.com/products/jdbc/, Sun Microsystems, Inc., 2001.

[MathML] David Carlisle, Patrick Ion, Robert Miner, Nico Poppelier, Mathematical Markup Language (MathML) Version 2.0 (Second Edition), http://www.w3.org/TR/2003/REC-MathML220031021/, W3C, 2003.

[MIMETYPES] , List of registered MIME types, ftp://ftp.isi.edu/in-notes/iana/assignments/mediatypes/, IANA, .

[OLE] Kraig Brockschmidt, Inside OLE, Microsoft Press, 1995, ISBN: 1-55615-843-2

[OOo] , OpenOffice.org XML File Format 1.0 Technical Reference Manual, http://xml.openoffice.org/xml_specification.pdf, Sun Microsystems, Inc., 2002.

[PNG] Thomas Boutell, PNG (Portable Network Graphics) Specification, http://www.w3.org/TR/REC-png-multi.html, W3C, 1996.

[RFC1766] H. Alvestrand, Tags for the Identification of Languages, http://www.ietf.org/rfc/rfc1766.txt, IETF, 1995.

[RFC2048] N. Freed, J. Klensin, J. Postel, Multipurpose Internet Mail Extensions (MIME) Part Four: Registration Procedures, http://www.ietf.org/rfc/rfc2048.txt, IETF, 1996.

[RFC2119] S. Bradner, Key words for use in RFCs to Indicate Requirement Levels, http://www.ietf.org/rfc/rfc2119.txt, IETF, 1997.

[RFC2396] T. Berners-Lee, R. Fielding, U.C. Irvine, L. Masinter, Uniform Resource Identifiers (URI): Generic Syntax, http://www.ietf.org/rfc/rfc2396.txt, IETF, 1998.

[RFC2616] IETF, Hypertext Transfer Protocol -- HTTP/1.1, http://www.ietf.org/rfc/rfc2616.txt, IETF, 1999.

[RFC2898] B. Kaliski, PKCS \#5: Password-Based Cryptography Specification Version 2.0, http://www.ietf.org/rfc/rfc2898, IETF, 2000.

[RFC3066] H. Alvestrand, Tags for the Identification of Languages, http://www.ietf.org/rfc/rfc3066.txt, IETF, 2001.

[RNG] James Clark, MURATA Makoto, RELAX NG Specification, http://oasisopen.org/committees/relax-ng/spec-20011203.html, OASIS, 2001. 
[SMIL20] W3C, Synchronized Multimedia Integration Language 2.0 (SMIL 2.0), http://www.w3.org/TR/smil20/, W3C, 2001.

[SVG] Jon Ferraiolo, (FUJISAWA Jun), Dean Jackson, Scalable Vector Graphics (SVG) 1.1, http://www.w3.org/TR/2003/REC-SVG11-20030114/, W3C, 2003.

[XForms] W3C, XForms, http://www.w3.org/TR/xforms/, W3C, 2004.

[XLink] Steve DeRose, Eve Maler, David Orchard, XML Linking Language, http://www.w3c.org/TR/xlink/, W3C, 2001.

[xml-names] Tim Bray, Dave Hollander, Andrew Layman, Namespaces in XML, http://www.w3.org/TR/REC-xml-names/, W3C, 1999.

[XML1.0] Tim Bray, Jean Paoli, C. M. Sperberg-McQueen, Eve Maler, François Yergeau , Extensible Markup Language (XML) 1.0 (Third Edition), http://www.w3.org/TR/2004/REC-xml20040204, W3C, 2004.

[xmlschema-2] Paul V. Biron, Ashok Malhotra, XML Schema Part 2: Datatypes, http://www.w3.org/TR/2001/REC-xmlschema-2-20010502/, W3C, 2001.

[XSL] W3C, Extensible Stylesheet Language (XSL), http://www.w3.org/TR/2001/REC-xsl20011015/, W3C, 2001.

[XSLT] James Clark, XSL Transformations (XSLT) Version 1.0, http://www.w3.org/TR/1999/REC-xslt-19991116, W3C, 1999.

[XSLT2] Michael Kay, XSL Transformations (XSLT) Version 2.0, http://www.w3.org/TR/2003/WD-xslt20-20031112/, W3C, 2003.

[ZIP] , .ZIP File Format Specification,

http://www.pkware.com/products/enterprise/white_papers/appnote.txt, PKWARE Inc., 2004. 


\section{Appendix C.MIME Types and File Name Extensions (Non Normative)}

The MIME types and extensions contained in this section are applicable only to office documents that are contained in a package (see section 2.1). See section 1.7 for the MIME type to use for documents that are not contained in a package.

The following table contains a list of MIME types and extensions for documents that conform to this specification, that, at the time this specification is published, have been registered according to [RFC2048]. Please see [MIMETYPES] for a current list of registered MIME types.

\begin{tabular}{|c|c|c|}
\hline MIME type & Ext. & Description \\
\hline No registered MIME types exist at the time this specification is published.
\end{tabular}

The following table contains a list of MIME types and extensions for office documents that conform to this specification where a registration according to [RFC2048] is in progress at the time this specification is published.

Please check [MIMETYPES] before using these MIME types. If a MIME type is not listed there, it is RECOMMENDED to use the MIME type that is the result of inserting " $x-$ " behind the "/" character (i.e. application/x-vnd.oasis.opendocument.text).

\begin{tabular}{|c|c|c|}
\hline MIME type & Ext. & Description \\
\hline application/vnd.oasis.opendocument.text & odt & Text document \\
\hline $\begin{array}{l}\text { application/ } \\
\text { vnd.oasis.opendocument.text-template }\end{array}$ & ott & $\begin{array}{l}\text { Text document used as } \\
\text { template }\end{array}$ \\
\hline application/vnd.oasis.opendocument.graphics & odg & $\begin{array}{l}\text { Graphics document } \\
\text { (Drawing) }\end{array}$ \\
\hline $\begin{array}{l}\text { application/ } \\
\text { vnd.oasis.opendocument.graphics-template }\end{array}$ & otg & $\begin{array}{l}\text { Drawing document used as } \\
\text { template }\end{array}$ \\
\hline $\begin{array}{l}\text { application/vnd.oasis.opendocument } \\
\text {-presentation }\end{array}$ & odp & Presentation document \\
\hline $\begin{array}{l}\text { application/ } \\
\text { vnd.oasis.opendocument.presentation- } \\
\text { template }\end{array}$ & otp & $\begin{array}{l}\text { Presentation document used } \\
\text { as template }\end{array}$ \\
\hline $\begin{array}{l}\text { application/vnd.oasis.opendocument } \\
\text {.spreadsheet }\end{array}$ & ods & Spreadsheet document \\
\hline $\begin{array}{l}\text { application/ } \\
\text { vnd.oasis.opendocument. spreadsheet- } \\
\text { template }\end{array}$ & ots & $\begin{array}{l}\text { Spreadsheet document } \\
\text { used as template }\end{array}$ \\
\hline application/vnd.oasis.opendocument. chart & odc & Chart document \\
\hline $\begin{array}{l}\text { application/ } \\
\text { vnd.oasis.opendocument. chart-template }\end{array}$ & otc & $\begin{array}{l}\text { Chart document used as } \\
\text { template }\end{array}$ \\
\hline application/vnd.oasis.opendocument.image & odi & Image document \\
\hline
\end{tabular}




\begin{tabular}{|c|c|l|}
\hline \multicolumn{1}{|c|}{ MIME type } & Ext. & \multicolumn{1}{c|}{ Description } \\
\hline $\begin{array}{c}\text { application/ } \\
\text { vnd.oasis.opendocument.image-template }\end{array}$ & oti & $\begin{array}{l}\text { Image document used as } \\
\text { template }\end{array}$ \\
\hline $\begin{array}{c}\text { application/vnd.oasis.opendocument.formula } \\
\text { vand.oasis.opendocument.formula-template }\end{array}$ & odf & Formula document \\
\hline $\begin{array}{l}\text { application/vnd.oasis.opendocument.text- } \\
\text { master }\end{array}$ & odm & $\begin{array}{l}\text { Formula document used as } \\
\text { template }\end{array}$ \\
\hline application/vnd.oasis.opendocument.text-web & oth & $\begin{array}{l}\text { Global Text document (see } \\
\text { section 2.3.1) }\end{array}$ \\
\hline $\begin{array}{l}\text { Text document used as } \\
\text { template for HTML } \\
\text { documents }\end{array}$ \\
\hline
\end{tabular}




\section{Appendix D.Core Features Sets (Non Normative)}

The OpenDocument specification does not specify which elements and attributes conforming application must, should, or may support. The intention behind this is to ensure that the OpenDocument specification can be used by as many implementations as possible, even if these applications do not support some or many of the elements and attributes defined in this specification. Viewer applications for instance may not support all editing relates elements and attributes (like change tracking), other application may support only the content related elements and attributes, but none of the style related ones.

Even typical office applications may only support a subset of the elements and attributes defined in this specification. They may for instance not support lists within text boxes or may not support some of the language related element and attributes.

The follow table provides an overview which element and attributes usually are supported by typical office application. It lists the chapters and sections contained in this specification and some typical office application classes. An " $X$ " in this table indicates that most (or at least a significant number) of the elements and attributes defined in a section usually are supported by a certain application classes. An "(X)" indicates that only a limited number of elements and attributes usually is supported.

\begin{tabular}{|r|l|l|l|l|l|l|l|}
\hline \multicolumn{1}{|c|}{$\begin{array}{c}\text { Sect- } \\
\text { ion. }\end{array}$} & Title & Text & $\begin{array}{c}\text { Spread } \\
\text { sheet }\end{array}$ & $\begin{array}{c}\text { Draw- } \\
\text { ing }\end{array}$ & $\begin{array}{c}\text { Presen- } \\
\text { tation }\end{array}$ & Chart & Image \\
\hline 2.2 & Document Metadata & $\mathrm{X}$ & $\mathrm{X}$ & $\mathrm{X}$ & $\mathrm{X}$ & $\mathrm{X}$ & $\mathrm{X}$ \\
\hline 2.3 & $\begin{array}{l}\text { Body Element and } \\
\text { Document Types }\end{array}$ & $\mathrm{X}$ & $\mathrm{X}$ & $\mathrm{X}$ & $\mathrm{X}$ & $\mathrm{X}$ & $\mathrm{X}$ \\
\hline 2.4 & Application Settings & $\mathrm{X}$ & $\mathrm{X}$ & $\mathrm{X}$ & $\mathrm{X}$ & $\mathrm{X}$ & $\mathrm{X}$ \\
\hline 2.5 & Scripts & $\mathrm{X}$ & $\mathrm{X}$ & $\mathrm{X}$ & $\mathrm{X}$ & $\mathrm{X}$ & $\mathrm{X}$ \\
\hline 2.6 & Font Face Declarations & $\mathrm{X}$ & $\mathrm{X}$ & $\mathrm{X}$ & $\mathrm{X}$ & $\mathrm{X}$ & \\
\hline 2.7 & Styles & $\mathrm{X}$ & $\mathrm{X}$ & $\mathrm{X}$ & $\mathrm{X}$ & $\mathrm{X}$ & $\mathrm{X}$ \\
\hline 2.8 & Page Styles and Layout & $\mathrm{X}$ & $\mathrm{X}$ & $\mathrm{X}$ & $\mathrm{X}$ & & \\
\hline 3 & Metadata Elements & $\mathrm{X}$ & $\mathrm{X}$ & $\mathrm{X}$ & $\mathrm{X}$ & $\mathrm{X}$ & $\mathrm{X}$ \\
\hline 4.1 & $\begin{array}{l}\text { Paragraphs and Basic Text } \\
\text { Structure }\end{array}$ & $\mathrm{X}$ & $\mathrm{X}$ & $\mathrm{X}$ & $\mathrm{X}$ & \\
\hline 4.1 & Headings & $\mathrm{X}$ & & & $\mathrm{X}$ & \\
\hline 4.2 & Page Sequences & $\mathrm{X}$ & & & & & \\
\hline 4.3 & Lists & $\mathrm{X}$ & & $\mathrm{X}$ & $\mathrm{X}(2)$ & & \\
\hline 4.4 & Text Sections & $\mathrm{X}$ & & & & & \\
\hline 4.5 & $\begin{array}{l}\text { Page-bound graphical } \\
\text { content }\end{array}$ & $\mathrm{X}$ & & & & & \\
\hline
\end{tabular}




\begin{tabular}{|c|c|c|c|c|c|c|c|}
\hline $\begin{array}{l}\text { Sect- } \\
\text { ion. }\end{array}$ & Title & Text & $\begin{array}{c}\text { Spread } \\
- \\
\text { sheet }\end{array}$ & $\begin{array}{l}\text { Draw- } \\
\text { ing }\end{array}$ & $\begin{array}{c}\text { Presen- } \\
\text { tation }\end{array}$ & Chart & Image \\
\hline 4.6 & Text Change Tracking & $x$ & & & & & \\
\hline 4.7 & Text Declarations & $\mathrm{X}$ & $(X)$ & $(X)$ & $(X)$ & $(X)$ & \\
\hline 5.1 & Basic Text Content & $\mathrm{x}$ & $X^{(1)}$ & $X^{(2)}$ & $X^{(2)}$ & $X^{(3)}$ & \\
\hline 5.2 & Bookmarks and References & $x$ & & & & & \\
\hline 5.3 & Notes & $x$ & & & & & \\
\hline 5.4 & Ruby & $x$ & & & & & \\
\hline 5.5 & Text Annotation & $x$ & & & & & \\
\hline 5.6 & Index Marks & $x$ & & & & & \\
\hline 5.7 & $\begin{array}{l}\text { Change Tracking and } \\
\text { Change Marks }\end{array}$ & $x$ & & & & & \\
\hline 5.8 & $\begin{array}{l}\text { Inline graphics and text- } \\
\text { boxes }\end{array}$ & $x$ & & & & & \\
\hline 6 & Text Fields & $x$ & $(X)$ & $(\mathrm{X})$ & $(\mathrm{X})$ & & \\
\hline 7 & Text Indices & $x$ & & & & & \\
\hline 8.1 & Basic Table Model & $x$ & $x$ & & & & \\
\hline 8.2 & Advanced Table Model & $x$ & $x$ & & & & \\
\hline 8.3 & Advanced Tables & & $\mathrm{x}$ & & & & \\
\hline 8.4 & Advanced Table Cells & & $x$ & & & & \\
\hline 8.5 & $\begin{array}{l}\text { Spreadsheet Document } \\
\text { Content }\end{array}$ & & $x$ & & & & \\
\hline 8.6 & Database Ranges & & $x$ & & & & \\
\hline 8.7 & Filters & & $x$ & & & & \\
\hline 8.8 & Data Pilot Tables & & $x$ & & & & \\
\hline 8.9 & Consolidation & & $x$ & & & & \\
\hline 8.10 & Table DDE Links & & $x$ & & & & \\
\hline 8.11 & $\begin{array}{l}\text { Change Tracking in } \\
\text { Spreadsheets }\end{array}$ & & $x$ & & & & \\
\hline 9.1 & $\begin{array}{l}\text { Enhanced Page Features for } \\
\text { Graphical Applications }\end{array}$ & & & $x$ & $x$ & & \\
\hline 9.2 & Drawing Shapes & $x$ & $x$ & $x$ & $x$ & & \\
\hline 9.3 & Frames & $x$ & $x$ & $x$ & $x$ & & $X^{(4)}$ \\
\hline
\end{tabular}




\begin{tabular}{|c|c|c|c|c|c|c|c|}
\hline $\begin{array}{l}\text { Sect- } \\
\text { ion. }\end{array}$ & Title & Text & $\begin{array}{c}\text { Spread } \\
- \\
\text { sheet }\end{array}$ & $\begin{array}{l}\text { Draw- } \\
\text { ing }\end{array}$ & $\begin{array}{c}\text { Presen- } \\
\text { tation }\end{array}$ & Chart & Image \\
\hline 9.4 & 3D Shapes & $x$ & $x$ & $x$ & $x$ & & \\
\hline 9.5 & Custom Shapes & $x$ & $\mathrm{X}$ & $\mathrm{X}$ & $\mathrm{x}$ & & \\
\hline 9.6 & Presentation Shapes & & & & $\mathrm{x}$ & & \\
\hline 9.7 & Presentation Animations & & & & $x$ & & \\
\hline 9.8 & $\begin{array}{l}\text { SMIL Presentation } \\
\text { Animations }\end{array}$ & & & & $x$ & & \\
\hline 9.9 & Presentation Events & & & & $x$ & & \\
\hline 9.10 & Presentation Text Fields & & & & $\mathrm{X}$ & & \\
\hline 9.11 & $\begin{array}{l}\text { Presentation Document } \\
\text { Content }\end{array}$ & & & & $x$ & & \\
\hline 10 & Chart Content & & & & & $x$ & \\
\hline 11 & Form Content & $x$ & $\mathrm{x}$ & $x$ & $x$ & & \\
\hline 12.1 & Annotation & $X^{(5)}$ & $X^{(1)}$ & & & & \\
\hline 12.2 & $\begin{array}{l}\text { Number Format for page } \\
\text { numbers, etc. }\end{array}$ & $x$ & $x$ & $x$ & $x$ & & \\
\hline 12.3 & Change Tracking Metadata & $x$ & $x$ & & & & \\
\hline 12.4 & Event Listener Tables & $x$ & $x$ & $x$ & $x$ & & \\
\hline 12.5 & Mathematical Content & $x$ & $\mathrm{x}$ & $\mathrm{X}$ & $x$ & & \\
\hline 12.6 & DDE Connections & $x$ & $x$ & & & & \\
\hline 13 & SMIL Animations & & & & $x$ & & \\
\hline 14.1 & Style Element & $x$ & $x$ & $x$ & $x$ & $x$ & $x$ \\
\hline 14.2 & Default Styles & $x$ & $x$ & $x$ & $x$ & $\mathrm{x}$ & $x$ \\
\hline 14.3 & Page Layout & $x$ & $x$ & $x$ & $x$ & & \\
\hline 14.4 & Master Pages & $x$ & $x$ & $x$ & $x$ & & \\
\hline 14.5 & Table Templates & $x$ & $x$ & & & & \\
\hline 14.6 & Font Face Declaration & $x$ & $x$ & $x$ & $x$ & $x$ & \\
\hline 14.7 & Data Styles & $x$ & $x$ & $x$ & $x$ & $x$ & \\
\hline 14.8 & Text Styles & $x$ & $X^{(6)}$ & $X^{(6)}$ & $X^{(6)}$ & $X^{(6)}$ & \\
\hline 14.9 & Enhanced Text Styles & $x$ & & & & & \\
\hline 14.10 & List Style & $x$ & & $\mathrm{X}$ & $\mathrm{X}$ & & \\
\hline
\end{tabular}




\begin{tabular}{|c|c|c|c|c|c|c|c|}
\hline $\begin{array}{l}\text { Sect- } \\
\text { ion. }\end{array}$ & Title & Text & $\begin{array}{c}\text { Spread } \\
- \\
\text { sheet }\end{array}$ & $\begin{array}{l}\text { Draw- } \\
\text { ing }\end{array}$ & $\begin{array}{l}\text { Presen- } \\
\text { tation }\end{array}$ & Chart & Image \\
\hline 14.11 & Outline Style & $x$ & & & & & \\
\hline 14.12 & Table Styles & $x$ & $x$ & & & & \\
\hline 14.13 & Graphic Styles & $x$ & $x$ & $x$ & $x$ & & \\
\hline 14.14 & $\begin{array}{l}\text { Enhanced Graphic Style } \\
\text { Elements }\end{array}$ & $x$ & $x$ & $x$ & $x$ & $x$ & \\
\hline 14.15 & Presentation Page Layouts & & & & $x$ & & \\
\hline 14.16 & Chart Styles & & & & & $x$ & \\
\hline 15.2 & $\begin{array}{l}\text { Page Layout Formatting } \\
\text { Properties }\end{array}$ & $\mathrm{x}$ & $x$ & $x$ & $x$ & & \\
\hline 15.3 & $\begin{array}{l}\text { Header Footer Formatting } \\
\text { Properties }\end{array}$ & $x$ & $(X)$ & & & & \\
\hline 15.4 & Text Formatting Properties & $\mathrm{x}$ & $x$ & $x$ & $x$ & $x$ & \\
\hline 15.5 & $\begin{array}{l}\text { Paragraph Formatting } \\
\text { Properties }\end{array}$ & $x$ & $x$ & $x$ & $x$ & $x$ & \\
\hline 15.6 & $\begin{array}{l}\text { Ruby Text Formatting } \\
\text { Properties }\end{array}$ & $x$ & & & & & \\
\hline 15.7 & $\begin{array}{l}\text { Section Formatting } \\
\text { Properties }\end{array}$ & $x$ & & & & & \\
\hline 15.8 & Table Formatting Properties & $(X)$ & $x$ & & & & \\
\hline 15.9 & $\begin{array}{l}\text { Column Formatting } \\
\text { Properties }\end{array}$ & $(X)$ & $x$ & & & & \\
\hline 15.10 & $\begin{array}{l}\text { Table Row Formatting } \\
\text { Properties }\end{array}$ & $(X)$ & $x$ & & & & \\
\hline 15.11 & $\begin{array}{l}\text { Table Cell Formatting } \\
\text { Properties }\end{array}$ & $(X)$ & $x$ & & & & \\
\hline 15.12 & List-Level Style Properties & $x$ & & $x$ & $x$ & & \\
\hline 15.13 & Stroke Properties & $X^{(7)}$ & $X^{(7)}$ & $x$ & $x$ & $x$ & \\
\hline 15.14 & Fill Properties & $X^{(7)}$ & $X^{(7)}$ & $x$ & $x$ & $x$ & \\
\hline 15.15 & Text Animation Properties & $X^{(7)}$ & $X^{(7)}$ & $x$ & $x$ & & \\
\hline 15.16 & Text Alignment Properties & $X^{(7)}$ & $X^{(7)}$ & $x$ & $x$ & & \\
\hline 15.17 & Color Properties & $X^{(7)}$ & $X^{(7)}$ & $x$ & $x$ & & $x$ \\
\hline 15.18 & Shadow Properties & $X^{(7)}$ & $X^{(7)}$ & $x$ & $x$ & & \\
\hline
\end{tabular}




\begin{tabular}{|c|c|c|c|c|c|c|c|}
\hline $\begin{array}{l}\text { Sect- } \\
\text { ion. }\end{array}$ & Title & Text & $\begin{array}{c}\text { Spread } \\
- \\
\text { sheet }\end{array}$ & $\begin{array}{l}\text { Draw- } \\
\text { ing }\end{array}$ & $\begin{array}{l}\text { Presen- } \\
\text { tation }\end{array}$ & Chart & Image \\
\hline 15.19 & Connector Properties & $X^{(7)}$ & $X^{(7)}$ & $x$ & $x$ & & \\
\hline 15.20 & Measure Properties & $X^{(7)}$ & $X^{(7)}$ & $x$ & $x$ & & \\
\hline 15.21 & Caption Properties & $X^{(7)}$ & $X^{(7)}$ & $\mathrm{x}$ & $\mathrm{X}$ & & \\
\hline 15.22 & 3D Geometry Properties & $X^{(7)}$ & $X^{(7)}$ & $x$ & $x$ & $x$ & \\
\hline 15.23 & 3D Lighting Properties & $X^{(7)}$ & $X^{(7)}$ & $x$ & $x$ & $x$ & \\
\hline 15.24 & 3D Texture Properties & $X^{(7)}$ & $X^{(7)}$ & $x$ & $x$ & $x$ & \\
\hline 15.25 & 3D Material Properties & $X^{(7)}$ & $X^{(7)}$ & $x$ & $x$ & $x$ & \\
\hline 15.26 & 3D Shadow Properties & $X^{(7)}$ & $X^{(7)}$ & $x$ & $x$ & $x$ & \\
\hline 15.27 & $\begin{array}{l}\text { Frame Formatting } \\
\text { Properties }\end{array}$ & $x$ & $(X)$ & $(X)$ & $(\mathrm{X})$ & $(\mathrm{X})$ & \\
\hline 15.28 & $\begin{array}{l}\text { Floating Frame Formatting } \\
\text { Properties }\end{array}$ & $x$ & $x$ & $x$ & $x$ & & \\
\hline 15.29 & Chart Formatting Properties & & & & & $x$ & \\
\hline 15.30 & Chart Subtype Properties & & & & & $x$ & \\
\hline 15.31 & Chart Axes Properties & & & & & $x$ & \\
\hline 15.32 & Common Chart Properties & & & & & $x$ & \\
\hline 15.33 & Statistical Properties & & & & & $x$ & \\
\hline 15.34 & Plot Area Properties & & & & & $x$ & \\
\hline 15.35 & $\begin{array}{l}\text { Regression Curve } \\
\text { Properties }\end{array}$ & & & & & $x$ & \\
\hline 15.36 & Presentation Page Attributes & & & & $x$ & & \\
\hline
\end{tabular}

(1) within table cells

(2) within text boxes

(3) within some chart objects

(4) only frames that contain images

(5) within text

(6) only automatic styles

(7) only for drawing shapes 


\section{Appendix E.Changes From Previous Specification Versions (Non Normative)}

\section{E.1. Changes from "Open Office Specification 1.0 Committee Draft 1"}

The following are the changes since the "Open Office Specification 1.0 Committee Draft 1":

- The name of this specification has been changed to "Open Document Format for Office Applications (OpenDocument) 1.0".

- The namespace URIs (section 1.3) have been adapted to the new specification name.

- The MIME type recommendations have been moved into a non normative appendix (appendix $\mathrm{C}$ ) and have been adapted to the new specification name.

- Various new definitions have been added. Among them are:

- Custom Shapes (section 9.5)

- SMIL Animations (section 9.8 and chapter 13)

- Support for XForms (section 11.2)

- Various errors in the schema and descriptions have been corrected.

- Some descriptions have been rewritten for easier understanding.

\section{E.2. Changes from "Open Document Format for Office Applications (OpenDocument) 1.0 Committee Draft 2"}

The following are the changes since the "Open Document Format for Office Applications (OpenDocument) 1.0 Committee Draft 2":

- Namespaces for compatible elements and attributes have been added as follows:

- urn:oasis:names:tc:opendocument:xmlns:xsl-fo-compatible:1.0 for attributes that are compatible with $[\mathrm{XSL}]$;

- urn:oasis:names:tc:opendocument:xmlns:svg-compatible:1.0 for elements and attributes that are compatible with [SVG];

- urn:oasis:names:tc:opendocument:xmlns:smil-compatible:1.0 for attributes that are compatible with [SMIL20].

- The following namespaces are not imported any longer:

- http://www.w3.org/1999/XSL/Format

- http://www.w3.org/2000/svg

- http://www.w3.org/2001/SMIL20/

- The attribute xforms: submission specified in section 11.3.11 has been renamed to form:xforms-submission. 
- Information for Custom Shapes (section 9.5), SMIL Animations (sections 9.8 and chapter 13) and Presentation Text Fields (section 9.10) has been added to the core feature set table in appendix $\mathrm{D}$; the chapter numbers in the table have been updated. 


\section{Appendix F.Notices}

OASIS takes no position regarding the validity or scope of any intellectual property or other rights that might be claimed to pertain to the implementation or use of the technology described in this document or the extent to which any license under such rights might or might not be available; neither does it represent that it has made any effort to identify any such rights. Information on OASIS's procedures with respect to rights in OASIS specifications can be found at the OASIS website. Copies of claims of rights made available for publication and any assurances of licenses to be made available, or the result of an attempt made to obtain a general license or permission for the use of such proprietary rights by implementors or users of this specification, can be obtained from the OASIS Executive Director.

OASIS invites any interested party to bring to its attention any copyrights, patents or patent applications, or other proprietary rights which may cover technology that may be required to implement this specification. Please address the information to the OASIS Executive Director.

\section{Copyright (C) OASIS Open 2005. All Rights Reserved.}

This document and translations of it may be copied and furnished to others, and derivative works that comment on or otherwise explain it or assist in its implementation may be prepared, copied, published and distributed, in whole or in part, without restriction of any kind, provided that the above copyright notice and this paragraph are included on all such copies and derivative works. However, this document itself does not be modified in any way, such as by removing the copyright notice or references to OASIS, except as needed for the purpose of developing OASIS specifications, in which case the procedures for copyrights defined in the OASIS Intellectual Property Rights document must be followed, or as required to translate it into languages other than English.

The limited permissions granted above are perpetual and will not be revoked by OASIS or its successors or assigns.

This document and the information contained herein is provided on an "AS IS" basis and OASIS DISCLAIMS ALL WARRANTIES, EXPRESS OR IMPLIED, INCLUDING BUT NOT LIMITED TO ANY WARRANTY THAT THE USE OF THE INFORMATION HEREIN WILL NOT INFRINGE ANY RIGHTS OR ANY IMPLIED WARRANTIES OF MERCHANTABILITY OR FITNESS FOR A PARTICULAR PURPOSE. 